

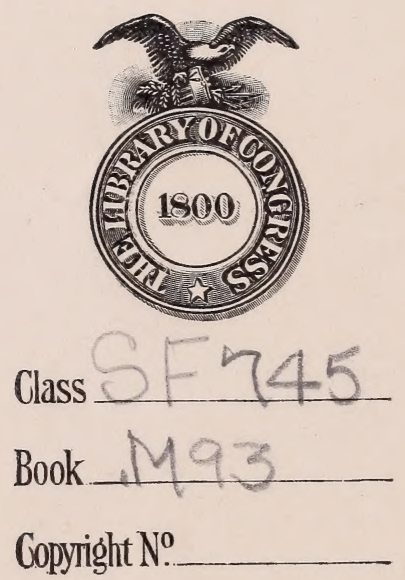

COPYRIGHT DEPOSIT. 


$$
-
$$





$$
\text { ? }
$$




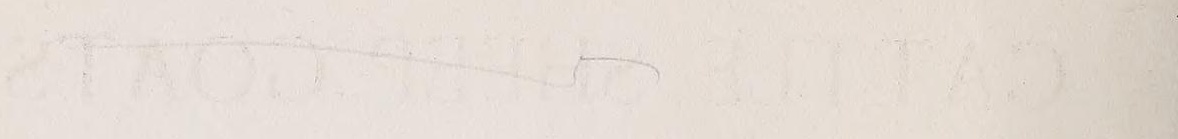




\section{DISEASES OF}

\section{CATTLE, SHEEP, GOATS}

\section{AND SWINE}

BV

\section{G. MOUSSU}

Professor at the Veterinary College of Alfort; Doctor of Medicine;

Doctor of Science, etc.

AND

JNO. A. W. DOLLAR, M.R.C.V.S., F.R.S.E., M.R.I.

President of the Royal College of Veterinary Surgeons; Vice-President of the Royal

Institute of Public Health; Corresponding Member of the Central Society of

Veterinary Medicine of Paris; Associate of the Society of Veterinary

Medicine of Brabant (Belgium); Life Member of the Royal

Italian Society of Hygiene, etc.

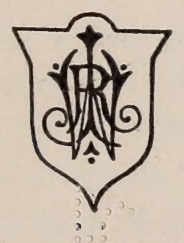

NEW YORK

WILLIAM R. JENKINS

VETERINARY PUBLISHER AND BOOKSELLER

85I AND 853 Sixth Avenue

$$
1905
$$

[All Rights Reserved] 

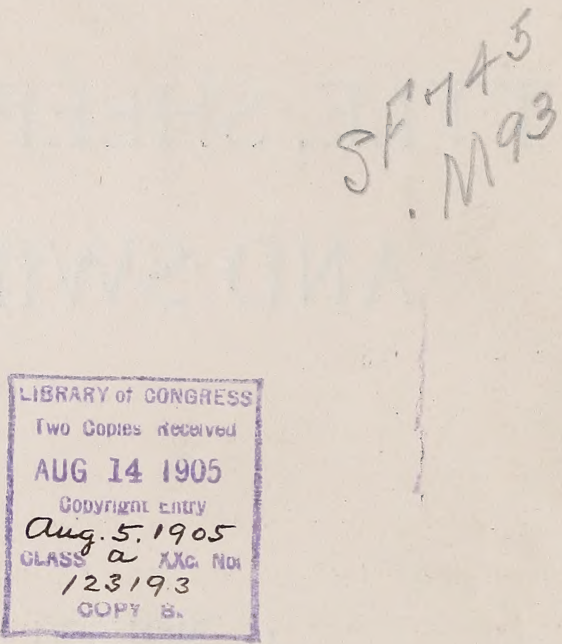

Copyright, 1905, by William R. Jenkins

All Rights Reserved 


\section{PREF A C E.}

No apology seems called for in presenting to English-speaking veterinary surgeons and students a treatise on the diseases of cattle. To those entrusted with the onerous task of preventing or curing disease in cattle, sheep and swine the scantiness of permanent literature dealing with the subject must always have proved a matter of some embarrassment, while to teachers and students alike the want of a concise and modern text-book has long been a difficulty of the first order. It is hoped that the present volume may go some way towards remedying this state of affairs.

As on previous occasions, the writer has freely availed himself of foreign sources of information. Two years ago he purchased the literary rights in Professor Moussu's "Maladies du Bétail," which had even then attained an European reputation, and which forms the backbone of the present volume. To obtain further information, the more important German treatises have been laid under contribution, while all accessible English, American, and Colonial literature of recent date has been referred to. (The references practically extend up to the moment of writing-the latest being June, 1905.) In this way the work may in some degree claim to have assumed an international character. The extent of the additions is indicated by an increase in the number of illustrations of 140, and of the text of nearly 50 per cent.

Professor McQueen has performed the greatly-valued service of reading proof-sheets and advising the writer as the book passed through the press.

To Dr. Salmon, of the United States Department of Agriculture, special thanks are due for his generous permission to quote from the annual reports of that body.

Other acknowledgments will be found in the text. 
Once again the writer, who on this occasion chances also to be the President of the Royal College of Veterinary Surgeons, appeals for lenient judgment on work performed under no common stress of duties, professional and political.

JNO. A. W. DOLLAR. 


\section{CONTENTS.}

\section{SECTION I.}

CHAP.

\section{DISEASES OF THE ORGANS OF LOCOMOTION.}

Methods of Examination

I. DISEASES OF BONES. . . . . . . . . . 3

General Diseases . . . . . . . . . . 4

Rachitis . . . . . . . . . . . 4

Osseous Cachexia $\quad . \quad$. $\quad . \quad$.

Local Affections . . . . . . . . . . . 20

Fractures . . . . . . . . . . 20

Fractures of the horns . . . . . . . . 21

Detachment of the horns . . . . . . . . 23

Fissuring of the horns . . . . . . . . 24

Fractures of the horns . . . . . . . . . 25

Exostoses . . . . . . . . . . . 27

Spavin in the ox. . . . . . . . . . . 27

Ring-bone . . . . . . . . . . . 28

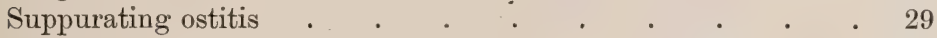

Bone tumours . . . . . . . . . . . . 30

II. DISEASES OF THE FOOT . . . . . . . . . . 31

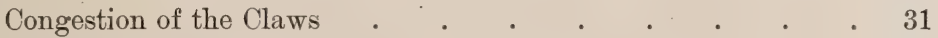

Contusions of the sole $\quad . \quad$. $\quad . \quad$. $\quad . \quad$. $\quad . \quad$. 31

Laminitis . . . . . . . . . . . 32

Sand crack . . . . . . . . . . . . . 34

Pricks and stabs in shoeing . . . . . . . . . . $\quad$. 36

Picked-up nails, etc. ("Grathered nail") . . . . . . 37

Inflammation of the interdigital space (Condylomata). . . 38

Canker . . . . . . . 40

Grease . . . . . . . . . . . 41

Panaritium-Felon-Whitlow . . . . . . . 41

Foot rot . . . . . . . . . . . 43

III. DISEASES OF THE SYNOVIAL MEMBRANES AND OF THE ARTICULATIONS

i. Synovial Membranes and Articulations . . . . 45

Synovitis . . . . . . . . . . . 45

Infllammation of the patellar synovial capsule : , , , 45 
CHAP.

III. DISEASES OF THE SYNOVIAL MEMBRANES AND OF THE ARTICULATIONS-continued.

I. Synovial Membranes and Articulations-continued.

Distension of the synovial capsule of the hock joint . . . 46

Distension of tendon sheaths in the hock region . . : 46

Distension of the synovial capsule of the knee joint . . . . 47

Distension of the synovial capsule of the fetlock joint . . . 48

Distension of tendon sheaths . . . . . . . . 448

Distension of tendon sheaths in the region of the knee . . 49

Distension of the bursal sheath of the flexor tendons . . . 49

Traumatic synovitis_-"Open synovitis" . . . . . 49

Traumatic tendinous synovitis . . . . . . . 50

Traumatic articular synovitis - Traumatic arthritis - "Open arthritis" . . . . . . . . . . . 51

II. Strains of Joints . . . . . . . . . . 52

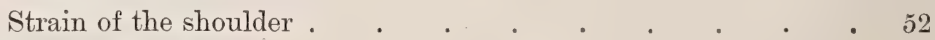

Strain of the knee . . . . . . . . . . . 53

Strain of the fetlock . . . . . . . . . . . . 54

Strain of the stifle joint . . . . . . . . . 54

Strain of the hock joint . . . . . . . . . . $5 \tilde{5}$

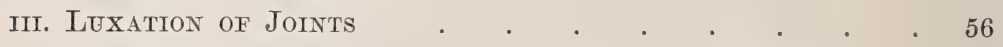

Luxation of the femur . . . . . . . . . 56

Luxation of the patella.$\quad$. . . . . . . 58

Luxation of the femoro-tibial articulation . . . . . . 61

Luxation of the scapulo-humeral joint . . . . . . 63

IV. Hygromas . . . . . . . . . . . . 64

Hygroma of the knee . . . . . . . . . . 65

Hygroma of the haunch . . . . . . . . 67

Hygroma of the trochanter of the femur . . . . . 67

Hygroma of the stifle . . . . . . . . 67

Hygroma of the point of the hock . . . . . . 68

Hygroma of the point of the sternum . $\quad . \quad . \quad . \quad . \quad 696$

IV. DISEASES OF MUSCLES AND TENDONS . . . . . 70

Rupture of the external ischio-tibial muscle (Biceps femoris) . 70

Rupture of the flexor metatarsi . . . . . . . 72

Parasitic Diseases of Muscles . . . . . . . . 73

Cysticercus disease of the pig . . . . . . . . . 73

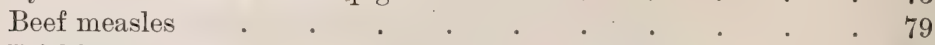

Trichiniasis-Trichinosis . . . . . . . $\quad . \quad 84$

V. RHEUMATISM . . . . . . . . . . . . . . $\quad . \quad 89$

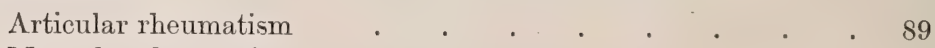

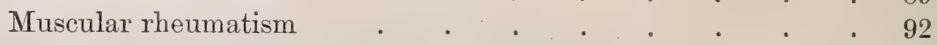

Infectious Forms of Rheumatism or Pseudo-Rheumatism a 94

Infectious rheumatism in young animals . . . . . 94

Infectious pseudo-rheumatism in adults $\quad . \quad$. $\quad . \quad$. $\quad .99$

Scurvy-Scorbutus : : : : , : : , 104 


\section{SECTION II.}

\section{DISEASES OF THE DIGESTIVE APPARATUS.}

CHAP.

Semiology of the Digestive Apparatus

I. DISEASES OF THE MOUTH . . . . . . . . 121

Stomatitis . . . . . . . . . • . 121

Simple stomatitis . . . . . . . . 121

Caturrhal stomatitis in sheep . . . . . . . 122

Necrosing stomatitis in calves . . . . . . . . . 123

Mycotic stomatitis in calves . . . . . . . . . . 124

Ulcerative stomatitis in sheep $\quad . \quad$. . . . . . . 125

General catarrhal stomatitis in swine . . . . . . 126

Ulcerative stomatitis in swine . . . . . . . 127

Mercurial stomatitis . . . . . . . . 128

Glossitis . . . . . . . . . . 130

Superficial glossitis . . . . . . . . . 130

Acute deep-seated glossitis . . . . . . . . 131

Chronic glossitis . . . . . . . . . 132

II. DISEASES OF THE SALIVARY GLANDS, TONSIIS AND PHARYNX . . . . . . . . . . . . 134

Parotiditis (Parotitis) . . . . . . . . . 134

Acute parotiditis . . . . . . . . . . 134

Chronic parotiditis-Parotid fistula . . . . . . 136

Inflammation of the submaxillary salivary gland $\quad . \quad$. $\quad .137$

Tonsilitis in pigs . . . . . . . . . . . . 138

Pharyngitis. . . . . . . . . 138

Pseudo-membranous pharyngitis in cattle . . . . 141

Pseudo-membranous pharyngitis in sheep . . . . 142

Pharyngeal polypi . . . . . . . . . . 143

III. DISEASES OF THE ESOPHAGUS . . . . . . . . 145

Esophagitis . . . . . . . . . . 145

Stricture of the œesophagus . . . . . . . . . 148

Dilatation of the œesophagus _ . . . . . . . . . 149

Esophageal obstructions . . . . . . . . . 152

Ruptures and perforations of the œesophagus . . . . . 157

IV. DEPRAVED APPETITE-THE LICKING HABIT-INDIGESTION $1 \tilde{8}$

Depraved appetite in the ox. . . . . . . . 158

Depraved appetite in calves and lambs . . . . . . 160

Colic in the ox . . . . . . . . . . . . . 162

Colic due to ingestion of cold water-Congestive colic . $\quad 162$

Colic due to invagination . . . . . . . . 163

Colic as a result of strangulation . . . . . . 167

Diseases of the stomach _ . . . . . . . . . 169

Indigestion . . . . . . . . . . . . 170

Gaseous indigestion . . . . . . . . . . . 170 
CHAP.

IV. DEPRAVED APPETITE-THE LICKING HABIT-INDIGESTION -continued.

Indigestion-continued.

Impaction of the rumen-Indigestion as a result of overeating

Impaction of the omasum (third stomach) . . . . 179

Abomasal indigestion . . . . . . . . . 182

Acute gastric indigestion in swine $. \quad . \quad . \quad . \quad 18 \check{5}$

V. INFLAMMATION OF THE GASTRIC COMPARTMENTS . . 186

Rumenitis-Reticulitis-Gastritis . . . . . . 186

Acute gastritis . . . . . . . . . . . 188

Catarrhal gastritis in swine . . . . . . . . 190

Ulcerative gastritis . . . . . . . . . 191

Chronic tympanites . . . . . . . . . 194

Gastric disturbance due to foreign bodies . . . . . 198

Tumours of the gastric compartments . . . . . 202

VI. ENTERITIS . . . . . . . . . . . . 203

Acute enteritis . . . . . . . . . . . . 203

Hromorrhagic enteritis . . . . . . . . . . 206

Chronic enteritis (Chronic diarrhøea) . . . . . . . . 207

Dysentery in calves . . . . . . . . . 210

Diarrhoic enteritis in calves . . . . . . . 212

VII. POISONING . . . . . . . . . . . . . 215

Poisoning due to food . . . . . . . . . 215

Poisoning by caustic alkalies . . . . . . . . 216

Poisoning by caustic acids . . . . . . . . . 217

Poisoning by common salt . . . . . . . . . 217

Poisoning by the nitrates of potash and soda . . . . . 217

Poisoning by tartar emetic . . . . . . . . 218

Poisoning by arsenic . . . . . . . . . . . 218

Phosphorus poisoning . . . . . . . . . . . . 219

Mercurial poisoning . . . . . . . . . . . . 219

Lead poisoning: Saturnism . . . . . . . . . 220

Copper poisoning . . . . . . . . . . . . . . 221

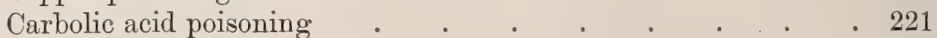

Poisoning by aloes . . . . . . . . . . . . 221

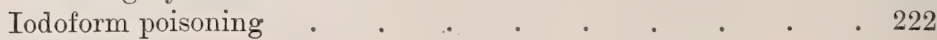

Iodine poisoning: iodism . . . . . . . . . 222

Strychnine poisoning . . . . . . . . . . . . 222

List of plants poisonous to stock . . . . . . . . 223

Colchicum poisoning . $\quad . \quad$. . . . . . . . 256

Poisoning by annual mercury . . . . . . . . 256

Poisoning by bryony . . . . . . . . . . 256

Poisoning by castor oil cake . . . . . . . . 257

Poisoning by cotton cake . . . . . . . . . . 257

Poisoning by molasses refuse . . . . . . . 258

Diseases produced by distillery and sugar factory pulp . . 259

VIII. PARASITES OF THE DIGESTIVE APPARATUS . • • . 263

Gastro-intestinal strongylosis in sheep . . . . . 263

Lumbricosị of calyes : : : : : : , , 267 
Strongylosis of the abomasum in the ox . . . . . . 268

Parasitic gastro-enteritis, diarrhœa, and anæmia in cattle, sheep and lambs

Intestinal coccidiosis of calves and lambs (Psorospermosis, hæmorrhagic enteritis, bloody flux, dysentery, etc.) . . . . 271

Intestinal helminthiasis in ruminants . . . . . . 275

IX. DISEASES OF THE LIVER . . . • . . . . 279

Congestion of the liver . . . . . . . . . . 280

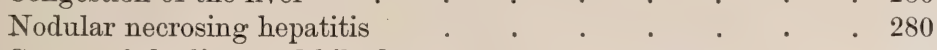

Cancer of the liver and bile ducts $\quad$. $\quad$. $\quad$. . . 282

Echinococcosis of the liver. . . . . . . . . . 283

Suppurative echinococcosis . . . . . . . . . . . 288

Cysticercosis . . . . . . . . . . 290

Distomatosis-Liver fluke disease-Liver rot . . . . 293

\section{SECTION III.}

\section{RESPIRATORY APPARATUS.}

I. EXAMINATION OF THE RESPIRATORY APPARATUS • . 311

II. NASAL CAVITIES . . . . . . . . . . . . 319

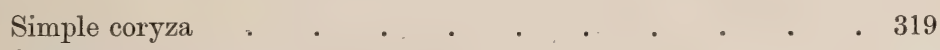

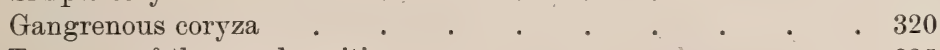

Tumours of the nasal cavities . . . . . . . . . . 325

Purulent collections in the nasal sinuses. Nasal gleet . . 326

Purulent collections in the frontal sinus . . . . . 327

Purulent collections in the maxillary sinus . $\quad$. $\quad$. $\quad$. $\quad . \quad 329$

Estrus larvæ in the facial sinuses of sheep. . . . . 330

III. IARYNX, TRACHEA AND BRONCHI : . . . . . 333

Laryngitis . . . . . . . . . . . . . . 333

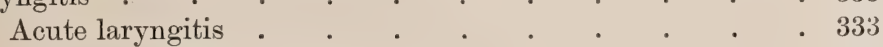

Pseudo-membranous laryngitis _. . . . . . . . $\quad$. 333

Tumours of the larynx $\quad . \quad . \quad . \quad . \quad . \quad .335$

Bronchitis . . . . . . . . . . . . 336

Simple acute bronchitis . . . . . . . . 337

Chronic bronchitis . . . . . . . . . 337

Pseudo-membranous bronchitis . . . . . . 339

Verminous bronchitis in sheep and cattle (Husk, hoose, etc.) 340

IV. LUNGS AND PLEURA . . . . . . . . . . 343

Pulmonary congestion. . . . . . . . . . 343

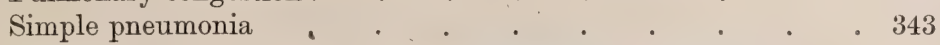

Pneumonia due to foreign bodies-Mechanical pneumonia . . 347

Pneumonia due to the migration of foreign bodies from the

reticulum . . . . . . . . . 348

Pneumomycosis due to Aspergilli . . . . . . . 350

Gangrenous broncho-pneumonia due to foreign bodies . . 351

Infectious broncho-pneumonia . . . . . . . 354

Broncho-pneumonia of sucking calves, , . , . , 3956 
CHAP.

IV. LUNGS AND PLEURA-continued.

Sclero-caseous broncho-pneumonia of sheep . . . . . 358

Pulmonary emphysema . . . . . . . . 359

Diseases of the pleura $\quad$. $\quad$. $\quad$. $\quad . \quad$. $\quad$. . . 361

Acute pleurisy . . . . . . . . . 361

Chronic pleurisy . . . . . . . . . . . 362

Pneumo-thorax . . . . . . . . . . 362

Hydro-pneumo-thorax and pyo-pneumo-thorax . . . 366

V. DISEASES OF STRUCTURES ENCLOSED WITHIN THE MEDIASTINUM . . . . . . . . . . 368

Tumours of the Mediastinum . . . . . . . 369

\section{SECTION IV. \\ THE ORGANS OF CIRCULATION.}

Semiology of the Organs of Circulation. . . . . . . 370

I. CARDIAC ANOMALIES . . . . . . . . . 374

Ectopia of the heart . . . . . . . . . 374

II. PERICARDITIS . . . . . . . . . . . 375

Exudative pericarditis due to foreign bodies . . . . 376

Chronic pericarditis . . . . . . . . . . . 389

Pseudo-pericarditis . . . . . . . . . 390

III. ENJOCARDITIS . . . . . . . . . . . 394

IV. DISEASES OF BLOOD-VESSELS . . . . . . . . . 396

Phlebitis . . . . . . . . . 396

Accidental phlebitis . . . . . . . . . . 396

Internal infectious phlebitis (Utero-ovarian phlebitis). . . 398

Umbilical phlebitis of new-born animals . . . . . 399

Umbilical phlebitis or omphalo-phlebitis . . . . . 402

V. DISEASES OF THE BLOOD . . . . . . . . . 406

Septicæmia of new-born animals . . . . . . 406

Takosis : a contagious disease of goats . . . . . . . 412

Blood-poisoning (Malignant œedema) in sheep and lambs in New

Zealand . . . . . . . . . . 41 .

Piroplasmosis . . . . . . . . . . . . . 416

Bovine piroplasmosis . . . . . . . . . 416

Bovine piroplasmosis in France . . . . . . . 424

Ovine piroplasmosis . $\quad . \quad . \quad . \quad . \quad . \quad . \quad .425$

Diseases produced by trypanosomata . . . . . . 426

Louping-ill . . . . . . . . . . . . 429 Braxy

Suggested meaşures for prevention . . . . . . 435

Bilharziosis in cattle and sheep . . . . . . . . 439

Heat stroke-Over-exertion , , : . : , , 442 
CHAP.

VI. DISEASES OF THE LYMPHATIC SYSTEM

The lymphogenic diathesis . . . . . . . . . . 448

Caseous lymphadenitis of the sheep . . . . . . 453

Goitre in calves and lambs . . . . . . . . . . 453

\section{SECTION V. \\ NERVOUS SYSTEM.}

Cerebral congestion . . . . . . . . 456

Meningitis . . . . . . . . . . 4506

Encephalitis . . . . . . . . . . 458

Cerebral Tumours . . . . . . . . . . . . 459

Insolation . . . . . . . . . . . 460

Post-partum paralysis-Milk fever-Mammary toxæmia-Parturient apoplexy-Dropping after calving . . . . 461

Coenurosis (Gid, sturdy, turn-sick) . . . . . . . 467

"Trembling," or Lumbar prurigo, in sheep . . . . . 475

\section{SECTION VI.}

DISEASES OF THE PERITONEUM AND ABDOMINAL CAVITY.

I. PERITONITIS

Acute peritonitis .

Chronic peritonitis

Ascites.

Peritoneal cysticercosis

II. HERNI $\mathrm{E}$

Congenital herniæ

Perineal hernia of young pigs

Umbilical hernia . . . . . . . . . 488

Acquired herniæ .

Hernia of the rumen

Hernia of the abomasum

Hernia of the intestine

Treatment of herniæ

$. \quad . \quad \cdot 495$

Diaphragmatic hernia . . . . . . . . . 496

Eventration. . . . . . . . . . . 499

Fistulæ of the digestive apparatus . . . . . . . $\quad$. 00

\section{SECTION VII.}

\section{GENITO-URINARY REGIONS.}

Diseases of the Urinary Apparatus

I. POLYPI OF THE GLANS PENIS AND SHEATH . . . 506

Inflammation of the sheath . . . . . . . . . 506

Persistence of the urachus . . . . . . . . . 508 
СHAP.

II. DISEASES OF THE BLADDER • . • • • • . 511

Acute cystitis . . . . . . . . . . . 511

Chronic cystitis . . . . . . . 513

Urinary lithiasis. Calculus formation . . . . . 514

Calculi in bovine animals . . . . . . . . . . 515

Urinary calculi in sheep . . . . . . . . . 518

Paralysis of the bladder . . . . . . . . . . 519

Eversion of the bladder . . . . . . . . . . 519

Hæmaturia . . . . . . . . . . . 520

III. DISEASES OF THE KIDNEYS . . . . . . . 527

Congestion of the kidneys . . . . . . . . . 527

Acute nephritis . . . . . . . . . . . . . 528

Chronic nephritis . . . . . . . . . . . 530

Hydro-nephrosis . . . . . . . . . . . . 531

Infectious pyelo-nephritis . . . . . . . . . . 533

Suppurative nephritis and perinephritis . . . . . . . 537

The kidney worm (Sclerostoma pinguicola) of swine _ . . . 539

IV. GENITAL APPARATUS . . . . . . . . . 542

Vaginitis . . . . . . . . . 543

Acute vaginitis . . . . . . . . . . . . 544

Contagious vaginitis . . . . . . . . . . . . 545

Croupal vaginitis . . . . . . . . . . . . . . . . .

Chronic vaginitis . . . . . . . . . . . 546

Metritis . . . . . . . . . . . 547

Septic metritis . . . . . . . . . . 547

Acute metritis . . . . . . . . . . 550

Chronic metritis . . . . . . . . . 552

Epizootic abortion in cows . . . . . . . . . 5 503

Salpingitis-Salpingo-ovaritis . . . . . . . . 55 j

Torsion of the uterus . . . . . . . . . . . . . 556

Tumours of the uterus. . . . . . . . . . 5

Tumours of the ovary . . . . . . . . . . . . 5

Genital malformations . . . . . . . . . . 560

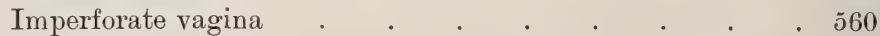

Nympho-mania . . . . . . . . . . 562

V. ITSEASES OF THE MAMMARY GLANDS . . . . . 56

Physiological anomalies . . . . . . . . . 567

Wounds or traumatic lesions . . . . . . . . . . 568

Chaps and cracks . . . . . . . . . . . . 568

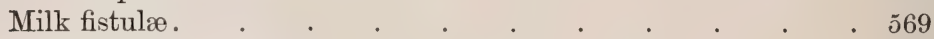

Inflammatory diseases. . . . . . . . . . 570

Congestion of the udder $\quad . \quad . \quad . \quad . \quad . \quad . \quad . \quad 570$

Mammitis . . . . . . . . . . 571

Acute mammitis . . . . . . . . . 573

Contagious mammitis in milch cows . . . . . . . 580

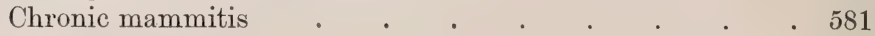

Gangrenous mammitis of milch ewes . . . . . . 583

Gangrenous mammitis in goats . . . . . . 584 
CHAP.

V. DISEASES OF THE MAMMARY GTANDS-continued.

Cysts of the udder . . . . . . . . . . 5 . . . . . . . .

Tumours of the udder . . . . . . . . . 585

Verrucous papillomata of the udder . . . . . . . . 586

VI. DISTURBANCE IN THE MILK SECRETION AND CHANGES IN THE MILK. . . . . . . . . . 587

Microbic changes in milk. Lactic ferments . . . . . j88

VII. MATE GENITAL ORGANS . . . . . . . . . 594

Tumours of the testicle . . . . . . . . . . . 594

Accessory glands of the genital apparatus . . . . . . . 597

\section{SECTION VIII.}

\section{DISEASES OF THE SKIN AND SUBCUTANEOUS CONNECTIVE TISSUE.}

I. ECZEMA

Acute eczema

Chronic eczema

Sebaceous or seborrhoic eczema

Eczema due to feeding with potato pulp . . . . . 603

Impetigo in the pig. . . . . . . . . . . . . 605

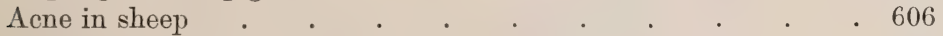

Fagopyrism (Buckwheat poisoning) . . . . . . . 606

II. PHTHIRIASIS . . . . . . . . . . . 608

SCABIES-SCAB-MANGE . . . . . . . . . 611

Scabies in sheep . . . . . . . . . . . . 611

Sarcoptic scabies . . . . . . . . . 612

Psoroptic mange-Sheep scab . . . . . . 614

The tobacco and sulphur dip . . . . . . . 626

Lime and sulphur dips . . . . . . . . 627

Arsenical dips . . . . . . . . . 632

Carbolic dips . . . . . . . . . . . 633

Chorioptic mange-Symbiotic mange-Foot scab 。 . 636

Mange in the ox . . . . . . . . . . . . 638

Sarcoptic mange . . . . . . . . . . . 638

Psoroptic mange . . . . . . . . . . 639

Chorioptic mange . . . . . . . . . 640

Mange in the goat . . . . . . . . . . 641

Sarcoptic mange . . . . . . . . . . 641

Chorioptic mange . . . . . . . . . 642

Mange in the pig. . . . . . . . . . . . . 642

Demodecic mange . . . . . . . . . . . 643

Demodecic mange in the ox . . . . . . . . 644

Demodecic mange in the goat . . . . . . . 644

Demodecic mange in the pig. . . . . . . 644

Non-psoroptic forms of acariasis . . . . . . . . . . . . . . . . . . .

Hypodermosis in the ox (warbles) . . . . . . 646 
CHAP.

III. RINGWORM

Ringworm in the sheep, goat, and pig. . . . . . 653

IV. WARTS IN OXEN . . . . . . . . . . 655

Urticaria in the pig . . . . . . . . . . . . 656

Scleroderma $. \quad . \quad . \quad . \quad . \quad . \quad . \quad .657$

V. SUBCUTANEOUS EMPHYSEMA . . . . . . . 659

\section{SECTION IX.}

DISEASES OF THE EYES.

Foreign bodies . . . . . . . . . . 661

Conjunctivitis and keratitis . . . . . . . . . . . 662

Verminous conjunctivitis . . . . . . . . . . . 662

Verminous ophthalmia of the ox . . . . . . . . 663

\section{SECTION X.}

\section{INFECTIOUS DISEASES.}

Cow-pox-Vaccinia . . . . . . . . 665

Cow-pox and human variola-Preparation of vaccine . . 669

Tetanus. . . . . . . . . 670

Actinomycosis . . . . . . . 672

Actinomycosis of the maxilla . . . . . 673

Actinomycosis of the tongue . . . . . . $67 t$

Tuberculosis

Actinomycosis of the pharynx, parotid glands and neck 675

Tuberculosis of the respiratory apparatus . . . . 690

Tuberculosis of the serous membranes . . . . . 694

Tuberculosis of lymphatic glands . . . . . . . . 696

Tuberculosis of the digestive tract . . . . . . 699

Tuberculosis of the genital organs . . . . . . 700

Tuberculosis of bones and articulations . . . . 701

Tuberculosis of the brain . . . . . . . 702

Tuberculosis of the skin . . . . . . . 703

Acute tuberculosis-Tuberculous septicæmia . . . 704

Swine fever-Verrucous endocarditis and pneumonia of the pig . 710

Swine fever . . . . . . . . 710

Verrucous endocarditis of the pig. . . . . . . 713

Pneumonia of the pig . . . . . . . 714

Hæmorrhagic septicæmia in cattle . . . . . . 716

\section{SECTION XI.}

OPERATIONS.

I. CONTROL OF ANIMALS .

Control of oxen . . . . . . . . . . . . 720

Partial control . . . . . . . . . . 720

Control of the limbs . . . . . . . . . . 720 
CHAP.

I. CONTROL OF ANIMALS-continued.

Control of oxen-continued.

General control

Control by casting

Control of sheep and goats . . . . . . . . . 725

Control of pigs . . . . . . . . . . . . 725

Anresthesia . . . . . . . . . . 726

II. CIRCULATORY APPARATUS . . . . . . . . 727

Bleeding . . . . . . . . . 727

Bleeding in sheep. . . . . . . . . . . 727

Bleeding in the pig . . . . . . . . . 728

Setons, rowels, plugs, or issues .

III. APPARATUS OF LOCOMOTION . . . . . . . 730

Surgical dressing for a claw $\quad . \quad$. $\quad . \quad . \quad . \quad$. $\quad .730$

Amputation of the claw or of the two last phalanges . . . 730

IV. DIGESTIVE APPARATUS . . . . . . . . . 734

Ringing pigs . . . . . . . . . . . . . 734

CEsophagus . . . . . . . . . . . . . . 734

Passing the probang . . . . . . . . . 735

Crushing foreign bodies in the osophagus . . . . . 73 j

Esophagotomy . . . . . . . . 736

Sub-mucous dissection of the foreign body . . . . . 736

Rumen . . . . . . . . . . 737

Puncture of the rumen . . . . . . . . . 737

Gastrotomy . . . . . . . . . . . . 739

Laparotomy . . . . . . . . . . . . . 740

Herniæ. . . . . . . . . . . . 741

Inguinal hernia in young pigs . . . . . . . 741

Imperforate anus . . . . . . . . . . 742

Prolapsus and inversion of the rectum . . . . . . 743

V. RESPIRATORY APPARATUS . . . . . . . 74

Trephining the facial sinuses . . . . . . . . 740

Trephining the horn core . . . . . . . 74 .

Frontal sinus . . . . . . . . . 745

Maxillary sinus . . . . . . . . . 74 . 74

Tracheotomy . . . . . . . . . . . 746

VI. GENITO-URINARY ORGANS . . . . . . . . 747

Urethrotomy in the ox . . . . . . . . . 747

Ischial urethrotomy . . . . . . . . . . 747

Scrotal urethrotomy . . . . . . . . . . 748

Passage of the catheter and urethrotomy in the ram . . . 749

Passage of the catheter in the cow . . . . 750

Castration . . . . . . . . . . . 751

Castration of the bull and ram . . . . . . . . . 751

Bistournage. . . . . . . . . . . 751

Martelage . . . . . . . . . . . 756

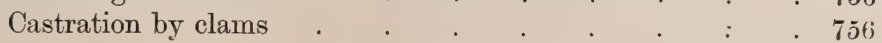

D.C. 
CHAP.

VI. GENITO-URINARY ORGANS-continued.

Castration-continued.

Castration by torsion . . . . . . . 757

Castration with the actual cautery . . . . . . 758

Castration by the elastic ligature . . . . . . 758

Castration of the ram . . . . . . . . . . 759

Castration of boars and young pigs . . . . . . . 759

Castration of cryptorchids . . . . . . 760

Female genital organs . . . . . . . . . 760

Castration of the cow . . . . . . . . . 761

Castration of the sow . . . . . . . . . 765

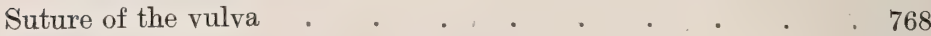

Trusses . . . . . . . . . . . 769

Section of the sphincter of the teat . . . . . . 770

Dilatation of the orifice of the teat . . . . . 770

Ablation of the mammæ $\quad$. . . . . . . 771 


\section{DISEASES OF CATTLE, SHEEP, GOATS AND SWINE.}

\section{SECTION I.}

\section{DISEASES OF THE ORGANS OF LOCOMOTION.}

\section{METHODS OF EXAMINATION.}

Accidental and local diseases of the apparatus of locomotion are matters of less urgency in the case of cattle than in that of the horse. On the other hand, general affections, such as rheumatism and osseous cachexia, demand a larger share of attention, and are of the utmost importance.

As the accurate diagnosis of any disease demands careful and systematic examination, the practitioner usually observes a certain order in his investigations, as indicated below :-

(1.) Inspection, from the side, from the front and from behind, reveals the existence of deformities of bones, limbs, muscles and joints, articular displacements, and irregularities of conformation or of gait.

By inspection of an animal as it walks various forms of lameness, and their particular characteristics, are rendered visible.

(2.) Palpation and pressure will detect changes in local sensibility, the softness or hardness of tissues, the existence of superficial or deep fluctuation, œdematous swelling, and abmormal growths like ring-bones and exostoses, as well as the exact character of articular enlargements.

(3.) Percussion is of little value in examining the apparatus of locomotion. Nevertheless, percussion of the claws, and of certain bones of the limbs, or of flat bones, may afford valuable information in cases of laminitis, ostitis, and periostitis. Percussion along the longitudinal axes of the limb bones is also useful in diagnosing intra-articular fractures, sub-acute arthritis, osteomyelitis, etc.

(4.) The gait. Lame animals should be made to move, in order to assist both in discovering the cause, and in estimating the gravity of the condition. Sometimes it is advisable to turn the animal loose, but most frequently it is moved in hand, either in straight lines or in circles.

D.C. 
Information so obtained should always be supplemented by local manipulation and by passive movement, such as flexion, extension, abduction, adduction and rotation of the joints.

A knowledge of the characteristics of normal movement in any given joint, renders it comparatively easy to detect abnormality, such as increased sensibility, articular crepitation or friction, and to diagnose fractures with or without displacement, ruptures of tendons or ligaments, etc. 


\section{CHAPTER I.}

\section{DISEASES OF BONES.}

The diseases affecting bony tissues may broadly be divided into local and general. Local diseases like ostitis, periostitis, necrosis, fracture, etc., are somewhat rare, and are less important in cattle than such general diseases as rachitis and osseous cachexia.

Rachitis is a disease of young animals, and occurs during the growing period. Osseous cachexia is a disease of adults. Nevertheless, there is a relationship between these two morbid conditions, for they frequently co-exist in one family. Moreover, brood mares and cows suffering from osseous cachexia give birth to foals and calves, which, if left with their mothers, almost inevitably become rachitic.

The general characteristic common to both rachitis and osseous cachexia consisting in diminution in the normal proportion of mineral salts entering into the constitution of the bone, numerous theories have been advanced to explain this irregularity in nutrition.

The theory of insufficiency is one of the oldest. It presupposes that the young animals' food contains insufficient mineral salts necessary for building up the skeleton, hence rachitis; or again, that the daily food of the adults does not afford sufficient mineral salts to compensate for the normal transformation which is continually going on within the organism, and for the direct losses which occur through the medium of the urine, milk, etc.

This extremely simple theory appears perfectly logical, but unfortunately does not fit in with all the observed facts. In reality, rachitis attacks children whose supply of milk, from a chemical point of view, leaves nothing to be desired. The same is true of animals, particularly of young pigs. The so-called "acid theory" has therefore been advanced to explain the points left obscure by its predecessor.

The acid theory. According to this theory, the food may contain more than sufficient mineral material without, however, preventing the development of rachitis or of osseous cachexia.

In animals suffering from digestive disturbance the alimentary tract may become the seat of excessive fermentation or of changes in secretion. There is thus produced an excess of lactic acid which passes into the 
circulation and accumulates in the tissues, checking the processes which end in ossification or, in the case of adults, even leading to decalcification.

It seems fairly well established that experimental administration of lactic acid to animals causes diminution in the quantity of calcium salts contained in the bones (Siedamgrotsky, Hofmeister). On the other hand however Arloing and Tripier failed to produce rachitis experimentally.

Bouchard revived this theory in a somewhat modified form. He considers that calcium salts are absorbed as carbonates and chlorides and phosphoric acid as phospho-glyceric acid. The reaction which these compounds undergo within the organism ends in the formation of the phosphate of calcium necessary to ossification, but this "phosphate of ossification " cannot be deposited if the organism contains an excess of lactic acid.

Theory of inflammation. A third theory which until now has received very little support is that called the theory of inflammation. The general lesions which characterise rachitis are regarded as resulting from primary attacks of ostitis and osteo-periostitis. The cause of these forms of inflammation is not suggested.

To the above views may be added that more recently emitted by Dr. Chaumier, according to which rachitis is of an infectious nature. Unfortunately no proof of this has yet been adduced.

\section{GENERAL DISEASES.}

\section{RACHITIS.}

Rachitis is a disease of youth, and is common both to the human species and to all domestic animals. It is characterised by irregularities in development and by imperfect consolidation of the bones. T'he boundary between rachitis and osseous cachexia is difficult to define and in fact at the present moment the two diseases can scarcely be defined with exactitude. Rachitis again is often complicated with softening of the bones, disease of the limbs, arrested development, etc., but it must not be forgotten that although the irregularities in ossification and development of the skeleton are the symptoms most striking to the eye, they do not stand alnne, and that from the point of view of development all the tissues, including the muscles, are more or less affected and that most of the physiological functions such as digestion and the secretion of urine are deranged.

Etiology. One of the principal causes suggested is that of heredity, and so far as human beings are concerned, one seldom fails to discover the rachitic taint. Certainly the offspring of individuals marked by any debilitating disease like alcoholism, tuberculosis, syphilis, etc., are poorly 
equipped for their future development. Their tissues lack the necessary qualities and, cceteris paribus, their physiological functions are performed less perfectly than are those of normal individuals.

It is difficult to apply such information to domestic animals, because badly developed subjects are not used for reproduction and the importance assigned to heredity can therefore scarcely be sustained. The conditions of life, on the contrary, have an unquestionable influence, and if rachitis is so frequent in young animals living near towns, for example, it is undoubtedly due to that want of air, light and liberty, which first affects the mother's health and later that of her offspring.

The same may be said of insufficient and improper food; for in this connection quality is of even greater importance than quantity. Even free feeding is insufficient if the fodder does not contain the material necessary for sustaining and building up the developing frame, a point which readily explains the occurrence of rachitis when young animals receive a diet deficient in certain chemical constituents.

This occurs in young lambs and pigs where the mothers are given too little variety or too small a quantity of food.

In calves and foals rachitis is rare but occurs when the mothers are exhausted or cachectic or are debilitated by chronic wasting diseases like tuberculosis or osseous cachexia. The milk is then no longer of normal chemical constitution.

One fact appears to dominate the whole subject of the causation of rachitis, viz., the failure to assimilate sufficient of the mineral salts required in building up the skeleton. This failure to assimilate may be caused by too meagre feeding, but even when the food is sufficiently rich, some digestive disturbance may reduce the amount absorbed below normal. This appears the only plausible explanation unless we admit $\mathrm{Dr}$. Chaumier's theory that the disease is of an infectious character.

Symptoms. The onset is absolutely insidious and the diagnosis of rachitis is never made until nutrition has long been abnormal.

This disturbance of nutrition is revealed by irregularity and abnormality in appetite, by difficulty in rising and moving about, and by the animals lying down for long periods. The subjects are feeble, sluggish and badly developed.

Next supervenes the second phase characterised by deformity of bones. This is of two kinds-deformity in the neighbourhood of joints (deformity or enlargement of the epiphyses) and deformity of the diaphyses. The former results from irregularity in ossification of the articular cartilages. The latter is followed by loss of rigidity in the bones of the limbs which, under the influence of the body weight and of muscular contraction, bend in different directions.

The bones appear of increased thickness principally towards the 
articulations. The latter are deformed, and on palpation are found to be surrounded by uneven and irregular growths.

The front limbs are distorted. In young pigs, lambs, and less frequently in foals, calves and dogs, the jaws become deformed, and mastication is rendered difficult.

The vertebral column may also be affected, and lordosis (bending downwards of the back) or skoliosis (lateral bending of the back) is somewhat frequent.

Cyphosis, or upward bending of the back, seldom occurs, and when seen, sometimes results from disease other than rachitis.

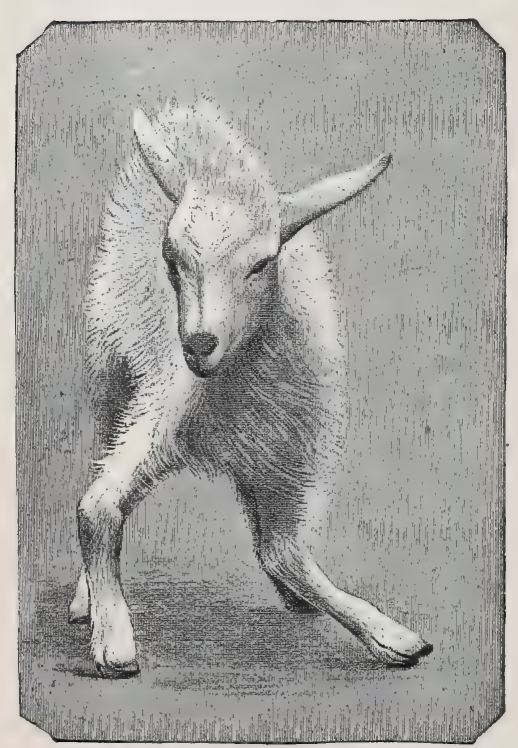

FIG. 1.-Rachitis in a young goat.

General development is always interfered with and the young creatures are generally dwarfed.

The digestive apparatus is disordered, the appetite is irregular and sometimes depraved, while indigestion, gastritis, and enteritis are not exceptional. Physiological and pathological research has shown that the quantity of phosphoric acid eliminated in twenty-four hours in a rachitic child is double the quantity passed by a healthy infant. The amount of urea in the urine (which is a criterion of nutrition, and usually varies in proportion to the amount of food ingested) is, on the contrary, diminished even when highly nitrogenous food is given, thus suggesting diminution in nutrition.

Lesions. The lesions are represented by abnormal and irregular thickening around the inter-articular cartilages. The cartilage is thickened, compressible, very spongy and without regular ossification. Diffused periostitis exists principally towards the extremities of the bone. Beneath the periosteum the surface of the bone appears rough and softened. On section the medullary canals are seen to be enlarged and filled with marrow of a gelatinous character. The Haversian canals are dilated, and the entire tissue appears very vascular. Chemical analysis proves that the mineral constituents of the bone, particularly the phosphates, have diminished by one-half; the organic constituents on the other hand are increased in a similar ratio, but the ossein is abnormal. Ossification has, in a word, been incomplete. 
Diagnosis. Diagnosis presents no difficulty except in the early stages before deformity has occurred.

Rachitis can scarcely be mistaken for any other condition except perhaps infectious rheumatism, but the rapid course of the disease in the latter case, the persistence of fever and the swelling of the joint cavities sufficiently differentiate the conditions provided care is exercised.

Prognosis. From an economic point of view the prognosis is very grave for if the lesions are extensive there is nothing to be gained by keeping the animal.

Treatment. Treatment differs very little, whether the animals are still being suckled or have been weaned. In the former case it is necessary to improve the quality and chemical constitution of the mother's milk by giving food, richer both in mineral salts and in nitrogenous material.

Cooked grains, milk, and forage of good quality should be given freely. When the mothers are exhausted and anæmic it is better to feed the little animals artificially or to change them to a foster-mother. Those already weaned should be given good rich milk, eggs, boiled gruel, and drugs, such as the phospho-chlorate of lime, 1 to $1 \frac{1}{2}$ drachms per day (for a calf) ; lacto-phosphate of lime, 1 to $1 \frac{1}{2}$ drachms ; bi-phosphate of lime, 1 drachm, or simply ordinary phosphate of lime. Oil containing 1 per cent. of dissolved phosphorus may be given in doses of 1 to $2 \frac{1}{2}$ drachms, according to the size of the calves, but its use calls for much care, and it should only be given for alternate periods of a fortnight. The glycerophosphates are not very active. Beef meal in doses of 6 drachms to $1 \frac{1}{2}$ ounces and chloride of ammonium in doses of 30 to 60 grains have also been used advantageously. The above drugs, but particularly the bi-phosphate of lime and chloride of ammonium, stimulate nutrition and diminish the quantity of phosphoric acid eliminated.

\section{OSSEOUS CACHEXIA.}

"Osseous cachexia" is a general disease which develops slowly and progressively, producing its most marked effects on the bony tissues. It has received a great many different names, such as osteoporosis, osteoclastia, osteomalacia, fragilitas ossium, enzootic ostitis, bone softening, etc., but none of these appears so appropriate as the term osseous cachexia, suggested by Cantiget.

All the above-mentioned names are applicable to some phase of the disease, but none to the disease in its complete development. Thus the name "osteoporosis," accepted by German authors, is quite applicable to the phase of rarefying ostitis seen at the commencement, but this condition occurs in other diseases. The expressions "osteoclastia" and 
"fragilitas ossium" suggest the fragility of the bones and the commonness of fracture. The term "osteomalacia" is warranted during the period of bone softening. The term "gout," though in practice confusing, has been held to be justified by the frequent appearance of synovitis and arthritis; while that of "enzootic ostitis" indicates the appearance of the disease in all the stables in one district, without however pointing to its nature. It is possible that under certain circumstances the train of symptoms might be incomplete, and then the terms above indicated would be quite inappropriate. "Osseous cachexia," on the other hand, is very comprehensive, and appears to cover the entire development of the disease, for which reason it here receives preference.

Law defines the disease as "a softening and fragility of the bones of adult animals, in connection with solution and removal of the earthy salts." He describes it as an enzootic disease of mature animals-mainly cows-in which the decalcifying process proceeds most actively in the walls of the Haversian canals and cancelli of the affected bones. In consequence of the removal of the earthy salts the bones become soft and more or less fragile.

The disease has been observed in England, Scotland, United States, France, Belgium, and Jutland, and generally in districts with lowlying damp pastures. It attacks cows which are heavy milkers. Susceptibility appears to increase with advancing age.

History. Having been described by Vegetius, the disease was again observed about 1650 in Norway where it was treated by the administration of crushed bones. It is fairly frequent in some parts of Germany and Belgium. In France it was studied in 1825 by Roux, and in 1846 by Dupont, but Zundel in 1870 was the first who gave a good description of it, founded partly on the authority of German authors and partly on observations made by himself in the Valley of the Lower Rhine. Since that time it has successively been reported in the Yonne by Thierry, in the Nièvre by Vernant, in the Aube by Collard and Henriot (1893), in the Indre by Cantiget, as well as in La Vendée by Tapon in 1893. In that and the succeeding year Moussu also saw numerous cases in the districts of Indre-et-Loire, Loire-et-Cher, Berry, Sologne, and in some parts of Beauce.

Symptoms. The first symptoms are difficult to detect and interpret, especially at the commencement of an outbreak and in parts where the disease is rare they may lead to confusion and errors in diagnosis. On the other hand, in regions where the disease is common the practitioner will be able to form his diagnosis from the appearance of the first signs.

To render clear the mode in which the symptoms develop we may divide the progress of the disease into four phases, though this grouping is somewhat arbitrary. 
1. The initial phase is not well marked, and is announced by digestive disturbance and by wasting. The former of these symptoms may be referred to some other cause, but consists in irregularity, diminution and sometimes perversion of the appetite. These earlier signs are soon followed by loss of spirits, and some interference with movement, but the symptoms only become of importance or attain their full development when the animals remain lying for a long period in the stable.

2. The second phase is characterised by more precise signs, which become almost pathognomonic. Difficulty in rising is added to the

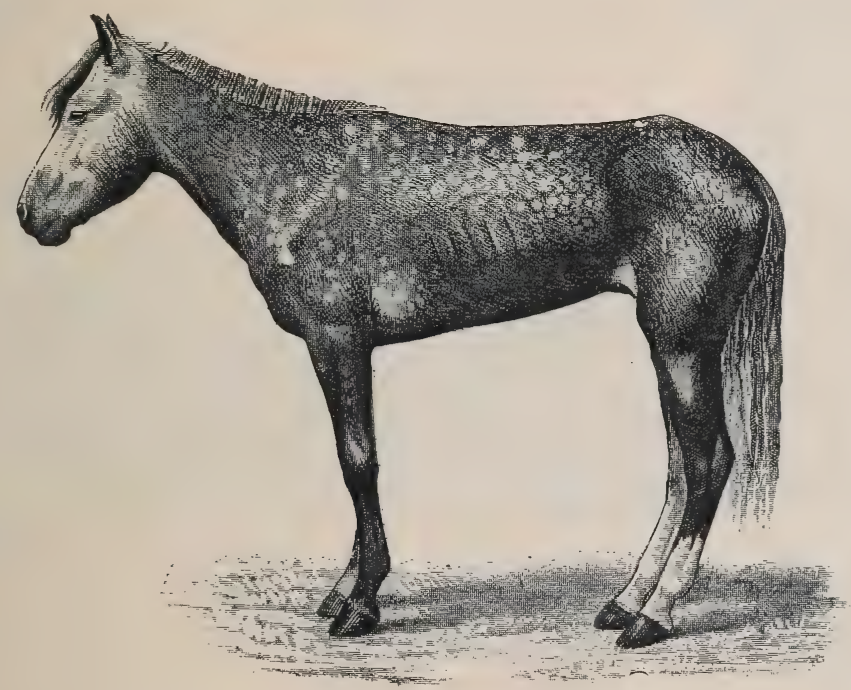

FIG. 2.-Horse suffering from osseous cachexia.

already existing tendency to remain lying, and to the interference with movement.

When lying down the patient no longer responds to the trifling stimulus, which a healthy animal needs to cause it to rise. It remains languid and apparently lazy, though in reality it experiences pain and difficulty on attempting to get up. The least muscular effort when lying down often causes it to moan, as do efforts to change its position or to walk. Even when standing still, it may appear to be in pain, and patients often assume a position similar to that of a horse suffering from laminitis.

At the end of this second phase, swellings appear, due to synovitis or arthritis of the extremities, synovitis of the sesamoid or navicular sheaths or to inter-phalangeal arthritis or arthritis of the fetlock joint. Weakness becomes marked, and the appetite is very irregular. 
Secretion of milk diminishes or ceases and abortion is not uncommon.

3. The third phase is characterised by fractures, and it is this peculiarity of the disease which has procured for it the names of fragilitas ossium, and osteoclastia. These fractures may affect any portion of the skeleton. Animals so suffering sometimes break a leg whilst trotting or the pelvis in simply jumping over a ditch; a collision with a fixed object like the jamb of the stable door, or a fall on the ground, may result in the fracture of one or several ribs.

Such shocks would be of no importance to a healthy animal, but to one suffering from osseous cachexia, any violence, or even the slightest

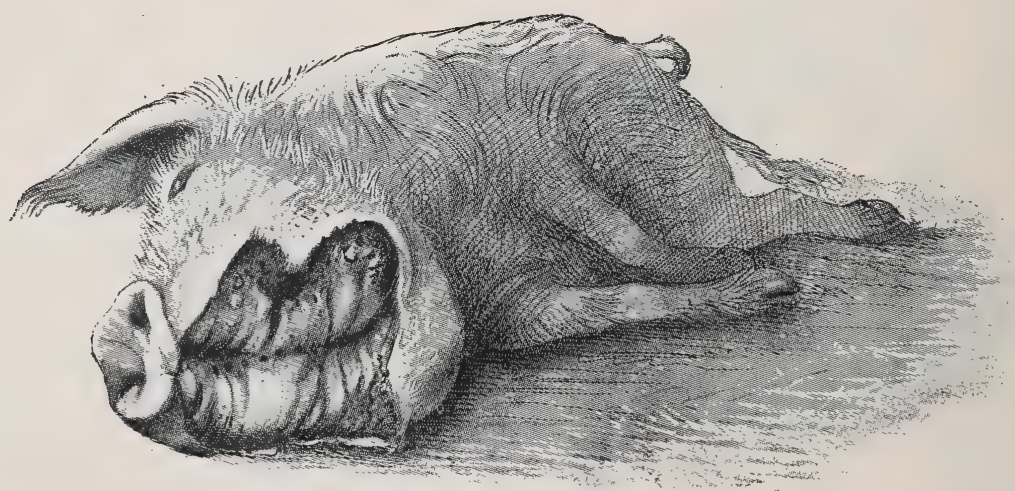

Fig. 3.-Pig suffering from osseous cachexia (fourth stage).

muscular effort may be followed by fracture of the gravest character, involving even the vertebral column. In cows the pelvis, femur, and tibia are most frequently injured.

In horses, particularly in riding horses, fractures are commonest in the region of the forearm, cannon bone, and anterior phalanges. So extremely fragile are the bones at this stage that the horse represented herewith broke twelve ribs at one time by simply falling on its side. It is interesting to note that such fractures are never accompanied by any extensive bleeding. They have little tendency to repair, no real callus formation occurs, and on post-mortem examination one often finds the ends unconnected by temporary callus, worn, and rounded by reciprocal friction.

At this stage but under other circumstances, the animals show great reluctance to rise, remaining down for twelve to twenty-four hours without shifting their position. If forced to get up, they stand as though fixed in one position, the respiration and circulation become rapid, and they soon grow tired and fall, 
4. The fourth phase, or period of osteomalacia, i.e. softening of the bones, is also the last. It is rarely seen in large animals like horses and oxen, because accidents so often accompany the preceding stages and necessitate slaughter; but it is common in goats and pigs.

In this phase the bones become elastic, soft and depressible, yielding to the pressure of the operator's fingers.

The flat bones are particularly liable to this change, which is common to domesticated animals. The bones of the head are the first to suffer; later those of the pelvis. The lower jaw becomes swollen, particularly about the centre of the branches which may attain three, four, or five times, their normal thickness.

The depression in the submaxillary space disappears. The upper jaw undergoes similar changes, becoming deformed and thickened until the cavities of the sinuses and the hollow appearance of the palate are lost, while the face is so changed that it cannot be recognised as that of a horse, goat, etc.

The molar teeth are almost buried, their tables alone being visible at the bottom of a depression, the edges of which rise above the neighbouring parts (pig).

Mastication is clearly impossible, the jaws appear paralysed, the muscles powerless, and only swallowing is possible, a fact which explains why life is only prolonged to this stage in animals which can be fed with a spoon or bottle (pigs and goats). The bones of the

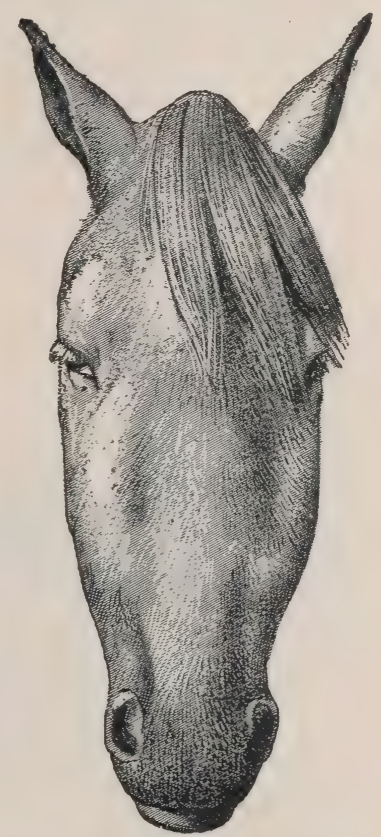

FIG. 4.-Deformity of the face in the horse shown in Fig. 2. cranium, although greatly changed in texture, are always less deformed than those of the face.

The changes are such that it is often easy with a mere post-mortem knife to cut the head completely in two. Osseous tissue, properly so-called, has disapppeared.

All the constituent tissues, with the exception of the skin and muscles, i.e., the bone, periosteum and aponeuroses, have the appearance and consistence on section of the fibro-lardaceous tissue seen in chronic inflammation.

The following is a condensed description of the disease as given by Law :-

Symptoms. Poor condition or even emaciation, with very visible projection of the bones. The coat is rough, skin tense, inelastic and 
hidebound, appetite variable, sometimes impaired, and nearly always perverted (or depraved) so that the patient will lick the manger continually or pick up and chew all sorts of objects: bones, leather, clothing, wood or iron, stones, etc. The amount of food consumed may, however, be up to the normal. The most marlied feature is the difficulty and stiffness of locomotion. . . . Temperature and yield of milk may remain normal.

"Later, appetite and milk secretion fail, temperature rises a degree or two, the animal refuses to rise, remaining down twelve to twenty-four hours at a time, and . . . when rising . . . remaining on the knees for a time, moaning and indisposed to exert itself further. At this stage many cases begin to improve and may get well in five or six weeks. Some will remain down for several weeks and finally get up

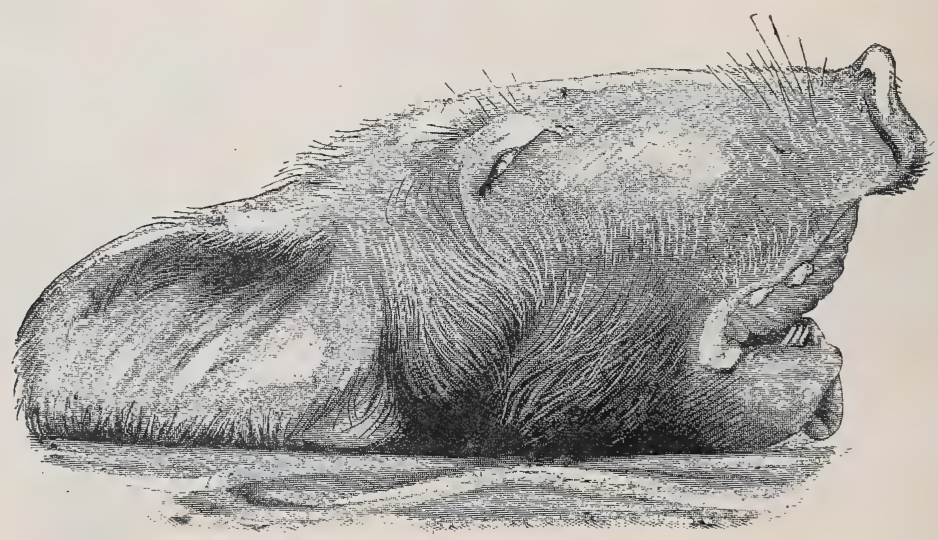

FIG. 5.-Head of a pig suffering from osseous cachexia.

and recover. With constant decubitus, however, the animal falls off greatly, becoming emaciated and weak, the appetite may fail altogether, and the patient is worn out by the persistent fever, nervous exhaustion and poisoning from the numerous bed-sores . . . which are common over the bony prominences. It is in these last conditions, above all, that fractures and distortions of the pelvic bones, and less frequently of the bones of the legs occur."

"The disease may advance for two or three months, and in case of pelvic fractures and distortions, there may be permanent lameness, and dangerous obstruction to parturition, even though the bones should acquire their normal hardness through the deposition of lime salts."

In horses, the different phases of the disease develop precisely as in bovines. The apparent differences between affected horses and cattle result in reality from differences in their capacity for continuing work. In the first phase, horses are incapable of work, their movements being 
badly co-ordinated. They are inclined to stumble, and appear as though suffering from strain of the lumbar muscles.

In the second phase pain referable to the bones sets in. Lameness develops without visible lesions and is rapidly followed by synovitis and arthritis in the lower portions of the limbs, and by wasting and anæmia.

The animals seem unable to move rapidly, or if forced to do so may sustain fractures even at a trot: the limb bones sometimes break or ligamentous insertions in the neighbourhood of joints are torn away, resulting in sudden falls on the ground and fracture of ribs or even of the vertebral column. This corresponds to the third phase, osteoclastia, in oxen.

From then onwards, horses become useless and, if not destroyed, may, after a few weeks or months, develop the condition known as osteomalacia, in which the flat bones become softened, the head, the branches of the lower jaw and the face become deformed, while mastication and other functions are impeded.

Germain gives the above symptoms as characteristic of the mode of development of the disease in French and Algerian horses imported into Tonquin, and his description, written several years ago, is fully confirmed by more recent observations. Since Tonquin was taken over by the French, however, improved methods of culture have resulted in the production of better cereals and forage; the fodder plants have been vastly improved, to the great benefit of imported animals.

In the goat, the disease shows some slight peculiarities. 'Ihus, in the second phase, during which goats and sheep suffer so markedly from lameness and pain in the bones, goats often

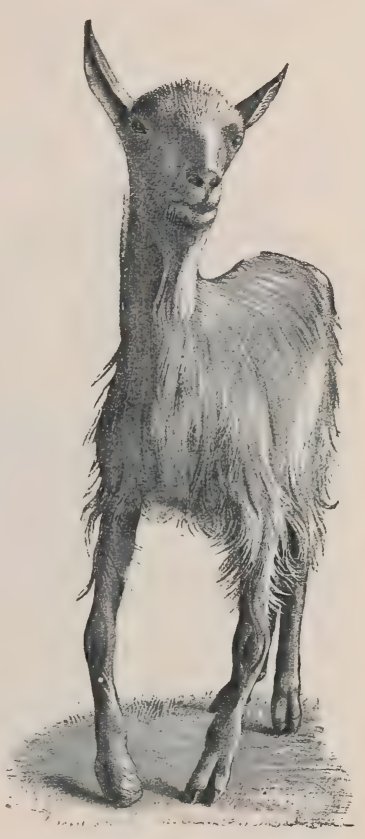

Fig. 6.-Osseous cachexia. This condition developed in two months, the last month of gestation and the first of lactation. walk on the knees. The disease, however, is uncommon in these animals. The phase of osteoclastia is also less marked and fractures are rare, because the animals weigh less and also because they are less exposed to falls and violent shocks. The bones, nevertheless, are extremely fragile and fractures may be produced at will.

Osteomalacia, on the other hand, is always well marked.

Regarding the development of the disease in pigs, we may repeat what has just been said respecting the goat. Walking on the knees is often one of the first signs, fractures are somewhat rare, and the period of softening and deformity is always very noticeable. 
Course. The development of the disease is slow, lasting from one to three months as a rule, and is little influenced by hygienic conditions. Good milking cows, however, seem to be most frequently attacked, probably because of the great losses of nutritive material which occur through the milk. The calves borne by such animals are often rachitic. Oxen are less commonly attacked. Horses rarely suffer from the disease in France, but frequently in Tonquin. Pigs reared on very poor soil seldom escape attack.

If treated from the beginning, or even before the second phase has become well developed, the disease may be cured, but after this period little improvement need be expected.

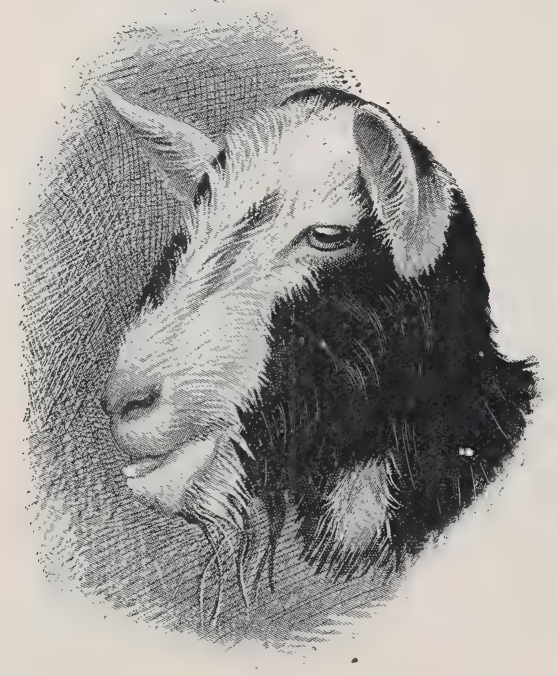

FIG. 7.-Osseous cachexia : softening of the maxillæ.

Causation. The problem of why osseous cachexia occurs has naturally given rise to numerous explanations, some plainly inadmissible, others, however, of greater or less plausibility.

The fact which, from the earliest times, appears to have attracted most attention is the relation defective nourishment bears to development of the disease. In Norway, as early as the year 1650 , the plant known as sterregraes (which renders animals dull and heavy) was thought to be the cause of the disease ; two centuries later, in 1846, the Anthericum ossifragum was similarly regarded. Zundel, in 1870, claimed that the Germans first referred the development of the disease to chemically incomplete forms of nourishment. This opinion seems fully confirmed by the remarkable observations of Germain on European horses imported into Cochin-China, and it is finally placed beyond question by the work of Cantiget. Basing his researches on analysis of the soil, he proved that osseous cachexia only occurs in cattle depastured on land which is too poor in phosphoric acid and calcium phosphate, and that it can be banished by enriching the soil with suitable manures up to a point when the proportion of phosphoric acid becomes normal. In good land, suitable for raising cattle, the proportion of phosphoric acid, according to the best exponents of agricultural chemistry, should not fall below 4,000 kilograms to the hectare. Cantiget and 
Brissonet have shown that where the soil contains less than 1,500 kilograms to the hectare, osseous cachexia is almost permanently present. As soon, however, as this proportion is raised above 2,000 kilograms by suitable culture, the losses diminish, and the cachexia finally disappears.

This view was greatly strengthened by fodder analyses, which showed that in all cases where the soil is poor in calcium phosphate, the forage is poor in phosphoric acid, and vice versâ. The food is too poor in mineral salts, firstly for normal development; and secondly for the proper nutrition of the skeleton.

Germain is of a similar opinion with regard to the occurrence of osseous cachexia in horses in Cochin-China, where the soil is very poor in lime. The fodder and cereals are poor in mineral salts, and even when given in large quantities do not furnish proper (chemical) nutrition. Clear proof of the correctness of this view is afforded by the fact that feeding with forage and cereals obtained from France or Algeria prevents the disease appearing, or diminishes and finally removes the previously existing symptoms. Furthermore, Germain shows that Europeans, living solely on the products of the country, to some extent suffer like the horses.

This theory though based on sufficiently solid foundations to carry conviction, has been questioned, and it may be desirable to record briefly the criticisms advanced against it.

One of the most important is as follows:-

As osseous cachexia of oxen occurs in certain well-defined districts in France, and seems due to the feeding, why does it not attack horses in the same regions in an enzootic form? The answer appears to be that horses receive a greater amount of rich food, particularly of cereals, which contain much larger amounts of mineral salts, including phosphates, than does ordinary forage.

The most serious objection was made by Tapon, who states that in 1893 he saw osseous cachexia in oxen on farms in La Vendée where superphosphate had been used for years, whilst the disease did not exist on other farms where such chemical manures were not employed. Before attaching much weight to this objection, however, it would be necessary to know the richness in phosphoric acid of the soil on the respective farms, for it is possible that, in consequence of natural conditions and in spite of the use of certain mineral manures, the richness of the soil on the first-mentioned farms, though manured with superphosphates, was still below that of the others which had received no artificial, manure.

The system of culture is also of importance, for at the present day, even with the use of artificial manures, cropping would rapidly impoverish soils which were not suitably and sufficiently enriched. 
Abundance or apparent richness of food signifies nothing if quality is lacking.

It may also be asked : if the question of nourishment is of such prime importance why are animals of European origin in Cochin-China affected, whilst the indigenous races prove immune? The answer would appear to be that, in addition to the defective quality of food, other factors, such as adaptation to environment and relative digestive power, play a considerable part in the production of the disease.

Fayouring causes. Whilst conceding that the disease is due to one determining cause, viz. the food, it is unquestionable that other causes may favour its appearance. Abundant milking is one, so that the disease most frequently appears six to eight weeks after calving. Gestation may also determine an attack. The disease is rarer in oxen than in milch cows. Starvation and bad hygienic conditions also have a certain influence; it is well known that during dry years, particularly when fodder is scarce, osseous cachexia makes the greatest ravages. Law states that the disease has been attributed to excess of organic matter in the soil, to succulent watery foods, as rank watery grasses, potatoes, turnips and other roots deficient in nutritious solids. Some agentmicrobe or toxin-swallowed with the food has been suspected but not yet isolated.

Other explanations have been advanced but up to the present time they scarcely deserve to be regarded even as hypotheses. Thus Anacker in $\mathbf{1 8 6 5}$ declared that the disease commenced as muscular rheumatism, was succeeded by destructive or atrophic ostitis, and ended as osteoporosis. So far as the order of the osseous lesions is concerned, this view is quite correct, but the ossific changes are consequences and not causes.

The idea that the disease was due to an infectious agent has been advocated by Leclainche, without, however, having been proved. Pétrone is the only person who has hitherto suggested that osteomalacia in man is due to infection with a nitric ferment (Micrococcus nitrificans). According to him, pure cultures of this organism injected into dogs, produce osteomalacia. These statements, however, require confirmation.

Lesions. The chief lesions are to be found in the bones. They consist in rarefaction of the compact tissue, increase in size of the medullary cavity and Haversian canals, and enlargement of the areolæ of the spongy tissue. The bone marrow loses its fatty constituents, appears red and gelatinous, and contains a greatly exaggerated number of blood-vessels. When heated, the bones do not yield oil as in healthy subjects, and when dry, they seem abnormally porous. In the osteoclastic phase, the bones become very friable and even the shafts assume 
a spongy appearance. They diminish in density. These changes correspond to the stages of eccentric rarefying ostitis and osteoporosis of German authors.

The flat bones often show well-marked periostitis, but the great thickening sometimes seen in certain of the bones of the head appears to be the result of a special osteo-periostitis. It is quite certain that the disease is due to something more than a mere want of mineral constituents in the bone, and poverty in this respect certainly does not explain the hypertrophic changes. The nutrition of the bones as a whole is disturbed, resulting in alterations both in the ossein and

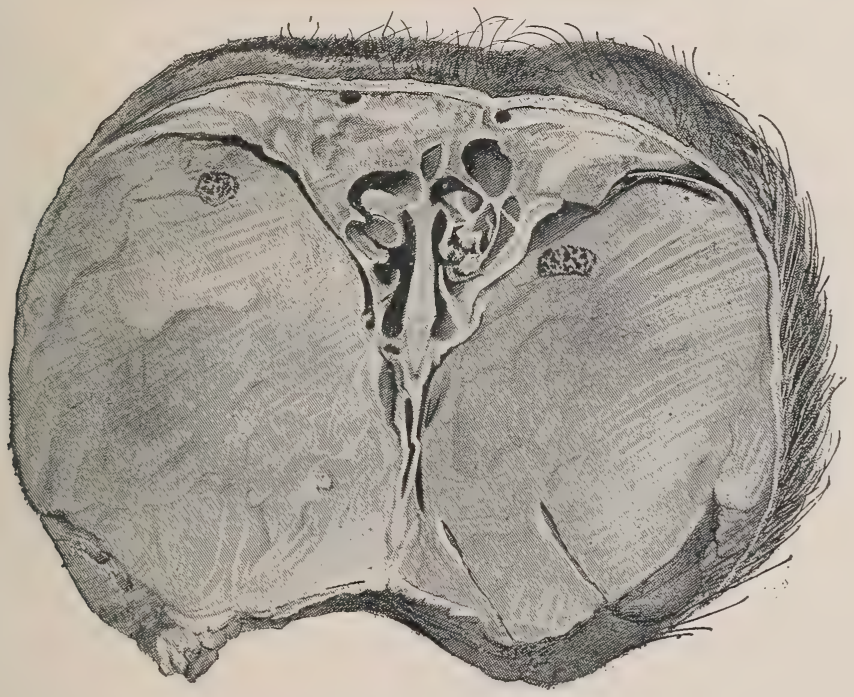

FIG. 8.-Transverse section through the middle region of the face in a pig suffering from osseous cachexia.

in the mineral salts, the whole process being accompanied by symptoms of osteo-periostitis.

The fractures which occur so frequently during the osteoclastic phase have well-marked peculiarities. The extravasation of blood is trifling, and no callus forms, even when the ends of the bones are immobilised by external aid; if the ends are left free, they soon become worn and polished by rubbing against one another.

In the naighbourhood of the articulations and ligamentous insertions the periosteum soon undergoes change, and it is not uncommon to find sub-periosteal and intra-osseous extravasations of blood.

Germain has also noted in horses the disappearance of the intervertebral and articular cartilages, and the frequent accurrence of anchylosis, true or false.

D.C. 
In the final stages, the bones may be cut with a knife, and a time arrives when bony tissue seems completely to have disappeared; thus, as shown in Fig. 8 herewith, it was possible to cut the entire head of a pig into thin slices without the slightest difficulty. All parts of the head had been affected by the softening change.

From the chemical point of view, the diminution in mineral salts and in phosphate of calcium has long been recognised, but the degree of this change varies according to the phase. In human beings the proportions have been estimated as follows: Normal bone, 50 to 80 per cent. of phosphate of calcium; bone in persons suffering from osteomalacia, 5 to 20 per cent. of phosphate of calcium. The changes in the ossein have not been carefully studied. We only know that histologically the ossein becomes fibrillar, and that chemically it no longer retains its normal composition.

The diagnosis is difficult, particularly on the first occasion of seeing the disease, and especially if this is of an enzootic character. The practitioner may also have some hesitation in diagnosing isolated cases in regions where the disease seldom occurs.

Otherwise, diagnosis is usually easy, as soon as lameness or synovitis, or arthritis of the lower regions of the limbs appears. Only in isolated cases are the lesions likely to be mistaken for accidental injuries, and it is also fairly easy to differentiate them from the localised lesions of rheumatism. The latter disease seems more frequently to attack the upper joints of the limbs, and is often accompanied by intense fever and cardiac disturbance.

Prognosis. In a general sense the disease is very grave, because it appears as an enzootic, and, in dry years and those during which there is a scarcity of forage, inflicts enormous losses on the breeders of certain countries. When advice is sought towards the end of the second phase of the disease the prognosis is therefore very grave. Under such circumstances it is often better to slaughter rather than to treat, provided that the affected animals, like cows, pigs, or goats are still of some value.

The prognosis is much more hopeful if treatment is attempted at an early stage, when improved diet and the use of suitable drugs sometimes lead to recovery.

Treatment. We know that in the Middle Ages this disease was often treated by the administration of crushed bones, and even at the present day ground bones are frequently recommended. Treatment must be subordinated to proper feeding, no system of medication being of any value whatever unless the food is suitable.

Germain states that imported horses in Cochin-China recover if simply returned to their former diet, i.e. to cereals and forage obtained from France or Algeria. Cantiget shows that such improvements in 
cultivation as the free distribution of superphosphate manures on impoverished soils modify the chemical composition of the forage, and render it capable of building up and sustaining the organism and bony tissues; treatment should therefore be essentially prophylactic in character.

Animals suffering from osseous cachexia should be fed on cereals and forage obtained from rich districts where the disease has never occurred; but, as in times of scarcity questions of expense almost always receive first consideration, it may be necessary to substitute bran for such products, or give oats, maize, beans, rice, and oil or cotton cake, etc., all of which can be obtained commercially, and are of sufficient nutritive richness. It is often advantageous to give such food cooked and slightly salted.

Commercial ground bones and calcium phosphate (bi- or tri- basic), in doses of 1 ounce per day for oxen and $1 \frac{1}{4}$ to 2 drachms for pigs or goats, have given excellent results in the hands of most practitioners. Some recommend the addition of iron salts or bitter tonics like gentian or nux vomica in doses of $2 \frac{1}{2}$ drachms per day for a full-grown ox.

Law declares that the treatment should be varied "with the predominance of the causes, essential or accessory. . . . Green clover, alfalfa, and other leguminous products, ground oats, beans, peas, linseed or rape cake . . . and vetches may be especially recommended. . . The free access to common salt and a liberal supply of bone meal are helpful. . . . Apomorphia is especially valuable in correcting the perverted appetite and stimulating digestion. A change of pasture is always advisable. In all cases where possible the water should be changed as well as the food. Attention to the housing, grooming, and general care of the animals should not be neglected. Finally, every drain upon the system should be lessened or stopped. The milk may be dried up, and the animal should not be bred."

Meat meal also renders good service, but the use of cod liver oil, suggested by Zundel, is too expensive, and phosphorised oil is too dangerous to be adopted in ordinary treatment.

Local treatment for synovitis and arthritis has been recommended. It is ineffective unless accompanied by good feeding and internal medication. On the other hand, the lesions often diminish rapidly or totally disappear under the influence of general medication alone. 


\section{LOCAL AFFECTIONS.}

\section{FRACTURES.}

Although oxen, sheep, goats, and pigs are much less subject to fractures than the horse and dog, nevertheless, they do suffer from such accidents. Repair is perfectly possible, but the cases are often not worth treating, unless the subjects are young or of considerable value. On the other hand, in fat and heavy subjects, it is difficult to fix the parts in position. Slinging produces bad results, and generally should not be encouraged.

Apart from fractures accompanying general chronic diseases, like rachitis and osseous cachexia, the vertebræ, the pelvis, the ribs, or any of the limb bones, may be fractured in consequence of accident.

Such fractures may be either complete or incomplete (fissures), simple or compound.

The general signs which indicate fracture are always the same, viz., loss of function, local pain, abnormal mobility, crepitation, due to rubbing together of the ends of the bones, and deformity of the part. Diagnosis is generally easy; prognosis on the other hand is very variable.

The vertebral column may be accidentally fractured in the region of the neck in consequence of the animal falling on its head; in the dorsolumbar region, from falling into ditches or ravines, or, in the case of bulls fighting, from violent muscular efforts. Fractures of the first kind are immediately fatal; those of the second result in paraplegia of the hind limbs, and necessitate immediate slaughter.

Fractures of the pelvis comprise :-

1. Fractures of the angle of the haunch, resulting from external violence and characterised by sinking of the external angle of the ilium, deformity of the hip, and lameness without specially marked characters. This fracture is rarely complicated. The symptoms of lameness diminish with rest, but deformity continues.

2. Fractures of the floor of the pelvis, usually extending from the anterior margin of the pubis to the foramen ovale and from the posterior margin of the foramen ovale to the end of the symphysis. They result from obstetrical manipulation, as in forcibly removing a fœetus which is too large, or a monstrosity. As a rule, the animals cannot rise, or if they succeed in doing so, are incapable of moving. Diagnosis is made by exploration through the rectum. Such fractures always necessitate slaughter.

Fractures of the neck of the ilium and of the base of the cotyloid cavity, even in cases of dislocation, are rare despite what has been said to the contrary. 
In the fore limb, fractures of the scapula and humerus are usually of traumatic origin, are seldom accompanied by marked displacement, and are capable of uniting if a long rest at grass is allowed. Pitch bandages should be applied to the surface, covering all the surrounding regions, viz. the withers, upper portion of the forearm, girth and chest, to assist in immobilising the region of fracture, and to promote union.

Fractures of the forearm are more difficult to treat, because the bandage applied must extend as far as the hoof. In this case displacement often occurs. It is therefore necessary, firstly, to reduce the fracture, and bring the ends in perfect contact, for which purpose it may be requisite to cast the animal, and give an anæsthetic; and, secondly, to apply a pitch plaster in the form of a shallow gutter, leaving the inner surface of the limb uncovered along a line about two inches wide following the course of the veins of the forearm.

Fractures of the metacarpus and metatarsus usually heal well in all animals of moderate weight, such as heifer's, steers, goats or sheep, provided a simple plaster bandage, covering the entire limb or preferably with an opening in the position above indicated, is applied and continued downwards as far as the claws.

In sheep and goats it is sometimes even sufficient to use a splint formed of straw-boards, and in the case of oxen, of wood, applied over a cotton-wool padding and retained in position by straps, or in the case of the heavier animals by dextrine or pitch bandages.

In the hind limb, fractures of the femur are more serious, because the apparatus that can be used to secure immobility is seldom or never effective; excepting in young animals, it is therefore usually better to slaughter.

Fractures of the tibia are treated like those of the forearm when it appears desirable to keep the animals alive.

Plaster bandages can very easily be prepared by saturating tarlatan in a mixture of equal parts of thoroughly dry plaster and water. Six to ten thicknesses of tarlatan, arranged alternately longitudinally and transversely, are sufficient. When adjusted they can be kept in position until the plaster has hardened by means of dry bandages applied from below upwards, which can be removed after a lapse of half an hour to an hour.

\section{FRACTURES OF THE HORNS.}

Anatomy of the horns. The horns form organs of defence, and project on either side of the frontal bone at the poll. Each consists firstly of a bony basis generally known as the horn core ; secondly, of a horn-secreting membrane; thirdly, of a horny sheath, the horn properly so called. 
(1.) The horn core projecting from the frontal bone does not develop until after birth. About the third month a little prominence appears under the skin, which, as it develops, assumes a conical shape, and may be seen to be covered with a horny substance. In proportion as the horn core grows, there develops within it a cavity which may either be of a simple character or divided by a longitudinal partition. This communicates with the frontal sinus, a fact which explains the collection of pus in the sinuses as a result of injuries to the horns. The sinus of the horn core does not exist in young animals, and is not completely developed before the third or fourth year of life.

(2.) The horn-secreting membrane is formed by the skin, which undergoes special development around the base of the horn and comes to

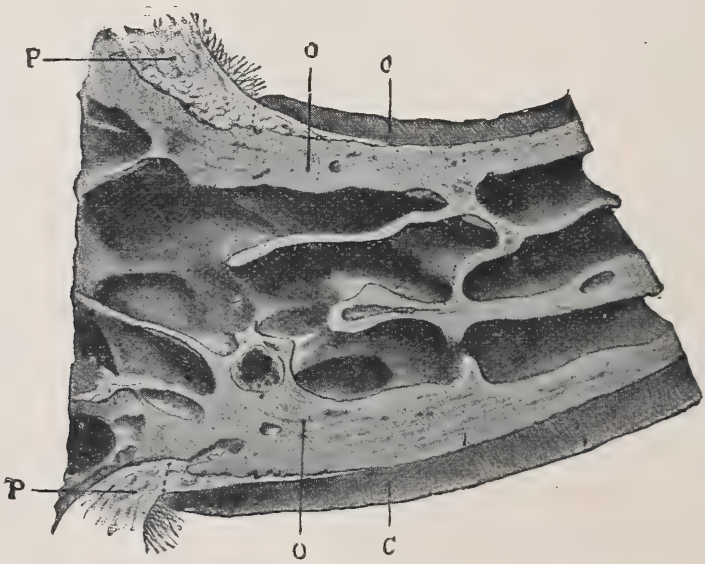

FIG. 9.-C, horn; $\mathrm{P}$, modified skin forming the keratogenous membrane; $\mathrm{O}$, horn core, exhibiting a double sinus.

resemble that of the coronary band, from which the hoof or claw is secreted. The band is about one-fifth of an inch in breadth. The papillæ of the dermis are specially developed at this point, and the epithelium which they secrete eventually forms the horn.

The internal surface of the growing horn is adherent to the horn core through the medium of another tissue formed by a specially differentiated periosteum which is continuous with the periosteum covering the frontal bone. It is not a true periosteum, but a vascular tissue formed of papillary layers analogous to those of the podophyllous tissue of the ox's claw or horse's hoof.

This keratogenous membrane receives a rich vascular supply from the arterial circle formed at the base of the horn core by a division of the external carotid, the blood conveyed by which is freely distributed to the enlarged papillæ. The great vascularity of these parts 
explains why lesions of the horns are often followed by such profuse bleeding.

(3.) The horn secreted by the papillæ of the horn band (which is analogous to that of the coronary band of the horse) forms a cone varying in its curve in various breeds. Its base is hollow, and contains little depressions holding the papillæ from which the horn is secreted. From its base up to the end of the horn core the walls pro. gressively increase in thickness. From this point it is solid; in a fully-grown horn the bone does not extend more than one-half or two-thirds of the entire length.

In the adult, the development of the horns varies with different breeds and is affected by sex. In the bull the horns are short, but in the cow and ox long. Short and fine in animals of improved breed like the Durham, they are long and thick in breeds of working oxen.

Injuries affecting the horns are of three classes, determined by the part affected.

1. Detachment of the horn or sheath.

2. Laceration:-

(a) Of the horny sheath alone.

(b) Of the horny sheath and of the horn core.

(c) Of the horn core alone, the horny sheath remaining intact.

3. Fractures:-

(a) Of the terminal half of the horn.

(b) Of the lower half.

(c) Of the base.

\section{DETACHMENT OF THE HORNS.}

When the yoke is badly fitted or padded, it is liable to cause a continual strain or a succession of shocks producing chronic inflammation of the keratogenous membrane. Should the end of the horn then be struck heavily, it is quite possible that the horn will either partially or wholly be detached. In this case it falls away without there necessarily being any important lesion of the horn core:

Such accidents are not infrequently caused by the driver striking the ox on the horn with the yoke in order to keep it quiet while it is being harnessed.

The prognosis of this condition is not grave, except for the fact that working animals cannot be used until the horn is completely regrown.

The treatment simply consists in thoroughly cleansing and disinfecting the horn core and then applying a protective dressing. The bony basis is surrounded with a mass of tow saturated with an antiseptic 
solution, like 2 per cent. creolin or carbolic acid solution, which is kept in position by a spiral bandage passed around the horn, and secured in a figure of 8 on the opposite horn. Instead of applying such a dressing, some practitioners content themselves with using an antiseptic ointment or even a simple dressing of tar.

\section{FISSURING OF, THE HORNS.}

Causation. In a general sense fissures may result from any violence affecting the centre portion of the horns, such as blows with the yoke or accidental bruises inflicted by the animals themselves in fighting with their neighbours.

Symptoms. Whether the fissure is confined to the horny covering itself or whether it extends to both the portions constituting the horn, that is, the horny covering and the horn core, two very noticeable symptoms are always present: 1. A straight fissure resembling a sand crack, and appearing usually on the convexity of the horn, and, 2. A very trifling hæmorrhage, which does not appear until some hours or even a day after the accident.

Diagnosis. If the lesion only affects the horn core, diagnosis is always difficult, for one can hardly perceive any sensitiveness of the horn near the fissure.

Prognosis. Provided that the horn core is not injured, the prognosis is favourable; but in the contrary case, it should be reserved; for hæmorrhage extending to the interior of the frontal sinus not infrequently causes suppuration in that eavity.

Treatment. Attempts should first be made to check hæmorrhage by applying masses of tow saturated with cold water and frequently wetted with slightly antiseptic solutions, such as 2 per cent. creolin or carbolic acid. If hæmorrhage persists in spite of this simple treatment, astringents may be employed, which, by causing the formation of a clot, mechanically arrest further extravasation of blood. These astringents vary considerably in value, and we should particularly warn practitioners against perchloride of iron, which causes necrosis of the tissues, and later, formation of pus. A 5 per cent. solution of gelatine is hæmostatic and excellent for the purpose named, as also is hydroxyl. solution. When once hæmorrhage is arrested, the keratogenous membrane rapidly heals in consequence of its vascularity, and soon secretes fresh horn. 


\section{FRACTURES OF THE HORNS.}

Etiology. Fractures of the horns, like fissures, are produced by violence, but of a more marked character. They are termed complete or incomplete, according as the entire thickness of the horn or only a portion of that thickness is involved.

The fracture may affect either the terminal half or the basilar half; or, again, it may hare its seat in the frontal bone below the origin of the horn core, in which case a flake of bone will be detached. Such fractures assume varying forms, and may either be deeply excarated, oblique, smooth, regular or dentated.

Symptoms. The symptoms are extremely simple. They consist mainly in the mobility of the fractured end, and such phenomena as sensitiveness, hæmorrhage, etc. When the fracture extends to the frontal bone, crepitation may also be noted.

Prognosis. The prognosis is not grave unless the fracture extends to the basilar half of the horn or affects the frontal bone.

Treatment. (1.) If the fracture is confined to the horn core, it is only necessary to bring the fragments into regular apposition, after having removed the broken end of the horn itself.

(2.) In treating a fracture affect-

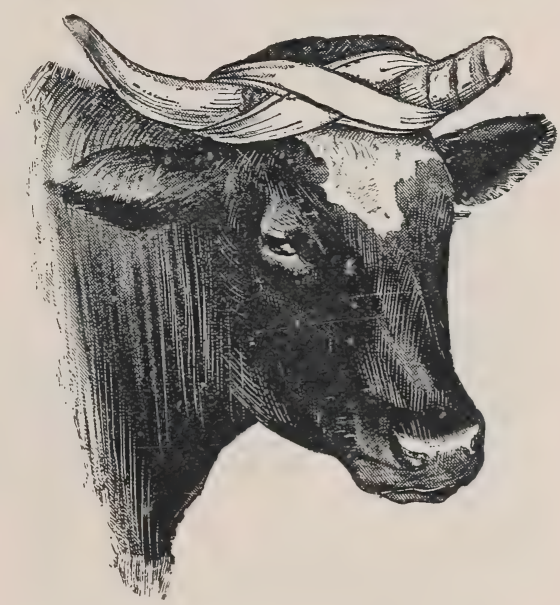

FrG. 10.-Dressing for fracture of the horn. ing the middle portion of the horn or in treating animals destined for the butcher, the best method is to make a simple wound by dividing the parts with a saw below the fracture. This is a painful operation, necessitating anæsthesia, and requiring the animal to be cast or firmly fixed to a post or placed in a trevis. To diminish the painful stage of the operation, it was formerly recommended to make a circular incision extending through the entire thickness of the horn proper, and then to remove with a fine, very sharp saw the portion of the horn core. 'This, horrever, is scarcely practicable, and it is much better to make a direct section. Hæmorrhage is checked with compresses, moistened with cold water, after which a dressing known as the "Maltese cross dressing" (Fig. 10) is applied according to general principles.

The surface of the section, after washing with an antiseptic solution, is powdered with iodoform or a mixture of iodoform and boric acid, 
covered with a mass of tow or cotton wool, saturated with liquid antiseptic, and then surrounded with a flat pad of wadding, which extends completely around the horn as far as its base. A second pad of larger size, intended to protect the wound against external violence, is arranged around the free extremity of the horn. This is kept in position by two small cross bandages. Another bandage, the loop of which is fixed to the base of the opposite horn, is then applied in spiral turns, completely enveloping the former pads and extending from the base to the point. Arriving at the free end, the operator reverses the bandage, draws it tight, and continues down to the base of the horn, fixing it by figure of 8 turns passed around the base of both horns.

(3.) In dealing with fractures of the lower third of the horn in working oxen, it is necessary to seek consolidation of the horn by callus formation,

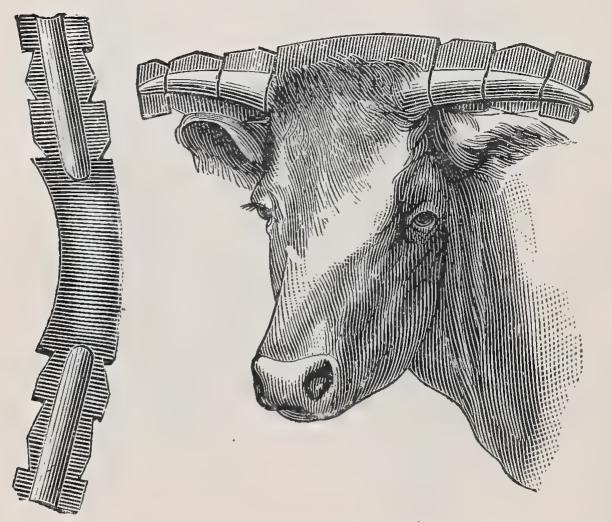

FIG. 11.- Splint for fractured horn. so that the animals may again become useful in the yoke. Very great difficulty accompanies attempts to immobilise the horn in such cases, since the least shock to the extremity of the horn destroys the union, on account of the length of the lever represented by the horn itself.

The first method of treatment consists, after the wound has been carefully disinfected, in fixing the ends in place by tightly applied pads, surrounded by splints, curved to fit to the horn. These are supported by several turns of a spiral bandage.

Were one certain of the cleanliness of the wound and of its perfectly aseptic condition, it would be better at once to have recourse to a fixed bandage, strengthened by plaster or silicate of soda.

These bandages are applied longitudinally and should extend a considerable distance on either side of the fractures. If the horn is long and thick, it is best to use a fixed dressing of this kind secured by bandages in the form of a Maltese cross.

All these methods, however, are more or less inconvenient, and the most practical procedure often consists in removing the horn. Treatment should only be attempted when the owner specially requests it in order to render the animal useful for working or show purposes.

Moreover, however strong the dressing, accidents are frequent, for accidental shocks to the fractured horn interfere with the co-aptation of 
parts, and diminish the chance of perfect, union. To aroid these drawbacks, the apparatus shown in Fig. 11 has been invented.

This apparatus consists of a splint, the middle of which fits the back of the animal's poll, the sides being gouged out to receive the lower half of the horn and notched, to enable the fractured horn to be well supported by bandages.

(4.) Fractures of the base of the horn are more serious, because a fragment of the frontal bone is usually torn away with the horn core. As a rule, the fracture is subcutaneous or without external wound. The horn is displaced, and swings loosely. On examination, a characteristic crepitation sound is easily detected. In such fractures hæmorrhage is subcutaneous and often extends to the frontal sinus, in consequence of which it is not uncommon for pus to form in the sinus as a complication.

Treatment includes reduction of the fracture, and the application of a fixed plaster or silicate bandage covering the fronto-occipital and

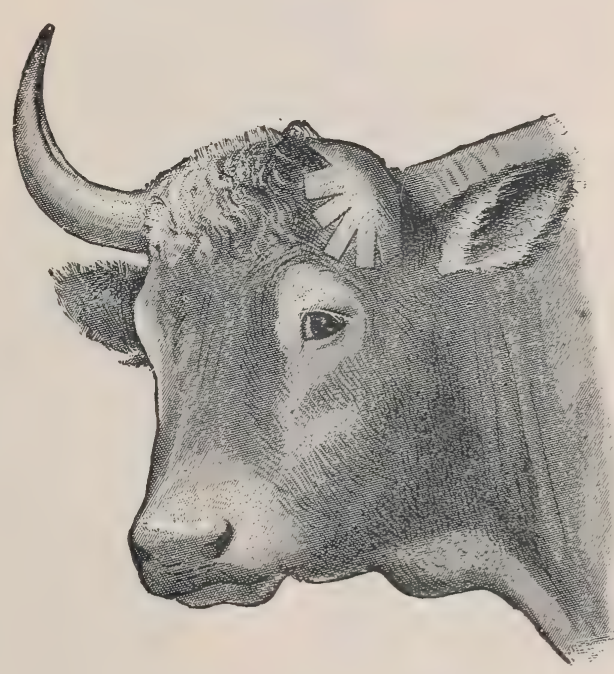

FIG. 12.-Dressing for fracture of the base of the horn. superior auricular regions.

When a skin wound exists, it is better to remove the horn and bony fragment, and to apply an antiseptic dressing in order to prevent infection of the frontal sinus.

\section{EXOSTOSES.}

\section{SPAVIN IN THE OX.}

Exostoses are somewhat uncommon in the bovine species, and when they occur are rarely of great clinical interest. Nerertheless, in cows and old working oxen one sometimes sees metatarsal spavin. Its gravity, however, appears to be very much less than in the horse, on account of its position. Very commonly there is only trifling lameness.

Treatment by application of biniodide of mercury ointment or the actual cautery gives good results. The prineipal precaution required is to prevent the animals licking the parts. 


\section{RING-BONE.}

Ring-bones only occur in working oxen, and particularly in aged animals used in hilly regions. They result almost exclusively from wounds, ligamentous and tendinous strains, and articular injuries.

They are preceded (as can usually be proved by dissection of limbs) by

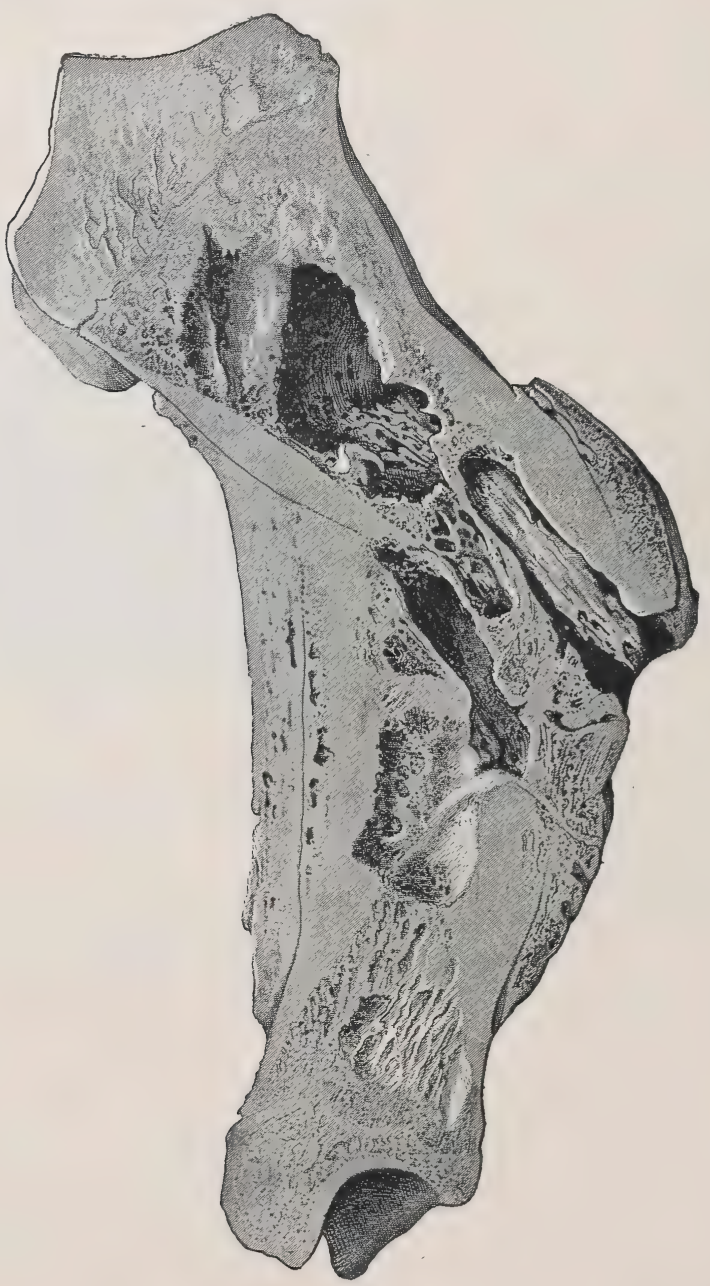

FIG. 13.-Tibia. Lesions due to open fracture and displacement. Irregular callus formation and segnestrum.

fibrous or fibro-cartilaginous induration in or about the coronet or one of the phalanges. These thickenings increase the diameter of the pastern in all directions. Ring-bones are seldom very large; but as they 
partially or entirely surround the insertions of the lateral ligaments, inter-phalangeal articulations or insertions of the digital extensors, they are painful, and produce lameness of varying intensity.

Diagnosis is easy, partly because the tension of the skin and the fibrous thickening render palpation painful.

Prognosis is grave, because the effect of ring-bone is sometimes to render working animals useless.

Treatment. To relieve the diseased claw of pressure due to its bearing on the ground, the shoe should be removed and the claw freely pared.

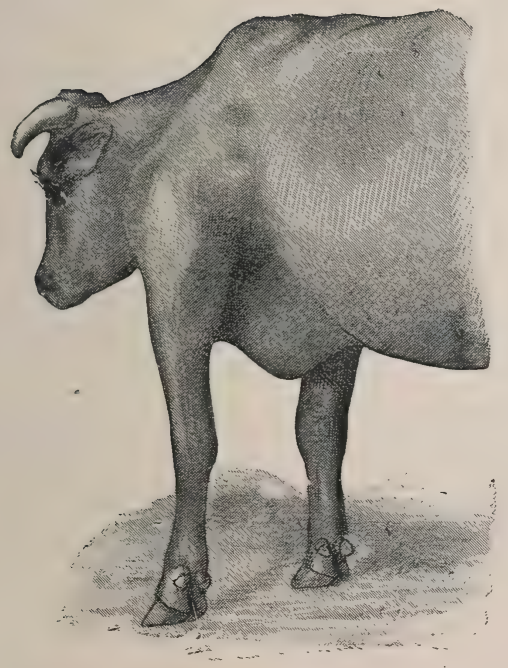

FIG. 14.-Sarcoma of the periosteum beneath the scapula.

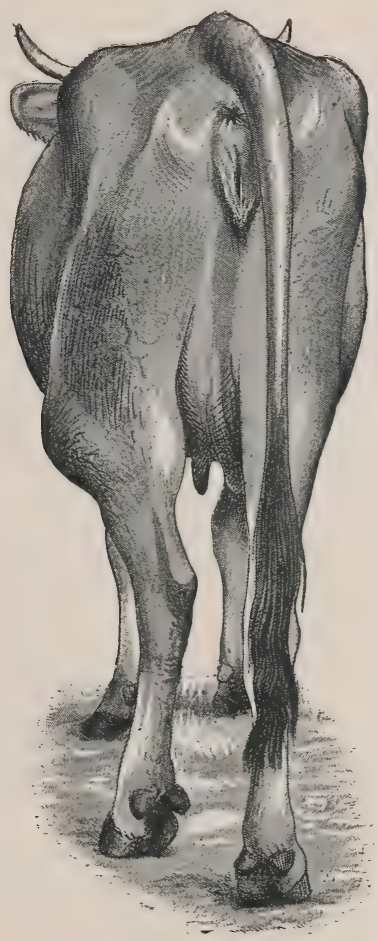

FIG. 15.-Sarcoma of the periosteum covering the upper end of the tibia.

If necessary, the healthy claw of the same foot may be raised by placing a piece of thick leather between the sole and the shoe. It is advisable at once to apply an energetic plaster, or, better still, to resort to firing in points.

\section{SUPPURATING OSTITIS.}

In addition to the changes in bone resulting from rachitis, osseous cachexia, tuberculosis, and actinomycosis, one sometimes sees cases of periostitis or ostitis pure and simple. As a result of external injury or 
direct wounds, the bone may be contused and injured, becoming the seat of diffused periostitis, necrosis, suppurating ostitis or osteomyelitis. Open fractures may produce the same results.

Treatment comprises disinfection of wounds, antiseptic injection of fistulæ, the application of antiseptic pencils, curettage, the removal of sequestra, and vesicant or resolvent complications. When such conditions extend to neighbouring joints and produce suppurative arthritis, the animals ought to be killed.

\section{BONE TUMOURS.}

The only bone tumours of real importance from a practical point of view are malignant growths represented by rapidly spreading epitheliomata or sarcomata, originating in the periosteum. Fortunately such tumours are rare.

They are not difficult to diagnose, as they develop rapidly, are accompanied by pain and lameness ending in diminution or loss of the power of movement, and frequently attack neighbouring lymphatic glands. Even when in good condition, animals lose flesh and appetite, and finally die of general wasting. The diagnosis is sufficiently guided by the deformity of the parts, the bosselated appearance of the tumours, the absence of fluctuation, the hæmorrhage which follows exploratory puncture, the character of the little fragments of tissue removed through these punctures, and finally the leukocytosis, which accompanies the development of malignant tumours.

The prognosis is grave, for it is usually difficult or out of the question to have recourse to removal, resection or amputation, when the tumours have acquired any considerable size. Success is impossible unless intervention is early, and the growth is in a readily accessible part. In other circumstances early slaughter is indicated. 


\section{CHAPTER II.}

\section{DISEASES OF THE FOOT. CONGESTION OF THE CLAWS.}

Congestion of the claws is not infrequently confused with contusion of the sole. It is, however, essentially different, and presents closer analogies with laminitis. The condition is characterised by congestion of the entire vascular system of the claw and principally of the velvety tissue. Like laminitis, it affects all four limbs; in rare cases the two front or two hind.

Congestion of the claw results almost exclusively from enforced movement on hard, dry and hot ground. It is commoner in animals unaccustomed to walking, and in heavy beasts which have been travelled considerable distances to attend fairs or markets. It is commonest in the bovine and porcine species, and less common in sheep.

The symptoms appear after animals return from a long journey by road. They are characterised by unwillingness to bear weight on the feet and difficulty in movement. Standing is painful, and the animals resist being moved; as soon as released they lie down.

Diagnosis presents no difficulty, though the condition is sometimes mistaken for slight laminitis.

Prognosis is favourable.

Absolute and prolonged rest is always followed by recovery, no internal medication being necessary, though this result is promoted by enveloping the claws in wet compresses or by using cold foot baths, etc.

\section{CONTUSIONS OF THE SOLE.}

Contusions of the sole are only seen in animals which work without shoes or in such as are badly shod.

Work on rocky ground, movement over newly metalled roads, and wounds produced by sharp stones, are the principal causes of contusion of the sole. Badly applied shoes, flat or slightly convex on their upper surfaces, may also produce bruising in the region of the sole. The anterior angle of the claw is rarely affected. 
Lameness is the first symptom to attract attention. It is slightly marked, unless the bruising has been overlooked until suppuration has set in. It affects only one or two limbs, and is rarely accompanied by general disturbance, such as loss of appetite, fever, exhaustion during work, etc.

Locally the claw or claws affected are abnormally sensitive to percussion of the wall, and particularly to compression of the sole.

The parts are hot to the hand, and thinning the sole with a knife shows little perforations, irregular points and crevices in the horn. One may also find softening, infiltration and hæmorrhage within the horn similar to those of corn in the horse, undermining of the sole over limited areas, and sometimes suppuration, if the animals have been forced to work when lame.

Complications like necrosis of the velvety tissue or of the bone, though comparatively common in the horse, are rare in oxen.

Diagnosis is not difficult provided the history of the ease is known. Confusion with laminitis is scarcely possible, for the gait of this lameness and the local symptoms are all different. Examination of the sole will usually dispel any remaining doubt.

Prognosis. The prognosis is favourable. When the horn is simply softened and a blackish liquid transudes, the lesion is trifling; if the discharge is reddish grey the lesion is graver, and implicates all the velvety tissue; finally, separation of the horn from the secreting membrane and the discharge of true pus point to death of the keratogenous tissue or of the bone.

Treatment should be commenced by carefully thinning the sole around the wound and applying moist antiseptic dressings or cold affusions. Removal of loose portions of horn hastens repair by allowing discharge, which has accumulated between the living tissues and the horn itself, to escape freely. The extirpation of necrotic tissue and the application of surgical dressings are only called for in specially grave cases.

This treatment usually gives good results. The acute complications which are so common and so dangerous in the horse seldom occur in the ox.

Most of these operations can be performed without casting, provided the animal is placed in a trevis or is sufficiently secured.

\section{LAMINITIS.}

Laminitis is characterised by congestion, followed by inflammation of the horn-secreting tissues of the foot. It is now rare in oxen and very seldom assumes an acute form. The slow pace at which animals of the bovine species move may sufficiently explain this rarity; 
nevertheless, prolonged travel on stony roads with heavy vehicles, rapid and repeated marches to towns or important fairs, are sufficient to produce attacks. Before the days of railways, and for some time after their introduction, in Britain cattle were travelled by road, and laminitis was common.

Long journeys in crowded railway trucks may also produce the disease, although the animal has not been forced to walk. Persons engaged in exhibiting cattle at shows are well aware of this. Prolonged maintenance of the standing position will produce the trouble, to which the joltings of the railway journey may also contribute their share. Prolonged standing on board ship may induce laminitis.

"Show condition" and the consumption of highly nitrogenous, and particularly of farinaceous, foods favour the occurrence of laminitis.

Breed is also considered to have some influence, and laminitis is said to occur more frequently in animals raised in flat districts, because in their case the space between the digits is larger than in mountain-bred cattle. In this connection the body weight may perhaps play a certain part.

The symptoms vary somewhat, depending on whether laminitis is general and affects all four feet, or restricted to the two front or the two hind feet.

The internal claws always seem more severely affected and more sensitive than the external. In very rare cases the animal remains standing, but usually it lies down, and will only rise under strong compulsion.

When standing, the symptoms are similar to those noted in the horse; the animal appears as though absolutely incapable of moving. If all four feet are affected the animal assumes a position as though just about to rise; if the front feet alone are affected the animal kneels in front whilst it stands on its hind legs, a very unusual position for the ox to assunce f finally, if the hind feet alone are affected, the animal seems to prefer a position with the feet under the body both in front and behind. (See Veterinarian, 1894, case by Bayley, and note by Nunn.)

It is always difficult to make the animal move. Walking seems painful, and most weight is thrown on the heels. The body swings from side to side as the limbs are advanced, and each limb is moved with a kind of general bodily effort.

The claws are hot, sensitive to the slightest touch, and painful on percussion.

Throughout the development of laminitis the general symptoms are very marked. The appetite falls off early, fever soon appears, and in grave cases the temperature rises to $105^{\circ}$ or to $106^{\circ}$ Fahr. Thirst is marked, and the animal seems to prefer cold drinks. The muzzle is dry, the face anxious and expressive of pain. Wasting is rapid.

'The ordinary termination is in resolution, which occurs between the D.C. 
eighth and fifteenth day, provided the patient has been. suitably treated. The disease rarely becomes chronic. On the other hand, the claw occasionally separates, as a consequence of hæmorrhage or suppuration, between the horn proper and its secreting membrane. Should this complication threaten, the pastern becomes greatly swollen, the extremities become intensely congested, and separation commences at the coronet. Loss of the claws, however, like suppuration, is rare.

Diagnosis. Congestion of the sole, the early stage of infectious rheumatism and osseous cachexia may, at certain periods of their development, be confused with laminitis; but the history and the method of development of the above-mentioned diseases always allow of easy differentiation.

It should, however, be added that, in certain exceptional conditions (suppurating echinococosis, producing chronic intoxication, tumours of the liver, and tumours of the pericardium and mediastinum), symptoms may be shown that suggest the existence of laminitis, although it is not really present. In these cases pain may possibly be felt in the bones of the extremities.

The prognosis is usually favourable, but necessarily depends on the intensity of the disease. Fat stock always suffer severely:

The treatment varies in no important particular from that prescribed for the horse, and is usually followed by rapid improvement. The chief indications are free bleeding from the jugular, the application of a mustard plaster over the chest, and the administration of a smart purgative (1 to $2 \mathrm{lbs}$. of sulphate of soda, according to the size of the animal) at first, followed by laxatives. This treatment may be completed by giving salicylate of soda per os in doses of 5 to 8 drams, or arecoline in subcutaneous injection, 1 to $1 \frac{1}{2}$ grains. Local treatment consists in cold affusions or poultices to the feet.

Failing cold baths, clay plasters applied to the feet are useful. To ensure success all these methods should be utilised simultaneously. In cases of separation of the claw, antiseptic dressings, with a thick pad of tow placed under the sole, become necessary.

Chronic laminitis may perhaps occur in the ox as in the horse, but, as a rule, oxen are slaughtered before the disease can assume this form. In dealing with fat, or even with fairly well-nourished, oxen it would clearly be more economical to slaughter early, and so prevent wasting and the resulting loss from disease.

\section{SAND CRACK.}

Sand crack-that is to say, the occurrence of vertical fissures in the wall of the claw-is not absolutely rare in bovines. It is commonest in 
working oxen drawing heavy loads, though in very exceptional cases it affects animals which have never worked. (Moussu describes one case in a young ox where four sand cracks existed simultaneously.) It may also result from injuries to the coronet. In contrast to the case of the horse, and owing to the different conditions under which the ox performs its work, the disease is commoner in front than in hind feet. In drawing, the ox's front limbs play the principal part, and the animal pivots, so to speak, on the claws of the front limbs.

The position of the crack may vary. It is commonest on the inner surface of the claw, rare at the toe, and still rarer at the quarter. It is often superficial and complete, extending throughout the entire height of the claw, but not throughout its thickness; sometimes it is complete and profound, the fissure then extending to the podophyllous tissue.

The symptoms are purely local in the case of superficial lesions. When the injury is deep seated, or when it originates in a wound of the coronet, lameness is present. Intense lameness, swelling of the coronet, and blood-stained or purulent discharge point to grave injury and probable complications.

Diagnosis is easy. The prognosis naturally varies with the symptoms. It is favourable when the fissure is merely superficial, but becomes grave when it is deep seated and the animal is exclusively used for heavy draught.

Treatment. When the lesion is superficial and unaccompanied by lameness, no surgical interference is necessary. Rest or very light work is alone required. As soon as lameness appears, rest is obligatory. The application of antiseptic poultices, containing $2 \frac{1}{2}$ to 3 per cent. of carbolic acid, creolin, etc., usually alleviates pain in a short time, and facilitates healing in the depth of the fissure.

In exceptional cases, where complications have occurred in consequence of suppuration beneath the fissure, suppuration in the coronary region, or necrosis of the podophyllous tissues, an operation becomes necessary, and is of exactly similar character to that performed under like circumstances in the horse.

Over a space of 1 to $1 \frac{1}{2}$ inches on either side of the fissure the horn is thinned "to the blood," and the subjacent dead tissue removed. The claw is then thoroughly cleansed with some antiseptic solution, the wound freely dusted with equal parts of iodoform, tannin and boric acid, and covered with pads of tow or cotton wool, fixed in position by appropriate bandages. After such operations a long rest is essential for complete recovery, during which, however, the animal may be fattened.

The object of operation is to prevent complications, like chronic 
suppuration and necrosis, which would endanger the animal's life, rather than to effect perfect restoration of usefulness for the work previously done.

\section{PRICKS AND STABS IN SHOEING.}

The wall of the ox's claw is so thin that shoeing is always somewhat difficult, more especially as nails can only be inserted in the external wall. Moreover, as very fine nails must be used, they are apt to bend, penetrate the podophyllous tissue, and cause injuries of varying importance. The ox is often very restless when being shod, and, even though firmly fixed, usually contrives to move the foot every time the nail is struck. The farrier, therefore, may easily overlook the injury which he has just caused, and by proceeding and ignoring it may transform a simple stab into a much more dangerous wound.

Symptoms. In most cases lameness appears immediately the animal leaves the trevis, but, although this is more difficult to explain, lameness is sometimes deferred until the day after, or even two days after, shoeing. Though little marked at first, lameness may become so severe that the animal cannot bear the pain caused by the foot touching the ground. When this stage is reached general disturbance becomes marked, fever sets in, rumination stops, and appetite is lost.

These symptoms point to the occurrence of suppuration. The pus, confined within the horny covering of the foot, causes very acute suffering and sometimes grave general disturbance; later it burrows in various directions, separating the podophyllous tissue from the horn, and ends by breaking through "between hair and hoof " in the region of the coronet. In exceptional cases, complications such as necrosis of the podophyllous tissue extending to the bone, and suppuration of its spongy tissue, may be observed.

Diagnosis. When the farrier suspects he has pricked an animal the immediate withdrawal of the nail will remove any doubt, because bleeding usually follows. If the condition is only detected at a later stage, the early lameness having been misinterpreted, examination of the claw and tapping the clenches of the nails will cause the animal to show pain at a given point, thus indicating the penetration of the nail. Removal of the offending nail is painful, and is often followed by discharge of pus or blood-stained fluid, which clearly points to the character of the injury. In obscure cases the shoe should not be reapplied.

When the horn wall is separated from the sensitive structures, there is marked general disturbance, and pus is discharging at the coronet, it is practically impossible to err in diagnosis.

Prognosis. In cases of simple nail puncture the prognosis is hopeful, provided that the condition is at once diagnosed. The longer it remains 
unrecognised, particularly if complication like necrosis has occurred, the graver becomes the outlook.

Treatment. In cases of simple puncture the nail should immediately be withdrawn and the animal placed on a perfectly clean bed to prevent the wound becoming soiled or infected. If lameness appear and become aggravated, the shoe should be removed and antiseptic poultices applied. In the majority of cases the lameness will then diminish, and in a few days completely disappear.

In cases of discovery within the first few days the same treatment is applicable, and is often sufficient. If, on the contrary, pus is discharging at the coronet, if lameness is intense and the general symptoms marked, it may be needful to operate.

The stages of operation comprise: thorough thinning of the horn in the shape of an inverted $\mathbf{V}$ over the affected portion of the wall, removal of the loose necrosed parts, disinfection of the wound, and the application of a surgical dressing covering the entire claw.

\section{PICKED-UP NAILS, Etc. ("GATHERED NAIL.")}

Penetrating wounds of the plantar region are, as in the horse, usually included under the heading of "Picked-up Nails." They are only seen in oxen or cows which are not shod. Pointed objects, like nails, harrow teeth, sharp fragments of wood or glass, etc., may produce injuries of the character of that now in question.

In considering the position of such wounds we may for convenience divide the plantar region into two zones, one extending from the toe of the claw to the point of insertion of the perforans tendon, the other comprising the region between this insertion and the bulb of the heel.

Symptoms. Lameness occurs immediately, and varies with the intensity of the existing pain. If the offending body has not remained fixed in the wound, this lameness may in a few moments disappear, either for good or merely for a time. The recurrence of lameness on the following day or a couple of days later marks the commencement of inflammatory changes in the deeper seated tissues. This lameness in many instances is accompanied by a movement suggestive of stringhalt, the foot being kept on the ground only for a very short time, or sometimes not being brought into contact with the ground at all.

The depth to which the offending object has penetrated, and the direction it has taken, may sometimes be discovered by a mere casual examination of the sole. In other cases only the orifice by which it has penetrated can be found. If the injury has existed for several days, the discharge from the puncture will be thin and blackish, purulent, or blood-stained, according to the case. Fever and general systemic disturbance suggest an injury of a grave character. 
Diagnosis. The diagnosis is easy, inasmuch as the lameness almost directs examination to the foot.

Prognosis is rarely grave. The direction, the situation and mode of insertion of the flexor tendon, which forms the plantar aponeurosis, ensure this aponeurosis being rarely injured by objects penetrating from without. The points of the offending bodies usually pass either forwards to the phalanx or backwards in the direction of the plantar cushion.

Treatment. The first stage in treatment consists in removing the foreign body and thoroughly thinning the neighbouring horn. An antiseptic poultice consisting of linseed meal saturated with 3 per cent. carbolic acid or creolin solution is then applied. Considerable and progressive improvement usually takes place in a few hours. If lameness persists, surgical interference becomes necessary; in the anterior zone it is confined to removing any dead portions of the velvety tissue and to extirpating the fragment of bone which has undergone necrosis. In the posterior zone the sinus must be probed and laid open, so that all the diseased parts can be treated as an open wound.

If, as happens in exceptional cases, the plantar aponeurosis is found to be severely injured, the complete operation for picked-up nail, as practised in the horse, may be performed, or the claw may be amputated. In the former operation the horn covering the sole is first thinned "to the blood."

The stages of operation are as follows:-

(1.) Ablation of the anterior portion of the plantar cushion. Transverse vertical incision at a distance of $1 \frac{1}{4}$ inches in front of the heel; excision of the anterior flap.

(2.) Transverse incision and ablation of the plantar aponeurosis by the same method.

(3.) Curettage of the point of implantation of the aponeurosis into the bone.

(4.) Antiseptic dressing of the claw.

Finally, if the primary lesion, wherever it may have started, has become complicated by arthritis of the interphalangeal joint, it will be necessary to remove the claw, or, better still, to remove the two last phalanges, the latter operation being easier than the former, and providing flaps of more regular shape and better adapted for the production of a satisfactory stump.

\section{INFLAMMATION OF THE INTERDIGITAL SPACE. (CONDYLOMATA.)}

Condylomata result from chronic inflammation of the skin covering the interdigital ligament. Any injury to this region causing even superficial damage may result in chronic inflammation of the skin 
and hypertrophy of the papillæ, the first stage in the production of condylomata.

Injuries produced by cords slipped into the interdigital space for the purpose of lifting the feet when shoeing working oxen are also fruitful causes.

Inflammation of the interdigital space is also a common complication of aphthous eruptions around the claws and in the space between them. Continual contact with litter, dung and urine favour infection of superficial or deep wounds, and by causing exuberant granulation lead to hypertrophy of the papillary layer of the skin. When the animal stands on the foot the claws separate under the pressure of the body weight and the condylomata are relieved of pressure. When, however, the limbs are rested, the claws mutually approach, compress the

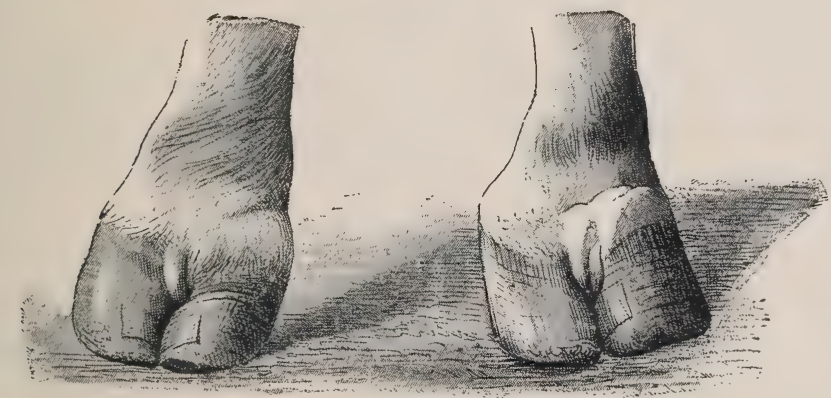

FIG. 16.-Condylomata of the interdigital space and sidebones,

abnormal vegetations, flatten, excoriate, and irritate them, thus favouring their further development.

The symptoms are easy to detect. The animals appear in perfect health, but have difficulty in walking, and show pain. They walk as though on sharp, rough ground, and lameness is sometimes severe. Locally, the anterior surface of the claws and the interdigital space are markedly congested and sensitive, or painful on pressure. The growths are of varying size, isolated or confluent, bleeding, excoriated, or covered with horn, and are visible between the claws when the animal stands on the limb. In many cases they form a perfect cast of the vertical interspace. When the superficial layers have undergone conversion into a horn-like material, lameness diminishes or disappears.

Diagnosis presents no difficulty.

Prognosis is only grave in so far as the condition interferes with animals working, but it may render working oxen entirely useless.

Treatment in the early stages is of a preventive character, and consists in placing animals which have been accidentally injured or attacked with foot-and-mouth disease on a perfectly clean bed. 
Surgical treatment is the only reliable method in cases where hypertrophy of the papillary layer is well marked, and is extremely simple.

The animal should be fixed in the trevis, the foot to be operated on separately secured, and the growths completely removed with sharp scissors or with a bistoury and forceps. When bleeding has subsided the wound is covered with a mixture of equal parts of iodoform, tannin, and powdered boric acid, and an interdigital dressing is applied. The dressing is removed after five to ten days, according to circumstances. If the cicatrix shows signs of exuberant growth it is dusted with powdered burnt alum, and the parts are treated as an open wound. When the growths are covered with horn and no longer painful it is not desirable to interfere with them.

\section{CANKER.}

Canker-i.e., chronic suppurative inflammation of the podophyllous or velvety tissue - is accompanied by hypertrophy of the papillæ and progressive separation of the horn of the sole. It is much rarer in the ox than in the horse, although it occasionally occurs.

Prolonged retention in dirty stables, where the bedding is mixed with manure and continually moistened with urine, is the principal cause of the disease. Individual predisposition and the action of some specific organism may also have some influence.

Canker in oxen, like the same disease in horses, is recognised by softening and separation of the horn of the sole, and by progressive extension of the process towards neighbouring parts. The usual course consists in invasion of the podophyllous tissue, separation of the wall and of the heels, and pathological hypertrophy of the horn-forming tissues, producing condylomata.

The new growths do not attain the same dimensions as in the horse, but, on the other hand, the disease very frequently takes a progressive course, involving the whole of the claw. A trifling accidental injury may be followed by infection of the subungual tissues, and thus become the point of origin for canker.

Canker may attack only one claw; on the other hand, it may extend to both claws of one foot, or to the claws of more than one foot in the same animal.

Diagnosis. Diagnosis is easy. The separation of the horn, the presence of a caseous, greyish-yellow and offensive discharge between the separated parts and the horn-secreting tissues, the appearance of the exposed living tissues, etc., leave no room for doubt.

Prognosis. The prognosis is grave; for, as in the horse, the disease is obstinate. 
Treatment consists in scrupulously removing all separated horn, so as fully to expose the tissues attacked by the disease. The parts should then be thoroughly disinfected with a liquid antiseptic, and a protective pressure dressing applied.

As a rule, cauterisation with nitric acid, followed by applications of tar or of mixtures of tannin and iodoform, iodoform and powdered burnt alum, etc., effect healing, without such free use of the knife as has been recommended in the horse during the last few years.

\section{GREASE.}

Grease in the ox seems only to have been described by Morot and Cadéac, and even in these cases the descriptions appear rather to apply to elephantiasis or fibrous thickening of the skin than to grease proper. The descriptions are not sufficiently clear, and the symptoms described differ too much from the classical type seen in the horse to convince us without further confirmation of the occurrence of the disease.

\section{PANARITIUM-FELON-WHITLOW.}

Any injury in the interdigital space or flexure of the pastern may, under unfavourable circumstances, be complicated by death of the skin, necrosis of the interdigital ligament, of the fibro-fatty cushion in the flexure of the pastern, and of the terminal portions of the tendons.

These lesions are sometimes regarded as panaritium. In reality, they correspond exactly to what, in the horse, are known as "cracked heels" and "quittor." The primary injury becomes infected with organisms which rapidly cause death of the skin or the formation of a deep-seated abscess and necrosis of the invaded tissues.

Causation. Neglect of sanitary precautions and filthy stables constitute favouring conditions, the feet being continually soiled and irritated by the manure and urine. Animals reared on plains, and having broad, flat, widely-separated claws, are more predisposed than animals from mountainous regions, in which the interdigital ligament is stronger and the separation of the claws less marked. Any injury, abrasion, or cut may serve as a point of origin for such complications.

Panaritium may even occur as an enzootic with all the characters noted in isolated cases. In Germany it has received the name of "contagious foot disease." These enzootic outbrealis of panaritium follow epizootics of foot-and-mouth disease, with lesions about the claws. Through the superficial aphthous lesions the parts become inoculated with bacteria, and the severity of the resulting injury is in some measure an indication of the virulence of the infecting organism. 
Symptoms. The first important symptom consists in intense local pain, rapidly followed by marked lameness. The affected region soon becomes swollen; the coronary band appears congested; the skin of the interdigital space projects both in front and behind; the claws are separated, and all the lower portion of the limb appears congested and oedematous. The engorgement usually extends as high as the fetlock, and the parts are hard and extremely sensitive. The patient is feverish, loses appetite, and commences to waste. After five to ten days sloughing occurs at some point--if the ligament is affected, in the interdigital space; if the tendons, or the fibro-fatty cushions, the slough appears in the flexure of the pastern. The dead tissue may separate and fall away, or remain in position macerated in pus. Separation is generally slow, requiring from twelve to fifteen days, and, unless precautions are taken, complications occur. If only the interdigital ligament or fibro-fatty cushion be necrotic, recovery may be hoped for; but, on the other hand, if the tendons, tendon sheaths, ligaments, or bones are affected, complications like suppurating synovitis, suppurating ostitis, arthritis, etc., supervene, with fatal results. Death may occur from purulent infection, unless the animal is slaughtered early.

The diagnosis is easy. The intensity of the lameness, separation of the claws, swelling of the pastern region, sensitiveness of the swollen parts, and absence of lesions in the ungual region sufficiently indicate the nature of the condition.

The prognosis is grave, for complications may result, in spite of proper treatment.

Treatment. Treatment consists, first of all, in thoroughly cleansing the affected limb and placing the animal on a very clean bed. The parts are next subjected to antiseptic baths containing carbolic acid, creolin, sulphate of zinc, or sulphate of copper. It is often more convenient, and quite as efficacious, to apply antiseptic poultices to the foot and pastern, and to allow them to remain for some days, being moistened several times daily with one of the solutions indicated. The effects are: rapid diminution of the pain, delimitation of the necrotic tissues is hastened, and the abscess is more readily opened.

Many practitioners recommend early intervention in the form of deep scarification in the interdigital space or pastern region. The local bleeding, and the drainage which takes place through the wounds so made, is said to hasten recovery or to prevent complications.

When the abscess has opened, and the dead tissue separated, the abscess cavity or wound should be regularly washed out with a disinfecting solution, to prevent complications, in case fragments of necrotic tissue have been retained. If, however, complications have occurred, no hesitation should be felt in freely incising the parts, and, if necessary, in 
removing one or both phalanges. When both joints of one foot are affected, and arthritis threatens to or has set in, there is no object in treating the animal, and early slaughter is to be recommended.

In cases where the disease follows foot-and-mouth disease, and threatens to become enzootic, it can generally be prevented spreading by keeping the foot-and-mouth subjects on very clean beds, and frequently washing the feet with antiseptic solutions. Disinfection of the sheds is also very desirable.

\section{FOOT ROT.}

Foot rot is a disease of sheep, and, like canker, is confined to the claws.

Thanks to the progress of hygiene, it tends to become rarer, but is still seen in the enzootic form in some portions of England and Scotland, in the mountains of Vivarais, the Cévennes, and the Pyrenees.

It affects large numbers of animals at once, animals belonging to one flock or to neighbouring flocks in one locality, and when it invades a sheep farm, all the animals may successively be attacked at intervals, according to the local conditions.

Symptoms. The disease develops rather insidiously, and the patients always retain an excellent appetite. It begins with lameness, which is at first slight, later becomes accentuated, and in the last periods is very intense. On examination, the coronet and lower part of the limb as high as the fetlock are found to be swollen. Palpation reveals exaggerated sensibility, and on direct examination, a fotid discharge is discovered in the interdigital space. This discharge, which is peculiar to the onset of the disease, only continues for a week or two, and is succeeded by a caseous exudate which is always offensive, which moistens and macerates the horn, the skin, the tissues in the interdigital space, and the region of the heels. From the 20th to the 30th day after onset the claw separates above in the interdigital space. The separation extends towards the heel, then to the toe, exposing ulceration of the subjacent podophyllous tissue.

From this time the patients experience very severe pain, and, as in other diseases of the feet, remain lying for long periods. Movement becomes extremely painful, and the animals frequently walk on the knees. The subungual lesions become aggravated, separation of the claw extends, necrosis of the podophyllous tissue and of subjacent tissue becomes more extensive, and the interphalangeal ligaments and the extensor or flexor tendons become involved. Finally, the claws are lost, and synovitis and arthritis are added to the complications already existing.

In an infected locality the development is always the same. The 
animals lose flesh, become anæmic, and, unless vigorously treated, soon die. The ordinary duration of the disease is from five to eight months, sometimes more. If, however, patients are isolated and well treated they recover.

Causation. The specific cause of foot rot still remains to be discovered, although everything points to the conclusion that it consists in an organism capable of cultivation in manure, litter, etc., for foot rot is transmissible by cohabitation, by mediate contagion through infected pasture, by direct contact and by inoculation.

The chief favouring influences are bad drainage, filthy condition of the folds, and herding in marshy localities.

Diagnosis. The condition can scarcely be mistaken, for the sheep suffers from no other disease resembling it, excepting, perhaps, foot-andmouth disease.

Prognosis. The prognosis is grave, for the disease usually assumes a chronic course, affects entire flocks, and the patients require individual attention.

Treatment. The primary essential to success in treatment consists in separating and isolating the diseased animals in a scrupulously clean place and providing a very dry bed.

In the early stages the disease may be checked by astringent and antiseptic foot baths. It is then sufficient to construct a foot-bath at the entrance to the fold, containing either milk of lime, 4 per cent. sulphate of iron, copper sulphate, creolin, etc. Through this the sheep are passed two or three times a week. These precautions rarely suffice when the feet are already extensively diseased; and when the horn is separated to any considerable extent, surgical treatment is indispensable. All loose portions of horn should be removed and antiseptic applications made to the parts.

When a large number of sheep are affected the treatment is very prolonged, but it is absolutely indispensable, and the numerous dressings required necessarily complicate the treatment. It would be valuable to experiment with small leggings, which would retain the dressings in position, and, at the same time, shelter the claws from the action of the litter, while favouring the prolonged action of the antiseptic.

When the lesions are not extensive, a daily dressing is sufficient.

Among the materials most strongly recommended are antiseptic and astringent ointments containing carbolic acid, iodoform, or camphor. Vaseline with 5 per cent. of iodine is very serviceable, and much to be preferred to applications like copper sulphate, iron sulphate, etc. Its greatest drawback is its expense. 


\section{CHAPTER III.}

\section{DISEASES OF THE SYNOVIAL MEMBRANES AND OF THE ARTICULATIONS.}

\section{I.-SYNOVIAL MEMBRANES AND ARTICULATIONS.}

\section{SYNOVITIS.}

Inflaniration of the synovial membranes, or synovitis, may affect the synovial sacs either of the joints or of the tendon sheaths. It may be acute or chronic and occur either idiopathically or follow the infliction of an injury. Its two chief forms are simple, or "closed," synovitis and suppurative, or " open," synovitis, the essential distinction between which is that in the latter micro-organisms are present, whilst in simple synovitis they are absent. In all cases the disease is characterised by distension of the sac affected.

Synovitis produced by a wound communicating with the outer air may be complicated by suppuration, and if the synovial membrane of a joint be involved the primary synovitis is almost always followed by traumatic arthritis.

The commonest forms of chronic simple synovitis are:-

\section{INFLAMMATION OF THE PATELLAR SYNOVIAL CAPSULE.}

Inflammation of the synovial membrane of the femoro-patellar joint is most commonly seen in working oxen as a consequence of strains during draught. It is also found in young animals which have injured the synovial capsule through falls, slips, or over-extension of the limb.

Symptoms. Development is slow and progressive, and injury may not be discovered until the lameness which follows has become fairly marked. This lesion is characterised by swelling in the region of the stifle. On palpation, fluctuation may readily be noted both on the outer and inner surfaces of the joint. The exudate is sometimes so abundant and distension so great that the straight ligaments, the neighbouring bony prominences, and the ends of the tendons are buried in the liquid swelling.

Lameness, which is at first marked, often diminishes with exercise. The length of the step is lessened. 
Diagnosis. The diagnosis presents no difficulty, but the lesions must be distinguished from those due to tuberculosis in this region, rheumatic arthritis, and the specific arthritis seen in milch cows.

The prognosis is grave, for the disease renders animals useless for work.

Treatment. Rest, cold moist applications, and massage constitute the best treatment in the early stages. Should swelling persist, one may afterwards apply a smart blister or even tap the joint aseptically, drawing off the fluid and then applying the actual cautery. Irritant injections must be avoided.

\section{DISTENSION OF THE SYNOVIAL CAPSULE OF THE HOCK JOINT.}

Bog Spavin in the $O x$.

Bog spavin is frequent in working oxen and in oxen from three to five years old. It is due to strain in draught or to strain produced in rearing up at the moment of covering. Old bulls, heavy of body, and stiff in their limbs are predisposed to it.

Symptoms. The symptoms usually develop gradually and without lameness, but sometimes declare themselves more rapidly with lameness, accompanied by marked sensitiveness on palpation. At first the hock shows a generalised doughy swelling, soon followed by dilatation of the articular synovial sac. Somewhat later four different swellings appeartwo in front, separated by the tendons of the common extensor and flexor metatarsi, and two at the back, extending inside and outside to the flexure of the hock.

Diagnosis. The only precaution required in diagnosis is to avoid confusion with articular rheumatism.

Prognosis. The prognosis is rather grave in the case of working oxen, and even of bulls; often slaughter is preferable to treatment.

Treatment differs in no respect from that of distension of the stiflejoint. In young bulls aseptic puncture and drainage of the joint, followed by the application of the actual cautery, probably give the best results.

\section{DISTENSION OF TENDON SHEATHS IN THE HOCK REGION.}

Like the preceding, this condition is rarely seen except in bulls and working oxen. It is characterised by dilatation of the upper portion of the tarsal sheath, one swelling appearing on the outer side, the other on the inner.

The differential diagnosis is based on the position of these synovial sacs, which are quite close to the insertion of the tendo-Achillis, and on the absence of any swelling in front of the joint. 
Treatment is identical with that indicated in the last condition.

Massage and cold water applications should be employed at first, to be followed by aseptic puncture and withdrawal of fluid, supplemented if necessary by firing in points.

\section{DISTENSION OF THE SYNOVIAL CAPSULE OF THE KNEE JOINT.}

This is one of the rarest conditions now under consideration, because the synovial membranes of the knee joint are everywhere strongly

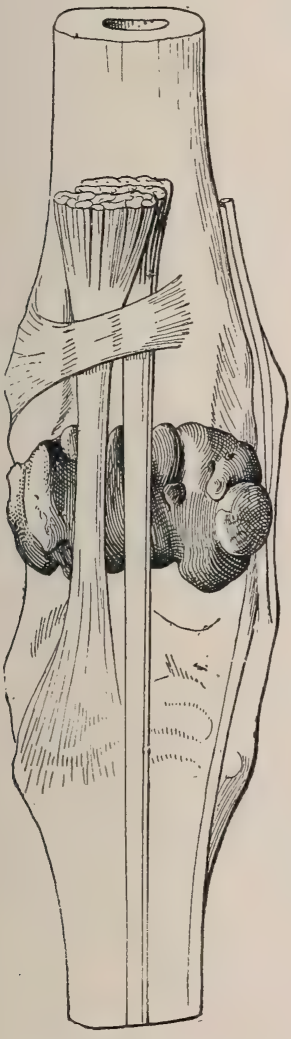

FIg. 17.-Front view of the ox's hock, showing the relations of the tendons and synovial sacs.

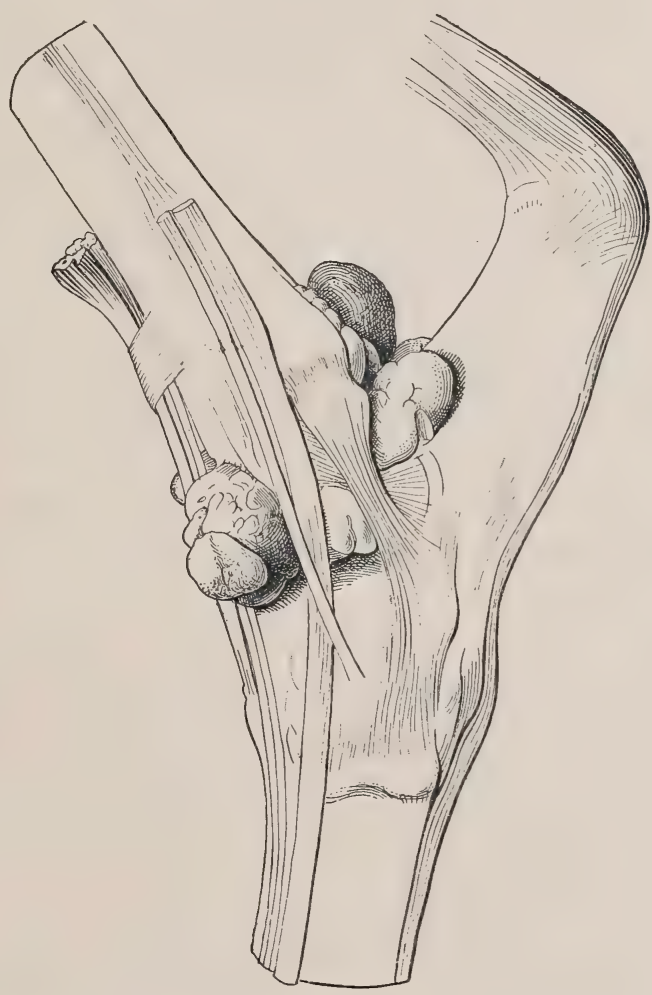

FIG. 18. - Side view of the ox's hock. The synovial sac of the true hock joint has been injected to show the relations of the sacs.

supported by very powerful ligaments. The synovial capsules of the carpo-metacarpal and inter-carpal joints are incapable of forming sacs of any size. On the other hand, the radio-carpal may become moderately prominent in front, especially towards the outside above the superior carpal ligament. When weight is placed on the limb, the excess of synovia is expelled from the joint cavity towards this little sac, which 
then becomes greatly distended. If, on the other hand, the knee is bent, the sac shrinks or disappears.

Treatment. Treatment is restricted to the application of a blister or to firing in points.

\section{DISTENSION OF THE SYNOVIAL CAPSULE OF THE FETLOCK JOINT.}

The synovial capsule of the fetlock joint in the ox is strongly supported in front and at the sides, but may protrude under the anterior ligament, producing a swelling behind the metacarpus under the five branches of division of the suspensory ligament and slightly below the sesamoid bones. These distensions, like bursal swellings, are commoner in hind limbs and in old working oxen. Their development is always followed in time by a certain degree of knuckling over. At first the metacarpus and phalanges come to form a straight line, but later the fetlock joint itself is thrust forward.

The diagnosis necessitates careful manual examination of the region of the fetlock joint.

The prognosis is somewhat grave, for the disease sooner or later necessitates the destruction of certain animals.

Treatment is practically identical with that used in all such conditions: friction with camphorated alcohol, cold affusions and massage in the earlier stages, followed if needful by blisters or firing in points.

\section{DISTENSION OF TENDON SHEATHS.}

Distension of the synovial capsule which surrounds the superior suspensory ligament, like distension of the articular capsule of the fetlock, occurs in working animals, and most commonly affects the front limbs. It is indicated by two swellings, one situated on either side of and behind the branches of division of the suspensory ligament and in front of the flexor tendons. These two swellings extend higher than the articular swellings, which, however, they sometimes accompany. The surface of the fetlock is then swollen, doughy on pressure, and somewhat painful.

These enlargements may produce more or less marked lameness and cause knuckling.

The diagnosis is clear from local examination.

The prognosis is unfavourable, as the animals after a time become useless for work.

Treatment. The beginning of the disease may often be cured by baths of running water, combined with massage. At a later stage, local stimulants, blisters, or firing are necessary. The best treatment 
probably consists in puncturing the parts with antiseptic precautions, washing out the synovial cavity with an antiseptic, and immediately afterwards lightly firing the surface of the region in points.

\section{DISTENSION OF TENDON SHEATHS IN THE REGION OF THE KNEE.}

Any of the numerous tendon sheaths which facilitate the gliding of tendons in the neighbourhood of the knee may become inflamed and give rise to a chronic synovial swelling. The commonest of such swellings is due to distension of the sheath of the extensor metacarpi magnus, which appears as a vertical line in front of the knee, extending from the lower third of the forearm and slightly to the outer side of the central line. This synovial enlargement arises in oxen working on broken roads, in clay or marshy soils, where the animals are liable to stick fast, and are often obliged to struggle vigorously in order to extricate themselves.

The diagnosis is based on the position and direction of the dilated synovial sheath.

Treatment is identical with that of other cases of chronic synovitis.

\section{DISTENSION OF THE BURSAL SHEATH OF THE FLEXOR TENDONS.}

This condition is rare. It is announced, as in the horse, by a dilatation of semi-conical form, the apex of which is situated opposite the lower margin of the carpal sheath, the base extending as high as the infero-posterior third of the radius.

The dilatation is more marked on the inner than on the outer side of the limb.

Distension of the synovial sheath of the common extensor of the digits in the fore limb and of the extensor of the external digit is still rarer than the preceding conditions.

\section{TRAUMATIC SYNOVITIS-" OPEN SYNOVITIS."}

When an injury in the neighbourhood of a joint penetrates deeply, it may implicate either the synovial sheath of a tendon or the synovial membrane of a joint. If the body inflicting the wound is aseptic, a condition which in accidental wounds is rare, the wound may have no grave consequences. Usually, however, the body producing the injury is infected, and the infection rapidly extends throughout the tendon sheath or synovial sac. In the first case, traumatic suppurating synovitis of a tendon sheath is the result; in the second, a suppurating articular synovitis arises, which soon becomes complicated with injury of the articular cartilages, ligaments, etc. (traumatic arthritis).

D.C. 
The primary lesion may only affect the periarticular region, not directly extending to the synovial membranes, and only after an interval of some days may symptoms of suppurating synovitis or suppurating arthritis appear, in consequence of progressive invasion of the parts by specially virulent microbes.

\section{TRAUMATIC TENDINOUS SYNOVITIS.}

Suppurative inflammation of the synovial bursæ of tendons in consequence of wounds most commonly affects the sesamoid sheaths of the front or hind limbs; more rarely, the tendon sheaths of the hock or knee; and, exceptionally, the small synovial sheaths of the extensors of the metacarpus and phalanges, etc.

Such inflammation follows injuries with forks, harrow teeth, or any sharp foreign body. It is characterised by the existence of a fistula or wound, indicating the course taken by the body inflicting the injury, from which at first normal synovia escapes. Later, however, the discharge becomes turbid, and after the second day gives place to a clotted, serous, or purulent fluid.

A diffuse, œdematous, warm, painful swelling very rapidly develops around the injury. The animal is more or less feverish and lame. The swelling soon extends throughout the entire length of the infected synovial sheath. The patient loses appetite, and unless treatment is promptly undertaken, complications supervene which often necessitate slaughter. The prognosis is always grave.

Treatment. Continuous irrigation has long been recommended. It is worthy of trial, but in the majority of cases occurring in current practice it cannot be carried out.

Moussu prefers a form of treatment which he claims has always succeeded in horses and oxen-viz., irrigation of the parts, followed by injection of sublimate glycerine solution.

He first washes out the infected synovial cavity with boiled water cooled to $100^{\circ}$ Fahr. A counter-opening may become necessary, and the washing should be continued until the escaping water appears perfectly clear. Immediately after each such irrigation he injects from 7 to 14 drams of glycerine containing 1 part in 1,000 of corrosive sublimate. He repeats this treatment daily.

By reason of its affinity for water and for the liquids in the tissues or suppurating cavities into which it is injected, the glycerine penetrates in all directions, reaching the finest ramifications of the synovial sacs, a fact which explains its superiority over aqueous antiseptic solutions.

Suppuration is rapidly checked and repair becomes regular. The pain and lameness progressively diminish, and recovery may be complete. 
It is advisable to assist this internal antiseptic treatment by external stimulants and by the use of a blister. Solutions of greater strength than 1 part of sublimate to 500 of glycerine are only required during the first few days of treatment and until suppuration diminishes. Later, they prove irritant, and interfere with healing.

\section{TRAUMATIC ARTICULAR SYNOVITIS-TRAUMATIC ARTHRITIS- "OPEN ARTHRITIS,"}

It has been described above how primary inflammation of the articular synovial membrane produced by a wound may rapidly develop into suppurating arthritis.

Symptoms. The pain is very marked at the moment when the accident occurs, but this pain, due to the mechanical injury inflicted, diminishes or completely disappears after some hours. Soon, however, synovial discharge sets in, announcing the onset of traumatic synovitis. At first limpid, it soon becomes turbid, then curdled, and finally grumous, purulent and greyish in colour.

Pain then returns, rapidly becomes intense, continuous and lancinating. It produces lameness, sometimes so severe that no weight whatever can be borne on the limb. A diffuse, œdematous, warm and extremely sensitive swelling then rapidly develops around the whole of the injured joint.

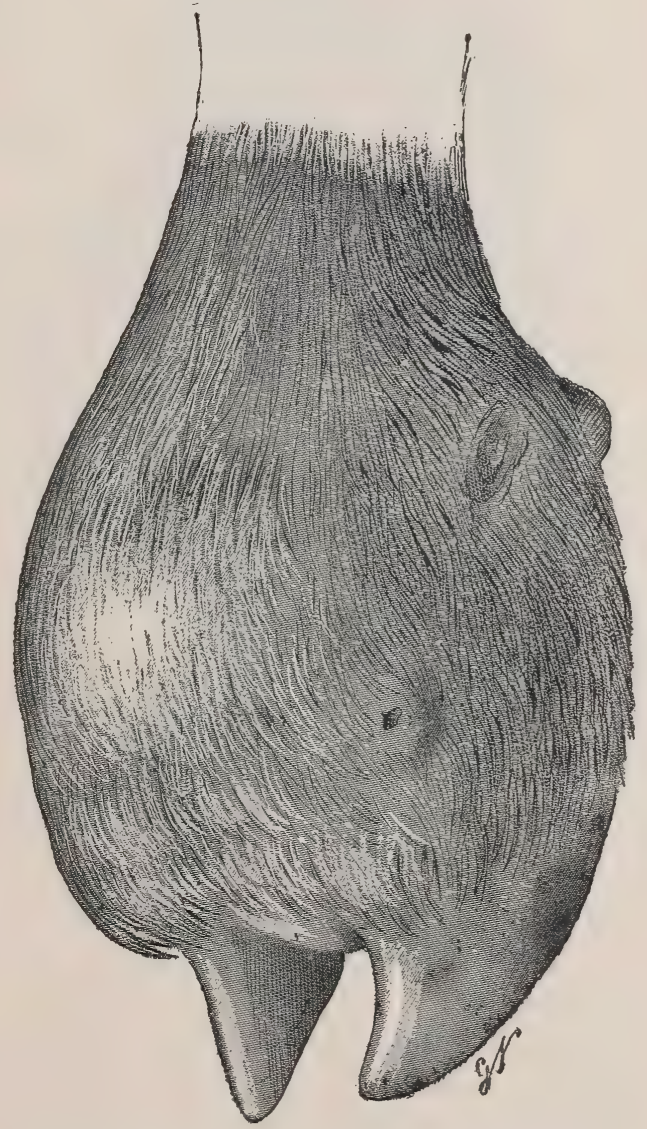

FIG. 19.-Suppurative arthritis of the fetlock. General disturbance, with fever and loss of appetite, appears, indicating a very alarming condition.

It is sometimes a little difficult to differentiate between this condition and that due to injury of a tendon sheath, but as a rule diagnosis is easy.

Prognosis is very grave. Life is threatened, and wasting occurs very 
rapidly. Infection of the synovial membrane is soon followed by necrosis and erosion of the articular cartilages, as well as by softening and inflammatory change in the ligaments, etc.

Treatment. Should the patient be in reasonably good condition, but otherwise of no particular value, it should at once be slaughtered. If, on the other hand, it possess a special value, and the owner wish to preserve

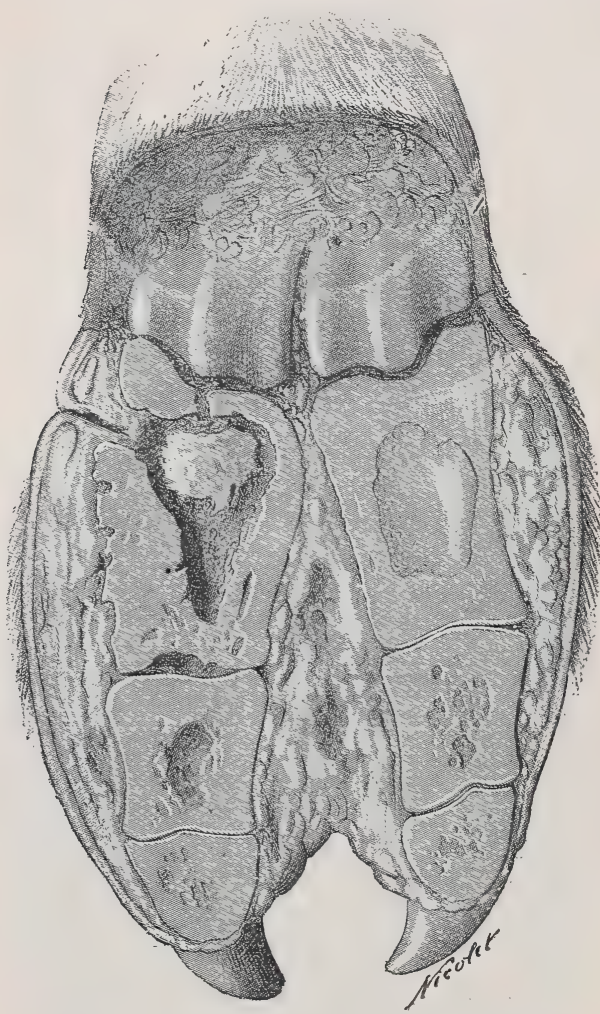

FIG. 20.-Suppurative arthritis following traumatic osteo-periostitis. its life, the same antiseptic treatment should be adopted as was indicated in dealing with suppurating synovitis of tendon sheaths. The loss of the articular cartilage of the joint is followed by free granulation and union of the apposed surfaces of bone resulting in anchylosis of the joint and qualified recovery. Such results, however, are only likely to follow in relatively light animals which are capable of standing for considerable periods.

\section{II.-STRÄINS OF JOINTS.}

When, in consequence of muscular contraction or external violence, the bony surfaces constituting a joint are displaced so as to stretch, lacerate, or partially tear the ligaments, synovial capsule or tendons supporting the joint, so-called sprain, or strain, or

wrench results. The articular surfaces are not sufficiently displaced to cause luxation, but a series of periarticular injuries result, and are followed by the symptoms which we recognise as those of strain. Joints in which movement is restricted within narrow limits, such as the hock or fetlock, are most liable to such injuries, which also occur in very freely movable articulations like the shoulder and stifle.

\section{STRAIN OF THE SHOULDER.}

This accident is produced by the animal falling on its side, by slipping at the moment of landing, the limb being extended and in contact with 
the ground, by the foot slipping at the moment when it is leaving the ground and the limb is at its extreme limit of backward extension, by side slips, etc. The periarticular lesions will then be found on the front, back, or internal surface of the articulation, depending on the manner in which the accident has occurred. According to some authors, violent muscular efforts, as well as work in soft clay soil, bogs and rice swamps, are also capable of causing strain of the shoulder.

Symptoms. At first moving appears difficult, the patient lies down a great deal, then lameness becomes characteristic. Novement of the scapulo-humeral joint being painful, the patient endeavours as far as possible to aroid it; the limb is advanced stiffly in an abducted position and with a mowing movement. This semi-circular movement avoids the necessity of flexing the scapulo-humeral angle, but diminishes the length of the stride.

Locally, the region of the scapulo-humeral angle is swollen, doughy, and extremely sensitive on manual examination. If the practitioner endeavours to flex it by lifting the limb the animal flinches.

Diagnosis. The diagnosis is not very difficult, although the peculiar mowing movement is also seen in other cases, such as cracked heels and mallenders.

Prognosis. The prognosis is favourable, for, provided there has been only a moderate strain of the tendons or isolated injury to the muscles, recovery is almost certain.

Treatment. Treatment consists in resting the animal and in assisting repair.

The parts can be immobilised by the application of a vesicant or mild blister. A week or ten days later dry friction, muscular and periarticular massage are indicated, and gentle exercise should be commenced. The same treatment may be employed in strain of the elbow joint and in strains of muscles or tendons in the neighbourhood of joints. Such lesions are, however, very rare in the ox.

\section{STRAIN OF T'HE KNEE.}

Strain of the knee is commoner in the ox than in the horse, because of the special conformation of the knee in the ox and the mode of working under a yoke. 'This mode of working gives less individual liberty to the fore quarters, and sometimes interferes with the animal's efforts when moving a load. Strains of ligaments and periarticular injuries occur as a general rule on the inner side of the limb.

The symptoms consist in lameness, exaggerated sensibility on pressure, pain on forced flexion of the knee, and swelling of the entire periarticular region.

The prognosis is somewhat serious in working oxen. 
Treatment, when applicable, should consist of stimulant dressings, massage, and douches.

\section{STRAIN OF THE FETLOCK.}

This is one of the commonest accidents of draught oxen.

Causation. Working on rough, irregular, rocky ground, or on roads with deep ruts, is the commonest cause of this condition. When the animal moves on irregular surfaces the two claws do not bear an equal share of weight; sometimes the whole weight is for a moment thrown on one claw.

The phalanges, therefore, are displaced inwards or outwards, or are twisted around their vertical axis, causing the fetlock joint and its supporting ligaments to be more or less severely strained. The internal or external ligaments of the joint or the suspensory ligament or flexor tendons may even be lacerated.

The fetlock may also be strained by the animal making violent efforts to free the claws or pastern which have become fixed in a hole in the ground, in bogging, in hobbling, or in leaping a fence.

Symptoms. Lameness is noticeable from the beginning, but is unaccompanied by any visible lesion. On examination of the limb, the entire region from the fetlock downwards is found to be sensitive to pressure, and painful when forcibly extended or flexed from side to side. This sensitiveness is particularly marked when the phalanges are rotated on the shank. A few days later the entire fetlock becomes the seat of diffused swelling.

Diagnosis is facilitated by the fact that the fetlock has an entirely different appearance from that seen when tendon sheaths or the synovial capsules of joints are distended. •

Prognosis. The prognosis varies considerably, according to the extent to which deep-seated structures are involved, and the gravity of the lesions is usually proportioned to the intensity of the symptoms.

Treatment. Frequent cold applications, cold foot baths for an hour or two night and morning, and even cold poultices are useful. When the pain has somewhat diminished, which usually occurs in from three to four days, vesicants may be employed, and, at a later stage, massage. Failing improvement by these methods, the injured region may be fired in points.

Strain of the hind fetlock occurs under precisely similar conditions to those above described in the case of front limbs.

\section{STRAIN OF THE STIFLE JOINT.}

Strain of the stifle joint results from over-extension of ligaments without displacement of the patella, and also (and probably more 
frequently) from injuries to the aponeurosis and tendons of insertion of the abductor muscles of the femur and tibia.

Causation. Strain of the stifle may follow violent collisions, such as occur in entering or leaving the stable, from falls on rough ground, from direct blows, from slips, etc., or even from the sudden and violent contraction of the muscles of the antero-external surface of the quarter.

Symptoms. Lameness follows immediately or soon after the accident, and is of a peculiar character; to avoid using the injured joint, the animal advances the hind limb with a mowing movement.

The injured region exhibits diffuse inflammatory swelling, which impedes palpation, and makes it difficult to determine the exact nature of the local lesion. The thigh at the stifle is painful.

Diagnosis and prognosis, Diagnosis presents no considerable diffculty. The prognosis must be based on the intensity of the symptoms, and becomes grave if the tendons or aponeurotic insertions be injured, or extensive damage have been done to ligaments.

Treatment. At first, continued cold applications, douches, and massage are most useful ; blisters are more effective in the grave cases, and comprise not only the ordinary blisters, but the powerful mixed blister containing tartar emetic, powdered hellebore, bichromate of potash, etc. If for any special reason it is of importance to preserve the animal's life, the parts may be fired; but for economic reasons it is usually better to fatten it for the butcher.

\section{STRAIN OF THE HOCK JOINT.}

Causation. Strain of the hock joint is commonest in young oxen which are being trained to work. Their hind quarters are necessarily more or less free, and the animals are apt to make side movements to avoid the goad, thus exposing the hock to irregular strain.

The internal ligaments are more frequently strained than the external, a fact due to the conformation of the hocks.

Symptoms. Strain is accompanied by lameness, most marked when the animals endeavour to turn, by exaggerated sensibility of the entire hock region, and, in grave cases, by subcutaneous oedema.

Diagnosis and prognosis. Diagnosis is simple. The prognosis is sometimes grave, because a spavin or a permanent chronic enlargement of the capsule of the true hock joint may form.

Treatment. It is often desirable at once to apply an extensive blister over the whole hock and to supplement this at a later stage by cold applications, or by continuous cold irrigation for half an hour or more both night and morning.

In exceptional cases the use of the actual cautery becomes necessary. 


\section{III.-LUXATION OF JOINTS.}

Luxation consists of permanent displacement of the bony surfaces forming a joint, and may follow violent mechanical injury or any other cause.

Luxations have been divided into congenital, i.e., such as exist from the time of birth; spontaneous, i.e., those which result from some defect of conformation or constitution; and acquired or accidental, which occur as results of falls, wounds, accidents, etc.

From the point of view of their duration, luxations are termed temporary when they do not necessitate reduction, progressive when the tendency is towards greater and greater displacement of the surfaces, or permanent when reduction is impossible.

Those commonest in bovines are luxations of the femur, luxation of the patella, femoro-tibial luxation, and luxation of the scapulo-humeral joint.

\section{LUXATION OF THE FEMUR.}

Luxation of the head of the femur with displacement beyond the cotyloid cavity is very frequently congenital. The condition also occurs with some frequency in adults or aged animals in consequence of relaxation of the articular ligaments and the absence of the subpubic ligament (pubio-femoral ligament).

Causation. Luxation may be congenital, the head of the femur being displaced backwards and carried above the cotyloid cavity. This form is of no practical interest, because the animals are not usually reared. More frequently in young or adult animals it assumes the spontaneous progressive form, in consequence of degenerative changes in and relaxation of the coxo-femoral interosseous ligament. The head of the femur presses on the upper margin of the cotyloid cavity, which it injures, and eventually becomes lodged in the neighbourhood of the neck of the ilium, in the great sciatic notch.

This luxation is also found as a purely accidental occurrence in animals which have suffered from falls and from slipping of the hind limbs backwards or sidewards, as occasionally follows awkward leaping movements. The slipping outwards of the limbs, which causes this form of luxation, is comparatively easy, because of the absence of the pubiofemoral ligament. The accident may also be followed merely by subluxation, that is to say, tearing of the inner portion of the capsular ligament and rupture of some portion of the adductor muscles of the thigh without rupture of the interosseous fibres, the head of the femur not quitting the cotyloid cavity. This accident occurs in stables with smooth, slippery floors, and in railway trucks. It may affect one side or both. The latter condition is exceptional. 
Finally, luxation may be either complete (in which case the capsular and interosseous ligaments are both ruptured) or incomplete. In the former case, the head of the femur becomes displaced uprrards and forwards towards the great sciatic notch, more rarely backwards in the direction of the ischium, and in exceptional cases downmards and inwards below the pubis into the foramen ovale.

Symptoms. The symptoms vary, depending on whether the luxation is of the spontaneous, progressive order or, on the contrary, is accidental. In progressive luxation, the animals are able to rise and walk with difficulty. The affected limb swings when the animal is advancing, not as though it rere paralysed, but simply as though displaced at its upper

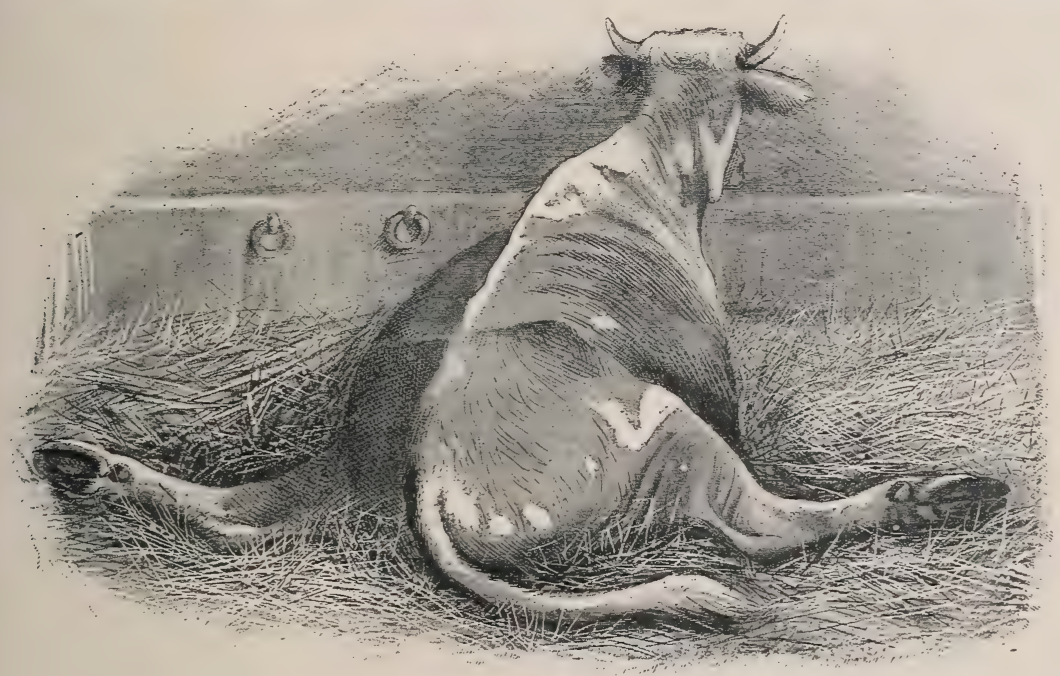

FIG. 21.-Accidental luxation of the hip joint.

part. Pain is exhibited when weight is placed on the limb, and there is difficulty in movement. The limb appears shorter than its neighbour when the animal stands on it, and the prominence representing the trochanter is more marked. When a false joint has formed, the limb is rigid, is moved stiffly and abducted, and the stride is shortened.

In accidental luxations, either of one or both limbs, the attitude assumed by the animal is often characteristic. One of the limbs is extended at right angles to the longitudinal axis of the body, and sometimes both limbs assume this position, an attitude which would be absolutely impossible under normal conditions. The animal cannot rise. It lifts the front part of the body by rising on its knees, but the hind quarters do not follow. The ruptured adductor muscles are unable to bring and hold the limb parallel to the longitudinal axis of the body; the abductors act unopposed, and at the moment when the animal makes 
its greatest effort to rise the limb (or limbs) is carried outwards and the body comes to the ground on the udder or groin.

Inspection discovers a depression where the great trochanter should be. On applying the open hand over the hip joint, whilst an assistant who grasps the pastern moves the limb in different directions, one can feel and hear, in spite of the mass of muscle covering the part, a modified crepitation due to sero-sanguinolent effusion in and around the joint. This crepitation can also be detected by pushing the hand as high up as possible on the internal surface of the thigh, and thus exploring the neighbourhood of the luxation.

Diagnosis. The position which the animal assumes when one endeavours to make it rise is characteristic. Diagnosis of progressive luxation, however, is more difficult.

Prognosis. The prognosis is extremely grave; for, even though reduction can be effected, one is practically unable to ensure that the parts shall remain in position.

Treatment. Treatment comprises reduction of the luxation and fixation of the parts. Reduction can be effected without very much difficulty by casting the animal on the affected side, placing a block of wood, or a pail, between the thighs, and manœuvring the limb in the desired direction. As, however, subluxation is the condition most commonly seen, there is frequently no reduction to effect. Moreover, fixation of the joint after reduction is almost impossible in such heavy subjects, and luxation is very liable to recur. From an economic standpoint, slaughter is advisable or imperative.

\section{LUXATION OF THE PATELLA.}

Luxation of the patella is not uncommon in working oxen, and is occasionally seen in cows as the result of a slip when entering or leaving the byre, the reason being the peculiar anatomical arrangement of the femoro-patellar articulation.

The internal lip of the trochlea of the femur is very high, the external, on the other hand, being only slightly developed. The patella itself is smal'er than that of the horse, and possesses a small cartilaginous prolongation, principally on its inner face, in marked contrast with the large cartilage found in the horse. As in the latter animal, the outward displacement of the patella is only prevented by the internal patellar ligament and the femoro-patellar aponeurosis. Under the influence of varying causes to which the joint is exposed, these supporting structures often prove insufficient to prevent the patella being displaced outwardly.

Causation. From an anatomical standpoint, luxation inwards seems impossible. It certainly must be very rare and be preceded by rupture of the external ligament. On the other hand, as in the horse, it seems 
possible that the patella may be caught on the summit of the internal lip of the trochlea, especially if the trochlea happens to present a flattening: at that point.

Outward luxation may be spontaneous or accidental. It is termed spontaneous, when produced by relaxation of the ligaments of attachment or by irregular muscular action; accidental, when resulting directly from any external mechanical cause. Violent contraction of the triceps cruralis, by lifting the patella beyond its normal limit of travel, helps, or at least permits, the patella to be displaced outwardly at the moment when the muscle relaxes.

Pathological relaxation of the ligaments and muscles, by allowing the patella to descend too far on the trochlea, also renders displacement possible, hence spontaneous luxation sometimes occurs even while the animal is at rest in the stable. This luxation is certainly only of a temporary character, or perhaps only of the nature of subluxation, and is often reduced by mere muscular contraction when the animals are forced to move.

Should the hind limb slip in a backward direction the angle of the joint becomes more obtuse and the lips of the trochlea are turned downwards, thus greatly favouring lateral displacement of the patella, which under these circumstances is no longer immobilised on the trochlear pulley; displacement outwards then occurs, constituting the condition termed luxation.

Various forms of mechanical violence, like blows, collisions of the stifle with the jambs of doors, falls, etc., may also bring about this luxation.

The symptoms of fully-developed accidental luxation are characteristic. Immediately the accident occurs, the limb is immobilised in a state of complete extension; neither the stifle nor the hock joint can be flexed, and only the fetlock joint retains any degree of mobility.

Movement is very difficult. The hind limb appears rigid, as though formed of one bone. The pastern is directed backwards and dragged along the ground, and when weight is placed on the limb the anterior surface of the pastern may almost be in touch with the ground. The limb is advanced, but the foot cannot be placed properly on the ground.

Locally the patella is found to be outside the external lip of the trochlea, and its internal ligaments are extremely tense.

If the luxation is spontaneous and of muscular origin, or a consequence of relaxation of the tendons, it is usually noticeable immediately the animal leaves the stall. The animal cannot move without great difficulty. It grows steadily, worse with the lapse of time, because the synovial membrane becomes irritated and chronic arthritis is set up.

The symptoms are identical with those of traumatic luxation, but are only temporary. 
Diagnosis. The position of the limb and the displacement of the patella are sufficiently distinctive to render diagnosis fairly easy, and to allow of this accident being differentiated from luxation of the femorotibial joint or hooking of the patella in the ischio-tibial muscle.

Prognosis. The prognosis varies greatly. If the luxation is of traumatic origin and the accompanying symptoms are not grave, reduction may be followed by permanent recovery. On the other hand, in spontaneous luxation recurrence is almost inevitable.

Treatment. The indications for treatment may be comprised in a single phrase: reduction, with immobilisation of the parts for a sufficient time. To effect reduction, a strip of webbing is fixed around the pastern of the affected limb, passed over the withers, in front of the shoulder of the opposite side, and brought round in front of the neck or between the front limbs. By means of this an assistant exercises strong traction on the limb until the fetlock is raised as high as the elbow of the same side. The operator then applies strong inward pressure to the

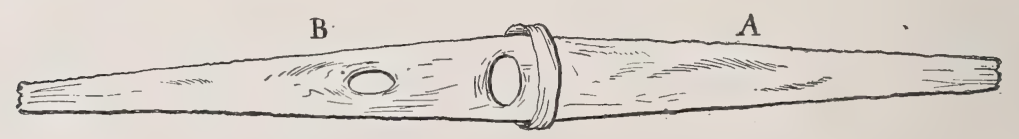

FIG. 22.-Bandage for luxation of the patella.

patella, which usually slips back on to the gliding surface of the trochlea at the first or second effort.

After-treatment comprises the application of a smart blister, producing cedematous infiltration of all the tissues around the joint, and thus impeding movement and recurrence of luxation. For this purpose various preparations are used-e.g., cantharides, biniodide of mercury, croton oil, etc. It is also advisable to fix the animal so that for a time it cannot lie down, and to secure the pastern to the neck by means of a side-line.

As an experiment, cases of simple fixation of the patella on the summit of the internal lip of the trochlea might be treated by Bassi's methodi.e., subcutaneous division of the internal lateral ligament of the patella which holds the bone in its abnormal position.

Finally, in spontaneous luxation, occurring in young animals in which blisters have been ineffectually tried after reduction, Bénard's bandage may be used, though it is not generally regarded as very practical. It consists of a piece of cloth of elongated lozenge form about four feet in length, six inches in breadth at its centre and two inches at its ends. Its centre is pierced by a transverse opening intended to surround the patella, and carries a loop for the purpose of supporting the turns of bandage. A second longitudinal opening is situated about eight inches from the centre. 
Reduction being effected, the whole region of the stifle joint is covered with Burgundy or ordinary pitch and the bandage then applied. The patella projects through the central opening. The end $\mathrm{A}$ is passed backwards around the thigh, and through the aperture B; the two cross ends are then brought forward, crossed again at the end, the loop over the patella carried a second time backwards, again crossed, and finally fixed in front under the patella. The bandage should be firmly applied, without, however, being so tight as to interfere with circulation, and must be left in place from eight to ten days.

Van Denmoegdenberg recommends placing the patient on an inclined plane, with the hind quarters a foot higher than the front, so as to cause permanent contraction of the anterior muscles of the quarter, and thus

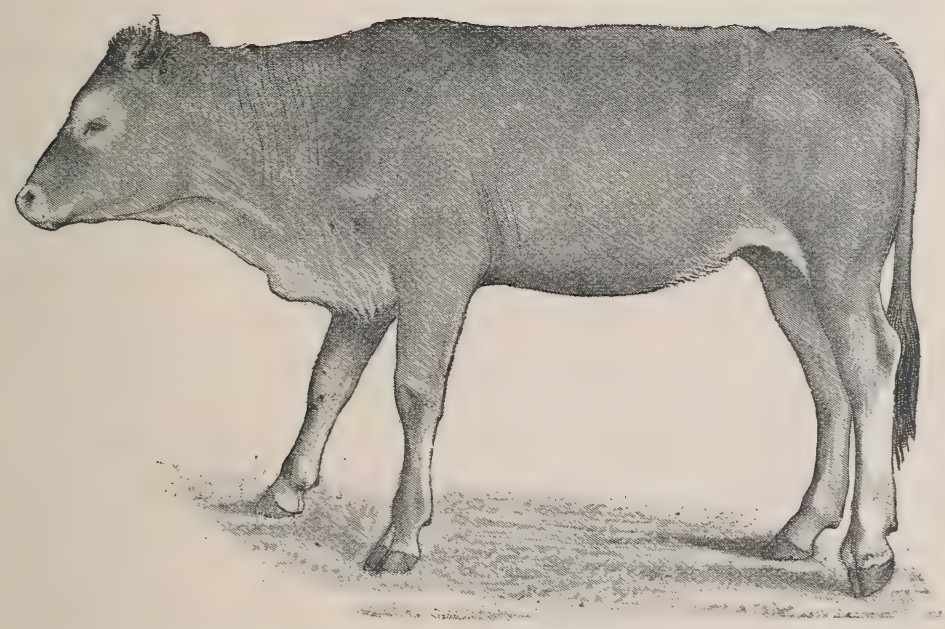

Fig. 23.-Backward luxation of the femoro-tibial joint. (From a photograph by Professor Besnoit.)

immobilise the patella. Simple cold baths, frequently repeated, friction with camphorated alcohol or essence of turpentine, complete this original but somewhat questionable treatment, and are said to result in recovery in a fortnight.

\section{LUXATION OF THE FEMORO-TIBIAL ARTICULATION.}

This form of luxation is rare, a fact explained by the strength of the lateral ligaments of the joint, and of the cruciform interosseus ligaments. It may assume different forms, according as the head of the tibia is displaced in front of, behind, to the inside, or to the outside of the lower . extremity of the femur. In all, therefore, it may appear in four different forms. The commonest is backward luxation. 
Causation. With the sole exception of luxations or subluxations due to tuberculosis (lateral luxations, either inwardly or outwardly, occurring during tubercular arthritis, with more or less marked destruction of the condyles), these luxations are always accidental or the result of mechanical violence.

They result from leaping into hollows, falling into deep ditches or ravines, or galloping through broken or steep places. Any violent shock affecting the femur, either in front or from the outside, is capable of causing luxation backwards or inwards.

Symptoms. The most frequent condition is backward luxation of the upper extremity of the tibia. Movement becomes difficult, the limb

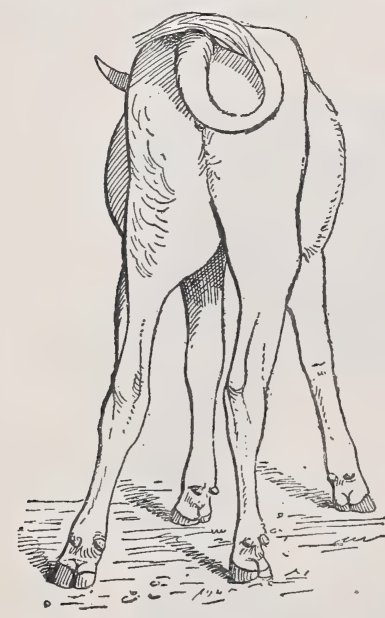

FIG. 24.-Luxation backwards and inwards of the femoro-tibial joint. (From a photograph by Professor Besnoit.) is held rigidly, and all the lower part of the leg is extended. None of the joints can be flexed. The leg is dragged forward, without the animal being able to place the foot flat on the ground, and the claws are trailed over the litter or the toe grazes the soil.

On local examination the stifle is seen to be deformed. The lower extremity of the femur and the patella appear prominent. The upper part of the tibia is thrust backwards, and seems to have disappeared, leaving a depression below the femoro-patellar region. The muscles forming the back of the thigh at this level are thrust out of position, and appear to project abnormally.

Viewed from behind, the inner line of the thigh appears more or less convex when the upper extremity of the tibia is luxated inwardly. On local examination the displacement of the bones can be readily detected. In luxation forwards the prominence of the stifle is caused by the summit of the tibial crest and by the patella, whilst the lower extremity of the femur cannot be felt. In outward luxation the upper extremity of the tibia forms an abnormal prominence, above which a horizontal digital depression appears.

Diagnosis. Provided that the examination is made soon after the occurrence of the accident, little difficulty will be found in coming to a conclusion, but the diagnosis necessitates more care when examination is deferred for two or three days, because extensive effusion then exists. Luxations or subluxations of tuberculous origin are generally consecutive to old-standing destructive tuberculous arthritis.

The prognosis is grave-firstly, because reduction is difficult; and, 
secondly, because it is often impossible to maintain the reduction and to preserve complete immobilisation of the injured joint.

Treatment should not be undertaken except in young animals which have not yet attained full development.

In attempting to reduce outrard or inmard luxation of the tibia the animal should be cast on the side opposite to the lesion. Counterextension is practised by passing a length of webbing around the limb in the region of the groin; extension in the direction of the length of the femur by means of a loop of webbing fixed to the cannon bone: the operator uses both hands in endearouring to replace the head of the tibia.

In forward luxation of the tibia counter-extension is effected by means of a loop of webbing passed above the hock and dramn forward. Extension is made backirards in an oblique direction, the operator again being left free to effect reduction with both hands.

Reduction of backward luxation of the tibia is still more difficult, in consequence of the contraction of the mass of muscle at the back of the thigh.

Plaster bandages are the most convenient means of immobilising the parts after reduction.

\section{LUXATION OF THE SCAPULO-HUMERAL JOINT.}

This luxation, like that of the femoro-tibial articulation, is exceptional. It may assume one of two forms, depending on whether the head of the humerus is displaced intrardly, or towards the back of the glenoid cavity; but as a rule luxation occurs intrardly. Forrard luxation of the head of the humerus is almost impossible, in consequence of the resistance offered by the tendons of the flexor brachii and antea spinatus muscles. Similarly, luxation outwards is very difficult, the tendon of the postea spinatus being very porrerful and offering enormous resistance.

Intrardly, on the other hand, the insertion of the subscapularis is much less porrerful, and there is no real opposition to movement of the head of the humerus.

Causation. Tiolent mechanical shocks transverse to the upper third of the arm may, by sheer force, displace the head of the humerus in an inward direction, causing rupture of the internal wall of the capsular ligament and of the subscapularis muscle. Jumping from high to low ground and falling on the front limbs tend to displace the glenoid cavity in front of the head of the humerus, and often result in luxation of the bone in a backward direction, a luxation, herrever, which almost always assumes a postero-internal direction. The commonest causes of these luxations are the siderard falls of animals which have attempted to 
cover others. Whether the subject be a bull or a cow, if the stationary animal suddenly moves to one side, or if the moving animal is frightened by the appearance of a dog, one of the front limbs may be violently dragged away from the body; the resistance of the capsular ligament and internal muscles of the shoulder may be overcome and luxation produced.

Symptoms. Symptoms are immediately apparent : no weight can be placed on the injured limb, and the animal moves on three legs. All muscular action is avoided, the limb is slightly shortened as a consequence of the head of the humerus slipping behind the shoulder, which is held rigidly during movement; the points of the claws are dragged along the ground.

On local examination the point of the shoulder appears to be deformed and outwardly displaced, in consequence of the pressure exercised by the displaced head of the humerus. Below the glenoid cavity and coracoid process lies a depression, at the base of which the displaced humerus can be felt. This depression, however, is soon filled up by the serosanguinolent effusion consequent on luxation.

Diagnosis. Bearing in mind the conditions to which the accident is due, the diagnosis presents no great difficulty.

Prognosis. The prognosis is grave, for although it is relatively easy to reduce the displacement, it is very difficult to keep the joint fixed in position.

Treatment. To effect reduction, the animal should be cast on the sound side, and a loop of webbing passed under the arm, in order to provide for counter-extension. The limb is extended by direct traction on the cannon bone or fetlock, whilst the operator endeavours to effect reduction with one hand placed in front and one behind the joint.

In young animals of trifling weight immobilisation may be attempted, the best method being the application of pitch plasters. If, on the other hand, the subject is heavy, there is so great a chance of displacement when lying down and rising, that such cases are usually sent to the butcher, or abandoned to chance. Relative recovery, sufficient to permit of growth or fattening, may take place without professional assistance.

\section{IV.-HYGROMAS.}

Hygromas result from chronic inflammation of serous bursæ, naturally existing, or of serous bursæ which form at prominent points where the skin is exposed to repeated friction, blows, shocks, or over-extension.

They usually develop slowly, without producing marked pain or alarming symptoms, and therefore the practitioner is seldom consulted until the swelling has attained a considerable size. 
The hygroma is usually characterised by its non-painful character and by regular fluctuation throughout. The walls of the serous bursæ are merely thickened, so that palpation is easy.

Should the hygroma become infected and inflamed, it assumes the same characters as an abscess: it becomes highly sensitive, is surrounded by œedematous infiltration, shows more marked fluctuation at some specialised point, and eventually breaks, discharging pus.

Long-standing hygromas often have thickened fibro-cartilaginous and extremely hard walls, which render examination more difficult.

Where the hygroma is much exposed to friction the skin covering it undergoes complete transformation, the layers of epidermis becoming converted into a substance resembling horn. The entire substance of the wall of the hygroma then undergoes change, and is often infiltrated with lime salts or encrusted with plates of bone of varying thickness.

\section{HYGROMA OF THE KNEE.}

This condition is very common in bovines, a fact explained by the manner in which these animals rise. Whilst the hind limbs are being lifted, the entire burden of the body weight is transmitted to the knees and the tissues covering them; so that, if the ground is rough, the skin may be sufficiently displaced to produce laceration of the subcutaneous connective tissue, serous effusion in the layers of connective tissue, and the immediate production of

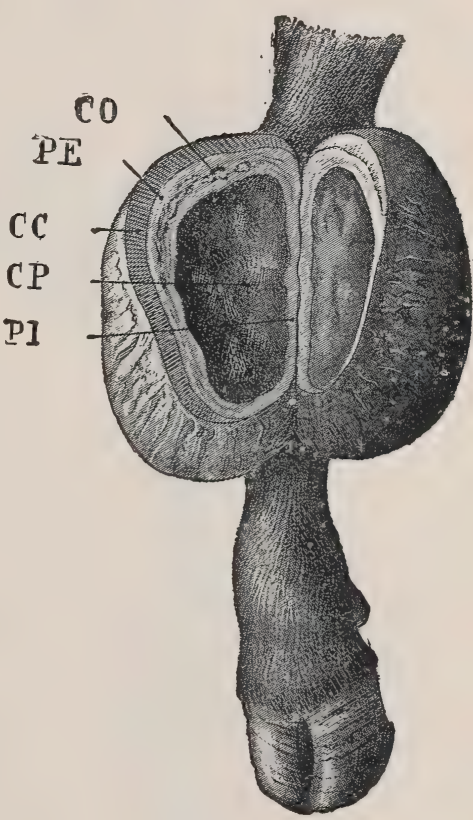

FIG. 25. - Old-standing hygroma of the knee. PE, external coat; $\mathrm{CC}$, cornified coat; $\mathrm{CO}$, osseous coat; CP, pus cavity; PI, internal coat. an hygroma beneath the skin and in front of the synovial sheaths of the extensor tendons.

Hygroma is principally caused by falls on the knees, roughness of the stable floor, prolonged decubitus during the course of a serious disease, or after an attack of foot-and-mouth disease.

Hygromas may be no larger than a turkey's egg or a man's clenched fist, but sometimes assume the dimensions of a child's head. Calcification and ossification of the walls and cornification of the skin are commonest in old hygromas of the knee.

The sensibility and uniform fluctuation make mistakes in diagnosis D.C. 
difficult. The condition can only be confused with distension of the synovial sheath of the extensor metacarpi magnus; but this (synovial) swelling extends in the same direction as the tendon, i.e., vertically, attains the lower third of the radius, and is broadest above. Hygromas must also be distinguished from tumours. Moussu only mentions a single case of this kind, the tumour being very slightly bosselated and, naturally, revealing no fluctuation.

The prognosis is not grave, though the condition may prove troublesome, because the original injuries may be continued even during treat-

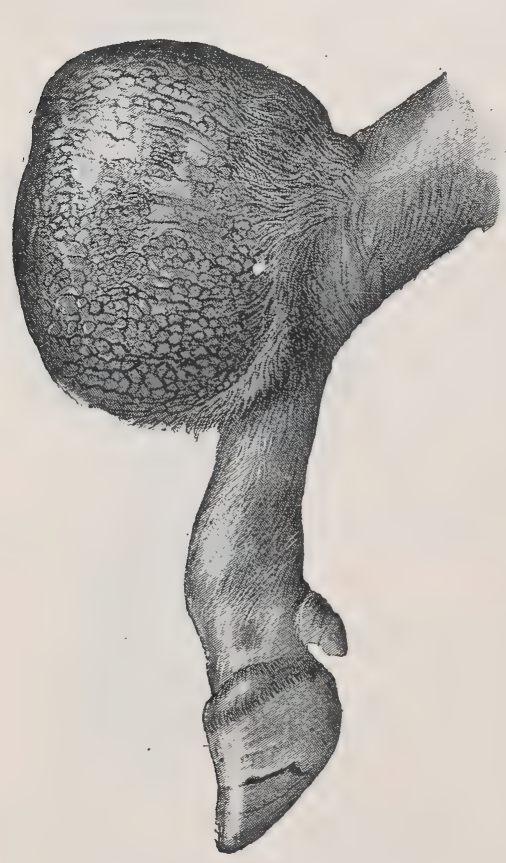

FIg. 26.-Hygroma of the knee. The skin has undergone conversion into a substance resembling horn. ment and prevent recovery.

Treatment. Success rarely follows cold applications or blistering, which are only of value at the commencement. It is better to puncture the cavity aseptically, remove the fluid contents, and fire the growth in points. Free opening of the lowest portion of the swelling is followed by discharge of liquid, but almost inevitably by infection at a later stage, and by suppuration. Recovery certainly may occur, a slight thickening of the anterior surface of the knee remaining; but the process is often very prolonged. Some authors prefer to pass a seton or drain vertically through the swelling. The results are identical with those following free opening and drainage, suppuration being unavoidable.

If the animal be sufficiently valuable to warrant surgical intervention, the entire hygroma, together with its indurated wall, may be excised. An elliptical fragment of skin is removed from the front of the swelling, and the whole mass separated by dissecting away or tearing through the connective tissue. Considerable care is necessary to avoid injuring the synovial sheaths of the extensor tendons. This treatment, which is only applicable in valuable animals, is completed by firmly suturing the lips of the wound, and applying an antiseptic surgical dressing or a plaster bandage similar to that used in operating on broken knees in horses.* The animal must be prevented from lying down until the wound has firmly united.

* See Dollar's "A Surgical Operating Table for the Horse." (London: Gay and Bird.) 


\section{HYGROMA OF THE HAUNCH.}

With the exception of hygroma of the linee, hygromas are commoner on hind than on front limbs. That of the haunch is limited to the external angle of the ilium. It follows violent falls or collisions with door posts, and results from laceration of the layers of subcutaneous connective tissue and separation of the skin from subjacent parts.

The effusion is often of a sero-sanguinolent character. It is more frequent in animals occupying narrow or irregularly shaped stalls, the hygroma being developed through repeated collision of the angle of the haunch with the wall. Finally, it may follow prolonged decubitus.

Diagnosis is easy, but the prognosis has a certain element of gravity, because, should suppuration occur, it may be succeeded by necrosis of the aponeurosis inserted into the external angle of the ilium.

Treatment should first be directed to removing the cause. Of the various modes of intervention, the best probably consists in disinfecting the parts, puncturing the swelling, and injecting some irritant of an antiseptic character, or simply washing out the cavity. Iodine and carbolic solutions are most commonly employed. Firing is contraindicated.

\section{HYGROMA OF THE TROCHANTER OF THE FEMUR,}

This condition is rare, except in thin milch cows kept under bad hygienic conditions and insufficiently supplied with bedding. Continual bruising of the prominences of the quarters whilst the animal is lying is the usual cause.

This hygroma forms a hemispherical swelling covering the trochanteric prominence. Movement is interfered with, and the stride is shortened.

The condition can only be confused with the diffuse swellings due to periarthritis in the coxo-femoral region, which frequently occur in corrs suffering from infectious pseudo-rheumatism.

The prognosis is somewhat grave, for in case of suppuration the insertions of tendons and fascia into the summit of the trochanter may become necrotic.

Treatment. The first point is to supply the animal with ample clean bedding. The swelling may be repeatedly blistered. If considered necessary, a puncture may be made under antiseptic precautions, the fluid drawn off, and the cavity washed out; but it is better to avoid opening the parts with a bistoury, on account of the danger of suppuration and of necrosis of the tendons and aponeurotic tissues in the neighbourhood.

\section{HYGROMA OF THE STIFLE,}

Hygroma of the stifle or of the patella appears under the skin, outside the external ligament of the femoro-tibial articulation. It usually follows 
repeated abrasion when lying down, especially if the paving of the stalls is rough or irregular.

The swelling varies in size from a hen's egg up to that of a child's head, and exhibits fluctuation throughout.

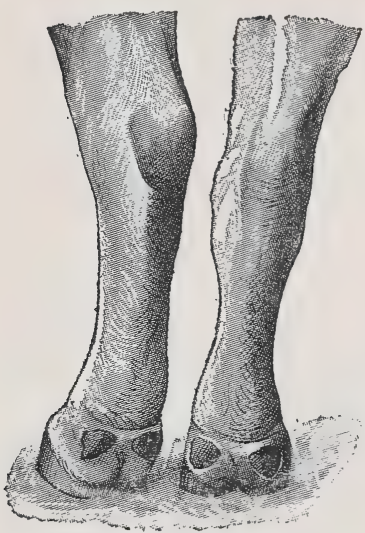

FIG. 27.--Capped hock.

The prognosis is somewhat serious, for here again complications may result from necrosis of neighbouring aponeuroses.

Some authors recommend passing a seton dressed with some irritant material through the swelling. Aseptic puncture, however, seems preferable, followed by washing out of the cavity and the application of a blister.

\section{HYGROMA OF THE POINT OF THE HOCK.}

This hygroma usually results from blows with the ox-goad, which cause inflammation of the subcutaneous connective tissue and œdematous infiltration extending down the leg. Afterwards the slightest

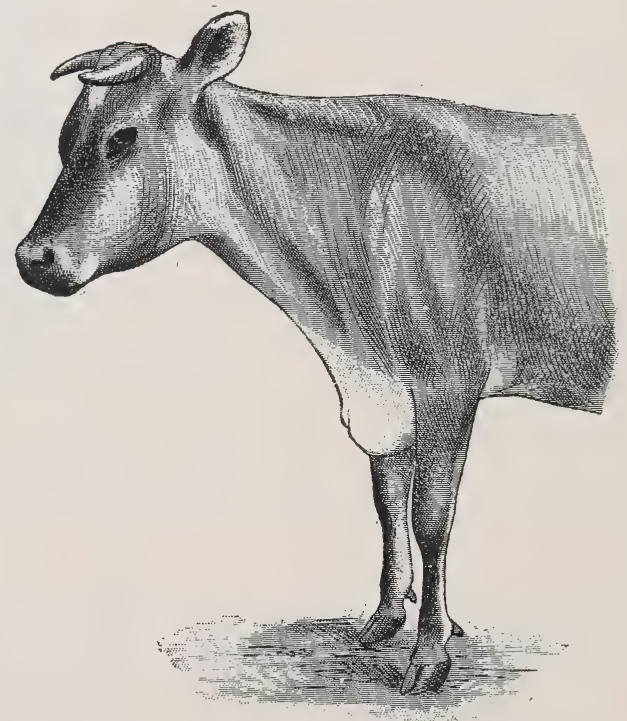

Fig. 28.-Hygroma of the point of the sternum.

injury, or even the friction due to the animal lying down, causes liquid to collect and an hygroma to form.

This hygroma is readily infected and often suppurates; it then becomes very sensitive, producing intense lameness. More frequently, however, under the influence of prompt treatment, the liquid is 
absorbed, the layers of subcutaneous connective tissue become hardened, and undergo more or less extensive induration.

\section{HYGROMA OF THE POINT OF THE STERNUM.}

Hygroma of the point of the sternum is a rarity. It only occurs in thin animals in which the point of the sternum is prominent, and which are confined to stables with rough floors and provided with insufficient bedding.

The swelling should not be opened, for the tissues in front of the sternum readily become the seat of suppuration.

If treatment is desirable, the fluid may be drained off by means of an aseptic puncture. 


\section{CHAPTER IV.}

\section{DISEASES OF MUSCLES AND TENDONS.}

\section{RUPTURE OF THE EXTERNAL ISCHIO-TIBIAL MUSCLE (BICEPS FEMORIS)。}

The biceps femoris muscle extends from the superior spinous process of the sacrum to the region of the patella and the external surface of the thigh. Above, it is inserted into the sacrum and the posterior margin of the ischium, below into the supero-external surface of the tibia. It completely covers the coxo-femoral articulation, and its passage over the summit of the trochanter is lubricated by a serous bursa. The whole of its anterior margin is connected with the fascia lata by an aponeurotic expansion. From varying causes this aponeurotic layer may become fissured; during the backward and forward movements of the limb the summit of the trochanter may enter the fissure and become fixed there by the tension and resistance of neighbouring tissues. This accident has been described as "displacement or rupture of the biceps femoris muscle."

According to Cruzel, fixation of the biceps femoris may occur, in very thin animals, without rupture of the musculo-aponeurotic layer, the process being then simply confined to stretching of the aponeurotic layer over the summit of the trochanter. Under such circumstances the musculo-aponeurotic layer presents a cup-like depression, into which the summit of the trochanter fits, and thus effectually prevents movement of the muscle.

Whether the accident is due to an actual fissure, or only to stretching of the aponeurosis, the symptoms are the same.

Causes. The principal predisposing causes are thin condition and malformation of the limbs (turning outward of the hind toes).

The accident may occasionally be caused by a slip backwards, or by extreme efforts in draught when ascending hills.

Symptoms. Immediately the accident occurs the limb becomes fixed in a position of maximal extension. The trochanter being caught, the femur can no longer be flexed, and the lower joints are also fixed in such a way that the limb can only be moved as a whole. The claws 
are dragged along the ground, and the affected limb, which can never be completely advanced, is brought forward with a mowing movement.

On local examination, the trochanter appears to be very prominent, and situated directly above a rigid cord which extends parallel with the anterior margin of the affected muscle.

If the accident consists merely in the formation of a depression in the muscle, in which the summit of the trochanter is fixed, and if there is no fissuring, the muscle and the femur are certainly immobilised, but

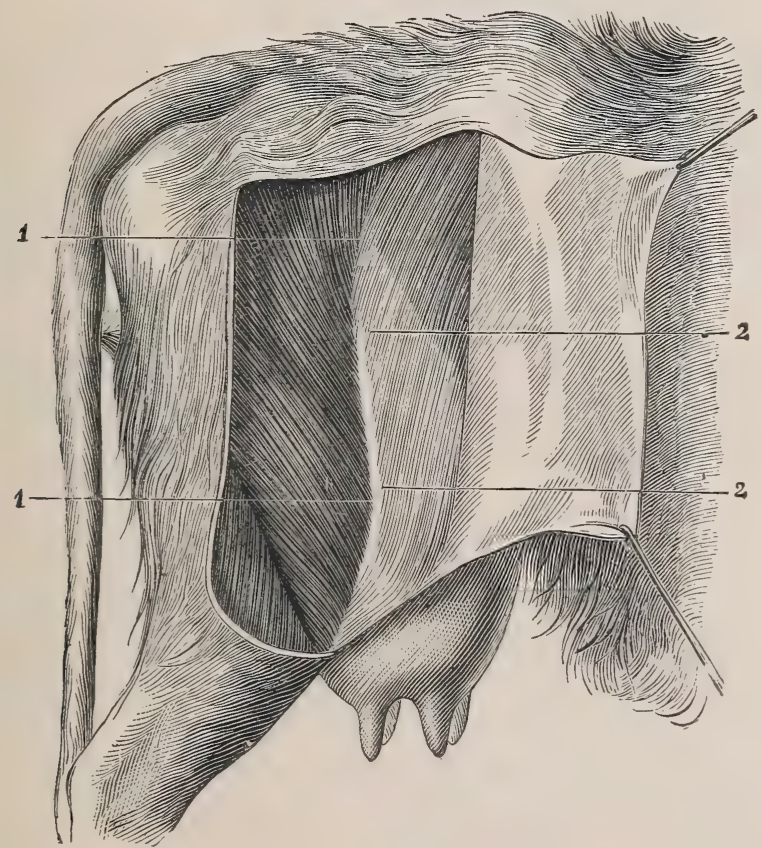

FIG. 29.-Arrangement of the external ischio-tibialis muscle. 1, Anterior margin of the muscle; 2 , fascia lata.

the limb can be moved to a certain extent, the "mowing" movement is less marked, and there is no well-defined rigid cord along the anterior margin of the muscle.

Diagnosis. The condition can only be confused with luxation of the patella; but manual examination of the affected parts revealing the presence of a rigid cord below the trochanter at once removes any doubt.

Prognosis. The prognosis is only grave in working animals. Moreover, the accident is now much rarer than formerly, if only because animals are better looked after and better fed.

Treatment. If the accident results simply from the formation of a depression in the musculo-aponeurotic layer which replaces the muscle 
at the point where it passes over the trochanter, there is nothing to be done. Reduction will occur spontaneously, and entire liberty of action will be regained. Cruzel states that it is sometimes sufficient to force the animal to move down a slope, in order to withdraw the trochanter from the depression in which it has been lodged, and to restore its normal mobility.

Rest and good feeding favour the deposition of fat, and soon alter the conditions responsible for the accident; the muscles of the quarter become surrounded with fat, the external ischio-tibial muscle (biceps femoris) is thrust outwards on account of its superficial position, and then cannot be ruptured by the summit of the trochanter. If, on the other hand, the musculo-aponeurotic layer is fissured and the summit of the trochanter firmly fixed in the opening, operation becomes necessary. This consists in incising the anterior margin of the muscle over the afore-mentioned rigid cord. The margins of the wound retract, the tension of the cord is diminished, the trochanter released, and the normal play of the limb restored.

Numerous methods of operation have been described and a number of special instruments invented. The earlier methods consisted in simple subcutaneous section of the rigid cord formed by the musculo-aponeurotic layer and the muscle. Subcutaneous section is carried out exactly like tenotomy, using straight and curved tenotomes. The seat of operation is about three inches below the summit of the trochanter. In the absence of tenotomes, section may be performed with a bistoury introduced from below the muscle by means of a grooved director, which has been inserted through a cutaneous puncture made at the point indicated over the anterior margin of the prominent cord.

In better nourished subjects, in which this cord is less prominent, the operator may, to ensure greater accuracy, make a vertical incision an inch or two in length at the point selected over the anterior margin of the muscle, isolate this muscle by means of the director, and afterwards perform the section. Considerable hæmorrhage occasionally follows division of some small muscular vessel, but is of no consequence unless the wound has been infected.

\section{RUPTURE OF THE FLEXOR METATARSI.}

The rupture of this tendon-muscle is exceptional, and, according to the description given by Furlanetto, is attended by the same symptoms as in the horse-i.e., flexion of the stifle joint is not accompanied by flexion of the hock or of the metatarsus on the tibia. The cannon-bone hangs vertically when the limb is moved.

Recovery follows prolonged rest. 
Wounds and sections of tendons in the region of the cannon bone, sections of the tendo-Achillis, etc., have been seen and described. All such injuries may heal under antiseptic treatment and after aseptic suture of the divided ends, provided the sutures and dressings are applied immediately. If, on the other hand, suppuration, infection, necrosis of tendons, synovitis or arthritis occur as complications, such injuries become extremely serious, and from an economic standpoint render it better to sacrifice the animal rather than attempt treatment.

Strains of tendons and tendinitis occur in the front limbs of oxen, particularly of those used in carts. The chief indications are swelling in the region of the cannon bone and fetlock, uneven contour of the flexor tendons, sensibility on pressure, and lameness of varying intensity.

Treatment consists in continuous cold irrigation, massage, the application of a blister or even of the actual cautery. As a rule, however, it is better to rest and fatten the animal.

A frequent complication of such injuries of tendons consists in knuckling over at the fetlock.

\section{PARASITIC DISEASES OF MUSCLES.}

\section{INFECTION WITH CYSTICERCI.}

Infection of the connective and muscular tissues with cysticerci results from the entry into the body of embryos of Ternia solium and Tcenia saginata of man. It occurs in man and almost all animals, but is only of grave clinical importance in the pig and ox.

The following table shows the chief cystic (cestode) parasites of animals, though the cysts are not always confined to muscular structures:-

\begin{tabular}{|c|c|c|c|}
\hline \multicolumn{2}{|l|}{ Adult. } & \multicolumn{2}{|c|}{ Larva. } \\
\hline Name. & Host. & Name. & Host. \\
\hline $\begin{array}{l}\text { Tania saginata } \\
\text { Tania solium } \\
\text { Tania marginata: } \\
\text { Tania conurus } \\
\text { Tania echinococcus }\end{array}$ & $\begin{array}{l}\text { Man } \\
\text { Man } \\
\text { Dogs } \\
\text { Dogs } \\
\text { Dogs }\end{array}$ & $\begin{array}{l}\text { Cysticercus bovis . } \\
\text { Cysticercus cellulosa. } \\
\text { Cysticercus tenuicollis : } \\
\text { Conurus cerebralis . } \\
\text { Echinococcus polymor- } \\
\text { phus }\end{array}$ & $\begin{array}{l}\text { Cattle. } \\
\text { Swine and man. } \\
\text { Cattle, sheep, and swine. } \\
\text { Cattle and sheep. } \\
\text { Cattle, sheep, swine, man, } \\
\quad \text { etc. }\end{array}$ \\
\hline
\end{tabular}

\section{CYSTICERCUS DISEASE OF THE PIG.}

This disease of the pig is due to Cysticercus cellulose, the cystic form of the Trenia solium or Tcenia armata of man. As a disease of the pig it has been recognised from the most ancient times, and is stated to be 
the cause of Moses and Mohammed having prohibited the consumption of pork by their disciples. In the Niddle Ages it formed the subject of legislation. It was, howrever, only when the investigations of Van Beneden and Kuchenmeister had completed those of the zoologists of the seventeenth and eighteenth centuries that the evolution of tæniæ became well known and the importance of the cystic phase clearly established.

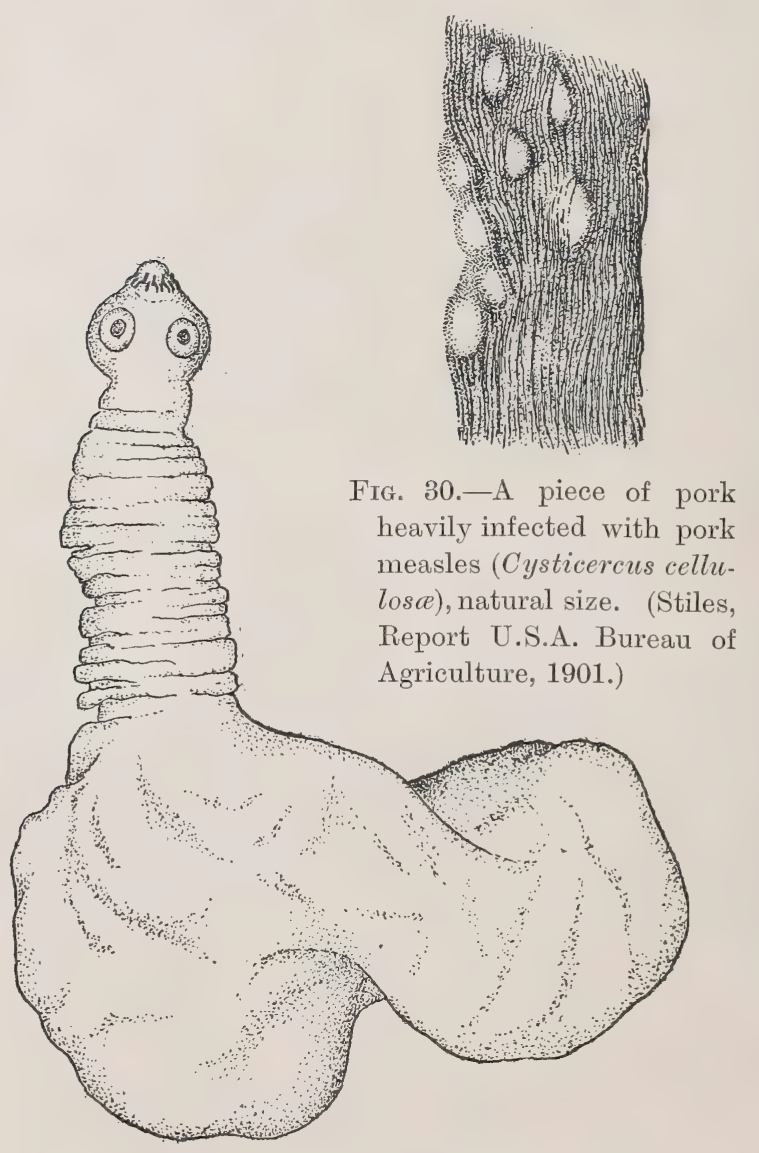

FIG. 31.-An isolated pork-measle bladder worm (Cysticercus cellulos $e$ ), with extended head, greatly enlarged. (Stiles, Report U.S.A. Bureau of Agriculture, 1901.)

Causation. The cause of cysticercus disease in the pig may be summed up in one phrase-viz., ingestion of eggs or embryos of T'cnia solium.

Young animals alone seem to contract the disease. After the age of eight to ten months they appear almost entirely proof against it.

It is very rare in animals reared in confinement, but is relatively 
common in those roaming at liberty ; because they are much more likely to discover human excrement and the embryos of tænia. The eggs having been swallowed, the sixhooked embryos are set at liberty in the intestine, perforate the tissues, enter the vessels, and are carried by the blood into all parts of the body. Those alone develop well which reach the interstitial and intermuscular connective tissue. The others in the viscera usually disappear. Their presence in the depths of the muscles produces slight general disturbance and signs of local irritation, due to the development of the cyst itself. At the end of a month the little vesicle is large enough to be visible to the naked eye; in forty to fortyfive days it is as large as a mustard seed, and in two months as a grain of barley. Its commonest seats are the abdominal muscles, muscular portions of the diaphragm, the psoas, tongue, heart, the muscles of mastication, intercostal and cervical muscles, the adductors of the hind legs, and the pectorals.

Symptoms. The symptoms of invasion are so little marked as usually to pass undetected. Occasionally, when large quantities have been ingested, signs of enteritis may occur, but these are generally ascribed to some entirely different cause. In some cases there is difficulty in moving, and the grunt may be altered.

Certain anthors declare that the thorax is depressed between the front limbs, but this symptom is of no particular value, and is
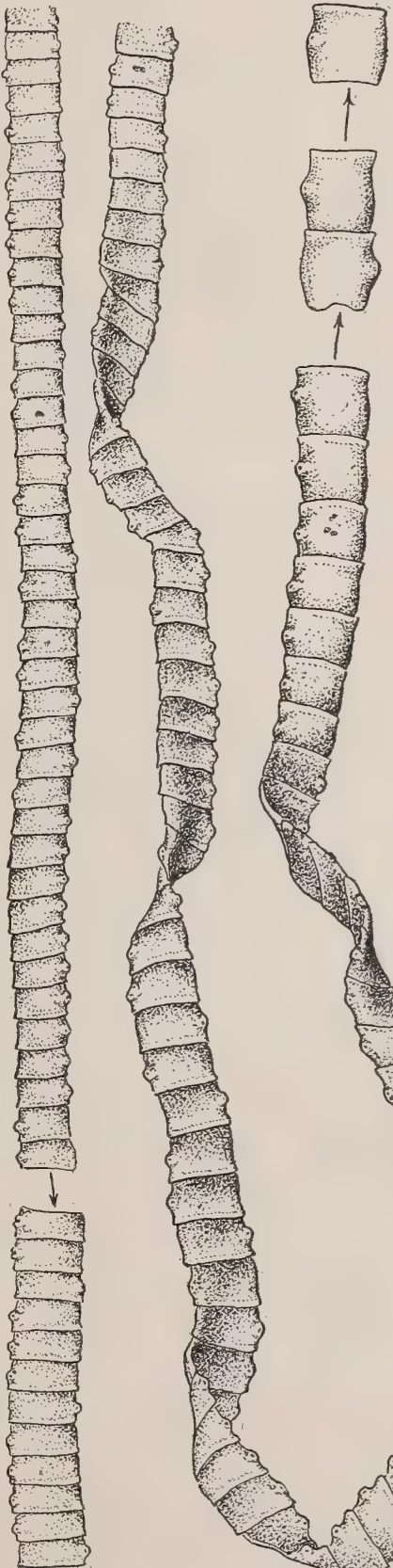

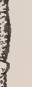

\section{.}


also common to osseous cachexia and rachitis. Paralysis of the tongue and of the lower jaw is of greater importance. In exceptional cases, where the cysticerci are very numerous and penetrate the brain, signs of encephalitis, vertigo, and turning sickness (gid, sturdy) may be produced. These signs, however, disappear, and the cysticerci undergo atrophy. Interference with movement may give rise to suspicion when the toes of

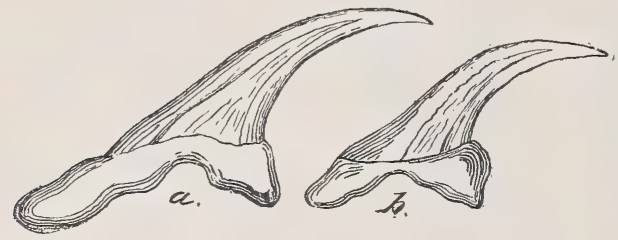

Fig. 33.-Large $(a)$ and small (b) hooks of pork-measle tapeworm (Tania sotium). $\times 280$. (After Leuckart.)

the fore and hind limbs are dragged along the ground, and thus become worn. This peculiarity is due to the presence of cysts in the muscles of the limbs, but it occurs in an almost identical form in osseous cachexia.

One symptom alone is pathognomonic, and it appears only at a very late stage-viz., the presence of cysts under the thin mucous membranes which are accessible to examination, such as those of the tongue and eye.

Visual examination then reveals beneath these mucous membranes the presence of little greyish-white, semi-transparent grains the size of a grain of barley, or even larger. Unfortunately, in an animal so difficult to handle as the pig, this visual examination is decidedly troublesome, and is usually replaced by palpation. In many instances the disease does not attract attention during the patient's life, and is only discovered on slaughter in consequence of the lesions by which it is characterised.

Diagnosis. As the characteristic lesions of cysticercus disease are to be found in the depths of the muscular and connective tissues, and

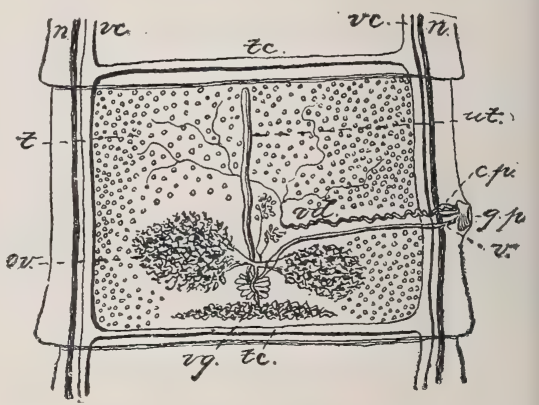

FIG. 34.-Mature sexual segments of pork-measle tapeworm(Tania solium), showing the divided ovary on the pore side. $c p$, Cirrus pouch; $g p$, genital pore; $n$, nerve ; $o v$, ovary; $t$, testicles ; $t c$, transverse canal; ut, uterus; $v$, vagina; $v c$, ventral canal; $v d$; vas deferens; $v g$, vitellogene gland. $\times 10$. (After Leuckart.) as the external symptoms may be regarded as of doubtful significance, the diagnosis can only be confirmed during life by manual examination of the tongue. This examination of the tongue has been practised since the earliest times. Aristophanes even speaks of it, and in the Middle Ages it was performed under sworn guarantees. The regulations concerning the inspection of meat have finally led to the suppression of this calling. 
In this method of examining the tongue, the operator commences by throwing the animal on its side, usually on the right side, and holding: it in this position by placing his left knee on its neck. He then passes a thick stick between the jaws and behind the tusks, opens the mouth obliquely, raising the upper jaw by manipulating the stick. Finally he fixes one end of this last by placing his foot upon it, and holds the other extremity by slipping it under his left arm. In this position he is able to grasp the free end of the tongue and by digital palpation to examine the tongue itself, the gums, the free portions of the frænum linguæ, etc.

If he discovers cysts, the diagnosis is confirmed, but failure to do so

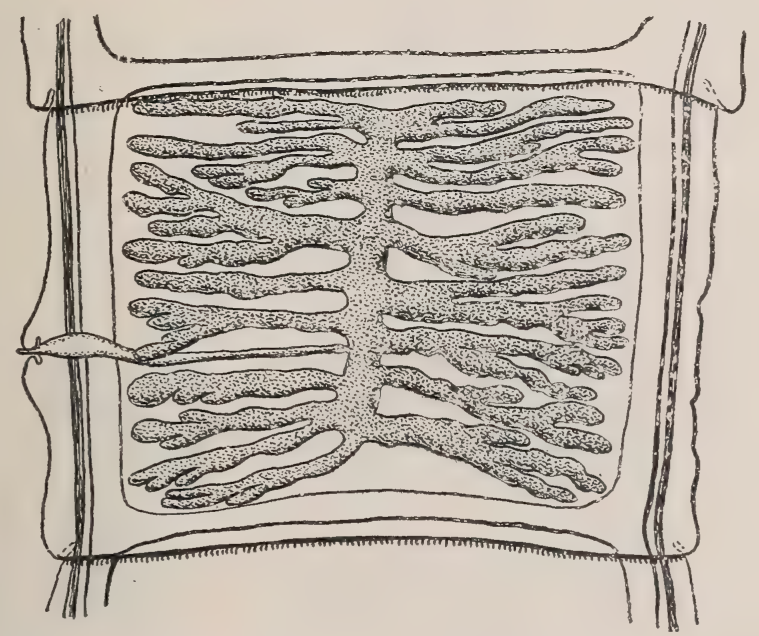

FIG. 35.-Gravid segment of pork-measle tapeworm (Tania solium), showing the lateral branches of the uterus enlarged. (Stiles, Report U.S.A. Bureau of Agriculture, 1901.)

by no means disposes of the possibility of infection. Railliet declares that about one animal in four or five shows no cysts beneath the tongue, and, moreover, fraud is possible in this connection, it being quite possible to prick the little cysts with a needle so that the liquid contents escape, and examination gives no positive result. For these reasons intra-ritam examination alone is now discounted, and the chief reliance is placed on post-mortem search.

Prognosis. The prognosis is very grave, not on account of danger to the lives of the infected, but because infected meat may be offered for human consumption. Should such meat, in an insufficiently cooked condition, be eaten by man, its ingestion is followed by the development of Trenia solium. If cooking were always perfect it would destroy the 
cysticerci, but the uncertainty in this respect should prevent such meat
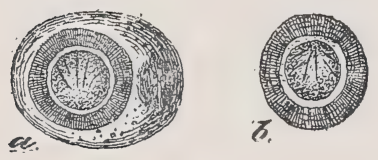

FIG. 36. - Eggs of porkmeasle tapeworm (Tania solium): $a$, with primitive vitelline membrane; $b$, without primitive vitelline membrane, but with striated embryophore. $\times 450$. (After Leuckart.) being consumed. The cysticerci are killed at a temperature of $125^{\circ}$ to $130^{\circ} \mathrm{Fahr}$.

Lesions. The lesions are represented by cysts alone--i.e., by semi-transparent bladders, each of which contains a scolex or head armed with four suckers and a double crown of hooks. The little bladders are most commonly found in the muscles, lodged in the interfascicular tissue, which they slightly irritate.

The number present varies extremely, depending on the intensity of infestation and the number of eggs swallowed. Whilst in some cases difficult to discover, in others

they are so numerous that the tissues appear strewn with them.

They are commonest in the muscles of the tongue, neck, and shoulders, in the intercostal and psoas muscles, and in those of the quarter.

The viscera-viz., the liver, kidneys, heart, lungs, etc.-are less commonly infested, and in these organs the cysts degenerate very rapidly. In animals which have been infested for a long time, the cysts may even have undergone caseocalcareous degeneration, the liquid being absorbed and the lesions presenting the appearance of little oblong firm nodules.

On cutting through masses of muscle the vesicles protrude from between the bundles.

In young animals, infestation with cysticerci causes wasting and ill-health; subsequently the patients improve in appearance, later on fatten, and gain marketable condition.

Of the carcases examined in Prussian slaughter-houses between $1876-82$, one in every 305 was found infested; between 1885-93, one in every 537.

Treatment. There is no curative treatment. Only preventive measures are of

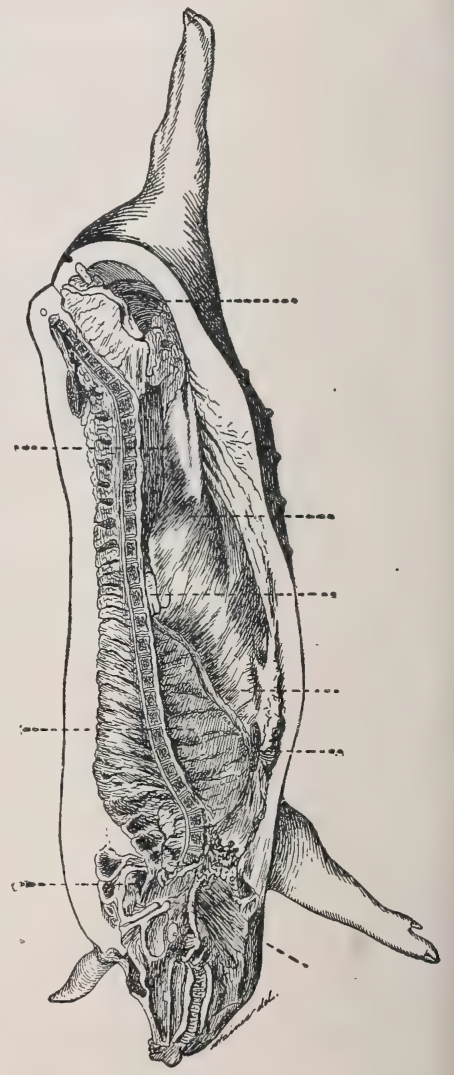

FIG. 37.--Half of hog, showing the portions most likely to become infested with pork measles. (After Ostertag.) 
value. These are confined to rendering it impossible for animals to ingest eggs of the Trenia solium.

Cysticercus disease is rare in the north, centre, and east of France, and in districts where animals are reared in confinement. It is commoner where pigs are at liberty, such as Limousin, Auvergne, and Perigord. It is frequent in North Germany, where the custom of eating half-cooked meat contributes to the propagation of T'enia solium. It is also frequent it Italy.

\section{BEEF MEASLES.}

Causation. The disease of beef measles is due to the penetration

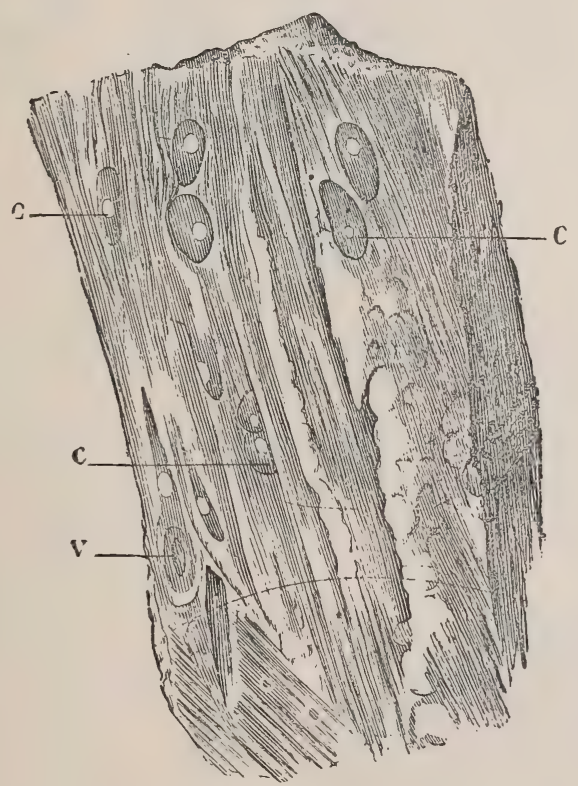

Fig. 38.-Cysticercus cellulosa in pork. $c$, Cysts ; $v$, fibrous tissue capsule which forms around the cyst.

into the connective and muscular tissues of embryos of the T'enia saginata, or unarmed tænia of man.

This disease, unlike that of the pig, has only been recognised within comparatively recent times, and only after Weisse's experiments (St. Petersburg, 1841) on feeding with raw flesh was attention drawn to it, although as early as 1782 the Trenia saginata had been described by Goëze.

Measles in the ox is rarely seen in France, but is common in North and East Africa. Alix has found it in Tunis, Dupuys and Monod in Senegal, and it is common in the south of Algeria. The disease is due 
simply to oxen swallowing eggs or embryos of the unarmed tænia, a fact which explains the frequency of the disease in places where the

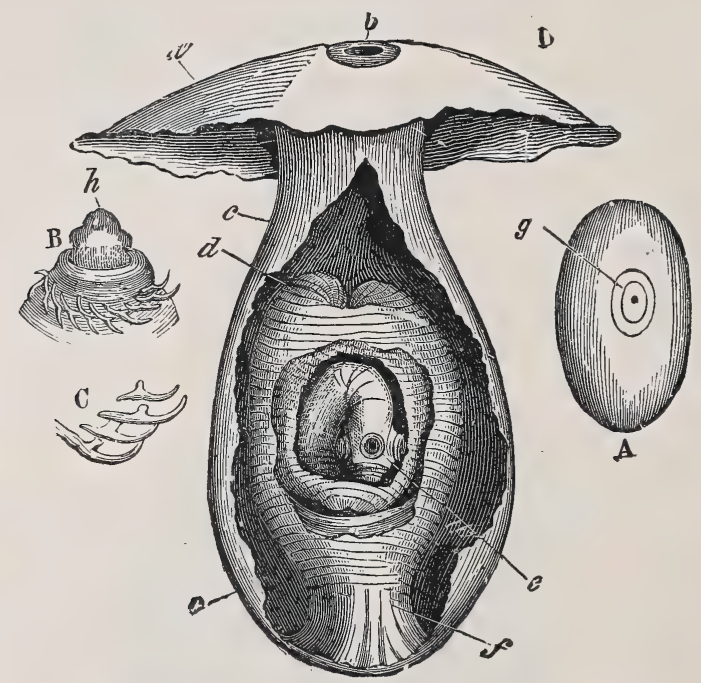

FIG. 39.-Anatomy of the Cysticercus cellulosa (after Robin). A, Cyst;

B, scolex with hooks; C, hooks ; D, magnified fragment of cyst.

inhabitants are of nomad habits, and consequently disregard the most elementary rules of public and general hygiene.

Furthermore, cattle in the Sahara, in Senegal and in the Indies,

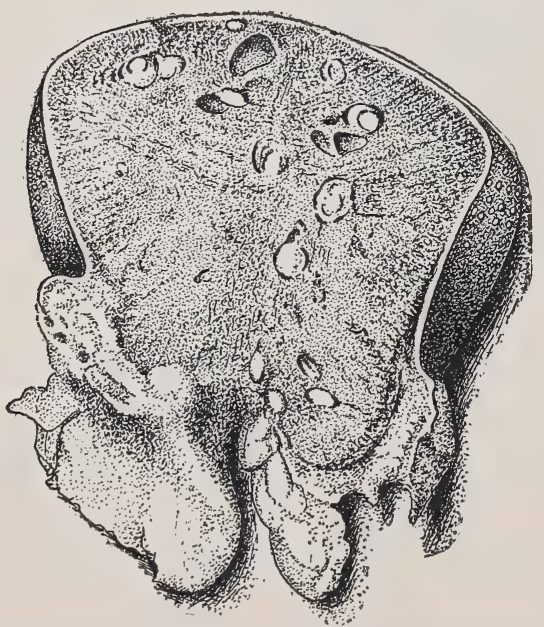

FIG. 40.-Section of a beef tongue heavily infested with beef measles, natural size (Stiles, Annual Report U.S.A. Bureau of Agriculture, 1901). have a very marked habit of eating ordure, and as no attempts are made to prevent it, the risk to these animals is greatly increased.

As in the pig, the embryos which reach the stomach and intestine penetrate into the circulatory system, and are thereby distributed throughout the entire organism.

The development of the cysticercus is complete in forty days, and if swallowed by man in infected meat after this period it again gives rise to the Trenia saginata.

The age of the animals seems of less importance than in the case of the pig, for Osterag and Morot have seen cases of beef measles in animals of ten years old. 
Symptoms. The symptoms are still less marked than in the pig, and in ordinary cases of infection always escape observation. Stiles, however, gives the following account of a case experimentally infected :-

"Symptoms. Four days after feeding segments of $T$. saginata to a healthy three-monthsold calf, the patient showed a higher temperature (the normal temperature was $39.2^{\circ}$ C.). The calf ate but little on that day, showed an accelerated pulse, swollen belly, staring coat, and upon pressure on the sides showed signs of pain. The next day the animal was more lively, ate a little, and for nine days later did not show any special symptoms except pain on pressure of the abdominal walls, and a slight fever. Nine days after the infection the temperature was $40.7^{\circ} \mathrm{C}$., pulse 86 , respiration 22 ; the calf laid down most of the time, lost its appetite almost entirely, and groaned considerably. When driven it showed a stiff gait and evident pain in the side. The fever increased

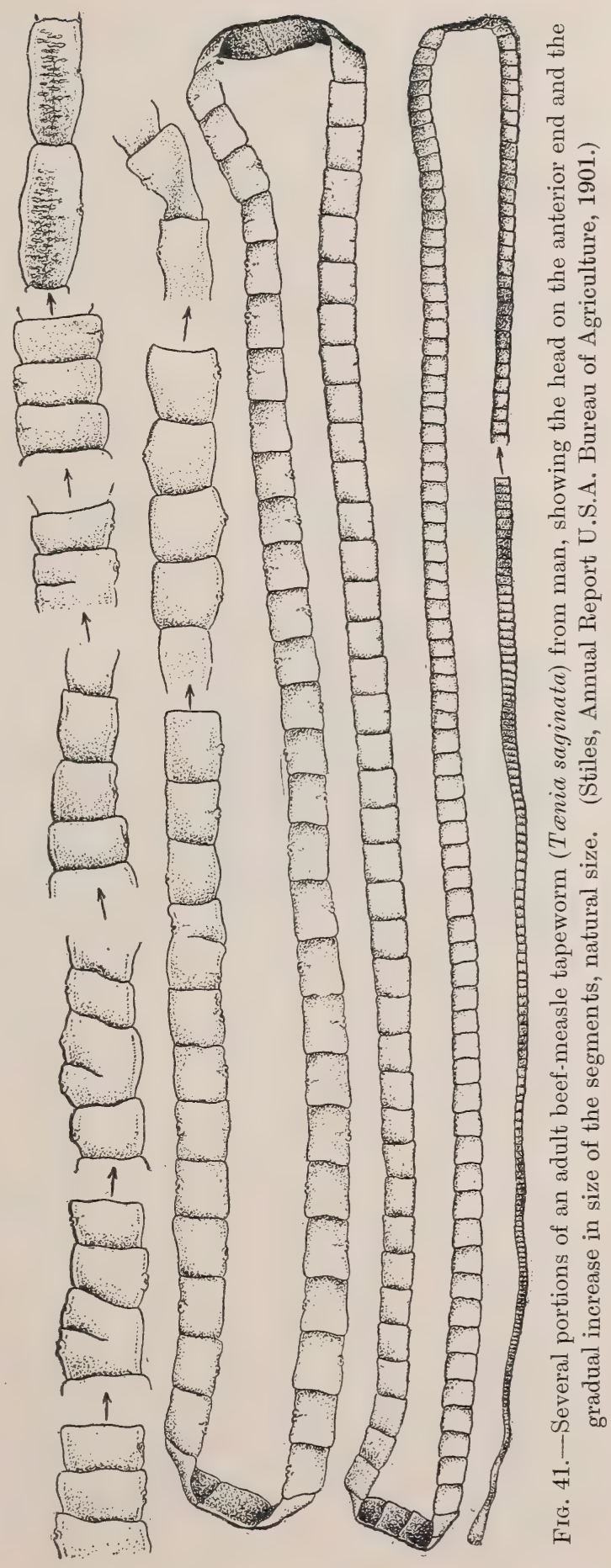
D.C. 
gradually, and with it the feebleness and low-spiritedness of the calf, which now retained a recumbent position most of the time, being scarcely able to rise without aid, and eating only mash with ground corn. Diarrhoea commenced, the temperature fell gradually, and on the twenty-third day the animal died. The temperature had fallen to $38 \cdot 2^{\circ} \mathrm{C}$. During the last few days the calf was unable to rise; in fact, it could scarcely raise its head to lick the mash placed before it. Pulse was reduced by ten beats. On the last day the heart-beats were very much slower, yet firm, and could be plainly felt. Several days before death the breathing was laboured, and
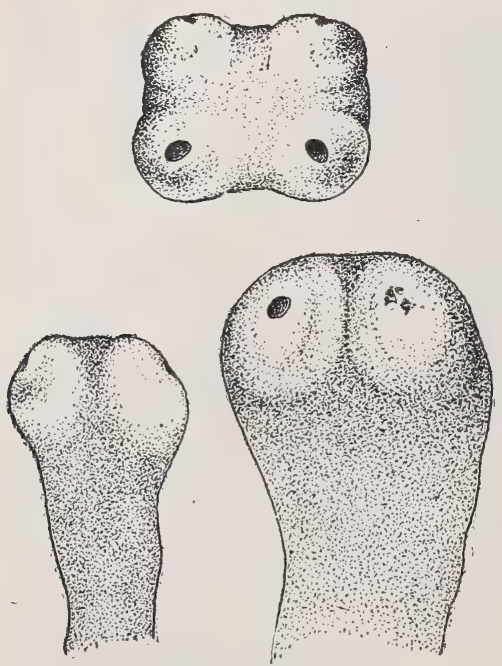

FIG. 42.-Apex, dorsal, and lateral views of the head of beef-measle tapeworm (Tania saginata), showing a depression in the centre of the apex. $\times$ 17. (Stiles, Report U.S.A. Bureau of Agriculture, 1901.) on the last day there was extreme dyspnœea."

Diagnosis. In forming a diagnosis we meet with the same difficulty as in the case of the pig. It is always easy to examine the tongue; but when visible lesions are absent diagnosis in the case of the ox remains doubtful and problematical even more than in the pig.

In the carcase, diagnosis is much easier. The cysts are sought for, as in the pig, by making sections of muscle, those usually selected being the pterygoid, cervical, cardiac, and psoas muscles, and those of the quarters.

Prognosis. The prognosis is grave, not indeed for the infected animals, which seem little injured by the parasite, but for human beings, who run the risk of contracting Trenia inermis by eating insufficiently-cooked meat.

A temperature of $115^{\circ}$ to $120^{\circ} \mathrm{Fahr}$. destroys the cysticerci, but in roast meats the central temperature of the mass always remains below this figure.

Salting for fifteen to twenty days destroys the vitality of the parasite.

Lesions. The lesions are confined to the presence of the cyst and of two little zones of chronic inflammation immediately surrounding it. Unless heavily infested the subjects fatten just as well as others.

The vesicles are semi-transparent, $\frac{3}{16}$ inch to $\frac{1}{4}$ inch in length, slightly ovoid in form, and contain a tænia head with four suckers, but without hooks. 
In seven to eight months the cysts undergo degeneration, the liquid is absorbed, and calcium salts are deposited throughout the mass. The lesions which remain have, in the ox, the appearance of interstitial disseminated tuberculosis.

There is no curative treatment. The infested animal recovers spontaneously with the lapse of time, for the cysticerci undergo degenerative processes, but the flesh of such animals is of little commercial value.

From a preventive standpoint we can only hope to improve matters by a gradual and progressive change in social and public hygienic conditions.

When the life of the nomad shall have been entirely replaced by that of the highly-civilised European and private hygienic precautions

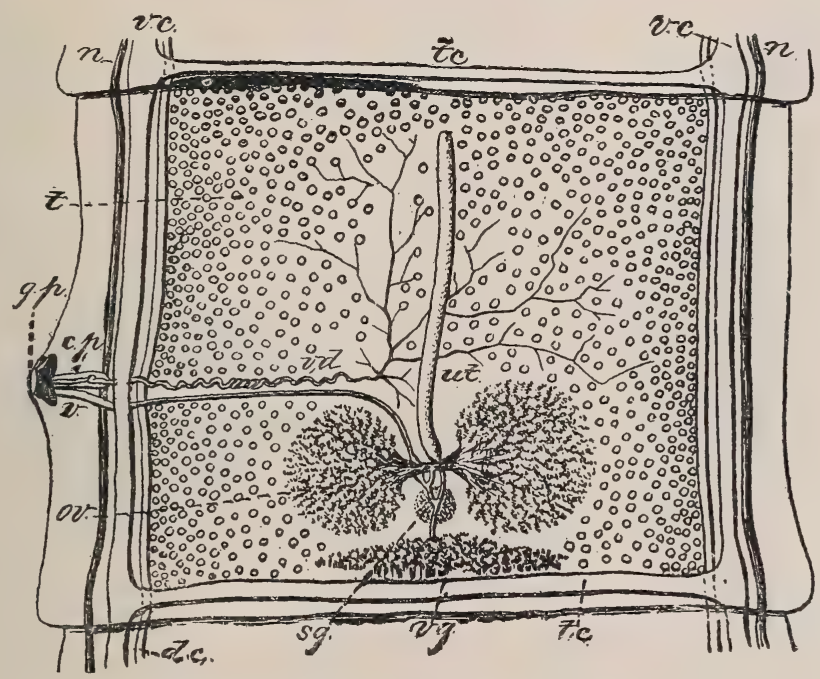

FIG. 43.-Sexually mature segment of beef-measle tapeworm (Tania saginata). c.p., Cirrhus pouch with cirrhus; d.c., dorsal canal; $g . p$. , genital pore; n., lateral longitudinal nerves; ov., ovary; $s g$., shell-gland; $t$., testicles ; $u t$., median uterine stem, enlarged (in part after Leuckart) ; v., vagina ; v.c., ventral canal, connected by transverse canal ; $t c, v d$., vas deferers ; vg., vitellogene gland.

have rendered it impossible for animals to obtain access to segments or eggs of the Tenia saginata, beef measles will disappear.

At present, in the countries where the disease is common, one experiences a feeling of astonishment that it is not far more frequent; for experiment has shown that a person infected with one unarmed tapeworm expels with the fæces an average of four hundred proglottides per month, each proglottis or segment of the worm containing about 30,000 eggs, each of which is capable of developing into a tapeworm. 
Beef measles is rather common in Germany, but rare in France, Switzerland, and Italy.

\section{TRICHINIASIS-TRICHINOSIS,}

Trichinosis is a disease caused by the entrance into the body of the Trichina spiralis. This parasite is swallowed in the larval form, and undergoes sexual changes in the intestine, at first producing intestinal trichinosis, which represents the first phase in the development of the disease.

The trichinæ breed rapidly. The embryos penetrate into or are directly deposited in the blood-vessels, which convey them to all parts of

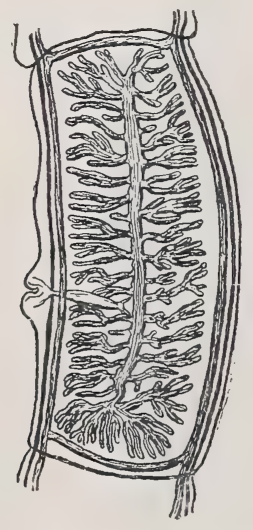

FIg. 44.-Gravid segment of beefmeasle tapeworm (Tania saginata), showing lateral branches of the uterus, enlarged. (Stiles, Annual Report U.S.A. Bureau of Agriculture, 1901.)

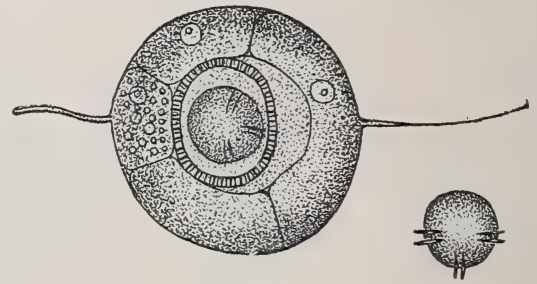

Fig. 45.-Egg of beef-measle tapeworm (Tania saginata), with thick egg-shell (embryophore), containing the sixhooked embryo (oncosphere), enlarged. (After Leuckart.)

the body, thus setting up the second phase of the disease, known as muscular trichinosis.

Trichinosis as a disease has long been recognised. Peacock in 1828 and J. Hilton in 1832 mentioned the existence of the cysts of trichinæ; Owen in 1835 gave the name of Trichina spiralis to the parasites contained in the cysts. Trichinosis being common in Germany at that time, Virchow and Leuckart undertook its investigation, but mistook other nematodes of the intestine for the Trichina spiralis. In 1847 Leydy recognised that trichinosis occurred in American pigs.

In 1860 Zenker found muscular and intestinal trichinosis on postmortem examination of a girl who had been suspected of suffering from typhoid fever, and a carefully conducted inquiry revealed the fact that 
this girl had some time previously eaten a quantity of raw ham. Virchow and Leuckart returned to their investigations, and the life history of the parasite soon became definitely known.

Causation. Trichinosis is capable of attacking all mammifers without exception, from a man to a mouse; and most animals which can be made the subjects of experiment contract the disease in varying degrees.

The intestinal form is seen in birds, but the muscles do not become infested by the embryos.

Cold-blooded animals are proof against the disease.

After the ingestion of meat containing cysts of the parasite, the processes of gastric and intestinal digestion set the larræ at liberty. These larvæ become sexual at the end of four to five days, and the females, which are usually twice as numerous as the males, begin laying eggs from the sixth day, continuing for a month to six weeks. Each female lays approximately from 10,000 to 15,000 eggs. The embryos perforate the intestinal walls, pass into the circulation, and are hurried into all parts of the system. This period of infestation constitutes the first phase of the disease.

Askanazy, in 1896, suggested that it was not the embryos which perforated the intes. tinal walls and thus reached the blood-vessels, but the fertilised female trichinæ themselves, which entered the terminal chyle vessels and laid their eggs directly within them.

This observation is of great interest, for it contradicts the view held by Leuckart and proves that treatment is useless even in the

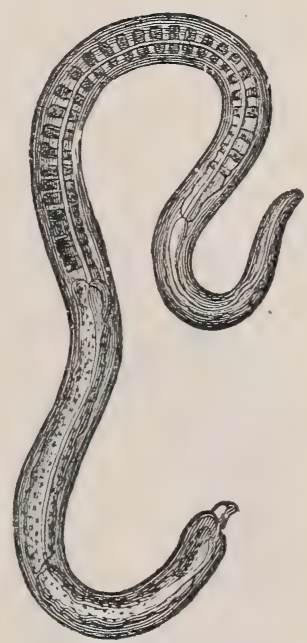

Fig. 46.-Male trichina from the intestine. (Colin.) first phase.

The males are about $\frac{1}{16}$ inch in length, the females $\frac{1}{8}$ inch to $\frac{5}{32}$ inch, and are ovoviviparous.

Symptoms. The symptoms lack precise character, even when the disease is known to be developing, and moreover they have only been carefully observed in experimental cases. As soon as the laying period begins, signs of intestinal disturbance may be observed, possibly due to embryos perforating the intestinal walls (if we accept Leuckart's view), or, according to Askanazy, to adult females penetrating the chyle vessels and disturbing intestinal absorption.

These symptoms are only appreciable in cases of "massive" infestation. If slight, the disturbance passes unperceived. In serere cases the symptoms consist of diarrhœa, loss of appetite, grinding of the teeth, 
abdominal pain in the form of dull colic, and sometimes irritation of the peritoneum. The embryos carried by the circulation then escape into the tissues and, like the cysticerci, become encysted, preferably in the muscles, in the interfascicular connective tissue towards the ends of the bundles. Each (asexual) parasite plays the part of a foreign body, causing infiltration of serum and exudation of leucocytes in its neighbourhood, and soon becoming encysted in the interior of a little ovoid space surrounded by a fibro-fatty wall. Fat granules accumulate at each end of the eyst.

The parasite, which at first appeared straight, soon assumes a bent form, then that of a figure " 6 ," then of a figure " 3 ," and preserves a

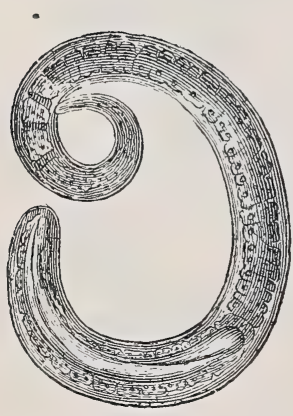

FIG. 47. - Free larval trichina. (Colin.)

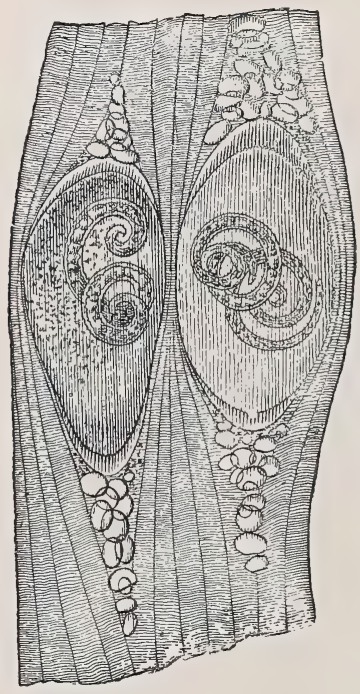

FIG. 48.-Trichinæ encysted in the muscular tissue. (Colin.)

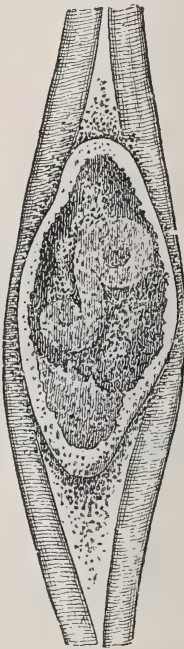

FIG. 49.-Old (degenerated) trichina cyst. (Colin.)

latent vitality throughout the entire period of encystment. These cysts are of very small dimensions, invisible to the naked eye, and their discovery necessitates the use of the microscope. They are about $\frac{1}{64}$ th inch in length and $\frac{1}{120}$ th inch in width. Very frequently two or three cysts may be found arranged in line, presenting the appearance of beads on a string: more rarely two parasites may be found in one cyst; exceptionally, as many as six or seven. The appearance of "beads on a string" is due to the fact that the parasites follow the interfascicular capillaries.

In animals which are kept for a long time and fattened the cyst walls 
undergo fatty infiltration. The change is commonest in pigs. In the same way calcareous infiltration sometimes occurs, but only when the parasites have lost their vitality. This calcareous degeneration consists in the deposit of carbonate and phosphate of lime in the walls of the cyst; it never begins before the seventh or eighth month after infestation, and is sometimes much longer delayed.

No man or animal ever becomes infested except by the ingestion of meat or drink containing larval trichinæ. The pig and small rodents are most frequently attacked. Man contracts trichinosis by eating insufficiently cooked infected pork. The fact that small rodents, particularly rats, eat the bodies of their kind explains the persistence of trichinosis in certain regions. Pigs roaming at large, and thus liable to find and eat the dead bodies of such rodents, may contract trichinosis in this way or from eating ordure.

For some weeks after the larvæ have penetrated the muscular tissues the animals show stiffness of the limbs, difficulty in moving, and in mastication, etc., but these troubles disappear in a short time.

The above facts explain why trichinosis in the pig is almost unknown in France, Italy, and Spain. It is commoner in Germany and in certain States of Europe, such as Holland and Russia, although investigations had previously shown that in Paris about 7 per cent. of the sewer rats were sufferers from trichinosis and that in Germany the percentage rose as high as 15 to 20 . In Chicago and Cincinnati, U.S.A., the proportion of rats suffering from trichinosis has been as high as 50 to 70 per cent., and as in some of the Northern States pigs were bred in complete freedom, it follows that at one time very large numbers of American pigs must have suffered from trichinosis.

In consequence of sanitary precautions this proportion has since greatly diminished.

Diagnosis. During the animal's life diagnosis is a difficult matter, though, on the other hand, simple microscopic examination of suspected meat is sufficient at once to settle the question. In dealing with the living animal, however, it is necessary, as in examining suspected meat, to obtain a fragment of muscle in order to submit it to microscopic examination. This fragment can be obtained by the method known as "harpoonage" - a trocar provided with a cutting hook, or" a trocar the canula of which has a sharp-edged opening near its end, being thrust into the muscle. On removing the trocar the elasticity of the tissues causes a fragment to project into the opening in the canula, and on withdrawing the latter a fragment sufficient for examination is obtained. One may proceed in the same way by harpoonage when examining large masses of suspected meat the surface of which reveals no lesion.

The specimen having been obtained, a few fragments of the 
muscular fasciculi are crushed between two glasses and examined with a low power.

The trichinæ will be found towards the ends of the muscle near the region of the tendons; few or none exist in the fat. These parasites are most readily discovered in the diaphragm, in the muscles of the shoulders and quarters, and in the psoas muscles.

Prognosis. The prognosis is relatively favourable so long as infestation is only moderate. But it is very grave from the point of view of public hygiene, on account of the possibility of persons becoming infected by eating the diseased meat.

Treatment. There is no curative treatment. Formerly it was believed that, provided the condition were early diagnosed, the intestinal form might possibly be cured by administering purgatives and vermifuges so as to prevent the embryos penetrating the system.

After Askanazy's discoveries this view had to be abandoned, and the practitioner is necessarily powerless in dealing with the muscular form. Time alone effects improvement and a relative cure by causing caseo-calcareous degeneration of the cysts. With a prophylactic object, every precaution should be taken to prevent the possibility of pigs being contaminated. This question particularly interests America, because of the extreme prevalency of pig trichinosis there.

From the point of view of public hygiene all infected meat should be seized and destroyed, despite the fact that perfect cooling destroys the vitality of the parasites, which perish at $120^{\circ} \mathrm{Fahr}$.

Ordinary salting but slightly affects their vitality, which explains why from time to time the importation of meat has to be prohibited and why meat should always be scrupulously inspected. 


\section{CHAPTER V.}

\section{RHEUMA T IS M.}

IN bovine pathology the term "Rheumatism " is applied to a number of different morbid conditions, the sole connection between which is that they seriously affect the organs of locomotion. This reason may perhaps be accepted as sufficient for including the study of rheumatism amongst diseases affecting locomotion.

The disease is of considerable importance, and for this reason the study of rheumatism itself necessarily precedes the description of pseudo-rheumatism, secondary rheumatism, or infectious rheumatism in young and adult animals.

\section{ARTICULAR RHEUMATISM.}

Acute rheumatism has a clearly marked predilection for the articulations. Sometimes the great serous membranes are simultaneously affected (pleura, pericardium, endocardium), but only in very exceptional circumstances are they primarily attacked. That form of rheumatism known as visceral is as a general rule secondary in comparison with articular rheumatism. Several joints and tendon sheaths may be attacked at the same time. Under such circumstances rheumatism may be defined as a febrile disease, probably of an infectious nature, revealing itself by simple or multiple inflammation of joints and the tissues surrounding them, and capable of becoming complicated with inflammation of the pleura, pericardium, endocardium, meninges of the brain, ete.

Causation. All authors agree in recognising the influence of heredity, of wet and cold, of sudden changes in temperature, draughts in the stable, prolonged exposure to low temperatures, or the chilling of animals saturated with perspiration. These are and cannot be otherwise than occasional causes; but the determining cause remains at present unknown.

In human pathology it has been prover beyond dispute that a certain relationship exists between arthritism, or the "uric acid diathesis," and rheumatism. This fact is so well recognised that doctors have said that rheumatism was to arthritism what scrofula is to tuberculosis. That, 
however, does not advance our knowledge of the question in the smallest degree, and it may simply be that arthritism represents one of the principal favouring conditions in the development of rheumatism.

In domestic animals the uric acid diathesis is little known, renal lithiasis is no more a rarity than gravel; but at the present time no one appears clearly to have established the relationship between these diseases and the development of rheumatism. What, however, we must all admit is that rheumatism exhibits all the phases of development of a rapidly progressive infectious disease.

Numerous attempts have been made by doctors during the last few years to discover the presence of a microscopic agent and to demonstrate its pathological characteristics. Several microbes have been described, but one is forced to confess that the results have until now been very contradictory and uncertain; and yet there is little room to doubt that the disease is of an infectious character.

Symptoms. The symptoms are generally well defined and well developed. The onset is sudden; an animal which one day before appeared perfectly well is attacked in one or several joints. Usually the upper joints of the limb are involved-the shoulder, elbow, knee, haunch, stifle, hock.

Nevertheless, invasion is probably not as sudden as it appears to be, and, as in the human species, the subject begins by feeling erratic pains, which, however, pass unnoticed. The animal moves with diffculty, as though it were suffering from laminitis, and has pain when placing weight on the limb, while the joint attacked soon shows a swelling which extends to the tendon sheaths and the neighbouring serous burse. The local temperature is higher than that of surrounding parts, sensibility becomes very marked, and pain attends the slightest pressure on, or even movement of, the affected joint. Intense lameness follows, which may even at first give rise to the suspicion of fracture. The animals remain lying for long periods, groan from time to time, and suffer great pain when rising.

In some cases the local manifestations appear to be transferred from one joint to another.

These local symptoms are accompanied by high fever. The temperature rises to $105^{\circ}$ or $106^{\circ}$ Fahr., the pulse to 80 or 90 , and the breathing is enormously accelerated if the patients are forced to move.

Loss of appetite is very marked. Rumination may be suspended, and these grave symptoms are accompanied by constipation, rapid wasting, cessation or marked diminution of the milk supply, decrease in the quantity of urine passed, etc.

A few days after the onset, visceral complications may occur, though 
fortunately such complications are far from being constant. Auscultation and percussion sometimes reveal the lesions of pleurisy, endocarditis, pericarditis, etc.

The development of articular rheumatism varies greatly, and may occasionally continue for weeks or months, the condition of one joint improving only to be followed by inflammation of another.

The risceral lesions rarely disappear completely, and it is not uncommon to note symptoms of chronic valvular endocarditis. Relapses are somewhat frequent, and the disease may continue in a chronic form after the acute symptoms have disappeared.

Lesions. The joint itself is not alone affected. All the tissues surrounding it are congested, swollen and painful, particularly the sheaths and insertions of the tendons. Within the inflamed synovial capsules of the joints an increased quantity of turbid synovia accumulates, distending the joint and producing a condition of hydrarthrosis.

In animals slaughtered during the course of the disease one finds congestive infiltration of the limbs.

The temperature of the parts near the affected joints is higher than that of neighbouring regions. Sensibility is much more acute, and the slightest external pressure gives rise to pain.

In favourable cases the joint may appear scarcely injured. The principal symptoms are those of pain. In old-standing cases certain permanent changes may occur, such as thickening and hardening of the wall of the synovial capsule, fibrous infiltration of the tissues around the joint, or even diffuse and irregular calcareous infiltration.

Cases of false or true anchylosis are rare, the animals usually being slaughtered before such conditions can develop.

Complications. The commonest complications are endocarditis and pericarditis. Valvular endocarditis localised in the auriculo-ventricular valves is revealed by a systolic sound, and by tumultuous or irregular beating of the heart when the animals are forced to move. Pericarditis, which seems rare in bovine animals, is much commoner in sheep. This pericarditis, however, produces none of the external signs of pericarditis due to a foreign body. Like tuberculous pericarditis, it is only accompanied by a trifling amount of exudate, and is recognised by increased cardiac dulness and diminution in the cardiac sounds on auscultation.

Simple pleurisy associated with pericarditis is frequent in sheep, but unknown or little known in the ox.

If in animals other visceral complications occur, affecting the peritoneum, meninges of the brain or intestines, they are at present little recognised.

Diagnosis. Articular rheumatism can only be confused with osseous cachexia or laminitis. Osseous cachexia, however, possesses symptoms 
peculiar to itself, and generally extends to an entire district, whilst rheumatism appears in an isolated form. Again, the arthritis peculiar to osseous cachexia most commonly affects the joints of the extremities (fetlock and phalanges). The disease may be differentiated from laminitis by simply manipulating the joints, which are painful in rheumatism but not in laminitis, and by percussing the claws, which are painful in laminitis but not in rheumatism, and by noting the character of the gait.

Prognosis. The prognosis is grave, as in all acute diseases which are capable of assuming a protracted chronic form. It is also necessary to take into account the loss of condition; the possibility of relapse, and the complications due to prolonged decubitus.

Treatment. The first indication is to place the patients in a nearly constant temperature, to supply bedding generously, and to arrange for the animal being undisturbed. Among drugs salicylate of soda gives the best results if administered in sufficient doses -6 to 7 drams per day for oxen or cows of medium size, 45 to 75 grains for sheep.

Diuretics, like bicarbonate of soda, nitrate of potash and hay tea or infusion of couch grass, pellitory, etc., also give good results.

The joints attacked may be blistered, but it is often preferable to use mild ointments, containing camphor or belladonna, because, as soon as pain diminishes, moderate massage of the affected parts, which favours rapid absorption of the effusions, can then at once be resorted to. The diet should consist of easily digested food and of lukewarm hay tea, etc. When the animals are suffering from kidney disease in any form salicylate of soda is contra-indicated.

Antipyrin may also be of service in doses of 45 to 75 grains for bovines and 15 grains for sheep. Preparations of methyl salicylate can only be used for animals of value.

\section{MUSCULAR RHEUMATISM.}

Muscular rheumatism is due to causes similar to those of articular rheumatism. The symptoms, moreover, often occur simultaneously, or may alternate with the articular manifestations, with which they are seldom entirely unconnected.

Moist cold seems to be the predetermining cause, whether it acts indirectly on the nerve trunks or affects the capillary circulation in the muscles, through the medium of the vaso-motor supply. The results are revealed by the development of neuralgia, neuritis or interstitial myositis; and these diseases, by producing more or less intense pain, cause difficulty in movement or distinct lameness. Attempts have been made to explain the development of these lesions by ascribing a certain action to the uric acid (which is said to be in excess in the body), and to the 
lactic acid, which accumulates in the muscular tissue after fatigue or over-exertion, and may occasionally produce temporary myositis. Up to the present time, no satisfactory proof has been furnished enabling us to identify the myositis of rheumatism with the myositis of over-exertion, which, moreover, appears to differ from it in essential particular's.

Symptoms. Muscular rheumatism is often ill-recognised in veterinary medicine, and closer observation would appear to suggest that it is much less frequent than has been stated. Generalised muscular rheumatism is rare; patients stand stiffly as though incapable of moving; the limbs and the back appear rigid, and the animal seems only capable of changing its position by a single movement of its whole body. One might readily believe at first sight that the case was one of generalised laminitis or slight generalised tetanus. The animal has difficulty in rising; when moving the limbs are dragged, and the patient is cautious in lying down.

Most frequently the disease is localised in one region, such as the shoulder, the loins, or the quarters. The affected part is stiff, tense, painful, hard, and as though in a state of cramp. Palpation and pressure reveal the presence of very exaggerated sensibility, which varies within wide limits, according to circumstances, changes in atmospheric conditions, etc. These local signs are accompanied by a general reaction of varying severity, somewhat resembling that seen in articular rheumatism. Appetite is diminished or suppressed, as is rumination ; the muzzle is dry and hot; the temperature may rise as high as $103^{\circ}$ or $104^{\circ} \mathrm{Fahr}$.

Lesions. The lesions are imperfectly recognised, because those who might most easily observe and study them have often neither the means nor the leisure for the purpose. Possibly one would at times discover lesions of neuritis; but in any case it is not so very rare to discover lesions of interstitial sclerosing myositis in the depths of the muscles of the quarter, loins, shoulders, etc., a condition rarely found in any other disease. Naturally these are only the ultimate lesions of muscular rheumatism; for slight attacks leave no traces visible to external examination.

Diagnosis. The commonest error is that of mistaking the condition for laminitis of all four limbs. The history often suffices to eliminate this disease from consideration, while palpation and percussion of the claws remove any remaining doubt.

The prognosis is not usually grave, and recovery sometimes follows the adoption of good hygienic conditions. On the other hand, certain patients lose flesh rapidly.

Treatment. Salicylate of soda and antipyrin still form the two most efficacious drugs, particularly the first, which may almost be regarded as a veritable specific in rheumatism. The doses vary with the size of the 
patients, from $2 \frac{1}{2}$ to 7 drams per day. These doses are continued for six to eight days consecutively. Some authors prefer salicylic acid, which, however, is more irritant, in doses of 15 to 75 grains. Tartar emetic, in doses of $2 \frac{1}{2}$ to 4 drams per day until purgation is established, is also said to have proved of great value in the hands of the older practitioners. Local treatment comprises stimulating frictions with camphorated alcohol, ammonia, and oil of turpentine. Such applications are usually of great service, particularly when associated with methodical massage of the affected parts. These modes of treatment should be supplemented with a proper dietary and the administration of tepid, diuretic fluids as required.

\section{Infectious Forms of Rheumatism or Pseudo-Rheumatisu.}

Under the head of infectious rheumatism or pseudo-rheumatism may be grouped joint diseases of a rheumatic type accompanying various general or local diseases in young animals and adults: diseases of the umbilicus, rachitis, peri-pneumonia, retention of the fœtus, dysentery, etc. These diseases are characterised by articular symptoms, which sometimes appear early, sometimes only when the disease itself is declining, and develop suddenly or gradually, the joint cavities themselves either being directly invaded by the agents of the primary disease or remaining exempt. These pseudo-rheumatic attacks are due to the localised action of microbic toxins on the articular synovial membranes. Sometimes the serous membranes of the large body cavities are also implicated.

This theory explains the development of acute arthritis without the presence of gonococci during the course of an attack of blenorrhagia in man, and may be applied in respect of certain forms of arthritis or synovitis without the presence of microbes in domestic animals.

\section{INFECTIOUS RHEUMATISM IN YOUNG ANIMALS.}

Causation. To explain the occurrence of infectious rheumatism in young animals a variety of causes has been invoked, such as bad feeding, the absence of that purgation which usually follows the action of the mother's first milk (i.e., colostrum), and clears the bowel of meconium, the effect of heredity, of chills, of insufficient food, and of unduly abundant or very rich food, which has been said to produce indigestion and its various complications.

All these causes may play a certain part in favouring the development of infectious rheumatism, but none constitutes the direct cause.

Lecoq and Loiset in their investigations regarding this disease in 
colts mentioned the almost invariable existence of lesions in the umbilical region. Bolinger in 1869 recognised the possibility of infection by way of the umbilical vein. Roll and Guillebeau are of the same opinion, and Morot's excellent study shows that here must be sought the most frequent point of origin of the articular symptoms. In animals born in dirty stables the umbilical cord becomes infected at the time of birth, or its cicatrix a few days later. The result is the development of rapidly fatal septicæmia, suppuration in the wound, omphalitis, omphalo-phlebitis, or umbilical arteritis; and to this infection are due the rarious complications, which may appear almost immediately, as in the case of septicæmia of calves, or may be deferred for a shorter or longer interval, for so long indeed that the umbilicus may appear to have healed externally (infectious pneumonia and endocarditis, infectious arthritis, etc.). The umbilical cord and the tissues surrounding the cicatrix form excellent culture grounds for those micro-organisms which always exist in such abundance in litter and manure; and there is, therefore, no difficulty in understanding why in dirty stables infection so readily occurs. The infective agents may be of very varying kinds, a fact which explains the difference in the symptoms which follow umbilical infection; although ovoid bacteria, streptococcus pyogenes, and the bacilli of necrosis seem most common.

Omphalitis and omphalo-phlebitis are not the only diseases capable of producing infectious rheumatism in young animals. Certain infections resembling dysentery and diarrhœic enteritis are also its frequent forerunners. In young animals even rachitis, which is accompanied by various digestive disorder's, may serve as the point of origin for infectious rheumatism and all its complications.

In older animals-i.e., in animals from fire to six months, or eren twelve to fifteen months-infectious rheumatism may occur without a clearly defined cause. It then develops with the symptoms and lesions of that condition known as "osteomyelitis of adolescence" in human pathology. These forms of osteomyelitis are due to infection with streptococci and staphylococci. In reterinary medicine the pathogeny has not yet been accurately ascertained.

Symptoms. Infectious rheumatism in young animals assumes one of two clinical appearances, possibly due to different causes, viz.-plastic or suppurative arthritis following umbilical infection, and simple exudative arthritis. In the former variety symptoms appear soon after birth, rarely after the age of two months, and as an exception in animals of six to eight months affected with rachitis. The onset is sometimes sudden; the patient, though healthy on the previous erening, is unable next morning to rise or move. Hence in France this disease has received the titles, amongst others, of laminitis and paralysis of the newly born. 
Certain joints, often a pair, appear swollen, hot and painful. The synovial sacs are distended, and in the upper joints of the limb appear much more prominent than in the lower. When the patients are still

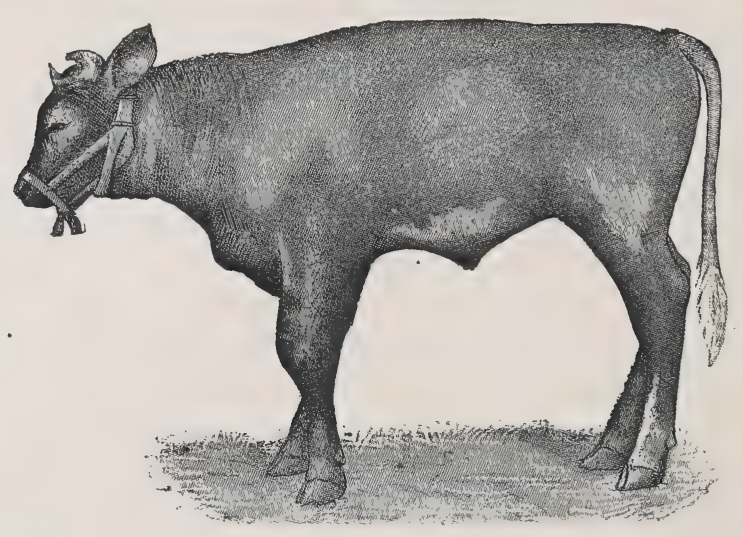

FIG. 50.-Young bull suffering from infectious rheumatism,

able to move they walk on three legs, but usually they remain lying permanently, and if aroused show great difficulty in rising and very acute pain in moving.

General disturbance is also very marked; the temperature varies between $103^{\circ}$ and $105^{\circ} \mathrm{Fahr}$. The animals are dull, have no appetite, and

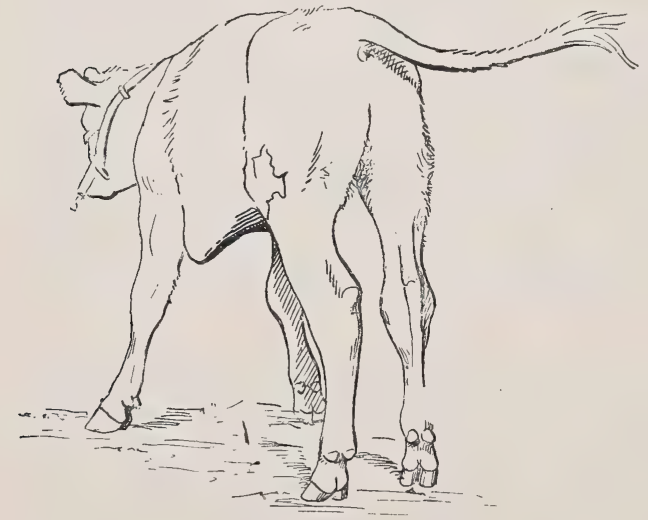

FIG. 51.-Attitude when walking of a calf suffering from infectious rheumatism.

exhibit intense thirst. The pulse rate and respirations are increased, and not uncommonly symptoms of grave visceral complications, such as endocarditis, pleurisy, pneumonia, etc., can be detected. Diarrhœic enteritis sometimes appears as a secondary development.

The animals remain permanently lying down. They can only be induced to rise with difficulty, and, when moved, exhibit very acute pain.

In many cases the disease then takes a rapid course and ends fatally. Death is the usual termination whenever any of the abovementioned visceral complications exist. Recovery is exceptional. In 
certain lucky cases, sometimes without any treatment whatever, the symptoms become less acute, the appetite persists or improves, fever diminishes, the condition of the joints remains stationary, and after the lapse of several weeks there is ground for anticipating recovery. In all cases, however, the convalescents remain thin and sickly, exhibit pain and capricious appetite, and in very few cases indeed is there any economic reason for keeping them alive.

More frequently infectious rheumatism terminates by abscess formation in the joints. The articular cavity becomes filled with pus, the tissues covering one of the synovial sacs soften, and the abscess opens, discharging fibrinous clots, thin watery pus mixed with synovia, and débris of articular cartilages or ligaments. Pyæmia is the final complication when the patients are not slaughtered.

In the cases comprised under the second heading the symptoms appear more slowly and develop insidiously, the chief, viz., a tendency to remain lying and difficulty in movement, long preceding the appearance of exudative arthritis. The course depends chiefly on the nature of the infective agent. In calves suffering from peripneumonia, calves from stables in which epizootic abortion rages, rachitic calves or calves suffering from severe diarrhœic enteritis, the joints do not become the seat of suppuration.

In such cases the arthritis is of a simple exudative character, but without microbic infection of the joint cavity. This form is less dangerous, and is often curable provided the original cause be kept in mind.

Lesions. The lesions are always very marked, and are quite different from those of simple rheumatism. The synovial membranes and the periarticular tissues are always thickened, injected, inflamed, and infiltrated.

In more benign cases the synovial exudate from the joint cavities is simply cloudy, contains no infectious germs, and proves sterile on attempts at cultivation. In such cases there is no abscess formation. But most frequently this stage of serous exudation is only temporary, and the articular fluid, which at first seems sterile, may, when tested some days later by means of cultures, reveal the presence of organisms. The synovia accumulated within the joint sometimes contains fibrinous flocculi, which are at first small, but later form veritable coagula, filling up and thickly coating all the prominences of the joint and moulding themselves on the extremities of the bones constituting the articulation. Sometimes the cartilages undergo very rapid ulceration, the subjacent layers of bone become inflamed, and the osteoarthritis which develops is so severe and painful that the patients are forced to remain absolutely still, and are quite incapable of rising. The lesions may D.C. 
remain stationary at this point. In other cases suppuration occurs in the articulation itself, the wall of the synovial cavity, the periarticular tissues, and the skin soften; then the abscess breaks, giving rise to open suppurating arthritis. Animals are rarely kept long enough to become so gravely affected; many die before this stage, and the others are usually slaughtered. Moreover, they rapidly perish from exhaustion and from visceral complications of a pyæmic character.

In many cases post-mortem examination reveals nothing whatever in the region of the umbilical cord (through which infection has occurred), but the germs of infection may be found in the blood or general circulation; or again, careful investigation may show ulceration of the umbilicus, lesions of omphalitis, of ascending umbilical arteritis in

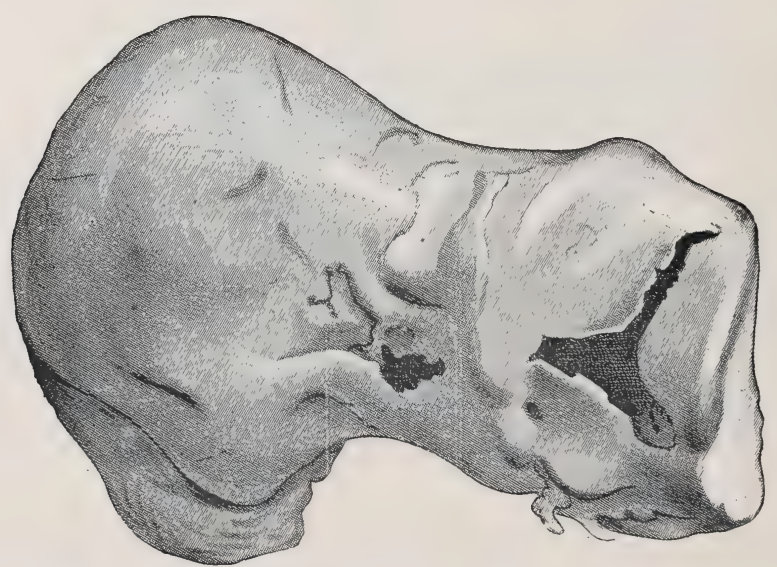

FIG. 52.-Ulceration of the articular cartilage in infectious rheumatism.

consequence of infection of the thrombus, of umbilical phlebitis, or of infective peritonitis, etc. The infectious agent reaches the liver through the blood-vessels, then attains the posterior vena cava, after which the infection assumes the gravest possible character, producing complications like arthritis and purulent infection, with the formation of multiple abscesses in the depths of the viscera.

The Staphylococcus aureus and various streptococci are the most frequent but not the only causes of these infections.

The diagnosis is not difficult, provided the disease be not mistaken for true rheumatism. As true rheumatism is very rare in young animals, and as, on the other hand, attention is aroused by the presence of lesions of the umbilicus and by the existence of diarrhœic enteritis, rachitis, etc., there is seldom room for doubt.

Prognosis. The prognosis is extremely grave whenever the case 
results from infection of the umbilicus. French statistics place the mortality at 90 per cent. and German at 75 per cent.

Curative treatment can only be attempted with any chance of success in the simple exudative form. Even then it is necessary to simultaneously treat the primary disease, such as rachitis or diarrhœic enteritis.

The recommendations of former practitioners as to the use of saline purgatives, cream of tartar, etc., were probably due to their having recognised that diarrhœa is sometimes the primary cause.

Moussu has seen simple exudative arthritis in rachitic subjects disappear, together with the rachitis, under proper treatment.

The indications therefore are, firstly, to take measures against the primary disease, treating the local lesions separately with blisters, douches, or simply cold applications and massage. Provided the general condition can be improved, recovery may follow.

Unfortunately, this treatment is useless against infectious rheumatism with suppurative arthritis resulting from infection of the umbilicus. In such cases treatment, if undertaken, should be directed towards perfectly disinfecting the umbilical wound or any existing sinuses.

Injections of strong carbolic solution, the application of antiseptic ointments or of antiseptic pencils containing iodoform, salol, etc., into the sinuses, followed by a surgical dressing covering the umbilicus, form the basis of this primary treatment, which, it need scarcely be said, has little chance of checking the course of already existing arthritis. The use of internal antiseptics and of antipyretics like camphor, salicylate of soda, etc., is worthy of trial. On the other hand, prophylactic treatment in an infected area has every chance of succeeding. The use of dry, clean litter under the mother and the new-born calf, thorough cleansing of the umbilical cord or umbilical cicatrix, and the application to the umbilicus of a small surgical dressing or even a smear of tar, almost always suffice to prevent the occurrence of these forms of arthritis.

\section{INFECTIOUS PSEUDO-RHEUMATISM IN ADULTS.}

The infectious pseudo-rheumatism of adults differs from infectious rheumatism in young animals in that it never becomes complicated with suppurative arthritis, and rarely affects more than one joint at a time. 'I'he hind limbs are the parts usually attacked, and the joints seem predisposed to disease in the following order of frequency: the femoro-tibial, coxo-femoral, and hock joints.

On account of its greater frequency in cows, it has been termed "arthritis of milch cows" and "infectious arthritis of milch cows," etc. In reality it may also attack bulls and oxen, but such cases are exceptional. 
Causation. First mentioned by Coulbeaux in 1824, and by Pauleau in 1832, this disease has been well-studied by $\mathrm{Ph}$. Heu. Old works mention it as attacking good milkers in the best dairies around Paris, and Heu declares it to be the most deadly disease after peripneumonia and tuberculosis.

This form of arthritis usually appears in an insidious form after abortion, retention of the fœetus, or post-partum metritis. In cases of epizootic abortion infectious rheumatism sometimes assumes an epizootic form, and completes the devastation begun by abortion. Under other and much rarer circumstances it may follow enteritis of adults or attacks of mammitis, etc. The pathogeny of these forms of arthritis is not

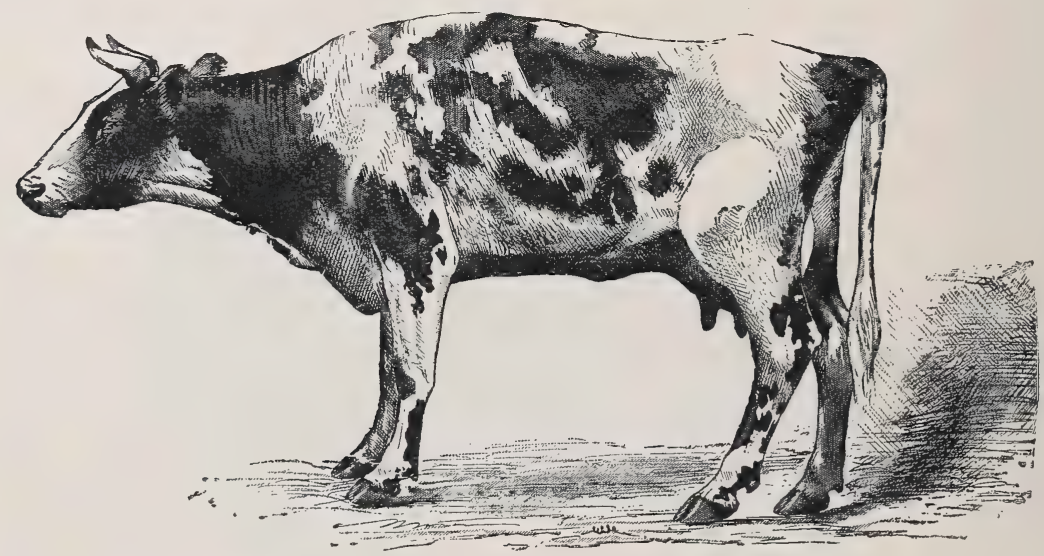

FIG. 53.-Infectious rheumatism. Arthritis of the left stifle joint.

difficult to understand, for in the greater number of instances they form delayed consequences of local uterine infection.

The soluble products secreted by infectious organisms multiplying within the uterus are absorbed by the uterine mucous membrane, causing slow intoxication; and in consequence of the special elective affinity which the toxins show for the articular serous membranes, and in many cases also for the visceral serous membranes, the special characteristics are developed. Under certain circumstances the joint cavity may even become the seat of true microbic infection.

Symptoms. The appearance of the first symptoms is difficult to identify, for many cows abort, fail to "cleanse," or become affected with metritis without infectious rheumatism supervening. It is a long-delayed condition, which may be postponed for weeks or even for several months after an abnormal parturition, and to a time when the symptoms of metritis have almost entirely disappeared. The onset is characterised 
by difficulty in rising, and soon afterwards by lameness, or by the animal failing to place weight on one of the hind limbs. The affected joint, usually one of the articulations of the stifle, appears markedly enlarged, is not appreciably hot to the touch, but reveals a certain amount of

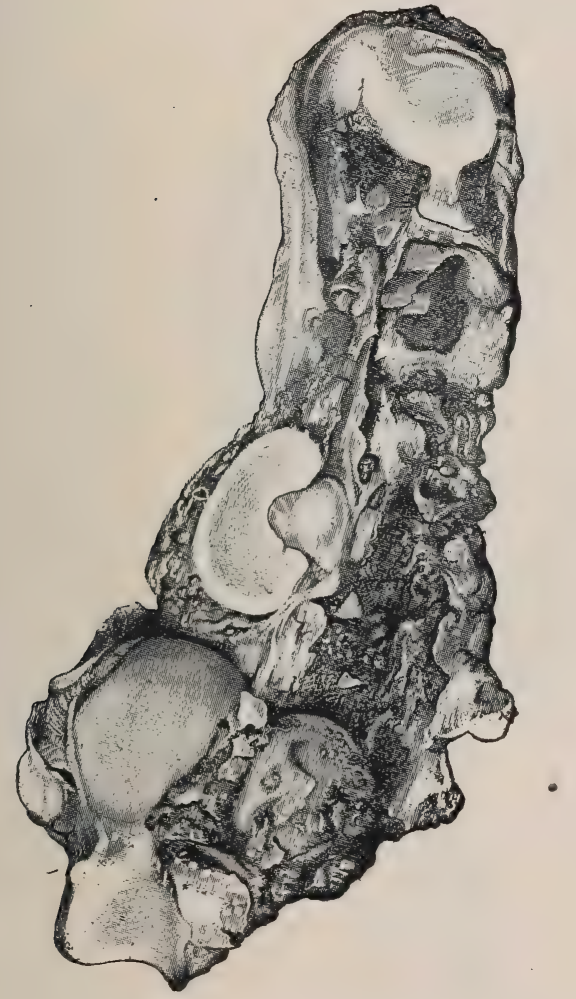

FIG. 54.-The femoro-tibial joint in a case of infectious rheumatism. All the articular surfaces of one side have undergone change (the condyle of the femur, interarticular cartilage, and the upper extremity of the femur).

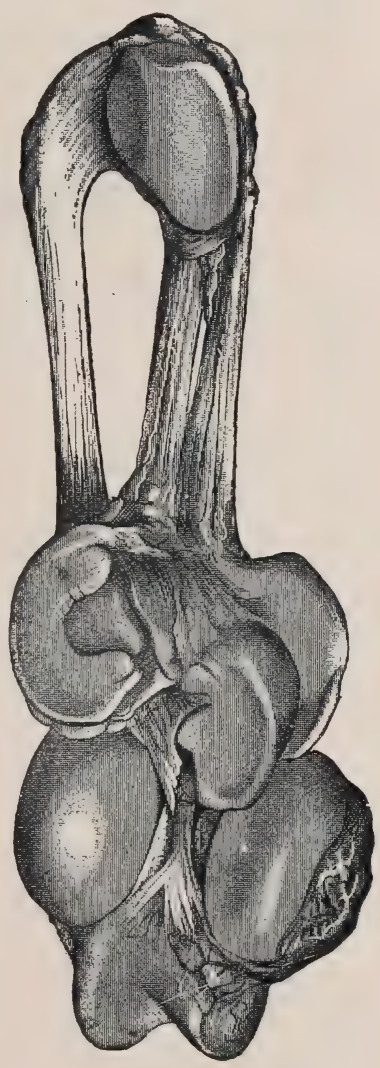

FIG. 55.-Normal femoro-tibial joint.

painful sensibility on pressure. The periarticular tissues are infiltrated and the synovial sacs slightly distended.

After a few days, a week or two at most, the periarticular swelling diminishes, and the condition appears to remain stationary.

Appetite is normal or only diminished. The pain continues, and causes progressive wasting and diminution in the yield of milk.

If at this time the practitioner makes a careful digital examination of the diseased region, he may find one of two conditions. In the first, the 
synovial sacs appear distended, fluctuating and in exactly the same condition as in ordinary bog-spavin. This is what has been termed the exudative form of infectious arthritis. In the second, the enlarged joint remains very sensitive, the walls of the synovial sac are thickened, fluctuation is either absent or only slightly marked, but induration is very manifest. This constitutes the plastic form.

Exudative arthritis is the form usually seen at first. It may preserve

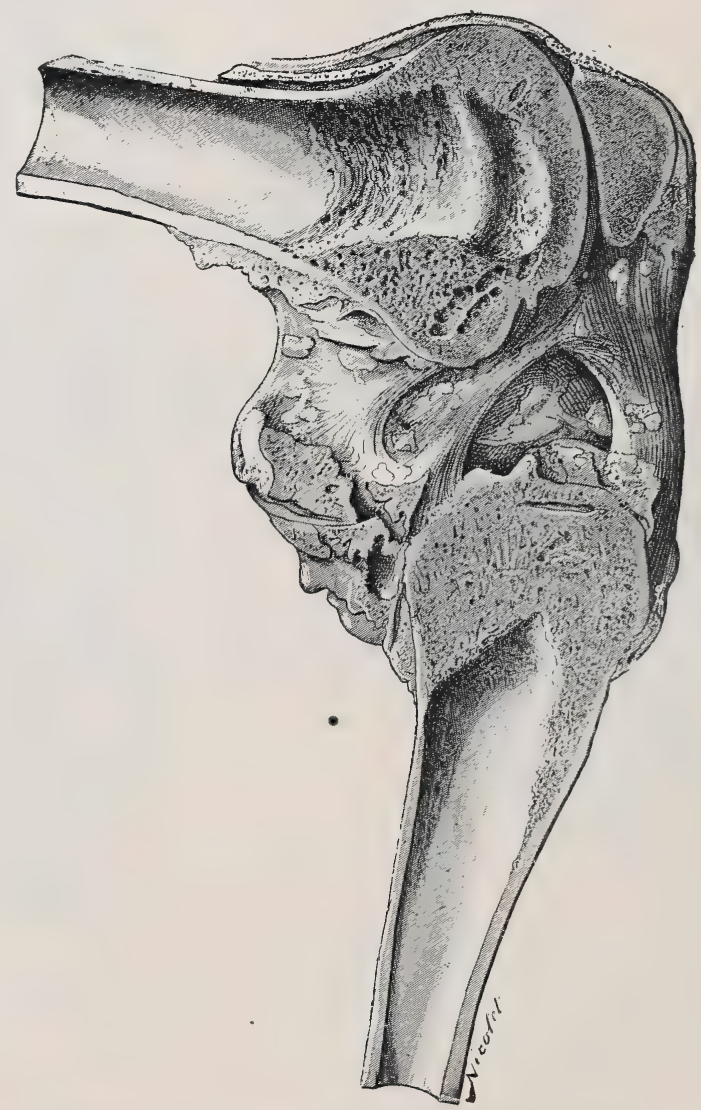

FIG. 56.-Plastic form of infectious rheumatism (pseudo-anchylosis).

its primary characteristics, but only too often proves to be the forerunner of the plastic form, which develops with the lapse of time. If nothing is done wasting becomes more marked, and is accompanied by cachexia. The animals are unable to rise, the complications inseparable from decubitus occur, and wasting or secondary purulent infection sets in.

Lesions. In the exudative form the changes are confined to inflam- 
mation and thickening of the synovial membranes, intra-articular exudation, and sometimes grooving of the cartilages without ulceration of the articular surfaces and without disorganisation of the joint.

The plastic form, on the other hand, leads to destruction of the cartilages, ligaments, and sub-cartilaginous bony layers, induration and calcification of the walls of the synovial cavity, and even periostitis of the ends of the bones, with the formation of false joints. The internal surface of the inflamed synovial membranes begins to granulate, the fibrous clots distending the articular dilatations are perforated by these extending granulations, and fibrous tracts soon develop even in the articulation itself, undergo calcification, and produce complete anchylosis.

Diagnosis. The diagnosis is easy. The animal's history and symptoms, and the stationary character of the pain in the earlier stages are sufficient to prevent any error.

Prognosis. The prognosis is grave, but not fatal. In the exudative form recovery may follow early treatment. In the plastic form, however, the chances of success are extremely meagre.

Treatment. It is easier to prevent than cure, particularly in these forms of arthritis. The means are simple, and consist in always treating the post-partum infection as soon as it is recognised. The animals can be effectively safeguarded against later articular complications by the free use of intra-uterine antiseptic injections, until the uterine injuries have wholly healed, and by the administration of saline purgatives and diuretics.

When infectious arthritis is diagnosed, it is still necessary to resort to the same methods if the uterine symptoms persist, and to complete the treatment by local applications.

The best method of local treatment seems to consist in puncturing the articulation aseptically, removing almost all of the liquid exudate, and immediately thereafter firing the skin covering the joint in points or lines.

If treatment has been invoked too late, if plastic arthritis with the formation of fibrous bands within the joint and destruction of the cartilages and calcification of ligaments, etc., already exists, there is no economic object in undertaking treatment. Fattening may be attempted, or the animals may be handed over to the butcher, if wasting is not too far advanced.

The use of cold douches, plaster bandages, blisters containing nitrate of mercury, painting with sulphuric acid, etc., are too inconvenient and too inefficient to be recommended in actual practice. Similarly, the salicylate of soda, which is so useful in simple rheumatism, has no real superiority over diuretics in this condition. 


\section{SCURVY-SCORBUTUS.}

Definition. "Scurvy is a subacute or chronic trophic disorder characterised by debility, inanition, anæmia, swelling and bleeding of the gums, gingival ulceration, dropping of the teeth, and petechial or more extensive hæmorrhages and exudations in the skin, serosa, and solid tissues."

Causes. "Among the lower animals, pigs especially suffer, when kept in close, foul quarters and fed on a monotonous and insufficient ration. Formerly scurvy . . . was attributed to an exclusive diet of salt food; to excess of sodium and deficiency of potassium salts; to the absence of fresh vegetables; to tainted food, etc. In pigs the food and environment are usually chiefly at fault, the subjects have been kept . . . in foul buildings, in a hot, moist atmosphere, and with an uniform diet of maize or other unvarying and insufficient ration. Röll attaches great importance to putrid food. Benion has found the affection mainly in obese swine, the forced feeding and intestinal fermentations manifestly operating as factors. Hess and others attribute the disease in pigs to the germ of erysipelas. Stengel has produced purpuric disease in animals by inoculation of the extravasated blood from human scurvy patients. Müller and Babes found a slender bacillus and streptococci in the tissues of scorbutic gums. . . . There is considerable presumption of the existence of a microbian cause, the efficiency of which is dependent on the unhygienic conditions above stated, while these unwholesome conditions are equally non-pathogenic in the absence of the microbe."

Lesions. "The blood is black and incoagulable or clots loosely, rigor mortis is slight, changes may be found in the number and character of the white and red blood globules, but are not constant; there is usually an excess of sodium salts and deficiency of potassium ones, and there is marked petechiation of the skin, mucosæ and serosæ. The bone marrow may be abnormally red and the bones fractured at the epiphyses, or carious. . . . The gums are softened, swollen, red and uneven, with hæmorrhagic discoloration, erosions, necrotic areas and ulcers."

Symptoms. "Anorexia or fastidious appetite, prostration, debility and sluggish, indifferent movements, are followed by the local lesions on the skin and gums. On the skin appear petechiæ and extravasations, which often implicate the bristles, so that they may be shed or pulled out with ease, the bulbs appearing dark and bloodstained (bristle rot). These may be followed by necrotic sloughs, and deep ulcers that are slow to heal. The gums are red and swollen, with hæmorrhagic spots, and bleed on the slightest touch. Erosions, sores, and ulcers are not uncommon; the tongue is dry and furred, and the mouth exhales a fœetid odour. The teeth may become loose in their sockets. Swelling of the 
joints . . . may be noticed, and lameness or stiffness from muscular or inter-muscular extravasation. Blood effusions into . . . the eye have been noticed, and paralytic or comatose symptoms from similar effusions on the brain. In the absence of improvement the patient becomes more and more debilitated and exhausted, and death may be preceded by profuse exhausting diarrhœa."

"Prognosis is unfavourable in advanced cases, and when the faulty regimen cannot be corrected."

Treatment. "The first consideration is to correct the unwholesome conditions of life, purify the building and its surroundings, and allow a free range on pasture. Subject each patient to a thorough soapy wash, and if possible allow clean running water in which a bath may be taken at will. Access to green food and invertebrates (slugs, larvæ, etc.) is important, or a varied diet of grain, middlings, bran, roots, fruits, tubers, cabbage, silage, etc., must be furnished. Iron and bitters (gentian, nux vomica) are useful, and sometimes small doses of arsenite of soda solution or cream of tartar are useful. Acorns or horse-chestnuts are recommended. For the mouth a wash of potassium chlorate, soda biborate, or potassium permanganate may be resorted to. . . . In the case of fat pigs it is more profitable to butcher at once, as soon as early symptoms appear." From Law's "Veterinary Medicine," p. 558, Vol. III. 


\section{SECTION II.}

\section{DISEASES OF THE DIGESTIVE APPARATUS.}

\section{SEMIOLOGY OF THE DIGESTIVE APPARATUS.}

The group of diseases which affect the digestive aparatus is one of the most important in bovine pathology, because almost all animals of the bovine species are bred with the object of utilising to the full their powers of digestion and assimilation.

Whether we consider adult fat animals, calves intended for slaughter or milch cows, the object sought is always the same-i.e., to secure the greatest possible economic return through the medium of the digestive functions.

Even although in working oxen there is no tendency to overfeeding, the animals remain none the less predisposed to diseases of the digestive apparatus; the meal-times are often too short, and rumination has to be performed under the yoke or during work-in a word, under unfavourable physiological conditions.

Semiology. To ensure correct diagnosis it is necessary here, perhaps more than in any other department of pathology, to be capable of grasping the symptoms or syndromes and signs afforded by the different parts of the digestive apparatus; to know how to co-ordinate and group them so as logically to deduce the final synthesis, the diagnosis. The diagnosis proving correct, the prognosis becomes easy, and this is the chief object from the economic standpoint. The practitioner who undertakes treatment knows how to deal with the case, and the owner likewise knows what he undertakes to do.

Although this classification may appear arbitrary, we shall consider successively diseases of the mouth, of the pharynx, œsophagus, stomach, intestines, etc., firstly describing the symptoms characterising these diseases. At the same time we should state that many symptoms are common to a large number of diseases and in themselves have absolutely nothing characteristic. They are simply sign-posts capable of showing the way.

Mouth. External examination reveals the condition of the muzzle, the lips and their commissures, and the surroundings of the buccal 
opening, and detects the existence of any desquamation, rents, eruptions, ulcerations, etc., which may be present.

In quiet animals the practitioner can examine the cavity of the mouth single-handed, but in troublesome animals it becomes necessary to have an assistant, who seizes the muzzle with one hand and the tongue with the other, or who simply fixes the animal's head. In exceptional cases it will be necessary to secure the patient to a post, tree, or wall. The mere attempt at examination will show whether there is trismus or absolute freedom of movement in the jaws.

By introducing the fingers between the commissures and applying them to the bars or to the free portion of the tongue, the practitioner will be able approximately to estimate the local and general temperature. The sensations experienced will also inform him of the degree of moisture or dryness of the mouth and of its sensibility.

On separating the jaws, he will note the odour exhaled and its possible abnormalities -its acid, sourish, fotid, or putrid character. He will directly observe any anæmia or hyperæmia of the mucous membrane, from the inner surface of the lips and cheeks up to the soft palate, although owing to the thickness of the buccal epithe-

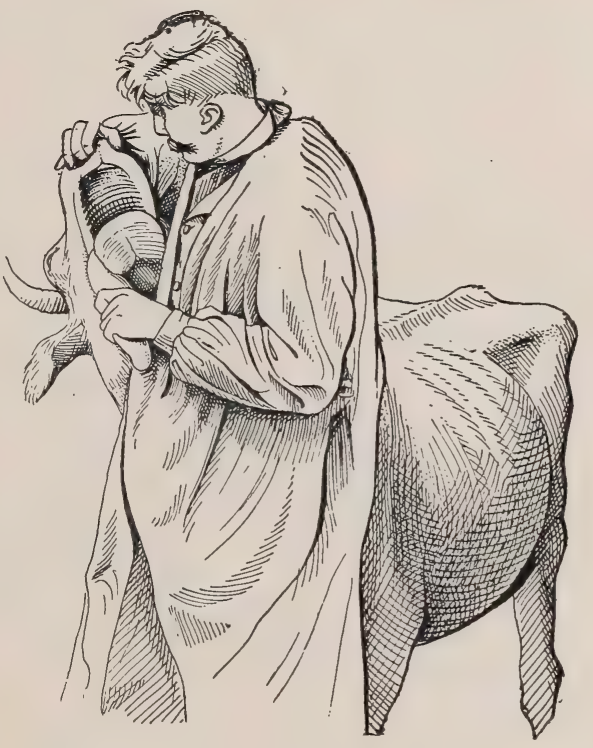

FIG. 57.-Examination of the mouth. lium it is not always easy to estimate anæmia or hyperæmia in the ox. The surface of the tongue should also be examined, and a note made whether it appear dry, pasty, dusty, sooty, etc., though these appearances are occasionally apt to lead one astray. The observer should also inquire regarding want of appetite, depraved or exaggerated appetite, etc.

Even the manner in which the animal picks up its food will serve to direct his attention to the development, or possible existence, of some disease of the mouth, although want of appetite is not always characteristic of a lesion in the pharynx or œsophagus, but sometimes of a lesion in its neighbourhood, like hypertrophy of the retropharyngeal or bronchial lymphatic glands.

This examination will also detect the existence on the lips of wounds, 
cuts, injuries or specific eruptions (aphtha, tuberculous ulcerations, the ulcerations of gangrenous coryza, etc.) on the gums indications of gingivitis, periostitis, mercurial poisoning, actinomycosis of the maxilla, and ulcerations of all kinds; on the tongue, of wounds, of simple or specific inflammatory eruptions (aphtha, the ulcerations of actinomycosis, tuberculosis, gangrenous coryza, etc.), as well as the swellings due to superficial or deep-seated glossitis. By the same method of examination, though with somewhat more difficulty, one can detect abnormal mobility, irregularity of development, caries, etc., of the teeth, the condition of the excretory ducts of the salivary glands, the state of the hard and

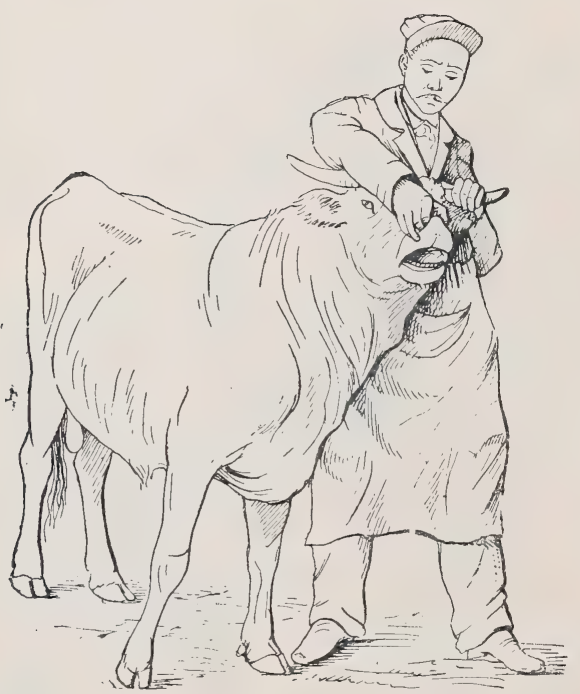

FIG. 58.-Examination of the mouth. soft palate, and the existence of fissures, vegetations, polypi and tumours.

Salivary glands. The salivary glands, particularly the parotid and submaxillary, should be examined by direct inspection and palpation.

Direct inspection reveals the existence of swellings, deformity of parts, increase in salivation, or ptyalism, which sometimes occur in conjunction with foot-andmouth disease, actinomycosis, acute stomatitis and mercurial poisoning, as well as increase in size of the salivary ducts.

Palpation reveals the degree of sensibility of the parts, the existence of cedema, induration, cysts, and, more frequently, distension of the salivary ducts as well as the presence of calculi, tumours, the direction of fistulæ, etc.

Difficulties may occur, particularly when the submaxillary and parotid glands are affected; but methodical and complete examination will usually enable one to differentiate the conditions.

Pharynx. The pharnyx may be examined externally by inspection and palpation, and internally by direct digital palpation. Inspection reveals possible deformities of the region of the gullet, palpation the condition of the tissues as well as abnormal sensibility and infiltration. Internally, digital examination must be cautiously conducted, and after a strong gag has been securely inserted in the mouth. Under such conditions it is without danger. The hand being inserted exactly in the median line will detect obstructions which may already have been partly identified by external palpation, as well as the existence of inflammation 
with or without the formation of false membranes, and of ulcerations, polypi, ete.

Esophagus. In consequence of its anatomical formation, sitụation and course, the cesophagus may be divided into two distinct parts-viz., the cervical, which can be examined from the outside, and the thoracic, which cannot so be examined.

The cervical part may be examined by inspection, by palpation from one side, or by palpation with both hands and from both sides.

Inspection leads to the detection of changes in the shape of the œsophagus and of the jugular furrow. In fat subjects, however, it is of

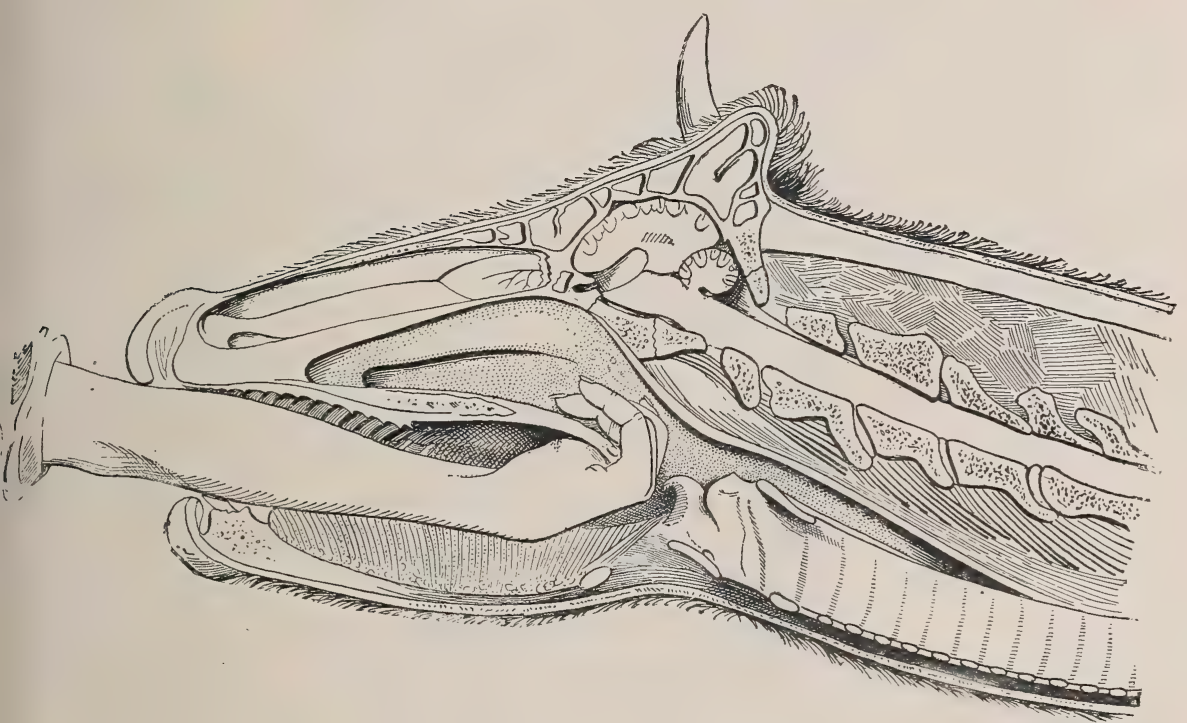

FIg. 59.-Examination of the pharynx.

little value. As the position and the course of the osophagus are known, unilateral palpation, or, better still, bilateral palpation, employing both hands, is of very much greater service. These methods reveal the presence of swellings, infiltrations, changes in shape and sensibility, the presence of foreign bodies, the existence of dilatations or contractions of the tube, etc.

Auscultation and percussion, though recommended by some practitioners, are not of much service.

Inability to swallow, due to change in the cesophagus, is also detected by inspection. Its existence suggests a number of possible conditions, such as fissure or ulceration of the esophagus, compression in the mediastinal region as a result of tuberculous or other disease, contraction or dilatation of the œsophagus, etc. Furthermore, inspection will betray 
the existence of dilatation of the tube, to which vomiting and regurgitation of food are sometimes due.

Internal exploration is the only method of detecting changes in the thoracic portion, and may also be utilised to locate lesions in the cervical region. It is practised by passing a sound of small calibre or any flexible cylindrical object, such as a cart rope, etc. The patient must be fixed with the head extended on the neck and a proper gag or speculum introduced into the mouth. Exploration assists us in recognising the existence of inflammation of the osophagus, true or false contraction, dilatation and the presence of obstructions.

In animals of the bovine species all these lesions-viz., inflammation of the œsophagus, fissuring and ulceration, obstructions, compressions, dilatations and contractions of the tube-although not very frequent, are nevertheless from time to time encountered.

Stomach. Exploration of the stomach or of the different gastric compartments presupposes an exact knowledge of the respective positions of the different reservoirs. Topographical anatomy shows that the rumen is situated in the left flank, and that it occupies the whole of the left abdominal region from the diaphragm to the pelvic cavity. As a consequence, it may be explored from the region of the twelfth rib; it is inclined slightly from above downwards, and from left to right, its extreme right border extending as far as, or a little beyond, the white line.

The reticulum, the smallest of the four reservoirs, is situated in the sub-ensiform region at right angles to the median plane of the body. On the left it touches the rumen and the diaphragm; on the right side it is in contact in front with the diaphragm, above with the omasum, and to the right and towards the rear with the abomasum. The omasum is situated above the reticulum and conical right portion of the rumen; in front it touches the liver, and towards the back and left the rumen. The abomasum is situated obliquely in the right hypochondriac region, its anterior surface resting on the lower wall of the abdomen towards the middle and right side of the body, its pyloric portion extending upwards, behind the right hypochondriac region.

Rumen. The rumen can be examined by inspection, palpation, percussion, and auscultation. The use of the cesophageal sound and of the trocar and canula is also of value in diagnosis.

Inspection affords information of a varying character, according to the moment when it is practised, even in a condition of health. It only extends to the condition of the flank before or after a meal, etc., emptiness of the rumen being accompanied by hollowness of the flank, and distension, following an abundant meal, by fulness in this region.

When digestion is not proceeding normally, the flank may be distended unduly by gaseous accumulations or by the presence of solid food. In 
abdominal and mediastinal tuberculosis and in gastro-enteritis there may be simple tension or slight dilatation. When indigestion or enteritis is

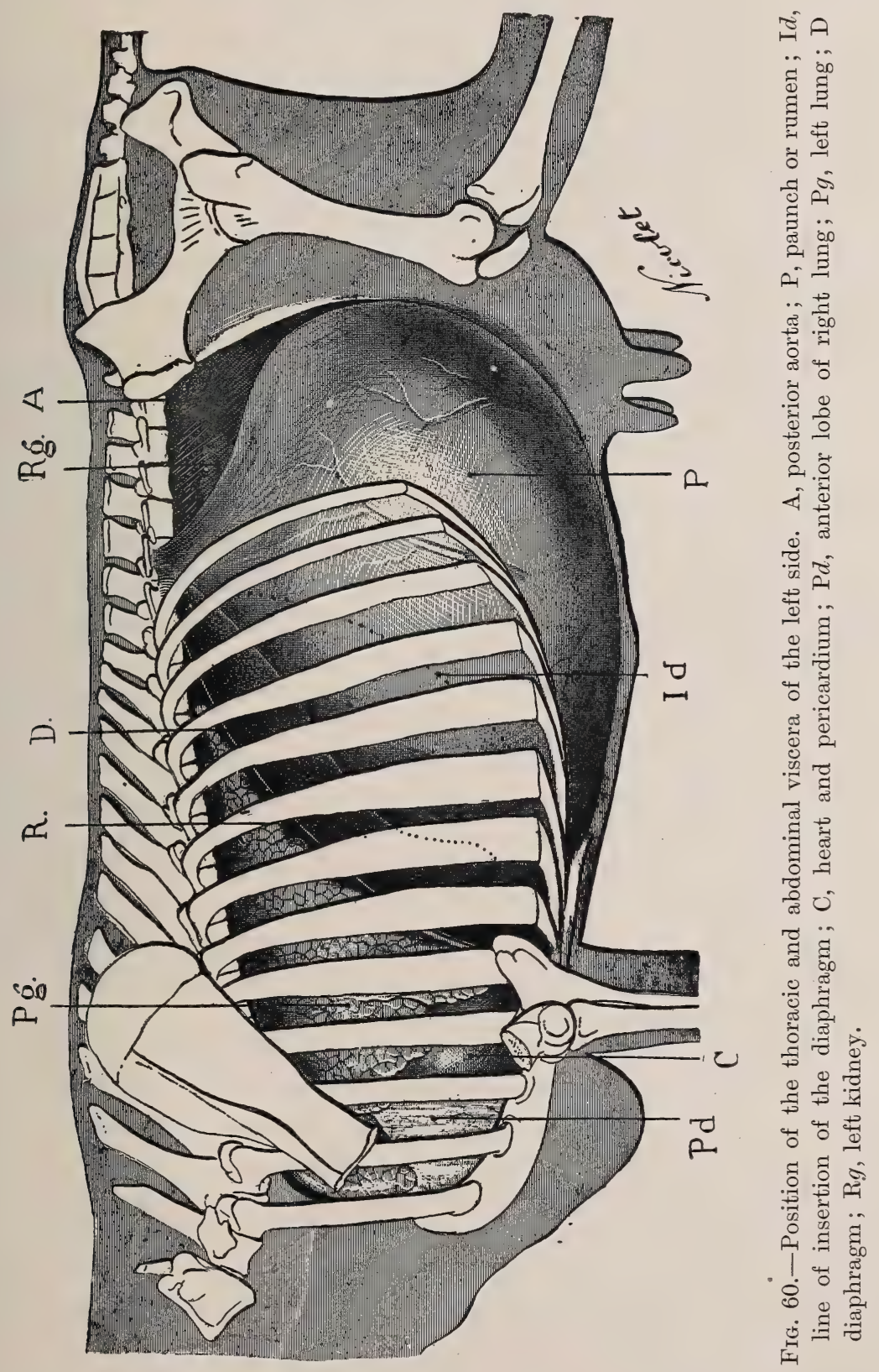

entering on a favourable stage, the flank may appear hollow, and in cases of chronic diarrhœa it may appear retracted. 
Digital examination or palpation may be practised over the entire region of the flank. It shows whether the rumen is full or empty, reveals the consistence of the contained food in cases of chronic indigestion, the sensibility of the walls, and the rate and order of the muscular contractions. Direct or indirect percussion may be carried out on a horizontal line from the twelfth rib as far back as the flank, and vertically from the lumbar vertebræ to the white line. In health one discovers in young animals an upper zone of normal resonance due to gas, a zone of semi-dulness and an inferior zone of absolute dulness, due to the liquids in the rumen. The spleen, which is attached to the supero-anterior surface of the left side of the rumen, does not seriously restrict the area open to percussion.

In pathological conditions percussion from above downwards may produce a tympanitic sound, due to gaseous indigestion or a clear sound throughout the greater portion of the vertical diameter suggestive of acute gastro-enteritis with the formation of gas in the rumen, or of adhesive peritonitis preventing the rumen from collapsing. Indigestion due to excess of solid food, on the contrary, is characterised by a dull sound throughout the entire region from above downwards. Percussion along a horizontal line permits of the delimitation of certain zones which vary a great deal in area, according to the case.

Auscultation is more instructive than percussion. Like percussion, it may be practised throughout the entire depth of the abdomen, from the transverse processes of the lumbar vertebræ as far as the white line, and in a horizontal direction from the eleventh rib to the region of the flank.

Auscultation of the upper zone enables one to detect sounds of deglutition, gurgling sounds (glou-glou), and a sound resembling falling water, due to the movement of solids or liquids in the rumen and reticulum. The sounds heard vary in different cases, and depend on the state of repletion or of emptiness of the rumen.

Auscultation of the middle zone reveals:

Firstly, a very special crepitation sound, which may be compared to the deflagration of a handful of salt thrown on burning coal. It is believed to result from the bursting of bubbles in the contents of the rumen under the action of normal digestion.

Secondly, a churning sound produced by the rhythmic peristaltic contractions of the rumen, by which the substances ingested are very intimately mixed. By applying the ear over the flank region or by palpation the rhythmic contractions of the rumen, two per minute in most cases, can readily be perceived.

In practice examination of the rumen is confined to these four methods.

Puncture. From an exclusively scientific point of view, exploration of the rumen also comprises analysis of the gas collected through puncture 
and analysis of the liquids removed by aspiration (first stages of gastric digestion). Normally, these gases, in the order of their abundance in the mixture, consist of the following: Carbonic acid, carburetted hydrogen and nitrogen.

In disease, and in most cases of abnormal fermentation, the carburetted hydrogen is greatly in excess of the carbonic acid. In chronic gastro-enteritis, ammonium sulphide and other offensive gases are found in addition.

Chemical analysis. In the rumen the ingested food is macerated in an alkaline liquid at a temperature of $100^{\circ}$ to $101^{\circ} \mathrm{Fahr}$. (the alkalinity is due to the saliva). This process markedly modifies the composition of the ingested matter. Nevertheless, the upper portion in contact with the gas sometimes presents a slightly acid reaction, probably due to carbonic acid. The sugary and fatty materials contained in the food respectively undergo lactic and butyric fermentation. Only a small quantity of the starch, however, is transferred into sugar. In the calf, and in very young animals, the reaction of the rumen is acid throughout the entire period of sucking. In disease, when rumination has long been suspended and chronic loss of appetite or gastro-enteritis exists, the reaction is generally acid. The sugars, gums, and soluble salts of forage, roots, etc., are dissolved in the rumen, but fatty materials undergo no modification.

The reticulum, which is the smallest of the gastric compartments, is situated in the sub-ensiform and retro-diaphragmatic regions, extending right and left of the middle line to a nearly equal distance. Above and to the left it communicates freely with the rumen, to the right with the omasum.

In practice it can only be examined in two ways: inspection and palpation.

By inspection changes in the configuration of the ensiform region may sometimes be detected. Such changes are rare, and must be distinguished from congenital deformity. They sometimes accompany inflammation of the reticulum produced by a foreign body, when the lower abdominal wall is directly perforated by such body.

In cases of inflammation of the reticulum due to foreign bodies, abscess formation, perforation, etc., it is possible to detect œedematous infiltration, abnormal sensibility, fluctuation and increased heat, etc., by manipulating the parts with the fingers or the clenched fist.

If the evidence pointing to the presence of a foreign body in the reticulum is considered sufficient, gastrotomy may be performed and the interior of the viscus examined with the hand, but although the operation is possible it is very rarely practised.

Omasum. The omasum occupies, so to speak, a position inverse to that D.C. 
of the reticulum, lying deep down on the right side, behind the diaphragm, under the hypochondrium, and above the abomasum and reticulum.

It is the only gastric compartment which cannot be examined, although when impacted it may be felt on the right side.

Abomasum. The abomasum is lodged in the lower part of the right flank under the circle of the hypochondrium. It extends obliquely from below, upwards from the sub-ensiform to the sub-lumbar region. The smaller curvature is turned towards the rumen on the left side; the larger curvature is in contact with the abdominal wall. In spite of what has so often been stated by those who have never seen it, the abomasum can be examined and is accessible along the circle of the hypochondrium.

In adults useful information can rarely be obtained by inspection; but in sucking calves the abomasum, if distended by indigestion, gastroenteritis, etc., sometimes appears prominently in the right abdominal region. Palpation with the fingers or with the fist will detect exaggerated sensibilty, irritation, inflammation, or distension.

Percussion and auscultation furnish no very precise information. The information obtained by the above-described examination of the stomach is in practice amplified by a search for certain symptoms which are usually easy to detect. They comprise :

(a) Suppression or irregularity of rumination. This very important symptom suggests the degree of gravity of the digestive disturbance, and to some extent the gravity of the general condition. Suppressed rumination is a common symptom in many diseases, some of which are purely digestive, though all are not. It is, however, a grave sign in most cases.

(b) Eructation, which is usually frequent, may be regarded as normal provided the exhaled gas preserves the fresh odour of grass or of the food swallowed, like brewers' grains, turnips, etc. Sometimes the gas is sour, acid, fœetid, or putrid, all of which conditions indicate disease.

(c) Yawning is not common. It becomes frequent and attracts attention in certain abnormal conditions; in others, again, it may be completely suppressed.

(d) Nausea and vomiting are rare. Vomiting is commoner in calves, and results from inability to digest the milk, or simply to over-distension of the abomasum. The matter vomited by adults usually consists of partly masticated food, and is derived from the rumen; while the contents of the abomasum are occasionally rejected, in which case the material is of pulpy consistence and has an acid smell.

(e) Digestive disturbance is sometimes accompanied by various modifications in the breathing, such as immobilisation of the hypochondriac region and of the diaphragm; abnormal sensibility and reflex coughing on palpation, and, in inflammation of the reticulum due to foreign bodies, costal respiration. 
It is by methodically observing, grouping, and classifying the symptoms presented that one is enabled to detect the links connecting them.

Intestine. The intestinal mass is contained in the right half of the abdomen above the compartment of the stomach. The large intestine occupies the upper zone, corresponding externally with the hollow of the flank from the thirteenth rib to the haunch. The small intestine occupies the middle zone from the thirteenth rib to the entrance to the pelvis and the stifle region; the inferior zone is occupied by the rumen and abomasum, and in pregnant females by the gravid uterus.

Notwithstanding these indications it is somewhat difficult to examine the mass of the large intestine, separated as it is from the abdominal wall by the $U$-shaped inflection of the duodenal loop, of which the deep retrograde branch is in contact with the terminal portion of the floating colon.

Inspection of the right flank furnishes no information of value in diseases of the intestine, nor is auscultation of much service beyond enabling one to detect the frequency, diminution, or absence of borborygmus. Palpation alone is really of service. Practised gently and superficially with the tips of the fingers it detects abnormal sensibility in acute cases of enteritis; when with more energy, palpation reveals whether the bowel be full or empty, provided that the muscular resistance be not too marked.

Colic. In colic the clinical signs, their varieties, and the lesions which give rise to them are of much more importance. When it results from intestinal congestion à frigore (due, for example, to the ingestion of cold water), colic is usually violent, sudden, and of relatively short duration. In other cases it is violent and prolonged for several hours, a whole day, or even two days, and may be followed by coma and suppressed peristalsis ; it then indicates invagination, volvulus, or strangulation. Sometimes, on the contrary, it remains dull and is slow and continued (acute gastroenteritis, hæmorrhagic gastro-enteritis, etc.).

Finally, colic of the latter character may, in addition, be accompanied by icterus, in cases of retention of bile, biliary calculi, hepatitis, etc.

Anus. Examination of the anus is easy. Simple inspection reveals its presence or absence, and consequently the existence of congenital rectal atresia, which is somewhat common in calves and colts. Digital exploration is, however, sometimes useful, for in occasional cases an anus may exist, which externally appears normal, but terminates in a sac, the rectum being closed by a membranous partition.

Nothing is easier to recognise than tenesmus; it occurs in cases of profuse diarrhœa, diarrhœa of calves, and dysentery in newly-born animals.

Rectal exploration. Exploration of the rectum is a last and most 
valuable means of confirming the diagnosis in all visceral diseases of the
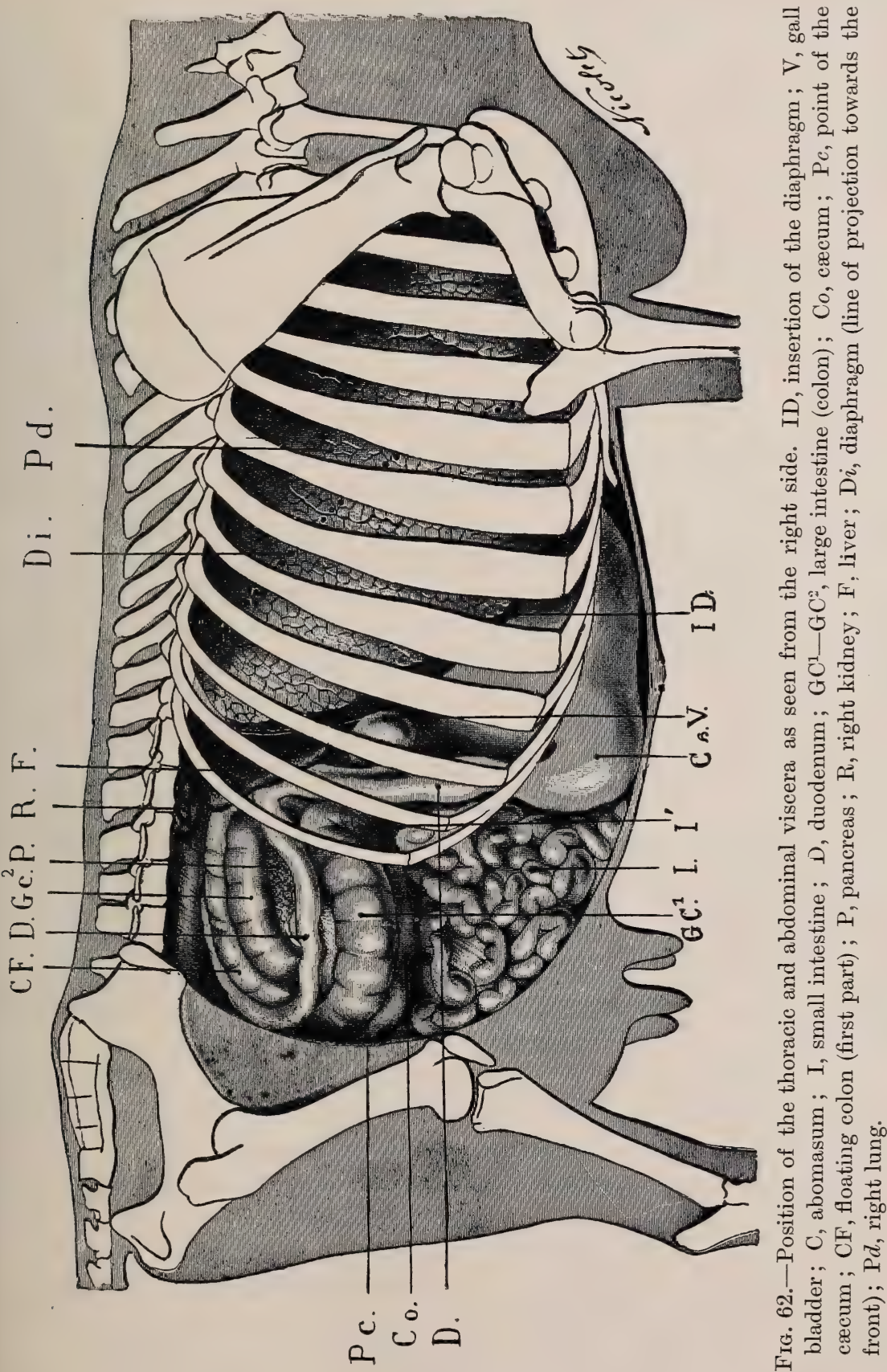

pelvis and abdomen. To utilise this method to the full, the rectal pouch 
should first be thoroughly emptied by the free use of enemata, the subsequent examination being made with great care. The animal's hind legs being secured, the operator smears his hand and forearm with some fatty substance, and, forming the fingers into a cone, introduces them with gentle pressure through the anus, the palm of the hand being turned downwards. Passing the hand gently along the rectum, the operator will be able to distinguish the conical posterior pouches of the rumen, the loop of the duodenum, the mass of convolutions of the small intestines and of the colon, etc. Next, he will examine the vagina, uterus, bladder, ureters, kidneys, aorta, and the pelvic and sub-lumbar lymphatic glands. He may be able to recognise distension of the rumen with food, twists of the intestine, herniæ, mesenteric or diaphragmatic invagination or volvulus of the bowel, etc.

In other cases he may be able to discover lesions of the kidney, of the uterus, of the broad uterine ligaments, of the ovaries, or of vessels.

In all cases it is desirable to make a methodical and complete examination, whatever the primary object may have been. Such an examination may be carried out as follows: The operator having introduced his hand into the rectum, begins by examining the state of the pelvic organs, the rectum, base of the vagina, the body and horns of the uterus, the bladder and the lymphatic glands and ligaments of the pelvis.

By laying the hand flat in the rectum and pressing gently downwards the anterior border of the pubis may be felt, somewhat more deeply placed. The rectum is then thrust slightly to the right, and the ascending branch of the right ilium, as high as the sacro-iliac articulation, and the lower surface of the sacrum, are directly examined; lastly, the hand is directed towards the left, gliding down the left ilium, and returning to the point of departure. In this way the state of the pelvic floor, of the arteries, veins, and lymphatic glands, etc., the degree of mobility, tension, or fulness of the uterus, as well as the condition of the broad ligaments, are all ascertained.

Still more deeply placed, and at the extreme limit to which the arm can be introduced, will be found some or all of the above-mentioned organs-viz., the small intestine, large intestine, kidney, etc.

Defæcation: Examination of the fæcal material. The character of the fæces is very important in certain diseases ; e.g., diarrhœa assumes a varying importance, according as the discharges are of an alimentary, serous, mucous, or sanguinolent type, and are slight, temporary, intense, profuse, or continued. In other cases defæcation is slow, becomes difficult, and various degrees of constipation exist. Defæcation may be completely suppressed, as in invagination or strangulation of the intestines; on the other hand, one may observe diarrhoea, dysentery (microbic 
or sporozoic diarrhœa), and intestinal hæmorrhage. The last-named may be of varying degrees of acuteness, from the passage of simple drops or streaks of blood, distributed over almost normal excreta, to the passage of unchanged blood in liquid jets or in clots.

Macroscopic examination. Macroscopic examination takes cognisance, firstly, of the quantity (40 to 80 lbs.), consistence (firmness or softness), colour (olive green, blackish green, greyish black, sooty, or tarry) and odour (normal, fœetid, putrid, etc.) of the fæces.

Sometimes the excreta are moulded and covered with glairy mucus, or contain such abnormal products as undigested food (a sign of chronic diarrhøea), false membranes, false membranes due to pseudomembranous enteritis, fibrinous clots, or parasites like liver flukes, tæniæ and strongyles.

Microscopic examination. Microscopic and bacteriological examination is sometimes useful; and even when macroscopic examination has revealed nothing, it is possible to detect the presence of the eggs of parasites like flukes, strongyles, hooked worms, etc., the presence of sporozoa (as in intestinal coccidiosis) and of specific microbes, as in the diarrhœa of calves, etc.

It is only by the synthesis of methodically collected signs that one finally succeeds in exactly diagnosing the numerous diseases which may affect the intestine: intestinal congestion, invagination, volvulus, intestinal strangulation (mesenteric or diaphragmatic herniæ, etc.), atresia of the anus, acute or hæmorrhagic enteritis, or intestinal helminthiasis.

Liver. The liver is situated in the right sub-lumbar region. It is fixed behind the diaphragm and under the hypochondriac region, and extends from the ninth to the thirteenth rib. It can be examined by palpation through the last intercostal spaces and behind the thirteenth rib. In health it is difficult to pass the fingers sufficiently under the hypochondriac circle to reach the liver; but in case of morbid hypertrophy it extends more or less beyond the last rib, and palpation between the last ribs sometimes reveals abnormal sensibility.

Percussion better than palpation enables one to delimit the space occupied by the liver, particularly towards the back, where there is no interposed layer of lung. Percussion is especially useful in detecting hypertrophy due to cancer, tuberculosis, echinococcosis of the liver, etc., or hepatic atrophy. In isolated cases icterus may exist, confirming the conclusions otherwise arrived at.

Pancreas. The pancreas is situated rather deeply in the right sublumbar region, below the kidney, behind the liver, above the floating colon, and within the duodenal loop. It is therefore very difficult to examine; moreover, the diseases which affect it are still little understood. 
The point most prominently brought forward by this demonstration of the topographical anatomy and semiology of the digestive apparatus is the difficulty of accurately diagnosing digestive diseases in the ox when one confines oneself to a superficial examination. To have any chance of arriving at an exact diagnosis, methodical and thorough examination is indispensable. Given this condition, accurate diagnosis becomes possible, despite all difficulties. 


\section{CHAPTER I.}

\section{DISEASES OF THE MOUTH.}

\section{STOMATITIS.}

Definition. By stomatitis we mean inflammation of the buccal mucous membrane. Stomatitis may be simple-i.e., due to accidental causes, to varying local forms of irritation, or to wounds; or, on the contrary, it may be specific, of infectious origin, like the stomatitis of foot-and-mouth disease, gangrenous coryza, cattle plague, etc.; or, finally, it may be of toxic origin, like the stomatitis of mercurial poisoning.

Here we shall only study the simple forms of stomatitis, the gangrenous stomatitis of calves, and mercural stomatitis. The others will be noticed in speaking of the diseases of which they form one of the symptoms.

*

SIMPLE STOMATITIS.

Causation. Simple stomatitis of bovine animals is often due to feeding with rough forage, or forage containing prickly or spiny plants, like thistles, sea holly, eryngo, etc. Sometimes it follows prolonged irritation by rough teeth, premolars or stumps, or accompanies the shedding of the temporary molars. Ingestion of irritant plants like nettles, certain specimens of the orders Labiate and Umbellifere, leaves covered with vesicant insects, cabbage and turnip leaves infested with aphides, oil beetles, etc., or the swallowing of hot liquids, may also, though more rarely, produce it. Finally, in grave diseases of the digestive apparatus, the buccal mucous membrane may secondarily become affected.

Symptoms. The primary symptoms are usually represented by ptyalism and a certain difficulty in grasping food. In other cases the mucous membrane appears slightly dry for some time before salivation sets in. On introducing the fingers into the animal's mouth some elevation of temperature may be noted, and on direct inspection the non-pigmented regions are seen to be abnormally vascular, a fact which 
has earned for this form of stomatitis the names of "erythematous" and "erysipelatous stomatitis."

If the stomatitis is due to local multiple irritation, such as results from spines and prickles in the food, the abnormal vascular appearance is confined to the neighbourhood of the abrasions or punctures, and the affected regions are of very varying size. In some cases, principally as a consequence of trifling local irritation and of burns of the first and second degree, blisters of varying size may develop and break, leaving behind ulcerations, which, however, always heal rapidly. The aphthous non-contagious stomatitis mentioned by certain authors seems most probably to pertain to this class.

Diagnosis. The diagnosis is usually easy, and a little attention to the accompanying symptoms is sufficient to avoid confusion with the various forms of symptomatic stomatitis.

The prognosis is favourable.

Treatment is based on removal of the determining cause, if this can be recognised, withdrawal of rough forage, removal of sharp points on the teeth, extraction of stumps, etc. As a rule, recovery follows rapidly. It can be hastened by washing out the mouth directly, or by means of a syringe, with water containing honey, vinegar, decoctions of brier twigs, oak bark, barley or rice. This treatment is completed by supplying nourishing gruel and food demanding little mastication.

\section{CATARRHAL STOMATITIS IN SHEEP.}

"The more delicate buccal mucosa in these animals would render them more subject to inflammations, but this is more than counterbalanced by the mode of prehension of aliments, not by the tongue, but by the delicately-sensitive lips, and further by the daintiness and care with which these animals select their food. The treatment would not differ materially from that prescribed for the ox." (Law's "Veterinary Medicine," Vol. II. p. 15.)

[The treatment referred to consists of simple astringent and antiseptic washes, borax given in the drinking water, or mixed with honey or treacle and smeared occasionally on the tongue. Washes of sodii hyposulphis or sulphis, or even weak solution of carbolic acid, may be used after the irritant cause has been removed. Vinegar or diluted mineral acids may be used alternately with decoctions of blackberry bark or other vegetable astringents. When there are symptoms of gastric disorder a laxative, followed by vegetable bitters and other tonics, may be prescribed. Foreign bodies-thorns, wire, etc.-fixed in the tongue, cheek, or palate should be searched for and removed at the first examination of the patient.] 


\section{NECROSING STOMATITIS IN CALVES.}

Definition. This name is applied to a special stomatitis, which in very young animals produces superficial necrosis of more or less extensive areas of the buccal mucous membrane, and sometimes of subjacent parts.

The disease, although somewhat rare in France, has been mentioned by Lafosse and well studied by Damman and Lenglen.

Causation. Its cause is still imperfectly understood. Some regard it as a consequence of insufficient nourishment, of disturbance resulting from dentition, of general exhaustion, and of bad hygienic conditions. These explanations are scarcely sufficient, however, and at the present time there is a tendency to regard it as a complication of primary grave, debilitating diseases, like the diarrhoea of calves, omphalitis, omphalophlebitis, etc. Moussu has never seen it apart from omphalitis, and he considers the lesions due to the action of the bacillus of necrosis. Infection occurs through the umbilicus becoming soiled by contact with the litter.

The early symptoms consist in loss of appetite, congestion of the mucous membranes, and salivation. Early examination of the buccal cavity reveals the presence everywhere, except on the palate, of whitishgrey or yellowish patches, whose aspect is markedly in contrast with that of neighbouring parts. These are fragments of the mucous membrane undergoing necrosis. They are numerous, are surrounded by a narrow inflamed zone, and may be from $\frac{1}{2}$ inch to 1 inch in diameter.

Necrosis advances rapidly, and extends throughout the thickness of the mucous membrane; the slough soon becomes delimited and separates. The odour of the mouth is then absolutely fœetid, and the saliva appears streaked with pus and blood.

The ulcerations exhibit a livid base, and show no tendency to heal. Necrosis extends in depth, and affects the muscles, tendons, and even the periosteum and the bones. The teeth are frequently loosened.

Grave complications, such as pharyngitis, broncho-pneumonia, infectious enteritis, and septicæmia soon appear, and the animals are carried off by septic infection and intoxication in a few days-at the longest in a week.

Diagnosis. The diagnosis is only difficult at first; and, at a later stage, the only mistake possible is that of confounding the condition with a very grave attack of aphthous stomatitis. Local sanitary conditions are sufficient to secure the avoidance of this error.

Prognosis. The prognosis is extremely grave; the disease usually results in death; recovery is exceptional. Very luckily the disease seems to become rare in direct proportion as the hygienic conditions of cattle breeding and keeping are improved. 
Treatment. Curative treatment always appears to be ineffectual when a grave primary disease has already enfeebled the animal before necrosing stomatitis appears. In those cases where the stomatitis constitutes the primary condition, attempts should be made as far as possible to cleanse the wounds. The buccal cavity should be thoroughly washed out, and the wounds then cauterised with solution of carbolic acid of 6 per cent. strength, nitric acid of 10 per cent. strength, hydrochloric acid of 7 to 8 per cent. strength, or chromic acid of 20 per cent. strength.

This local cauterisation is performed with a tampon of cotton wool fixed to a handle; for necessarily it is impossible to apply any dressing whatever of a permanent nature. The cauterisations may be repeated twice a day. The stump of the umbilical cord should never be forgotten, for it sometimes contains a mass of necrotic tissue the size of a man's thumb. It should be thoroughly cleansed and the wound plugged with a mixture of iodoform and boric acid.

This treatment will not avail unless the functions are stimulated by rich food that can be easily masticated and digested, and by giving milk of good quality, or boiled milk, eggs, meat-powder, cooked beans, etc. Aromatic infusions and hay tea containing coffee and small quantities of alchohol or tincture of quinine are also of service.

\section{MYCOTIC STOMATITIS IN CALVES.}

\section{Thrush. Muguet.}

"This is a form of stomatitis manifested by a raised white patch on the mucous membrane and determined by the presence of the Oidium albicans (Saccharomyces allicans), a cryptogam discovered by Berg in 1842 in thrush in children. It is closely allied to the mucor, and attacks only the young and feeble. The white crust consists of epithelial cells intermingled with an abundance of the white mycelium and oval spores of the fungus. Andry in his artificial cultures found that it was pearly white when grown on gelatine, dirty white on potato, and snow white on carrot."

Symptoms. "Buccal mucosa, red, congested and tender, shows here and there white curdy looking elevations, or red erosions caused by the detachment of such masses. These bear a strong resemblance to the crusts seen on this mucosa in rinderpest, but are easily distinguished by the absence of the attendant fever, and by the discovery, under the microscope, of the specific microphyte. The eruption may extend to the pharynx and œesophagus and interfere fatally with deglutition, but usually it merely renders sucking painful and is not serious."

Treatment. Cleanse and disinfect the sheds, "and invigorate the young animals by sunshine, free air and exercise. Locally . . . borax, which 
arrests the growth of the parasite, whether in artificial cultures or in the mouth. The powder may be rubbed into the sores, or it may be mixed with . . . molasses and used as an electuary. As substitutes boric acid, salol, thymol, potash chlorate, or Condy's fluid may be used." (Law's "Veterinary Medicine," Vol. II. p. 36.)

\section{ULCERATIVE STOMATITIS IN SHEEP.}

The name of ulcerative stomatitis of sheep has been given to a disease which is characterised by the appearance of a pultaceous deposit on the surface of the buccal mucous membrane and later by the development of ulcerations and of vegetative growths.

Causation. The cause is imperfectly understood. In certain years the disease appears to attack lambs at the time of weaning, but it may also affect flocks of animals as old as fifteen or eighteen months, two years, or even more. It is contagious, and may extend to one-half or twothirds of the entire number in the flock. Full-grown and old animals appear to be immune.

It was formerly thought, à priori, and in consequence of the character of the buccal deposit, that the disease was identical with thrush, and that the lesions were produced by Oidium albicans. Neumann in 1885 declared, however, that he could not find the fungus in question in scrapings from the mucous membrane.

Moussu had similar negative results in the experiments he made during 1894, when he examined both young and old animals belonging to flocks in the departments of Berry and of La Brie.

In addition it has been suggested that the disease affects badly caredfor and badly fed lambs, and subjects suffering from "watery cachexia." This seems correct in many cases, and Moussu has seen ulcerative stomatitis decimate flocks which had previously been attacked with intestinal helminthiasis and verminous broncho-pneumonia; but, on the other hand, in the environs of Melun he saw it in animals which had previously been quite free of disease and were kept under perfect hygienic conditions.

The conditions in which animals are reared, the use of common drinking ponds, and the method of supplying flocks with food, are the chief causes of the distribution of the disease.

Symptoms. The symptoms consist in loss of appetite, or rather in difficulty in grasping and masticating food, wasting, a certain degree of dulness, and salivation. Somewhat later one often sees appear on the free margin of the upper and lower lips an eruption of small vesicopustules, which quickly become covered with yellowish-brown crusts and bleed on the slightest touch. The lips swell, become sensitive and painful, so that examination of the cavity of the mouth should be made with 
care. The mucous membrane is then seen to present a very varying number of greyish-white or greyish-brown points, each of which is due to the destruction of the epithelium and to the production of a pultaceous deposit, which can very readily be removed, leaving uncovered superficial ulcerations, which bleed if very lightly touched. These ulcerations are of irregular shape, and are distributed most freely over the gums, the internal surface of the lips and cheeks. The palate and tongue are only invaded in the last stages. Still later one notes, instead of these ulcerations and as a consequence of abnormalities in the process of repair, reddish violet turgid vegetations varying in size from that of a millet seed to that of a pepper-corn or even of a small mulberry.

The total duration of the disease varies with its degree of intensity. In favourable cases it may not exceed eight to ten days; in grave cases it continues for fifteen to twenty days. Recovery is usual when the subjects are vigorous and well-nourished lambs, or well-kept sheep, previously free of disease. On the other hand, Moussu has noted a mortality of 15 to 20 per cent. in thin animals of bad bodily condition and already depressed by verminous broncho-pneumonia and intestinal helminthiasis. When the attack is about to prove fatal, complications such as gastro-enteritis, resulting from septic infection or intoxication, and accompanied by fœtid diarrhœe, often make their appearance.

Diagnosis. The diagnosis presents no difficulty, the characteristics of this stomatitis being entirely different from those of the aphthous form. The claws are never affected, and there is no lameness.

Prognosis. In this connection the practitioner will do wisely to avoid committing himself, and to closely study the conditions under which the disease has developed. In all cases the appearance of fotid diarrhœa must be regarded as of very unfavourable augury.

Treatment. The first precaution to take consists in separating all the healthy animals in the flock, and placing them in a non-contaminated spot.

The diseased are then treated one by one ; each day the buccal cavity is washed out with boiled water or with a solution of borax or boric acid. After each washing the ulcerations should be touched with tincture of iodine or with some other rather active antiseptic solution. Finally it might be useful, and would be of value, to add to the gruel or water a small quantity of common salt and of salicylate of soda at the rate of 15 grains to the pint.

GENERAL CATARRHAL STOMATITIS IN SWINE.

Causes. "Swine suffer from simple stomatitis when exposed to thermal, mechanical, or chemical irritants. Such irritants comprise food that is too hot, or is hard and fibrous, or which contains spikes and awns, 
capable of entering and irritating gland ducts or sores, or food which is fermented or putrid, food or medicine of an irritant character. The habit of catching and holding swine with a running noose over the upper jaw, and the forcing of the jaws apart with a piece of wood in search of the Cysticercus cellulosa are further causes. In several specific infectious diseases inflammation of the mucous membrane, with eruption or erosion, is not uncommon. This aphthous fever is marked by vesicular eruption, muguet by epithelial proliferation and desquamation, hog cholera and swine plague by circumscribed spots of necrosis and erosion. Patches of false membrane are not unknown, and local anthrax, tubercle, and actinomycosis are to be met with. Inflammation may start from decaying teeth.'

"Symptoms resemble those in other animals, refusal of food, or a disposition to eat sparingly, to select soft or liquid aliments, to swallow hard materials half chewed, or to drop them, to champ the jaws, and to seek cold water. Accumulation of froth around the lips is often seen, and the mouth is red, angry, dry, and hot, and exhales a bad odour."

"Treatment does not differ materially from that adopted in other animals: Cooling, astringent, antiseptic lotions, honey and vinegar, and in case of spongy or eroded mucosa, tincture of myrrh daily or oftener. Soft feeding, gruels, pulped roots, . . . and clean water should be constantly within reach. In case of . . . indigestion a laxative, followed by vegetable tonics, will be in order." (Law's "Veterinary Medicine," Vol. II. p. 17.)

\section{ULCERATIVE STOMATITIS IN SWINE.}

"This is the scorbutus of Friedberger and Fröhner, the glossanthrax of Benion."

Causes. "It has been attributed to insufficient or irritant food, to damp, close pens, and to chronic debilitating diseases, and all these act as predisposing causes. In gastritis and in infectious fevers like hog cholera, swine plague, and rouget (hog erysipelas) the spots of congestion and petechiæ on the buccal mucous membrane may become the starting points for ulcerative inflammations. These conditions appear, however, to be supplemented by infection from bacteria present in the mouth or introduced in food and water, and, as in the case of other domestic animals, the most successful treatment partakes largely of dis-
infectant applications."

Symptoms. "Loss of appetite, grinding of the teeth, champing of the jaws, the formation of froth round the lips, fœtor of the breath, redness of the gums and tongue, and the formation of vesicles, or white patches, which fall off, leaving red, angry sores. These may extend, forming deep unhealthy ulcers, with increasing salivation and fœetor. 
As the disease advances the initial dulness and prostration become more profound, and debility and emaciation advance rapidly. Unless there is early improvement an infective pharyngitis or enteritis sets in, manifestly determined by the swallowing of virulent matters from the mouth, and swelling, redness, and tenderness of the throat, or colics and offensive black diarrhoa, hasten a fatal issue. Rachitis may be a prominent complication, as it seems in some instances to be a predisposing cause."

Treatment. "Isolate the healthy from the diseased, and apply disinfection to all exposed articles and places. Employ local antiseptics as in other animals. Sulphuric or hydrochloric acids, in fifty times their volume of water, or tincture of iron, chlorate of potash, or chloride of ammonia, or borax have been used successfully. Bitters and aromatics have also been strongly recommended." (Law's "Veterinary Medicine," Vol. II. p. 29.)

\section{MERCURIAL STOMATITIS.}

This form of stomatitis possesses certain distinguishing characters, and develops after severe or trifling mercurial poisoning.

Causation. Sheep sometimes suffer from mercurial poisoning as a result of the use of baths containing corrosive sublimate or mercurial ointment for acariasis or other cutaneous parasitism. Animals of the bovine species seem predisposed to the disease as a consequence of their special sensitiveness to the action of mercury, which is not shared by other species.

Mercurial poisoning may occur accidentally, but is usually the result of some attempt at treatment. Any preparation containing mercury or mercurial salts may produce it. In domesticated animals it most frequently results from the action of the ordinary mercurial blister or mercurial ointment of the pharmacopœia, or again of calomel. Sometimes it follows the use of mercurial salts in uterine douches, or in lotions used to wash out large abscess cavities or wounds.

The application of blisters or of antiparasitic dressings, or infriction with grey ointment over extensive surfaces, favours this intoxication. It may result from direct local intra-cutaneous absorption, from vapour given off by mercurial applications obtaining entrance into the body through the broncho-pulmonary and digestive tracts, from vapour given off by metallic mercury (as in ships' holds), or from ingestion of mercurial compounds licked off the skin, as certainly occurs. Hitherto in all discussions, even the most recent, on the mechanism of poisoning, partisans of different views do not appear to have given sufficient attention to these now clearly proved facts. The conclusion to be drawn is that in animals of the bovine species mercurial preparations ought to be used with caution, 
and that even under such conditions stomatitis may appear. Finally, it should be remembered that all lesions of the kidney indicated by albumi. nuria and other signs, and all lesions of the liver, favour poisoning by checking or preventing the elimination of mercury by the kidney, or by interfering with its transformation in the hepatic cells.

Nature. Regarding the essential nature of stomatitis, it would appear (according to the work carried out in 1890 by Gallipe on mercurial stoma titis in man), that we should regard it as a septic stomatitis, and not as a primary toxic stomatitis. The mercury absorbed by the body not only produces salivation, but a very important change in the chemical composition of the saliva. The vitality and toxicity of the saprophytic microbes normally present in the buccal cavity appear greatly to increase, and although only the most trifling erosions may exist in the mucous membrane, true intra-mucous inoculation takes place, and forms the point of origin for septic stomatitis.

It has been found that it is not even necessary to have lesions in the buccal mucous membrane ; in fact, this is the weak point in the theory emitted. Nor is a modification in the chemical composition of the saliva sufficient; for when a mixture of iodine and the iodides, for example, is being given, the saliva is chemically modified, and yet stomatitis, properly so-called, does not occur.

What seems most probable is that mercurial stomatitis is a toxiinfectious stomatitis, in the development of which mercury acts primarily by its toxic effect on the salivary glands, whose secretion it modifies, and on the buccal epithelium, the renewal of which it checks. Infection of the mucous membrane is thereby favoured, even in the absence of any previous lesion, and stomatitis develops.

Symptoms. The symptoms consist in abundant salivation with discharge from the mouth, suggesting the existence of foot-and-mouth disease. In grave cases the saliva appears streaked with blood, even from the beginning. The buccal cavity exhales an intense fotid odour which, during the following days, becomes more marked; the mucous membrane is pale in colour, and coated with a greyish exudate. The mouth is hot and sensitive, the gums are swollen, reddish-violet in colour, and painful. Alveolar periostitis soon sets in, the teeth become loose, and mastication is rendered impossible, especially as the inflammation causes the tongue to swell and lose its mobility. These symptoms are unaccompanied by fever.

In the last stage ulcerations and local necroses appear on the gums, on the inner surface of the lips and cheeks, and around the commissures of the lips. The patients are almost unable to feed, rapidly lose flesh, become anæmic, and die from septic infection. The temperature is often below normal.

D.C. 
A toxi-infectious gastro-enteritis, with fœtid, blood-stained diarrhœa, is grafted on the primary stomatitis. Complications in connection with the respiratory, circulatory, and urinary apparatus appear, and the patients die in a condition of absolute exhaustion.

Naturally this termination is not inevitable; trifling cases of poisoning, and even grave forms, when properly treated, may, and should, recover.

Diagnosis. The diagnosis is mainly based on the history, except in cases of accidental and unsuspected poisoning.

Prognosis. The prognosis is grave, for even when the disease does not prove fatal the animals remain anæmic and exhausted for long periods.

Treatment should principally be directed towards combatting the local complications. When poisoning is due to external applications of mercury or its salts the skin should be washed with soap and water, and afterwards dressed with a soluble sulphide, which renders the mercury insoluble. 'The mouth should frequently be rinsed with boiled water, with decoctions of barley or marsh-mallow, with a 30 per cent. solution of boric acid or alum, or with a 1 to 2 per cent. solution of salicylic acid.

Fully grown cattle receive daily doses of $1 \frac{1}{4}$ to 2 drams of chlorate of potash internally. This drug appears to owe its favourable action to the fact that it is partly eliminated by the salivary glands. Lastly, with the idea (which may, perhaps, be illusory) of minimising and checking the bad effects of the mercury introduced into the body, certain practitioners have recommended the administration of eggs, flowers of sulphur ( $2 \frac{1}{2}$ to 5 drams), sulphate of iron ( $1 \frac{1}{4}$ to $2 \frac{1}{2}$ drams), and of iodide of potassium ( $1 \frac{1}{4}$ to $2 \frac{1}{2}$ drams), drugs which are alleged to form insoluble compounds with mercury.

\section{GLOSSITIS.}

The term "glossitis" is applied to all inflammations of the tongue, whether superficial or deep seated. These inflammations may result from trifling causes, in which case they are termed "simple, acute, or chronic golossites"; or, on the other hand, from well-recognised causes, like tuberculosis or actinomycosis, in which case they receive the name of "specific glossites." Here only ordinary glossites are investigated, the others being more particularly described in chapters specially reserved for the description of the primary diseases of which these form symptoms.

\section{SUPERFICIAL GLOSSITIS.}

This condition is characterised by lesions in the mucous membrane or in the immediately subjacent tissues, deeper seated structures not being involved. 
The causes are similar to those of simple stomatitis, and as in animals of the bovine species the tongue is the principal and almost the only organ of prehension, it is particularly exposed to the chance of injury.

As mechanical injury done by rough fodder first makes its effects felt on the tongue, superficial glossitis is often due to the action of sharp or prickly plants like furze, wrest-harrow, thistles, sea-holly, etc. The glumes, awns, and spikelets on certain plants play a similar part.

Caustic medicinal draughts, hot drinks, and sharp points on the molar teeth may also produce the disease without the rest of the buccal mucous membrane being involved.

Symptoms. These are extremely simple. There is, first of all, some difficulty in grasping the food and some diminution of appetite, which, however, is more apparent than real, the digestive organs acting well. The second symptom consists in moderate salivation without special characters.

The local symptoms alone are characteristic. The mucous membrane covering the tongue appears red, swollen, locally inflamed, and painful. The inflamed areas are usually located on the free part near the frænum or opposite the molar teeth.

Thorns, foreign bodies, awns or spikelets of rough grasses can often be seen implanted in the tongue, and if the disease has existed for a short time, little ulcerations may not improbably be discovered.

Diagnosis. The characteristics of this superficial glossitis are sufficiently marked to allow of easy diagnosis, and to prevent it being confused with the lesions of actinomycosis.or tuberculosis or with the extensive desquamation which accompanies foot-and-mouth disease.

Prognosis. The prognosis is never grave. Recovery occurs in six to eight days, provided the primary cause be removed.

Treatment consists in avoiding the use of rough food, in removing: foreign bodies implanted in the mucous membrane or in rasping rough and irregular teeth. For the rest, as in stomatitis, mere attention to cleanliness suffices. The mouth is washed out with boiled water, boric acid solution, mixtures of vinegar and water, or with water containing a small percentage of alcohol.

\section{ACUTE DEEP-SEATED GLOSSITIS.}

This disease has also been termed parenchymatous and interstitial glossitis, because all the deep-seated tissues, including the muscles and connective tissue layers are involved in the inflammation.

Causes. The disease may be due to a neglected attack of superficial glossitis, to some grave microbic infection, or to excoriation and ulceration of the mucous membrane. Very often it is of traumatic 
origin, and is due to violent traction on the tongue by herdsmen or others when administering draughts of their own composition. This traction causes rupture of the muscle and slight interstitial hæmorrhage.

The symptoms develop somewhat rapidly. Whilst at first the animal shows difficulty in grasping food, it is soon totally incapable of doing so. The tongue loses its mobility, is no longer protruded from the mouth, and swallowing becomes so painful that salivation sets in. On direct examination the tongue is found swollen, thickened, immobile, painful, and occupying the whole of the oral cavity, sometimes projecting beyond the region of the incisors, and preventing the mouth being closed. Inflammation may become so intense that the point of the tongue hangs out of the mouth. It becomes blackish, bleeding, swollen, and excoriated by contact with foreign bodies or simply with the row of incisor teeth. The saliva becomes fœetid, blood-stained and purulent, and contains large quantities of broken down epithelial cells. It is not uncommon to note more or less extensive necrosis.

Diagnosis. The diagnosis of this form of glossitis presents no difficulty, for if under certain conditions it may resemble, for example, the glossitis of actinomycosis, it differs essentially from that disease by its rapidity of development, by its complications, and also by the absence of the specific ray fungus.

Prognosis. The prognosis is grave, not only on account of the possible complications, but also because the animals are unable to feed and therefore lose flesh with very great rapidity.

Treatment. The first steps in treatment are in the nature of local disinfection, in order to prevent general infection. The mouth therefore should be washed out five or six times per day with boiled water, followed by antiseptic injections until improvement commences. Solutions of boric acid or borax (3 per cent.), chlorate of potassium (2 to 3 per cent.), or salicylic acid (3 to 4 per cent.), are useful, but the free employment of 1,2 , or 3 per cent. solutions of chloral is even preferable.

With the idea of protecting the organ from external injury, Lafosse formerly suggested the use of a suspensory bandage for the tongue, fixed to the base of the horns by small bandages. Guittard recommends scarification of the free part, and the application of a support perforated at the bottom to allow the blood, saliva, pus, etc., to escape. In spite of careful attention the disease may last from two to three weeks.

\section{CHRONIC GLOSSITIS.}

This form of disease has also been termed "sclerosing glossitis" and "non-actinomycotic wooden tongue," because it is anatomically characterised by induration of the tissues, and because apparently it resembles 
true "wooden tongue,", with which it was confused until within the last few years. Imminger in 1888 and Pflug in 1891 described two forms. The first, termed "superficial sclerosing glossitis," is said to be the more frequent, and most commonly affects young animals suffering from irregularities in dentition. It seems due to a superficial acute glossitis assuming a chronic form. The submucous connective tissue in time undergoes hyperplastic changes, so that the tongue at first becomes swollen and afterwards absolutely rigid. One never finds ulcerations or actinomyces.

Diagnosis. A close examination will always enable the case to be diagnosed at the first visit, or under any circumstances after a short time.

The prognosis is very grave, because the animal has the greatest difficulty in grasping and masticating food, or may even be unable to do so. Patients lose flesh very rapidly, and end by dying of hunger.

Treatment is of little service. The administration of iodine, the only drug which appears indicated, has proved of very questionable value. Economically, it is better to slaughter the animal.

The second variety is rare, and has been termed "deep-seated sclerosing glossitis." It is simply a chronic form of ordinary deepseated glossitis. The layers of connective tissue separating the muscles are represented by very hard, inelastic vertical partitions. As a consequence, the whole tongue becomes indurated and more or less completely immobile. In this condition, again, there is neither ulceration nor actinomyces.

Diagnosis. The diagnosis necessitates a careful examination. In the living animal it is very difficult to establish a distinction between this and the preceding form, though after death the task becomes much easier.

The prognosis is grave, complete restoration of the parts being impossible.

The treatment differs in no respect from that given in the preceding instance. It is of little value.

Imminger and Pflug have also described a deep-seated nodular sclerosing glossitis, characterised anatomically by the existence in the depth of the tongue of fibrous nodules, varying in size between a small nut and a fowl's egg. The tongue is only slightly increased in size.

This disease may perhaps be due to actinomycosis. 


\section{CHAPTER II.}

\section{DISEASES OF THE SALIVARY GLANDS, TONSILS AND PHARYNX.}

\section{PAROTIDITIS (PAROTITIS).}

THE term "parotiditis" indicates an inflammatory condition of the parotid gland. Of this disease several forms exist. The disease is termed simple when due to accidental causes or infections, specific when resulting from some special disease germ like the ray fungus. Anatomically, these diseases consist in inflammation of the glandular parenchyma and connective tissue stroma which surrounds the acini.

\section{ACUTE PAROTIDITIS.}

Causation. The causes of acute parotiditis are varied. Mechanical violence or contusions (due to collisions with fixed bodies, horn thrusts, or blows from the ox-goad) may produce it, the glandular parenchyma and connective tissue separating the acini or the peri-glandular tissue being torn, crushed, lacerated, and often also directly infected in consequence of the injury. Ascending infection through the medium of the salivary ducts represents a second possible cause of the disease.

Finally, parotiditis may, in some cases, constitute only a localisation of a general disease. It seems a fact that in rare circumstances parotiditis may assume an epizootic character, and attack a large number of animals in a particular stable or in neighbouring stables; and if, under these circumstances, we are unable to blame the food (which has not been done), we are forced to admit the influence of infection and contagion.

Symptoms. Whatever the cause, the symptoms are generally well marked. In many cases the first indication of the disease is apparent or real want of appetite, always complicated with difficulty in swallowing, and often accompanied by trifling fever.

Salivation, resulting from irritation of the gland and inability to swallow, becomes abundant, and at once draws attention to the buccal cavity and adjacent parts. Inspection of the patient then reveals the existence in the parotid region of a diffuse swelling, which on palpation is found to be hot and painful, and to occupy the whole of 
the parotid region between the lower jaw and the upper extremity of the neck. The lesion is usually unilateral, but occasionally bilateral.

Parotiditis may terminate in resolution, suppuration, or necrosis. The suppuration may either be simply subcutaneous and extra-glandular, or may involve a portion of the salivary gland and of the parotid lymphatic gland in addition.

Necrosis is exceptional, though Moussu saw double and total gangrene of both parotids, complicated with septicæmia, in the animal, of which a sketch is given herewith.

If the disease is due to violent injury by a foreign body, traces of a wound may be found, but it is often useless to search for these, even when the parts have been pricked with a sharp goad. When the inflammation has resulted from ascending infection of the salivary ducts, exaggerated sensibility may sometimes be detected throughout the whole length of Stenon's duct, particularly at the point where the duct crosses the jaw. There is always marked difficulty in moving the head, particularly towards the side, and sometimes in a vertical plane. The head is extended on the neck, and is held stiffly in such a way as to suggest

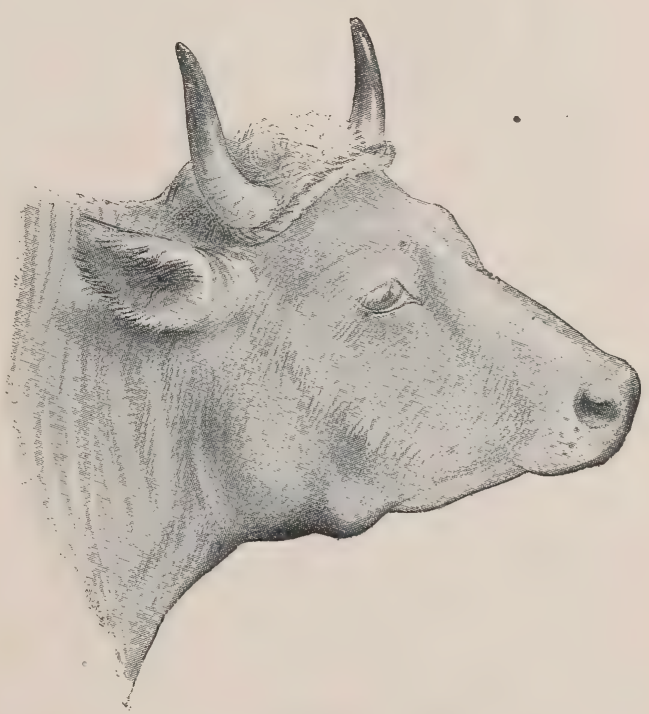

FIG. 63.-Acute parotiditis. the possibility of tetanus. Some observers have described as an important symptom marked swelling of the orifice of Stenon's duct. It is certainly difficult to detect, and furthermore is of no great significance.

Diagnosis. Although diagnosis is easy, it is a difficult matter to detect the point of origin of the disease. The salivation and difficulty in swallowing might seem to suggest pharyngitis, a condition which sometimes exists simultaneously. The distinction between this disease and the forms of chronic parotiditis, or tumour formation in the parotid (due to actinomycosis, lymphadenoma, melanoma), is also easy, on account of the slow development of the last-named conditions. The only condition liable to be confused with that under consideration is abscess of the sub-parotid gland.

Prognosis. The gravity of the disease varies greatly. When 
inflammation is slight, resolution usually occurs in eight to fifteen days. The onset of suppuration is announced by renewal of the fever, by more marked swelling, which becomes localised at a given point, and by the existence of deep or superficial fluctuation. Care, however, is required to detect the last named.

Necrosis may occur suddenly when the infecting organism is specially virulent, and may affect one-third, one-half, or the whole of the gland. The prognosis then becomes extremely grave, and if diagnosis has not been prompt and treatment energetic, death may follow in a short time from septic infection.

Treatment. Unless some well-marked sign foreshadows a complication, treatment should be directed to ensuring resolution. Bleeding has been recommended; its good effects, however, are open to doubt, though one cannot entirely forbid it. All practitioners agree in recognising the value of vesicant applications. The affected parts may be freely dressed with an ointment containing 2 per cent. each of pulverised tartar emetic and bichromate of potash, with the ordinary cantharides blister, or even with a weak mercurial blister, provided that the animals can be prevented from licking the wound.

Some practitioners prefer vesicants prepared with cantharides and croton oil. Whatever be the vesicant chosen, it is best after three or four days to apply emollients of slightly antiseptic character, such as ointments containing camphor, boric acid, salol, etc. When abscess formation is recognised the abscess should be opened as early as possible. Some precautions are necessary to avoid injuring important nerves and vessels; in dealing with a deep-seated abscess it is necessary to use the knife for dividing the skin alone, to seek the abscess by blunt dissection with the finger or with round-pointed scissors, and to open it with a similar instrument. The cavity should then be freely washed out with a warm antiseptic solution-3 per cent. carbolic solution, or 1 per cent. iodine solution, etc. If necessary a drain composed of iodoform gauze can be inserted, or a counter-opening made.

In the case of partial necrosis, all the necrotic tissue must be carefully removed, injury to vessels, which would favour septicæmic infection, being avoided. Afterwards free antiseptic irrigation should be employed several times per day.

In necrosis of the entire parotid extirpation may seem indicated; but the greatest prudence is demanded, for the operation is extremely serious and delicate.

CHRONIC PAROTIDITIS-PAROTID FISTULA.

When a case of acute parotiditis is not treated, and does not end in suppuration, it is usually succeeded by chronic inflammation and fibrous 
induration of the gland. Any obstruction of Stenon's duct, whatever the originating cause (foreign bodies like wheat awns, oat grains, calculi, etc.) stops the flow of saliva throughout the excretory apparatus, and produces over the entire parotid region a doughy swelling, which might seem to indicate the existence of indolent parotiditis. The collections of liquid thus produced have improperly been termed "salivary abscesses." If ascending infection fails to occur, or if infection is unimportant and does not lead to suppuration, a relatively painless chronic parotiditis develops, and in this case movements of the head and mastication and deglutition alone are impeded.

The salivary ducts, however, may become so distended that the main superficial collecting duct undergoes softening, and the skin covering it becomes ulcerated, just as would occur had a true abscess formed. Under such circumstances the skin soon yields and a salivary fistula is established.

The symptoms consist in swelling or induration of the gland, interference with movement of the head and with mastication; the whole developing slowly without pain or fever.

The distinction of this condition from actinomycosis of the parotid may sometimes present some difficulty until a fistula develops.

The prognosis is grave, because there is no hope of normal conditions being restored.

Treatment. Treatment often proves unsuccessful. Should the condition have resulted from an obstruction, it is first necessary to attempt the removal of such obstruction, whether it be a foreign body or calculus, and so to re-establish the normal channel for the saliva. Local stimulation may be tried, though in cases of fistula without much hope of success. Smart blistering of the parts surrounding the opening and firing in points have been recommended; but rather than persist in prolonged treatment of doubtful value, it is often better to prepare the animals for slaughter.

\section{INFLAMMATION OF THE SUBMAXILLARY SALIVARY GLAND.}

Inflammation of the submaxillary gland is rare in the ox. As in the horse, it is usually caused by the penetration of foreign bodies, such as glumes or awns of grain, fragments of straw, thorns, etc., into Wharton's duct.

The difficulty in grasping food and the restricted movement of the tongue are the first symptoms to attract attention. On examination, the region of the "barbs," usually on one side, appears injected, swollen, inflamed, and sensitive.

The submaxillary space is effaced by the swelling of Wharton's duct: 
the corresponding submaxillary gland is doughy and painful on pres. sure. The symptoms rarely become more threatening than this.

The diagnosis presents no difficulty.

The prognosis is favourable.

Treatment consists primarily in removing the obstruction from the duct. Steady pressure from behind, forwards along the course of the duct, may sometimes cause the foreign body to be ejected into the mouth, along with a jet of offensive saliva. The distended and inflamed duct soon becomes emptied, and all the symptoms rapidly diminish. In other cases, when the obstructing body is firmly fixed in position, it is necessary to open the duct within the mouth by a stroke of the bistoury.

\section{TONSILITIS IN PIGS.}

"Acute and chronic forms are seen. The former has the general causes and symptoms of pharyngitis-fever, dulness, a disposition to lie with head extended and buried in litter, drooping ears, watery eyes, indifferent appetite, painful deglutition, and sometimes vomiting. Mouth red and hot, breath foetid, tonsils swollen, their alveoli filled with mucopurulent matter or with fœtid cheese-like masses. Cough at first dry and hard, later loose and gurgling.

In chronic form there is general swelling of the tonsils with distension of follicles by above-mentioned putty-like masses, which are often calcareous. These are due to the proliferation of microbes, which find in these alveoli a most favourable field for their propagation. The affection usually ends in recovery, but may go on to grave local ulceration and general infection.

Treatment. Astringent antiseptics to buccal mucous membrane. Electuaries of honey or treacle and borax, sal ammoniac, chlorate or permanganate of potash and externally stimulating embrocations to the skin of the throat. In some cases solutions of iron chloride or tincture of iodine may be used with advantage and as a wash for the mouth and fauces. Attend to general health. If constipated give Glauber's salt or jalap, . . . and elimination through the kidneys must be sought through the use of nitrate of potash or other diuretic." (Law's "Veterinary Medicine," Vol. II. p. 46.)

\section{PHARYNGITIS,}

Inflammation of the mucous membrane of the pharynx is less frequent in the ox than in the horse, a fact probably due to its less sensitive character in the ox and to differences in the methods of working oxen.

Causation. The causes are numerous and varied; and although local microbic infection undoubtedly plays the most effective part in the development of the disease, it is none the less certain that external 
influences are of considerable importance. For this reason chills, sudden rariations in temperature, sudden arrest of perspiration, and currents of cold air have always been invoked as causes. Cruzel thinks that the ingestion of ice-cold water in winter is sufficient to produce acute pharyngitis. The action of rough forage may of itself explain the development of pharyngitis in animals constantly kept indoors under excellent hygienic conditions. In such cases the disease may be regarded as of traumatic origin. Furthermore, it is necessary to mention direct injuries of the mucous membrane caused by clumsy examination, awkwardness in passing the probang, and attempts on the animal's part to swallow sharp foreign bodies, which scratch, tear, lacerate, or penetrate the mucous membrane and become fixed in it.

Finally, another series of causes, and not the least important, remains to be considered-viz., the forced administration of irritant substances like ammonia, tincture of iodine, oil of turpentine, very hot liquids, etc.

To sum up, the four great series of causes consist in direct irritation, intra-pharyngeal wounds, variations in temperature, or primary or secondary microbic infections.

The symptoms are characteristic. They consist in loss of appetite, difficulty in swallowing, consequent on the condition of the pharynx, and fever, which is often marked from the first. Pharyngeal dysphagia can easily be distinguished from that due to injury of the cesophagus, inasmuch as it occurs on the first attempt to swallow.

Urged on by hunger, the animal grasps food, which it cherrs and attempts to swallow, but immediately allows it to fall back into the manger, or ejects it by a painful coughing effort. In the case of liquids, even of lukewarm drinks, the same accident occurs, the food or liquid being returned by the nostrils. Slight salivation results from this difficulty in swallowing. The animal's attitude is similar to that in parotiditis. The head is held stiffly, extended on the neck, in order to aroid compressing the region of the pharynx, and can only be moved slowly and with much pain. There is no apparent swelling of the parotid region, but on manipulating or pressing on the gullet the animal sometimes manifests the very acute tenderness of the parts by coughing and endeavouring to thrust away or to kick the examiner. Finally, examination of the mouth sometimes shows reddening and excessive sensibility of the soft palate and of the pillars of the fauces.

These symptoms often assume a more alarming character, or are complicated by others; in fact, the condition very rarely stops at pharyngitis, but is usually accompanied by inflammation of the larynx, of the soft palate, and of the naso-pharynx. The whole throat is then inflamed; the nose and sometimes the eyes discharge, and there is difficulty in 
swallowing, interference with respiration or noisy respiration, and intense fever.

Diagnosis. The diagnosis presents no difficulty, the symptoms mentioned being easy to identify, whether the condition be simply inflammation of the pharynx or be of a more extensive character. Nevertheless, cases occur where the symptoms are alarming, but in which one might hesitate between the diagnosis of ordinary acute sore throat and the sore throat which ushers in gangrenous coryza. A definite expression of opinion must then be deferred to a later date; for one cannot be absolutely certain whether or not the other signs of gangrenous coryza will appear.

When there is only difficulty in swallowing one might à priori suspect traumatic injury of the mucous membrane, with or without the presence of a foreign body. It is also necessary to bear in mind the possibility of difficulty in swallowing being occasioned by reflex irritation without local lesions, originating in enlargement of the retropharyngeal lymphatic glands as a consequence of tuberculosis or other disease.

The prognosis is favourable; even without treatment acute pharyngitis usually tends to recovery in eight to twelve days, and rarely becomes complicated. Nevertheless, some reserve ought to be exhibited in cases of pharyngitis due to the action of rough forage, the removal of the cause being here indispensable to any improvement. Similarly in cases of pharyngitis due to foreign bodies having penetrated the mucous membrane, which are chiefly characterised by inability to swallow, the disease may continue for a very much longer time than above indicated, unless the foreign body is discovered and removed. Inflammation is limited to a zone surrounding the point of implantation. It extends more deeply with movements of the foreign body, and may end in the formation of an abscess. Of this variety is Hopsomer's remarkable case, in which a darningneedle finally obtained exit through the submaxillary space, in which it had caused the formation of an abscess.

Treatment. The treatment is the same whether we are dealing with a simple acute pharyngitis or with a more widely distributed inflammation. It consists in smartly stimulating the region of the throat with mustard, cantharides oil, or ointment, or with an ointment containing 2 per cent. of tartar emetic and of bichromate of potash, and then covering the parts with a flannel hood. This mode of treatment seems preferable to that recommended by German authors-viz., the application of cold compresses to the throat, the administration of cold gargles, etc. Moderate bleeding, to the extent of two or three quarts, has the great advantage, as in all similar cases, of lowering the temperature.

This treatment may, if necessary, be completed by the internal administration of 3 to 5 drams of Kerme's mineral (oxysulphuret of 
antimony) in electuary, according to the animal's size. Medicated inhalations diminish local irritation, render swelling less painful, and facilitate the separation of false membranes and the discharge of adherent mucous secretions.

The ordinary food should be replaced by cooked roots, lukewarm drinks and gruels, all rough fodder being prohibited.

If difficulty in swallowing alone continues, the operator should examine the mucous membrane of the pharyngeal cavity with the hand, in order to discover and remove any foreign body which may have become implanted there.

\section{PSEUDO-MEMBRANOUS PHARYNGITIS IN CATTLE.}

In addition to the above acute forms of pharyngitis, a pseudo-membranous, croupal, or pseudo-diphtheritic pharyngitis has been described in the ox. It is due to polymicrobic infection, and is characterised by the formation of false membranes on the pharyngeal mucous membrane. The condition seems to be a pharyngitis of exceptional intensity, varying markedly from the classic type and being most nearly related to severe sore throat, laryngitis, gangrenous coryza, etc.

It rarely attacks aged cattle, but is readily transmissible to calves and young stock. Cadeac failed to inoculate it on guinea-pigs and rabbits. Damman succeeded with sheep and with rabbits, the latter dying in twenty-four hours after inoculation with hæmorrhage at the seat of puncture. Löffler hypodermically infected mice and produced extensive infiltration of the abdominal walls, and often of the peritoneum, surface of the liver, kidneys and intestine, on which formed a thick yellowish exudate containing the organism.

Causes. Löfler found filaments of a long delicate bacillus about half the thickness of the bacillus of malignant œedema. The bacillus did not grow in nutrient gelatine or in sheep's blood serum, but readily grew in blood serum of the calf. Cadeac gives as predisposing causes: sudden chills, rapid alterations of temperature, inhalation of irritant vapours, suppression of cutaneous secretion, swallowing irritant liquids, and injuries.

Symptoms. The nasal membrane is reddened, thickened and covered in patches with false membrane, causing snuffling and wheezing breathing. The throat is tender and swollen, cough is frequent, gurgling, and followed by expulsion of false membrane, muco-pus and some blood. Shreds of false membrane adhere to the nose and lips. Other symptoms are: fever, accelerated pulse, dark mucous membranes, haggard countenance, mouth open, hanging tongue, stringy salivation, and constipation or diarrhœe.

The disease runs a rapid course, and death may occur in twenty-four hours. Recovery may be equally rapid, but often convalescence is protracted. 
Lesions. Intense congestion of mucosæ of nose, mouth, pharynx, larynx, and bronchi, with here and there patches of false membrane, which may be soft or tough, according to the duration of the attack. The deep surface of the false membranes is blood-stained; and, according to Preitsch, false membranes sometimes occur in the osophagus, rumen, and omasum, which in consequence may show patches of ulceration.

Treatment (as for the horse). This includes poultices, counter-irritants, laxatives, febrifuges, alkalies and antiseptics. Inhalations of medicated vapour, warm water to which has been added some antisepticcarbolic, lysol, creolin, camphor, sulphurous acid ; or for calves, iodoform, oil of turpentine, calcium sulphide, silver nitrate, coal tar. To detach the false membranes ipecacuanha and potash chlorate, or soda sulphate, or magnesia sulphate may be tried. Papayin and pepsin have been suggested as appropriate remedies. Anyodnes-digitalis, belladonna, morphia and aconite-may be useful. Tracheotomy is indicated as a last resort.

\section{PSEUDO-MEMBRANOUS PHARYNGITIS IN SHEEP.}

Roche-Lubin states that this is common in flocks as a result of moving the sheep in dusty enclosures. The dust is supposed to excite the intense croupous inflammation of the mucous membrane. The disease has been noticed in spring in young lambs shortly after weaning. Damman states that he transmitted the disease to the sheep from the exudate of the calf.

Symptoms. Frothy salivation with constant movements of the jaws, viscid nasal discharge, difficult deglutition, panting, snuffling breathing, throat swollen and very tender, frequent cough, discharge of exudate. The head and neck are extended, the eyes dull, appetite is lost, the mucous membranes are red or cyanotic, and the animal appear's weak and listless. As respiration becomes more difficult the mouth is held open, the tongue is protruded, and with each cough shreds of false membrane are expelled. Death from suffocation is not uncommon.

The lesions are not different from those seen in the calf.

The treatment is similar to that for the calf. Tepid drinks containing hydrochloric acid, or sulphate of soda (1 lb. to 50 sheep) in the drinking water, has been recommended. Fumigation with sulphurous acid or chlorine may be tried. Small numbers may be treated by swabbing the throat with solution of sodii hyposulphis or weak caustics and antiseptics.

In young and in adult pigs pseudo-membranous pharyngitis is often only a manifestation of pnemmo-enteritis. It therefore calls for no special 
description at this point. No exact investigation of the organisms which produce these forms of pharyngitis with false membrane formation has been made in veterinary surgery. We only know that these diseases are not true diphtheria due to "Klebs' bacillus." Treatment should be very energetic from the commencement, but otherwise it differ's in no respect from that ordinarily adopted.

Tonics and stimulants, like alcohol, wine, coffee, etc., are indicated.

[The following account of the disease is summarised from Lan's "Veterinary Medicine," Vol. II.]

"Pseudo-membranous pharyngitis has long been recognised as a contagious disease of swine, attacking especially swine liept in herds or in close, insanitary pens. Young pigs are more liable to attack than older animals, perhaps, owing to the older animals having suffered the disease in early life.

Modern observation shows that pharyngitis with false membranes is common in swine plague, and the present tendency is to refer all such cases to that category. It is, however, altogether probable that the occurrence of local irritation, with the addition of an irritant or septic microbe altogether distinct from that of swine plague or hog cholera, gives rise at times to this exudative angina. Certain it is that septic poisoning with the food is not at all uncommon in the hog, in the absence of these infectious diseases.

Symptoms are those of sore throat, with much prostration, a croaking cough, yellow discharge from nose and mouth, and marked muscular weakness. The tongue, tonsils and soft palate are red, swollen, and studded with patches of false membrane. The identification of swine plague may be made by the history of the outbreak, the number of animals affected, the tendency to pulmonary inflammation, the enlarged lymph glands, the presence of the non-motile bacillus, which does not generate gas in saccharine media, and which readily liills rabbits and pigs with pure cultures of the germ.

Treatment. Isolation, cleansing and disinfection. Locally antiseptics and generally a febrifuge regimen will be advisable."

\section{PHARYNGEAL POLYPI.}

The term "pharyngeal polypi" includes tumours of varying character, which affect the polypus form, and oceur with considerable frequency in the bovine species. Many of these polypi are simply actinomycotic growths springing from the pillars of the fances, from the upper parts of the palate or from its posterior surface. Less frequently they arise from the lateral walls or the free surface of the hard palate.

Symptoms. The symptoms are so characteristic that the diagnosis rarely presents much difficulty. They may shortly be described as 
indicative of repeated obstruction in the pharyngeal, œsophageal or laryngeal region. At the moment of deglutition, the polypus is thrust towards and obstructs the œsophageal orifice.

Reflex stimuli are thus excited, which prevent deglutition; an attack of coughing occurs, and food mixed with saliva is ejected from the mouth and nostrils. The attack of coughing displaces the polypus either in a forward or lateral direction, and swallowing then again becomes possible, until by changing its position the growth produces fresh signs of obstruction.

In other cases the polypus may only be of such small size as to impede the food passing through the pharynx on its way into the asophagus or to cause difficulty in respiration by partially blocking the pharyngeal portion of the nasal cavities. In such cases deglutition is only checked and rendered slower.

Or again, the pedicle of the polypus may be sufficiently long to allow the growth at certain moments to fall in front of the laryngeal opening. Respiration is then painful, difficult and noisy. Unless the growth is displaced during the subsequent attack of coughing, asphyxia may appear imminent, or may even occur unless assistance is afforded.

Guided by these symptoms, the operator will explore the pharynx manually, and thus discover the position and size of the tumour. Tumours of the naso-pharynx produce very similar symptoms.

The prognosis is based on the information obtained by manually exploring the pharynx. It is relatively favourable if the polypus has a well-marked neck, but is very grave if the tumour is largely sessile and cannot be removed.

Treatment. Medical treatment appears useless except in cases of polypi due to the presence of actinomyces. The administration of iodine and iodide of potassium, in large doses, may then lead to resorption; but extirpation is often preferable.

In other cases extirpation is the only rational treatment. The operation necessitates the performance of provisional tracheotomy in order to avoid risk of asphyxia. The growth may be directly removed through the buccal cavity without incision, provided that it prove possible to pass the chain of an ecraseur around the pedicle; or through the buccal cavity, with incision, after vertically or obliquely dividing the soft palate; or, lastly, through the larynx, after performing median laryngotomy, thus obtaining access to the pharynx.

Only the first method of intervention is to be recommended; the last two are more delicate. They necessitate after-treatment, and when the patients are in a condition for slaughter it is frequently preferable to send them to the butcher. The essential point is not to act without a full knowledge of the causes. 


\section{CHAPTER III.}

\section{DISEASES OF THE ESOPHAGUS.}

THE œsophageal tube is of very simple anatomical construction, and performs an equally simple physiological function; nevertheless, in the ox it is liable to a large number of diseased conditions. These conditions may affect only a circumscribed area of the mucous membrane or the entire extent of the tube. Again, both the muscular and mucous tissues may be affected, as in inflammation of the cesophagus accompanied or followed by contraction, and in the formation of oesophageal abscesses and tumours; or the muscular tissue alone may be affected, as in cases of dilatation. Even where no lesion is apparent the normal rhythm of deglutition may be interfered with, either by the presence of a foreign body (obstruction) or by spasm of the muscular layers (œsophagismus) or by compression due to tissues surrounding the oesophagus (false contractions).

We shall successively study the different forms of oesophagitis, contraction, and dilatation, together

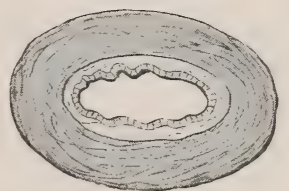

Fig. 64.- Schema il. lustrating anatomy of the œsophagus: a strong external layer of muscle, intermediate cellulo. elastic layer, inner layer of mucous membrane lying in folds and capable of great dilatation.

with their complications; then obstructions, ruptures of the œsophagus, œsophagismus, and false contractions.

\section{ESOPHAGITIS.}

Inflammation of the csophagus may be due to many different causes, and may occur in one of three different degrees of severity. It may be either superficial, i.e., limited to the epithelial layer of the mucous membrane; or deep, affecting the entire thickness of the mucous membrane (epithelium, corium, and œsophageal glands); or, finally, it may attack both the mucous and muscular layers. German authors recognise various divisions, such as erythematous, catarrhal, follicular, and phlegmonous osophagitis. In reality these are not always different forms, but simply successive stages in the evolution of a single morbid condition.

D.C. 
Here we shall only study the ordinary forms of oesophagitis, leaving on one side those which occur symptomatically during foot-and-mouth disease, cattle plague, gangrenous coryza, actinomycosis, etc.

Causation. The causes of asophagitis may be divided into three groups of different character:- (a) Rough fodder (clover containing wrestharrow, thistles, thorns, furze, or splinters of wood, etc.) must be placed in the first rank, for its repeated action abrades and irritates the mucous membrane to such an extent as finally to produce inflammation. This inflammation usually remains superficial and of moderate intensity; its occurrence can be anticipated during years of scarcity, when the animals feed on rough and irritating material like fern, broom, heather, furze, etc.

(b) Hot drinks, whether in the nature of beverages or medicinal dranghts, are a frequent cause of œsophagitis if administered by careless or inexperienced persons. The mucous membrane is scalded over a varying area and with different degrees of severity, or is destroyed by the chemical action of such drugs as ammonia, dilute acids, iodine solution, etc.

(c) Rough or clumsy manipulation in withdrawing or displacing foreign bodies, or merely passing the probang, produces that variety of œsophagitis termed traumatic. In clumsy hands œsophageal sounds or catheters may abrade or even tear the mucous membrane and subjacent tissues.

Symptoms. These vary to some extent, according to the intensity of the inflammatory phenomena. If the lesions are superficial and only implicate the epithelium, as in catarrhal œsophagitis, the symptoms often pass unnoticed, and only produce difficulty in swallowing. When inflammation has involved the entire thickness of the mucous membrane the immediate consequence is loss of appetite due to pain during swallowing. After the bolus of food has been masticated, and has passed into the pharynx, the animal stretches out its head and neck and seems to be making efforts to force it down the œsophageal canal. The progress of the bolus is slow and clearly difficult.

In œsophagitis due to scalding the blisters are soon broken by the passage of food, the corium is exposed, and the animal has equal difficulty in swallowing either solids or liquids. The reflex action provoled by the passage of the food over these lesions may be so violent that the ingesta never arrive at the stomach, but are violently rejected by a sudden and unexpected antiperistaltic contraction. Even saliva is returned. Moreover, in these cases the history is generally clear, and the animal is feverish or greatly depressed. These objective symptoms are very significant, and when, in addition, an abnormal and exceptional degree of sensibility is detected at some point by palpation, they unmistakably indicate the existence of œsophagitis. 
The irregularity in deglutition, and therefore also in rumination, sometimes excites moderate tympanites without any very apparent cause. Should the condition still appear doubtful the cesophageal sound may be passed, but with great care. It generally aggravates the pain and pro. duces intense antiperistaltic movements, which the practitioner should not attempt to overcome.

Complications. If œesophagitis is moderate, recovery is the rule. The symptoms of pain gradually diminish.

When, on the contrary, inflammation is very intense, as in certain cases of traumatic osophagitis, the injured spot may become infected and suppuration follow. The existing fever then persists or becomes more marked; the animal is extremely depressed; respiration may be difficult and accelerated, and appetite is entirely lost.

If the osophageal abscess remains submucous the diagnosis is difficult, but it is often problematical, even when the abscess develops in the cervical region. The jugular furrow (usually on the left side) becomes the seat of a severe diffuse inflammatory swelling, the course of which clearly indicates the development of the symptoms. In exceptional. cases fluctuation may be detected.

If from the first the abscess develops around the osophagus or in the course of suppuration comes to occupy this position, swelling in the jugular furrows is more apparent and easier to detect, and in this case fluctuation may be localised. When the lesions are within the thorax no tangible symptoms can be detected. Death may oceur in a few days, when an abscess in the lower cervical region breaks into the anterior mediastinum, or when an abscess in the thoracic region opens into the pleural cavity. In oesophagitis produced by scalding and from swallowing hot or caustic liquids the mucous membrane, and sometimes the muscular tissue, is destroyed, and ulcerations and cicatrices result, or the œsophagus may even be perforated, with rapidly fatal results; even when recovery occurs, cicatrices form and cause very grave contraction.

Diagnosis. The diagnosis is generally easy, provided that the symptoms noted are methodically analysed and the history of the case is taken into consideration.

Prognosis is favourable in ordinary cases. On the other hand, it may be very grave when general symptoms become marked, when the vital functions are disturbed and a deep-seated abscess appears to be forming.

Lesions. In the first degree the lesions are confined to inflammation and desquamation of the epithelium; in the second, to inflammation of the corium and of the mucous membrane; in the third, to infiltration of the submucous layers and of the muscular and periosophageal tissues. Sloughing and perforation follow the administration of caustic liquids. 
Treatment. As the direct application of medicines to the inflamed mucous membrane can only be of a momentary character, treatment is confined to administering emollient, anodyne, and slightly astringent drinks, the action of which is assisted by feeding with milk, farinaceous or mucilaginous foods. Under these circumstances recovery occurs in ten to fifteen days. The application of stimulant or blistering ointments along the jugular furrow may have a good effect.

When the general condition of the patient becomes aggravated, and the formation of an abscess appears certain, it is best to recommend slaughter. In the case of a submucous abscess the passage of the probang may, however, predispose or cause the abscess to open into the œsophagus, and thus lead rapidly to recovery, but this is exceptional. The "pointing" of the abscess and its opening towards the jugular furrow may be followed by temporary improvement, but at a later stage is followed by fistula formation, or by contraction of the oesophagus itself. From an economic standpoint it is better to slaughter.

\section{STRICTURE OF THE CESOPHAGUS.}

Under normal conditions the cavity or lumen of the œsophageal tube is, so to speak, imaginary: the walls of the tube lie flatly together, and the mucous membrane is in folds. During the act of swallowing the tube becomes dilated to a degree varying with the size of the bolus of food, and again retracts as soon as deglutition is effected. Whenever the dilatability of the tube is markedly diminished by changes in its walls, and, in a much higher degree, when this dilatability has disappeared, true stricture exists. In the former case small boluses of food and liquids alone succeed in passing the stricture; in the latter, liquids alone can pass.

Causation. Strictures are never primary. They result from intense attacks of oesophagitis, ending in sclerosis of the mucous coat, extensive ulceration consequent on scalding, or interstitial inflammation affecting the muscular coats, which then become thickened or sclerosed.

Internal injuries due to attempts to withdraw or propel foreign bodies along the cesophagus may also cause strictures.

Lesions. In simple strictures the lesions are confined to the development in the depths of the mucous membrane and in the muscular layers of inflammatory tissue, which becomes denser with lapse of time. This alters the character of the walls and the structure of the tissues, and causes them to lose their elasticity. After extensive ulceration the tissue of the cicatrix contracts and hardens to a very varying degree.

Symptoms. The apparent symptoms are very clearly marked; the appetite is good, and the animal masticates as usual, but in the act of deglutition is seen to extend the head on the neck, and to make 
efforts to swallow, which prove unavailing when the contraction is too marked. A reflex antiperistaltic movement often causes the substances ingested to be at once rejected. These violent efforts, however, in time provoke dilatation above the stricture. A quantity of food accumulates in this dilatation, and the symptom so characteristic of œsophageal stricture then appears-viz., regular regurgitation. The second constant symptom associated with compression or obstruction of the osophagus is tympanites after feeding, however trifling may be the amount swallowed. Rumination is suspended, and even eructation of gas is difficult. Finally, the characteristic sign of stricture is noted on passing the probang, which reveals the existence of the condition, indicates its position, and suggests its degree of development.

Diagnosis. Strictures only develop progressively and slowly, a fact which enables them to be differentiated from osophagitis. It is more difficult to differentiate them from dilatations, because the stricture always ends by becoming complicated with dilatation; but this distinction is of little practical importance, the consequences being identical.

Prognosis. The prognosis is very grave, and there is no economic reason for attempting treatment except in special cases; the indications are in the direction of slaughter.

From the economic standpoint there is no treatment. Basing their actions on
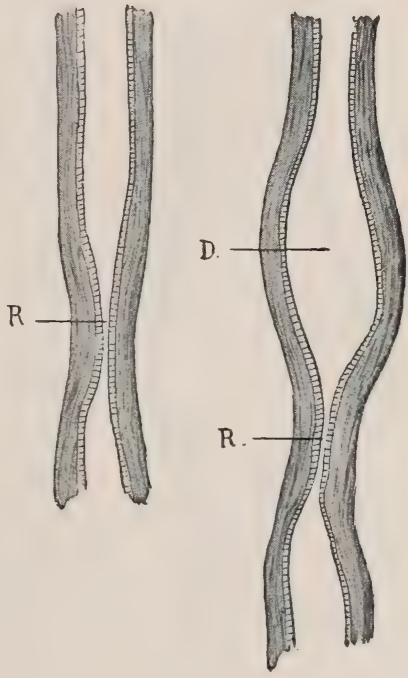

FIG. 65.-Schema of recent and old-standing contraction of the œesophagus. R, simple contraction; D, secondary dilatation. human practice, the Germans have recommended progressive dilatation of the lumen of the osophagus by passing a series of catheters of gradually increasing size. What, however, is justifiable in human medicine, where the only object is to keep the patient alive at any cost, may be highly objectionable in veterinary practice; and in the present instance this is the case. Except in very rare instances, which the practitioner alone can appreciate, dilatation is contra-indicated, and the owner's interest lies in slaughtering the animal before it has lost much condition.

\section{DILATATION OF THE CESOPHAGUS,}

Dilatations are more frequent than strictures. Their mode of origin is easily understood. When the muscular tissue has lost its tonicity and 
contractile power at a given point, or when, as a consequence of any form of inflammation, it has begun to undergo atrophy, the mucous membrane becomes herniated, because its circumference is not supported regularly during deglutition. The ectasia, which at first is of small size, becomes more marked in consequence of the tendency that exists for the food to accumulate in the dilated region. Dilatation is thus set up.

Localised attacks of esophagitis, accidental injuries and fissuring of the œsophageal muscular tissue, produced by clumsy efforts to displace foreign bodies with the probang, are the principal

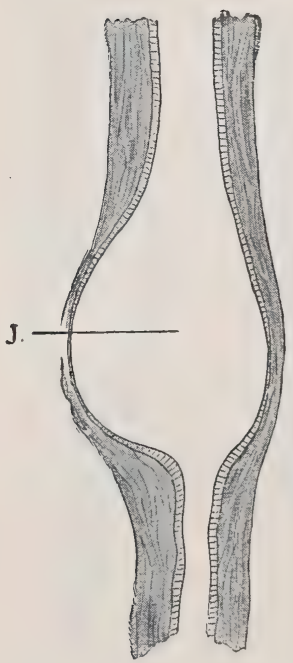

FIG. 66.-Schema of œsophageal stricture (the muscular layer above the stricture has undergone atrophy; the mucous membrane is dilated). causes of dilatation. When the probang is imprudently or clumsily manipulated, it may press excessively at any point where the œesophagus makes a slight bend, and thus split the contracted muscular coat without injuring the lax mucous membrane.

Esophageal contractions, as we have seen, may form the point of origin of dilatations, but in this case the dilatations are more regular in form, and affect the entire circumference of the tube. The muscular tissue is still everywhere normal, and becomes dilated in consequence of equally-applied excentric pressure.

Symptoms. When the dilatation develops slowly and progressively, as a consequence of muscular atrophy, the symptoms remain unnoticed for a long time, and the owner only begins to be anxious when the animal loses condition, or when the driver or cowman detects masses of half-chewed food mixed with the saliva in the manger.

Certain signs are pathognomonic; others may be regarded as of secondary importance. By carefully watching an animal which is feeding the following symptoms may be noted: As a general rule hunger is very marked, and the animal chews its food and swallows the first few mouthfuls in a perfectly normal way. Three, five, eight, or even ten mouthfuls may be swallowed; then the animal suddenly stops, appears a little anxious, extends its head and neck, an antiperistaltic contraction occurs, and one or two masses of food are rejected and fall into the manger. 'The discomfort being thus momentarily relieved, the animal, which is dying of hunger, although faced with food which it is unable to swallow, returns to its meal, swallows one, two, or three boluses of food, regurgitation again occurs, and the whole process is repeated.

What is going on under these circumstances is easy to explain. 
At the commencement of the meal the dilatation is usually empty, or nearly empty. A mouthful of food is swallowed. It descends the cesophagus until it arrives at the diverticulum, into which it partially or wholly passes, the peristaltic wave of contraction ceasing at this point. The second mouthful follows with the same result, then a third, a fourth, etc. The diverticulum soon becomes filled to repletion, and no more food can enter it. The food therefore accumulates in the upper portion of the œsophageal tube until the latter becomes nearly filled; but as this tube, provided its innervation is intact, is intolerant of the presence of any foreign body, and as efforts to swallow prove fruitless, a sudden antiperistaltic wave of contraction occurs, with the result that all the material contained in the tube above the dilatation is ejected into the mouth, whence it falls into the manger. The same result follows any further attempts to swallow during a particular feeding time. From this it will be seen that the animal can ingest at a given time only as much as the dilatation will contain.

In the intervals between meal times and under the action of the saliva and warmth, the food collected in the dilatation becomes softened, breaks down, and slowly moves onward towards the rumen. When the next feeding time arrives the dilatation is almost empty, and the same set of symptoms recurs.

If, instead of forage, the animal begins by taking gruel or very fluid material, deglutition appears normal, or at least fairly easy ; but if drinking is deferred until after taking hard food, it becomes almost impossible, because the passage is obstructed. These symptoms are, so to speak, pathognomonic. Under any circumstances they are so significant that error in diagnosis is unlikely.

By careful examination œsophageal regurgitation can very easily be distinguished from true vomiting ; the character of the rejected material shows that it has not come from the stomach, while the boluses of food preserve their cylindrical form, and are still saturated with saliva.

Some secondary signs also deserve to be mentioned, such as the animal's anxiety and restlessness whilst its neighbour's are feeding, the existence of trifling and intermittent tympanites due to suppressed eructation, suppression or irregularity of rumination, constipation, etc. At a later stage there is rapid wasting and disordered appetite, and finally the patients die slowly of hunger, whatever efforts are made to feed them.

When the seat of dilatation is in the cervical portion of the cesophagus, there are other symptoms which leave no doubt as to the condition. When empty the pouch cannot be detected ; but during a meal the accumulation of food causes it to assume the appearance of a doughy, diffuse, indolent swelling, which alters the outline of the jugular furrow, yields 
to pressure, and sometimes produces respiratory disturbance by pressing on the trachea, the pneumo-gastric and inferior laryngeal nerves, etc.

When the dilatation is intra-thoracic and the above-described symptoms have been observed, the dilated spot can only be detected and localised by using the probang. The greatest possible prudence, however, is required in manipulating the instrument, in order to avoid rupturing the thin walls of the dilated portion.

The diagnosis is not always easy; when food is regurgitated, and one finds by auscultation that the sound usually produced by the passage of solids or liquids into the rumen is absent, there need be no hesitation in diagnosing either a dilatation or a stricture. The clinical consequences being the same, the possible error would be of little importance.

Prognosis. 'The animal's life is rarely in immediate danger, but from the economic point of view the prognosis is extremely grave, and it is in the owner's interest to slaughter the animal as soon as possible in order to avoid loss. Even in cases of dilatation in the cervical region, surgical interference is not advisable.

Treatment. As foreshadowed by what has been said, there is no rational economic treatment. When the dilatation is in the cervical region, one might in exceptional cases attempt to restore the regular calibre of the oesophagus by removing an elliptical portion of mucous membrane, and bringing the muscular tissue together with sutures; that is to say, when the rupture or fissuring of the muscular coat has been accidental. But as one is usually unable to remove the primary cause, to which the change in the muscular tissue is essentially due, the dilatation would recur without the operation having conferred any benefit.

When an exact diagnosis has been made, the only useful indication is to confine the animal to very fluid food, which will not obstruct the resophagus. Ordinary forage should be withheld.

\section{CESOPHAGEAL OBSTRUCTIONS.}

In this chapter we shall only consider such obstructions as occur in consequence of the animal having attempted to swallow without sufficiently chewing objects which become arrested in the œsophagus.

Obstruction is termed "total" or " partial," according as the obstructing body fills the entire calibre of the osophagus at the point of obstruction, or only occupies a part of the space. Partial obstructions produced by beet and turnip tops, etc., are usually but momentary; liquids and saliva are still able to pass between the obstruction and the walls of the tube, and as soon as the arrested food becomes a little softened it is displaced and the œsophagus again becomes patent.

Causation. The circumstances under which this accident occurs are extremely easy to understand. Obstructions are produced by apples, 
potatoes, turnips, carrots, cabbage-stalks, beetroots, ete., which, whether sliced or not, are swallowed gluttonously. Not having been sufficiently comminuted, and being of larger size than the osophagus can readily accommodate, they become arrested at some point between the commencement of the œsophagus or a few inches behind the pharynx, or just in front of the point of entry of the gullet into the stomach. The latter is the commonest position, though not infrequently the obstruction occurs in the intra-thoracic portion.

It may occur in the stable, but is commoner in animals which, having broken loose, have entered orchards, gardens or potato or turnip fields and attempted to swallow apples, cabbages, potatoes, etc., found there.

In sheep, obstruction of the œsophagus is due to similar causes, but in their case the above-mentioned objects are replaced by small wild apples, turnip shells, Jerusalem artichokes, horse-chestnuts, carrots, etc.

The symptoms may be divided into general and local.

General symptoms. As soon as the foreign body becomes fixed in position, the animal begins to make exceptional efforts to swallow. The head is extended on the neck, and the osophagus and the muscles surrounding the trachea are violently contracted. These efforts proving fruitless, feeding is necessarily stopped, and the animal at once appears slightly anxious.

Very soon afterwards salivation sets in, saliva being continuously secreted. If the obstruction is total, the saliva cannot be swallowed, and is either returned in quantities by antiperistaltic movements or escapes in frothy filaments from the mouth.

I'ympanites is not long in appearing. It is progressive, and results both from arrest of eructation and from continued fermentation in the rumen. It may eventually come to a standstill, or may continue and threaten to produce asphyxia.

Local symptoms. The local symptoms are difficult to appreciate, except in cases of cervical obstruction. Sometimes the foreign body produces a local swelling, which changes the outline of the jugular furrow, most frequently on the left side. In many cases it can only be detected by manipulating the parts between the trachea and the lower surface of the cervical vertebræ. When the obstruction is within the thorax, the probang alone can detect its position.

Diagnosis. The diagnosis is usually easy. The history and the observed symptoms are often very clear, and the suddenness with which the obstruction has made its appearance prevent the condition from being confused with dilatation or stricture.

The prognosis is very variable. It is often easy to remove the obstacle; in other cases intervention is difficult, and death may occur rapidly. 
Treatment is confined to one essential point-removal of the obstruction. The chief difficulty lies in choosing the mode of intervention. Moreover, success depends on several factors, which, in the order of their importance, are as follows: the size of the obstructing body; the time which has elapsed since the accident occurred; the bodily condition of the animal-i.e., whether it be fat or thin-and the extent to which tympanites has developed.

The first thing to do (and in favourable cases all that is required) is to puncture the rumen and leave the canula for some time in position. The onward progress of the foreign body, especially when the obstruction is in the intra-thoracic portion of the tube, is often impeded by the tympanites, which tends to thrust the object towards the pharynx, or at least to fix it in position. In consequence of a sudden change in the conditions of pressure the foreign body may move and pass into the rumen; all danger is then at an end.

Even though the obstruction does not immediately cease, puncture of the rumen, by removing the danger of asphyxia, allows one at least to wait for several hours, sometimes until next day, during which time the object may pass into the rumen without further extraneous assistance. The other methods may be grouped into four series:

I. External taxis. This is directed towards loosening the foreign body and thrusting it towards the pharynx and buccal cavity. It can only be used against obstructions in the cervical region. 'T'wo methods, although very ancient, are still practised.

(a) 'The first is carried out in the following way: the animal is fixed to a post or tree so that it cannot struggle, its head being drawn up as high as possible. The operator stands on the left side, with his back turned towards the patient's head, his left hand is pressed into the right jugular furrow, his right hand is placed on the left jugular furrow immediately below the foreign body. By using the fingers the foreign body is moved, and is progressively thrust towards the pharynx, in spite of the animal's efforts to swallow. In carrying out this manipulation it is absolutely indispensable not to let slip the obstructing body for a single instant, otherwise the peristaltic action will immediately return it to its former place. When it has been raised as far as the pharynx, an assistant passes his hand into the back of the mouth, as indicated in a former chapter, seizes the object and withdraws it; or, instead, the assistant takes over the operator's duties, while the latter himself extracts the foreign body.

(b) In the second method the animal is fixed in a different position, the head being held about 10 to 12 inches from the ground, with the neck lowered and inclined towards the earth. As in this position the resophagus is longitudinally relaxed, and can be dilated to its fullest 
extent transversely, the difficulty of displacing the obstacle should be very much less. In this case the operator always stands on the left side of the neck, but with his back towards the animal's body. The right arm is passed around the neck and the right hand pressed into the right jugular furrow, the left hand being similarly engaged in the left jugular furrow. The method of employing the fingers is identical, or instead of the fingers the thumbs may be used.

When the obstructing object has been lifted as far as the pharynx it has a tendency to fall out of the mouth, and if it fail to do so it can be fixed in position and removed as in the preceding case.

II. Extraction. These methods are applicable to cases where the foreign body has become fixed in the cervical region, but more especially to obstructions in the intra-thoracic part of the cesophagus. In the majority of cases they are dangerous, and may lead to pinching, rupture, or perforation of the osophageal mucous membrane. They should therefore be regarded as exceptional measures. Theoretically, the instruments described are perfect, but practically they do not secure the results anticipated, because one can never prevent displacement, wrinkling, and involution of the osophageal mucous membrane.

The forceps probang has the drawback of seldom grasping smooth foreign bodies with sufficient firmness to permit of their extraction.

The corkscrew sound exposes one to the great danger of completely piercing the œesophagus, because it has to be managed blindly, and because one never knows at what depth the corkscrew portion should be protruded in order to obtain a proper hold of a foreign body.

III. Passage of the probang. When taxis fails or

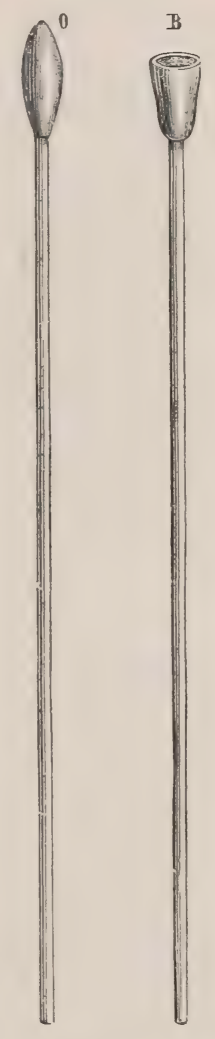

Fig. 67: - Esophageal sounds. Probangs.

is inapplicable, we are forced to attempt thrusting the foreign body onrards. The method is much safer than the preceding, but, nerertheless, demands great tact, prudence, and gentleness. Suitable œsophageal sounds are made with cupped extremities, though in cases of emergency an instrument can often be successfully improrised from a cane, whip handle, or flexible stick, about $4 \frac{1}{2}$ to 5 feet in length, securely wrapped at one end with cloth or tow and freely coated with some greasy material such as lard, vaseline, or oil.

'The end of the sound having arrived in contact with the obstacle, 
the operator exercises moderate but permanent pressure. The obstacle may not move immediately, because of spasm of the cesophagus, which grasps it. It is therefore necessary to wait and to take advantage of a moment when the resistance is less, and even then the obstacle may not move.

Rough manipulation with improvised sounds may tear, fissure, or perforate the muscular and mucous coats, producing the gravest consequences.

IV. Crushing. The crushing of an obstruction in the cervical region was long ago suggested, and is still greatly commended by empirics and farriers. It is performed by means of a little mallet and a piece of board. The method is barbarous, and exposes the animal to such grave complications as crushing of the œsophageal walls, followed by necrosis, laceration of the connective tissue, and interstitial hæmorrhage, injuries of the superficial or deep-seated jugulars, of the carotid artery, pneumo-gastric nerve, etc. It should never be practised, even although attempts have been made to improve it by replacing the mallet and board by specially formed forceps intended for crushing potatoes or roots. Only in the rare cases where one is certain that the foreign body consists of a very ripe fruit could crushing be justified, and in this case there is no need to have recourse to special instruments, for the hands alone suffice.

Injection of alkaloids. The practitioner occasionally finds himself in the embarrassing position of having vainly tried all the above methods. Before adopting the last resource, viz., œsophagotomy, it is then worth while to test the action of certain alkaloids, injected subeutaneously, after having punctured the rumen.

We know that pilocarpine and eserine stimulate secretion and the action of the bowels. Injected under the skin they cause frequent swallowing efforts, and intense peristalsis extending throughout the length of the digestive tract. Doses of $1 \frac{1}{2}$ to 2 grains of pilocarpine and 1 to $1 \frac{1}{2}$ grains of eserine, according to the size of the animal, sometimes produce excellent results, and rapidly remove obstructions.

Apomorphine, the effects of which are, so to speak, inverse, because they tend to produce anti-peristalsis and vomiting, may be tried in doses of 2 or 3 grains.

Esophagotomy. The last resource is œesophagotomy, which, however, is only applicable in cases of obstruction of the cervical portion of the œsophagus. It should be performed as described in the section hereafter on operative manipulation. (See also Möller and Dollar's "Regional Surgery," p. 166.)

The point selected is necessarily governed by the position of the obstacle. There is no need to enter into full details. We may remark that it is not always necessary to perform the complete operation, and 
the third and fourth stages can sometimes be avoided by substituting for them attempts to break down the foreign body by submucons manipulation. The œsophagus, having been exposed and isolated, is punctured with a straight tenotome immediately below the obstacle. A curved tenotome is then introduced, and the root, potato, or fruit divided. As a rule, a little pressure from the outside then causes one or other of the fragments to move onwards and deglutition becomes normal.

Attempts have also been made to divide the obstructing body directly without previous incision and without isolating the oesophagus. It is much more difficult, for the least movement of the patient changes the relationships of the super-posed layers and introduces obstacles to the manipulation of the blunt-pointed tenotome which is employed. More success often attends attempts to puncture the object with a fine trocar.

\section{RUPTURES AND PERFORATIONS OF THE CESOPHAGUS.}

Causation. Wounds of the osophagus caused by external violence are rare, or at least secondary; lacerations produced from within, on the contrary, as a result of clumsy manipulation are relatively frequent. They may extend throughout the length of the tube, but in a far greater number of cases are found near the entrance to the stomach at the point where the osophagus turns towards the left.

The passage of the osophageal sound or probang is apt to exaggerate this curvature, and if pushed violently the instrument may produce first a flexure, then a partial rupture or even a perforation of the tube.

In other cases a rough, irregular, infected foreign body may when swallowed penetrate the wall and cause inflammation, necrosis and perforation of the œsophagus.

The symptoms are always very grave, and of rapid development. They consist in local œdematous swelling, sero-sanguineous infiltration at the entrance to the chest, in the pretracheal region and along the jugular furrows.

The pneumo-gastric and inferior laryngeal nerves being compressed, dyspnœa results. If the œsophagus is perforated in the thoracic cavity septic pleurisy at once sets in.

Diagnosis. The diagnosis is easy, provided the history point to perforation of the csophagus.

The prognosis is fatal whenever the perforation is within the thorax. It is sometimes possible to intervene in cases of perforation in the cervical region, but from the economic standpoint such intervention is of little value. 


\section{CHAPTER IV.}

\section{DEPRAVED APPETITE-PICA-THE LICKING HABIT.}

Depraved appetite, causing animals to swallow bodies which cannot properly be described as food, is frequent. The condition is commonest in adult animals of the bovine species, in calves and in lambs. The consequences are sometimes very serious, so that although depraved appetite does not represent a well-defined morbid entity, it is important to be in a position to remedy it.

Depraved appetite does not appear under the same conditions in young and in old animals. In adults it often results from faulty feeding, or from some wasting disease which develops insidiously, or remains unrecognised; in young animals it is the result of insufficient nourishment.

Roloff \& Röll hold that pica is the first symptom of osteomalacia (which see).

\section{DEPRAVED APPETITE IN THE OX.}

Causation. In the bovine species depraved appetite occurs in adult, debilitated animals, which are often, though not always, suffering from some well-marked digestive disturbance.

The frequency of this symptom, and the peculiarities in its occurrence, have caused it to be referred to a large number of different causes, among which may be mentioned bad hygiene, chronic gastro-enteritis, tuberculosis, osseous cachexia, pasteurellosis, gestation, ete.

It is very certain that the peculiarity in the appetite is, above all, the result of incomplete and irrational alimentation. The animal has certain special requirements, to meet which the food must be of suitable composition. If these alimentary and digestive conditions are not fulfilled, depraved appetite may occur, even in animals which appear well nourished. Certain authors refer the appearance of this condition to want of certain soda salts in the daily ration, and, in support of this opinion, they point to the frequency of the disease in mountainous regions where the geological formation is chiefly granite, as in the Black Forest. Alluvial soils are supposed not to produce it. It. certainly seems more common on soils lacking in certain constituents or exhausted by 
repeatedly growing certain crops. Nevertheless, in France it might be urged that pica occurs equally on all kinds of soil, and a German author, Lemke, ascribes this perversion of nutrition to the want of phosphorus. Haubner and Siedamgrotsky attribute it to a nervous disorder. All causes which exhaust the organism, especially all chronic diseases of digestive origin, may induce aberration of appetite.

Permanent stabling, confinement, absence of sunlight, want of exercise and pure air contribute to the general debility which predisposes to attack. Dry seasons, by reducing the supply of food, have a similar effect.

In tuberculosis and in pasteurellosis, it is the general organic decline which produces these puzzling changes in appetite. Similarly the influence of gestation depends on the superadded demands on the organism caused by the development of the fotus.

Symptoms. The symptoms may be divided into two phases.

In the first phase, the animals still preserve their appetite, but whenever they have an opportunity they eat earth, sand, manure, litter saturated with urine, plaster, etc. They lick the walls, the boarding, the mangers and the trees, and they chew and swallow linen spread out to dry.

'This phase may continue for a very long time, three to four months or more, provided no acute complication results from the eating of such foreign material. There is no fever, but the appetite, although well preserved, is often capricions, and the ordinary food is eaten slowly.

In the second phase, which frequently marks the development of complications produced by the passage, contact, or prolonged sojourn of various materials in the digestive tract, fever appears, little marlied as a rule, but continuous in character.

The appetite is diminished. The animal wastes; the secretion of milk diminishes, and signs of chronic gastro-enteritis may be noted. The perversion of appetite still continues; rags, decomposing or filthy materials, pieces of old shoes, etc., are eaten, and it is not surprising that such substances should have an unfavourable effect on the mucous membrane of the digestive tract.

The wasting process slowly leads to marked emaciation, and after an interval of from six months to a year, or even two years, the patients die in a state of complete exhaustion. The lesions found on post-mortem examination are those of various diseases capable of producing depraved appetite or simply lesions of chronic gastro-enteritis.

Diagnosis. The diagnosis presents no difficulty. The important point is to discover whether or not there exists some previously unrecognised primary disease.

Prognosis. The prognosis of this condition is grave, because deprared appetite is frequently only a symptom of some incurable disorder, 
or because the changes in the digestive mucous membrane are already too far advanced to permit of much improvement.

The lesions comprise: general emaciation, presence of a yellow serum in the fatty tissue, muscles pale and flabby, catarrh of the mucous membrane of the stomach and bowel. The blood seems less in quantity and coagulates feebly or not at all.

Treatment. The treatment should be directed against the primary cause, if such exists (osseous cachexia, pasteurellosis, gestation, etc.).

In other cases a change in management and in feeding, and the administration of food rich in mineral salts like chlorides, carbonates, and phosphates of lime, soda or potash, produces the best possible results. The leguminous foods, sainfoin, clover and lucern, are to be recommended. The animal, if formerly stabled, should be turned out and its living conditions entirely altered.

It is often useful to place a block of rock salt in the manger; when hyperacidity of the stomach is suspected lime water, chalk, or magnesia should be given. Where digestion is weak or slow $\mathrm{HCl}$, pepsin and vegetable bitters are indicated. Nevertheless, one sees cases which refuse to yield to any of the ordinary methods. In treating these, Lemke has recommended the subcutaneous injection of chloride of apomorphine, a drug which may be regarded as a true specific. The doses vary between $1 \frac{1}{2}$ and 3 grains, and an injection is given once a week for three weeks in succession. After this the tendency to pica is said to disappear and the general condition to improve. The treatment must be repeated every three months in countries where depraved appetite appears general and permanent.

It is difficult to understand by what mechanism this drug produces the effects attributed to it, but those who have employed it speak very highly of its action.

We may add that in addition to the different modes of treatment, it is not infrequently necessary to hastily perform gastrotomy in order to avoid fatal consequences, which would otherwise follow indulgence in this habit. When an animal has swallowed a considerable quantity of linen, for example (and Moussu has seen cases in which many pounds weight had been devoured), immediate intervention is required to avoid intestinal obstruction. Furthermore, when the history is quite clear gastrotomy allows the entire mass of foreign bodies, ingested at different times, to be removed.

\section{DEPRAVED APPETITE IN CALVES AND LAMBS.}

Causation. Depraved appetite is commonest in calves and lambs when the animals are insufficiently nourished, or when the mothers are suffering from chronic debilitating diseases and are therefore yielding 
milk poor in fat and in mineral constituents. In a few rare cases it is impossible to discover what causes the young animals to devour these foreign materials. Even fully-grown sheep, when shut up together in winter, acquire the habit of chewing each other's wool, sometimes to the extent of virtually depilating their fellows and accumulating wool balls in their stomachs.

Symptoms. Calves have a tendency to lick themselves or their neighbours, and thus little by little collect a varying quantity of hair which they swallow. When this habit of licking is little marked the quantity of hair ingested may not be dangerous; but in the contrary case the hair (which cannot be digested) accumulates and is permanently retained in the abomasum. It soon becomes converted into masses, cemented together with mucus, and forms round balls, to which the name of œgagrophiles has been given. If these œgagrophiles, or hair balls, are of small size, they prove of trifling importance; but too frequently they attain considerable dimensions and obstruct the pylorus or the intestine. The young calves then refuse all nourishment, and die in twenty-four to forty-eight hours in a state of complete exhaustion or after a series of epileptiform attacks.

In lambs the complications due to depraved appetite develop in a similar way, but the wool swallowed is obtained from the mothers. The lambs first suck the locks of wool, then tear them off and swallow them. So long as these peculiarities of appetite are little marked no bad results follow; but if the shepherd is careless, and fails to note the condition of his young flock sufficiently early, accidents occur.

The wool is not so easily converted into balls as is hair, but it soon accumulates in the pyloric region or in the intestine, and forms obstructing masses. The little patients lose appetite and lie down in corners, where they are found dead after twenty-four to forty-eight hours. The masses of wool or of hair are rarely passed with the excrement; more frequently they are vomited, but this again is exceptional; usually they become arrested at the entrance to the pylorus. The lambs show colic, tympanites of the abomasum, and attempts at vomiting, though unfortunately these are often overlooked. The quantity of wool found in the abomasum and intestine on post-mortem examination may be considerable, in relation to the size of the digestive compartments. Death results from intestinal obstruction, exactly as in the case of calves.

These aberrations of appetite in lambs have been considered as due to the want of sufficient mineral salts in the mother's milk; and it has been stated that the lambs practise this habit because of the laxative result of the fat contained in the wool swallowed. The explanation seems very logical, though it is by no means perfectly proved. It is certain that this habit becomes particularly common after years in which forage has D.C. 
been scarce and among flocks in bad bodily condition. The force of example also plays a certain part, and animals probably imitate one another, and so acquire the disease. This explains the importance of early segregation.

Diagnosis. The diagnosis of depraved appetite, pica, or the licking habit presents no difficulty; but it can only be arrived at by the cowman or shepherd, for the symptoms can only be detected by continued watching.

The diagnosis of pyloric or intestinal obstruction is very difficult in the absence of information. It becomes easy after the first post-mortem examination has been made.

Prognosis. The prognosis is grave. In calves, obstruction of the bowel by hair-balls inevitably causes death, and in sucking lambs the mortality may be high : as much as 15 per cent. to 20 per cent. according to the observations of several observers. The mortality occurs about the age of six weeks to two months, whilst the licking habit may begin towards the end of the second week.

Treatment. Prophylaxis demands that the mothers (whether cows or ewes) be well fed. An excellent precaution consists in adding to the food a sufficient quantity of salt and of phosphate of lime (2 drams to $2 \frac{1}{2}$ drams of each). This treatment of the mothers is necessary as soon as the tendency to licking becomes manifest.

In calves the best method of avoiding fatal results is to prevent the young animals licking one another; and the method now usually practised on well-managed farms consists in applying a simple muzzle of wicker work immediately after each meal.

In lambs treatment is more difficult. As soon as the shepherd sees any tendency to depraved appetite the lambs should only be left with their mothers whilst being suckled. The flock should be exercised in the open, and ordinary salt should be placed at a number of points on the ground occupied by the animals.

\section{COLIC IN THE OX.}

COLIC DUE TO INGESTION OF COLD WATER. CONGESTIVE COLIC.

Causation. Congestive colic occurs in the stable, in animals which have been doing heavy work, and, returning in a heated condition, drink large quantities of cold water. It is commoner when animals have not eaten for a considerable time, and when, therefore, the stomach is nearly empty. Under these circumstances chill of the digestive viscera is direct and immediate.

Symptoms. This form of colic occurs suddenly, soon after the water has been swallowed, and is characterised by violent pain. At first the 
animals show uneasiness, stamp, and conținually move about striking themselves in the flank with the feet or horns, swishing the tail, etc. They refuse food, lie down and rise frequently, and paw the ground.

As a general rule this form of colic lasts from half an hour to one hour, and terminates in recovery. In some rare cases where death occurred Cruzel found on post-mortem examination congestion of the abomasum, and, in a few, congestion of the small intestine, with or without rupture.

The diagnosis is easy, on account of the suddenness of onset, rapid development and history of the disease, discovered on questioning the owner or herdsman.

The prognosis is not grave. This form of colic generally cures itself. Nevertheless precautions are required against possible complications, such as intestinal hæmorrhage and invagination.

The necessary preventive measures are self-evident. Animals returning from work should not be allowed to drink freely of cold water, but should first receive a little food and afterwards water at the temperature of the atmosphere.

When colic has set in, the patient can be walked about. If pain persists, the region of the abdomen may be dressed with oil of turpentine, mustard, or similar counter-irritants. The application of warm clothing is also useful. Finally, in grave cases, a moderate quantity (three, four, or five quarts) of blood may be withdrawn from the jugular. The administration of stimulants like wine, alcohol, etc., is also indicated.

\section{COLIC DUE TO INVAGINATION.}

Invagination consists in the passage of one portion of the intestine into the next-following portion. When once the condition has been set up it tends to become aggravated, the invaginated part being drawn further and further forwards. Invaginations therefore may vary in length between a few inches and sixteen to twenty inches.

Law states (Vol. II. p. 347) that in cattle and swine invagination of the large colon is almost impossible owing to the relation of the bowel with the layers of the mesentery. The anatomical arrangement is opposed to the formation of invagination, yet this accident is not uncommon in cattle and swine. The small intestine can be invaginated into the cæcum or into itself. The cæcum may become invaginated, or it may pass into the colon or rectum.

Cartwright, Veterinarian (1829), reports a case of invagination in a bull calf, and Youatt gives particulars of a similar case which was followed by sloughing and discharge per anum of the intussuscepted portion of bowel. (See also Möller and Dollar's "Regional Surgery," p. 328.) 
Causation. This variety of colic is due to a number of somewhat obscure causes. In a general sense we may say that anything which increases intestinal peristalsis increases the risk of invagination. The accident may follow intestinal congestion, but is most frequent in animals suffering from intestinal worms, or in animals used for heavy work. Under the influence of violent tractive efforts the peristaltic movements are stimulated, and the intestine being in an oblique

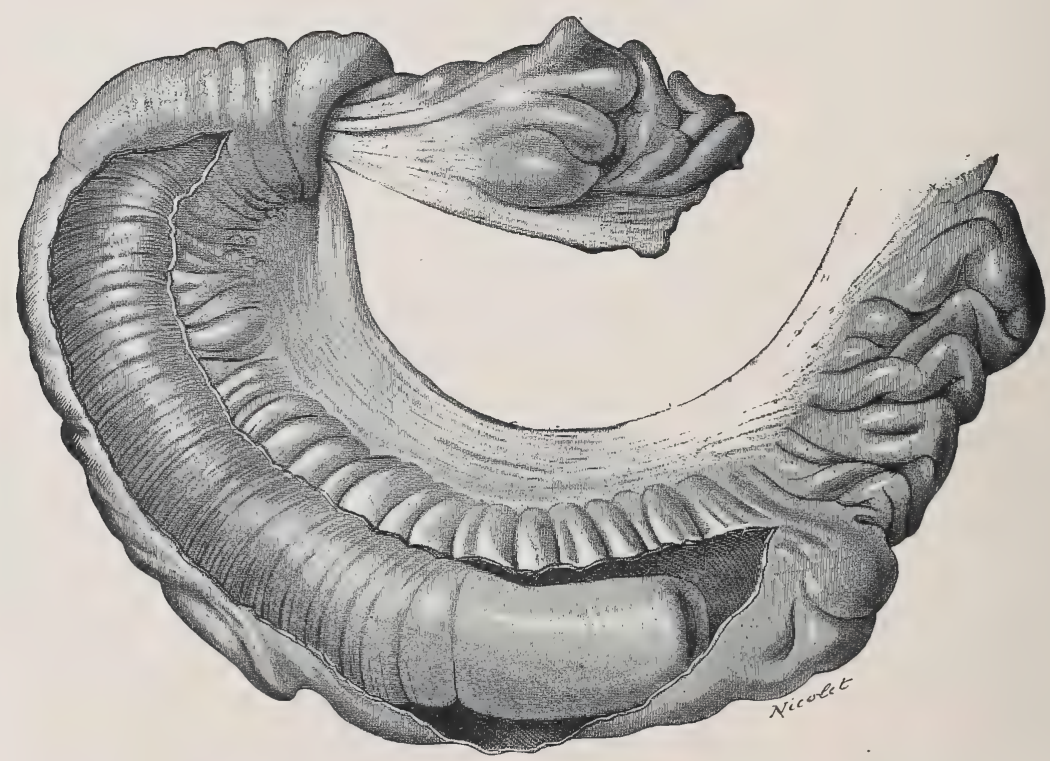

FIG. 68.-Invagination of the intestine in an ox (the constricting portion has been incised longitudinally).

position on a plane inclined backwards, the contracted portion may slip into the dilated section behind it.

Invagination may also occur without any apparent cause, even in animals standing in the stable.

Symptoms. The attack always occurs suddenly, develops rapidly, and is of an extremely grave character.

Colic comes on while the animal is working, moving about, or resting, according to circumstances, and at first resembles that due to congestion. It afterwards becomes very violent; the animals paw, stamp, show great uneasiness, throw themselves violently down, and rise suddenly, only to again lie down as before. The face expresses anxiety, suffering and depression; the tail is often kept lifted, and efforts are continually made to defæcate, mucus being passed. By passing the hand into the rectum the invagination may occasionally be discovered. 
Colic persists with great intensity for ten to trrelve hours, interrupted only by rare periods of calm. At the end of this time, howerer, it may suddenly disappear, and the animal may fall into a semi-comatose state. This indicates the onset of necrosis in the invaginated section, the painful reflexes no longer being transmitted to the sympathetic system. The disappearance of colic is sometimes regarded as a sign of improvement, but this improrement is illusory. From this time onwards the animals stand stolidly, obstinately refusing both food and drink. If they lie down, it is with great care. Palpation of the right side of the abdomen is painful, and the animal actively resents it. One of the most important and constant signs at this stage is the absence of defæcation, due to obstruction of the intestine, which is occluded. The animals may survive for ten, trrelve, or eren fifteen days (see also Möller and Dollar's "Regional Surgery," loc. cit.). The invaginated, necrosed portion may even be passed with the frees, and recovery may occur, the continuity of the intestinal tube being secured by the adhesion of the serous surfaces; but such spontaneous recoreries are exceptional. Lsually after a few days death results from peritonitis.

Occasionally, trifling invaginations may become reduced spontaneously. Diarrhoa, with the passage of blood-stained material is then seen for a time, a sign which alone at this stage would justify the diagnosis of invagination.

Diagnosis. The intensity of the colic and the absence of defrcation for several days afterwards, justifies the diagnosis of invagination. Purgatives then remain without effect. In addition, rectal exploration offers a valuable means of diagnosis. The last portions of the intestine are found absolutely empty, and the arm when withdrawn is found to be covered with viscous blood-stained mucus, resulting from the serosanguinolent exudate, due to compression of the blood-vessels.

In cases of this kind accompanied by the above-mentioned symptoms abdominal exploration by the rectum should always be practised, but it rarely gives exact information. The hand, when passed towards the right flank, may sometimes reach the invaginated part, which conveys the impression of a cylindrical strelling. The invagination, however, can rarely be reached. If the operator is successful, he will find that as he displaces this cylindrical mass or attempts to grasp it, the animal shows signs of exaggerated sensitiveness.

The prognosis is of exceptional gravity. Apart from the rare cases where the invaginated portion becomes necrotic and is eliminated, death is inevitable. Unless an operation is performed, septic peritonitis may develop about the fifth or sixth day.

Treatment. The only treatment consists in surgical intervention. Some practitioners have recommended giving large doses of purgatives 
with the idea of causing changes in the neighbourhood of the invaginated part; but such treatment presents little chance of success. The same is true of the administration of large doses of olive oil, either in the form of draught or of enema.

Siebert attempted reduction by generating $\mathrm{CO}_{2}$ from soda bicarbonate dissolved in water and diluted $\mathrm{HCl}$, injected successively per rectum. In time fæces and $\mathrm{CO}_{2}$ escaped, and the patient recovered. Siebert claims to have cured by this method a cow with invagination of five days' duration; but the effect of his treatment may be doubted, as afterwards a portion of bowel was found in the cow's dung.

When diagnosis is certain, the only treatment that can be recommended consists in performing laparotomy followed by enterotomy. One cannot, however, operate in all eases, nor do all cases offer the same chances of success. If the invagination is situated in the first portion of the small intestine, and is hidden beneath the circle of the hypochondrium, intervention is out of the question, but if it has been detected by rectal exploration in the last portion of the intestine, operation may prove successful. Only in cases of the latter description should it be attempted.

Laparotomy is performed in the right flank according to the usual method (see Möller and Dollar's "Regional Surgery," p. 313). After opening the peritoneal cavity, the invaginated loop of intestine must be sought. It is not always easy to discover amongst the mass of intestines present, but can be recognised by its hardness and by the congestion of neighbouring parts. After withdrawing it through the abdominal opening, the operator may then proceed by one of several methods.

(1.) Some authors recommend grasping the two ends, drawing them apart, and thus reducing the invagination. The actual manœuvre is not difficult, but even when unattended by accident or tearing of the intestine it is by no means always followed by recovery. Although the intestine may not appear gangrenous externally, necrosis often occurs eventually.

This method should only be practised during the first twenty-four hours after the appearance of colic, and even then one must always bear in mind the possible consequences just mentioned, and the chances of rapidly fatal septic peritonitis.

(2.) The second method consists in removing the invaginated portion of intestine. It is best to apply bichromatised catgut or silk ligatures to all the arteries which pass from the mesentery into the loop to be removed; after which the loop itself may be simply divided an inch or two above and below the invagination, in order to be quite certain that one is operating on healthy tissue, the divided ends being held meanwhile by an assistant. The intestine is afterwards sutured with a fine needle and 
bichromatised catgut or boiled silk. The form of suture will be found described in Dollar's "Operative Technique." It may be valuable to test the efficacy of decalcified bone tubes for uniting the ends of the intestine.

The operation is long, delicate and difficult, and it is imperative not to infect the abdominal cavity during its performance. To prevent this the liquid and solid materials present in the bowel may be thrust upwards and dormmards away from the diseased part before the section is made; and in this way the wound and the operator's hands are preserved from infection. The intestine should be kept closed during the application of sutures by means of flat clamps cautiously applied. In their absence the ends may be held by an assistant, whose hands should previously have been carefully disinfected.

(3.) In cases where the serous coats of the two portions of bowel constituting the invagination are to some extent adherent, another operation of a less perilous character may be performed. This consists in liberating the invaginated part by means of longitudinal incision, without previously disengaging the parts, and without resection. The invaginated (external) portion of intestine is divided longitudinally; the gangrenous part immediately becomes visible, and may be removed. The operator has then only to suture the longitudinal wound, an operation which is much easier and demands much less time than any circular intestinal suture whatever. These operations must not be attempted except in response to the express wish of the owner, who should be fully informed of the dangers to which they expose the animal; for after the second day of invagination local peritonitis has often developed and one is then operating on injured or infected tissues, in itself a very unfavourable modifying condition. The current formula that " the operation was very successful" is not accepted in veterinary practice when the patient dies three or four days afterwards. From the economic standpoint it is better to slaughter animals of any value, for unless secondary peritonitis has occurred, and the animal is not feverish, the meat is fit for consumption. "Volvulus," or twist of the intestine, is said to be almost unknown in eattle, though Reichert records a case of volvulus of the ileum.

\section{COLIC AS A RESULT OF STRANGULATION.}

The symptoms of this colic differ very little from those of the preceding with which they are often confused. But in regard to its causation the condition is essentially different.

Causation. Strangulation of the intestine in the ox may be produced in several different ways: by the passage of a loop of intestine through a tear in the epiploon, through the diaphragm, mesentery, broad ligament of the uterus, the serous layer surrounding the spermatic cord, etc., 
or by strangulation of an intestinal loop by fibrous bands resulting from chronic peritonitis, etc. Of these various causes, the three principal may here be described:-

(1.) Tearing of the mesentery. As a result of mechanical violence the epiploon or mesentery becomes fissured, and the peristaltic movements cause a loop of intestine to pass through and become fixed in the fissure. If the opening is narrow, as is usually the case, the base of the intestinal loop, riding on the lower lip of the slit, becomes constricted by the margins of the opening through which it has passed.

(2:) In pelvic hernia a loop of intestine passes between the spermatic cord and the walls of the pelvis. The fissure in this case is in the serous fold which supports the large testicular arteries and the vas deferens. The fold is often ruptured during castration, especially during the practice of " bistournage," in consequence of traction exercised on the cord.

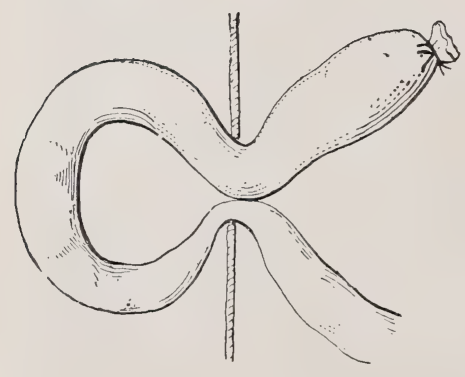

FIG. 69.--Schema of hernial strangulation.

(3.) Pseudo - ligaments and fibrous bands due to chronic peritonitis.-In local, subacute or chronic peritonitis false membranes may become organised, forming fibrous cords or folds connecting the parieto-visceral or inter-visceral surfaces. If by accident a loop of intestine insinuates itself beneath one of these fibrous bands, the passage of digestive material is first impeded and then stopped. The intestine becomes engorged, and symptoms of strangulation soon follow.

The symptoms appear suddenly, and are similar to those of invagination. They consist of very acute colic, which disappears after ten to twelve hours.

The peristaltic movements drive the semi-digested food, whether liquid or gaseous, towards the lower (strangulated) end, from which it cannot escape. It therefore distends the herniated loop and sets up intestinal engorgement. This constitutes the first stage of strangulation, and is accompanied by severe disturbance in the local circulation. The mucous membrane of the intestine becomes swollen and infiltrated, so that it alone soon fills the entire neck of the hernia. Necrosis of the loop of intestine is then only a matter of time.

The diagnosis of colic by strangulation is difficult. The condition cannot often be recognised at an early stage, and may easily and excusably be confused with invagination. Only in rare cases will rectal and abdominal examination enable one to detect a pelvic or mesenteric hernia. 
The prognosis is even graver than in cases of invagination. Intestinal hernia progresses very rapidly, necrosis soon sets in, and is followed by fatal consequences if the condition be not relieved.

The treatment is exclusively surgical. As a general rule, whenever colic is recognised as resulting from strangulated hernia, it is immediately necessary to perform laparotomy in the right flank, and after having discovered the cause of strangulation, to divide the mesentery, epiploon, serous fold supporting the testicular cord, or accidental fibrous bands, so as to free the herniated loop and avoid necrosis. If necrosis already exist, the intestine may be resected, exactly as in invagination.

\section{DISEASES OF THE STOMACH.}

In ruminants diseases of the gastric compartments are numerous, and, although they have been recognised since the earliest times, much remains to be discovered concerning at least some of them. This fact results from the imperfect state of our linowledge concerning the essential phenomena of gastric digestion in ruminants. Digestion really consists of a number of different acts-some mechanical or neuro-motor, some chemical; in addition to which must be reckoned the phenomena of sensation, concerning which patients cannot give any information.

The mechanical phenomena, consisting in the constant movement of ingested material through the different compartments, rumination, eructation, evacuation towards the intestine, etc., are rell known to us; and a careful examination of diseased animals enables us to estimate the importance of changes in them.

On the other hand, the chemical phenomena are little understood. It has hitherto been considered that the rumen, reticulum, and omasum are only simple diverticula, with mechanical functions, and that the abomasum is the reservoir in which the chemical changes take place. Another view, which is perhaps not altogether justified, presupposes that the chemical transformation of the food in the abomasum takes place as in other animals, and in particular as in man, in whom the chemistry of gastric digestion has been the object of extremely careful research by certain French and other pathologists. We do not believe (for reasons too long to be explained here) that the gastric digestion of ruminants, or even of herbivora in general, can be identified with that of omnivora.

The nature of the food being totally different, the chemical reactions in the stomach and intestines are also different; in proof of which we need only cite the single fact that ptyalin is absent from the saliva. Straw and oats are not digested in the same way as a mutton cutlet.

But even supposing that the broad outlines of physiological action 
are the same, nothing has hitherto been discovered in veterinary surgery respecting possible variations in the chemical processes taking place in the stomach during different gastric diseases; and it appears not improbable that in this direction causes might be discovered which veterinary practitioners have hitherto sought elsewhere. Excess or insufficiency of hydrochloric acid, and variations in the quantity of the organic acids, play so important a part in the theory of gastric pathology in man, that it is scarcely surprising to find similar ideas recurring in the pathology of domestic animals. The correctness of these views remains to be proved; and without wishing actually to classify dyspeptic conditions as in man, we may assert that diseases described under other names stand in direct relation to variations in the gastric secretion or to disturbance of gastric movements-e.g., simple chronic tympanites, which, without a doubt, is often a neuro-motor dyspepsia.

The classification we shall adopt in studying the diseases of the gastric compartments is, therefore, extremely simple. In the first series we shall consider sudden, accidental, and temporary forms of indigestion, and in the second series, acute or chronic forms of gastric inflammation.

\section{INDIGESTION.}

GASEOUS INDIGESTION.

Gaseous indigestion, also described as indigestion of the rumen, is characterised by the rapid accumulation of gases (chiefly carbon dioxide, carbon monoxide, and marsh gas), due to fermentation in the upper part of the rumen. It is common in oxen and sheep, and has received the names of mephitic indigestion, acute tympanites, meteorism, etc. It occurs during or immediately after feeding.

Causation. Numerous causes have been invoked to explain the sudden occurrence of gaseous indigestion.

The most important is the particular condition of the animal at the moment when it has been attacked. For if external influences alone were responsible, there is no reason why all the animals of a given herd or flock, or of a particular stable, which are under similar conditions as regards feeding, etc., should not be affected in the same way.

That the external causes cited (cold, excessive heat, stormy weather, etc.) may affect different animals differently and unfavourably is beyond doubt. But the temporary morbid condition of the animal itself is the essential condition to the development of indigestion.

In all probability the animal has in every case been more or less unwell, except in those attacks of indigestion resulting from progressive poisoning during the course of a meal, such as occur when toxic plants 
like belladonna, veratrine, colchicum, poppies, tobacco, hemlock, etc., have been eaten. In such temporary abnormal states movement of the rumen is partly abolished, or at least is markedly retarded, and, as a consequence of vaso-motor disturbance, the mucous membrane is probably not so abundantly covered with mucus nor so freely irrigated with secretion, as usual. Under these conditions, if the animal, which may appear perfectly well, is allowed to partake of soft, wet, fermentescible food, gaseous indigestion is very likely to develop.

Cultivated grasses, like lucern, sainfoin, clover, and especially grasses gromn on artificially manured fields, are regarded as particularly liable to cause gaseous indigestion. This conclusion seems justified by experience, particularly by the fact that young shoots or young, tender after-growths are very liable to fermentation.

This exaggerated tendency to fermentation of tender grasses has even been held exclusively responsible for indigestion, and the cessation of peristalsis in the rumen has been considered a secondary phenomenon, due to distension.

Whether atony of the rumen be the primary condition and abnormal fermentation secondary or inversely, whether fermentation be primary and atony secondary, is not of importance; for either view may be adopted without altering the results, and without the theory being invalidated by the objection that other animals subjected to similar influences had not contracted the condition

We have already dramn attention to the importance of the condition of the animal's health for the time being. Digestive peristalsis being diminished, eructation, admixture of food in the rumen, and its onward movement being impeded, fermentation proceeds rapidly. As a consequence the rumen becomes distended, and, cause and effect changing places, the distension in its turn arrests peristalsis, which had previously only been checked.

Local chills, produced by ingestion of food covered with rime, hoarfrost, or simply with dew, may favour gaseous indigestion; such conditions retard or suspend the peristaltic movements by direct local action, and probably by producing vaso-motor disturbance of the mucous membrane. In very rare cases chill has an undeniable influence, either by provoking general vaso-motor disturbance, which reacts on the secretions, or neuro-motor trouble. Gaseous indigestion is not uncommon in animals living on dry winter food, which have been moved from their ordinary quarters and sent on railway journeys or to fairs, etc. As a general rule this form of indigestion is commonest in spring, when the transition from dry winter food to grass, etc., has not been carefully effected. It is also frequent during stormy weather in full summer. Marked barometric changes seem to have an influence on the general 
health, and particularly on the nervous system, thus favouring organic fermentations.

Symptoms. The earlier symptoms of indigestion escape observation, but they soon begin to develop rapidly, and are then very easy to follow. They always exhibit the same characters, developing, however, with more or less rapidity in different cases. Soon after they commence feeding animals appear to experience special discomfort, which causes those at grass to stop grazing; even when stabled they stop feeding. From this time they show eructation, repeated yawning, restlessness, and some anxiety.

In a quarter of an hour, or less, the left flank begins to project, both laterally and vertically, so that eventually the walls of that part of the abdomen may project above the transverse processes of the lumbar vertebræ. The right flank also becomes swollen, as a consequence of the intestine being thrust out of position. The animal very rapidly shows general disturbance; the nostrils are dilated, the mucous membranes congested, respiration becomes rapid, and asphyxia threatens. The respiration soon becomes panting, for the distended rumen paralyses the diaphragm and compresses the lungs. To ease respiration the animals open the mouth, extend the neck, and stand with the front limbs spread apart; but this fails to prevent dyspnœa becoming more intense and asphyxia imminent.

The heart beats more rapidly, the superficial veins appear swollen, and the mucous membranes cyanotic. T'he rhythmic contractions of the rumen can no longer be detected by manual examination of the left flank; and on auscultation one neither hears the liquid nor the rolling sound, but only exaggerated crepitation. Finally, there is marked tympanitic resonance on percussion.

In cases of very grave tympanites the gaseous pressure in the interior of the rumen appears to stop the crepitation sound. The animals soon become unable to walk or even to move, suddenly fall to the ground, and die rapidly from asphyxia.

The rapidity with which gaseous indigestion develops varies greatly. Sheep and oxen may die from tympanites, within an hour or even half an hour of their arrival in the field; but more frequently the symptoms develop slowly, only becoming alarming after some hours and continuing for twelve or even twenty-four hours without causing death.

As a rule, the gas is voided by a series of eructations which empty the rumen, and recovery follows; but when distension is extreme eructation cannot occur, and gaseous indigestion then ends in asphyxia and death.

Lesions. It might be imagined that this form of indigestion would only appear when the rumen contains a large quantity of food; but, in point of fact, the rumen often " contains very little. 
On post-mortem examination the rumen is found to contain an enormous quantity of gas, which, when collected and submitted to analysis, reveals approximately the following composition:-Carbonic acid, 74 per cent.; carburetted hydrogen, 24 per cent.; sulphuretted hydrogen, 2 per cent.; nitrogen, traces. The composition of this mixture varies within certain limits, according to its origin; but carbonic acid always predominates.

Lungwitz, after elaborate experiments with different foods kept in closed vessels at the body temperature and with similar agents fed for days as an exclusive aliment to oxen provided with a fistula of the rumen for purposes of collection, found carbonic dioxide the predominating gas in all cases, though the proportion varied with the nature of the food.

Marsh gas varied from 16 to 39 per cent., being especially abundant in cases of abstinence. Hydrogen sulphide was found only in traces. Oxygen and nitrogen were present in small amount, and were attributed to air swallowed with the food. In fermentation the oxygen may be completely consumed.

The abdominal organs, particularly the intestine, are congested, as a result of impediment to the venous circulation. The thoracic organs exhibit the lesions of asphyxia.

Pathogeny. Death is due to carbonic acid poisoning, brought about in two different ways-viz., progressive asphyxia, caused by inability to inflate the lungs, and absorption of carbonic acid gas from the rumen; by virtue of the laws of diffusion, part of the gas contained in the rumen passes into the blood.

The diagnosis is always very easy, and even farm servants may recognise the condition.

The prognosis varies, according to the rapidity with which the disease develops. In rapid cases, where the condition is fully established in thirty minutes to one hour, asphyxia may be threatened from the beginning; but in others, e.g., when the attack follows consumption of dry food, tympanites may develop slowly, only attaining its maximum intensity after a considerable lapse of time. In general one may say that tympanites is grave in proportion to the rapidity with which the gas is generated.

Treatment. From the prophylactic point of view, it is necessary to avoid suddenly changing animals from dry to green food; the transition should be effected by giving mixtures of dry and green food.

Curative treatment comprises a large number of methods.

The latest, and one of the most practical, consists in massage of the left flank. The open hand is applied to the left flank and sharply pressed directly downwards, care being taken not to injure the parts. This manipulation excites reflex action, awakens the dormant contractility 
of the rumen, and leads to restoration of peristaltic movement. The gases pass into the omasum and abomasum, or in many eases make their way into the œsophagus. The sudden impulses sometimes cause food to be returned into the mouth, eructation recommences, and the gas accumulated in the rumen is partially and progressively evacuated. This manipulation is often practised in breeding districts, particularly in the case of sheep, in which the disease occurs with the same characters. The shepherd fixes the animal between his legs, and, thrusting the extended fingers of either hand into the flanks, makes sudden, sharp movements, which again set up eructation and get rid of the excess of gas.

In Germany cold douches are often applied to the flanks. These excite vaso-motor action and reflex peristaltic movements, which result in eructations and in the evacuation of the rumen. But this is not a very practical method, and necessitates arrangements which seldom exist on sheep farms.

The action of massage may be completed by administering stimulants like wine, alcohol, or infusions of such aromatic plants as cummin, fennel, peppermint, camomile, etc. These act first of all mechanicaliy, by clearing the terminal portion of the œesophagus. Furthermore, they stimulate the mucous membrane of the rumen, causing reflex peristaltic contractions, and, as a consequence, circulation of the partly digested food; finally, the majority of them arrest fermentation.

With the latter object, ether and assafœtida are also given. The use of these drugs, however, entails disadvantages, and if the animal has finally to be slaughtered renders the flesh unfit for consumption.

The giving of absorbents is probably most widely practised. The ammonia which many of them contain absorbs carbonic acid, thereby diminishing the pressure of gas contained in the rumen, and therefore the distension of the first gastric reservoirs. Unfortunately this action is only temporary, and if the drug is given in too concentrated a form, the mucous membrane of the mouth, of the oesophagus, and sometimes even of the rumen and reticulum, may be irritated and inflamed, producing lesions of stomatitis, pharyngitis, œesophagitis, contraction of the oesophagus, etc., which after recovery from the acute condition may gravely affect the animal's general health. A further drawback is that the flesh rapidly acquires an ammoniacal odour.

Perhaps the best internal treatment consists in administering purgatives such as hyposulphite or sulphate of soda or sulphate of magnesia, in doses of 10 to 20 ounces, according to the animal's size, or, in the case of pregnant animals, in small frequently repeated doses. 'These check fermentation, and so arrest the evolution of gas, whilst by their purgative properties they excite contraction of the gastric reservoirs and cause eructation. 
None of these methods of treatment, therefore, should be used exclusively, but all may be utilised as auxiliaries to mechanical or surgical measures, and all should be preceded by the use of the probang and puncture of the rumen.

The first of these operations, the technique of which scarcely requires description, is often of little value; for the solids and liquid contents of the rumen being permeated with gases, rise as a fermenting mass into the upper portions of the rumen, and continually obstruct the open end of the catheter, so that very little gas escapes.

Puncture of the rumen is much more effective and easier to perform. The owner himself often operates with an ordinary pocket knife, sometimes introducing a couple of fingers or a short length of elder-wood tube into the wound thus produced.

The incision should be made at one stroke, for any hesitation may cause the wall of the abdomen to recede from the rumen, which lies immediately below. Should gas escape under the skin, emphysema, which often extends to the loins and along the quarters, may be produced, and may be followed by diffuse subcutaneous suppuration, re. sulting from pyogenic germs entering the subcutaneous tissue.

Large quantities of gas escape from the puncture, sometimes with such force as to drive out the canula. The flow of gas then ceases. In other cases the tube becomes blocked; because, as the pressure within the rumen diminishes, the gases dissolved or mixed with the partially digested food are freed, and the whole contents of the rumen become converted into an aerated, bubbling mass. Liquid or semi-liquid materials may be ejected to some distance, or may pass between the skin and the muscles, or between the walls of the rumen and the abdomen, producing various complications, like necrosis, abscess formation, etc. Such accidents can be avoided by exercising firm pressure with the fingers on the tissues surrounding the canula.

Even when the rumen has resumed its normal size recovery is not certain, and may not occur for several hours, or even several days, afterwards. The patients should therefore be kept under observation for some time, and it is usually best to leave the canula in place for one or two days, and to put the animal on low diet.

Necrosis of aponeurotic tissues, fistula formation, and local peritonitis only occur if the instrument is dirty or is introduced in a wrong direction.

IMPACTION OF THE RUMEN. INDIGESTION AS A RESULT OF OVER-EATING.

In this condition the rumen is over-distended with food. The symptoms are principally due to abnormal fermentation, the peristaltic 
action of the rumen being in abeyance, and the food failing to pass towards the omasum and abomasum. Rumination is generally suppressed.

The disease usually follows change of diet. When the diet has long been restricted, as occurs during years of bad harvests, and animals are afterwards set at liberty in rich pastures, they eat greedily, distend the rumen with large quantities of green fodder, and set up all the necessary conditions for this form of indigestion. Similar results follow when gluttonous animals are freely supplied with rich food. Working oxen also suffer if withdrawn from work and fed with roots, beetroot refuse, brewers' grains, or other manufacturing residue for the purpose of fattening. These materials can only be absorbed in moderate quantity, and the large amount of water, etc., they contain is apt to disturb the animal's digestive powers, while owing to its fine state of division such food cannot be returned to the mouth for secondary mastication, and rumination therefore remains incomplete: the food accumulates in the rumen, distending and eventually paralysing it. This is a common result of feeding on semi-liquid pulp, which in order to be ruminated should be mixed with rough forage.

Insufficiency of drinking water is another and more frequent cause, especially during the winter, because the ox-herd or cowman is often too lazy to give a regular and sufficient supply unless water is laid on in the stable itself. The dry food becomes compacted into a mass, which cannot be returned to the mouth for rumination. Moreover, less saliva is then secreted, and Colin has shown that rumination is impossible when the parotid ducts are ligatured.

Symptoms. As may readily be imagined, the symptoms vary, according to the quantity and digestibility of the food swallowed. In the first place the appetite falls off : animals suffering from commencing indigestion only take part of their food; later on appetite ceases, and with it rumination. Trifling colic sets in, resembling that due to congestion, and is indicated by unrest, switching of the tail, lifting of the hind legs, slight groaning, moving from side to side, and lying down and rising at short intervals. The animals seem oblivious of their surroundings, anxious, and at times semi-comatose.

When the case has been neglected for several days the animal may masticate without having any food in the mouth, and may attempt to eructate and to regurgitate food; but such attempts always fail. It then absolutely refuses food, and animals which have eaten large quantities of green forage may show tympanites. If called in at this period of the disease the veterinary surgeon finds nothing positive except signs referable to the digestive apparatus. By methodically examining the digestive tract, and in particular the stomachs, one discovers during palpation of the left flank that the rumen is distended. This is characteristic. By 
deep palpation it is even possible to detect marked resistance and a certain characteristic firmness resulting from accumulation of food. The percussion sounds over this region are dull, and pressure causes pain, as though the rumen and peritoneum were inflamed. When the open hand is laid flat on the rumen and thrust downwards, no peristaltic movement can be discovered. Finally, on auscultation the normal sounds, including crepitation, fermentation, and rolling sounds are all absent.

There are no well-marked general symptoms. Respiration and circulation are hardly accelerated, nor is the artery particularly tense.

Course and Termination. The course of the disease varies, and the condition may be divided into two forms, acute and chronic. The first develops in a single day, and may cause death by the same mechanism as acute tympanites-i.e., asphyxia or carbonic acid poisoning; the other continues for five, ten, or even twenty or thirty days, according to the promptitude with which treatment is undertaken.

In protracted cases, however, the indigestion itself ceases to be as important as the complications. Sometimes spontaneous recovery occurs, the food passing away towards the intestine, or even being vomited, though the latter conclusion is rare. Recovery may also follow from treatment. If the disease is neglected it may become complicated with gastro-enteritis.

The diagnosis is not very difficult. Indigestion resulting from impaction is distinguished from acute tympanites by its less rapid course and by the less marked distension of the rumen (in this case due to solid food), and from acute gastro-enteritis by the varying degree of fever which accompanies the latter condition.

Prognosis. The prognosis is always grave, even in cases of acute indigestion resulting from eating green food.

In this case gaseous indigestion occurs as a complication, and necessitates immediate intervention. The other forms may rapidly yield to proper treatment, or, in spite of every care, may give rise to prolonged complications.

Lesions. On post-mortem examination of animals which have died of complicated forms of the disease, we find certain lesions peculiar to gaseous indigestion associated with impaction of the rumen.

If death has followed the consumption of root pulps, we see signs of poisoning. As a consequence of prolonged stagnation of food in the rumen, there follows an exaggerated organic fermentation, whose products are absorbed through the stomach or intestine and pass into the circulation.

These various fermentations, which may be of the lactic, butyric, and even putrid order, produce changes in the mucous membrane of the rumen; wide tracts of the epithelium may be shed, exposing the corium,

D.C. 
and producing enormous ulcerations, which in certain cases implicate the entire inner surface of the rumen.

The treatment must be varied, according to the cause, symptoms, and immediate complications. When the disease is of an acute type, such as that produced by over-gorging with lucern and green food, it is best to proceed as in gaseous indigestion, i.e., to puncture the rumen and give frequent large doses of purgatives until the stomach and bowels have been freely unloaded. The animals should then be kept for some days on small quantities of easily digested food, and should be allowed lukewarm, mucilaginous drinks.

When tympanites and impaction occur simultaneously, immediate surgical intervention becomes necessary, and gastrotomy may then be performed by a very simple method.

Two loops of cord are passed around the abdomen, one behind the hypochondriac circle, the other in front of the angle of the haunch. Assistants placed on the right side draw these loops tight, so as to immobilise the left flank. A bistoury is then thrust directly through the walls of the abdomen and rumen. As a consequence of the pressure exercised by the ropes, if not of the pressure of gas itself, the food material contained in the rumen is often expelled in a powerful stream. As the superposed tissues cannot very readily change their mutual relations, the author of this suggestion claims that there is little danger either of infectious materials passing into the subcutaneous connective tissue, or of peritonitis; but this rude treatment can only be resorted to in cases of extreme urgency, and it appears by no means without danger.

Injections of 10 to 15 centigrammes of pilocarpine and 5 to 10 centigrammes of eserine are also useful.

When impaction of the rumen assumes a less acute form, moderate doses of purgatives may be given and repeated daily, or twice a day, until the peristaltic action of the rumen is restored and resumes its normal rhythm. In certain cases, however, recovery is only apparent. The food in contact with the walls of the rumen breaks down, and passes away into the abomasum and intestine, while appetite returns. The animals then resume feeding, and some days afterwards show all their former symptoms. Low diet should therefore always be continued for some time.

In spite of treatment, or in consequence of treatment being too long delayed, no improvement may follow. The ingested food is not expelled. Putrid fermentation results, auto-intoxication sets in, and the temperature rises to $40^{\circ}$ or $41^{\circ} \mathrm{C}$. Unless gastrotomy is performed death is then certain.

This operation should be undertaken whenever the fever rises to $40^{\circ} \mathrm{C}$., and two-thirds of the contents of the rumen removed. The rumen should 
not be completely emptied, as there is danger of collapse of its walls. Complications in the region of the wound can be avoided by drainage.

If the operation succeeds, the patients must be placed on very low diet or on milk for some days, and should be given lukewarm farinaceous drinks, and a little hay of good quality to excite rumination. In old milch cows this operation is seldom followed by a satisfactory recovery. Apart from the loss of milk, the animal loses condition, refuses to feed, and gradually succumbs to exhaustion.

\section{IMPACTION OF THE OMASUM (THIRD STOMACH).}

Definition. "A form of indigestion, of which the prominent feature is the drying and impaction of the ingesta between the folds of the third stomach. It may seem to be a primary disease, but in very many cases it occurs as a result of some acute febrile or inflammatory affection." (Law's "Veterinary Medicine," Vol. II. p. 123.)

Synonyms. Dry murrain, clew-bound, fardel-bound, stomach staggers, grass staggers, vertigo, chronic dyspepsia, chronic indigestion.

Causes. Torpidity of the omasum, suppression of salivary secretion, with absence of "waves of liquid floating the finely divided food from the mouth or rumen to third stomach, are prime conditions of desiccation of the contents." The third stomach, like the first and second, has no provision for liquid secretion, and depends for its supply on constant flushing by swallowed fluids. Therefore, if feeding and rumination are arrested and salivary secretion is suppressed, and if movements of the rumen and resulting overflow into the third stomach are checked, the ingesta of the third stomach, compressed between its folds, becomes drained of liquid and converted into a powder or dry mass. All febrile and inflammatory affections tend to this end, and more or less drying, with impaction of the contents of the omasum, is a constant feature in such cases. But in the majority of cases this condition is to be looked on as a secondary or subsidiary affection, and the real disease must be sought elsewhere.

The explanation of the susceptibility of the third stomach in constitutional troubles has been sought in the source of its innervation. Electric stimulation of the vagus rouses the movements of the first and second stomachs, but not those of the third. Action of the third stomach is excited by stimulation of the spinal cord, and of the sympathetic nervous branches going to the ganglionic cells in the ralls of the omasum (Colin and Ellenberger). Its nerve supply coming from a different source, derangement of its function may occur independently of antecedent disorder of the first or second, and its motor supply coming from a source so closely related to the vaso-motor centres, perhaps affords some explanation of the 
connection of disorders of the omasum with febrile and inflammatory diseases.

Food is an important cause. Impaction of the omasum is a winter disease-the time of dry feeding. Dry, fibrous, innutritious fodder, and scarcity of water contribute to its production. It attacks cattle in spring or autumn on pastures in which fresh grass grows among the dead, dried, or withered stems of a previous growth. It occurs when stock are fed on corn or corn stalks (maize stalks) affected with smut or ergot, or on cereals or grasses similarly damaged, and in both cases especially when the water supply is deficient or restricted.

Sheep and goats, which habitually drink little, suffer less than do cattle, which drink freely.

Other causes. Fermented foods, microbian ferments and their products, which tend to induce torpidity of the omasum, fever, and lessened secretion of saliva, with diminished supply of liquid from mouth or rumen.

Pericarditis, by causing vascular stasis in the omasum, may induce torpor and impaction.

Lead poisoning paralyses action and favours impaction. Finely divided food stuffs - meal and bran-eaten greedily, may pass in quantity directly into the omasum and induce impaction. "The most acute and fatal forms occur in connection with a sudden change from dry to rich, luscious, green food in spring, the unwonted stimulus giving rise to general irritation of the whole gastric mucosa, with disordered and impaired function of all four stomachs, but especially of the third. Such cases are usually congestive and inflammatory, and the suspension of the gastric movements is a grand cause of impaction. In such cases, too, the brain or spinal cord, or both, are seriously involved, and the early death is preceded by torpor, paralysis, violent delirium or convulsions, following largely the type of acute lead poisoning." (Law, loc. cit.)

The symptoms depend on the degree of impaction, and vary from simple, irregular, or suspended rumination to severe gastric and nervous disorder. The less acute cases are marked by failure to re-establish regular rumination or partial convalescence from fever or inflammation. The fever subsides, but the appetite remains eapricious, the muzzle dry, eyes dull, spirits low, and breathing accelerated; the condition is sometimes accompanied by moaning. Slight tympanites may appear, and the contents of the rumen may feel solid, the mouth hot, clammy, and fotid. The bowels are constipated, the fæces small in quantity, hard, covered with mucus or blood-streaked, and containing particles of undigested food; in other cases diarrhœa may set in, to be followed later by constipation. Alternations of constipation and diarrhoa may be repeated again and again. Exploration by pressure of the closed hand over the omasum will give an impression of solid resistance. There may be slight 
shivering, the ears and limbs are cold, the hair is erect in patches, dry and lustreless.

In cases occurring independently of previous disease, diarrhœea may be the first symptom observed, the malady being preceded by local irritation and congestion; but this soon gives place to constipation or diarrhoea and the symptoms above mentioned. The animal is found lying apart on its left side, with its nose in its right flank, the pulse and breathing quickened, the eyes congested; expiration is accompanied by a grunt. The patient walks with its back arched and dragging its limbs. The appetite may continue, but only in an impaired and irregular form, and as rumination ceases grinding of the teeth becomes common. The secretion of milk is diminished or arrested, emaciation advances day by day. Fœtid eructation may be a marked symptom. This form may last from ten to fourteen days, and merge finally into paralysis of the hind limbs, drowsiness and stupor, or delirium and convulsions.

"In more acute cases (from sudden access of green food, change of water, or ingestion of irritant plants), the affection partakes more or less of the nature of congestion or inflammation of the viscus (omasitis), and may run a rapidly fatal course" (Law, loc. cit.). The animal is seen apart from the herd in a characteristic recumbent position, the eyes are red and glassy, the eyelids semi-closed, the patient shows much drowsiness and stupor, but when raised may still feed in a sleepy, listless manner. The bowels are loose or confined, the pulse and breathing accelerated, the right hypo-chondrium is firm and tender, and the sound of fermentation absent or subdued over the omasum. Soon nervous disorder appears, the eyes glare wildly, the animal seeks relief in motionsometimes in a straight line, sometimes to one side-and being blind and unconscious of obstacles, may fall into pits or ditches, knock against trees, fences, gates, or buildings, and continue pushing against resisting objects, breaking its horns or teeth, and otherwise sustaining injury through violent muscular contractions.

Course. Chronic cases may continue indefinitely, with symptoms of poor health, impaired digestion, and gradual loss of condition. After death the omasum may contain dried food which the animal consumed several months before the attack.

In cases ending in early recovery there occurs abundant diarrhœea, "the fæces are mixed with flattened, dark, solid, and polished masses, the impacted ingesta from the omasum. Tympany subsides; movement in rumen and omasum and rumbling in bowels can be heard. Appetite returns." (Law.)

Diagnosis. The condition of the pulse and respiration, and the grunting. with expiration may lead to confusion with pneumonia.

At first there is no fever, tenderness is confined to the right flank; 
there is an absence of pulmonary crepitation, of pleural effusion, and of movement in the rumen and omasum. Signs of gastric and intestinal disorder can be detected.

Lesions. The omasum is gorged - it may be twice its normal sizesolid, resistant, almost stony. The spaces between the leaves are packed with dried food, which, when removed, carries a layer of epithelium from the mucous membrane. (This (layer on contents) is not inconsistent with health.)

The rumen contains ingesta packed in masses, more or less offensive from putrefaction.

The abomasum is empty of food, but contains much mucus. Its mucous membrane is congested.

The small intestine is red in places, empty and collapsed.

The larger intestine contains a quantity of dry, glistening pellets, and much mucus.

Treatment follows the lines of impaction of rumen, though the response is usually less certain, and always slower. Flax-seed tea, several bucketfuls per day, will often succeed.

Epsom and common salts, with sol. ammoniæ, excite thirst; liquids should be supplied freely.

In obstinate cases, and in absence of gastric or cerebral congestion, 20 croton beans, or 20 drops of croton oil, may be added to the purgative. Nux vomica stimulates the nervous supply. Enemata may be given freely.

Other remedies, stimulating contractility and secretion, are: Eserine, $1 \frac{1}{2}$ grains ; veratrine, 1 grain ; barium chloride, 10 to 15 grains; or pilocarpin, 3 grains, hypodermically.

The patient may be days or even a week without alvine discharge and recover.

If fever and symptoms of gastric congestion appear, a blister may be applied to the right side over the omasum.

Nervous symptoms, such as dilated pupils, blindness, congested mucous membrane, hot horns and ears, drowsiness or excitement, are combated by applying cold water or ice to poll, etc.

When free action of the bowels is restored, laxative diet, roots (pulped), green food, plenty of common salt, and free access to drinking water should be prescribed.

During convalescence a course of tonics, including nux vomica, is advisable to help in restoring normal gastric functions.

\section{ABOMASAL INDIGESTION.}

Primary indigestion in the abomasum appears to be rare in adults, for until the present time no one has given a sufficiently characteristic 
description of this disease to enable it readily to be recognised. On the other hand, it is to be presumed, although final proof has certainly not been furnished, that in cases of gaseous indigestion, or of impaction of the rumen, the abomasum, whose physiological action is predominant, must simultaneously suffer.

Primary abomasal indigestion, on the contrary, is common in young animals before weaning, so that the condition has been given the name of "milk indigestion." It could not very well be otherwise, for the abomasum is the only one of the gastric divisions which in ruminants is active during the first few weeks of life. At this period it is larger than the other gastric reservoirs; and the rumen, the reticulum, and the omasum do not undergo great development till weaning begins.

Causation. Milk indigestion attacks young animals under varying conditions.

In animals suckled by the mother the disease rarely occurs, but yet when the mothers are good milkers, like the Flemish, Norman, Jersey, and Holland breeds, and when there is too long an interval between the feeds, calves, which are naturally greedy, and in addition are hungry, are apt to take too large a quantity of milk-in fact, they often gorge to the fullest possible extent. Owing to its over-distended state the abomasum either fails to secrete sufficient of the rennet ferment necessary for coagulating the milk or secretes an insufficiently active ferment. The first stage of digestion remains incomplete, giving rise to so-called "milk indigestion."

When the cows are employed in ploughing, etc., or in drawing carts, not only are the calves fed at long intervals, but the milk is not always of proper chemical composition even in the udder. As a result of work, fatigue, over-exertion and irregular feeding, the cow's yield of milk for the time is less digestible than the normal supply, or may even prove irritant to the calf's stomach. Milk indigestion is thus set up.

When the cows are fed on factory waste, like beetroot-pulp or brewers' grains, toxic or irritant products may even find their way into the milk, which then irritates the little creature's abomasum and produces gastric indigestion. Just as in the production of congenital alcoholism in man, the young animal is then ingesting, unknown to those responsible for its well-being, chemical substances which produce various pathological changes.

But milk indigestion is commonest of all in calves fed by hand. The food usually given is a mixture of milk from the previous night, and skim milk or even butter milk. It contains lactic ferments and various microbes, some capable of producing toxic principles.

When swallowed and brought directly in contact with the mucous membrane these cause abomasal indigestion. 
Symptoms. Soon after feeding, the little animal appears dull and somnolent, and shows moderate abdominal pain, suggesting trifling colic.

This stage is soon followed by nausea; the breathing and the heart's action become rapid, vomiting efforts are made, and finally milk, in the form of firm or partially softened curds, depending on the time which has elapsed since the last feed was taken, are vomited. The quantity ejected varies. Pressure over the right side of the abdomen produces pain, and tympanites of the abomasum may sometimes be detected on percussion.

The sensitiveness and gaseous inflation are confined to the middle and lower zone of the hypochondrium. Soon after vomiting the animal begins to improve. The patient seems brighter, relief is very marked, and in some cases proves permanent; but more frequently a certain degree of depression persists, the mouth emits a sourish odour, and for a time the appetite remains poor. This temporary irritation of the abomasum has a tendency to become permanent; or even to extend to the intestine, in which the conditions appear more favourable to the development of micro-organisms than do those in the stomach. Indigestion then becomes complicated with diarrhœic enteritis.

The diagnosis presents no difficulty.

The prognosis is not serious, provided that the young animals are carefully attended to ; but such complications as diarrhœic enteritis may become very grave if neglected.

The treatment. To prevent recurrences :

(1) The periods of feeding should be regulated;

(2) The cows should not be worked, or should be worked as little as possible ;

(3) Mixed milk, or milk which has already undergone lactic or other fermentation, should be avoided.

If the calves must be reared by hand, the mixed milk should at least be boiled or relatively pasteurised by heating to $70^{\circ}$ or $80^{\circ} \mathrm{C}$., and the buckets used for feeding should be kept scrupulously clean. These precautions become absolutely necessary when diarrhœa exists amongst the calves. Curative treatment consists in placing the animals on low diet for two or three days after the attack of indigestion, or in giving them boiled milk diluted with from one-half to two-thirds of boiled water.

The addition of a mild saline purgative like sulphate of soda, in doses of one-half to three-quarters of an ounce, usually ensures a cure. Infusions of lime-tree flowers, peppermint, camomile, etc., may advantageously be used to replace boiled water in diluting the first foods. 


\section{ACUTE GASTRIC INDIGESTION IN SWINE.}

The causes comprise putrid food, swill, spoilt turnips, potatoes, apples, succulent vegetables, frozen food, and the admixture of caustic alkaline powders (used in washing table dishes) with the swill. Indigestible matters-hoof, horn, hair, bristles, tree bark, etc.-when not rejected by vomiting, cause gastritis and indigestion. Lastly, medicinal substances and poisons, paint and lead, sometimes produce the disease.

Among the symptoms may be mentioned dulness, arching of the back, standing with the feet brought together, erection of the bristles, hiding under the litter, grunting, uneasiness, shifting from place to place, tenseness of the abdominal wall, borborygmus; these may be followed by diarrhœa and recovery. Speedier relief is afforded by copious vomiting of irritant matters.

The treatment should commence with the free administration of emetics. To combat alkaline poisoning vinegar may be given, followed by a laxative. Prophylaxis calls for greater care in feeding. 


\section{CHAPTER V.}

\section{ACUTE INFLAMMATION OF THE GASTRIC COM- PARTIMENTS.}

\section{RUMENITIS-RETICULITIS-GASTRITIS.}

Causation. Acute primary inflammation of the first gastric reservoirs, viz., the rumen and reticulum, is not common. It sometimes accompanies such infectious disorders as foot-and-mouth disease, gangrenous coryza, etc., but then constitutes an added phenomenon which should be studied along with the original disease itself. Rumenitis or reticulitis may however follow the ingestion of irritant foods or plants, of very hot liquids, and more frequently still of unskilfully compounded medicines. In such cases the mucous membrane is directly attacked, and pathological congestion, infiltration, and desquamation may follow, or even vesicles and ulcerations may rapidly be formed.

Symptoms. Inflammation of the rumen or reticulum is announced by loss of appetite, suspension of rumination or of regular peristalsis, slight tympanites, and particularly by excessive sensitiveness to palpation. This sensitiveness is general, but is more specially marked in the left lower third of the abdominal cavity, and in the retro-ensiform region which corresponds to the position of the reticulum. Moderate fever is present.

These symptoms, which indicate the gravity and intensity of the inflammation, may persist, become aggravated, provoke vomiting from the rumen, and leave as a legacy motor dyspepsia, or even more serious consequences. On the other hand, they may progressively diminish and disappear for good.

Lesions. The lesions comprise hyperæmia of the walls of the rumen and of the mucous membrane, extensive local exfoliation of epithelium, and sometimes true ulceration of the mucous membrane.

Diagnosis. The diagnosis is based on the exceptional sensitiveness of the gastric compartments on palpation, and also on the history, provided reliable information can be obtained.

Prognosis. The prognosis should be reserved, because it is never possible to foretell whether acute lesions may not give place to chronic 
disease, which, though apparently unimportant, may terminate in grave consequences.

Treatment. Owing to their local action demulcent drinks and teas are indicated. Cooked food is useful, because it makes little demand on the digestive powers; steamed hay and farinaceous substances are given, both on account of their nutritious qualities and of the slight local irritation they cause when swallowed.

Lukewarm drinks and saline laxatives, such as the sulphate of soda, and carbonate of soda or Carlsbad salt in small doses of $1 \frac{1}{2}$ to 2 ounces, seem most useful in combatting the reflex atony of the digestive compartments.

Inflammation of the omasum, like that of the rumen and reticulum, occurs as a secondary phenomenon in conditions like rinderpest, Texas fever, foot-and-mouth disease, anthrax, and gangrenous coryza (malignant catarrh); but primary inflammation is much rarer even than that of the rumen and of the reticulum.

This is accounted for by the deep position of the omasum, which is thus sheltered from external violence, early contact with irritant foods and from the effect of chills, etc. It can only become inflamed by the prolonged action of irritant food and drink, which have already produced lesions in the rumen and reticulum; or as a consequence of the prolonged stagnation of dry food in cases where animals have been deprived of water.

Under these conditions inflammation of the omasum develops slowly, and from the clinical point of view is identical with what was formerly known as obstruction of the omasum. Obstruction or impaction is probably much rarer than has been stated, in so far at least as it constitutes a primary condition, for in the great majority of cases it is consecutive to impaction, inflammation of the rumen, or inflammation of the abomasum. Obstruction of the omasum, which was formerly invoked in all doubtful and ill-defined cases of digestive disturbance, seldom occurs as an isolated disease.

It has been suggested that the omasum, being supplied with nerves solely by the sympathetic system, and provided with a relatively weak muscular coat, was more susceptible than the other reservoirs to the reaction of abdominal reflexes, and therefore more subject to inflammation, indigestion and obstruction. We do not hold that view, because, as a result of its general situation and the position of its orifices of communication, this compartment is easily able to expel its contents so long as they are liquid. Its function appears chiefly to be to complete the trituration of food after rumination.

We do not consider that inflammation of the omasum never occurs, for we are well aware of the contrary, and that the inflammation assumes a subacute course and is accompanied by stasis of the semi-digested food between the mucous leaves which partly fill the cavity. We simply wish 
to emphasise the view that the condition is not a primary and isolated inflammation.

Symptoms. The symptoms are always vague and very difficult clearly to define.

Inflammation of the omasum is indicated by relative loss of appetite, marked thirst, general atony, and diffuse and vague sensitiveness in the inferior half of the right hypochondrium (zone of the asternal ribs). There are no pathognomonic symptoms.

Obstruction has also been described as accompanied by loss of appetite, constipation, the passage of black, coated, fœtid and sometimes blood-streaked fæces, symptoms of chronic tympanites with fotid eructations and sometimes vomiting. These sometimes accompany hydrochloric acid dyspepsia, a form of chronic gastritis, and, from our standpoint, the stagnation of food in the omasum is only secondary. We therefore interpret the facts in quite a different manner, and believe that only by a rational and physiological interpretation of the symptoms observed can one diagnose the condition.

Diagnosis. The diagnosis of inflammation of the omasum can only be made by a process of exclusion; and although we are admittedly dealing with a condition secondary to disturbance of the rumen and reticulum, or on the other hand consecutive to inflammatory states or to modifications in the secretion of the abomasum (dyspepsia), the diagnosis does not present insuperable difficulties.

The prognosis is only grave when the primary acute or chronic conditions of the other gastric compartments are serious.

The lesions comprise abnormal vascularity of the mucous membrane and desquamation, and even gangrene, of the leaves. The partially digested food is not passed on, becomes dry and hard, and in time aggravates the local condition.

The treatment does not essentially differ from that of other gastric inflammations. The object to be attained is to evacuate as completely as possible, not only the omasum, but all the gastric reservoirs, for which purpose one may freely administer demulcent drinks-linseed gruel, bran mashes and lukewarm liquids containing laxatives. At first such alkaloids as arecolin and pilocarpine may be subcutaneously injected to ensure energetic and speedy evacuation.

Later on slightly stimulant aromatic infusions, like infusions of sage, peppermint, hyssop, thyme, etc., stimulate the functions of the stomach and hasten the return of normal conditions.

\section{ACUTE GASTRITIS.}

The term "acute gastritis," sometimes "gastro-enteritis," is used in bovine pathology to indicate inflammation of the abomasum. If this 
inflammation is confined to the superficial epithelial layers it is defined as superficial catarrh of the abomasum; if, on the contrary, it extends to the deep epithelium of the gastric glands and to the mucous corium, it is termed deep-seated gastritis.

Clinically it is impossible to make these distinctions. We simply recognise degrees of gravity, and only in this way can one diagnose acute gastritis, phlegmonous gastritis, ulcerative gastritis, etc.

Causation. The abomasum frequently becomes inflamed as a consequence of irritant foods, apart altogether from lesions of the rumen or reticulum, the mucous membrane lining the abomasum being so much more delicate than that of either of the two first compartments.

Irritant plants, parasites, acid drinks, very cold water, certain acid or toxic industrial residues like mouldy brewers' grains, fermented vegetable pulp, decomposed beet, etc., and mouldy or spoilt forage of any kind may all produce acute gastritis.

Intense feeding-i.e., feeding with farinaceous materials, with large quantities of beans, roots, peas, given regularly-may also cause gastritis by overtaxing the functions of the organ. Frozen or fermented roots and sudden changes in feeding produce similar results. Chills have also been blamed, but it is probable that they only act as favouring causes.

Symptoms. It is necessary to consider these very carefully in order to arrive at a correct diagnosis.

Inflammation of the abomasum is attended with moderate fever, diminution in appetite, irregularity in rumination and some tension of the rumen, without, however, true tympanites.

At first the bowels are constipated, but in time fœtid diarrhœa sets in. Examination of the digestive apparatus on the left side and in the right posterior abdominal region reveals nothing abnormal, but pressure over the lower portion of the abdomen and along the cartilages of the right hypochondriac region produces, on the contrary, well-marked pain. This region corresponds to the position of the abomasum.

The conjunctiva appears reddish yellow, as in most visceral inflammations.

Some authors have described attacks of extreme excitement, but these are no more pathognomonic than is grinding of the teeth, which is a constant symptom, or the metallic sound noted on auscultation of the rumen. This sound occurs in all cases of inertia of the rumen, and indicates distension and emptiness of the viscus (acute peritonitis, chronic adhesive peritonitis, inflammation of the reticulum as a consequence of the presence of foreign bodies).

Dull colic and groaning are not uncommon. Finally, Thierry and others have all mentioned an alliaceous smell of the eructations as pathognomonic. 
Acute gastritis develops regularly in ten to fifteen days, after which the symptoms diminish and disappear, giving place to normal health. In grave cases, despite proper treatment, acute gastritis more frequently ends in a chronic condition, finally leading to gastric atrophy, and the insufficient secretion of hydrochloric acid, with all the consequences of these conditions. The glands of the stomach degenerate; the secretion becomes abnormal and dyspepsia is set up.

Diagnosis. The diagnosis is rather difficult, for the condition is very apt to be confused with primary dyspepsia, or with inflammation of the gastric compartments. It might also be mistaken for acute enteritis of the first part of the small intestine; but as gastritis is very often complicated with duodenitis, such a mistake is without serious consequences.

Prognosis. The prognosis is grave, not because death is a frequent termination, but because the disease very often leads to chronic incurable lesions.

The lesions consist of congestion of the vascular network of the mucous and subepithelial coats, serous infiltration of the corium and submucous connective layers, desquamation, and later in excessive proliferation of the epithelium.

When the inflammation is deep seated the epithelium of the gastric glands becomes swollen and cloudy, and undergoes a kind of atrophic degeneration. In very grave cases, petechiæ, superficial capillary hæmorrhages, and slight ulceration may be noted. The mucous folds are always thickened and infiltrated.

Treatment. In cases of gastritis or acute gastro-duodenitis moderate bleeding (three to four quarts) and local stimulation were formerly recommended. This practice certainly has its advantages, provided it is not pushed to excess. Sinapisms give good results, but as they must be left in position for a considerable time, it is often better to apply vesicants over the lower right hypochondriac region. At first purgatives are useful, because they unload the digestive tract, arrest the organic fermentation which results from stagnation in the movement of food along the alimentary tract, and diminish the tendency to intoxications or infections.

At a later stage small doses of laxatives and bicarbonate of soda should be given daily, the diet being of an emollient character, and consisting of milk, starchy or farinaceous foods, and small quantities of good hay.

Linseed, bran, cooked grain, decoctions of pellitory, barley and various cereals may also be administered with advantage.

CATARRHAL GASTRITIS IN SWINE.

Definition. Inflammation of the gastric mucosa, with muco-purulent discharge. 
The causes comprise irritants, fermented or putrid swill, decomposed food, excess of brine, alkalies (washings from table dishes, hotels, etc.), gastric parasites.

The condition occurs also in hog cholera, swine plague, rouget, etc.

The chief symptoms are: Inappetence, vomiting, uneasiness, colic, constipation or diarrhœa, fever, stiffness, tense and tender abdomen, arched back, frequent grunting, limpness of the tail. Sudden vomiting may bring about a rapid recovery.

Treatment calls for a complete change of food, which may include freshly cooked roots, linseed or meal, butter milk, boiled milk, etc.

As an emetic 30 grains of ipecacuanha may be given. Constipation can be relieved by a dose of calomel or jalap. If diarrhoea is persistent small doses of grey powder should be given, and to combat the irritation of the gastric mucous membrane bismuth subnitrate is also useful.

The piggery should be cleansed and disinfected, and the litter frequently changed.

\section{ULCERATIVE GASTRITIS.}

Ulcerative gastritis (ulcer of the abomasum) is recognised after death, but hitherto it has been impossible to so clearly identify the symptoms as to permit of diagnosis during the animal's life. In has been found after death in adults and in calves (Ostertag).

Causation and Pathogeny. The cause of gastric ulceration is decidedly obscure, though we know that certain forms occur during infectious diseases like cattle plague, foot-and-mouth disease, gangrenous coryza, and as a consequence of certain direct local infections; other forms result from the administration of drugs; and finally some are of secretory origin.

In human medicine at the present day there is a tendency to refer the development of round ulcer and ulcerative gastritis to the secretion of an excess of hydrochloric acid. Probably the same cause may be at work in domestic animals, but the proof has not yet been given.

With regard to the pathogeny, the theories of embolism or of thrombosis of capillary vessels find favour with few authorities at the present day. Yet these explanations are logical enough, for if we prevent physiological irrigation of any given part, it is possible to conceive that ulcer formation may follow from auto-digestion, i.e., from the simple action of the gastric juice on a surface which is no longer protected.

The theory of microbic origin has been advanced; but although it may be accepted in relation to the intestine, where the most varied organisms abound, it is scarcely so applicable to the stomach, in which acidity is always very marked and must exercise a very energetic antiseptic action. In sucking calves, however, this theory appears the most plausible. 
It is quite certain that ulceration may result from the unwise use of drugs, like tartar emetic or arsenious acid, especially if these be administered for long periods; but such ulceration always occurs at the same points, viz., at the deepest portion of the rumen, reticulum, or abomasum.

On the other hand, ulceration due to secretory disturbance occurs at different points, and the figure opposite shows that the mucous folds themselves may be injured and perforated.

Lesions. Ulceration of the abomasum varies in severity. The case referred to showed excavated ulcers from the site of which a portion or the whole of the epithelium and glandular layer had been shed; true round ulcers, which had destroyed the entire depth of the mucous membrane and had produced chronic inflammation and sclerosis of the muscular layer; and finally perforations resembling cleanly punched-out holes.

The Symptoms are those of a mild form of ordinary acute gastritis, without marked fever, and without special injection of the conjunctiva. The appetite is diminished and irregular, but more as a consequence of excessive reflex sensibility of the injured organ than from absence of hunger. This excessive reflex sensibility of the abomasum causes relative or absolute gastric intolerance, so that only a small amount of the food ingested passes towards the intestine.

Absolute intolerance on the part of the abomasum may even occur, as in a case described by Moussu in 1895, which produced a very special form of impaction of the rumen, absolutely different from primary impaction.

Intolerance of the abomasum for food already ingested and ruminated may extend to the omasum. Peristalsis of the rumen then ceases, and slight tympanites occurs. The most characteristic condition is the existence of obstinate constipation. If ulceration takes place without producing any important vascular lesion, which, however, is rare, the fæces are hard and coated, but without other peculiarity; if, however (and this appears to be the rule), local hæmorrhage occurs, the extravasated blood is modified by the gastric and intestinal juices, and the fæces appear of a black, tarry colour. This coloration is very significant, and differs from that produced by the bile. It occurs only in gastric hæmorrhage, and at intervals.

Diagnosis. The diagnosis of gastric ulcer is difficult, and can only be arrived at with confidence when the above-mentioned coloration of the fæces can be detected.

Prognosis. From an economic standpoint the prognosis is grave. The patient may recover ; the ulcers may heal, but cicatrisation is always prolonged, and as, on the other hand, the glandular apparatus of the abomasum is generally more or less injured, complete recovery is impossible. 


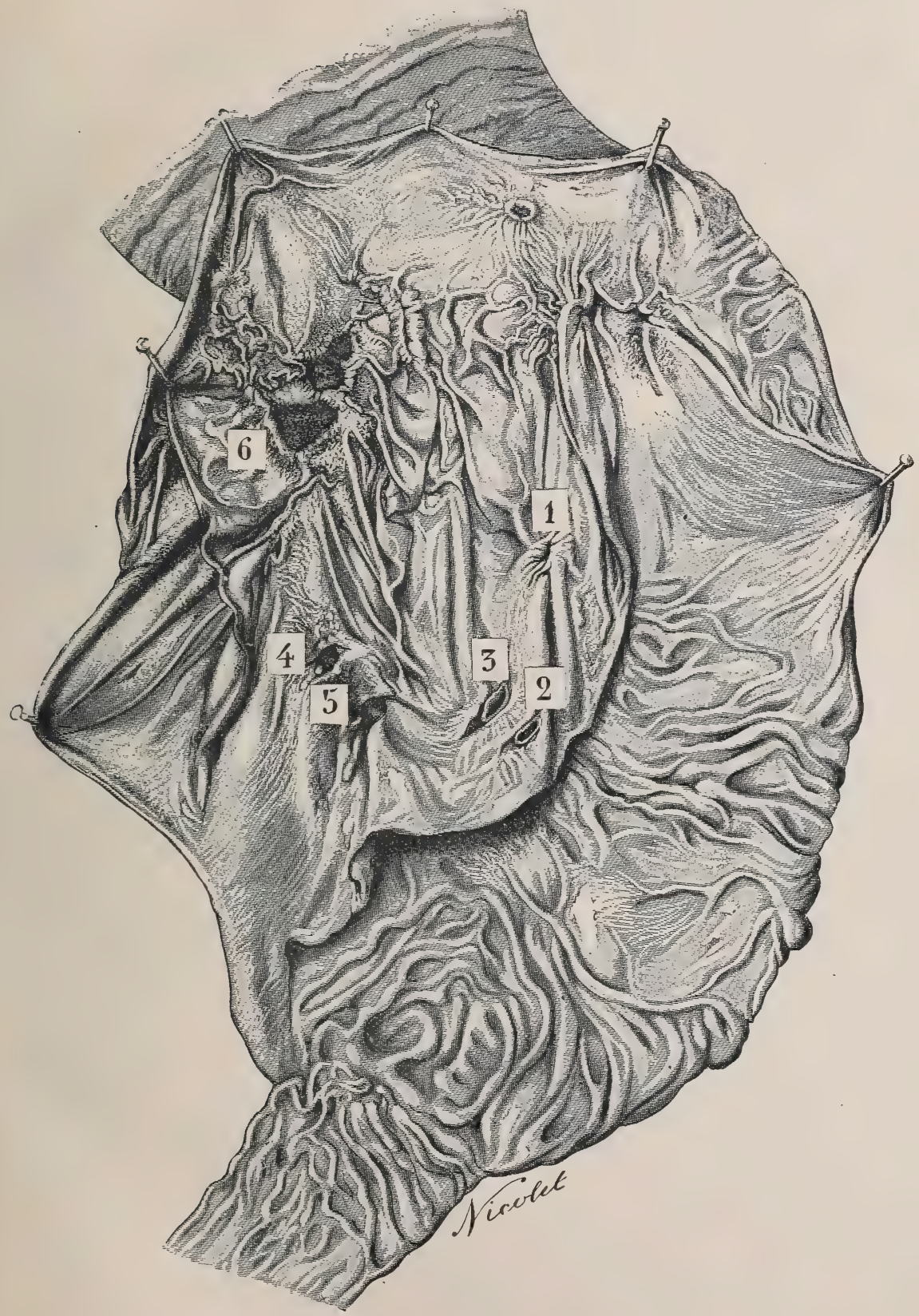

FIG. 70.-Ulcerative gastritis. 1, Typical round ulcer; 2 and 3, ulcers perforating folds of the abomasum ; 4,5 , and 6 , old-standing ulcers with sclerosis of the walls of the abomasum and adhesive perigastritis.

D.C. 
Treatment should be directed towards checking hæmorrhage, diminishing intolerance to food, and assisting the healing of the lesions. These objects are facilitated by prolonged complete rest in the stable, by injecting ergotine, or, $\cdot 7$ per cent. saline solution, at the time when the hæmorrhage occurs, and, if possible, by placing the animals on milk diet or on emollient food, like gruels, emollient infusions, barley, milk, cooked roots, etc.

Stimulation over the region of the abomasum is also of value. At a later stage, when the acute symptoms have diminished, Carlsbad salts, in doses of 1 to 2 ounces per day, can be given. Bicarbonate of soda is also of considerable value.

\section{CHRONIC TYMPANITES.}

\section{Chronic Indigestion--Obstruction of the Abomasum-Chronic Gastritis- Dyspepsia.}

Among pathological conditions of the stomach in ruminants a certain number are clinically marked by one constant symptom, viz., chronic tympanites, a fact which was formerly recognised as indicative of chronic indigestion.

It is evident that such titles have only the significance accorded them, and the term chronic indigestion used only to mean that gastric digestion was badly performed, and that the condition was more or less permanent. It being granted, on the other hand, that the term indigestion is used to characterise temporary conditions during which digestion is suspended, and produces immediate disturbance, it would appear that the term gastric dyspepsia is more exact and more in conformity with the present state of our knowledge of general physiology. In studying this question it is clear a number of facts still require explanation, for, as has been previously indicated, we know almost nothing concerning the variations in the chemical phenomena of gastric digestion under different morbid conditions; nevertheless, the dominant fact, the imperfect or irregular digestion, is easily appreciable. Future discoveries will no doubt enable us more exactly to differentiate several dyspeptic conditions due to chemical or mechanical causes and with or without anatomical lesions. At present it is sufficient to indicate the limits of inquiry.

Causation. The symptom of chronic tympanites accompanies a great number of very different conditions-some due to disease of the digestive tract itself, others to general diseases or lesions of neighbouring parts. In the latter cases the tympanites is only indicative of secondary dyspepsia; in the former, on the other hand, the dyspepsia is primary.

Secondary dyspepsia occurs very commonly during tuberculosis, 
diseases of the liver, subacute or chronic peritonitis, gestation, lesions of the mediastinum, etc., etc.

(a) Secretory or Chemical Dyspepsia. In primary dyspepsia it is impossible to discover any lesion sufficient to explain the disturbance. Chronic tympanites, for instance, follows prolonged consumption of rough or bad food (in years when forage has been scarce or winter food has been lacking), and too short a supply of water for weeks in succession. It also occurs as a consequence of acute inflammation of one or other of the gastric compartments-rumenitis, reticulitis, gastritis. Sometimes it assumes an insidious, slow, progressive form, without any apparent cause whatever.

In these various conditions, the mucous membrane of the gastric compartments suffers from the deferred results of the bad feeding or want of water. Its secretory powers and anatomical structure becoming modified, it is no longer able regularly to elaborate the juices necessary for digestion, and chronic indigestion, imperfect digestion, or dyspepsia result. Similar results follow acute inflammation of the omasum, reticulum, or rumen. Integral repair becomes impossible. Anatomical injury is done, disturbance of secretion follows, and dyspepsia is a necessary consequence.

(b) Motor Dyspepsia. Finally, it would appear that general bad health, abstinence and exhausting work, may produce a form of dyspepsia, unconnected with secretory disturbance, but resulting from mechanical disturbance due to general enfeeblement and to atony of the muscular walls of the gastric compartments. The rumen ceases to perform its work of mixing the food, the reticulum also acts badly, and the abomasum receives imperfectly prepared material. The result is what might be described as motor dyspepsia, in opposition to those forms which are of chemical origin.

The condition may vary in degree, peristalsis being diminished (one contraction every two or three minutes instead of two per minute), or being simply intermittent and occurring only for a few hours during the day, or finally being altogether suppressed. Suppression is never absolute, but atony may be so marked that the mixing of the food is very imperfectly performed.

Symptoms. The most constant symptom present in all dyspeptic conditions is chronic tympanites, indicated by a certain degree of tension or by permanent dilatation of the rumen.

Rumination is impeded and irregular, the distended rumen loses its power of contraction, and no longer causes eructation nor passes gas into the intestine. It becomes progressively inert, whether the inertia be primary and occur suddenly, as a result of some particular condition of the sympathetic system (motor dyspepsia), or secondary and of slow 
development in consequence of disturbance in the gastric secretions and of abnormal organic fermentation (secretory dyspepsia).

This symptom of tympanites is always accompanied by irregularity, diminution, and frequently also by depravity of appetite.

Wasting occurs, but to a very varying degree, according to the nature of the primary condition and the method of feeding.

These general symptoms are accompanied either by constipation or by diarrhœa; and as stagnation of food leads to fermentation, which always forms products differing from those of normal digestion-toxic materials in fact-a chronic auto-intoxication results, which in its turn, if not remedied, becomes a cause of irritation, and aggravates the bad general condition.

In many cases fever is absent, except during the final complications, in animals in the last stages of wasting; but some signs always exist on which the diagnosis may be founded.

The most frequent clinical type of these dyspeptic conditions is motor dyspepsia, consisting in relative atony of the rumen without disturbance in the secretion of the gastric mucous membranes. Luckily, this is the most easily curable form, and is only marked by distension, dulness and constipation.

Dyspeptic diseases of secretory origin are little understood. Their essential causes have been badly described, and their clinical symptoms are ill-recognised.

We cannot prove whether the forms said to be due in man to excess of hydrochloric acid and insufficiency of hydrochloric acid really occur or are well defined in domestic animals: nor are we better informed as to the exact part played by the organic acid of fermentation (lactic, butyric, acetic acid, ete.) ; but the most complete investigations which have yet been made justify our supposing there is some parallel.

Moussu described primary ulcerative gastritis in 1895 ; and as this form is almost certainly associated with excess of pepsine, the occurrence of an excess of hydrochloric acid also seems possible, the more so as the symptoms noted resemble the general symptoms of that condition-preservation of appetite and of the motor power of the rumen, accumulation of food in the rumen as a consequence of reflex intolerance of the abomasum, constipation, and vomiting.

In-addition to these two morbid conditions, a third occurs with some frequency. It is characterised by chronic tympanites, alimentary diarrhoa (the food being badly digested), and progressive wasting. This condition seems due to an insufficiency of hydrochloric acid, brought about by chronic gastritis, the epithelial cells of the mucous membrane appearing incapable of producing sufficient hydrochloric acid for digestion. 
Diagnosis. In the present state of our knowledge regarding digestion in ruminants the precise diagnosis of these pathological conditions must always remain difficult; but it is indisputable that with the above grouping of symptoms we are more likely to succeed than by confining ourselves to the diagnosis formerly common, viz., that of chronic tympanites or chronic indigestion.

The difference between primary and secondary dyspepsia should always be borne in mind, and careful examination will often reveal the special condition which has served as the point of departure for gastric disturbance. Thus generalised tuberculosis, or tuberculosis of the liver or mediastinum, should always be. sought for, and the possible existence of such conditions of the liver as echinococcosis, cancer of the bile ducts, tumours, etc., and diseases of the kidneys should be borne in mind. The influence of gestation, which so frequently causes gastric disturbance complicated with albuminuria, should never be overlooked, and in these secondary forms of dyspepsia the determining cause, and not the objective symptoms should receive chief attention.

Prognosis. The prognosis of secondary forms of dyspepsia varies with the gravity of the primary disease. The prognosis of primary dyspeptic conditions varies greatly, and that state in which hydrochloric acid is too sparingly secreted is certainly the gravest.

Lesions. The lesions have not been carefully studied, but it is probable that in many cases they might afford a key to the symptoms noted. Like all mucous lesions, they are difficult to demonstrate histologically. Infiltration and thickening of the mucous corium and submucous layers have been described. Such lesions indicate nothing; but in some cases new growths have been found in the gastric compartments or contractions about the pyloric orifice, the essential importance of which cannot be doubted.

Treatment. If our knowledge is still insufficient to enable us precisely to diagnose what we have termed "gastric dyspepsia," or what is still currently described as "chronic dyspepsia," the difficulty is even greater when attempting to lay down lines of treatment, because of the lack of known facts and the want of a base for reasoning. Thus we find, without being able to explain why, that some prescribe tartar emetic, others rapid and energetic purgatives, others, again, laxatives; whilst German authors, apparently without any justification, recommend oil of turpentine. It seems to us, however, that one might do better than this and attempt to lay down some rational indications for treatment.

(a) In cases characterised only by chronic tympanites, without diarrhoea, without manifest constipation (motor dyspepsia), and without any other apparent organic disturbance, drugs calculated to stimulate peristalsis of the rumen seem indicated. 'The most promising comprise 
ipecacuanha in doses of 1 to 2 drams per day, tincture of nux vomica in similar doses, powdered nux vomica in doses of 45 to 75 grains, and laxatives like Carlsbad salts in doses of 8 to 10 drams.

Little by little peristalsis returns, becomes regular, and the chronic tympanites disappears for good. This is frequently the case during gestation, when the condition simply consists in motor dyspepsia without anæmia.

(b) If, on the contrary, chronic tympanites is accompanied by constipation, and the fæces are hard or, as is usually the case, covered with mucus, suggesting the probability of excess of hydrochloric acid, salines are indicated - not to the point of producing purgation, which would not lead to any lasting improvement, but still as laxatives, continued daily for ten, fifteen, or twenty days, or, if necessary, even longer.

Carlsbad salts in doses of 8 to 10 drams, or one-ounce doses of sulphate of sodium associated with $2 \frac{1}{2}$ drams of bicarbonate of potash after each meal, are to be recommended in preference to bicarbonate of soda alone, because they act on the secretions, on the muscular system, and also on the liver.

(c) Finally, when chronic tympanites is accompanied by diarrhœa, a condition which usually indicates insufficient secretion of hydrochloric acid, the administration of that acid tends to arrest or check organic fermentation and to facilitate digestion in the abomasum by supplementing the diminished physiological secretion. The dosage is an important point. At first small quantities should be given, $2 \frac{1}{2}$ drams per day, divided into two doses and freely diluted in the drinking water; but this amount may, if necessary, afterwards be doubled or trebled. The drinking water is not rendered irritant by these doses, for it is admitted, and Moussu has confirmed the fact by analysis, that the quantity of $\mathrm{HCl}$ in the gastric juice may rise as high as $\cdot 2$ to $\cdot 3$ per cent. Chloride of sodium, the excito-secretory action of which on the gastric mucous membrane is well known, may be given for long periods in doses of 1 to $1 \frac{1}{2}$ ounces per day.

In these various chemico-pathological states the food should receive the closest attention. The forage, which should be good, may be supplemented by the addition of cooked roots, demulcent drinks, and, if possible, milk.

\section{GASTRIC DISTURBANCE DUE TO FOREIGN BODIES.}

These morbid conditions are extremely complex, but the facts that they are due only to one cause, and that they possess certain symptoms in common, permit of a certain grouping. It would obviously be illogical to speak of traumatic indigestion of the rumen, reticulum, and abomasum, as has been done in more than one book on this subject; for 
the gastric disturbances described below should be considered as complications, and not as diseases.

Causation. In young animals foreign bodies may be composed of hairs, wool, bristles, cotton, and clover hairs.

Hair balls are common in the rumen, and are sometimes met with in the fourth stomach. They cause irritation, indigestion, sometimes pyloric obstruction, dilatation, and eventually death.

Wool balls in lambs, bristle balls in young pigs, cause much gastric irritation. Cotton balls occur in lambs fed on cotton-seed cake; the fibre constitutes a foreign body. The hairs of clover leaves may form a ball in the abomasum of lambs.

Under the influence of depraved appetite animals of the bovine species consume, apart from their regular food, the most varying substances, such as linen, fragments of wood, nails, stones, gravel, sand, etc. Moreover, forage, even when of good quality, often contains foreign bodies like nails and pins (when the fields are near factories), sewing or knitting needles (when the animals are looked after by women), fragments of iron wire derived from bales of compressed forage, etc., etc. The ingestion of such objects is followed by various consequences, which may be studied in three divisions, in the first of which the foreign object is soft in character, in the second is blunt at one extremity and pointed at the other, and in the third is pointed at both ends.

(1.) Soft objects. The movements of the rumen, the warmth and the action of the digestive fluids, may cause soft objects to be broken up; the disturbance they produce is then insignificant.

Of such substances, however, some are quite incapable of digestion (clothing, sacks, linen, etc.), and may produce obstructions; others are both indigestible and heavy (gravel and sand), and may fall into the depressions of the compartments, where they remain, or, if passed into the reticulum, may become arrested in the deepest lying part. They then produce atony of the muscular coats, slowing of peristaltic movements, diminution in the frequency of eructation, and, as an additional consequence, chronic tympanites, sometimes visible at the flank.

The symptoms are vague and common to a number of the digestive diseases already described. The animals masticate without having anything in the mouth; rumination becomes irregular or is altogether suppressed, but this is not characteristic, being a symptom common to many visceral diseases.

Later, as a result of auto-infection, diarrhœa sets in; under the influence of abnormal fermentation in the gastric compartments the eructations become fotid; the animals fall into a condition of marasmus. Death usually results after a varying time-when large quantities of foreign substances have been ingested, in twenty to thirty days. 
The diagnosis chiefly rests on the history, and can only be of a confident character when one knows what quantity and what kind of foreign body has been swallowed.

The prognosis is grave, because the animal usually dies of progressive exhaustion.

Treatment. There is only one rational form of treatment-viz., gastrotomy, followed by examination of the rumen and reticulum and removal of the foreign body. Before undertaking operation the surgeon should be fully informed as to the canse and the probable results to be expected.

(2.) Foreign bodies with one pointed extremity. These usually consist of large-headed nails, or fragments of iron wire rolled up at one end, which have been swallowed during primary mastication along with forage.

When ingested, they may become implanted at any point in the gastric apparatus without necessarily penetrating deeply. When fixed across the division of the reticulum, they cause slowing of its physiological action. Should they penetrate the wall either of the reticulum or of the rumen, they may attack on the right the liver, or on the left the diaphragm or spleen, producing suppurating hepatitis, splenitis, or respiratory disturbance. The hypochondriac region then appears sensitive. 'The muscular portion of the diaphragm is partly paralysed, and costal respiration set up, while frequent coughing of reflex origin is provoked by irritation of the pneumo-gastric and diaphragmatic nerves, and may give rise to suspicion of some thoracic disease, from which, however, it is distinguished by the absence of discharge, expectoration, and pulmonary symptoms.

Finally, if implanted in the lower wall or sides of the rumen or reticulum, foreign bodies may carry with them infectious agents and set up localised or generalised peritonitis.

Early diagnosis is a matter of great difficulty, as it can only rest on the diaphragmatic disturbance or on the symptoms of peritonitis.

Lesions. Small-sized sharp bodies cause lesions of trifling extent, which in most cases are only indicated by retardation of movement of the gastric compartments, between which and the diaphragm, and between the diaphragm and the posterior portions of the lung, various adhesions are set up. In such cases the peripheral inflammation ends by producing a fibrous sleeve, which prevents the pleural cavity becoming infected. Other cases show patches of adhesive peritonitis or signs of generalised peritonitis, the real cause of which often evades discovery during life.

Treatment. Gastrotomy is the sole means of effecting a cure, but we are forced to admit that it only gives good results when the operator 
knows what he is trying to find. Without this information he acts in the dark, is obliged to abandon himself to chance, and although luck sometimes favours him, it more often leaves him in the lurch.

(3.) Foreign bodies pointed at both ends. Bodies like needles, pins, straight fragments of iron wire, knitting-needles and broken hairpins, become implanted in the gastric walls and travel in the most diverse directions, in obedience to the varied movements of the organ injured. They produce results similar to those just described. Most frequently they fall into the lower part of the gastric compartments, pass near the ensiform cartilage, between the pleura and the triangularis sterni into the thickness of this muscle, or into the mediastinum, and there produce either an abscess in the region of the ensiform cartilage, an abscess of the thoracic wall, or a collection of pus in the sub-pericardial or subpleural region (pseudo-pericarditis). They may even reach the pericardium, causing pericarditis, and sometimes, when adhesions are set up between the heart and pericardial sac, carditis.

By deviating to the right or left, the foreign body may produce pleurisy or even pneumonia. If it moves towards the right, it involves the liver and produces suppurative hepatitis; if to the left, suppurative splenitis. Travelling in a downward direction, it encounters the abdominal wall, and after producing an abscess may be eliminated; passing backwards, it falls into the peritoneum, and may lead to peritonitis. In those exceptional cases in which foreign bodies reach the abomasum they generally become implanted towards the greater curvature, producing in the abdominal wall an abscess which breaks externally, and through which the foreign body is discharged; gastric fistula is then a common sequel.

The symptoms vary, according to the complications. The earliest comprise digestive disturbance, which coincides with the passage of the sharp object through the rumen or reticulum, and depends on whether such passage produces local peritonitis and pain, rendering movement of these compartments impossible. Later, when the diaphragm has been penetrated, respiratory disturbance occurs, and is succeeded by apparent improvement, which in its turn may be followed by the occurrence of pericarditis, pleurisy, or abseess formation.

In other cases where suppurative hepatitis or splenitis, or even peritonitis may be present, the symptoms are extremely vague and very difficult to refer to their real cause.

Diagnosis is difficult, unless the owner is able to supply exact information that at some previous time the animal had swallowed such and such an object.

The prognosis is grave, though cases occur where a foreign body is tolerated, and may for a long time be retained without producing accidents. 
Treatment. Gastrotomy should not be performed unless the operator is possessed of very precise information. In such case the rumen and reticulum should be emptied and the foreign body sought for and removed.

When the symptoms strongly point to the presence of a foreign body exploratory gastrotomy may be performed, but the operator will do well to employ the operation only as a last resort.

Many complications, like septic peritonitis, hepatitis, and splenitis, are practically hopeless; but others show a tendency to recovery. This is the case when abscesses form in the thoracic or abdominal wall, or beneath the pleura or pericardium. The entire difficulty consists in diagnosis, for when once this is clearly defined intervention is fully justified. As, however, the surgical measures vary in every case, the exact course to be adopted must be left to the initiative of the surgeon.

\section{TUMOURS OF THE GASTRIC COMPARTMENTS.}

Papillomata result from hypertrophy of normal papillæ; they resemble those of the pharynx and œsophagus. The growth may attain the size of a fist. It often resembles a cauliflower in appearance. When very large, such growths may cause obstruction. A very striking illustration of a papilloma of the mucous membrane is given on p. 180 of Möller and Dollar's "Regional Surgery."

Sarcoma has been noted by Paule, Kitt, and Schütz as forming in the subserous tissue of the omasum, and later bulging out as a wounded swelling of irregular size.

Actinomycosis of the abomasum has been reported by Professor Axe. 


\section{CHAPTER VI.}

\section{EINTERITIS.}

Enteritis consists in inflammation of the intestine, or, more precisely, in inflammation of the intestinal mucous membrane. All the constituent portions of the intestinal tube may be affected (duodenum, jejeunum, ileum, colon, cæcum); but clinical distinctions and localisation of inflammation in the various parts are very difficult in the domesticated animals, and at present it is impossible to describe with any accuracy the differences between duodenitis, enteritis of the jejeunum and ileum, colitis or typhlitis. Without doubt certain symptoms suggest that some regions are more affected than others; but clinically we are only able to distinguish between acute and chronic enteritis. Acute enteritis may assume different forms, according to its intensity, rapidity of development, and lesions, so that it is possible to distinguish between such conditions as simple acute enteritis and hæmorrhagic enteritis.

Chronic enteritis, an abstraction founded on our knowledge of such specific forms of enteritis as are due to tuberculosis, distomatosis, helminthiasis, etc., usually assumes the diarrhœic form.

\section{ACUTE ENTERITIS.}

As acute enteritis, whether localised in one portion of the intestine or involving the whole intestinal tube, is produced by varying causes, and assumes very varying degrees of intensity, its clinical symptoms are equally diverse.

Causation. The various forms of enteritis result from two great series of causes: infections and intoxications. Normally the intestine contains an extremely large number of different microbes, which may prove of service so long as circulation, secretion, and peristalsis continue normal; but as soon as any perturbation occurs, either in the blood supply or in the movement of the bowel, normal secretion is impeded; abnormal organic fermentation commences, producing irritant principles or toxins which at once set up local irritation, or, being absorbed, produce that complex of symptoms which we recognise as enteritis, intoxication of intestinal origin, or even infection. 
Bearing in mind these facts, we are better able to understand the part played by cold, by damaged fodder, by intense, stimulating feeding, or sudden changes in the food, as well as by the action of drastic purgatives, which modify beyond physiological limits the condition of the glands, or even cause local desquamation of epithelium.

Toxic substances or plants act similarly by modifying either the circulatory, secretory, or motor systems.

Symptoms. The first appreciable external symptoms appear to result from fever--loss of appetite, suspension of rumination, dryness of the muzzle and of the mouth, earthy-red colour of the conjunctival mucous membrane, etc.

On manipulating the left flank one notes neither tympanites nor sensitiveness-in a word, there is no indication of functional disturbance of the rumen.

On the right side, on the contrary, palpation causes the animal to resist and to show signs of pain. According as this sensitiveness is more marked in the middle or upper region or towards the hypochondriac circle, we infer that the inflammation is most acute in the large or small intestine, either in the middle portions or, again, in the most anterior portion. The temperature always rises at the commencement, attaining $103^{\circ}$ to $104^{\circ} \mathrm{Fahr}$. $\left(39 \cdot 5^{\circ}\right.$ or $40^{\circ} \mathrm{C}$.), but rarely a higher point, a fact which negatives the idea of a rapidly progressive infectious disease. Slight colic appears, and is accompanied by constipation; the fæces are covered with mucus, or false membranes, or are completely enclosed in fibrinous tubes. After four or five days the fæces change in character. Constipation gives place to a liquid, blackish, very fotid diarrhœa. Finally the mouth exhales a stercoraceous odour, resulting from foetid eructation.

The passage of flat or tubular false membranes continues for a certain time.

When false membranes constitute the chief symptom, the condition is termed "pseudo-membranous, croupal, or diphtheritic enteritis." This form is only a variety of acute enteritis, but is grave, because complications due to hæmorrhage or infection are very liable to occur.

In certain cases movement causes groaning. In the slighter forms the patient may recover spontaneously. If fed with easily digested materials, the symptoms diminish in intensity. Towards the eighth day the frees become normal, appetite and rumination reappear, and the secretion of milk which had fallen off rises to its normal amount. Resolution has occurred.

More frequently, either because the animals have less power of resistance or because decomposition is taking place more actively in the intestine, or, again, because intoxication is setting in, the disease 
becomes aggravated. Constipation is more marked, and the fæces passed are in small masses, covered with layers of epithelium, or sometimes streaked with blood. In other cases the diarrhœa becomes exaggerated and assumes a mucous or sero-mucous character ; the temperature rises, and death results from exhaustion and infection, micro-organisms passing from the lumen of the intestine through its walls and invading the general circulation.

Lesions. To study the lesions to advantage the autopsy must be performed as soon as possible after death.

In slight cases they consist of trifling generalised congestion of the intestinal mucous membrane. The points most affected show infiltration of the submucous coat, and sometimes of the muscular coat; the wall of the intestine is double its normal thickness, but the thickening never equals that seen in cases of rapidly fatal intestinal congestion.

At a more advanced stage the mucous membrane clearly is inflamed, its surface is covered with a fibrous exudate, and the glandular and epithelial cells proliferate, assume the embryonic form, and produce the new tissue which one recognises under the form of false membranes.

The false membranes are generally but slightly adherent, and are readily expelled.

In other cases, however, they adhere firmly, and when detached by the friction caused by movement of semi-digested food through the bowel, produce rupture of capillary vessels ; this explains the presence of blood streaks in the fæces.

The diagnosis is relatively easy, especially at the onset, on account of the special character of the fxces and the sensitiveness of the right flank.

The prognosis is seldom grave. If the enteritis is taken at the beginning, recovery is usual; but if the animal has been ill for eight or ten days and is exhausted, and if fever and diarrhoea are intense, the prognosis should be guarded.

The treatment is that of all acute inflammatory diseases. Mustard plasters may be applied to the chest and abdomen and left in position for some hours, or may be repeated. Some practitioners prefer hot blankets or dry friction, or, again, rubbing with essence of turpentine, but this substance should be spread over large surfaces, in order to prevent injury to the skin. Bleeding is only justifiable in plethoric animals, and should never be exhaustive.

To relieve the digestive symptoms purgatives are at first given, even though diarrhoea is marked from the onset; for purgatives still constitute the best intestinal antiseptics, because they get rid of the intestinal contents and microbes. The diet should be carefully selected, and may consist of gruel, mucilaginous materials, linseed tea, cooked roots, etc. 
Of drugs, sulphate of sodium in doses of 10 to 15 ounces is probably the best. It can gradually be replaced by 2 to 3 drachm doses of bicarbonate of soda or of ordinary salt per day. Laudanum, camphor, and bismuth relieve persistent colic and diarrhœa. Pilocarpine, veratrine and eserine, though recommended by some authors, present no advantage in our opinion. The first two of these substances certainly cause purgation, but the action is quite temporary. The last induces violent contraction of the striped muscular tissue, and may produce grave lesions or invagination when the bowel is diseased, thickened or infiltrated.

\section{HÆMORRHAGIC ENTERITIS.}

This form of enteritis derives its name from the dominant symptom, which consists in the passage of unaltered or clotted blood in the fæces. In the former case the blood is bright in colour, as if it came directly from an open vessel. In the second it is coagulated, and assumes the form of fibrinous clots, which seem to result from the superposition, in the intestinal tract of their constituent elements, viz., serum, blood corpuscles, and fibrin.

Causation. Hæmorrhagic enteritis is rarely seen except during the hottest days of summer, and in young animals which have previously shown nothing abnormal. The high temperature seems to favour its appearance, but is always supplemented by another cause, viz., the ingestion of irritant food, particularly of weeds and toxic plants or herbage of bad quality; amongst such may be mentioned dog's mercury, and plants of the order Papaveraceæ, Euphorbiaceæ, etc. Otherwise the often rapid manner in which the disease develops indicates toxic enteritis.

In other cases, more benign in appearance, but quite as grave in reality, blood is passed continually, and the disease assumes a chronic form. It is then of parasitic origin, and is due to intestinal psorospermosis.

Symptoms. The primary symptoms are similar to those of acute enteritis, and consist of fever, dryness of the muzzle and of the mouth, colic and constipation. This is soon followed by loose motions containing blood or blood clots, according as the hæmorrhage occurs at a greater or less distance from the rectum. The fæces are then ejected violently to a considerable distance, on account of the exaggerated intestinal peristalsis.

The disease may produce death in twenty-four hours, though usually the end is deferred for several days, or, in cases due to sporozoa, for a considerably longer time. In these cases there is some chance of recovery, provided that treatment be prompt.

The diagnosis is very easy. 
The prognosis is in all cases grave.

The lesions are ill-recognised. The animals die rapidly, and if not immediately examined show no characteristic lesions. The changes to be looked for consist in intense congestion or ulceration of the intestine, or even in ulceration of arterioles and of arteries of considerable size.

In parasitic enteritis one finds localised inflammation and, in the epithelial interstices, sporozoa, which cause the rupture of blood-vessels.

Treatment. It is necessary to act energetically from the outset, and to arrest hæmorrhage by acting on the intestine, on the vascular system, and on the skin. Mustard plasters or other external stimulant applications are therefore indicated. Internally, small doses of astringents like tannin, preparations of opium, 25 per cent. solution of sulphuric acid in alcohol, etc., are given to produce constriction of the vessels, though they seldom arrest the discharge of blood for long. In most cases it is necessary to have recourse to subcutaneous injections of ergotine, in doses of 5 to 10 grains in young animals, and 15 to 45 grains in adults. The dose may be given in two parts to prevent an unduly severe action. The smooth fibres of the small vessels are thus directly excited, and hæmorrhage ceases in consequence of clotting in the contracted vessels.

One may give for the same purpose injections of arseniate of strychnine, which has the advantage of sustaining the tone of the heart and preventing syncope. The dose should not exceed 1.5 grains in large animals.

Finally, a stimulating diet containing milk, alcohol, soup, cooked vegetables, and small quantities of vegetable pulp is useful.

With early treatment animals sometimes recover in a few hours.

Intra-venous or subcutaneous injections of physiological salt solution ( 2 drachms of sodium chloride in one quart of water) may prove of value when hæmorrhage has been abundant and vascular pressure is low.

\section{CHRONIC ENTERITIS (CHRONIC DIARRHAEA).}

Chronic diarrhœe is common in byres. It appears suddenly, often fails to attract attention, and assumes the form of simple diarrhœa, a fact which accounts for it frequently being described as chronic diarrhoea, dysentery, etc.

Causation and pathogeny. This form of diarrhœa occurs sporadically throughout France, and under exceptional circumstances may permanently attack a number of animals in a given place.

The immediate cause is not known, but without doubt the disease is of microbic origin. Like Lignières, Moussu at one time believed that this disease was very probably identical with that known in 
Argentina under the names of diarrhœa, entéqué, or bovine pasteurellosis. The hypothesis has not been verified, and Lignières' treatment, said by him to have succeeded in Argentina, always failed in Moussu's hand.

The only point which seems admissible is that this disease, which Moussu considered to have analogies with chronic sporadic dysentery in man, is due to one or several organisms, which develop in the

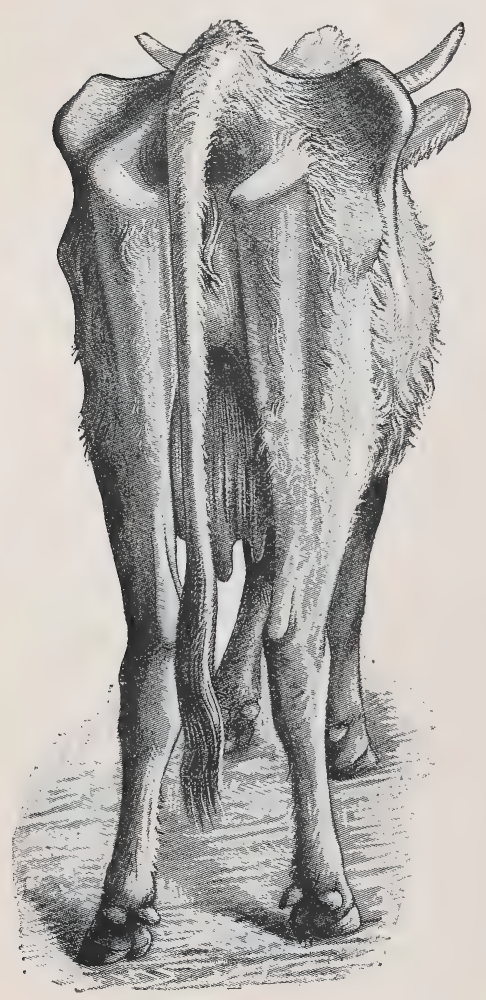

FIG. 71.--Appearance of a patient suffering from advanced chronic diarrhœa. intestine and produce toxins, causing diarrhœa, without, however, marked inflammation of the intestinal mucous membrane.

Symptoms. The onset is often overlooked. The diarrhœa gradually increases without appearing to be very serious; but it persists in varying degrees of intensity. The patients do not appear to suffer, and do not lose their appetite or spirits, but in time the diarrhœa becomes exhausting; they waste, and after some months become excessively thin and poor.

Intestinal peristalsis becomes exaggerated without the existence of colic or tympanites. The evacuations are frequent, and little by little the abdomen retracts, until, in horseman's parlance, "the belly is up to the back," even in cows of four, seven, and eight years' bearing.

The diarrhœa is serous, always fœetid, and without tenesmus.

The fæces may either be very soft or be passed in veritable jets. They are always a little discoloured, and frequently contain grain or undigested forage. They always contain numerous bubbles of gas.

The wasting during later periods of the disease is absolutely characteristic, and different from that of other wasting diseases, such as chronic broncho-pneumonia, tuberculosis, etc. The patients finally become walking skeletons. The red corpuscles of the blood progressively decrease, until the number may fall as low as 800,000 or even 500,000 red corpuscles instead of six millions, the normal figure. The oedema common to wasting conditions appears, and the animals die without suffering, in a condition of absolute exhaustion. 
Complications are rare, though occasionally intestinal hæmorrhage or broncho-pneumonia occurs. The temperature, which remains normal or shows very slight changes throughout the course of the disease, may then oscillate between $101^{\circ}$ and $103^{\circ}$ Fahr. $\left(38^{\circ}\right.$ and $39 \cdot 5^{\circ} \mathrm{C}$.).

Lesions. At the first glance no lesions can be detected on post-mortem examination except those of generalised wasting, but when the autopsy is carried out immediately after death all the in-

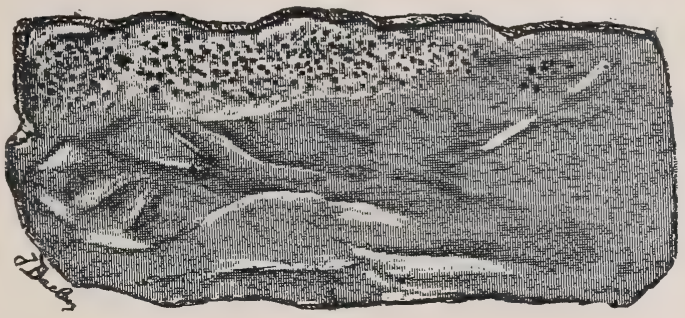

FIG. 72.-Appearance of a portion of the small intestine in chronic diarrhoea.

terior of the intestine appears affected. The mucous membrane of the abomasum and the mucous folds appear infiltrated, thickened, and with moderate sub-epithelial congestion. The intestine seems friable, and tears with the slightest traction. The sub-epithelial portions of the

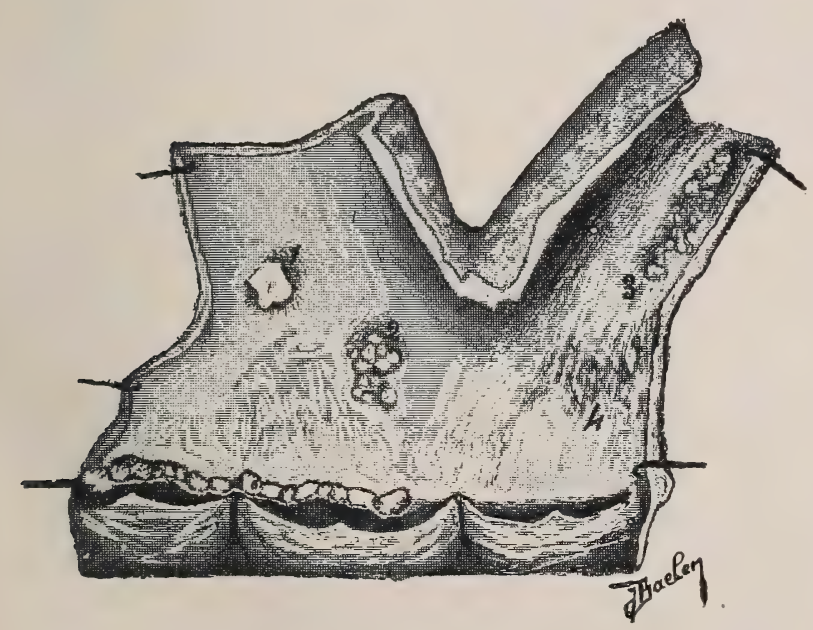

FIG. 73.-Atheromatous lesions of the aorta in chronic diarrhœa. mucous folds are infiltrated and congested, while the more prominent parts of the folds, which are exposed to the friction of semi-digested food, become eroded.

Throughout the length of the jejeunum and ileum the mucous membrane exhibits multitudes of small ulcerations.

Histologically, the epithelium and the glands seem atrophied, without any inflammatory change having occurred in the mucous or submucous coats.

The colon and the cæcum show similar lesions, and in addition brown deposits of pigment under the mucous membrane and along the course of the small blood-vessels. This lesion resembles that found in chronic dysentery in man, and suggested to Moussu a possible connection between the two diseases.

D.c. 
The liver is less than the normal size. In the heart Moussu has seen one case of sclerosis of the auricles and calcareous infiltration of the sub-endothelial lining of the aorta.

In several instances he has noted calcareous infiltration of the mesenteric lymphatic gland.

Diagnosis. It is easy to diagnose this condition, and quite possible to distinguish it from the diarrhœa of tuberculous enteritis, infectious hepatitis, and other conditions.

Prognosis. The prognosis is very grave, and the disease almost always proves fatal.

Treatment. At the present moment no curative treatment is known. Lignières' treatment-viz., the injection of physiological salt solution and serum from healthy oxen, and saline solution or defibrinated blood - has never given permanently successful results.

All the drugs usually employed against diarrhœa, the antiseptics, astringents, etc., fail, or confer merely momentary benefit. Economically, nothing is to be gained by keeping the patients alive. With great care existence may be prolonged for months, or even for several years, but the animals never regain condition, and are never of any use.

The most rapid and lasting good effects follow the administration of $2 \frac{1}{2}$ to 8 fluid drachms of hydrochloric acid per day, given in two portions and very freely diluted.

\section{DYSENTERY IN CALVES.}

This disease sometimes appears on the first day after birth, frequently on the second or third. It may be mistaken for septicæmia of umbilical origin.

Symptoms. The young animal may be born vigorous and in good condition, though this is exceptional. More frequently it is puny and below normal weight. The first evacuation (of meconium) may exhibit the diarrhœic character ; in other cases this peculiarity only appears on the second or third day, when half-digested milk is passed. The fluid is greyish, extremely foetid, and rapidly becomes brownish and bloodstained. Evacuation is frequent and accompanied by tenesmus.

The patients at once become very dull, refuse to suck, and resist efforts to feed them by hand. The temperature rises, and the diarrhœa; which at first was of an alimentary character, becomes mucous, serous and blood-stained. The little patients appear "tucked up," the flanks sink in, the strength diminishes, and in twenty-four hours, or two or three days at most, they die of exhaustion.

Recovery is exceptional, and when the disease assumes this character it usually attacks a considerable proportion of the other animals in the byre. 
Causation. The exact cause of this form of dysentery in new-born calves has not yet been identified, but the disease is undoubtedly a microbic enteritis, and may even be a primary septicæmia of puerperal origin.

For a very long time this dysentery was mistaken for simple diarrhœa, though it exhibits neither the characteristics, course, nor termination of the latter disease.

The co-existence of epizootic abortion and dysentery in certain byres has led some authors to believe that a connection exists between the two diseases, and that the young are infected with dysentery at birth. Evidently, intra-uterine infection is not exceptional, and it seems quite natural that the new-born calf may equally suffer when the foetal envelopes and fluids are infected before parturition. Epizootic abortion is probably not the only disease which may produce this condition.

The diagnosis is very simple. The course of the disease and its rapid development prevent it from being mistaken for ordinary diarrhoea. It is more difficult to distinguish from septicæmia of umbilical origin, although this disease also has well-marked characteristics.

The prognosis is not hopeful. Statistics show that almost all the affected animals die, and that those exceptional cases which survive remain puny and sickly. There is no economic advantage in attempting to save them.

The mothers of affected calves seldom show any sign of illness, though the after-birth is often retained.

Lesions. The macroscopic lesions are of very trifling importance compared with the gravity of the disease. The digestive tract appears congested throughout. The intestinal mucous membrane is moderately swollen, but without gross lesions. The intestinal contents exhale a sickly, fœtid odour. 'The smaller vessels and capillaries forming the peripheral vascular network appear distended, as in septicæmia. 'The carcases putrefy with extreme rapidity.

Curative treatment is very uncertain. Varying results have been obtained with doses of 4 to 5 drams of slight purgatives like borotartrate of potassium, sodium sulphate, and magnesium sulphate; small doses of intestinal antiseptics like salicylic acid, 15-grain doses of salicylate of soda, 45 to 75 grains of carbolic acid, 2 per cent. carbolic solution in doses of 7 to 12 drams; 1 per cent. Lysol solution in doses of 2 to 4 ounces; benzo-naphthol, lactic acid 45 to 75 grains, tar water, lime water, etc. Although all these preparations, when carefully used, generally give good results in the early stages of simple diarrhoa, they appear to fail in dysentery of new-born calves.

Prophylactic measures are more reliable. They consist :

(1.) In scrupulous disinfection of the byres when the first case of abortion occurs ; 
(2.) In successively isolating cows which are about to calve in a small specially detached stable;

(3.) In carefully disinfecting the genital organs of cows which have aborted, firstly with boiled water at a temperature of $100^{\circ}$ Fahr., and then with 1 per cent. iodine solution.

Calves which are infected when born cannot be saved, but abortion can be prevented and dysentery so stamped out.

\section{DIARRHCEIC ENTERITIS IN CALVES.}

This disease is usually called "simple sporadic diarrhcea." It may appear at any time before weaning, and can usually be cured if treated early before the patients show bodily wasting.

Causation. Indigestion from failure of the abomasum to deal with the milk usually precedes diarrhœic enteritis; it may terminate without complications, but very often is followed by diarrhœea. Anything which produces milk indigestion, therefore, favours the occurrence of enteritis. Such predisposing influences include over-distension of the abomasum, milk of bad chemical composition, milk tainted by keeping or by storage in dirty and infected pails, etc. The addition to the milk of nutritive substances which the abomasum and intestine are not yet capable of digesting, such as wheat, rye, barley, or maize meals, very often produces diarrhœe even when the meal is well cooked.

Chills, privations, irregular feeding, and badly-managed weaning may facilitate its development, but none of these causes, however important they may be, seem to play any other part than that of favouring the multiplication of the numerous varieties of microbes to be found in the intestinal tract. Vascular disturbance occurs, either as a result of direct irritation of the intestinal mucous membrane or of the action of toxic products contained in milk which has served as a culture medium for these microbes; this is followed by secretory disturbance, and the intestinal contents being modified in character, the microbes normally present undergo changes in number and quality. Inoffensive organisms assume pathogenic qualities and secrete toxic principles, normal digestion is disturbed, the intestinal defence becomes less perfect, toxic principles which the liver is incapable of destroying are absorbed, and diarrhœic enteritis is set up.

Symptoms. Diarrhœic enteritis appears during the second week of life, towards the end of the first month, or even later. It is characterised by the passage of fæces consisting of mucus and containing little clots of milk.

This is the first stage of alimentary diarrhoa, also termed "white diarrhœa" or "white scour." It may prove unimportant; it may last a 
day or two and then cease. Most commonly diarrhoa increases and assumes a mucous and then a serous character, whilst the dejecta exhale a very characteristic repulsive smell. The excrement becomes greenish brown, and after several days or a week or more may appear bloodstained. The number of evacuations varies enormously, depending on the gravity of the condition. The ejected material is irritant, and the parts soiled by it, like the perineum, hocks, and back of the cannon bones, become slightly inflamed as though blistered; later, the hair falls away.

The general health then begins to suffer. Fever remains moderate, but the mouth is pasty, the tongue coated, and the breath foetid.

The patients become thin, and lose their appetite and spirits. Palpation of the abdomen, especially of the right flank, is slightly painful, and the pulse is accelerated.

The diarrhœa may spontaneously diminish if the animal's constitution prove sufficiently strong; but if it follows its course, the little patient becomes weaker, eats less, the evacuations increase and are accompanied by tenesmus. In seven to eight days, in rare cases in four to five, the animal dies from toxi-intoxication of intestinal origin, or from infection resulting from intestinal germs, particularly the bacterium Coli communis, obtaining entrance into the circulation. On the other hand, the diarrhœa may continue for weeks.

The diagnosis is easy, and there should be no difficulty in distinguishing this disease from dysentery and from umbilical phlebitis, which are also accompanied by diarrhœa.

The prognosis is grave, unless treatment is early undertaken. In the latter case there is a good chance of recovery.

Lesions. The macroscopic lesions are not of much interest, being confined to congestion of the intestinal mucous membrane, superficial desquamation of the epithelium, smali vascular erosions, and general wasting.

The mesenteric lymphatic glands are swollen and œdematous.

After death from general infection, it is not uncommon to find pleural, peritoneal, and pericardial exudation or even endocarditis.

Even in cases where no post-mortem change has had time to occur cultures from the blood yield varieties of the bacterium Coli communis.

Treatment. The meals should be given at fixed hours, and regulated both as regards quantity and quality. These precautions alone suffice to prevent the appearance of diarrhœic enteritis.

Curative treatment has every chance of succeeding when undertaken at the outset. Delafond and Trasbot recommend mild purgatives, which act more rapidly than intestinal antiseptics. These comprise borotartrate of potash in doses of 4 to 5 drachms, sodium sulphate in doses 
of $2 \frac{1}{2}$ to 4 drachms, sulphate of magnesia, etc. By evacuating the bowel and removing a large number of the germs which have multiplied there, they arrest intoxication and prevent infection. Nevertheless, they should not be administered for long, and after one or two doses should be followed by antiseptics like benzo-naphthol, in doses of 15 to 30 grains, salicylic acid in doses of 5 to 10 grains, or salicylate of soda 45 to 60 grains. Mucilaginous and sweetened drinks eontaining lactic acid in doses of 45 to 75 grains per day may be given between the meals, or at intervals if the calf is sucking.

Laudanum in doses of 6 to 10 drops per day administered in rice water, extract of opium, weak solutions of tannin, etc., are also of value. Filliâtre has successfully used tar water in the first stages. The solution consists of vegetable tar 6 drachms, boiling water 1 quart. This solution is used tepid in the proportion of 1 part to 3 parts of warm milk.

Decoctions of spiked purple loosestrife, willow bark, etc., are also of great value in certain districts.

The drug which appears least dangerous, however, is that so often successfully used in young children-viz., subnitrate of bismuth. It can be given in doses of 30 to 45 grains per day, with lactic acid in doses of 75 to 150 grains, according to the size of the patients. If the animals are greatly exhausted and have been ill for some time there is less chance of recovery, and under these circumstances Dr. Lesage's anti-colic serum might be used.

It gives excellent results in infants, and it has proved successful in simple diarrhœea of calves. 


\section{CHAPTER VII.}

\section{POISONING.}

Accidental poisoning is frequent in domesticated animals. It may present no visible lesions, and it is therefore very important to recognise the symptoms which indicate the secret lesion.

\section{POISONING DUE TO FOOD.}

Under this title are included all forms of poisoning resulting from the ingestion of bad fodder. Such expressions as "intestinal typhus" and "typhic gastro-enteritis" only indicate a special stage in the condition, which is never twice the same.

Causation. The most important changes in the food ingested do not consist in a mere modification in its chemical composition, but in the presence of various parasites which develop in grain and forage, after moistening, or after abnormal fermentation in the interior of the grains. These parasites are chiefly represented by fungi belonging to the genus Mucor: Aspergillus or Penicillium; blight-Puccinia graminis, Uredo linearis; smut-Tilletia caries, Ustilago segetum, Ustilago maydis; yeasts of different kinds resulting from the fermentation of brewers' grains; and, finally, unrecognised microbes which act by means of the poisons they secrete.

The symptoms are always very vague. At first the only marked symptom is loss of appetite, accompanied by dryness of the mouth and muzzle, depression and constipation. The animals never clearly show signs of gastro-enteritis; nevertheless, the changes in general health point very clearly to a digestive origin.

In cases of acute poisoning the symptoms develop rapidly. Torpor becomes more marked, the movements of the heart tumultuous, and the temperature rises to $105^{\circ} \mathrm{Fahr}$. $\left(40.5^{\circ} \mathrm{C}\right.$.), diminishing later until death occurs.

In chronic forms of poisoning constipation is present at first, but is soon followed by profuse, fœtid, blackish diarrhœa, sometimes containing streaks of blood and accompanied by abdominal pain.

In exceptional cases these digestive symptoms are amplified by the presence of broncho-pneumonia, pleuro-pneumonia, nephritis, and cystitis, 
as in poisoning by tannin and essential oils. These complications are of infectious origin.

In young animals, like lambs and young pigs, still with their mothers alimentary intoxication may also occur though the mothers show no signs of illness. The passage of poisonous principles into the milk cannot be disputed. Moussu has seen numerous cases of alimentary intoxication in lambs whose dams were fed with decomposed beet pulp, and in sucking pigs whose mothers had received bad maize, turnips, etc.

Diagnosis. Careful examination of the substances with which the animals are fed, and consideration of the history, prevent confusion with ordinary poisoning. Anthrax as a cause can easily be eliminated.

The prognosis is grave, unless the practitioner is summoned early.

The lesions are those of acute gastro-enteritis-congestion of the mucous membrane, abomasum, and intestine, submucous infiltration, shedding of the epithelium, which sometimes attains the stage of ulceration, suffusion and intestinal or superficial hæmorrhage, dilatation of the capillaries, etc.

The symptoms of poisoning are produced by the absorption of toxic products, which pass from the intestine into the blood current.

Poisoning is frequently complicated by infection produced in a similar manner.

Treatment. The first point is to change the food. This alone is often sufficient to dispose of the digestive disturbance in a week or two. In addition, mustard plasters may be applied, and purgatives, stimulants and mucilaginous drinks may be given. Finally, diureties are useful in eliminating the toxic products accumulated in the blood. They comprise general stimulants like wine, alcohol, tea, coffee, etc. Subcutaneous or intravenous injection of physiological salt solution is indicated.

\section{POISONING BY CAUSTIC ALKALIES.}

The cause consists in the administration of insufficiently diluted solutions of ammonia in cases of tympanites, or the ingestion of quicklime, used for disinfecting stables, by animals suffering from depraved appetite.

The symptoms indicate injury to the anterior part of the digestive tract. They consist in salivation, loss of appetite, colic, indigestion, diarrhœea, and progressive loss of strength.

Diagnosis is only possible when the history is clear.

The prognosis is grave if the doses swallowed have been so large as to cause severe burning of the mouth, œsophagus, rumen, etc. The local lesions are grey and soft.

Treatment consists in immediately giving acidulated draughts containing vinegar or 1, 2 or 3 per cent. of hydrochloric acid, and emollient, 
mucilaginous drinks containing opium for the purpose of calming the irritation.

\section{POISONING BY CAUSTIC ACIDS.}

Cases of this kind are rare. Gerlach described a case of poisoning by straw which had been removed from sulphuric acid carboys. Abadie saw a number of cases which were traced to the unskilful treatment of two empirics.

The symptoms point to the existence of stomatitis, œsophagitis, and gastro-enteritis. Death occurs rapidly, with a running-down pulse. On post-mortem examination one discovers more or less deep burning of the mucous membrane of the digestive tract.

The diagnosis is difficult in the absence of information.

The prognosis is grave.

Treatment comprises administration of alkaline draughts, solutions of bicarbonate of soda, calcined magnesia, etc., and of mucilaginous drinks containing opium, chalk, etc. This may produce temporary relief.

Water mixed with whipped whites of eggs is also extremely valuable, but it is often better to slaughter the animal as soon as the condition is diagnosed.

POISONING BY COMNON SALT.

This form of poisoning is rare in oxen on account of the large quantity of salt which can be ingested without producing bad effects. It is commonest in animals to which old brine has been given or which have received rough salt containing toxic substances (sheep and pigs). Beef, pork, or fish brine, four or five months old, is especially dangerous because of the toxins it contains. Half a pint is a fatal dose for a pig (Reynal). The symptoms include marked thirst, vomiting and diarrhœa ; at a later stage motor and nervous disturbance appears, resulting from poisoning of the cerebro-spinal system. Paralysis, epileptiform convulsions, trismus, coma and death characterise extremely acute cases.

To the naked eye, the lesions are those of acute gastro-enteritis; and, in many cases, of marked congestion of the brain and medulla and of the mucous membrane of the bladder.

The treatment is prophylactic and hygienic. Old brine and salt of doubtful purity should be avoided. The symptoms should be treated by administering diuretics, preferably soda bicarbonate, which does not irritate the kidney, and by giving mucilaginous drinks with anodynes.

\section{POISONING BY THE NITRATES OF POTASH AND SODA.}

This form of poisoning has frequently been described as following the ingestion of water used for washing sacks which have contained chemical 
manures. Occasionally it results from the administration of medicines containing excessive doses of nitrate of potash. The symptoms vary in severity with the purity of the salt, with its nature, and with the degree of concentration of the solution: nitrate of potash is more dangerous than nitrate of soda.

The chief symptoms may be grouped as follows :-

Salivation, indigestion and tympanites, nausea, vomiting, diarrhœa, and (especially) intense polyuria : the kidney may be irritated to such a degree as to produce albuminuria and hæmaturia. Intense dulness and general weakness precede death, which sometimes occurs in four to twelve hours after the poison has been taken.

The lesions are to be found in the digestive and urinary apparatus. The kidneys are congested and hypertrophied, or present changes indicating epithelial nephritis. The ureters and the bladder may show similar lesions.

Treatment comprises removal of the cause, and the administration of emollients, narcotics, and diffusible stimulants.

POISONING BY TARTAR EMETIC.

Tartar emetic is sometimes given for the purpose of favouring secretion and restoring rumination; occasionally the proper dose is exceeded and poisoning occurs. Given repeatedly, tartar emetic is apt to accumulate in the deeper portions of the gastric compartments and to produce general symptoms of super-purgation, and such local symptoms as ulceration and even perforation of the walls of the stomach, which in turn is followed by abscess formation in the abdominal wall.

Diagnosis is easy. The prognosis is grave.

Treatment is confined to the administration of mucilaginous and diuretic fluids. Tannin has been recommended.

\section{POISONING BY ARSENIC.}

Overdoses of Fowler's solution produce rapidly fatal results, in twenty-four to forty-eight hours. Lesions are little marked.

Arsenious acid acts like tartar emetic by accumulating and producing local gastritis; it may also cause acute poisoning, being in that case characterised by severe colic with tympanites, salivation, and fœtid, sometimes blood-stained, diarrhoea. The urine becomes albuminous, and remains scanty. Incomplete paralysis is sometimes produced, and various forms of hæmorrhage result from changes in the constituents of the blood.

If the gastric compartments are perforated, an abscess may develop in the abdominal wall. The lesions are those of acute gastro-enteritis. The contents of the stomach exhales an odour resembling garlic. The 
parenchymatous organs-the liver, kidney, and heart-show fatty degeneration in chronic cases.

In prescribing arsenic, one should begin with small doses, which can gradually be increased as the animal becomes accustomed to the drug. Curative treatment consists in administering antidotes, such as hydrated oxide of iron, sulphate of iron, and calcined magnesia.

Haubner described a form of chronic arsenical poisoning produced in the neighbourhood of blast furnaces near Freiberg. Similar forms of poisoning may perhaps occur in the neighbourhood of chemical and smelting works in England.

\section{PHOSPHORUS POISONING.}

Though the chronic form is common in men, phosphorus poisoning only occurs accidentally in animals through eating phosphorus paste, used as a poison for rats, or as a consequence of excessive doses given medicinally. Some cases have been described by Maury.

The symptoms comprise salivation, loss of appetite, alliaceous odour of the buccal cavity, arrest of intestinal peristalsis, indigestion, colic, diarrhoea, exhaustion and death in a state of coma. Albuminuria and icterus also occur.

The lesions are the same as those of arsenical poisoning-viz., stomatitis, pharyngitis, and gastro-enteritis. The specific changes consist in fatty degeneration of the liver and kidneys, and the peculiar odour resembling garlic exhaled by the flesh.

Death is a consequence of deoxygenation of the blood, which appears black, and when spectroscopically examined reveals only the lines of reduced hæmoglobin.

Treatment. Oil and milk dissolve phosphorus and render it more easily assimilable; they should never be administered. Large doses (up to six fluid ounces) of essence of turpentine have been recommended. This prevents the phosphorus absorbing oxygen at the expense of the blood. Saline purgatives assist in eliminating the poison.

\section{IIERCURIAL POISONING.}

Mercurial poisoning may result either from the administration of medicines or from accident. Cases of the former kind follow the internal use of sublimate or calomel. Doses of 2 to $2 \frac{1}{2}$ drams of calomel, if repeated for a certain time, may prove toxic in the ox. Accidental poisoning results from the use of mercurial ointment as a parasiticide over large surfaces. Poisoning, however, rarely occurs unless the animal is able to lick the parts. Some authors have tested this point by freely applying mercurial ointment over parts likely to absorb it readily, without having seen any bad effect. 
Symptoms. Free salivation, the saliva gradually becoming fœtid and blood-stained. The slight irritation of the buccal membrane first seen is soon replaced by congestion of the gums, then by gingivitis and periostitis, with local ulceration and hæmorrhage. The portion of the gums surrounding the teeth becomes violet, and suppurative alveolitis may follow.

As a consequence of disturbance in the digestive secretions digestion ceases, defæcation becomes irregular, the fæces are often hard and coated, and can only be passed with difficulty, though sometimes there is profuse fœtid diarrhœa.

Respiration is difficult, jerky, or even dyspnøic, and is accompanied by discharge from the nose and by expectoration. The gait is irregular, and paralysis may follow. Finally the skin shows an eruption resembling that of impetiginous eczema; vesico-pustules, covered with yellowish crusts, appear over the entire surface of the body.

Lesions. The lesions of hæmorrhagic gastro-enteritis are sometimes accompanied by catarrhal tracheo-bronchitis, and even intra-pulmonary hæmorrhage. The muscles are discoloured, appear as though boiled, and are covered with ecchymoses. Blood effusions occur beneath the eczematous crusts, but the rest of the skin is anæmic.

Treatment includes administration of raw eggs, or, better still, of white of egg beaten up in water; the albumen in coagulating imprisons the mercury. Failing these, other bodies, like flowers of sulphur and iodide of potassium, which with mercurial salts form soluble and harmless compounds, may be given. Chlorate of potash has also been recommended.

Complications like stomatitis and gastro-enteritis are treated by the usual methods.

\section{LEAD POISONING: SATURNISM.}

Lead poisoning is very rare, and seldom occurs except near camps or factories. It then results from swallowing lead "spray" mixed with the grass or from inhalation of lead vapour.

The symptoms comprise salivation, nausea, colic, obstinate constipation, tympanites, and arrest of milk secretion. A peculiar form of trembling affects the head; epileptiform convulsions, amaurosis, and paralysis may also occur. General sensibility diminishes, and death follows.

The disease may assume a chronic form, characterised by a blue line around the gums, changes in the joints, albuminuria, and bodily wasting.

The lesions are those of ulcerative stomatitis, anæmia of the mucous membranes, and fatty degeneration of the epithelium. In the chronic form the kidneys are atrophied. 
Treatment consists in giving substances which form insoluble compounds with lead. It comprises the administration of dilute sulphuric acid, solutions of sodium sulphate or magnesia, milk, eggs, and iodide of potassium.

\section{COPPER POISONING.}

This is a rare form of poisoning. It may follow ingestion of food stored in copper vessels, licking of ointments containing copper salts, or ingestion of vine leaves, or leaves of other plants which have been sprayed with sulphate or acetate of copper to prevent " mildew."

The symptoms comprise vomiting of green-coloured material, colic, diarrhœa, muscular weakness, and convulsions. The urine contains dissolved albumen and hæmoglobin.

The lesions are those of acute enteritis and dilatation of the stomach. The essential lesion consists in decomposition of the blood with the formation of methrmoglobin. Nephritis and granular degeneration of the muscles form secondary symptoms.

Treatment consists in administering raw eggs, albumen, milk, mucilage, flowers of sulphur, or calcined magnesia.

\section{CARBOLIC ACID POISONINA.}

Carbolic acid poisoning sometimes follows the use of carbolic acid solutions in the form of injections, enemas, or baths.

Symptoms. Administered in over-doses or for too long a time, carbolic acid produces stomatitis, œsophagitis and vomiting.

True poisoning is characterised by changes in the kidneys and bladder ; the urine becomes brown and turbid, and possesses a well-marked carbolic odour, the animals suffer from severe trembling and appear stupid, and coma and paralysis precede death.

The specific lesions consist in parenchymatous nephritis, sometimes accompanied by renal hæmorrhage, cystitis and hyperæmia of the lung and brain. The flesh has an odour of carbolic acid, which renders it unfit for human consumption.

Treatment consists in giving stimulants and slight diuretics, such as ether, alcohol, wine, coffee, saline sulphates, or Glauber's salt. The last named forms phenyl-sulphuric acid, which is not toxic. Olive, rape, or linseed oil has been recommended. All these drugs are useless if the kidney lesions have become too pronounced.

\section{POISONING BY ALOES.}

This form of poisoning is caused by administering over-doses of aloes.

Apart from the accidents possible in pregnant female animals, large doses of aloes produce symptoms of super-purgation-profuse diarrhoa, running down pulse, and nervous prostration. 
The lesions are those of gastro-enteritis, the intestine being empty, and its mucous membrane of red colour.

Treatment is confined to administering drugs like camphor, rice, bismuth, opium, chloral, and emollients, which diminish peristalsis and lessen secretion.

\section{IODOFORM POISONING.}

The causes are limited to the licking of wounds which have been dressed with iodoform.

The symptoms include gastric disturbance, somnolence, coma, and the signs of iodism. liver.

The only lesions are those of fatty degeneration of the kidneys and

Treatment comprises the exhibition of vomitants, stimulants, and diuretics.

IODINE POISONING : IODISM.

Death by iodine poisoning is absolutely exceptional : the complications described under the name of iodism are rather to be referred to saturation of the organism than to true poisoning.

The cause of iodism is unduly prolonged administration of iodide of potassium, or of iodine in solution.

The symptoms include discharge from the eyes, coryza, hypersecretion from all the mucous membranes, and gastric disturbance. The cutaneous eruption exhibits special peculiarities, including desquamation of the epidermis in the form of bran-like scales, and pruritus.

The first indications in treatment are to discontinue the drug and to administer stimulants and nutrients like milk, cod-liver oil, etc.

\section{STRYCHNINE POISONING.}

Strychnine poisoning is most commonly due to over-doses of the drug accidentally given during treatment.

The symptoms comprise tetanic convulsions, hyperæsthesia and dyspnœa. As a result of muscular rigidity, the ribs cannot be moved, and death by asphyxia follows.

Treatment comprises the use of anæsthetics, and the administration of chloral hydrate, as long as the contractions continue. Bromide of potassium, tobacco, tannin, etc., are also useful.

The chief part of the botanical descriptions in the following list have been extracted, by permission, from the Annual Report for 1898 of the United States Bureau of Animal Industry, p. 392. To Dr. D. E. Salmon, 
who gave this permission, and who also kindly supplied the blocks, the writer (Mr. Dollar) desires to express his very sincere thanks. Considerable modifications have been made, and in the case of the more important poisonous plants, details of symptoms and treatment have been added.

\section{LIST OF PLANTS POISONOUS TO STOCK.*}

PERISPORACEÆ (ROT-MOULD FAMILY).

Aspergillus glaucus.-This is the common flocculent woolly mould which sometimes develops to a dangerous extent on corn, oats, and other food grains which have either been harvested before full maturity or been stored in a damp place. The mouldy growth is pure white at first, but changes with the ripening of the spores to gray and then green. The spores are apparently the cause of the so-called enzootic cerebritis, or "staggers," of horses, which, during the winter of 1898-99, was reported as having caused very heavy losses in the southern portion of the United States. European investigators have shown that the spores of this mould will grow within the living body if they are introduced into the blood. Death is probably caused by some poison which is simultaneously produced with the mould in the body of the animal.

\section{HYPOCREACEE (ERGOT FAMILY).}

* Claviceps purpurea.-This, the most common species of ergot, infests various species of native and cultivated grasses. It sometimes causes great losses of stock.

\section{POISONING BY ERGOT OF RYE: ERGOTISM.}

Causation. This form of poisoning is due to the presence of ergot in grain or forage.

Symptoms. The symptoms are seldom very marked. In mild cases pregnant females may abort; grave cases are indicated by local gangrene of the mucous membrane and gangrene of the extremities, particularly in poultry, in consequence of the constrictive action of the drug on the peripheral blood-vessels, on the vaso-motor centres, and on unstriated muscular fibres.

Treatment. The use of chloral and morphine has been suggested in this condition; but as the lesions when discovered are established and permanent, this treatment seems illusory. More is to be expected from preventive measures and the avoidance of ergotised food.

* The names of plants proved beyond doubt to be poisonous to stock are marked with an asterisk. The evidence in regard to the toxic properties of others is not in every case entirely convincing, though there is good ground for strong suspicion. 


\section{USTILAGINACE (SMUT FAMILY).}

Ustilago maydis.-The black powdery fungus known as corn smut is common throughout the corn-producing districts of the central United States, and is occasionally reported as being fatal to stock. Experiments made in Wisconsin and elsewhere show that it is not a very active poison when eaten in moderate quantity. When given in gradually increasing amounts up to $2 \mathrm{lbs}$, no effect was noted, but $4 \mathrm{lbs}$. on each of two successive days caused the sudden death of one cow. Since corn smut

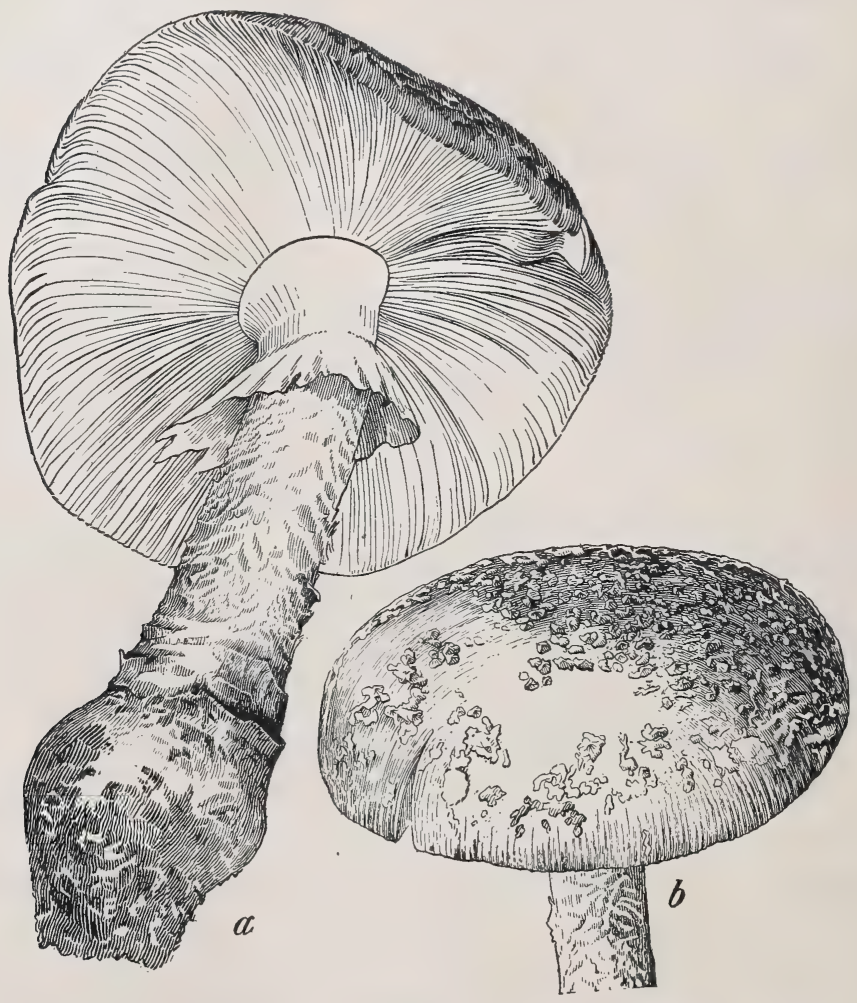

FIG. 74.-Fly amanita (Amanita muscaria). $\quad a$, Mature plant; $b$, top view of cap showing corky patches-both one-half natural size.

has been shown to be less fatal when wet, it seems probable that its physical and not its chemical character may be responsible for the death of cattle which have eaten it in considerable quantity.

POISONING BY SMUT OF BARLEY.

Causes. The presence of smut in straw, in millers' grains, or in damaged kiln-dried grains which have fraudulently been added to grain 
sold for feeding, represents the principal source of this form of poisoning.

The symptoms are very vague. There is indigestion, with a tendency to nausea, and vomiting. Diarrhœa may be present: At a later stage the heart and nervous system are affected. The gait becomes hesitating, the animals show general dulness and spasm of the pharynx; death, when occurring, is by cardiac or respiratory syncope.

Treatment consists in giving an entire change of food, and administering stimulants and purgatives.

\section{AGARICACE⿸ (MUSHROOM FAMILY).}

Amanita muscaria.-The well-known fly amanita (fly fungus ; deadly amanita) may be found from spring to early winter in pine forests throughout the United States. Cows are supposed to be killed by eating it, and almost every year the daily papers chronicle the death of several human beings who were led to eat the fungus through mistake for some edible species. The fresh cap is frequently rubbed up with milk and used to poison flies.

\section{PHALLACEE (STINK-HORN FAMILY).}

* Clathrus columnatus.-In an article published in the Botanical Gazette (Vol. XV. p. 45), Dr. Farlow, of Harvard University, gives an account of an investigation of a case of poisoning in hogs which was caused by eating this peculiar fungus. It grows in patches in oak woods and openings, and is quite common throughout the Southern States.

\section{POLYPODIACE (FERN FAMILY).}

Pteris aquilina.-In July, 1895, nineteen cattle died in Maryland, which were supposed to have been poisoned by eating the common bracken fern. Very few similar cases are on record, but one European authority cites one in which five horses were killed by eating hay contaminated with this fern, and another states that cases are quite frequent among cattle in England.

\section{EQUISETACEA (HORSETAIL FAMILY).}

Equisetum aryense.-The field horsetail was reported from Connecticut in 1871 as being poisonous to horses. Cases are very rare, and it is probable that this plant is deleterious only when eaten in considerable quantity, and then perhaps only on account of its physical character. Experiments made in Europe show that a similar species (E. palustre) is fatal to horses when fed in considerable quantity with hay.

D.C. 
TAXACEA (YEW FAMILY).

Taxus minor.-The common yew, or ground hemlock, is called poison hemlock in some places. The leaves of this shrub are probably poisonous to stock, as are those of the European yew. This species is more accessible to stock than the western yew (Taxus brevifolia), which grows only in deep cañons.

\section{YEW POISONING (TAXUS BACCATA).}

The leaves of yew, when eaten in any considerable quantity, are very poisonous. Two ounces to eight ounces, according to the size of the animal, slow the heart's action, produce weakness, staggering, and apoplectic congestion, which may have a fatal result in less than an hour. The bark is less injurious, and the fruit is scarcely poisonous at all.

The early symptoms, which are only seen in chronic cases, consist in excitement, followed by somnolence and muscular weakness, with slowing of the respiration and circulation.

\section{POACEE (GRASS FAMILY).}

Lolium temulentum.-The seed of the darnel, or poison rye grass, an introduced annual especially abundant on the Pacific Slope, is considered poisonous to both man and animals.

Stipa robusta.-This is a perennial plant which is known in Arizona and New Mexico as sleepy grass. It produces a narcotic effect on horses and cattle that feed upon it, but stock bred in that region rarely touch it.

Zea mays.-The numerous deaths that are frequently attributed to Indian corn are mostly due, not to any poison inherent in the plant, but rather to parasitic or saprophytic fungus growths, as noted under Aspergillus and Ustilago. The green fodder is very apt to cause severe and even fatal tympanites if the animal's diet is not properly regulated. Death has also been attributed to the presence of nitre (potassium nitrate) in the growing stalks. It is supposed that in very rich soil this substance will sometimes accumulate in the stalks in considerable quantity during prolonged drought.

\section{POISONING BY THE MALE TUFTS OF MAIZE.}

The green tufts alone are toxic; the toxicity disappears after drying. Prolonged administration produces nephritic colic and renal lithiasis.

\section{POISONING BY SWEET SORGHUM GRASS.}

This form of poisoning is characterised by attacks of trembling, tympanites, and frequent attempts to urinate. The heart beats feebly, 
and the pulse grows progressively weaker, until at last it becomes imperceptible.

\section{MELANTHACEE (BUNCH-FLOWER FAMILY).}

* Chrosperma muscætoxicum.-The bulbous portion of the fly poison, or crow poison, an eastern plant, is sometimes eaten by cattle with fatal

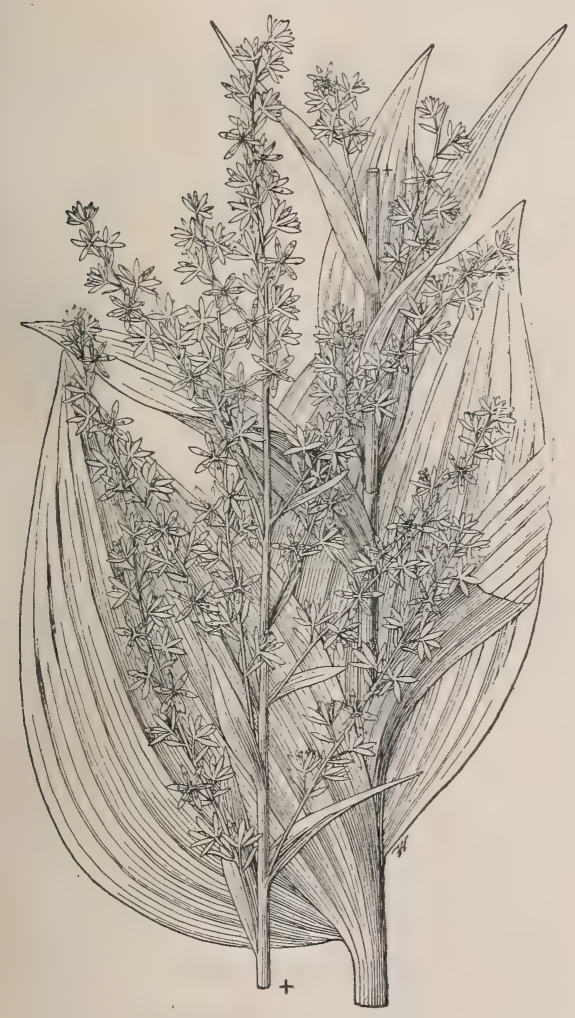

FIG. 75.-False hellebore (Veratrum viride), one-third natural size.

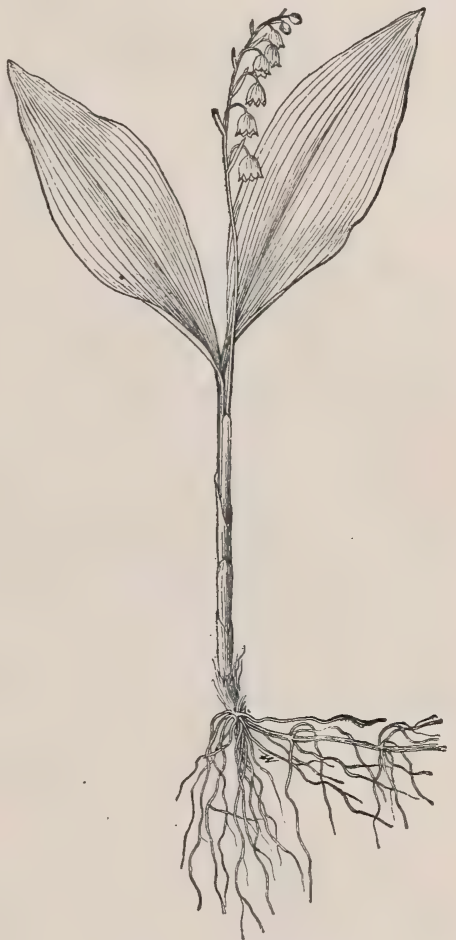

FIG. 76.-Lily of the valley (Convallaria majalis), one-third natural size.

results. The bulbs, when crushed with molasses, are used to stupefy flies.

* Yeratrum viride.-The leaves of the common swamp hellebore (American white hellebore; false hellebore; Indian poke) of the eastern and northern portions of the United States have proved fatal to man and to horses. Sheep eat the young leaves and shoots with apparent relish. The seed is poisonous to chickens.

Zygadenus venenosus. - The name death camas has been applied to this plant in the North-Test of America to distinguish it from the true camas (Quamasia quamash), which is highly esteemed for food by 
the Indians. In Oregon it is erroneously called "lobelia" by most, stockmen and farmers. Horses, cattle, and sheep, as well as man, are poisoned by eating the bulb.

Zygadenus elegans. - The bulbs, and perhaps the leaves, of the Glaucous zygadenus, or alkali grass, are poisonous to cattle.

\section{CONVALLARIACE (LILY OF THE VALLEY FAMILY).}

Convallaria majalis.-All parts of the lily of the valley are powerfully poisonous, and are liable to injure cattle and horses.

\section{HÆMODORACEE (BLOODWORT FAMILY).}

Gyrotheca capitata.-This is the red root, or paint root, of the Atlantic coast and Cuba, so called on account of the red colour of its sap. White hogs are supposed, throughout the Southern United States, to be particularly subject to the poison contained in the plant.

\section{FAGACEÆ (BEECH FAMILY).}

Quercus sp.-In Europe the acorns of various species of oak cause sickness and death in hogs and cattle. This effect may possibly be due to tympanites, but may also be due in some way to the tannin or the bitter principle which they contain.

\section{POISONING BY ACORNS.}

The acorn, or fruit of the oak (Quercus robur and Quercus sessiliflora), is much valued as a food for swine. "A peck of acorns a day with a little bran will make a hog, 'tis said, increase a pound weight per diem for two months together." Though largely consumed by swine with no apparent ill effects, acorns constitute a dangerous food for young cattle, especially when eaten before they are ripe and when herbage or other feeding is scanty or restricted.

The symptoms comprise dulness, loss of appetite, constipation, followed by diarrhœa, with straining and colicky pains, head carried low, eyes retracted, with mucus about the eyelids and blood-stained discharge from the nose. Frequently the abdomen is distended. Temperature normal.

The lesions are, abrasions of the buccal membrane on the palate, cheeks, etc.; impaction and intense congestion of the omasum.

Treatment. Change of pasture. Alkalies-potash or soda bicar bonate, magnesia; tonies and stimulants. 


\section{URTICACEÆ（NETTLE FAMILY).}

* Urtica gracilis.-The slender nettle covers thousands of acres of reclaimed swamp land in Michigan and Wisconsin, which is made nearly worthless by its dense growth, horses refusing to pass through it to cultivate the soil.

\section{CHENOPODIACEÆ (GOOSEFOOT FAMILY).}

Sarcobatus vermiculatus.-Black greasewood, or chico, is a scraggy shrub which grows in strongly alkaline soil in the south-western and western portions of the United States. A correspondent in New Mexico states that on one occasion he counted as many as 1,000 sheep that had been killed by eating the leaves of this plant. It is claimed that cows are not affected by eating it at any time, and that sheep can eat it quite freely in winter. Death is perhaps due more to tympanites rather than to any poisonous substance which the plant contains.

PHYTOLACCACEA (POKEWEED FAMILY).

* Phytolacca decandra. - The leaves of the common pokeweed (poke; garget; American nightshade) of the eastern half of the United States is occasionally eaten by cattle with fatal results.

\section{ALSINACEA (PINK FAMILY).}

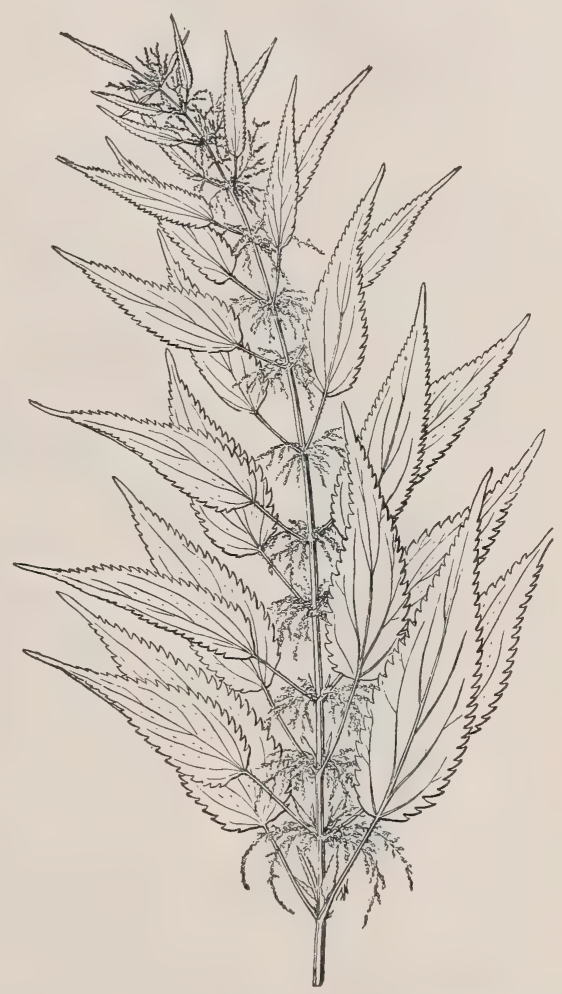

* Agrostemma githago. - The Fig. 77.-Slender nettle (Urtica gracilis). common corn cockle (cockle; mullein pink) is a weed common to both the United States and Europe. Poultry and household animals are occasionally poisoned by eating the seeds or the bread made from wheat contaminated with the seeds.

\section{MAGNOLIACE在 (MAGNOLIA FAMILY).}

Illicium floridanum. - The leaves of this species of anisetree are supposed to be poisonous to stock. 
RANUNCULACEE (CROWFOOT FAMILY).

* Aconitum napellus.-Aconite (monkshood; wolfsbane) is very commonly cultivated in gardens, and is therefore capable of doing great damage to stock. Horses and cattle have frequently been poisoned by eating the leaves and flowering tops.

* Aconitum columbianum.-The Western aconite, or monlshood, is native in the north-western portion of America, where it sometimes poisons sheep.

Anemone quinquefolia.-The common wind flower, which grows throughout most of the United States, is extremely acrid and poisonous. Cattle seldiom touch it. The plant loses most of its poison in drying.

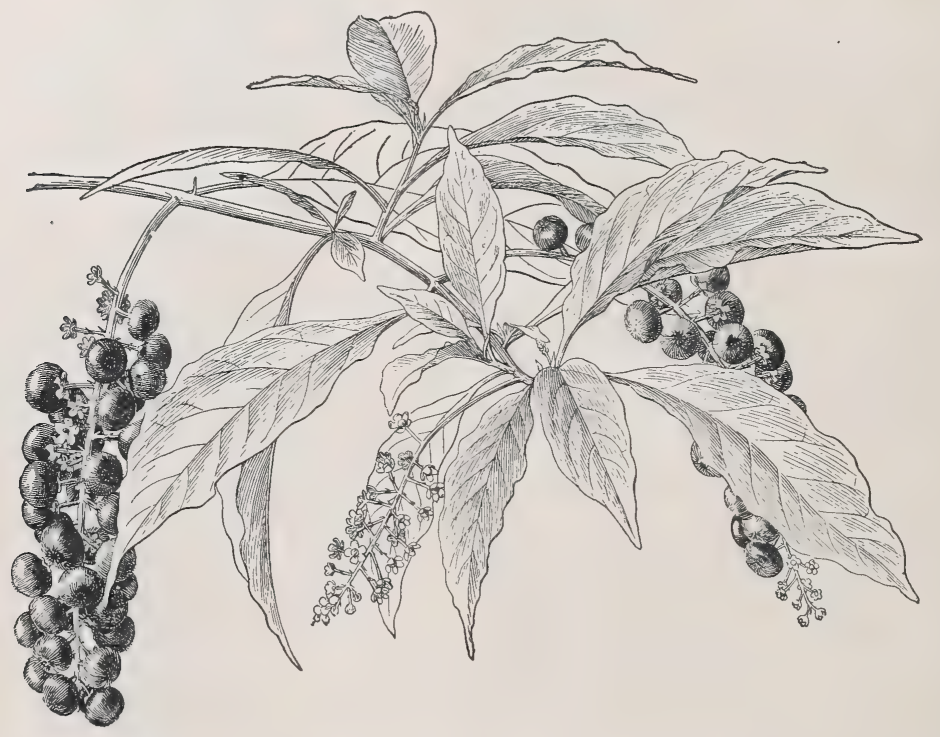

FIG. 78.-Pokeweed (Phytolacca decandra), one-half natural size.

* Delphinium tricorne.-The dwarf larkspur, or stagger weed, of the north-eastern quarter of the United States has been especially reported from Ohio as fatal to cattle in April, when the fresh leaves appear.

* Delphinium consolida. - The seeds of the commonly introduced field larkspur are well known to be poisonous; the leaves are known in Europe to be fatal to cattle.

* Delphinium menziesii.--The purple larkspur of the north-western quarter of the United States is very common throughout Montana. In one case of poisoning reported by Dr. E. V. Wilcox, of the Montana Experiment Station, over 600 sheep were affected, 250 of which were claimed to have been killed by the weed. An experiment made by 
Dr. S. B. Nelson, Professor of Veterinary Sciences in the Washington State Agricultural College, shows that it is possible to feed as much as $24 \frac{3}{4} \mathrm{lbs}$. of the fresh leaves to a sheep within a period of five days without any apparent ill effect taking place. An experiment made by Dr. Wilcox shows that the extract from less than an ounce of the dried

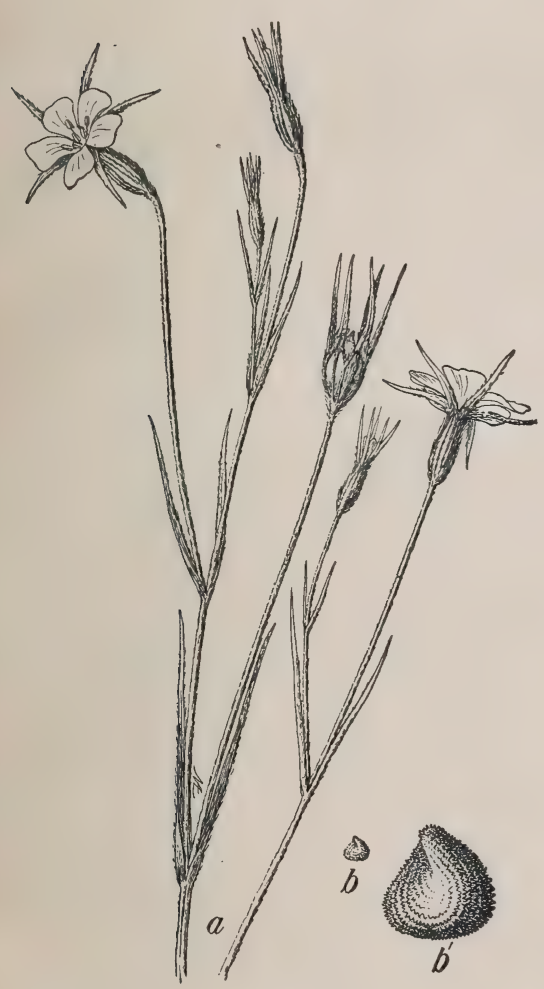

Frg. 79.-Corn cockle (Agrostemma githago). a, Sprays showing flowers and seed eapsule, one-third natural size; $b$, seed, natural size; $b$, seed, four times natural size.

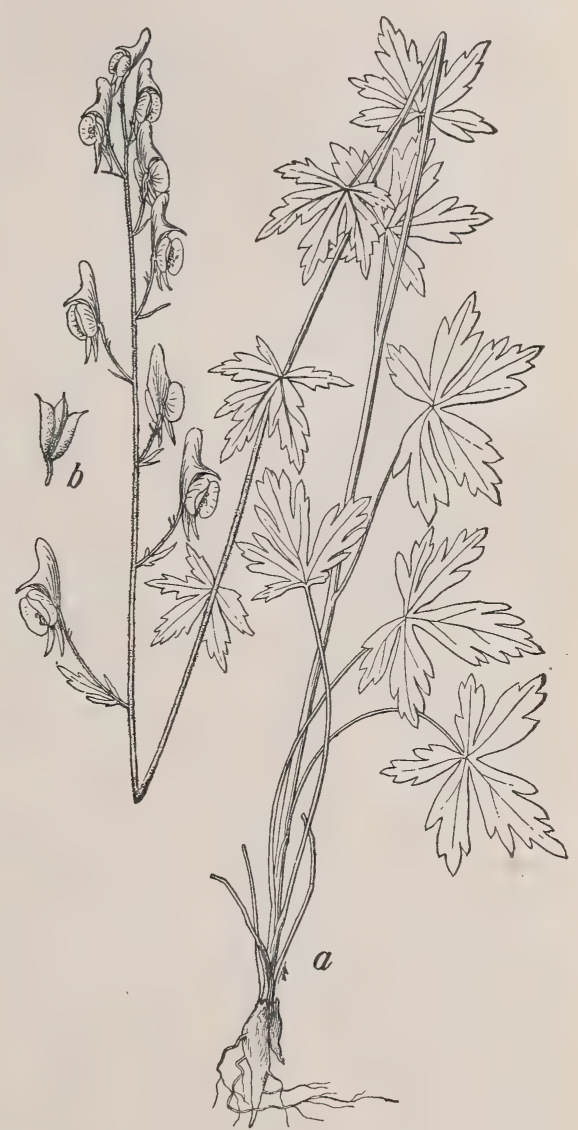

FIG. 80.-Aconite (Aconitum columbianum). $a$, Flowering plant ; $b$, seed capsule-both one-third natural size.

leaves killed a yearling lamb in two hours, the dose having been given by way of the mouth.

\section{LARKSPUR POISONING IN SHEEP.**}

Severe losses have from time to time been recorded, especially in America, from larkspur poisoning, the number of animals lost amounting

* The following account is summarised from a bulletin of the Montana Experiment Station by Dr. Wilcox. 
to thousands. The first signs of poisoning are slight general stiffness and straddling gait, especially of the hind legs. The stiffness becomes more and more pronounced, until walking is difficult and evidently painful. Soon there are manifested various involuntary twitchings of the muscles of the legs and sides of the body, and loss of control or co-ordination of the muscles. Ordinarily there is no increase in the quantity of the saliva, no dribbling of saliva from the mouth, no champing of the jaws or attempts at swallowing. The sheep manifest none of the mental disturbances frequently seen in cases of poisoning from other sources, as for example loco-weed and lupine. There is no impairment of the special senses. The sheep seem to hear and see as well and as correctly as under normal conditions of health.

No indications of any disturbances of the digestive functions are to be seen. The appetite remains good, and the sheep eat up to the very last. They were observed eating industriously during the intervals between the attacks of spasms which they have during the last stages.

At first the frequency of the pulse and of the respiratory movements is lessened and the temperature is lowered. The pulse remains very weak, but in the later stages becomes very rapid, in some cases 130 per minute. 'Toward the last also the respiration is very shallow and rapid. During the final convulsions the respiration is sometimes 120 per minute, but so shallow that the air is simply pumped up and down the windpipe. The air in the lungs is therefore not renewed, and the animal dies by asphyxia or suffocation.

So long as the sheep can stand on its feet, or walk, it keeps up with the flock as nearly as possible. The exercise, however, excites it, makes its respiration more rapid, and it has frequently to lie down for a moment and then get up and hobble along after the flock. The worst cases can thus easily be detected, since they straggle behind the rest of the flock.

The later stages follow rather rapidly. The involuntary movements become more frequent and more severe. All four legs tremble and shake violently. In fact, all the muscles of the body contract spasmodically until the animal totters over on its side and dies in the most violent spasms.

Larkspur has the effect of arresting the heart's action and respiration and of paralysing the spinal cord.

Treatment. Place the animal by itself in a cool, quiet, shaded place and avoid all excitement. Of the drugs tested, atropine sulphate dissolved in camphor water has given the best results. Wilcox (Bull. 15, Montana Ex. Station) recommends for sheep from $\frac{1}{20}$ to $\frac{1}{15}$ grain in the earlier, and $\frac{1}{6}$ to $\frac{1}{4}$ grain in the later convulsive stages. Cattle require from four to five times these doses. Inhalations of ammonia vapour, and small doses of alcohol and ether, are also useful.

In other cases very good results have been obtained from giving 


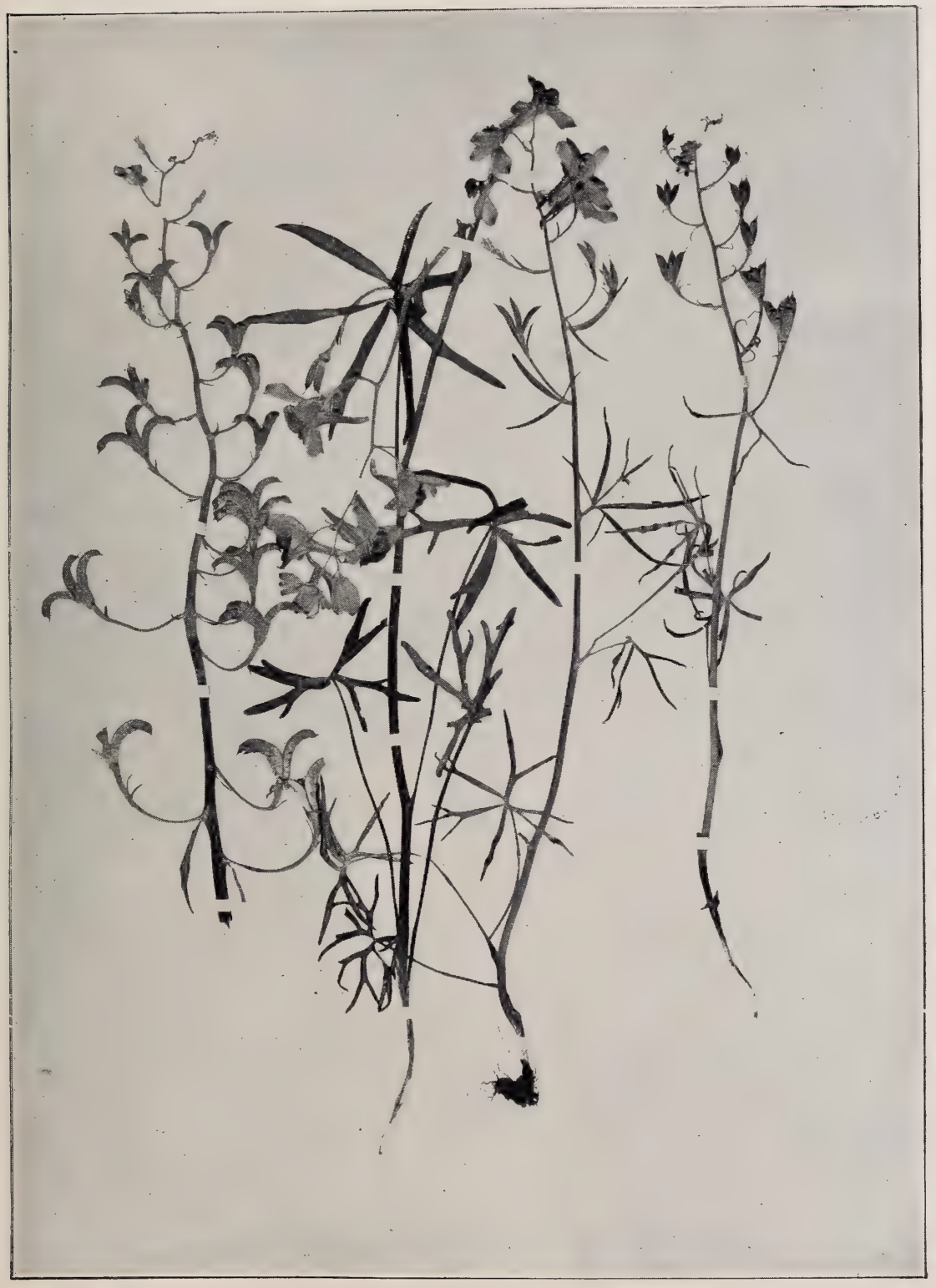

FIG. 81.-Delphinium menziesii.

(To illustrate "Larkspur Poisoning." From the Annual Report, U.S.A. Department of Agriculture, 1898.) 



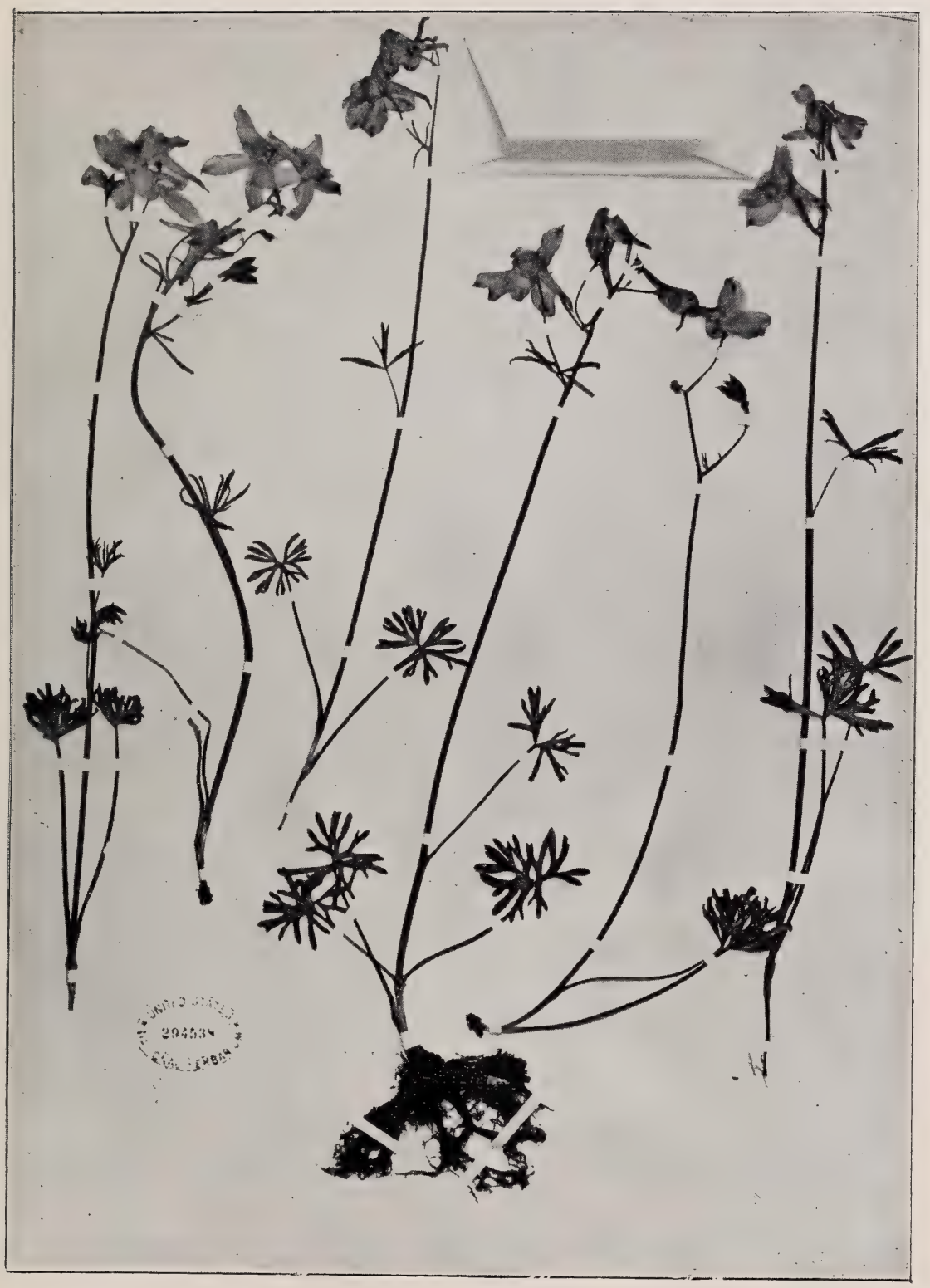

FIG. 82.-Delphinium menziesii.

(To illustrate "Larkspur Poisoning." From the Annual Report, U.S.A. Department of Agriculture, 1898.) 



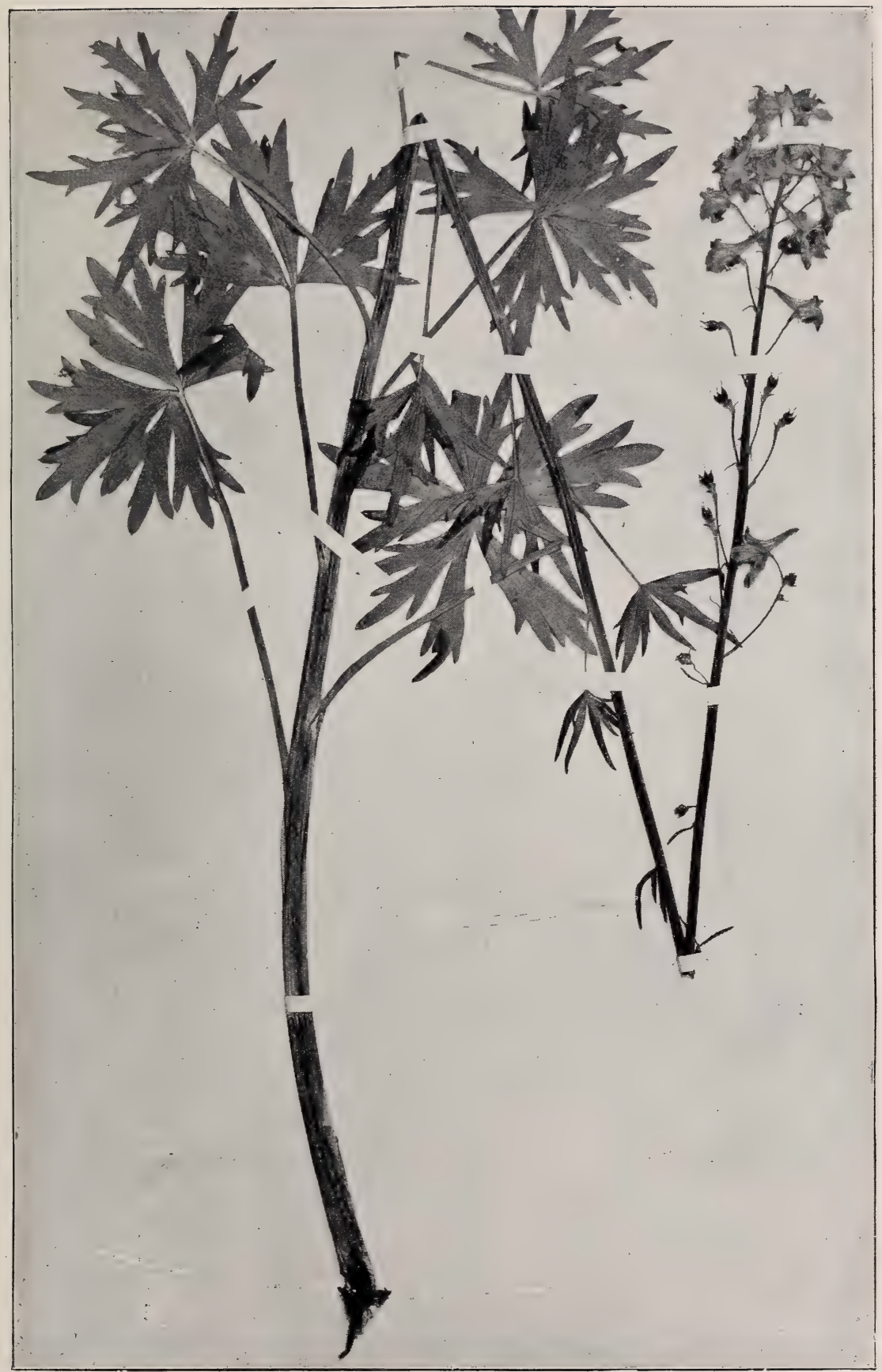

FIG. 83.-Delphinium scopulorum.

(To illustrate "Larkspur Poisoning." From the Annual Report, U.S.A. Department of Agriculture, 1898.) 

permanganate of potash in the form of a drench: 5 to 10 grains for an adult sheep or pig, 15 to 20 grains for a horse, and 30 to 50 grains for an ox, dissolved in a pint or two pints of water.

* Delphinium geyeri.-The Wyoming larkspur is well known throughout Wyoming, Colorado, and Nebraska under the name of poison weed.

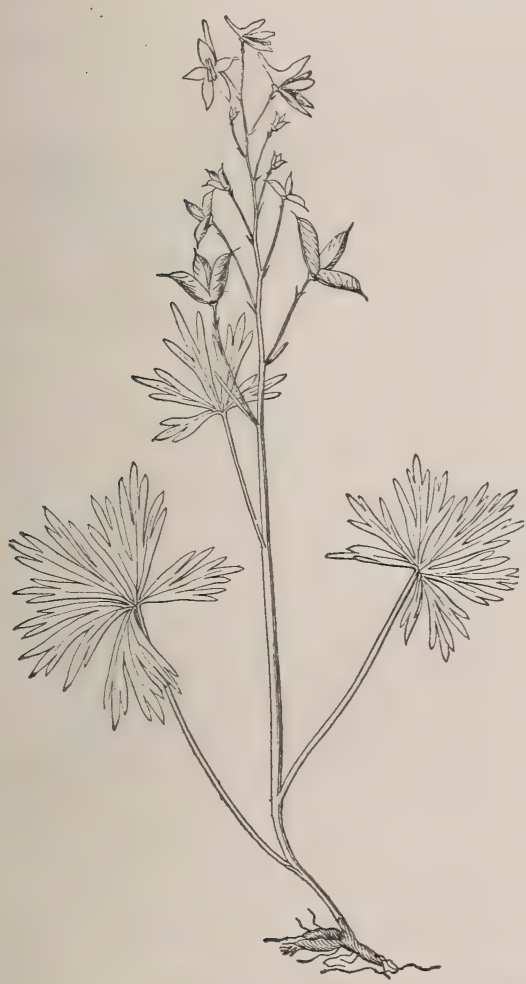

FIG. 84.-Dwarf larkspur (Delphinium tricorne), one-third natural size.

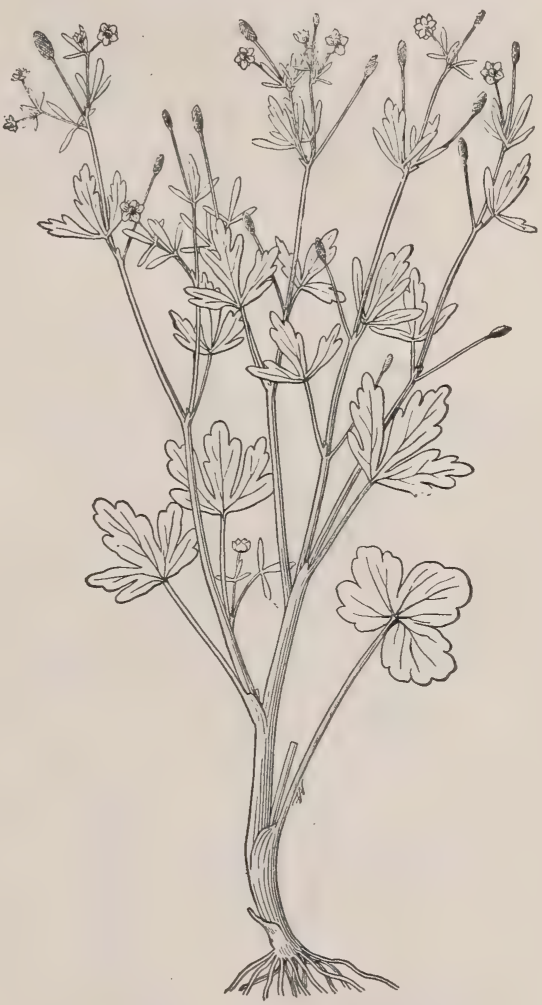

Fig. 85.-Cursed crowfoot (Ranunculus sceleratus.)

It is reported to be the most troublesome plant to stock in Wyoming, the dark-green tufts of foliage being especially tempting in spring when the prairies are otherwise dry and barren.

Delphinium recurvatum.-This species of larkspur grows in wet subsaline soil in the southern half of California. It has been reported from San Luis Obispo county as fatal to animals.

Delphinium scopulorum.-The tall mountain larkspur of the Rocky Nountains has been reported to the Canadian Department of Agriculture as poisonous to cattle in the high western prairies of Canada.

Delphinium trolliifolium.-This plant is common throughout the coast region of northern California, Oregon, and Washington. In 
Humboldt County, Cal., it is known as cow poison, on account of its fatal effect on cattle. Its toxic character has been questioned. Perhaps it is not equally poisonous throughout all stages of its growth.

* Helleborus viridis.-The green hellebore is a European plant, sometimes self-sown from gardens. All parts of the plant are poisonous. Cattle have been killed by eating the leaves.

POISONING BY HELLEBORE.

This form of poisoning is of slow progress, the plant producing irritation of the digestive mucous

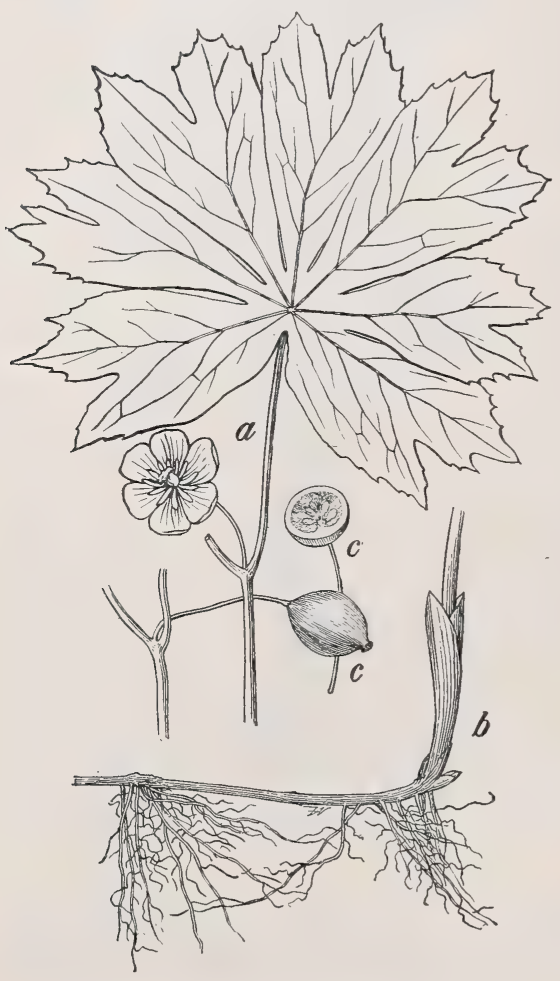

FIG. 86.-Mandrake (Podophyllum peltatum). membrane. The symptoms consist in loss of appetite, blackish, glairy diarrhœa, and intermittence of the pulse.

* Ranunculus sceleratus.The cursed crowfoot, or celeryleafed crowfoot, is found throughout the eastern half of the United States and also in Europe. Cattle generally avoid all of the buttercups, but fatal cases of poisoning from this plant are recorded in European literature. When dried in hay, the plant appears to be non-poisonous. The bulbous crowfoot (R. bulbosus) and the tall crowfoot $(R$. acris) are well-known to be very acrid in taste, and it is probable that all of the species which grow in water or in very marshy land are poisonous.

\section{POISONING BY RANUNCULACEA.}

Poisoning only occurs when the green plants are eaten. Drying causes certain essences contained in them to disappear, and thus destroys their toxicity.

This form of poisoning is indicated by yawning, colic, blackish, fœetid diarrhœa, and rapid loss of strength.

The animals suffer from stertorous breathing, weakness of the pulse, and aberration of vision. They die in convulsions. 


\section{BERBERIDACEÆ (BARBERRY FAMILY).}

Podophyllum peltatum.-The leaves of the common mandrake, or May apple, of the eastern half of the United States, are sparingly eaten by some cattle. Cases of poisoning are very rare, but the experience of one correspondent shows that the milk from a cow that had been feeding on the plant off and on for about three weeks was so extremely laxative as to be positively poisonous. The accident occurred to a baby, fed exclusively on cow's milk. The physiological effect of the milk was precisely like that of mandrake. It was shown that the cow ate the plant, which was abundant in one pasture, and when the animal was removed to a pasture free from the plant the child's illness stopped at once.

\section{BUTNERIACEE (STRAWBERRY-SHRUB FAMILY).}

Butneria fertilis.-The large oily seeds of the calycanthus, or sweetscented shrub, contain a poisonous alkaloid, and are strongly reputed to be poisonous to eattle in Tennessee.

\section{PAPAVERACEE (POPPY FAMILY).}

Argemone mexicana.-The Mexican poppy is reputed to be poisonous to stock both in the United States and in New South Wales. The seeds are narcotic, like opium.

* Chelidonium majus.-The yellow milky sap of the celandine, an introduced weed common in the eastern United States, contains both an acrid and a narcotic poison. Both are powerfully active, but cases of poisoning are rare, as stock refuse to touch the plant. Reeks, of Spalding, however, describes (J.Comp. Path. and Therap., Dec. 1903, p. 367) an outbreak of poisoning by common celandine in which twenty-one valuable cows were affected and three died. The symptoms comprised excessive salivation and thirst, convulsions, unconsciousness and epileptiform movements.

* Papaver somniferum, opium poppy, or garden poppy: P. rhœas, field poppy, red poppy, or corn poppy.-These plants are sometimes self-sown from gardens. Both contain acrid and narcotic poisons, and European literature records the death of various animals from eating their leaves and seed pods.

\section{POISONING BY POPPIES.}

The consumption of poppies causes arrest of peristalsis, secretion of foamy saliva, colic, depression, coma, and in severe cases death by stoppage of respiration. 
PRUNACEA (PLUM FAMILY).

* Prunus caroliniana.-The laurel cherry, or mock orange, is native in the south-eastern quarter of the United States, and is there often cultivated for hedges. The half-withered leaves and the seeds yield prussic acid, and are poisonous when eaten by animals.

* Prunus serotina. - The wild black cherry is a valuable forest tree which ranges throughout the eastern half of the United States. Cattle are killed by eating the partially

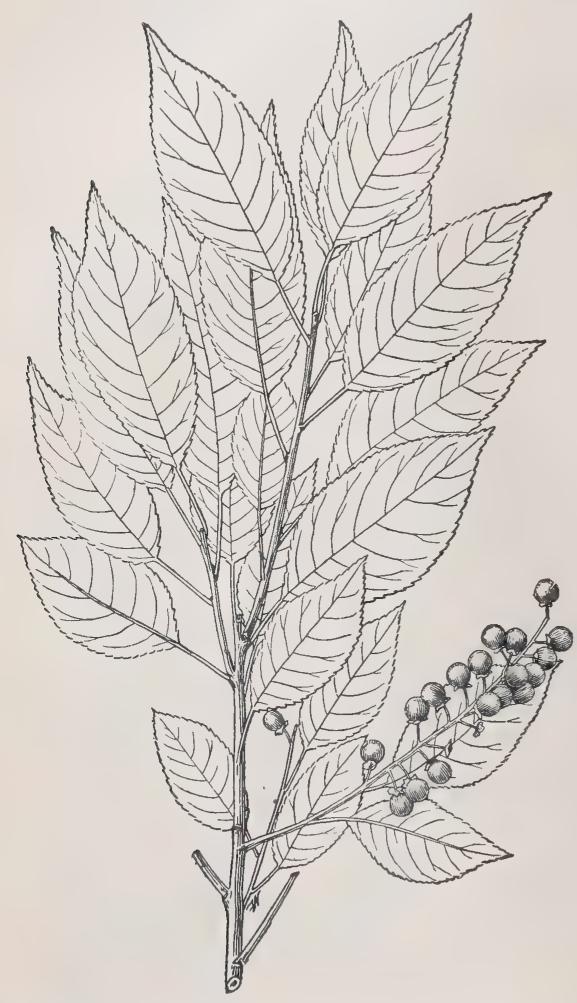

FIG. 87.-Black cherry (Prunus serotina), one-third natural size. withered leaves from branches thrown carelessly within their reach or ignorantly offered as food. The leaves of various other wild and cultivated cherries are probably poisonous to cattle in the same way.

\section{VICIACEA（PEA FAMILY).}

Aragallus lambertii. - The Lambert, or stemless loco weed, is, next to the following species, the best-known representative of a large group of closely related plants which are native to the western half of the United States, and are known as loco weeds on account of the peculiar excited condition which they induce in animals that eat of their leaves. Horses and cattle are both affected, but the chief damage is done to horses. After being permitted to graze on any of these plants the animal acquires an unnatural appetite for them, and soon refuses all other kinds of food. It rapidly becomes unmanageable, shows brain symptoms, and finally dies from lack of proper nourishment.

Astragalus mollissimus.-This, the woolly loco weed, is perhaps the best known of all the loco weeds. It is the species most abundant in Colorado, where from 1881 to 1885 nearly $\$ 200,000$ was paid out in bounties in an attempt to exterminate it. The plant is still abundant in that State, and reports of the damage done by it continue frequent. 


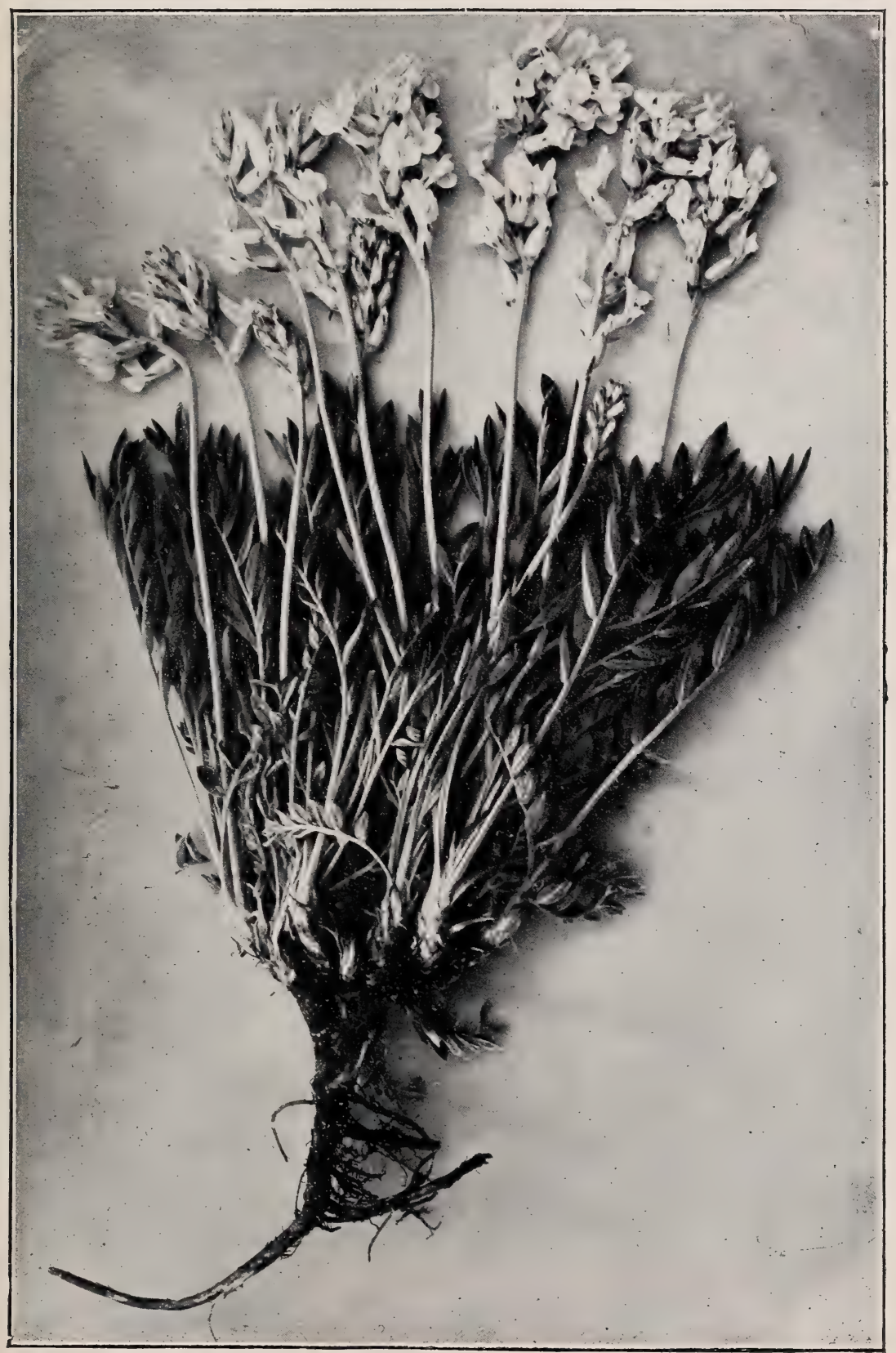

Fig. 88.-White loco weed (Argallus spicatus) in flower.

(From the Annual Report, U.S.A. Department of Agriculture, 1900.) 



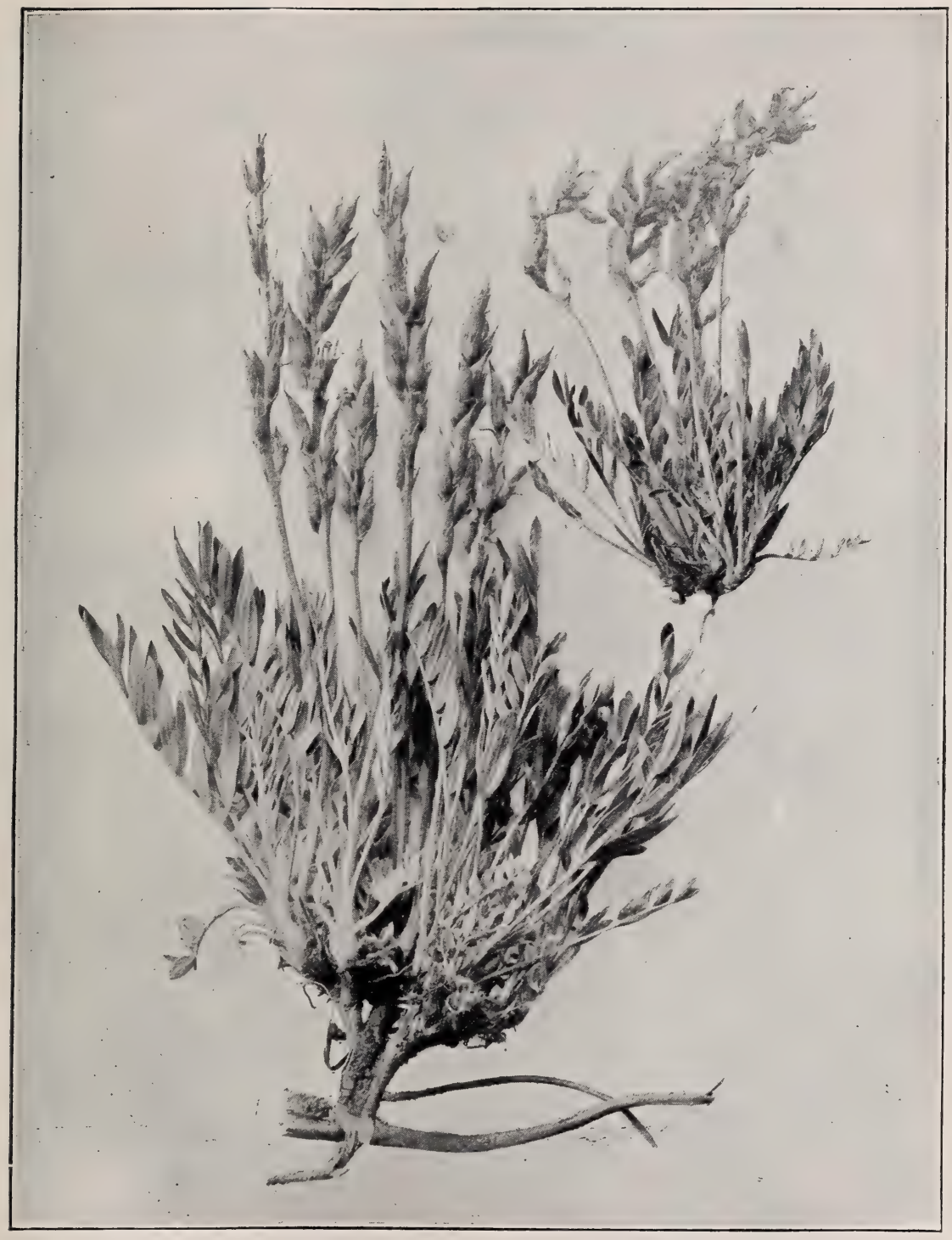

FIG. 89.-White loco weed (Argallus spicatus), showing seed pods.

(From the Annual Report, U.S.A. Department of Agriculture, 1900.) 



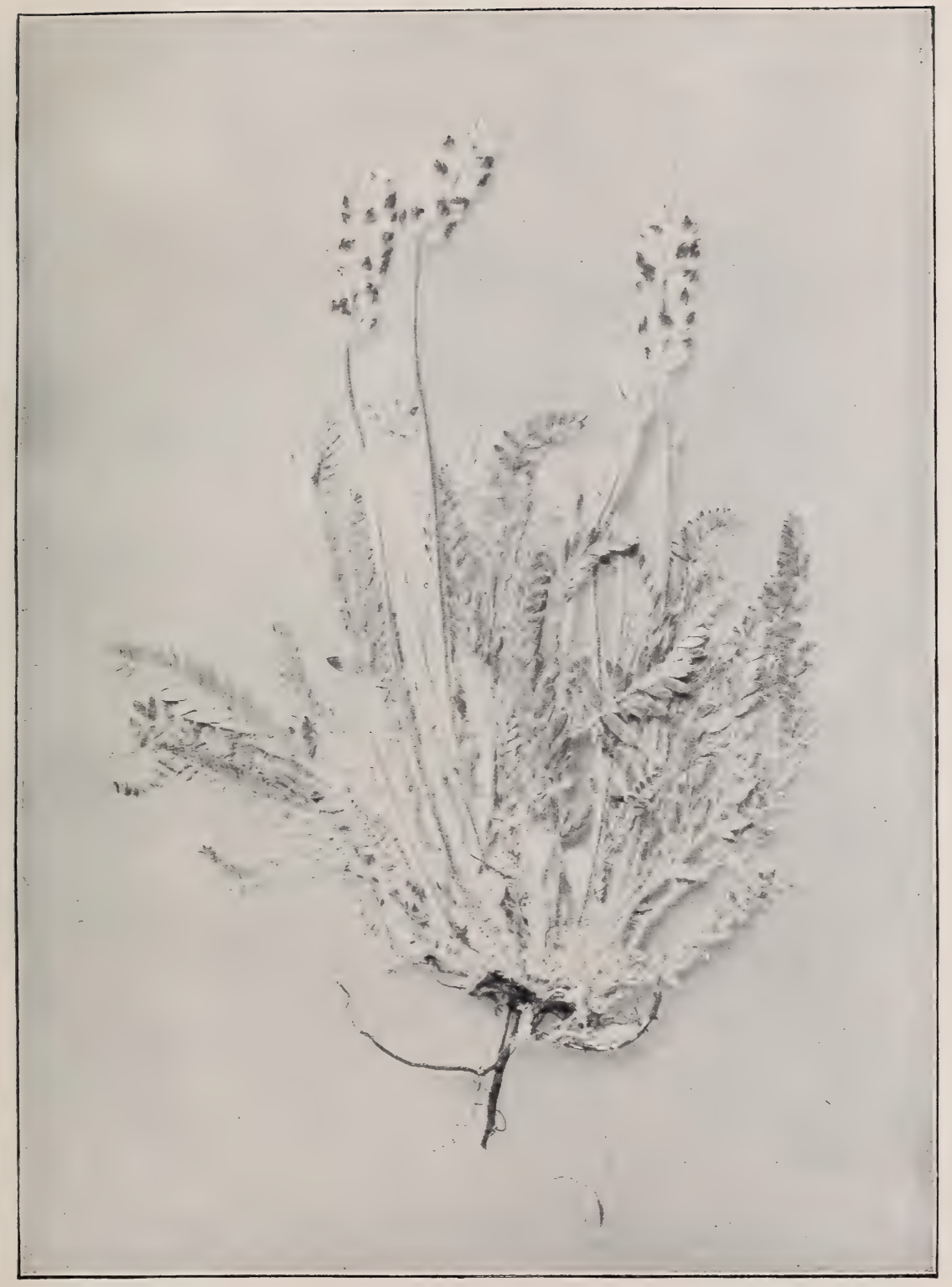

Fig. 90.-Loco weed (Astragalus splendens).

(From the Annual Report, U.S.A. Department of Agriculture, 1900.) 

Specimens of the three following species of Astragalus have been forwarded to the Division of Botany with the information that they were causing great financial loss in the districts noted. It is quite probable that other species are dangerous also.

\section{POISONING BY WHITE LOCO WEED (ARAGALLUS SPICATUS).}

This is an erect tufted perennial, 4 to 18 inches high, with pinnately

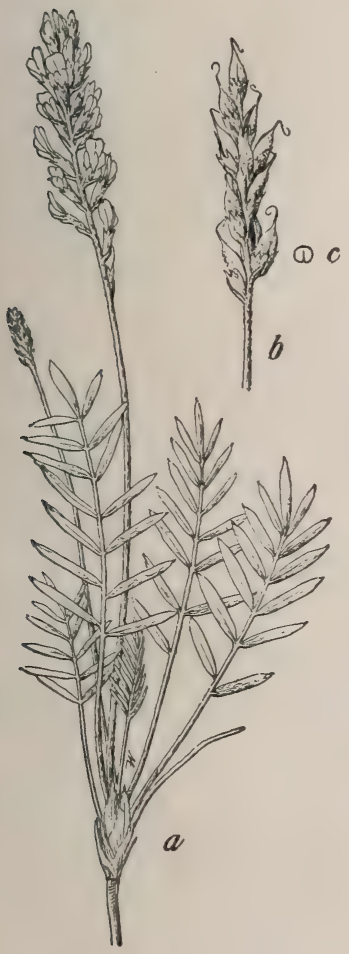

FIG. 91.-Stemless loco weed (Aragallus lambertii). a, Flowering plant; $b$, seed pods; $c$, cross-section of seed pod-all one-third natural size.

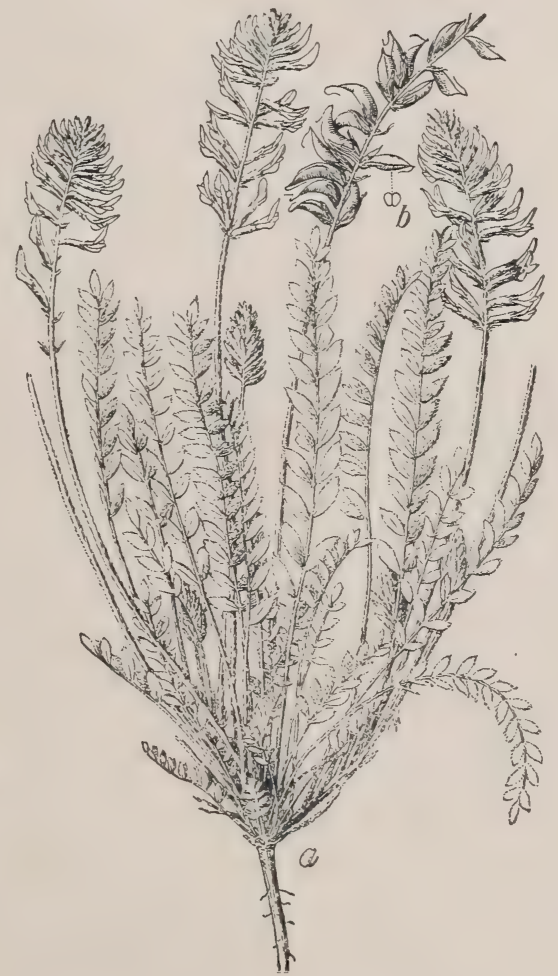

FIG. 92.-Woolly loco weed (Astragatus mollissimus). $a$, Whole plant; $b$, section of pod-both one-third natural size.

divided leaves and spikes of white or cream-coloured flowers, shaped like those of the pea. The pod is one-celled, and when shaken produces a rattling sound, which gives the plant the name of "rattle weed" in some localities. The white loco weed is exceedingly common throughout Montana. It occurs most abundantly on the northern slopes of foothills up to an altitude of about 8,000 feet. Its preferred habitat is for the most 
part in rather dry situations. The habit of the plant varies in different parts of Montana. In some localities the flowers are pure white, while in others they are decidedly yellow.

In Colorado the plant which is most ordinarily known as loco weed is Astragalus mollissimus, while in Montana the species already named is perhaps most important; but there are others which have a rather wide distribution and are known to produce the same effects. Among these may be mentioned A. splendens, A. lagopus, and A. besseyi.

The losses caused from the loco disease are very heavy in nearly all the Rocky Mountain States. The locoed condition is so commonly observed among sheep and horses that cases are not reported, and it is practically impossible to learn the exact extent of the disease. In the Judith Basin one prominent stockman was nearly ruined financially by the prevalence for a number of years of the loco habit among his sheep. In another instance the raising of horses was abandoned over a large tract of country on account of the loco weeds.

The loco disease occurs under two forms-an acute and a chronic. An acute case of loco disease was observed by Dr. Wilcox in a two-yearold ewe with a lamb at its side. The ewe was observed eating large quantities of white loco weed on May 22nd, 1900. During the afternoon of the same day it became unmanageable, and the lamb was badly affected. An examination of the ewe at this time showed that it was completely blind and was affected with dizziness. It walked around in long circles to the right, and after a short period remained standing for a few moments in a sort of stupor. At the beginning of each attack the head was elevated and drawn to the right; eyelids, lips, and jaws were moved rapidly. Each attack lasted from one to two minutes, and the intervals between the attacks lasted about five minutes. The second day the attacks became more severe and of longer duration, the head being turned more decidedly to the right and the animal sometimes falling upon the ground. Similar symptoms, accompanied by digestive disturbances, were manifested by the lamb during the second day, and it died during the afternoon. On the morning of the third day it was found that the ewe was pushing against the fold, and had apparently been in that position during the greater portion of the night. The animal then began to whirl round to the right. Later it became unable to stand, and the spasmodic movements were largely confined to the legs. On the morning of the fourth day it died. The pupil of the eye was at no time dilated, and the expression was nearly normal. The pulse was at first very irregular, but on the second day became again regular and of normal frequency. The only remedy which was tried was frequent injections of one-quarter grain doses of morphine, but this was without effect. Two other ewes ate smaller quantities of loco weed at the same 
time and were similarly affected, but less severely. In these cases morphine was tried with better success. The lambs, however, died from the poisonous properties contained in the milk of the mother.

The general symptoms of loco disease are quite familiar to all stock raisers. Perhaps the most characteristic are those of cerebral origin, and are shown in peculiarities of gait and action, which may be compared to a drunken condition. The brain disturbances may consist in impairment of the special senses or in irregular motor impulses, which produce incoherent muscular action. In some cases the animal becomes blind. More frequently the animal makes errors in judgment of the size and distance of objects. These visual disturbances are often quite ludicrous. The animal often takes fright, apparently at imaginary objects, or at objects which under ordinary circumstances would cause no alarm. Locoed horses are somewhat dangerous for driving purposes on account of their tendency to run away. Such horses are frequently attacked with kicking fits without any apparent cause. The sense of hearing is often affected, and the response to sounds is irregular and out of proportion to the volume and character of the sound. Irregularities in muscular movements of sheep may assume a variety of forms. The animal may simply carry its head in an extended or otherwise unnatural condition. In some cases the back is arched. Trembling is a characteristic symptom. In locoed horses a great difficulty is sometimes experienced in persuading them to go backward. Locoed sheep are exceedingly difficult to manage. The different members of the flock may suddenly take a notion to run away in different directions, with the result that it is almost impossible for the shepherd to prevent their becoming separated. In cattle the disease appears to be rare, although symptoms, so far as observed, are essentially the same as those in sheep and horses. Occasionally locoed cattle manifest dangerous symptoms, and attack men and other animals.

In chronic cases of loco the animal gradually becomes more emaciated and crazy. In sheep the fleece may be shed in patches or as a whole. The animal becomes unable to care for itself, and is apt to fall into the water while attempting to drink. Fits of trembling are of frequent occurrence, and the animal finally dies of inadequate nutrition and total exhaustion. In chronic cases of loco disease in horses the animal is usually left to its own resources on the range. During the later stages it may remain for weeks at a time upon a small area of ground without taking water. Dr. Wilcox saw a number of such cases in horses that were almost unable to walk. Under such circumstances the animals seldom or never lie down. One horse which was seen remained for a period of two weeks, in 1897, upon a piece of ground about 150 feet square. During this time the horse had no water. 
Numerous autopsies on locoed sheep and horses revealed slight congestion of the brain membranes in all cases. The lungs and heart were in normal condition. Fatty tissue was considerably reduced in quantity, and the muscles were paler in colour than under normal conditions.

The most serious mistake in connection with loco disease is made in allowing locoed sheep to remain with the rest of the flock. The loco habit is apparently learned by imitation of locoed animals, and so long as locoed sheep are allowed to remain with other sheep the loco habit rapidly spreads. An experienced sheep raiser, after being nearly ruined financially through the loco disease, adopted the method of immediate isolation and the feeding of locoed sheep for mutton. His stock was replaced with sheep that were free from the loco habit, and the trouble has been entirely eradicated from his range.

No specific remedy for the loco disease has been discovered, and in the nature of the case no such remedy is likely to be found. In the present state of knowledge concerning the subject the only rational treatment to be recommended is that of confinement and feeding with a nutritious diet. By separating the locoed sheep at once from other sheep the spreading of the habit will be prevented, and the locoed animals may be fattened and thus prevented from becoming a total loss. Although locoed animals may readily be fattened and sold for mutton, their recovery from the loco habit is apparent only, and is due to their inability to obtain the loco weed. Such animals when allowed to run upon the range again almost invariably return to their old habit of eating loco weed. Animals which have once been locoed are, therefore, unsuitable for stocking the range.

In combatting the loco disease the most rational methods include providing salt for the sheep, the immediate removal of locoed sheep from the band, confining them in a fold, and feeding them upon a nutritious diet. They may thus be fed for market, and their pernicious habit will not spread to other sheep. In the case of locoed horses, an apparent recovery takes place if they are confined in a stable and fed on ordinary cultivated forage or allowed to run in pastures where no loco weeds are found. Such horses are always somewhat dangerous, and more apt to run away or become unmanageable than horses which have not become affected with this disease.

* Crotalaria sagittalis.-The rattlebox (rattle weed; wild pea) is an annual weed which grows on sandy soil throughout most of the eastern half of the United States. In some years it is especially abundant in the bottom lands of the Missouri Valley. Horses and sometimes cattle are killed in this region by eating grass or meadow hay which is contaminated with the plant. 
Lupinus leucophyllus.-This herbaceous shrub is a representative of a very large genus of plants, many of which are widely and abundantly distributed throughout the western United States, and are generally known as lupines. The above species is very abundant in Montana, where it is said to have caused the death of a very large number of sheep. There is some question whether the animals are killed by a poisonous constituent of the plant or merely by tympanites. The seeds of all the lupines are probably deleterious in the raw state. In Europe, however, the seeds of Lupinus albus, after the bitter taste has been removed by steeping and boiling, are eaten by human beings as well as by cattle.

\section{POISONING BY LUPINES (LUPINUS \\ LEUCOPHYLLUS ; L. SERICEUS ; L. CYANEUS).}

These plants are commonly known by the names blue pea, blue bean, and wild bean. They are coarse, silky-haired perennial herbs, with blue flowers arranged in conspicuous terminal racemes, which blossom in June and July, with long-stemmed leaves, which are divided into from seven to eleven leaflets radiating from a common point. The fruit is a hairy, several-seeded pod, and the seeds are small and somewhat flattened.

As a rule these plants do not

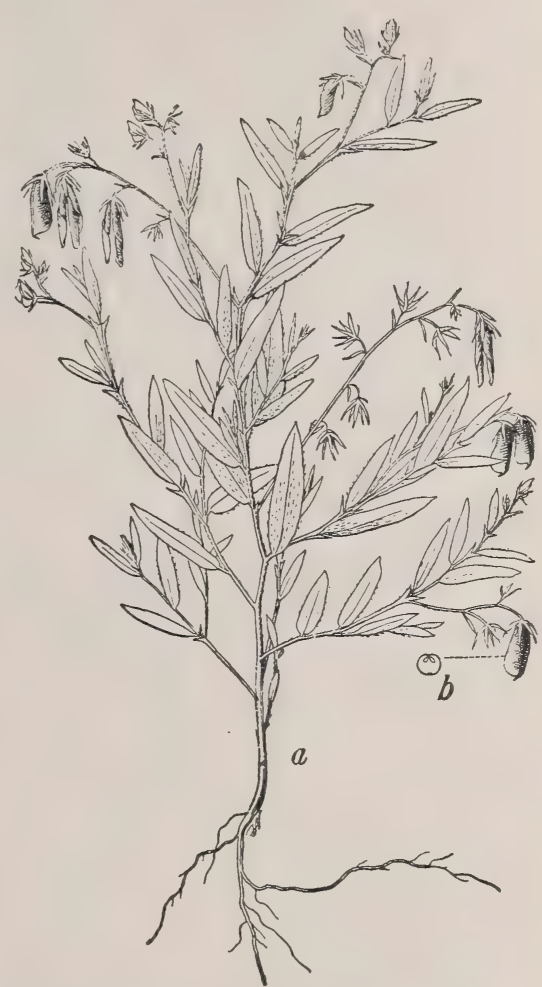

FIg. 93.-Rattle box (Crotalaria sagittalis). $a$, Whole plant; $b$, cross-section of seed pod-both one-third natural size. occur in the flat river bottoms. They occur most abundantly on the foothills and mountain ranges at moderate elevations.

During the season of 1900 the lupines in Montana began to bloom about May 20th, and the first full pods were collected on June 5th. Lupines are not very extensively eaten by sheep during the spring and summer, except when they are unusually hungry or are being driven from one range to another. Lupines are more often eaten by sheep in summer on the mountain sides, and in the fall and early winter after 
early frosts have opened the pods and the seeds have fallen out. Lupine hay is greedily eaten by all kinds of stock during the winter, and large quantities of this hay have been fed for the past fifteen or twenty years. Lupine hay is cut in different years at dates ranging from the 1 st of July to the middle of September. When cut during the first half of July the newly ripe pods, full of seeds, are secured in the hay. When, how. ever, the harvesting of lupine hay is postponed until September, the pods become ripe and split open, and the majority of seeds fall out. A striking variation in the quantity of pods containing seeds is noted during different years. During seasons in which May and June are wet the quantity of pods is usually large. When, however, these months are dry only a few pods are found on each plant, and a vast majority of the flowers fail to be fertilised.

Dr. Wilcox has observed that sheep are especially fond of the pods of various leguminous plants before they become mature and while they are still in a succulent condition.

Dr. Wilcox saw a flock of sheep which while being driven from one range to another, in a hungry condition, was allowed to feed upon an area of lupines in a nearly ripe condition. Within two hours the sheep manifested violent symptoms of poisoning, and ultimately 100 out of the lot of 200 died. He afterwards saw many hundreds of fatal cases in sheep and a number in horses, both from eating green lupines and lupine hay.

As an experiment two sheep were given each 150 medium-sized lupine pods (L. leucopliyllus) which were entirely full of ripe seeds. The sheep ate the pods readily. Both sheep became frenzied within about forty-five minutes after feeding upon the lupine pods, and died about one hour later. The symptoms in these cases were the same as those observed in poisoning under natural conditions.

The symptoms of lupine poisoning are so well known in Europe that chronic lupine poisoning has been given the name lupinosis. It is characterised by loss of appetite, fever, dyspnœea, constipation, and yellowness of the visible mucous membranes. Diarrhœa, sometimes of a sanguinolent type, appears later. The urine becomes albuminous, tinted with bile products or stained red by hæmoglobin, and the head shows odema. Death occurs in a few days. In America the chronic form has not been observed. In cases of lupine poisoning in Montana there was noted acute cerebral congestion, accompanied with mental excitement. The sheep rushed about in different directions, butting one another and other objects. The first stage of frenzy was soon followed by a second stage, characterised by pronounced irregularity of movement, spasms, and falling fits. In the majority of cases death occurred in from one-half to one and one-half hours. In extensive cases of lupine poisoning it was 


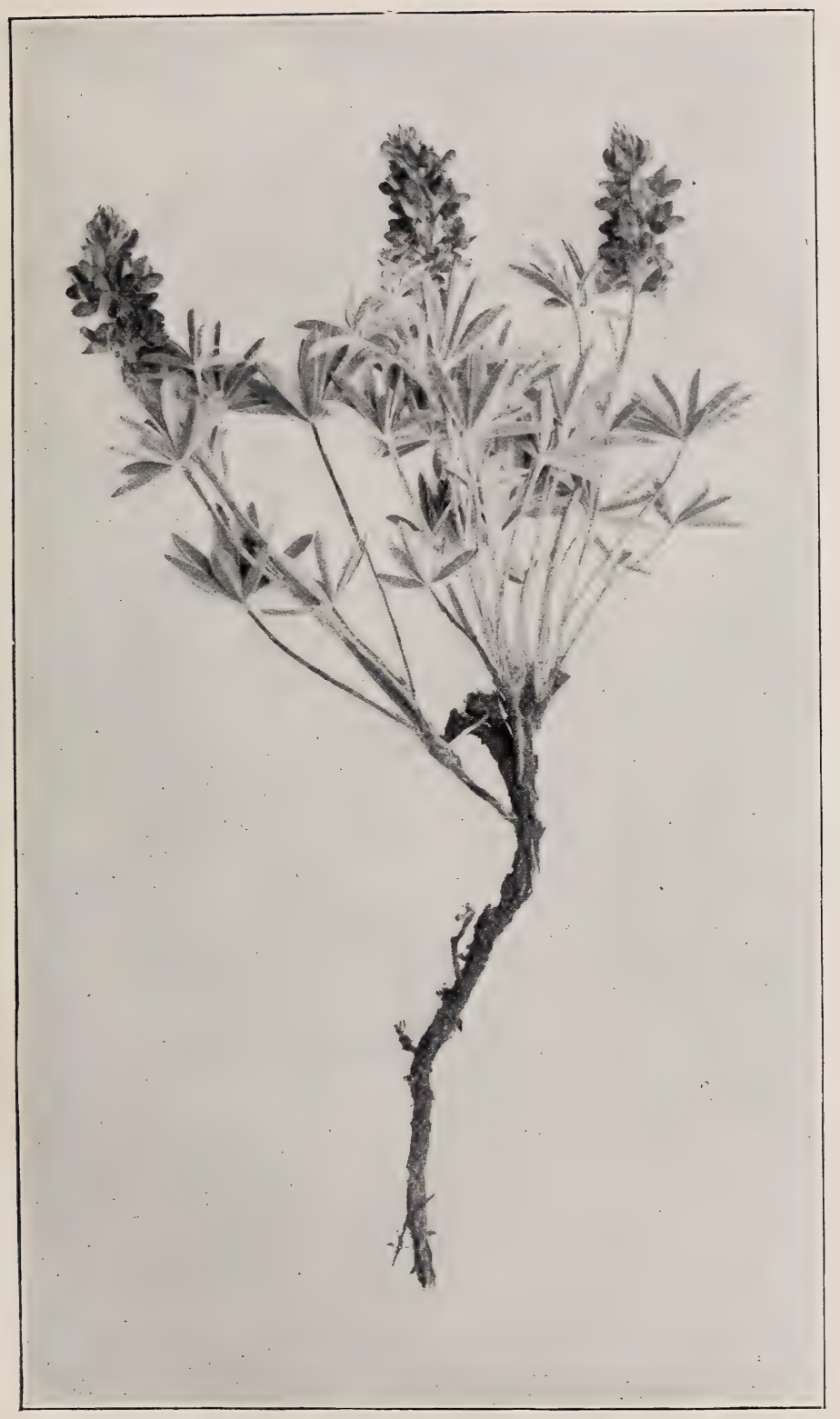

Fig. 94.-Lupine (Lupinus leucophyllus).

(From the Annual Report, U.S.A. Department of Agriculture, 1900.) 



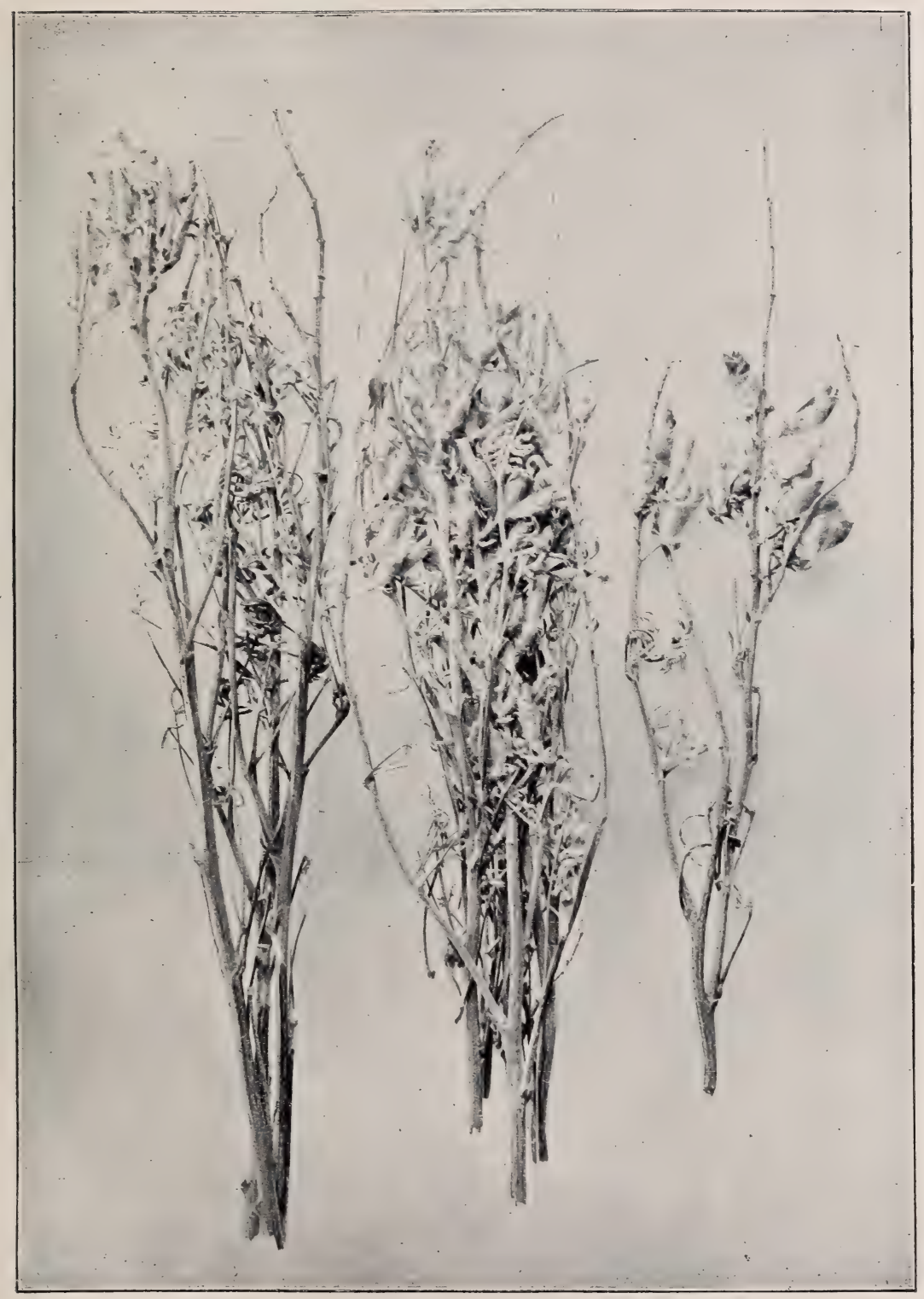

Fig. 95.-Lupine (Lupinus leucophyllus) in hay.

(From the Annual Report, U.S.A. Department of Agriculture, 19c0.) 

uniformly observed that a number of the sheep lingered on from two to four days before they died. The muscular convulsions resembled those caused by strychnine. The excretion of the kidneys was much increased and frequently was bloody. Post-mortem examinations of sheep poisoned by lupines revealed conditions similar to those in acute forms of loco disease, with the addition of a congested condition of the kidneys.

No remedies have been tried in cases of poisoning from American species of lupine, but it seems reasonable to suppose that potassium permanganate would probably destroy the lupine alkaloids in the stomach if administered promptly after the first signs of poisoning. Experience and observation indicate that lupine hay is always dangerous for sheep if cut at a time when the seeds are retained in the hay. The evidence thus far collected regarding this matter indicates that the seeds are the most poisonous part of the plant.

\section{POISONING BY VETCHES (LATHYRUS SATIVUS): LATHYRISM.}

In the horse this disease is due to feeding on grain containing the seeds of vetches, but in the ox to eating the green portions of the plants. Feeding has to be continued for at least a month to produce accidents.

The earliest symptoms consist in suppression of milk secretion, and somnolence. Nervous symptoms-from which alone the horse sufferssoon make their appearance. The neuro-muscular system is attacked. Interference with the nervous system is followed by inco-ordination of movement, and later by paraplegia of the hind quarters. Roaring is not noticeable, probably because the patients rarely move rapidly.

The lesions have been little studied, but appear to consist in congestion and infiltration of the meninges, cord, and roots of the lumbosacral plexus.

Treatment. If the animals are paralysed, treatment is rarely of value; otherwise it is sufficient to remove the cause and to administer purgatives and diuretics, with the object of eliminating toxic products. Recovery follows in three to four weeks.

Robinia pseudacacia.-The common locust tree is native in the central and eastern parts of the United States, and is extensively cultivated for ornamental purposes throughout the Union. The bark and leaves contain a powerful poison, and persons have been killed by eating these parts.

* Sophora secundiflora. - The beautiful bright-red beans of the frijolillo, or coral bean, of southern and western Texas contain a powerfully poisonous alkaloid. The plant is said to have poisoned stock in Texas and in northern Mexico. 


\section{LINACEE (FLAX FAMILY).}

Linum rigidum.-The large-flowered yellow flax is reported from Pecos Valley, Texas, as poisonous to sheep. An investigation made by the Bureau of Animal Industry, U.S.A., showed that the plant is poisonous.

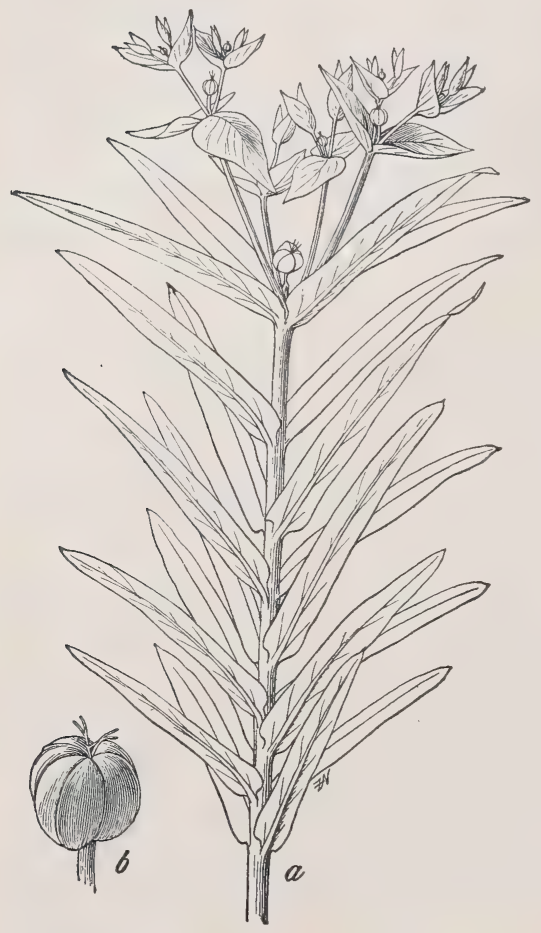

FIG. 96.-Caper spurge (Euphorbia lathyris). a, Upper half of plant, one-third natural size; $b$, seed capsule, natural size.

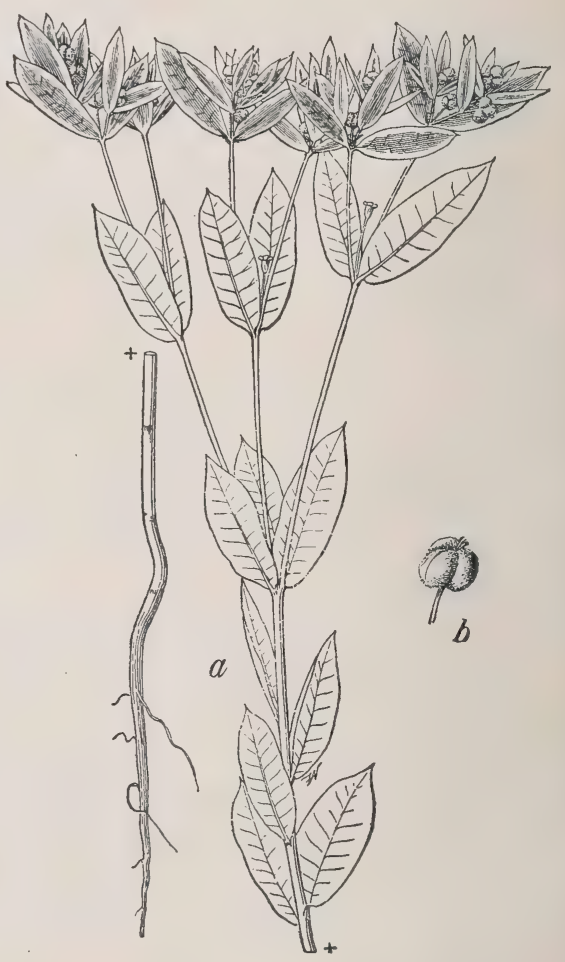

FIG. 97.-Snow on the mountain (Euphorbia marginata). a, Whole plant, one-third natural size; $b$, seed capsule, natural size.

\section{MELIACEE (UMBRELLA-TREE FAMILY).}

* Melia azedarach.-The Chinese umbrella tree is much cultivated for ornament, and sometimes grows wild in the South. A correspondent from Arizona stated that three of his hogs were poisoned by eating the seeds, which were ignorantly offered to them for food.

\section{EUPHORBIACE更 (SPURGE FAMILY).}

Euphorbia.-There are many species of spurge native to the United States, nearly all of which contain an acrid milky juice. Stock generally 
avoid them, but cattle have been poisoned by drinking water into which the plants have been thrown. The juice of E. marginata and E. bicolour is used to some extent in Texas to brand cattle, it being held to be superior to a red-hot iron for that purpose, because screw worms will not infect the fresh scar and the spot heals more readily.

* Jatropha stimulosa.-The seeds of the spurge nettle of the Southern States are extremely poisonous. Stock avoid the plant on account of its stinging hairs.

* Ricinus communis. - The castor oil plant is quite commonly

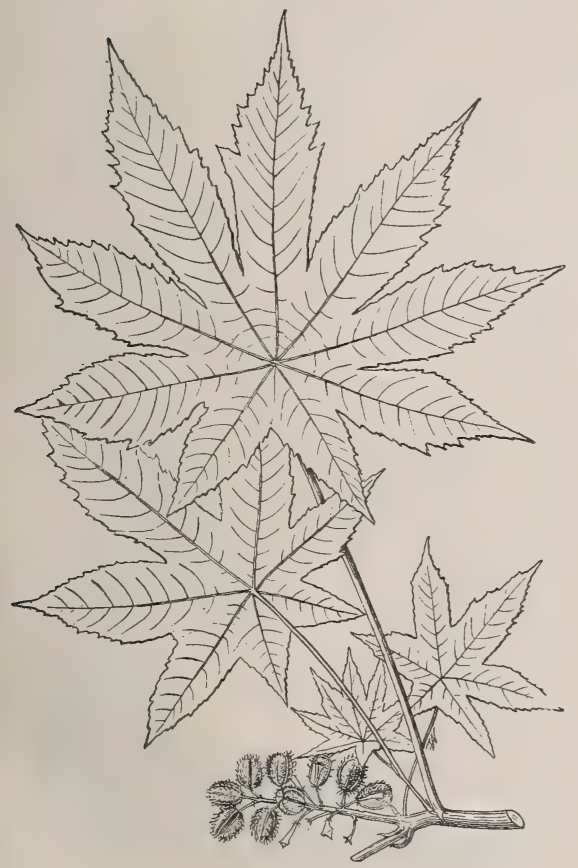

Fig. 98.-Castor oil plant (Ricinus communis).

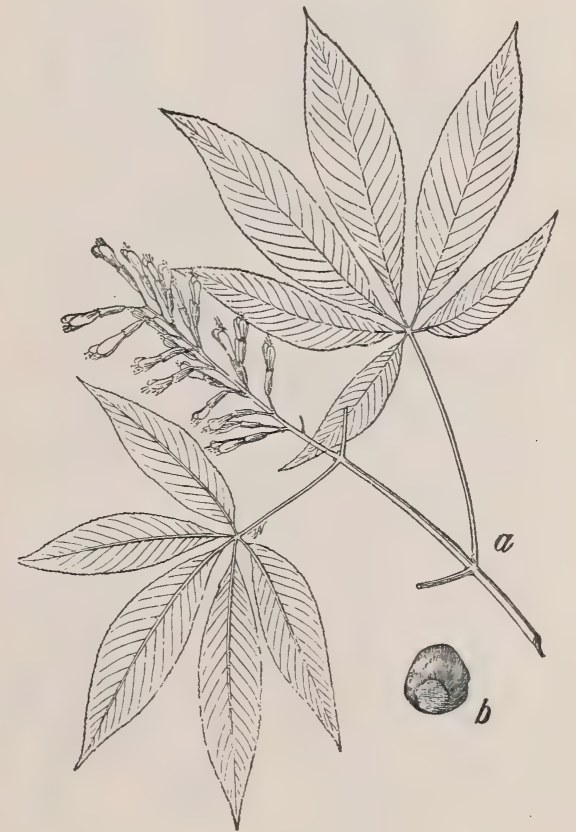

Fig. 99.-Red chestnut (Esculus pavia). $a$, Flowering branch; $b$, seed-both two-ninths natural size.

cultivated in the warmer portions of the United States, and grows wild in the South. The seeds have been accidentally eaten by horses with fatal effect, and they have been strewn on pasture lands in the North-Test for the purpose of killing sheep that were trespassing thereon. A Frenchman has discovered a method of making cattle immune to the effects of the toxalbumin contained in the seeds, so that they may be fed to stock without causing any apparent ill effect. A note on poisoning by castor oil cake will be found hereafter. 
BUXACEA (BOX FAMiLY).

* Buxus sempervirens.-The leaves of the common box, cultivated for hedges, are poisonous to all kinds of stock.

ÆSCULACE (HORSE-CHESTNUT FAMILY).

Esculus californica, California buckeye: ÆE. glabra, Ohio buckeye; fœtid buckeye: AE. hippo-

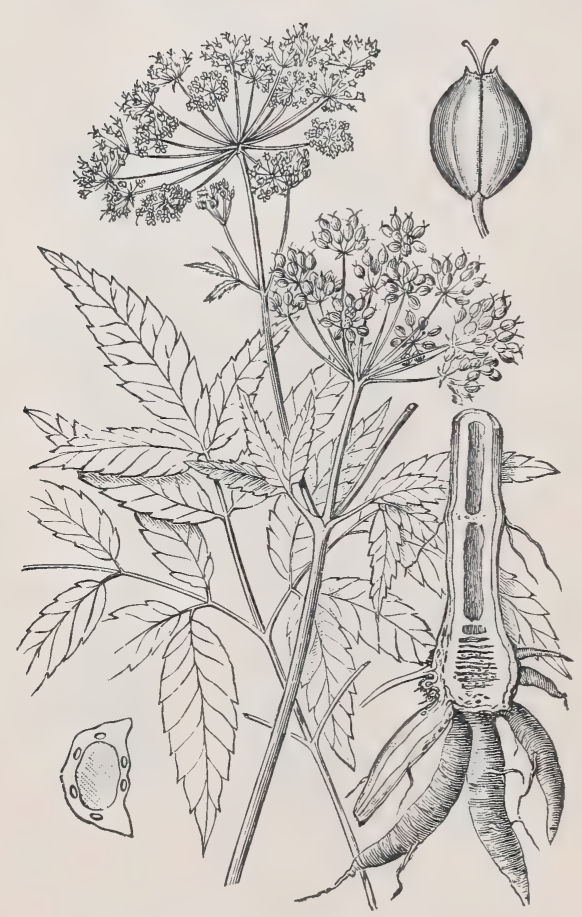

FIG. 100.-Water hemlock (Cicuta maculata), showing section of spindle-shaped roots and lower stem, the leaves, flowers, and fruit, one-half natural size; also fruit and cross-section of seed, enlarged five times.

which contained nearly 50 per cent. of these plants. One horse died from the effects of the poison, and two were killed to prevent their further suffering.

POISONING BY ST. JOHN's WORT.

The ingestion of St. John's wort produces excitement followed by dulness, interference with vision and hearing, and by visual hallucinations 
with a tendency to lean backwards, the front limbs remaining fixed in position. The patient often sits down on the hind quarters like a dog.

\section{APIACEA (CARROT FAMILY).}

* Cicuta maculata. This is the water hemlock (spotted hemlock; beaver poison; cowbane), which grows most abundantly throughout the

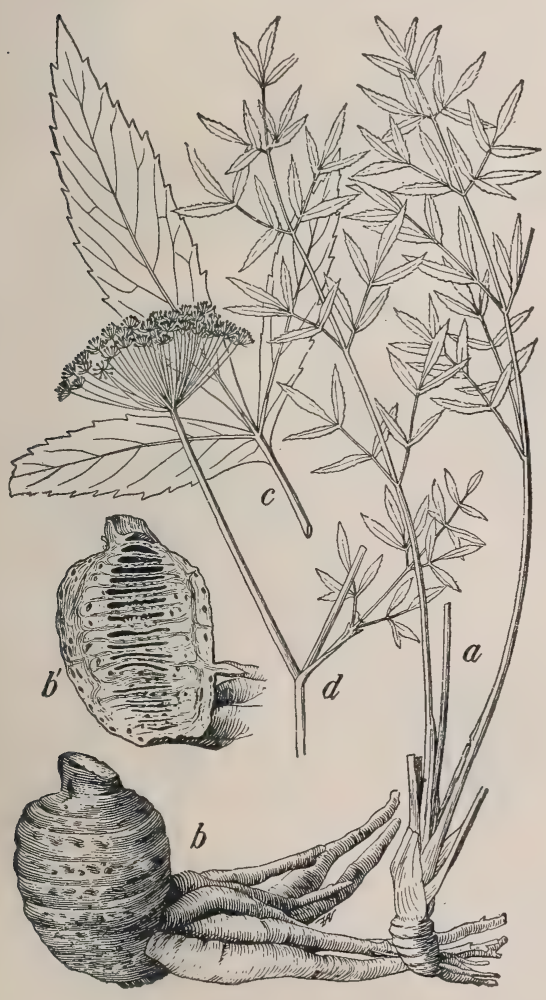

Fig. 101.-Oregon water hemlock (Cicuta vagans). $a$, Plant with leaves, one-sixth natural size; $b$ and $b^{1}$, rootstock and horizontal roots, showing section, halfsize; $c$, terminal leaflets, one-sixth natural size; $d$, flowering spray, full size.

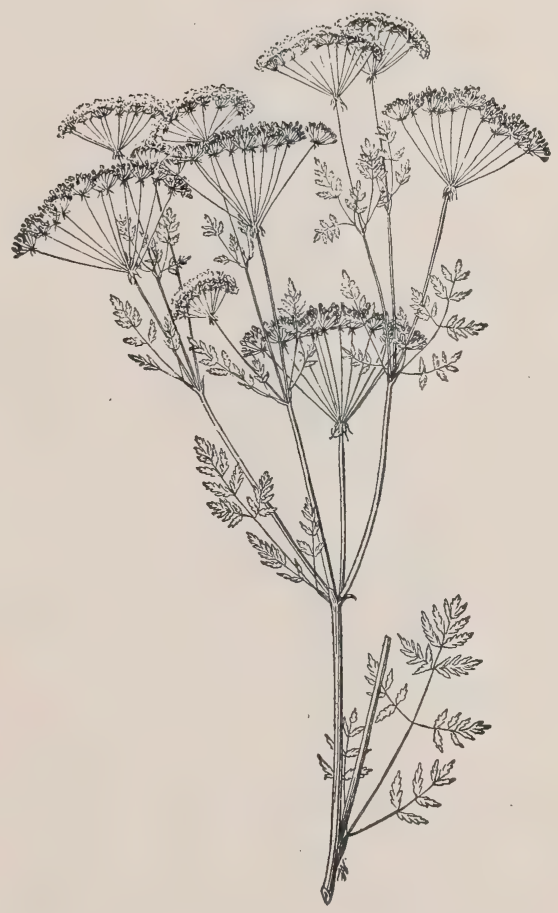

FIG. 102.-Poison hemlock (Conium mac. ulatum), showing upper portion of plant with flowers and seed, one-third natural size.

United States. It is one of the best known poisonous plants. Stock are not infrequently killed by eating the fleshy roots or hay with which the plants are mixed.

* Cicuta vagans. - Cattle are frequently killed in Oregon and Washington by eating the large fleshy rootstocks which have been 
washed, frozen, or dug out of the soil, or by drinking water in marshes where the roots have been trampled upon. The roots of the other species of Cicuta are undoubtedly poisonous, but cases have been reported against one other species only, namely, $C$. bolanderi. It grows in marshy land in California.

* Conium maculatum.-The well-known poison hemlock, or spotted hemlock of Europe, is an introduced weed not uncommon in the northeastern section of the United States and in California. The plant is

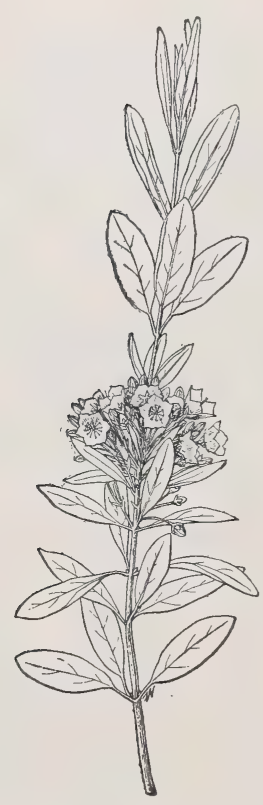

FIG. 103. - Narrow-leaf laurel (Kalmia angustifolia), showing flowering branch, onethird natural size.

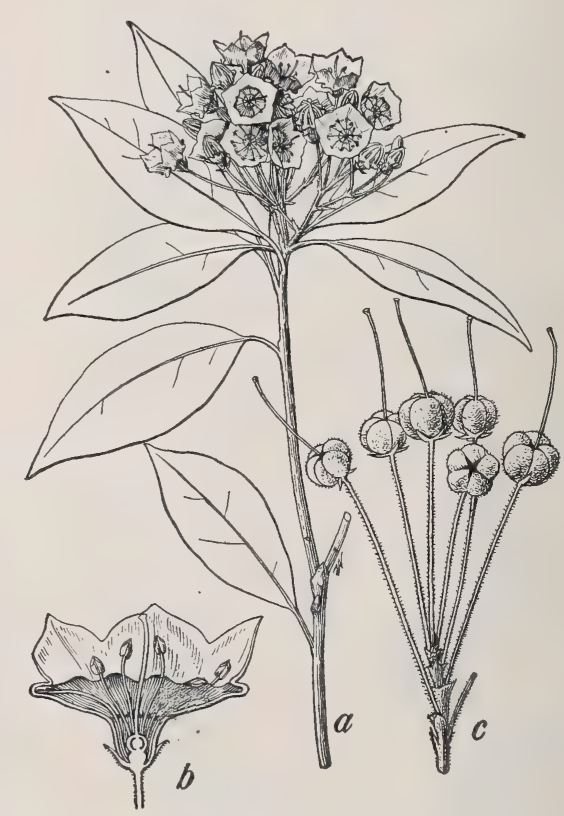

FIG. 104.-Broad-leaf laurel (Kalmia latifolia). $a$, Flowering spray, one-third natural size; $b$, vertical section of flower showing peculiar attachment of stamens, natural size ; $c$, fruiting capsules, natural size.

generally avoided by stock on account of its bad odour, but animals have been killed by eating it in the fresh state. Since the poisonous constituent is volatile, the dry plants are not so dangerous.

FOISONING BY HEMLOCK AND WILD CHERVIL (ANTHRISCUS SYLVESTRIS).

Poisoning only results from ingestion of the green plants. It is characterised by salivation, nausea, dyspnœa, generalised trembling and vertigo, paraplegia, and symptoms of gastro-enteritis. 
This disease, seen in Algeria, and recently studied by Brémond and Bojoly, need only be mentioned. The information at present arailable is indefinite, and the symptoms so closely resemble those of Texas ferer that there seems a possibility of confusion having arisen.

The lesions are those of hæmorrhagic gastro-enteritis.

Treatment consists in giving tannin, opium, and emollients.

\section{Oxypolis rigidus.-The cowbane} is natural in swamps throughout the eastern half of the United States. The leaves and roots are reputed to be poisonous to cattle.

Sium cicutæfolium.-The leaves of the hemlock water parsnip, which is more or less common throughout the United States, are said to be poisonous to stock.

\section{ERICAEA (HEATH FAMILY).}

Andromeda polifolia. - The wild rosemary, or moorwort, is a plant native to the northern regions of Europe, Asia, and America, entering the United States only in the extreme north-east. The leares, which have been eaten by sheep

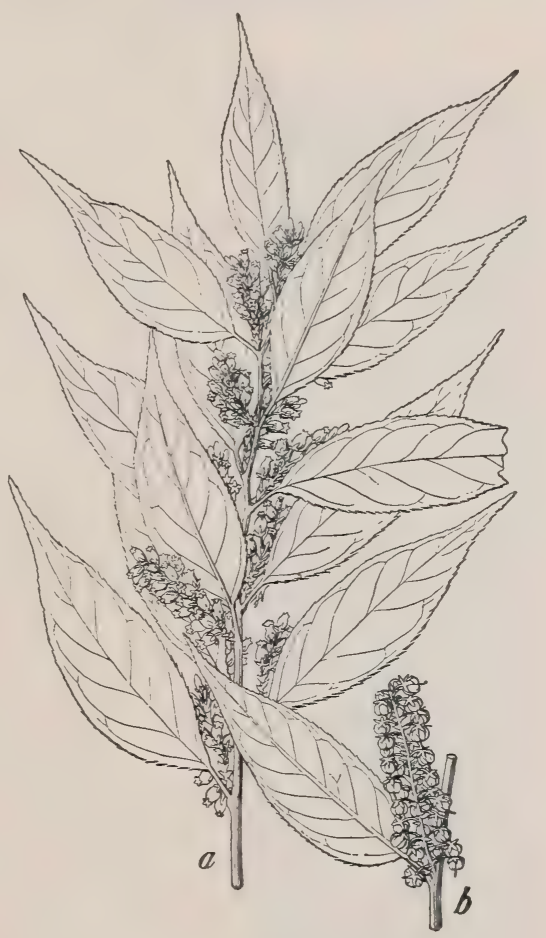

Fig. 105.-Branch iry (Leucothö̈ catesbai). a, Flowering branch; $b$, fruiting capsules. with fatal effect, contain a narcotic poison known as andromedotoxin. The plant is not very dangerous in its native habitat, because it grows in bogs which are inaccessible to stock.

* Azalea occidentalis. - The California azalea is very much dreaded by sheep men who drive their flocks into the southern Sierras for pasture. Investigation has shown that the leaves contain a poisonous substance.

* Kalmia angustifolia.-The narrow-leaf laurel is abundant in the north-eastern section of the United States, where it is also well known as sheep laurel and lamb-kill. The leaves contain andromedotoxin, and sheep and calves are frequently poisoned by eating them.

* Kalmia latifolia.-The broad-leaf laurel is native throughout the greater part of the eastern half of the United States, and is known by 
a great variety of common names, the most important of which are laurel and ivy. The latter name is-most commonly used south of Maryland. Scores of cattle and sheep are poisoned annually by eating the plant. It is probably the most dangerous of all the shrubs belonging to the heath family.

* Leucothoë catesbæi.--This is the branch ivy, hemlock, or calf-kill,

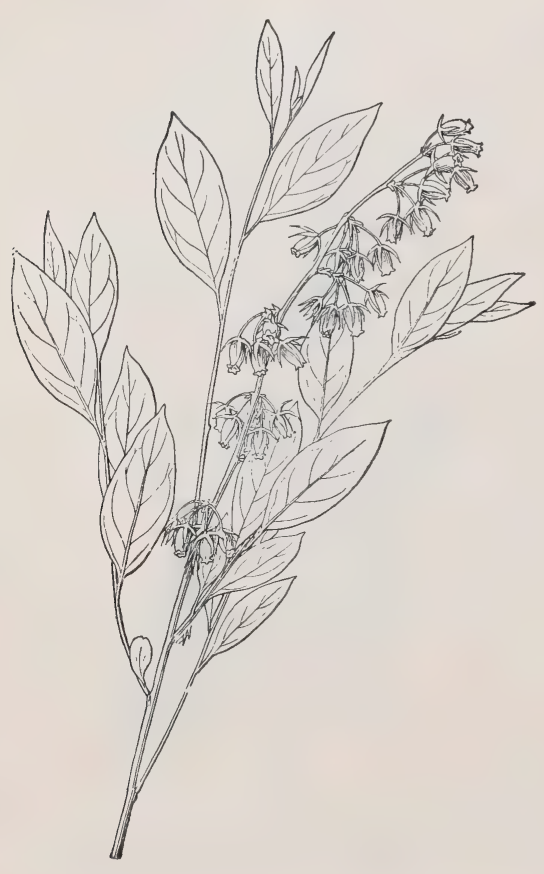

FIG. 106.-Staggerbush (Pieris mariana), showing flowering branch, one-third natural size.

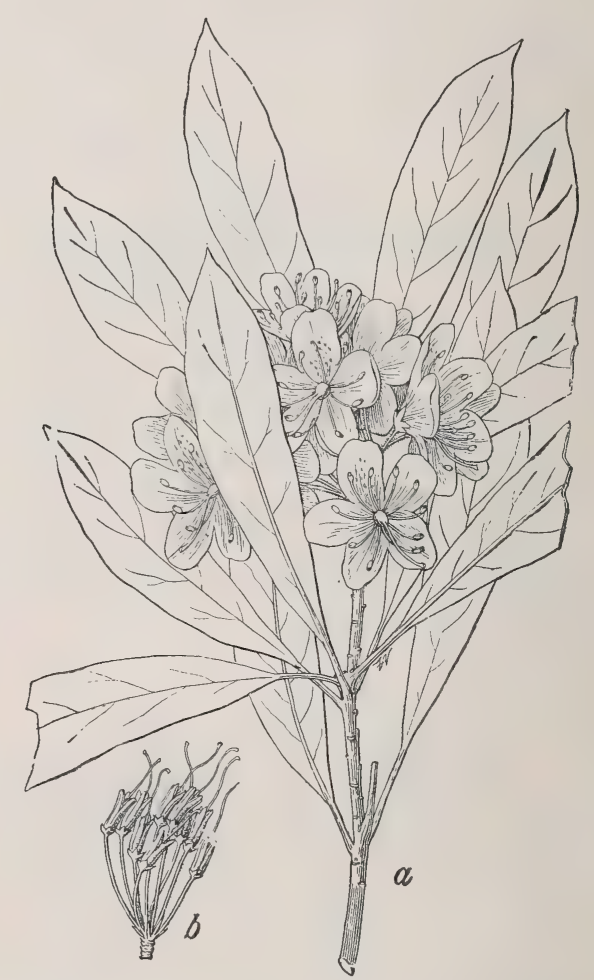

FIG.107.-Great laurel (Rhododendron maximum). $\quad a$, Flowering branch; $b$, fruiting capsules-both one-third natural size.

of the Allegheny Mountains. It is well known in that region to be fatal to all kinds of stock.

* Leucothoe racemosa.-The swamp Leucothö of the Atlantic and Gulf States has been reported from New Jersey as especially fatal to calves.

* Pieris mariana.-The stagger bush of the Atlantic Coast region, Tennessee, and Arkansas is commonly known to be poisonous to calves and to sheep. The name stagger bush was applied to the shrub on account of the peculiar intoxicating effect of the leaves.

* Rhododendron californicum. - The California rhododendron is 
native on the Pacific Slope from San Francisco to British Columbia. The plant is reported from Oregon as poisonous to sheep. It is quite probable that the leaves contain andromedotoxin, but they have not been tested.

* Rhododendron maximum.-The great laurel (rosebay; mountain laurel ; rhododendron) is a large evergreen bush or small tree which is quite commonly cultivated for ornament, and is found native in the Allegheny Mountains. The leaves contain andromedotoxin, and they are occasionally eaten by stock with fatal effect.

\section{PRIMULACE (PRIMROSE FAMILY).}

Anagallis aryensis. - The pimpernel is a European plant which has obtained a specially strong foothold in California, where it grows luxuriantly and is sometimes known as poison weed. It is suspected of having caused the death of a horse at Santa Ana. Chemists have isolated a powerfully poisonous oil and a strongly active ferment from the plant.

\section{OLEACE (OLIVE FAMILY).}

Ligustrum vulgare.-The privet, or prim, is a garden shrub, introduced from Europe and Asia, which is much used for hedges, and has escaped from cultivation in western New York and southward to North Carolina. Accidents have been occasioned in children both by the fruit and the leaves. The plant is to be suspected in cases of poisoning in animals.

\section{APOCYNACEA (DOGBANE FAMILY).}

Apocynum androsæmifolium, spreading dogbane: A. cannabinum, Indian hemp. - These plants are generally distributed throughout the United States. Stock generally avoid them in pasture fields on account of their acrid milky juice. When dry they are not so poisonous as when in the fresh state.

Nerium oleander.-The oleander is a common house plant throughout a large portion of the United States. It grows freely out of doors in the Southern and Western States, and has probably escaped from cultivation in some places. It grows wild in northern Mexico. The leaves 
are well known to be most powerfully poisonous, and stock are occasionally killed by eating them.

\section{ASCLEPIADACEÆ (MILKWEED FAMILY).}

* Asclepias eriocarpa.-This is the plant with broad mullein-like leaves which is known as milkweed in California. Several authentic accounts of the poisoning of

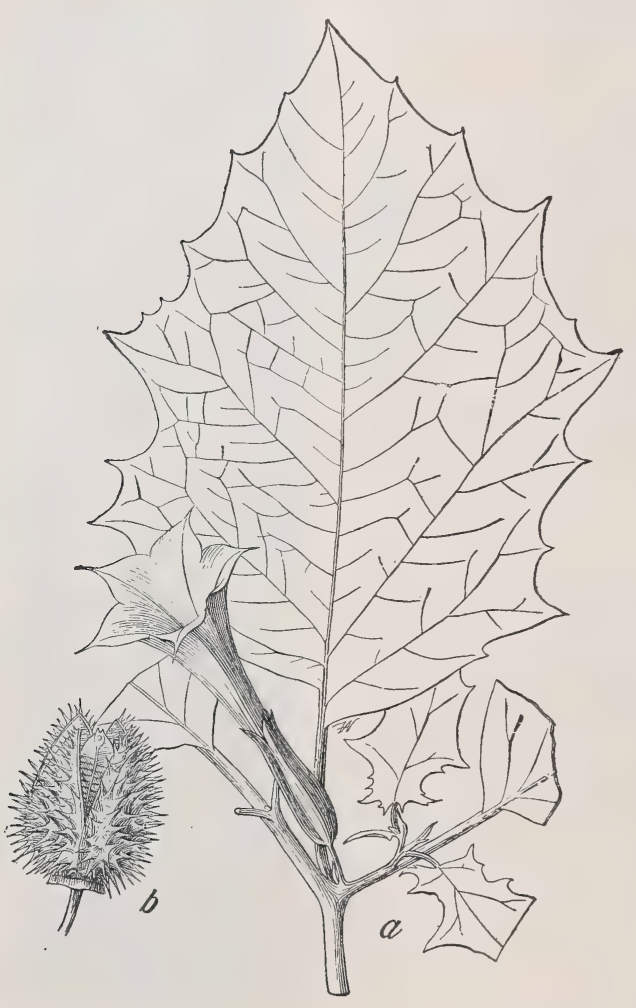

FIG. 109.-Jimson weed (Datura stramonium). $a$, Flowering spray ; $b$, fruiting capsule - both one-third natural size. weed. They are European plants which have become weeds in waste grounds and about dwellings throughout the greater portion of the country. One or two instances are recorded in which cattle have been poisoned by eating hay containing the young leaves.

* Hyoscyamus niger.-The black henbane is an ill-smelling plant, a native of Europe, now naturalised in Michigan, and from New York northward. One or two cases are recorded in European literature in which stock 
have been poisoned by eating the plant of their own accord, but there is very little danger from it, on account of its ill odour and harsh texture.

* Nicotiana tabacum.-This is the tobacco most commonly cultivated in the United States. It is native to South America and has escaped from cultivation to some extent in the Southern States. According to some authorities stock are not always disposed to shun this plant on account of its characteristic ill odour and taste, but, on the contrary,

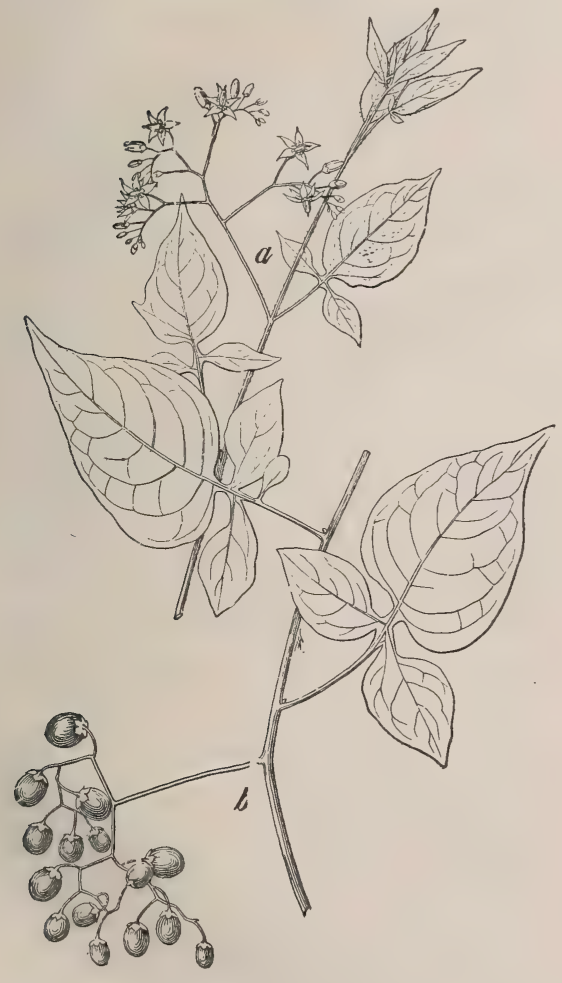

FIG. 110.-Bittersweet (Solanum dulcamara). $a$, Flowering spray; $b$, fruitboth one-third natural size.

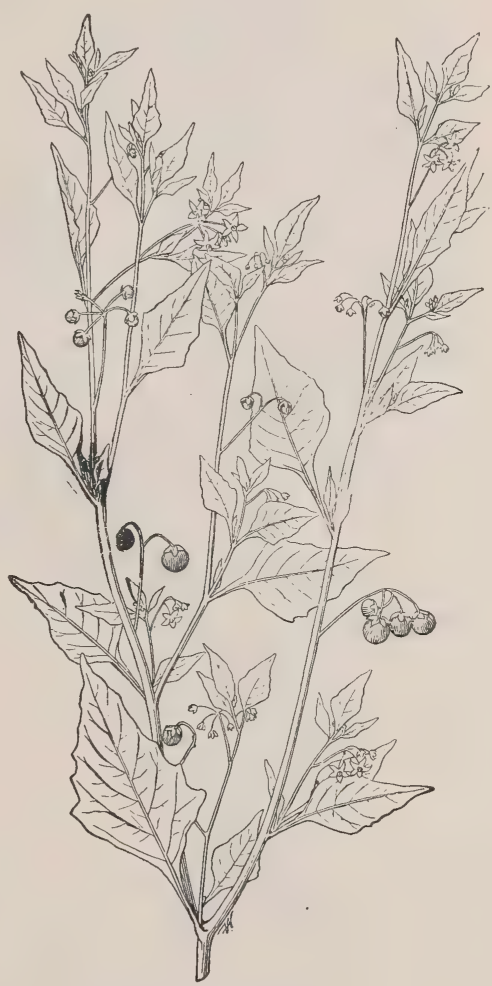

FIG. 111.-Black nightshade (Solanum nigrum), one - third natural size.

will eat a small amount of the leares with apparent relish, especially when they are somewhat fresh. Stock have, however, been poisoned by eating leaves which were placed within their reach to dry, and also by eating food contaminated with the juice of the leaves. Considerable precaution should be used in applying tobacco juice to fresh cuts or bruises in stock, as the poison is easily absorbed into the system and may prove fatal. There are several native species of tobacco in the 
western half of the United States, all of which are undoubtedly poisonous if eaten even in moderate quantity.

TOBACCO POISONING.

Tobacco poisoning may be produced by baths or lotions containing tobaceo juice, which is often used as a parasiticide. The ingestion of tobacco leaves in forage may also

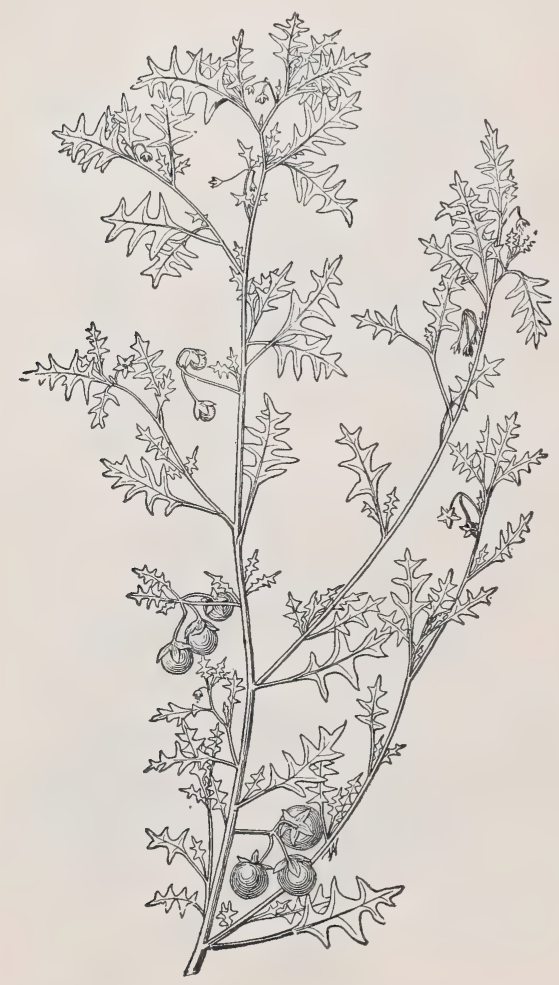

FIG. 112.--Spreading nightshade (Solanum triflorum), one-third natural size. produce poisoning. Doses of 1 ounce in the goat and 10 ounces in the ox are toxic.

The symptoms consist in salivation, vomiting, nausea, diarrhœa, cardiac palpitation and dyspnœa.

The lesions are those of gastroenteritis with cerebral congestion.

Treatment consists in giving tannin, black coffee, etc.

Solanum dulcamara.-The bittersweet, or climbing nightshade, is a European weed, now introduced in the north-eastern quarter of the United States. The leaves are suspected of being poisonous to stock.

* Solanum nigrum.-The black nightshade (common nightshade; garden nightshade) is a common weed in cultivated fields throughout the greater portion of the United States. Cattle seldom eat the plant, but a few cases of poisoning are recorded for calves, sheep, goats, and swine.

* Solanum triflorum.-'The spreading nightshade is a native of the Great Plains (United States), and also a common garden weed from Arizona and T'exas to British America. Complaints of the poisoning of cattle by this plant have been sent to the Department of Agriculture from Nebraska. Experiments show that the berries are poisonous.

Solanum tuberosum.-The small, immature tubers of the common cultivated potato and those that have turned green from exposure to the sun are slightly poisonous. The green fruit and the white sprouts 
from mature potatoes are likewise poisonous. In all of these cases the deleterious substance may be removed or destroyed by thorough boiling.

\section{SCROPHULARIACEE（FIGWORT FAMILY),}

Digitalis purpurea.-The purple foxglove is a common garden plant which has sparingly escaped from cultivation and is naturalised to some extent on Cape Breton Island. Horses are occasionally poisoned in Europe by nipping the plants from gardens or by eating hay contaminated with it.

Gerardia tenuifolia.-The slender gerardia is native to the eastern half of the United States, and has been specially reported as poisonous to sheep and to calves in the Southern States.

Gratiola officinalis.-The hedge hyssop of the Southern States contains an acrid poison. The same plant grows in Europe, and is there regarded as poisonous to stock.

Pedicularis.-The plants of this genus are commonly called lousewort. In Europe several species are suspected of being slightly poisonous to stock. One of these, $P$. palustris, occurs in Labrador, and there are over thirty species

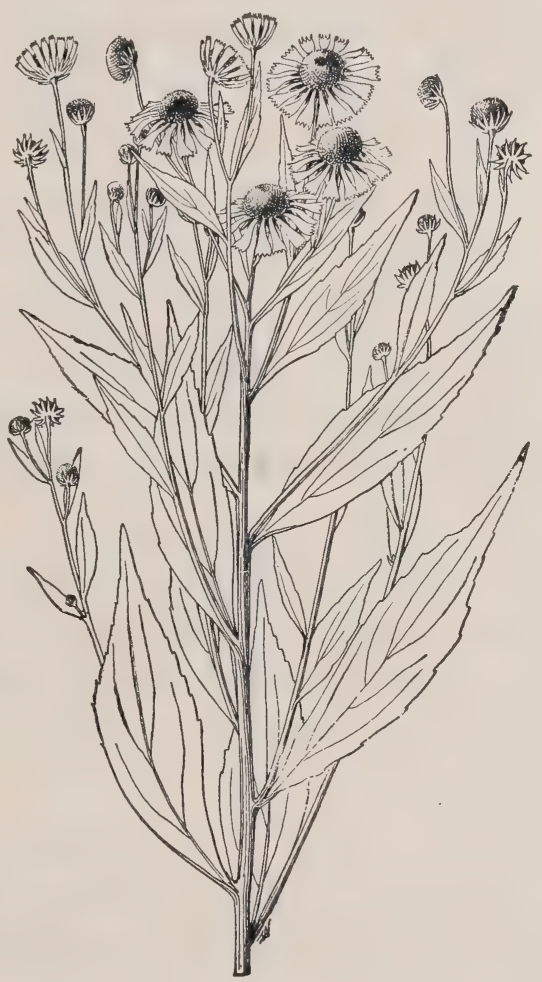

Fig. 113.-Sneezeweed (Helenium autumnale), one-third natural size. native to the United States, largely Western. They should all be suspected of being poisonous.

\section{CAMPANULACE (BELL-FLOWER FAMILY).}

Lobelia inflata, Indian tobacco: L. kalmii, brook lobelia: L. spicata, pale-spiked lobelia: L. syphilitica, great lobelia.-All of the species in this genus contain an acrid and usually milky juice, and are poisonous. None has been specially reported as poisonous to stock, but the abovenamed species are to be suspected, because they frequently occur in grass and are sometimes found in meadow hay. 
AMbrosiaceE (RAGWEED FAMILY).

Xanthium canadense.-The young seedlings of the American cocklebur are reported from Texas as being rapidly fatal to hogs.

Xanthium spinosum.- The spiny clotlur is suspected of being poisonous, but few cases have been definitely recorded against it. 'The seeds apparently contain a toxic compound.

Xanthium strumarium.-The young seedlings of the broad cocklebur are reported from Georgia as being fatal to hogs. Experiments seem to show that the seed is poisonous.

\section{CARDUACEE (THISTLE FAMILY).}

*Helenium autumnale.-Sneezeweed (sneezewort; autumn sneezeweed; stagger weed; false sunflower) is found throughout the greater portion of the United States, being most abundant in the Southern and Eastern States. Sheep, cattle and horses that are unfamiliar with the plant are often poisoned by it when driven to localities where it is abundant. Stock avoid it, as a rule, but it is claimed that they sometimes develop a taste for the plant, and are killed quickly by eating it in large quantity.

Senecio jacobæa.-The tansy ragwort, or stagger wort, is a European plant which grows as a weed in ballast about New York and Philadelphia. Farther north, in Nova Scotia, it has become extensively naturalised, and it is there regarded by stock men as poisonous. It is interesting to note that $S$. guadalensis of Mexico is also considered fatal to stock.

\section{COLCHICUM POISONING.}

Fodder of bad quality often contains leaves, flowers, and particularly seeds of colchicum, which produce nausea, vomiting, colic and diarrhœa. The colchicine acts particularly on the kidney and heart, producing specific disturbance, indicated by hæmaturia, polyuria, and cardiac palpitation, with lowering of the body temperature.

Even when poisoning is not fatal, it is very apt to produce abortion.

POISONING BY ANNUAL MERCURY.

Annual mercury given in green fodder is stated to produce indigestion, diarrhoa, vesical and intestinal hæmorrhage, and early death. Some authors, however, deny that it has such toxic properties.

POISONING BY BRYONY.

In large doses all parts of the bryony plant are toxic - the root, stalk, and leaves. 
Bryony is sometimes used as a purgative. Poisoning is characterised by nausea, sweating, diuresis, frequent action of the bowels, and, in grave cases, by tetaniform convulsions followed by death.

\section{POISONING BY CASTOR OIL CAKE.}

Causation. Excessive use of this form of cake is the usual cause of such poisoning, though bad quality is also an important factor. The castor oil beans are often insufficiently crushed and compressed, so that a considerable amount of oil is contained in the cakes as sold; but the most dangerous constituent is undoubtedly the material known as ricin, which, in some specimens of cake, may exist in highly dangerous quantity.

The oil contained in the cake, like every other fatty substance, favours intestinal peristalsis and the onward movement of the digested food. The laxative principle excites secretion, and if the cake be given for considerable periods, the most serious consequences may ensue.

Cakes prepared from mixed rape seed and castor beans act in a similar way, though in a longer or shorter time, according to their richness in ricin.

The earliest symptom consists in purgation, which gradually develops into superpurgation, and is followed by direct irritation of the muscous membrane, indicated by serous, fœetid, and sometimes sanguinolent, diarrhœa. The symptoms may appear in twenty-four hours. They are usually accompanied by a rise in temperature of $2^{\circ}$ to $3^{\circ}$ Fahr. Secretion of milk ceases, and animals heavy with young sometimes abort. In exceptional cases death follows.

The lesions are those of hæmorrhagic enteritis.

Treatment is principally of a preventive character. The cakes should be examined, and if they contain insufficiently crushed seeds or beans should be discontinued or given in smaller quantities. The proportion of ricin in mixed rape and castor cakes should be determined.

Curative treatment consists in removing the cause and treating the enteritis. The latter is best controlled by giving emollients, diuretics, and mucilaginous drinks prepared from linseed, marsh-mallow, barley, etc.

\section{POISONING BY COTTON CAKE.}

Cotton cake forms a rich food, which fattens animals very rapidly, but given in excess may produce true poisoning, and if prepared from undecorticated seed may produce mechanical irritation ending in obstruction of the bowel.

The latter accident occurs only in the sheep. It consists in obstruction of the omasum (œsophageal gutter), and particularly of the D.C. 
abomasum, by the woody seed covering, the fibres of which become agglutinated and close the pyloric opening, just as do the fragments of wool or the hairs in animals affected with the licking habit (pica, depraved appetite). The mass thus formed passes into the intestine, and is apt to become fixed at some point and to cause death.

In the ox, as in the sheep, true poisoning may result from the action of an injurious principle which Cornevin discovered in the seed and particularly in the meal. The relative rarity of such accidents is explained by the composition of the cakes, which are rich in husks but poor in meal.

In the first series of accidents the symptoms resemble those produced by the intestinal obstructions peculiar to the licking disease; in the second they appear about the eighth to the fifteenth day, and are indicated by sensitiveness of the abdomen and by efforts to pass urine. The urine is albuminous; at a later stage it becomes darker in colour, reddish, and stained with hæmoglobin. The mucous membranes exhibit a sub-icteric tint.

Lesions. The liver shows interstitial hepatitis, consequent on changes in the hepatic cells due to the poisonous principle. The kidney first shows lesions of interstitial, but afterwards of epithelial, nephritis; the endothelium of the tubes appears to be undergoing proliferation.

Treatment should only be undertaken when the organic lesions seem trifling, and suggest the possibility of cure without excessive outlay. Under such circumstances it is sufficient to remove the cause and to supply proper diet.

\section{POISONING BY MOLASSES REFUSE.}

Molasses refuse is much used about Paris and in the department of Le Nord for fattening or simply for feeding animals. Added to rough fodder, even of poor quality, the refuse renders it palatable, and thus forms an economic food; it also improves the condition of animals with broken wind. Its poverty in nitrogenous materials (the refuse consists of 60 per cent. of hydrocarbons; 10 to 12 per cent. of potash and soda salts) renders it necessary to enrich it in this respect. Moreover, only a limited quantity should be given. If given in larger amounts than 2 to $2 \frac{1}{2}$ parts per 500 parts of body weight it may produce bad effects. In this case the earlier symptoms point to interference with the urinary apparatus, the digestive apparatus being affected later; both accidents are due to the potash and soda salts present in the refuse, and may become so well marked as to constitute true poisoning.

The symptoms consist in abundant diuresis, resulting from the excess of potash and soda salts, and are followed by albuminuria. Superpurgation is usually present. 
Lesions. On post-mortem examination one finds lesions of irritant gastro-enteritis, and of chronic nephritis.

Treatment consists in withdrawing the molasses refuse, and giving milk, mucilaginous fluids, barley water, and cereals, which soothe the kidney.

\section{DISEASES PRODUCED BY DISTILLERY AND SUGAR FACTORY PULP.}

This disease, which is very common in France and Germany, results from feeding on distillery and sugar factory residues, consisting for the most part of beet pulp.

In 1860 Guionnet described it under the name of disease of the abomasum, and more recent work by Butel, Rossignol, and Arloing has thrown a great deal of light on its exact nature.

Causation. Guionnet attributed the injurious action of beet pulp, etc., to excess of acidity, due to the addition of sulphuric acid during manipulation in the factory; but it has since been shown that this acidity, if existing, is specially due to various fermentation products, the results of lactic, butyric, and acetic fermentation, etc.

Rossignol regarded the symptoms as wholly due to the excessive proportion of water, viz., 90 per cent.; but this does not explain the general symptoms of poisoning.

The real cause is to be sought in the manner of preserving the pulp in simple earth silos or in cemented silos, where it undergoes fermentation and putrefaction. 'The contained liquid is then extremely toxic. Filtered through porcelain and injected under the skin, it produces vaso-motor and vaso-paralytic disorder, identical with that seen in acute forms of the disease; in other cases it excites abnormal secretion, and leads to permanent diarrhøea and chronic gastro-enteritis.

This liquid, if injected intravenously, may prove toxic in doses of 2 to 3 cubic centimètres per kilogram of bodily weight. Its injurious effect is due to toxins secreted by special bacilli, which were isolated and studied by Arloing. The toxicity diminishes as the pulp becomes older, and can be avoided by adding antiseptics like common salt, which prevent fermentation. These experiments of the Lyons professor are certainly very interesting, and, although perhaps not identical with what occurs in practice, sufficiently indicate the way in which poisoning occurs.

Pathological disturbance only follows the use of decomposed pulp.

Animals reared on farms where distillery and sugar factory pulp is regularly given become accustomed to it, and are rarely affected. The chief sufferers are those recently imported, or recently placed on such food; in them the disease may assume either the acute, nervous, subacute, or chronic form. 
Acute form: Symptoms. This form is exceptional in the ox, but is more frequent in the sheep. In oxen the earlier symptoms point to digestive disturbance, and consist of dulness, loss of appetite, colic, sensibility of the abdomen, cessation of rumination (without tympanites), and constipation. The excreta are hard, coated, and blackish in colour, but not blood-stained.

Diarrhœa follows, is accompanied by aggravation of the general symptoms, the temperature rises to $104^{\circ}$ or $106^{\circ} \mathrm{Fahr}$. ( $40^{\circ}$ or $41^{\circ} \mathrm{C}$.), and exhaustion is pronounced. Other, less characteristic, symptoms, such as grinding of the teeth and mastication without food being present in the mouth may accompany the above and arouse fears of peritonitis. In sheep the dulness and prostration shown at first suggest the existence of anthrax - a view strengthened by the fact that the respiration becomes very frequent and the fever intense, whilst death may be rapid, and may sometimes occur with startling suddenness.

Lesions. Bacteriological examination, or even a naked-eye examination, made immediately after death enables one easily to differentiate between the two conditions. When the animals have died very rapidlyin one night-lesions of enteritis alone are present. More marked cases exhibit thickening and intense congestion of the mucous membrane of the abomasum, which may be of a deep mahogany colour.

The intestine itself is affected, and even though the glandular epithelium is little changed, the intercellular spaces show ecchymoses and multiple hæmorrhages, which give the contents of the digestive tract the appearance of wine lees.

The abdominal viscera scarcely present any characteristic lesions. The liver has the appearance of having been boiled, as in many forms of poisoning. The kidney is congested and blackish; the spleen only appears hypertrophied when post-mortem examination has been delayed and micro-organisms from the intestine have invaded the circulatory system. After death the kidneys and spleen very rapidly undergo softening.

Neryous form: Symptoms. Whilst in the first form the symptoms appear especially due to diastatic ferments present in the pulp liquid, in the nervous form they appear rather to result from the convulsing and paralysing action of ptomaines.

The ox seems more particularly susceptible to the action of the latter. It shows symptoms resembling those of horses suffering from a severe abdominal form of influenza: profound depression, mahogany-coloured conjunctiva, lachrymation, infiltration of the cornea, high temperature ( $104^{\circ}$ to $106^{\circ} \mathrm{Fahr} .-41^{\circ}$ to $41.5^{\circ} \mathrm{C}$.), strong action of the heart, but small pulse. The cerebral symptoms are especially marked. The animal suffers from vertigo, and when excited, or when attempts are made to administer medicine, it thrusts its head against the wall, as though 
suffering from a cerebral tumour. It also shows hyperæsthesia, slight colic, and sensitiveness of the abdomen.

In sheep the symptoms consist in alternate extreme depression and extreme excitability.

In both species the termination is alrays rapid: death occurs in a few days.

The abdominal lesions are identical with those of the acute form. They consist in gastro-enteritis, or, rather, intense congestion of the abomasum and intestine, with extravasation of blood around the acini of the glands and beneath the mucous membrane, etc., and in more or less marked desquamation of epithelium.

The annexed organs sometimes present secondary changes: in the nervous centres the lesions are more marked; the meninges are congested, in some cases inflamed, and cerebro-spinal fluid is present in increased quantity.

Subacute or Chronic form: Symptoms. This form is equally frequent in the ox and sheep; it develops insidiously, and for a time may escape detection.

The symptoms are those of slight gastro-enteritis without tympanites; but this condition is succeeded by serous, fœetid, uncontrollable diarrhœa, which weakens the animal and causes death from excessive wasting and hydræmia.

The sensitiveness of the entire right side of the abdomen, the special diarrhœa, the cardiac disturbance, and the widely-distributed œedema, usually suffice to prevent confusion with the ordinary forms of gastroenteritis. In sheep the diarrhœa is blackish, sometimes blood-stained, and is accompanied by a sub-icteric or icteric tint of the mucous membranes, of the skin, and of all the tissues. The intensity of coloration affords a guide to the rapidity of development of the disease. The urine is also bile-stained, and there appears to be an exchange of functions between the two great depurative organs, the liver and kidney. The urine may become sanguinolent, because it contains either unchanged blood or simply dissolved hæmoglobin.

The lesions are similar to those previously described, but with modifications of intensity. When diarrhœa has been marked and persistent, the digestive mucous membrane is hardened, indurated, and appears as though tanned. This is due to chronic inflammation, probably to the gastro-enteritis with which the condition begins. The liver appears as if cooked; the fat, the majority of the tissues, and especially the conjunctiva, exhibit a light yellow tint, pointing to hepatic disturbance.

The diagnosis is generally easy in all three forms, provided that the food be examined, 
The prognosis varies; the acute and nervous forms are usually fatal. But when the disease develops slowly, recovery may occur.

Pathogeny. Practical observation and laboratory researches show that the above conditions result from poisoning. Histological examination of livers from sheep which have died rapidly shows complete degeneration of the hepatic cells, which become incapable of performing their function. 'I'he biliary acids, no longer being withdrawn from the circulating blood, produce general intoxication, destruction of the blood corpuscles, and the appearance of hæmaphæic icterus and hæmoglobinuria.

Treatment. Being convinced that acidity alone caused this disease, the older practitioners suggested the administration of salines. In reality it is necessary to check fermentation. Drainage of the silos in which beet pulp is stored is often sufficient for this purpose; but if badly arranged, drainage rather assists the growth of moulds and various organisms in the mass, which affords a medium favourable to their multiplication. Complete desiccation would undoubtedly give much better results, but cannot economically be effected. The best practical measure consists in storing the pulp in special silos, divided into compartments by lattice work partitions. The escape of liquid is facilitated by forming the floors of the silo with a sufficient slope. Preservation, however, is not perfect, and some compartments are always found damaged. Excessive fermentation can be checked by adding ordinary salt to the pulp in the proportion of $\cdot 2$ per cent.

Curative treatment comprises restricted diet for several days, and the administration of milk, carbonate of soda, and stimulants, which favour excretion of the toxins. Some patients may be saved by subcutaneous injections of large quantities of normal salt solution. When poisoning is pronounced, and the viscera are clearly injured, it is more economical to slaughter the animals, provided that the icterus does not render the flesh useless for sale. 


\section{CHAPTER VIII.}

\section{PARASITES OF THE DIGESTIVE APPARATUS.}

Parasites of the digestive apparatus are extremely common in ruminants, some, like the infusoria of the rumen, being of no importance, others, on the contrary, appearing to play a predominating part in the development of certain forms of anæmia and serious wasting diseases, such as the various forms of gastro-intestinal strongylosis, coccidial enteritis, etc.

\section{GASTRO-INTESTINAL STRONGYLOSIS IN SHEEP.}

PARASITIC GASTRO-ENTERITIS. OVINE PASTEURELLOSIS.

Verminous diseases of the abomasum are common in the sheep and goat, but (in France at least) appear rare in the ox.

Causation. Gastro-intestinal strongylosis is produced by various parasites of the genus Strongylus, such as Strongylus contortus, Strongylus convolutus, Strongylus instabilis, Strongylus circumcinctus, Strongylus filicolis, which occur not only in the abomasum, but also in the small portions of the first intestine, sometimes in very large numbers.

The first is a threadlike worm, pointed at both extremities, and from $\frac{1}{2}$ to 1 inch in length. It exhibits a red tint, which zoologists refer to the presence of blood in its digestive apparatus, derived from the host's intestinal mucous membrane. Some doubt has been thrown on the nature of this coloration, however; and certain Italian authors, in common with Lignières, have declared it due to a certain red pigment developed in the tissues of the parasite, the spectroscopic reaction of which differs from that of hæmoglobin.

The strongyles are said not to penetrate the mucous membrane, but simply live on the intestinal contents. This may be true of certain varieties, but it is none the less certain that others penetrate the mucous membrane deeply, even to the extent of becoming half-embedded in it. Particularly is this the case with Strongylus circumcinctus, found in the epizooty which occurred at Grignon, and also found by Moussu in grave epizooties which he has from time to time investigated.

According to Lignières this gastro-intestinal strongylosis only plays a 
trifling part in the development of the above-mentioned disease, which he declares to be due to infection with a cocco-bacillus of the Pasteurella group, the development of which alone, he states, explains all the symptoms.

In France the disease now under consideration has never extended beyond limited regions, but in Argentina, where Lignières carried out his investigations, it is said to be very fatal. Lignières bases his opinion on the following facts:-

Firstly, that experimental verminous infection of young animals does not produce either anæmia or cachexia.

Secondly, that animals in excellent condition may prove to be infested with large numbers of parasites.

Thirdly, that animals which have succumbed to this disease, hitherto regarded as verminous, sometimes prove to harbour few or no parasites.

Fourthly, that this grave and fatal disease, and the symptoms by which it is clinically recognised, can be produced with cultures of the cocco-bacillus, which he has isolated.

These statements are very precise, but Mossu declares that he has never yet been convinced of the reliability of the last statement referring to experimental transmission, any more than of the data regarding alleged protective vaccination.

The symptoms of gastro-intestinal strongylosis or ovine pasteurellosis point to a progressive pernicious anæmia of chronic or subacute form. Rapidly progressive cases are exceptional.

In France the form seen is almost invariably chronic. The animals appear dull, sluggish, and feeble; they lose appetite, waste, become anæmic, then cachectic, and, after several months, die of exhaustion and wasting, after having shown diarrhœa during the later stages.

The fæces are pasty, and exhale a very marked putrefactive odour. There is little room to doubt that auto-intoxication from resorption of intestinal products is continually going on.

The animal's general appearance is bad, the ears are pendant, the wool is dry and dull, and can be removed in handfuls by the slightest pull. There are no other external symptoms, and the diagnosis can only be arrived at by discovering the eggs of the parasite in the fæces.

Lesions. Post-mortem examination reveals all the general lesions of advanced cachexia and of gastro-enteritis of varying intensity. The abomasum and first portions of the small intestine usually contain a considerable number of strongyles; tæniae are often present in the intestine, and Moussu declares that he has always found a certain number of hooked worms and osophagostomes.

The peritoneal, pleuritic, and pericardial exudates common in most wasting conditions are always present, but the quantity of exudate in 
each cavity varies within wide limits. The liquid may even resemble that due to inflammation or infection; sometimes it is light pink or red in colour.

Pulmonary lesions usually exist. Moussu has almost always found gastric strongylosis associated with pulmonary or tracheo-bronchic strongylosis, but Lignières asserts that the Argentine cases shorred nodules of hepatisation which had nothing to do with the pulmonary strongylosis, and which appeared to result from areas of pneumonia produced by the specific cocco-bacillus and other organisms. He has even found abscesses and cavernous spaces in the lung.

Pathogeny. According to Lignières the specific agent of pernicious anæmia is a cocco-bacillus which stains well with fuchsin, violet, blue, safranin, etc., but does not take Gram, and which in cultures assumes either the strepto-bacillary form or occurs in barrel-shaped masses. It grows in simple bouillon at $38^{\circ} \mathrm{C}$., but better still in peptonised bouillon, which turns turbid for five or six days, afterwards becoming limpid in consequence of the organisms falling to the bottom of the vessel. It does not coagulate milk. On agar the culture is thin, bluish, shows an iridescent reflection, and when old appears whitish.

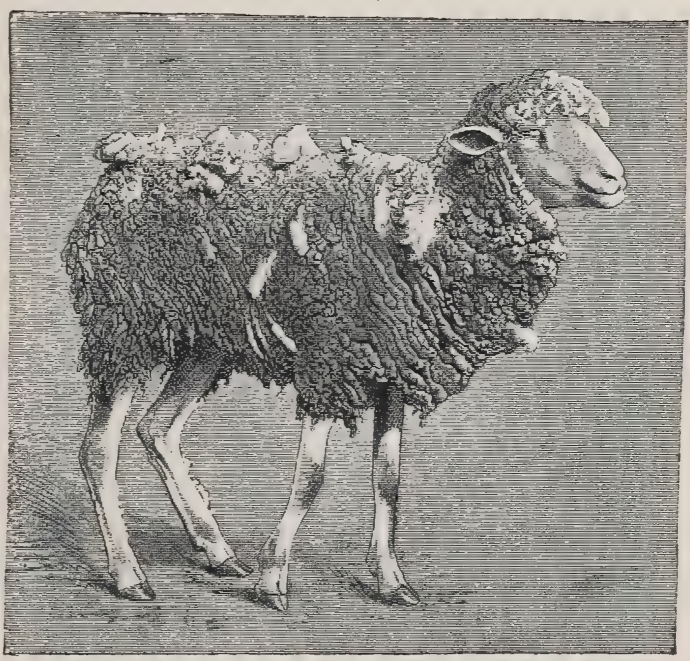

FIG. 114.-Wasting due to gastro-intestinal strongylosis. Grown on gelatine, the appearances are similar-the gelatine is not liquefied; on serum the pellicle is scarcely visible.

'The organism is said to be pathogenic for guinea-pigs, rabbits, dogs, and, of course, for sheep. Moussu, however, does not consider that the reported cases of transmission through the blood stream or by subcutaneous injection are really convincing or characteristic. He does not question the fact that Lignières discovered a special pathogenic agent in all cases and in all his patients; but what appears to him debatable is the exclusive part which Lignières attributes to that agent.

The fact that in Argentina, just as in France, strongyles have always been discovered in epizootics of this nature of itself constitutes something; and causes the second fact, that it has never yet been proved 
that any heavy mortality occurred in the absence of parasitic infestation, to assume considerable importance. Moussu regards these two facts as the greatest obstacles to Lignières' theory. He states that in his view the verminous affection is the essential, primordial and primitive affection, and that microbic infection is only secondary, and an almost inevitable result of grave verminous infestation.

It is quite certainly wrong to think that verminous affections may continue with scarcely any injury to the animal, especially when such a belief is based on observation of a few parasites which are not of a predatory character. In most cases of the kind now under consideration, the various parasites found (Strongylus circumcinctus and filicolis, Anchylostomes, etc.) cause more or less grave lesions.

These intestinal wounds facilitate the infection to which the fatal course of the disease is due. The disease, then, is not a special unvarying infection, but consists of multiple superposed infections.

It is wrong to believe and to teach that the parasitic disease is of no importance, and Moussu declares his belief that the proposed vaccinations will prove unavailing. $\mathrm{He}$ is of opinion, on the other hand, that when the parasitic invasion can be overcome the mortality will be checked, and only those animals which are already suffering from severe infections will succumb. Is not this precisely what has been observed in human pathology regarding miners' anæmia or miners' worm disease (anchylostomiasis)?

The diagnosis presents no difficulty for those who have had a little practice with the microscope, since the presence of eggs of the parasite can always be detected in the fæces in cases where external signs have given rise to suspicion of gastro-entestinal strongylosis.

The macroscopic diagnosis on post-mortem examination is not so easy as might be supposed, and when very small varieties are in question it is sometimes necessary to examine the mucous membrane of the abomasum or intestine very carefully in order to discover the parasite.

The prognosis is grave, because before attention has been drawn to many patients, the entire herd may be more or less infested. It is also grave because the pastures are infested with eggs or embryos, and the power of increase of these parasites is enormous.

Treatment. The earliest and most energetic means should be adopted in all cases. Treatment comprises :-

Drainage of swampy pastures.

Dressing of the pastures with chemical manures, preferably with iron sulphate, at the rate of 40 to $80 \mathrm{lbs}$. per acre.

Disinfection of manure to destroy the contained eggs or embryos.

The use of chalk, iron sulphate, various acids, etc.

As regards curative treatment, the diseased animals should be grouped 
and isolated as far as possible, and should receive doses of the following vermifuge :-

Powdered areca nut ..

Arsenic
2 ounces.

30 grains.

The above is sufficient for ten animals, and a dose should be given daily for a period of six days in a small quantity of bran. Treatment is completed by abundant nourishment, and by distributing about the pastures pieces of rock-salt suitably protected.

Many other vermicides or vermifuges have been suggested, but are less easy to use. They comprise essence of turpentine, mixtures of oil with essence of turpentine and benzine, picrate of potash in doses of 7 to 20 grains per day, ethereal extract of male fern, etc.

\section{LUMBRICOSIS OF CALVES.}

Following the example of human medicine, we apply the term "lumbricosis" to a disease caused by ascarides in calves, although Neumann separates the ascarides of calves from the lumbricoid ascarides with which they are usually confused in current practice.

Causation. The disease is exclusively due to infestation with embryos of the parasite, which in young calves afterwards develop in the first portions of the intestine and in the abomasum, interfering with secretion, and producing mechanical disturbance, colic, and digestive irregularity, eventually followed by marked loss of condition. Death may even follow, either from rupture of the pylorus or duodenum, or from secondary septicæmia of intestinal origin, due to the parasites burrowing into the mucous membrane and facilitating infection. In adults of all species lumbricosis is rare. It occurs principally in young animals from the time of weaning up to the age of eighteen months or two years.

The diagnosis cannot usually be formed until the parasites are found in the fæces, but microscopic examination sometimes rêveals the presence of the eggs, and thus excites suspicion.

Provided the condition is diagnosed early, the prognosis is not grave; but when patients have become exhausted and anæmic they require a long time to recover, even when freed from parasites.

Treatment. Guittard recommends empyreumatic oil as very efficacious, and gives it in doses of $2 \frac{1}{2}$ to 3 drachms diluted with ordinary oil, or emulsified with any kind of mucilage.

Calomel gives good results, and may be administered in doses of 15 to 60 grains, according to the animal's age and size.

Powdered areca nut would probably be easier to administer with the food. Oil of turpentine is given mixed with ordinary oil, but its action is less certain. 


\section{STRONGYLOSIS OF THE ABOMASUM IN THE OX.}

Although well studied by Stadelmann and Ostertag in Germany, and by Stiles in America, this disease has not yet been regarded in France as giving rise to accidents.

It is produced by the Strongylus convolutus vel Ostertagi, which becomes embedded under the epithelium of the mucous membrane and causes the formation of small nodules, the size of a pin's head or lentil, which can be detected on palpation. The cavity thus formed beneath the epithelium communicates with the gastric cavity by a little orifice, through which the cephalic end of the parasite passes.

\section{PARASITIC GASTRO-ENTERITIS, DIARRHCEA, AND ANEEMIA IN CATTLE, SHEEP AND LAMBS.}

A disease characterised by anæmia with wasting and diarrhœa is sometimes produced in cattle by the presence in the fourth stomach of small strongyles varying in size between 3 and 9 millimètres in length, according to the variety encountered. One variety of the smaller size has been named by McFadyean Strongylus gracilis. Penberthy, who described the disease in the Jour. of Comp. Path. and Therap. for 1894, p. 249, states that in certain cases he also found the Strongylus ventricosus, the Tricocephalus affinis, and minute straight worms about $\frac{2}{25}$ of an inch long, which he regarded as anguillulæ. Neumann declares that pernicious anæmia with catarrh of the abomasum in young animals is due to Stronaylus convolutus.

The symptoms comprise anæmia, wasting, and diarrhœea of varying severity. In acute cases, which are common between the ages of six months and two years, husk is sometimes (accidentally) present. The disease is rarest in summer. Certain animals lose flesh rapidly, though appetite is retained. Acute fœetid watery diarrhœa follows. The animal shows tenesmus, appears dejected, and has a temperature of $103^{\circ}$ to $105^{\circ}$ Fahr. 'I'he mucous membranes become pale, the pulse small and weak, the appetite capricious, the eyes sunken, belly tucked up, coat harsh and dry and hide tight. Wasting is rapid. The animals are listless, and often lie down for long periods. Death occurs from exhaustion.

The parasite. Scrapings from the abomasum and intestine when diluted with water and viewed under a lens show minute bodies resembling short, fine hairs. When isolated these are easily visible with the naked eye. In situ they appear to be lying on the mucous membrane, sometimes with the head fixed in the latter. They vary in length from $\frac{3}{25}$ to $\frac{6}{25}$ of an inch, and are brownish in colour. For a detailed description see Penberthy loc. cit. McFadyean (Jour. of Comp. Path. and 
Therap. for 1896, p. 314) also gives a very full description, illustrated by plates, of the two species of strongyles which he regards as the cause of gastritis in cattle in England.

The lesions are those of wasting diseases. The first three gastric compartments may be thin, but usually show no abnormality. The mucous membrane of the abomasum shows evidences of catarrh, is sometimes covered with a loose croupous material, or is denuded of its epithelium and even extensively destroyed. In acute cases it is more or less deeply reddened over spots or extensive patches. Occasionally it appears jelly-like, owing to effusion into its substance. Except for the catarrhal condition. the small intestines may be healthy, but the large, especially the colon and cæcum, show lesions similar to, but more pronounced than, those of the abomasum.

The treatment comprises administration of the usual vermifuges, of which Penberthy prefers turpentine. The diet should be nutritious and easily digestible. Among drugs, non - irritant iron salts, cod liver oil, bitter vegetable tonics and com-

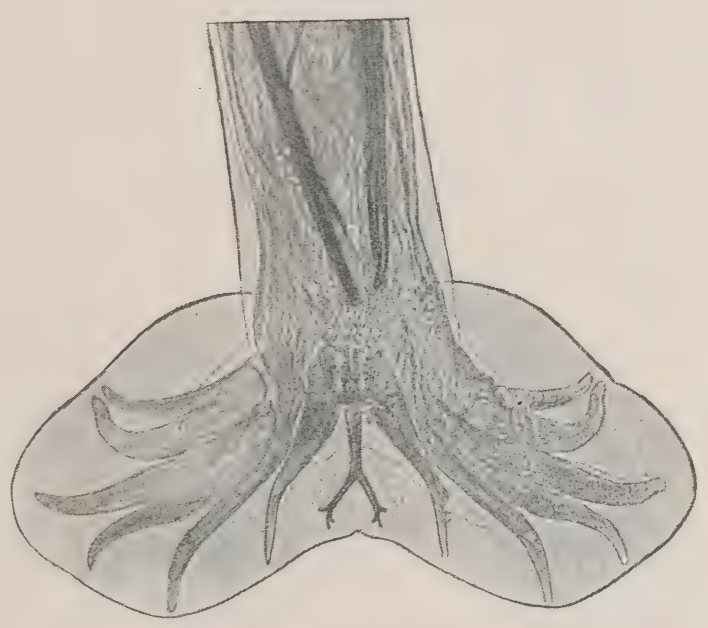

Fig. 115.-Caudal extremity of male Strongylus cervicornis. (Block kindly lent by Prof. McFadyean.) mon salt are recommended. The animals should be housed and liept warm; the litter, containing parasites, should be destroyed. The pastures may be dressed with salt and lime; those worst infected should be ploughed. An uninfected water supply is essential.

Parasitic Gastro-enteritis in Sheep and Lambs. A disease in seven to eight months old lambs, closely resembling the above, was described by McFadyean in the Jour. of Comp. Path. and Therap. for 1897, p. 48. Sheep over one year old were not affected.

The symptoms comprised diarrhœa, rapid wasting, impaired appetite, thirst, a tendency to lick and swallow sand or earth, dulness, and continued fever $\left(105^{\circ}\right.$ to $108^{\circ} \mathrm{Fahr}$.). There was no evidence of acute pain or of marked cough.

Duration. The disease sometimes proved fatal in one or two days, but sometimes extended over several days or weeks. The mortality 
varied from 10 to 20 per cent., but in many cases almost all the members of a flock exhibited diarrhoea and loss of condition.

The cause appeared to be the presence of small nematode worms in the fourth stomach, which generally exhibited gastritis with inflammatory congestion of the mucous membrane, though in a considerable number of cases the lining membrane of the stomach was markedly anæmic. In a large number of cases the irritation of the mucous membrane was continued into the duodenum, but as a rule the remainder of the small intestine was not inflamed.

The worms named by McFadyean Strongylus cervicornis are from 10 to $12 \mathrm{~mm}$. in length, so that although not of microscopic dimensions they cannot be seen when suspended in the stomach contents. They are readily detected in microscopical preparations under a low power.

The treatment is similar to that of gastro-enteritis in cattle, but chief

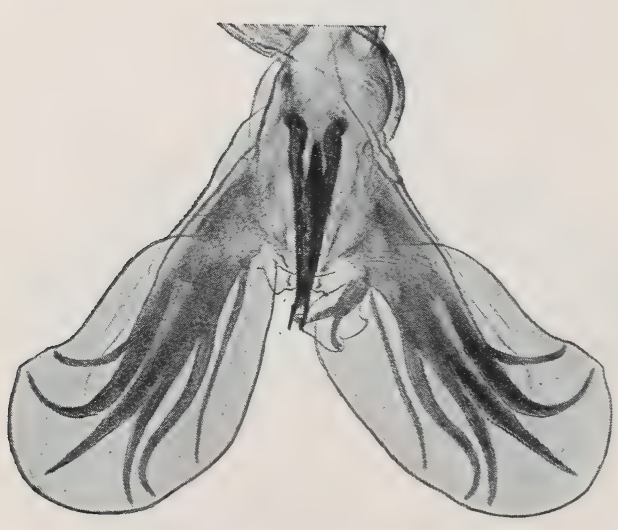

Fig. 116. - Caudal extremity of male Strongylus contortus. (Block kindly lent by Prof. McFadyean.) attention should be directed to prophylaxis.

A verminous disease, closely simulating the above, and affecting cattle, sheep, and goats in Texas, is described by Ch. Wardell Stiles in the Annual Report of the United States Department of Agriculture for $1900, p$. 356. The disease was of mixed character, and consisted in various degrees of verminous gastritis, verminous enteritis, and verminous bronchitis. In the stomach were found the common twisted wireworm (Strongylus contortus) and Ostertag's encysted wireworm (Strongylıs Ostertagi). It appeared to be present in every calf, steer and cow examined (post-mortem), and was undoubtedly the chief agent in causing death. The sheep and goats were very similarly affected. In the bowel of cattle were found the hookworm (Uncinaria radiata), nodular disease worm (Esophagostoma columbianum) : in that of sheep the hookworm (Uncinaria cernua) and nodular disease worm (Esophagostoma columbianum), and the fringed tapeworm (Thysanosoma actinioides). In the lungs of the cattle Strongylus micrurus (the small-tailed lungworm), and of sheep the threadworm strongyle (Strongylus filaria) were detected.

Treatment. Sulphate of copper, gasoline and coal-tar creosote were tried, but the best results were obtained from doses of 30 grains 
(for a lamb) up to 100 grains (for a two-year-old sheep) of thymol in 1 per cent. coal-tar creosote solution.

\section{INTESTINAL COCCIDIOSIS OF CALVES AND LAMBS (PSORO. SPERMOSIS, HEMORRHAGIC ENTERITIS, BLOODY FLUX, DYSENTERY, ETC.)。}

History. This disease is very common in the Avalon and surrounding districts, sometimes assumes the characteristics of a true epizootic, and affects young bovine animals between the ages of six months and two years, but is commonest and most contagious in animals of from ten to eighteen months old.

It attacks animals in good or bad condition, without distinction of breed or species. It begins towards the end of July, attains its maximum development towards the end of August and September, and disappears in October, though occasionally it continues until November. In exceptional cases Degoix has seen it during January and February in animals which had returned from the fields to the byres about the end of November. It develops earlier than verminous bronchitis, in conjunction with which, however, it often occurs. It is commonest in warm, moist, rainy years, and amongst animals pastured on swampy ground containing numerous springs and streams. Year after year it attacks animals occupying particular pastures in summer. The soil of these pastures is undoubtedly infested with the germs of the disease, just as in places the soil is infested with anthrax bacilli. The appearance of symptoms is preceded by an incubation period of one or two months. The length of this incubation period is fixed by the observations which Degoix has made during the past twenty years, and depends on the time which elapses between the animals being turned into infected pastures and the appearance of the preliminary symptoms.

Symptoms. The disease commences with liquid, serous, fœetid, greenish-black diarrhœa, the material being voided without special straining and the animals losing neither their spirits nor appetite. Fever can scarcely be detected, the temperature ranging between $38^{\circ}$ and $39^{\circ} \mathrm{C}$. On the second or third day the diarrhœea changes in character. Though it always remains foetid, it now becomes mucous, reddish-black, or sanguinolent, and contains more or less frequent blood clots of varying size.

'The passage of this material causes violent straining, which becomes more and more common, and is accompanied by very pronounced rectal tenesmus. The animals stand with their backs arched for one or two minutes, sometimes longer, and the liquid escapes in large quantities, soiling the quarters and hocks. The animals are dull, show a certain 
degree of colic, and frequently grind the teeth. Appetite is lost, thirst is severe, and rumination ceases. Wasting makes rapid strides, the coat stares, the animals have difficulty in standing on account of their weakness, fever sets in, and the temperature rises to $40^{\circ} \mathrm{C}$. This condition may last from five to ten days and terminate either in recovery or death. Recovery is frequently rapid in animals which have continued to eat, and in which the acute period has been of short duration-five to seven days at most. On the other hand, it is slow if the appetite has disappeared and the acute period has been prolonged beyond ten days.

The diarrhœa, which has lost its sanguinolent character towards the sixth or eighth day, may continue for somewhat longer. The attacks of straining become rare, and cease between the tenth and fifteenth days. The appetite remains capricious for a long time.

Convalescence is marked by alternate improvement and retrogression. The animals are weak, and only recover quickly under energetic treatment and forced feeding with concentrated digestible foods like milk, soup, cooked grain, etc., administered for three weeks or more.

Death may occur towards the tenth or fifteenth day from exhaustion. The patients become very anæmic and thin, the eyes are withdrawn into the orbits, and the animals appear indifferent to what goes on about them. 'They still groan feebly, occasionally grind the teeth, and lie continually on the chest with the head extended. 'l'he body temperature falls and death follows.

In well-bred animals in good condition the disease sometimes assumes a much graver and more rapidly progressive form, with peracute symptoms, and makes as many, if not more, victims than that previously described.

The process is as follows: After suffering for a day from serous diarrhoea, to which the owners pay little attention, the animals show sanguinolent diarrhoa and pass blood clots. This is almost immediately followed by very violent convulsive attacks-true eclampsia. The animals are then unable to stand, lie on the side with the head outstretched and resting on the ground, the eyes withdrawn into the sockets and often showing pirouetting movements (nystagmus), the neck drawn upwards and backwards (opisthotonos), and the limbs rigidly extended. From time to time the whole body is shaken by extremely violent convulsive movements.

This condition, which is sometimes preceded by weakness of the hind quarters and symptoms of locomotor ataxia and inco-ordination, may continue from six to thirty-six hours; in nine cases out of ten it terminates in death.

Causation. On microscopic examination of the serous dejections one finds distributed throughout the liquid mass very small numbers of 
ovoid corpuscles having a double outline, and contents of varied appearance; these are the coccidia.

When the diarrhœa has become sanguinolent and muco-fibrinous, the fluid contains these coccidia in considerable quantities, and large numbers of them may be found in the mucus, where they are mixed with epithelial débris, blood corpuscles, and lymphatic cells, etc. They are rarer in the clots. Coccidia cannot be found in the fæces of healthy animals, even in those occupying the same pastures with the diseased. Should the clinical symptoms be thought insufficient of themselves clearly to identify the disease, a simple microscopic examination of the fæces will remove any doubt.

Lesions. Post-mortem examination immediately after death enables one exactly to identify the habitat of the parasite and the lesions it produces. These lesions are to be found throughout the large intestine, from the cæcum to the anus.

The large intestine is almost or entirely empty, the mucous membrane is reddish-brown in colour, lies in folds, is œedematous, and everywhere covered with a coating of mucus. This coating varies in character at different points; in places it forms more or less thickened patches of greyish or yellowish colour, and of a resistant character, as though mixed with coagulated fibrin. These patches are fairly well defined, they are irregular in form, and vary in width from some millimètres up to several centimètres. They are more or less adherent to the mucous membrane, from which they can easily be stripped away. The mucous membrane thus exposed is slightly depressed, and of a whitish colour, thus markedly contrasting with the surrounding red coloration. This depression represents a slight ulceration, which, though superficial, is clearly visible to the naked eye.

Microscopic examination of the mucus patches reveals the existence, both superficially and in the depths, of epithelial cells derived from the mucous membrane, of vesicular cells derived from the Lieberkuhnian follicles, of numerous blood and lymphatic corpuscles, and, distributed irregularly throughout this mass of cells, of coccidia, resembling those found in the dejections.

In thin sections of the intestine, made through the ulcerated mucuscovered patches, and in a direction perpendicular to the mucous membrane, one finds that the epithelial covering of the intestine has disappeared.

The Lieberkuhn's follicles are shortened, their orifices are irregular and partly blocked with epithelial débris. In a large number of these follicles the blind extremity is dilated, and more or less filled with coccidia, varying in appearance according to their stage of development. The epithelial cells normally lining these blind ends seem to have disappeared, D.C. 
and to have been replaced by the parasites. This, however, is not really the case. It is easy to prove by suitable dissection (after maceration of the sections in 30 per cent. alcohol) that the coccidia are lodged in epithelial cells which have become modified in shape and undergone hypertrophy as the parasite has grown, whilst the nucleus has been pushed to one end and undergone atrophy.

Alongside the deceased glands may be found others which are quite healthy. In the parts which are most markedly affected the interglandular connective tissue is infiltrated and slightly thickened. Degoix has never discovered coccidia in the cells of the intestinal mucous membrane itself. The lesions may be met with throughout the large intestine, but are more numerous and grave as the rectum is approached.

This disease is characterised by extensive inflammation, affecting the entire mucous membrane of the large intestine, by more or less extensive ulceration of this bowel, the ulcers being covered with muco-fibrinous patches rich in coccidia, by localisation of the parasites in the epithelial cells of the glandular cul-de-sac, by the appearance of the disease at a special time of year and in special districts, where it assumes the form of an enzooty, and by the fact that it always appears in the same pastures. The mortality varies between 12 and 25 per cent. from year to year.

Microscopic appearances. The protozoa which produce this disease assume the form of ovoid cysts, varying in length from 18 to $25 \mu$, and measuring at the widest part about $13 \mu$. They possess a hyaline envelope whose existence is proved by its double contour line, and yellowish, granular, highly refractile contents. This granular material does not always occupy the whole of the cavity. At a certain stage of development it collects towards the centre, forming a nucleus and leaving clear spaces at the poles of the cell. At a later stage this nucleus divides into four portions which afterwards separate.

Treatment. The protozoa, in their cystic condition, are very resistant to destructive influences. The most common natural cause of their destruction is drying in the open air. Unfortunately, in the localities where the patients usually live, that is, filthy byres, wet pasturages, etc., this destruction only takes place to a very limited extent.

The disease can be attacked by prophylactic and therapeutic measures.

Prophylaxis consists-firstly, in destroying the parasites contained in the dejections by the free use of 3 per cent. sulphuric acid solution; and, secondly, in removing the young animals from infected fields during the months between June and September.

Therapeutic treatment comprises-firstly, stimulant applications to the abdomen; secondly, disinfection of the intestine by the administration of salol, benzo-naphthol, very diluted solutions of creolin, etc; thirdly, intestinal irrigation by the administration of mucilaginous drinks 
containing bicarbonate of soda, supplemented by general tonis treatment and the supply of concentrated, very nourishing, and easily digested food (Degoix, Rerue générale de Médecine Vêtérinaire, No. 28, February 15th, 1904, p. 177).

McFadyean describes a similar disease to the above in two to three months old lambs (Jour. of Comp. Path. and Therap., March, 1896, p. 31). The mortality reached 10 per cent. The lambs at the time the disease broke out in the flock were closely folded together with the ewes on growing roots, which, needless to say, were much soiled with earth and fæces before they were completely consumed.

\section{INTESTINAL HELMINTHIASIS IN RUMINANTS (Ox, Sheep, Goat).}

Verminous disease of the intestine is often accompanied by similar disease of the stomach (gastro-intestinal strongylosis of the sheep, lumbricosis of the calf), but it also occurs apart from the presence of gastric parasites. Parasites are more frequent in the intestine than in the abomasum, because the alkaline intestinal juices prove a much more favourable medium for their development than do the acid juices of the stomach.

The actual parasites may include ascarides, strongyles, hooked worms, œsophagostomes, tricocephales, sclerostomes, and various tæniæ (Trenia expansa et $a l b a)$. Many of these have already been, or will hereafter be, referred to.

Helminthiasis due to round worms like strongyles, and the various forms of hooked worm, is

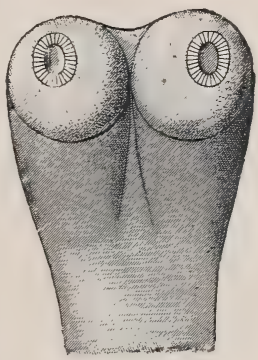

FIG. 117.- - Head of Tania alba of the ox and sheep. (After Neumann.) graver than that due to flat worms, but most extraordinarily varied collections are sometimes met with. Speaking generally, however, helminthiasis more particularly affects young animals like calves, lambs, and yearling sheep, is rarer in adults, and in all cases the complications it produces are of trifling importance in adults as compared with those caused in the young.

The persistence of verminous diseases in certain infested countries, districts, farms, or pastures is explained by the enormous number of eggs or embryos passed with the fæces and disseminated with manure, as well as by the high degree of resistance of the eggs and embryos to destructive influences.

Causation. The various forms of intestinal helminthiasis are all due to embryos or eggs of worms obtaining entrance to the stomach or bowels 
of susceptible animals. Nevertheless, external conditions largely favour

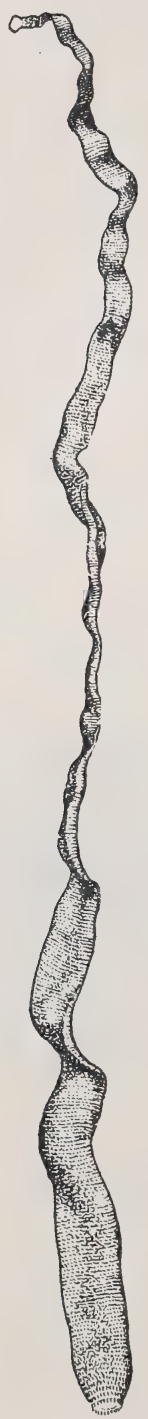

FIG. 118.-Adult specimen of the fringed tapeworm (Thysanosoma actini. oides). (After Stiles, 1893.) infestation. The existence of marshes, ponds, and stagnant water on certain pastures, heavy annual rainfall, the occurrence of wet seasons, etc., favour the existence and growth of embryos, and, as a consequence, the infestation of animals.

The symptoms of intestinal helminthiasis are always very vague, whether the victims be oxen, sheep, or goats. They point to the slow and progressive development of a pernicious anæmia, characterised primarily by interference with appetite and digestion, then by anæmia, and finally by cachexia. The patients are dull, lose condition, suffer from depraved appetite, and, during the cachectic period, from diarrhœa, and die in a condition of exhaustion. These forms of helminthiasis are much more frequent in sheep than in oxen.

The diagnosis is only arrived at by discovering parasites in the fæces, or, in those doubtful cases where one has been led to make a microscopic examination of the excrement, by the detection of large numbers of eggs.

From an economic standpoint the prognosis is grave, because severely infected animals recover their condition very slowly, whatever treatment be adopted.

Treatment includes the measures previously indicated regarding the destruction of germs, eggs, or embryos distributed over the pastures, and the drainage of wet grounds and stagnant pools. Where such diseases are common, the pastures should be dressed with iron sulphate, and the stable manure disinfected or burned.

Curative treatment comprises the administration of such anthelmintics as can be given with the food. They should not require to be administered by force, as this proceeding would not only necessitate a great loss of time, but might result in accidents.

Arsenious acid, in doses of 15 grains per day, and tartar emetic, in doses of 75 to 150 grains, according to the animal's size, are among the best drugs for oxen, and may be given for four or five days running. Benzine, oil of turpentine, and empyreumatic oil are more difficult to administer.

In the case of sheep, preference should be given to areca nut, in doses of 75 to 120 grains, according to the animal's size, and to 
tansy, which are convenient to give, and can be added to bran, oats, or beetroot.

Adult tapeworms of oxen are of relatively minor importance, but one tapeworm of sheep, viz., the fringed tapeworm ('Thysanosoma actinioides), also known as Tania fimbriata; Moniezia fimbriata deserves notice,
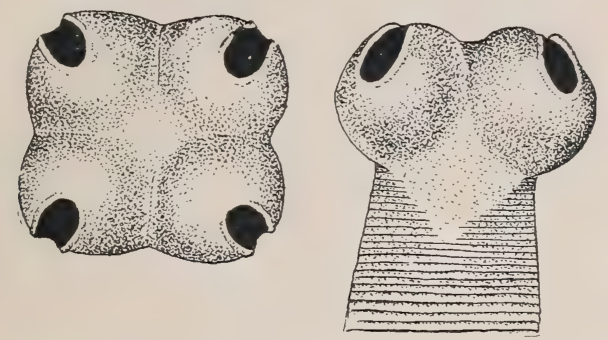

Fig. 119.-Ventral and apex views of the head of the fringed tapeworm

(Thysanosoma actinioides). $\times$ 17. (After Stiles, 1893.)

as at times it forms a veritable scourge to the sheep industry of North America and South America.

Disease. The disease in sheep caused by the fringed tapeworm has been studied in detail by Curtice, who considers that next to scab it is the most important sheep disease of the western plains of North America. The financial loss it causes is extensive, and results from the failure of the lambs to fatten, the small crop of wool, and the weakening of the animals, so that they cannot withstand cold winter weather. The parasites develop slowly, and are present in considerable numbers before their

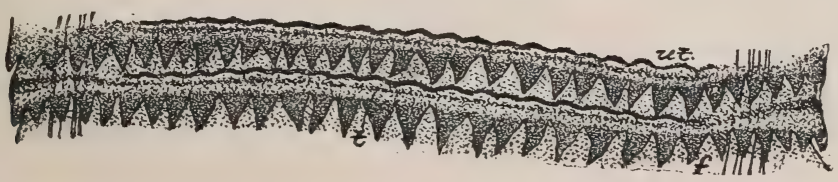

Fig. 120. - Segments of the fringed tapeworm (Thysanosoma actinioides), showing canals and nerves, and $(f)$ fringed border, $(t)$ testicles, and $(u t)$ uterus. Enlarged. (After Stiles, 1893.)

presence is suspected. Toward September the lambs fail to grow as they should; in November the symptoms are marked. First, the worms produce local irritation of the intestine, which finally develops into a chronic catarrhal inflammation; their presence in the gall-ducts produces similar results, and obstructs the flow of bile ; infected lambs are large-headed, under-sized, and hide-bound ; their gait is rheumatic, and they appear more erratic than the other sheep, standing oftener to stamp at the sheep-dogs or herds, and lagging behind the flock when driven; the 
general symptoms are those of malnutrition, and Curtice considers them nearly identical with the symptoms of the "loco" disease; in fact, he states that it is extremely difficult to distinguish between the two diseases, and believes that the fact that the worms " may tend to produce depraved appetites and a morbid craze for a particular food is also reason for suspecting that the loco disease may depend on the tapeworm disease." General systemic disturbances result from malnutrition; the usual fat is absent; serous effusions are noticed in the body-cavities, serous infiltration in the connective tissue.

Treatment is similar to that of parasitic gastro-enteritis of sheep and lambs (which see). 


\section{CHAPTER IX.}

\section{DISEASES OF THE LIVER.}

Frour the physiological standpoint the liver is an organ of such importance that its pathology should be studied as completely as possible. Furthermore, it is often the seat of a number of varying lesions, either of parasitic, toxic, infectious, or cancerous origin.

In animals of the bovine species the liver is placed in the right retrodiaphragmatic region, so that it is somewhat difficult to examine by any of the ordinary methods, like palpation and percussion. Under normal conditions it is entirely concealed beneath the hypochondrium, except towards the upper margin of the thirteenth rib, where it can be examined by palpation. When, as in various morbid conditions, it is considerably increased in size, it extends as far as the margin of the hypochondriac circle, thus becoming directly accessible to palpation and percussion. Sometimes it even enters the hypochondriac region, passing outside the omasum and abomasum, which it then thrusts towards the middle of the abdominal cavity.

The margin of the liver exhibits a depression lodging the gall bladder nearly opposite the centre of its vertical depth.

As the liver is so deeply situated, percussion is found to be the method of examination which gives the best results. Beyond the limits of the zone of auscultation, percussion gives above a semi-dull sound, then, proceeding downwards, a dull sound due to the liver, the omasum, and the collection of liquid in the bowel. When this dulness is well defined, clear, broad from above downwards, and extends to or beyond the hypochondrium, it indicates hypertrophy of the liver. By deep palpation of the posterior margin of the hyposhondrium the liver can then be sounded, and its excessive size detected.

The symptomatology of the liver is still very imperfectly understood, for in practice the urine is rarely tested for bile pigments, nor attempts made to ascertain whether the glycogenic function is normal by the test for alimentary glycosuria.

In this connection nothing has yet been done to assist in diagnosing certain hepatic conditions. Fortunately, those diseases of the liver which we have to study are more often of a parasitic nature than true diseases of the hepatic tissue. 
The reported cases of venous or biliary cirrhosis, moreover, are too illdefined and too incomplete to be taken as a type for description. We leave them on one side. In a similar way, apart from parasitic cholangeitis and cholecystitis, inflammations of the biliary ducts are little known, and are rare.

\section{CONGESTION OF THE LIVER.}

In bovine pathology only passive congestion of the liver, often a result of various primary affections with cardiac lesions, is well recognised.

Active congestions probably occur during infections or intoxications of various kinds, but have not been made the object of special research.

Among diseases likely to produce passive congestion must be included all those which interfere with the return circulation through the posterior vena cava. All cardiac affections with lesions of the valves or orifices of the right heart, all forms of pericarditis, tumours or lesions of the mediastinum compressing the posterior vena cava, produce stasis, passive congestion, and progressive development of what is called "cardiac liver."

Symptoms. The liver is considerably hypertrophied, as a consequence of the stasis of blood and progressive dilatation of the portal system. Its zone of dulness increases in size, whilst on palpation its borders may sometimes be detected. This condition is always accompanied by digestive disturbance.

The function of the liver is more or less interfered with; the urine is scanty in amount and charged with deposit. Ascites of varying intensity frequently occurs; cardiac disturbance accompanies or usually precedes the above symptoms.

The lesions of passive congestion are represented by progressive dilatation of the entire portal venous system (nutmeg liver). In time this dilatation may produce biliary cirrhosis, as a result of chronic irritation of the blood-vessels and perivenous inflammation. This condition is known as "cardiac cirrhosis of the liver."

The diagnosis of this pathological condition is generally easy, provided that the primary disease which causes it be recognised.

The prognosis is always grave, and the practitioner is limited to treating the primary affection, such as endocarditis, pericarditis, etc.

\section{NODULAR NECROSING HEPATITIS.}

This form of inflammation of the liver is somewhat rare in animals of the bovine species. The disease is difficult to diagnose, and is often only recognised on post-mortem examination. 
Isolated tracts of the liver become inflamed, between which the rest of the tissue preserves its normal character; the parts affected appear to undergo complete degeneration, the cause of which is difficult to explain. On examining affected animals after death, the liver is found to be greatly enlarged, and apparently invaded by multiple tumours. On section, the parenchyma generally is of normal colour, but the diseased parts are represented by dirty greyish-yellow tissue of a lardaceous character, somewhat resistant to the knife.

The affected spots vary in size, between that of a lentil or hazel nut and an egg, and are formed of necrotic tissue.

The periphery is the seat of true chronic fibro-plastic inflammation.

Causation. According to Stubbe, these lesions are produced by microbes, originating in the intestines, and carried to the liver by the

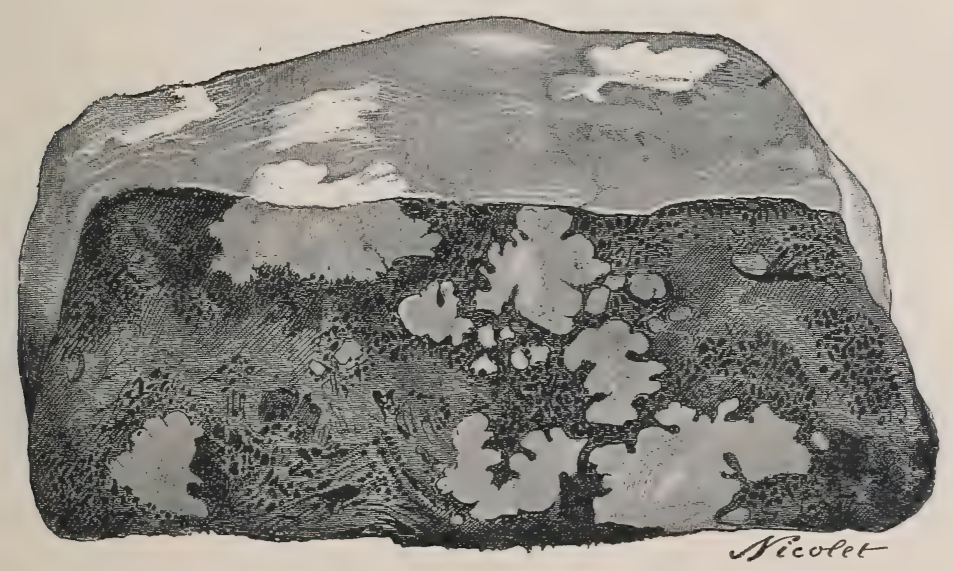

FIG. 121.-Appearance of the liver in necrosing hepatitis

mesenteric veins. The lesions and blood of the liver yield cultures of a microbe resembling that of necrosis; nevertheless, such lesions have not been experimentally reproduced.

According to Berndt, infection from the uterus is possible, and indeed probable. Moussu has only seen three cases of this particular condition of the liver in living animals. Two of these were in a working ox and a bull respectively, so that Berndt's view would not seem to be exclusively applicable. Moussu is convinced that infection is of intestinal origin, and that it takes place through the mesenteric veins; he claims to have found the proof of this in the existence of multiple pylephlebitis and complete obliteration of the subhepatic veins in some cases.

Symptoms. The symptoms are so vague as to render diagnosis diffcult. Berndt, on the other hand, regards it as fairly easy. He states 
that the disease occurs in old cows, which after parturition show loss of appetite, polydipsia, fever, dyspnoea, and short, feeble cough, suggesting pneumonia. After a few days the animals appear extremely weak, remain lying for long periods, and exhibit icterus. Percussion of the liver detects abnormal sensibility and hypertrophy.

The three cases seen by Moussu showed only slight yellowness of the membranes, general weakness and difficulty in walking, which at first glance appeared to suggest laminitis, marked hypertrophy of the liver, sensitiveness over the hepatic region, and, as complications, uncontrollable diarrhœa and peritonitis. But these symptoms are also noted in suppurating echinococcosis, and even in cancer of the biliary ducts, so that diagnosis does not appear easy. Nevertheless, there is always marked fever, and on post-mortem examination it is not unusual to find, in addition to the hepatic lesions, a certain amount of perihepatitis, partial peritonitis, and even pleurisy in the region of the diaphragm. The question is of little practical importance, for the gravity of the disease just described is such that economically no treatment is possible. The great point lies in correctly diagnosing disease of the liver, and that is relatively easy.

\section{CANCER OF THE LIVER AND BILE DUCTS.}

Cancer of the liver, that is, broadly speaking, the development in the liver of malignant tumours, capable of becoming generalised throughout the organism, is comparatively rare when compared with parasitic diseases of the same organ. It may be primary or secondary in character, but is much more frequently secondary. In bovines primary cancer assumes the forms of adenomata, trabecular epitheliomata, or adeno-carcinomata. Moussu describes a case in which the growths assumed the form of papillomata or adeno-papillomata extending throughout the biliary ducts, and partly obstructing the common bile duct, which was greatly dilated.

The real cause of these, as of all other primary tumours, remains shrouded in mystery.

Secondary cancer is more frequent; it occurs usually in the form of little isolated tumours (nodular cancer) of varying size and greyish colour.

Symptoms. Clinically the description, or rather the identification, of cancers of the liver is difficult, and the diagnosis particularly troublesome in cases of primary cancer.

In secondary cancer (following tumour of the testicle in oxen castrated by the method of bistournage, for example) the general condition, on the other hand, is usually so affected that attention is pointedly drawn to the 
seat of the secondary growths. The patients lose appetite, the fæces become fœtid, and diarrhœa sets in without clear signs of enteritis.

Examination of the liver almays reveals hypertrophy, and sometimes sensitiveness. The patients rapidly lose flesh, become cachectic, and the proportionate number of red blood corpuscles diminishes. From the normal of six or seven millions the number may fall to one million or less, while that of the white corpuscles considerably increases. This leucocytosis, which accompanies all forms of visceral cancer, enables one to distinguish between cancer and chronic forms of diarrhœic enteritis; it must not be confused with leucæmia. Moderately developed ascites is common, in consequence of obstruction in the porto-hepatic circulation.

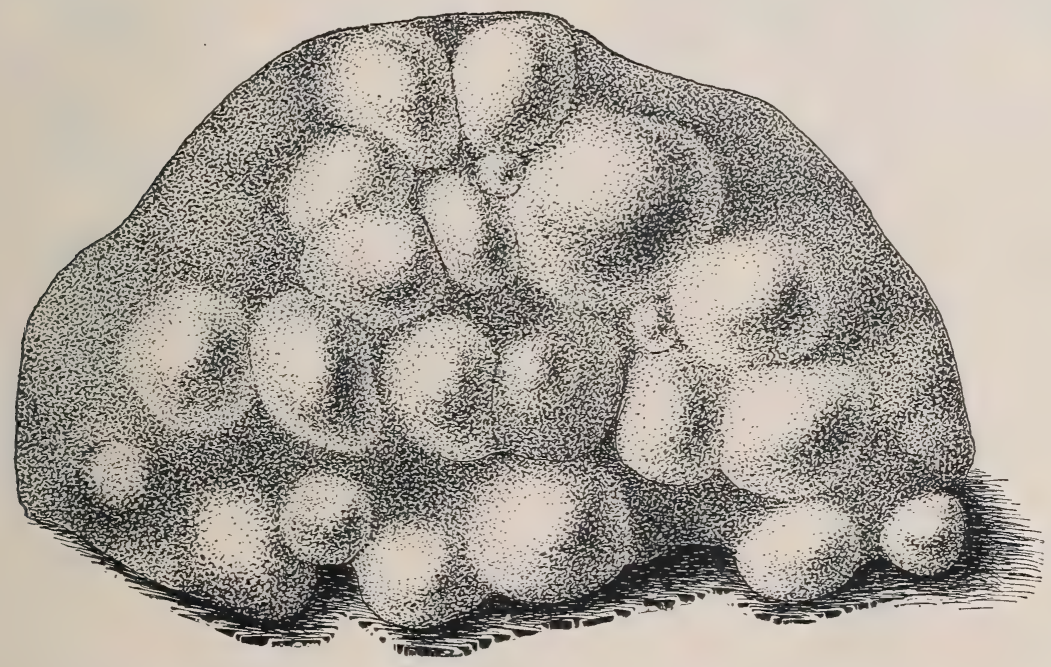

FIG. 122.-Portion of pig's liver infested with echinoccocus hydatid, natural size. (Stiles, Annual Report, U.S.A. Department of Agriculture, 1900.)

The diagnosis of cancer of the liver or biliary ducts is surrounded with difficulty, and the prognosis is extremely grave, because no treatment is possible.

\section{ECHINOCOCCOSIS OF THE LIVER.}

This term is applied to the development in the depths of the hepatic parenchyma of hydatids of Trenia echinococcus.

The echinococcus hydatid is found in man, cattle, sheep, swine, etc. It represents an intermediate stage of development of the echinococcus tapeworm of dogs. Since this parasite develops its larval stage in man also, and further, since it is the most dangerous animal parasite found in man, it is important to thoroughly understand its life history in order to guard against infection.

Adult stage (T'enia echinococcus). 
Hosts. Dog, dingo, jackal, wolf.

Life history. Starting with the adult tapeworm (Fig. 124) in the small intestine of the dog or wolf, the eggs are scattered over the ground and are swallowed by the intermediate host with the fodder or water. Upon arriving in the stomach the egg-shell is destroyed, and the six-

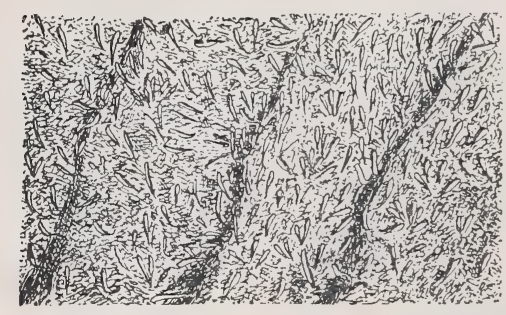

FIG. 123.--Portion of the intestine of a dog infested with the adult hydatid tapeworm (Tania echinococeus, natural size. (After Ostertag, 1895.)

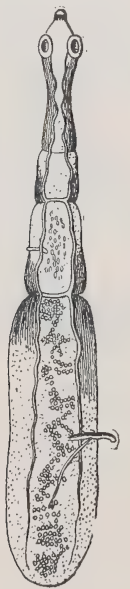

FIg. 124.-Adult hydatid tapeworm (Tania echinococcus) enlarged. (After Leuckart, 1880.)

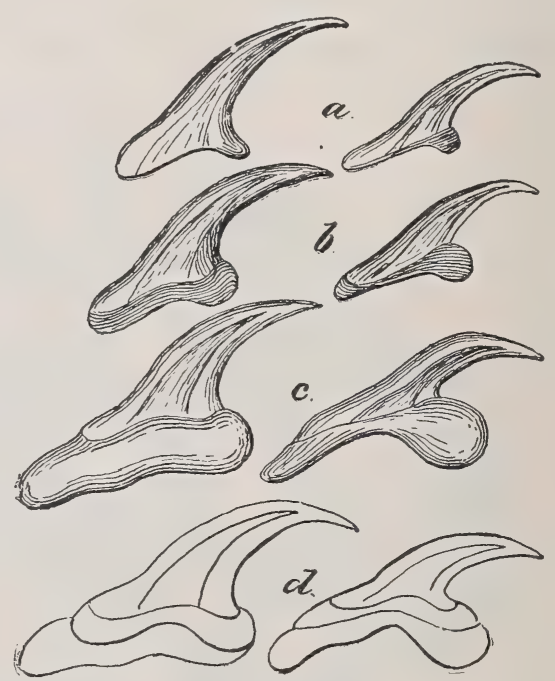

FIG. 125.-Hooks of adult hydatid tapeworm. $a$, From a hydatid; $b$, three weeks after feeding to a dog; $c$, from an adult; $d$, combined figures of $a-c$, showing the gradual changes in form. $\times$ 600. (After Leuckart, 1880.)

hooked embryo, which is thus freed, bores its way through the intestinal wall, and wanders, actively or passively (that is, carried along by the blood), to various organs of the body-liver, lungs, ovaries, bones, skull, etc.-where it develops first into an acephalocyst, which may develop further, as shown by the accompanying illustrations. The heads which are formed, upon being devoured by a dog or.wolf, then develop into adult tapeworms. 
Young animals are most exposed to this disease; in adults or aged animals the migration and derelopment of the embryo are more difficult.

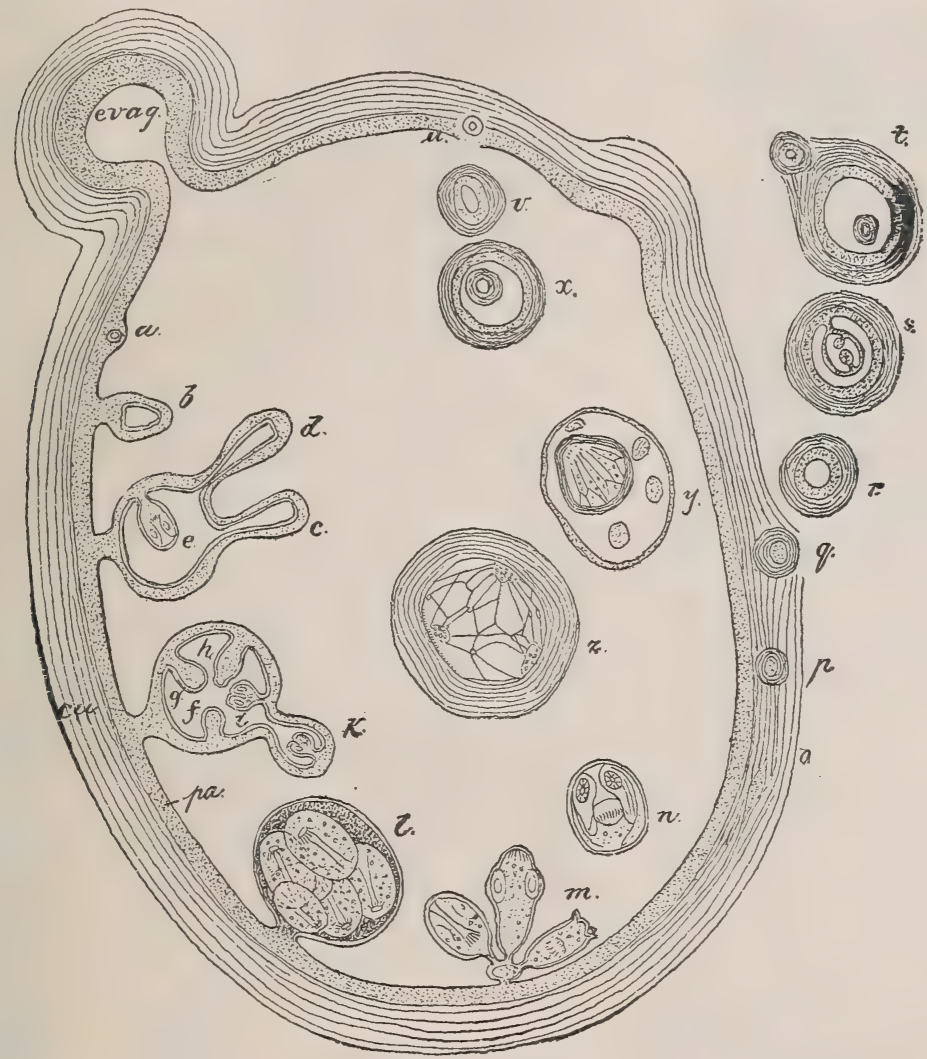

FIG. 126.-Diagram of an echinococcus hydatid. $c u$, Thick external cuticle ; $p a$, parenchym (germinal) layer ; $c, d, e$, development of the heads, according to Leuckart; $f, g, h, i, k$, development of the heads according to Moniez; $l$, fully-developed brood capsule with heads; $m$, the brood capsule has ruptured, and the heads hang into the lumen of hydatid; $n$, liberated head floating in the hydatid; $o, p, q, r, s$, mode of formation of secondary exogenous daughter cyst; $t$, daughter cyst with one endogenous and one exogenous grand-dạughter cyst; $u, v, x$, formation of endogenous cyst, after Kuhn and Davaine; $y, z$, formation of endogenous daughter cysts; after Naunyn and Leuckart; $y$, at the expense of a head, z, from a brood capsule; evag, constricted portion of the mother cyst. (After R. Blanchard, 1886.)

These embryos perforate the tissue of the liver, become fixed in it, and derive from it the nourishment necessary for their conversion into cystic bladders of varying size, either sterile or fertile.

The number of vesicles is rarely large, and when only one or two are 
present they seldom produce sufficient disturbance to attract attention. On the other hand, when numerous they deform the liver, produce glandular atrophy, increase the total size of

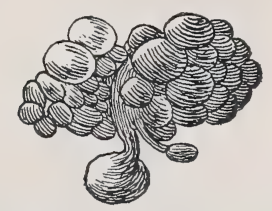

FIG. 127. -A racemose echinococcus, natural size. (After Leuckart, 1880.)

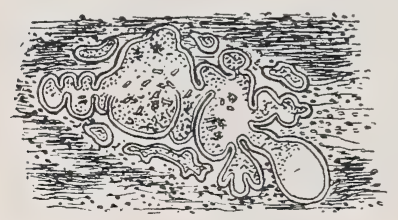

Fig. 128.-Section through a multilocular echinococcus. $\times$ 30. (After Leuckart, 1880.) the organ, and lead to the appearance of clearly-marked symptoms.

The cystic vesicles contain a clear, limpid, transparent fluid, in which float secondary, daughter, or granddaughter vesicles.

Symptoms. Echinococcosis of the liver has no well-marked symptoms, and is therefore difficult to diagnose in animals whose liver is deeply seated, and therefore beyond palpation. The signs which may characterise the period of penetration of the embryos through the intestine and into the depths of the liver, and which are probably represented by slight colic, vague pain and diarrhœa, usually pass unnoticed. But later, when the liver is extensively invaded appetite becomes irregular without apparent cause, animals show intractable diarrhœa, general feebleness, dulness, and wasting.

These symptoms do not point with sufficient clearness to a special

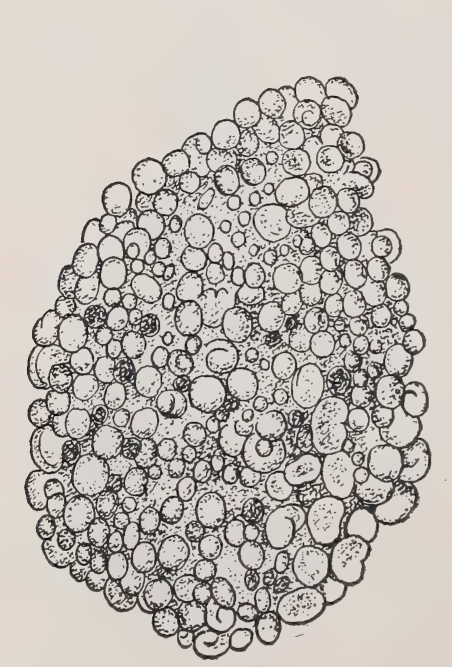

FrG. 129.-A multilocular echinococcus from the liver of a steer, natural size. (After Ostertag, 1895.)
9
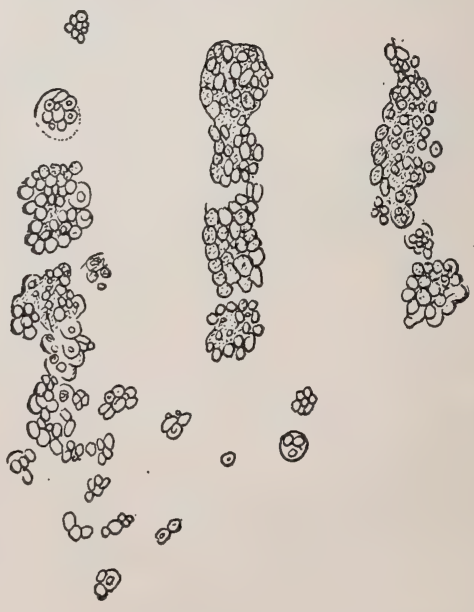

FIg. 130.-A multilocular echinococcus from the pleura of a hog, natural size. (After Ostertag, 1895.) 
visceral lesion, but as they call for a complete examination, the practitioner is almost forced to a certain conclusion by the fact that the examination remains negative except in regard to the liver. The liver seems large and sensitive, and may sometimes be considerably hypertrophied, for cases have been seen in the ox where the normal weight of 10 to 12 lbs. has been increased to 60 or even 100 lbs., while in the pig, whose liver normally weighs 4 lbs., the weight has been as high as 20 or 40 lbs. In such cases percussion and palpation show that the liver extends beyond the right hypochondriac region and invades a large portion of the corresponding flank. But such great enlargement is exceptional, and when only a dozen vesicles are present, al-

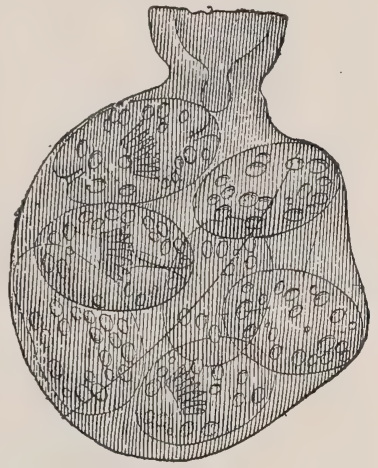

FIG. 131.-Echinococcus bladder worm or hydatid. though the functions of the liver may be seriously disturbed, the information obtained by physical examination is seldom sufficient to justify an exact diagnosis. The liver is found to be enlarged and thickened; otherwise the examination gives negative results.

The diarrhoa may result from failure of the liver to secrete sufficient bile to destroy intestinal toxins, or to carry on its glycogenic function; but it may possibly be the direct result of chronic intoxication by the contents of the vesicles.

Experience has shown, in fact, that in man, when a superficial vesicle becomes ruptured, the peritoneal cavity is flooded with the contents of the cyst; the daughter cysts adhere to the peritoneum, and that almost invariably vascular disturbance occurs, accompanied by itching of the skin and an eruption resembling that of urticaria.

The liquid of the vesicles contains an active toxalbumin.

Diagnosis. In certain cases, diagnosis is possible, and even easy, but in others it is extremely difficult and almost impossible.

Prognosis. The prognosis is always grave, for if the

FIG. $132 . \quad-$

Taniaechinococcus. lesions in the liver do not produce death, as usually happens, they so profoundly affect the animals' general state, that it is no longer worth while to keep them alive.

No practical treatment exists. In exceptional cases it certainly might be possible, although in the large herbivora always difficult, to expose 
the liver and to puncture and evacuate the contents of some of the cysts; but the result would be illusory, because some vesicles would always be inaccessible, and economically intervention would be incomplete and useless.

Although there is no useful method of treatment, prophylaxis is possible and valuable. It consists in preventing the development of tæniæ in farm and sporting dogs. For this purpose it is sufficient to prevent their obtaining raw offal containing vesicles of echinococci from sheep, oxen, or pigs, and also to free them from any helminths which they may harbour. In this way they no longer spread eggs of tæniæ

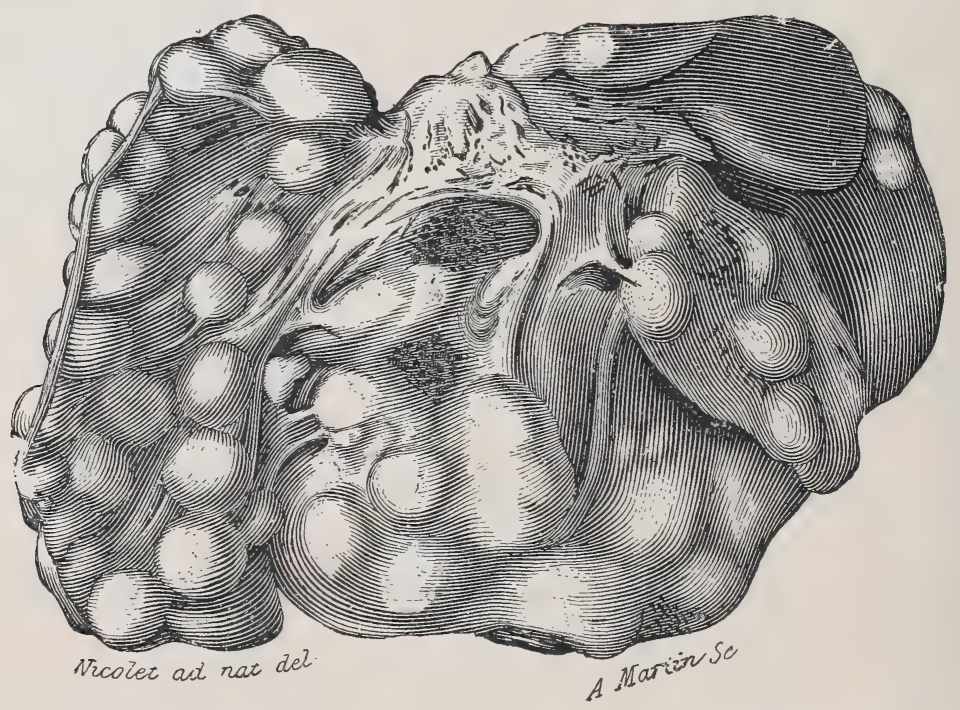

FIG. 133.-Pig's liver with echinococcus cysts. (After Railliet.)

with their fæces in the neighbourhood of ponds or drinking places, and the cattle do not ingest the embryos.

\section{SUPPURATIVE ECHINOCOCCOSIS.}

Causation. Simple echinococcosis may remain undetected for a long time, and young animals affected with it may grow up without exhibiting marked general disturbance. The old echinococci end by degenerating, the wall of the cyst becomes modified, the liquid it contains, turbid, lactescent, then caseous; the vesicle becomes wrinkled, and finally nothing resembling the primary vesicle remains. The liquid is soon absorbed, and the primary cyst is only represented by a caseous magma, which undergoes calcareous infiltration and progressive atrophy.

Under other circumstances the development of the echinococcus 
vesicles is less regular; they may become accidentally infected and transformed into encysted abscesses, constituting suppurative echinococcosis of the liver. The membrane of the vesicles usually resists the passage of microbes, but the fibrous tissue surrounding the cyst is very vascular; and if, in consequence of vascular disturbance in the liver (which may result simply from feeding, trifling infection or other visceral disease), the blood should for a short time be infected, microbes penetrate through solutions of continuity in the wall of the vesicle, which becomes a centre of suppuration. The liquid becomes turbid, the primary cyst is transformed into an abscess, and suppurative echinococcosis is set up.

Symptoms. The general condition resulting from the development of suppuration in echinococcus cysts is very different from that of true echinococcosis. If the abscess develops rapidly, acute generalised peritonitis or localised peritonitis of the right anterior abdominal region may almost immediately occur, producing all the characteristic symptoms of ordinary peritonitis. In all cases, even in the absence of well-marked peritonitis, perihepatitis occurs, and the liver becomes adherent to the posterior surface of the diaphragm, to the hypochondriac region, to the abdominal wall, or to one of the gastric compartments.

This perihepatitis is indicated by exceptional sensitiveness in the right hypochondriac region, and by respiratory disturbance due to fixation of the diaphragm.

In certain cases these abscesses seem to develop like "cold" abscesses -i.e., without fever, and this without producing very marked digestive disturbance; but the patients waste rapidly, become weak, show slight subicteric coloration of the membranes, and appear to lose their strength. Movement is slow and hesitating, as though the animals were suffering from laminitis, the anæmia becomes more marked from day to day, and examination of the blood reveals abundant leucocytosis, the existence of which often assists in the diagnosis of internal suppuration. In a few months, at least in the cases we have seen, the animals become cachectic.

In other and still more obscure cases suppuration of the liver is accompanied by total hypertrophy, excessive sensitiveness in the right hypochondriac region, progressive loss of appetite, excessive thirst, and uncontrollable diarrhoea and fever, although in the case mentioned above there was little fever and no diarrhœa. The course of these cases, which probably result from intestinal infection, is much more rapid. In a fortnight or three weeks, sometimes less, the patients are carried off by intoxication, generalised purulent infection, or septicæmia.

Diagnosis. The diagnosis of suppurative echinococcosis and of primary abscess of the liver is difficult to establish. It is attained D.C. 
chiefly by a process of exclusion, though the signs furnished by percussion of the right flank, and by

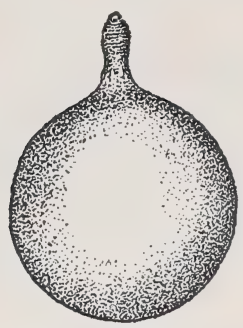

FIG. 134.-Thin - necked bladder - worm (Cysticercus tenuicollis), with head extruded from body, from cavity of a steer, natural size. (Stiles, Annual Report, U.S.A. Bureau of Agriculture, 1901.) examination of the blood, are of some assistance.

Prognosis. The prognosis is extremely grave.

Treatment is of little value. Even supposing that the diagnosis has been exact, surgical intervention is out of the question, and only this would appear theoretically to offer a chance of success. The abscesses are multiple, deeply placed, separated from one another, and sometimes surrounded by enormous tracts of inflamed tissue. In fact, the condition is of such a character as entirely to prohibit active measures.

\section{CYSTICERCOSIS.}

This disease is produced by the thin- or longnecked bladder-worm (Cysticercus tenuicollis) found

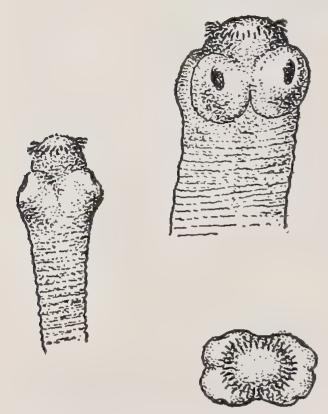

Fig. 136.-Head of the marginate tapeworm (Tania marginata). $\times$ 17. (Stiles, Annual Report, U.S.A. Bureau of Agriculture, 1901.)

in cattle, sheep, and swine. The cysticercus represents an intermediate stage of development of the marginate tapeworm (Tania marginata) of dogs and wolves.

It is by no means uncommon in Europe and America, and occurs in the body cavity of cattle, sheep, swine, and other animals, attached to the diaphragm, omentum, liver, or other organ.

When eaten by dogs or wolves, it develops into the marginate tapeworm, which was formerly confused with $T$. solium of man, and gave rise to the
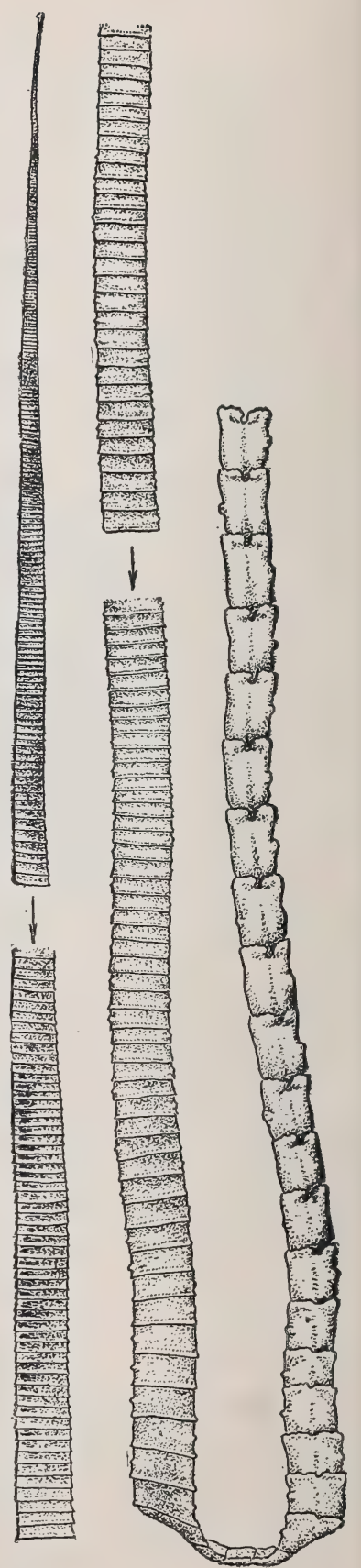

FIG. 135.-The marginate tapeworm (Tania mar. ginata), natural size. (Stiles, Annual Report, U.S.A. Bureau of Agriculture, 1901.) 
erroneous idea that the pork-measle tapeworm occurs in dogs as well as in man.

Life history. In tracing the life history it is best to begin with the

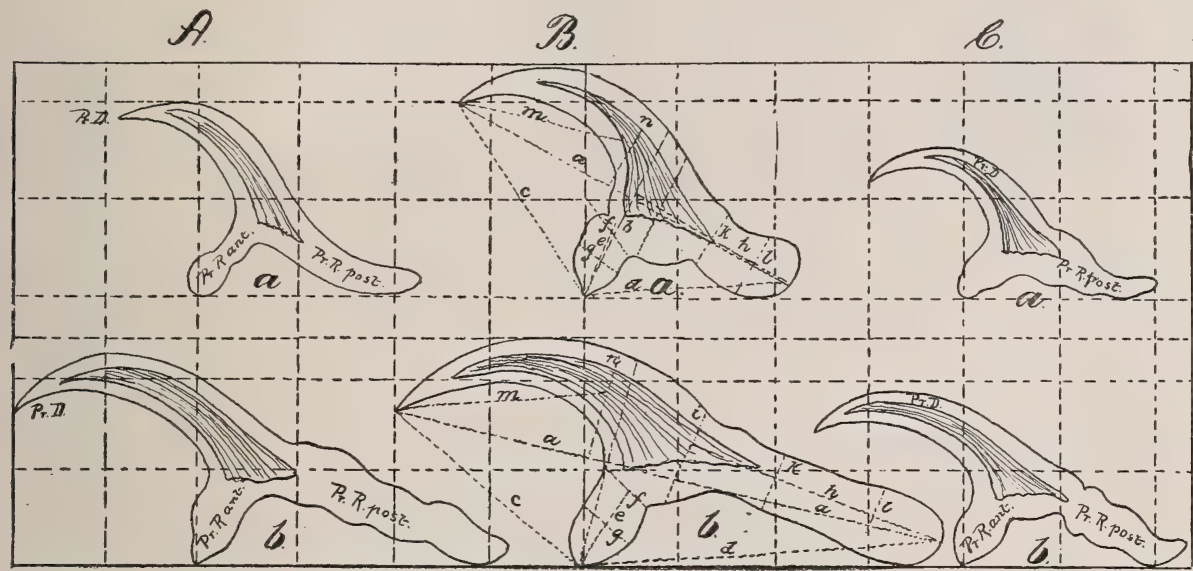

FIG. 137.-Small and large hooks of (A) Trenia marginata, (B) T. serrata, and (C) T. conurus. $a$, Small hooks; $b$, large hooks. $\times 480 . \quad$ (After Deffke.)

egg, produced by the adult tapeworm in the intestine of dogs. These eggs, containing a six-hooked embryo, escape from the dog with the

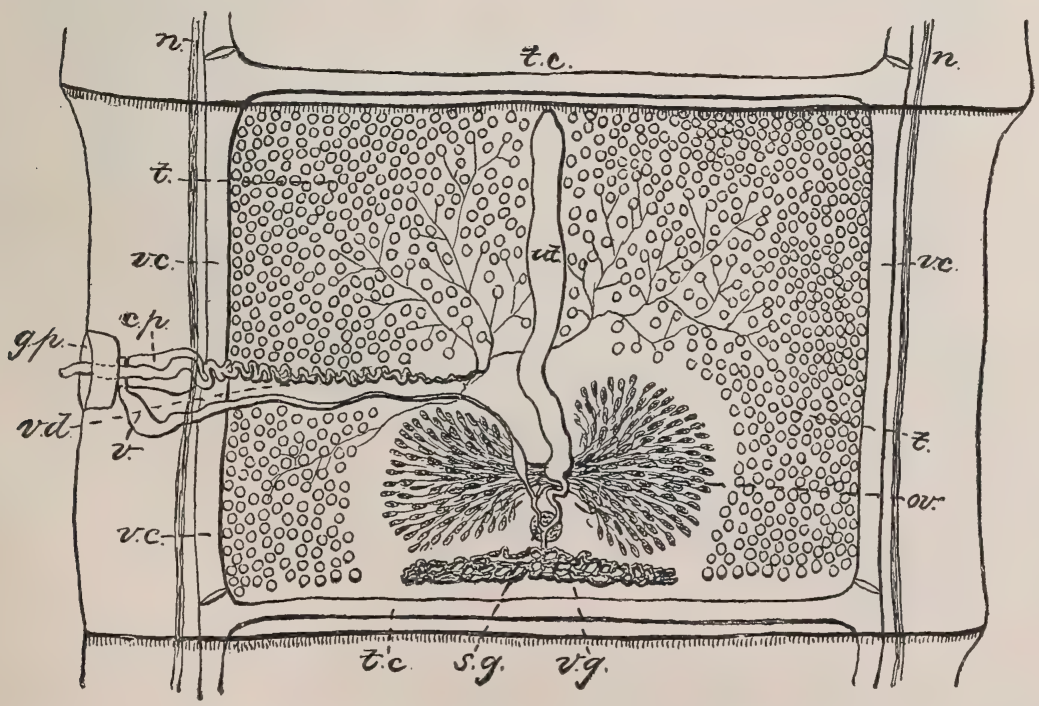

FIG. 138.-Sexually mature segment of the marginate tapeworm (Tania marginata). $c p$, Cirrus pouch; $g p$, genital pore ; $n$, nerve ; ov, ovary ; $s g$, shell gland ; $t$, testicles ; $t c$, transverse canal; $u t$, uterus ; $v$, vagina; $v c$, ventral canal ; $v d$, vas deferens; $v g$, vitellogene gland. Enlarged. (After Deffke.) 
excrements, and are scattered on the ground, either singly or confined in the escaping segments of the tapeworm. Once upon the ground,

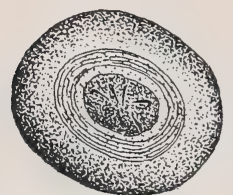

FIG. 139.-Egg of the marginate tapeworm (Tanix marginata), with six-hooked embryo, greatly magnified. (Stiles, Annual Report U.S.A.Bureau of Agriculture, 1901.) they are easily washed along by rain into the drinking water, ponds, or brooks, or scattered on the grass. Upon being swallowed with fodder or water, they arrive in the stomach of the intermediate host (cattle, sheep, etc.), where the eggshells are destroyed and the embryos set free. The embryos then traverse the intestinal wall, and, according to most authors, arrive either actively, by crawling, or passively, by being carried along by the blood, in the liver or lungs, where they undergo certain transformations in structure. While still in the finer branches of the bloodvessels of the liver, which they transform into small, irregularly-shaped tubes about 12 to $15 \mathrm{~mm}$. long and 1 to $1.5 \mathrm{~mm}$. broad, the embryos lose their six hooks, and develop into small, round kernels, which are generally situated at one

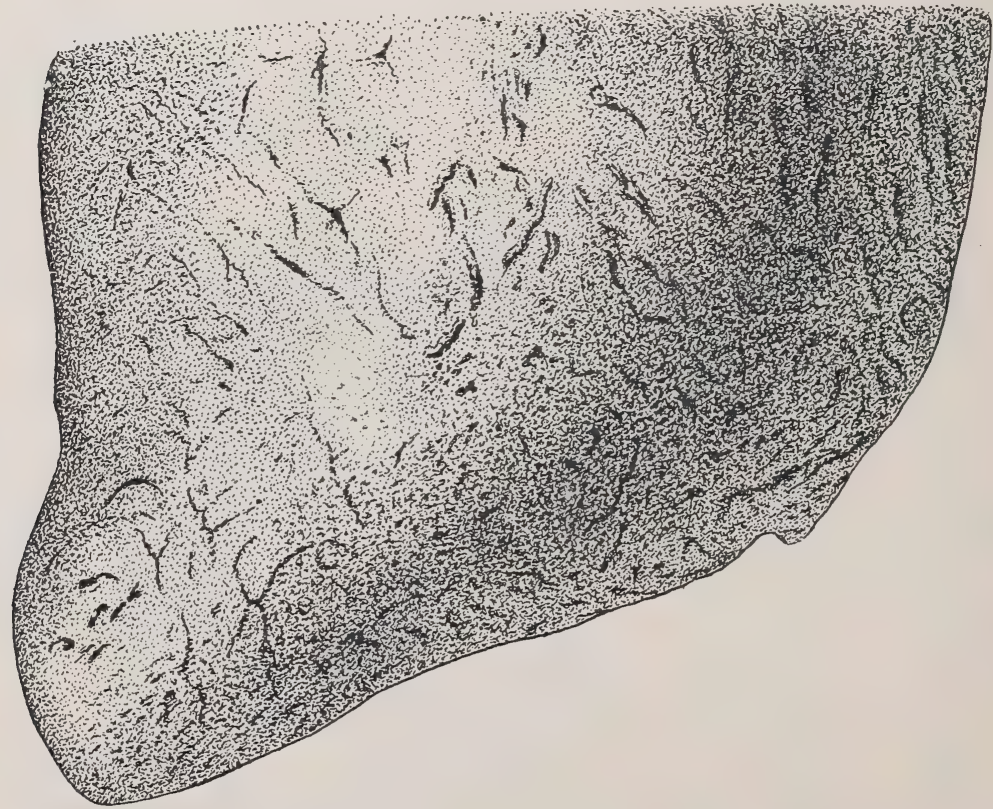

FIG. 140.-Portion of the liver of a lamb which died nine days after feeding with eggs of the marginate tapeworm (Tania marginata), with numerous "scars," due to young parasites. (After Curtice.)

end of the tubes. The embryo can first be seen about four days after infection. The "scars" (Figs. 140 and 141) described in the liver of 
animals infested with Cysticercus tenuicollis are nothing more nor less than these tubes, or altered blood-vessels, caused by the growth and wandering of the parasites.

Curtice takes a somewhat different view-that is, he considers the liver as a place of destruction for the young parasites, rather than a normal place for their development; he also claims that the embryos, which may even travel the entire length of the intestine of the intermediate host, traverse the intestine and arrive directly in the position where they complete their larval development without first passing: through the liver.

After developing into the full-grown bladder worm, the parasites remain unchanged until they are devoured by a dog or wolf, or until,

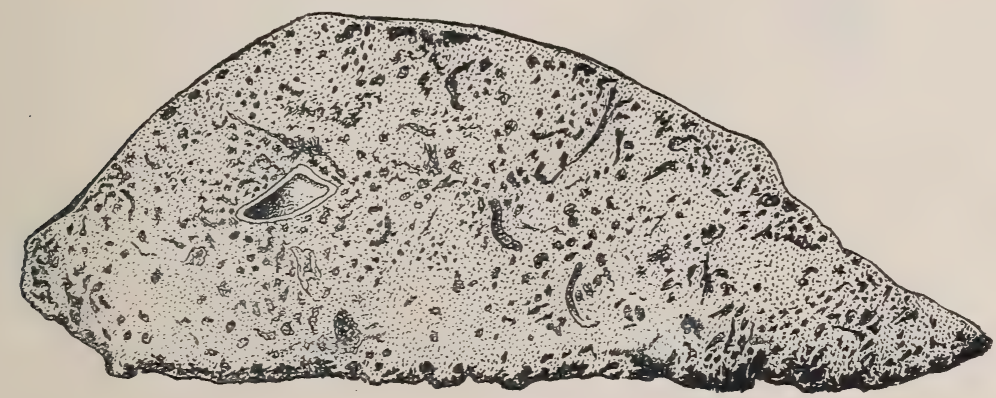

FIG. 141.-Cross-section of the liver of a lamb which died nine days after feeding with eggs of the marginate tapeworm (Tania marginata). (After Curtis.)

after an undetermined length of time, they become disintegrated and more or less calcified.

If the hydatid is devoured by a dog or wolf, either when the latter prey upon the secondary host or when the dog obtains the cyst at a slaughter-house, the bladder portion is destroyed, the scolex alone remaining intact in the digestive fluids. The head holds fast to the intestinal wall with its suckers and hooks; by strobilation (transverse division) it gives rise to the segments, which as we have already seen, together with the head, go to make up the adult tapeworm. Reproductive organs of both sexes develop in the separate segments, and eggs are produced, within which are developed the six-hooked embryos, the point from which we started.

\section{DISTOMATOSIS-LIVER FLUKE DISEASE-LIVER ROT.}

In France the name of distomatosis has been given to a disease caused by the presence of distomata in the bile ducts. It is the "liver rot" of England, the Eberfäule of Germany, and is produced by the growth in 
the biliary ducts of oxen, sheep, and goats of two species of distomata, viz., the Distoma hepaticum or Fasciola hepatica, and the Distoma lanceolatum.

In 1875 Zundel established the causative relation between the presence of distomata in the liver and the development of progressive fatal cachexia in most of the animals affected. This

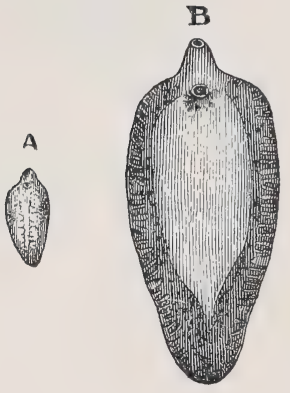

Fig. 142.-Fasciola hepatica. A, young; B, adult parasite. (After Railliet.) opinion was emphasised by the works of Leuckart and Thomas on the development of distomata, and at the present day the parasitic theory is accepted as beyond question.

Fasciola hepatica (Distoma hepaticum).--The common liver fluke of cattle, sheep, swine, etc.

Life history. The adult parasite, instead of producing young similar to itself and capable of developing directly into adults in cattle, produces eggs which develop into organisms totally different from the adult form, living a parasitic life in other animals. In scientific language, the parasite is subject to an alternation of generations, together with a change of hosts. The following summary of the life history will make this point clear :-

(a) The adult hermaphroditic worm (Figs. 144 and 145) fertilises itself (although a cross fertilisation of two individuals is not impossible) in the biliary passages of the liver, and produces a large number of eggs.

(b) Eiggs (Figs. 143, 146 and 147).-Each egg. is composed of the
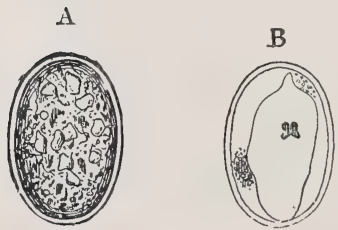

Fig. 143.--Eggs of Fasciola hepatica. A, from the bile duct; $\mathrm{B}$, embryonic; $\mathrm{C}$, after opening. (After Railliet.)

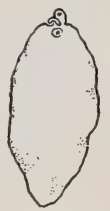

FIG. 144.-Common liver fluke (Fasciola hepatica), natural size. (Stiles, Annual Report, U.S.A. Bureau of Agriculture, 1901.)

following parts: (1) A true germ cell, which originates in the ovary and is destined to give rise to the future embryo; (2) a number of vitelline or yolk cells, which are formed in a specialised and independent portion (vitellogene gland) of the female glands-instead of developing into embryos the yolk cells form a follicle-like covering for the true germ cell, and play an important role in the nutrition of the latter as it undergoes further 


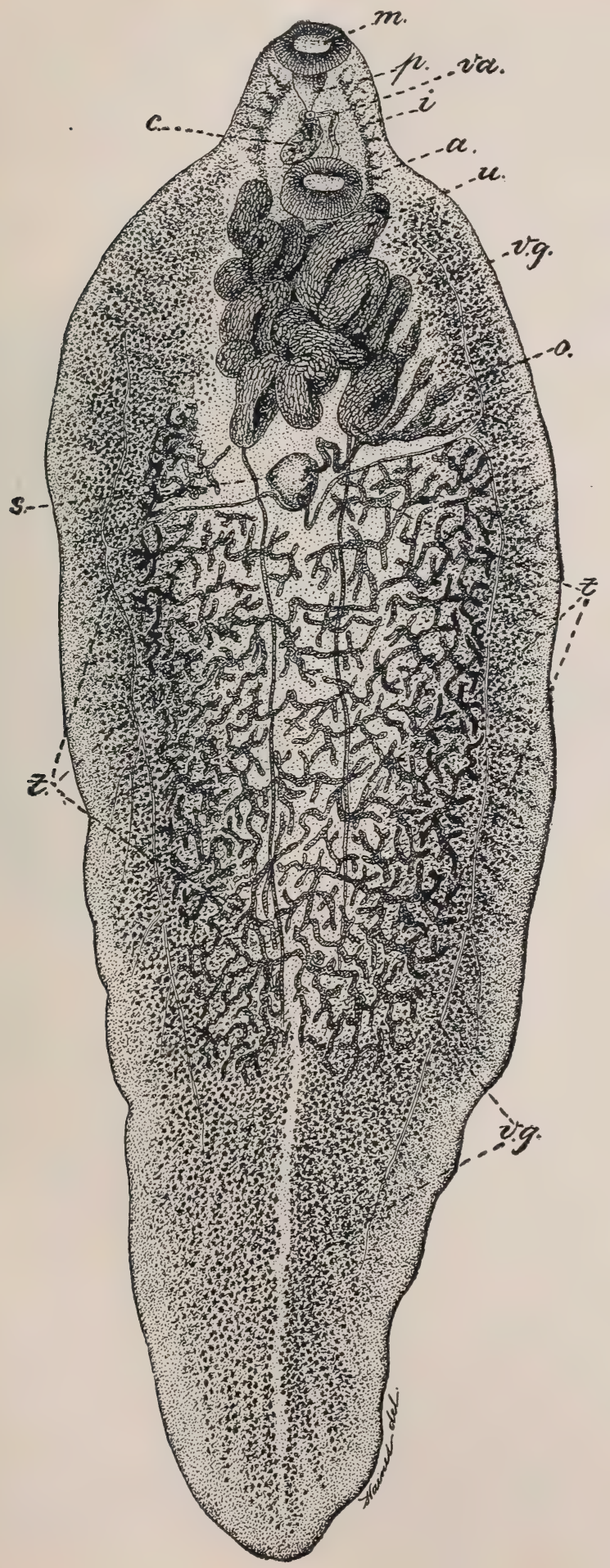

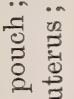

$$
\begin{aligned}
& 3= \\
& \text { है } \\
& \text { ० } \frac{1}{0} \\
& \text { ‥ } \\
& \text { छ } \\
& \text { క్ }
\end{aligned}
$$

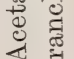

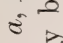

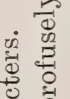

$$
\begin{aligned}
& \text { है } \\
& \text { స్ } \\
& \text { ० 을 } \\
& \text {. } \frac{\pi}{30} \\
& \text { 긍 } \\
& \text { है चू } \\
& \text { o की } \\
& \text { क्ष } \cdots \\
& \text { 츨 } \\
& \text { 은. } \\
& \text { का } \\
& \text { - } 00 \\
& \varnothing_{0} \overrightarrow{10} \\
& \text { क्ञ है क्षै } \\
& \text { क) } \\
& \text { क } 2 \\
& \text { हु } \\
& \text { 胥 } \frac{7}{6} \\
& \text { है। } \\
& \text { \&. } 08 \\
& \text {. } \\
& \text { के गे } \\
& \text { 政 }
\end{aligned}
$$

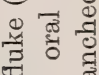

$$
\begin{aligned}
& \text { 다 월 } \\
& \text { 허는 } \\
& \therefore \geqslant \\
& \text { 등 왈 일 } \\
& \text { ฮี ฮี } \\
& \text { है के } \\
& \text { 0... } \\
& \text { E } \\
& \text { मे }
\end{aligned}
$$

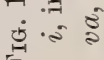


development ; (3) a shell surrounding the germ cell and vitelline cells, and provided at one end with a cap or operculum. The eggs escape from the uterus of the adult through the vulva, are carried to the intestine of the host with the bile, then pass through the intestines with the contents

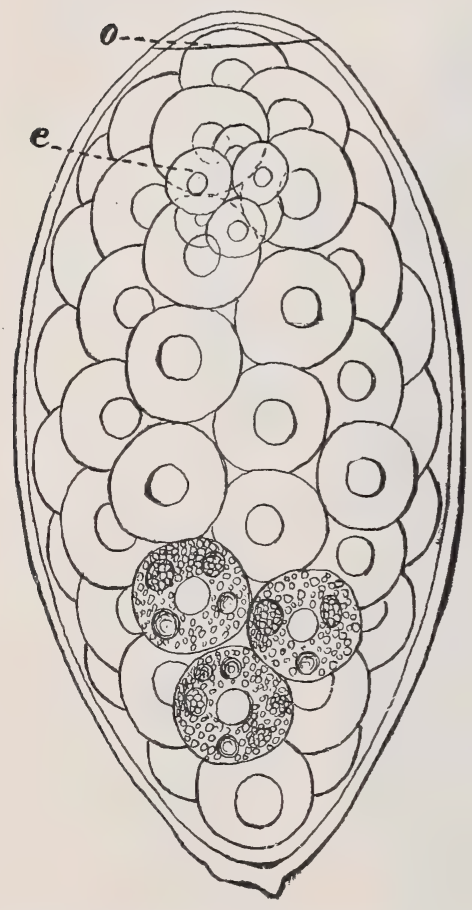

Fig. 146.-Egg of the common liver fluke (Fasciola hepatica), examined shortly after it was taken from the liver of a sheep. At one end is seen the lid or operculum, 0 ; near it is the segmenting ovum, $e$; the rest of the space is occupied by yoke cells which serve as food; all are granular, but only three are thus drawn. $\times 680$. (After Thomas, 1883, p. 281, Fig. 1.)

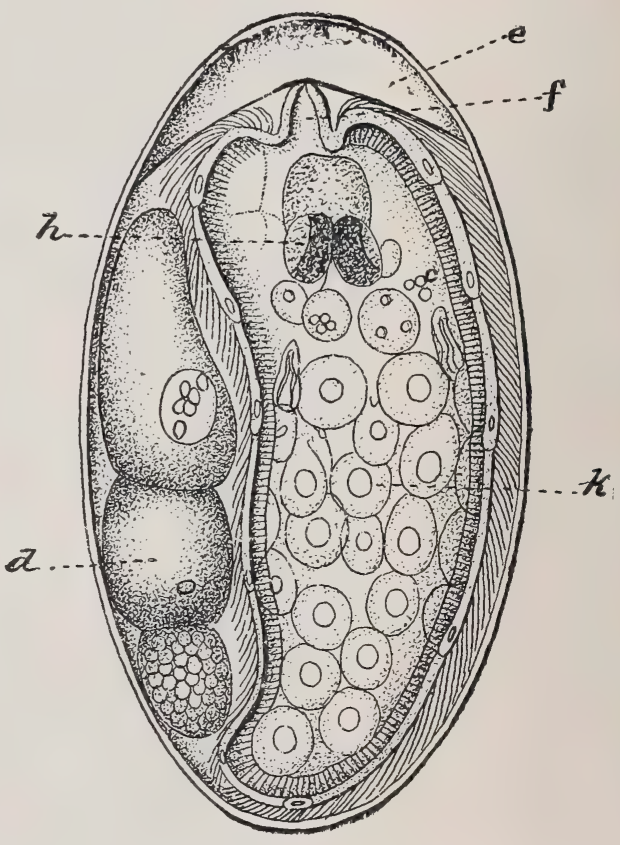

FIG. 147.-Egg of the common liver fluke containing a ciliated embryo (miracidium) ready to hatch out: $d$, remains of food; $e$, cushion of jelly-like substance; $f$, boring papilla; $h$, eye-spots ; $k$, germinal cells. $\times 680$. (After Thomas, 1883, p. 283, Fig. 2.)

of the latter, and are expelled from the host with the fæcal matter. Many of them become dried, and then undergo no further development; but others are naturally dropped in the water in marshes; or, being dropped on dry ground, they are washed into water by the rain, or are carried to a more favourable position by the feet of animals pasturing or passing through the fields. After a longer or shorter period of incubation, which 
varies with the temperature, a ciliated embryo (miracidium) is developed. At a temperature of $20^{\circ}$ to $26^{\circ} \mathrm{C}$. the miracidium may be formed in ten days to three weeks; at a temperature of $16^{\circ} \mathrm{C}$. the development takes two to three months; at $38^{\circ} \mathrm{C}$. it ceases entirely. Experiments have shown that as long as these eggs remain in the dark the miracidium will not escape from the egg-shell; accordingly it will not escape during the night. When exposed to the light, however, or when suddenly brought

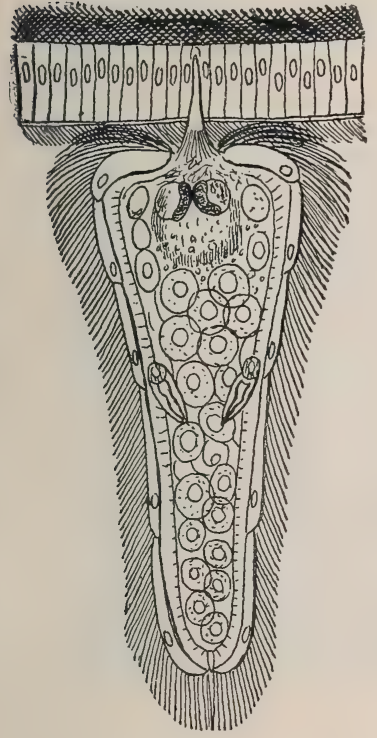

FIG. 148.-Embryo of the common liver fluke ( $F$ asciolahepatica), boring into a snail. $\times 370$. (After Thomas, 1883, p. 285, Fig. 4.)

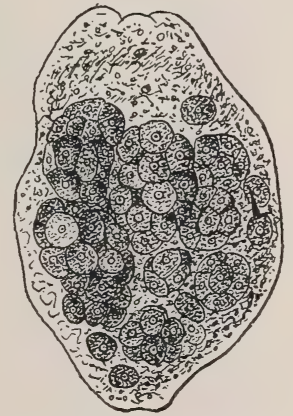

FIG. 149.-Sporocyst of the common liver fluke which has developed from the embryo, and contains germinal cells. $\times 200$. (After Leuckart, 1889, p. 109, Fig. 67 B.)

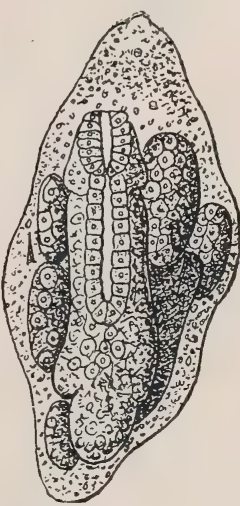

FIG. 150.-Sporocyst of the common liver fluke, somewhat older than that of Fig. 149, in which the germinal cells are giving rise to rediæ. $\times 200$. (After Leuckart, 1889, p. 109, Fig. 67 C.)

into contact with cold water, the organism bursts the cap from the egg-shell, crawls through the opening, and becomes a-

(c) Free-swimming ciliated miracidium (Fig. 148).- As already stated, this organism is entirely different from its mother. It measures about $0.15 \mathrm{~mm}$. long; is somewhat broader in its anterior portion than in its posterior portion; on its anterior extremity we find a small eminence, known as a boring papilla; the exterior surface of the young worm is covered with numerous cilia, which by their motion propel the animal through the water; inside the body we find in the anterior portion a simple vestigial intestine and a double ganglionic mass provided with a peculiar pigmented double cup-shaped eye-spot; in the posterior portion 
of the body cavity are found a number of germ cells, which develop into individuals of the next generation.

Swimming about in the water, the miracidium seeks out certain snails (Limnce truncatula, L. oahuensis, L. rubella), which it immediately attacks (Fig. 148). The miracidium elongates its papilla and fastens itself to the feelers, head, foot, or other exterior soft portion of the body of the snail;

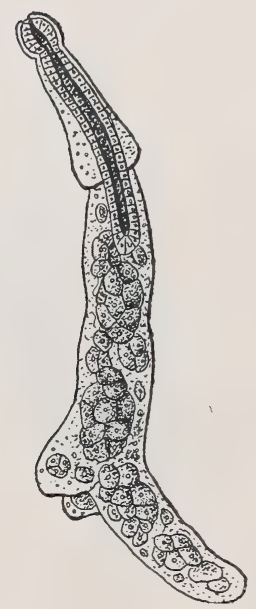

FIG. 151.-Redia of the common liver fluke (Fasciola hepatica), containing germinal cells which are developing into cercariæ. $\times 150$. (After Leuckart, 1889, p. 269, Fig. 129 A.)

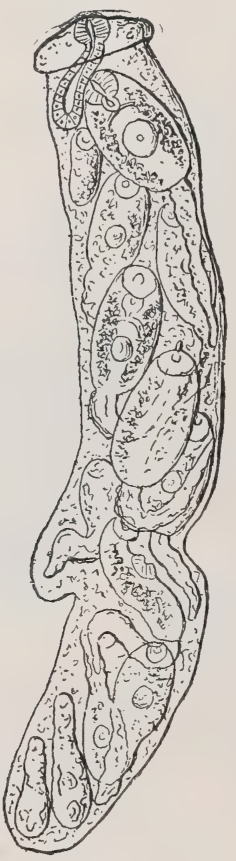

FIG. 152.-Redia of the common liver fluke, with developed cercariæ. $\times 150$. (After Leuckart, 1889, p. 270, Fig. 130.)

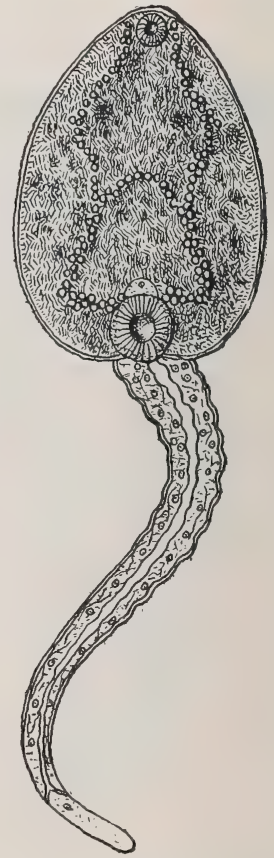

Frg. 153.--Free cercaria of the common liver fluke, showing two suckers, intestine, large glands, and tail. (After Leuckart, 1889, p. 279, Fig. 137.)

some of the parasites enter the pallial (lung) cavity and attach themselves there. After becoming securely fastened to the snail the miracidium discards its ciliated covering, and shortens to about half its former length $(0.07 \mathrm{~mm}$. to $0.08 \mathrm{~mm}$.). The parasites now bore their way into the body of the snail, and come to rest in the liver or near the roof of the pallial cavity, etc.; the movements gradually cease, and we have before us the stage known as the-

(d) Sporocyst (Figs. 149 and 150).-The eye-spots, ganglionic swellings, and vestigial intestine become more and more indistinct, and are finally 
lost. The sporocyst grows slowly at first, then more rapidly, and at the end of fourteen days or so measures $0.5 \mathrm{~mm}$. The germ cells mentioned as existing in the posterior portion of the miracidium now develop into individuals of a third generation, known as-

(e) Redice (Figs. 151 and 152).-The rediæ escape from the sporocyst when the latter are from two weeks (in summer) to four weeks (in late fall) old. Upon leaving the body of the sporocyst they wander to the liver of the snail, where they grow to about $2 \mathrm{~mm}$. long by $0.25 \mathrm{~mm}$. broad. Each redia consists of a cephalic portion, which is extremely motile, and which is separated from the rest of the young worm by a ridge; under the latter is situated an opening, through which the next

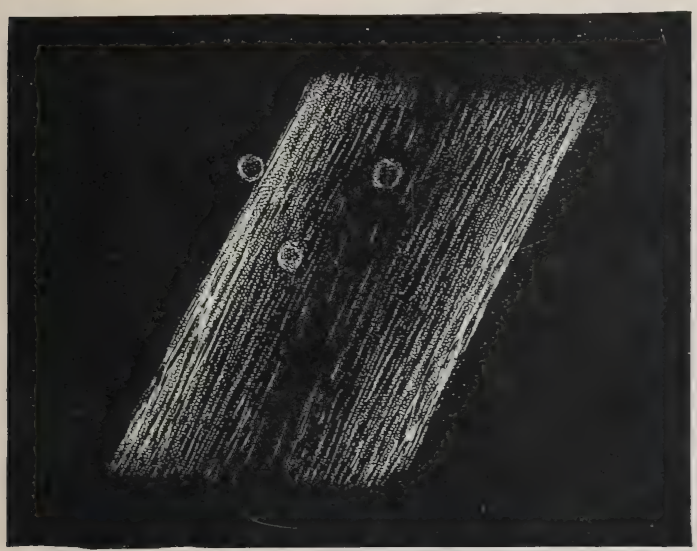

Frg, 154.-Portion of a grass stalk with three encapsuled cercariæ of the common liver fluke (Fasciola hepatica). $\times$ 10. (After Thomas, 1883, p. 291, Fig. 13.)

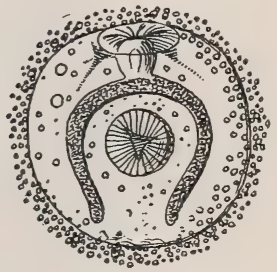

FIG. 155.--Isolated encysted cercaria of the common liver fluke. $\times 150$. (After Leuckart, 1889 , p. 286, Fig. 142.)

generation (cercariæ) escape. The posterior portion of the worm is provided, at about the border of the third and the last fourths of the body, with two projections. There is a mouth with pharynx situated at the anterior extremity, the pharynx leading to a simple blind intestinal sac. The redia, as well as the sporocyst, may be looked upon as a female organism, and in its body cavity are found a number of germ cells, which develop into individuals of the next generation, known as-

( $f$ ) Cercarice (Figs. 153-155). - These organisms are similar to the adult parasites into which they later develop. The body is flat, more or less oval, and provided with a tail inserted at the posterior extremity. The oral sucker and acetabulum are present as in the adult, but the intestinal tract is very simple; on the sides of the body are seen two large glands, but the complicated genital organs of the adult are not visible. The cercaria leaves the redia through the birth opening, remains 
in the snail for a longer or shorter time, or passes out of the body of the snail and swims about in the water. After a time it attaches itself to a blade of grass (Fig. 154) or some other object, and forms a cyst around itself with material from the large glands, at the same time losing its tail. It now remains quiet until swallowed by some animal. Then, upon arriving in the stomach-of a steer, for instance-the cyst is destroyed, and the young parasite wanders through the gall-ducts or, as some believe,

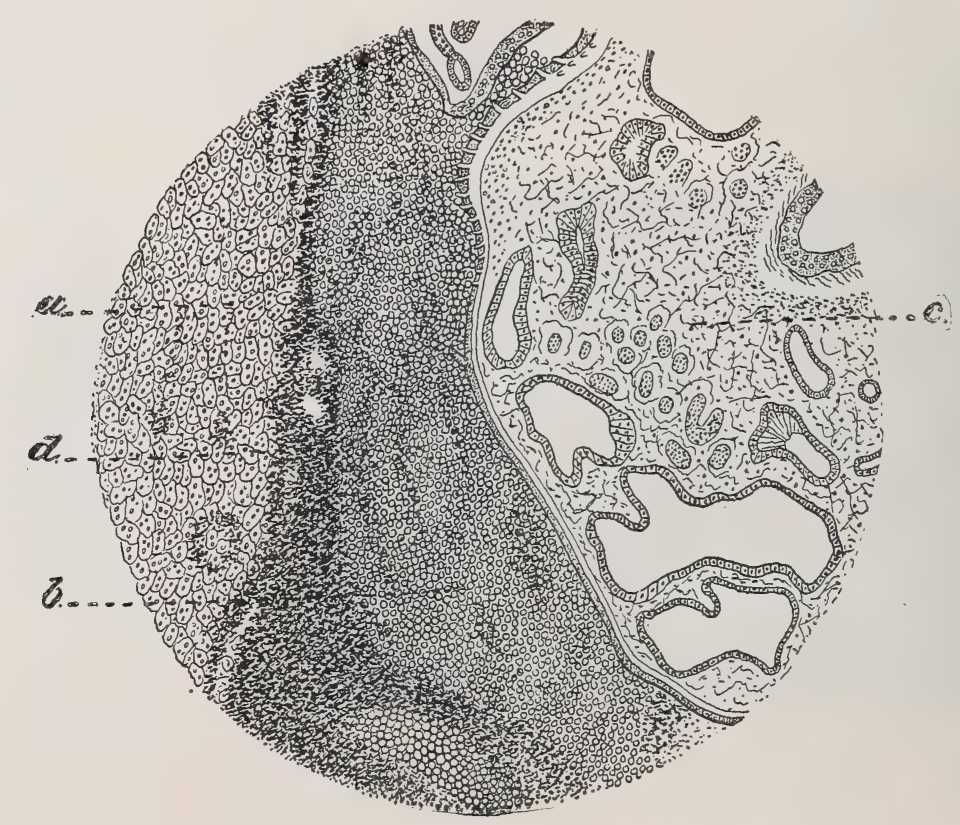

FIG. 156.-Drawing from a microscopic preparation, showing a hæmorrhage in the parenchyma of the liver caused by the common liver fluke (Fasciola hepatica). $a$, Atrophic liver tissue; $b$, round-cell infiltration; $c$, a portion of the parasite; $d$, hæmorrhage. (After Schaper, 1890, Pl. I., Fig. 1.)

through the portal veins to the liver, where it develops into the adult hermaphrodite.

From the above we see that this parasite runs through three generations, namely:

(1.) Ovum, miracidium, and sporocyst . . . first generation.

(2.) Redia . . . second generation.

(3.) Cercaria and adult . . . third generation.

During this curious development, which lasts about ten to twelve weeks, there is a constant potential increase in the number of individuals, for each sporocyst may give rise to several (five to eight) rediæ, each redia to a larger number (twelve to twenty) cercariæ, and each adult to an 
enormous number $(37,000$ to 45,000$)$ of eggs. This unusual fertility of the parasite is necessary because of the complicated life history and the comparatively small chance any one egg has of completing the entire cycle.

Hosts. An interesting and, from an agricultural standpoint, an important matter connected with this fluke is that it is found in a large number (about twenty-five) of domesticated and wild animals, and this

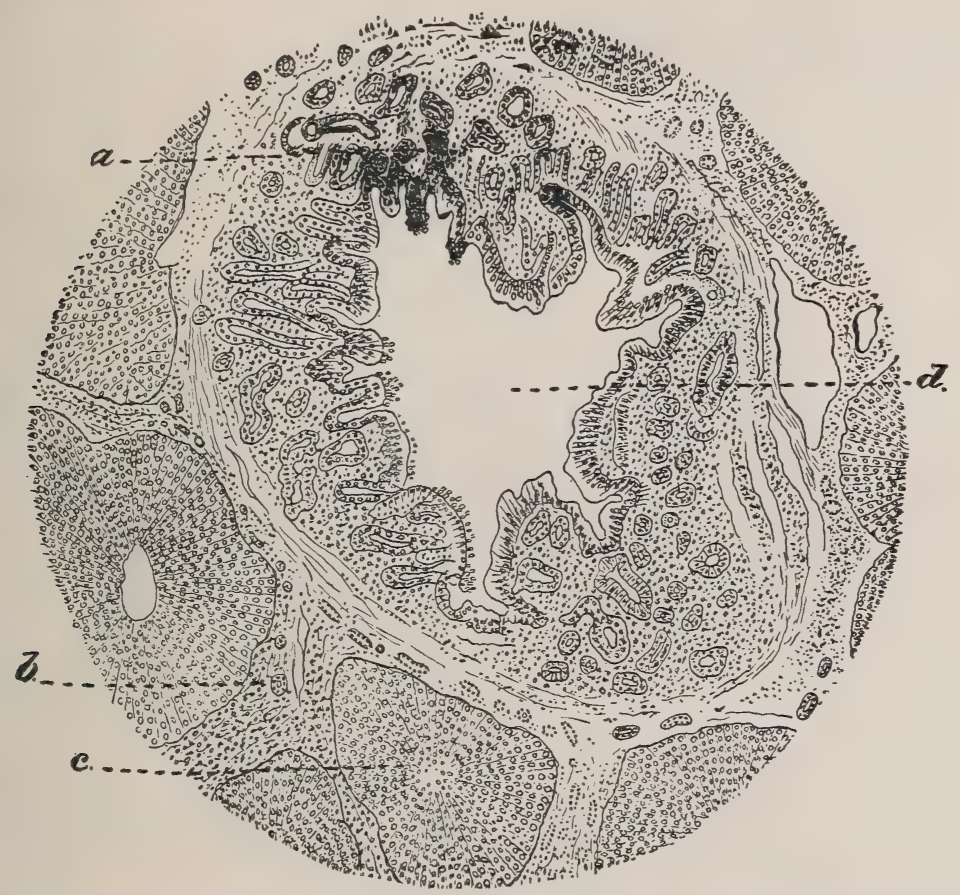

FIG. 157.-Drawing from a microscopic preparation, showing the glandular hyperplasia of the mucosa of a gall-duct caused by the common liver fluke (Fasciola hepatica). $a$, Hypertrophied submucosa ; $b$, interstitial connective tissue ; $c$, compressed lobule; $d$, lumen of the gall-duct-thickened fibrous wall of the gall-duct. (After Schaper, 1890, Pl. I., Fig. 2.)

fact probably explains to some degree the wide geographical distribution of the parasite.

Symptoms. The symptomatology of this disease may clinically be divided into three well-marked periods :

I. Primary period. The primary phase commences with the penetration of the embryos of the parasite into the body, firstly into the intestine, and then into the liver by ascending the bile ducts. This phase occurs during the last months of the year, October, November, and December, and is rarely accompanied by alarming symptoms. At this time the sheep appear in good health, the summer being over, and the 
animals, being well nourished and fat, are able to resist the first attacks of the parasite, so that even an observant shepherd only notices a little dulness, want of condition, and muscular weakness. It requires a carefully trained eye to note these very general symptoms, for the bodily condition only changes very slowly and progressively, the appetite remaining good. Experienced butchers, however, in the districts where distomatosis is common, readily detect this condition. The animals make little resistance when handled.

Nevertheless, even in this primary phase, the conditions are not always

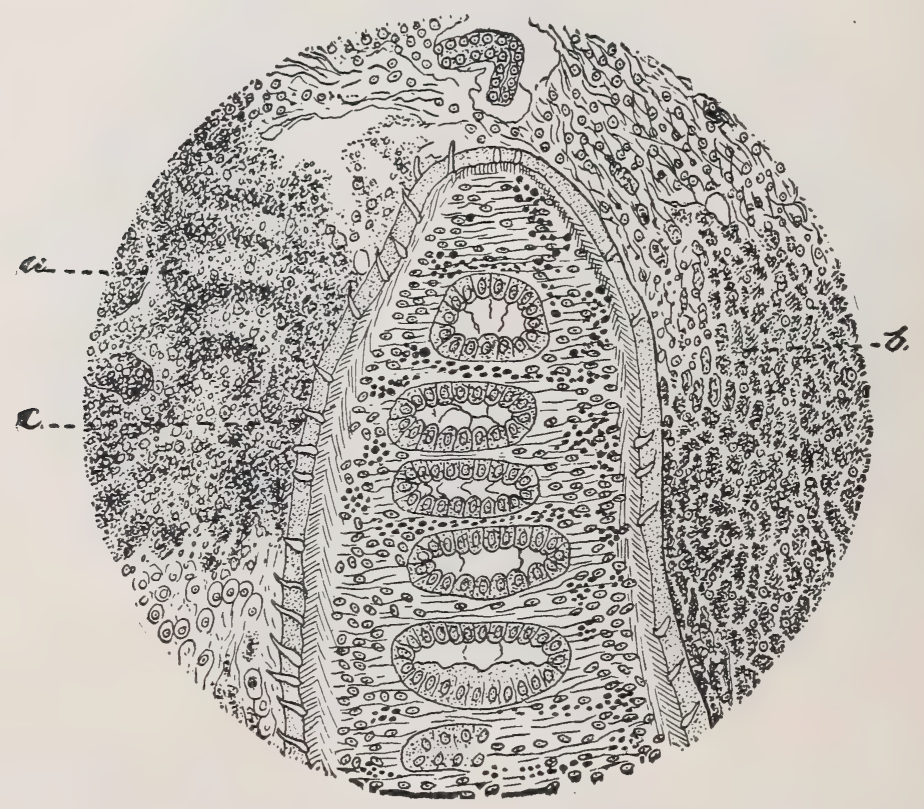

FIG. 158.-Drawing from a microscopic preparation showing a fluke in the tissue of the liver. $a$, Necrotic liver tissue ; $b$, atrophic liver cells; $c$, spines on the fluke, showing the outline of the body. (After Schaper, 1890, Pl. III., Fig. 5.)

as above sketched, and a certain number of deaths may occur. Gerlach has mentioned the possibility of death by cerebral apoplexy, in consequence of the young distomata penetrating to the brain. Moussu has certainly never seen such a complication, but has seen death from hepatitis, perihepatitis, and secondary pericarditis in animals gravely infested. The young embryos, whether they penetrate only by the bile ducts, as has been stated, or are carried to the liver by the blood stream, often excavate canals in the substance of the gland before establishing themselves in the bile ducts. They make their way as far as Glisson's capsule, and may even penetrate it; and as they carry with them innumerable intestinal germs, when they arrive vici the bile ducts, they set up 
hepatitis, perihepatitis, with the formation of numerous false membranes, or even infectious fibrinous peritonitis. Should the patients die during this phase one finds young distomata at the surface of the liver, or even in the thickness of the false membranes.

Jan. Feb. Mar. Apr. May Jun. July. Aug, Sept. Oct. Nov. Dec.
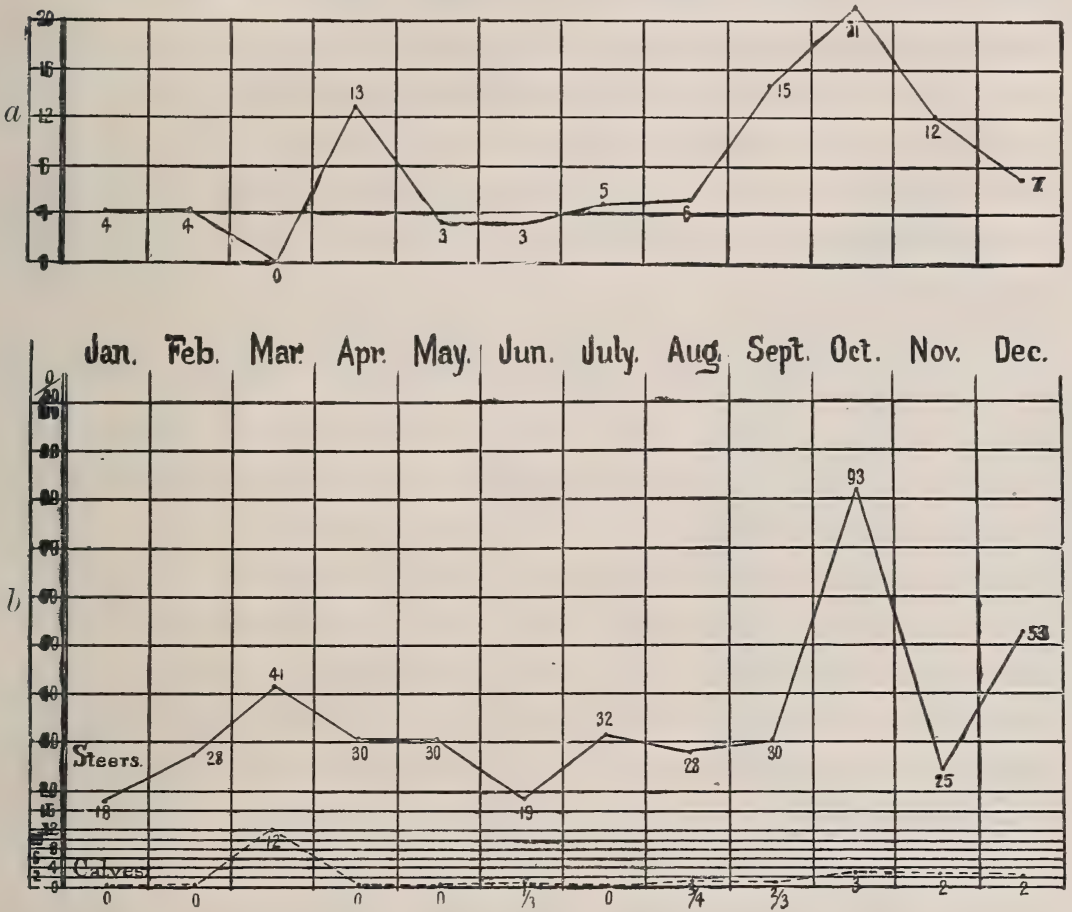

Jan. Feb. Mar. Apr. May. Jun. July. Aug Sept. Bet. Nov. Dec.

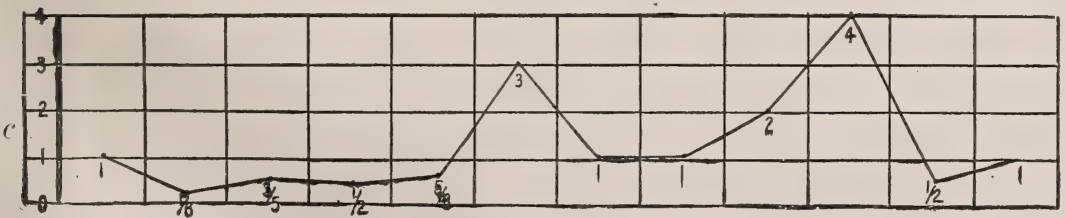

FIG. 159.-Tabular diagram of the occurrence of the common liver fluke (Fasciola hepatica) during different months of the year. $a$, Cattle; $b$, sheep; $c$, swine. (After Leuckart, 1889, p. 301, Fig. 147.)

When infestation is discrete the appearances are quite different. Careful breeders have even stated that at this period the young sheep appear to show a greater tendency to fatten.

II. Second period. In the primary phase deaths are exceptional; they 
only become common towards the end of the winter. During the second or middle period (December and January) the patients lose flesh, appear less active, show less regular appetite and greater thirst. The conjunctiva becomes pale and swollen, the sclerotic has a bluish tint, and the eyelids are somewhat infiltrated. The wool appears drier and less curly; locks of wool part readily from the skin, and the individual fibres become dry and fragile.

This phase is accompanied by very marked anæmia, rapid exhaustion

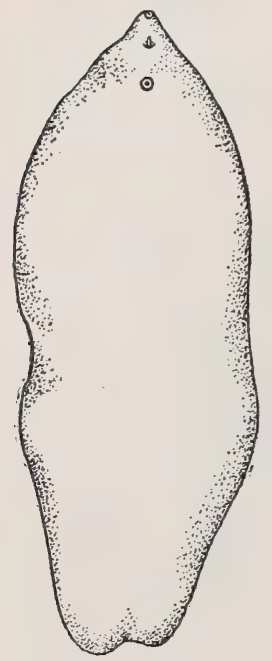

FIG. 160.-The large American fluke (Fasciola magna), natural size. (Stiles, Annual Report, U.S.A. Bureau of Agriculture, 1901.) during movement, and inability to run for any length. of time.

The different methods of examination reveal nothing specially striking, except that the valvular sounds of the heart are sharper, and that trifling œdema occurs under the thorax and abdomen.

Microscopic examination of the fæces reveals the presence of eggs of distomata. The sheep rapidly become thin from about the end of January, even although the appetite persists and nourishing food is given.

III. Third, or wasting, period. The decline, which sets in about February, appears extremely obstinate, and resists all treatment.

The patients become feeble, eat less, and digest badly. Submaxillary œdema, common to advanced wasting diseases, then appears. If the sheep are removed from the fold to pasture, the swelling of the submaxillary space is very noticeable. It consists in an indolent œdematous tumefaction, which disappears when the animals are travelled, but reappears when grazing on account of the low position in which the head is then held.

The condition then becomes complicated with diarrhœa, and soon grows alarming. On examination, extensive dropsy may often be found in the thorax, pericardium, and abdomen.

Death results from exhaustion; the animals do not appear to suffer, but become extraordinarily anæmic, and perish without a struggle. The blood is simply rosy in colour, like gooseberry syrup: the clot is soft and gelatinous: the number of red blood corpuscles has fallen from about seven millions to a few hundred thousand.

Icterus is rare, though certain cases have been described where it has appeared during the last and even during the middle stage.

When animals begin to die in a district which has long been infested, the losses are enormous, the condition sometimes constitutes a perfect 
scourge. It should be remarked, however, that all those affected do not die; animals kept under good conditions may even survive for several months, although greatly wasted.

Towards March and April the parasites leave their position, and are conveyed by the current of bile towards the intestine, to be rejected with the fæces. This is the period of convalescence and recovery; but recovery is only relative, for the parasites are never entirely evacuated. The distomata then recommence their life cycle outside the animal body.

Unfortunately the mortality caused by distomata is accidentally aggravated by other diseases, and the scourge then becomes an absolute disaster for the districts where such complications occur. Thus Besnoit and Cuillé, of Toulouse have shown that distomatosis may become complicated with a form of very rapidly fatal hæmorrhagic septicæmia, produced by an ovoid bacterium.

Distomatosis, already sufficiently grave, then becomes infinitely more serious, if only from the fact that it may prove the point of origin of an absolutely fatal complication.

In bovine animals the symptoms develop exactly as in sheep, though the cachectic period is uncommon and the injury done is often less important than in sheep. The patients exhibit irregular appetite, wasting without appreciable cause, anæmia, and even diarrhœa. In spite of excellent winter feeding they do not regain condition, and relative re-

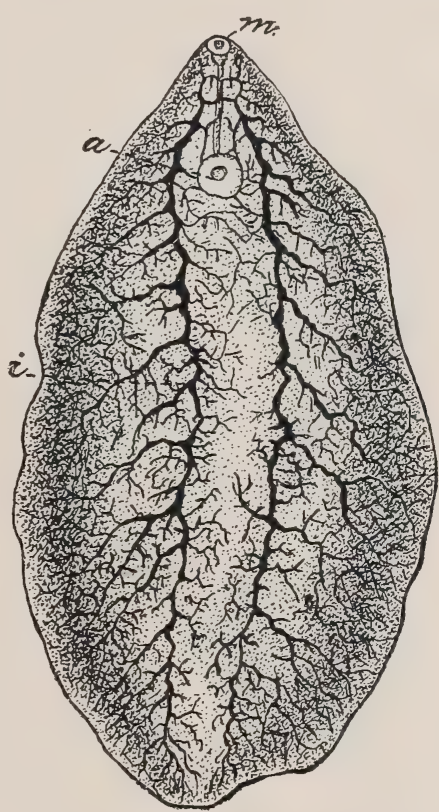

Fig. 161.-Macerated specimen of large American fluke, showing the digestive system and acetabulum. $\times 2$. (After Stiles, 1894, p. 226, Fig. 2.) covery only sets in with the approach of spring. Death from simple distomatosis is exceptional, but in animals so predisposed enteritis develops more easily, as do all forms of infection of intestinal origin.

The disease is, however, also grave for bovines because successive reinfection occurs, and the disease may be prolonged for years.

Causation. Distomatosis is due to one cause, viz., the entrance of embryo flukes into the digestive apparatus of herbivora.

The adult distomata in the biliary ducts continually discharge large quantities of eggs, though the process is most active between February and June or July. The eggs are carried away with the bile and fæces

D.C. 
and pass on to the pastures, where they continue their life cycle, thanks to moisture and the presence of stagnant water. The embryos, having escaped from the egg, enter the bodies of the snails found in or near stagnant water (Limncea truncatula), become converted into sporocysts, and afterwards into rediæ and cercariæ. The cercariæ become encysted on the lower surface of blades of grass in damp pastures, whence they are transferred to the animals' stomachs along with the grass itself.

As the Limncea truncatula lives not only in marshy regions, but also in all damp situations, the embryos of distomata are distributed over

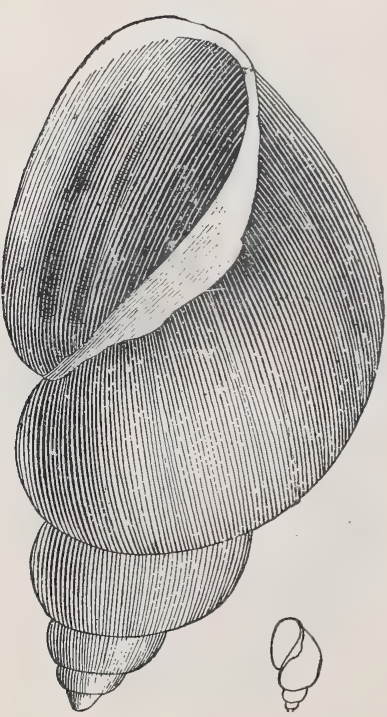

FIG. 162.-Limncea trunca. tula. Natural size and magnified. (After Railliet.) enormous areas, and the disease itself is equally widespread. The embryo, after ingestion, is set at liberty, and passes from the intestine into the innermost recesses of the liver, being guided up the bile ducts by the current of bile. At this point it attaches itself to the wall of the bile duct, passes through its various stages of evolution, and attains the adult form. It then begins laying eggs, and thus starts a new evolutionary cycle.

The life cycle of Distoma lanceolatum is not yet known, and this variety, moreover, is less widely distributed than the Distoma hepaticum.

The bile ducts are more easily penetrated by the distoma in young animals, a fact which explains why ealves and lambs are particularly affected. Adults present a less favourable nidus, a fact which renders them less easily infected, but does not entirely prevent the parasites from attacking them. Old animals, although unable to resist entirely, seldom harbour many of the parasites.

Wet years appear to favour the extension and propagation of distomatosis in an extraordinary fashion, a fact which is easily understood, if we regard the phases of evolution of the parasite. The autumn appears particularly favourable to the infection of herds. This is explained by the fact that, during the summer, the dryness of the fields entirely prevents the development of such eggs as may be distributed over them; whilst wet periods during the autumn favour this development.

On the other hand, the grass becomes eaten down in autumn, so that the animals gather it almost level with the ground. As the cercariæ attach themselves to the lowest leaves they are then ingested in much 
larger quantities. The bad effects of wet seasons are not immediately apparent, but appear during the following spring.

Distomatosis is common throughout almost the whole of Europe, Africa, and America. In France it is most serious in the moister regions of Sologne, in Berry, the mountainous and wet districts of the great central plateau, and particularly in the Pyrenees. It particularly attacks oxen in the valley of the Meuse, the marshes of Picardy, the lower regions of Normandy, and in all the mountainous pastures of the central plateau.

Lesions. The lesions of distomatosis vary with the stage of development of the parasites. During the primary phase of invasion of the bile ducts by young distomata one finds interstitial diffuse hepatitis, due to perforation of the gland by young parasites, adhesive perihepatitis, with the formation of false membranes, and not uncommonly slight peritonitis.

Zoologists state that the young distomata penetrate the liver by passing upwards against the current of bile. It does not appear impossible, however, that they may penetrate by another path, particularly as so-called "erratic" forms of distomatosis like distomatosis of the lung, heart, lymphatic glands, and various other tissues are not uncommon. It has been suggested that the young distomata, arriving in the bile ducts, perforate the gland, giving rise to these lesions of perihepatitis, peritonitis or erratic distomatosis; but this view is scarcely in harmony with the fact that the parasites are usually found in the bile ducts.

During the second phase, corresponding to the development of almost adult distomata, the perihepatitis and peritonitis set up either produce fatal results by secondary infection or diminish and disappear. The parasites develop in the bile ducts, in which they attain the adult condition. They steadily ascend towards the origins of the ducts, dilating them in their passage in an extraordinary way. The number of parasites varies greatly: sometimes there are but few, and they are only discovered on post-mortem examination; in other cases the bile ducts are crammed with them, as many as six or seven hundred or even a thousand being present. The distended bile ducts always show chronic peripheral inflammation, which steadily becomes aggravated, producing pericanalicular atrophying sclerosis. This condition is followed by change in and disappearance of a certain quantity of hepatic tissue, and by various forms of vascular and secretory disease.

This is the period of greatest disturbance, not only in consequence of the actual presence, but also of the mode of living, of the parasites.

Moussu declares that the parasites live principally on blood, at least during the first and second stage of their sojourn in the liver, adducing as proof that if one completely injects the vascular system of the liver 
(arteries and veins), some of the injected matter will be found a day afterwards in the digestive apparatus of the parasites.

The disturbances which they produce are therefore due to their actual presence and its consequences, to their mode of life, and to the intercurrent infections of which they are sometimes the initial cause.

It is idle to object that the part played by these parasites is less important than has been suggested, and that the mortality results from intercurrent infection, and not from the parasites themselves. It is equally idle to point out that carcases of animals suffering from severe infection with distomata, particularly the carcases of sheep, are frequently found in slaughter-houses, in perfectly fat condition, and with the appearance of not having suffered in any way. These observations are perfectly correct and well founded. But it matters little that death results from an infection superadded to the distomatosis, if the presence of distomata is the determining factor in causing the superadded infections, and if such infection is, as Moussu believes, almost inevitable in animals already exhausted by the action of the parasites.

The fact that animals suffering from distomatosis and slaughtered for food are well nourished is not a valid objection; for it has long been known that wasting and anæmia are not immediate consequences, and that before they are clearly apparent the distomata must have been present in the liver for several months. Bakewell and the Marquis of Behague have shown that in moderately infected animals there is a tendency to lay on flesh during the first and a portion of the second stage of development of the disease.

If the animals are slaughtered before the period of progressive decline sets in, it is quite possible to form entirely wrong views regarding the importance of these parasites.

The wasting process commences towards the end of the second phase of the disease, and then makes rapid progress. The parasites, which have then been continuously drawing on the blood for their nourishment for a long time, produce anæmia, and some infection of the bile ducts, and usually a certain degree of icterus.

The third phase is accompanied by general signs of cachexia, which need not again be described. They are similar to those of all progressive cachexias. In animals which survive this phase and are ultimately slaughtered the liver always shows very marked sclerosis, commencing around the biliary ducts. Even after the parasites have been evacuated, these ducts appear indurated, thickened, fibrous, and sometimes encrusted with biliary deposits or obstructed with true calculi. These calculi may or may not contain parasites; sometimes they simply contain eggs : they are open, tubular, and perforated, but always irregular on the surface. 
When in addition complications have appeared, one usually finds general lesions of septicæmia and blood infection.

In erratic distomatosis, which is of no importance clinically, distomata may become encysted in the lung or other viscus, and in time die. The cysts, which only contain one and rarely two parasites, present a fibrous shell, enclosing a blackish, pultaceous, grumous magma, which sometimes has undergone a certain amount of calcareous infiltration. The parasite may be entirely destroyed.

Diagnosis. Early diagnosis is difficult, and can only be established by microscopic examination of the excreta and the discovery of eggs. On an average one may find one egg in each preparation when the liver contains 80 to 100 flukes. When wasting is very marked, and particularly when there has already been a number of deaths, diagnosis becomes extremely easy. It is sufficient to find flukes in any form (Distoma hepaticum vel lanceolatum) to be assured as to the cause of disease.

Prognosis. In severely infested cases the prognosis is extremely grave, because no efficient method of treatment exists. Embedded in the liver, the parasites resist the action of all drugs, and we know of no anthelmintic eliminated by the bile which in any way affects their vitality. When the disease is recognised early, the most economical method is to fatten the animals as rapidly as possible and prepare them for slaughter.

Treatment. There is no reliable curative treatment. The drugs which one might employ would kill the animal before poisoning the parasites embedded in the liver. Various mixtures containing sulphate of iron, juniper leaves, etc., have been recommended; but rich food constitutes the best of all treatment, both from a curative and a prophylactic standpoint.

With the view of preventing the disease, however, and protecting flocks from attack in places where the disease is common, certain precautions should be adopted. They comprise-(a) providing a free supply of rock-salt, either in masses placed in the mangers or distributed with the food; the salt increases gastric secretion, and has a slight action on the parasites: (b) adding to the food during the first months of winter branches of birch, juniper, willow, and broom; the leaves of these plants contain aromatic or resinous principles which act on the liver, are eliminated by the bile, and may have valuable results.

But of all preventive measures the most effective consist in draining, cleansing, and drying low, moist, or marshy lands, because the molluses which are essential to the life cycle of the parasites are unable to develop where the soil is dry. The manure containing the eggs of 
the parasites may be disinfected by adding to it lime, sulphate of iron, or common salt. Common salt and lime spread over the pastures has a double beneficial influence, acting both as a manure and as a parasiticide. From May to August is the best time for spreading this dressing.

The fluke embryos are destroyed by 1 to 2 per cent. solutions of common salt, and by $\frac{3}{4}$ per cent. solutions of lime.

In over-stocked fields the animals are obliged to graze very close to the ground, and are thus more exposed to infection. Over-stocking should therefore be avoided. Animals should not be left too long on the same ground. If infected they should at once be driven to higher pastures. Raised water tanks can be placed in the pastures-they are less likely to become infected. Animals from known infected flocks or herds should not be purchased. Livers from infected animals should be cooked or destroyed. If eaten raw by dogs the eggs pass uninjured through the dog's intestine and infect fresh pastures.

Sulphate of iron distributed in quantities of 250 to $400 \mathrm{lbs}$. per acre is valuable as a manure, and would probably have a greater effect in destroying the embryos. 


\section{SECTION III. \\ RESPIRATORY APPARATUS.}

\section{CHAPTER I.}

\section{EXAMINATION OF THE RESPIRATORY APPARATUS.}

From the clinical standpoint a study of the respiratory apparatus implies the examination of the nostrils, nasal cavities, frontal and maxillary sinuses, larynx, trachea, and, for the purpose of ascertaining the condition of the lungs and pleura, of the chest.

Nasal cavities. Examination of the external portion of the nasal cavities is extremely simple. The observer notes the degree to which the orifices are dilated, the frequency of the respiratory movements and the condition of the muzzle. He may find various eruptions, crusts due to discharge, etc., and will remark any peculiarities presented by these. The depths of the nasal cavities can only be examined through the nostrils to a very limited extent; but the condition of the pituitary mucous membrane, its degree of vascularity, and the existence of ulcerations or of vegetations can be observed.

Digital examination is sometimes useful in discovering the condition of the lower portions of the turbinated bones. The electric light does not greatly assist the observer, on account of the narrowness of the air passages. On the other hand, examination of the face, and palpation and percussion over the region of the frontal sinuses is of considerable value. By inspection, one discovers deformity caused by tumours, by ossific inflammation, or other lesions; and detection is rendered easier by the fact that deformities are usually asymmetrical, only occurring on one side.

Palpation reveals the degree of resistance and flexibility of the external bony wall as well as the condition of the subcutaneous tissues.

Percussion sometimes discloses absolute dulness, due to tumours of the mucous membrane, of the turbinated bones, or of the bones of the face.

The pharyngeal portion of the nasal cavities is difficult to reach, 
but can be examined by passing the hand, palm upwards, into the pharyngeal cavity, when the fingers may be slipped behind the soft palate and thus introduced into the posterior nasal chamber. The animals should first be very carefully secured and a strong gag introduced into the mouth.

Sinuses. Frontal sinus.- The frontal sinus occupies the greater part of the anterior cranial region and the summit of the head, extending from the highest point of the poll as far forward as a line drawn between the two orbits. Above, it directly communicates with the sinus of the horn core. In this upper region, where it abuts on the sinus of the opposite side along the median line, it is of greatest

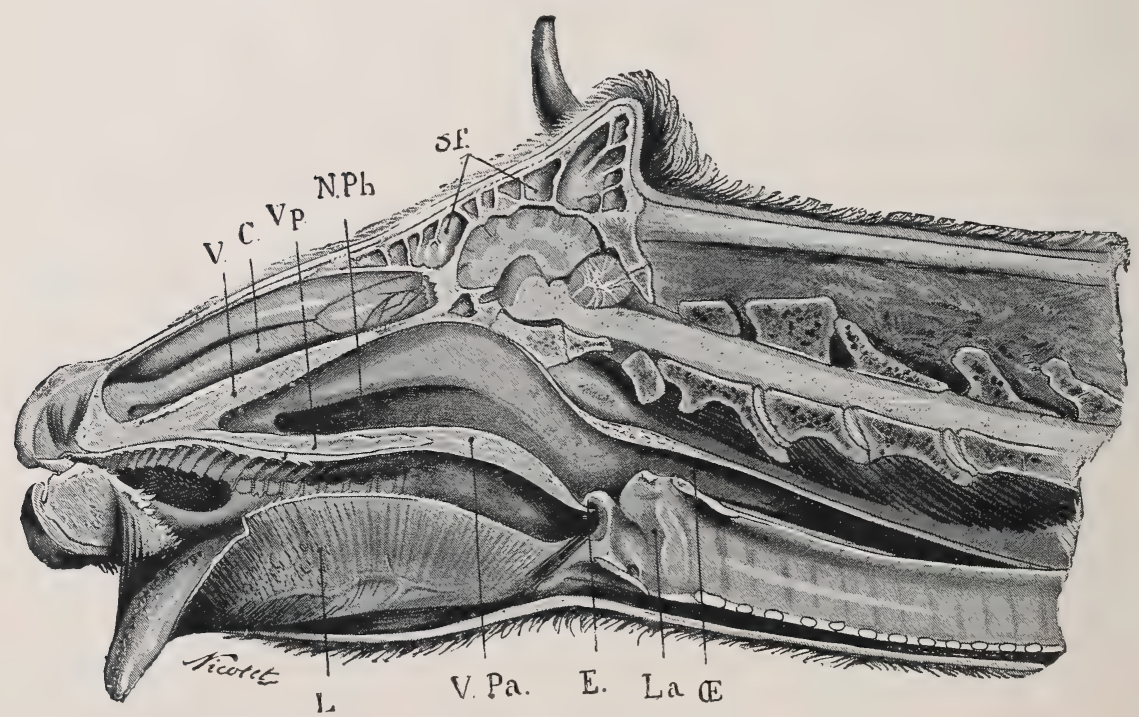

FIG. 163.-Median section through an ox's head. Sf, Frontal sinus; N.Yh. naso. pharnyx; Vp., hard palate ; C., turbinated bones; V., vomer ; L., tongue ; V. pa., soft palate ; E., epiglottis ; La., larynx; (E., œsophagus.

size. Below, on the other hand, it is very narrow, much broken up and incompletely divided into a series of cells, by thin, bony plates running in all directions.

It is in direct communication with the nasal cavity.

The frontal sinus, then, occupies all the supero-lateral portion of the cranial box, which is thus provided with a double wall. At one point, however, over a lozenge-shaped area, the cranial cavity is only protected by a single wall. It is in this region that the slaughterman strikes the animal when killing it.

The frontal sinus may be examined by inspection and palpation, 
Inspection sometimes reveals deformity of the external wall of the sinus. Such deformity, however, is rare, and Moussu has only observed it in two cases of tuberculosis of the bones forming the cranial wall.

By percussion one detects abnormal sensibility, and partial or complete dulness over certain areas.

Maxillary sinus.--The maxillary sinus occupies the whole lateral

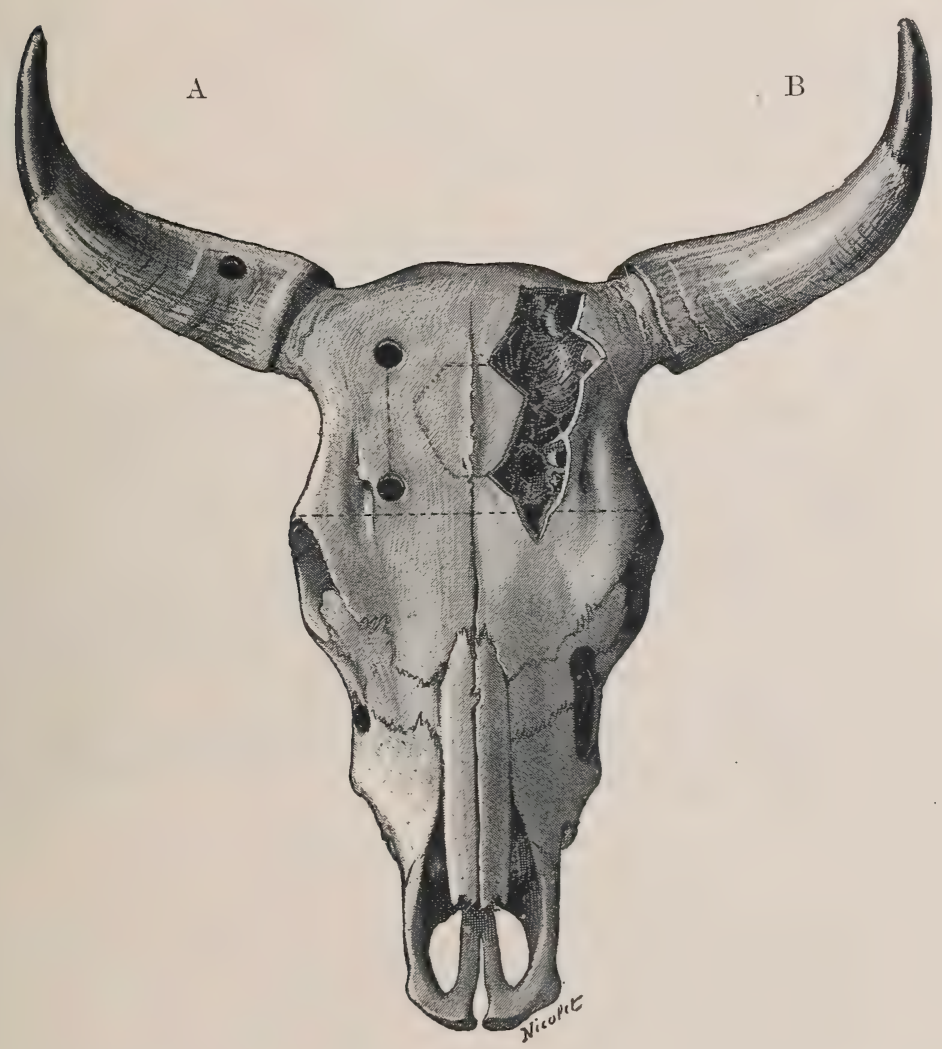

FIG. 164.-General arrangement of the sinuses. The circular openings indicate points at which trepanation is performed.

region of the face, from the inferior extremity of the maxillary spine as far as the sub-orbito-palatine region. In front its external wall is very solid, but behind, below the orbit, is extremely thin. Externally the sinus is largely protected by the anterior insertion of the external masseter muscle, a fact which explains how rarely it is injured.

Larynx. In consequence of its deep situation, the larynx can 
scarcely be examined except by inspection, external palpation, internal digital palpation, and auscultation.

External examination presents no difficulty, and enables one to detect lesions around or near the larynx.

Palpation reveals the existence of œdematous swellings, enlargements of the retro-pharyngeal lymphatic glands, inflammatory engorgements, etc.

Internal digital examination, like examination of the pharynx, can only be performed after very firmly fixing the animal in position, and inserting a gag in the mouth.

The hand is introduced, palm downwards, and passed as far as the

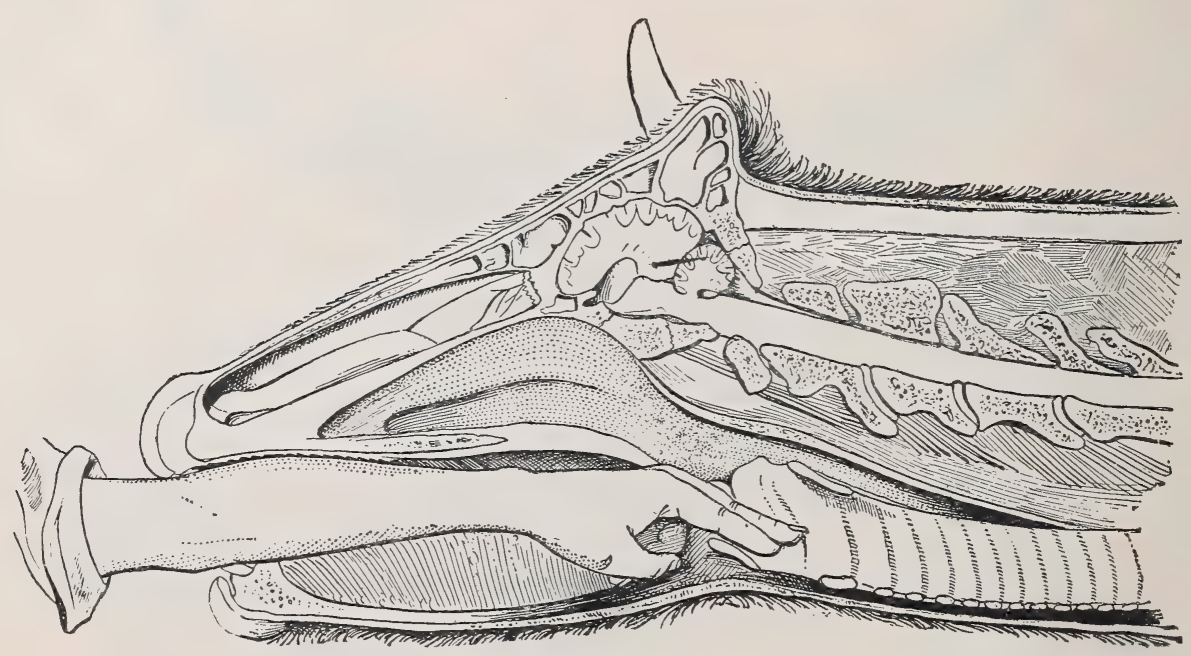

Fig. 165.-Manual examination of the larynx in the ox.

pharyngeal cavity. The index finger can then easily be slipped into the glottis. Exploration must be effected rapidly and without violence. It reveals the condition of the glottis, the presence, position and form of growths like myxomata and of new tissues, due to the presence of actinomyces, as well as tuberculous vegetations or ulcerations.

By auscultation we discover the presence of normal or abnormal laryngeal sounds-roaring, whistling, or bubbling sounds, etc.

Trachea. The trachea may be examined by palpation and auscultation.

Palpation reveals the degree of sensitiveness, abnormalities in position or form, the presence of peripheral inflammations, fractures of rings, etc.

Auscultation indicates whether the tracheal sound be normal or otherwise, or accompanied by abnormal sounds, like mucous râles, or by pathological sounds conveyed from the chest. 
Thorax. The thorax can be examined by inspection, palpation, percussion, and auscultation.

By inspection we discover whether the formation of the thorax as a whole is normal, or whether there exist congenital or acquired deformities, asymmetry like that produced by pneumo-thorax or deviations of ribs from the normal line, etc. One also notes the breathing movements, the manner in which the sides expand and contract, the respiratory rhythm, and any special peculiarities of inspiration or expiration.

Palpation reveals the degree of sensitiveness of the thoracic wall and of the intercostal spaces, the existence of more or less extensive local œdematous infiltration, and the presence or disappearance of thoracic conditions like hydro-thorax.

Percussion indicates the degree of sonority of the chest in different parts. It can either be practised directly with the hand or through the medium of a pleximeter. The latter method is preferable when dealing with fat animals. Percussion, however, gives slightly different results, according to the degree of fatness of the subjects. It should be practised both in a vertical as well as in a horizontal direction.

At all points where the muscles are thick or well developed the results produced are negative, in the sense that only a dull sound is obtained. This is the result obtained in auscultating the areas marked 1, where one meets with the ileo-spinal and common intercostal muscle, and in that marked 4, where the olecranian muscles are encountered (Fig. 166). Over the middle and inferior zones, however, the results are much more instructive.

On the right side percussion of the middle zone gives, under normal conditions, a clear sound and perfect resonance from above downwards, and from in front backwards, between the fourth intercostal space and the ninth rib. Beyond this point lies the liver, which gives a partial dull sound, and absolute dulness from the ninth to the twelfth rib, in consequence of its position, and of the projection of the diaphragm towards the thoracic cavity.

Percussion of the inferior zone produces less marked resonance, which diminishes more and more towards the base, in consequence of the thinness of the pulmonary lobes at this point. This resonance does not extend as far as the hypochondriac region, because the lower part of the abomasum insinuates itself beneath the costal cartilages and causes a region of dulness.

On the left side percussion gives precisely similar results, except in the upper portion of the middle zone. Beyond the ninth intercostal space the sound changes, and has a tympanic character; because here the anterior and upper portions of the rumen are encountered, 
as they are lodged beneath the hypochondrium. Below, the sound is dull, on account of the gastric compartments generally being full of food.

Certain trifling modifications of this normal condition may be noted, depending on the degree of fatness or thinness of the subjects. The pathological changes which may occur are as follows:-

A tympanic sound, with or without metallic character, may be found at a point where normally one would expect a clear sound (pneumothorax, diaphragmatic hernia). A dull sound may be met with in the same regions, all resonance being lost (pneumonia, broncho-pneumonia, pleural exudate, etc.). Partial dulness and partial loss of resonance may occur in regions which ought to give a resonant sound (deep pneumonia, tuberculous lesions, the presence of echinococcus cysts, etc.).

Auscultation-i.e., examination by means of the ear-is the most valuable method of discovering and localising pulmonary, pleural, or cardiac lesions.

Various sensations are conveyed to the ear, depending on the method in which the normal or pathological sounds are produced.

The deductions to be drawn as regards the nature of existing disease are based on the intensity, character, duration, and special attributes of the sounds noted.

Direct auscultation is the most certain method, but the ear cannot be applied with equal facility at all points. Under such circumstances a simple or binaural stethoscope, or the phonendoscope, may be used with advantage.

To properly appreciate the sounds heard it is essential to be exactly acquainted with the relation between the lung and thorax. On the left side (Fig. 60) the anterior pulmonary lobe occupies the space between the first and fourth ribs, in front and above the base of the heart. The middle, or cardiac, lobe covers the left upper and postero-lateral part of the heart from the fourth to the sixth rib. The posterior lobe occupies all the region beyond the sixth rib as far as the twelfth.

On the right side the arrangement is similar, but the anterior lobe and the cardiac lobe are more developed (Fig. 62).

Under ordinary circumstances the extensive movement of the lung which occurs during inspiration produces a special sound known as the respiratory or vesicular murmur. Contrary to what has been written and said, this sound in animals possessing absolutely sound lungs ends with inspiration. Expiration is silent, though it is easy to estimate its duration.

In auscultating the lung, we may distinguish four zones, a superior zone, a middle zone, an inferior zone, and a scapular zone.

The superior zone is bounded by the vertebro-costal gutter, descends 
approximately as far as the inferior line of insertion of the common intercostal muscle, and extends from the summit of the scapula in front to the hypochondrium behind.

Auscultation of this region through the ileo-spinal and common intercostal muscle will always reveal, except in very fat animals, the vesicular murmur to a point as far back as the eleventh intercostal space. Nevertheless, this vesicular murmur is relatively feeble, and becomes imperceptible beyond the eleventh rib.

The middle zone comprises the most convex portion of the ribs, and at this point the wall of the thorax is thinnest, while the lung below is thickest.

For these reasons the vesicular murmur is heard at its maximum

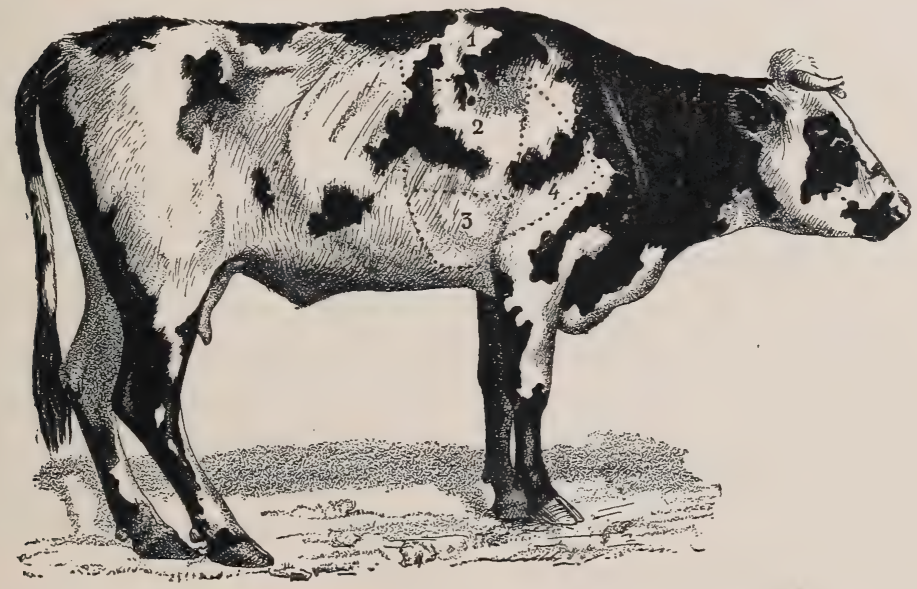

FIG. 166.-The areas over which auscultation of the chest may be performed, showing their extent, position and relations to the thoracic wall. 1, Upper zone; 2 , middle zone; 3 , inferior zone ; 4 , subscapular zone.

intensity at this point. Towards the upper and lower limits of this zone are found the great bronchial divisions, so that auscultation should always be practised with the greatest care at this point. The middle zone occupies approximately one-third of the total depth of the thorax. The vesicular murmur becomes weaker as one passes backwards, and finally disappears at a considerable distance from the angle of the hypochondrium, following a curved line the convexity of which is directed forwards, and which is continuous with that limiting the upper zone.

These peculiarities are due to the anatomical arrangement and mode of insertion of the diaphragm on the internal surface of the hypochondrium. 
The inferior zone is very limited, and corresponds externally to the inferior third of the thorax, and topographically to the cardiac lobe or middle lobe of the lung and to the inferior portion of the posterior lobe.

As these pulmonary lobes are of comparatively slight thickness, the vesicular murmur is feeble. It can be heard over a trapezoidal space, forming a prolongation of the middle zone, but not below in the region of the sternum or pectoral muscles.

The fourth zone extends over the mass of the olecranian muscles. It is of triangular form, in consequence of the inclination of the scapula and humerus. Except in very fat animals the vesicular murmur is readily audible through the muscular mass on the right side better than on the left, on account of the development of the right anterior pulmonary lobe.

On the left side the beating of the heart is heard above the pulmonary sounds.

Clinically one may hear an exaggeration of the ordinary respiratory murmur whenever the lung is actively exercised, as, e.g., immediately after trotting. This exaggeration, however, is often pathological. It is known as "juvenile or supplementary respiration," when due to the fact that some other portion of the lung is not acting.

The respiratory murmur may be lessened in certain morbid conditions, such as emphysema and congestion of the lung, and may completely disappear in pneumonia or broncho-pneumonia, a fact which is even of greater significance.

In various pathological conditions the respiratory murmur may also be modified. On the other hand, the movement of air in the bronchi also produces various sounds of importance.

A number "of different bronchial sounds may be distinguished; these include both inspiratory and expiratory sounds, for sometimes an expiratory sound may become audible and clearly appreciable, or may acquire characters of the greatest importance.

The inspiratory sound may be strong, rough, rasping, painful, moist or rattling. The pathological expiratory sounds may vary between audible, strong, rough, prolonged, or rattling. The varieties of souffle, or rattle, are the tubal souffle of inspiration or expiration (met with in pneumonia or broncho-pneumonia); the soft, deep-seated pleuritic souffle (peripneumonia); the continuous cavernous souffle (met with in tuberculosis); the broad amphoric souffle, in which the vibrations are extensive and of metallic character (met with in pneumo-thorax).

As to the varieties of riles which usually accompany these souffles, they may all be met with in tuberculous animals, and comprise crepitant and sub-crepitant, mucous, cavernous, snoring and sibilant râles. 


\section{CHAPTER II.}

\section{NASAL CAVITIES.}

\section{SIMPLE CORYZA.}

Srmple acute coryza, or inflammation of the mucous membrane of the nasal cavities, is of comparatively trifling importance in bovine animals, and, were it not for the possibility of gangrenous coryza being confused with it, there would scarcely be any necessity for a special description.

The onset of coryza is indicated by repeated sneezing and coughing, by congestion of the pituitary mucous membrane, which soon begins to secrete abnormally, and by difficulty in respiration, which becomes snoring or whistling.

T'he discharge, transparent at first, then mucous and muco-purulent, is abundant in quantity; the inflammation is arrested at this point or extends towards the facial sinuses, the pharynx and larynx; the eyes are swollen and watering, and almost all the characteristic symptoms appear which mark the onset of gangrenous coryza. Two signs, however, are wanting. The appetite is fairly well maintained, and the temperature little above normal. Simple coryza occurs at all times of the year in consequence of sudden chills, but is commonest in spring and autumn.

At first the distinction between this condition and gangrenous coryza can only be ascertained after taking the temperature.

The prognosis is absolutely favourable, and often in forty-eight hours every symptom disappears.

Treatment is confined to keeping the animals in stables at a suitable temperature, sheltered from draughts. Emollient fumigations and inhalations of turpentine, creosote, or eucalyptus oil rapidly check the more alarming symptoms. Warm drinks and foods and cooked roots are recommended.

In sheep, acute coryza as a consequence of chills, etc., occurs in autumn, but is more commonly due to a parasitic cause, viz., invasion of the nasal cavities by larvæ of certain cestridæ. During the succeeding winter it continues under the form of chronic coryza, as a result of the larvæ retaining their position in the sinuses. 
Treatment of this parasitic coryza consists in trephining the sinuses and destroying the larvæ.

\section{GANGRENOUS CORYZA.}

Gangrenous coryza is a grave disease of diphtheritic character, which at first seems to be localised in the mucous membrane of the upper respiratory passages, but which exhibits a tendency to affect all the mucous membranes of the system.

The term "gangrenous coryza," adopted in France, has been replaced in other countries, especially in Germany, by such descriptions as "contagious disease of the head," and "malignant catarrhal fever of the ox." Old writers describe gangrenous coryza as a disease common in the Jura, the eastern parts of France generally, and in the valley of the Saone. In reality this disease occurs everywhere, both in the centre, west and north of France, as well as in the eastern regions. Serious outbreaks frequently occur in Germany and Italy.

Symptoms. Gangrenous coryza assumes three different forms, which, however, only represent successive degrees of intensity of the attack. In the peracute form death occurs in three to five days, even when the characteristic signs are not all as yet apparent. In the acute, and by far the most frequent form, the disease lasts from fifteen to twenty days, and also ends in death in by far the greater number of eases. Finally, in the form usually termed chronic, the disease lasts from four to eight weeks, and most frequently ends in recovery.

Acute and peracute forms. - The onset is marked by very striking symptoms, which precede the local symptoms by some hours, or by a day or more.

The temperature rises rapidly from the normal to $103^{\circ}$ or $105^{\circ}$ Fahr. $\left(39.5^{\circ}\right.$ to $41^{\circ}$ C.), or even higher. Appetite and rumination are entirely suspended; the respiration becomes rapid and difficult, while the heart beats strongly and tumultuously; the muzzle is dry, the mouth hot, and salivation so abundant as to suggest an attack of footand-mouth disease. Fæces and urine are only passed at long intervals, and dysuria is present.

At first everything seems to indicate the development of an acute infectious disease; but soon afterwards appear local indications affecting the respiratory, ocular, digestive, urinary, nervous and cutaneous systems.

The respiratory symptoms are most important, and almost characteristic. Respiration becomes difficult, rough as in acute coryza, but soon assumes a snoring character, and is accompanied by a discharge, containing false membranes, from both nostrils.

The serous and muco-purulent discharge becomes rusty or reddish- 
brown, soon acquires a very fœetid smell, and is found to contain epithelial débris and yellowish-green false membranes. After the least effort to cough or the slightest touch on the membranes themselves-sometimes without any visible cause at all-epistaxis sets in, the blood being mixed with the discharge or simply escaping in the form of reddish strings, like that occasionally seen in glanders.

The mucous membrane of the nasal cavities is red, turgid, apt to bleed, and painful to the touch.

Percussion of the nasal cavities, sinuses, and even of the horns reveals everywhere exceptional sensibility.

Sometimes, but only in certain subjects, the lower portions of the head, including the muzzle, nostrils, lips and forehead, become infiltrated, as though the case were one of purpura. Thoracic complications are rarely absent, unless the disease is treated. Towards the end of the first week the respiration, still painful and snoring, becomes more rapid; and auscultation reveals at various points in the lungs areas of bronchitis and of broncho-pneumonia, indicated by bronchial rales, rattling breathing, and tubal souffles, etc. These complications are accompanied by attacks of coughing, which increase the discharge, and may threaten to end in suffocation. This happens when large masses of false membranes from the bronchi are thrown into the larynx and cannot readily be ejected through the glottis, which has been reduced in size by œdematous infiltration and inflammation.

Percussion is generally useless. The appearance of the eyes is also very significant. These symptoms develop simultaneously with the respiratory disturbance, and are marked by infiltration of the eyelids, œdematous conjunctivitis, and ophthalmia. The cornea becomes whitish, infiltrated, opaque, and sometimes shows ulcerative keratitis; or, on the other hand, it remains simply semi-transparent, and through it the media of the eye may be seen to have become opalescent. Ulcerative keratitis may develop rapidly and end in perforation of the cornea.

In certain rare instances examination with the ophthalmoscope has revealed the existence of exudative iritis; this condition may be complicated with syncchia, intra-ocular hæmorrhage, and result in permanent loss of vision.

These ocular symptoms are accompanied by continuous, abundant and prolonged discharge of tears, intense photophobia, and exceptional sensitiveness to manual examination, ete.

Digestive disturbance appears less important, and may be regarded as consequent on the febrile reaction, the general disturbance, or the condition of the respiratory apparatus. But complete examination will show that from the onset of the disease a special form of stomatitis occurs. From the first the mouth is hot and dry: soon afterwards abundant D.C. 
reflex salivation occurs, and the discharge, like that from the nose, becomes excessively foetid. This stomatitis differs entirely from ordinary forms of stomatitis and from the stomatitis peculiar to foot-and-mouth disease, and is characterised by the necrosis of fragments of epithelium forming false membranes. These on being shed leave exposed numerous ulcers distributed over the tongue, cheeks, and lips. Neither vesicles nor pustules are produced, but merely false membranes of small dimensions.

The false membranes and ulcerations occur on the soft palate and in the pharynx.

When the patients survive for a certain time, croupal enteritis and ulcerative enteritis, sometimes accompanied by hæmorrhage, develop. The administration of enemata is followed by the passage of fæces containing considerable fragments of epithelium or of streaks of blood. From the outset these digestive complications are indicated by failure to ruminate, by cessation of peristalsis and by constipation, which is usually succeeded by abundant fœetid diarrhœa.

Functional disturbance of the genito-urinary apparatus is rarer, or at least more difficult to detect. The animals refuse drink; micturition seems to be suspended or very difficult. The urine may be albuminous or rose-coloured, in consequence of the presence of hæmatin ; more rarely it is purulent or sanguinolent. There may also be urethritis, cystitis, pyelitis, and nephritis, with the passage of hyalin cylinders in the urine, although this is not always the case.

In females the mucous membrane of the vagina and lips of the vulva usually seem congested and odematous; but it is rare to find diphtheritic false membranes, as on the buccal and nasal mucous membranes, etc. On the other hand, vaginitis and exudative metritis are common.

Cutaneous outbreaks also constitute important symptoms by which this disease is recognised. At points where the skin is fine, on the inner surface of the thighs, around the girth, on the inner surface of the forearm, and on the mammæ, etc., an exanthematous eruption occurs, followed later by the development of pustules, which at first sight might suggest cow-pox.

These pustules are prominently apparent, and can readily be detected on palpation. They are more or less confluent, hard, and without a peripheral œdematous zone.

In the case of the mammæ these pustules occur most commonly on the teats, are round or slightly oval in form, bright red in colour, and sometimes violet-red. They never become converted into vesico-pustules, as in cow-pox, or into vesicles; and in no way resemble the skin eruption peculiar to foot-and-mouth disease.

Certain nervous symptoms have also been described, comprising trembling, epileptiform convulsions, and paraplegia of the hind quarters. 
Moussu has never seen nervous disturbance assume the form of epileptiform convulsions, and it is possible that the paraplegia referred to simply marks the last stage of the disease.

Causation. The essential cause of gangrenous coryza has not yet been definitely ascertained. Within recent years teachers of the highest authority have represented the disease as a general affection belonging to the hæmorrhagic forms of septicæmia (Nocard and Leclainche). Nocard has found ovoid bacteria in the false membranes of the larynx, and Leclainche a paracoli-bacillus in the mesenteric ganglia and the intestines, but the disease has never been reproduced in a characteristic and complete form similar to the clinical type.

Other microbes have also been described as occurring in the blood or discharges; but attempts to transmit the disease by using cultures or the different morbid products which observers have collected have invariably failed, and it has therefore been concluded that the disease is not contagious, but merely infectious.

Moussu does not regard this disease as a hæmorrhagic septicæmia, because the blood proves sterile unless grave pulmonary, intestinal or renal complications occur, and because the disease appears capable of being cured in a short time by simple methods. In the present state of knowledge he prefers to regard it as an infectious disease of diphtheritic form, at first localised in the upper respiratory and digestive tracts, always tending towards a grave toxæmia, and towards complications due to various other infections.

Even though direct contagion has not been proved, it is impossible to doubt that stables may become infected. This is sufficiently proved by the continued appearance of the disease when disinfection is neglected after the occurrence of the first case.

It is possible that the causes formerly assigned-viz., chills, the influence of draughts, and a morbid predisposition-may increase susceptibility in animals otherwise well cared for, but it is quite certain that infection of the stable is an important factor.

Lesions. The lesions vary with the complications, but those shown in the beginning are always identical. The mucous membrane of the nasal cavities is congested, inflamed, sphacelated, and ulcerated at different points. The turbinated bones and the ethmoid cells may become necrotic; in the larynx the region of the glottis is always most markedly affected; the mucous membrane becomes ulcerated in the neighbourhood of the vocal cords, and the tissues may become more deeply attacked.

In the trachea and bronchi the mucous membrane undergoes desquamation, and may become ulcerated at the points where false membranes have formed. The mucous membrane of the sinuses is always affected, but is rarely ulcerated. 
Such complications as capillary bronchitis, broncho-pneumonia, and gangrene of the lung may be noted.

The mucous membrane of the mouth presents a violet-red or blackish-red colour; the tongue and gums are swollen, and ulcers as large as a lentil or a halfpenny may occur either singly or in a confluent form.

The genito-urinary apparatus reveals signs of croupal cystitis, submucous effusions of blood, vaginitis with the formation of false membranes, acute pyelitis, etc.

Diagnosis. When the symptoms are fully developed the diagnosis of gangrenous coryza is extremely simple, but the absence of some of these may justify hesitation in forming an opinion. If the examination is carefully carried out, it is always possible to distinguish this disease from simple coryza, which is only accompanied by trifling fever, and in which appetite is preserved; from foot-and-mouth disease, with its very characteristic buccal eruption and absence of lesions from the upper portions of the respiratory tract; and from contagious ophthalmia.

Prognosis. Up to the present time the prognosis has always been regarded as extremely grave, the mortality being sometimes as high as from 90 to 100 per cent., and moreover the cases which recover are invariably those of what is considered the chronic form. Sudden sinking of temperature during the disease is an unfavourable sign. From 1894 to 1900 Moussu never cured a single case, whatever his method of treatment; nevertheless, it now seems possible to regard the condition a little more hopefully, provided that no incurable complication occurs before the beginning of treatment.

Treatment. Of all the modes of treatment suggested-viz., antifebrile agents, general stimulants, purgatives, diuretics, external stimulant applications, etc.-none have succeeded. Antiseptics injected into the nasal cavities, antiseptics given internally, milk diet, and all the methods hitherto suggested are equally useless.

The only treatment which appears to have achieved any degree of success is that of injecting physiological salt solution in large doses (up to six quarts per day, divided into three parts). All the animals treated by this method recovered, provided they presented no broncho-pulmonary complications.

The sole inconvenience of this treatment is the difficulty in carrying it out when the animals are at a distance from the practitioner. It is indispensable that they should be close at hand, in order that he himself may make the injections at the proper time. There is some reason for hoping, however, that serum from animals which have recovered will prove to be more active than saline injections.

This method of treatment should be followed up by the most rigid 
hygienic precautions. The mouth, nasal cavities and eyes should frequently be washed with antiseptic solutions. The stables should be freely ventilated, and the floors and bed kept in the cleanest possible condition, etc.

Whenever a case of gangrenous coryza is observed it should be isolated, and the stables should most carefully be disinfected.

\section{TUMOURS OF THE NASAL CAVITIES.}

Apart from actinomycosis of the upper jaw, tumours of the nasal cavities or of the sinuses are not frequent in bovine animals. They are, however, occasionally met with, and present symptoms which must be

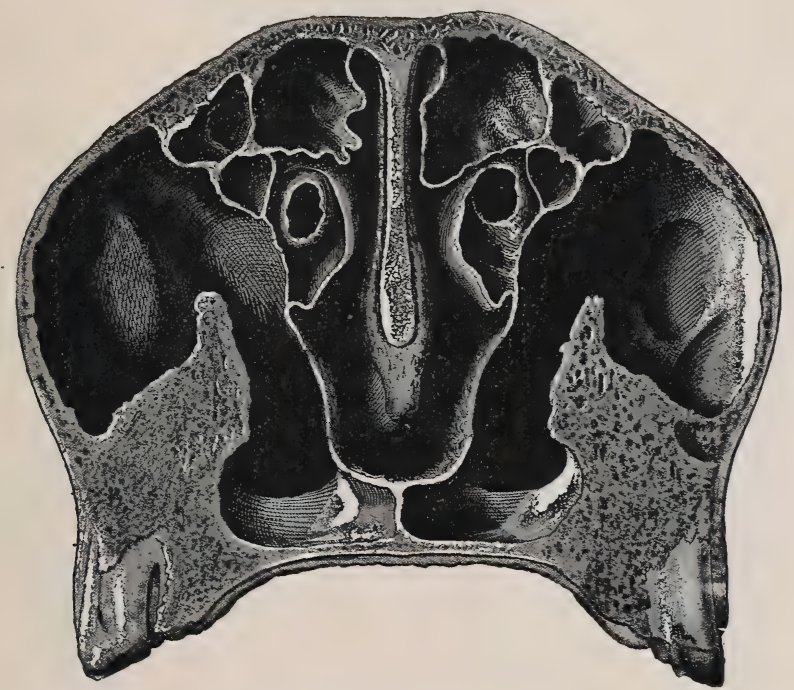

FIG. 167.-Transverse section through the nasal cavities near their centre (normal).

carefully studied in order to avoid errors of diagnosis. Usually they are of the nature of myxomata, more rarely of fibro-myxomata.

Symptoms. The dominant symptom is difficulty in breathing, both when moving and eating, a difficulty which is sometimes so considerable as to cause snoring respiration or roaring. Nevertheless, examination of the trachea and of the lung, visual examination of the lower portions of the nasal cavities, and manual examination of the pharynx, larynx and glottis give only negative results. It may even happen, as occurred in the case from which the illustration herewith was taken, that percussion of the maxillary sinus reveals normal resonance.

In the case of tumours of small size the forehead is not deformed. When, on the other hand, the tumour partly obstructs the nasal cavities it 
may thrust on one side the septum nasi and externally cause well-marked asymmetry of the face. Sero-mucous or muco-purulent discharge then occurs.

The diagnosis is somewhat difficult, for continuous or temporary roaring (or at any rate difficulty of respiration), being the dominant symptom, must be distinguished from roaring due to a laryngeal lesion like paralysis or tumour formation, from perilaryngeal compression due to enlarged retropharyngeal glands, and from tracheal or pulmonary lesions; and its origin must be located in the nasal cavities.

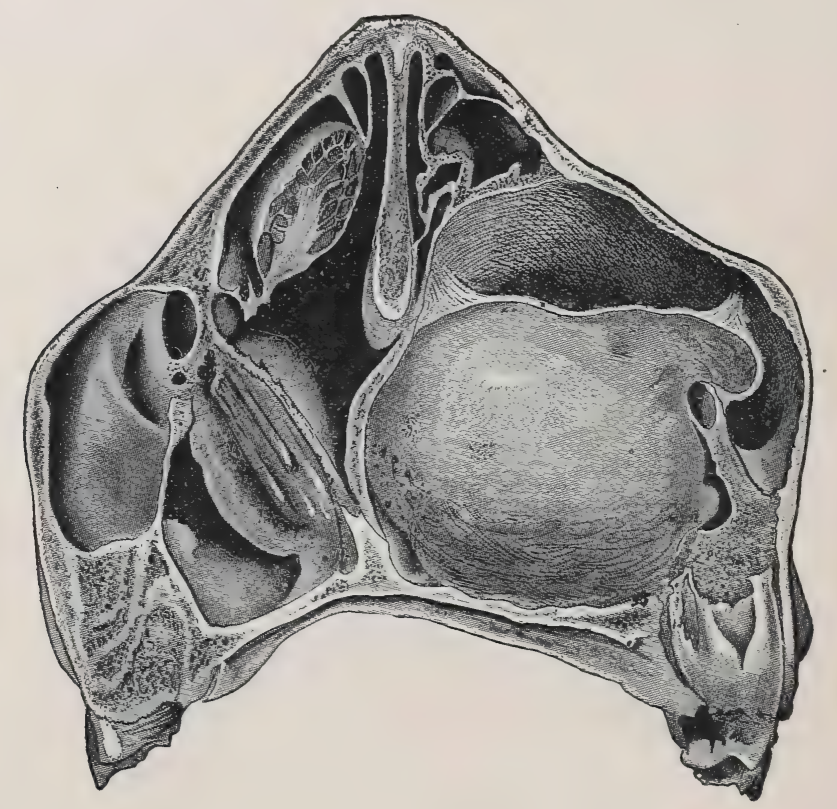

FIG. 168.-Transverse section through the nasal cavities : myxoma of the right side and of the maxillary sinus. Deformity of the forehead and face.

The prognosis is grave, in consequence of the difficulty of exploring the depth of these cavities and of the possible nature of the tumour. Nevertheless, in the case of simple myxomata permanent recovery usually follows extirpation.

Treatment is confined to extirpation, which is quite possible in the case of pedunculated tumours; in the case of sessile tumours free trepanation of the roof of the nasal cavities becomes necessary. The operation is quite safe.

PURULENT COLLECTIONS IN THE NASAL SINUSES. NASAL GLEET.

From the clinical point of view two varieties of this condition may be distinguished-inflammation of the mucous membrane of the maxillary 
simus and inflammation of the mucous membrane of the frontal sinus and of the horn core. These forms of inflammation frequently lead to suppuration. The pus collects in the depressions and divisions of the frontal or maxillary sinus.

\section{PURULENT COLLECTIONS IN THE FRONTAL SINUS.}

Causation. In the majority of cases inflammation of the mucous membrane of the frontal sinus is produced by external causes: fractures of the horns and horn core accompanied by hrmorrhage into the horn core; fractures of the horn with exposure of the sinus of the horn core; wounds and violent blows on the occipital region or the frontal bone; cracks or depressions of the external wall of the sinus, etc.

In all these cases, whether blood is effused or the mucous membrane of the sinus is merely infiltrated with serum, infection may be produced by germs being deposited from the air passing through the nasal cavities and causing suppuration.

Purulent collections in the frontal sinus may result from continued irritation, like that due to a badly fitting yolie. They also occur as an accidental complication of general diseases like gangrenous coryza.

Symptoms. The catarrh or purulent collection in the sinus may be unilateral or bilateral, and the symptoms rary, according to the form which it assumes.

Unilateral collections. - Nasal hæmorrhage is often the first symptom, but this is often regarded as of little importance, because the formation of pus does not occur until very much later. The animal shows ill-defined pain, loses appetite, remains dull and sommolent, and carries its head on one side. The horn on the injured side is hot and sensitive, and at a later stage the eye becomes affected by contiguity of tissue. It is then swollen, closed, and ratery; the conjunctiva is infiltrated, and somerhat inflamed. Sensibility and partial or complete dulness of the affected side may be detected by palpation and percussion. On the animal being made to cough, a yellowish or greyish-white discharge of rery foetid and sometimes putrid character escapes.

Bilateral collections.-Catarrh is rarely bilateral at first; but if the unilateral lesion is not treated, it affects the median septum dividing the tro carities, and the inflammation extends to the second sinus. The animal then shors dull pain, and exhibits marked depression; sometimes it appears quite prostrate. The head is carried low and inclined towards the ground, while the above-mentioned ocular symptoms and the indications furnished by palpation and percussion extend to both sides. Coughing produces momentarily a double discharge, which the animal disposes of after the fashion of horned cattle. 
Diagnosis. The diagnosis only presents difficulty in the early stages. Later the warmth and sensitiveness of the horns, the partial dulness, offensive character of the discharge, etc., render diagnosis easy.

The disease is not likely to be mistaken for gangrenous coryza, despite the condition of the eyes, because it develops slowly, progressively, and without marked fever.

Prognosis. If treated early, unilateral or bilateral collections of pus in the sinuses are capable of cure, but later when bodily health is impaired and the local lesions of the mucous membrane very pronounced, there is less chance of success.

Lesions. The initial lesions consist in cracks, fissures, or fractures of the bones of the face or exostoses of traumatic origin. In other cases the mucous membrane alone is affected. As a result of chronic irritation it becomes thickened, inflamed, and ulcerated, and granulates freely. The depressions in the sinuses contain grumous, fœetid pus, which irritates the surrounding tissues and produces pain and general symptoms of cerebral irritation, which are sometimes very disquieting.

Treatment. Numerous methods of treatment were formerly recommended, such as absolute rest, bleeding, cold affusions, perforation of a horn, section of a horn, etc. None of these is of any value.

At first, provided only a certain degree of sensitiveness and simple catarrh without suppuration exist, antiseptic fumigations with tar, carbolic acid, thymol, etc., are useful; but later, when pus has formed, they are useless. At this period the only rational and efficacious treatment consists in trepanation. In unilateral collections three openings are necessary.

The first is an opening into the sinus of the horn core. It is made $\frac{3}{8}$ to $\frac{3}{4}$ of an inch above the horn-secreting band of the horn. It must not be forgotten, however, in planning such an opening that the sinus of the horn core only exists in a rudimentary condition in young animals, and that it is scarcely possible to trephine the horns before the patient is three years of age.

The second opening is made towards the upper part of the frontal sinus about $\frac{3}{4}$ inch below the horn-secreting ring at the base of the horn and in a line with the axis of the horn core itself. Whatever the animal's age and however little the sinuses may be developed, this opening is certain to expose the cavity of the frontal sinus.

In old animals where the frontal sinus is enormously developed, and where very large depressions exist in the orbital region, a third opening should be made just above a transverse line uniting the upper margins of the two orbits and inside the suborbital suture.

These openings having been made, treatment consists-firstly, in completely washing out the cavity with boiled water, cooled to $95^{\circ}$ or 
$100^{\circ}$ Fahr.; and, secondly, in injecting antiseptic and astringent solutions so as to check the formation of pus. Among such may be mentioned 3 per cent. carbolic solution, 5 per cent. carbolic glycerine, 2 per cent. solution of iodine in iodide of potassium, etc.

Whatever the drugs employed, the cavities should be washed out every day, first with plain sterilised water, and then with antiseptic solutions at the body temperature, since cold solutions often cause inflammation of the mucous membrane of the opposite sinus.

\section{PURULENT COLLECTIONS IN THE MAXILLARY SINUS,}

This disease is much rarer than that of the frontal sinus, and only within the last few years (Ries, 1899) has a really good description been given of it.

The causation is imperfectly understood. Injuries to the suborbital region and maxillary ridge, caries of the molar teeth, and inflammation occurring during the development of general diseases represent the principal causes.

The dominating and characteristic symptom of the presence of pus in the maxillary sinus consists in incessant snorting, accompanied by violent movements of the head and the discharge of purulent or mucopurulent material.

At the beginning of these attaclis of snorting, which are produced by the reflux of pus from the sinus towards the nasal carities, the respiration becomes snoring and rapid, and the animal makes sniffing movements as though the nasal cavities were partially obstructed. After these crises, the respiration again becomes silent.

Contrary to what has been observed in purulent collections in the frontal sinus, the discharge is unilateral, reddish yellow in colour, viscous in consistence, and is accompanied by clots of a gelatinous material or even of blood.

During the attacks of snorting, the discharge resembles that of croupal or pseudo-membranous bronchitis, but the material discharged is not moulded on the internal shape of the bronchi. The masses of discharge are irregular in form, and appear as though made up of fibrous tissue matted together. Attention having been attracted by the discharge, exploration of the trachea and chest reveals nothing; on an examination of the sinuses, horrever, palpation and percussion betray a certain amount of sensitiveness, together with partial or complete dulness, and the lesion is discovered.

Diagnosis. Confusion between pus formation in the maxillary and frontal sinuses can be avoided by careful examination.

Prognosis. The prognosis is not very grave; the animals maintain 
their appetite, but become thinner, and the condition shows no tendency to spontaneous cure.

Treatment. The only rational treatment consists in trephining, an operation practised immediately above the maxillary tuberosity and over the lowest part of the sinus (Fig. 164). This opening allows the cavity to be washed out and the sinus drained.

Antiseptic treatment exactly resembles that of purulent collections in the frontal sinus. Injections of astringents, dilute carbolic acid and iodine solutions, etc., are recommended.

\section{CESTRUS LARVE IN THE FACIAL SINUSES OF SHEEP.}

(FALSE STURDY.)

Causation. This disease of sheep, which sometimes produces vertigo resembling that shown in gid or sturdy, is produced by the growth of larvæ of Estrus ovis in the frontal sinuses. The œstrus of the sheep assumes perfect insect form during the fine days of summer from July to September. The females swarm around the flocks and attempt to alight on the animal's head close to the nostrils, where they deposit their eggs or larvæ. The larvæ crawl into the nostrils, thence into the nasal cavities, the meatus, and finally the sinuses, where they become fixed. In these sinuses they undergo complete development, increasing from a length of about $\frac{1}{10}$ inch to from $\frac{3}{4}$ to 1 inch before their transformation into the nymph and perfect insect. They remain in the sinus for eight to ten months. When numerous and well developed they may fill the whole of the cavity.

Symptoms. It is easy for a careful observer to note the time at which the larvæ penetrate the sinus. During the hottest hours of the day the adult insects are continually hovering over the flocks, and on watching carefully one sees sheep suddenly become excited, tap with their feet, rub their faces against any hard, resisting object in the neighbourhood, plunge their nostrils into the dust, and snort violently.

When the larvæ have penetrated the nasal cavities they produce frequent attacks of sneezing by irritating the mucous membrane, and cause an intense sero-mucous and afterwards a moderate muco-purulent coryza. As long as the larvæ remain of small size, the apparent results they produce are insignificant, as during the first months of winter; but when they are numerous, and have become of considerable size, they cause symptoms which might suggest an attack of gid or sturdy.

Thus the bodily movements become spasmodic, the gait irregular, and the animals show attacks of vertigo. They stagger and fall, making convulsing movements, grinding their teeth and rolling their eyes, while frothy saliva escapes from the mouth, etc. 
Death may occur during such attacks, which, however, are happily very rare. Most commonly the animals are simply dull and somnolent. They feed badly, carry their heads low, and sometimes hide themselves under the mangers or in corners.

In exceptional circumstances they bury their heads in the wool or carry them high in the air in walking, while they lift their front legs high, with a stepping movement.

Diagnosis. The diagnosis of parasitic invasion of the sinus in the

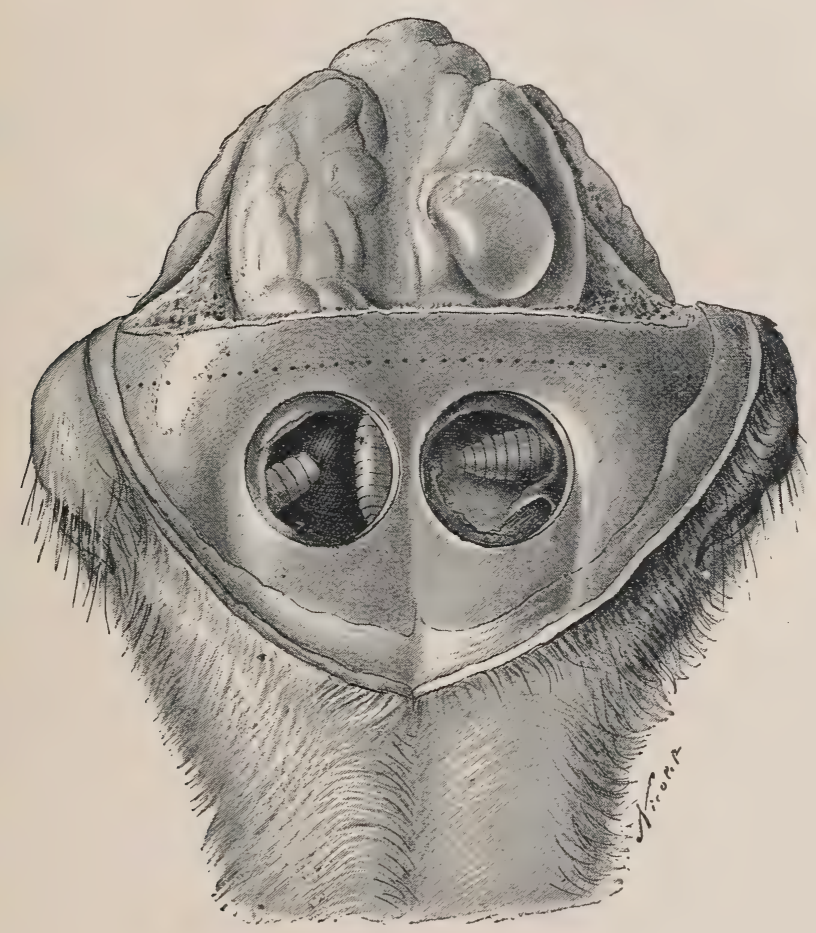

FIG. 169.-Parasitic invasion of the sinuses and conurosis (showing the seat of operation in either condition).

sheep is rather difficult, because certain of the symptoms suggest gid. One never finds the signs of true gid, however, and, on the other hand, a certain amount of discharge and attacks of snorting always exist. Finally, gid (cœnurosis) only attacks young animals, whilst the larvæ of oestridæ are commonest in adults.

To confound the disease with verminous bronchitis is still less likely, for although a discharge exists in both cases, this is accompanied by cough in bronchitis, and only by sneezing attacks in infection of the sinuses. 
Finally, in bronchitis, histological examination leads to the discovery of eggs or embryos of the strongyles. The diagnosis becomes very easy on post-mortem examination, the identification of the larvæ of œstridæ being extremely simple.

Prognosis. The prognosis is only grave when infestation is very pronounced. Most commonly the parasites complete their development without producing disquieting symptoms; as summer approaches they are expelled and recovery occurs.

Lesions. The only lesions consist in very active inflammation of the mucous membrane of the sinus, which appears excessively hypertrophied, and in the existence of larvæ. These develop in the midst of a magma of purulent, foetid discharge. The number of parasites usually ranges from two to twenty, although Zürn declares that he has found as many as eighty in one subject.

Treatment. Many precautions have been suggested for preventing infestation. Many are impossible or difficult to carry out in current practice, even the soaking of the nostrils in empyreumatic oil, and the majority are useless. T'he only suggestion to which we attribute any importance consists in trying to prevent the perfect insects from obtaining a lodgment in cavities in the walls or roofs of sheep-sheds.

The really efficacious modes of treatment are also few in number, for the larvæ are so firmly lodged in the mucous membrane of the sinuses that they can only be detached after these have been trephined. 'The use of nasal injections, embrocations and powders, with the object of making the animal sneeze, are ineffectual.

Trepanation should not be performed until it is quite clear that the flock is badly infested, and when serious symptoms appear to threaten the lives of some of the animals.

The operation, although very simple, requires care on account of the thinness of the bones.

The anatomical directions are the middle line of the head, and a transverse line uniting the upper margin of the two orbits. The orifices are made in the two lower angles produced by the intersection of these lines (Fig. 169).

It is then easy to remove with the fingers or forceps the larvæ situated immediately below the point of trepanation, and afterwards to wash out thoroughly the cavities of the sinuses. If some lie at points which cannot be directly reached, they can be killed by injecting a little benzine and water. I'his proceeding is quite safe. 


\section{CHAPTER III.}

\section{LARYNX, TRACHEA AND BRONCHI.}

\section{LARYNGITIS.}

LARYNGEAL diseases are common, but are usually only the local expression of some grave general infection, such as foot-and-mouth disease, gangrenous coryza, or tuberculosis. Pathological conditions such as these can be disregarded for the moment, as they will receive attention under special heads.

The two current forms of laryngeal disease are acute laryngitis and stridulous laryngitis.

\section{ACUTE LARYNGITIS.}

Acute laryngitis, like simple coryza, of which it is often only an accompaniment, is caused by chill, by irritant vapours, by smoke, etc., or by external traumatic causes.

The cough is dry and painful at first; afterwards it is accompanied by a discharge or by the swallowing of mucus or muco-purulent products. The respiration sometimes appears accelerated and difficult, but roaring or whistling and marked fever are rare. The slightest pressure over the laryngeal region causes pain and attacks of coughing. The respiration is normal while the animal is at rest, provided that the laryngitis remains localised; frequently, however, it is complicated with bronchitis.

The appetite is somewhat diminished, but all these symptoms very rapidly improve.

The diagnosis is based on the frequency of the cough and the sensitiveness of the throat region.

The prognosis is favourable in cases of simple laryngitis.

Treatment consists in the administration of steam inhalations, warm drinks, the application of mustard plasters or blisters around the laryngeal region, and the administration of expectorants to facilitate mucous discharge.

PSEUDO-MEMBRANOUS LARYNGITIS.

Pseudo-membranous laryngitis, also termed by German authors croupal or diphtheritic laryngitis, because it bears a certain resemblance 
to human croup, is characterised by the formation of false membranes on the vocal cords, arytenoid cartilages, and subglottal region, etc.

In France it has been described under the name of stridulous laryngitis, because its dominant symptom consists in attacks of intense dyspnœa, during which the respiration is accompanied by whistling. Without making any attempt to prejudge the nature of the disease, which resembles the pseudo-diphtheritic forms of laryngitis in man, we prefer the term pseudo-membranous laryngitis. Moreover, this pseudo-membranous laryngitis very frequently accompanies pseudo-membranous sore throat, tracheitis and bronchitis, with formation of false membranes.

Causation. The causes suggested are similar to those of simple sore throat: they include chills, the ingestion of ice-cold water, or the inhalation of irritant gases, during outbreaks of fire, etc., etc. ; but it is quite certain that here, as in many similar cases, a primarily simple laryngitis is complicated by a well-marked infection.

Symptoms. The general symptoms seen during the early stages consist in loss of appetite, general depression, rigors, and a rise in temperature of $1^{\circ}$ to $2^{\circ}$ Fahr.

Then, after twenty-four or forty-eight hours, the respiration becomes more rapid, difficult, whistling and dyspnœic, with intermittent attacks of suffocation.

Examination of the chest gives negative results, but, on the throat being manipulated, the slightest pressure exercised over the larynx produces attacks of coughing. During the first few days these attacks are loud, spasmodic, and difficult, but on succeeding days they lead to the discharge, from the nostrils or mouth, of masses of false membrane accompanied by whitish and sometimes blood-stained fluid. Secondarily the mucous membrane of the nasal fossæ appears inflamed to a varying degree. The conjunctiva is also affected, the eyes are watering. An important symptom consists in the fact that this watering is accompanied by internal ophthalmia, as in gangrenous coryza.

In consequence of the respiratory difficulty, the animal takes up a characteristic attitude, holding its neck stiffly in a horizontal position and its head completely extended; the nostrils are widely dilated. When the disease is fully developed rumination is suppressed, the bowels are constipated and the fæces are coated as in grave cases of enteritis, the yield of milk diminishes, the heart beats feebly, the pulse remains small; death may occur from asphyxia, and probably also from intoxication.

The disease usually lasts from eight to ten days, but death may occur earlier. In the majority of cases, however, the animal can be saved. All the symptoms diminish, the temperature falls, rumination again appears and with it appetite, and the whole condition becomes normal. 
Many animals, however, remain thin and recover slowly; these are principally cases which have suffered from pseudo-membranous bronchitis.

Lesions. The lesions may be confined to the larynx, but may also invade neighbouring cavities. They consist in the formation of mucoalbuminous and fibrinous exudates, covering the mucous membrane in superposed layers, penetrating the epithelial stratum, and adhering so strongly to the corium that attempts to loosen them cause the deeper seated structures to bleed.

Diagnosis. The symptoms are sufficiently well marked to prevent any confusion arising except with gangrenous coryza, but in this case there is no ophthalmia lesion of the nasal cavities, cutaneous eruption, or eruption over the claws.

The prognosis is grave when the disease attacks debilitated or exhausted animals.

The treatment is confined entirely to treating symptoms. As in all acute inflammatory affections, moderate bleeding, sinapisms over the region of the larynx, repeated as often as necessary, or applications of antimonial ointment have been recommended. Applications of moxas or setons in the neighbourhood of the dewlap might possibly prove of value.

Internally tartar emetic, in doses proportioned to the size of the animal ( $2 \frac{1}{2}$ to 3 drachms in adults), has been recommended; also iodide of potassium.

General stimulants, like alcohol, coffee, tea, acetate of ammonia, and suitable hygienic conditions suggest themselves. Tepid drinks can be given freely. Nourishing and easily digested food, and a ration of milk complete the treatment.

Emollient and antiseptic fumigations are also of value, as in ordinary sore throat or laryngitis, for they favour the separation and discharge of the false membranes.

\section{TUMOURS OF THE LARYNX.}

Acute forms of pseudo-membranous laryngitis, or even tuberculous laryngitis, are not the only diseases which affect the larynx. It is by no means exceptional to discover intra-laryngeal tumours, mucous polypi, tuberculomes, and growths due to actinomyces, etc.

The presence of these tumours is indicated by difficulty in respiration, fits of coughing, and threatened suffocation, accompanied by discharges of varying character.

When the tumour is largely sessile, the respiration may simply be snoring or whistling, without any suggestion of suffocation; but if, on the other hand, it is pedunculated, displacement of the polypus produced 
by the currents of air during inspiration and expiration causes spasm of the glottis, fits of coughing and threatened suffocation.

The diagnosis is not always easy, though examination of the nasal cavities, the sinuses, trachea and chest gives negative results. Auscultation of the larynx may suggest the existence of the lesion, but an exact diagnosis can only be attained by digital examination of the larynx through the pharynx.

The prognosis of these tumours is grave, because death from

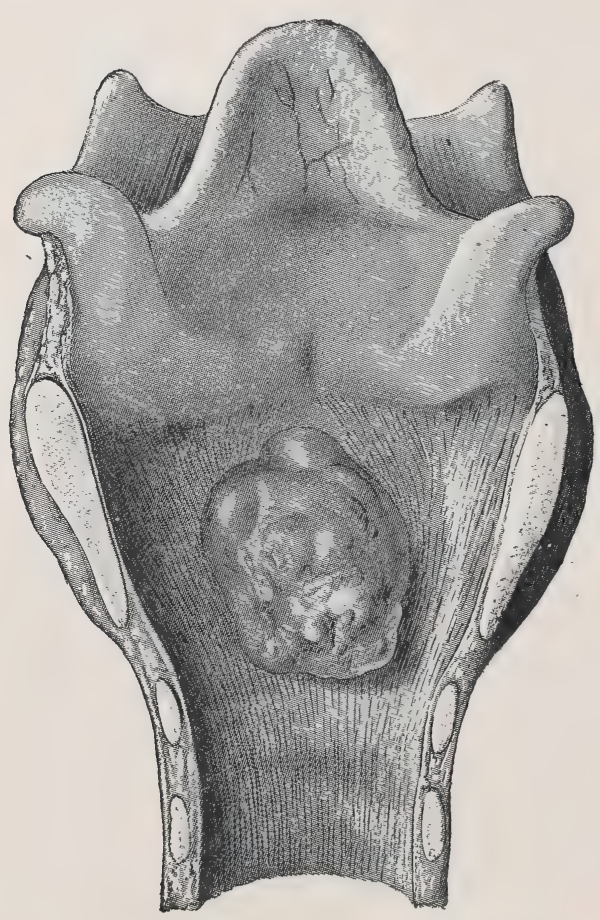

FIg. 170.- Sessile form of intra-laryngeal polypus. asphyxia may occur during an attack of coughing or as a consequence of the fits of suffocation.

Treatment. As it is usually extremely difficult, if not impossible, to discover the exact nature of the tumour, medical treatment, except for instance in the case of actinomycosis, is of very uncertain value.

Surgical treatment alone suggests itself. Before attempting an operation tracheotomy should be performed and a metal canula inserted. The animal having been cast, and the upper laryngeal region anæsthetised by means of cocaine, a vertical incision is made in the median line below the larynx, passing through the skin, the vertical junction between the neck muscles and the three first circles of the trachea, and access is thus obtained to the larynx and subglottal region. The operation should only be performed in exceptional cases, such as that of a valuable stud animal.

Provided that the new growth has a well-developed pedicle it can be removed through the mouth by the use of an écraseur or simply by tearing out.

\section{BRONCHITIS.}

Diseases of the bronchi in bovine animals reveal very different characters, according to the nature of the primary cause, for which 
reason cases occur of simple acute bronchitis, verminous bronchitis, simple chronic bronchitis, pseudo-membranous bronchitis, tuberculous bronchitis, etc.

\section{SIMPLE ACUTE BRONCHITIS.}

Simple acute bronchitis coexists with, or is often only the logical and inevitable complication of, coryza and acute laryngitis. It is commonly associated with inflammation of the mucous membrane of the trachea.

It accompanies cold and wet seasons, and usually appears with the autumn frosts, in animals still at grass. In animals under shelter it occurs after undue exertion followed by chills, and after sudden rain storms. It also attacks working oxen, which are much exposed to the weather.

The symptoms follow very rapidly on the determining cause. They are ushered in by rigors, trembling attacks, diminution or loss of appetite, arrest of rumination, acceleration of breathing, and the appearance of a rough and spasmodic cough.

In ordinary cases these symptoms rapidly diminish, even without treatment. The appetite again becomes moderate, rumination returns, but the cough remains more or less rough and spasmodic, ending in the discharge, or more often in the swallowing, of abundant bronchial mucus.

This is the condition at the period of crisis. Percussion of the thorax reveals normal resonance. On auscultation of the sides during the period of onset, rough rales are heard, which at the period of crisis are replaced by mucous rîles. The cough diminishes in frequency, and after a fortnight everything again becomes normal.

The diagnosis is very easy, the important point being not to confuse common bronchitis with tuberculous bronchitis, which very often assumes a chronic form.

Prognosis. The prognosis is not grave, even though the disease may assume a chronic condition.

Treatment does not differ from that of acute bronchitis in the horse. It consists in antiseptic and steam fumigations, tepid drinks, the administration of doses of 2 to 3 drachms Kerme's mineral in adult animals, and of 1 to $1 \frac{1}{2}$ drachms of iodide of potassium, given in a mash or in honey electuary. During convalescence tar water should be administered.

CHRONIC BRONCHITIS.

Chronic inflammation of the mucous membrane of the large bronchi and trachea may follow acute bronchitis, but it is also a frequent D.c. 
termination of verminous bronchitis. It is found in fully developed animals, adult or old, and particularly in those inhabiting wet, cold valleys.

It is characterised by frequent paroxysms of coughing, which appear on the slightest provocation, such as the action of cold air on leaving the stable or of the air of a confined space on animals returning from the open; concussion of the chest by the pleximeter, squeezing of the loins, rapid movement, etc.

This coughing is accompanied by the discharge of mucus, which rarely arrives at the nostrils, but is swallowed in passing through the pharynx. Such mucus is always thick, greenish yellow in colour, and without smell.

'The respiration, although regular whilst the animal is at rest, becomes accelerated on moving, and after attacks of coughing. It is sometimes rapid and whistling.

Percussion discloses neither partial nor complete dulness, but everywhere irregularly distributed mucous rattling and sibilant râles are revealed by auscultation.

There is no fever, the appetite is maintained, and, what is an even more important point, animals in good condition preserve their flesh. Interlobular pulmonary emphysema and emphysema resulting from dilatation are inseparable accompaniments of chronic bronchitis, for which reason the flank respiratory movement is frequently very marked.

The diagnosis is of only moderate difficulty, because although in certain conditions the disease may be mistaken for tuberculosis or emphysema, it can be distinguished by bacteriological examination of the discharge, by an injection of tuberculin, by careful auscultation, and by consideration of the general condition.

Lesions. The walls of the bronchi are thickened, the submucous connective tissue is sclerosed, the muscular fibres are modified in structure, and have become fibrous, while the epithelial layer is desquamated and suppurating. The peribronchial tissue also undergoes sclerosis, and in certain cases the smaller bronchi present marked dilatations resembling small caverns (bronchi-ecstasis).

Treatment can never be more than palliative; the aim should be to prevent the lesions becoming aggravated, and to check the pathological secretion from the bronchi, but the lesions already existent can never be removed. 'Tar water should be perseveringly administered. Essence of turpentine in doses of 2 to $2 \frac{1}{2}$ drachms per day in electuary (adults), creosote in doses of $1 \frac{1}{4}$ to $1 \frac{1}{2}$ drachms, and terpine in doses of $\frac{3}{4}$ to 1 drachm give the best results, and produce a marked improvement. 


\section{PSEUDO-MEMBRANOUS BRONCHITIS.}

The pseudo-membranous forms of bronchitis, formerly termed "croupal or diphtheritic bronchitis," are rare. They develop suddenly or follow pseudo-membranous laryngitis. Like the latter, they are due to a specific infection, possibly aided by accidental causes.

Their causation is imperfectly understood, and they cannot be compared, still less homologated, with diphtheritic disorders in man. They are characterised by the formation of false membranes, which develop on the mucous surface, mould themselves over the internal surface of the large bronchi, and ramify throughout the bronchial channels like branches of trees. They are of greyish-yellow colour, and appear to be formed of fibrin, coagulated albumen, and epithelial débris cemented together with mucus.

Symptoms. At the outset these pseudo-membranous forms of bronchitis have the same characters as acute bronchitis, which at the crisis would be marked by the expulsion of fragments of false membrane by coughing. Most frequently it seems that the bronchitis follows its regular course, and in such case it is only during convalescence or a considerable time afterwards that the membranes begin to be discharged during paroxysms of coughing.

The patients are subject to intense dyspnoea, appear about to suffocate, and during the efforts then made the false membranes are discharged in the form of half-organised layers, or, on the other hand; in branched masses, resembling twigs.

The dyspnoa at once ceases. Despite the development of these false membranes in the bronchi, no alarming symptoms are produced, which is explained by the fact of the false membranes being adherent only to the inner surface of the principal conduits, without closing or even markedly obstructing them or the smaller passages leading to the pulmonary alveoli. When, however, they are displaced, violent reflex spasms are produced as soon as the fragments approach the larynx.

Diagnosis. The diagnosis rests entirely on examination of the expectorated material.

So far as the prognosis is concerned, it is less grave than might be supposed from the symptoms. The gravity arises from the fact that this disease has a certain tendency to become chronic.

Treatment scarcely differs from that of ordinary bronchitis. Tar, creosote in doses of $2 \frac{1}{2}$ to 5 drachms given in oil; terpine in doses of $\frac{1}{2}$ to $\frac{3}{4}$ drachms per day can be recommended. Iodide of potassium also has certain advantages. 
VERMINOUS BRONCHITIS IN SHEEP AND CATTLE (HUSK, HOOSE, ETC.).

Lambs, young sheep, and calves sometimes suffer severely from infestation with lung worms, which set up great irritation in the bronchial passages, leading to chronic bronchitis. The animals show frequent attacks of paroxysmal coughing, during which some of the parasites may be expelled. The irritation produced causes serious loss of condition, and if not alleviated may lead to death. The parasite of the sheep is known as Strongylus filaria (sheep lungworm), that of the calf Strongylus micrurus. The worms are from 2 to 4 inches long, whitish in colour, and of the diameter of a hat-pin.

Treatment. According to generally accepted views among veterinarians and zoologists, it is a comparatively simple matter to kill worms in the bronchial tubes, and a number of cases of the disease are reported in literature which are alleged to have been cured. These views, however, are open to very serious doubt.

Neumann (1892b, pp. 590, 591, 593, 594) summarises the subject of treatment as follows :-

Two different procedures in treatment are pursued. In one, substances are passed into the digestive canal, which, being diffused in the blood, are believed to be capable of attacking the worms in the bronchial tubes. With this view, the picrate of potash $(0.20$ to $0.40 \mathrm{gram}$ per head). is given, dissolved in thin gruel or mucilage; creosote; oil of turpentine; a mixture of equal parts of oil of turpentine and tincture of camphor-a teaspoonful every day to each lamb in a mucilaginous fluid; a mixture of creosote 120 grams, spirits of wine 500 grams, and water 700 gramsan ordinary spoonful every day to each animal; or creosote 60 grams, benzine 300 grams, water 2 litres - an ordinary spoonful given every day for eight days to each sheep. Hall states he has successfully employed prussic acid in ten-drop doses, morning and evening.

But experience has shown that, while such treatment is troublesome to carry out, its efficacy cannot be relied upon.

Success is more certain with fumigations, as they penetrate directly to the worms, stupefy them, and induce fits of coughing that cause expulsion. They are practised in buildings from which all forage is previously removed, and which are well closed. Into these the diseased [animals] are introduced, and on a red-hot shovel are placed rags, horns, feathers, hair, old pieces of leather, empyreumatic oil, tar, juniper berries, asafetida, etc. 'The intensity, duration, and number of these fumigations are graduated as the sheep become accustomed to them. At first once a day may suffice, and then the intensity should be moderate and the duration about ten minutes; afterwards two, and finally three, may be given during the day, each lasting for twenty minutes. Kowalewsky 
says he has obtained very good results from similar fumigations. Fumigations with chlorine, sulphur, and sulphuret of mercury or cinnabar have been recommended, but they are dangerous.

(Stephen recommends as follows: Put about forty lambs at a time into an air-tight house, and place tar, sulphur, and turpentine in a pot of burning coals, suspended by a chain from the ceiling and brought as near to the heads of the animals as possible; the fumes are to be allowed to fill the house, and more ingredients are added as required, the lambs being kept in the place for twenty-five minutes each time, and the process to be repeated on three occasions.)

Tracheal injections in the verminous bron hitis of calves are of great utility; but for a flock of sheep they would be troublesome and difficult to administer. However, Nieman, has successfully employed them on 384 sheep belonging to several small owners. He used a solution of 2 parts iodine and 10 parts iodide of potassium in 100 parts of distilled water. This fluid was mixed, in equal parts, with oil of turpentine, and made into an emulsion with olive oil; each sheep received 5 to 8 grams of the mixture, and the number of the injections varied according to the gravity of the disease-from two to three at two days' interval. The worms were killed and expelled during the paroxysms of coughing, and the bronchitis was modified.

The medical treatment should be assisted by very nourishing food, and by bitter, stimulating, and ferruginous tonics, which arouse the digestive functions and allow those animals which are least exhausted to reach the period of elimination of the parasites.

At the commencement of any kind of treatment it is well to have an examination of the flock, with the object of sending the worst cases to the butcher.

The same medicaments have been employed in treating this malady in calves as in that of sheep, and no better results have been obtained. Numann and Janné have, however, been successful with asafetida (30 grams), Chabert's empyreumatic oil (60 grams), and a mucilaginous decoction (500 grams) - a spoonful of this mixture being given in a half litre of milk, and the treatment continued for about a month.

The results are less uncertain if the worms lodged in the bronchial tubes are directly acted upon, either by means of injections of the same kind as those employed for sheep, or fluid medicaments introduced directly into the bronchi.

Read says he has cured calves worn down almost to skeletons by verminous bronchitis by the following procedure: The head of the calf is slightly elevated, and about 2 drachms of ether, chloroform, oil of turpentine, or rectified oil of amber-single or combined-are poured into each nostril and allowed to vaporize there; it will then, by the 
respiration, be carried into the air passages, and thus destroy the filariæ. In some cases it must be repeated two or three times, but once has frequently the desired effect.

The method of treatment by intra-tracheal injection, introduced by Levi, of Pisa, has yielded very satisfactory results. Levi has been completely successful with a sheep. Éloire has employed it in sixteen calves affected with the disease, and all were cured. He used the following mixture: Black poppy oil, 100 parts; oil of turpentine, 100 parts ; carbolic acid, 2 parts; purified cade oil, 2 parts. Each calf received 10 grams of this mixture daily for three days.

The injection, which should be given slowly, is followed by a fit of coughing, and the expired air has the odour of turpentine. This treatment has also been successful at Milan. Similar favourable results have followed Hutton's treatment of eight calves, some of which were in the last stage of verminous bronchitis. He employed a mixture of oil of turpentine, tincture of opium, pure carbolic acid, and water-the oil of turpentine forming one-half of the mixture. 'The dose was $\frac{1}{2}$ an ounce, and in the serious cases this was given every day for three days, and in other cases every second or third day. Kriwonogow has likewise cured twenty-two calves by giving each of them two tracheal injections of 8 grams of the following mixture: Essence of cloves and oil of turpentine, 360 parts of each; carbolic acid and olive oil, 30 parts of each.

(Williams speaks highly of the administration of prussic acid. Penhale gives-by intra-tracheal injection, and slowly-oil of turpentine 2 drams, carbolic acid 20 minims, and chloroform $\frac{1}{2}$ a dram.) 


\section{CHAPTER IV.}

\section{LUNGS AND PLEURE.}

\section{PULMONARY CONGESTION.}

Besides passive congestions of the lung, which it is unnecessary to describe here, and which result from cardiac or pericardiac affections or the compression of important vessels, there sometimes occur, particularly among young animals, cases of active congestion of the lung. Such cases are produced by over-exertion on the part of animals which have escaped from control or have been chased by dogs.

They are most common in animals usually kept in stables, but which have accidentally escaped, or in very fat animals.

Dyspnœa and cough are the chief symptoms. The animals stop as though exhausted, extend their neck and head, dilate their nostrils and thrust their limbs out on either side of the body, while at the same time they appear in a condition of terrible distress.

The respiration is rapid and short, the patient can scarcely breathe, and asphyxia seems imminent. On auscultation it seems that the respiratory murmur has disappeared over almost the entire extent of the lung.

Death may occur very quickly.

The diagnosis is extremely easy, provided that the history is known.

The prognosis is grave.

One of the most successful methods of treatment consists in free bleeding. In a great majority of cases this causes the symptoms to abate as though by enchantment. Cutaneous stimulation by mustard and similar irritants, as well as ablutions of cold water, are useful. The animal should be placed in a very airy spot.

\section{SIMPLE PNEUMONIA.}

History. Veterinary surgeons have long been divided in opinion on the question whether simple pneumonia occurs in animals of the bovine species. Whilst some affirm it, others think that all lesions of the lung in the ox, apart from pneumonia due to foreign bodies, should be regarded as of the nature of peripneumonia. 
Some ten years ago two veterinary surgeons of the department of the Aisne, Coulon and Ollivier, practising in a district where peripneumonia rages, made some extremely interesting observations on pneumonia in the ox. Their object was to distinguish between contagious peripneumonia and simple pneumonia during life, simple pneumonia having formerly been regarded as a non-contagious peripneumonia. Despite the rather unfavourable conditions in which ordinary practitioners are frequently placed, these gentlemen performed a work of great value. The facts which point to the occurrence of simple pneumonia are as follows :--

The disease is not contagious. One may allow affected animals to mix with normal subjects without the disease being communicated. Pulmonary exudate from cases of simple pneumonia can be injected into the dewlap and hind quarters of young and adult animals, without pathological results.

The lesions and course of simple pneumonia entirely differ from those of peripneumonia.

Causation. Simple pneumonia is not common, and only occurs quite exceptionally in fat stock, or in milch cows kept in stables at a regular temperature, as in the north of France and near Paris.

It occurs most commonly in working animals, which are exposed to variations in temperature and to chills. By causing vascular disturbance, chill favours microbic infection and visceral inflammation. Trasbot has described the case of an ox which, after having worked hard, and whilst freely sweating, was left exposed to the wind under a shed for about three hours. This animal contracted unilateral pneumonia the following day.

Coulon and Ollivier have seen the disease in animals living in damp, low-lying valleys, or valleys exposed to the north wind, which are exposed in consequence to great variations in temperature.

The symptoms follow almost the same course as in the horse, and one may distinguish three periods:-

I. Period of onset. The symptoms which mark the onset of the disease are moderate fever, which progressively increases, and acceleration of respiration and of circulation. The number of the respiratory movements rises to twenty or twenty-five per minute, those of the pulse to fifty, sixty, or eighty. The conjunctiva becomes injected, and then of a yellow tint. At this period the appetite never disappears completely, rumination is regular, and there is neither tympanites nor colic.

These general symptoms, which are not of special significance, are supplemented by more precise local symptoms--an abortive, difficult and painful, cough which is easily induced, and a whitish discharge. T'he 
rusty expectoration which is characteristic of simple pneumonia in the horse and in man has never been observed.

Percussion discloses partial dulness, usually on one side, in the lower region of the chest: the respiratory murmur in this region is ascertained by auscultation to have diminished, whilst in the upper part and also on the opposite side the respiratory murmur is increased.

II. Period of exacerbation. This period is characterised by accentuation of all the symptoms: the temperature rises, and may attain $104^{\circ}$ Fahr.; the submaxillary artery is tense; the dulness becomes more marked, whilst crepitant and mucous râles are heard. In the portions still unattacked the function of the lung is exaggerated in order to make up for the defect of the diseased parts, and the respiration becomes juvenile.

The appetite, which previously had been maintained, diminishes considerably, without, however, entirely disappearing, and intense thirst sets in, as a consequence of the fever.

III. Period of crisis. The general symptoms remain stationary for four or five days; the respiration, which is always affected, sometimes becomes as rapid as thirty to forty per minute; the tubal souffle which invariably occurs in pneumonia of the horse is not always clearly audible.

Terminations. (1.) Resolution.-This is indicated by the attenuation of all the symptoms and the disappearance of fever, which gradually sinks from $105^{\circ}$ to $101^{\circ} \mathrm{Fahr}$. The respiratory movements become fuller and fewer in number, the pulse slower, and the artery softer and more compressible. The cough changes its character, is stronger, more sonorous and prolonged, and is accompanied by the free discharge of muco-pus. The dulness descends, and the tubal souffle, if previously existing, is replaced by the returning crepitant rîle. In general the disease runs its course in eight to ten days in young and in fourteen to fifteen days in aged subjects.

(2.) Death by asphyxia is almost the only fatal termination of pneumonia in the ox. It occurs in one-third to one-fourth of the subjects attacked. Its approach is announced by a deep mahoganyred coloration of the conjunctiva. The pulse becomes very rapid, 100 to 110 per minute, thready, small, and almost imperceptible, whilst the beating of the heart is strong and tumultuous. Respiration is rapid and very laboured (50 to 70 per minute). The animal's attitude is typical; it stands with its limbs thrust out, its head extended, its nostrils dilated, and its mouth half open, discharging foamy and viscous saliva. Throughout the greater portion of the lung gurgling sounds and crepitant mucous râles can then be detected.

(3.) Cases ending in gangrene and suppuration are excessively 
rare, and others resulting in chronic pneumonia have not been authoritatively described.

Pneumonia is distinguished from broncho-pneumonia by the existence of dulness at the period of crisis, whilst in the case of bronchopneumonia this period is only marked by partial dulness, which is even then sometimes slight. Moreover, broncho-pneumonia usually develops much more slowly.

The disease, then, is distinguished from peripneumonia by the following points :-

(a) By the character of the temperature curve, which is regular in pneumonia, only attaining its highest point at the period of crisis, whilst in peripneumonia it ascends suddenly, and presents sudden oscillations.

(b) The appetite remains, although diminished.

(c) Sensitiveness in the region of the ribs is but feebly marked, or is entirely absent, simple pneumonia not being accompanied by pleurisy.

(d) The dewlap never shows odema, a symptom which usually accompanies the period of crisis in peripneumonia, when the jugular veins and the anterior vena cava are compressed.

(e) These signs alone are almost sufficient on which to base the diagnosis, but they are often supplemented by two others, of somewhat less importance (for in exceptional cases they may also be observed in simple pneumonia), viz.- the absence in most instances of a membranous sound, and of a well-marked souffle.

Prognosis. Two-thirds of the cases recover. This proportion might be increased if the veterinary surgeon were called in at the beginning.

Lesions. Post-mortem examination reveals neither pleural exudate nor pleural lesions. The lung is large and of increased weight, hepatised along its lower borders, and congested in its upper part.

The sero-hæmorrhagic infiltration of the interlobular spaces varies, according to the region examined: the upper regions are engorged and black, owing to capillary hæmorrhages and blood clots, which completely surround the pulmonary lobule, the latter being violet or brownish-red in colour. In the hepatised portions the lobules are of a washed-out reddish tint, and the interspaces of a whitish colour.

The bronchi are filled with frothy, whitish mucus; the small bronchi sometimes contain fibrous concretions and the mucous membrane is injected, and may be destroyed in places. The bronchial lymphatic glands are enlarged, congested, and contain small hæmorrhages.

It is important in making a post-mortem examination to be able to distinguish pneumonia from peripneumonia. This is comparatively easy if one bears in mind that in the latter pleurisy always exists, that the interlobular connective tissue spaces are always greatly distended with a citrine-coloured serosity, that on section the peripneu- 
monic lung resembles a mosaic; and that, finally, the course of hepatisation is centripetal, the inflammation commencing at the periphery of the lobule, and progressively extending towards the centre. In pneumonia, on the contrary, pleurisy is always absent; the interlobular connective spaces are only distended slightly, if at all, and always contain a brownish-red serosity: the course of hepatisation is centrifugal; it commences in the pulmonary alveoli, and extends towards the periphery and the interlobular divisions. The following table gives a résumé of the other differences between the two diseases:-

\section{Peripneumonia.}

Edema of the dewlap.

Pleural exudate.

Centripetal lobular hepatisation.

Extreme infiltration of the interlobular connective tissue spaces (primary yellow infiltration).

\section{Pneumonia.}

No ødema of the dewlap.

No pleural exudate.

Ascending centrifugal lobar hepatisa. tion.

Moderate infiltration of the interlobular connective tissue spaces (secondary reddish-brown infiltration).

Treatment. Good hygiene, regular ventilation, moderate warmth, and the administration of tepid drinks facilitate recovery.

Certain German author's recommend cold compresses to the thorax, douches, and cold enemata in pneumonia. We do not think that such treatment has proved very successful, although it has been well tried.

The classic treatment commences with moderate bleeding, the free application of mustard to the sides, the application of moxas, frictions with antimonial or blister ointment, and the administration of draughts containing 2 to $2 \frac{1}{2}$ drachms of tartar emetic per day, or considerable doses of alcohol; and this treatment seems to have given the best results. Antithermic agents, like acetanilide, phenacetin and quinine sulphate, are too costly to be greatly used in bovine medicine. Salicylate of soda is preferable.

In order to assist circulation, support the tone of the heart and avoid engorgement of the lung and asphyxia; digitalis should be given in doses of $\frac{3}{4}$ to 1 drachm per day, or digitalin in subcutaneous injections of 5 to 6 milligrammes, continued for five or six days. Finally, iodide of potassium may be given in doses of 1 to $1 \frac{1}{2}$ drachms, to reduce inflammation and as an expectorant.

\section{PNEUMONIA DUE TO FOREIGN BODIES-MECHANICAL PNEUMONIA.}

It may happen that in examining a patient pneumonia is diagnosed under circumstances which seem to forbid its being regarded as simple or primary. This may be explained by the fact that ruminants are very 
apt to suffer from pneumonia produced by foreign bodies. The lung may be penetrated either by some sharp object making its way forwards from the rumen or recticulum or by liquid or solid material passing into the trachea. These are two common methods by which this form of pneumonia is produced.

PNEUMONIA DUE TO THE MIGRATION OF FOREIGN BODIES FROM THE RETICULUM.

Causation. The conditions under which food is swallowed by ruminants after preliminary mastication permit indigestible objects, such as stones, fragments of wood, nails, needles, bits of iron wire, etc., to enter the rumen, whence they reach the reticulum in consequence of peristaltic movements. Sharp, perforating objects, like needles or fragments of iron wire, penetrate the walls of the gastric compartments, and, impelled by the movements of these organs, pass through the intervening tissues, usually in the direction of the heart. Under conditions which cannot precisely be defined, these foreign bodies make their way towards the pleural cavity (usually the right, in consequence of the situation of the reticulum), traverse the diaphragm, and directly penetrate the base of the lung.

As the migrating object is usually infected, its passage through the diaphragm always produces a localised patch of diaphragmatic pleurisy. Although possible, it is only rarely that the pleural sac becomes generally infected, or that rapidly fatal septic pleurisy is set up. Usually the localised pleurisy causes the base of the lung to become adherent to the anterior surface of the diaphragm. The foreign body continuing its movements, passes into the lung, and there sets up pneumonia.

Symptoms. When the practitioner is first consulted he often finds only indications of the crisis period of a localised pneumonia at the base of the affected lung. The symptoms include fever, accelerated breathing, moaning, loss of appetite, cough without discharge, dulness over the base of the lung on percussion, disappearance of the respiratory murmur in the dull area, souffle opposite the inferior bronchi, and normal or juvenile respiration towards the front, i.e., in the anterior lobe, and sometimes in the cardiac lobe.

The temptation under such circumstances is to deliver a diagnosis of simple pneumonia with prognosis of probable recovery. It should be remembered, however, that in all cases of basilar pneumonia without affection of the anterior lobes there is a considerable chance of the condition being due to the presence of a foreign body. On more careful examination it is found that the intercostal spaces opposite the affected region are very sensitive, and that the circle of the hypochondrium is 
correspondingly sensitive. The owner, moreover, almost always informs the practitioner that for several weeks his animal has coughed, shown tympanites, diminished appetite, etc.

Compression of the roots of the corresponding diaphragmatic nerve at the base of the neck always produces coughing.

These symptoms rarely accompany the development of simple pneumonia. Furthermore, the course of this accidental pneumonia is entirely different. Instead of developing regularly according to the abovedescribed cycle, pneumonia due to foreign bodies develops slowly, and only becomes well defined after several weeks, whilst its tendency is to grow more and more aggravated. The zone of dulness extends both in a forward and upward direction. The souffle extends forwards. Auscultation and palpation sometimes reveal the formation of an abscess or local gangrene; while there is slight œedema of the wall of the chest, as well as a gurgling sound at the moment when the lung is displaced, high fever, intensely coloured urine, and very marked leucocytosis, etc. Death is inevitable, and when gangrene exists it sometimes occurs suddenly.

Diagnosis. The diagnosis is based on the information furnished with regard to the course of the disease, the localisation of the hepatised zone, and the progressive character of the affection.

The diagnosis, nevertheless, is always a little doubtful, but may be so far assured as to attain the position of a quasi-certainty.

Prognosis. The prognosis is unequivocal.

Treatment. No practical treatment, either to extract the foreign body or to combat the special pneumonia which it has produced, can be attempted. All the interlobular connective layers and the lobules themselves are invaded by various micro-organisms carried by the foreign body. Numerous fragments of tissue serve as centres of suppuration and gangrene, and the only chance would lie in attempting resection of the lung. Such intervention has no practical interest in veterinary surgery. It is true that when the existence of an abscess is suspected, an aseptic exploratory puncture may be made, and, in the event of the diagnosis being so far confirmed, the abscess might be opened through an intercostal space. Under such circumstances, however deep the point of penetration of the foreign body, the development of the resulting abscess causes local pleurisy and adherence between the pleura and lung, so that there is no immediate danger of producing purulent pleurisy and pneumo-thorax. If small the foreign body might possibly be discharged through the passage thus afforded.

In practice the best plan is to recommend slaughter as soon as the diagnosis becomes certain, provided that the meat can still be utilised. 


\section{PNEUMO-MYCOSIS DUE TO ASPERGILLI.}

The term pneumo-mycosis, or pulmonary aspergillosis, is used to denote a condition due to the growth in the respiratory apparatus of a fungus of the order aspergillus (family, Perisporice; sub-order, Perisporiacece; order, Ascomycetes).

In ruminants, as in all other animals, pulmonary aspergillosis occurs accidentally, and may often pass unperceived, in spite of the indications given by Lucet and Bournay regarding its development and symptoms.

It seems most frequently to be caused by Aspergillus niger and Aspergillus fumigatus, particularly by the latter, which, according to Rénon's work, also appears to be the most pathogenic. It only develops in animals whose respiratory apparatus is injured and is the seat of such lesions as those of chronic bronchitis, bronchi-ectasis, and of parasitic lesions or those containing cavernous spaces resulting from abscess formation, etc., etc.

The fungi, or more properly the spores, which have accidentally penetrated into the respiratory channels germinate and develop in the pathological dilatations, causing disseminated areas of pneumonia and some mechanical disturbance, but not producing intoxication by liberating toxins.

Causation. Infection occurs through the air passages, in consequence of the inspiratory current carrying spores of the fungi into the ramifications of the bronchi, where they develop if the soil is favourable. Development is favoured if the animals exposed to contamination are in bad condition, or if, as sometimes happens, the walls of the stables are not kept clean, and are covered with various forms - of fungi.

Prolonged feeding on musty fodder may also favour respiratory infection; but it seems highly improbable that under ordinary conditions infection can occur through the digestive tract. Infection of the lung must also be regarded as exceptional, if one bears in mind the frequency with which oxen are fed on musty or mildewed fodder and the small number of accidents recorded.

Symptoms. 'The symptoms are obscure, and pulmonary aspergillosis is often only discovered on post-mortem examination. A cough is the chief symptom. It is dry at first. Afterwards it becomes spasmodic and frequent.

Respiration is difficult, dyspnœic, effected only with effort, and sometimes even discordant. Expiration is sighing.

Percussion reveals zones of partial or complete dulness when the lesions are near the surface of the lung, which, however, is rare. 
Bournay has noted the occurrence of a musical sound resembling that obtained by tapping a small crystal or glass bell.

Auscultation is said to reveal rough or sibilant râles, but as the lesions occur only in animals whose respiratory apparatus has already been impaired, it is difficult to offer an opinion on the subject.

These signs are invariably accompanied by a certain amount of general ill-health, wasting, and irregularity of appetite and of rumination.

On post-mortem examination the lung of the affected animal appear's strewn with nodules, varying in size between a hazel-nut and a walnut. On section, Bournay claims to have found a wall or fibrous shell covering a greenish cryptogamic growth, in the centre of which was a yellowish, sharply defined kernel formed of masses of fungi (Mycelium, sterigmata and spores). In cases of rapid development, the pulmonary tissue around the parasitic lesion is completely hepatised.

Diagnosis. Diagnosis is impossible without recourse to microscopic examination of the discharge. This examination, which, however, is somewhat difficult, may, after staining, result in the detection of débris of the mycelial filaments and of spores, with or without bacilli of tuberculosis. The diagnosis as regards fungi can only be assured by preparing cultures.

Prognosis. The prognosis is grave, because the disease is always superadded to lesions, which of themselves would justify a sombre view.

Treatment. In consequence of the small number of observations published and the difficulty in diagnosis, no rational treatment has hitherto been laid down. From a purely theoretical standpoint, fumigations with tar and essence of turpentine and the inhalation of carbolic spray have been recommended. Life in the open air would, without doubt, be equally or more efficacious. Preventive treatment consists in withholding musty fodder and keeping the stables clean.

\section{GANGRENOUS BRONCHO-PNEUMONIA DUE TO FOREIGN BODIES.}

Foreign bodies which find their way into the trachea instead of the asophagus provoke in most cases broncho-pneumonia, which very rapidly terminates in gangrene and death.

Causation. Forced feeding of sick animals which have lost their appetite is one of the principal causes of this grave condition. In order to administer food such as mashes, gruel, hay tea, etc., the oxherds have a bad habit of lifting the entire head and drawing forward the tongue whilst they pour the concoctions into the animal's mouth. The liquid cannot then be divided into portions, deglutition in the pharynx is badly effected, and the substances administered find their 
way partly into the larynx and partly into the œsophagus. In the case of astringent, bitter, or highly stimulating drugs, a similar accident may be caused by spasm of the pharynx or esophagus, where the tongue has not been left free and excessive quantities have been given.

Again, during the course of diseases complicated with paralysis of the pharynx (cow-pox, parturient apoplexy), obstruction of the pharynx and œsophagus (tympanitic indigestion), intense pharyngeal dysphagia (footand-mouth disease), etc., the risk of broncho-pneumonia due to foreign bodies is much greater still. It may even occur spontaneously in animals in the enjoyment of complete freedom (foot-and-mouth disease).

Lastly, cases of broncho-pneumonia have been described as a consequence of inhaling foreign bodies, when the animals are fed, for example, with meal made from undecorticated cotton-seed. Under such circumstances the lesions produced are similar to those of pneumoconiosis in man (the chronic forms of pneumonia of miners, charcoal-burners, quarrymen, stonemasons, etc.).

Symptoms. The symptoms of gangrenous broncho-pneumonia become apparent immediately after the foreign body has entered the trachea. They commence with a violent, spasmodic cough, produced by reflex action, which in its turn is due to the laryngeal mucous membrane having been touched. But this cough is now too late to be of use, for the food, drug or liquid has passed into the depths of the trachea, and cannot be ejected. The cough soon ceases, and the animals may even return to their food. These appearances, however, are deceptive, for twelve, twenty-four or forty-eight hours later the cough reappears, whilst appetite diminishes. The attacks of coughing are succeeded by the discharge of a greyish or reddish-grey offensively smelling material; respiration becomes more rapid, the heart's action violent, and the temperature rises to $103^{\circ}$ or even $105^{\circ} \mathrm{Fahr}$. (39.5 to $40.5^{\circ} \mathrm{C}$.).

The patients soon refuse all solid food, and if the chest is then examined by percussion one finds partial dulness, rarely simple dulness, over the cardiac lobes opposite the point where the girth passes. The partial dulness may rise to a varying height on both sides; sometimes it is confined to one side.

On auscultation the respiratory murmur in the upper two thirds of the lung appears exaggerated on both sides, and is found to have greatly diminished, or disappeared altogether in the inferior zone.

Auscultation through the scapula almost always shows that the anterior lobes are affected; but, at all events, in the examinations we have made, checked by post-mortem examination, the cardiac lobes have always proved to be most affected, a fact attributable to the direction of the principal bronchi. The lower portion of the posterior lobes may also be affected, but this is rarer. All the lower zone is irregularly 
hepatised, and on auscultation one hears large moist râles, whilst respiration sometimes appears of a blowing character, and divided by a pause, but there is no tubal souffle. If the patient survive for a certain time, the sounds heard on auscultation undergo change; gurgling noises and sometimes true cavernous souffles are heard, as a result of suppuration in the bronchi and gangrene of one or more areas in the lung. Diffuse gangrene is rare, and the inferior zone is usually the only portion affected.

During this phase the expired air has an absolutely characteristic gangrenous odour.

Death occurs by asphyxia and intoxication, but some animals hold out for a fortnight and more.

Lesions. Post-mortem examination reveals a suppurative but secondary inflammation of the mucous membrane of the nasal cavities, pharynx, larynx, and trachea.

In the bronchi, sometimes very deeply placed, remains of foreign bodies are found in cases where some solid material has been inhaled. The mucous membrane of the bronchi is violet in colour, in places appears to be sloughing, and is covered by gangrenous patches immersed in a reddish-grey putrid fluid of offensive odour. In places the pulmonary tissue has undergone gangrene; and incision of the diseased centres discovers irregular cavities, filled with a pultaceous, greyish material, which often makes its way into the bronchi. These are the irregular cavities which give rise to the gurgling sounds. The walls of these cavities are formed of disintegrating pulmonary tissue, which again is surrounded by a zone of grey hepatisation. The gangrenous areas may unite, forming vast caverns. If near the surface they cause adhesive or septic pleurisy.

Diagnosis. The diagnosis is not very difficult, provided that an exact account can be obtained of the circumstances which preceded the appearance of the disease. The signs furnished by the discharge, the expired air, percussion and auscultation are sufficiently significant to remove any doubt.

Prognosis. The prognosis is extremely grave, and in the great majority of cases fatal.

Treatment. There is very little chance of recovery, no matter what treatment may be employed. The most favourable termination consists in the gangrene remaining limited to the bronchi and to a small fragment of the lung, so that the damaged tissues, being gradually delimited and sloughed off, may finally be discharged by coughing.

This is an exceptional termination, but attempts may be made to assist its evolution by giving alcohol in doses of 8 to 10 ounces per day, and salicylate of soda in doses of 4 to 5 drachms. When the condition

D.C. 
can be early diagnosed before intense and continued fever has set in, and when the animal's condition is good, it is often preferable to slaughter the patient.

\section{INFECTIOUS BRONCHO-PNEUMONIA.}

The ox's lung is liable to so many and such extremely varied diseases that it seems desirable to add to the above descriptions some remarks on infectious broncho-pneumonia of external or internal origin.

Anatomically these forms of broncho-pneumonia are characterised by the occurrence of "islands" of pulmonary hepatisation, more rarely by extensive (massive) hepatisation; in all cases the hepatisation is irregular, and in no way resembles that of simple pneumonia.

Causation. The internal causes are numerous and varied. They are due to primary infection of an organ whence arises a general infection, sometimes even true septicæmia. Some form of broncho-pneumonia, such as simple broncho-pneumonia, purulent broncho-pneumonia, gangrenous broncho-pneumonia, etc., then follows as a complication. These broncho-pneumonias are therefore only manifestations of purulent infection or septicæmia. They frequently follow postpartum infections, vaginitis, metritis, and suppurative mammitis.

Symptoms. The general symptoms first attract attention, and are extremely acute. They comprise high fever, loss of appetite, cessation of rumination and of milk secretion, breathlessness, blowing, etc.-all signs of grave and rapidly progressive infection.

Sometimes at this period nothing more than the primary lesion, such as metritis or mammitis, can be detected. It may even happen that the uterus seems little affected, and, despite the accelerated respiration, neither partial nor complete dulness of the lung is discovered.

Hepatisation only occurs some days afterwards, and with it irregular partial dulness localised in the lower zones, disappearance of the respiratory murmur in the corresponding regions, exaggeration in the infected regions, an expiratory sound which is barely perceptible or may be of a blowing character, or, again, after several days may be transformed into a tubal souffle.

The cough then becomes frequent, generally difficult, paroxysmal, feeble, and easily provoked. The appetite suffers, the patients seem to prefer fluid nourishment and lose flesh very rapidly.

If the broncho-pneumonia is about to terminate in suppuration or gangrene, the respiration becomes sighing, the breath fœetid, and the cough is accompanied by a greyish muco-purulent or gangrenous discharge. 
When the abscesses are deep-seated, the alarming symptoms retain their primary degree of intensity for weeks, until the animals are completely exhausted. Abscesses, originating in deep-seated parts, may even extend towards the surface of the lung, and produce either adhesive pleurisy that can be detected by palpation, or exudative pleurisy, easily recognised on percussion.

When the infective microbes are not pyogenic the general condition appears less grave, the animals exhibit only moderate fever, appetite is diminished but not lost, wasting is slower, and may continue for months, but the affected portions of lung become converted into fibrous masses or a material resembling spleen pulp.

The duration of infectious broncho-pneumonia, therefore, varies with the nature of the infecting organism. In cases which terminate in gangrene, the animals may survive for three or four weeks; in those where suppuration occurs, for several months. In short, recovery is the rule in simple broncho-pneumonia; but from an economic standpoint there is little reason for keeping the animals alive.

Diagnosis. The diagnosis is not generally very difficult; for if at first the case may be mistaken for one of simple pneumonia, the persistence or prolonged aggravation of the symptoms and the irregularity in position of the lesions revealed by percussion and auscultation enable the condition to be distinguished at an early period from simple pneumonia.

Confusion with acute or chronic pleuro-pneumonia may easily be avoided by noting the absence of pleural effusion, and of the soft pleuritic souffle of peripneumonia, etc.

Where auscultation is chiefly relied upon it is more difficult to differentiate between this disease and acute tuberculosis, and between. it and broncho-pneumonia produced by foreign bodies, although the latter disease develops differently.

Prognosis. The prognosis is always extremely grave and, in cases where there is gangrene or abscess formation, fatal. From the economic standpoint the chronic form is also very grave.

Treatment. As broncho-pneumonia is frequently of a secondary character, treatment should at first be particularly directly against the primary condition, whether in the mammary gland, uterus or elsewhere. Early treatment of suppurative mammitis, metritis, etc., is therefore necessary.

Broncho-pneumonia is treated by free vesication of the walls of the chest, the administration of tonics and antiseptics, alcohol in small doses, acetate of ammonia in doses of 1 to 2 drachms, salicylate of soda in doses of 5 to 8 drachms per day, salicylic acid in doses of 1 drachm, and creosote in doses of $1 \frac{1}{2}$ to 5 drachms, given in electuary, etc.

Diuretics, farinaceous gruels, etc., may be used freely, and are of value. 
If the symptoms persist or become aggravated, and suggest the development of an abscess or gangrene, it is better to slaughter the animal.

\section{BRONCHO-PNEUMONIA OF SUCKING CALVES.}

Young animals still with the mother, particularly calves during the first few weeks of life, are liable to broncho-pneumonia of a specialised character, as regards not only its causes, but its development and duration.

Causation. The causes may be grouped under two principal heads :-

(a) In slow or difficult cases of parturition, the fœetus may be injured whilst being delivered, as a consequence of direct compression of the great blood-vessels, etc. (particularly of compression of the umbilical cord, compression of the thorax in the cardiac region, or partial premature separation of the envelopes), and may thus by reflex action make automatic inspiratory movements.

Respiration being impossible, inasmuch as the thorax has not yet passed the posterior passages, such inspiratory efforts made during the passage through the pelvis may cause amniotic liquid to pass into the bronchi. This accident is particularly liable to occur during deliveries with breech presentation. If, as happens frequently, the amniotic liquid has become infected either prior to or as a consequence of obstetrical manipulation, the result is fatal; for the passage of infected amniotic fluid into the bronchi develops a bronchopneumonia of a degree of gravity depending on the character of infection.

(b) By an entirely different mechanism broncho-pneumonia may occur in sucking calves during the first few weeks of life, even in the case of animals born in a vigorous condition, and kept in warm and well-arranged stables. 'This form follows diarrhœa, and constitutes a final complication which is always of very marked gravity, and in most cases fatal.

Such secondary broncho-pneumonia only occurs when the diarrhœa has resisted treatment, and it is important to note that the pectoral lesions appear at a time when the intestinal mischief seems to have diminished, the diarrhoa having lessened or disappeared. This variety of broncho-pneumonia of young animals is by far the most frequent. It has been termed broncho-pneumonia of intestinal origin, and exactly resembles, so far as its development and gravity are concerned, the broncho-pneumonia in young infants described by Sevestre and Lesage.

The term broncho-pneumonia, moreover, is not strictly correct, or at least is not exclusive; for the rapid forms often exhibit lesions other than those of broncho-pneumonia. Post-mortem examination reveals pleurisy and pericarditis. 
Pathogeny. At the outset of these attacks of broncho-pulmonary disease, a careful bacteriological examination of the organisms to be found in the discharge of bronchial mucus leads to the discovery of bacilli which do not stain with Gram, and which resemble varieties of the colon bacillus; in other cases of streptococci. At a later stage, when the animal has become weak, micro-organisms are present in much greater variety. Nocard found in lung abscesses the bacillus of epizootic lymphangitis. It seems that the development of various lesions in the thoracic cavity may be due to auto-infection, i.e., to the penetration from the intestine of germs which, after passing through the circulation, establish themselves at some point in the lung. The pleura is attacked at a later period as a consequence of continuity and contiguity of tissue.

In a similar way pericarditis and even valvular endocarditis may be produced.

Symptoms. The symptoms are similar to those of all forms of broncho-pneumonia. Where diarrhœa has been neglected, the conditions may apparently improve without evident cause, whilst the respiration becomes more frequent. The patient soon suffers from cough, and in a few hours the existence of broncho-pneumonia is clearly apparent. Acceleration of breathing is the dominant symptom. The respirations may rise to fifty to sixty per minute, at which they continue, while fever sets in. On percussion the thorax may appear of normal resonance throughout; but when pleural lesions and exudates exist, resonance gives place to partial or complete dulness. Should pericarditis or small cardio-pericardial adhesions exist, they may escape observation, but if the exudate is abundant or the adhesions multiple or of large size the usual symptoms of pericarditis develop progressively.

On auscultation the respiratory murmur is always found to be greatly exaggerated in the healthy parts, usually the upper portions of the lung. On the contrary, it is attenuated or suppressed in the affected regions. The other signs vary greatly, according to the extent, intensity, and more or less advanced condition of the lesions. Crepitant and bronchial râles, blowing respiration and tubal souffles, etc., are among the symptoms.

The duration of the disease varies; some patients may be carried off in five or six days, while others survive for one or two months, or even longer. A few recover, but they remain thin, puny, and atrophied, and are not worth keeping alive.

Lesions. The lesions extend to the bronchi, the pulmonary tissue, and sometimes the pleura and pericardium. They consist in lesions of diffuse broncho-pneumonia, pleurisy with false membranes and parietopulmonary adherences, and pericarditis with partial cardio-pericardial adhesions. 
In rare cases abscesses caused by pyogenic streptococci may be found.

The anterior lobes, cardiac lobes, and lower part of the posterior lobes are those singled out for attack.

Diagnosis. The diagnosis is not difficult, provided that the circumstances preceding the appearance of the pulmonary lesions are known.

Prognosis. The prognosis is very grave.

Treatment. Treatment very often proves useless, because the patients have little resisting power and are exhausted, and also because they are suffering from a slowly progressive septicæmia. It may, however, be worth while in the early stages to apply blisters to the chest and administer general stimulants : alcohol in doses of 8 to 12 drachms per day, divided into two parts and mixed with milk; acetate of ammonia in doses of $\frac{1}{2}$ to 1 ounce; and tinctura digitalis 5 to 6 drops.

The primary disease of the intestine is masked by the pulmonary symptoms, but should not be overlooked. Rice water, sub-nitrate or salicylate of bismuth may be added to the milk or albuminous solutions constituting the diet. When an epizootic of broncho-pneumonia complicates the diarrhoea it is necessary to take all the preventive measures which have been suggested in connection with white scour and umbilical diseases in calves. These comprise disinfection of the premises and local disinfection of the animals affected.

\section{SCLERO-CASEOUS BRONCHO-PNEUMONIA OF SHEEP.}

The sheep suffers from a special form of broncho-pneumonia, which is seldom seen except in isolated cases, but which, under exceptional circumstances, may nevertheless attack a certain number of animals in a particular flock. It was first noticed and described by Liénaux in 1896, and has more recently been studied by Sivori (1899). Moussu has only seen it in flocks in the north of France.

Causation. The causes of this disease are still imperfectly understood.

Sivori's researches show that the disease may be referred to a microorganism, but we do not yet know exactly by what path infection occurs.

The agent of sclero-caseous broncho-pneumonia in the sheep appears similar to that described by Preisz and Guinard in 1891, and identical with the microbe of ulcerative lymphangitis of the horse (Nocard, 1897). It is probable that infection occurs through the respiratory apparatus.

Symptoms. The clinical development of the disease is difficult to describe, because its course is slow and unaccompanied by well-marked external signs.

The animals lose flesh, pant for breath when moved, drop to the rear of the flock cough frequently, feed badl and end by becoming 
cachectic. Many suffer from the disease and yet remain in fair bodily condition.

On post-mortem examination the lungs are found not to collapse, having lost their elasticity, and are of a yellowish-white colour, which is only seen in this disease. On section the pulmonary tissue appears dense, hard, and of a fibrous and lardaceous character. At various points nodules with fibrous envelopes and caseous, yellowish or greenish contents are found.

When the caseous nodules are near the surface the pleura may be chronically inflamed and thickened. The liver and kidney frequently contain caseous lesions.

Diagnosis. The diagnosis becomes easy after the first post-mortem examination, for the lesions discovered cannot be mistaken for those of parasitic broncho-pneumonia, degenerated pulmonary echinococcosis or tuberculosis. In the living animal, on the contrary, the diagnosis is extremely difficult.

Prognosis. The prognosis is grave. No special method of treatment is known.

\section{PULMONARY EMPHYSEMA.}

Pulmonary emphysema, i.e., exaggerated dilatation of the pulmonary tissue by air, is not uncommon in the bovine species, and occurs under the two classical forms-(1) alveolar or intra-lobular emphysema limited to dilatation of the alveoli; and (2) interlobular emphysema, produced by the entrance and diffusion of air in the interlobular spaces in consequence of rupture of the lobules.

These two forms are very frequently associated:-

(1.) Emphysema by dilatation usually begins in the right pretracheal lobe; also in the cardiac and even in the posterior lobes.

(2.) Interlobular emphysema begins in the same regions, but it spreads readily in a backward direction, remaining interstitial ; or, on the other hand, becoming sub-pleural at the periphery of the lung.

In both cases the pulmonary tissue is pale, the blood-vessels are partially obliterated by compression; circulation and aeration of the blood are impeded-hence the appearance of the disturbance noted.

Causation. Emphysema is seen in adult working oxen; also, and to an even greater'degree, in aged cows. It is produced by excessive strains in draught, or more often by the paroxysms of coughing so common during simple or parasitic bronchitis, broncho-pneumonia, pneumonia, chronic broncho-pneumonia, etc. Successive gestations also produce it.

All these pathological conditions also interfere with the nutrition of the bronchial mucous membrane, particularly of its deep-seated muscular layer, which is then incapable of regulating the distribution of air in the 
bronchial channels. The distribution being no longer regulated by reflex action, air accumulates at certain points as a result of the expiratory efforts made during coughing, and dilatation of the vesicles or lobules occurs.

Diseases of the digestive apparatus, acute or chronic tympanites in particular, may play a certain part by compressing the diaphragm, causing expiratory efforts and fits of coughing.

Furthermore, swelling of the lymphatic glands at the entrance to the chest, by compressing the pneumo-gastrics, provokes reflex cough and finally emphysema.

Symptoms. Pulmonary emphysema is marked by accelerated respiration due to diminution in the respiratory capacity, which is often very seriously affected; to insufficient absorption of oxygen in consequence of diminution in the space available for exchange of gases in the lung, and to insufficiency of expiration. This acceleration in breathing, though little marked during repose, becomes very pronounced after exercise, or during hot weather; and under these circumstances is accompanied by a paroxysmal, feeble but shrill cough, without discharge. This cough without discharge is frequently followed by swallowing.

Percussion reveals an important point, viz., increase in the normal resonance of the thorax.

On auscultation the vesicular murmur is found to be diminished, the respiration assumes a rough and rasping character, inspiration is difficult, expiration painful, and often divided into two periods, as indicated by a slight double movement of the flank. Expiration is clearly audible. Its duration is generally less than that of inspiration, although in some cases it is equal or even longer. It is accompanied by sibilant and snoring râles, sometimes even mucous ráles, of an intermittent character. In rare cases there may be difficulty of respiration, as in broken-winded horses.

Diagnosis. The diagnosis may suggest a doubt as to whether emphysema or tuberculosis is present, but in the latter there is fever, the general condition is poor; on percussion the thorax reveals areas of partial dulness; and expiration is rough and prolonged, sometimes of a blowing character, a peculiarity which is exceptional in emphysema.

Prognosis. The prognosis is not very grave, except where emphysema is only an accompanying symptom of another disease, such as chronic bronchitis, tuberculosis, etc.

Treatment. Little can be done to check the development of the above described pulmonary lesions; but the cough may be relieved, and the pulmonary circulation improved by assisting the heart.

The most prompt and efficacious assistance is given by digitalis in doses of $\frac{1}{2}$ to 1 drachm per day for adults, iodide of potassium in doses of 
1 to $1 \frac{1}{2}$ drachms, and bromide of potassium in doses of 1 drachm to guard against reflex excitability of the pneumo-gastric. This treatment, however, should not be followed for more than five or six days, and should then be replaced by the administration of arsenious acid in doses of 15 grains per day, ground horse-chestnuts in doses of 3 ounces per day, etc., etc.

\section{DISEASES OF THE PLEURA.}

Primary inflammation of the pleura is very rare in animals of the bovine species, but secondary diseases of this membrane, on the other hand, are frequent.

\section{ACUTE PLEURISY.}

Cruzel, Fabry, and a number of practitioners have described the occurrence in working animals of acute pleurisy à frigore or serofibrinous pleurisy in consequence of severe, sudden variations in temperature or prolonged chills. At the present day it seems fairly well established that pneumonia, and not pleurisy, is commonest under such conditions, and Moussu disclaims ever having seen primary pleurisy. On the other hand, pleuritic effusions are very common in contagious pleuro-pneumonia, secondary pleurisy due to pericarditis produced by foreign bodies, septic broncho-pneumonia or broncho-pneumonia due to foreign bodies, and the pleurisy which accompanies septicæmia consequent on parturition, etc. These forms of disease, however, are not simple sero-fibrinous pleurisy, but septic or suppurative pleurisy, still little understood in veterinary surgery.

T'uberculosis of the pleura, although very frequent, is rarely accompanied by marked exudation. Like secondary disseminated pleural carcinoma, it usually assumes the vegetative and adhesive form, with adhesions of greater or less extent between the lung and wall of the chest.

Symptoms. In all these morbid conditions the symptoms vary greatly, and it would be difficult to give an accurate general description of them.

In acute pleurisy à frigore shivering attacks, moderate fever, dulness, loss of appetite, interference with rumination, dryness of the skin, rapid wasting and intercostal pain, first indicated by dull colic, constitute the usual symptoms.

The respiration is short and irregular, interrupted when the exudation is abundant. Pressure over the intercostal spaces produces pain, as does strong percussion. Percussion reveals an area of dulness bounded above by a horizontal line.

Auscultation shows the respiratory murmur to have disappeared throughout the zone of dulness, and reveals the presence of a soft 
pleuritic souffle (a soft tubal souffle quite different from that of contagious pleuro-pneumonia) when pleural exudation is abundant. In septic or suppurative pleurisy fever is higher, loss of appetite more marked, wasting more rapid, and depression extreme, with, however, identical local symptoms.

Diagnosis. The diagnosis of pleural exudation presents little difficulty, because of the peculiar characters of the dulness and the pathognomonic indications obtained by auscultation. The exudation is usually unilateral, the mediastinum being very resistant and not perforated in the ox.

By passing the needle of a Pravaz's syringe with antiseptic precautions through the intercostal space a little fluid may be drawn off and the diagnosis formed, the form and nature of the pleurisy being simultaneously established. The liquid extracted can be examined bacteriologically, and can be grown on nutritive media, or inoculated into experimental animals.

The prognosis is grave, because in the ox pleurisy is very often of a secondary character. The outlook varies, however, with the form of the pleurisy and the nature and virulence of the infecting organism.

Treatment. Treatment consists firstly in applying an energetic vesicant like antimonial ointment or liquid cantharides blister; internally diuretics such as soda bicarbonate, nitrate of potash, resin, and decoctions of pellitory, dogs' grass, etc., may be given. If thought desirable the chest may be tapped and the pleural cavity washed out with an antiseptic solution.

CHRONIC PLEURISY.

Chronic pleurisy is frequent in aged animals, but usually assumes the form of local adhesive pleurisy. The adhesions between the lung and pleura are more or less extensive; they result from verminous bronchopneumonia, echinococcosis, external injuries, etc. Clinically this form is of no importance, and is almost impossible to diagnose. During the development of pleural tuberculosis, on the contrary, adhesive dry pleurisy is frequent, and sometimes becomes so well marked that almost the whole of the opposing pleural surfaces may become united.

\section{PNEUMO-THORAX.}

The name pneumo-thorax is given to the condition produced by the entrance of air or gas into one of the pleural cavities.

The accident is usually produced by rupture of the parenchyma of the lung and of the pleura, a rupture which produces a communication between the alveoli or a bronchus and the corresponding pleural cavity. 
As soon as the rupture occurs air passes from the lung into the pleural cavity, and the lung collapses in consequence of the resilience of its elastic constituents.

Under other, much rarer, circumstances pneumo-thorax occurs in consequence of gas generated in the digestive tract passing into the pleural spaces. The condition then makes rapid progress, and death occurs in a few days.

Symptoms. The symptoms are well defined. As soon as the accident occurs the animal exhibits extremely marked and sudden dyspnœea, accompanied by heaving at the flank or general agitation of all the muscles of the body. One of the lungs, in fact, has suddenly been called on to perform the functions of both, and at first it naturally has great difficulty in meeting this demand.

The heaving at the flank and the general agitation of the body muscles is due to the fact that the regularity and rhythm of contraction of the diaphragm are disturbed, and the mechanical conditions have become different on the two sides. From the first, respiration is moaning and expiration becomes rapid, stertorous and deep, while the face is anxious-looking, and the nostrils are dilated as though the animal was on the point of suffocation. On examining the animal from in front or behind, the thorax is easily seen to be wanting in symmetry,

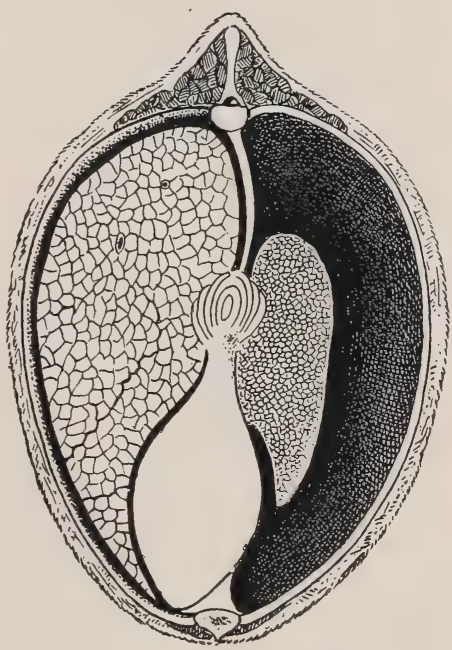

FIG. 171.-Schemaillustrating open pneumo-thorax. Right lung collapsed; pericardium and heart displaced towards the right. the side on which the pneumo-thorax has occurred being immobile as compared with the sound side. The latter, moreover, is dilated in order to compensate for the loss of function of the collapsed lung.

Percussion reveals greatly increased resonance on the side of the pneumo-thorax. On the other hand, the opposite side yields a normal sound.

Auscultation reveals an increase of the respiratory murmur on the side which is still acting and, on the contrary, complete and total suppression of the respiratory murmur on the affected side. On applying the ear to the chest wall, a large soft, amphoric souffle of well-marked metallic character is heard. This is particularly clear on respiration, giving the impression of the existence of a large cavity beneath the ear. 
The sighing sound heard on auscultation of the chest wall is louder than that heard externally or over the region of the nostrils or larynx; and it seems to be reinforced, as though by the resonance of a large cavity with thin metallic walls. Once or twice per minute, moreover, a sound may be heard like that of dropping water. It is of a very special character, resembling that produced by drops falling to the bottom of a hollow metallic vase, and setting up prolonged vibration.

As secondary symptoms the heart's action is accelerated, the number of beats rising to 80 or even 120 or 130 beats per minute; appetite is lost; slight tympanites develops as a result of rumination and eructation being suspended; the peristaltic movements of the rumen are interrupted, and constipation develops.

Diagnosis. The diagnosis of pneumo-thorax is easy, and the condition can scarcely be mistaken for any other except diaphragmatic hernia; but the indications derived from percussion and auscultation are so different in the two cases that they need not be further emphasised.

The task becomes more difficult, however, when an attempt is made to identify the exact form of pneumo-thorax, for three principal varieties are recognised.

In open pneumo-thorax, the first and most frequent form, air passes from the lung into the pleura at each inspiration, and flows back from the pleural cavity towards the bronchus at each expiration. The intrapleural pressure is then approximately equal to the intra-bronchial pressure, and undergoes similar oscillations. (It should be noted that the aperture in the lung is seldom sufficiently large to establish an absolute equality of pressure between the bronchus and the pleural cavity. Respiration, therefore, though very seriously impeded, generally continues in a modified form.)

In a second variety, termed "valvular pneumo-thorax," air passes freely from the lung into the pleural cavity, but is unable to return from that cavity towards the lung, because a flap of tissue acts as a valve and closes the orifice at the commencement of expiration. As soon as intra-pleural pressure rises above that of the inspiratory effort, the valve remains permanently closed.

In the third variety, called "closed pneumo-thorax," the orifice of communication is obstructed by some mechanism, and the pleural sac only contains a film of air.

In practice, valvular pneumo-thorax is recognised by the movement of the thoracic wall (which in open and closed pneumo-thorax remains depressed), as well as by extreme intensity of the dyspnœea and attacks of threatened suffocation. Closed pneumo-thorax, which is only a termination and a stage in the cure of open pneumo-thorax and of valvular 
pneumo-thorax, is suggested by progressive improvement in the symptoms. Scientifically it is very easy to make this diagnosis by putting a manometric apparatus in communication with the pleural cavity by means of a simple hollow needle provided with a thick-walled rubber tube.

In open pneumo-thorax the liquid column in the manometer undergoes rhythmic oscillations corresponding to the respiratory movements; in valvular pneumo-thorax the intra-pleural pressure increases progressively until it becomes higher than the external pressure; and finally, in closed pneumo-thorax, the column of the manometer assumes a certain level at which it rests.

Prognosis. The prognosis is very variable, according to the primary cause of the accident. Animals might recover, but economically there is little advantage in preserving them when the diagnosis is assured, except in cases of animals of great value, and when the primary disease admits of it.

Causation. Pneumo-thorax may be produced by various causes. The most frequent cause in large animals is pulmonary echinococcosis, during the course of which a peripulmonary vesicle, after having injured several lobules, one of the air passages or even a bronchiole, may break through the pleura, thereby setting up direct communication between the bronchi and the pleural cavity.

To pulmonary tuberculosis, with peripheral softened tubercles, perforating simultaneously into an alveolus or a small bronchus and into the pleura, must be assigned the second place.

Vesicular and interstitial sub-pleural pulmonary emphysema is also a frequent cause of pneumo-thorax, the pleura being ruptured over the emphysematous points.

Finally, and exceptionally, an abscess of the lung may open into the pleura and form sinuses, which may establish a communication between the digestive reservoirs and pleural-sacs; but such accidents produce pyo-pneumo-thorax and septic pleurisy of a rapidly fatal character.

The diagnosis of pneumo-thorax, and even of its varieties, does not, however, enable one to form a prognosis; the important point is to ascertain the original cause.

Treatment. It may be said of pneumo-thorax that no treatment exists, and that the position is one of expectancy. In fact, we possess no means of directly dealing with such diseases as echinococcosis, tuberculosis, or emphysema. For this reason it is best as a rule to advise slaughter. Nevertheless, when the condition is due simply to pulmonary echinococcosis of a discrete character, there is some chance that after several months the animal may recover spontaneously. The communicating orifice becomes closed by reparative processes (cicatricial contraction, the formation of a false membrane, limited adhesion between the 
two walls of pleura, etc.) ; the layer of air imprisoned within the pleural cavity is progressively absorbed, provided that it has not been accidentally infected; the collapsed and partially splenised lung progressively regains its function under the inspiratory efforts, and after some months complete recovery may occur. This termination cannot always be confidently predicted, because complications may arise at any moment; under no circumstances can complete recovery be anticipated when the primary disease is tuberculous.

In cases of valvular pneumo-thorax with extreme oppression, attacks of suffocation threatening death as a consequence of excessive intrapleural pressure, displacement of

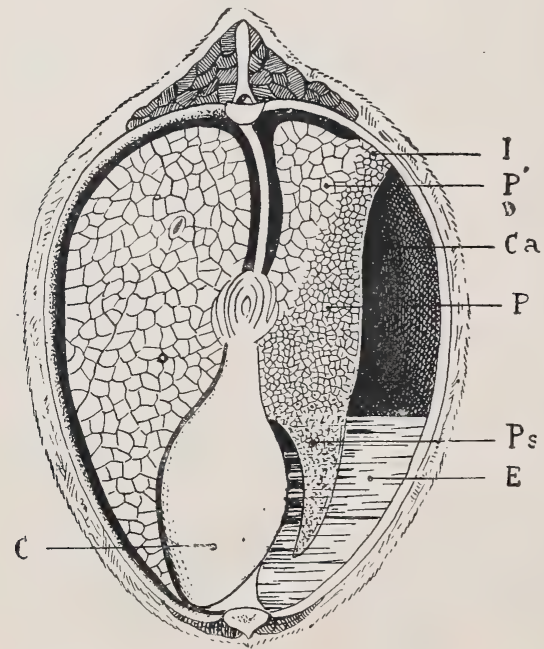

FIG. 172.--Hydro-pneumo-thorax. I, Point of adhesion of the pleura; P, healthy lung; $\mathrm{P} s$, splenised lung; $\mathrm{E}$, liquid or purulent exudate; $\mathrm{C} a$, air cavity constituting pneumo-thorax; $\mathrm{C}$, heart. the mediastinum towards the opposite side, compression of the heart, and functional disturbance of the sound lung, it may be worth considering whether the attacks of suffocation and threatened asphyxia can be modified or removed by preventing the excess of intra-pleural pressure. By simply passing a stout hollow needle through one of the intercostal spaces, the intra-pleural pressure may be reduced to that of the external atmosphere, and the effects of compression removed. This, however, is a last resort, and has no permanent effect.

\section{HYDRO-PNEUIMO-THORAX AND PYO-PNEUMO-THORAX.}

When pneumo-thorax is set up, it rarely remains simple. In the great majority of cases the pleura becomes infected, either directly, by the lesion which has determined the pneumo-thorax (tubercle, superficial abscess, actinomycotic lesion, etc.), or secondarily, by the penetration of germs from the air or from the bronchus (echinococcosis, emphysema). Simple pneumo-thorax then becomes converted into hydro-pneumo-thorax or pyo-pneumo-thorax, according to circumstances - that is to say, whether the exudation into the pleural cavity is of a simple character or is of the nature of pus.

Symptoms. Hydro-pneumo-thorax is characterised by the signs common to true pneumo-thorax, which constitutes the primary lesion, viz., sudden difficulty in breathing, exaggerated unilateral resonance, 
amphoric souffle accompanied by a sound like that of drops of water falling into a metallic vessel, and by the signs of secondary exudative pleurisy, viz., moderate fever, dulness over the lower zones of the chest, limited above by a horizontal line, slight splashing sound, and a soft distant pleuritic souffle.

All the secondary symptoms-loss of appetite, suppressed rumination, sighing, accelerated pulse, etc.- -are found in a more or less accentuated form.

In pyo-pneumo-thorax fever is more marked, while the signs noted on auscultation and percussion are identical, and are accompanied by digestive disturbance and marked œedema of the wall of the chest, which can be seen or detected by palpation.

Diagnosis. The diagnosis is relatively easy when the lesion is secondary; but the difficulty (as in simple pneumo-thorax) is to identify the exact character of the primary affection.

On the other hand, pyo-pneumo-thorax and hydro-pneumo-thorax are not always complete; adhesions of very varying character may exist between the lung and the chest wall; hence it is impossible to group together all the possible symptoms.

Diagnosis is facilitated by aseptically puncturing the chest with a Pravaz's syringe.

Prognosis. The prognosis is extremely grave even in cases of hydropneumo-thorax. Treatment is useless, for even allowing that the primary disease might be cured, this process of cure, after reabsorption of the transudate, would be extremely tedious, and the animals would long remain in poor condition.

Treatment. In hydro-pneumo-thorax no treatment is advisable. Nothing is gained by thoracentesis, at least at an early stage, or before the lesion causing the pneumo-thorax has closed.

In pyo-pneumo-thorax, on the contrary, the theoretical course is to evacuate the pus and completely wash out the pleural sac with lukewarm non-irritant solutions of antiseptics. 


\section{CHAPTER V.}

\section{DISEASES OF STRUCTURES ENCLOSED WITHIN THE MEDIASTINUM.}

The mediastinum is a space enclosed in the median plane of the thorax by the approach of the two opposite layers of pleura. Needless to say, at those points where the layers are in apposition, the space is theoretical only. It extends from the suprasternal region to the dorsal subvertebral region, and encloses all the vessels which pass from or to the base of the heart, the trachea, the oesophagus, the pneumo-gastric, diaphragmatic and cardiac nerves, etc., as well as the pericardial sac and the heart. The organs most frequently affected are the lymphatic glands lodged in the thickness of the mediastinum, the glands placed at the entrance to the chest, the bronchial glands, and the glands situated in the posterior mediastinum.

Inflammation of the mediastinum may coincide with inflammation of the mediastinal layers of the pleura; but this can only be detected on post-mortem examination. The lesions which can be recognised during life are simple inflammation of glands, resulting from pulmonary or pleural diseases, tuberculous inflammation of glands, and the presence of cancerous tumours of the mediastinum and hypertrophy of glands due to lymphadenitis.

Simple inflammation of the lymphatic glands is secondary and consecutive to broncho-pneumonia, verminous bronchitis, infectious bronchitis, etc.

It produces reflex irritation by compressing the pneumo-gastric and laryngeal nerves, and is indicated by loud, spasmodic coughing.

Treatment consists in administering iodide and bromide of potassium, terpine, in doses of 1 drachm per day for adults, essence of turpentine and tar water.

Tuberculous inflammation of glands, inseparable from pulmonary tuberculosis, has very special characteristics peculiar to tuberculosis.

Inflammation due to lymphadenitis is also very easy to diagnose as a rule, in consequence of the symmetrical enlargement of lymphatic glands elsewhere. 


\section{TUMOURS OF THE MEDIASTINUM.}

Sarcomata, carcinomata, lymphomata, and lympho-sarcomata all occur in the mediastinum. They attack young healthy animals, and sometimes develop with such rapidity that in a few weeks they become generalised and invade the heart, lungs, and principal viscera. Their cause is at yet unknown.

Symptoms. At first sight the symptoms are much like those of pericarditis caused by foreign bodies. They consist in deformity of the presternal region, swelling of the jugulars, submaxillary œdema, irregular pretracheal tumefaction, etc.

The tumour, whatever its nature, commences in the mediastinum, develops towards the entrance to the chest, where it projects, and before long produces in the pretracheal region clearly marked œdematous swelling.

Between the two first ribs the tumour compresses the carotids, the jugulars, the nerve trunks, and also the trachea and osophagus, producing difficulty in the return circulation, especially in the jugulars, swelling in the submaxillary space, loss of appetite and dyspnœe.

Palpation affords indication of a tumour of soft consistence, bosselated, more or less adherent to the skin, usually painless on pressure, and of irregular development. Compression of the œsophagus interferes with the deglutition of rough forage, impedes rumination, prevents eructation, and thus produces trifling but permanent tympanites.

The heart is affected reflexly or directly as a result of generalisation of the tumour, and the pulse may rise to 70 or even 120 per minute.

During the first stages neither auscultation nor percussion points to any pulmonary lesion. At a later stage the lung itself may be affected. The other important functions are normal.

Animals suffering from sarcoma, carcinoma, or lympho-sarcoma of the mediastinum waste very rapidly, lose appetite, become feverish, and soon develop cachexia.

Diagnosis. The diagnosis of tumour of the mediastinum is easy, because of the well-marked character of the apparent symptoms.

Prognosis. The prognosis must be regarded as extremely grave, and in most cases fatal, for there is no active method of intervention, extirpation being impossible.

There is no treatment. The animal should at once be slaughtered. 


\section{SECTION I.V.}

\section{THE ORGANS OF CIRCULATION.}

\section{SEMIOLOGY OF THE ORGANS OF CIRCULATION,}

The semiology of the circulatory apparatus comprises the clinical examination of the heart, arteries and veins, and the examination of the pulse and blood.

Heart. In animals of the bovine species, the heart is situated in the thoracic cavity opposite the third, fourth, fifth and sixth ribs, nearly in the median plane of the thorax, and inclined from front to back at an angle of 70 degrees.

The pericardial sac touches the extremity of the sternum where it is in immediate contact with the lower insertion of the diaphragm. This peculiar arrangement favours the development of pericarditis due to foreign bodies.

On the left side the pericardial sac may come in direct contact with the internal surface of the thoracic cavity opposite the lower extremity of the third, fourth, and sometimes fifth ribs. At all other points the pulmonary lobes, as expanded during inspiration, separate it from the thoracic wall.

Although the pericardium and heart are situated in the median plane, percussion and auscultation should be performed on the left side, since the anterior and cardiac lobes of the left lung are less developed than those of the right; but the heart can be auscultated on the right side, as is advisable at times.

In the healthy ox there exists an area of the left thoracic wall which may be called the cardiac zone, on a level with which are heard the normal heart sounds. In diseased conditions this zone or area may vary in size, and the sounds may be modified.

The heart can be examined by inspection, palpation, percussion, and auscultation.

Under ordinary conditions inspection reveals nothing in well-nourished animals; but in very thin subjects and in those suffering from recent cardiac lesions or pseudo-pericarditis, a rhythmic movement of the chest wall is sometimes detected. 
Palpation is performed by placing the open hand on the cardiac zone. In this way the cardiac shock can be felt, its degree of intensity judged, and, in an imperfect manner, its rhythm.

Percussion by means of the fingers or a pleximeter discloses the extent of the physiological area of partial dulness, due to the presence of the heart, as well as its variations in pathological conditions, particularly in pericarditis with marked exudation.

In such cases there may even be complete dulness when the distended pericardium thrusts upwards the corresponding pulmonary lobe, and comes in contact with the internal surface of the thoracic wall, or, in cases of pericardial pneumatosis, exaggerated resonance, and a tympanitic sound.

Auscultation is carried out either directly or by the stethoscope or the phonendoscope. The normal or pathological sounds of the heart are thus ascertained, as well as the intensity of the cardiac beats and sounds, the frequency of the rhythm, ete.

When injuries have occurred which cause murmurs, it is best to use the stethoscope and to apply it at the points where murmurs are heard at their maximum intensity, i.e., exactly at the spot where the cardiac shock is noted, and towards the base, in the region where the great arterial trunks begin.

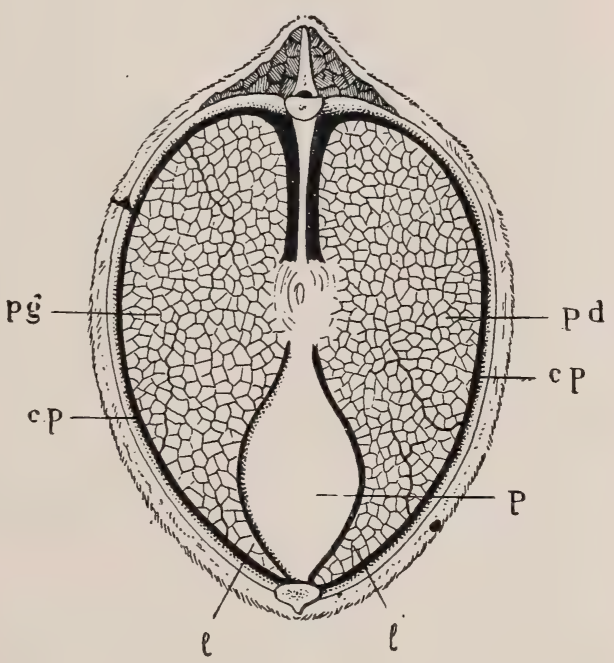

Fig. 173.- Schema of a section through the chest cpposite the heart. Pg, Left lung; $\mathrm{P} d$, right lung; $c p$, right and left pleural cavities; $\mathrm{P}$, pericardium ; $l, l^{1}$, cardiac lobes of the lung interposed between the pericardium and thoracic wall.

In the latter case it is often useful to draw the left fore leg forward.

Arteries. The arteries are rarely the seat of lesions that can be detected by examination, and therefore such examination is usually limited to noting the state of the pulse.

Arteritis and thrombosis of arteries are rare, and although lesions of atheroma have been discovered in certain chronic diseases, such as tuberculosis, chronic diarrhœa, etc., they are difficult to detect, even on examination per rectum of the bifurcation of the aorta.

Pulse. The examination of the pulse, on the contrary, is of great importance. In animals of the bovine species the pulse may be taken at 
a number of different points, such as the submaxillary artery, on the side of the lower jaw: in the case of very thin animals at the radial within and in front of the elbow joint; at the internal saphenous artery, at the height of the mamma or scrotum; or at the coccygeal artery, at the base of the tail.

This examination reveals the frequency ( 50 to 60 per minute), the quality, whether strong, feeble, imperceptible, etc., the regularity, etc., ete.

Veins. The veins are more easily examined than the arteries, on account of their superficial position in most cases.

Inspection and palpation are the only means of examination.

Inspection shows the degree of fulness or collapse, and also the existence or absence of what has been termed venous pulse.

Venous pulse occurs only at the lower extremity of the jugulars. It is very frequent in animals of the bovine species, and in thin animals is not necessarily a pathological symptom. It is due to reflux of blood in the anterior vena cava under the influence of the expiratory effort; sometimes to emphysema, tuberculosis, etc.; in other cases to the return of blood towards the vena cava and jugulars at the moment of auricular systole, as a result of lesions of the tricuspid or auriculoventricular orifices.

By palpation of the veins their permeability can be estimated, also the degree of distension or obstruction, and the condition of their contents.

Capillary system. Among methods of arriving at the state of the circulatory system must be included an examination of the vascular condition of the accessible mucous membranes, such as those of the eye, mouth, nostril, vulva, etc. This examination is easy to carry out, and is of value in diagnosing congestive states, pneumonia, and local inflammation.

Blood. Examination of the blood is sometimes necessary for the exact diagnosis of certain diseases, and therefore should be carried out whenever occasion requires. The physical state, coloration, and rapidity of coagulation afford valuable data in certain diseased conditions, and indicate the approximate richness in hæmoglobin, the normal or abnormal composition of the plasma, and the richness of the blood in white corpuscles.

Microscopic examination is still more valuable, whether carried out by the moist method, in which a drop of blood is compressed under a cover glass, or the dry method with or without staining. In the latter case the specimen is fixed with a mixture of equal parts of alcohol and ether or by immersing it in a 1 per cent. solution of osmic acid.

By this means it is possible to detect the condition of the red and 
white blood corpuseles and hæmatoblasts; the existence or non-existence of leucocytosis and its degree, as well as the existence, for instance, of lèucocythæmia.

The blood corpuscles may also be counted.

Histological examination, supplemented by suitable staining, reveals the presence of normal or abnormal blood corpuscles, parasites such as piroplasma, or microbes such as bacteria.

Such examination necessarily presupposes a knowledge of what should be looked for in the normal state.

In normal blood the red blood corpuscles predominate. They are all similar in form and, with few exceptions, of the same size. They stain strongly with acid solutions such as eosine. In pathological conditions, large or giant corpuscles may be found (macrocytes), as well as those of medium size (normal) and small size (microcytes). Some are vigorous and stain deeply; others, on the contrary, are degenerating or dead, and have no greater affinity for one constituent than for another of the double or triple stains commonly employed.

In pathological conditions the hæmatoblasts occur in very varying numbers.

The white blood corpuscles found in health may be classified as follows :-

Large and small lymphocytes, each of which has a round voluminous nucleus and a narrow border, and contains a non-granular protoplasm; their proportion varies between 22 per cent. and 25 per cent. :

Polynuclear leucocytes or polymorphous leucocytes with a single nucleus, which originate in bone marrow, stain best with neutral colours, and are present in the proportion of 70 per cent. to 72 per cent. :

Mononuclear leucocytes with an ovoid eccentric nucleus stain best with basic colours, and form about 1 per cent.:

Polynuclear leucocytes stain best with eosine or acid colours, and form about 1 per cent. to 2 per cent.

When these white blood corpuscles are in larger number the condition is known as leucocytosis, and when one or other variety is in very great excess the condition is known as leucæmia. 


\section{CHAPTER I. \\ CARDIAC ANOMALIES.}

\section{ECTOPIA OF THE HEART.}

Eсторгі of the heart, i.e., congenital malformation in which the heart is displaced from its normal position and thrust sometimes completely beyond the thoracic cavity, is not very rare. The heart may be well developed, but it is not enclosed by the thoracic walls when the thoracic cavity closes during the first stages of embryonic life. The sternum, which is cartilaginous and becomes ossified only at a later period, remains fissured along the median line, and the fissure, usually of oval form and with rounded margins, surrounds the auricles and the vessels at the base of the heart. The ventricles form a hernia projecting beyond the thorax, which then only contains the two pleural sacs and a complete mediastinal partition. The pericardium remains undeveloped.

Despite this malformation, the embryo develops. The fœetus may in due season be brought forth living, but as a rule death occurs in a few hours.

The diagnosis is easy, but this malformation cannot be treated. All that can be done is to protect the ectopiated organ against external violence in cases where the young creature is born alive. 


\section{CHAPTER II.}

\section{PERICARDITIS.}

Pericarditis consists in inflammation of the pericardial sac. It is attributable to different causes, varying in importance and in causation.

Specific pericarditis may be produced by the tubercle bacillus, or it may develop during an attack of contagious peripneumonia. Tuberculous or peripneumonic forms of pericarditis as a rule form only complications of chronic pulmonary tuberculosis or peripneumonia. They are very rarely primary in character, and, like the allied forms of pleurisy, assume a vegetative and adhesive form in tuberculous cases.

Moussu has never seen the true exudative form either in acute or chronic tuberculosis, but only vegetative and caseous forms.

Simple acute pericarditis. Cases of simple acute exudative pericarditis have been described, and have been referred to chills, wounds, or injuries in the region of the heart, and in a few cases to the rheumatic diathesis.

Such forms of pericarditis may occur, but probably are very rare, for Moussu has seen but two cases. As the symptoms correspond exactly to those of exudative pericarditis produced by a foreign body, it is unnecessary to describe them specially.

The only important detail to bear in mind with this disease is the possibility of cure.by suitable treatment, such as the application of stimulants or vesicants to the cardiac zone, the administration of salicylate of soda or diuretics, and complete rest.

The diagnosis, moreover, should be confirmed by making an aseptic exploratory puncture with the capillary trocar. The nature of the liquid withdrawn will indicate whether the case is one of simple acute pericarditis or pericarditis due to a foreign body.

Cancerous pericarditis is generally secondary, and is caused by development of tumours on the pericardial serous membrane, and in the myocardium. Moussu, however, has seen one case of primary cancerous pericarditis, the tumours being found only on the periphery of the myocardium. The growth assumes a vegetative form with moderate exudation. The symptoms, however, so closely approach to those of exudative pericarditis due to foreign bodies that only the 
latter variety, which is by far the most frequent in animals of the bovine species, need be described.

\section{EXUDATIVE PERICARDITIS DUE TO FOREIGN BODIES.}

This condition has been erroneously described as traumatic pericarditis, but the latter term would suggest that the disease was due to an injury acting from without. It may be defined as a disease produced by the discharge into the peri-

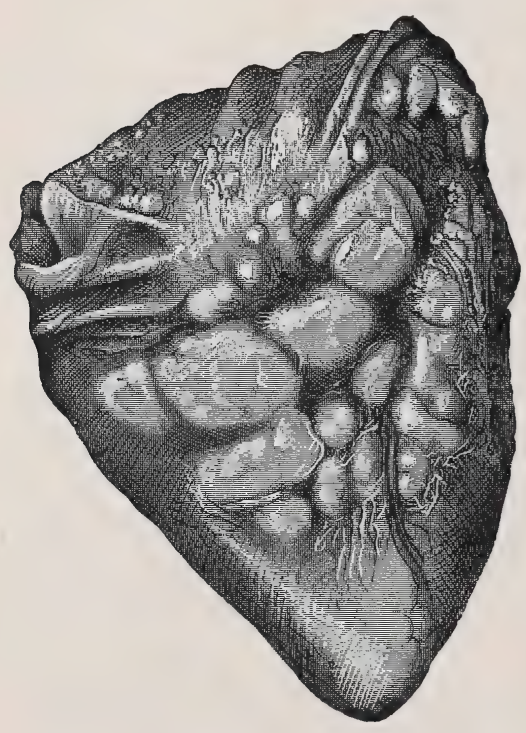

FIG. 174.-Tumours of the surface of the heart. Primary cancerous pericarditis and myocarditis. cardial cavity of some foreign body from the gastric compartments.

Boizy in 1858 described several cases of this kind of pericarditis. Hamon in 1866 gave an excellent table of symptoms. Roy in 1875 supplemented this with numerous observations showing clearly the possibility of recognising the disease by clinical examination. Pericarditis due to foreign bodies is to-day one of the best characterised diseases of the ox, and it is easy to diagnose.

Before approaching the etiological side of the question, it is necessary to recall in a few words the anatomical arrangement of the pericardium and its relations to neighbouring organs.

In the ox the diaphragm presents a marked concavity directed towards the abdomen. The pericardium, situated exactly in the median plane, is fixed by its point to the sternum. A fold of adipose tissue directly connects it with the anterior surface of the diaphragm. On the abdominal side the conical right compartment of the rumen is in free communication with the reticulum, which is closely applied to the posterior surface of the diaphragm on the median line opposite the spot occupied by the pericardium on the anterior surface (Fig. 176). As a result of this arrangement any object passing through the reticulum and diaphragm in the median plane would enter the pericardial cavity. These particulars indicate clearly how this form of pericarditis is produced.

Causation. One of the chief causes of pericarditis by a foreign body is connected with the way in which oxen feed. They rapidly swallow their food and any foreign bodies that may be concealed in it, submitting it later on to a second mastication in the course of rumination. This 
method of feeding results in bolting the food almost without mastication, hence the possibility of swallowing foreign bodies.

The proximity of the reticulum to the pericardium is also an important factor, because the foreign bodies fall into the reticulum as soon as the bolus of food begins to break up. It is important to notice, moreover, that pericarditis is commonest on farms where the oxen are attended by women, or in regions where sharp objects are to be found on roads or pastures frequented by the animals, such as the vicinity of needle, nail, and rivet factories.

The sole cause is the penetration of a foreign body into the pericardial sac.

Pathogeny. All kinds of foreign bodies are swallowed by oxen, as is abundantly shown by post-mortem examinations. These indigestible bodies pass with the food into the rumen, and accumulate in the deepest portions of that receptacle. Owing to physiological contractions the lower wall of the rumen rises to the level of the orifice of communication with the reticulum, and so passes much of the material accumulated within it into this organ.

Soft foreign bodies fall towards the lower parts of the reticulum, but sharp objects may lodge in its walls. Very often the bodies penetrate in this way without causing reticulitis or grave inflammation. The functions of the reticulum are not impeded. The commonest of such objects are needles, pins, nails, or fragments of iron wire. On account of their form, needles are the most dangerous. The sharpness of one extremity ensures its passing readily through the tissues, and as the point is the part that offers least resistance, the needle continues gradually to penetrate.

If the foreign body becomes implanted vertically in the lower wall of the rumen or reticulum it may be expelled directly through the medium of an abscess. This is a favourable termination, though it usually results in permanent gastric fistula.

More often the objects penetrate the anterior wall of the reticulum and gradually work their way towards the diaphragm, impelled by the movements of the reticulum and the other digestive compartments. They perforate the muscle and pass into the thoracic cavity, either in the direction of the pericardium or of the pleural sacs.

First as to the penetration of the pericardium. The foreign body, whatever it may be, produces by its presence alone very marked irritation, and as in addition it is always infected in consequence of its having passed through the digestive compartments, inflammation is set up to a degree proportionate to the pathogenic qualities of the infective agent.

Symptoms. The early symptoms are those of indigestion, and not of pericardial disease, a fact which is easy to understand, because at first the 
whole mischief is in the abdominal cavity. The patients are dull, restless, and seem to be suffering from an obscure ailment. They remain standing more than usual, show more than ordinary deliberation in lying down, lose appetite, cease to ruminate regularly, and exhibit intermittent tympanites.

The cause of these symptoms is as follows: At first the reticulum is partly immobilised by the local inflammation, and at a later stage movement of the diaphragm is checked by reflex action when the sharp body has progressed far enough to touch it. The rhythmic movements of the reticulum and the diaphragm are interfered with, rumination is disturbed, eructation ceases, and tympanites appears.

The patient often utters slight groans, particularly when forced to move; but as this is a sign common to all grave diseases it can only give rise to a suspicion as to what has occurred. In ten to fifteen days this primary phase may have terminated; but it is impossible to say how long it lasts, for it varies with each animal as with each variety of foreign body, and it may be prolonged for months.

From the moment it reaches the thoracic cavity the foreign object makes its way towards the channel formed on either side by the ribs and below by the sternum, and therefore towards the point of the heart. This is the second phase of development.

The passage of the foreign body through the diaphragm occupies a more or less considerable time, depending on its length; the beginning of this second phase is characterised by relative immobility of the circle of the hypochondrium during respiration. The abnormal sensibility and pain impede contraction of the diaphragm.

Palpation of the region of the xiphoid cartilage then reveals abnormal sensibility, and sometimes causes the animal to resent being handled.

From this time the pericardial symptoms proper commence, the foreign body having come in contact with the pericardium. This phase, unlike those which precede it, presents well-defined symptoms. The irritation of the heart and its ganglionic system by a foreign body in the pericardium is shown by considerable acceleration of the heart beats even before there is any exudation into the pericardial sac. Instead of 60 to 70 beats, the normal number, the pulse may rise to $80,90,100$, or even 110 beats per minute. The heart sounds are tumultuous, dull and ill-defined, while the pulse appears bounding and strong.

But this period of cardiac excitement while persisting is soon complicated by other symptoms. As soon as the foreign body penetrates the pericardial sac, there is infection, which produces an active form of inflammation and abundant exudation. From this time the pulse becomes weaker and weaker, until, under the steadily increasing pressure on the heart, it is almost imperceptible. 
There is only moderate fever. As soon as the exudation becomes considerable, the symptoms of pericarditis grow very marked: they may be grouped in the following order, according to their importance.

A. Cardiac symptoms. On palpation of the cardiac zone on the left the impulse of the heart is no longer felt. Percussion, which under normal circumstances reveals only partial dulness, now seems to give pain, and indicates abnormal dulness distributed in a vertical plane. The pulmonary lobes between the pericardium and thoracic walls are thrust upwards. The distended pericardial sac approaches the parietal layer of the pleura and may adhere to it, hence the dulness. This dulness extends as far back as the xiphoid appendix of the sternum, and can be detected on both sides, marginated above by a convex line.

In rare cases the dulness is absent, being partially replaced by tympanitic resonance, due to the presence of gases in the distended pericardial cavity, which gases originate in the digestive reservoirs or result from putrid fermentation of the pericardial exudate.

Simple or double pleurisy, or even pneumonia of the cardiac lobes resulting from infection by contiguity, may complicate cases of rapid pericarditis. The dulness then appears modified, as do the signs observed on auscultation.

Auscultation furnishes valuable indications. From the outset it reveals acceleration of the heart. At a later stage, but only for a short time, it permits of the detection of the pericardial rubbing sound which precedes serous exudation, and which may persist for several days when large quantities of false membrane are produced.

If exudate is present in considerable quantities a liquid sound is heard at each heart beat. The heart appears to be beating in water, but the liquid note varies considerably. It has been termed the "claclaque" sound (Lecouturier, 1846), in allusion to the sound produced by the meeting of water ripples; "clapotement" sound (Boizy, 1858), with reference to the sound produced under the influence of a light breeze on the borders of a stream; "glouglou" sound (Roy, 1875), suggested by the noise of liquid escaping from an inverted bottle into a resonant vessel, etc. It is important, however, to remember that cases occur (principally when the pericardium is greatly distended and entirely filled with liquid) where, with the animal at rest, these sounds are difficult to detect. To render them noticeable the patient must be walked for a few yards.

Vernant, again, has described a sound as of dripping water, of quite special character; he compared it to that resulting from the fall of drops of liquid on to a marble table or into a half-filled vessel. So far as can be ascertained this sound of dripping water greatly resembles that heard in pneumo-thorax, but it is less resonant and less prolonged. 
It appears to be characteristic of the presence of air in the pericardial cavity, and its special quality varies with the quantity accumulated in the pericardium. Masked by these pericardial sounds the beating of the heart seems dull, badly defined, distant and stifled.

B. Jugular symptoms. The "jugular" symptoms are secondary, and result from the accumulation of liquid in the pericardial cavity. No intra-pericardial exudate can exist without exerting pressure on the heart, and as the auricles have very thin walls and are more compressible than the ventricles, this pressure immediately causes difficulty in the return circulation, whence venous stasis, varying in intensity, but clearly visible and appre-

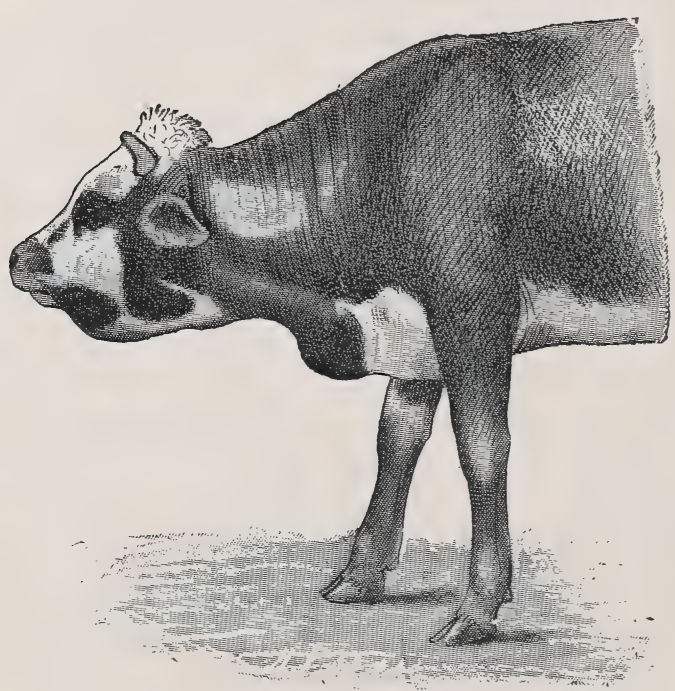

FIG. 175.-Appearance of a patient suffering from fully-developed pericarditis. ciable on account of the distension of the jugulars.

The venous stasis is general, for the pulmonary veins are as much compressed as the posterior and anterior venæ cavæ, but it is only apparent in the large superficial veins. This stasis is accompanied by venous pulse, and particularly by peripheral or internal œdema, œdema of the lung, intestine, mesentery, etc., of the submaxillary space and of the dewlap and entrance to the chest. Edema of the submaxillary space is specially characteristic, for it appears almost

first amongst external signs. That of the dewlap follows at a later stage, and extends backwards as far as the umbilicus, rising above this point as high even as the entrance to the chest and the axillary region.

C. Pulmonary symptoms. The pulmonary symptoms result from difficulty in the return circulation and from the venous stasis. They are due to passive congestion and œdema of the lung or to hydro-thorax. At rest the respiration may appear fairly regular, but at the least movement it is accelerated, and may rise to 40 or even 60 per minute.

Percussion reveals lessened resonance of the parts, and in the case of hydro-thorax dulness marginated by a horizontal line, as in pleurisy.

On auscultation the vesicular murmur may sometimes have diminished or even disappeared, while the respiration may be blowing, as in 
active congestion, and in exceptional cases a tubal souffle may be observed. In most cases the animal has a paroxysmal, somewhat frequent cough, due to reflex irritability of the pneumo-gastric.

Cruzel in addition mentions a double respiratory movement like that produced in the horse by broken wind. This is really the result of hydro-thorax, and is not a constant symptom.

D. General symptoms. When the disease has lasted a certain time the patients show certain well-marked general symptoms: they remain standing in one position for long periods, with the head and neck extended, the front legs thrust outwards from the trunk and the body rigid, as though the least movement caused them pain. The general attitude expresses anxiety, the animals lie down with great care and seldom remain long in this position, which interferes with the functions of the heart and lung. In the last stages the animals remain constantly standing, appetite is almost entirely lost, and they waste rapidly.

The course of pericarditis due to foreign bodies is very variable. Sometimes death occurs in eight or ten days. In other cases the animal may survive for weeks, provided it is well tended. Everything depends on the rapidity with which the foreign body moves and on the character of the infectious organisms which it introduces into the pericardium. Death is the inevitable termination, and occurs as a consequence of cardiac and respiratory syncope. It may follow suddenly as the result of a simple forced movement, even when the animal still seems to retain some amount of strength. When the organisms introduced into the pericardium are of marked virulence, complications such as septic pleurisy and pneumonia may be observed, and death soon takes place.

It has been suggested that recovery might follow a return of the foreign body towards the reticulum. This view can only have been advanced as a consequence of errors in diagnosis, either as to the existence of pericarditis or as to its nature. Pericarditis due to cold or rheumatism sometimes becomes cured spontaneously.

Death, again, may suddenly occur by syncope when the foreign body penetrates the myocardium, passes through it, and enters the ventricular cavities.

The return of the foreign body is not conceivable, at all events after it arrives in the pericardial cavity. Up to that time the only disturbance is of a digestive character; no pericarditis exists. But when for example the disturbance is due to long fragments of iron wire which may extend from the reticulum as far as the pericardium, it is clear that the pericarditis is of a kind which cannot be cured without leaving traces. In our opinion, natural recovery is impossible.

Diagnosis. The diagnosis of pericarditis cannot be made until such 
pericarditis actually exists, i.e., until the disease has arrived at the third stage of development mentioned above.

As long as the symptoms point only to the first or second stage, the logical diagnosis is reticulitis produced by a foreign body. At this time the development of pericarditis, although possible, is not inevitable.

When, on the other hand, one knows how the digestive disturbance has originated and developed and thereafter notes signs of cardiac irritation, disappearance of the cardiac impulse, dulness of the heart sounds, venous stasis, etc., the diagnosis is easy even thus early.

Mistakes are not very likely. Only in some cases are they liable to occur, as in acute peripneumonia of the anterior pulmonary lobes, causing compression of the pericardium of the anterior vena cava and producing secondarily venous stasis and oedema of the dewlap. Cases of specific pericarditis due to peripneumonia also occur, and under such circumstances a mistake would be even more excusable. Nevertheless, the temperature curve in itself is a sure indication, for whilst in peripneumonia the fever is always very marked, it is scarcely noticeable in pericarditis due to a foreign body.

When the diagnosis of pericarditis has been arrived at it is desirable to determine the exact nature of the disease, for whilst cases of pericarditis due to foreign bodies are incurable and in the interest of the owner the animals should be slaughtered, pericarditis due to cold or rheumatism may be successfully treated. Rheumatism generally affects the synovial membranes even before it produces pericarditis, and this indication, supplemented by the history of the case usually ensures one against mistakes regarding the initial cause.

It is much more difficult to distinguish pericarditis due to a foreign body from pericarditis due to carcinoma and from the forms of pseudo-pericarditis produced by lesions in the neighbourhood of the heart. When considering the latter we shall deal with this particular point.

Prognosis. The prognosis is always fatal.

Lesions. When the foreign body is very thin and sharp, the reticulum may not become attached to the diaphragm. In such cases its passage has been rapid and the tissues have healed.

Usually the reticulum, diaphragm and pericardium are united by a mass of fibrous tissue as thick as a man's arm. It resembles a fibrous sleeve surrounded by an œedematus zone, usually of slight extent. This mass of new fibrous tissue is traversed by a sinuous tract resulting from the irritant action of the foreign body on the surrounding tissues. All writers describe this fibrous sleeve, which, however, only occurs in cases where a very long foreign body has occupied a considerable time in passing from the reticulum to the eavity of the chest. 
In very exceptional cases the sinuous tract is ramified, possibly as a result of displacements of the foreign body.

The orifices of the tract are to be found, one in the reticulum, the other in the pericardium. On the side of the reticulum there is never more than one opening, and in many instances the tract is already closed on that side, either by exuberant granulations or by a cicatrix.

On the contrary, the fistula is more frequently open in the pericardial cavity. Its walls are of very varying appearance, depending on their age :

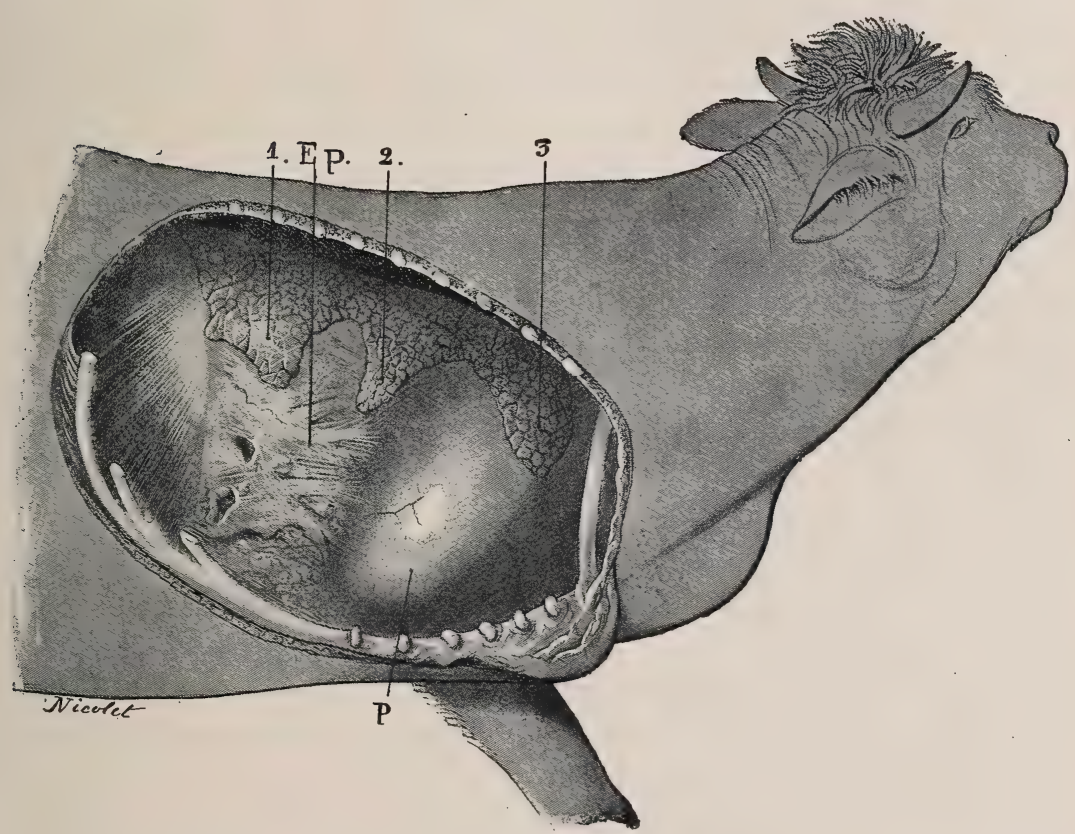

FIG. 176.-Appearance of the lesions in a case of fatal pericarditis. P, inflamed pericardium, distended with exudate and adherent to the neighbouring pulmonary lobes; 1, posterior lobe ; 2 , cardiac lobe; 3 , anterior lobe; $\mathrm{F} p$, pleural false membranes.

they may be red, greyish, soft or hard, and when the lesion is of old standing they may have been converted into a sclerotic tissue.

The pericardium appears distended with a considerable quantity of liquid of a special character-sometimes sero-sanguinolent, sometimes almost or entirely purulent; sometimes yellowish, or greenish-grey; sometimes frothy, inodorous, or very foetid.

These characters depend on the nature and number of the germs which have invaded the pericardial cavity. They also vary with the gravity and number of the hæmorrhages produced by the action of the foreign body on the myocardium. 
The quantity of liquid also varies within very wide limits. There may be scarcely any exudation. In that case the pericarditis is of a partially adhesive character, with abundant false membranes. As a rule the quantity of fluid exudation varies between seven and eight quarts, but sometimes the quantity is much greater. Trasbot described an instance in which the united weights of the heart and pericardium exceeded $36 \mathrm{lbs}$. Hamon mentioned a case of pericarditis in which the liquid exudate exceeded twenty quarts.

When inflammation is first set up the liquid is serous, yellowish, or reddish-yellow. It contains fibrinous flocculi in suspension. Little by little this exudate becomes purulent, whilst the internal layers of the pericardial serous membrane undergo desquamation. These are next covered with false membranes of varying appearance; the fibro-albuminous exudation is wrinkled, villous and tufted. The two layers of serous membrane are connected at certain points by this exudation, the adhesions being sometimes very extensive. The pericardial sac properly so called becomes the seat of marked lardaceous thickening, due to inflammation. The heart appears entirely covered with a layer of greyish or earthy-coloured granulation tissue, which appears as though baked, and was compared by Hamon to the back of a toad. It is atrophied as a consequence of prolonged compression.

Under the influence of the excentric pressure of the liquid the pericardial sac is distended and comes in contact with the walls of the chest, to which it may adhere. The foreign body, especially if small, is not always easy to find.

The myocardium often displays interesting lesions. At first there is thickening, or more commonly sclerous degeneration, of the superficial layers covering the ventricles, and then appears a crop of little miliary abscesses. Abscesses of considerable size have several times been detected in the walls of the ventricles and in the interventricular septum.

The foreign body, moreover, may not only injure the myocardium, but may even perforate it completely and produce ulcerative endocarditis (Cadéac). In this case infectious germs very rapidly invade the circulation and all the tissues, and the animal dies of pyæmia.

These essential lesions are accompanied by others of varying importance. Thus the lung is congested throughout, and by contiguity of tissue inflammation may extend from the pericardium to the lower part of the pulmonary lobes and to the pleura.

Interference with the return circulation induces lesions due to venous stasis: dropsy of the chief serous membranes, œdema of the connective tissue, pleural and peritoneal exudations, etc. If the hind limbs never become swollen it is because the skin covering them is very resistant 
and does not readily yield. The liver becomes hypertrophied, congested and engorged with blood, and when the animals live for some weeks, shows the appearances known as cardiac or nutmeg liver.

Treatment. The treatment of pericarditis due to the presence of foreign bodies is at present merely palliative. Often the only thing to be done is to slaughter the animal.

We need not go back to the methods formerly recommended. All are illusory or mischievous, such as the use of purgatives to arrest or reverse the progress of the foreign body, removal of the foreign body after opening the rumen, puncture of the pericardium, etc.

In 1878 Bastin successfully opened the pericardium and extracted the foreign body through a window produced in the thoracic wall.

This operator recommends that after drawing the left limb forward and incising the skin and muscles, the operator, with his hand bound round with a cloth, should perforate the pleura, and then having found the foreign body, proceed to extract it. By this method it seems difficult to cause perforation of the pericardium, which would certainly lead to the production of pneumo-thorax complicated with fatal septic pleurisy.

It must be borne in mind that the two pleural sacs, right and left, descend as far as the sternum (Fig. 173), and that it is not possible to touch the pericardium directly without perforating the pleura.

Moussu has drained the pericardium through the pleura in the hope of relieving the pressure on the heart and facilitating the reabsorption of the odema, in order to permit of the subsequent slaughter of the animal, but has had unsatisfactory results. Lastly, he has practised median trepanation of the sternum in the infra-pericardiac region. Here again the operation is difficult, because of the odematous infiltration of all the substernal region, while it is so dangerous to the patient, which must be cast and may suddenly succumb, that it is of no use in ordinary practice.

There is probably only one condition in which it would be possible to attempt intervention with a fair chance of success, that is, when there exists a fibrous connection between the pericardium, lung, and wall of the chest on the right or left side.

In such cases aspiratory puncture or incision of the pericardium in an intercostal space might prove of service, because it would not expose the animal to the danger of pneumo-thorax.

The only difficulty lies in ascertaining beyond all question the existence of such an adhesion before attempting operation, and this is really very great, even having regard to the form of the dulness and the absence of all respiratory sound in the lower third of the thoracic cavity and cardiac zone. The pulmonary lobe between the heart and chest wall may be thrust upwards and be partially adherent to the pericardium and to the parietal pleura, and at the same time it may be impossible to avoid

D.C. 
producing operative pneumo-thorax when the cartilages are resected to admit of incising the pericardium.

The only logical method seems to be puncture of the pericardium through the xiphoid cartilage, as described below.

The topographical anatomy of the thoracic viscera shows that the point of the pericardium extends along the sternum to a point close to the lower insertion of the diaphragm, and that the pericardial sac is

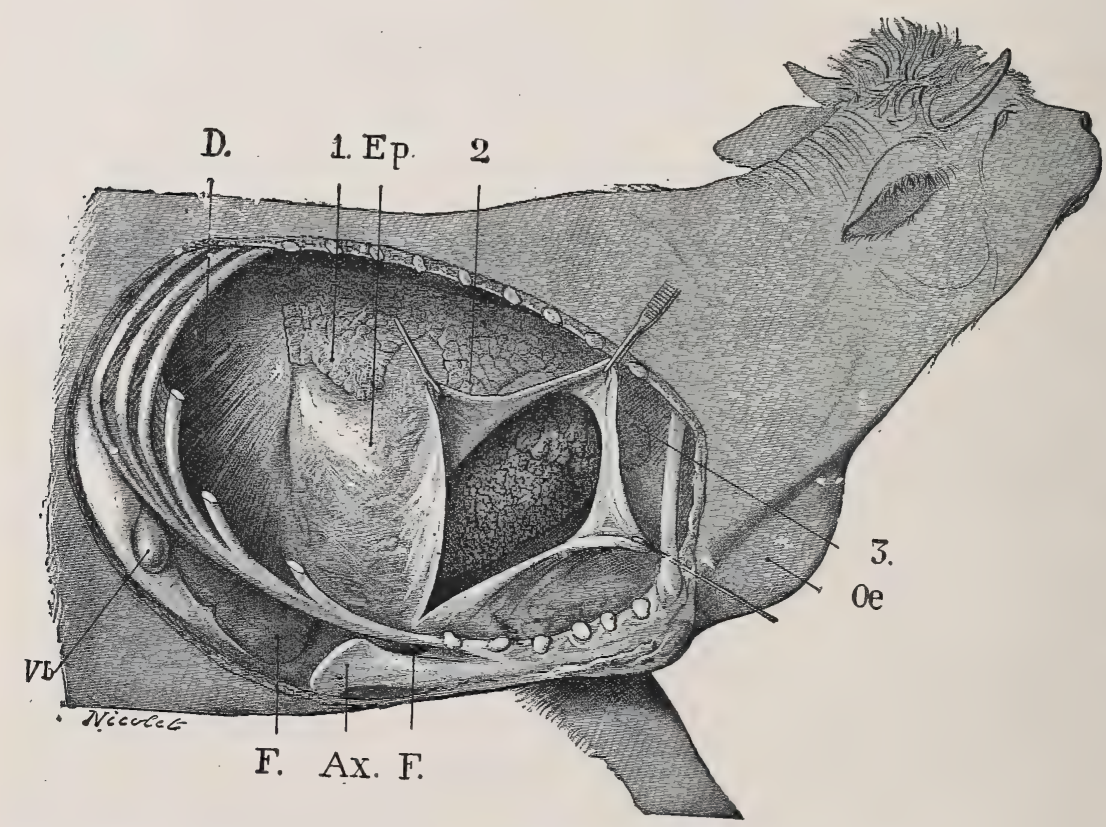

FIG. 177.-Lesions of exudative pericarditis produced by a foreign body. Relation of the pericardium to the sternum and ensiform cartilage. Pericardium opened. $\mathrm{D}$, diaphragm ; $\mathrm{E}$, œdema of the dewlap, $\mathrm{A} x$, ensiform cartilage ; $\mathrm{F}$, liver ; V $b$, gall bladder ; 1 , posterior lobe of the lung, drawn backwards ; 2, cardiac lobe ; 3 , anterior lobe; E, spot where the foreign body penetrated, towards the point of the pericardium, between the neck of the ensiform cartilage and the circle of the hypochondrium.

only separated from the xiphoid region, or rather from the region of the neck of the xiphoid appendix of the sternum, by the fatty cushion at the point of the heart.

A glance at the annexed diagram (Fig. 177) will show this.

The diagram, carefully reproduced from an anatomical preparation of an animal which succumbed to pericarditis, shows that the distended pericardium extends close to the neck of the xiphoid cartilage.

First stage. Identify the three following anatomical guiding points:(1.) Xiphoid appendix and white line. (2.) Point at which the circle of 
the hypochondrium becomes attached to the sternum. (3.) Point at which the external mammary vein penetrates the abdominal wall (Fig. 178).

Lines uniting these three points enclose a right-angled triangle, which the operator must imagine to be bisected by a third line.

The incision, which should be about 8 inches in length, follows this bisecting line at an equal distance between the white line and the circle of the hypochondrium, to a point within about 8 inches of the anterior margin of the mamma. All these points are readily observable before the animal is cast.

The cutaneous incision affords exit to large quantities of fluid, and the

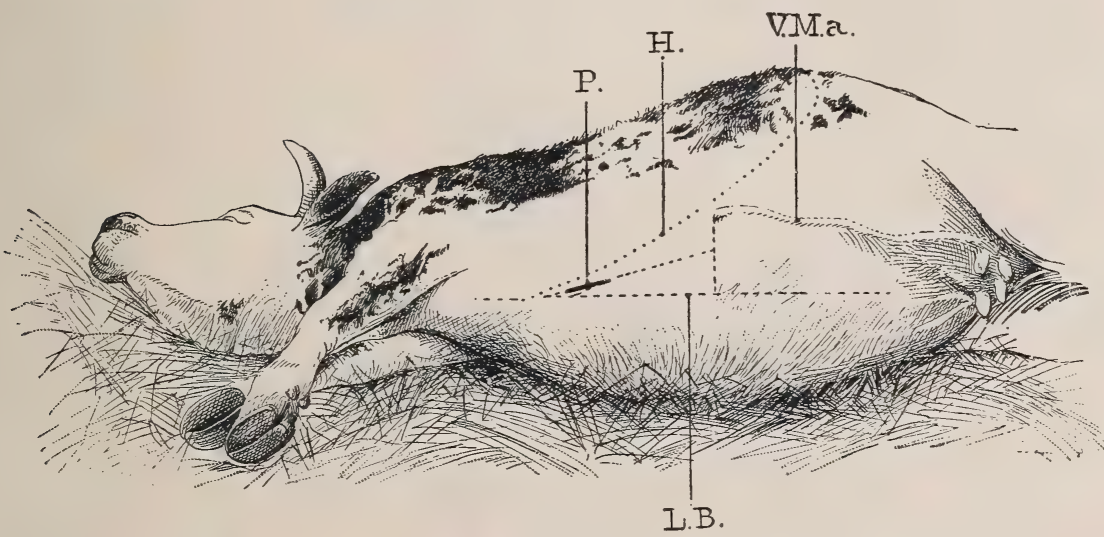

FIG. 178. - Seat of operation for puncturing the pericardium by way of the ensiform cartilage. L B, White line; H, line of the hypochondrium; V. M.a., anterior mammary vein; $\mathrm{P}$, point where the pericardium is punctured through the incision.

pectoral muscles attached to the neck of the ensiform cartilage can then be divided with the bistoury. The area of operation is thus uncovered.

Second stage. The second phase comprises incision of the tissues opposite the neck of the ensiform cartilage, about 8 inches in front of the base of the triangle and at equal distances from the points Nos. 1 and 2 ; incision through the skin for a distance of 8 inches, and dissection of the muscles of the ensiform region exposed at the neck of the cartilage.

By means of the index finger or the index and middle fingers of the right hand the mediastinal space is explored, and the fatty masses round the base of the heart broken through. If the pericardium is greatly distended, the point of the sac can be felt with the tip of the finger, or its position can be recognised, even from a little distance, on account of the fluid contents transmitting the impulse of the heart. The sensation conveyed to the finger is very clear. 
The right index finger is then replaced by the left, and, a trocar about 10 inches long and $\frac{1}{4}$ inch in diameter being introduced along the index finger used as a director, the pericardial sac is reached. The exudative fluid transmits the impulse due to the beating of the heart, and the pulsations can be clearly distinguished when grasping the handle of the trocar.

Third stage. Digital exploration of the course of the puncture and fatty cushion at the base of the heart, with the object of discovering the position of the pericardium.

Fourth stage. Puncture with a trocar about 10 to 12 inches in length, puncture of the pericardium, irrigation and dressing.

The trocar is inclined in a slightly oblique direction from without

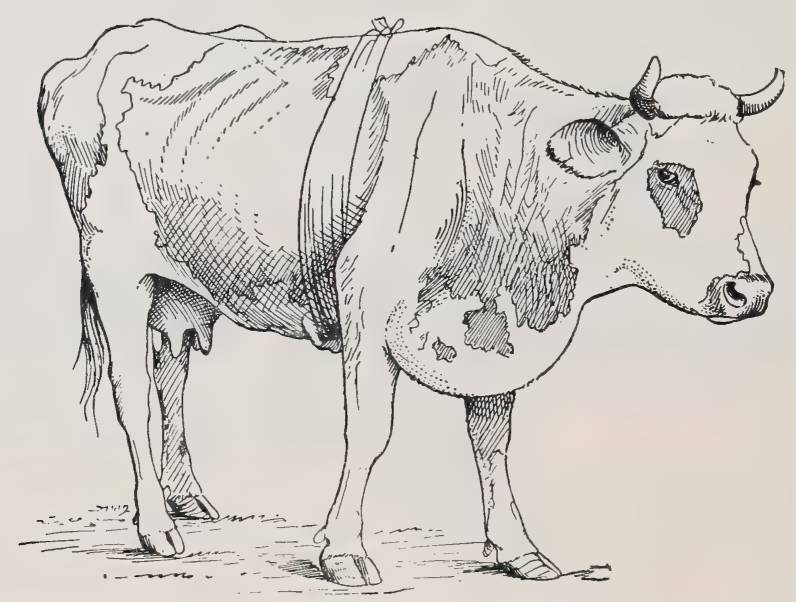

FIG. 179.-Photograph of a patient immediately after operation. Extensive œedema of the dewlap and neck.

inwards and forwards towards the median plane, in order that the point may not deviate towards the left pleural sac; the left index finger is then withdrawn, and by a sharp thrust of the right hand the trocar is pushed forward about 1 to $1 \frac{1}{2}$ inches and the pericardial sac is entered.

The position of the canula should not be altered whilst liquid is escaping, for if it is thrust in too far a considerable quantity of fluid may remain in the deepest portion of the sac.

The cavity having been drained, a long strip of iodoform gauze is introduced into the track and a protective surgical dressing applied over the incision in order to prevent infection by the litter.

In consequence of the introduction of the fingers into the track caused by puncture and the escape of pericardial liquid along the canula or after removal of the canula, the operative wound is necessarily infected; but this 
is of little importance, because the opening is inclined downwards, and also because it is impossible to hope for aseptic healing.

The dressing is renewed after forty-eight hours, and every three or four days afterwards.

The redematous infiltration about the front portion of the body disappears rapidly in two to three days, and should the animal be slaughtered the meat is quite sound in àppearance.

'I'his operation does not aim at effecting a cure, but is simply for the purpose of allowing animals which would otherwise be valueless to be slaughtered and sold.

\section{CHRONIC PERICARDITIS.}

Pericarditis when due to tuberculosis may assume the chronic form. Tuberculous pericarditis, at least in a large number of cases, is only accom-

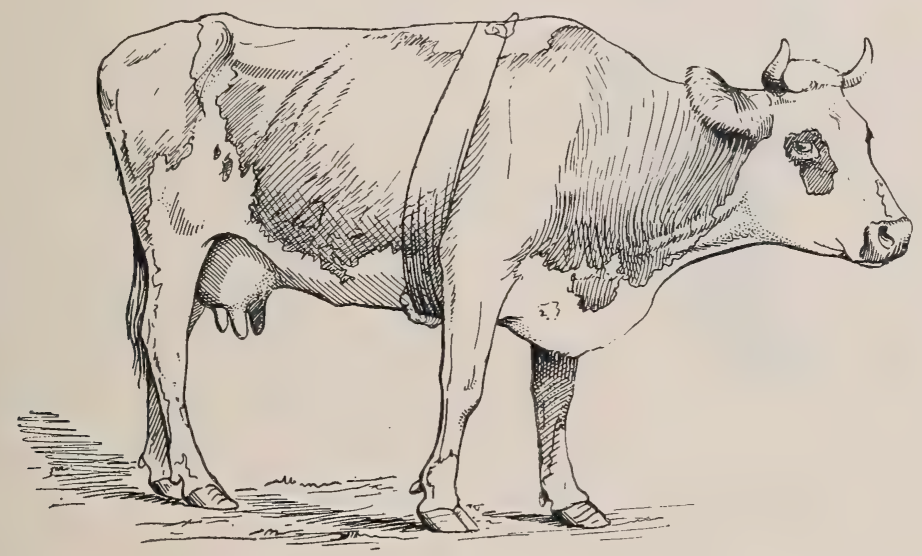

FIG. 180.-Photograph of the same subject as Fig. 179, taken six days later.

panied by slight exudation, which might remain unnoticed unless the animals were carefully examined; but it causes the internal surface of the pericardial sac and the surface of the myocardium to become covered with exuberant vascular growths, which by setting up adhesions lead to partial or generalised union of the heart and pericardial sac. Between these adhesions, which form partitions, are found little cavities filled with sero-sanguinolent, grumous, or caseous liquid. In time the adhesions increase in number, pericarditis obliterates the free space, and the heart becomes wholly adherent to the pericardium.

As in acute pericarditis, the fibrous layer undergoes thickening and hardening processes. The superficial layers of the myocardium undergo sclerous transformation, and the tissues forming the adhesions them. selves may assume the characters of fibrous tissue. 
In one solitary case Moussu saw another form of chronic pericarditis with complete adhesion of the heart and pericardial sac, without any exudation and almost without any false membranes. He was unable to determine the exact cause, but was strongly inclined to regard the disease as having followed pericarditis à frigore or pericarditis of a rheumatic character.

Adhesions between the heart and pericardial sac are also said to be the inevitable though delayed result of all punctures of the pericardium through the ensiform carti-

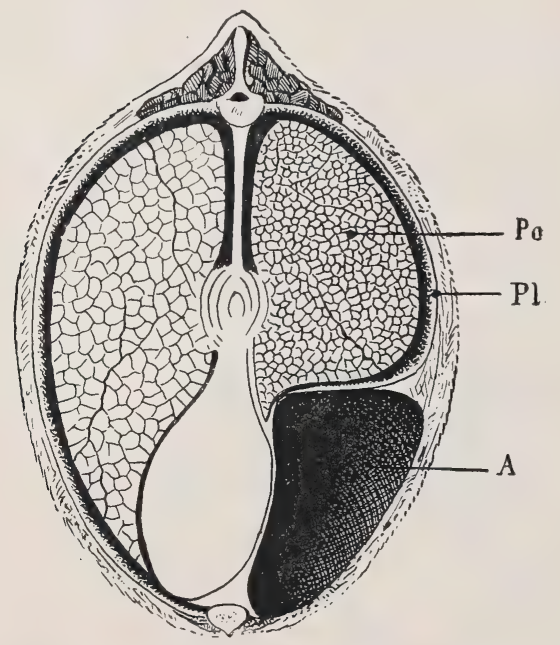

FIG. 181. - Schema of a subpleural abscess on the right side which produced symptoms of pericarditis (pseudopericarditis). Po, Right lung, partly splenised and thrust upwards; $\mathrm{Pl}$, parietal pleura separated from the wall of the lower half of the chest; A, cavity of the subpleural abscess.

Sudden death is a frequent consequence. lage in cases of pericarditis due to a foreign body.

Symptoms. If the chronic pericarditis is limited to a few partial adhesions, it remains unnoticed; but when it is more marked it offers certain signs of acute pericarditis, such as partial dulness of the cardiac area, which is more extensive than usual, disappearance of the cardiac shock, weakening of the sounds, feebleness of the pulse, very marked venous pulse, moderate stasis, extremely rapid and aggravated dyspnoea when the animal is forced to walk, threatened asphyxia if exercise is prolonged, and complete asystole.

All these symptoms are due to the existence of adhesions between the heart and pericardium, which, by destroying the interpericardial space, interfere with diastole while preventing regular systole.

The diagnosis of chronic pericarditis is very difficult. The prognosis is extremely grave, and we do not possess any means of dealing with the condition.

\section{PSEUDO-PERICARDITIS.}

Under this title we purpose grouping a certain number of pathological accidents due to different causes, but manifesting themselves by identical symptoms, which symptoms offer so marked a resemblance to those of pericarditis produced by foreign bodies as to suggest the presence of that 
disease. This refers to accidents by which the foreign body closely approaches, without actually touching, the pericardium, the lung or the pleural sacs, but in which it causes purulent collections which displace the pericardium, indirectly compress the heart, and finally cause symptoms of an apparently pericardial character.

Causation. During the development of pericarditis the foreign body perforates the reticulum and diaphragm, passing along the middle line of the body, without which it would not come in contact with the pericardium. If the perforation, however, occurs to the right or left of the median plane, the foreign body moves forward just as easily, but it misses the pericardium and passes either into the lung, where it causes fatal pneumonia; or the pleura, where either it sets up septic pleurisy in the subpleural connective tissue or produces an abscess.

The abscess is generally lateral, situated in the right subpleural region, or it may develop below the pericardium. These are the two varieties of pseudo-pericarditis seen by Moussu.

There is, however, a third variety, which might be called "parasitic pseudo-pericarditis." It is extremely rare, and Moussu has only seen one case. It was due to the presence of an enormous hydatid cyst of the right lung as large as a man's head, which was situated towards the mediastinal plane of the lung and pressed on the supero-posterior surface of the heart and pericardium. In consequence of the permanent downward pressure which it exercised it interfered seriously with the heart's action and caused symptoms of pseudo-pericarditis.

Symptoms. The general and external symptoms are those of pericarditis-viz., dulness, diminution in appetite, irregular rumination, wasting, œdema of the dewlap, distension of the jugulars, marked venous pulse, great anxiety and dyspnœa when the patients are forced to move, etc.

But the cardiac symptoms differ notably, and moreover vary, according to the nature of the lesions. Speaking generally percussion reveals complete dulness on one or both sides, and auscultation always indicates the absence of sounds due to extravasated fluid in the pericardial sac.

When the abscess is situated below the pericardium, a condition difficult to diagnose, the dulness seldom extends very high on either side of the chest, and the sounds heard over the cardiac area, while much weaker than usual, are audible above the normal points.

An abscess developing beneath the pleura on one side displaces the heart in the opposite direction. The cardiac beat is weakened by the compression, but, nevertheless, transmits an impulse to the purulent fluid, which in its turn conveys it outwards through the intercostal spaces in the form of movements corresponding in rhythm with the beating of the heart, so that at first glance one might imagine an 
aneurism existed at the base of the large arterial trunks. The lower pulmonary lobe is thrust upwards, and over the area of dulness pulmonary sounds completely disappear.

When the heart is compressed by a large hydatid cyst or other lesion, the general and external symptoms are similar to those above described.

Finally, one last symptom, which appears of some importance, may be mentioned. When animals suffering from pericarditis due to a foreign body are forced to move, the beating of the heart becomes so tumultuous that it can no longer be counted, and even in a state of rest it may rise to

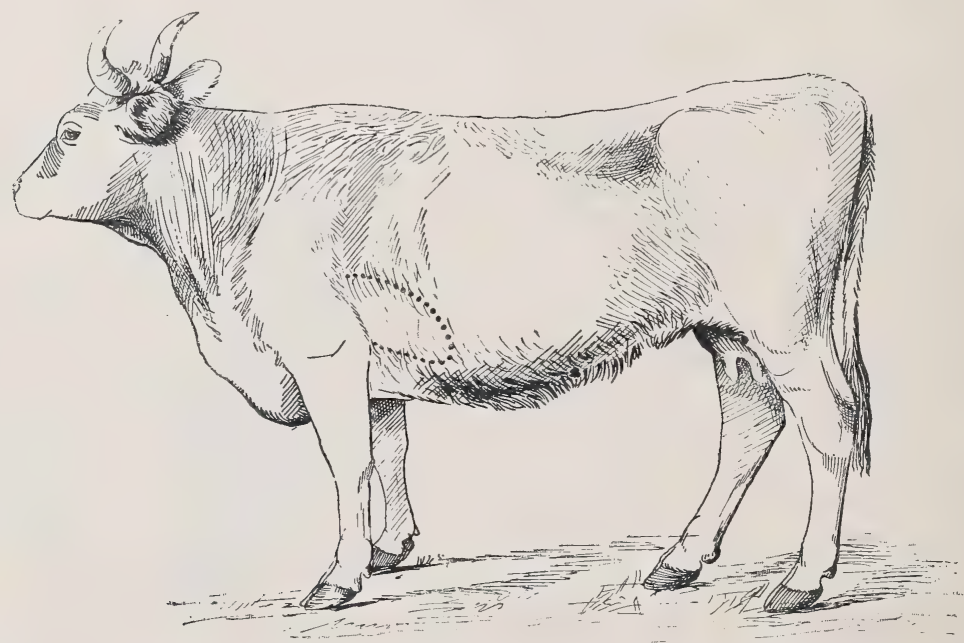

FIG. 182.-Appearance of an animal suffering from pseudo-pericarditis (infrapericardial abscess). The dotted outline indicates the space occupied by the heart, which is thrust upwards.

140 or 150 beats per minute. In cases of pseudo-pericarditis it rarely rises above 90 or 110 .

Diagnosis. The attempt to diagnose this condition accurately must not be regarded merely as a result of scientific curiosity. Under certain circumstances the diagnosis may be of very great importance. While the patient affected with pericarditis due to a foreign body is beyond all hope of recovery, certain cases of pseudo-pericarditis appear amenable to treatment.

The diagnosis, therefore, is of great importance, and the practitioner should spare no effort to confirm it, bearing in mind the symptoms enumerated, and remembering that the normal sounds of the heart never completely disappear.

An aseptic exploratory puncture with a long, fine needle will sometimes prove of great assistance. 
Prognosis. Although grave, the prognosis is less so than in true pericarditis.

Treatment. If clearly recognised, both subpleural and subpericardial abscesses seem curable. By freely puncturing the pus-filled cavity through an intercostal space, the liquid may be evacuated and recovery may occur. Healing is favoured by carefully washing out the cavity with a non-irritant disinfectant.

The only precaution required in making such punctures is to avoid the internal thoracic artery and vein, the intercostal artery, and the lower cul-de-sac of the pleura. 


\section{CHAPTER III.}

\section{ENDOCARDITIS.}

If the symptoms of pericardial diseases are well defined, we cannot say the same of diseases of the heart, properly so-called. Such affections often pass unnoticed, being detected only on post-mortem examination. Moreover, cardiac diseases are rare. Very frequently they are only of a secondary nature, accompanying or following better recognised conditions, such as infectious diseases, post-partum infections, etc.

Causation. Endocarditis, i.e., inflammation of the endocardium and valves, is rarely primary, simple and benign. It was formerly thought to be the result of chills or of the rheumatic diathesis. These simple forms of endocarditis usually escape observation, though careful examination in the first instance reveals them.

Much more frequently, however, endocarditis is secondary, malignant, infectious and infecting. This variety occurs as a complication of postpartum infection or of very serious general conditions, such as peripneumonia, gangrenous coryza, aphthous fever, tuberculosis, etc. To detect it, not only must the original disease be accurately diagnosed, but all the changes the disease is producing in important organs must be followed.

While it is generally admitted that all forms of endocarditis, even of the most benign character, are originally due to infection, it is certain that in those of the second group the organisms which have entered the bloodstream through a lesion of the uterus, lung or other tissue, are endowed with very great virulence. They attack some point on the endocardium, and produce either ulcerations which become covered with fibrous clots, or exuberant new growths of a pathological nature, which generally are papilliform, fragile, and prone to become detached by rupture of their pedicle and thus to be launched into the general circulation and to form emboli. The surface of these infected vegetations, like that of the ulcerations, becomes covered with fibrinous clots, which are readily loosened, form emboli in their turn, and infect distant organs.

Symptoms. The general symptoms of infectious endocarditis are by far the most important. They consist of prostration, loss of appetite, severe thirst, and high temperature. The local symptoms consist 
principally of murmurs: soft murmurs due to insufficiency of the auriculo-ventricular valves, heard during systole, particularly opposite the point of the heart where the cardiac shock is most clearly felt. This fact differentiates them from the murmurs of chronic endocarditis, which are usually due to aortic contraction, and are accompanied by a systolic sound heard at the base of the heart, more in advance and at a higher point than those now under consideration.

These murmurs or souffles furthermore vary in intensity and in character, according to whether the endocarditis results from postpartum infection, pyæmic disease, or some other cause.

Diagnosis. The diagnosis of endocarditis has not yet been the object of really careful study in bovine pathology, but there is no doubt that it can often be detected by patient examination.

Prognosis. The prognosis is very grave, and patients may die in a few days.

Treatment comprises vigorous local stimulation over the cardiac area, the administration of antithermic and antiseptic drugs, such as salicylate of soda, or of digitalis, sparteine or other cardiac tonics.

Pathologists have also described, chiefly as post-mortem curiosities of interest to pathological anatomists, various diseases and lesions due to insufficiency or contraction of the auriculo-ventricular, aortic, and pulmonary openings, lesions due to infectious myocarditis, to the presence of parasites and to other causes.

The symptoms of these various diseases or lesions in bovine animals are still too imperfectly understood to permit of more than a very limited description.

In the present state of our knowledge, diagnosis would always be of an uncertain character, and for this reason we do not propose to deal with them at present. 


\section{CHAPTER IV. \\ DISEASES OF BLOOD-VESSELS.}

Diseases of vessels, arteries or veins, in animals of the bovine and ovine species are frequently nothing more than localisations of grave general disorders, and rarely admit of treatment. This is specially the case in regard to arteries, but a study of the diseases of veins has some practical importance.

\section{PHLEBITIS,}

Phlebitis, i.e., inflammation of a vein, is of interest only in the case of bovine animals. In them certain conditions may oceur which the practitioner should understand, with a view either to prevention or treatment. Inflammation of the veins may be due to external causes, such as surgical or accidental wounds (phlebotomy wounds, accidental wounds, local inflammations, etc.), or to internal causes of infectious origin (general infection, puerperal infection, etc.).

\section{ACCIDENTAL PHLEBITIS.}

The jugular vein may become inflamed as a result of accidental wounds or of phlebotomy, but the mammary vein in cows is much more frequently affected. In both cases the disease is due to infection of the clot which seals the vessel; it may assume the form of either adhesive phlebitis or suppurative phlebitis. Whether produced directly by the use of infected instruments or whether it is of a secondary character, traceable to the clot being infected by germs entering from without being conveyed to the wounds by the head-stall chains, by litter, manure, etc., the result is the same. The inflammation, at first confined to the endothelium, extends to the wall of the vein and causes fibrin to be precipitated over the inner wall of the inflamed vein for a distance varying with each case.

If the micro-organisms do not produce suppuration, the vein appears simply thrombosed and inflamed, the phlebitis remains of an adhesive character, and may disappear spontaneously, provided the animal be kept quiet. If, on the other hand, suppuration is set up, the clot gradually breaks down, the internal surface of the vein develops granulations and 
undergoes suppuration, and the phlebitis is then said to become suppurative. The clot may even become entirely detached, transforming the suppurative phlebitis into a very grave form of hæmorrhagic phlebitis.

The jugular is the commonest seat of adhesive phlebitis, the mammary vein of suppurative phlebitis.

Symptoms. The symptoms are easy to recognise. The accidental or instrumental wound is the seat of a painful œedematous swelling. It discharges a reddish offensive serosity, or exhibits blackish-violet bleeding granulations surrounding a little central sinus.

The affected vein, whether the jugular or mammary, soon becomes swollen, is sensitive to the touch and very rapidly becomes indurated in the direction of its origin for a greater or less distance.

Phlebitis has then set in, and according as one or other complication predominates, it is described as suppurative or hæmorrhagic.

Diagnosis and prognosis. The diagnosis presents no difficulty. In phlebitis of the jugular the neck is held stiffly, and the jugular furrow is partly obliterated.

The prognosis is somewhat serious, particularly in phlebitis of the mammary vein, for obliteration of the vein interferes with the function of the venous plexus from which it springs, and, although there may be a limited vicarious circulation, the secretion of milk is indirectly and secondarily checked owing to difficulty of irrigation.

The extension of phlebitis of the jugular towards the head and the venous sinuses of the cranial cavity, is quite exceptional.

When the mammary vein is inflamed it appears collapsed in the direction of the heart and swollen, indurated, and painful in that of its origin in the mammary gland.

Treatment. The first point requiring attention is so to fix the animal as to prevent the clot from being pressed upon or crushed, though, unfortunately, this cannot always be properly done. The diffculty is obviated by applying vesicants, which cause swelling and pain, and so reduce natural movement of the parts to a minimum.

At first, when the parts surrounding the operative wound are simply swollen and phlebitis is threatened, repeated application of tincture of iodine or a liquid vesicant is useful, and may prevent the disease developing.

In existing cases a blister applied over and around the whole of the hardened tract may prevent the mischief from proceeding beyond the adhesive stage. In such case the clot becomes organised, the vein remains obliterated, and recovery follows.

Similar treatment may also be employed in suppurative phlebitis, but as the clot gradually breaks down in consequence of the action of bacteria it is useful and almost indispensable to disinfect the vessel. For 
this purpose the opening of the sinus must be enlarged, and, by means of a sterilised or very clean syringe with a curved nozzle, the parts washed out daily with warm boiled water, followed by an antiseptic injection containing 2 per thousand of iodine, 3 per cent. of carbolic acid, or, better still, glycerine containing 1 per thousand of sublimate.

If in spite of this treatment the phlebitis extends towards the origin of the jugular or mammary vein, a counter-opening may be made at the point where the clot still remains adherent, and a strip of iodoform gauze saturated with tincture of iodine or with blistering ointment diluted to one-eighth with oil may be passed. Needle firing is also of value. Finally, as a last resource, a ligature may be applied to the vein above or beyond the clot.

This operation, which in the horse is confined to hæmorrhagic phlebitis, is especially applicable to phlebitis of the mammary vein in the cow. As the vein is subcutaneous, the operation may easily be performed in the standing position; the successive stages are as follows :-

The patient is firmly secured and its hind limbs hobbled by passing a rope around the hocks in a figure of eight. It is steadied on one side by an assistant who presses on the quarter.

One cubic centimètre of a 10 per cent. solution of cocaine is subcutaneously injected on each side of the vein at the point chosen. Ten minutes later a button-hole incision is made through the skin and a loop of thick catgut passed around the vein by means of a curved needle. The ligature is tied firmly with a surgical knot and the little wound afterwards covered with a mass of cotton wool secured by collodion.

\section{INTERNAL INFECTIOUS PHLEBITIS (UTERO-OVARIAN PHLEBITIS).}

The internal forms of phlebitis of parasitic or infectious origin are as yet little understood, but mention may be made of phlebitis of the uteroovarian veins which frequently follows parturition and post-partum infection. This is probably in many instances the real cause of the postpartum paraplegia without gross-or apparent material lesions.

This form of infectious phlebitis may extend to the large internal and external iliac veins and produce embolism and septicæmia, as is shown by recorded cases.

The mechanism of the disease is easily understood. The infective agents penetrate the veins of the uterine mucous membrane and pass from the lumen into the wall of the vein. Here they cause inflammation of the vascular endothelium, followed by the deposit of a fibrous clot of cylindrical form, which sets up partial thrombosis of the vein. This thrombosis becomes complete by the formation of a central clot due to venous stasis. 
It is not necessary for the germs to penetrate at a number of points. The thrombosis progresses until it gains a large trunk beyond the original point of infection.

Symptoms. Phlebitis of the veins of the pelvis is frequently misunderstood or overlooked, because the practitioner is apt to confine his attention to external signs, the paresis and paraplegia of the hind quarters.

The symptoms usually appear from five to eight days after normal parturition or parturition in which there is retention of the after-birth followed by metritis. The animals show fever and lose appetite, signs which may be due to metritis, but soon after they experience difficulty in rising, and some days later remain permanently recumbent.

The circulation is weak, and the entire intra-pelvic region painful; the large nervous trunks are affected, exertion becomes difficult, and the animals refuse to rise. At this stage they should not be forced to do so.

In two to three weeks improvement may occur and lead to recovery but in many instances various complications in the nature of purulent infection or septicæmia set in, or the animals are previously slaughtered.

Diagnosis. The diagnosis can only be determined after the symptoms develop. Confirmation might in some cases be obtained by rectal exploration made methodically and gently.

Prognosis. The prognosis is grave.

Treatment. Treatment should be based on disinfection of the uterus by injections of boiled water or warm iodised solutions and drainage by means of strips of iodoform gauze. The animals should be placed on a thick and scrupulously clean bed, and as far as possible be spared any considerable exertion for a fortnight. By changing their position once or twice a day complications may be avoided.

\section{UMBILICAL PHLEBITIS OF NEW-BORN ANIMALS.}

One of the most serious conditions met with in practice is that known as umbilical phlebitis of new-born animals. Whilst in fact it is easy to deal with phlebitis of the jugular or mammary vein, surgical or medical assistance becomes extremely difficult in this case, because the inflamed vein is deeply situated in the abdomen and passes through one of the most important internal organs, viz., the liver. When it is added that umbilical phlebitis is in 95 per cent. of cases of a suppurative character, the reader may form some idea of its gravity.

Unless the condition is early diagnosed and measures are at once taken, such complications as infectious hepatitis, purulent infection, and septicæmia cannot be avoided. Death is then inevitable.

In order clearly to understand this phlebitis, however, it is necessary 
to recall the anatomical formation of the umbilical region in the newborn animal.

At birth the umbilical cord is represented by a cylindrical mass, surrounded by the terminal portion of the amnion. It enters the abdomen through a circular perforation in the abdominal wall known as the umbilical ring. This ring may be divided into two parts, one deeply seated, the fibro-aponeurotic ring, consisting of an aperture in

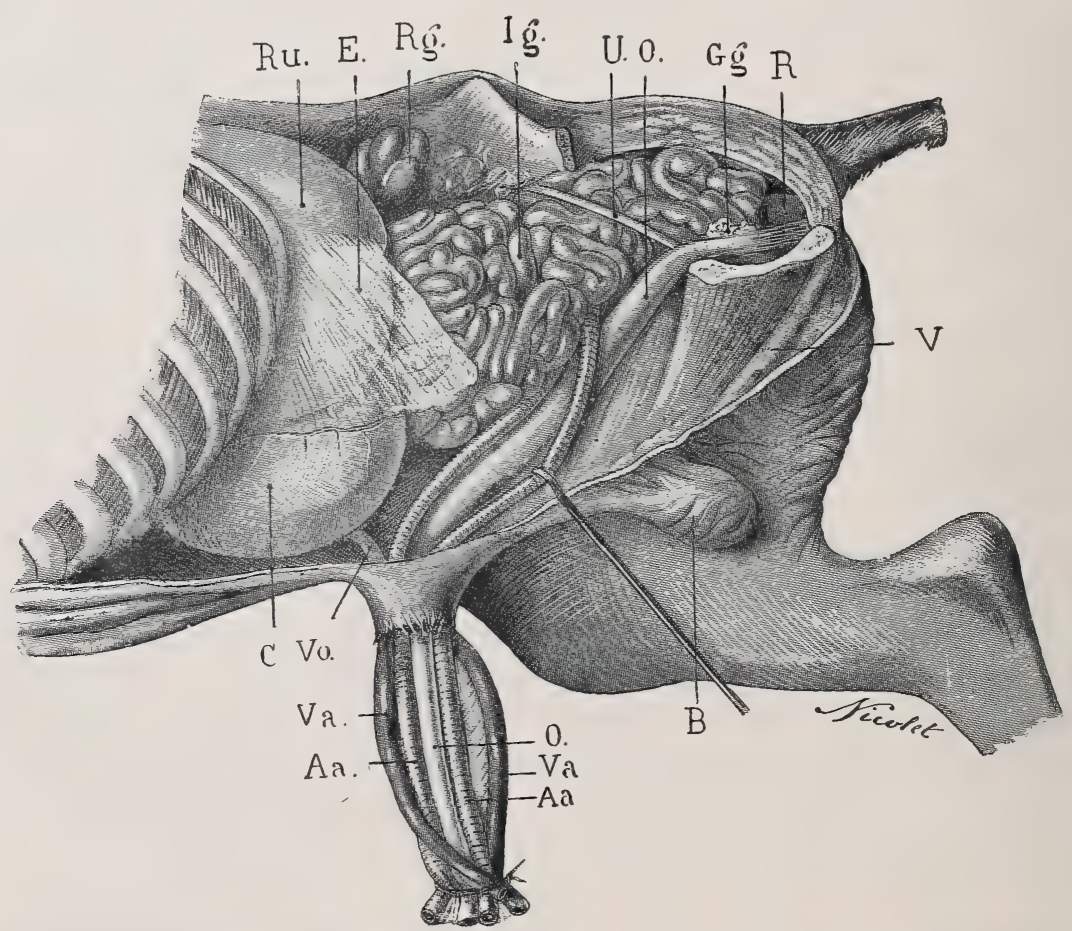

FIG. 183.-Position of the abdominal viscera in a new-born animal: $\mathrm{R} u$, rumen; $\mathrm{E}$, epiploon; Rg, left kidney; I $g$, small intestine; C, abomasum; U, ureter ; $\mathrm{O}$, urachus; $\mathrm{R}$, rectum. Umbilical cord: Vo, Umbilical vein ; $\mathrm{A} a$, allantoid arteries; $\mathrm{V} a$, allantoid veins; $\mathrm{O}$, the urachus.

the white line; the other the superficial or cutaneous ring, formed by the skin, which is wrinkled all round it, and constitutes a kind of sleeve about an inch in length. This cutaneous sleeve is continuous with the amniotic tissues. The entire umbilical cord is therefore enveloped in an amniotic-cutaneous sheath.

It is composed of four principal structures-the umbilical arteries, the umbilical vein, the urachus, and the interstitial mucous tissue.

The umbilical arteries and vein consist of two parts-the extra-fœtal part, which co-operates in forming the cord, and the intra-fotal part. 
The first is formed of two arteries and two veins, in contra-distinction to the condition in solipeds, where the cord only contains one vein. In the second, the arrangement is as follows: The two umbilical allantoid arteries on entering the abdomen curve backwards towards the entry of the pelvis, passing over the sides of the bladder enveloped in the lateral ligaments, and extend upwards towards the bifurcation of the aorta, finally pouring their contents into the internal iliac arteries. In the adult they may still be traced as annexes of these latter vessels. The two umbilical veins on passing through the ring unite to form one within the abdomen. This vessel passes forwards, rising along the lower abdominal wall, then becomes lodged in the thickness of the inferior middle ligament of the liver, and finally penetrates that organ where it unites with the portal vein. It is also connected with a vessel known as "the vein of Arantius," which places it in communication with the posterior vena cava, a vein not found in solipeds.

The fotal blood is purified by exchanges between it and that circulating in the maternal placenta, and when re-arterialised it returns by the umbilical vein.

The urachus, found in the embryo and fœtus, eventually gives rise to the bladder. In new-born animals this viscus is therefore open at its base, and communicates with the allantoid cavity through the urachus. 'The urachus starts from the base of the bladder, and, extending along the median plane of the lower abdominal wall between the two umbilical arteries as far as the umbilical opening, takes its place in the cord alongside the vessels. Through it the secretions of the fœetal kidneys drain into the allantoid cavity. The interstitial mucous tissue, also called "Wharton's jelly," is a gelatinous material which unites these different vessels and helps to support and protect them in the umbilical cord. It is particularly abundant opposite the umbilicus.

Immediately after birth the umbilical cord ruptures of itself as a result of the fall which the young animal experiences or of movements made by the mother, as for instance when she attempts to rise. In certain other cases it is divided by the mother biting it, or it may be ligatured by some person present. However the rupture may be brought about, it always occurs at a distance of 2 to 4 inches from the umbilicus. The immediate result is to produce thrombosis of the umbilical ressels and obstruction of the urachus. The tro umbilical arteries rarely bleed, for hæmostasis is brought about by stretching, and these arteries, being very elastic, almost immediately retract and close. The umbilical veins simultaneously become blocked, and the single intra-abdominal vein having no further raison d'être, gradually becomes obliterated. The urachus should normally be obliterated at the moment of delivery (Colin and Saint-Cyr), or at any rate soon D.C. 
afterwards, as a consequence of rupture of the cord (Chauveau and Zundel).

Immediately after delivery another change sets in. The extra-fœtal portion of the cord, which remains attached to the umbilicus, dries on contact with the air, the Wharton's jelly retracts, the whole undergoes a kind of necrosis, assumes the appearance of a dry scab, and in eight or ten days falls away, leaving in its place the umbilicus, which should be half cicatrised on the fall of the cord. Thus the umbilical cord presents an extra-fotal degenerated portion and a persistent portion about $\frac{1}{2}$ to 1 inch only in length, buried in the cutaneous ring of the umbilical region.

If all the changes indicated occur normally and physiologically, the little wound in the region of the umbilicus cicatrises in a perfectly regular way. But unfortunately this is not always the case. At times the cicatrix becomes contaminated by manure, urine or dust, suppurates, and may then become the seat of various complications, such as umbilical phlebitis, omphalitis or persistence of the canal of the urachus.

\section{UMBILICAL PHLEBITIS OR OMPHALO-PHLEBITIS.}

History. Umbilical phlebitis, and in a more general sense all pathological conditions of the umbilicus, in new-born animals have been the object of numerous investigations by Lecoq, Bénard, Loiset (1843), Bollinger (1874); and more recently by Morot (1884), Uffredizzi (1884), Chassaing (1886), etc.

Omphalo-phlebitis may occur as a primary condition or may appear as a complication of omphalitis and of persistence of the urachus. It consists essentially in suppurating inflammation of the umbilical vein, but is not infrequently accompanied by omphalitis, arteritis, peritonitis, and cystitis.

Causation. The disease results from infection of the (normal) clot and of the wound resulting from severance of the cord. The infection may only cause simple phlebitis of the umbilical vein, but if the organisms are virulent the phlebitis almost inevitably degenerates into suppurative phlebitis.

Formerly omphalo-phlebitis was thought to be caused by the mother licking the foal, by irregular tearing of the cord, by crushing and separation of the obliterating clot, etc. The truth is that all these causes favour infection of the umbilical wound, which is the primary cause, suppurative phlebitis being secondary only.

When the cord is ruptured both the veins and arteries become plugged, and bleeding ceases. This plugging should end in organisation of the clot and obliteration of the vessels. If, however, the wound is 
infected, micro-organisms make their way between the clot and walls, and extend along the inner surface of the vein, infecting first the clot and then the vein, and thus setting up suppurative phlebitis.

If suppuration does not continue, recovery may occur spontaneously. Infection may be confined to the clot, producing simple phlebitis, but it often extends along the umbilical vein to the liver, causes infectious hepatitis and purulent infection or septicæmia. Similar results may be produced by infection of the arteries, the organisms making their way as far as the bifurcation of the aorta, and thus gaining the general circulation. Moussu believes that this is the commonest method by which septicæmia is produced in calves.

Symptoms. In these cases it is usually the general symptoms which first attract attention, the local lesion passing unnoticed for a greater or less time.

The animal shows intense fever, due to either suppurative phlebitis, infectious hepatitis, or, as often happens, to generalised infection. Appetite is lost, diarrhoea is abundant, the respiration and circulation are accelerated, and the temperature rises to $104^{\circ} \mathrm{Fahr}$., or even $105^{\circ} \mathrm{Fahr}$.

The local symptoms are those usually associated with omphalitis or phlebitis. An examination of the umbilical ring reveals an cedematous, hot, sensitive swelling, the lower part of which exhibits a chronic, suppurating, fungoid, blackish wound of unhealthy appearance.

This wound is the seat of one or more sinuses which penetrate the vein, arteries, or urachus. If only one sinus exists, it always passes upward and forward into the umbilical vein. The utmost precaution should be employed in examining the parts. Should it be thought desirable to probe the sinus in order to discover its direction, the probe must be very cautiously introduced, and only for a short distance, because rough handling would tear the tissues and carry infective material to deeper seated points.

Complications. These are numerous and very grave. Long ago Lecoq described a disease suggestive of laminitis, which beyond doubt was only a form of purulent infection. At a later date Loiset studied a disease following omphalitis, in which interstitial abscesses developed in the cord. This also was simply purulent infection.

More recently complications such as pleurisy, pneumonia, infectious endocarditis, diarrhœic enteritis, and especially suppurative polyarthritis of young animals have been referred to omphalo-phlebitis. All these complications result from infection. The micro-organisms themselves or the toxins they secrete appear to have a particularly injurious action on the serous membranes, a fact which throws light on the frequency of such complications as pleurisy, peritonitis, endocarditis and arthritis. 
Intoxication also plays a certain part, and microbic toxins are responsible, at least at first, for the uncontrollable diarrhœa, arthritis with sterile exudations, etc.

Diagnosis. This presents no difficulty. The alarming general symptoms seen at the outset immediately suggest in the case of young animals the possibility of disease in the umbilical region.

Prognosis. The prognosis is grave, it may be said very grave, because treatment is difficult to apply, and dangerous complications, which almost always prove fatal, may already have been set up.

One must always distinguish, however, and take into account in forming the prognosis, the special characteristics of the phlebitis, and weigh carefully the signs of complication. The fistula should be cautiously explored, and its depth, etc., noted, while the temperature, circulation, respiration, etc., should be carefully studied.

Treatment. A very important item of treatment consists in regularly and scrupulously cleansing the region of the umbilicus after the cord has separated and until the wound has completely cicatrised. 'The parts are washed with boiled water and dusted with boric acid, iodoform, ete.

A still better plan, and one that almost certainly guards against this disease, is to apply an antiseptic dry dressing as soon as the new-born animal has become dry. This need only consist of a small sheet of antiseptic cotton wool fixed to the umbilicus by four pitch bandages or by two pieces of webbing passed over the back. In this way contamination of the cord and the risk of infection are avoided.

In cases of fully-developed phlebitis the old generation of practitioners used to recommend local dressings with adhesive plasters, astringent and vesicant applications, etc. All such methods are useless, because they only act on a part of the diseased structures and cannot reach the blind ends of the sinuses. The classic treatment of suppurative phlebitis also is out of the question.

All that can be done, therefore, is slightly to open up the sinuses and wash them out frequently with antiseptic solutions, such as boiled water, sublimate-glycerine, carbolic glycerine, etc., afterwards applying antiseptic dressings. 'These methods, however, are scarcely likely to put an end to infectious complications such as suppurative polyarthritis.

There is no danger in using strong carbolic solution, 3 per cent. creolin, 4 per cent. chloride of zinc, sulphate of eopper, etc. Should there be several sinuses and should one of them extend in a backward direction, it is necessary to make certain that no communication exists between the urachus and the bladder. For this purpose some boiled water may be injected into the sinus. If a communication exist, this water will 
fill the bladder and distend the urachus. The treatment necessary in this case is similar to that of persistence of the urachus.

It is well in all cases to be guided by the following principle: never to resort to treatment unless suppuration has occurred and the sinus is blind. To check suppuration a blister may be applied around the umbilical region while means are taken to prevent the animal licking the parts.

Chassaing in 1886 suggested a rather original method of operation which deserves description. It is founded on the permanent treatment of sinuses, and consists in introducing a flexible osier stick, a kind of bougie, enveloped in tow and moistened with the following mixture:

$$
\begin{array}{lllllllll}
\text { Collodion } & \ldots & \ldots & \ldots & \ldots & \ldots & \ldots & \ldots & 3 \text { parts. } \\
\text { Sublimate } & \ldots & \ldots & \ldots & \ldots & \ldots & \ldots & \ldots & 1 \text { part. }
\end{array}
$$

This is introduced for a distance of 3 to 4 inches into the fistula, and is fixed to the skin with gutta-percha or pitch. The dressing is renewed every five or six days, and healing takes place, it is said, in one, two, or at most three weeks.

It is very likely that if the sinuses were previously cleared and simply plugged with antiseptics or treated by introducing pencils of salol, nitrate of silver, sulphate of copper, iodoform, etc., at least as good results might be obtained. 


\section{CHAPTER V.}

\section{DISEASES OF THE BLOOD.}

\section{SEPTICEMIA OF NEW-BORN ANIMALS.}

The above title is given to that exceedingly fatal disease commonly known as "white scour," etc., the mortality in which often rises to 95 per cent.

The disease was studied by Poels in Holland in 1889, Dèle in Belgium in 1891, Perroncito in Italy, Galtier in the centre of France in 1891-92, and quite recently by Nocard in Ireland in 1901.

It occurs throughout all the breeding districts of France, and in some parts causes enormous losses, the mortality comprising two-thirds or even three-fourths of all new-born calves. In certain breeding establishments in Normandy all the new-born animals without exception die unless special precautions are adopted.

In foals, septicæmia of the new-born is very rare, because horsebreeding establishments are much better cared for, and breeding mares are segregated. In byres, on the other hand, the greatest promiscuity exists. The disease is equally uncommon in lambs, although it makes numerous victims in folds which have once been attacked. It is, however, quite common in young pigs.

Symptoms. The development and course of the disease are in certain respects characteristic.

The disease usually appears within two or three days after birth, and only in rare cases after the second week. Calves which at birth appeared vigorous and in good health are found dull on the second day; after the second or third meal they suffer from diarrhœa, and from that time refuse all nourishment, lie down as though exhausted, and sometimes die very rapidly.

Some even perish in ten to twelve hours without showing diarrhœa; although apparently well at night, they are found dead or dying the next morning. This is the peracute form.

Most frequently the young creatures suffer for two or three days, sometimes a week. Appetite is partly preserved; at first the diarrhœa resembles that due to inability to digest milk, but the fæces soon 
become greyish or blackish and very fœetid. The hair of the tail, quarters and hocks is soiled and matted, the skin irritable and reddish; the patients lose strength, appear unsteady on their limbs, and develop rapid respiration and tumultuous action of the heart.

They take little food, become weaker by degrees, and die in a con. dition of exhaustion.

Fever, well marked at first, frequently diminishes, and the tempera. ture may remain normal for several days, falling to $97^{\circ}$ Fahr., or even $95^{\circ}$ Fahr., twenty-four hours before death.

This is the commonest form of the disease. It lasts three to five days, and is always grave.

Cattle-men recognise the disease chiefly by the diarrhœa and loss of appetite.

Lastly, a third and rarer form occurs during which appetite is maintained in spite of the diarrhœa. The animals remain thin, develop poorly, but survive for a month, six weeks or two months. The diarrhœa diminishes or disappears, but its disappearance is followed by complications such as broncho-pneumonia, pleuro-pneumonia, endocarditis, acute arthritis, etc., a fact which led Prof. Galtier to give the disease the name of "septic pleuro-pneumonia in calves." These complications, again, are extremely grave, and generally prove fatal after a period of varying length. They are due to local development of micro-organisms of the kind which produce septicæmia, and similar to those described under the name of broncho-pneumonia of intestinal origin in sucking calves.

They differ, however, as regards their cause, from the primary affection, and may be due to very varied organisms, the commonest being those of suppuration. These organisms, in fact, are alien to the primary disease, and obtain entrance from without, very probably by the tracheobronchial tract.

In young pigs septicæmia assumes the same forms as in the calf. In lambs the chronic form seems more frequent than the peracute and the ordinary forms.

Causation. The septicæmia of calves, and possibly of all new-born animals, of whatever species, is produced by a microbe which flourishes in the manure and litter of stables, and which Nocard included in the group of Pasteurella. It can be found in the blood from the moment the first external symptoms appear until the time of death. During the last hours, however, the bacterium Coli communis also invades the circulation in many instances, and if cultures are not made until sume hours after death, the colon bacillus and bacteria of putrefaction are more particularly discovered.

The microbe of calf septicæmia can be readily cultivated in jelly or in ordinary liquid media. Injected into the veins of experimental animals, 
it reproduces the clinical symptoms, and causes death more or less rapidly, according to the dose injected.

The virulence of cultures grown in defibrinated calf's blood seems more intense, and Moussu has been able to reproduce the clinical form of the disease by applying to the umbilical cord of a new-born animal a pledget of cotton wool saturated with such a culture, and covering it with a dressing. The germs of the disease are spread throughout the byres through the medium of fæces. When the umbilical cord has become dry, that is, after the third day, the application of virulent cultures to the stump no longer causes infection.

Pathogeny. The pathogeny of this septicæmia of calves and of newborn animals is easy to explain.

At birth the young animals fall on the litter, and the umbilical cord becomes contaminated. The infective agent, finding an excellent culture medium in the tissues of the cord, at once begins to develop, increases in enormous numbers, steadily ascends along the cord, and sets up septicæmia. It grows in the gelatinous Wharton's jelly and in the fibrinous plug closing the arteries and umbilical vein, and soon enters the true circulation. Septicæmia is then fully established, general disturbance sets in, and with it the diarrhœa by which it is externally indicated.

It is important to remember, however, that infection occurs most readily through the medium of the cord, and during the first few days after birth: it may occasionally be brought about towards the eighth or tenth day, when the shrivelled portion of the cord falls; in this case its entrance is effected through the little umbilical wound.

Lesions. The lesions are sometimes so obscure that the practitioner may hesitate to deliver an opinion.

In acute cases, where death occurs in two or three days, or even in ten to twelve hours, post-mortem examination reveals only increased vascularity of the serous membranes - the peritoneum, pleura, pericardium, etc.; and it may be almost impossible to discover anything abnormal in the cord, for although the clots closing the arteries and veins are infected, they are neither separated from the walls of the vessels nor broken up.

On the surface of the urachus, at the base of the bladder, and in the depths of the peritoneal folds supporting the allantoid arteries (sometimes also the hepatic vein), unequivocal signs of local ascending infection may, however, almost always be found, together with intense injection of the capillaries, little hæmorrhagic spots, and commencing formation of false membranes, etc.

The infection extends also by the lymphatic vessels contained in these peritoneal folds, and finally attains the sublumbar region.

When the disease develops less rapidly the peritoneal cavity 
contains a certain quantity of blood-stained serosity, as do the pleuræ and pericardium, whilst vascular engorgement of the serous membranes is extremely marked. The intestine shows traces of congestion and inflammation throughout its length, and its contents contain the specific organism in very large numbers.

Finally, in the chronic forms, the serous membranes and the intestine seem only slightly attacked, possibly because the lesions have undergone retrogressive changes. The striking features are the secondary lesions, such as those of pneumonia, broncho-pneumonia, pericarditis, and abscess formation in the lung.

Nocard gives the following description of the lesions found during his investigation of "white scour" of calves in Ireland (Veterinarian, April, 1902, p. 171; see also Prof. Mettam's paper, Veterinarian, June, 1902, p. 307) :-_"The lesions found on autopsy vary according to. whether the evolution of the disease has been rapid or slow. One lesion, however, is never absent-that of the navel and the navel vessels. In all the calves attacked we found a large umbilicus with hardened coats enclosing a clot easily broken down, sometimes soft and purulent. In every case, also, we observed blood suffusions, often very extensive, along the course of the umbilical vessels and of the urachus, invading often the posterior third of the bladder. In cases where the evolution had been rapid we found the lesions of true hæmorrhagic septicæmia. All the viscera were congested to excess; their surface was studded with petechiæ, ecchymoses, or subserous blood suffusions. The capillary network of the peritoneum, pleura, and pericardium appeared strongly injected. This lesion was especially marked on the epiploon. The intestine was the seat of intense congestion, especially at the level of the "floating colon.'

"The mucous membrane was thickened, gorged with blood, and friable; the solitary glands, thick and protruding, were sometimes transformed into a kind of bloody magma, or they were ulcerated, as in anthrax; the contents of the bowel were mixed with a large quantity of blood. The mucous membrane of the fourth stomach was altered nearly to the same degree; it was studded with interstitial hæmorrhages, especially above the level of the open edge of its folds. The mesenteric glands-especially those of the colon-were enormous, gorged with blood, reddish, and often hæmorrhagic. The mucous membrane of the bladder was often covered with petechiæ, the urine which it contained was clear and limpid, but always rich in albumen. [In one sample which was analysed, the urine contained more than 4 grammes of albumen to the litre.] The lungs were gorged with blood, like the intestines; sometimes they were manifestly œedematous, but generally their tissue was still supple, elastic, permeable, and without apparent lesion. 
"In the subacute forms the lesions are much less marked. The mucous membrane of the intestine is less congested; sometimes adema of the submucous tissue exists. The mucous membrane of the fourth stomach is often punctuated with brownish-red patches, traces of the capillary hæmorrhages which were produced at the onset of the disease. The mesenteric glands are swollen, gorged with serum, but not hæmorrhagic; the liver is large and of a yellowish tint; the spleen is little altered; the urine always contains albumen; the lungs are seldom quite sound; they usually contain here and there small diffuse centres of catarrhal pneumonia, of nodular bronchial pneumonia, or simply of atelectasis.

"These lesions are more constant and more dense if the animals have resisted the disease for some time; they then constitute the transition stage between the simple collapse at the beginning of the disease and the suppurating lesion of lung disease. The joint lesions when they exist are very interesting. At the beginning all the periarticular tissues are infiltrated with a yellowish gelatinous serosity. The synovial membrane is covered with vascular aborisations of an extreme richness, which extend on to the articular cartilages. The synovial capsules are distended by a considerable quantity of thick synovia of a deep yellow or brownish tint, holding in suspension flakes of fibrous exudate more or less dense and abundant. When the lesion is older the synovia is replaced by a thick fibrous exudate, which fills sacculations, and extends between the articular surfaces. In this case the lesion appears identical with that of the arthritis seen in pleuropneumonia of sucking calves."

Diagnosis. The diagnosis presents no difficulty, for the development and acute course of the disease (the majority of patients die within a week of birth) leave little room for doubt.

This disease is easily distinguished from dysentery in new-born animals, which appears at birth, as also from simple diarrhœic enteritis; in the latter disease the symptoms are delayed, sometimes occurring only when the animals are weaned; moreover, the disease is never so grave as that now under consideration.

Should, however, the post-mortem appearances seem indecisive, the diagnosis can be based simply on the high mortality.

Prognosis. The prognosis is extremely grave. About 95 per cent. of the animals attacked die, and among those which survive many show thoracic complications, that render them useless.

Treatment. Treatment of animals already affected is useless, and, moreover, too costly. Drugs administered through the digestive apparatus to a large extent miss their mark, because the digestive symptoms are secondary, primary infection having occurred through the 
circulation. The administration of purgatives and internal antiseptics can, therefore, only prove illusory.

On the other hand, prophylactic treatment is of the greatest value; all that is necessary is to prevent the umbilical cord from becoming infected.

The great mortality, which causes such severe loss to breeders, is simply due to want of proper care of new-born animals. Even in carefully kept byres the mortality may be high, for the specific agent develops in litter contaminated with fæcal matter, by lying on which young animals become fatally infected.

To check or prevent this septicæmia in breeding establishments, it is merely necessary to take the same precaution as is taken in dealing with young children, i.e., to apply an aseptic or antiseptic dressing to the stump of the cord after ligation. As soon as the young animal has been dried by the mother or by artificial means, a carefully boiled ligature is applied to the cord at a distance of about 1 inch from the umbilical ring. The portion of the cord below the ligature is snipped off, the remaining part is carefully washed with boiled water or boric solution, and is surrounded with a mass of iodoform wool, kept in place by a bandage passed over the back.

The cord will shrivel a little less rapidly than it

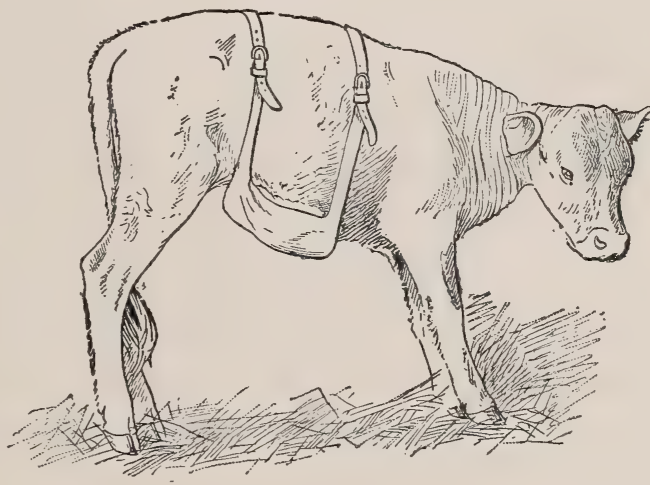

FIG. 184.--Dressing for umbilicus of newborn calf. would if exposed to the air, but will be protected from all infection. The young animal should be separated from the mother to prevent her from displacing the dressing by licking the parts.

In a few days all danger is at an end. This method is very simple, and can be carried out even by the breeder and in an infected byre. Nocard recommends the use of umbilical dressings containing collodion, and the practitioner can choose whichever method he pleases.

In grave outbreaks involving large establishments, the byres should be rigorously disinfected, and it is sometimes well to segregate cows about to calve in a special byre, from which the calves are not allowed to pass until the umbilicus is cicatrised. 


\section{TAKOSIS: A CONTAGIOUS DISEASE OF GOATS.*}

This disease has been seen in Angora goats brought from Texas into Pennsylvania, U.S.A.

Symptoms. The disease presents many of the symptoms usually accompanying a parasitic invasion, and is characterised by great emaciation and weakness, with symptoms of diarrhœa and pneumonia. In the early stages of the affection there is usually little to indicate that anything is seriously amiss with the animal. The first observable symptom manifested is the listless and languid appearance of the animal, evidenced by its lagging behind the flock, and is usually accompanied by a drooping of the ears and a drowsy appearance of the eyes. The pulse is slow and feeble, and the temperature is elevated slightly at first, but becomes subnormal a few days before death. The highest temperature observed in the natural disease was $104^{\circ} 1^{\circ}$, and the lowest, in a prostrated animal a few hours before death, registered $99 \cdot 7^{\circ}$ Fahr. Snuffling of the nose, as in a case of coryza, with occasional coughing is sometimes in evidence.

As the disease advances the animal moves about in a desultory manner, with back arched, neck drawn down toward the sternum, and with a staggering gait. Rumination is seldom impaired. The appetite, while not so vigorous, is still present, though capricious, and the affected animal shows plainly that the ravages of the disease are rapidly overcoming the restorative elements derived from the food. The fleece is usually of good growth, and presents a surprisingly thrifty appearance when the condition of the animal is taken into consideration. All the exposed mucous membranes appear pale, and the respirations are accelerated and laboured. The goats finally become so weak that they are readily knocked down and trampled upon by their fellows. If picked up they may move off slowly and eat a little, but within a few hours are down again, and in this way linger for several days, shrinking to about half their natural weight, and occasionally bleating or groaning, with head bent around on the side or drawn down to the sternum. A fluid discharge from the bowels of a very offensive odour is usually observed in the last few days of life, but this symptom is not constant.

Course and Susceptibility. This disease may assume a subacute or chronic type, usually the latter. The animal dies of inanition in from eight days to six or eight weeks. Several owners have reported deaths after only two or three days of illness, but the goats doubtless had been affected for a longer period, although not noticed on account of their mingling in the flock. Many of the animals live for weeks, but gradually

* Annual Report, U.S.A. Bureau of Animal Industry, 1902, p. 354 (Mohler and Washburn). 
become weaker and more debilitated, finally dying in a comatose condition. In no instance has the natural recovery of an animal after once the symptoms of takosis were noticed been observed or heard of.

The younger goats seem to be the most susceptible to the disease, although the old animals are by no means immune.

Pathological Anatomy. As already indicated, the general appearance of the carcase simulates that produced by a wasting disease. The visible mucous membranes are pale and anæmic, while the fleece, which appears somewhat dry and lustreless, furnishes a shroud for the extremely emaciated condition, that becomes plainly perceptible on skinning. The same anæmic condition of the subcutaneous and muscular tissues is observed on eviscerating the carcases. The lungs in most cases are the seat of a peculiar diversified inflammation, never of a remarkable extent. The external appearance of these organs is at times mottled, caused by a few congested areas, several patches of an iron-grey colour similar to areas of pneumonia during the process of absorption, and normal tissue. On section through the reddened patches, a frothy mucus may exude from the bronchioles, and in one case numerous punctiform hæmorrhages were observed on the sides of the incision. This tissue, while not so buoyant as a normal portion would be, nevertheless floats when placed in water.

The heart in all cases is pale and dull, its tissue soft and flabby, while inflamed areas, more or less penetrating, are present at times on the epicardium about the auricular appendages, and at other times on the endocardium, especially that lining the ventricles. These hæmorrhagic patches consist of either pure extravasated blood or blood mixed with serum, which gives them a more diffuse appearance and a gelatinous consistence. The pericardium is slightly thickened, and usually contains a small increase of fluid tinged with blood. The liver usually appears normal, although the gall bladder is frequently distended with pale-yellow watery bile. The kidneys are anæmic and softened. The cortex appears slightly thicker and paler than normal, and contrasts strongly with the darker pyramids. The capsule strips off easily from the parenchyma of the organ. In one instance several pale areas simulating anæmic infarcts were observed under the capsule extending into the cortex, which probably resulted from the compression of the capillaries by the swollen parenchymatous cells. The presence of albumin in the urine was detected by the nitric-acid test. The spleen appears atrophied and indurated, and on section the fibrous tissue far exceeds the splenic pulp. Attachments by fibrous adhesions may fix the spleen to the diaphragm or the neighbouring organs. The intestines may contain normal fæcal matter or semi-fluid fæces of a disagreeable odour. The surface of the mucous membrane is at times covered with a slimy 
mucus or plastic exudate, and the appearance is that of a chronic catarrh associated with necrosis of the mucosa.

Bacteriology. Examination of cultures and slides showed the presence of a micrococcus, usually arranged in the form of a diplococcus, which was found in pure cultures from the heart's blood, spleen, kidneys, and pericardial fluid, and essentially so in the tubes inoculated from the other organs.

The specific organism of takosis appears in fresh bouillon cultures as a spherical or oval micrococcus with a diameter of 0.8 to $1 \mu$. In these cultures it is single or in chains of two, three, or four elements, but most frequently in pairs, as diplococci, with a diameter transverse to the axis of the chain greater than the longitudinal diameter.

Treatment: Prophylaxis. Sudden climatic changes should be avoided as far as possible, and when shipments of goats for breeding purposes are to be made which necessitate their transportation over considerable distances the changes should be made during the months of summer or late spring, and not in the fall or winter, when the contrast of temperature will be so much greater.

Angora goats should be provided with stables that are thoroughly dry, erected upon ground that has perfect natural drainage.

As a third measure of prevention may be mentioned careful feeding.

The segregation or isolation of all affected animals as soon as they evince any symptoms of the disease will be found a most valuable means of protection for those that remain unaffected, and a strict quarantine over all of the diseased members of the flock should be maintained so long as the disease remains upon the premises.

Medicinal treatment has proved unsatisfactory in many of the cases of takosis to which it has been applied. The best results have been derived from the administration of calomel in 0.1-gram doses twice daily for two days, followed by arsenic, iron, and quinine, as follows :

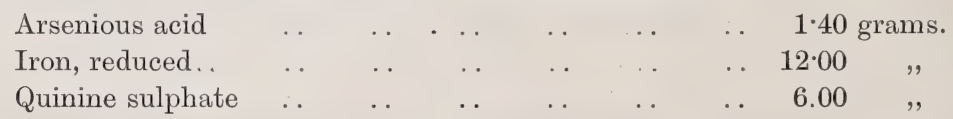

Mix and make into twenty powders, giving one to each adult goat morning and evening at the conclusion of the administration of calomel. After an interval of two days this treatment is repeated. In case the diarrhœa persists, the sulphate of iron has been substituted for the reduced iron, with beneficial effects.

Conclusions. After preliminary investigation, the following conclusions have been reached:-

(1.) The disease described as takosis has appeared in many parts of America, but particularly in the Northern States, where it has caused great loss to many breeders of Angora goats. 
(2.) It is a progressive, debilitative, contagious disease, characterised by great emaciation and weakness, with symptoms of diarrhœa and pneumonia, and causes a mortality of 100 per cent. of those affected and from 30 to 85 per cent. of the whole flock.

(3.) From the carcases of numerous animals that have succumbed a new organism, Micrococcus caprinus, has been recovered in purity, and is presumably the etiological factor.

(4.) This micrococcus possesses pathogenic properties for goats, chickens, rabbits, guinea pigs, and white mice, but not for sheep, dogs, or rats.

(5.) Medicinal treatment was attempted with varying success, while the immunising experiments thus far conducted (although too few to permit of any conclusive statement or accurate estimate as to their protective value) have shown highly encouraging results. When accompanied with measures of isolation and disinfection, the treatment may prove of great assistance in the suppression and eradication of the disease in an infected flock.

\section{BLOOD POISONING (MALIGNANT CEDEMA) IN SHEEP AND LAMBS IN NEW ZEALAND.}

This disease, which occurs during the operations of shearing sheep and of castrating and docking lambs, is the cause of considerable loss annually to sheep breeder's in several districts of New Zealand. In 1893 J. A. Gilruth, Chief Veterinarian for New Zealand, issued a leaflet dealing with the disease and the preventive measures to be adopted. Generally the first thing that draws the owner's attention seriously to the condition of his flock is the discovery, in from thirty-six to fortyeight hours after docking or shearing, of a few dead sheep lying in various parts of the paddocks. Next morning he finds a few more dead, and so on for three or four days, when, as a rule, the mortality ceases.

Symptoms. In the early stages of this disease the animal seems listless, disinclined to move about, and, if the sun is shining strongly, prefers to lie in the shade. If forced to move, the hind legs are drawn forward with a peculiar stiff, dragging motion, as if there were no joints. There are slight muscular tremors all over the body, which become spasmodic as the disease progresses. If the flock be driven about much, the diseased animal soon shows signs of great fatigue, ultimately dropping to the ground thoroughly exhausted. The breathing is fast and painful, being maintained more by a series of spasmodic jerks than by any regular act. The pulse is quick and weak; the temperature is very high, registering $106^{\circ}$ to $108^{\circ} \mathrm{Fahr}$., showing acute fever; the eyes close, and the whole face is expressive of pain. Gradually the spasms cease and coma sets in, resulting in death. The scrotum and surrounding skin 
right along the floor of the abdomen and between the hind legs become swollen and black. This gangrenous tissue, when present before death, can be peeled off without pain to the animal. On post-mortem examination various conditions are met with. The animals are generally found to be among the best of the flock and in fairly good condition. The scrotal and perineal regions in lambs (between hind legs and below tail) are always, or almost always, gangrenous, this condition extending along to the floor of the chest, and sometimes implicating the tail. Many of the muscles, generally those of the shoulders, haunches, and loins, are dark in colour and infiltrated with a black, watery fluid. The intestines are generally healthy, though sometimes the peritoneum is inflamed. The spleen and liver are in the usual condition after death, due to febrile disturbance. In the chest, either the pleuræ (coverings of the lungs) or pericardium covering of the heart) are often inflamed, with occasionally a fibrinous exudation, causing surfaces to adhere.

Cause. In Gilruth's report for 1900 he demonstrated the cause of blood-poisoning to be a microbe known as the malignant œdema bacillus (Vibrion septique of Pasteur). This organism, which is found in many dirty yards, swampy soils, ete., on gaining entrance to the system of almost any animal by means of a wound, rapidly increases in numbers, producing gangrene, or death, of the part affected first, and ultimately the death of the animal.

Curative treatment is practically useless.

Preventive measures. Destruction of the carcases of animals which have succumbed to this disease by efficient burial or by fire. Disinfection of surface soil of yards, etc., by quick-lime. Cleansing of floors and walls of sheds with strong hot lime wash containing crude carbolic acid in the proportion of 1 to 50. Disinfection of flesh cuts made by the shears or the docking or castrating knife. Boiling of docking and tailing knives before use. Observance of antiseptic applications even when temporary yards are employed. Sheep and lambs after operation to be kept in a paddock free from swampy patches.

\section{PIROPLASMOSIS.}

Under the title piroplasmosis is included a group of diseases caused by hæmosporidia, and found in animals of the bovine and ovine species. These affections are far from having the same importance in temperate as they have in tropical countries; nevertheless, it is very important to be able to recognise them.

BOVINE PIROPLASMOSIS.

Bovine piroplasmosis has been described under different names, such as hæmoglobinæmia, hæmoglobinuria, Texas fever (U.S.A.), tick fever 


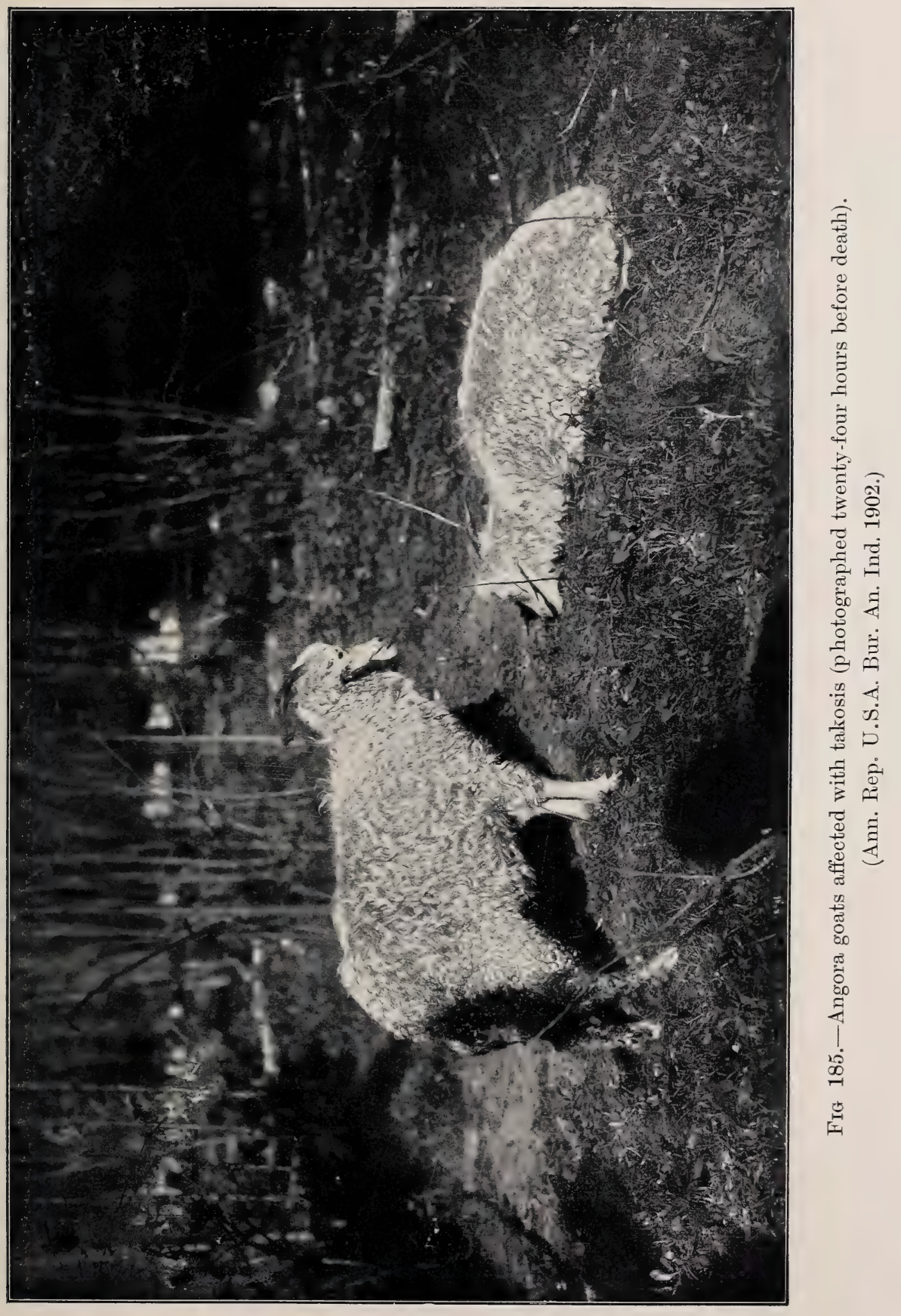





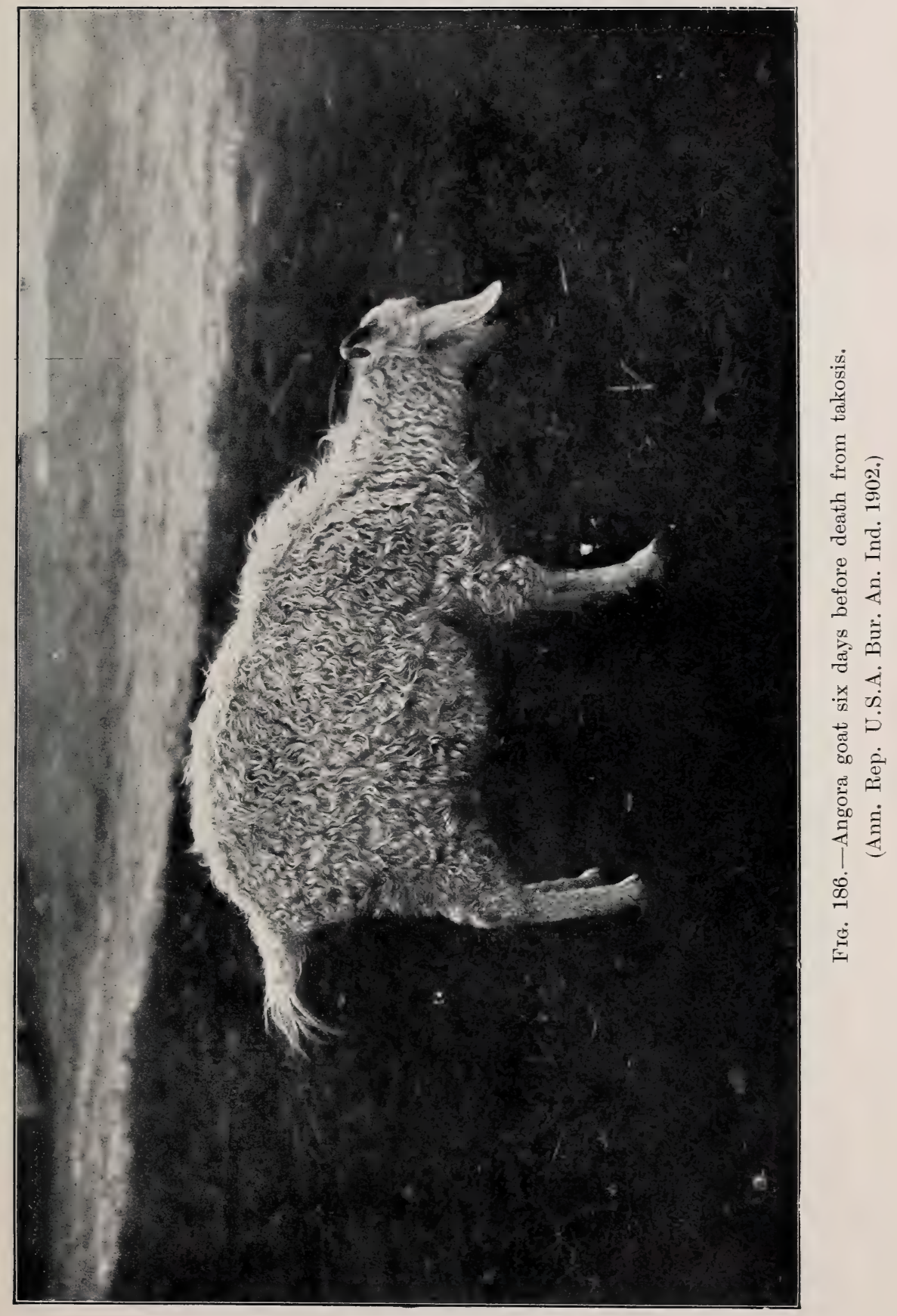





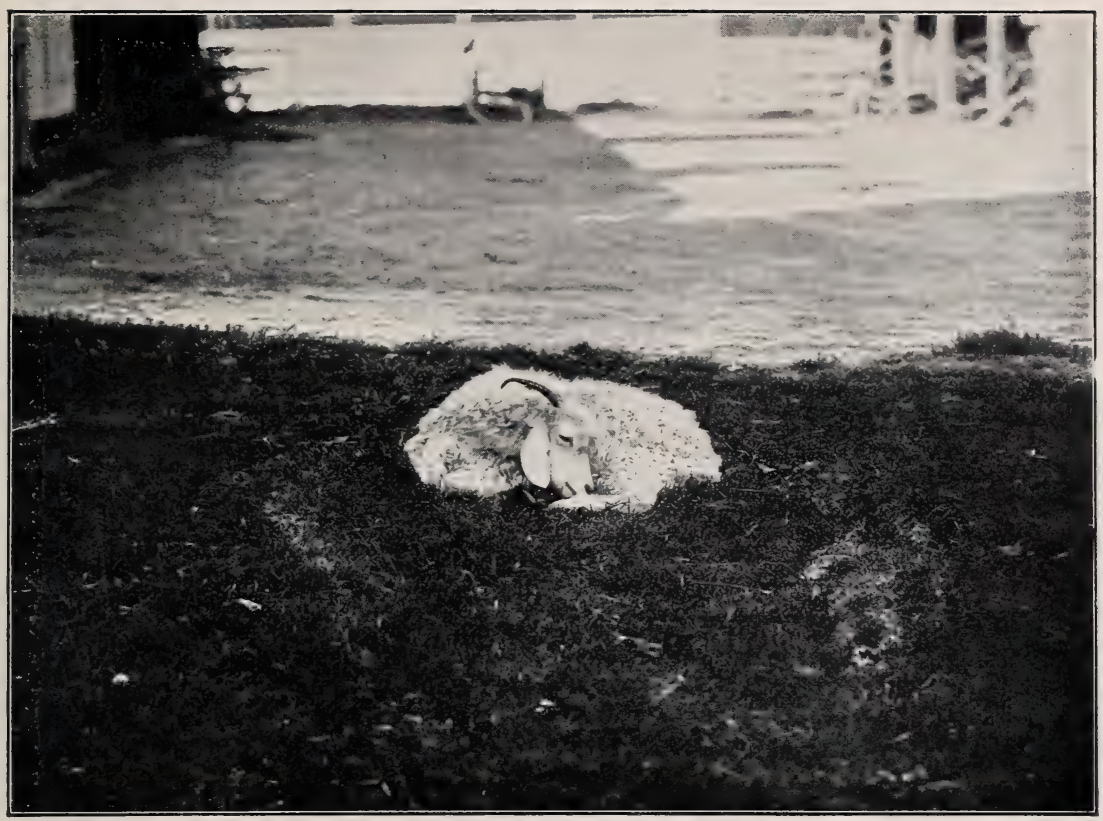

FIG. 187.-Angora goat (photographed three days before death). (Ann. Rep. U.S.A. Bur. An. Ind. 1902.)

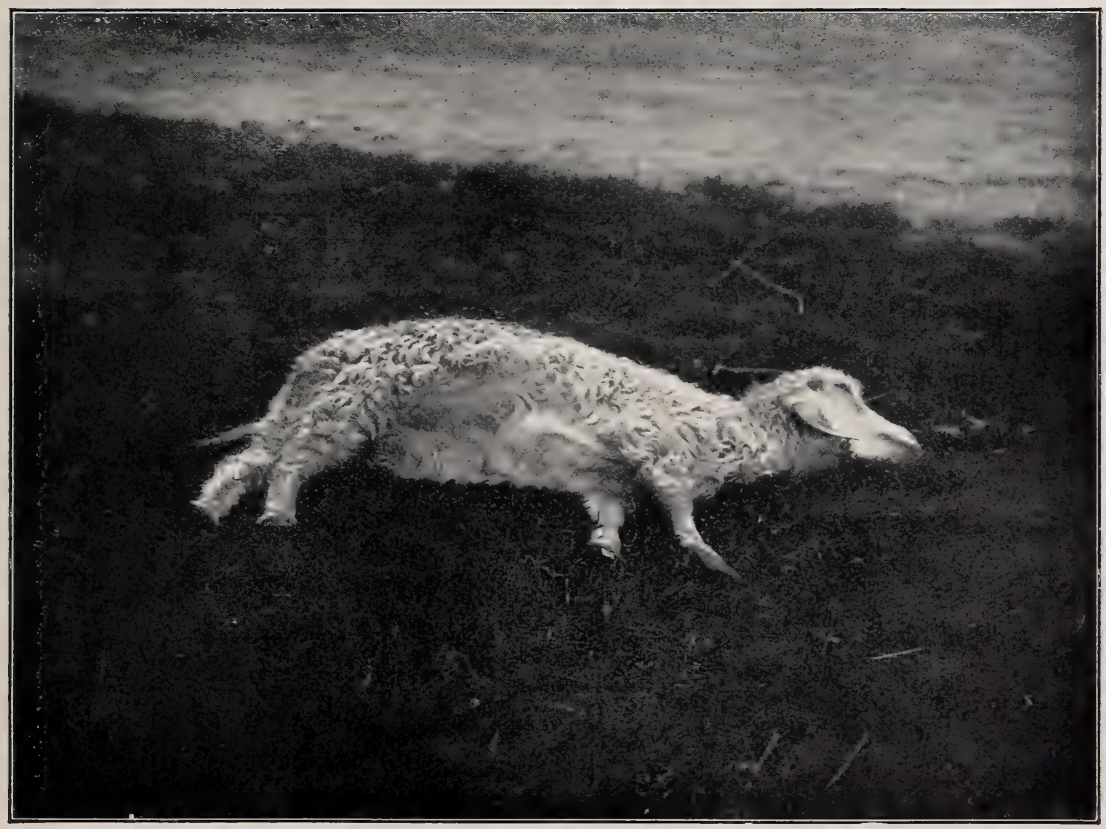

FIG. 188. - The same goat as shown in Fig. 187. Position assumed after exhaustive efforts to regain its feet. (Ann. Rep. U.S.A. Bur. An. Ind. 1902). 



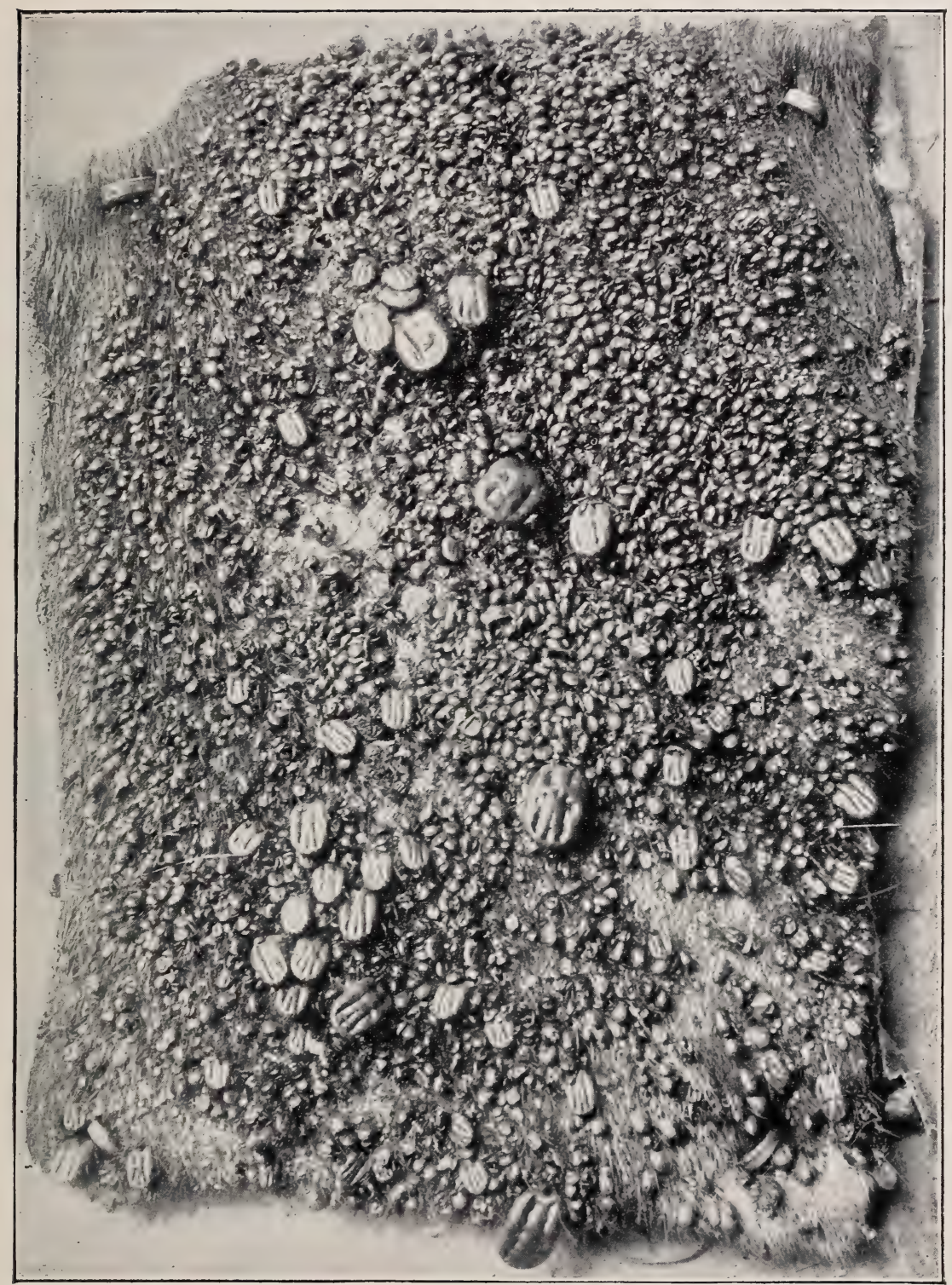

Fig. 189.-Portion of a steer's hide, showing the Texas fever tick (Boophilus annulatus) of the United States. Natural size. (Styles, Ann. Rep. Bur. An. Ind. 1900.) 



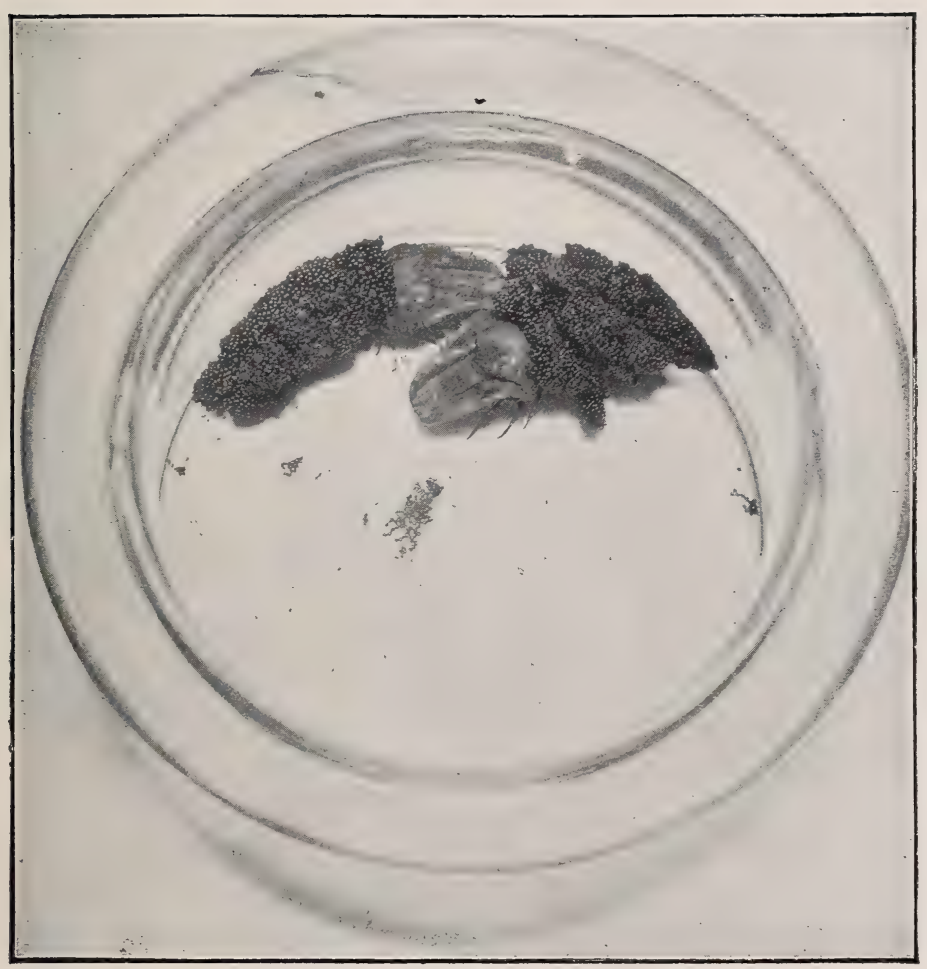

Fig. 190.-Two female ticks (Hyalomma agyptium) ovipositing. Natural size. (Photograph by J. E. Emery. Annual Report, U.S.A. Bureau of Animal Industry, 1900.) 

(Australia), tristeza (Argentine Republic), African coast fever, East Coast fever, redwater, Rhodesian fever (Cape), and bovine malaria.

It was first described by Babés in 1888, in animals inhabiting the Danube Valley, and was termed by him bacterial hæmoglobinuria of the ox. Afterwards it was well described by Smith and Kilborne (in 1889) under the title of Texas fever. It was re-discovered in Finland by Krogius and Van Hellens in 1894; in Sardinia by San Felice and Loi ; in Australia in 1895 by Pound; and it has been the object of remarkable investigations by Koch in South Africa (1898-1904).

Nicolle and Adil-Bey (1899) state that it exists in a latent form in European Turkey and Asiatic Turkey; and Lignières (1900), after a

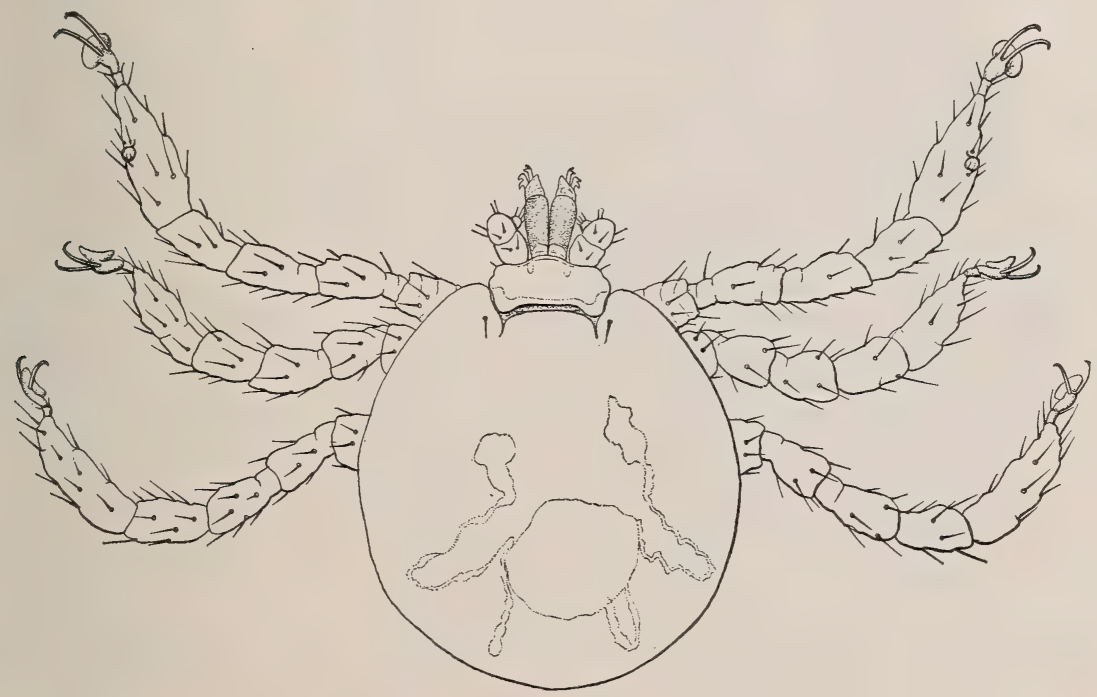

FIG. 191.-Dorsal view of larva of Boophilus annulatus of North America. Greatly enlarged. (Stiles, Ann. Rep., U.S.A. Bur. An. Ind., 1900, p. 388.)

series of researches in Argentina, suggests the final solution of the questions which it raises.

Symptoms. Babès describes it as an acute febrile disease, clinically distinguished by the passage of blood-stained urine. The urine is coloured by dissolved hæmoglobin; red blood corpuscles are not found. Smith and Kilborne, and afterwards Stiles, described two forms: an acute, rapidly fatal form, in which the Piroplasma bigeminum is found in the blood of the general circulation, in the spleen and kidneys; and a chronic form, in which, notwithstanding the absence of clinical signs, the parasites may be discovered in the blood under the form of diplococei.

Lignières describes a grave and a benignant form. The grave form D.C. 
is indicated by dulness, loss of appetite, and considerable fever. In twenty-four hours the temperature rises to $104^{\circ}$ or $105^{\circ}$ Fahr., and the pulse to 100 or 120 per minute, while the respiration is greatly accelerated.

The urine is of a light-red or brownish-red colour, resembling coffee-grounds, but it contains no blood corpuscles. The animals die in from three to eight days with symptoms of asphyxia; but this termination is not inevitable, and recovery may occur spontaneously. Improvement is indicated by a fall in temperature, disappearance of

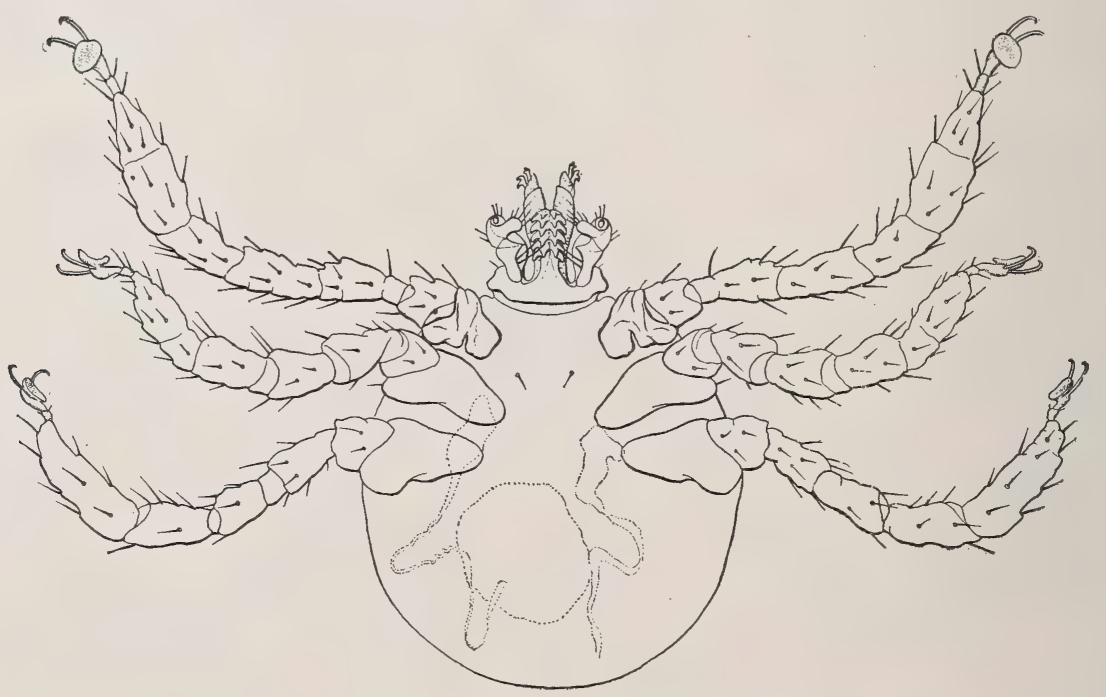

FIG. 192.-Ventral view of larva of Boophilus annulatus of North America. Greatly enlarged. (Stiles, Ann. Rep., U.S.A. Bur. An. Ind., 1900, p. 388.)

the blood-stained urine, and a return of appetite, together with marked thirst.

Drs. Smith and Kilborne describe the symptoms as follows:--."The beast when first observed to be amiss appears to be dull and sluggish, with a disinclination to move, and hence it is generally found apart from the rest of the herd. The hair stands erect like that of an animal on a cold day (a staring coat), the ears hang, and the eyes have a dull and lustreless appearance. In some cases the animals cease to feed, or ruminate, in others they continue to nibble at the herbage until nearly the last, but in a languid, indifferent manner, indicating that they have little relish for their food, and they fall off very rapidly in condition. There is generally a dribbling of saliva from the mouth, the muzzle may appear quite moist during the early stages of the disease, but it invariably becomes dry and crusty as the disease 
advances. Later on the animal manifests a strong reluctance to move, and when compelled to do so, it wallss with a dragging, straddling gait, as if weak across the loins. In severe cases, when the sick beast is left undisturbed, it will remain almost constantly in one place, standing with its head depressed and ears hanging in a drowsy semi-comatose condition, looking the very picture of complete nervous prostration. Other animals will lie down the greater part of the time and scarcely move, and when dead the limbs will be found in their natural position, and the head doubled round on the shoulder as if asleep. On making a post-mortem examination of some of these cases the carcase was found to be pale and bloodless, as if the animal had been bled to death.

"In other acute cases a twitching and quivering of the muscles will be observed, especially of those situated in the flank and behind the shoulder. The pulse and breathing are much quickened, and the animal will stand and grind its teeth and curl up its upper lip, indicating great uneasiness and pain. The fæces during the early stages of the disease are very often soft, with a tendency to diarrhœea, more

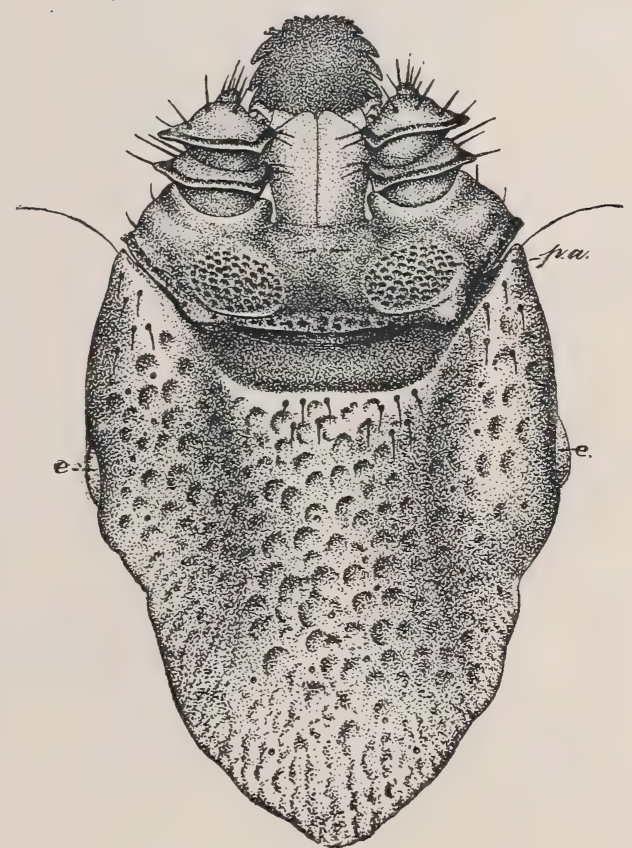

Fig. 193.-Scutum and scutellum of female Boophilus annulatus, showing mouth parts, porose areas $(p . a$.$) , and eyes (e)$. Greatly magnified. (Stiles, Ann. Rep., U.S.A. Bur. An. Ind., 1900, p. 392.) especially in transport oxen on the road, but they invariably become hard as the disease advances; but whether hard or soft, they have generally a brownish tinge, and often mixed more or less with blood and mucus. In some severe cases which recover, the favourable crisis is often ushered in by a salutary diarrhœa."

In the benign form the animal for the space of about a week shows indifference to its surroundings, loses its appetite, wastes, and, less frequently, has slight feverish symptoms, without discoloration of the urine. The only reason for the belief that this trifling 
disturbance is due to piroplasmosis is furnished by examination of the blood, in which the parasites may be found in very small numbers in certain blood corpuscles.

Calves seldom take the disease except in the benign form.

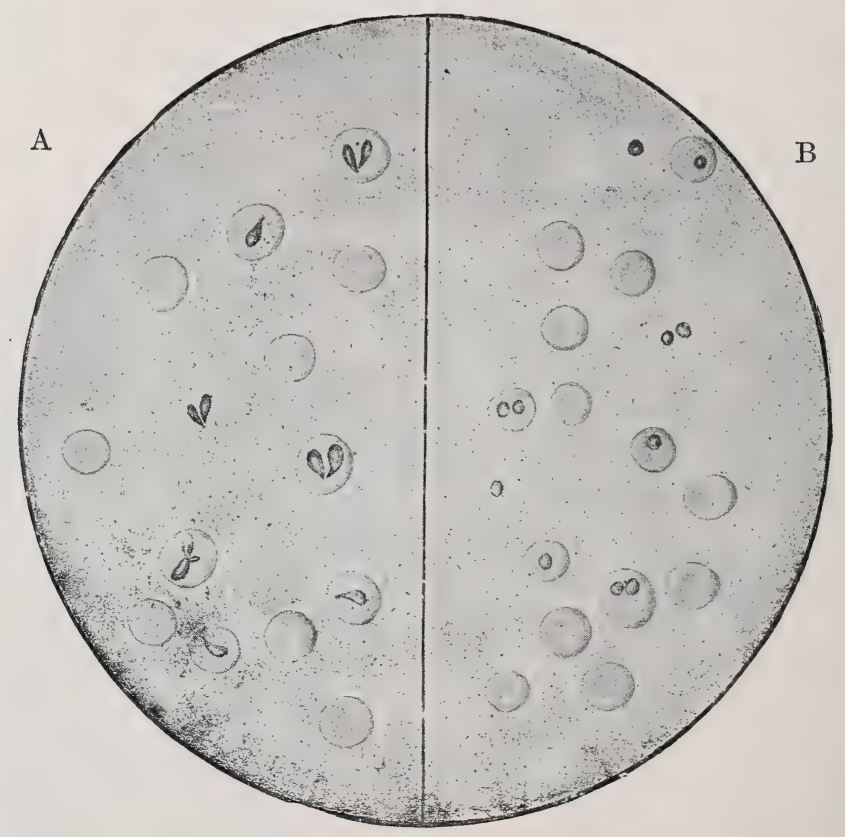

Fig. 194.-A, red blood corpuscles containing pear-shaped Piroplasma.bigeminum (the typical form); B, red blood corpuscles containing round Piroplasma bigeminum. (After Lignières.)

Lesions. At first glance the lesions appear to resemble those of anthrax, but may be differentiated from them in many details.

The skin is covered with ticks or shows traces of their punctures.
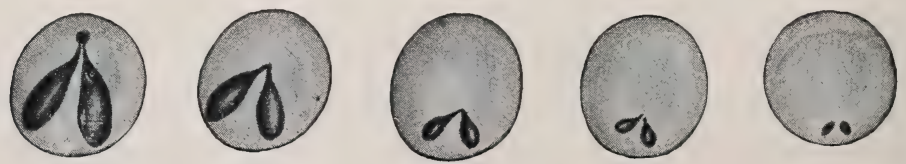

FIG. 195.

The myocardium appears as if boiled, the spleen is invariably hypertrophied and two or three times as large as in the normal state.

The kidneys are violet in colour and congested, and the adipose layer surrounding the kidney is infiltrated with a yellowish serosity. 
The urine may present a variety of tints, derived from hæmoglobin. The liver is often engorged with blood and the gall bladder always distended.

When convalescence sets in, icterus appears; but it is a special kind of icterus, depending on changes in the hæmoglobin-in fact, a hæmaphæic icterus. Histological examination of the blood furnishes the explanation of the disease by revealing the presence of the parasite.

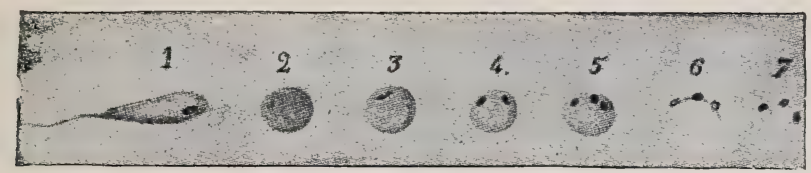

Frg. 196.-Slow formation of free germs. 1, Pear-shaped organisms with large chromatic element and a flagellum; 2, newly-developed round cell without chromatic element; 3,4 and 5 , formation of the chromatic elements ; 6 , the germs about to escape ; 7 , the germs freed. (After Lignières.)

Pathogeny. The parasite is the Piroplasma bigeminum, which is easily demonstrated by drying the blood, fixing it and staining with very weak methylene blue. The blood is light in colour and pale, and the serum is tinted by the dissolved hæmoglobin. The blood

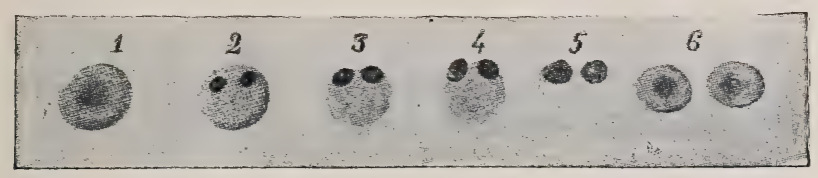

FIG. 197.- Kapid formation of free germs. 1, Newly-developed round parasite without distinct germs ; 2 , round parasite with two germs ; 3 , the germs approaching the surface and undergoing development; 4 , the germs beginning to grow outwards preparatory to forming new round parasites; 5 , the process previously noted is becoming more marked; the protoplasm of the cell is undergoing degeneration, and scarcely stains at all ; 6, round parasites. This completes the eycle. These parasites (6) are similar to (1). (After Lignières.)

corpuscles diminish in number with extreme rapidity while the parasites are developing, and in twenty-four or forty-eight hours may fall from some six millions and a half, the normal number, to one million or even to two or three hundred thousand red blood corpuscles per cubic millimètre.

This destruction is due to the action of the piroplasmata, as may be shown by staining with a 5 per cent. methylene blue or carbolised thionine. These parasites usually assume a simple pyriform shape, and two or three may be found in one blood corpuscle. The number 
of parasites and infected blood corpuscles is generally in direct ratio to the intensity of the infection. These parasites are found throughout the blood, but principally in that of the spleen, kidneys, and mesenteric veins. They are only abundant whilst the temperature is rising or at the moment when it reaches its highest point, and they often disappear before death or convalescence.

The pyriform shape is only temporary, and corresponds to the acute phase of the disease, but the parasite assumes the round form as soon as convalescence sets in. This round form gives birth to one, two or three spherical spores, which are set free in the plasma after the destruction of the maternal protoplasm, and are able after transference to a fresh red blood corpuscle to again assume the pyriform shape peculiar to the grave forms of disease.

The parasite can only be cultivated in defibrinated blood from a hæmoglobinuric subject, and the cultures do not always yield more than reproductions of the round form, the pear-shaped form only being produced with red blood corpuscles in the living animal body.

Regarding the method of growth of the piroplasma in the body and in cultures, Lignières believes that the parasite may produce two forms of spores differing in their nature. One, the active spore, has little resisting power. It soon degenerates outside the animal body, forms rapidly at the expense of one of the pear-shaped parasites, and may immediately reinfect another red blood corpuscle. The other, called the passive spore, is very resistant, and retains its vitality for a long time outside the body, being produced at the expense of spherical parasites already withdrawn and incapable of producing the disease.

The form of piroplasmosis at present under consideration is peculiar to the ox, and none of the other domestic animals or experimental subjects can be inoculated with it.

Subcutaneous or intravenous inoculation of the ox with 5 to 10 cubic centimètres always gives positive results when made with blood or active products, such as the pear-shaped parasites or active spores, but is ineffectual when the parasites have already begun to retract in order to form passive spores.

Calves seldom contract more than the benign form of the disease, and do not die.

In the grave form following experimental infection the temperature begins to rise between the third and sixth day, and corresponds with a marked increase in the number of parasites to be found within the red blood corpuscles. The urine at first becomes albuminous, then hæmoglobinuric, whilst the red blood corpuscles diminish in number to a very marked extent, falling from about six or seven millions to one million, or even a few hundred thousands, in the course of a few 
days. The temperature, which may previously have risen to above $105^{\circ}$ Fahr., suddenly falls, indicating the approach of death.

If an immediate autopsy is made, the spleen is always found to be enlarged, the intestinal mucous membrane reddish in tint or bloodstained, and the serous membranes, particularly the endocardium, covered with petechire.

Few or no parasites can be discovered except in the blood from the cardiac muscle and the kidneys.

The grave form may end in recovery. This end is indicated by the temperature remaining normal after defervescence, the appearance of hæmaphæic icterus of an obstinate character, and the progressive return of appetite.

The disease is usually transmitted by adult and larval ticks carrying: the parasite from infected animals. Lignières has proved that this transmission occurs through the medium of passive spores, which, though themselves incapable of producing the disease, become active and infective in consequence of the local irritation produced by the poisonous saliva of the ticks.

The pathogeny of Texas fever may be shortly summed up as follows:-Animals suffering from the disease carry in their blood a protozoan organism called the Piroplasma bigeminum, analogous to the parasite of human malaria; once introduced into the blood, this organism remains there in an active condition throughout the animal's life; it is transferred to susceptible cattle either within or without the infected district by the Southern (U.S.) cattle tick Boophilus anmulatus; Southern cattle, although carrying the protozoa, are harmless unless infested by this particular tick: the mature ticks and their eggs contain the protozoa, and the mystery of certain grounds over which infected animals have passed being first dangerous, then harmless, and again dangerous depends on- $(a)$ the infestation of the ground with mature infected ticks; $(b)$ the destruction or death of the mature ticks; and $(c)$ the hatching out of new (infected) ticks from the eggs laid on the ground by the mature female ticks.

Dr. Salmon states that in Texas a successful method of protection is in practice based on the observations that young cattle do not suffer so severely as adults, and that the disease always assumes a milder form in winter. Young animals introduced during the winter are inoculated with virulent blood. They contract a mild form of disease, and afterwards resist. In this way the losses, which previously amounted to 90 per cent. of all freshly introduced stock, have been reduced to about 10 per cent.

A remarkable and very interesting observation (if absolutely reliable) deserves to be mentioned, viz., that the ticks develop regularly in the 
natural prairie, but do not develop in parts artificially sown with grass such as lucerne, and that when contaminated or diseased animals are transferred to artificial meadows they do not convey the disease to other animals already there; the latter are proof against it.

Diagnosis. The disease is so typical that it cannot be mistaken for anthrax. In anthrax the urine is never hæmoglobinuric and very rarely hæmaturic, and the fæces are sometimes blood-stained, a symptom never present in piroplasmosis. Anthrax can be transmitted to experimental animals, but piroplasmosis cannot.

Prognosis. The prognosis is generally grave.

Treatment. Van Hellens recommends the use of quinine in large doses. He give 5 drachms in one dose, and repeats it for the next two, three, or four days.

Lignières says that he has never obtained the slightest success with quinine, though it is true he has never given higher doses than $2 \frac{1}{2}$ drachms.

Attempts have been made to confer immunity by injecting animals with serum from others which have recovered. Vaccination with the blood of patients arrived at the period of convalescence has also been tried. The results, however, have not been very satisfactory.

Lignières has formulated an efficient method of vaccination, of which he has not yet published the full details, but which appeared by reason of its simplicity likely to render great service. Nevertheless, his most recent reports seem to show that vaccination is not always efficacious, and that in the Argentine Republic alone several varieties of the disease exist, two being caused by allied but different parasites. The vaccine used against one variety is powerless against the other. The problem of vaccination would therefore appear to be much more complex than in the case where one form only occurs in any particular country.

The immunity arising from attacks of piroplasmosis is in direct ratio to the gravity of the disease, and according to Lignières' views this acquired immunity is due to the secretion by the piroplasma of a substance which is toxic for the red blood corpuscles. This toxic substance provokes, as in other diseases, an organic antitoxic reaction.

\section{BOVINE PIROPLASMOSIS IN FRANCE.}

Until recent years it did not seem that piroplasmosis occurred in France. It had been detected in Algeria, although its existence had not been conclusively proved. Mathis claims to have met with it in the department of the Loire in 1896 and in the Ain in consequence of the importation of Algerian cattle, but its ravages were comparatively trifling. 
Having good reason to suspect that certain morbid conditions, known as mal de Brou, might be due to piroplasmosis, Lignières endeavoured to verify his theory, and discovered that sometimes, but not often, this disease was mistaken in France for anthrax and mal de Brou. Piroplasmosis in France appears less grave than in America, and is rarely fatal

As regards its symptoms, it usually develops suddenly with fever, loss of appetite, acceleration of the pulse and respiratory movements, suppression of the milk secretion, and the passage of red hæmoglobinuric urine. In exceptional cases death may occur in from three to five days.

On post-mortem examination a varying number of ticks (Ixodes hexagonus) are found on the skin, the spleen is always increased in size, and the kianeys are black and hæmorrhagic.

The disease transmitted by ticks, as in Texas fever, seems due to the presence of a round parasite, different from the well-known Piroplasma bigeminum.

The elucidation of this disease, which occurs towards the northern frontier of France in the neighbourhood of Maubeuge, calls for further investigation. It never appears to be very fatal, and it attacks more especially animals imported into the infected region. A method of vaccination identical with that used by Lignières against one of the forms of the American disease may perhaps in the future prove available against the disease in France. Until then the best treatment would appear to consist in free subcutaneous injection of saline solution and the administration of evacuants, sulphate of quinine, and laxatives.

\section{OVINE PIROPLASMGSIS.}

Causation. The existence in France of this disease has not yet been clearly established, for in the only communication on the subject (by Leblane in 1899) the writer seems to have confused the toxic hæmoglobinuria produced by feeding on decomposed beet pulp with the parasitic hæmoglobinuria due to piroplasmosis.

In Italy ovine piroplasmosis was described by Bonomo in 1896 under the title of parasitic icteric hæmaturia of sheep. It is said to be due to a parasite of the red blood corpuscles (Amoba sporidium polyphagum), the said parasite being of oval form, very refractile, always occupying an outer position near the free margin of the corpuscle, and sometimes floating freely in the plasma.

According to Babès, who described it under the name of garceac du mouton, the same disease appears to occur in the islands and low parts of the Danube valley. 
Symptoms. The development of this parasite produces in the patient loss of appetite and high fever, accompanied by the passage of dark coloured hæmoglobinuric urine. Icterus is frequently present. The animals rapidly become exhausted, collapse and die.

On post-mortem examination carried out immediately after death the spleen is found to be large, the pulp being like wine lees. The liver is soft and yellowish; the kidneys are soft and black.

The disease is said not to be transmissible by direct transfusion (?).

It would appear that this disease has also been seen in Turkey by Nicolle and Laveran, near Constantinople, in 1899. The parasites (Piroplasma oris) are round or slightly elongated and occur near the periphery of the red blood corpuscles. It is to be hoped in the interest of breeders in localities where this disease rages that Lignières' method of vaccination against bovine piroplasmosis may prove reliable and equally applicable in the case of sheep.

\section{DISEASES PRODUCED BY TRYPANOSOMATA.*}

In 1904 Professor Koch delivered an address, from which the following is a summary, to the Berlin Medical Society regarding his experiences and observations on diseases produced by trypanosomata in Africa :-

A wide field of study has recently been opened by the discovery of various pathological protozoa. Three discoveries especially have directed attention to these special disease organisms-

(1.) Laveran's discoveries regarding malaria. Ross has shown that the malaria parasites are carried by mosquitoes (Anopheles claviger).

(2.) The discovery of the protozoa of Texas fever by Smith. In this case ticks (Rhipicephalus (boophilus) annulatus) convey the disease.

(3.) The discovery of the trypanosoma of the tsetse disease, which is conveyed by a stinging fly (Glossina morsitans).

These discoveries were followed by numerous others indicating protozoa as causes of disease.

The trypanosomata are morphologically distinguished by the existence of a flagellum. When fresh blood is examined it is scarcely possible to overlook the protozoa in the preparation, for attention is at once attracted by the energetic way in which the red blood corpuscles are continually being displaced. The peculiar form of the protozoa, however, can only be detected in stained preparations. Romanowsky's staining method is

* An interesting article and a series of figures on the "Evolution of the Trypanosoma Evansi" were published in the Jour. of Comp. Path. and Therap. for September, 1904, p. 210. The same number also contained articles on several piroplasmic diseases. 
probably the best. Trypanosomata stained by this method show a fishshaped body, the front end of which carries a flagellum. The body of the trypanosoma is coloured blue. At the anterior end may be seen a red-stained nucleus; at the opposite end a much smaller red spot, which has been termed the nucleolus, but is more properly described as the centrosome. From the centrosome a red thread extends along the outer margin of the body as far as the front extremity, where it becomes continuous with the flagellum.

The trypanosomata increase by longitudinal fission. The centrosome and then the nucleus divide, and finally a second flagellum is formed. Sometimes the young trypanosomata remain connected, producing the so-called "rosettes."

The disturbance produced by trypanosomata seldom becomes acute, but often continues for years. The only sign of disease consists in illdefined fever with long intermissions. The destruction of the red blood corpuscles causes anæmia, the animals or men become weak and waste away, œdema and sometimes erythema occur at varying points in the body, and occasionally the lymphatic glands become swollen.

The classical land of the tsetse disease is in the neighbourhood of the Zambesi. There it was seen and very well described by Livingstone; but, unfortunately, further investigations have shown that tsetse disease extends over the whole of Africa.

Whilst the trypanosomata of rats can only be conveyed to the one species, those of tsetse disease thrive in all mammals, particularly in the horse, mule, ox, dog, rat, and mouse.

The tsetse organism has been shown to kill both horses and mules, but to be less dangerous for oxen. A certain relative immunity exists in some races. As regards the ass, observers are not agreed; Koch failed to infect it. Sheep and goats are also but slightly susceptible. The conveyance of trypanosomata from the blood to uninfected animals occurs through the medium of a stinging fly (the Glossina morsitans).

Surra is endemic in the Philippines, Java, and the island of Mauritius. Koch regards the trypanosomata of surra as strictly analogous with the parasites of tsetse disease. Horses (and, in India, elephants) especially suffer from surra. Although the Glossina morsitans does not occur in India, other stinging flies replace it and convey the disease.

Another variety of trypanosomiasis is mal de caderas, seen in South America, particularly in Argentina and Brazil. It affects horses. According to Koch, the parasites of mal de caderas exactly resemble the tsetse and surra parasites. Other observers, however, declare that the mal de caderas parasites are distinguished from those before mentioned by their particularly small centrosome. Mal de caderas affects not only horses, but all the other animals which suffer from tsetse. 
Another variety of trypanosoma, the trypanosoma Theileri, is especially striking on account of its size. It is only found in oxen, and exhibits a very slight degree of virulence.

Koch divides trypanosomata into two great groups.

The grouping is based on three important peculiarities : firstly, the morphology of the parasite; secondly, its virulence; and, thirdly, its relation to the host.

The first group only exists in one species of animal. They have become so completely accustomed to this method of life that they cannot exist under other circumstances. Their virulence is slight but constant. This group comprises the trypanosoma of rats and the trypanosoma Theileri.

The second group (to which all other trypanosomata belong) shows great variation in virulence and in form. These trypanosomata are not peculiar to any one species, but may affect dogs, rats, horses, etc. Their morphological peculiarities also vary according to the animals in which they are found. Thus, the tsetse parasites when cultivated in the bodies of dogs and rats become much smaller than usual, and the centrosome appears near the end; when cultivated in horses the end appears pointed, and the centrosome lies near the centre; in the pig the parasites lose their peculiar short flagellum. Their virulence also varies within wide limits.

It has been found possible, as in the case of bacteria, to modify the virulence of trypanosomata by successive passages through different animals. By inoculating dogs with comparatively innocuous trypanosomata and conveying the disease from dog to dog the virulence is markedly increased. On the other hand, parasites which prove very virulent for oxen become much less active for these animals after passages through rats and dogs. This apparently trifling discovery laid the foundation for protective inoculation experiments. Parasites of the second group can also exist in the bodies of almost all mammals.

Koch is of opinion that the parasites of surra in India and of tsetse disease in Africa are absolutely identical. Laveran, on the other hand, states that he has protected animals against tsetse, and that they have nevertheless suffered from surra.

That this in no way disproves the identity of the two parasites is shown by other experiments.

Koch, whilst in Dar-es-Salaam, made some interesting experiments for the purpose of discovering a method of protective inoculation. $\mathrm{He}$ had found that the virulence of the ox parasites could be modified. $\mathrm{He}$ therefore inoculated oxen first with these weakened parasites and afterwards with others of high virulence. All the control animals died while those treated as above remained alive. 
Veterinary Surgeon Schmidt kept these animals under observation, and reinoculated them from time to time with highly virulent material, notwithstanding which they were still perfectly well six years after the first inoculation.

In practising this method, however, the trypanosomata used for the first inoculation must not be unduly weakened. The method would have appeared fully successful were it not for the fact that the protected and apparently quite vigorous animals still suffered from the presence of parasites in the blood. To extend its use, therefore, meant that one would not suppress, but would spread the disease. The effect would be to produce herds harbouring the parasite, which herds, though exhibiting no signs of illness, would nevertheless in a sense be propagating the active cause. Further observation has also shown that the protection so conferred is only relative. Dogs can always be infected with the blood of such animals. It has long been known in Africa that antelopes and buffaloes harbour trypanosomata in their blood without showing external signs of disease.

Another method of protection must therefore be sought, such as destroying the various stinging flies; but this offers little hope of success. Koch admits that he sees no method of dealing with them. The other method is directed against the parasite, and here he seems more hopeful. The disease can be rooted out by killing all diseased animals suspected of disease. The line of procedure is indicated by the experience gained in Mauritius and Java. When surra broke out in Mauritius almost all the oxen died in two years. In Java the nature of the disease was early recognised, and all suspected animals were at once slaughtered or isolated until slaughtered; in this way the disease was soon stamped out.

\section{LOUPING-ILL.}

The close analogy between the-convulsive form of the disease described as "trembling" (which disease is well known in France) and the condition known in Britain as louping-ill lead us to give here a short account of the latter condition. For a great part of what follows we are indebted to articles by Meek and Greig Smith, published in the Veterinarian, Vol. LXIX, Nos. 820 and 840.

Nature and Symptoms of the disease. The disease known usually as louping-ill or trembling has long been of annual and sometimes of biennial recurrence in certain parts of Great Britain. In these places sheep farmers look for the appearance about the middle of April, to its continuation during May, and to its gradual disappearance early in June. Lambs are most liable, but sheep are also quite susceptible to the disease, and in both the symptoms are the same. The disease under consideration 
is rendered quite distinct by certain well-known symptoms. Though these have been described in various ways, the disease can be recognised by the more or less complete paralysis of the body and limbs. Symptoms may succeed one another very rapidly, or may be spread over some length of time. The animal at first loses control over the muscles, which are seen to twitch convulsively. It may fall down and struggle on the ground, sometimes jumping up again, often to some height. Between the fits it is often seen to stand trembling. These symptoms are frequently accompanied by frothing at the mouth. Some such appearances are the usual onset to the disease, and are followed by a paralysis which usually affects the hind limbs, but may also include more or less of the body and the head and neck. The fore-limbs are often similarly paralysed. The affected limb or limbs become cold to the touch. The paralysis necessarily brings the animal to the ground, though it may be able to crawl about by the aid of the unaffected legs. When the head and neck are affected the former is usually drawn to one side, and the eyes often become oblique. Excitement is greatly increased when the animal is disturbed. The symptoms, then, in a few words are more or less complete paralysis, preceded as a rule by fits and trembling.

The small number which recover present "a wry neck, stiff joint, high back, or other deformity." During recovery swellings occur at the joints; these may be pierced with good results, giving a large discharge of pus. According to Fair, in the Veterinarian, Vol. VIII., "these abscesses usually appear in the neighbourhood of the joints, but sometimes above the arms, the brisket, or any neighbouring part of the body."

While the disease is characteristically a sheep ailment, other animals are also liable. Swine fed with the carcases or blood of sheep which have succumbed to louping-ill die with every characteristic of the disease in a short time. If the carcase has been boiled this does not occur. Swine will also frequently take the malady if allowed access to the grass of affected fields. Cattle are said to take the disease, and in the North Tyne district it is said that if a cow takes louping-ill, the milk will give the illness to a calf or lamb. One or two cases of horses being attacked are also reported.

Regarding the infectious character of louping-ill, the following is very well known. Sheep bred on diseased places are not nearly so liable to the disease as sheep which have been introduced from unaffected places. Louping-ill may be introduced into a new place, but in such cases, unless the importation from affected farms be continued, the malady may disappear.

Distribution of the disease. In Great Britain it is confined to the North Tyne district of Northumberland and to the contiguous border counties of Scotland, extending into Kirkeudbrightshire and certain 
valleys of Dumfriesshire. It is rare in Berwickshire, common in the north and west of Roxburghshire and the similar hilly districts of Selkirkshire and Peeblesshire. It occurs in Ayrshire, to a slight extent in Lanarkshire, and is found in the western parts and islands of Argyleshire and Inverness-shire.

Not only is the disease very circumscribed in its distribution as a whole, but locally in the places mentioned infected and non-infected spots are pointed out. These may be quite contiguous. The flocks in the North Tyne district feed up and down the hills in limited "cuts," and it is one of the features of the illness that certain "cuts" are very liable to it, while others, even on the same farm, are just as free. In many cases the nature of the pasture is such as to suggest to an experienced man the probability of its being subject to the disease. A dry and foggy pasture seems best suited for harbouring the cause of the malady. These infected places have remained wonderfully constant, but

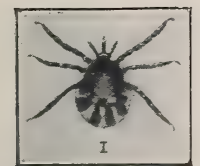

FIG. 198.-Larva of the grass tick.

Length, $\frac{1}{40}$ th of 1 inch.

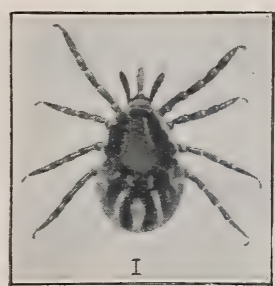

FIG. 199.-Pupa of the grass tick. Length, $\frac{1}{18}$ th of 1 inch.

a peculiar feature about them is that some may be very bad for loupingill one year, and others bad another year. Of two adjoining farms, one may be badly attacked and the other mildly, while in the following year the conditions may be reversed. Districts may present the same peculiarities. Thus, though the disease is essentially endemic, it is not absolutely constant in its recurrence. There seem to be certain circumstances capable of favouring or retarding it.

Lesions. The chief lesions are localised in the membranes of the brain and spinal cord, which are congested or inflamed, and contain an increased amount of cerebro-spinal fluid or a jelly-like, sometimes bloodstained exudation. Softening and hardening of the spinal cord have both been observed. Inflammation of the pleura and pericardium, with fluid or jelly-like exudation, are common ; lobar congestion of the lungs, endocarditis, gastritis, and enteritis have all been described; some observers have mentioned congestion of the kidneys and liver and swelling of the spleen. Lesions of the nerve-centres are the most constant and reliable. 
Etiology. Depressing and weakening influences of all kinds have been blamed for producing the disease, but the general consensus of opinion points in the direction of infection with micro-organisms carried and introduced into the sheep's system by the common sheep tick or "grass tick" (Ixodes redurius). The following remarks on, and illustrations of, this parasite are from an article by Mr. Wheeler, of Alnwick (Veterinarian, Vol. LXXIII., No. 867, p. 141).

Life History of the Grass Tick. Sheep ticks (which must not be confused with the sheep-ked, or keb, a wingless six-legged fly, universal on sheep everywhere) are allied to the spiders. They pass through four stages of existence: the egg-the six-legged larva-the eight-legged pupa-and, finally, the eight-legged adult male or female.

In each of the three stages of larva, pupa, and adult female, all

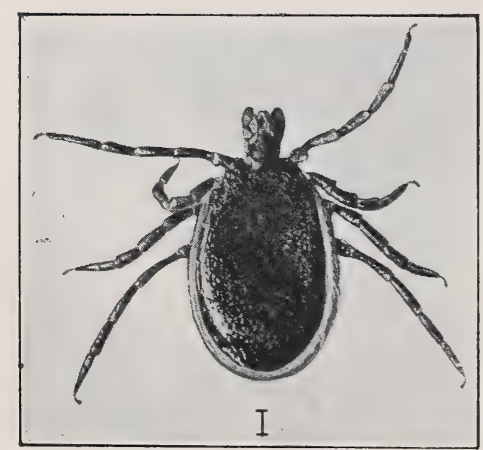

Fig. 200.-Adult male of the grass tick. Length, $\frac{1}{9}$ th of 1 inch.

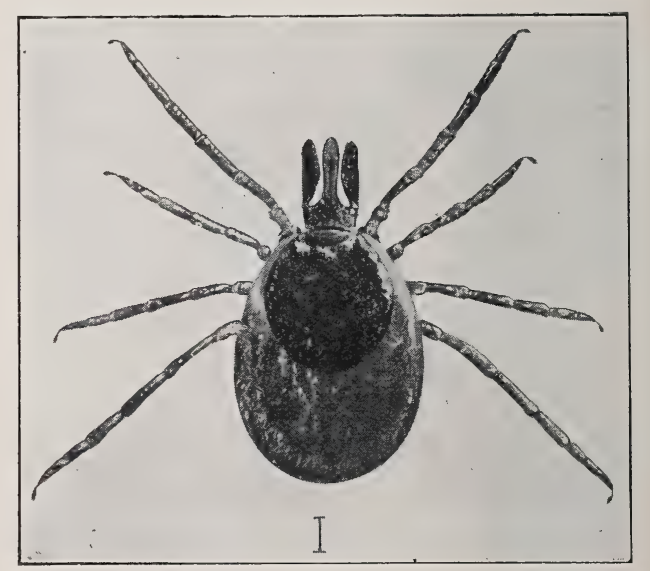

Fig. 201.-Adult female. Length, $\frac{1}{7}$ th of 1 inch.

species of ticks attack some "host" or animal, either beast, bird, or reptile, to which they attach themselves by the "rostrum" or beak, and become greatly distended by suction of the host's blood. When replete they fall to the ground-if a larva or pupa, in order to undergo its metamorphosis to the next stage of its existence, and afterwards seek a fresh host; if an adult female, to lay its eggs amongst herbage. The adult male is not capable of distension by suction, though it equally attaches itself to a host.

After undergoing metamorphoses, grass ticks, with the exception of males, are light in colour, soft and lethargic, and remain concealed for some time while recovering strength before seeking a fresh host.

Professor Neumann alludes to the fact that a fresh host is sought by ticks three several times during their existence. 
The Larya. When first hatched out from the eggs, which are supposed to be laid at the roots of coarse herbage, the young ticks are white and soft, but soon gain strength. Provided the weather is favourable, they climb up the stems, and, holding by their two posterior pairs of legs, await the passing of a host, employing their two front legs as insects use their antennæ.

In this, as in other "free living" stages of their existence, the young larvæ show great activity, attaching themselves and clinging tenaciously to any moving object. They appear to be more numerous on the rank rushes growing in damp, undrained places.

On finding a host, larvæ attach themselves by the rostrum, and remain there for about two days, by which time they are distended, black and globular. At this time they are easily detached from the host, and have lost their activity and clinging habits.

The Pupa. The possession of eight legs distinguishes the pupa easily from the larva. The extra pair are placed behind the others. After the metamorphosis, the pupa takes up its position on the stalks of herbage, just as the larva had done, for another chance of attachment to a host. But whereas adult grass ticks seem to confine themselves mostly to sheep, cattle, and deer, the larvæ and pupæ attach themselves very readily to various hosts, such as horses, dogs, and even human beings. After about four days the pupa is again replete with blood, black and opaque, and again drops to the

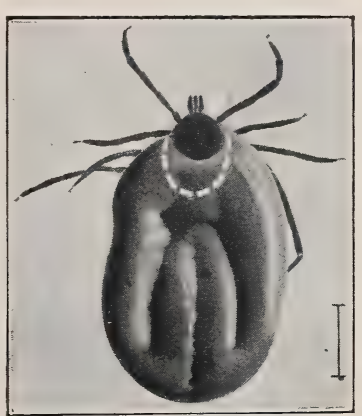

FIG. 202.-Partially distended female. The dotted white line represents the size of the tick before distension. ground to undergo its second and final change.

Adults. On reaching the adult age, both males and females again wait on herbage for a passing host. At this time, as well as after distension of the female on the host, an action which appears to be sexual intercourse freely takes place, even in confinement. On the host the females gradually distend (Fig. 202), and in the course of so doing vary much in colour and appearance. When fully replete, the female Ixodes reduvius becomes globular and black. One taken in this condition on April 15th commenced to lay on May 12th, and a few others taken at the same time commenced shortly afterwards.

Grass ticks never remain on the host to undergo metamorphosis or to lay eggs. They must therefore during their cycle of existence contrive to find a fresh host no fewer than three times.

In an article published in the Transactions of the Highland and Ag. Soc. for 1902 Mr. Wheeler draws attention to the close points of D.c. 
resemblance between louping-ill, Texas fever, tsetse fly disease, surra, heart-water, yellow fever, and malaria.

In the article previously referred to he summarises his conclusions as follows :-

One species only of tick, Ixodes reduvius, commonly known as the grass tick, has been found to carry the louping-ill bacillus to the sheep. It is easily recognised by the red body of the young females, the legs, shield, etc., being dark brown.

It lays its eggs, and undergoes its metamorphoses, in coarse herbage, and after each change seeks a fresh "host" on which to distend itself to a large size by suction of blood.

In all stages grass ticks abstain from all food except when on a host,

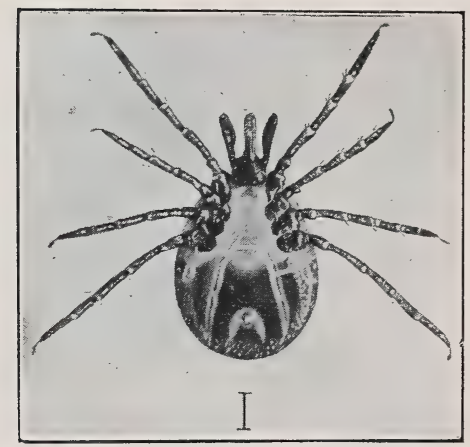

FIG. 203.-Female, under size.

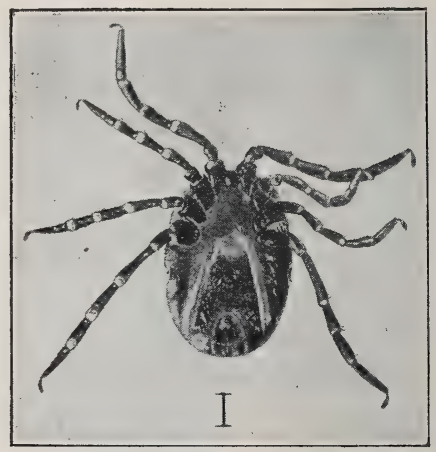

FIG. 204.-Headless female.

and they are endowed with extraordinary powers of fasting until a host is found.

Ticks soon die of drought where there is no good harbourage among rank vegetation.

Judging from analogy, it is probable-

That the bacillus can only be obtained from a diseased sheep, and inserted by the tick into another sheep.

That ticks convey the bacillus through their eggs to their offspring, as well as retain it through their metamorphoses.

That there is no danger in removing sheep from foul ground to cultivated lowlands, but that the disease is easily imported from one hill farm to another.

Strong and fat animals are nearly as susceptible to attack as weakly ones.

If the land is once free of disease, it can only be re-imported by diseased sheep, or ticks taken from them. 
SUGGESTED MEASURES FOR PREVENTION.

Burning and cutting of long grasses, bracken, rushes, etc.

Salt and sulphur given to the sheep.

Inoculation.

Removal of all diseased sheep to a separate inclosure, where handpicking and dipping are carefully attended to, the pasture is kept short, and damp places are drained. The sheep to be confined to this inclosure so long as the tick season lasts.

Immediate slaughter and burial of all affected sheep.

\section{BRAXY.}

[The following is a very condensed account of a paper published by C. O. Jensen on the above disease. It first appeared in English in the Veterinarian, Vol. LXIX., No. 825, p. 621, along with the original illustrations.]

The name Braxy is applied to a disease in some respects resembling anthrax, which appears as an epizootic, and is best known in Iceland, the Faroe Islands, and parts of Norway, though it also occurs in Scotland and Cornwall. Krabbe describes the disease as infectious, very acute in its course, and as proving fatal within a few hours of the appearance of certain characteristic swellings about the posterior parts of the body. Post-mortem reveals extensive dark purplish staining of the abomasum and distension of the digestive canal with gas, while decomposition of the cadaver occurs with excessive rapidity, the liver and kidneys undergoing softening, the skin assuming a bluish tint, the wool becoming loose, and the entire carcase giving off a most offensive stench. Krabbe states that the disease was regarded as a form of anthrax-a view, however, in which he does not coincide. Somewhat later Messrs. J. Sigurosson, S, Jōnsson, and Einarsson, all natives of Iceland, and the Norwegian State Veterinary Surgeon, Ivar Nielsen, carefully described the disease, throwing considerable light both on the conditions in which it appears and on its etiology.

According to them, braxy is an acute, or even exceedingly acute, infectious disorder, which begins as a hæmorrhagic inflammation of the mucous membrane of the abomasum, is accompanied by excessive development of gas in the digestive canal, especially in the stomachs, and proves fatal in some cases by a kind of general infection, in others by a specific intoxication, or by dyspnœa due to tympanites.

Braxy commits its chief ravages during the winter months: appearing first in autumn, the cases increase as winter approaches, to diminish again in spring; in summer they are exceedingly rare. This fact explains why 
the disease was so long regarded as due to climatic influences. Even at the present day, when it is known to be due to a specific organism, the action of temperature, etc., must still be regarded as probably playing an important part in infection. The disease is said not to occur in mild weather; but whether or not this be true, every one is agreed that it is principally seen during frost, especially when frost is unaccompanied by snow.

From experience gained both in Iceland and Norway, the disease appears to be often localised in certain districts and fields-a fact largely accounted for when we learn that up to the present little or no attempt has been made to prevent the spread of infection from the dead bodies.

Braxy chiefly attacks young animals, and is rare in those over three years of age. Hjaltelin estimates the number of deaths in a single district during the years $1849-1854$ at approximately 6,000, made up as follows:-

$\begin{array}{llllllllr}\text { Yearling lambs } & \ldots & \ldots & \ldots & \ldots & \ldots & \ldots & \ldots & 2,440 \\ \text { Two-year sheep } & \ldots & \ldots & \ldots & \ldots & \ldots & \ldots & \ldots & 2,460 \\ \text { Three-year sheep } & . & \ldots & \ldots & \ldots & \ldots & \ldots & \ldots & 1,020 \\ \text { Animals older than three years } & . & \ldots & \ldots & \ldots & \ldots & 80\end{array}$

The younger animals suffer most, and in Norway Nielsen directs attention to the heavy fatalities amongst lambs.

Symptoms. The sheep suddenly appears ill, is dull, lies about, and cannot be induced to rise; all movement seems to give pain, and from time to time the animal groans; the posterior parts of the body become swollen, and a little froth often escapes from the mouth. The pulse varies between thirty and thirty-five per minute, and is often imperceptible in the extremities; the temperature may rise to $105^{\circ}$ or even $108^{\circ} \mathrm{Fahr}$. This condition may last some hours, and always ends with the animal's death; sheep, which overnight had shown no signs of illness, are often found dead in the morning. The incubation period is from forty-eight to sixty hours, but ordinary cases seldom live longer than from five to eight hours after the symptoms declare themselves.

The striking post-mortem appearances, especially the hæmorrhagic inflammation of the abomasum, were early the subject of remark. This appearance is very characteristic.

If the animals are slaughtered, the most important change is found to be a purplish, dark, somewhat swollen patch in the abomasum; during the course of the disease this increases in size, and if the animal should be allowed to die of braxy the entire abomasum shows hæmorrhagic or sero-hæmorrhagic infiltration; the abomasum and the first part of the small intestine usually contain no food, but may often show a certain amount of bloody fluid. This hæmorrhagic inflammation may extend in 
a forward direction, implicating the other stomachs, or backward, invading the small or both small and large intestines. The other parts of the intestinal canal are congested. The pleural and peritoneal cavities contain a little serous fluid. The blood is dark in colour, but may be clotted; the spleen is at times somewhat swollen, at others normal. The liver is usually light-coloured, soft, and degenerated; occasionally this degenerative process is extremely marked, but due allowance should always be made for post-mortem change. The kidneys may appear degenerated; in many cases they are enlarged and soft, or almost fluid

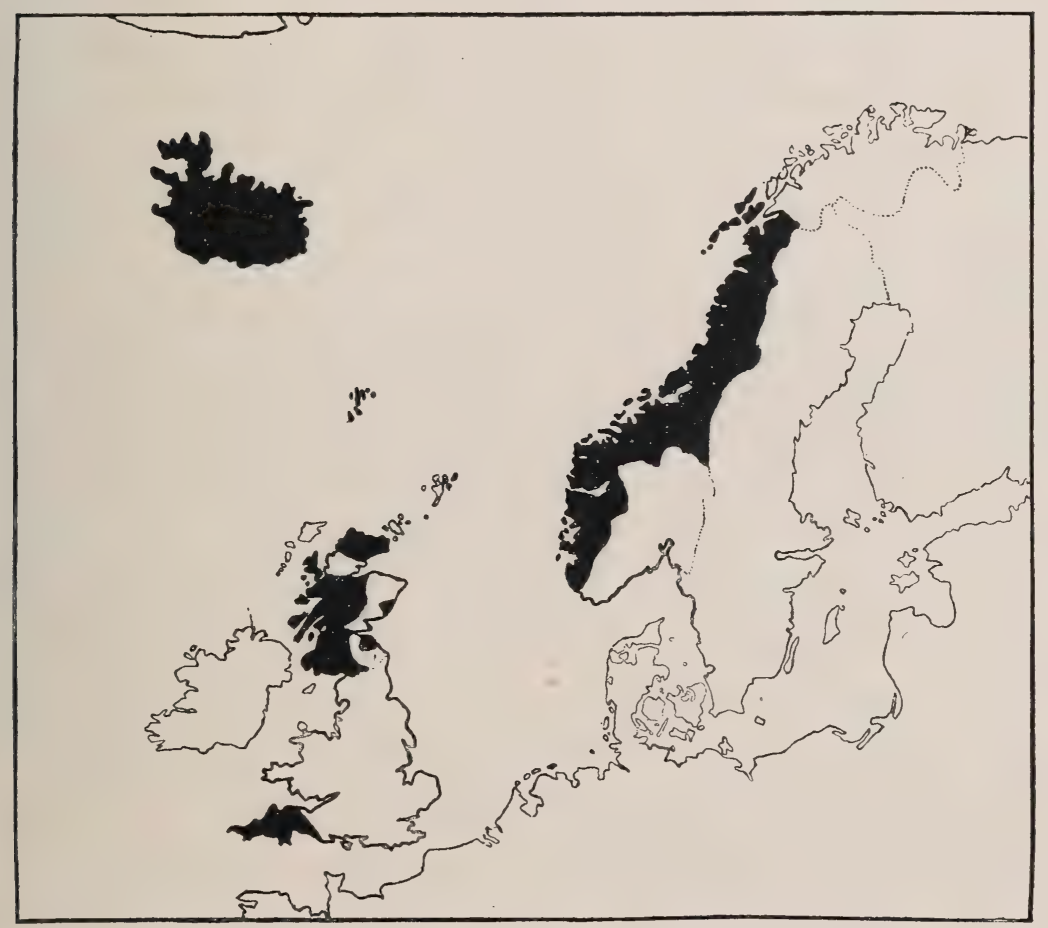

FIG. 205.-The shaded areas of the above map indicate the distribution of braxy.

in consistence. The carcase decomposes very rapidly; within a short time of death the belly is distended with gas, the rectum protrudes at the anus; the skin assumes a bluish colour in places, and the wool falls out; sometimes the skin bursts, revealing the presence in the subcutaneous tissue of a sero-hæmorrhagic fluid.

Braxy is, then, a primary violent hæmorrhagic inflammation of the abomasum, with or without secondary general infection.

From careful study it seems quite certain that the Scottish "braxy is identical with the Norwegian and Icelandic "bradsot"; it appears at 
the same season, and is intimately connected with climatic influences; it runs its course so rapidly that animals left healthy at night are found dead in the morning; and the pathological anatomy of braxy is the same as that of "bradsot."

To Ivar Nielsen, of Bergen, must be ascribed the honour of elucidating the etiology of braxy. During the course of investigations, published in 1888 , he found, partly in the local lesions of the intestinal track, partly in the capillaries of the internal organs, a special bacillus, easy to distinguish from that of anthrax, of which he gives the following description:

"The bacilli (B. gastromycocis-ovis) are oval, of a length varying from 2 to 6 micromillimètres, and a thickness of one micromillimètre. They are often in pairs, arranged in a straight line or meeting at an angle; in the former case, and especially if deeply stained, the pair may present the appearance of a single bacillus. Occasionally they form long chains. Near the centre of the bacillus, but not always centrally placed, may often be found a zone measuring more than half the total length of the bacillus, and exhibiting little or no colouration. It appears as though the stained portions gradually contracted, finally forming two deeply coloured masses at the poles of the lemon-shaped bacillus, which then somewhat resembles the bacillus of rabbit septicæmia, except that the unstained part of the braxy bacillus is larger and more rounded, appearing to be bulged out laterally. In dry preparations the bacillus is easily recognised on account of the highly refractile character of the colourless portion; but in sections careful search is often required, especially if the section be somewhat thick. Whether the colourless portion represents a spore cannot at present be said, though such appears probable. The bacillus is always found in the mucous membrane of the abomasum, and especially in the submucous and subserous connective tissue. In the other organs the bacillus may be present in considerable numbers, or, on the other hand, may be impossible to detect."

The same bacillus has been found in the tissues of affected sheep both in Norway and in Iceland; the bacillus, when subcutaneously injected, produces a violent hæmorrhagic inflammation of the same character as one finds in the abomasum in cases of spontaneous braxy, and the local changes at the point of inoculation may, just as in spontaneous braxy, be accompanied by a general infection with degeneration of different organs, and with softening of the kidney substance.

The bacillus of braxy is anaërobic. In cultures it develops considerable quantities of gas, just as it does when inoculated into the tissues. It is closely related to the bacillus of symptomatic anthrax, which it somewhat resembles in general appearance, and of which it reminds one by its ability to produce hæmorrhagic inflammation in the muscular tissues. It is distinguished from the last named, however, by being pathogenic to 
swine, mice, pigeons, and poultry, which are not killed by the bacillus of symptomatic anthrax.

The bacilli of braxy, malignant œdema, symptomatic anthrax, together with Ivar Nielsen's shortly described bacillus of whale's septicæmia, and Thoma's bacillus of malignant emphysema (found in extensive subcutaneous inflammation and emphysema in man), and certain others less well known, form a group of closely allied bacilli resembling one another in form, in being anaërobic, and in producing a sero-hæmorrhagic inflammation and emphysema, but differing in the manner of producing their effects.

Experience and analogy both seem to indicate that young animals occasionally suffer from mild attacks of braxy from which they recover. Such animals afterwards exhibit a well-marked immunity against the disease.

Ivar Nielsen attempted to vaccinate against braxy by a method resembling that used in black-quarter. He dried the diseased kidney tissue, and injected subcutaneously small quantities of the material thus obtained suspended in water. A slight local inflammation followed, which appeared to protect against later "spontaneous" infection. He has used this method in his own district, and states that it is also practised to some extent in Iceland. As far as one can judge-and of course a just opinion is very difficult to form-these inoculations appear of value.

The result of experiment, considered in conjunction with the good results of inoculation for black-quarter, would seem to indicate that Nielsen's method of vaccination against braxy may yet prove of the greatest possible value, although the method will doubtless require modification in its details.

These modifications Jensen enumerates at some length.

(Mr. Dollar has been informed that Professor Hamilton and Dr. McCall have been engaged in an investigation regarding the possibility of conferring immunity against braxy, and that a Government report will be issued on the subject. Up to the present time however-April, 1905-he has not been able to obtain this report or any advance proof sheets of it.)

\section{BILHARZIOSIS IN CATTLE AND SHEEP.}

This disease is caused by the bovine blood fluke (Schistosoma bovis of cattle and sheep. Synonyms: Bilharzia bovis; Bilharzia crassa; Gynacophorus crassus; Gynacophorus boris; Bilharzia hematobia crassa; Schistosomum bovis.

Geographical Distribution. Egypt, Italy, Sicily, India (?).

This parasite was discovered by Sonsino (1876) in Egypt in the portal veins of the ox, and later he found it in sheep, while Grassi 
and Rovelli afterwards found it in about 75 per cent. of the sheep slaughtered at Catania, Sicily.

Source of Infection. Clinical observation and analogy point to unfiltered drinking water as the source of infection.

Position of the Parasite. The worms are found in the veins of the abdomen, the vena porta, vena linealis, vena renalis, and the venous plexus of the bladder and of the rectum.

Symptoms. The young parasites appear to do no injury; in fact, even the adult worms seem to be inoffensive in themselves. The eggs, on the other hand, armed with a sharp point, are the exciting cause of the disease. The position of the parasite in the venous system, and

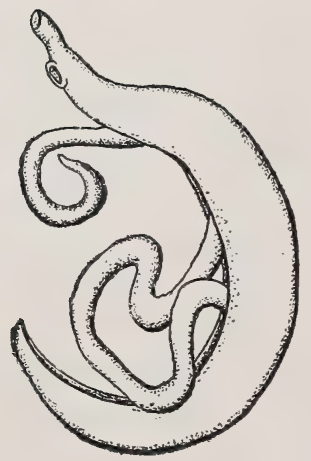

FIG. 206.-The bovine blood fluke (Schistosomabovis), male and female. $\times$ 9. (After Leuckart, 1894, p. 467, Fig. 204 A.)

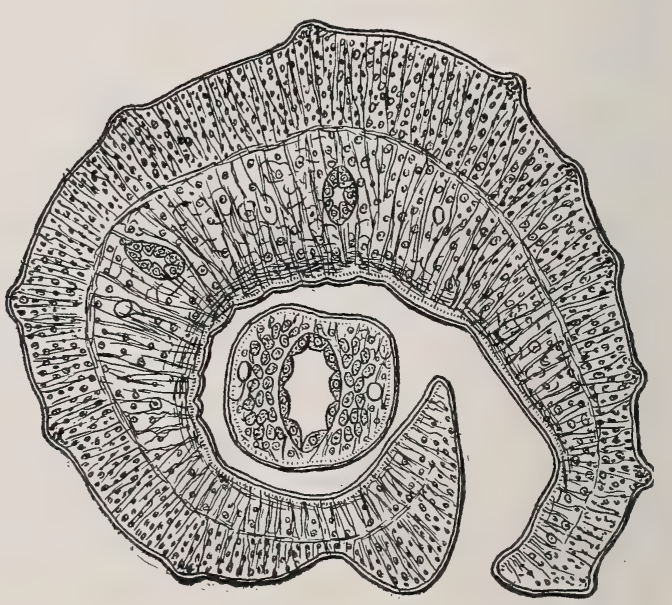

Fig. 207.-Cross section of bovine blood fluke (Schistosoma bovis), showing the position of the female in the gynæcophoric canal. $\times 200$. (After Leuckart, 1894, p. 472, Fig. 209.)

the consequent location of the agglomeration of eggs, determine the particular symptoms. Either the genito-urinary system is attacked, in which case hæmaturia is one of the first symptoms, or the large intestine is attacked and blood is noticed in the fæces.

If the parasites are lodged in the venous plexus of the genito-urinary system, the chief symptoms are : hæmaturia, pains in the lumbar region, the left iliac fossa, the thigh, or in the vulva, which may be spontaneous or may accompany micturition ; cystitis, vesical calculus, urinary fistulæ, vaginal verminous tumours, nephritis.

The eggs accumulate in the capillaries, which they rupture; they traverse the mucosa and fall into the bladder, thus causing more or less hæmorrhage; in this way the hamaturia is established, which is 
often the initial symptom. At first the urine is quite bloody, but it gradually becomes clearer, and it is only at the end of micturition that muco-purulent flakes are expelled, in which numerous eggs and even embryos are found; the urine contains also epithelial cells, more or less pus, eggs, and occasionally embryos. On micturition sharp pains are felt at the base of the penis or at the gland, possibly due to the passage of eggs. The passage of eggs through the walls of the bladder gives rise to cystitis; blood becomes more abundant in the urine after fatigue or coitus; clots may form and cause retention of urine; chronic urethritis may develop, evidently due to the presence of the eggs. In Egypt 80 per cent. of the cases of vesical calculus in man coincide with bilharziosis ; the formation of the calculi evidently results from the presence of the eggs, for the central nodule always contains one or more of these structures. Urinary fistulæ, opening on the perineum, more rarely into the rectum, occasionally form. The mucosa of the vagina, also of the uterus and bladder, becomes impregnated with calcareous salts. Nephritis develops in grave cases.

If the parasites lodge in the veins of the rectum the lesions caused are analogous to those described for the genito-urinary tract.

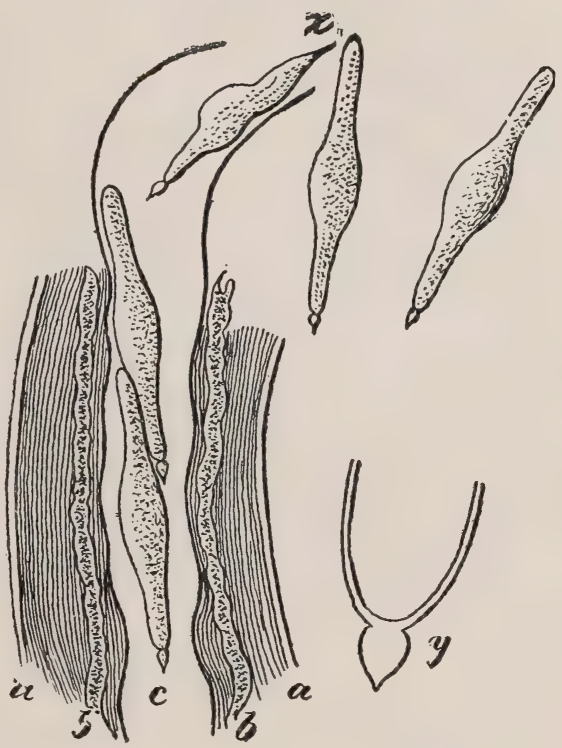

FIG. 208.-Eggs of bovine blood fluke (Schistosoma bovis), showing the peculiar process on the end. $a, b$, Layers of the oviduct; $c$, eggs in the oviduct $\times 180$; $x$, eggs deformed by pressure; $y$, spinous process on end of egg $\times 700$. (After Sonsino.)

The heart, lungs, and liver generally remain normal.

Pathology. The bladder is reduced in size, while its wall is greatly thickened, due chiefly to hypertrophy of the muscularis; the mucosa is also thickened, and at certain points it is indurated by uric or calcareous deposits, but the principal lesion consists in ulcerations covered with sanious pus. Lesions analogous to those of the bladder are also observed in the lower third of the ureters, and may extend as high as the kidney; the ureter is enlarged and tortuous; the mucosa irregular; its lumen may remain nearly normal in size, but its wall becomes very thick: the flow of urine may be obstructed; in short, a veritable 
hydro-nephrosis obtains, which results in atrophic lesions of the kidney, and may finally end fatally.

The mesenteric lymphatic glands may hypertrophy, their substance becoming tumefied, presenting small hæmorrhagic centres, and containing eggs. The liver may contain eggs and become somewhat cirrhotic; the eggs accumulate in the branches of the portal veins, or after piercing the walls they lie in the hepatic parenchyma. The lungs may also contain eggs.

Diagnosis. The diagnosis may easily be made by a microscopic examination of the urine to determine the presence of the egg.

Prognosis, etc. The severity of the disease varies directly with the number of parasites (and hence the number of eggs) in the body. Fortunately, in the majority of cases, the number of parasites is small, though it may increase from repeated infections to 500 or more. In cases of comparatively light infection the disease is reduced to a slight chronic cystitis, with now and then exacerbations, in course of which a slight amount of blood and pus is passed in the urine. The disease may last for years without apparent increase. In the most severe cases death may occur from various causes, rupture of the bladder, ascending pyelo-nephritis, uræmia, albuminuria ; the patient may die in marasmus, being exhausted by the dysentery or the anæmia.

Bilharziosis is accordingly not such a fatal disease as has sometimes been supposed.

\section{HEAT STROKE-OVER-EXERTION.}

In oxen and sheep heat stroke is rare as a primary accident, but it is frequently produced by over-exertion resulting from the combined action of the sun's rays, heat, and fatigue due to work or travelling.

It is commonest during the hottest months of the year in oxen doing hard work or in flocks which have been travelled considerable distances. It may also be seen during cooler seasons as the result of exceptional fatigue.

The disease results from a general intoxication which reacts most markedly on the cerebro-spinal centres. It is in fact a complex intoxication resulting from failure of the natural excretory organs to perform their function completely, and from excessive central heat acting on the nervous centres.

Fat animals out of condition are more readily attacked than working animals or sheep reared in the open air.

The symptoms are very characteristic. Oxen when attacked first of all show extremely rapid respiration and dyspnœa, announcing progressive asphyxia. They move with the nostrils dilated, the eyes prominent and injected, the mouth open and the tongue lolling out. 
Then all of a sudden they come to a stop beside a wall, or, if at liberty, in a ditch, and refuse to move. They may die rapidly with symptoms of asphyxia if they are forced to move until completely exhausted. In others, after a rest of several hours, the breathing becomes slower, the anxiety less and normal conditions return.

In sheep the same general signs may be seen: panting respiration, cyanosed mucous membranes and extreme anxiety, while death follows rapidly in the same way, with symptoms of asphyxia.

The diagnosis is extremely easy. The prognosis is grave.

Treatment consists in prompt and free bleeding to prevent pulmonary congestion. The animals should be rested in a shady, sheltered spot. They should have cool drinks and be sprinkled over the head, neck, or entire surface of the body with cold water.

To prevent such attacks, fat animals should not be moved for long distances, or during the hottest hours of the day, while difficult and prolonged exertion should be avoided. 


\section{CHAPTER VI.}

\section{DISEASES OF THE LYMPHATIC SYSTEM.}

Diseases of the lymphatics are numerous, highly important, and still imperfectly understood. They follow various accidents, local inflammations, certain specific diseases, such as tuberculosis, and may occur in an isolated form without involving any other part of the body.

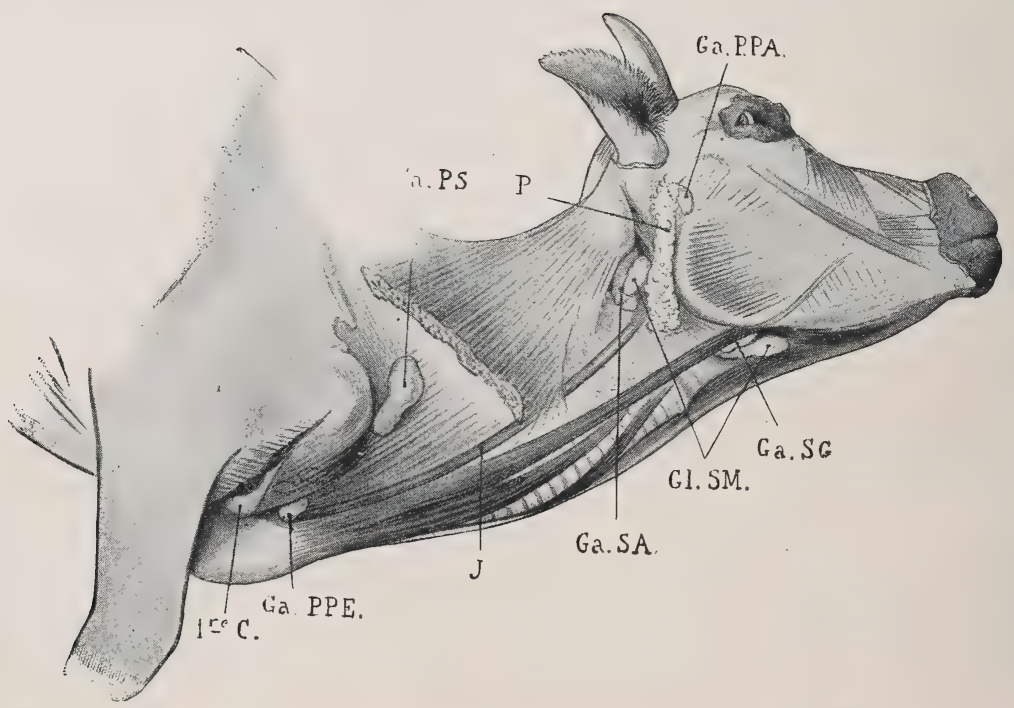

Fig. 209.- Superficial lymphatic glands of the head and neck. P, parotid gland; Gl.SM, submaxillary gland; GaSG, subglossal gland; GaPPA, preparotid gland; GaSA, subatloid gland; GaPS, prescapular gland; GaPPE, prepectoral gland; $\mathrm{J}$, jugular; $1^{\text {re }} \mathrm{C}$, first rib.

Inflammation, usually of infectious origin, may attack lymphatic vessels (lymphangitis) or lymphatic glands (adenitis), giving rise either to simple lymphangitis, suppurative lymphangitis, or again to simple or suppurative adenitis.

It is unnecessary to emphasise this point in general surgical pathology, for it is identical with that which is observed in other domestic animals, but in order properly to detect the glandular symptoms in certain 
diseases peculiar to the lymphatic apparatus, and in certain specific diseases, such as tuberculosis, farcy of the ox, etc., it is necessary to understand thoroughly the topography of the lymphatic system.

Topography of the lymphatic glandular apparatus : Examination. The lymphatic glands are in some cases superficial, in others deep seated, and are arranged symmetrically on either side of the body.

Beginning with the head and fore quarters, the lymphatic apparatus

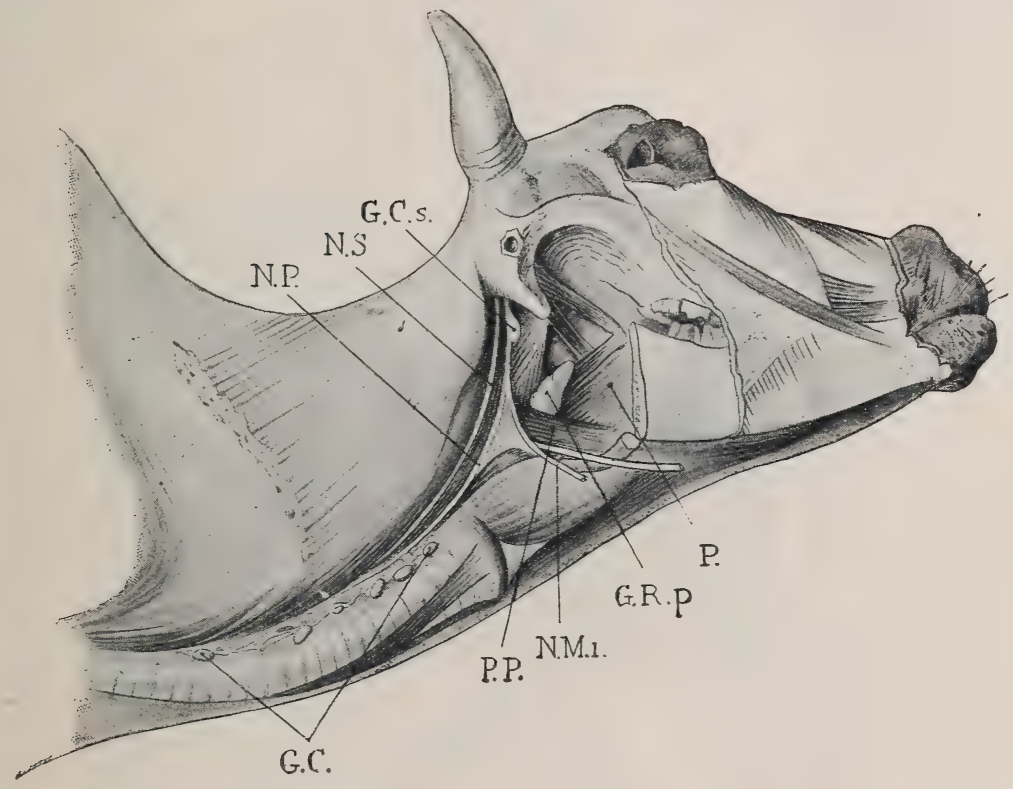

FIG. 210.-Deep-seated lymphatic glands of the head and neck. The posterior portion of the lower jaw removed. P, pharynx; GRp, retro-pharyngeal gland; GC, deep cervical glands (cervical chain); NS, spinal nerve; NP, pneumo-gastric nerve; $\mathrm{GC} s$, superior cervical nerve ganglion; $\mathrm{NM} i$, inferior maxillary nerve.

comprises a subglossal, a preparotid, a subatloid, a prescapular and several prepectoral glands (Fig. 209).

None of these glands are very deeply placed, and all are easily accessible to palpation, provided their exact position is known and the animals are not too fat.

The subatloid is a little more difficult to detect, but in thin animals the tips of the fingers can easily be passed under the wing of the atlas so that the condition of the gland can be examined.

In a normal condition, any gland on being examined conveys a sensation of softness and elasticity of a special character which never varies. Palpation is painless.

When, however, the gland is diseased, palpation causes pain in the case of all acute affections. It may, indeed, be impossible to reach 
the glands, as they are buried sometimes in œedematous swellings of varying size. On the other hand, they may be painless on being touched, but swollen, hypertrophied, indurated, hardened or caseous.

The deep-seated glands of the fore portion of the body comprise the retro-pharyngeal and the cervical chain running along the posterior border of the trachea. Normally these glands cannot be examined (Fig. 210); but when the seat of certain morbid processes, they may be so enlarged as even to be readily visible. The larynx and pharynx are then displaced downwards, the depression between the head and

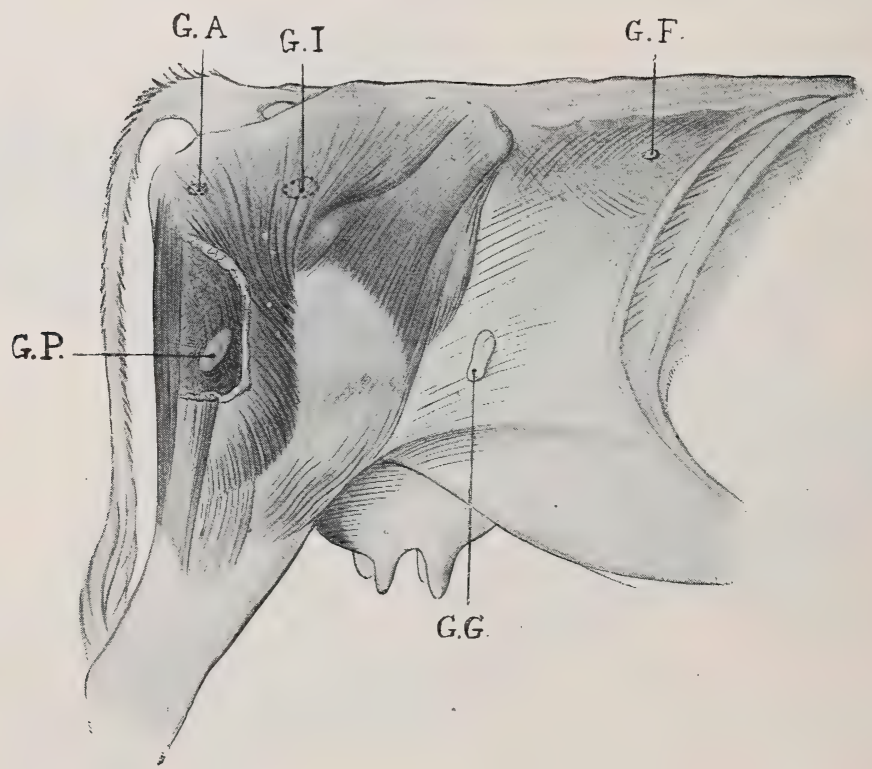

FIG. 211.-Lymphatic glands of the hind quarter. GG, Precrural lymphatic gland; GF, lumbar lymphatic; GP, popliteal lymphatic (deep-seated); GI, ischiatic lymphatic (deep-seated); GA, anal lymphatic (deep-seated).

upper extremity of the neck disappears, together with the depression known as the jugular furrow. Such deformities may be either perfectly symmetrical, as in lymphadenia, or (as is more commonly the case) asymmetrical, as in tuberculosis; and if inspection leaves any doubt, the glands may be further examined by palpation.

To obtain the fullest information both hands should be used, one arm being passed over the neck and the fingers engaged behind the trachea. The operator may also stand in front of the animal, whose head should be lifted so that the points of the fingers can be thrust deeply inwards on either side of the trachea in the direction of the vertebral column. 
In the posterior portion of the body the number of glands that can be examined is much smaller. The gland of the stifle, also called "gland of the flank," is, so to speak, the only one which can readily be detected by examination or palpation. Nevertheless, in cases of lymphadenitis, tuberculosis of the glands, etc., it becomes easy to

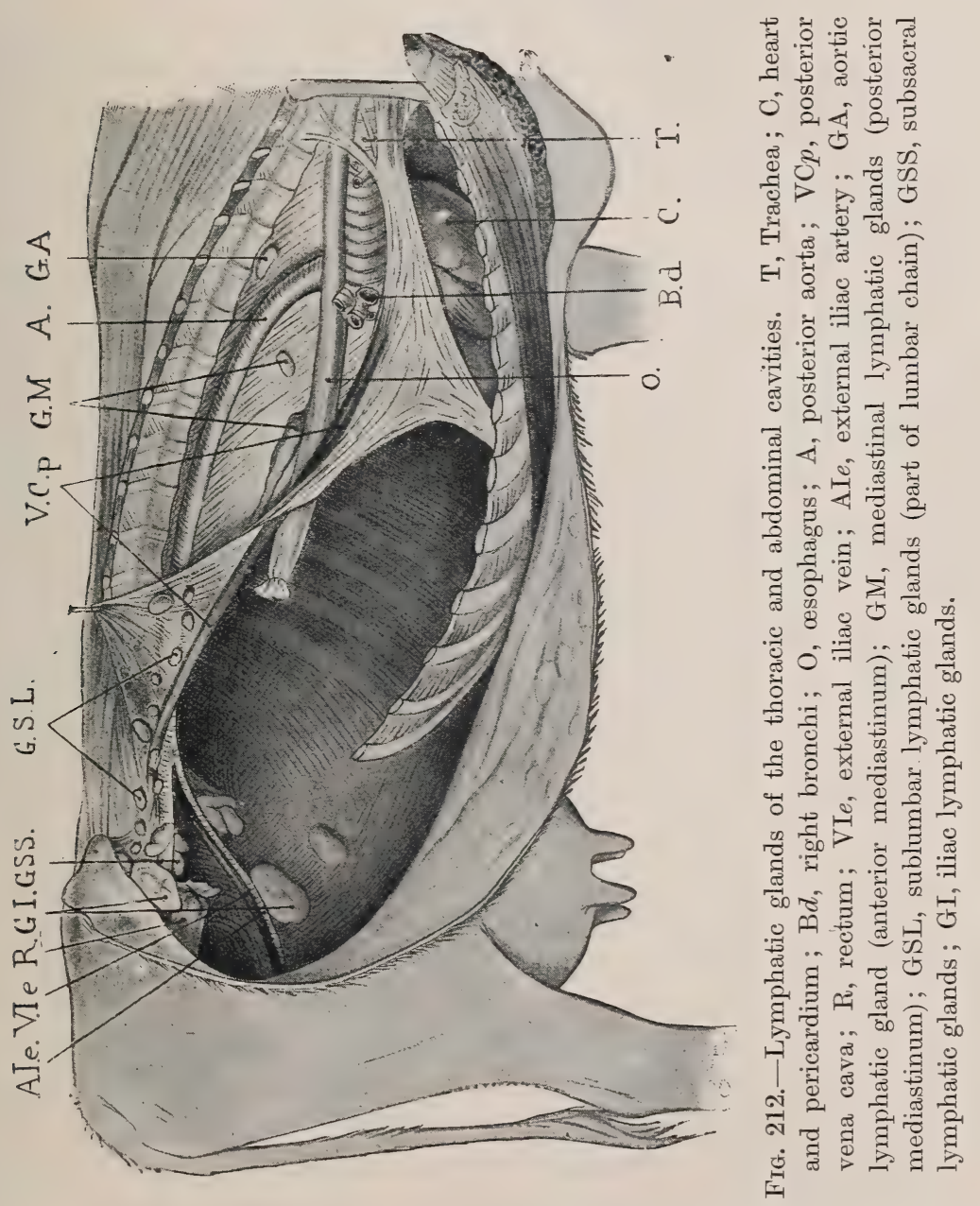

detect lymphatic glands in the loose fold of skin known as the flank. These glands are very small, and three in number. They are arranged in a triangle, one being much more prominent than the two others. In exceptional cases, little nodular glands, indistinguishable at ordinary times, may become hypertrophied. This is particularly true of the small glands in the neighbourhood of the last rib.

The retro-mammary glands need only be mentioned, but it is 
important to know that a deep-seated popliteal gland $\varepsilon^{\text {ilso exists }}$ above the semi-tendinosus and semi-membranosus muscle in the thickness of the muscles of the thigh; as also an ischiatic glan $\mathrm{d}$ opposite the ischiatic notch, which can only be examined by inter nal palpation from the pelvis, and an anal gland situated deeply or the sides and in front of the sphincter ani.

With the exception of those of the pelvis and of the sublumbar region, the glands of the thoracic and abdominal cavity eannot be examined, but change in them is indicated under certain circumstances by clearly defined clinical symptoms, and more ${ }^{\text {over }}$ it is necessary to be able to detect changes in these glands on p pst-mortem examination.

In the thoracic cavity the lymphatic apparatus comprises the mass of the prepectoral glands, which extends into the anterior mediastinum between the first ribs (glands of the entry to the chest), the aortic lymphatic gland situated beneath the dorsal porton of the spine opposite the bifurcation of the aorta, and the lymph atic glands of the posterior mediastinum, one of which is relatively sr ${ }^{\text {nall }}$ and is lodged in the concavity of the posterior aorta, the other large, elongated and situated immediately above the osophagus in $f^{\text {ront of }}$ its passage through the diaphragm.

In the abdominal cavity a sublumbar chain is found situated on the sides of the lumbo-sacral portion of the vertebral celumn, the mass of the subsacral lymphatic glands, and, at the entrance to the pelvis, extending on either side along the course of the external iliac arteries and veins and resting on the shafts of the iliac bones, the iliac glands.

All these glands are partly accessible to examination by the rectum.

Last of all, we may mention the gland situated on th ${ }^{e}$ hilum of the liver, the mesenteric glands, and the little lymphatic gl ${ }_{i}^{\text {ands }}$ above the sternum.

In the front limb the only glands of importance from a clinical standpoint are those of the internal surface of the should ${ }^{3 r}$, close to the divisions of the brachial plexus.

When enlarged or invaded by any specific organism, they may, by compressing the nerve trunks, cause lameness.

\section{THE LYMPHOGENIC DIATHESIS.}

(LYMPHADENITIS, LYMPHO-CYTHÆMIA, MYeLO-CYTHÆMIL.)

It has been questioned whether the term "lymphogenic diathesis," which was employed in human medicine by Jaccoud to descr ibe certain morbid conditions also found in animals of the bovine species, should 
continue in use. At the present moment it is difficult to determine the question. Under any circumstances it has the advantage of including diseases of the lymphatic system, indicated by hypertrophy of the lymphatic glands (adenitis) or by an exaggerated production of white blood corpuscles (leucæmia), and the passage into the general circulation of products derived from the lymphatic apparatus. For these reasons it may be employed here.

Clinical investigation long ago demonstrated that in man certain pathological conditions were characterised by a peculiar colour of the blood, due to the presence of white blood corpuscles in excessive quantities, whence the names "leucæmia" (Virchow) and "leucocythæmia" (Bennett). In the same way it has been shown that the change in the blood characterised by a superabundance of white blood corpuscles generally coincides with engorgement or more or less marked hypertrophy of the lymphatic system and of the adenoid tissue of the body (lymphatic glands, spleen, bone marrow, and, in exceptional cases, liver, kidneys, etc.)-leuco-cythæmic lymphadenitis; but that many cases also occur in which this hypertrophy of the adenoid tissue or of the lymphatic gland tissue may exist, without any excessive number of white blood corpuscles in the blood, whence the name "aleucæmic lymphadenitis or pseudo-leucæmia." Cases of true leucæmia without adenitis are much rarer, the lesions therein being confined to the adenoid tissue of the bone marrow.

These three morbid conditions-leucæmic lymphadenitis, or leucocythæmia; aleucæmic or pseudo-leucæmic lymphadenitis, or more simply adenitis; and true or simple leucæmia-are frequently found in the bovine species. Whilst stating that these diseases are frequent, we must, however, be understood to except the numerous cases of tuberculous lesions formerly included under the same head.

Jaccoud has shown that in reality the causes of these three morbid conditions are very similar, and that a case which at first appears to be of the nature of aleucæmic lymphadenitis may later become transformed into leucæmic lymphadenitis; or, inversely, that a case which at first appeared to be a simple leucæmia might often become complicated with lymphadenitis: hence the grouping of these different morbid conditions under the heading of lymphogenic diathesis.

Investigations have now thrown more light on the subject because of the more perfect recognition of the varieties of white blood corpuscles, and the above-mentioned morbid conditions may be defined as follows:-

(1.) The first variety consists of a more or less marked adenitis or lymphadenitis without leucæmia (aleucæmic lymphadenitis).

(2.) The second variety, consisting of leucæmic lymphadenitis, or leuco-cythæmia, is a lymphatic lucæmia or lympho-cythæmia, the D.C. 
anatomical characteristic of which is enlargement of lymphatic glands, and the histological characteristic increase in number of the large and small lymphocytes.

(3.) A third variety, formerly regarded as simplę leucæmia without lymphadenitis, is myelogenic leucæmia or myelo-cythæmia, the anatomico-pathological characteristic of which is to be found in myeloid hypertrophy of the bone marrow, giving to the bone marrow on postmortem examination a puriform appearance, and in the myeloid condition of the spleen.

Histologically this variety is characterised by an absolute increase in numbers of the large mono- and poly-nuclear eosinophile leucocytes.

Symptoms. Simple lymphadenitis begins in an insidious manner, and is characterised by weakness, anæmia, paleness of the mucous membranes, and wasting without apparent reason, although the appetite is preserved. It is only at a later stage that the glandular enlargements are discovered (adenitis), and often this discovery is not made until the veterinary surgeon is called in.

The existence of the disease is indicated by enlargement of the superficial glands, and this enlargement, which may commence at any point, extends along the course of the lymphatic vessels to the neighbouring glands, until in a shorter or longer time it involves all the lymphatic glands in the body.

The enlargement of the glands is usually symmetrical, and on clinical examination it is sometimes easy to detect at the outset an increase in size of the retro-pharyngeal glands, the glands of the neck, the prescapular glands, the glands of the flank, etc.

Rectal exploration reveals hypertrophy of the glands of the pelvis and of the sublumbar region, etc. The animals waste very rapidly, and sometimes in a few months become incapable of standing. They develop cachexia, and die in a state of exhaustion, with no other lesions than those of lymphatic hypertrophy. Neither do they exhibit any marked increase in the number of white corpuscles in the blood.

In lympho-cythæmia the beginning of the disease is often identical with that of simple lymphadenitis, the increase in the number of white blood corpuscles not occurring until later. In other cases, on the contrary, leucæmia appears first, and the enlargement of the lymphatic gland follows; but what characterises this form and allows of it being distinguished from myelo-cythæmia is the great increase in the number of large or small lymphocytes. The development is identical with, and sometimes much more rapid than, that of the preceding form. The animals waste away and become anæmic and cachectic, dying at last in a state of absolute exhaustion.

Post-mortem examination reveals, as in the previous condition, 
symmetrical hypertrophy of all the lymphatic glands; the spleen is very often enormous, and the liver is sometimes affected, as are also, in exceptional cases, the kidneys.

It may happen that the spleen alone appears affected, or at least that it has been first attacked, a fact which explains the existence of leucæmia before any enlargement of the lymphatic glands.

Causation. The causes of lymphadenitis and of lympho-cythæmia are unknown in veterinary as in human medicine. Apparently these diseases are more common in adults than in young animals. Some regard them as infectious in character, but this can hardly be the case, as all experimental attempts to transmit the diseases have failed. It is more plausible to compare the development of these morbid conditions with that of malignant tumours, and although some doubt still exists, simple lymphadenitis may be described as an aleucæmic lymphoma or lympho-cytoma, which has gradually become generalised, spreading by way of the lymphatic channels from the gland first affected through the surrounding glandular system. Lympho-cythæmia, on the other hand, may be said to be a leucæmic lympho-cytoma, which spreads both by the blood circulation and by the lymphatic paths (spleen, hæmatopoietic glands and organs).

This view of the development of the lesions enables us to class lympho-sarcomata with lymphomata and lympho-cytomata. The malignant character and extremely rapid development of lymphosarcomata appear due to its extending by contiguity of tissue, and simultaneously by the lymphatic paths.

This new grouping would consequently place on one side myelogenic leucæmia, also called myelo-cythæmia, which is perhaps a different morbid species. This would destroy the unity implied in Jaccoud's theory of the lymphogenic diathesis; but for all that this method of grouping might be justified by reference to specific cellular characteristics. In myelo-cythæmia the disease appears to commence as a lymphocythæmia, i.e., it is unaccompanied by enlargement of lymphatic glands or hypertrophy of the spleen or liver, though the blood appears leucæmic. The condition is not a leucæmia due to lymphocytes, but rather a leucæmia produced by mono- and poly- nuclear eosinophile leucocytes, i.e., leucocytes derived from the bone marrow.

The patients are carried off rapidly after persistent wasting, decline and cachexia, whilst on post-mortem examination the puriform aspect of the bone marrow is an extremely striking characteristic.

Diagnosis. There is rarely much difficulty as regards the diagnosis. The enlargement of the lymphatic glands, for instance, can readily be detected, and the only disease with which this can possibly be confounded is tubercular enlargement. 
With the means at present available for diagnosing tuberculosis, such as microscopic examination of the discharge, inoculation with discharge, examination of material from the glands, injection of tuberculin, etc., the nature of the disease can always be placed beyond doubt.

In lympho-cythæmia and in myelo-cythæmia, the whitish-violet lactescent appearance of the blood is of unmistakable significance, particularly when the manifest progressive wasting of the whole system is taken into account.

Histological examination of the blood after fixation and staining will in the former cases reveal the presence of very large numbers of lymphocytes, and in the latter an absolute increase in the number of the mono- and poly-nuclear lymphocytes. It should be easy, therefore, to distinguish the two diseases, especially as other symptoms vary.

In the early stages leucæmia may be mistaken for the leucocytosis seen in infectious diseases. These forms of leucocytosis are very common in animals of the bovine species. They occur in certain forms of tuberculosis, in uterine infections, in cases of internal suppuration, in tumour of the heart, the rumen, etc., and vary in so far as one style or another of white blood corpuscle predominates. The diagnosis, therefore, necessitates that the white blood corpuscles should be counted, and whenever it is found that their variations in number are no more than between 5,000 and 15,000 per cubic millimètre, the case may be regarded as one of temporary leucocytosis.

If, on the other hand, those corpuscles number more than from 15,000 to 20,000 , or, as may sometimes happen, they attain to from 200,000 to 300,000 per cubic millimètre (one white to two or three red blood corpuscles), the case is one of leucæmia, and, according to the predominance of the particular type of cell, it is a lymphocythæmia or a myelo-cythæmia.

In leucæmic conditions the red blood corpuscles are also present in fewer than the normal numbers. They are more irregular, assume giant and dwarf forms (macrocytes and microcytes), sometimes exhibit lacunæ, and are always polychromatophile, i.e., without special affinity for any particular constituent of double or triple stains.

Prognosis. The prognosis of diseases included in the lymphogenic diathesis is extremely grave, and in the present state of our knowledge it may be assumed that sooner or later death is inevitable.

Treatment. Treatment can scarcely be considered to exist, for at the best it can only delay the development of the disease. Nevertheless, and with this reservation, it is certain that preparations of iron, iodine and arsenic have a certain effect, probably by acting on the hæmatopoiesis. 


\section{CASEOUS LYMPHADENITIS OF THE SHEEP.}

In the sheep the lymphatic glands are sometimes the seat of peculiar changes, which do not appear to have any marked effect on the general condition. Thus a post-mortem examination or an examination of animals in the slaughter-house sometimes shows a certain number of isolated or symmetrical glands, such as the mediastinal, tracheal, inguinal, pelvic or sublumbar glands, to be greatly enlarged and completely degenerated. The precrural, prescapular, and popliteal glands are said to be most frequently affected. Their contents are caseous and yellowish, enveloped in a fibrous sheath, and show no signs of peripheral inflammation. The other organs and viscera may either be healthy or exhibit caseous lesions identical with those found in caseous broncho-pneumonia.

The causes of this disease are imperfectly understood, although Cherry and Bull (1899, the Veterinarian, Vol. LXXII., No. 860, p. 523) have isolated from the lesions an organism identical with Preisz's bacillus and with the microbe of ulcerative lymphangitis in the horse.

Norgaard and Mohler (Annual Report, United States Bureau of Agriculture, 1899, p. 638) have studied the disease. In June, 1897, Turski, at Danzig, found about 150 breeding ewes, from eight to twelve years old, suffering from nodules or abscesses the size of a child's fist in the inguinal and prescapular regions. They had been sold for slaughter, and many were in very poor condition. The disease occurs in Europe, Western America, South America, and Australia. Several thousand cases are annually seen in the slaughter-houses of the United States.

The symptoms generally escape notice, and it is only by accident that one sometimes detects marked enlargement of the lymphatic glands of the neck or of the superficial inguinal glands. The patients, moreover, may remain in very good bodily condition, so that the lesions are only discovered on the meat being inspected.

Having regard to our imperfect knowledge of this disease, it is impossible to express an opinion as to its importance or treatment.

\section{GOITRE IN CALVES AND LAMBS.}

Although not strictly relevant to the foregoing matter, a few remarks may here be made on the subject of goitre.

True goitre consists in hyperplasia of the follicles of the thyroid gland, with colloid change of their contents, which are chiefly albuminous. The swelling is mainly due to enlargement of the follicles, and is termed struma follicularis. It may attack the entire organ or only one-half; less frequently it is confined to certain sections. Other varieties of goitre are recognised, such as fibrous, varicose and cystic goitre. (For fuller details see Möller and Dollar's "Regional Surgery," p. 149.) 


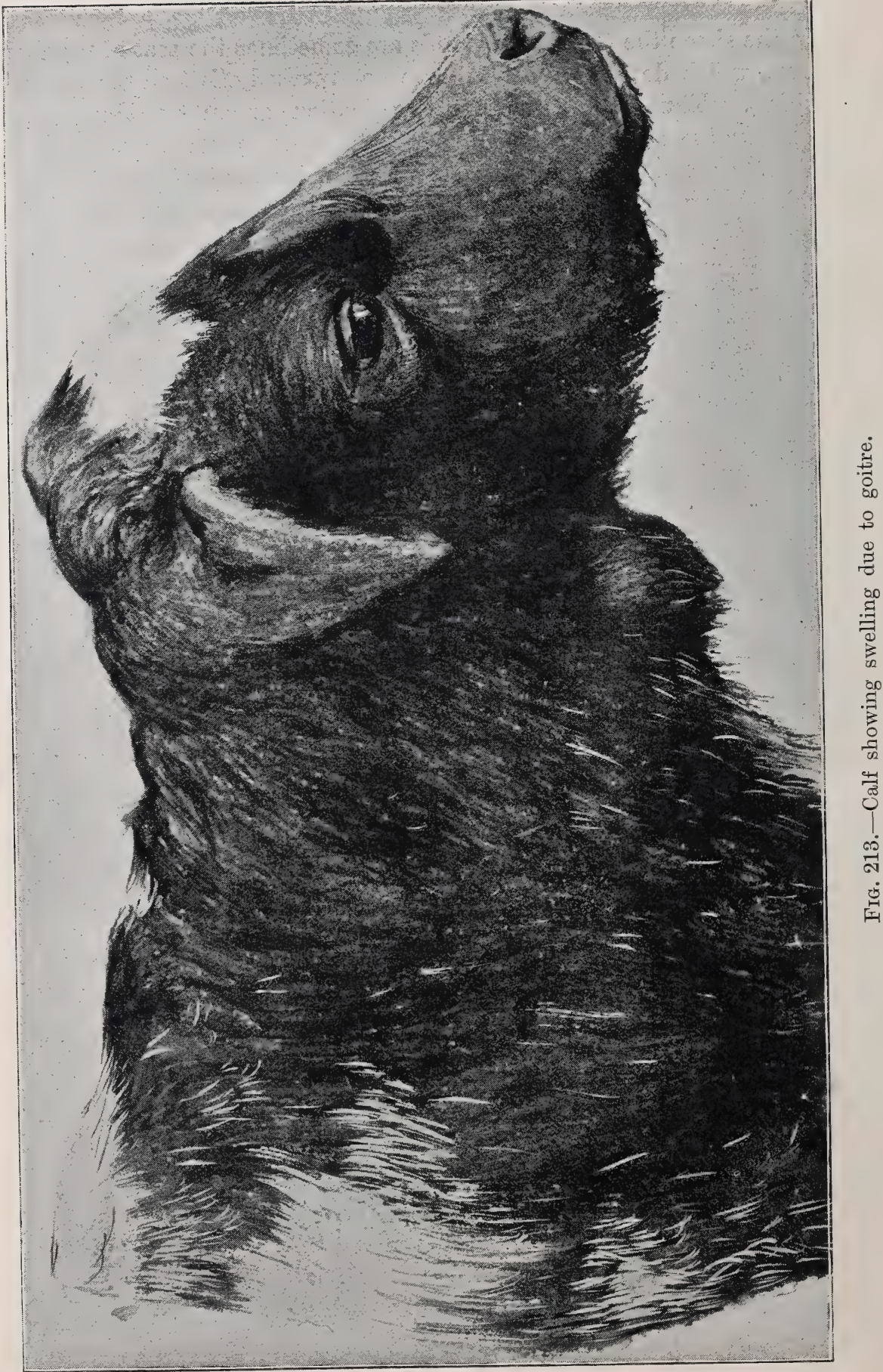


Treatment by injection of thyroid juice or by feeding on thyroid extract has given better results than drugs.

The following account of an outbreak in New Zealand is summarised from the Annual Report of the Chief Veterinarian of New Zealand, $1901:-$

The calves affected were born with enlarged thyroids. The farm is of rich alluvial deposit, and rather below the level of the river, which it borders. The land has been in occupation, however, for many years, and no similar condition had been previously noted. At first, as calves only were affected, it was thought possibly to be due to the bull, a twoyear-old animal, but when a foal was born suffering from a similar malformation this theory naturally fell to the ground.

The land had been ploughed with a special plough 20 inches deep, but this is no uncommon practice in the island.

About the same time, a similar disease was discovered affecting lambs at a farm near Outram. From 450 ewes, 150 lambs had been lost, the glands being enlarged to the size of a cricket ball. A few had been born dead, many only lived a few hours, others lived several days, and a considerable number recovered. There was no connection, directly or indirectly, between the two farms, they being at least fifty miles apart. A few of the calves died or were killed, the remainder recovered, and the foal grew rapidly better. The land on both farms is very similar in composition.

Mr. Wilkie states, from observation of previous cases in lambs, that "it appears to be always associated with malnutrition and a condition of anæmia in the parent, induced in most cases by feeding with watery, innutritious foods."

Specimens were forwarded, from a calf and from a lamb, of enlarged glands. The gland of the calf was enormously enlarged, being at least twice the size of an orange, dark in colour, flabby in consistency, and on section a mucous material exuded copiously from the cut surface. Micro-examination showed the acini to be larger than normal, filled with the usual mucous material, and lined with cubical epithelium. The connective tissue surrounding the alveoli was, however, crowded with round-cells, so much so that the whole parenchyma seemed to be practically composed of these cells.

A specimen of an enlarged thyroid from the lamb was about the size of a sheep's kidney, and very much the same shape and colour. Sections microscopically examined showed a different condition to that of the calf's thyroid. Here the acini were filled with epithelial cells loosely arranged as if the lining epithelium had been proliferating rapidly, while the connective tissue surrounding the acini was fairly normal. The section had a somewhat adenomatous appearance. 


\section{SECTION V.}

\section{NERVOUS SYSTEM.}

\section{CEREBRAL CONGESTION.}

According to Cruzel, cerebral congestion is somewhat frequent in working oxen subject to continued concussion from the yoke, especially among animals working on a rocky soil. The condition may also be produced by prolonged exposure to the sun, as well as by sudden and intense cold.

Passive cerebral congestion by stasis may be produced by any cause markedly interfering with the return circulation (pericarditis due to foreign bodies). Clinically it is of no importance.

The animals, previously in good health, suddenly appear comatose. They are insensible to stimulation of any kind, the head is rested on any convenient object or is held stationary, the animal looks drowsy, the gait is hesitating or vacillating, the respiration slow or irregular. Left at liberty, the animal does not seem to know where it is going; indeed, sometimes it is absolutely blind and strikes against any obstacle in its path, or falls and suffers from epileptiform convulsions. The cranial region is abnormally warm. The course of the attack is rapid, and the animal either dies in a state of coma or convulsions or else recovers rapidly.

Diagnosis. The diagnosis is decidedly difficult; and the prognosis should be reserved.

Treatment commences with free bleeding, the amount of blood drawn being proportioned to the animal's size. The sides of the body may then be stimulated and a purgative administered.

\section{MENINGITIS.}

The generic term "meningitis" includes all inflammations of the arachnoid, pia mater and internal surface of the dura mater.

These forms of inflammation oceur in diseases such as tuberculosis and in parasitic diseases of the brain. Under other circumstances, they are rare, and may be produced by very varying causes.

An epizootic cerebro-spinal meningitis of the bovine species has 
also been described, principally in Germany. It seems almost unknown in France, and French literature contains no well-authenticated case.

Furthermore, an epizootic cerebro-spinal meningitis of sheep, or rather of lambs, has been described in Germany, in Italy, and in France. These descriptions are all open to many objections. It seems that under the term "cerebro-spinal meningitis" have been grouped cases of enzootic tetanus, doubtful cases of poisoning, and particularly cases of conurosis in the first stage of development. We therefore discard these descriptions, which differ too much among themselves to be of any value.

Causation. Meningitis occurs in the ox and sheep as a complication of wounds in the cranial region, accompanied by fissuring of the bone, periostitis, abscess formation, etc.

It is also seen as a complication of fractures of the horns, and oldstanding catarrh of the facial sinuses. In the sheep it follows parasitic catarrh due to the larvæ of œstridæ.

The meningitis appears, according to circumstances, in the forms of local meningitis, anterior frontal meningitis, basilar meningitis, etc. Finally, it may develop as a complication of different diseases, such as gangrenous coryza, purulent infection, subparotid abscess, suppurative phlebitis, suppuration of the eye or of the orbit, etc.

Symptoms. It is difficult to detect and interpret the first symptoms shown, because these chiefly consist in dulness, want of appetite and constipation, without any particular fever. At a later stage, excessive excitability is produced by noises, by changes of light, or by handling. Careful examination of the patients shows a change in their expression, rapidly followed by contraction and inequality of the pupils or deviation of the visual axis (strabismus, squinting). The pulse becomes irregular, as also the respiration. The appetite is entirely lost, and it is not uncommon to note a contraction of the muscles of the neck and jaws, as well as inability to move about and symptoms similar to those of dropsy of the cerebral ventricles.

The chronic form is rare.

Lesions. The lesions comprise local or general hyperæmia and exudative inflammation of the pia mater and arachnoid, together with the formation of false membranes or of pus in the subdural space. 'The meninges are partially adherent, and the superficial layers of the brain are also inflamed by contiguity of tissue.

Diagnosis. The diagnosis must be based on the disturbance of vision, movement, and appetite, and on the course of the symptoms, as well as on the external signs in the case of such diseases as are prone to become complicated with meningitis. 
Prognosis. Sooner or later the ease is likely to end fatally, and there is no practical use in treating the patient.

Treatment. If in exceptional cases slaughter is objected to, setons and blisters may be applied to the poll or the parotid region, or the parts may be enveloped in ice bags or compresses of iced water frequently renewed.

\section{ENCEPHALITIS.}

Encephalitis, i.e., inflammation of the cerebral substance, is very closely allied to meningitis; in a great number of cases meningitis and encephalitis co-exist. In other cases encephalitis may be found apart from meningitis, and vice versâ. Moreover, many of the symptoms of meningitis are to be found in cases of encephalitis.

Encephalitis may develop as a complication of meningitis. Encephalitis may also follow abundant parasitic infestation, as in cœenurosis (which will be particularly studied as it affects sheep), or microbic infection, the commonest form of which in the ox is tuberculosis. The encephalitis may be diffuse or circumscribed, according to the cause, while the symptoms are varied and numerous. Very frequently, particularly in cases of tuberculosis, encephalitis assumes a chronic form.

Symptoms. The earliest symptoms are extremely difficult to detect, because they are scarcely characteristic and because it is impossible to ascertain the sensations of the animal.

It is only when the disturbances in walking, in the eyesight, in swallowing, etc., are noted that suspicion is aroused.

The symptoms may appear suddenly. Nevertheless it is beyond doubt that there are certain slightly marked prodromata, indicated by diminution of appetite, wasting, and changes in vision. Soon afterwards occur other forms of disturbance, which may be classified under the heading of "motor, visual, nervous, and impulsive." The patients appear stunned, their movements are slow and hesitating, they partially lose control over their limbs and display lameness, with spasmodic movements of one or two limbs. Examination of the joints shows no injury. The lameness may simultaneously affect two diagonal limbs or two fore and two hind limbs, or even three limbs. This lameness is of central origin.

The ocular disturbance is marked by diminution or loss of vision, by strabismus, or by frequent unconscious movements of the eyes and eyelids, and also more particularly by inequality, contraction or dilatation of the pupils.

Nervous, impulsive disturbance is most readily noted when the animals are at liberty. Even when the sight remains, they seem 
quite incapable of avoiding obstacles or as though absolutely forced to move to the right or left, etc.

Attacks of giddiness, moreover, are not unusual under the influence of the slightest excitement. During such attacks the animals thrust the head against a wall, or they involuntarily recoil or make lateral movements. In many cases these vertiginous attacks end by the animal falling and showing epileptiform convulsions, during which it may die.

The symptoms are never the same in two different animals, but they may easily be classed according to the above indications. The indications furnished by the condition of the eyes and by the peculiar impulsive movements are particularly significant.

On the other hand, there are modifications in breathing without apparent local cause, and difficulty or even impossibility of swallowing, etc., although there exists no material obstacle.

Diagnosis. The condition is often confused with meningitis, and the mistake is not serious, because meningitis and encephalitis frequently accompany one another.

Prognosis. The prognosis must be regarded as fatal. The patients very seldom recover, and there is no reason for keeping them alive.

Treatment. Here, again, blisters may be applied to the upper extremity of the neck, or setons may be passed. Cooling applications to the cranial region have also been suggested. None of these methods produces more than a temporary palliative effect.

\section{CEREBRAL TUMOURS.}

The brain may be injured and compressed by various tumours of other than parasitic origin. Such tumours may originate in the bones, the meninges or the choroid plexus, or they may simply be due to generalisation of a previously existing tumour. Whilst of very varied origin and nature, all tumours of the cranial cavity have one common effect, viz., to compress the brain. This continuous compression causes progressive atrophy of the brain, but its existence is not always suspected, because the lesions may not give rise to any marked symptoms.

The hind portions of the hemispheres and the white substance are generally very tolerant. The front portions, on the other handthe frontal lobes and the grey substance-resent compression, which provokes various symptoms in consequence.

The symptoms of compression and atrophy of the brain differ greatly, a fact which is easily understood, inasmuch as the seat of the change may vary, and therefore it is possible only to trace the chief manifestations, which suggest the existence of a cerebral tumour. 
The general changes are indicated by signs precisely similar to those so common in horses with dropsy of the ventricles (general depression, inability to back, long intervals between the prehension of successive mouthfuls of food, sudden cessation of mastication, etc.), by an impulsive or automatic gait, and by the assumption of strange attitudes (kneeling down in front, etc.). When at rest the animals appear to be in a state of continual torpor.

Special symptoms sometimes occur, which enable the seat of the injury to be localised in more or less exact fashion. These symptoms affect the vision (amblyopia, amaurosis, strabismus, nystagmus), general sensibility (hyperæsthesia, anæsthesia, etc.), and the power of movement (total, partial or crossed hemiplegia, want of co-ordination of movements, etc.).

Trifling stimuli almost always lead to marked and even epileptiform attacks.

The diagnosis of cerebral tumours is very difficult, particularly when attempts are made to indicate their exact seat, but that of other cerebral lesions is somewhat easier.

The prognosis is very grave, and in the case of domestic animals nothing can be done. In the ox intra-cranial operations are difficult, by reason of the presence of the sinuses which obstruct the approach to the brain cavity; economically surgical treatment is seldom advisable.

\section{INSOLATION.}

Insolation is an exceptional accident in animals of the bovine, ovine, or porcine species. If at liberty these animals move about, and always seek shelter when the sun is fierce. If, on the contrary, they are harnessed and kept standing for long, exposed to the full midday sun during June, July or August, they may suffer from insolation.

During the International Cattle Show attached to the Exhibition of 1900 in Paris, a considerable number of cases of insolation occurred in animals of one class, exposed to the full midday sun, in an illventilated spot. The other classes only received sunlight from the sides, and in them not a single case occurred.

Death may follow in a few hours; it is difficult to say precisely how it is brought about, but it is always accompanied by congestion of the cerebro-spinal centres and general blood stasis.

The symptoms of the development of insolation occur very rapidly. In animals of the bovine species there is accelerated respiration, which soon amounts to dyspnœa. The mucous membranes then become cyanotic. The animals attacked seem anxious, although not agitated, 
and soon afterwards the eyes water, the mucous membrane and the lips of the vulva display edematus infiltration and congestion, and areas of cutaneous congestion, closely resembling mud fever in the horse, appear over the mammæ. At this stage the animals move with difficulty, and show all the symptoms seen at the outset of gangrenous coryza.

All these symptoms develop in one, two, or three hours, and death may follow if nothing is done. They disappear, however, as rapidly as they appear. In an hour or less we have seen in some cases a complete return to the normal condition. Given the facts, the diagnosis is extremely easy.

Treatment. Treatment should be commenced by immediately removing the animal to a cool, airy, shady place. It may then be bled, and the head and neck should be freely drenched with cold water. The symptoms generally disappear as though by magic.

\section{POST-PARTUM PARALYSIS-MILK FEVER-MAMMARY TOXEMIA- PARTURIENT APOPLEXY-DROPPING AFTER CALVING.}

For a great part of the following short account we are indebted to an excellent report by J. J. Repp, V.M.D., in the Joumal of Comp. Medicine and Veterinary Archires, September, 1901 :-

The word "fever" in connection with the terminology of this disease is not very appropriate, because in the majority of cases fever is not present, but the animal has a subnormal temperature. The term milk fever is very misleading and indefinite, as it is also used by the laity to designate other diseases, such as parturient septicæmia and the various forms of mammitis. Parturient paralysis must be clearly differentiated from parturient septicæmia, which is a disease of an entirely different character and which may occur in any of the domestic species, whereas parturient paralysis occurs only in the cow.

Distribution. Parturient paralysis occurs wherever milch cows are kept. It is more prevalent in dairy districts, because it is the heavy milking strains of cows that are most subject to the disease.

Cause. No definite cause can be assigned for this disease. Schmidt's theory is that parturient paralysis is caused by the evolution in the mammary gland of a poisonous substance through the over-activity of the epithelial cells of this gland excited by the determination to the udder after birth of large quantities of blood which was supplied to the uterus and the foetus before birth, but which now goes to the udder because of the natural demand for milk secretion. This poisonous substance being carried in the circulation to various parts of the body, brings on the symptoms which characterise the disease. It is well recognised that 
living cells may, under certain circumstances, produce poisonous substances. Schmidt's theory, therefore, is in accord with an established principle.

Pathogenesis, or generation, of the disease. Parturient paralysis, as a rule, occurs in cows which give a heavy flow of milk and which are in a high state of nutrition. It may develop at any age, but is extremely rare in cows before they have reached adult age and have given birth to several calves. It is also rare in old cows. It occurs, then, in cows which are of middle age and in the full height of their activity as milk producers. The disease attacks the cow after she has given birth to a calf, usually within twenty-four hours thereafter, but in some cases not until a week or even a month after parturition. In a few cases the disease has its inception a short time before parturition. Cows which are stabled and deprived of exercise are said to be more prone to the disease than those which are permitted to exercise at will. There are many exceptions to this statement, although it is the usual teaching. Further observation may show that it is not correct. In Iowa more cows take this disease while at pasture than in any other circumstance. This doubtless arises from the fact that in Iowa cows are given more freedom than is customary in older dairy States. The disease may arise at any time in the year, but, on account of the fact that spring-time is pre-eminently the calving season, most cases originate at this season.

Morbid anatomy. The morbid alterations are limited and variable, and offer nothing characteristic. The blood is irregularly distributed, a condition which probably indicates marked vasomotor disturbance resulting from the profound interference with the nervous functions which accompanies the disease. The abdominal organs are usually filled with blood. 'I'he brain may be anæmic, œedematous, easily torn, and yellowish in colour. In other cases it shows hypersemia of the meninges and of the brain substance.

Symptoms. The disease usually appears within twenty-four to fortyeight hours after parturition. In extreme cases it may not occur until two months or even six months after parturition. It may rarely occur before birth. It usually follows an easy birth. At the onset of the disease the cow manifests some uneasiness; it moves about in a restless manner, stamps, strikes the abdomen with its hind legs, perhaps bellows, grinds the teeth, and may have spasms of groups of muscles or even a general convulsion. After this period, which may be unnoticed, the symptoms of paralysis come on. The cow shows weakness, staggers, and at last falls. As the paralysis advances it stretches on the ground, lying on its side usually with the neck bent to one side so as to bring the nose into the flank or the costal region. This is the characteristic position in parturient paralysis. If the head is brought 
into the normal position, it at once returns to the unnatural position in which it was found. The animal is in a state of partial or complete unconsciousness, does not respond to blows or calls, and takes no note of its surroundings. The eye is dull and not sensitive to the finger touch, sunken, pupil dilated, and the upper lid is drooping; the tongue is paralysed, saliva runs from the mouth, the pharynx and osophagus have lost the power of motion, so that the animal is unable to swallow ; the peristalsis of the stomachs and intestines is in abeyance, and as a result digestion is arrested, fermentation sets in, and the animal becomes tympanitic; the contents of the rectum and colon are hard and dry, and may be covered with mucus or blood, urination is suspended; the os uteri is almost invariably dilated if the disease occurs within a day of parturition; pulse small, often imperceptible, 60 to 120 per minute; temperature, usually normal or below normal, may be as low as $95^{\circ}$ Fahr., in some cases may be as high as $105^{\circ}$ Fahr. Such a high temperature probably does not occur in a case of pure parturient paralysis, but only when there is a complication of parturient septicæmia. The extremities are cold. The after-birth is sometimes retained. There may be accompanying prolapse of the uterus.

Course. Without treatment, and, indeed, with most kinds of treatment which have been applied in the past, the disease usually runs rapidly to a fatal issue. It lasts two to three days, and in some cases longer, the condition gradually becoming more and more aggravated. Death results from sudden failure of the heart or brain, and is often preceded by profuse diarrhoa. In milder cases the cow may linger as long as two to four weeks and then die of pneumonia, which results from the inhalation, or introduction through attempts at medication, of foreign substances into the lungs during the period of paralysis of the pharynx and œsophagus. If recovery occurs, the animal is entirely well in two to five days. In rare cases paralysis of the hind parts may persist for a long while.

Diagnosis. This is made by a study of the history and symptoms. It is comparatively easy.

Differential diagnosis. It must be distinguished from ante-partum paralysis, broken-back, parturient septicæmia; but one familiar with the character of these diseases will find no difficulty in maling this differentiation.

Treatment. This may be considered under two distinct subdivisions, viz., preventive treatment and curative treatment.

(a) Precentive treatment.-By considering what has been said under the head of "generation of the disease," one can easily infer what measures should be adopted to prevent the disease. Cows in the later stage of gestation should be fed moderately, grain especially being 
given sparingly or entirely withheld; the animals should be given an opportunity to take plenty of exercise; the bowels should be kept in good condition by the administration of such salines as magnesium sulphate, sodium chloride, and sodium bicarbonate. The after-birth should be removed soon after parturition and several uterine douches administered.

(b) Curative treatment.-The older methods of treatment comprised: warmth and friction to the mammary gland; the administration of sedatives, such as opium, chloral and bromide of potassium; stimulants, including ammonia, ether, turpentine and alcohol; washing out the uterus with water or disinfectant solutions; the relief of tympany by the use of the trocar and canula (by which instrument medicines may also be injected directly into the rumen); the removal of fæces from the rectum; warm clothing of the body and general attention to the animal's comfort, and to the teachings of hygiene. For all these widely diversified methods good results have been claimed, and, we may add, bad ones at times recorded. F. T. Harvey (Cornwall) estimates the average mortality at from 40 to 66 per cent., though he claims for his more recent practice a lessened mortality of only 20 per cent.

Schmidt does not claim that his method of treatment disposes bodily of the morbid condition, but that it does measurably assist Nature in her efforts to restore the animal to the normal physiological state. It is well known that after the beginning of the attack the animal, if left to itself, rapidly grows worse until the crisis of the disease is reached, at which time death occurs or convalescence begins, usually the former. It has been observed, however, that if the treatment is applied within a few hours after the inception of the disease its progress is modified in such a way that convalescence at once begins, as a rule, and the animal hastily recovers its health, usually within twelve hours, although in extreme cases it may be as late as forty-eight hours. The following is an outline of the plan of treatment of parturient paralysis suggested by Schmidt. The operator should disinfect his hands and the udder and teats of the cow by washing with a 5 per cent. solution of carbolic acid or creolin, or a $1 \frac{1}{2}$ per cent. solution of lysol or trikresol. The apparatus needed for the treatment consists of a small glass funnel, a rubber hose three feet long and one-eighth inch in calibre into which the funnel fits, and an ordinary milking tube over which the rubber hose fits. This apparatus should be sterilised immediately before it is used by boiling or soaking in such a solution as recommended for washing the udder. Dissolve from 2 to $2 \frac{1}{2}$ drachms of potassium iodide-the size of the dose depending upon the size of the cow and the character of the attack-in about one quart of clean water previously boiled to sterilise it, and allow the solution to cool to a 
little above body temperature, or $40^{\circ}$ C. or $10 t^{\circ}$ Fahr. The temperature may be determined with the clinical thermometer. Withdraw all the colostrum or milk from the udder. Then insert the milking tube, with hose and funnel attached, into one of the teats, elevate the funnel about two feet above the teat and slowly pour in one-fourth of the solution, allowing the funnel and hose to become empty several times during the process in order to permit the entrance of a liberal quantity of air. Repeat this infusion with the other three quarters of the udder. After all is introduced, knead the udder carefully so as to cause the solution to permeate the ducts and acini as much as possible.

As the condition of the cow is usually such as to call for additional treatment, the veterinarian should not be content with injecting the potassium iodide solution, but should resort to any and all other measures which promise assistance.

As the cow is usually unable to urinate, the bladder will be found filled with urine. This should be removed with the catheter, and its removal accomplished at intervals until the recovery of the cow renders this procedure no longer needful.

It may be advisable that catharsis be brought about. As the cow is usually unable to swallow, it is dangerous to attempt to give medicines by the mouth. This may be done if assurance that the cow can swallow is obtained. Some have given medicines successfully through a probang inserted into the stomach. The plan is feasible. Schmidt says that he usually resorted to an aloe powder. If this is done 1 ounce to $1 \frac{1}{2}$ ounces of aloes may be given. It would seem preferable to give the aloes in a bolus, capsule, or drench. Some have given linseed oil or Epsom. salts. If the animal cannot swallow and a probang is not at hand, one may administer $1 \frac{1}{2}$ to 2 grains of physostigmine salicylate subcutaneously, repeating the dose in about three hours if purgation is not produced. Rectal injections should be given at short intervals in order to get rid of the accumulation of hard, dry fæces in the rectum. These injections may be of linseed oil, cottonseed oil, or warm soap solution. Schmidt recommends, also, enemata of sodium chloride solution. Neanwhile the cow should be kept propped up on the sternum by means of bags of straw or pieces of wood. If the temperature is below normal, as it usually is, the cow should be thickly clothed with blankets and straw heaped up about it. Schmidt used powdered digitalis given by the mouth when the heart was rapid and weak. It would seem much better in every way to give the tincture of digitalis subcutaneously. He has also resorted to subcutaneous injections of camphor and caffeine. This is good treatment. If the cow does not show marked improvement within eight hours the potassium iodide infusion may be repeated. 
Schmidt has found that as much as 6 drachms may be injected into the udder without harm to the cow. Schmidt, in his first report, made in 1898, recorded 50 cases treated for parturient paralysis by this method with but two deaths from the disease. There were, however, only 46 recoveries, as two cows were slaughtered for beef during the first day of convalescence. A short time later a report was made by Jensen showing that in Denmark up to that time sixty-five veterinarians of that country had treated 412 cases by the Schmidt method, 90 per cent. of which recovered. Such results seem to indicate this as the treatment par excellence for parturient paralysis. It still remained to secure the introduction of this treatment into the United States and to determine what results could be obtained. In all 166 cases were reported; of these 166, 119 resulted in recovery, while 47 were fatal. Of the fatal cases, in eight of the cows death may be traced to some complication, such as prolapse of the uterus, foreign-body pneumonia, etc. In these cases the Schmidt treatment cannot be said to have failed, for it is not in any way intended that it shall be able to overcome such accidental conditions. If the cow has recovered from its condition of paralysis as a result of the Schmidt treatment far enough to be out of danger from that source and to promise recovery, but later falls a victim to some complication that is in no measure a part of parturient paralysis, but only a result of that disease, it may with justice be said that the Schmidt treatment was a success so far as the malady against which it was directed is concerned. Looking at the reports from this generous point of view, in 127 cases out of 166 , or 76.5 per cent., the Schmidt treatment was successful so far as the parturient paralysis was concerned.

In a paper published in the Berliner Thieräratliche Wochenschrift in August, 1902, Schmidt reviews the results of his treatment as evinced by 914 patients treated by thirty-one different practitioners: 884 , or 96.7 per cent., recovered, twelve died and six were slaughtered during the course of the disease. Twelve others were slaughtered at a later period in consequence of complications. Jensen reported the results of 1,744 cases.

Schmidt also found that the simple injection of air was in many cases sufficient to produce recovery, and subsequent observation tends to show that the fluid injected is of less importance than was first anticipated. A large number of unirritating solutions may be employed. Schmidt, however, still counsels the use of a quart of 1 per cent. solution of iodide of potassium, in which can be dissolved 5 grammes of caffein sodio-salicylate if the heart's action is weals. About 10 ounces of this solution are injected into each quarter, and are followed by a liberal injection of air. The parts should afterwards be freely massaged. 


\section{CCENUROSIS (GID; STURDY, TURN-SICK).}

Cœnurosis is a disease due to invasion of the animal body by embryos of larvæ of the Trenia conurus of dogs and wolves. These embryos only develop freely in the brain substance (Conurus cerebralis) and medulla oblongata. The hosts of the larvæ include the calf, sheep, goat, roedeer, reindeer and horse.

The disease was formerly erroneously called "turn-sick," for the turning is only a manifestation, and even a tardy manifestation, of the disease, while in addition it is not invariably present.

Cœnurosis principally attacks lambs of from three to six months, although it occurs up to eighteen months, and sometimes even two years. It is exceptional, however, in adults. Similarly in the bovine species it usually affects young animals up to the fourth or fifth year.

Conurosis with diffuse parasitic encephalitis often remains unrecognised, the animals being regarded as affected with epizootic meningitis of unknown cause or septic intoxication, and when they die the owners are ignorant as to the cause of death. The stage corresponding to turnsick, which is an advanced phase of the disease, is only seen in animals which have been infested to a slight extent, and in which three or four parasities only, sometimes only one, have attained the brain and developed there. Such cases exhibit all the classic symptoms of turnsick, viz., turning movements, heaviness, vertigo, ete.

Causation. Conurosis is due solely to one cause, viz., the ingestion of eggs or embryos in feeding or drinking.

The Tenia cœnurus lives in the dog, and fertilised segments are passed with the frees in yards, pastures and fields, and on the margins of roads, ditches and ponds. Amongst damp grass or in water the eggs, which contain more or less well-developed embryos, may retain their vitality for several weeks, and when swallowed the embryos are set at liberty in the intestine.

The six-hooked embryos perforate the walls of the intestine, pass into the blood stream or chyle ducts, and from these points are carried in all directions. Those which gain the nervous centres, the brain or spinal cord, continue to develop; the others, dispersed through different tissues, degenerate and disappear.

Experimental infection with these parasites shows that the brain is invaded after about a week's time. From the twentieth day the presence of embryos can easily be detected in the superficial layers of the convolutions. They make their way through the grey substance, leaving behind them greenish-yellow sinuous tracts with caseous contents.

The cyst or finn undergoing development can be found at the end of one of these tracts in the form of a little transparent bladder, of a size 
varying between that of a pin's head and that of a lentil or a small hazel-nut.

Later the tracts, with their caseous contents, disappear, and the development of many of the vesicles proves abortive. At the end of a month the vesicles, continuing to develop regularly, attain to about the size of a pea. Between the fiftieth and sixtieth days heads or scolices appear in the interior of the vesicle, which then reaches the

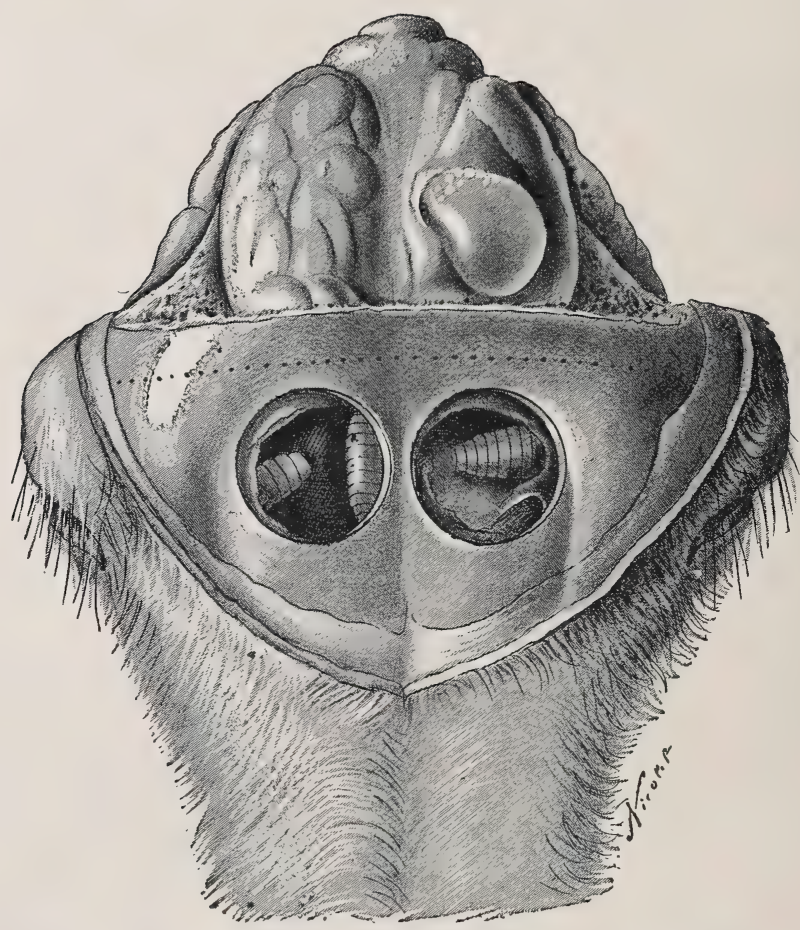

FIG. 214.-Brain of sheep. Coenurosis of the left hemisphere. Estrus larvæ exposed by trepanation.

dimensions of a hazel-nut. From this time the vesicles continue to increase in size until the death of the patient. Usually they become as large as a walnut, or even larger, and the interior contains hundreds of scolices, each showing a head.

The cystic phase only develops completely in animals whose brains contain a limited number of cysts, and in such the signs of turn-sick are well developed. In others, where the numbers are large (ten to fifteen embryos or more), death occurs during the primary stage, usually towards the end of the first month, in consequence of acute encephalitis and without any of the symptoms of turn-sick.

The number of animals attacked is sometimes enormous. Moussu 
has recorded cases where fifty, one hundred, and even four hundred lambs of one flock were affected. The enormous mortality in such cases is very apt to cause errors in diagnosis. Conurosis occurs most frequently during rainy seasons, moisture favouring the preservation of

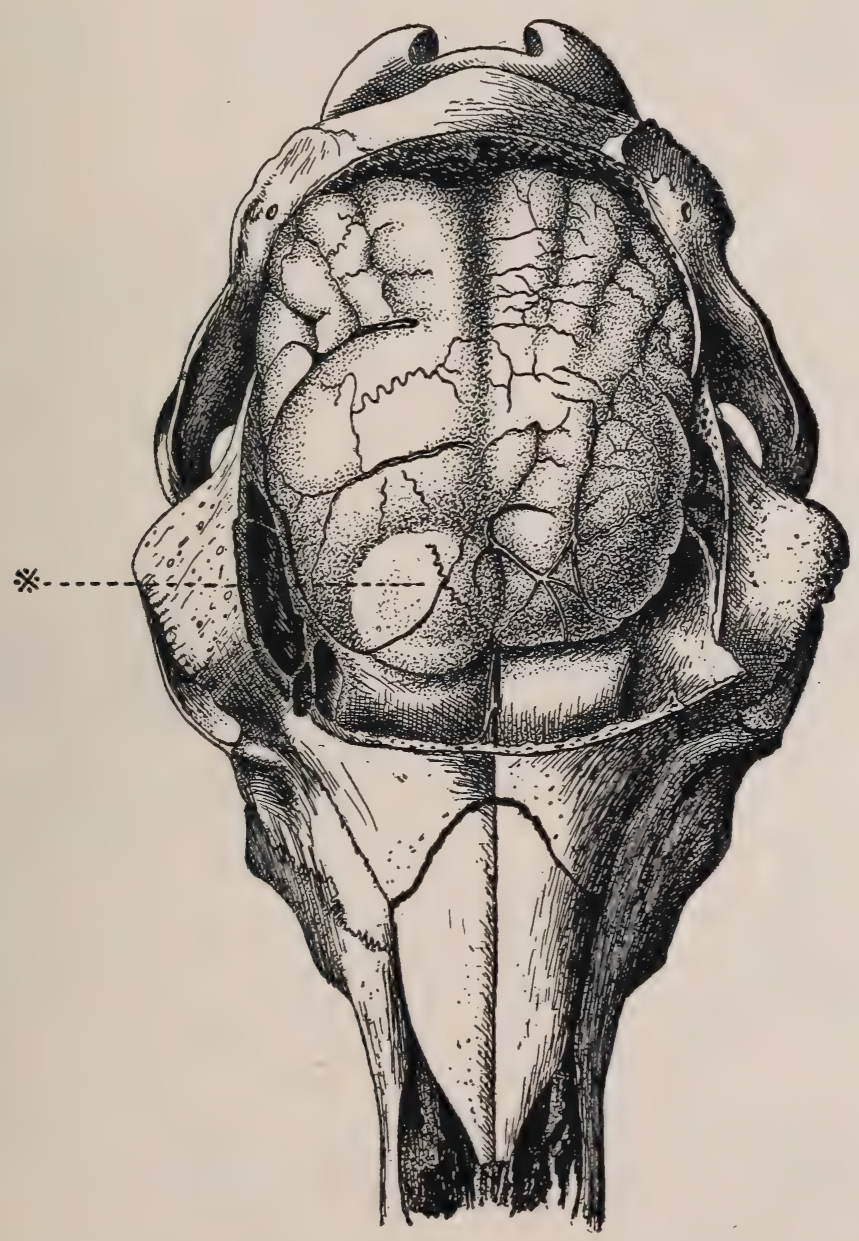

FIG. 215.-Skull of a sheep showing the brain infested with a gid bladderworm (Cœnurus cerebralis). Two-thirds natural size.

the eggs. Young animals become infected, particularly during the spring and autumn, more rarely in the summer, as prolonged desiccation, say for a period of twelve to fifteen days, destroys the vitality of the eggs, but animals may become infested at any time through drinking contaminated water. Moussu has seen cœnurosis (acute encephalitis) from the last-named cause in the middle of January. 
Symptoms. First phase-Disseminated enceplatitis.-The symptoms vary with the phases of evolution of the parasite and of the disease which it causes. After the six-hooked embryos have penetrated to the brain, the animals affected lose appetite and show a certain degree of dulness, which is all the more marked inasmuch as the animals usually affected are young, and therefore should appear bright and alert. Then follow wasting and depression; the animals remain stationary for whole

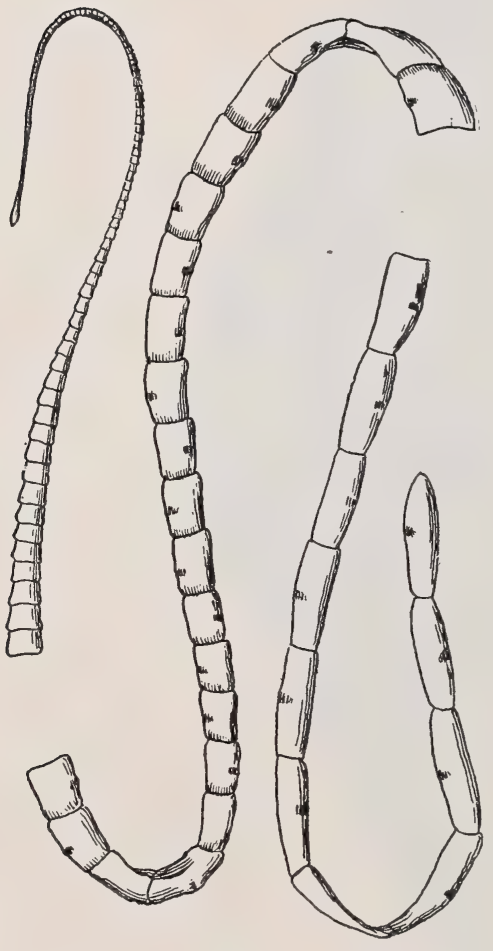

FIG. 216.--An adult gid tapeworm (Tæenia coenurus). Natural size. (After Railliet.)

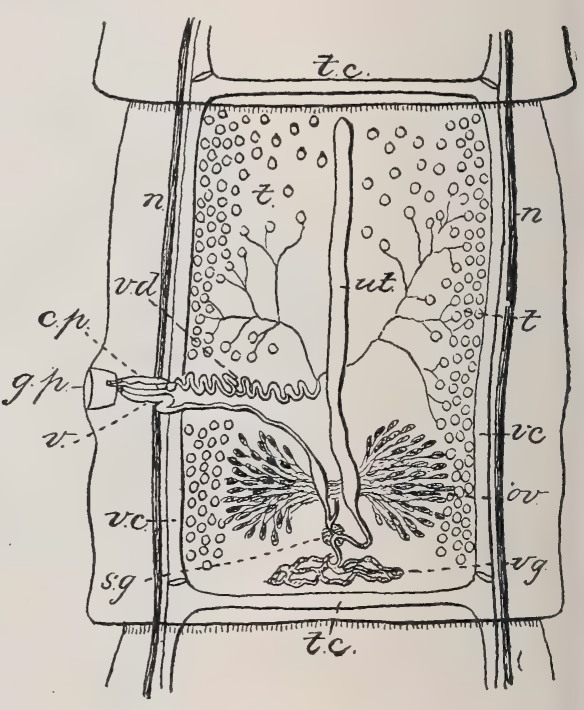

FIG. 217.- Sexually mature segment of the gid tapeworm (Tania conurus). $c p$, Cirrus pouch; gp, genital pore; $n$, nerve; ov, ovary; $s g$, shell gland; $t$, testicles; $t c$, transverse canal; $u t$, uterus; $v$, vagina; $v c$, ventral canal ; $v d$, vas deferens; $v g$, vitellogene gland. $\times 20$. (After Deffke.)

hours together, the head being carried low or inclined to one side. At this stage disturbance in vision and irregularities in movement may appear.

The eyesight is almost always affected, but the symptoms may vary widely. In some cases the patients seem to be absolutely blind, and strike against any obstacle in their way; in others the power of vision seems to be lost only on one side. All that can be discerned objectively is an inequality in the pupils, together with retraction or dilatation, convergent or divergent strabismus, nystagmus, etc. The humours of the 
eye appear infected, but examination with the ophthalmoscope reveals lesions of more or less extensive neuro-retinitis.

The visual disturbance is of central origin. The powers of movement may be affected in numerous ways, which at times are extremely difficult to estimate with accuracy. Sometimes the gait is uncertain, inco-ordinated, and hesitant; at others the animal shows lameness or loss of control over a front or hind limb, or over two limbs simultaneously (either the two front or hind limbs or the diagonal limbs), or it may be absolutely unable to stand.

It walks obliquely, or the front or hind limbs collapse; or again, it may persistently lie down, a fact which makes the shepherd think it is suffering from paralysis. On examination, however, no true indications of paralysis can be found; sensation and motor power are both preserved in a modified form.

Death is very frequent at this stage of the disease; the animals eat little or nothing, refuse drink, and die of exhaustion.

All this general disturbance is of central origin, and is due to disseminated parasitic encephalitis, but up to this point the seat of the disease is not yet clearly apparent.

Second phase.-T'urn-

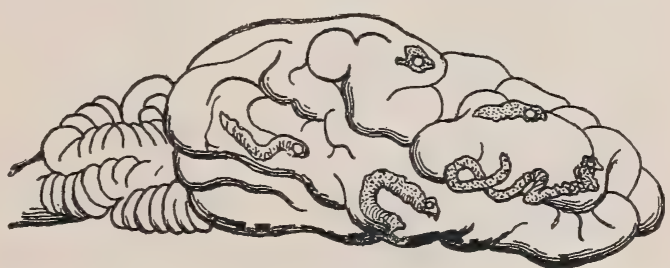

Fig. 218.-Brain of a lamb infested with young gid bladderworms (Conuruscerebralis). Natural size. (After Leuckart.)

sick.-The central symptoms are slow of development, and are due to the progressive growth of one or two, more rarely three or four, fertile vesicles. These are the true symptoms of turn-sick, and it is only after this phase of the disease has developed that the term becomes appropriate.

Left at liberty, the patient usually walks in a circle towards the right or left in an impulsive and irresponsible fashion. Sometimes it describes a circle, always of the same size. In other cases, on the contrary, it travels along a spiral track, getting further from or nearer to the centre as the case may be. The turning movement may become so accentuated that the animal appears to revolve as on a pivot, and if it is confined in a field or straw-yard its legs become caught in the litter and it falls to the ground.

Attempts have been made under these circumstances to discover the exact point of compression, i.e., the point at which the cyst exists, by noting the direction of the turning movement. The diagnosis, however arrived at in this way is frequently illusory, because it is not uncommon 
to find two or three vesicles, and in any case the most important information in regard to diagnosis is to be derived from the ocular symptoms.

When only one vesicle exists, the turning movement usually occurs towards the side on which it is situated, and the eye of the opposite side is affected with amaurosis.

If the cyst is situated near the olfactory lobes, the animal marches with a high-stepping movement and the head drawn back towards the

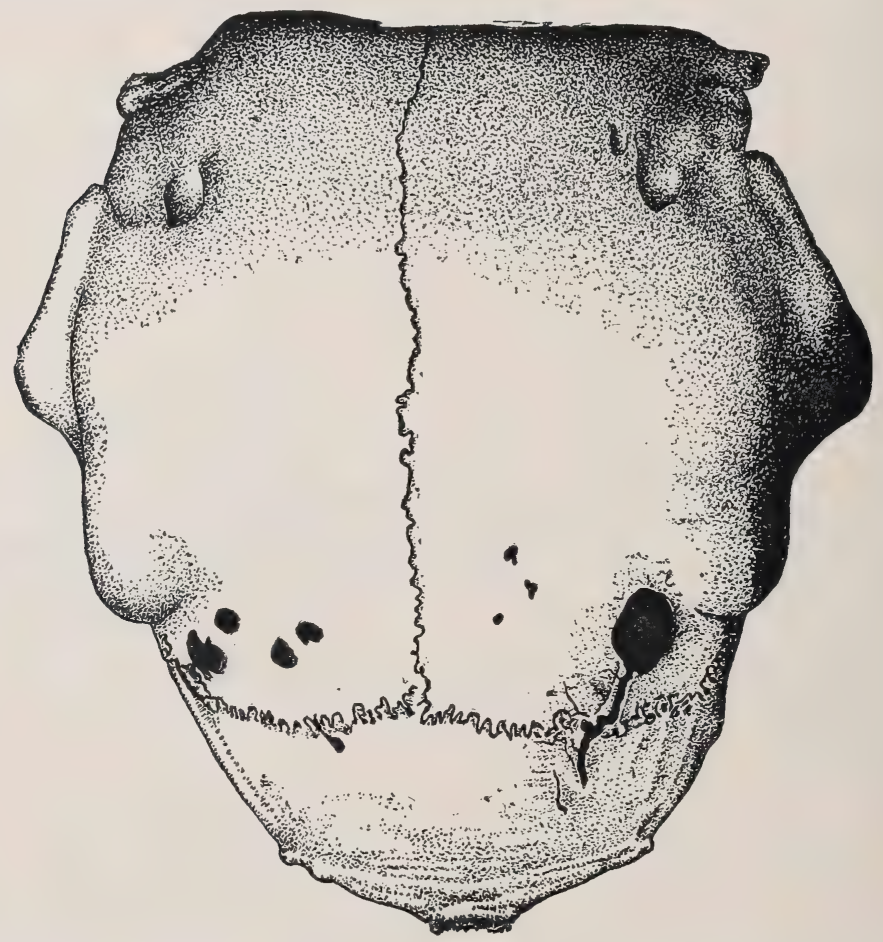

Fig. 219.- - sheep's skull, the hind portion thin and perforated, due to the presence of gid bladderworms (Conurus cerebralis). (After Dewitz.)

body. If the cyst is in the cerebellum the animal is incapable of moving, because it can no longer co-ordinate its movements. Finally, if the cyst develops in the occipital region, animals turn towards the wind, with the neck raised and the head extended.

At the moment when they fall to the ground they sometimes have epileptiform convulsions, grind their teeth, and salivate profusely. In a severe attack even death may supervene at this point.

Cœnurosis of the Medulla. The embryos may develop in the medulla oblongata as well as on the brain itself. Compression and atrophy of the medulla then give rise to true paralysis.

The animals exhibit paralysis of the hind quarters, unilateral paralysis 
only, or still less marked signs. Everything depends on the degree of development of the cysts.

Boyine Animals. Coenurosis in oxen is less important than in sheep. Moreover, it very rarely affects a large number of young animals belonging to one farm. Loss of appetite, dulness and depression are the earliest indications, as in sheep. The gaze seems fixed, the neck is held stiffly and almost rigidly, the animal shows a tendency to vertigo, pushes its head against a wall, or leans the head or neck on the manger or trough.

Inequality in the size of the pupils, amaurosis, hesitating and incoordinated movements may also be seen developed in different degrees, The animals have the appearance of horses suffering from "immobilité "that is, the very peculiar general condition produced by dropsy of the brain ventricles, or from encephalitis. They forget to eat or do not attempt to chew unless handfuls of food are thrust between the molars; they plunge the muzzle into a bucketful of water and do not drink, etc. They take little notice of what passes around them, although they may become greatly excited if an attempt is made to move them, to give them medicine from a bottle, or to set them at liberty, etc. Such attacks of excitement often end in vertigo and in the animals falling to the ground and showing epileptiform movements. All these symptoms may occur with extraordinary variations, due in reality to the peculiar position which the conurus occupies.

Second phase. If set at liberty during the first phase of the disease, the animal's gait appears only slightly disordered, but when a single vesicle has become well developed in one of the hemispheres (and this is usually the case with oxen), the symptoms of turn-sick appear as in sheep, and are equally varied. The patients seem impelled to move in a given direction, whatever obstacles may be in their way. It is not at all uncommon to see them thrusting their heads against walls or trees, falling into ponds or ditches, or attempting to force their way through blind alleys between hay or straw stacks.

After the cyst develops in the cerebellum, the animals are soon unable to move. They may be able to stand in one position, but on any attempt to move they fall.

Lesions. The lesions develop successively from the moment the embryos arrive in the mass of the brain. At first the six-hooked embryos only excite a slight disseminated encephalitis. Their course through the brain is marked by short, greyish-green caseous tracts, the thickness of 
a needle, which are readily discoverable in the superficial layers. Later these caseous deposits become absorbed, the lesions of disseminated encephalitis diminish and disappear, while a certain number of vesicles after partial development undergo atrophy and disappear. Before long nothing remains but local atrophic encephalitis caused by the development of the vesicles, and from this time the central symptoms begin to appear.

Diagnosis. When the turning movement has developed the diagnosis is generally easy, but it is more difficult during the first period, when encephalitis alone exists; or at least, it is very difficult at this period to discover whether the symptoms are attributable to encephalitis, meningoencephalitis, cœnurosis, tuberculosis, or to some injury.

Prognosis. The prognosis is grave, and very few animals recover.

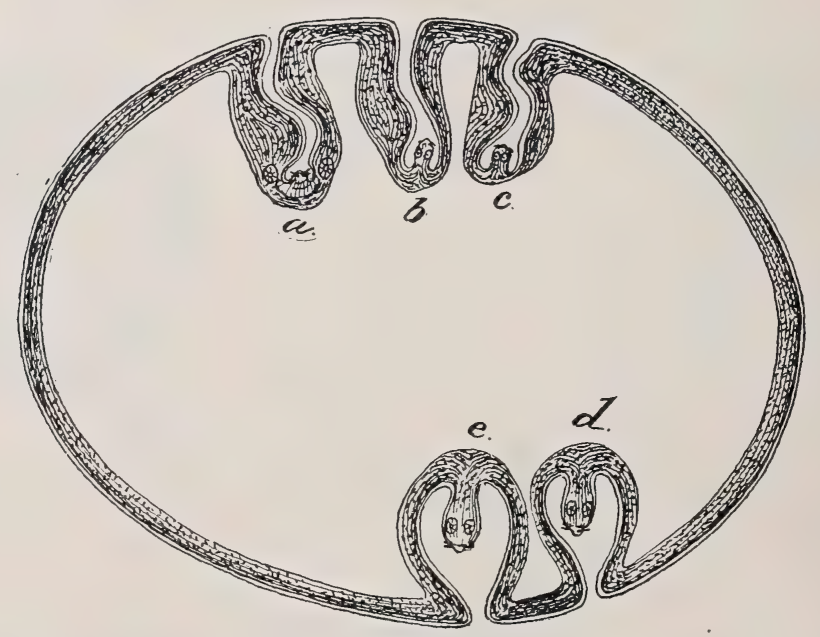

FIG. 221.-Diagrammatic section of a gid bladderworm (Ccenurus cerebralis). $a$, Normal disposition of scolex $; b, c, d, e$, diagrammatic drawing to show the homology between cysticercus and cœenurus. (After Railliet.)

Zürn estimated the cases of recovery in flocks at 2 per cent. In these cases the cysts degenerate and disappear.

Treatment. At the present moment there is no really practical curative treatment.

The best plan appears to be to trephine the skull and remove the cyst, provided its exact position can previously be determined. That, however, is the great difficulty. Its solution presupposes a perfect knowledge of the central nervous system and of the real purport of any apparent symptoms.

On the other hand, in sheep at least, the position of the cyst must always remain somewhat uncertain, because there are generally several, and the symptoms are of a mixed character. 
The only treatment, therefore, which would be likely to succeed is difficult to carry out, and more difficult in the ox than in the sheep because of the great development of the frontal sinuses.

It has been mentioned that under exceptional circumstances the cœnurus, if very superficially placed, may cause atrophy of the cranial wall in the sheep by the outward pressure it exerts, and that the points of least resistance may be detected by the touch. In such cases intervention is necessary and is greatly facilitated; but they must be extremely rare, and Moussu declares he has never seen one.

Hartenstein has suggested continuous cooling of the cranium by irrigation or by applications of ice, the local lowering of temperature serving to impede the derelopment of the coenurus. This method should be tried in animals of high value, and, if the symptoms have not yet become too alarming, recovery is possible. On the other hand, when the symptoms indicate the presence of an old-standing cyst, there is little chance of success. Moreover, the treatment could not be applied where a large number of animals are affected.

It is much better to send the animals to the butcher if they are in good condition. In Scotland, however-particularly in Caithnessoperation is said to be frequently practised with success.

Prophylaxis. In well-managed establishments it is easy to avoid the appearance of conurosis.

The development of this condition being due to the Trenia comurus of the dog, which passes ripe segments containing eggs with its fæces in the pastures, the first indication is to prevent the development of this tænia in sheep dogs, sporting dogs and house dogs, and the sole precaution required is to avoid giving them as food sheeps' heads containing the bladder rorms. But, as despite these precautions they may accidentally contract infection with Tcenia coenurus by eating the offal of slaughtered animals, it is a wise precaution to administer to all farm dogs, twice a year at least, a dose of some tænicide.

They should be starved and kept in for twenty-four hours, and should then receive a full dose of some energetic vermifuge, such as areca nut, kamala, kousso, powdered pomegranate root, extract of male fern, etc., followed by a purgative. The material passed and the fæces should be burned or mixed with quicklime. By these simple precautions the losses which formerly proved so heavy may entirely be prevented.

\section{"TREMBLING," OR LUMBAR PRURIGO, IN SHEEP.}

The above term is applied to a disease peculiar to sheep, and characterised by neuro-muscular disturbance, which always ends fatally after a longer or shorter period. 
Symptoms. Clinically the disease occurs in two forms, one termed the convulsive form, the other the pruriginous form. In the convulsive form the patients rapidly lose appetite, soon appear unable to stand, fall on their sides, and exhibit spasmodic contractions of certain groups of muscles. After a time the clonic contractions may give place to persistent rigidity. The patients are carried off rapidly in a week or two without other important symptoms. This disease appears to exhibit certain analogies with louping-ill, but these do not seem to be recognised in France, where it chiefly occur's.

In the pruriginous form the beginning of the attack is obscure, and only the shepherd is able to note anything unusual. 'The animals move in a jerky way, the hind limbs being lifted at times after the manner of a horse suffering from stringhalt. They are excitable, exhibit trembling movements when touched, with convulsive movements of the head, and present an anxious and vacant appearance. The development of these symptoms, which constitute what may be termed the first phase of the disease, is sometimes prolonged, and in winter may last from one to two months. In summer it rarely lasts longer than a week or two.

At this stage a special pathognomonic symptom develops, viz., intense and permanent pruritus of the hind quarters, which causes the animals to rub the croup against any projecting objects, walls, mangers, etc. 'l'hey thrust themselves backward against the object, and rub with such violence that the wool is torn away and the skin itself often excoriated, although the irritability appears to be in no wise diminished thereby. In the fold the animals sometimes rub against one another, making their sides raw, and bite themselves on the croup, the quarters, and the tail.

When the affected parts are touched with the hand, peculiar movements of the head and the lips are immediately excited, similar in character to those caused by mange. Up to this time the animals continue to feed, but they progressively lose condition, become weak in the hind quarters, and fail to keep up with the other animals in the flock. Their gait becomes hesitating; they move with a trotting step and appear semi-ataxic.

All these symptoms develop without fever, but become aggravated from day to day, until they end in paraplegia and death. This second phase lasts for a period of from two to four months in winter.

Lesions. No lesions can be detected on a simple examination of the dead bodies. Gilbert has mentioned a change in the blood; Trasbot, chronic inflammation of the pia mater and of the lumbo-sacral portion of the spinal cord. German writers have described sclerotic changes in the posterior portions of the cord. Moussu has carried out a large number of examinations, but has never found these lesions, either on anatomical 
or on histological investigation, and he considers that they are not by any means present in all cases.

Besnoit and Morel, who carried out a very remarkable anatomical and pathological study of the disease, used Nissl's staining method, but only found very discrete changes in the cord (vacuoles in the motor cells of the anterior horns). They found, however, significant lesions of neuritis in the peripheral nerves.

Causation. The cause is as yet little understood, and it is difficult to prove how the forms of neuritis described by Besnoit are brought about.

The disease was not known in France before the introduction of Merinos, and former investigators referred it to heredity, consanguinity, precocity, and even to sexual excitement. German writers declare that it seems more particularly to attack rams and the better-bred varieties. In reality, the disease occurs in all flocks, and in all kinds of sheep indiscriminately; it attacks ewe lambs and young castrated lambs as well as rams. Moussu is absolutely of this opinion, and for want of more precise information agrees with Trasbot, and, he believes, with Besnoit, that the symptoms shown may possibly be referred to a chronic intoxication.

Diagnosis. The diagnosis is difficult during the early stages, but when the pruritus becomes manifest there can no longer be room for doubt.

Cases of paraplegia might perhaps be mistaken for paraplegia due to cœnurosis, but in the latter case there is no pruritus.

Prognosis. The prognosis is extremely grave, observation having shown that all the patients die after a longer or shorter time.

Treatment. Until now treatment has proved absolutely useless, and it would seem the best course to slaughter the animals before wasting becomes marked. 


\title{
SECTION VI.
}

\section{DISEASES OF THE PERITONEUM AND ABDOMINAL CAVITY.}

\author{
CHAPTER I. \\ PERITONITIS.
}

Peritonitis, i.e., inflammation of the peritoneum, may attack any of the domesticated animals. It must, however, be regarded as an almost accidental and relatively infrequent disease. It is due to infection of very varying character, and from the clinical standpoint may assume one of two forms-acute peritonitis or chronic peritonitis.

\section{ACUTE PERITONITIS.}

The micro-organisms which produce peritonitis have not been the subject of special investigation in the domesticated animals, though the colon bacillus and streptococci, so frequently found in the female genital tract after parturition, seem to be the most frequent causative agents. Certain putrefactive organisms may also bring about the disease.

The peritoneum may become infected, and acute peritonitis ensue under various circumstances.

All operations in which the peritoneal cavity is opened, such as castration of the cow and of the sow, laparotomy, gastrotomy, enterotomy, etc., may be followed by acute peritonitis if performed without sufficient regard to aseptic precautions. Peritonitis then usually assumes an acute septic form.

Even simple puncture of the rumen, though usually quite harmless if carefully performed, may by followed by local or general peritonitis should food material escape from the rumen and find its way into the peritoneal cavity.

One of the most frequent causes is infection from the genital tract soon after parturition. Here the agents of infection are not introduced directly into the cavity, but find their way there in consequence of a 
diseased condition of the mucous membrane and the uterine walls. Ascending infections of this character and infections by contiguity of tissue may only give rise to local peritonitis, though in too many instances they become generalised.

Acute peritonitis may follow infection from the stomach or bowel, should a foreign body perforate the rumen or reticulum and pass backwards towards the peritoneal cavity, or a serious intestinal inflammation (enteritis, invagination, etc.) facilitate the passage of microbes through the thickness of the intestinal wall.

Abscess of the liver, suppurative echinococcosis, renal infection, pyelo-nephritis, acute cystitis, rupture of the bladder, etc., may in a similar way become complicated with acute peritonitis.

Finally, abdominal wounds may cause interstitial ruptures and lesions in the serous membrane, accompanied by local exudation (kicks, horn-thrusts, blows from cart-poles, ete.), and if microbic agents are brought within the region of the lesion by the general circulation or otherwise, peritonitis may follow.

Symptoms. At first the symptoms are vague and imperfectly defined, and diagnosis is always very difficult during the first few days, except in cases where there exists a lesion or a condition previously recognised as likely to become complicated with peritonitis.

The early symptoms comprise fever, loss of appetite, arrest of rumination, rigors, constipation, etc., but these symptoms only attain full significance when accompanied by what has been termed "peritonism."

The patient appears to be suffering from tympanites, as may really be the case, but the tympanites of the rumen and gaseous distension of the loops of bowel are not primary, and only result from the arrest of peristalsis. The primary condition is peritonism, i.e., distension of the peritoneal cavity, this being indicated by a symmetrical fulness of the right and left flanks.

The patients suffer from dull colic, and from this time always assume an attitude indicating pain. They remain in one position, with the back arched, the limbs gathered together, and the lower abdominal wall shortened. The face expresses suffering, the respiration is short and rapid and of the costal type, movement is painful and causes groaning, and the animals do not shrink when the lumbar region is pressed upon.

Palpation of the abdomen causes pain, and if practised at certain points may be followed by groaning. This method of examination, however, gives no further information, because the abdominal wall is rigid, tense, and as though tonically contracted.

Percussion is followed by tympanic resonance in the right and left 
upper zones, due to accumulation of gases of fermentation, and to distension of the peritoneal cavity itself. Towards the lower parts, however, percussion produces a dull sound. The presence of liquid can here be detected by the manner in which impulses are transmitted, particularly at the period of crisis and when much exudation exists.

Abdominal auscultation shows that the digestive movements are arrested. Peristaltic movement ceases, and the movements peculiar to the rumen and to the progress of food through the intestine are absent. Fermentation sounds, however, can be detected.

The heart beats are strong, rapid and violent, and yet the pulse remains feeble, though the artery is tense.

At a later stage, when the disease becomes aggravated, pain is less acute, depression is extreme, the animals no longer even drink, the abdominal wall becomes relaxed, and diarrhoea is succeeded by constipation. Palpation of the abdomen is less painful and does not cause groaning, but the pulse becomes feebler, much more frequent, imperceptible, and at last the animal dies from intoxication and exhaustion, caused by the fever and pain.

When peritonitis is due to rupture of the intestine or escape of alimentary material from the rumen into the peritoneal cavity, as may occur after puncture of the rumen or gastrotomy, etc., fever is not always very marked. The temperature may even fall below the normal point. Some cases vary greatly from the type described as regards their development, but the important features are always present, and the difference is chiefly found in the course of the disease.

Diagnosis. The diagnosis is rather difficult, but when there is colic, together with persistent peritonism, exaggerated sensitiveness to palpation and arrest of the functions of the digestive apparatus, there is little room for doubt.

Prognosis. The prognosis of acute peritonitis is very grave.

Lesions. The lesions vary with the primary cause (traumatism, metritis, suppurative echinococcosis, foreign bodies escaping from the digestive tract into the peritoneal cavity, etc.).

The parietal and visceral layers of the serous membrane are always inflamed, vascular, roughened, dull, and in places covered by vegetations. Between the loops of intestine and in the peritoneal pockets there are discovered more or less numerous and more or less thickened false membranes, presenting the characteristics of the false membranes seen in acute pleurisy.

The liquid varies in quantity and in colour, being sometimes lemonyellow, sometimes purulent, sanguinolent, or even blackish, and of putrid odour. 
The lesions may appear more marked at a particular point, such as the uterus, rumen, hypochondrium, etc., and the intestinal loops may become partly fixed in position by false membranes. In time these false membranes may solidify and undergo transformation into fibrous tissue.

Treatment. Treatment is generally useless in cases where peritonitis results from rupture of the bladder or intestine or from eventration. Complete and perfect cleansing of the infected abdominal cavity is impossible in large animals.

In other cases the animals should be left completely at rest, and purgatives should be avoided. Movement or the administration of purgatives provokes peristalsis, and, as a consequence, almost inevitably leads to generalisation of a lesion which might otherwise have some chance of remaining localised, as in pelvi-peritonitis and peritonitis due to foreign bodies issuing from the rumen or reticulum. If the movements of the intestinal loops disperse the septic liquids beyond the points originally injured, the whole cavity becomes inoculated and generalised peritonitis is set up.

Emollient and diuretic drinks containing opium, and oatmeal or linseed gruels, have the advantage of soothing the colic and preventing stagnation in the bowel. These should be given from the first and solid food entirely avoided.

The sides of the abdomen should be mildly stimulated, provided the operation does not give rise to undue pain and cause the animals to struggle. Vesicants are preferable to mustard, though mixtures of mustard and linseed meal may be used, and, if found advantageous, can be repeated.

Mercurial salts, though much used in earlier times, are now entirely given up. Diuretics, such as bicarbonate of potash, nitrate of potash, alcohol, and acetate of ammonia, should be used, according to circumstances.

Aseptic washing-out of the peritoneal cavity would be advantageous, but in large animals cannot easily be effected.

\section{CHRONIC PERITONITIS.}

Causation. Chronic peritonitis may occur as a termination of the acute form, but it may also develop gradually as a result of disease of the kidney (pyelo-nephritis), of the uterus or ovaries (chronic metritis, tumour of the ovary), of the liver (suppurative echinococcosis), or of any other lesion in neighbouring parts which is capable of setting up continued irritation.

It also accompanies tuberculosis of the peritoneum, cancer of the peritoneum, chronic disease of the bladder, etc. Further, it appears,

D.c. 
but more rarely, in certain chronic diseases, such as chronic dysentery and lymphadenitis.

Lesions. The lesions consist of local thickenings of the peritoneal layers, and numerous papilliform vegetations scattered very irregularly over the parietal peritoneum, mesentery, epiploon, etc.

If the disease has existed for a long time, fibrous bands or solid adhesions may be discovered, connecting various parts of the digestive apparatus with one another, or with the abdominal walls.

Sometimes the intestinal contents seem almost entirely adherent to the abdominal walls.

The primary lesions of the liver, spleen, kidneys, or genital organs, from which the disease originated, are also found.

The quantity of exuded liquid varies greatly; sometimes there is a great quantity of a transparent or lemon-coloured liquid, resembling that of ascites. In other cases the liquid is scanty, and may be confined between layers of bowel, which are connected by an inflamed layer of epiploon.

These old-standing lesions cause atrophy of the abdominal organs, contraction of the intestine, and sometimes true obstruction.

In chronic tuberculous peritonitis the adhesions between the intestine and the abdominal walls may be enormous. The peritoneum is generally covered with great masses of tuberculous new growth, while the mesenteric and sublumbar lymphatic glands are attacked.

Symptoms. The disease develops without marked fever or grave interference with the chief functions, and the first approach of the disease may, therefore, easily be overlooked. Chronic peritonitis, moreover, may remain strictly localised.

When the disease assumes the ascitic form the dominant sign is readily detected. Where new membranes form the principal lesions the symptoms are much less definite, and the existence of disease is chiefly indicated by digestive disturbance, such as diminished peristalsis, the occurrence of colic, diarrhøea, ete.

It is well to remember, however, that these troubles often follow an ascitic stage, which may gradually disappear owing to the fluid becoming absorbed. Even in the fibrous form, where the intestines appear completely glued together by adhesions, the volume of the abdomen is increased and the belly is deformed, as in ascites.

In time patients suffering from primary lesions of an important internal organ are affected in their digestion, lose flesh and become anæmic, and finally eachectic.

Diagnosis. The diagnosis is by no means easy, particularly in the fibrous forms, owing to the great difficulty of discovering the primary lesion. 
Prognosis. The prognosis is grave, though it must not be regarded as necessarily fatal. In cases resulting from genital diseases, and in localised chronic peritonitis resulting from persistent, but not excessive, mechanical violence, complete and perfect recovery may occur.

On the other hand, in cases of chronic lesions of the liver, kidneys, heart, etc., and in tuberculosis, carcinoma, ete., recovery cannot be expected.

Treatment. Treatment should be directed towards combatting the chronic inflammation. With this object resort may be had, when necessary, to persistent stimulation of the sides of the abdomen, mild blisters and mustard plasters, or friction with turpentine.

The food should be easy of digestion, and of first-rate quality. The most useful drugs comprise mild, unirritating diuretics, general stimulants, and tonics.

Animals affected with incurable lesions should not be treated.

\section{ASCITES.}

True ascites consists in dropsy of the peritoneum, unaccompanied by inflammation of that membrane, or by the presence of infectious microorganisms in the transuded liquid. Properly speaking, it is not a morbid entity, but only a symptom common to several very complex diseases.

Causation. The diseases which produce it may be set forth under five principal heads :-

(1.) Cardiac affections in general, particularly chronic lesions of the heart, interfering with venous circulation, and causing prolonged stasis of blood in an organ or tissue.

(2.) Pericarditis due to foreign bodies, and the various forms of pseudo-pericarditis, i.e., lesions in the neighbourhood of the heart, causing compression of that organ and of its vessels.

(3.) Generally speaking, all lesions which interfere with the return circulation, particularly lesions of the liver (distomatosis, echinococcosis, and interstitial hepatitis). These produce compression of the portal vein or other obstacle to circulation, and the transudation is exclusively localised in the abdominal cavity. The connective tissue does not become infiltrated.

(4.) Diseases of the kidneys (nephritis, pyelo-nephritis), which secondarily produce cardiac disturbance.

(5.) Gestation, which causes compression of certain digestive viscera, and of certain veins of the pelvic cavity.

Ascites was formerly regarded as always forming a complication either of anæmia or of hydræmia. We now know that the primary cause of these three collections of symptoms (ascites, anæmia, and 
hydræmia) is the development of certain chronic wasting diseases or chronic lesions of the heart, liver, and kidney, which act and react upon each other.

Symptoms. True ascites is unaccompanjed by fever. The condition develops slowly, insidiously, and therefore escapes notice at first. Only when the exuded liquid is present in considerable quantities is the condition apparent. The symptoms are similar to those of ascites following chronic peritonitis.

The transuded liquid progressively accumulates in the peritoneal cavity, the lower portion of which it distends. When the animal is viewed from behind the enlargement appears symmetrical, despite the position of the rumen. The intestinal contents float on the liquid and are thrust upwards towards the lumbar region. On palpation, the abdominal cavity seems unusually full, the tension differing in proportion to the quantity of liquid. The accumulation of liquid may become considerable and interfere with respiration, circulation, and movement. Very marked anæmia always exists, the mucous membranes are extremely pale, the respiration is rapid, the pulse feeble, all these symptoms being consequent on the primary disease of the heart or liver. Percussion of the lower part of the belly produces a dull sound. On the left side this dulness often extends from the linea alba as high as a horizontal line, uniting the external angle of the ilium and the hypochondriac circle. On the right it is bounded by a horizontal line. Percussion or, better still, palpation provokes on one side of the abdomen a wave or impulse of the liquid, perceptible to the touch or even to the view at the opposite side.

Diagnosis. In general diagnosis is easy, thanks to the slowness with which the disease develops.

Prognosis. The prognosis varies in each case, more especially according to the more or less marked debility of the animal. Ascites due to gestation is usually of a very simple character, but if it is the result of pericarditis produced by a foreign body, or of nephritis, the outlook is very gloomy; lesions of the kidney in particular showing little tendency to recovery. Finally, the prognosis varies when the ascites follows disease of the liver, for certain exceptional cases have been noted in which an attack of hepatitis has led to the disappearance of the transudate.

Lesions. T'he lesions peculiar to this disease are very trifling. Transudation takes place without inflammation of the peritoneum, although the veins of the abdominal cavity are abnormally dilated. The abdominal wall is thin and distended, and the tissues are colourless as though soaked in water. The cavity is distended with a clear lemon-coloured albuminous liquid free from blood corpuscles. 
Treatment. The treatment must vary according to circumstances, i.e., having regard to the primary cause. Ascites due to gestation, which is always slight, calls only for simple hygienic treatment; but when the disease is attributable to lesions of the heart, pericarditis, or chronic affections of the kidney or liver, it is generally incurable in common with the original lesions themselves.

If, finally, no clearly defined cause can be detected, or if the ascites is due to chronic peritonitis, treatment should be attempted. The first step may consist in evacuation of the liquid for the purpose of reducing the excessive pressure on the diaphragm and facilitating respiration. For this purpose an aseptic puncture is made with a fine trocar on the right side of the abdomen in the flank region, about equidistant from the umbilicus and the loose flap of skin in front of the stifle. The absorption of liquid may afterwards be assisted by administering diureties, such as digitalis, bicarbonate of potash or nitrate of potash, and by giving lukewarm drinks, tonics, etc. In Germany injections of pilocarpine have been suggested, but it is doubtful whether they have proved satisfactory.

\section{PERITONEAL CYSTICERCOSIS.}

The above name has been given to a parasitic disease caused by the infestation of young animals, such as calves, lambs and young pigs, with embryos of the Tania marginata of the dog.

Symptoms. Peritoneal cysticercosis is often of so mild a character, and the number of embryos which penetrate the body so small, that in the majority of cases there are no visible symptoms. It is not until the meat comes to be dressed by the butcher that little cysts (Cysticercus tennicollis are discovered in the abdominal cavity.

Unfortunately, in exceptional cases it may also happen that the number of embryos in the abdominal cavity is so great as to produce lesions of acute hepatitis, acute peritonitis, and sometimes pleurisy. These grave forms are more common in young pigs and lambs.

The animals appear dull, feeble, exhausted and without appetite, but exhibit marked thirst, lose flesh and become anæmic in a few days. Soon afterwards they show symptoms of acute peritonitis, with exudation of fluid, and death may follow in a week or two.

In cases where infestation is less marked, the animals may exhibit only progressive anæmia, without well-developed symptoms of peritonitis, until death occurs.

Lesions. On post-mortem examination a sero-sanguinolent exudation is seen, together with more or less numerous false membranes, and a varying number of young cysticerci floating freely in the liquid or enclosed in the folds of the mesentery. The cystic vesicles are spherical, 
ovoid, or elongated, and translucid or opalescent. They are some millimetres in diameter, and in some cases are very numerous, ranging from a few hundreds up to several thousands, but in others comparatively few.

The liver shows signs of intense hepatitis, caused by embryos burrowing into its tissue.

Causation. The causes are limited to a single fact, viz., ingestion of the eggs of Trenia marginata, which are spread over the fields in the excrement of dogs suffering from that parasite.

Diagnosis. The diagnosis can only be arrived at by a post-mortem examination, when cysticerci in various stages of development are discovered.

Prognosis. The prognosis is difficult to indicate, because everything depends on the intensity of the infestation.

Treatment. No curative treatment is possible, direct action on the developing parasites being impracticable. Nevertheless, some patients survive, and after having shown grave general disturbance may gradually improve.

The only efficacious treatment is of a prophylactic nature, as in cœnurosis and echinococcosis. Dogs suffering from tæniæ should periodically be treated and freed from their parasites. 


\title{
CHAPTER II.
}

\section{HERNIAE**}

\section{CONGENITAL HERNIE}

\author{
PERINEAL HERNIA OF YOUNG PIGS.
}

This variety is very common in young pigs, on account of their anatomical peculiarities ard of the persistence and enlargement of the inguinal canal. Loops of intestine, impelled by their own weight, accumulate at the lowest point and readily pass into the canal.

It is usually when the little pig begins to eat, i.e., a fortnight or three weeks after birth, that the symptoms become plainly apparent.

The hernia is indicated by a swelling which commences in front of the pubis and extends backwards behind the hind limbs. When the herniated loop of intestine is examined by palpation, the presence of liquid in it can be detected, particularly after a meal, while a characteristic gurgling sound is heard.

Diagnosis. To confirm the diagnosis, the animal is placed on its back, whereupon reduction as a rule is easily effected. As soon as the animal rises again the hernia returns.

The prognosis is not grave.

The treatment is exclusively surgical, and the hernia can be reduced and castration performed at one and the same time. The animal being placed on its back and firmly held, an incision of about 2 to 3 inches in length is made in the inguinal region, dividing the skin and subcutaneous connective tissue only. The vaginal sheath is then completely isolated, the hernia reduced, and a ligature applied to the sheath and the spermatic cord close to the abdominal wall. The testicle is then removed.

If adhesions have been set up, which is quite exceptional, the vaginal sheath is incised and the loop of adherent intestine liberated, when it can readily be returned. The vaginal sheath and spermatic cord are then twisted as high as the level of the inguinal ring and tied with catgut. To prevent this ligature becoming displaced, it should be fixed

* For a fuller description of herniæ and their treatment, see Möller and Dollar's "Regional Surgery," pp. 263-309. 
by passing a sterilised thread through it and through the mass of tissue; the hernial sac should be divided immediately below. In order to ensure greater security, it may even be desirable to pass a suture through the margins of the inguinal ring.

\section{Umbilical Hernia.}

Umbilical hernia is less common in young animals of the bovine, ovine and porcine species than in the foal, and when existing almost always disappears at the period of weaning. The rumen then assumes its full development, the loops of intestine are displaced and thrust towards the sublumbar region, and the hernia disappears. The same is true of the young pig, the development of the stomach producing the same favourable result.

In the rare cases where this hernia is not reduced spontaneously, it may be necessary to utilise the methods so frequently employed in the foal, and, despite the number of these, there are only two which can thoroughly be relied on to give good results.

In the first, irritants are employed.

Subcutaneous injections of concentrated solution of common salt, filtered and sterilised, or 10 per cent. solution of chloride of zine produce enormous engorgement of the connective tissue, which thrusts back the herniated loop of intestine and later causes the development of very resistant fibrous tissue, which prevents the hernia returning.

To ensure this result, however, it is indispensable that perfect asepsis should be observed in the injections, for if germs are introduced severe suppuration occurs at the point of injection. The injections are made at four opposite spots in the subcutaneous tissue surrounding the hernia, 1 to 2 drachms of saline solution being injected at each spot; of the chloride of zinc solution half a cubic centimètre is used. This method is only of value in small herniæ, which may sometimes be cured by the application of sinapisms alone.

The second method is applicable to larger herniæ, and aims at destroying the hernial sac.

The application of clams is simplest, and can be recommended. The patient is placed on its back, reduction is effected, the hernial sac is drawn upwards vertically, and the clams placed as near the abdominal wall as possible, after care has been taken that no portion of the intestine is included in the sac. The clams are kept in place by a suture passed through the neighbouring tissues.

In other cases where a radical cure is necessary, because of adhesions within the hernial sac, the patients are similarly placed on their backs, the hernial sac is opened aseptically, the adherent parts liberated, the herniated portions of intestine reduced, and the hernial ring sutured 
with sterilised strong silk, the skin being afterwards brought together with silk sutures after removal of the sac itself. A surgical dressing can then be applied to the umbilicus. The patients should be carefully dieted.

When the hernial ring is large and its lips widely dilated, the silk sutures, even when supported by secondary sutures, sometimes cut. through the tissues and do not achieve the desired result.

Degive's method (see "Möller and Dollar's Regional Surgery," p. 304) can then be employed. The hernial sac is opened under antiseptic precautions, in order to break down any existing adhesions, and the skin and edges of the hernial ring are transfixed with packing needles about 8 inches long. Above these is adjusted a clam, which is closed, by means of a screw and firmly secured. The packing needles are then replaced with horse-shoe nails, the points of which are bent round. In about a week the necrotic tissue falls away, and recovery occurs even in severe cases in which previous treatment had failed.

\section{ACQUIRED HERNIE.}

Acquired or accidental herniæ are not serious, and only deserve to be studied in so far as they affect organs contained within the

Fig. I

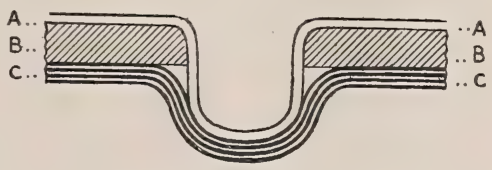

Fig. 2.

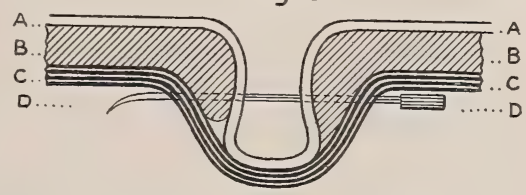

Fig. 3.

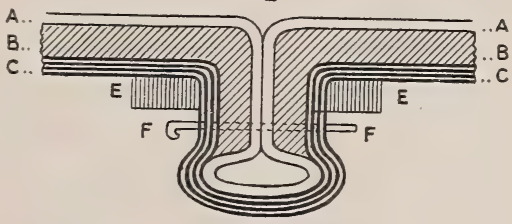

FIG. 222.--Schema illustrating Degive's operation for umbilical and ventral herniæ. A, Serous; B, musculoaponeurotic, and $\mathrm{C}$, cutaneous coats of the hernia; D, the special needle in place; EE, clams; FF, nails. The three figures show the successive stages of the operation.

abdominal cavity. They may result from violence, or may occur without the intervention of any external cause.

Traumatic herniæ may occur at any point in the abdominal wall. Under the influence of a violent blow from a waggon pole, a horn thrust, a kick, a fall, etc., the muscular tunic of the abdominal wall is injured and becomes fissured in the direction of its fibres. The peritoneum is rarely affected. Being pushed outwards by the digestive viscera, however, the peritoneum projects into the muscular layer, distends it, separates the layers of subcutaneous tissue, and finally forms a distinct hernia. 
The consequent disturbances are more or less marked and the lesions more or less variable, according to the part affected. In the lower region fissure of the abdominal wall affects the rectus abdominis, obliquus abdominis and transversus abdominis, and on the right side gives rise to hernia of the abomasum or small intestine, on the left of the rumen. In the lateral regions muscular fissures can be produced only in the transversus and obliquus abdominis muscles. Hernia of the rumen is rare on the left side. On the right side hernia of the intestine is more readily produced.

In all cases where hernia is suspected, the hernial orifice should be examined. Its situation will at once show which organ is affected.

Spontaneous herniæ are very rare in the domesticated animals. They occur only in aged animals, and various reasons have been suggested to explain their appearance. Certain herniæ of this character are only found in old female animals which have borne a considerable number of young. Repeated gestation produces elongation and relaxation of the muscular fibres from the weight of the foetus and its envelopes. In time, the abdominal walls become thinner and thinner under the weight of the viscera, and thus facilitate the slow formation of a hernia. Certain practitioners consider that some of these spontaneous herniæ are due solely to the pressure produced by the distended viscera, as for example in greedy-feeding animals. In such cases the weight of the viscera would cause, as in the previous case, a certain degree of anæmia and emaciation of the abdominal muscles.

These spontaneous ventral herniæ are due in reality to changes in the nutrition of the abdominal wall, the exact cause of which it is difficult to ascertain. The elastic tunic becomes atrophied, and ceases to act as an automatic girth; the muscular wall gradually becomes sclerosed from the white line towards the sides, and having lost its elasticity becomes distended and thinned.

These changes are not exclusively caused by old age, for they may be found even in young animals.

Nothing can be done in cases of spontaneous herniæ. The qualities originally pertaining to the abdominal wall cannot be restored, and treatment is confined to applying suspensory bandages, and, where possible, preparing the animals for slaughter.

\section{HERNIA OF THE RUMEN.}

Causation. Hernia of the rumen is, as a rule, of traumatic origin, and always occurs in the left flank, either in the lower or middle regions. Cases of spontaneous hernia of the rumen have been observed in very old and anæmic animals, as well as in females which have borne many 
young and which have suffered from spontaneous progressive hernia of the uterus.

Symptoms. The symptoms are the same in all herniæ. Immediately after the injury the abdominal organs show a tendency to escape in the direction of least resistance. A fold of the rumen passes through the muscular fissure, and a swelling soon becomes visible externally, which alters the contour of the abdomen. Most frequently at this time traces of the injury can be detected on the surface of the skin, either the linear trace due to a horn thrust, the ill-defined lesion due to a kick, or what not. There follows rapid swelling, which results from the inflammatory reaction due to rupture of small vessels within the muscle. A certain amount of sanguineous exudation and of cedematous swelling occurs, and may at first suggest the existence of an abscess of the abdominal wall. At the same time there is more or less fever, which may continue for a few days, but the swelling seldom lasts very long; in two or three days even, it becomes reabsorbed, commencing at the upper part and diminishing progressively downwards.

Henceforth the hernia alone remains.

It is soft, compressible, and sometimes susceptible of reduction. On palpation, the operator feels a rupture extending through the tunic and the abdominal wall, sometimes even through the muscular tissue of the rumen, in cases where the skin is neither perforated nor torn through. The mucous membrane of the rumen is rarely ruptured.

Whether or not the peritoneum is injured, the rumen presses between the lips of the wound, thrusts back the skin, and separates the connective tissue, thus setting up local irritation and oedematous swelling. The rumen may contract more or less close adhesions with the abdominal wall, and even with the subcutaneous tissues.

Afterwards, when the exudate has been reabsorbed, palpation reveals a different condition of things. The mass is uniformly fluctuating or semi-fluctuating, and is surrounded at the base by an indurated ring of very varying dimensions. The final indication-which, however, is not invariably seen-deserves attention, viz., the change in volume of the hernia at different moments, particularly during meals. This change in size only occurs if the hernial orifice is large.

In cases of spontaneous hernia of the rumen, the condition is not fully established at first. It is always progressive, and the lesion is situated in the lower abdominal region. It increases in size from day to day, from week to week, whilst the animals lose appetite and flesh. Spontaneous herniæ are never accompanied either by exudation, engorgement, fever, or traces of mechanical injury.

When only slightly developed, herniæ do not threaten life, a fact which often prevents the owners troubling about them. Progressive 
herniæ may become of considerable size, and two cases are recorded in one of which the opening of the hernial sac was $13 \frac{1}{2}$ inches in length and 18 inches in width, and in the other 28 inches in length and 24 inches in width. The latter is the largest ever recorded.

Complications. Complications are not always grave. If the hernia is little marked the function of the rumen is not greatly affected and its rhythmic contraction continues. When the original injury has caused rupture of the muscular tissue of the rumen, and the mucous membrane has passed into the opening, it may become strangulated and gangrenous.

Finally, if the mucous membrane has been torn at the same time as the muscular tissue (which is very uncommon), alimentary material may escape into the subcutaneous connective tissue, setting up either cellulitis and death by infection, or suppuration; abscess formation and rupture towards the exterior, followed by a persistent sinus; or again septic peritonitis, and death.

The same results may ocour when the hernia is in a very low portion of the abdomen ; food accumulates in it, becomes stagnant there, sets up local irritation and inflammation, and sometimes abscess formation with external discharge, followed by fistula of the rumen.

Gastric fistula without secondary complications is compatible with life, and even with fattening for slaughter, provided the peritoneum covering the rumen becomes attached to the opposing surface of peritoneum around the perforation. The fistula is then surrounded by a circular mass of fibrous tissue, forming a kind of sleeve.

Lesions. The lesions are the same in all herniæ. They consist primarily in rupture of the abdominal wall, and, later, of sero-sanguinolent infiltration of the margins of the wound, similar to that accompanying the formation of an abscess. Hernial swellings are of very varying size. Apart from cases similar to those above described, the swelling may be simply an inch or two in diameter, or it may attain the dimensions of a hen's egg or even of a man's fist.

When the abdominal tunic only has been ruptured, as is most frequently the case, the peritoneum is thrust outwards and forms a cavity, the hernial sac. This sac is absent when the peritoneum is ruptured. Little by little the surrounding connective tissue forms a pseudo-serous hernial sac. But, nevertheless, in some cases there may be found, immediately under the skin, the mucous membrane of the rumen in a state of congestion and ready to become gangrenous.

Diagnosis. Easy in all cases.

Prognosis. Very variable. In the case of small herniæ situated in the lateral regions of the abdomen the prognosis is not very grave. If, however, the rupture is wide, and situated in the lower portion of the 
abdominal walls in a dependant position, the hernial swelling steadily grows in size in consequence of the weight of the food which is constantly thrust in this direction by the contraction of the rest of the rumen, and recovery is impossible. The only resource is to fatten the animals as quickly as possible for slaughter.

\section{HERNIA OF THE ABOMASUM.}

Causation. This condition is due to causes similar to those above mentioned, including mechanical violence. It is rare in adults, but much commoner in young animals, especially in sucking calves, where the abomasum is the most highly developed digestive compartment.

Hernia of the abomasum is produced essentially and almost exclusively by horn thrusts inflicted when calves attempt to suck cows other than their own mothers.

Symptoms. Hernia of the abomasum always occupies a certain position in the lower part of the right flank, or, rather, in the space comprised between the white line and the lower part of the circle of the hypochondrium.

The immediate symptoms are similar to those of hernia of the rumen. They include: progressive swelling, formation of a peripheral œdematous ring, interstitial sero-sanguineous exudation, which becomes absorbed after a few days; finally, the development of a hernia, formed as a rule by the larger curvature of the viscus, which is in direct contact with the abdominal wall.

The lesions are those common to all hernia, and usually include a partially healed wound.

Diagnosis. The diagnosis is easy, particularly in calves, and the possible existence of the condition should always be borne in mind when dealing with injuries of the right pre-umbilical zone. An abscess of the lower abdominal wall may occur at or near the umbilicus as a result of omphalitis or umbilical phlebitis, but it is readily distinguished from a hernia.

The prognosis is graver than in the case of hernia of the rumen, for the displacement of the abomasum interferes with its regular function. 'T'he prognosis varies, however, in accordance with the size of the hernia. If the rupture is small, there is some chance that the abomasum, on account of its longitudinal position, many penetrate but slightly into the fissure.

If, on the other hand, the rupture is large, the prognosis becomes very serious. It is sometimes best to slaughter the animal, if in good condition; otherwise an operation is necessary. 


\section{HERNIA OF THE INTESTINE.}

Causation. This is due to the same cause as hernia of the rumena blow which, while injuring the skin only to a trifling extent, damages the abdominal walls, and even the intestine itself.

Symptoms. The hernia is situated in the lower or lateral zone of the right flank.

The symptoms present some peculiarities. The loop of intestine which has passed through the aperture in the abdominal walls becomes distended by the accumulation in it of semi-liquid alimentary material, and, acting by its own weight, produces a hernial sac, which steadily grows in size. The skin being very mobile, and the subcutaneous connective tissue very loose, they readily yield and become separated. The inflammatory symptoms disappear, and are followed by a swelling under the skin, which is compressible all over, and can readily be reduced, whereupon it gives forth a gurgling noise, or a sound as of borborygmus. Reduction is easier when the animal is lying on its left side, or on its back.

Complications. Strangulation of the small intestine is the only serious complication in this form of hernia, but it is very dangerous. It occurs frequently when the rupture is somewhat highly-placed on the lateral portion of the abdominal wall, because the loops of intestine have a tendency to descend, thrusting away the skin owing to the weight of material which they contain.

The partially digested food is apt to accumulate in the herniated loop, and hernial engorgement, the first phase of strangulated hernia, rapidly occurs.

Fermentation is set up in the half-digested food, and putrid gases are generated. Thus the hernial sac becomes distended, the vessels are compressed, circulation is arrested, and gangrene supervenes.

At this time gurgling sounds and a certain degree of tympanitic resonance may be noted. These are followed by all the symptoms of intestinal strangulation-namely, intense colic, which suddenly disappears when the intestine becomes mortified, absolute loss of appetite, stoppage of rumination, constipation, suppression of defæcation, tympanites, and peritonitis.

The diagnosis is comparatively easy at an early stage, owing to the peculiar character of the soft swelling, which is easily compressible. At first there may be difficulty in distinguishing it from a collection of serous fluid, but the facility with which the swelling can be reduced removes any doubt.

The prognosis is always serious, on account of possible complications, due to strangulation of the herniated loop. When the hernia is 
chronic, reduction is much more difficult, there being, as a rule, adhesions between the intestine and the hernial sac.

\section{TREATMENT OF HERNIÆE.}

Numerous attempts have been made to treat abdominal hernia in bovine animals.

Irritant and vesicant applications to the skin have been recommended, with the object of producing a large swelling, and thus thrusting back the herniated mass into its proper position.

One of the most popular of these applications is nitric acid of a strength of $36^{\circ}$ Baumé, applied to the skin twice at an interval of ten days. Skilfully used, it gives good results in umbilical herniæ, but its effects in ventral herniæ are less certain. It causes slow mortification of the skin, abundant subcutaneous swelling, and produces an eschar, which separates in about a fortnight.

An ointment of yellow chromate of potash (1 to 8) has been recommended, and can be applied two or three times at intervals of eight or ten days.

Bandaging and various forms of local dressing have also been employed from time to time. Serres employed simple bandages similar to those used in cases of inguinal or crural hernia in human beings. These bandages have a pad, which is applied over the hernial opening, but their action is strictly palliative. They simply allow of the animal being kept a certain length of time for fattening.

When the hernia has been reduced recourse may be had to bandages saturated in melted pitch, care being taken to extend the dressing a considerable distance beyond the limits of the hernial opening. Successive layers of bandage are superposed across and across, and, to make the dressing more solid, the pads may be reinforced with a sheet of solid cardboard. This method only succeeds when the swelling is slight and is situated elsewhere than in the lowest portions of the abdomen.

Some practitioners prefer a cloth bandage after reduction. The bandage is ten to fifteen yards in length, and should be considerably wider than the greatest measurement of the hernial opening. Such bandages can easily be applied to calves, whose bodies are of regular shape, but in adults, in which the body is of ovoid formation, they prove faulty, and tend to slide backwards or forwards.

All these measures are merely more or less palliative and of temporary effect.

The only rational and radical treatment is surgical. This is clearly indicated when the hernia is recent and of small size. At a later stage, when fibrous adhesions have formed between the various organs, and reduction has become difficult, caution must be observed. Surgical 
treatment is always a serious matter, and should only be attempted in the case of valuable breeding animals, or those which cannot be sold for slaughter.

Young animals are kept without food for twenty-four hours and are cast on the side opposite the hernia; they can be placed on the right or left side, or on the back, as seems most convenient. The site of operation is disinfected, and the operation carried out with aseptic precautions. The skin covering the swelling is incised and, the margins of the hernial orifice having been examined, the sac is isolated. Next, an incision is carefully made, any adhesions which may exist are broken down and the herniated parts are reduced. It only remains to suture the wound with silk or catgut, bringing the lips of the fissure together. Finally the skin wound is firmly united, and a large suspensory bandage tightly applied.

If the hernia is of long standing, and is irreducible on account of numerous adhesions, operation may still be attempted. In that case the incision must be an inch or two longer, all adhesions should be destroyed, and the margins of the orifice need to be freshened so as to insure their uniting.

During the days following operation, the animals should have light food, principally gruel, mashes and cooked roots. But it must be borne in mind that this operation is serious, and may possibly be followed by eventration.

\section{DIAPHRAGMATIC HERNIA.}

The term diaphragmatic or mediastinal hernia denotes a condition in which certain of the abdominal viscera penetrate into the thoracic cavity. This displacement may be congenital, acquired, or accidental.

The accidental hernix are of traumatic origin, and are often caused by fractured ribs, which injure the diaphragm. The hernia is then purely diaphragmatic.

Congenital or acquired herniæ are more frequently mediastinal ; they occur exactly in the median plane as a consequence of fissure of the diaphragm above the ensiform cartilage, and cause a separation between the two layers of serous membrane enclosing the posterior mediastinum.

The region immediately behind the diaphragm in the ox being occupied by the large viscera-namely, the anterior conical portions of the rumen, the reticulum, the omasum, and the liver-diaphragmatic or mediastinal hernia is far from being common, though occasionally it may be discovered or at least suspected.

Causation. The causes of diaphragmatic and mediastinal hernia are closely connected with injuries in the region of the hypochondrium; with arrest in the development of the diaphragm; or with accidental vertical fissuring consequent on gestation or acute tympanites. 
The fissure seems most commonly to occur between the point where the œsophagus passes through the diaphragm and the ensiform cartilage of the sternum, in which case mediastinal herniæ most commonly supervene. As, on the other hand, the rumen, owing to its size, form and position, cannot readily be displaced, the reticulum and omasum are the viscera which most commonly pass into the thorax.

Symptoms. In true accidental diaphragmatic hernia visceral displacement only occurs on the right side, and symptoms of this are immediately apparent. The passage of the liver, reticulum, or omasum

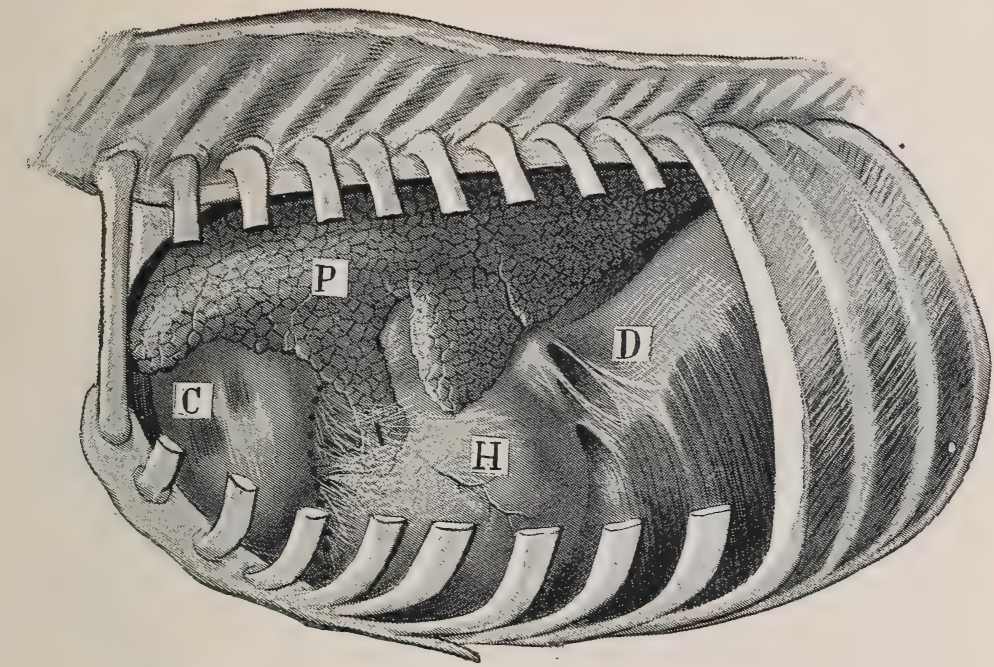

FIG. 223.--Intra-mediastinal diaphragmatic hernia (viewed in position from the left side). $\mathrm{P}$, Lung ; C, heart (displaced) ; D, diaphragm ; H, hernial mass.

into the right pleural sac compresses the lung, causes attacks of dyspnœa and acceleration of the heart's action.

Percussion may not reveal any important change, but on auscultation digestive sounds can plainly be heard within the chest.

The symptoms are far from being well defined. They may be more or less intense, and colic may or may not be present. Mediastinal hernia (Fig. 223) appears to develop slowly, and it is only by degrees that the viscera become displaced.

There is thèn no sudden change, no clearly marked disturbance, but simply a certain amount of digestive irregularity, together with loss of appetite, cessation of rumination, slight indigestion, and moderate tympanites. The disturbance is really due to obstruction in the alimentary canal and displacement of the reticulum and omasum, so that rumination and deglutition are affected.

Very often this condition may last for weeks, in either a stationary D.C.

$\mathrm{K} \mathrm{K}$ 
or more or less aggravated form, so that there is an appearance of chronic gastro-enteritis, motor dyspepsia, or chronic indigestion.

Though a diagnosis in this sense would be correct, the atony of the rumen is not primary, but of mechanical origin.

One indication is constantly present, which might suggest indigestion due to overloading of the rumen, and which is also seen in ulcerative gastritis, viz., progressive stasis of food in the cavity of the rumen. When the patients remain for some time under observation, this stasis becomes every day more marked, and, being recognised, the

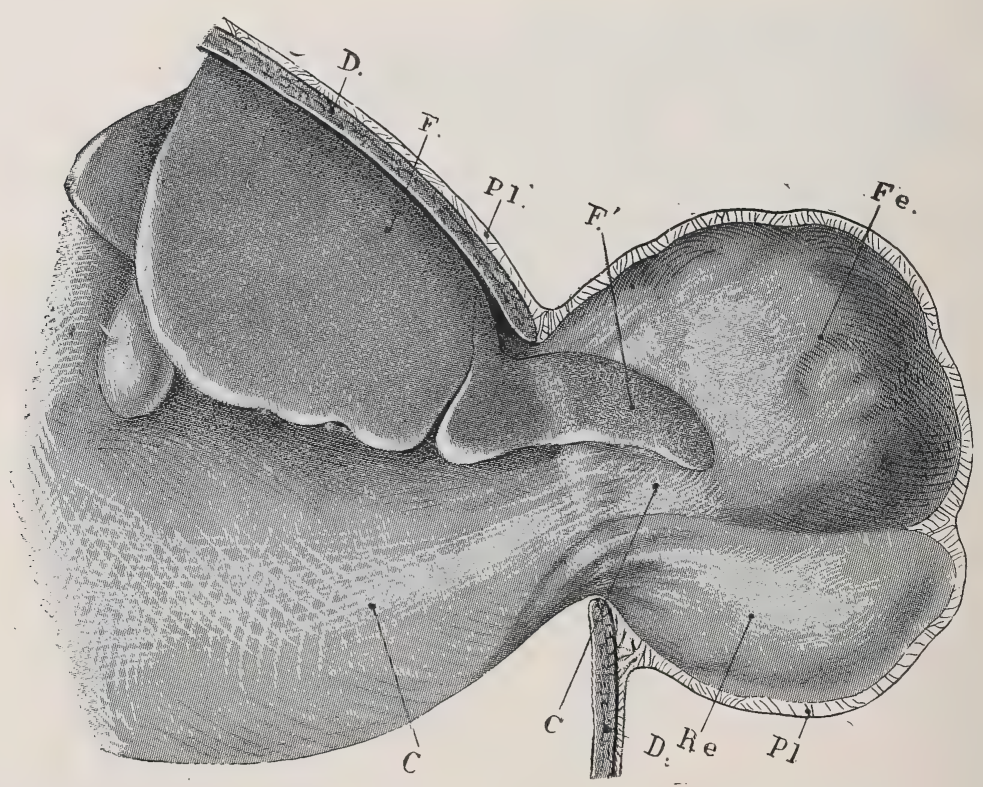

FIG. 224.- Schema of the position of the organs in the hernia represented by Fig. 223. D, Diaphragm; $\mathrm{FF}^{1}$, liver ; $\mathrm{Pl}$, pleura ; Fe, omasum ; Re, reticulum; CC, abomasum.

diagnosis becomes easier. Animals suffering from mediastinal hernia lose condition, waste away, and in the end may die in a state of cachexia.

Lesions. The lesions vary greatly. In accidental diaphragmatic hernia they are confined to rupture of the diaphragna, sometimes of the liver, and to changes in the reticulum or omasum.

In intra-mediastinal hernia the layers of the mediastinum form a true hernial sac, and if the lesion is of old standing the displaced viscera may become attached to it, compressed, and partially strangulated.

Diagnosis. The diagnosis is very difficult, at all events in mediastinal hernia, and can only be arrived at by a process of exclusion. 
The most significant symptom is progressive stasis of food within the rumen, suggestive of some obstacle in the alimentary canal.

Prognosis. The prognosis is extremely grave, because it is impossible to reduce the hernia.

Treatment. No treatment is possible. The essential point is to confirm the diagnosis as soon as possible and to slaughter the animal while it is yet in good condition.

\section{EVENTRATION.}

Eventration belongs to the same group of lesions as herniæ, of which it is merely a more serious form. It differs from them only in the fact that the entire abdominal wall is injured. The skin, muscle, and peritoneum are torn, and the digestive organs pass into direct communication with the external air.

The name eventration has also been given to enormous subcutaneous abdominal herniæ, in which the sero-muscular wall is injured over a large area and the viscera become displaced and separate the subcutaneous tissue layers while at the same time they alter the whole shape of the abdomen.

Causation. The cause is always the same-some grave mechanical injury to the abdominal wall, producing an extensive perforation. The injury may be due to a horn thrust or to the animal falling on some sharp-edged body.

The symptoms are very marked. Through the wound, the rumen, the abomasum, or the intestine protrudes more or less. Generally it is the small intestine which becomes displaced, because it is the most mobile of the abdominal viscera. These organs soon become dried by contact with the air, and may become infected, soiled, congested, thickened, torn, or gangrenous. The successive development of these changes causes serious and violent colic, accompanied by expulsive efforts; the animals throw themselves on the ground, and may tear the mesentery, the intestines, etc. At an advanced stage the animal may stand motionless, looking at its viscera. Death may also be caused in a very short time by the intense pain.

Diagnosis and Prognosis. The diagnosis is evident. The prognosis is always very grave, although, of course, it depends on the condition of the displaced viscera.

Treatment. It is often useless to attempt anything, and if the animal is in suitable condition it is best, as a rule, to slaughter it.

If the accident is quite recent, and the viscera only slightly injured, surgical treatment may be attempted. With this object, the displaced organs are carefully and thoroughly washed with lukewarm boiled water, 
or with some unirritating disinfectant, to guard against peritonitis, and are then reduced.

The abdominal wound must afterwards be carefully sutured. This is performed in two stages. The musculo-serous layer is first brought together with catgut, or better still with silk, and the skin joined by means of deep and closely-placed stitches. To prevent these sutures being torn out, and to support them, the abdomen is swathed in a broad cloth bandage, tightly applied.

\section{FISTULE OF THE DIGESTIVE APPARATUS.}

Fistulæ of the digestive apparatus are of accidental origin and of relatively small practical interest. In most cases they necessitate surgical and other treatment of too delicate a kind and too prolonged

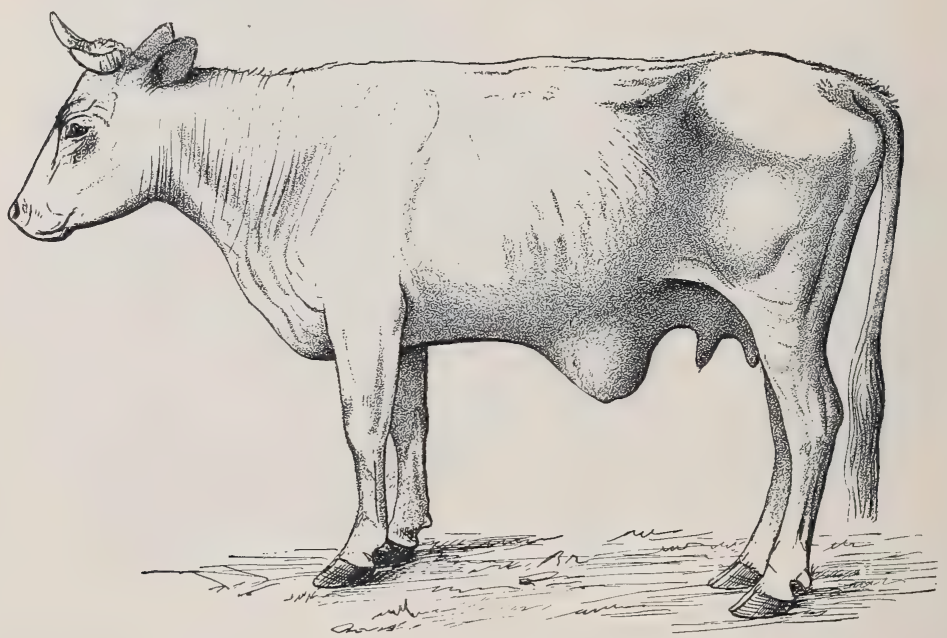

Fig. 225.-Fistula of the rumen.

a character to justify the necessary expense. Their nature and origin sufficiently suggest the course to be adopted.

These fistulæ are divisible into two varieties, gastric fistulæ and intestinal fistulæ. Gastric fistulæ comprise fistulæ of the rumen, reticulum, and abomasum. They may be of external origin, but in the majority of cases they are produced by foreign objects accidentally swallowed and eliminated through the medium of an abscess of the abdominal walls. Their position and direction indicate their point of origin. (Fistulæ of the rumen appear on the left side of the reticulum, near the ensiform cartilage and middle line; those of the abomasum on the right side, near the middle line.) In doubtful cases, chemical 
analysis of the liquid which escapes will afford valuable information. Acidity alone is a sufficient indication in fistula of the abomasum.

Fistulæ of the rumen and reticulum are difficult to close on account of their low position in the abdominal wall, but, if great care is exercised, they may be successfully treated. Those of the abomasum, on the contrary, only tend to increase in size, and any surgical interference still further favours the destructive action of the gastric juice. As a rule, therefore, they cannot be treated.

Fistulæ of the second variety comprise all intestinal fistulæ. They may be either accidental or artificial, and they are less grave than gastric fistulæ, because they are rarely situated in the lower portions of the abdomen. With time they may become closed either spontaneously or by means of simple treatment tending to regulate the passage of food through the bowel. 


\section{SECTION VII.}

\section{GENITO-URINARY REGIONS.}

\section{DISEASES OF THE URINARY APPARATUS.}

Symptomatology. The urinary apparatus comprises the organs of secretion (the kidneys) and those of excretion (the ureters, the bladder, the urethra).

A thorough examination of the urinary apparatus should include, firstly, that of the external organs (the sheath, glans penis and urethra) in the male, and, in the female, the vulva, meatus and urethra; secondly, that of the internal organs (the bladder, ureters and kidneys) in both sexes.

To carry out the external examination, the head must first be fixed and the hind limbs hobbled. If necessary, the animal can be attached to the side of a wall.

External examination comprises inspection and palpation, which is only possible in males. Inspection will reveal at once the existence of malformation, deformity, traumatic lesions, or tumours of the organs.

By palpation the sheath and glans penis can be examined, and cellulitis, abscess formation, calculi in the urethra, obstruction of the extremity of the canal by very fine gravel, as in the case of sheep, etc., can be detected.

The inner margin of the right kidney may also be examined by external palpation, though only in very thin animals. The examination is made from the flank region, behind the last rib, in an upward direction and towards the right (Fig. 227). The kidney, attached under the lumbar region, sometimes extends back beyond the last rib, under the transverse processes of the lumbar vertebræ. On the left the presence of the rumen prevents any examination.

In sheep this examination requires special care.

Examination of the internal urinary organs must be made through the rectum. It should be undertaken slowly and gently. In the male the hand detects the condition of the organs contained within the pelvic cavity, the fulness or emptiness of the bladder, or the existence of calculi within it. More deeply seated can be felt the entire length of the right 
ureter; its state of dilatation can be felt, and the existence of diverticula of inflammation, if any, can be ascertained. The left ureter cannot readily be examined, on account of the position of the rectum, except as regards the posterior part, close to the bladder and the rumen.

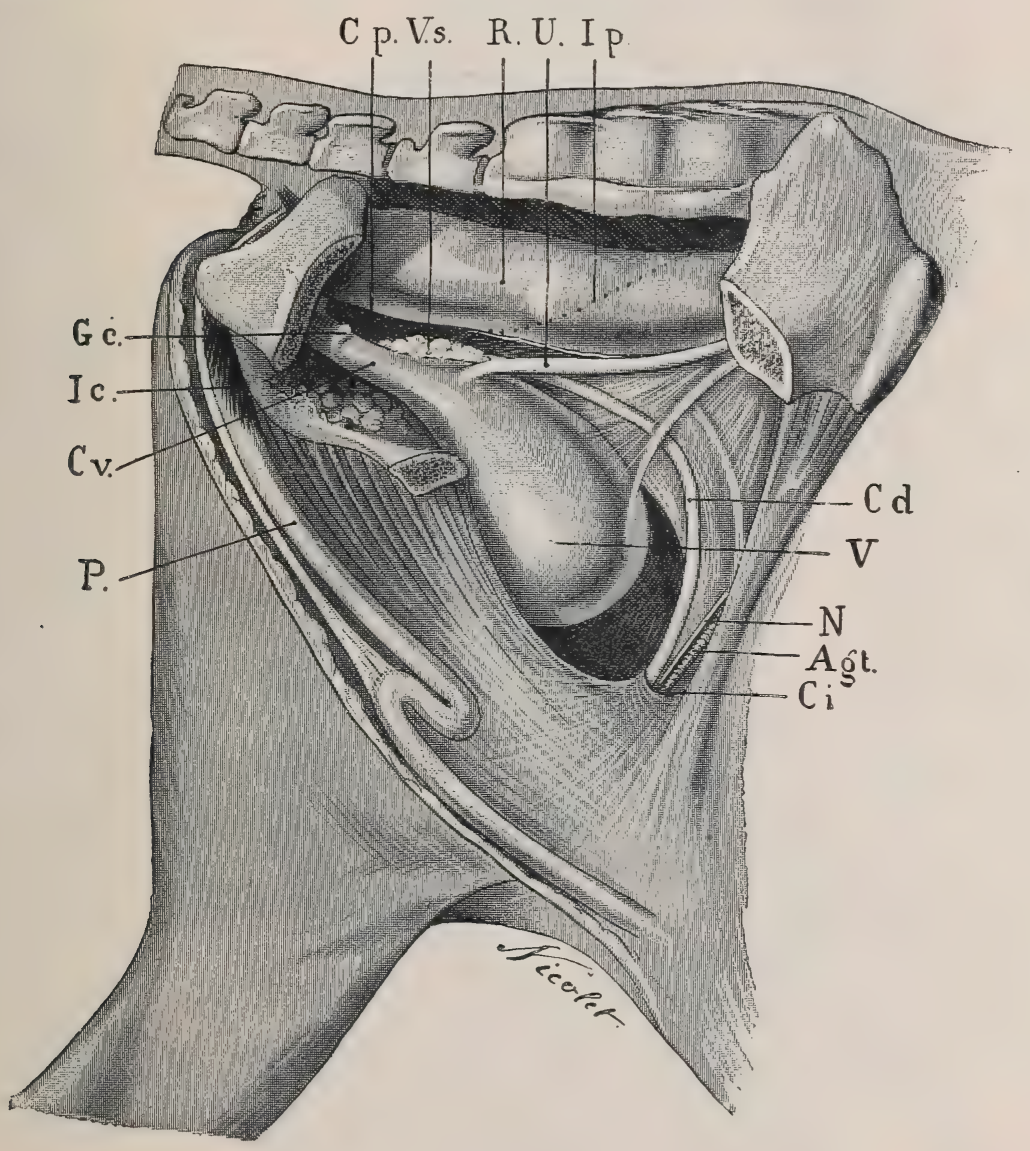

FIG. 226.-Genito-urinary organs in the male (pelvic cavity exposed). $\mathrm{C} p$, Peritoneal cavity (posterior cul-de-sac); Vs, vesicula seminalis; $\mathrm{R}$, rectum ; U, ureter; I $p$, line of insertion of the peritoneum; $\mathrm{C} d$, vas deferens; $\mathrm{V}$, bladder; Agt, testicular artery; $\mathrm{C} i$, inguinal canal ; $\mathrm{P}$, penis; $\mathrm{C} v$, neck of the bladder; $\mathrm{I} c$, accelerator urinæ muscle; Gc, prostate gland.

In the abdominal eavity the rectum, or rather the floating colon, turns to the right, in such a way that, in spite of the shortness of the meso-rectum and meso-colon, the hand can be passed as far as the right kidney. It is, then, easy to discover whether this organ is sensitive to pressure, hypertrophied, atrophied, cystic, etc. The paunch interferes with examination on the left side. 
In females the ureters and kidneys can be examined in the same way, but the bladder and the canal of the urethra must be reached
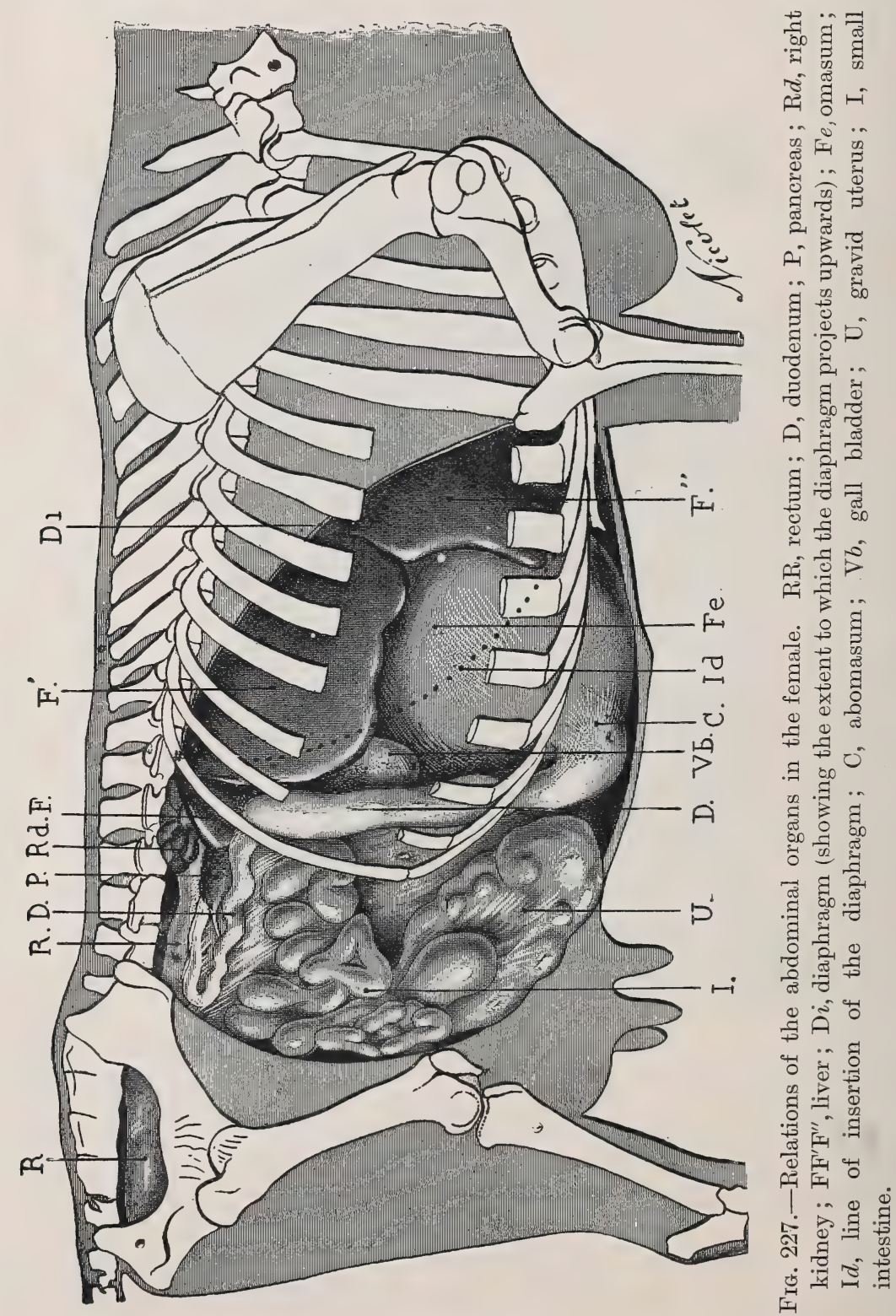

through the vagina. About 2 or $2 \frac{1}{4}$ inches from the vulva on the floor of the vagina is the entrance to the urethra, covered with a special valve. The meatus becomes visible by separating the lips of the vulva and the 
vaginal walls. For this purpose a speculum ad hoc can advantageously be used. It is clear that if it is thought desirable to examine the bladder through the rectum, which is not absolutely impossible, the vagina will be interposed between the arm and the urinary organs, and the sensations experienced will, therefore, be much less clearly defined (Fig. 226).

In passing a catheter for the purpose of emptying the bladder the end of the sound should be introduced under the valve of the meatus. By slightly lowering the back end of the sound, the front end is lifted above the cul-de-sac, and the operator at the same time pushes gently forwards. It is then only necessary to lift the hand, and the sound passes readily into the urethra and the bladder.

Examination of the urine and even chemical analysis are of great importance in diagnosing diseases of the urinary tract.

From the clinical point of view very complete analyses are not necessary, but the tests for albumen, sugar, bile pigments and indican are absolutely indispensable; and the same is true of microscopic examination for ascertaining the presence or absence of epithelial cylinders, blood corpuscles, pus corpuscles, etc. It is important also to note the colour and odour and the quantity passed.

These examinations are by no means difficult. Albumen is detected by adding a few drops of acetic acid to the urine, and heating, or by adding nitric acid and Esbach's liquid. Esbach's albumenimeter is sufficiently exact to discover the approximate amount of albumen present. Sugar is detected by the use of Fehling's solution, bile pigments by nitric acid, and indican by adding a few drops of 10 per cent. chloride of calcium solution and hydrochloric acid. Should indican be present a more or less deeply tinted indigo blue circle appears, resulting from its oxidation.

Hippuric acid is precipitated by pure hydrochloric acid. 


\section{CHAPTER I.}

\section{POLYPI OF THE GLANS PENIS AND SHEATH.}

IN young animals the extremity of the glans penis and the margins of the sheath are not infrequently studded with polypi, soft swellings of papillomatous or verrucous appearance, sometimes of considerable size, which interfere with micturition, and deform the glans. The existence of these growths is shown by very well marked signs-viz., difficulty in micturition, deformity of the sheath, deviation of the jet of urine, obstruction in the passage of the penis, and more or less marked deformity of the penis itself.

These polypi are of the same nature as those so common in dogs, viz., papillomata.

The diagnosis is very easy. On digital examination the growths are almost always found at the base of the sheath.

At an early stage the prognosis is benign, provided intervention is promptly undertaken. If the disease is of old standing, or is not treated, the animals may become somewhat thin. They suffer pain, caused by retention of urine and inflammation of the sheath; urethritis may even be set up.

The treatment is fairly easy, and is exclusively surgical; but as it necessitates casting the animals the bladder should first be examined per rectum, and operation should never be attempted until the bladder has been emptied so as to avoid possible rupture. The animal being fixed on its back, the penis is withdrawn from the sheath, and the polypi can then be snipped off with scissors, the wounds being slightly cauterised to arrest hæmorrhage: in the case of the sheath, rather extensive wounds must sometimes be produced; these may be sutured.

To prevent the wounds afterwards becoming infected and suppurating, the sheath should be regularly washed out with an antiseptic solution and the animal kept on a very clean bed.

\section{INFLAMMATION OF THE SHEATH.}

This condition is much more common in the ox than in the horse on account of the different anatomical structure of the parts, and the methods of keeping and using oxen. 
Causation. Several predisposing causes undoubtedly exist. The sheath is prolonged beneath the abdomen to a considerable distance beyond the glans. It is narrow, deeply seated, and, during micturition, not even the point of the penis passes beyond it. The urine, therefore, soils the interior, or a certain quantity may be retained, according to whether the orifice is more or less obstructed by urinary sediment, sebaceous material, manure, or other material. Moreover, experience shows that of all the large ruminants, those used for outdoor work are the most affected.

Of the occasional causes, if we except sebaceous and urinary products, the most important is mechanical violence, such as the lacerations or wounds produced by the bed-piece of the trevis when the animal is being shod. The working ox throws its whole weight on this bedpiece, on which it lies on one side or the other, according to the foot which is being lifted. The sheath is compressed, and if the animal is heavy and struggles, the parts may be abraded and torn, or the sheath and even the glans may be crushed. In less dangerous cases the connective tissue may be lacerated by the edge of the bed-piece. Any injury so inflicted is aggravated by dirt in the neighbourhood.

Symptoms. The first signs which attract attention are of a general character, and seldom very strongly marked. The animal shows slight fever, dryness of the muzzle, is restless and continually moves about, as though to get rid of the pain it feels. The hind limbs are frequently lifted. In this attitude it makes efforts to urinate, but urine is passed very slowly, and the act seems painful; then, later on, the appetite diminishes, rumination is suspended, and, as in all intense forms of cellulitis, complications may occur.

The local symptoms are more suggestive. The slowness and difficulty with which urine is passed at once attracts attention to the diseased parts. Examination immediately reveals extreme sensitiveness of the sheath, although as yet there is only slight engorgement. At a later stage a large swelling develops and extends along the abdominal wall on either side, sometimes upwards into the groin. In certain cases the sheath may be totally obstructed by sebaceous and inflammatory material, and in the absence of surgical assistance the bladder may become ruptured.

Inflammation most frequently ends in the tardy formation of an abscess, which shows little tendency to open spontaneously. It is usually accompanied by gangrene and by mortification of a mass of skin and subcutaneous tissue, sometimes of portions of the abdominal tunic. Such grave complications may even lead to the opening of an artery, and to fatal hæmorrhage.

All these symptoms develop comparatively slowly. Abscesses scarcely 
ever appear before the twelfth or fifteenth day, but when the disease is not treated it may continue as long as five or six months. On the other hand, resolution is the ordinary termination under suitable treatment.

Diagnosis. The diagnosis is easy when the exact facts can be ascertained. The difficulty in micturition is the chief indication. At a later stage, local swelling and acute sensitiveness are characteristic.

Prognosis. The prognosis is grave, having regard to possible complications and the chronic character which the inflammation tends to assume.

Treatment. When the sheath is filled with sebaceous material or foreign substances it may be necessary to cleanse it daily with mild antiseptic injections. Some practitioners recommend laying open the external orifice, an operation which may be performed in the standing position. This, however, is not without danger, and infection may easily occur.

When the subcutaneous connective tissue surrounding the sheath and the glans penis is infiltrated and inflamed, the inflammatory swelling must be examined every day, so as to detect the abscesses as early as possible, and open them without delay. Under some circumstances deep firing in points produces very favourable results.

When an abscess has caused partial necrosis of the sheath, it is well to pass a drain and wash out the parts frequently with antiseptic solutions. For this purpose a counter-opening is made through the skin opposite the fluctuating point. The mucous membrane of the sheath is then cautiously punctured, and a seton or strip of gauze is passed by means of a seton needle, so as to allow of constant drainage.

\section{PERSISTENCE OF THE URACHUS,}

This condition is an infirmity or congenital anomaly, rather than a morbid condition. Persistence of the urachus after birth and after separation of the cord is due to the fact that the canal which communicates with the bladder fails to close up. The urine, instead of escaping through the urethra, passes along the urachus, and the animal urinates through its umbilicus.

Causation. The causes are simple. Certain practitioners have suggested that sex has some influence, this being an anomaly occurring more frequently in males than in females. From investigations which have been carried out, it would appear that, in some cases, persistence of the urachus is due to an imperforate condition of the urethra; in other cases, to its obstruction by accumulations of mucus of a caseous appearance, which enter the urethra and completely block the passage. 
Finally, in other cases the urachus simply persists while no lesion exists on the side of the urethra.

Whatever the original cause, escape of urine by the umbilicus produces irritation, which may end in complications, infection of the unhealed umbilical wound, infection of the canal of the urachus itself and the bladder, or even infection of the peritoneum.

Symptoms. At first, persistence of the urachus is shown by permanent or intermittent discharge of urine through the umbilicus. Usually this discharge is only seen from five to eight days after birth, when the necrosed cord is detached; in most cases it is continuous, for the opening is abnormal and has no sphincter.

Contact with the air and the wound causes the urine to undergo a kind of ammoniacal fermentation and to irritate neighbouring tissues, such as the stumps of the umbilical vessels, the interstitial connective tissue, and even the skin. The wound constitutes an excellent culture medium for microbes. The umbilicus becomes swollen and œedematous, and soon exhibits a saccular swelling, 2 to 4 inches in diameter, which, on palpation, proves to be hot and painful; its centre is occupied by the opening of the urinary fistula. A probe passed into this fistula travels upwards and backwards (see Fig. 183).

At a later stage other complications, including omphalitis and omphalo-phlebitis, may set in. The most frequent of the delayed complications is purulent cystitis, with the formation of purulent concretions in the bladder, which may occur even after the fistula of the urachus has healed. In other cases the canal of the urachus contracts and becomes obliterated towards the bladder. A blind fistula then persists, with an opening at the umbilicus, or, the parts having healed externally, all that remains is a phlegmonous swelling with the characteristic symptoms.

Diagnosis. A thorough examination will invariably allow of urinary fistulæ being distinguished from other diseases of the umbilicus, the escape of urine at this point being so suggestive.

Prognosis. The prognosis is rather grave, on account of the complications, which are possible, and, in fact, usual, unless the disease is promptly diagnosed.

Treatment. Formerly as a preventive measure a ligature was applied to the whole mass of the cord at birth, but this ligature in no way prevents the canal of the urachus persisting after the atrophied portion separates. Cauterisation of the urinary fistula with Rabel's fluid or tincture of iodine can have no good result, unless the urethra is permeable.

If the urethra is obstructed it is evidently from that side that treatment should be attempted, either by displacing the muco-albuminous 
plugs with a catheter or in some other way. Such intervention is very delicate and difficult in the case of young animals. Sometimes it is better to allow the discharge of urine from the umbilicus to continue, and to recommend that the animal should be rapidly fattened for the butcher.

In practice, pervious urachus may be treated by suturing, by firing across the fistula, and sometimes by blistering. The first step in all cases is to see that the urethral passage is free. English veterinary surgeons claim to have had a fair measure of success from these methods.

If a radical cure were thought desirable, the abdomen would have to be opened, and the operator would proceed to isolate the urachus, resect it, and suture the base of the bladder by bringing together its external surfaces. The operation, though not perhaps impossible, is of no practical value. 


\section{CHAPTER II.}

\section{DISEASES OF THE BLADDER.}

\section{ACUTE CYSTITIS.}

Cystritis, or inflammation of the bladder, may be divided into two varieties: simple (acute or chronic) cystitis, and chronic cystitis due to the presence of calculi.

Simple acute cystitis occurs most commonly in the cow, less frequently in the ox, and rarely in the pig or sheep. Female animals are more subject to it than males.

It consists of more or less acute inflammation of the vesical mucous membrane. The inflammation sometimes extends to the muscular coat and the peri-vesical tissues, setting up local or general peritonitis.

Causation. The internal causes to which Cruzel attributes the disease are rather open to question. Retention of urine in particular is common in working oxen, which only pass urine when resting, and this would be more likely to produce distention, paralysis, or rupture of the bladder than true inflammation; it is doubtful whether inflammation would even follow the chronic irritation resulting from frequently repeated over-distention.

The ingestion of irritant plants certainly has a more marked action. Irritant principles eliminated by the kidneys might not injure them, although they would bring about changes in the vesical mucous membrane, with which they would remain in contact for some time.

More frequently cystitis is the result of inflammation due to continuity of tissue, and forms a complication of urethritis, vaginitis, and the conditions set up by retention of the after-birth. It may also result from ascending infection causing pyelo-nephritis, or ascending infection of any kind which eventually attacks the bladder. It is a very common consequence of the use of unclean catheters, but only in equines and females, as the catheter is not passed in the ox or bull until after urethrotomy.

In by far the majority of cases it is of infectious, and not of toxic, origin.

Symptoms. At first the symptoms are obscure. They commence with slight colic, and are afterwards characterised by frequent, difficult 
and painful micturition and by the small quantity of urine passed on each occasion.

The urine, moreover, is modified in appearance. At first it contains a small quantity of blood, and is reddish, or at least of a darker tint than is usual. Later it becomes thick and whitish, and contains greyish films of epithelium, and the débris of fibrinous coagula. The microscope reveals in it the presence of pus corpuscles, flat polygonal cells with large nuclei, and sometimes red blood corpuscles.

Locally almost all that can be detected in male animals is a little tenderness of the neck of the bladder on rectal examination, and in females on examination of the meatus urinarius by means of a speculum.

The vaginal mucous membrane then appears inflamed around the meatus, which itself is exceedingly sensitive.

In cases of very marked inflammation, accompanied by partial necrosis of the mucous membrane or the production of false membranes, the temperature rises as high as $104^{\circ} \mathrm{Fahr} .\left(40^{\circ} \mathrm{C}\right.$.), appetite disappears, colic is extremely acute, and violent efforts to pass urine are continually made until the animal is completely exhausted. The patient then refuses to walk about, but frequently lies down, arches its back, and constantly makes efforts to urinate, which are abortive or end only in the passage of little jets of fluid.

The urine passed contains little greyish necrotic fragments or débris of false membranes, suggestive of diphtheria. In female animals the canal of the urethra may also become obstructed, and rupture of the bladder, though rare, occasionally occurs.

In the ordinary forms inflammation persists for two to three weeks, then diminishes in intensity, and either ends in recovery or becomes chronic.

In the grave forms, where inflammation and infection extend to the peri-vesical tissues and the peritoneum, death by peritonitis is the rule.

On post-mortem examination, the mucous membrane is seen to be of a greyish colour, and sloughing or gangrenous over surfaces of varying size, whilst the surroundings are infiltrated, blackish and greatly thickened. The whole of the connective tissue ldyer which supports the peritoneum near the base of the bladder, and also the adipose tissue around the bladder, are markedly inflamed. At this stage pelviperitonitis or generalised peritonitis may occur as complications.

In the simple forms the mucous membrane is desquamating, infiltrated, and covered with granulations of apparent healthy appearance.

Diagnosis. The diagnosis is comparatively easy, the external symptoms being so clearly defined. There is a difficulty, however, in distinguishing this disease from cystitis caused by a calculus. In 
male animals this latter form of cystitis is characterised by frequent spasmodic contractions of the accelerator urinæ. In acute cystitis, on the contrary, the contractions are temporary only and of no importance. Lastly, in female animals cystitis due to calculus formation is quite exceptional, owing to the large diameter and shortness of the urethra.

When nephritis and cystitis co-exist certain signs indicate the fact.

Prognosis. The prognosis varies, according to the acuteness of the disease and the character of the urine and epithelial clébris, which afford valuable information.

Treatment. The treatment should be directed towards relieving the vesical and pelvic pain and modifying the local conditions.

Hot fomentations to the loins and flanks relieve pain. The administration of bicarbonate of soda and of cold drinks, such as barley-water, decoctions of couch-grass and pellitory, mucilage, etc., are of service. These materials are readily taken by the patients, and have a soothing effect. Camphor also produces good results, but benzoate of soda is most useful on account of the disinfectant action produced within the bladder, as a consequence of the benzoic acid being separately eliminated by the kidney. Repeated washing out of the bladder with antiseptic solutions has been recommended, but is open to criticism. Such treatment is difficult in male animals, owing to the special formation of the glans penis and urethra, and in female animals it is by no means easy. In all cases of acute cystitis, in fact, the passage of the catheter is painful, and as a metal, gutta-percha or hard rubber sound is used, the mere contact of the tip of the instrument injures the diseased mucous membrane, makes it bleed, and gives rise to danger of autoinoculation, with the possibility of serious results.

Those who recommend this method of treatment can never have followed closely the development of a grave case of acute cystitis, and if the practice is at all permissible, only a soft catheter should be used.

In chronic cystitis, on the other hand, washing out the bladder might be useful.

\section{CHRONIC CYSTITIS.}

Chronic inflammation of the bladder is still rarer than acute cystitis. It usually attacks females as a consequence of acute inflammation, though the condition may be chronic from the first, in which case the early stages are commonly overlooked.

Symptoms. The chief functions of the body do not appear to be disturbed, although the urine seems abnormal. Micturition is difficult, slow, and somewhat painful, and is followed by long-continued tenesmus.

'The urine appears whitish, purulent, slimy, or of a deeper tint, D.C.

L $\mathbf{L}$ 
rapidly becoming blackish. It is of ammoniacal or fœetid odour, and decomposes quickly.

The period of development may be prolonged, and recovery rarely occurs spontaneously. The condition often leads to ascending infection, inflammation of the ureters, pyelitis, and nephritis.

On post-mortem examination of animals slaughtered before complete wasting has occurred the vesical mucous membrane is found to be thickened, granulating, or suppurating. The muscular tissue shows infiltration and localised sclerosis, and is very irregularly thickened, inelastic, and wanting in contractile power. The peri-vesical tissues may be chronically inflamed.

Diagnosis. The diagnosis is easy, provided the peculiar characteristics of the urine are noted, and an examination is made through the vagina of the condition of the walls of the bladder, of the ureters and of the kidneys.

Prognosis. The prognosis is grave, because treatment would occupy too much of the practitioner's time to allow it to be rigorously carried out, and therefore animals are usually slaughtered.

Treatment. Treatment comprises the use of many of the drugs used in acute cystitis, particularly benzoate of soda, benzoic acid, and bicarbonate of soda. The medicines comprised in the balsamic group are also valuable, viz., turpentine, tar, and terpin.

In this chronic form the bladder should be irrigated, but this must be done with strict aseptic precautions, the fluids used being cooled boiled water, boric acid or borax solution of 3 per cent. strength, or solution of fluoride of soda of a strength of 15 grains to the quart.

We need not point out the difficulties of pursuing this treatment in ordinary practice. As a rule, treatment is confined to internal medication whilst the animals are fattened.

\section{URINARY LITHIASIS, CALCULUS FORMATION.}

Normally the urine contains in solution certain salts, such as urates, hippurates and phosphates of lime, magnesia or ammonia. Under certain circulistances, in animals predisposed to the condition, these salts are precipitated in the kidneys, ureters or bladder, and form powdery or sandy deposits known as sediments; or, on the other hand, calculi, produced by the adhesion of the powdery masses. This constitutes urinary lithiasis.

The sediments are of a greyish-yellow colour.

The calculi are generally rose-coloured, white or somewhat grey. They contain oxalates and carbonates of lime and magnesia, earthy phosphates, etc. In appearance and shape they vary greatly. 'They 
may resemble coral or may form growths of a rounded, polyhedric or raspberry appearance. Some are hard and resistant; others friable. They vary in size between that of a grain of sand and a hen's egg or more. A large calculus is usually solitary; the smaller sizes are often multiple.

Calculi occur in oxen and sheep, but more particularly in the latter species. They develop slowly without producing any marked external signs, and often it is only when the urethra becomes obstructed and urine is retained that the diagnosis is established. Calculi are rare in females in consequence of the dilatability of the urethra.

CALCULI IN BOVINE ANIMALS.

Causation. The older writers believed that calculi developed through winter feeding and a stinted supply of water. Nowadays this would not apply to well-managed establishments, water being provided regularly, and winter feeding comprising roots, etc., rich in water. Experience and observation have shown that the chief cause is excessive feeding, calculus formation occurring most frequently in animals which are most richly fed.

Nevertheless, it would be a mistake to overlook the effects of temperament and constitution. In human medicine the importance of hereditary predisposition and of the special diathesis is undeniable (uric or gouty diathesis). In veterinary medicine the same thing applies, for, apart from rich feeding, it is not uncommon to meet with cases of gravel in animals living under the most diverse conditions, although the issue of the same parents.

Certain infections of the urinary passages, though trifling at first, or at least of slow development, are also capable of causing mineral deposits in the urinary passages, a fact which Moussu considers to be proved by his success in experimentally reproducing given forms of pyelo-nephritis.

Symptoms. The symptoms are often overlooked, as long as the calculus deposits affect only the kidneys, their increase in that part not giving rise to alarming symptoms. It is certain that the eventual passage of the calculus through the ureter towards the bladder causes nephritic colic, but this form of colic is little understood, and has never been well described. Writers have simply mentioned cases of colic accompanied by great tenderness in the lumbar region, temporary suspension of the secretion of urine, and more or less marked dysuria.

Nothing resembling the extremely grave symptoms of nephritic colic in mankind has been described, though probably there is little difference in the complaint as it affects the bovine species. 
When the sediment or the calculus reaches the bladder there is a tendency for it to be passed during micturition. The signs then become more strongly marked, because they point to obstruction of the urethra. If the deposit is simply of the nature of sediment, there is merely a little difficulty in urination, accompanied by some moderate amount of pain, and sediment is afterwards found within the sheath or adhering to the groups of hair at its extremity.

If, however, the deposit is in the form of small calculi, these are pushed towards the neck of the bladder and the urethra, which then appears to be obstructed.

The obstruction may occur at the origin, at the ischial curve, or at the S-shaped curve of the penis (Fig. 226). Henceforth strongly marked and unmistakable symptoms rapidly develop. Vesical colic appears, owing to retention of urine, and rapidly acquires extreme intensity, though it instantly ceases with rupture of the bladder in cases where no treatment is attempted. This form of vesical colic is accompanied by continual but unsuccessful efforts to urinate and by spasmodic contractions of the accelerator urinæ.

Appetite and rumination cease, and the animal shows extreme anxiety. Palpation along the course of the penis reveals unusual tenderness, and the calculus can sometimes be felt near the $\mathbf{S}$ curve, though more frequently in the ischial arch. The litter is not soiled with urine.

Cautious rectal examination proves the bladder to be extremely distended, or, in the case of rupture having occurred, entirely collapsed. In the latter case the spasmodic contraction of the accelerator urinæ completely ceases soon after rupture, and the animal appears to be recovering. This deceptive calm is due to the disappearance of the vesical colic, but the animal's condition is still graver in consequence, and it must of necessity die. Rupture of the bladder is followed by inundation of the peritoneal cavity with urine, which is partly reabsorbed by the peritoneum, producing a kind of urinary intoxication, so that despite the elimination of certain volatile principles through the lungs (the breath has an odour suggestive of urine), the animal very soon dies.

In many cases, also, the urine is not aseptic, and after rupture of the bladder acute peritonitis supervenes and carries off the patient in from six to ten days.

Even when the urine appears to be aseptic, chronic exudative peritonitis is produced by the irritant action of the urine on the peritoneal endothelium. The exuded liquid mixes with the urine, and the animal soon shows marked ascites. Despite this condition, some animals have been known to survive as long as from three to six weeks without showing very marked disturbance. 
Death is the inevitable sequel after a longer or shorter time. When large calculi have been arrested, or rather developed, in the bladder the same symptoms occur should the calculus be thrust towards the neck of the bladder so as completely to obliterate the passage. This, however, is a very rare accident. As a rule the obstruction is merely temporary, and the resulting vesical colic and retention last but a short time. The displaced calculus falls back again into the lower: part of the bladder, where it is retained, and the urinary passages again become free.

Diagnosis. The diagnosis is sometimes extremely easy, but it may present serious difficulty.

When the urethra is obstructed, the symptoms are so striking that there can scarcely be any doubt; but the diagnosis of renal calculus, nephritic colic, calculus in the bladder, and rupture of the bladder demands more attention. Examination of the urinary organs through the rectum then proves of great service.

Prognosis. The prognosis is grave in all cases, because of the possibility of the urinary passages being obstructed, so that surgical interference is necessary.

Lesions. The lesions caused by urinary calculi may vary greatly. Though insignificant and scarcely apparent in certain cases, they are often very marked, and comprise simple or suppurative pyelitis, inflammation of the ureters, hydro-nephrosis, cystitis of varying intensity, urethritis, and inflammation of the sheath.

Treatment. All farmers who fatten their animals know that the use of alkaline drugs, such as bicarbonate of soda, together with diuretics, linseed, barley and pellitory diminish the danger of urinary calculus formation. Bicarbonate of soda is often given with this object, and is excellent in cases where lithiasis does not extend beyond the production of sandy or muddy deposits. By rendering the urine more alkaline it prevents the growth of sabulous deposits, and may even cause slow but progressive solution of concretions already formed. When, on the other hand, the urethra is obstructed, and urine is retained, early surgical treatment (urethrotomy) alone offers any chance of preventing rupture of the bladder.

Certainly it is possible, as recommended by the older practitioners, to try massage of the glans penis and urethra opposite the obstruction, and, after withdrawing the penis, to attempt to loosen and eject the obstructing matter. But such attempts very frequently fail, because the material is too firmly fixed, and no time must be lost.

Urethrotomy is usually practised at one of two points, according to circumstances-firstly, opposite the ischial arch; and, secondly, opposite the S-shaped curve. 
Ischial urethrotomy is the promptest method of affording relief, and should always be preferred whenever there is danger of rupture of the bladder.

It is performed in the standing position, but is only possible when the animal is not too fat.

In other cases urethrotomy is performed opposite the point where the calculus is fixed, and aims at removing the obstruction. It can only be practised after casting the animal; but, in this case also, it is indispensable that the animal should not be excessively fat, as in such cases a secondary urinary abscess is almost certain to form.

Should the animal be so fat as to render treatment difficult, it is best to slaughter it at once.

\section{URINARY CALCULI IN SHEEP.}

Urinary calculi are commoner in sheep than in oxen, and seem to depend more on the breed and on conditions of feeding. They are almost exclusively confined to animals which are richly fed, to show animals, and to males. In exceptional cases they are seen, under ordinary conditions of feeding, in aged subjects.

Calculus formation can moreover be induced experimentally, and in a relatively short time, by giving certain rations-e.g., $7 \mathrm{lbs}$. per day of maize, lentils and beans for adults, and 3 lbs. for lambs. The other favouring circumstances, viz., hereditary gouty diathesis and infection, are less well established than they are in the case of the ox.

In sheep the symptoms are still less characteristic than in oxen, for which reason gravel in sheep merits special description.

It shows itself in the passage of turbid urine, forming a deposit at the extremity of the sheath, which becomes somewhat inflamed. The colic resulting from retention of urine is shown by depression, want of appetite, dysuria, and generalised convulsive shivering fits.

The patients lie down in the sterno-abdominal or sterno-lateral position. They constantly suffer from attacks of general violent shivering, and die after twelve, twenty-four, or forty-eight hours.

On post-mortem examination the bladder is found to be ruptured, or the urethra obstructed.

Diagnosis. The diagnosis involves no difficulty, provided the method of feeding is understood. In many cases the shepherds themselves perfectly recognise the cause of the symptoms.

Prognosis. The prognosis is very grave, it being impossible to pass the catheter on account of the perineal valve in the urethra, while it is difficult to operate, the urethra being very small and deeply embedded in a thick layer of fat. 
Treatment. The only resource is massage along the urethra, which may sometimes break up the mass of sediment or move the obstructing calculus. One remark may, however, be made, viz., that in the majority of cases the urethra is obstructed at its extremity by local accumulations of sediment behind the spiral filiform prolongation of the penis.

It is then sufficient, and experienced shepherds have no hesitation in performing the operation, to remove the spiral filament, thus facilitating the expulsion of the sediment and affording relief. If both methods, viz., massage and section of the filament, fail, the animal should be slaughtered, so as to avoid rupture of the bladder, which would render the flesh useless as food.

From a preventive standpoint, all sheep which are richly fed should receive an allowance of some slightly alkaline drink.

\section{PARALYSIS OF THE BLADDER.}

Paralysis of the bladder is somewhat frequent in female, but very rare in male, animals. In the majority of cases it is the consequence of difficult parturition, or is a post-partum complication.

It is characterised by incontinence of urine or retention with overflow. The continuous discharge soils the hind quarters, hocks, shanks, pasterns, etc., and the urine decomposes and causes irritation; it soon sets up urinary eczema in all the parts with which it comes in contact, a condition which can only be successfully treated by removing the cause.

The prognosis is grave, for methods of treatment are few, and of doubtful efficacy.

Treatment. If the condition results from post-partum infection, this must naturally first receive attention. Should the infection have disappeared whilst incontinence of urine still continues, the administration of tonics, e.g., tincture of nux vomica in daily doses of $\frac{3}{4}$ to 1 drachm for ten days or so in the case of a bovine animal, and a stimulating application to the lumbo-sacral region, may bring about recovery.

But if, in spite of such treatment, the incontinence persists, it is better, from an economic standpoint, to treat the urinary eczema with astringents, etc., and quickly to fatten the animal.

\section{EVERSION OF THE BLADDER.}

Eversion of the bladder only occurs in female animals after difficult parturition. The viscus is turned completely inside out, as occurs in eversion of the uterus, the base of the bladder becoming invaginated in 
the cavity of the bladder itself, and afterwards passing into the urethra and vagina. The bladder thus becomes totally displaced, and appears between the lips of the vulva, resting on the inferior commissure, and forming a mass the size of an orange.

Eversion cannot occur unless the ligaments of the bladder have become relaxed, stretched or ruptured. Expulsive efforts and the pressure of the intestinal mass complete the process, the peritoneum and peri-vasicular layers of connective tissue being torn.

Diagnosis. The diagnosis of eversion of the bladder presents no difficulty. The everted mass appears to have a narrow neck opposite the meatus, and is seen to form a reddish, unctuous mass. The mucous membrane now forms the external coat and appears covered with mucus so long as inflammation does not occur.

The urine continually escapes from the ureters (which open on the surface of the mucous membrane) as it is formed, and flows away by the lower commissure of the vulva. The vulva is half open, and the prominence formed by the bladder projects beyond it.

Prognosis. The prognosis is grave, because reduction is difficult, and may be accompanied by rupture of the organ; also because even in favourable cases it is invariably followed by acute cystitis.

Treatment. Treatment is confined to reduction. Before attempting this, measures must be adopted to prevent straining, either by passing a rope round the animal's body, thus causing it to flex the vertebral column, or by puncturing the rumen or performing tracheotomy. The open hand is then applied to the surface of the swelling, which is gently compressed and thrust in turn through the meatus and urethra. The portions nearest to the urethra should first be returned. It is sometimes necessary to use both hands, and even to employ a catheter with a large round head, to reduce the eversion effectually. After reduction a truss or vulval clamp should be applied. Subsequent treatment consists in the administration of sedatives-e.g., laudanum, mucilaginous drinks, barley-water, pellitory, etc.

\section{HAEMATURIA.}

Hæmaturia, i.e., the passage of blood-stained urine, is in itself only a symptom, which may accompany very varying conditions, such as the congestion peculiar to the early stages of nephritis, traumatic lesions of the kidneys, ulceration of the uriniferous tubules, or of the pelvis of the kidney, lesions of the ureters, bladder, etc., etc. The term, therefore, does not indicate a disease, but nevertheless in bovine practice the term hæmaturia has acquired a special significance.

This hæmaturia of bovine animals is clinically indicated by the presence of blood in the urine; anatomically by lesions of the bladder, 
sometimes also of the ureters. It is probable that some forms at least of the condition will ultimately be proved to be due to the piroplasmata, but in the present state of our knowledge the disease can only be described from the clinical standpoint. The reader is recommended to refer to the article on "Bovine Piroplasmosis," ante.

Pichon in 1863 and Sinoir in 1864 introduced the name "hrmaturia" in the course of their remarkable investigations concerning the disease. Vigney in 1845 and Gillet in 1862 had previously described it, and it has since formed the subject of constant researches.

Detroye in 1891 termed it "essential hæmaturia," and Galtier in 1892 gave it the name of "hæmorrhagic cystitis." Boudeaud in 1894 also used the term "hæmaturia of bovine animals." In Germany the disease is known as "stallroth" (stable-red).

Geographical distribution. Hæmaturia is a perfect scourge in certain countries. It seems to have made its appearance in the departments of the West of France, the Mayenne and the Sarthe, afterwards spreading into the Maine-et-Loire and the Indre. At the present day, it inflicts great ravages in the Creuze, the Corrèze, Haut-Vienne, Cantal and Haute-Loire districts. It has been described in Germany, Belgium, and Italy. These forms are probably due to Piroplasma bigeminum.

Causation. The most varying opinions have been advanced regarding its cause. Pichon believed its appearance was due to changes in cultivation, which between 1830 and 1860 completely altered the general appearance of the country and the conditions of breeding in the old province of Maine. Land reclamations and the use of lime dressings have been mentioned, as weil as the introduction of the Durham breed of cattle. Sinoir practically adopts the latter view, for he considers that the crossing with the Durham breed, while increasing the precocity, has diminished the powers of resistance of the indigenous cattle.

But in course of time these ideas have become modified, and investigation has taken a new direction. Detroye regarded the disease as a microbic and easily transmissible disorder, while Galtier in the following year described it as merely a chronic hæmorrhagic cystitis, produced by the consumption of irritant plants in animals previously suffering from distomatosis. In Germany, Arnold attributed "stallroth" to coccidia developing in the epithelium of the vesical mucous membrane.

Cruzel considered the disease to be due entirely to poor feeding. Boudeaud thought the same. He says that hæmaturia affected onetenth of the whole of the oxen in the south of the Indre and the north of the Creuze, in parts where the arable soil is thin and poor in phosphoric acid. Furthermore, he suggests that dressings with lime and phosphates would result in the disappearance of hæmaturia. 
We cannot admit that poor forage and feeding alone are sufficient to produce hæmaturia, for one frequently sees poorly nourished animals pass through all the stages of wasting and most profound cachexia without ever showing signs of this particular ailment. Besides, hæmaturia may attack animals in good condition.

Detroye's early opinion as to the infectious or microbic nature of the disease seems scarcely more acceptable, for it now appears certain that the organism originally described is incapable of producing the disease.

Galtier's theory is still less admissible. According to the Lyons professor, hæmaturia occurs only in animals suffering from distomatosis. The liver, he says, being affected by the growth of liver flukes, no longer performs its proper work of destroying toxins, and if under these conditions the animals eat improper food containing ranunculaceæ, sedges, rushes, etc., the toxic principles of these plants are absorbed. Then, he adds, these principles being no longer destroyed, are eliminated by the kidneys, their stay in the bladder causes irritation, and hæmorrhagic cystitis is set up, this being afterwards maintained by microbic agents in the bladder.

This very specious theory, all the points in which may readily be refuted, in our opinion falls to the ground before the simple fact that hæmaturia occurs in animals which present no trace of distomatosis on post-mortem examination, and that, furthermore, it is not seen in the lower regions of the departments of the Nord, the Pas-de-Calais and the Somme, where ranunculaceæ and other irritant plants are common and distomatosis rages.

Moussu states that he has proved that hæmaturia is very rare in young animals and is exceptional before the age of two and a half years or three years; that it attacks oxen as often as cows; that it is particularly common in low regions; and that it is scarcely ever seen above a height of 800 yards. Careful investigation, moreover, shows that the passage of bloor occurs just as frequently in winter, when the animals are housed, as in spring, when at pasture.

Lesions. The lesions of hæmaturia are to be found in the bladder, though in exceptional cases they may also affect the ureters and kidneys. They have been described by Pichon and Sinoir, but as these observers regarded the condition as a disease of the blood due to poor feeding, etc., they did not attach much importance to them. Detroye has described the different appearances very well, though Moussu states that he has never met with the "blisters" which he mentions.

The first period is accompanied simply by abnormal vascularity of the bladder, which appears in the form of true varicosities of the sub-mucous vessels and intra-mucous capillaries. But if thị lẹsion 
is primary, it does not correspond to the period during which bloodstained urine is passed, and is not sufficient to explain it. It always appears in the form of a more or less abundant hæmorrhagic intramucous, sub-epithelial spotting.

Over the hæmorrhagic area, which may be of very varied dimensions, ranging from those of a small pin's head to those of a lentil, the epithelium is swollen and loosened, and so separated from the surrounding parts as to have lost its vitality. This patch of separated epithelium soon falls away, leaving an epithelial ulceration of the mucous membrane. The subjacent clot rapidly breaks up in contact with the liquid in the bladder, and is replaced by a small ulceration which becomes the seat of continual capillary hæmorrhage. Nevertheless, the neighbouring tissues react, and the process of repair may end either in true cicatrisation, which appears to be rare, or more frequently in the formation of exuberant granulations, which are also of the nature of a soft, bleeding vegetation. This vegetation is either sessile or pedunculated, and is of very varying size.

The wall of the bladder also reacts, becoming sclerosed and thickened beneath the granulations, so that, in animals which have long suffered from hæmaturia, it may entirely have lost its dilatability.

When the disease has existed for a certain time, sub-epithelial hæmorrhages, ulcerations, vegetations and points of sclerosis may all co-exist, a fact which shows that the disease does not develop all at once, but that, on the contrary, every little lesion develops separately and continuously. This fact also explains the length of time for which blood may be passed, despite the presence of old or healed lesions.

Finally, in very old standing cases dating from several years back (Moussu saw an animal aged twenty-eight years which had suffered from this disease for more than twenty years, but in a very intermittent fashion), it is not exceptional to find numerous papilliform vegetations 1 or 2 inches in length, either with a fine pedicle or largely sessile, invading one-half or two-thirds of the internal surface of the bladder.

These vegetations sometimes, though rarely, invade the ureters. When they occur towards the point where these conduits enter the bladder, they obstruct the passage of urine, and lead to the development of hydro-nephrosis or pyelo-nephritis.

Symptoms. The early symptoms often escape notice, because general disturbance is rare. The first appreciable signs are cystitis and frequent urination.

The urine passed is turbid, particularly towards the end of the act of urination; then it is of a pink or red colour, and all intermediate 
shades between a pale pink and a bright arterial red colour may be observed.

The patients sometimes seem to pass unaltered blood in the urine, but on microscopic examination this blood is found to be extremely diluted. Provided the bladder is not gravely infected by the (secondary) penetration of germs into its cavity the blood corpuscles remain normal, or are scarcely changed. As soon as the bladder, however, becomes secondarily infected an almost immediate change takes place; the red blood corpuscles become crenated, broken up and dissociated; the hæmoglobin is also partly dissolved and modified, and at this stage the urine is red-brown or coffeecoloured, according to the length of time it has been retained in the bladder.

In other cases, chiefly when hæmaturia has existed for some time, the extravasated blood coagulates in the bladder, and the urine passed contains filamentous clots the size of a man's thumb, a pigeon's egg, or more. If the clots formed are too large to be passed, which is often the case in the ox, they may obstruct the urethra, causing retention of urine and all the accidents which accompany this condition, even including rupture of the bladder. This, in the ox, is a frequent termination. In the cow the dilatability and shortness of the urethra render retention of urine much rarer. It is certainly possible, however, and it is not exceptional, to find from 4 to $6 \mathrm{lbs}$. of clotted matter in the distended bladder. All these conditions can be detected by rectal exploration, and by attention to the symptoms of obstruction of the urethra.

Whenever there is retention of clots dysuria is extremely marked and, so to speak, permanent, the animals having continual tenesmus.

Hæmaturia observes a slow, progressive course, which, in time, ends in death by exhaustion, though this is not invariably the case. Hæmaturia is frequently intermittent, and, after having been very marked for weeks or months, may suddenly or gradually cease, and only reappear a long time afterwards. This fact is explained by a study of the development of the lesions. When ulceration occurs the sub-epithelial vessels of the mucous membrane, which have contributed to the formation of the hæmorrhagic spot, are widely open, and a capillary hæmorrhage results; but as soon as a small clot forms in this position, or local capillary thrombosis occurs, the hæmorrhage ceases, with the result that the hæmaturia disappears. Unfortunately, however, the obliterating clots are not permanent, any more than the local thrombosis-or, in the event of their proving permanent, another small lesion develops at a different point, and this lesion may at any time cause the reappearance of the hæmaturia; the process goes on until the animal succumbs. 
Should the lesions heal successively, spontaneous recovery may take place, but such recovery is exceptional.

The animals may not appear to suffer from the passage of blood for weeks or even months, but after a time they become less capable of replacing the loss. They become anæmic, the number of corpuscles falls from the normal figure of from six to seven millions of red corpuscles per cubic millimètre to three millions, two millions, one million, and even to five hundred or eight hundred thousand.

The richness in hæmoglobin simultaneously diminishes; wasting progresses to the point of cachexia, and the appetite diminishes while diarrhœa appears; swellings are noticeable about certain parts of the body; and the animals, continuing to pass blood, die in a state of absolute exhaustion, without apparent suffering.

This termination is the most common, unless slaughter is determined on, and is very different from the premature end which follows the formation of clots and obstruction of the urethra.

Externally the patients only show feebleness, pallor of the visible mucous membranes, and difficulty in urination. The bunch of hair at the lower commissure of the vulva is always soiled with blood-stained urine or little clots.

Hæmaturia may cause death by exhaustion in from six weeks to two months, but not infrequently it lasts for months or even years.

Diagnosis. The diagnosis presents no difficulty when the urine can be examined; but in the periods of intermittence no opinion can be advanced. These intermittences are so frequent that in parts of the country ravaged by this disease it is a usual custom, when selling, to grant or refuse guarantees for a longer or shorter term.

The condition can be distinguished from parasitic hrmoglobinuria (piroplasmosis) or from Brou's disease (a febrile disease of rapid development) by simply examining the urine or blood.

Prognosis. The prognosis is extremely grave, for, up to the present, no really efficacious treatment has been discovered, and although some animals may live for years without their lives being in any way endangered, this cannot possibly be foreseen, and there is no economic advantage in keeping them.

Treatment. No curative treatment is known.

It is true that iron salts, tonics, Rabel's liquid, decoctions of certain plants, such as plantain, have been recommended, but apart from the fact that they are of doubtful efficacy, they cannot be used over long periods. All these preparations also tend to increase the coagulability of the blood; but considering that the disease is beyond question of a parasitic character, good results cannot always be expected of them. 
Preventive treatment appears more hopeful, although even in this connection, the best informed appear to have considerable doubts. All those who have studied the question agree in recommending drainage of the pasturages, and their improvement by the use of various manures, particularly superphosphates and lime. These improvements alter the character of the pasture, render the soil healthier, and may perhaps prove sufficient to diminish or prevent the local growth of the germs. Under such conditions, Boudeaud declares that he has seen hæmaturia disappear from farms where it had previously been in permanent possession. It has also been recommended that the affected cattle should be sent elsewhere to places where the disease does not exist, and experience shows that spontaneous recovery is more frequent under such conditions.

It is probable that, during attacks of hæmaturia in a contaminated country, successive parasitic infestations occur, which would explain the persistence with which blood is passed, a symptom which does not occur in a healthy country. This view, however, is still only an hypothesis. 


\section{CHAPTER III.}

\section{DISEASES OF THE KIDNEYS.}

\section{CONGESTION OF THE KIDNEYS.}

Congestion of the kidneys is not a morbid condition in the strict sense of the term, for it is merely the forerunner of nephritis caused by infectious diseases or intoxications (primary active congestions) or the final consequence of other diseases, such as diseases of the heart or liver, mechanical compression of the vena cava or renal veins (secondary passive congestion, cardiac kidney).

Nevertheless, under certain circumstances the development of nephritis may be arrested at the primary congestive stage, and it is only then that an opportunity occurs of studying it as a definite complaint.

Causation. All infections accompanied by lesions of the kidneys, and these are numerous (gangrenous coryza, anthrax, parasitic hæmoglobinuria), produce congestion of the kidneys.

Cold also acts directly under certain conditions, as do large doses of diuretics, irritant foods the principles of which are eliminated through the urine (fermenting or putrid sugar-pulp, for example), and foods rich in resins, essential oils, various glucosides, tannin, ete. (young shoots of trees during the spring-time).

Symptoms. The symptoms are difficult to define accurately, and the diagnosis can only be arrived at with the aid of the history.

Renal congestion produces pain, indicated by dull colic and repeated and ineffectual attempts to urinate, suggesting acute cystitis. The patients lose appetite, and present all the general symptoms of marked visceral inflammation, viz., fever, acceleration of breathing, somewhat tumultuous action of the heart, etc.

External or internal examination of the kidneys reveals abnormal sensitiveness. The urine is of a dark or bright-red tint, owing to the presence of red blood corpuscles. These blood corpuscles are precipitated on placing the fluid in a tall glass, and can be detected, together with renal epithelium, by microscopic examination.

The diagnosis is somewhat difficult, and it requires very careful attention to distinguish between congestion of the kidney and true nephritis. 
The prognosis should always be reserved until it is certain that acute nephritis will not ultimately develop.

The treatment consists in removing the cause of the congestion; rich foods, or foods containing irritant principles, should, therefore, be avoided, as also the administration of diuretics, etc.

Otherwise, the treatment is similar to that employed in all visceral inflammations: bleeding to the extent of two to four quarts, according to the size of the animals, warm poultices to the loins and flanks, dry friction, mucilaginous drinks and emollient decoctions of barley or pellitory. The animals should be kept in a warm place.

In cases of passive and secondary congestion, treatment must be directed towards improving the condition of the organ primarily affected, whether it be the heart, liver, or lymphatic glands.

\section{ACUTE NEPHRITIS.}

The term nephritis applies to inflammation of the renal tissues. Clinically, two forms only can be distinguished, the acute and the chronic.

As regards its pathological anatomy, the inflammation may principally affect either the interstitial tissue or the epithelial parenchyma, a fact which has suggested the division of the condition into epithelial nephritis, interstitial nephritis, and mixed nephritis. Clinically, such distinctions are impossible; and in reality all forms of nephritis are to a varying degree mixed, the lesions predominating in one or other of the constituent tissues. These lesions depend on the extent, intensity, and duration of the inflammatory attack, whatever the primary causes. All the constituent tissues of the kidney may be affected, simultaneously or individually: the Malpighian corpuscles, the convoluted tubules, the collecting tubules, or the interstitial connective tissue.

Causation. Cold seems to be an important factor. All acute or chronic intoxications in which the toxic principles are eliminated by the kidneys, such as poisioning by cantharides, fermented beet pulp, young shoots of trees or toxic plants, may cause acute nephritis.

Infectious diseases, such as gangrenous coryza, hæmoglobinuria, tuberculosis and post-partum infections, also play an important part, whether the nephritis be direct, that is to say, the result of the infecting agent itself, or indirect, i.e., produced by toxins generated in the body. In female animals gestation is an often unsuspected cause. Moussu believes that albuminuria is frequent during gestation, and although in most cases it is only of moderate degree, he thinks it is often associated with sub-acute nephritis, which might be aggravated by an accidental cause. 
Many forms of nephritis are overlooked in consequence of their slight character.

Symptoms. The early symptoms are similar to those of congestion of the kidney, viz., dull colic, excessive sensitiveness over the region of the loins, passage of pink urine, loss of appetite, and fever. At a later stage, in cases of acute nephritis due to cold, the animal stands with the limbs close together and remains stationary, arching the loins and back, which are held stiffly. The animal obstinately refuses to move in consequence of the pain produced by so doing.

The general condition becomes grave, respiration is rapid, the pulse frequent, the artery tense, the muzzle dry, the accessible mucous membranes are injected, and appetite is almost entirely lost.

Urine is frequently passed, but the act causes pain, and the quantity is small. Absolute anuria is rare, and does not last long.

The urine is generally sanguinolent, at least at first, but to a very varying extent. It is always albuminous, the quantity of albumen varying enormously, and on microscopic examination, is usually found to contain red and white blood corpuscles, epithelium from the kidney hyaline or epithelial cylinders, and, towards the end, pus corpuscles.

Edema or anasarca, though common in mankind, does not occur in a very marked form, except in intense acute nephritis. Epistaxis is also rare.

Diagnosis. The diagnosis requires some care, because unless the urine be examined the symptoms might lead to error. Nevertheless, it is always possible to distinguish between this condition and hæmaturia or accidental renal hæmorrhage.

Prognosis. The prognosis is grave, because absolute recovery is rare, and because the condition is very apt to become chronic.

The degree of anuria and the respiratory difficulty are of great service in confirming the prognosis. As soon as urine is freely passed the prognosis becomes more favourable.

Treatment. Among the most effective methods of treatment must be included bleeding, which always produces some improvement. Dry friction over the kidneys and flanks, hot moist applications, and the application of a sheep-skin to the loins are also of service. Internally, mucilaginous drinks, diuretic decoctions and milk give the best results. The proportion of albumen rapidly diminishes, dysuria becomes less marked, urine is passed in greater quantities, and in from eight to ten days all the alarming symptoms disappear. Bicarbonate of soda may then be given for a fortnight.

In very grave cases camphor, bromide of camphor, injections of camphorated oil ( 1 to $2 \frac{1}{2}$ drachms internally, or 1 to $1 \frac{1}{4}$ drachms in subcutaneous injections) give excellent results in modifying the pain and moderating the inflammation. 
From $\frac{1}{2}$ to 1 drachm of digitalis in powder, or better still an injection of from 5 milligrammes to 1 centigramme of digitaline may also be given when dyspnœa is very great and is accompanied by anasarca. Medicines such as oil of turpentine and considerable doses of nitrate of potash, however, are contra-indicated.

\section{CHRONIC NEPHRITIS}

True chronic nephritis, i.e., a condition strictly limited to the renal tissue, and unaccompanied by pyelitis, is still little known among our domestic animals. The symptoms characterising it have not always been carefully noted, and the diagnosis is very often uncertain. Nevertheless, one of the most common forms has been carefully studied by Seuffert, viz., chronic hypertrophic nephritis.

Causation. Chronic nephritis is the common sequel to the acute forms, whatever their origin, but it may also occur primarily from repeated chills produced by such conditions as exposure to heavy continued rain when at grass, chills contracted during cold nights and the great variations in temperature in spring and autumn. The conditions, however, thus produced are rather of the nature of subacute nephritis than of chronic nephritis, properly so called.

These forms of chronic nephritis may also occur primarily in con sequence of chronic hepatic lesions with pressure on the posterior vena cava, producing blood stasis in the kidneys. Finally, they may represent the delayed effects of slight lesions which have escaped notice and have developed during grave diseases or as a consequence of repeated gestation.

From the anatomico-pathological standpoint, the only conditions hitherto recognised are the chronic hypertrophic forms of nephritis (large, white sclerotic kidney with lardaceous degeneration and sometimes marbling). This is probably because the animals are slaughtered as soon as they suffer in condition, but if they were kept long enough they would undoubtedly suffer also from the atrophic chronic forms of nephritis found in man and in the dog. In the case of man observation has shown that these two forms only represent different stages in the development of one disease, the large, hypertrophied kidney of the early stages afterwards undergoing marked progressive atrophy.

The symptoms are at first so vague that diagnosis would be impossible on a single examination. Seuffert states that the condition develops as follows:-

The first sign, loss of appetite, is soon followed by constipation and dull colic, due to congestion of the kidney; the pain is often so great as to cause intermittent groaning. 
The urine passed is always turbid, and sometimes blood-stained, but this staining rarely lasts longer than a week. The urine then gradually resumes its normal appearance, is passed in small quantities, and contains more or less albumen. The yield of milk markedly and progressively diminishes.

If treatment is resorted to at this stage laxatives and diuretics appear to effect a real improvement. Unfortunately, however, the apparent improvement is but temporary; the kidneys become hypertrophied, and the right soon occupies the whole of the sublumbar space, its margin extending as far as the extremity of the transverse processes near the anterior angle of the hollow of the flank.

This hypertrophy and the extreme sensitiveness can be detected by external palpation. Internal examination confirms the facts so observed as regards both the kidneys.

The patients eat little and become thin, whatever treatment be adopted. They progressively waste, and die after some months in a state of marasmus, exhausted and intoxicated.

It is very probable that the digestive disturbances are complicated by respiratory and cardiac trouble, as in man and the dog; but neither cardiac nor uræmic disease of the kidney has been recorded.

Diagnosis. When the urine is analysed the diagnosis becomes comparatively easy. Persistent albuminuria and hypertrophy of the kidneys during the early stages are significant indications. There can be little hesitation except in so far as pyelo-nephritis and hydro-nephrosis are concerned, but the conditions are distinguished by the character of the urine in the two latter cases, together with the condition of the pelvis of the kidney, and of the ureters.

Prognosis. The prognosis is grave, and Seuffert believes that recovery never occurs. This is also true, generally speaking, as regards all forms of chronic nephritis.

Treatment. As the disease must be regarded as incurable there is really no justification for treatment. Nevertheless, if for special reasons the owner wishes to keep the animals for a certain time, as in the case of a cow near its time of calving, recourse may be had to the internal treatment suggested in acute nephritis, viz., mucilaginous drinks, diuretic infusions, milk, bicarbonate of soda, stimulating applications to the loins, etc.

\section{HYDRO-NEPHROSIS.}

Hydro-nephrosis, i.e., retention of urine in the pelvis of the kidney and in the collecting and secreting tubules, is a somewhat common malady of the bovine species. It is usually confined to one kidney. 
Causation Anything which obstructs the discharge of urine through the ureters may cause hydro-nephrosis. Thus, vesical tumours pressing on the orifices of the ureters, calculi which have become fixed in them, torsion or "kinking" of the ureters, may bring about hydro-nephrosis. The urine secreted by the kidney being unable to escape, accumulates in the pelvis of the kidney, in the ureter, and uriniferous tubules, producing dull colic, which escapes observation, or the exact cause of which is not discovered, because the second kidney vicariously acts for the one affected, and urination continues regularly. Secretion continu-

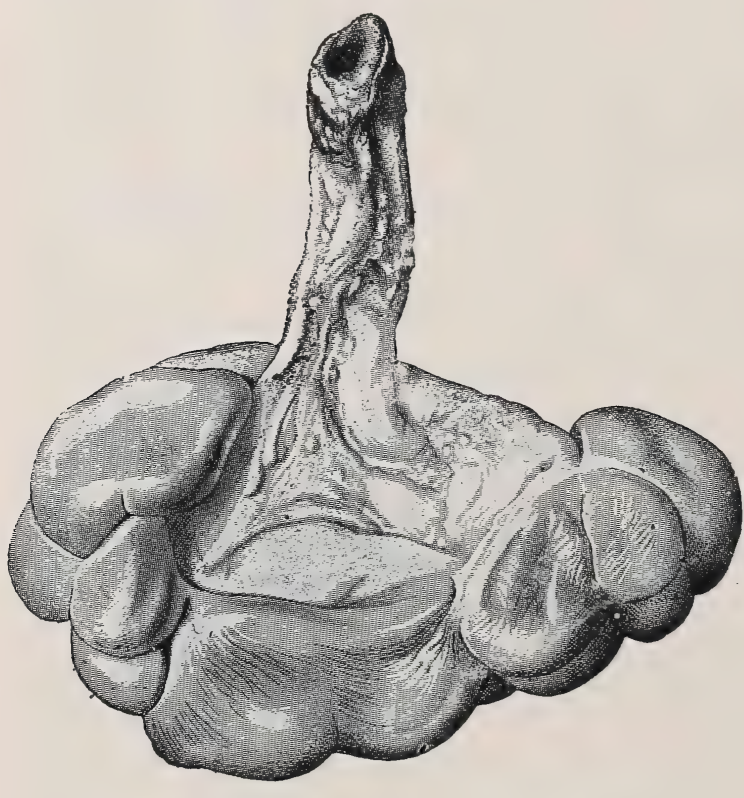

Fig. 228.-Hydro-nephrosis of the kidney. ing in spite of the obstruction, that portion of the ureter above the obstructed point, together with the pelvis and the uriniferous tubules, gradually becomes dilated, until the whole mass of the kidney is hypertrophied.

The ureter sometimes becomes enlarged to the size of a man's arm, the kidney double, treble, or quadruple its normal side: the interlobular divisions are lost, and each circumscribed lobule soon forms a cystic cavity varying in size. The pressure due to the accumulated urine causes the renal tissue, first the medullary substance and afterwards the peripheral zone, to undergo atrophy.

The kidney is represented by a vast cystic cavity, and the lobules by culs-de-sac; the cortical layer may become atrophied to such a degree as to form merely a fibrous sheath, the primary constituent elements of which are difficult to discover. From 20 to 40 pints of liquid may sometimes be found in the cystic kidney.

Diagnosis. The condition is rarely diagnosed, because, as one of the kidneys continues to act, no acute disturbance follows. Only in cases where the cystic kidney projects into the flank are suspicions aroused. Examination per rectum will then permit of the diagnosis being made.

Prognosis. Hydro-nephrosis being, as a rule, unilateral, the 
prognosis is not very grave as regards immediate danger. As the condition is hopeless, however, the lesions being irreparable, the animal should be prepared for slaughter.

Treatment. Practically there is no treatment. Puncture of the cystic cavity or even the removal of the hydro-nephrotic kidney certainly suggests itself, but, as such operations are usually opposed to the interests of the owner, they are rarely or never practised.

\section{INFECTIOUS PYELO-NEPHRITIS.}

The term "infectious pyelo-nephritis" describes an inflammation which may involve any portion of the mucous membrane of the

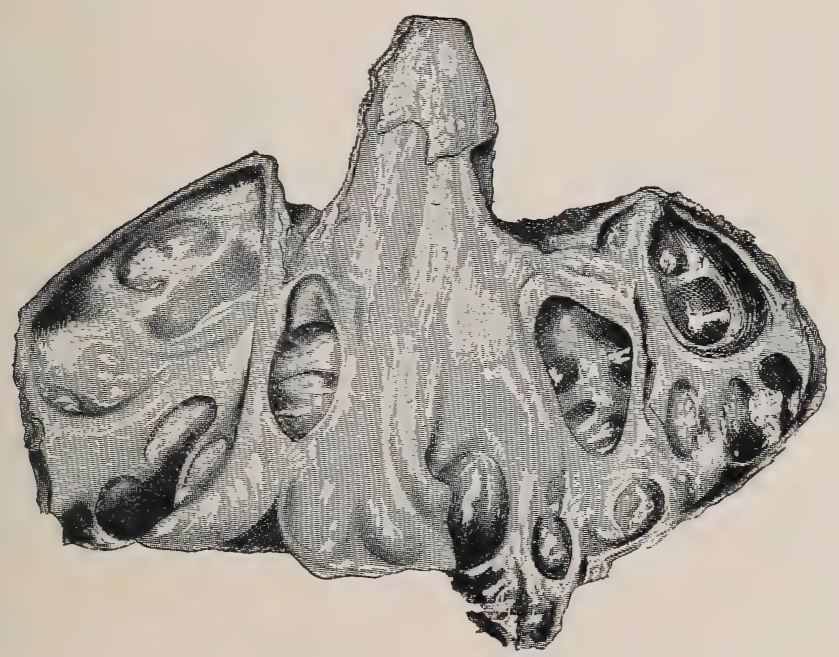

FIG. 229.- Section of a kidney affected with hydro-nephrosis. The gland substance is almost entirely atrophied, and each lobule shows marked dilatation.

urinary tract, and which is produced by a special bacillus. As a rule, this inflammation commences in the mucous membrane of the calices and pelvis (pyelitis). It afterwards extends into the depths of the uriniferous canaliculi (nephritis), but in grave and old-standing cases the mucous membrane of the ureters and the bladder may also be affected. The disease had long been known in France (Rossignol, 1848). It was afterwards described in Germany (Siedamgrotski, 1875; Pflüg, 1876), in Switzerland (Hess, 1888), and also in France (Lucet, 1892 ; Masselin and Porcher, 1895).

Causation. Female animals are more frequently affected than males, because the lesions are produced by an ascending infection, originating very frequently in genital infection after delivery. Never- 
theless, calculus formation is also an important factor in producing the disease.

Many different agents are capable of producing pyelo-nephritis. Hofflich in 1891 described a bacillus about 2 to 3 micromillimètres in length, which stained readily with aniline colours and with Gram solution. Lucet in 1892 found a short bacillus which did not stain with Gram, and later another thin bacillus which did. Kitt has described cocci, but no other organisms. Masselin and Porcher discovered a cocco-bacillus which stained with Gram and reproduced the disease in an animal lent by Moussu, after a single intra-vesical injection of the culture. Cadéac has met with staphylococci, and Moussu has discovered various bacilli, some resembling the colon bacillus, and pyogenic streptococci.

There is no doubt that many different organisms may produce pyelo-nephritis by ascending infection. The most common seem to be forms of paracoli, such as the Bacillus urea. Moussu nevertheless believes that Hofflich's bacillus, which was rediscovered by Porcher, is that which produces typical pyelo-nephritis. It grows in the bladder without producing cystitis, and is succeeded by an ascending infection of the ureters without causing primary ureteritis, the local inflammation occurring chiefly, it would seem, in the pelvis and the kidney. All the other organisms which Moussu has tested have caused lesions of cystitis and of ureteritis, together with those of pyelo-nephritis.

In these latter cases the pyelo-nephritis assumes the acute form, and is accompanied not infrequently by cellulitis and abscess formation in the tissue around the kidney.

Symptoms. Pyelo-nephritis develops in one of two principal forms, the slow chronic form, which is the most frequent, or an acute or subacute form, much more rapid in its development.

The chronic form for a time escapes notice. There is no doubt that at first some general disturbance occurs, such as diminution of appetite, disturbed nutrition, unhealthy general appearance, staring of the coat, tightness of the hide, wasting, etc., but such symptoms are in no wise characteristic, being found in all grave diseases.

The signs only become really significant from the clinical standpoint when the urine appears modified in character, and such modification does not occur until the pelvis of the kidney and the kidneys themselves are already gravely diseased.

The urine is then turbid, of a brownish colour, and charged with sediment, filaments of mucin, pus corpuscles, and earthy phosphates. On analysis it is found to contain more or less albumen.

At a late stage it may even become glairy, blood-stained, or of the colour of blood, and when the pelvis or the calices of the kidney 


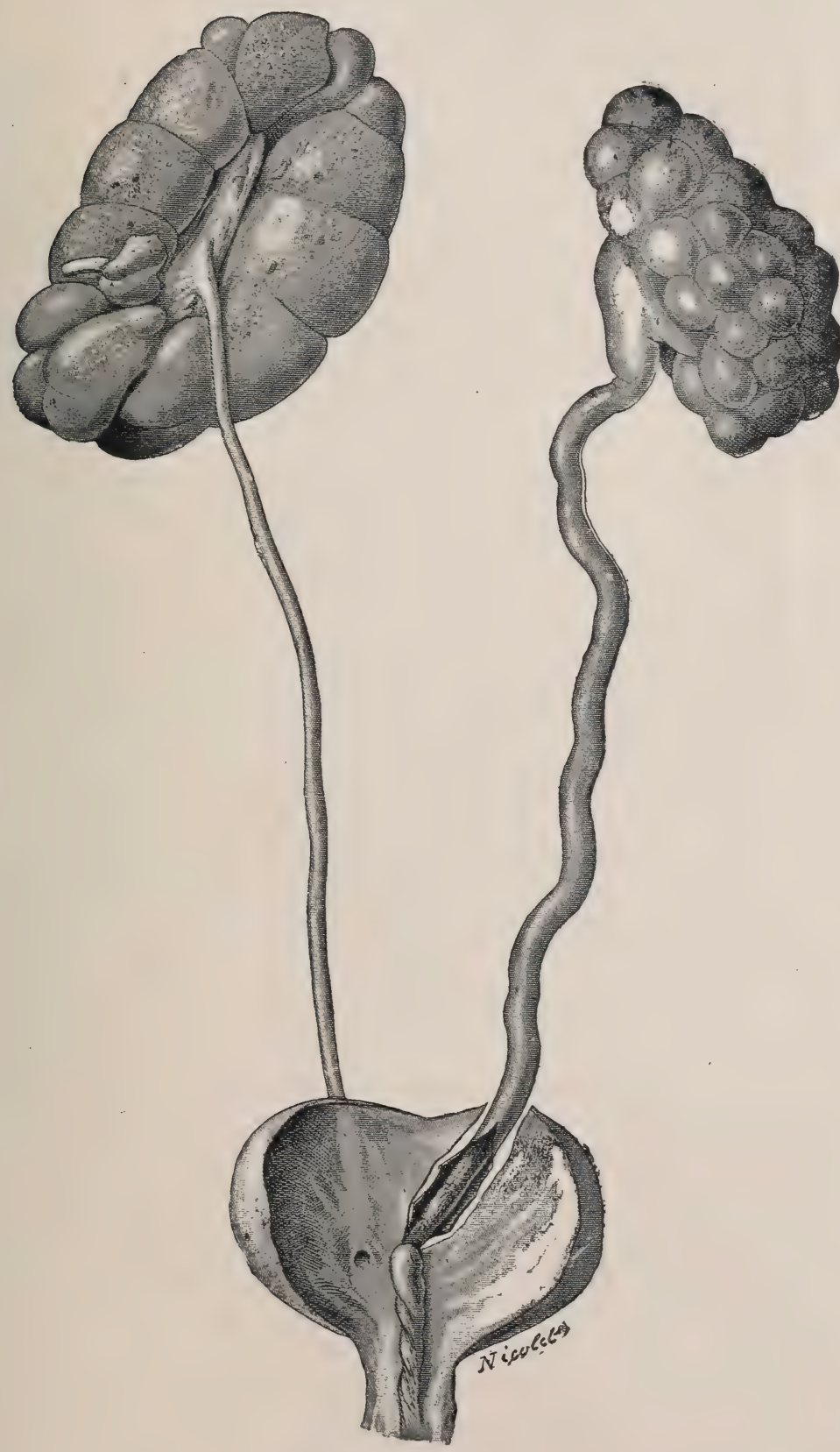

Fig. 230.-Pyelo-nephritis with hæmorrhagic pyo-nephrosis of one side. One ureter is dilated and blocked with a blood clot. 
are ulcerated may, on standing, deposit considerable quantities of red blood corpuscles.

Exposed to the air, the urine rapidly assumes a brown tint and smells strongly of ammonia.

Percussion of the loins in the region of the kidneys causes pain, as does external palpation by the flank. On rectal examination at this period the ureters are found to be distended and hard, and they give the impression of rigid or bosselated fibrous cords, sometimes as large as a child's arm. The corresponding kidney, often both kidneys, are enlarged, sometimes to double or treble their normal volume, and are painful on pressure and fluctuating, at least in the region of the pelvis. On vaginal examination the meatus urinarius is usually found to be inflamed, rough and turgid.

In this condition the animals rapidly lose flesh, the appetite becomes irregular, the general condition gradually gets worse, and they die as a result of continued uro-septic fever or uræmic troubles.

The acute form takes a much more rapid course, with fever, more marked general disturbance, acceleration of pulse and breathing, the passage of turbid and sometimes purulent urine with a strong ammoniacal smell. Pyo-nephrosis is the most frequent and characteristic end. Ordinary chronic pyelo-nephritis may also occur in these cases, and the acute course may be determined simply by accidental ascending infections.

Diagnosis. During the early stages diagnosis is extremely difficult, unless a careful examination of the urine be made. Afterwards it becomes easy, the appearance of the urine and the indications furnished by rectal exploration being perfectly characteristic. In very exceptional cases there may be some doubt, as where the urine remains normal, in spite of hydro-nephrosis, or where there is old-standing hæmaturia or renal tuberculosis. In simple hæmaturia the lesions are confined to the bladder and ureters, the kidneys not being affected, and in renal tuberculosis the diagnosis can always be confirmed by the use of tuberculin.

Prognosis. The prognosis is extremely grave, for the lesions produced are irreparable, and, moreover, local intervention is impossible.

Treatment. There is no curative treatment. All that is possible is palliative treatment with the object of facilitating the function of the kidney and of disinfecting the urinary passages by administering antiseptic substances which are excreted by the kidney. It is not possible, however, to administer active drugs of this kind (e.g., combinations of carbolic acid). As the kidney acts badly it soon ceases to eliminate such substances, and the condition would not be improved, but aggravated.

Benzoate of soda in doses of 2 to $2 \frac{1}{2}$ drachms per day dissolved in diuretic liquids is the most useful drug, and sometimes holds the disease in check for a sufficient time to allow of the animals being fattened. 
Treatment also comprises certain prophylactic precautions. As the infection which produces pyelo-nephritis originates in the genital tract, it is desirable to protect all animals in a receptive condition (those about to calve or having recently calved) from infection; hence, when the disease is detected in a cow-shed, the patients should be isolated, and the shed thoroughly disinfected.

\section{SUPPURATIVE NEPHRITIS AND PERINEPHRITIS.}

Suppuration of the kidney may occur under two conditions. In the majority of cases such suppuration occurs as a complication of pyelonephritis; less frequently it is the consequence of infection from within or infection of adjacent parts, leading to the formation of an abscess.

When it results from an ascending infection the kidney becomes swollen, congested and inflamed, and soon displays localised minute hæmorrhages. Pus then forms within the calices, in the large straight tubes, and diffuse suppuration invades all the uriniferous tubules. The enlarged kidney is yellowish, firm under the knife, and when sections are compressed pus exudes from the openings of the tubular canaliculi.

When suppurative nephritis has resulted from accidental infection of internal origin, an abscess is found to have produced more or less extensive atrophy of a portion of the kidney while not affecting the rest of the organ.

It is only in those favourable cases where the renal abscess opens into the pelvis that suppuration may invade the whole of the kidney, producing diffuse suppurative nephritis by secondary infection of the uriniferous tubules. Such complications are rare. Usually the abscess empties through the pelvis, and recovery may occur.

More frequently suppurative pyelo-nephritis develops, together with ureteritis, cystitis, dilatation of the ureters, dilatation of the pelvis of the kidney, and dilatation of the collecting tubules of the pyramids, the final stage resembling the lesions of pyo-nephrosis.

Perinephritis and perinephritic cellulitis, i.e., inflammation with or without abscess formation in the connective tissue and adipose layer surrounding the kidney, always occur in cases of suppurative nephritis or pyelo-nephritis. Such inflammations may also, in exceptional cases, follow direct mechanical injury, but they almost invariably represent complications, the organisms infecting the kidney passing through the tissues and the layer of fibrous tissue, or extending by the lymphatic paths, finally attaining the fatty tissue surrounding the kidney and there undergoing multiplication. The fatty tissue is infiltrated with reddish serosity, is inflamed, and may become the seat of large abscesses communicating with or separate from the abscesses of the kidney itself. 
Symptoms. Suppurative nephritis is characterised by fever, loss of appetite, arrest of rumination, and frequent attempts to urinate. These attempts are painful, are accompanied by groaning, and end in the passage of an insignificant quantity of blood-stained and purulent urine.

Palpation, more especially palpation of the right flank, percussion over the region of the loins, and examination of the kidneys through the rectum are painful. Wasting is rapid.

If the suppurative nephritis develops rapidly, and particularly if it be accompanied by perinephritis, the patients refuse to rise and appear to be suffering from paraplegia, although not really so, both sensation and motor power persisting in a greater or less degree.' Probably the condition is accompanied by reflex pain and irritation of the nerve-trunks of the lumbo-pubic plexus.

On the other hand, when suppurative nephritis tends to develop slowly and assume a chronic form, lesions of pyo-nephrosis gradually

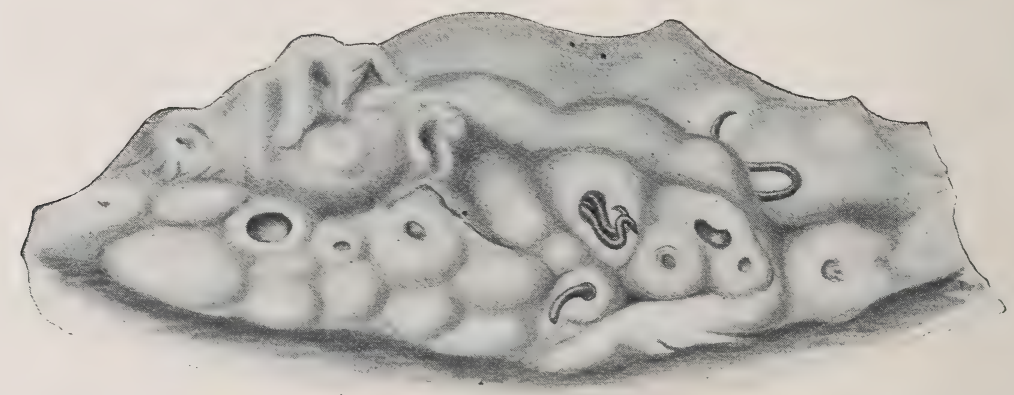

FIG. 231.-Leaf lard around kidney of pig.

develop, and are identical in appearance with those of hydro-nephrosis, except that the ureters, the pelvis and the dilatations corresponding to the lobules, are filled with pus.

Diagnosis. The diagnosis is not very difficult. The urinary trouble and the composition of the urine itself always arouse suspicion. The diagnosis is confirmed by careful and methodical examination per rectum; the inflammation of the fatty tissue surrounding the kidney can usually be detected.

Prognosis. The prognosis is extremely grave, and almost always fatal, particularly in cases of diffuse nephritis.

Treatment. No curative treatment can be absolutely relied on. Treatment, if attempted, is limited to the methods suggested for pyelonephritis. Mucilaginous, emollient, and diuretic drinks, and daily doses of 2 to 3 drachms of benzoate of soda given in the drinking water, cause some improvement. 
Stimulation of the region of the loins also undoubtedly has a favourable effect, and should always be practised, particularly where perinephritis is developing. It may check the course of the disease and prevent the formation of abscesses. On slaughtering animals suffering as above described the layer of tissue surrounding the kidney is found to be lardaceous and fibro-fatty.

Any treatment through the bladder is contra-indicated, for even the passage of a catheter may cause severe injury of the urethra or the vesical mucous membrane and produce a fatal aggravation.

If these conditions are diagnosed early, while the function of the kidney is more or less preserved, and if the animal is still in good condition, it should be slaughtered.

\section{THE KIDNEY WORM (SCLEROS- TOMA PINGUICOLA) OF SWINE.*}

In the United States of America a worm is frequently found in the fat surrounding the kidneys of pigs, and is supposed by farmers to be the cause of paralysis of the hind limbs.

This so-called kidney worm of hogs (Sclerostoma pinguicola) should not be confounded with the kidney

* From Report of the U.S.A. Bureau of Animal Industry, 1899, p. 612. (Louise Taylor.)

FIG. 232.-Sclerostoma pinguicola. External view of female. $a$, Male, natural size; $b$, female, natural size; $c$, mouth; $d$, buccal cavity ; $e$, œsophagus ; $f$, intestine; $g$, anus ; $h$, genital opening; $i$, genital tract; $k$, cephalic gland. (Louise Taylor, Annual Report, Bureau of Animal Industry, 1899, p. 614.)

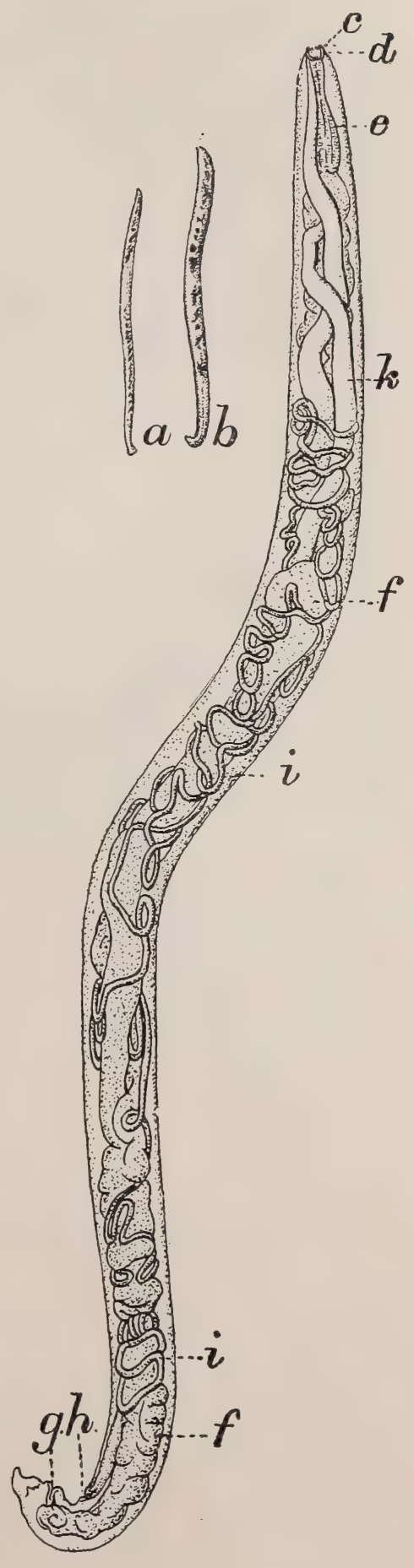


worm (Dioctophyme riscerale) of dogs and man. Both of these parasites belong to the same zoological family (Strongylidæ), but to different subfamilies and genera. The kidney worm of dogs grows to a length of 1 to 3 feet. The kidney worm of hogs is much smaller, attaining at most something less than 2 inches in length.

The body of the worm is plump, mottled in color-red, yellow, white, black-according to the organs visible beneath. The average female is about $37 \mathrm{~mm}$. and the average male $32 \mathrm{~mm}$. in length. The worms seem to occur in pairs, usually in cysts or canals; thus, upon the examination of two kidneys with their surrounding fat, fifteen specimens were
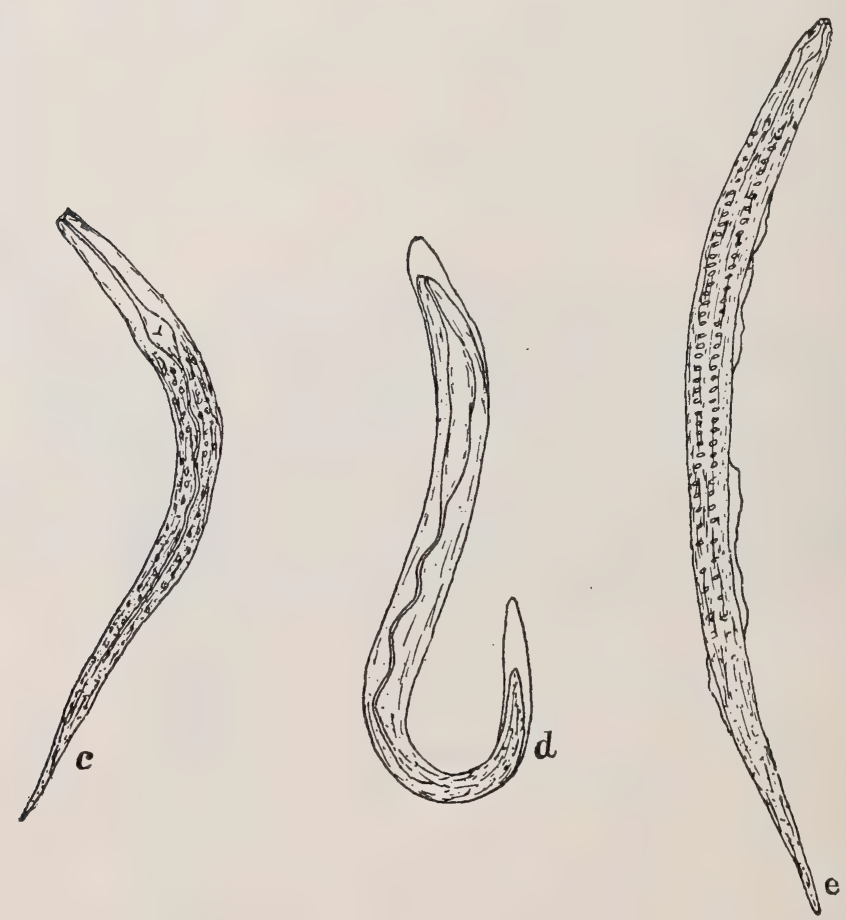

Frg. 233.-Embryos of Sclerostoma pinguicola. (Louise Taylor, Annual Report, U.S.A. Bureau of Animal Industry, 1899, p. 634.)

found, seven males and eight females. The connective tissue layers between the fat were found to be the most general seat of infection, and the cysts were numerous and closely packed together. Although a cyst usually contained two worms, a male and a female, sometimes three were found together, two females and one male, or just as often one female and two males. The cysts contained pus, which bathed the parasites, and in which were thousands of eggs in the segmentation stage. Still, 
other cysts, upon being cut into, were found without parasites and in a necrotic condition.

It will be noticed that Sclerostoma pinguicola is colloquially known as the kidney worm. In no case, however, has Miss Taylor found it in the kidney substance, but only in the tissue surrounding this organ; the lard appears to be its normal habitat, at least.

Just how the eggs leave the kidney fat or enter the bodies of fresh hogs has not been demonstrated, but it does not seem unreasonable to suppose that they eventually find their way out with the urine. Indeed, Dean reports eggs found in the urine. From analogy one is led to believe that no intermediate host is required, but that in all probability the embryos develop for a short time in water, casting several skins, and they eventually gain access to the hogs either through contaminated drinking water or food.

Because of the hog's habits, it is difficult to see any practical measures which can be adopted to prevent infection. Feeding from troughs and supplying plenty of pure drinking water will decrease but not exclude the disease. Leuckart's advice to the Germans, "Swine should be kept in a less swine-like manner," holds good in all countries and in connection with all diseases. It is equally impossible to suggest practical methods of treatment. This is all the more true because it seems probable that a number of distinct complaints are popularly grouped together by the farmer as kidney-worm disease. 


\section{CHAPTER IV}

\section{GENITAL APPARATUS.}

Semiology. The examination of the genital apparatus properly so called is easy in animals of large size, whether male or female, but is more delicate and difficult, and is sometimes partially impossible, in small creatures.

In male animals it comprises the examination of the testicles by inspection and palpation, of the vas deferens, and of the intra-pelvic genital organs (vesiculæ seminales, prostate, etc.).

Inspection and palpation of the scrotum reveals hypertrophy, atrophy, œdematous or sanguineous infiltrations, inflammation of the tunica vaginalis, and tumours of the testicle. Intra-pelvic examination partly covers the same ground as examination of the pelvic portion of the urethra, and, provided the anatomical relationships of the different organs encountered are known, there is no difficulty in detecting the position of possible lesions (Fig. 226).

In small male animals, such as he-goats and rams, rectal exploration is confined to the use of one or two fingers.

In female animals examination comprises inspection, intra-vaginal examination, and rectal examination.

Inspection reveals lesions of the vulva and clitoris.

Vaginal examination with the hand establishes the condition of the walls of the vagina, the neck of the uterus, and the vaginal culs-de-sac.

If a lesion is detected, its character can easily be ascertained by means of a speculum, which exposes the base of the vagina, the prominence formed by the uterus, or any particular part of the vagina itself. Examination with the speculum is the only useful method in young female animals, heifers in particular, on account of the narrowness of the genital tract.

In small female animals, such as she-goats, ewes and sows, the fingers alone can be employed.

As regards examination of the uterus, the direct method gives little exact information, and examination by the rectum is to be preferred. By passing the arm into the rectum and gently pressing downwards towards the base of the pelvis, the hand can be brought in contact 
with the body of the uterus, which can be moved and displaced from right to left; the horns of the uterus can be felt and followed from the body of the uterus as far as the Fallopian tubes and the ovaries. By this means the state of the uterus, its degree of sensitiveness and mobility, as well as the state of the Fallopian tubes and of the ovaries, can all be ascertained. The examination also reveals the existence or

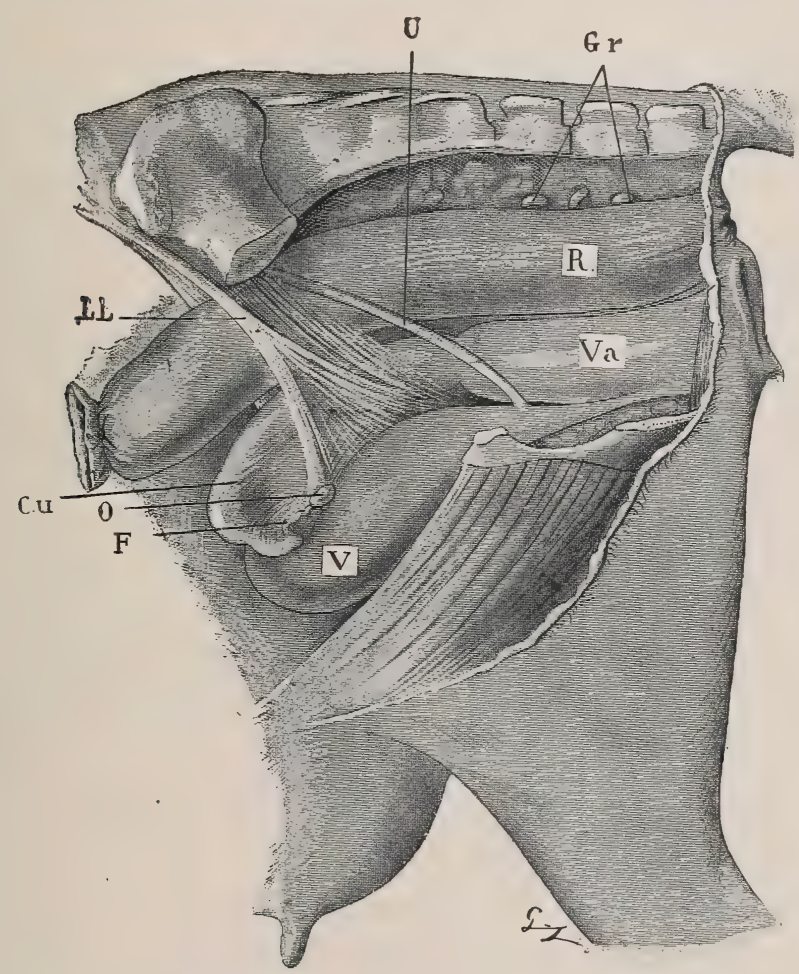

FIG. 234.-Genital organs in a cow, showing the anatomical relations. R, Rectum; $\mathrm{G} r$, meso-rectal lymphatic glands ; $\mathrm{U}$, ureter ; $\mathrm{LL}$, broad ligament; $\mathrm{V} a$, vagina ; $\mathrm{V}$, bladder ; $\mathrm{C} u$, uterine cornu ; O, ovary ; F, Fallopian tube.

non-existence of gestation, during which the uterus becomes hypertrophied and is displaced in a forward direction towards the right flank, at the same time descending in front from the base of the pelvis over the abdominal wall and under the mass of the intestinal convolutions.

\section{VAGINITIS.}

Inflammation of the vaginal conduit may be primary or secondary. It usually follows difficult parturition, but may occur under various 
circumstances. From the clinical standpoint three varieties are distinguished: simple or contagious acute vaginitis; croupal vaginitis; and chronic vaginitis.

ACUTE VAGINITIS.

Causation. Deep-seated genital injuries leading to metritis, excessive and prolonged strains due to painful labours, accidental injuries caused by obstetrical operations, etc., are followed by more or less acute vaginitis.

Suppurative inflammation of Gartner's canals, irritant and caustic injections, and foreign bodies likewise cause local irritation, which may become complicated by infection and eventually produce vaginitis. The infective organisms may be numerous and varied.

Symptoms. The vagina being closed to external inspection, the symptoms are not very apparent. At first, acute vaginitis is suggested by swelling of the vulva, pruritus, and dysuria. The lips of the vulva are odematous, injected, sensitive and of a brownishred or violet-red colour on the internal surface. Sometimes they are excoriated and torn.

The period of full development is accompanied by the escape from the vulva of a serous, mucous, muco-purulent or purulent discharge of varying odour. Urination is painful and defæcation difficult. Examination of the vagina by means of a speculum shows the mucous membrane to be excoriated, ultra-sensitive, ulcerating or suppurating at certain points. The parts are hot.

The general symptoms are little marked, and without importance. The usual termination consists either in recovery, which may be spontaneous, or in passage to the chronic form.

The diagnosis is easy, and the prognosis favourable, provided the vaginitis has not been caused by severe mechanical injuries, capable of setting up cellulitis or the formation of deep abscesses of the pelvis.

Treatment. One of the principal reasons why vaginitis persists is the retention of morbid products in the vaginal culs-de-sac. Treatment ought therefore to aim chiefly at removing these by soothing, astringent, and antiseptic injections. Soothing injections should first be tried. They consist of lukewarm water at body temperature, decoctions of black-cherry bark, poppy-heads, linseed, etc. After a few days, when the excessive sensibility has disappeared, antiseptic and astringent solutions may be used, such as crystallised alum, 150 grains to the pint; sulphate of zinc, 75 grains to the pint; carbolic acid, lysol, cresyl, etc., 150 grains to the pint.

Injections of permanganate of potash of the strength of 150 grains to the pint and of solutions of iodine at a strength of 1 in 2,000 are 
more active, but require more careful handling. Hydroxyl diluted with from 3 to 5 parts of water is also of great efficacy. Strong solutions should never be used, because they cause irritation and expulsive efforts.

All these injections may be made without difficulty by passing a simple perforated drainage tube to the end of the vagina, and connecting it with a syringe, or, better, with a small cistern hung from the wall, which allows the required pressure to be obtained.

When there are deep and severe wounds, the parts should be washed out once or twice daily and the vagina should be packed with surgical wool and iodoform gauze. The septic liquids are absorbed by the dressing, which acts continuously. This dressing is renewed until recovery takes place.

\section{CONTAGIOUS VAGINITIS.}

During the past few years certain observers have described a disease which has been termed "contagious vaginitis," in consequence of the facility with which it is transmitted.

This vaginitis may be transmitted by copulation, the bulls then serving as propagators of the disease. The bulls themselves are usually affected with balanitis.

The causative agent of the disease is unknown.

This contagious vaginitis is characterised by all the symptoms of acute vaginitis, and it is only from the fact of its appearing in all the animals served by one bull that its contagious character is established. A short time after service the vulva appears swollen and extremely sensitive; at the same time general disturbance appears, viz., diminution of appetite and of milk secretion, slackening of rumination, etc.

Vaginal exploration, which is somewhat difficult, reveals a papulovesicular eruption, accompanied by a muco-purulent discharge.

This vaginitis is easy to diagnose. It may disappear spontaneously, and the treatment differs in no respect from that of ordinary acute vaginitis.

\section{CROUPAL VAGINITIS.}

Croupal vaginitis is a form of acute vaginitis, from which it is distinguished by the formation of false membranes resembling those of diphtheria over the whole of the vaginal mucous membrane.

It was described by Baumeister. Moussu has only seen one case, and that at a period which rendered recovery out of the question.

Symptoms. The external symptoms are those of acute vaginitis, with greyish, foetid, purulent or sanguinolent discharge. On examination, the mucous membrane is found to be covered with yellowish, greyish false membranes, and with vegetations of a greyish, dirty,

D.C. 
verrucous appearance. The entire extent of the vaginal mucous membrane may be attacked, together with the neck of the uterus. In Moussu's case the uterus itself was entirely invaded.

These false membranes and vegetations are very adherent, and bleed freely at the slightest touch. They are apt to extend by degrees.

The cause of this infection has not yet been determined. It appears to obtain access to the parts during parturition, and develops insidiously for a week or two, when widespread lesions have already formed.

The diagnosis is extremely easy.

The prognosis is grave, for the lesions have a tendency to extend towards neighbouring organs. Moreover, the general health is severely, affected; there is rapid wasting, loss of appetite, and continued fever and death occurs from exhaustion, intoxication, and possibly infection.

Treatment. The treatment suggested for the ordinary acute forms appears to be useless in this condition. The new membranes show too many folds, depressions, and accidental culs-de-sac for simple injections to have any real effect. Better results might be expected from packing with antiseptic gauze or from the use of antiseptic ointments applied after washing out the cavity with permanganate of potash solution or hydroxyl.

Curettage, followed by the use of gauze dressings, might also be tried; considerable difficulty must necessarily be anticipated in operating in a cavity which has become inextensible and partly filled with vegetations and false membranes.

\section{CHRONIC VAGINITIS.}

Chronic vaginitis usually represents the last stage of some form of acute vaginitis, though it occasionally develops in a slow and progressive fashion as a primary condition in consequence of some deep-seated genital lesion. There is usually a constant discharge of irritant material.

The symptoms are not very striking, and are purely local.

Externally all that can be detected is a continuous or, much more frequently, an intermittent muco-purulent discharge from the vulva, which occurs only on urination, defæcation, coughing, etc.

Locally, examination with a speculum discloses the fact that the mucous membrane is of a greyish colour, thickened, less yielding than usual, and in places sclerosed. The entire thickness of the mucous membrane is affected, as also at times the muscular tissue, chronic irritation having caused sclerosis.

The diagnosis is very simple, and the prognosis of no particular gravity, because the animals can always be fattened. The condition is only grave as regards animals intended for breeding. 
Treatment is often very successful, but, as in all chronic diseases, it extends over a considerable time. Practically it is not often attempted. It does not differ greatly from that of ordinary acute vaginitis, but the best results seem to follow the use of astringents.

\section{METRITIS.}

Infectious or traumatic diseases of the uterus are of the greatest importance in bovine pathology, both on account of their frequency and gravity. They comprise septic metritis, acute metritis, and chronic metritis.

\section{SEPTIC METRITIS.}

Septic metritis is also termed "metro-peritonitis" and "parturient septicæmia." It may be compared with puerperal fever in woman.

These terms are sufficiently explicit to indicate that if at first the metritis is typical it frequently becomes complicated with peritonitis, and too often also with true septicæmia.

Causation. The disease only appears after parturition or abortion, and during the few days immediately succeeding delivery. Parturition may occur spontaneously in a perfectly regular and easy manner, and nevertheless be followed by fatal metritis as a consequence of infection. Usually the labour has been difficult, and the after-birth, or portions of the foetal membranes, have been retained. Septic metritis then develops in consequence of their putrefaction.

Infection with micro-organisms is therefore the essential cause, and the only one of importance. None of the conditions formerly invoked can do more than favour or check the course of this infection.

Moreover, the subsequent complications are entirely due to the special character of the infective agent.

These infective agents may be of various descriptions. They have been the subject of numerous investigations, on account of the gravity of puerperal fever in woman. Pasteur, Colin, Chauveau and Doléris were the first to take up this question. In veterinary medicine several inquiries have been instituted, but a great deal remains to be done. The most frequent agents are varieties of streptococci, of the colon bacillus, and of putrefactive bacteria.

Septic metritis may occasionally be purely accidental and only affect one animal, but infection of stables by a primary case is an obvious cause of propagation. Moussu has seen six animals successively die of septic metritis in one year, and in a stable which had not been disinfected after each death.

Symptoms. The first symptoms occur between the first and fourth days after parturition, when the uterine mucous membrane is still 
tender, discharging and bleeding, and the lochial discharge is abundant. The disease rarely appears after the first week. The earliest symptoms are dulness, depression, loss of appetite, and general weakness. The animals appear exhausted, the secretion of milk is diminished or altogether suspended, and all the chief functions of the body are interfered with.

The temperature varies in a peculiar and significant way. In some forms, due to infection with streptococci or to mixed infection, it rises to $104^{\circ}$ or $105^{\circ}$ Fahr.; in others it remains stationary or falls below normal. It might be thought that in these latter cases, which are usually due to infection with the colon bacillus, the general condition was not grave. This, however, would be a very serious error, for in such cases death follows as rapidly as in the others.

The patients, or some of them at least, have slight colic and peritonism when the infection extends to the peritoneal pockets at the entrance to the pelvis. At a later stage they appear prostrate, remain lying, and seem to be suffering from paralysis of the hind quarters.

None of these general symptoms are in themselves significant, and to appreciate them at their true value the local signs must be taken into account.

The external genital organs are moderately swollen, the vagina is infiltrated and sensitive, and is soiled by exudate of varied character.

The neck of the uterus is sometimes prematurely contracted after the first or second day, constituting a troublesome complication both in examining the parts and in treatment. When, bowever, it is dilated and the hand can be passed, it is found that the uterus itself is not contracted or is only half-contracted, and that it is filled with a reddish-grey liquid of putrid appearance, sometimes without smell, at others foetid or even putrid.

The uterine mucous membrane is infiltrated, thickened, and extremely fragile, partially destroyed, and breaks at the slightest touch. The cotyledons may become loosened by necrosis, and accumulate in the depression formed by the gravid horn of the uterus; otherwise they may be detached without difficulty.

When the neck of the uterus is prematurely contracted direct exploration gives no result, but rectal examination reveals a much enlarged uterus, filled with liquid or distended with putrid gas.

If, however, the after-birth has not come away, fragments of fœtal membranes may be removed from the deeper portions of the uterus or the surface of the cotyledons.

Death is inevitable unless treatment is early initiated. The animals succumb to infection produced by germs entering the vascular appa- 
ratus. When the infection extends by contiguity of tissue to the peritoneal cavity the immediate causes of death are infection and intoxication. In cases where the infection remains localised within the uterus the animal is poisoned by the absorption of toxins through the uterine mucous membrane.

The condition may prove fatal in from four to six days in the cow, but in a shorter period in the goat, ewe, and bitch.

Lesions. The uterus is excessively fragile, and can be torn at will. The mucous membrane in which the micro-organisms more particularly develop appears necrosed in places. Large areas are sloughing or ulcerated.

The vessels are thrombosed, and extensive portions of the organ may be affected by true capillary phlebitis.

The lymphatics are dilated and distended with pus in animals which have resisted for some days.

If there is peritonitis, the entire floor of the abdominal cavity is affected, and sometimes the peritonitis is generalised.

The lesions in the other tissues and viscera are similar to those found in septicæmia and in general intoxication, such as injection of the capillary system, and interstitial extravasations of blood in the pleura, pericardium and other tissues.

Diagnosis. The diagnosis of septic metritis is not very difficult, though something more is required than simple observation of external signs. From the clinical standpoint it is of no great importance to distinguish between the various forms or to determine the responsibility of micro-organisms for the infection. In every case the practitioner must utilise all the means at his disposal.

Prognosis. The prognosis is extremely grave, and death almost invariably occurs when treatment is not early undertaken.

Treatment. Treatment should be prompt and energetic. Infection of the genital organs being the cause of the symptoms, it is against this that remedies should be directed. The parts should first be thoroughly washed out with boiled water at the body temperature. A stiff drainage tube about 6 feet in length is passed to the base of the uterus and connected at its free end with a reservoir of liquid, which can be raised so as to obtain sufficient pressure. During this operation the animals should be placed with the front limbs higher than the hind.

When the liquid injected returns perfectly clear, antiseptics may be employed. Strong solutions containing mercury, carbolic acid, lysol or creolin should be avoided, partly because of their toxic action, but principally because they cause irritation and violent expulsive efforts. 
A 25 per cent. hydroxyl solution gives surprisingly good results. A 1 in 2,000 iodine solution (iodine 15 grains, potassium iodide 1 drachm, warm water 4 pints) is also of very great service.

As the first irrigations are difficult to carry out thoroughly, it is often advisable to cleanse the parts directly by means of a large disinfected sponge, which should be passed over the whole surface of the mucous membrane and into the depressions of the uterus, thus directly removing septic liquids. Thereafter irrigation will be easier and more efficacious.

This method, however, of cleansing the parts is dangerous for the operator unless he takes the antiseptic precautions necessary in every case of delivery.

When the neck of the uterus is prematurely contracted, the difficulties become much greater, and are sometimes insurmountable on account of the impossibility of dilating it. It then becomes necessary to use metallic canulæ or uterine sounds made of aluminium in order to pass through the neck of the uterus. Liquids can be evacuated by compressing the uterus through the wall of the rectum, but the method is very troublesome.

This local treatment should be practised twice a day at least until ali danger is over, and may be completed by the administration of diffusible stimulants, such as alcohol in doses of 6 to 10 ounces, acetate of ammonia in doses of 2 drachms, wine, coffee, and diuretic decoctions. These may be given with food or drink to whatever amount is considered necessary if the animals still retain their appetite. The food should be light and easily digested.

From 8 to 12 pints of physiological salt solution may be injected intravenously every day, the temperature at which this fluid is injected being varied according to the degree of fever. (Physiological salt solution consists of chloride of sodium 9 parts, sterilised water 1,000 parts.)

Prophylaxis. Should a case of septic metritis occur in a byre containing other cows about to calve the building should be disinfected.

\section{ACUTE METRITIS.}

The term "acute metritis" is used to indicate a variety of inflammation of the uterus of a sufficiently grave character, which, however, does not prove fatal in twenty-four or forty-eight hours.

Causation. In domestic animals acute metritis develops exclusively after difficult parturition and as a consequence of the tearing of tissues or accidental post-partum infection.

At one time it was the rule to recognise a traumatic form consequent 
on wounds by embryotomy hooks, crutches, cords, etc., etc. There is no reason for maintaining this distinction, because the essential condition for the development of metritis is the infection of the injuries.

Acute metritis follows non-delivery, incomplete delivery, or accidental infection.

Symptoms. The external signs are very few, and must be carefully studied, in order that wrong conclusons may be avoided.

Certain of these external signs suggest general disturbance such as one finds in all acute visceral inflammations, viz., loss of appetite, progressive wasting, irregular slight fever, diminution or cessation of the secretion of milk, dulness, etc.

The others are purely local. The discharge from the vagina is mucoid, muco-puralent, sanguinolent or fœetid, according to circumstances. It is small in quantity, and occurs only when the animal lies down or makes expulsive efforts. Examination with the speculum reveals the existence of slight secondary vaginitis and more intense inflammation of the neck of the uterus, which remains half open. Rectal examination shows that the uterus is abnormally large and more difficult than usual to displace. If acute metritis has existed for some weeks, the uterus is painful to the touch, and sometimes fixed in position in consequence of the development of parametritis and of slight pelvi-peritonitis, the occurrence of which is always indicated by temporary tympanites.

Cases of acute metritis may recover spontaneously, but they rarely do so. The condition usually tends to become chronic or to be complicated with peri-uterine diseases which may prove fatal.

Diagnosis. The diagnosis can be established without difficulty by rectal examination and direct examination with a speculum.

Prognosis. The prognosis is grave, because the patients are temporarily or permanently incapable of becoming pregnant, and because acute metritis may be complicated with pelvi-peritonitis, phlebitis of the intra-pelvic veins, etc.

Treatment. The uterus, and particularly the uterine mucous membrane, being affected, all our efforts should be concentrated on that organ. A careful study of the lesions shows that the glandular follicles are infected, and with them the entire thickness of the mucous membrane. The object to be attained, therefore, is the perfect disinfection of this tissue. The parts should repeatedly be washed out with warm water at blood-heat, followed by antiseptic injections containing 4 drachms of chloral per pint; a 1 in 2,000 iodine solution or 20 per cent. to 25 per cent. hydroxyl solution, etc. Despite such injections, the inflammation disappears slowly and with difficulty, and when the neck is sufficiently open it might perhaps be possible, as in human medicine, 
slightly to curette the mucous membrane of the uterus and plug the cavity with iodoform gauze.

Where, however, the neck of the uterus is so far contracted as no longer to admit a sound or canula for irrigation, the difficulties are very great. Nothing effectual can be done until the neck of the uterus is dilated, an exceedingly troublesome operation.

In the forms termed "post-partum traumatic metritis" antiseptic injections must not be made with any considerable pressure, because of the danger of rupture; plugging the cavity with antiseptic gauze is preferable.

\section{CHRONIC METRITIS.}

Chronic metritis is often the termination of acute metritis, though inflammation of the uterine mucous membrane may assume the chronic form from the first. All post-partum infections with pathogenic microbes may give rise to chronic metritis, as may the various forms of cystitis, vaginitis, rupture of the vulva, etc. Tuberculosis also leads to chronic metritis, which is easily diagnosed by a simple bacteriogical examination of the discharge.

Symptoms. Chronic metritis is accompanied by bad general health and persistent local disturbance. The animals show a permanent mucopurulent discharge varying in amount, or simply an intermittent discharge, which is then more abundant and only lasts some hours or some days, but reappears after irregular intervals. On examination the neck of the uterus is found to be half open, slightly hypertrophied, sometimes sensitive, and covered with vegetations.

Examination through the rectum may show the organ to be considerably hypertrophied, sensitive, and comparatively immobile. Cases are numerous, however, in which the examination reveals nothing very striking.

In other cases, vaginal examination by means of the speculum reveals nothing, except that the neck of the uterus is completely closed, and yet on rectal examination the uterus is found to be of large size, tense, uniformly fluctuating, and in exactly the position to be expected were the animal pregnant. This clinical form was formerly termed "hydrometritis," but it would be better named " pyo-metritis," inasmuch as it depends on chronic metritis. The neck of the uterus remains contracted, and the morbid products accumulate in the body and uterine horns, which are gradually dilated. Then suddenly the uterus is seized with reflex contractions overcoming the resistance of the neck and expelling the contents in one jet. The discharge may continue for some days, after which the neck again closes and the disease enters on a new phase.

Lesions. The lesions affect the mucous membrane, more particularly 
the glandular tissue and the interstitial tissue. From the anatomical and pathological point of view different forms are recognised, some with glandular and mucous atrophy, others with marked hypertrophy, the mucous membrane being covered in some cases with vegetations and fungus-like growths.

Diagnosis. From a clinical standpoint, it is only necessary to distinguish the ordinary forms from tuberculous metritis, which latter is of no clinical importance on account of the impossibility of treatment.

Prognosis. The prognosis is grave, as in all chronic diseases. Furthermore the animals are, for the time being, sterile and diffcult to fatten.

Treatment. One of the fundamental conditions of treatment is to attack the disease locally, and it is necessary, therefore, that the uterine neck should be dilated.

If the neck of the uterus is pervious, the parts must be washed out daily with antiseptic solutions, after having lightly curetted the mucous membrane with a blunt curette. Boiled water is first used, and is followed by solutions of chloral, iodine, hydroxyl, or permanganate of potash.

When the neck of the uterus is contracted, it must first of all be dilated. In practice such treatment is sometimes considered too costly, so that the animals are slaughtered or recovery is left to chance.

Moussu has seen several animals suffering from metritis, and even from salpingitis, recover spontaneously after six to eight months at grass.

\section{EPIZOOTIC ABORTION IN COWS.}

This disease, which was carefully investigated, first by Professor Nocard of Alfort, and afterwards by Professor Bang of Copenhagen, may be regarded as a specific uterine catarrh, determined by a definite species of bacterium.

It often affects large numbers of animals in one district or on one farm, and causes very serious loss. It is conveyed from cow to cow either by the bull or by litter or utensils used in the byre which have been soiled by the uterine discharges of an infected cow. As in many other infectious disorders, one attack of the disease seems to confer a certain immunity, and although some cows become sterile after an attack and others continue to abort, a certain proportion after aborting two or three times acquire relative immunity, so that they conceive and carry their calves the full time. This is probably why epizootic abortion usually ceases after some years in herds which are kept isolated and do not receive fresh recruits.

The microbe of epizootic abortion is a very small bacterium which stains well with Löffler's methylene blue. When massed together 
these bacteria resemble cocci, but isolated specimens are seen to be true bacteria containing one, two, or occasionally three roundish, elongated deeply-stained granules. They do not stain with Gram, and are non-motile.

These bacteria exhibit remarkable vitality. Bang relates cases which seem to prove that they may exist within the uterus for at least fourteen months, and in the uterine exudate outside the body for at least seven months, even at comparatively low temperatures.

On post-mortem examination one finds between the mucous membrane of the uterus and the fœtal envelopes an abundant odourless exudate, dirty-yellow in colour, somewhat thin, pultaceous, slimy, or lumpy in character. Under the chorion is found a thin, clear, gelatinous substance contained within the fine connective tissue lying between the chorion and allantois. The umbilical cord is often oedematous. All these exudates contain the specific minute bacterium.

The above exudate forms the peculiar dirty, reddish-yellow, slimy, flocculent, pus-like odourless fluid which escapes from the vagina during or immediately after the act of abortion.

The results of infection of the uterus with Bang's bacterium may be delayed for a considerable time. In two cases where he injected pure cultures into the vaginæ of pregnant cows no apparent local results had been produced at the end of thirty-three and thirtyfive days respectively when the cows were slaughtered; but in the case of two other pregnant cows, inoculated three months after conception, signs of abortion became apparent, and one cow in fact aborted in about ten weeks; post-mortem examination revealed the characteristic local changes, and microscopical and cultural preparations clearly established the presence of the specific organism.

Although the sexual organs form the usual channel of infection, it, seems possible that the organism may in some cases enter the body through the respiratory or digestive tract.

The treatment in this condition is chiefly of a prophylactic character. Bulls which have served cows belonging to herds known to be infected should not be allowed to cover healthy cows. They should undergo careful local disinfection, and for a time be withdrawn from the stud.

Cows which show signs of impending abortion should at once be removed to a separate shed. The fotus and its envelopes should be buried or burnt, and the person who attends the diseased cow should be prohibited from entering the common cow-shed.

Where space does not admit of this the affected cows should be removed as far as possible from those still healthy and placed in a separate row. When they abort the after-birth should be removed 
by hand, and the uterus daily washed out with some non-irritant but effectual disinfectant. Even after apparent recovery a period of probation should elapse before the cow is again put to the bull.

The genital organs and vaginæ of the still healthy animals may also be irrigated with a disinfectant solution, in order, if possible, to ward off infection. For disinfecting the channels and floor of the stable quick-lime will be found clean, non-odorous, cheap and effective.

In dealing with this disease one must always bear in mind the great vitality of the bacterium, the relatively long time it may persist either in the animal's body or in the infected sheds, and the considerable period which may elapse before its effects become evident.

The same or a similar organism seems capable of producing abortion in sheep and mares.

\section{SALPINGITIS-SALPINGO-OVARITIS.}

This section will be brief, because the condition is very far from having been thoroughly elucidated. Moussu himself has only studied a single case of simple suppurative salpingo-ovaritis.

Salpingitis and salpingo-ovaritis, i.e., inflammation of the Fallopian tubes and of the ovaries, can only develop as a consequence of ascending infection, as a complication of acute or chronic metritis, by auto-infection during the course of tuberculosis, or as an accident during what is known as tubal gestation.

Tuberculous salpingitis is frequent, and exists in a very large majority of cases of genital tuberculosis. Accidental salpingitis as a consequence of tubal gestation is extremely rare, and is usually overlooked or mistaken for some other condition.

From the clinical standpoint, therefore, we recognise two varieties of salpingitis - the one suppurative, the other tuberculous.

Symptoms. The external symptoms are similar to those of metritis, because salpingitis develops as a complication of metritis after parturition, abortion, or retention of the after-birth. The only external symptom is a discharge of varying quantity from the vulva. This may be intermittent or permanent, and it is accompanied by frequent expulsive efforts in no respect characteristic.

The nature of the lesions is ascertained by rectal examination, and as lesions of the uterus, of the Fallopian tube, and sometimes of the ovary often co-exist, the examination must be carried out methodically and gently in order to distinguish between the parts touched. The normal relationships may be modified by uterine lesions, inflammatory adhesions, local peritonitis, etc.

Diagnosis. The diagnosis requires care. 
Prognosis. The prognosis is grave. The lesions are too deepseated to be directly attacked, and, moreover, salpingitis may terminate in pyo-salpynx, i.e., in encysted abscess of the Fallopian tube.

Treatment. The treatment is similar to that of metritis. The natural opening of the Fallopian tube into the uterus allows pus and morbid products to escape, and when the metritis disappears the salpingitis may diminish and recovery may take place.

Treatment therefore is quite indirect, for in veterinary surgery it is useless to attempt to repeat on large domestic animals the brilliant operations of human surgery. The relations between uterine diseases and those of the Fallopian tubes are so close that this method of treatment gives excellent results. Moussu has seen a case of chronic metritis complicated with salpingitis recover after simple uterine treatment.

\section{TORSION OF THE UTERUS.}

Although torsion of the uterus is a condition more particularly pertaining to the domain of obstetrics, a few remarks on the subject may not be altogether out of place at this point.

The accident is commonest in the cow, but it has also been described in the mare, ewe, bitch and cat, and it probably occurs, though less frequently, in the other domestic animals. In the cow it is commonest during the last month of pregnancy.

Very little is known as to its cause, though the consensus of opinion -if any consensus can be said to exist in face of the existing divergent views-appears rather to indicate that it follows falls in awkward positions, sudden efforts, severe prolonged exertion, or tympanites.

In pregnant cows the uterus assumes the appearance of a pendulous organ the body and horns of which constitute the bob of the pendulum, whilst the ligaments represent the cords by which it is suspended. The fixed points are formed by the insertions of the two ligaments in the neighbourhood of the two external iliac angles.

The uterus, however, is also steadied in position by the vagina and by the cellular tissue surrounding it; in fact, in non-pregnant animals it can scarcely be regarded as pendulous, but rather as freely floating and readily yielding to the movements of the surrounding organs.

As soon as the uterus is occupied by a fœetus, however, the conditions become changed. In consequence of the increased weight of its contents the uterus exerts a pull on the broad ligaments and sinks lower in the abdominal cavity. The vagina and the surrounding connective tissue are rendered tense to a degree depending on the increasing weight of the calf. The uterus then more closely resembles 
a pendulum, the bob being represented by the fœtus and its envelopes. The suspensory apparatus can be divided into three parts, viz., the two broad ligaments and the tissue connecting the uterus to the vagina.

The pull on the vagina increases greatly as soon as the gravid uterus is twisted either to the right or left, for, torsion being attended with more or less extensive displacement towards the lower abdominal wall, the tension on the vagina must become more marked.

Considering now how the spiral folds and the constrictions which are of such importance in diagnosis are formed, we find that both structures, viz., the wall of the uterus and the ligaments, are implicated, though to different degrees. Whilst the spiral folds are more particularly formed by the wall of the uterus, the broad ligaments are chiefly responsible for the constrictions, though to some extent the spiral folds also contribute to their production. The spiral folds of the body of the uterus are formed solely by twisting of its own walls. This can easily be shown by taking any tubular organ whose walls are not too rigid, and twisting it round its horizontal axis.

The broad ligaments contribute less to the formation of the spiral folds, though they play a more important part in producing constrictions and thus in compressing the wall of the uterus.

The symptoms are ill-defined. Sometimes there is difficulty in micturition, but as a rule little evidence exists of any abnormal condition until the advent of labour pains. The first pains, which are usually feeble and separated by rather long intervals, are succeeded by colic. The succeeding efforts steadily become more violent and frequent, but the "water-bag" fails to appear, and in a period_varying between twelve and forty-eight hours the pains subside. Rumination is at first suspended, the pulse and respiration are accelerated, and the surface temperature is irregular.

If treatment is not undertaken similar symptoms, but of exaggerated intensity, may again appear in from one to six days. Failing relief death always follows after a varying interval.

The diagnosis is not difficult, provided the maternal passages be examined. On passing a carefully lubricated hand into the vagina the operator discovers, at a varying distance from the os uteri, signs of collapse and twisting of the canal. In cases of quarter twist it is often possible, by rotating the hand so as to follow the spiral folding of the vagina, to introduce the fingers as far as the os uteri; but in half or complete rotation only one or two fingers can be passed so far, or it may be altogether impossible to reach the os.

In the Berliner Archiv for 1902 Lempen gave a summary of the extensive literature dealing with this disease and of the varying views 
held regarding its origin and treatment, particularly as to the direction in which the animal should be rolled in order to reduce the torsion.

In common with the majority of authors, Lempen rightly concluded that the rolling should be in the same direction as the torsion. $\mathrm{He}$ also proposed to describe the torsion as being to right or left, according to the direction of the spiral folds to be found on the upper wall of the dilated cervix uteri when the examiner stands behind the animal. This mode of describing the changes seems least likely to cause misunderstanding.

In describing the degree of torsion Haase takes as his index the upper wall of the uterus. Where this has moved through an angle of 90 degrees he speaks of quarter torsion; when through an angle of 180 degrees (in which case the upper wall will have become the lower) of half or semi-complete torsion; when through an angle of 270 degrees as three-quarter, and when through 360 degrees (the upper wall having then described an entire circle and returned to its former position) as complete torsion.

In forming a diagnosis, the extent to which the maternal passages seem fixed in position, the amount of resistance they offer to the hand, and the degree of tension in the spiral folds to some extent indicate how far torsion has proceeded. Where the spiral folds are very tense and the passages completely immovable, so that the operator cannot reach the fœetus, torsion is usually complete; in cases of less complete torsion (one-quarter to three-quarters) the cervix uteri is closed and displaced to a proportionate extent, and the resistance to the passage of the hand is in keeping.

The prognosis is very grave.

Treatment is difficult, and of the numerous methods suggested (laparotomy and direct abdominal taxis, vaginal hysterotomy followed by abdominal taxis, vaginal taxis, ete.) most have fallen into desuetude or are looked on as of so desperate a character as only to be justified in extreme cases. That which most merits attention and has been attended by most uniformly favourable results consists in the rotation of the animal's whole body. The best results are said to be obtained by casting the animal, or causing it to lie down, on a sloping surface with the hind quarters higher than the fore and then to roll it down hill, in the same direction as the spiral twists discovered in the vagina. It is possible to follow the course of the manipulation by retaining the hand in the vagina, but failing this the vagina should be examined after each attempt. Even though the first attempts fail hope should not be abandoned, for Haase has succeeded in effecting delivery after rolling the animal twenty times. The operation should be performed smartly and the animal's body be rolled as a whole, the fore limbs turning along with the hind. 


\section{TUMOURS OF THE UTERUS.}

The study of tumours of the uterus is still so incomplete that it would be impossible to give a precise description of them. This is to a great extent due to the fact that as treatment is difficult the animals are usually slaughtered as soon as an assured diagnosis can be given.

The general symptoms of tumours of the neck, body, or horns of the uterus resemble those of chronic metritis, viz., permanent or intermittent discharge from the vulva, wasting, expulsive efforts, dysuria and sterility. The position of the tumour, its form, point of insertion, size, consistency, mode of attachment, etc., can be detected by vaginal or rectal examination.

The diagnosis having been made, the only question is as to treat. ment. Should the tumour prove mobile, clearly delimited, and with a well-marked pedicle, it can be removed either by tearing away or by breaking into fragments, or again simply by dividing the pedicle and applying antiseptic pads to check bleeding. But if the tumour proves largely sessile and ill-defined and it extends into neighbouring tissues the animal should be slaughtered, as such patients can neither be fattened nor used for reproductive purposes.

\section{TUMOURS OF THE OVARY.}

We might repeat in regard to tumours of the ovary what has just been said as regards those of the uterus, though the former are much commoner than the latter.

Clinically, ovarian tumours may be grouped under two heads, solid tumours and cystic tumours-the first represented by the fibromata, fibro-sarcomata and epitheliomata, the second by uni- or multi- locular cysts.

All these tumours are dangerous; they may develop rapidly, and they rarely fail to produce disturbance, the animals presenting various genital troubles, among which may be mentioned sterility and nymphomania.

The cystic tumours; which develop at the expense of epithelial invaginations of the peritoneal covering or at the expense of Pflüger's tubes, and not, as was formerly believed, by the morbid development of the Graafian vesicles, constitute dangerous growths, true cystoepitheliomata or cystic epitheliomata, capable of producing fatal complications (vascular disturbance, local or general peritonitis, compression of the ureters, etc.).

The diagnosis must be arrived at by vaginal and rectal examination. 
It is usually possible to distinguish the condition from disease of the kidney, bladder, or pelvic lymphatic glands.

Treatment. The only possible treatment is removal of the diseased ovary and of the ovarian tumour, but much depends on circumstances. If a large tumour has formed extensive adhesions, ablation may be impracticable or so dangerous that under the circumstances in which veterinary practitioners are forced to operate it cannot be undertaken. If, on the contrary, the ovarian growth is free and pedunculated, even though of large size, extirpation is possible.

The method is exactly similar to that of castration of the cow, and follows the same rules, but the vaginal incision has to be much longer, so as to allow the entire hand to be passed as far as the tumour. The pedicle is divided by means of the écraseur, which should be worked very slowly. In removing very large tumours, however, it is possible to operate from the flank.

\section{GENITAL MALFORMATIONS.}

IMPERFORATE VAGINA.

Many forms of genital malformation occur, but only those which produce sterility are pathologically important.

One alone causes very marked disturbance, viz., imperforate vagina. This condition may be accidental or acquired, and may follow either difficult parturition, with circular lesions of the vagina, or burns or cauterisation of the vagina, followed by adhesion of its walls.

It is generally of congenital origin, and the obstruction as a rule is in the region of the hymen, as a consequence of some anomaly in development, and not of abnormal development of the hymen itself.

This imperforate condition of the vagina is not attended by grave consequences during early life; but later, when the generative functions become active, all the products of secretion of the uterine and vaginal mucous membranes accumulate in the closed cavity, giving rise first to muco-metritis, then to muco-kolpitis, similar in its development to the hæmato-kolpitis of young girls. The uterus gradually becomes distended with liquid, the neck is dilated, and a portion of the vagina may attain enormous dimensions, so much so as to suggest pregnancy.

Symptoms. The symptoms become appreciable only after a time - about one year or fifteen months in heifers-and they seem to be associated with the appearance of cestrum. The animals make continued expulsive efforts, which when the genital canal is much distended may become extremely violent. There is also dysuria as a consequence of 
compression, together with uterine and vesical colic, loss of appetite and wasting.

Diagnosis. The diagnosis requires care, and can only be arrived at after examination of the vagina and examination per rectum. In young females this examination is extremely difficult, because of the narrowness of the genital tract and of the rectum. For vaginal examination we prefer to use a small speculum, which exposes the depths of the vagina or the transverse septum without necessitating other manipulation. On rectal examination the uterus and vagina are

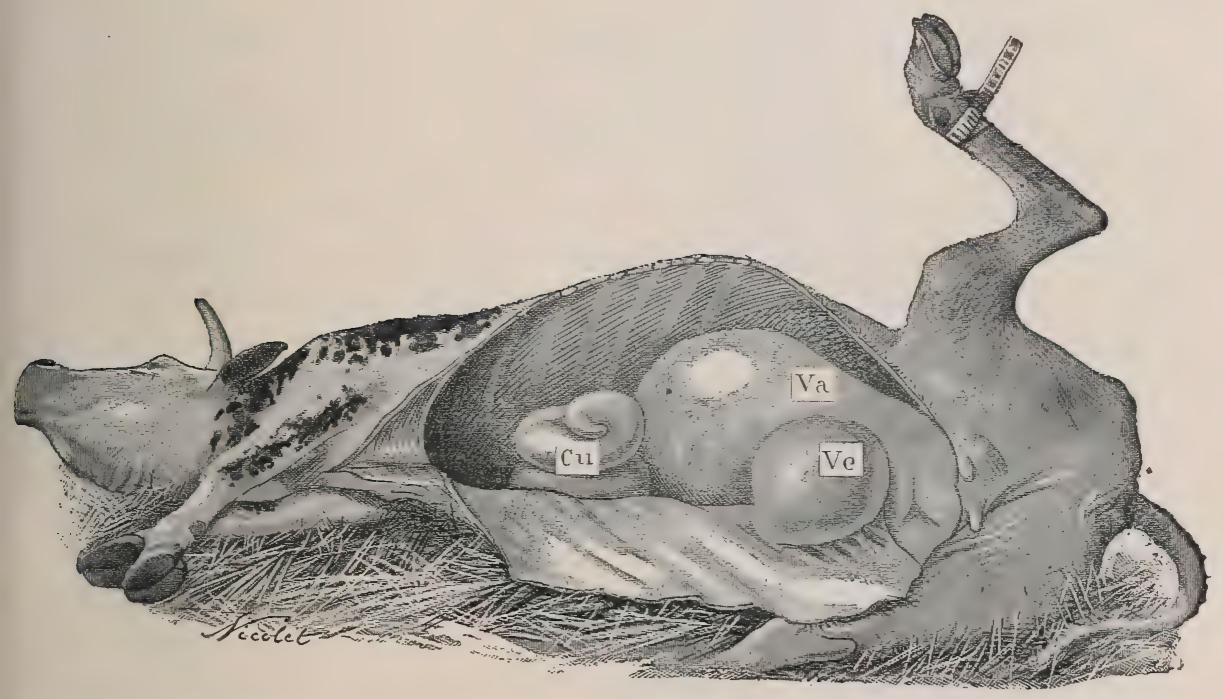

FIG. 235.-Imperforate vagina: position and appearance of the genital organs. Cu Distended uterine horns (muco-metritis); $\mathrm{V} a$, dilated extremity of the vagina; $\mathrm{V} e$, bladder, distended with urine, owing to compression of the urethra. The hymen was situated about $1 \frac{1}{4}$ to $1 \frac{1}{2}$ inches in front of the meatus urinarius.

sometimes found to be enormously enlarged, and to contain a quantity of fluid, but no foetus.

Prognosis. The prognosis is grave. Unless treatment is undertaken the animals die in consequence of exhaustion or secondary peritonitis.

The treatment is simple, and consists in aseptic puncture of the septum and evacuation of the contents. The operation is carried out with a long, large-sized trocar, which is passed through the centre of the most prominent portion of the transverse septum where it projects towards the vulva. Five, ten, or fifteen quarts of mucous fluid escape, and the constitutional disturbance disappears almost instantly.

D.C

O O 
Antiseptic precautions are necessary in order to avoid the development of secondary pyo-metritis. The artificial orifice can afterwards be gradually dilated to allow free exit to the discharges, but in practice, as the animals cannot be used for breeding purposes, they are usually fattened for slaughter.

\section{NYMPHO-MANIA.}

The term "nympho-mania" is employed to describe a special condition in female animals which is manifested by continual sexual excitement. The animals are almost always sterile. The disease is most frequent in cows.

Causation. This general condition may depend on one of many causes, but is rarely due to a true neurosis, as was once believed. Some morbid influence of genital origin is always responsible for the appearance of the symptoms.

Nympho-mania, therefore, often co-exists with lesions of the ovaries (simple ovaritis, cystic ovaritis, tumours of the ovary), with lesions of the Fallopian tubes and of the uterus (salpingitis, chronic metritis and tumours of the uterus), with chronic vaginitis and lesions of the clitoris (hypertrophy and tumour formation), and even with perivaginal or peri-uterine lesions (cysts or tumours).

In exceptional cases it may be found occurring as a simple nervous disturbance without genital lesion, and it would then appear to be due to some temporary genital affection having produced nervous irritation.

In short, nympho-mania may be considered as almost invariably the result of a genital lesion.

Symptoms. The symptoms are very clearly marked. They consist in persistence of the sexual appetite, which is quite abnormal in female domestic animals. The patients lose flesh, feed badly and irregularly, annoy their fellows, cause accidents, and sometimes become dangerous.

Diagnosis. The diagnosis of nympho-mania is so simple that the condition is generally recognised by the owners or cow-herds. The only difficult point lies in discovering the exact cause. Complete examination of the genital organs per rectum and per vaginam is absolutely necessary to settle this question.

Prognosis. From an economic standpoint the prognosis is gene. rally grave.

Treatment. The treatment varies considerably, according to the nature of the lesion.

In mild cases where nympho-mania is due to some lesion of the clitoris (balanitis, hypertrophy, or tumour formation), the radical treatment consists in clitoridectomy. The operation is comparatively 
slight, the organ being removed with forceps and scissors, or with a bistoury after the animal has been hobbled or placed in a trevis. The hæmorrhage which follows removal of the clitoris is of little importance, and after-treatment simply consists in keeping the parts clean.

Animals so treated can sometimes be preserved for breeding.

When nymphomania co-exists with, and is a delayed consequence of, either chronic vaginitis or metritis localised in the neck of the uterus, or, again, chronic metritis of the cavity of the uterus, etc., the treatment must be directed against these diseases, and the nervous condition may be sufficiently modified to render the animals useful for breeding, or at least for slaughter, while fattening is easy.

Similarly, when the nervous condition results from a lesion of the ovary, improvement will only follow removal of the diseased part. The

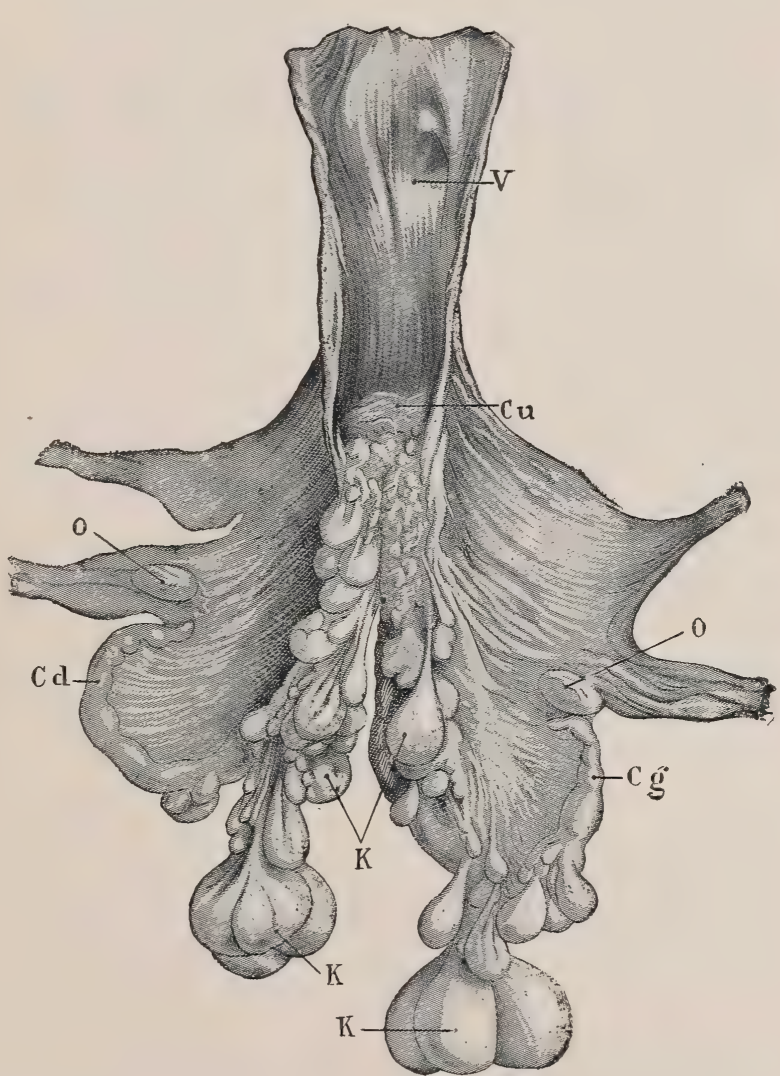

FIG. 236.-Specimen of lesions found in nympho-mania. $\mathrm{V}$, Vagina laid open; $\mathrm{C} u$, neck of the uterus; $\mathrm{O}, \mathrm{O}$, ovaries; $\mathrm{C} d$, right horn of the uterus; $\mathrm{C} g$, left horn of the uterus; $\mathrm{K}, \mathrm{K}, \mathrm{K}$, peri-uterine cysts.

operation is similar to that of castration of the female. It presents, however, certain added difficulties, in consequence of the size of the organs and of the abnormal adhesions which often occur. Nevertheless, these difficulties are seldom insurmountable.

In the case of peri-uterine disease operation is difficult, and it is better to slaughter the animal. 
Finally, as may occasionally happen, should there be no congenital lesion capable of explaining the appearance of nympho-mania, the disease may be regarded as a neurosis, and may then be treated by such sedatives as the bromides of potassium, sodium and strontium, in doses of 2 to 3 drachms per day, divided into two or three portions. Bromide of camphor also gives excellent results by acting simultaneously on the nervous system and calming excessive genital irritation.

The above method of treatment is much preferable to performing clitoridectomy, or ovariotomy as a kind of panacea, although certain writers have suggested these operations without taking into account the special local conditions. 


\section{CHAPTER V.}

\section{DISEASES OF THE MAMMARY GLANDS.}

Is animals used for the purpose of providing milk, viz., cows, goats, and milch ewes, diseases of the mammary glands are of daily occurrence, but are rare in those in which the mammary function is limited to the nourishment of the young, such as the mare, female ass, sow, etc.

In order clearly to understand the development of these diseases, it is necessary to bear in mind the anatomical construction of the organs, for which purpose we may take as a type the mammary gland of the cow, which is the most complicated.

The udder of the cow is of hemispherical shape. It is situated in the inguinal region, and is composed of two parts, the right and left, which are absolutely independent and can easily be isolated from each other along the median plane throughout their extent. The mass of parenchyma is enveloped in a fibrous envelope, which is covered with a very loose layer of subcutaneous connective tissue. Each half is subdivided into two quarters, an anterior and a posterior quarter. Each quarter again represents a distinct gland, although anatomical separation between the anterior and the corresponding posterior quarter would be almost impossible, the separating fibro-connective partition being common to both glands.

In very good milkers it sometimes happens that two small supplementary glands may be found behind the posterior quarters, bringing up the total number to six.

Parenchyma. Each of these glands is provided with a teat containing a large sinus. Anatomically the mamma consists of glandular tissue arranged like a bunch of grapes, in which the active tissues of the acini deliver their secretion into little excretory canals, which unite, forming a large collecting plexus. The collecting canals, or galactophorous canals, open into the galactophorous sinus, which occupies the entire depth of the teat and communicates with the exterior by a small pore provided with a sphincter. The interacinous connective tissue of the udder and the subcutaneous tissue of the teat, which envelops the galactophorous 
sinus, is extremely rich in elastic fibres, enabling the organ to undergo great changes in volume without injury.

Yessels.-The mammæ are supplied by two great arteries, the mammary arteries, which are given off from the prepubic arteries, pass into the inguinal canal, and penetrate the gland by its upper, deep face. Each principal lateral artery divides into two trunks, one for the anterior, the other for the posterior quarter.

The veins which collect the blood from the mammæ form two systems, the first accompanying the mammary arteries, the second,

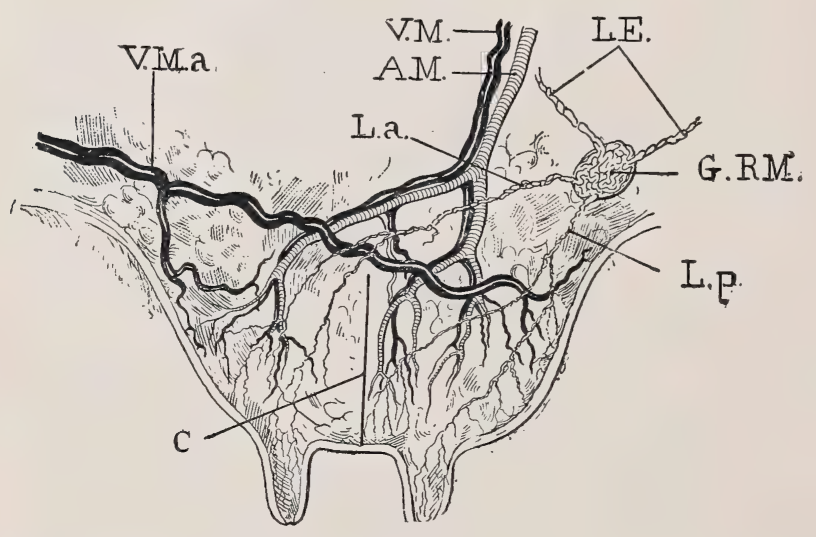

FIG. 237.- Schema showing the structure and organisation of the udder. Antero-posterior section showing the arrangement of the anterior and posterior quarters and the teats, skin, transverse partition, etc. GRM, Retro-mammary lymph gland; Lp, lymphatics of the posterior quarter; La, lymphatics of the anterior quarter; LE, efferent lymphatics; AM, mammary artery; VM, mammary vein; VMa, anterior mammary vein (subcutaneous abdominal vein); C, transverse inter-mammary septum. more superficial, giving rise to the anterior subabdominal mammary veins. The arterio-venous plexus of the udder, which represents the vascular pedicle of the organ, penetrates the gland, near a line dividing the posterior and middle thirds of the upper surface, an inch or so in front of the mammary lymphatic gland.

\section{Lymphatics.-} On either side of the middle line lies

an extremely rich lymphatic plexus, the origin of which is to be found near the ends of the teats and in the peri-acinous spaces.

The superficial collecting vessels are dispersed under the skin, perforate the fibrous sheath towards the base of the teat, and anastomose with one another on the surface of the gland, the anastomosis being most intimate between those of the same quarter, finally emptying separately by two large trunks into the retro-mammary lymphatic gland of the same side.

The vessels of the anterior quarter enter the lymphatic gland at its most anterior point; those of the posterior quarter join it a little below. 
The retro-mammary lymphatic glands are two in number, and are situated very high and towards the back, above the posterior quarters and close to the perineum, outside the fibrous envelope of the gland. They are sheltered in a recess excavated within the depths of the gland itself. The main collecting lymphatics from the anterior and posterior quarters enter it separately.

The lateral efferent vessels are divided into two groups, one of which ascends vertically in the perineal region, towards the lymphatic glands round the anus; the other passes through the inguinal canal towards the sub-lumbar region, together with the bloodvessels.

The mammary nerves are two in number. The anterior has a downward course outside the fibrous envelope and supplies the teat; the posterior nerve is similarly distributed. In other domestic female animals which have only two mammæ the general arrangement is precisely the same.

\section{PHYSIOLOGICAL ANOMALIES,}

Imperforate condition of the Teat.-It sometimes happens that although the udder is otherwise well formed, the teats, or more frequently a single teat, proves to be imperforate. Between the galactophorous sinus and the exterior, opposite the sphincter, a little membrane may be found which closes the teat and entirely prevents the contents of the udder from escaping. Its existence is only discovered when the animal first calves and lactation commences. Not a drop of milk can be withdrawn, although the udder is swollen. Local examination readily reveals the defect.

Treatment is very simple and effective, the membrane being perforated with the end of a milk catheter.

Contraction of the Sphincter (Atresia of the Extremity of the T'eat). - Under other circumstances the teat may present a distinct perforation, and yet milking may be impossible, or at all events may be extremely difficult. This is sometimes due to contraction of the sphincter, or possibly to atresia of the orifice.

The diagnosis of this condition is easy, but the outlook is not promising.

Treatment is rather difficult. Some operators recommend dividing the terminal sphincter with a small, specially-formed bistouri caché, provided with two cutting points. The operation has very satisfactory immediate results, but after the little wounds so produced have healed, cicatricial contraction takes place around the orifice.

Forcible dilatation is far preferable. It is carried out in the same way as in human medicine, where the sphincter ani or the orifice of 
the uterus has to be dilated. No superficial lesion and no incision is produced; the result is therefore more permanent (see "Operative Technique").

\section{WOUNDS OR TRAUMATIC LESIONS.}

CHAPS AND CRACKS.

These injuries consist in little transverse or oblique wounds of the teat.

Causation. In free milkers the udder appears completely relaxed after milking. In the intervals between the different milkings, however, the quarters become swollen, and are sometimes so distended as to overcome the resistance of the sphincter at the base of the teat. The teats are then greatly elongated, and, despite the richness of the tissues in elastic fibres, this distension leads to little superficial epidermic fissures.

These small lesions are unimportant, but if they become infected by contact with the litter they granulate and suppurate, so that grave complications may eventually follow.

The wounds caused by the calves' teeth when sucking, or simply by the rough way in which the little animal seizes the teat, may produce similar accidents.

Symptoms. The teat shows one or more little transverse fissures, a few millimètres to a centimètre or more in length. The base of the fissure appears of a reddish or brownish-red colour, and has thickened, indurated, painful, discharging or suppurating margins. Local sensitiveness may be either slight or very pronounced. In the latter case, the patients resist being milked, and even refuse to let the calf suck.

Diagnosis. The diagnosis is extremely simple.

In a general sense the prognosis is favourable, but nevertheless the local infection may extend and become generalised, thus giving rise to interstitial mammitis, sometimes of a very grave character. On the other hand, the sensitiveness may of itself render milking difficult or impossible, and thus cause serious distension of the gland with milk.

Treatment. As both sucking and milking aggravate the lesions, they should be prevented by the insertion of a millk catheter.

The surface of the udder and the wounds should be cleansed with an antiseptic solution and be dressed with a 20 per cent. camphorated vaseline or with carbolic or iodoform ointment, to favour healing. If the cracks produce excessive sensitiveness a small quantity of orthoform may be added to the camphor ointment. Before the milk catheter is inserted, the teat should be very carefully cleansed with boiled water and the catheter sterilised by boiling. Neglect of these precautions may result in infection of the galactophorous sinus and mammitis. 


\section{MILK FISTULE}

Causation. Any accidental injury to the udder which establishes connection between the galactophorous canals or the galactophorous sinus and the exterior may give rise to milk fistulæ, if the injury occur during lactation.

Apart from lactation these wounds may be grave, though if carefully treated they heal without complication. During lactation, on the contrary, the milk escapes permanently from the injured spot, cicatrisation cannot occur, and a fistula forms.

Symptoms. The principal symptom is the permanent discharge of mill. The fistula may be large or small, according to circumstances. In rare instances it is situated on the udder itself, but it is commonest on the teat. Milk may escape in mere drops or, on the other hand, in considerable quantities.

Diagnosis. The diagnosis presents no difficulty.

Prognosis. The prognosis is grave so far as the loss of milk is concerned, although the lesion has no
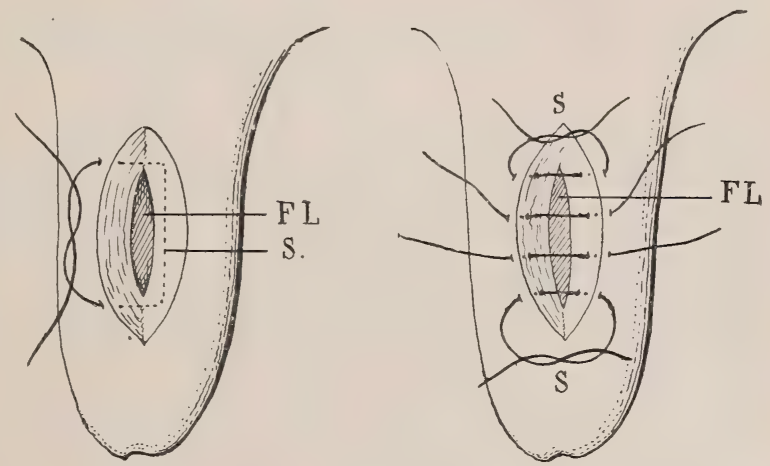

FIG. 238.-Milk fistulæ. 1, Deep suture-schema showing the course of the suture; FL, base of the fistula; $\mathrm{S}$, suture ; 2, superficial interrupted suture.

effect on the general health. It is particularly serious, however, because it may cause the interior to become infected, and an acute parenchymatous mammitis may thus be set up. It must also be borne in mind that old fistulæ are much more difficult to obliterate than recent ones.

Treatment is much more troublesome than might at first be thought, the great obstacle to repair being the continual secretion and discharge of milk.

At first, attempts should be made to re-establish and render permanent the natural method of discharge. This can be effected by inserting an aseptic milk catheter and fixing it in position with a little pitch bandage.

The course of the fistula is then cleansed, curetted, and rendered aseptic in some way, as for example by washing with boiled salt solution and dilute hydroxyl.

As there is little hope of obliterating the fistula by merely suturing the skin, its course should first be closed ly passing one or two deep 
sutures without touching the external orifice and without passing over it (Fig. 238). The discharge of fluid being then entirely stopped, the external portion of the fistula is thoroughly cleansed, powdered with iodoform, and finally closed with external, closely-applied sutures. These sutures are protected with a little cotton-wool or collodion dressing, and healing then almost invariably occurs.

The animal should be given a very clean bed, and closely watched to prevent it tearing out the milk catheter. On the fourth or fifth day the catheter is removed, and is afterwards only used at intervals.

As all the sutures can be of aseptic catgut or silk, there is no necessity to trouble about their removal. The dressing can be left until it falls away of itself.

\section{INFLAMMATORY DISEASES.}

\section{CONGESTION OF THE UDDER.}

Congestion-i.e., distension of the vascular plexus as a consequence of momentary stasis, vaso-motor disturbance, or paralysis of the little vessels in the udder-can only be regarded as pathological in cases when it precedes marnmitis or when it results from prolonged neglect to milk the animal, external irritation, etc. It was studied long ago by H. d'Arboval, Gellé, Delafond, Trasbot, Lucet, etc.

It also occurs, but in a form which might almost be termed physiological, after the first parturition in the large milch breeds, where the rush of blood which precedes secretion is very great.

Symptoms. The udder is, swollen, tense, doughy, shining, and œdematous, not very painful on pressure, but sufficiently so to interfere with movement. The general condition is little altered, but the temperature of the udder is abnormally high.

Manipulation reveals the existence of more or less cedema, the parts preserving the imprint of the finger. Sometimes this odema extends along the abdominal wall in front of the udder. The milk may be grumous or even sanguinolent. The congestive state continues for some days, eight to ten at the most, and may gradually disappear by resolution. Not infrequently it terminates in acute mammitis after forty-eight hours.

Lesions. In simple mammary congestion the lesions are confined to excessive dilatation of the peri-acinous capillary vessels, and extravasation into the connective tissue. On section the tissue has a dark-red appearance.

Diagnosis. The diagnosis is simple.

Prognosis. The prognosis is less alarming than might at first sight be supposed. 
The treatment consists simply in hygienic precautions, frequent milking, emollient, sedative applications to the udder, and frequent washing. As far as possible the use of milk catheters should be avoided.

Boric vaseline and belladonna ointment may be recommended. In very serious cases blood can be withdrawn from the jugular. This is better than bleeding from the mammary vein, which always entails the risk of thrombus formation.

\section{MAMMITIS.}

Under the heading "mammitis" are included different forms of inflammation of the mammary tissue, whether such inflammation attack the parenchyma of the gland or the interstitial tissue. Generally the whole gland is invaded at the end of a few days, whatever the point of origin, and the inflammation is therefore of a mixed character.

Mammitis has been recognised from very early times. In his researches on "contagious mammitis" Nocard in 1884 showed that infection was the principal factor in its evolution.

Numerous classifications, based on the causes or on the pathological anatomy of the condition, have been suggested; but most appear too rigid, and therefore, without discussing them, we confine ourselves to giving the following résumé:-

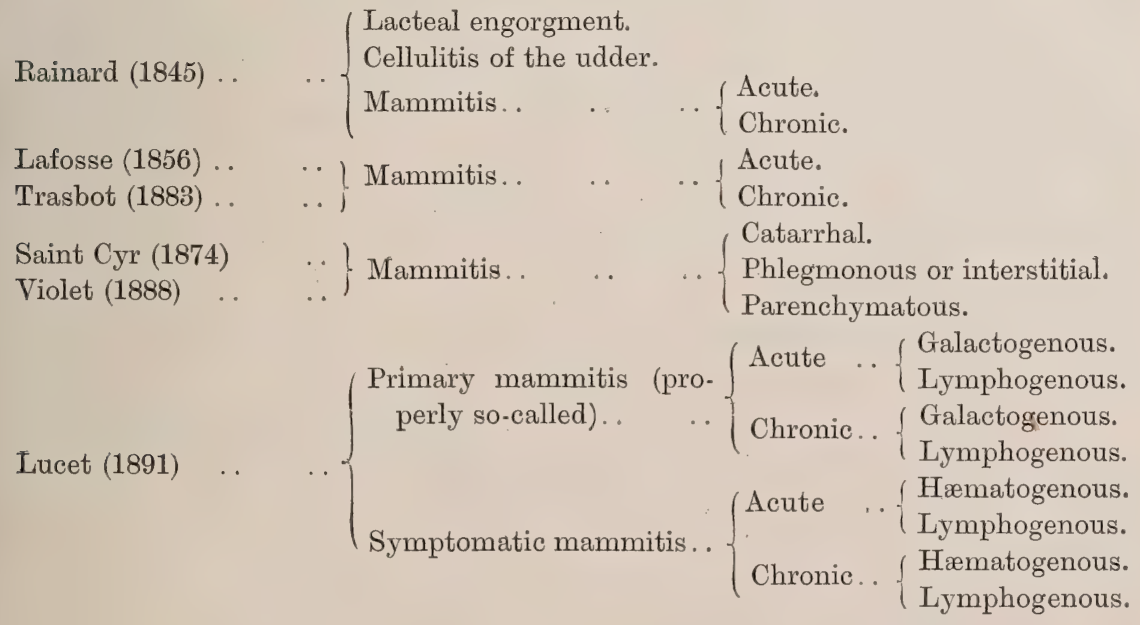

All these classifications are justified by the guiding ideas of the writers, yet, as in every case of attempted systematisation, they have the disadvantage of not being in entire agreement with clinical experience.

For instance, the differences between catarrhal and parenchymatous 
forms of mammitis are only of degree, and it is difficult, therefore, to see why they should be divided into two distinct varieties. The difference is in regard to the prognosis.

Similarly in practice it is difficult and sometimes impossible to distinguish between an interstitial and a parenchymatous mammitis, because all the tissues of the gland may be involved at a given moment. The only factor which allows of differentiation is the discovery of the point from which infection took place. Finally, it is sometimes so difficult to distinguish between galactogenous and lymphogenous mammitis that the attempt has had to be abandoned. In gangrenous mammitis of milch ewes, for example, the infective organism is found not only in the sinus and the galactophorous canals, but also in the serosity of the interstitial tissue and of the perimammary œdema.

Without doubt the causative agent of mammitis may enter the gland by three principal channels--the galactophorous sinus, the lymphatic plexus (after some injury), and the blood circulation. But from the clinical standpoint it is not at all necessary to identify all the causes in order to establish the classification.

The symptoms allow of a division only into acute and chronic mammitis. Careful examination of the general condition of the patients will afterwards allow cases of primary mammitis to be distinguished from secondary or symptomatic mammitis such as occurs in tuberculosis. Finally, consideration of the conditions under which a particular case of mammitis has appeared, and study of the symptoms in detail (peculiarities of the milk, local temperature, hardness of the tissues, œdematous infiltration, etc.) will in most cases indicate whether the mammitis be parenchymatous or interstitial.

This system really differs little from that adopted by Lucet in his work on Mammitis.

The classification adopted in the following pages is as follows:--

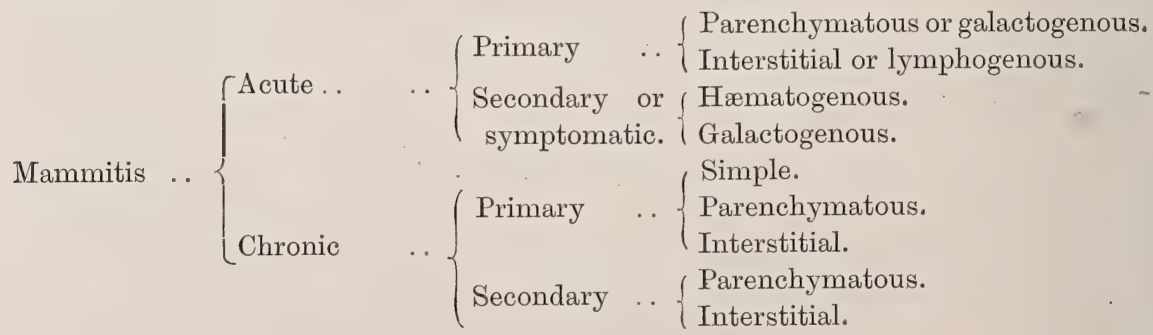

We shall leave on one side everything concerning secondary symptomatic mammitis, the study of which merges into that of the general diseases from which it arises. 


\section{ACUTE MAMMITIS.}

Causation. The general cause of acute mammitis, like that of chronic mammitis, is infection by pathogenic organisms, whether such organisms enter by the usual natural path, viz., the galactophorous sinus and excretory apparatus, by the lymphatic path, owing to some accidental injury, or, again, by the blood circulation.

Infection of the lymphatics undoubtedly plays a part in superficial and interstitial inflammations, and it is proved that certain microorganisms may pass into the milk, as it has been proved that they pass through the kidney.

But if infection is the determining cause, certain secondary favouring influences must not be overlooked.

Thus lactation is an almost indispensable condition. It is true that some cases of mammary inflammation apart from lactation have been described, but they have been the result of violence, accidental or mechanical.

Accumulation of milk in the udder (overstocking) has unquestionably a certain influence in the large milk-yielding animals, not because it directly produces inflammation, but because milk then escapes spontaneously; and as the udder cannot be entirely evacuated without external assistance, the entrance is kept permanently open for the passage of germs, which are freely transferred to the teat from the litter and surrounding objects.

Cold, or rather chills, also act in a complex manner, particularly by disturbing vaso-motor control. Different forms of mechanical violence, such as blows, crushing strains, wounds, etc., may immediately and directly set up local or general inflammation.

Bacteriological investigation has proved that numerous and varied micro-organisms can be found in the milk or interstitial exudates in cases of mammitis, but only a few special forms have been proved to be specific: streptococcus of contagious mammitis of milch cows, and micrococcus of contagious gangrenous mammitis of ewes (Nocard).

Pathology. The pathogenic results produced by infective organisms depend on their number and power of reproduction, and on the activity of their life products.

The most immediate and regular result of acute mammary infection is coagulation of the milk within the udder by decomposition of the lactose, and the formation of lactic or even of butyric acid. The acini and excretory canals are dilated by coagula, and can no longer expel their products of secretion, so that the colonies of micro-organisms develop there in full security. The active epithelial cells undergo granular degeneration and disappear, whilst the walls of the glands 
become infiltrated and large numbers of leucocytes are poured forth around the glandular culs-de-sac.

The tissues being thus affected, the virulent organisms penetrate from the acini into the interstitial tissue, and from this time onwards the lesions become mixed.

Inversely, should infection originate in the lymphatic spaces, a time arrives when the organisms make their way from the interstitial tissue into the acini, with a similar result in the end.

The development of the lesions may be arrested or may pass on to suppuration, or even gangrene, of the parenchymatous lobules. Cases happen in which infection is so rapid and severe that the successive stages cannot even be identified, and gangrene appears without any preliminary stages at all. Luckily the commonest forms are less serious.

Symptoms. Acute mammitis is characterised by its sudden appearance, more or less acute general symptoms (dulness, fever, and loss of appetite), and variable local symptoms. When the practitioner is able to follow the development of the disease throughout, he may sometimes distinguish well-marked signs, which permit the two clinical varieties to be distinguished.

A. Interstitial Mammitis.-This form, which might perhaps-also be termed peri-mammitis when it primarily affects the subcutaneous lymphatic spaces, has also received the names of phlegmonous and lymphogenous mammitis.

It is characterised by alarming general symptoms, and particularly by a rise in temperature of $2^{\circ}, 4^{\circ}$, or even $5^{\circ}$ Fahr., with all its consequences, such as loss of appetite, stoppage of rumination, acceleration of breathing and circulation, slight tympanites, constipation, and by the thrusting of the hind limb on the affected side away from the centre line. The animals groan when forced to move.

These symptoms sometimes precede by a considerable interval the appearance of the local changes, which consist in painful swelling of one or two quarters, rarely of more.

The peri-mammary subcutaneous tissue is infiltrated, œdematous, painful on palpation and preserves the imprint of the finger. The teat is tense, swollen, very tender, and of reddish colour: In the grave forms the swelling extends forwards under the abdomen in the direction of the umbilicus, and backwards towards the perineum. The local temperature is abnormally high, the secretion of milk in the diseased gland is modified or checked, and sometimes this phenomenon extends by reflex action to the neighbouring quarters, although the latter may not themselves be affected. The inflammation rarely extends from one quarter to another, because the lymphatic plexuses do not anastomose (Fig. 237). 
The animals lose appetite and fall away rapidly.

Resolution may occur after from five to eight days. By degrees all the symptoms then become less marked. The appetite returns, pain diminishes, the fever drops, and the lesions gradually disappear, but the yield of milk rarely regains its former amount.

Suppuration may occur; sometimes a superficial subcutaneous abscess forms, more rarely, a deep-seated, interstitial abscess, originating in the connective tissue or lymphatic spaces. With a superficial abscess, the local symptoms again revive to a slight extent; these are present in a more marked degree where the abscess is deep-seated. An extremely sensitive odematous swelling forms, the skin covering which is at some point of a deep-red tint, whilst fluctuation gradually appears.

In cases of deep-seated abscess formation the general condition becomes alarming; the affected quarter is tense throughout, hard and very sensitive.

Deep-seated suppuration is difficult to detect, and exploratory punctures with a fine needle may be necessary before the diagnosis can be made.

Local or diffuse gangrene forms a rare complication. It is due to the vessels of one or several glandular lobules becoming obliterated or thrombosed.

Such a termination is indicated by extreme aggravation of the general symptoms, feebleness of the heart and great weakness of the patients, who fall into a condition of coma. Locally the udder remains odematous, the skin becomes of a blackish-violet colour, whilst the local temperature falls and the animals die from exhaustion and intoxication.

Parenchymatous Mammitis. - Parenchymatous mammitis when mild is also termed catarrhal mammitis. It is in reality true primary mammitis; interstitial being primarily and practically perimammary lymphangitis.

In this case infection occurs through the teat, and may be localised in the sinus or excretory apparatus, giving rise to galactophoritis, but it usually extends to the acini. Inflammation of the mammary tissue is therefore direct and primary. It rapidly extends, however, through the glandular wall into the interstitial tissue, thus setting up (from the anatomo-pathological standpoint) a mixed mammitis. Clinical distinction between this form and interstitial mammitis is at first easy.

The symptoms usually occur in the following order:- Swelling of the affected quarter or quarters; appreciable increase in size and sensitiveness; the presence at first of curdled milk in the galactophorous sinus, then of clots mixed with slightly red tinged serosity; 
complete cessation of the yield of milk, and suppuration in the secreting portions of the gland.

The general symptoms appear only after the objective signs, and vary greatly in intensity, according to the case. As in the interstitial form, there may be marked fever, loss of appetite, cessation of rumination, groaning, and difficulty in walking.

In some grave forms, where development is peracute, infection spreads rapidly from the glandular to the interstitial tissue, and subcutaneous, sub-abdominal, or perineal interstitial œedema occurs secondarily.

The udder is turgid, tense, shining, and of reddish-violet colour in places, as if a deep-seated abscess were developing.

Pressure on the galactophorous sinus causes the flow of reddish-grey milk, sometimes fœtid or of gangrenous odour. The animals seem exhausted, show signs of profound intoxication, are unable to rise, and appear paralysed.

But besides these grave forms are others, in which the patients seem scarcely to suffer: appetite is preserved and all the vital functions are in full activity. Only the local signs are of importance.

This variability in the clinical symptoms of acute forms of mammitis is entirely due to differences in the pathogenic infecting organisms.

Parenchymatous mammitis may end in resolution in three to four days, with progressive but slow return to the physiological condition. This termination is announced by the gradual disappearance of all the symptoms and the return of milk secretion. It is, however, quite exceptional for the former condition to be fully restored, and in many cases the affected quarter must be regarded as lost from the physiological standpoint.

It gradually becomes hardened, sclerotic and atrophied.

Suppuration is very common. It attacks the galactophorous sinus, the excretory canals, and even the acini. If obstructions occur in the course of the collecting vessels, or if evacuation is not artificially stimulated by milking, the pus collects in the depths of the gland, and enormous diffuse abscesses may form at the expense of the mammary tissue.

Circumscribed or diffuse gangrene, as a primary condition, is rarer. Infective organisms rapidly invade even the depths of the gland, the interstitial and subcutaneous tissue, and thrombosis due to infection or intoxication occurs, followed by gangrene: Death results from infection or intoxication.

Complications such as necrosis of the abdominal tunic, of the fibrous tissue enveloping the mamma, and of the muscular layers on the inner surface of the thighs, may occur in the suppurative forms. 
Diagnosis. The diagnosis of acute mammitis is easy, and the interstitial forms (mammary lymphangitis) can be distinguished from the parenchymatous forms very early in the attack.

Careful examination suffices to differentiate between this condition and mammary congestion or primary chronic mammitis. The examination, however, must be much more thorough and searching when a specific disease (such as tuberculous mammitis) is in question.

Prognosis. The prognosis of acute mammitis is always grave, whatever form the disease may assume, for, if the animal's life is not invariably endangered, its economic value is always affected. Moreover, should superficial or deep-seated abscesses form, prolonged suppuration may follow, resulting in loss of condition and enormous depreciation.

Lesions. The lesions of interstitial mammitis are similar to those of ordinary lymphangitis, the condition originating near the teat and gradually extending to the layers of connective tissue between the acini, mammæ, etc.

In the parenchymatous form the inflammation may remain partial, and be localised in particular tracts of glandular tissue. The secreting epithelium, when infected, exhibits cloudy swelling, becomes loosened, and disappears; the margin of the gland and the interstitial divisions become infiltrated with enormous numbers of white blood corpuscles, and are the seat of suppurative processes which end in the production of small acinous abscesses. By the union of neighbouring abscesses large branching collections of pus are produced, and lead to partial or total destruction of tracts of the parenchyma, of the connective tissue divisions, vessels and aponeuroses.

The abscess tends to break through the skin, which becomes inflamed and ulcerated, or, when the micro-organisms are of slight virulence, the tissues may react, so that the abscess becomes surrounded with a thick indurated wall, and finally encysted.

Treatment. Very numerous methods of treatment have been proposed, an admission which, in itself, suggests that no perfect one has been discovered. No infallible system, in fact, exists of arresting the disease and restoring the parts to their normal condition.

From a prophylactic standpoint, mammitis can be avoided by placing the animals under proper hygienic conditions, paying special attention to cleanliness, avoiding over-stocking, and treating excoriations or injuries to the teat or udder as soon as they appear.

Once acute mammitis has developed, general and local treatment must both be attempted.

The older practitioners were in the habit of bleeding from the mammary or jugular vein. Since their time, however, objections have D.C. 
been made to bleeding because acute mammitis has been proved to be of an infectious character, and, therefore, it is undesirable to lower the patients' resisting power.

This reasoning, however, appears to be erroneous. Little by little the advantages of bleeding, both in intoxications and infections, have been recognised, and one thing at least is beyond dispute, namely, its action on fever. Undoubtedly, it must not be resorted to without judgment, nor should it be freely employed in debilitated animals; but in well-nourished patients its effect on fever and on the accompanying respiratory and circulatory disturbance is immediate.

We, therefore, recommend moderate bleeding from the jugular.

Bleeding from the mammary vein entails too great a risk of infection to be commendable.

Purgatives and diuretics diminish or prevent accidents such as intoxication and the complications resulting from temporary suspension of the digestive function.

Local treatment is more or less efficacious in mammary infection. To relieve pain and check infection it should be of an emollient and antiseptic character. Ointments containing 10 per cent. of carbolic acid, boric acid or iodine, or $12 \frac{1}{2}$ per cent. of camphor, opium or belladonna, are of real service during the first stages, particularly of mammary lymphangitis and interstitial mammitis.

Repeated applications of 10 per cent. carbolic-glycerine have similar advantages.

In the less acute forms originating in the parenchymatous tissue, mild ointments of plumbic iodide, Goulard's extract, or mercury may also be used if precautions are taken to prevent the animals from licking, and so poisoning themselves.

When the tendency to suppuration is marked, vesicants hasten the development of the abscess and facilitate puncture. The most commonly used are the 33 per cent. tartar emetic ointment or the 10 per cent. biniodide of mercury ointment.

If, on the other hand, the mammitis is of the interstitial type, with severe subcutaneous cedema, extending over the belly and towards the perineum, good results often follow deep firing in points over the swollen region. The points should be widely spaced, venous branches being avoided. In this way numerous ducts are formed by by which the toxic and septic liquid which causes the œedema is enabled to escape.

This method of treatment can be supplemented by the simultaneous use of antiseptic ointments.

Finally, in mammitis of the parenchymatous type, where there is no marked tendency to invade the interstitial tissue, the most 
important point is to wash out the interior of the gland, and even the acini as far as possible, with antiseptic fluids. Practically this is difficult to effect, because such irrigation must be performed aseptically, and cannot properly be left to the cowmen.

In current practice, therefore, one often has to be content with stripping the udder every hour. Milk clots which accumulate in the sinuses and galactophorous canals are broken down by soft pressure, and withdrawn with more or less difficulty. By repeated milking they are prevented from accumulating in the galactophorous sinus and canals, a very important point. Neglect of this precaution enables the colonies of micro-organisms to develop uninterruptedly in the culs-de-sac, whereupon the coagula formed of caseine obstruct the excretory channels and complications develop despite all external treatment.

By repeated friction of the udder as in milking the advantages that would be produced by washing out the gland from the direction of the acini are secured, and thus the ascending infection is checked.

The diseased udder must always be emptied before making antiseptic injections, which would otherwise be useless.

Should the practitioner decide to face the practical difficulties of injections, he must take care that his instruments are aseptic; that the solutions employed are always at or about bodily temperature; that these solutions are incapable of irritating even tissues so tender as the epithelium of the acini or of the galactophorous canals; and, finally, that the drugs employed will not coagulate the milk within the gland.

Bearing in mind these points, the practitioner will do well to restrict himself to the use either of boiled water, physiological salt solution ( 9 per cent.) ; alkaline 3 per cent. solution of borate of soda; or 05 per cent. of fluoride of sodium. Every precaution having been taken, from 12 to 20 ounces of liquid can be injected into each quarter, according to its size. The solutions should be made to penetrate as far as possible into all portions of the gland by gently manipulating the parts, and should again be withdrawn in about a quarter of an hour.

It must always be remembered that failure to observe the above precautions may make matters worse instead of better, and therefore that intra-mammary injections can only be of value when carried out by a skilled person.

In otherwise hopeless cases there remains as a last resort total or partial ablation of the mamma. This operation is advisable in cases of diffuse gangrene, or of intense massive suppuration, where there is imminent danger of death from infection.

Directions for its performance will be found in Möller and Dollar's "Regional Surgery" (uniform with the present volume), p. 454. 


\section{CONTAGIOUS MAMMITIS IN MILCH COWS.}

Although its cause was unknown before the investigations of Nocard and Mollereau in 1884, this disease is of very common occurrence in England, in large dairies in the environs of Paris, and is also seen, though more rarely, in Normandy, in Brie and the Soissonnais, causing serious losses on account of its transmissibility:

It had been described in Germany by Gerlach as early as 1854, and Kitt in 1885 recognised it as common, and proposed for it the title of "contagious catarrhal agalaxia." It also occurs in Denmark, Italy, and England.

Contagious streptococcic mammitis of cows always assumes a chronic form, during the course of which indurated centres appear, varying in size between that of a hazel-nut and a walnut.

Causation. The cause is always to be found in contagion, the disease being due to a streptococcus, which has been carefully studied by Nocard. Its transmission from a diseased to a healthy udder is explained by the fact that the milkers are careless as to cleanliness, and thus directly convey the germs to healthy teats and facilitate infection.

Symptoms. As in chronic mammitis, general symptoms are not well marked, though some cases are ushered in with cough, slight nasal discharge, and offensive diarrhœe. The first appreciable indication is a change in the milk, which diminishes in quantity, and, although normal to all appearance, coagulates rapidly if left undisturbed. Infection has already taken place, although nothing can be detected on examining the udder.

Next appears a nodule of hardened tissue above the teat. This nodule is of rounded or ovoid shape, ill-defined towards its periphery, and it becomes progressively enlarged without any sign of acute inflammation. The milk becomes watery and of a bluish colour. Microscopical examination reveals numerous streptococci. The hardening process proceeds slowly. At the end of several months the sclerosis may only have extended to one-third of the height of the infected quarter. With the appearance of these lesions the character of the milk again changes. It becomes yellowish in colour and foetid, and contains a fibrous reticulum, whilst its reaction is distinctly acid. The lesions, which at first had been confined to one quarter, successively extend to the others unless precautions are taken.

Lesions. The lesions consist in mammary sclerosis, with nodules which progressively increase in size. This sclerosis originates in the galactophorous canals, and extends first to the periphery, then to the interstitial connective tissue; the latter undergoes hyperplasia and confines within its tracts the true glandular elements, destroying their secretory power. Locally, catarrhal mammitis exists. 
The lesions can easily be recognised on microscopic examination, and, when the section is suitably stained, numerous streptococci can be detected in the acini, which are blocked with proliferating epithelial cells. These lesions are due to the invasion of colonies of micro-organisms, which spread from below upwards.

The streptococcus causing this mammitis colours readily with thionin and methylene blue. It can be cultivated in liquid and solid media containing sugar or glycerine, but growth is impeded by the presence of peptone or common salt. Under ordinary circumstances the culture dies after some weeks, but if the acidity of the medium is neutralised by the addition of powdered carbonate of lime, it preserves its vitality for six or eight months. The culture when injected into the udder of a healthy cow or she-goat reproduces the disease. The micro-organism is not pathogenic for any of the smaller animals used for purposes of experiment.

Diagnosis and prognosis. The diagnosis is easy, the presence of the sclerotic nodules being characteristic, whilst in doubtful cases a microscopical examination can always be made.

The treatment should be prophylactic and curative.

Prophylactic treatment comprises disinfection of the milkers' hands, which are the ordinary vehicles of contagion, and disinfection of the cow's udder. Diseased animals in a byre should be milked last of all, and the milk should be destroyed.

Curative treatment is confined to local antiseptic injections. Warm concentrated boric solution gives good results. The injections may be repeated three or four times a day after milking, the liquid being left for a certain time within the udder. In this way animals suffering only from a slight attack may be cured, but when the disease is already somewhat advanced injections lose their effect.

Injections of fluoride of sodium ( 1 to $\cdot 5$ per cent. strength) appear to be much more effectual. Moussu claims to have cured by this means several old-standing cases where all four quarters of the gland were diseased.

These injections necessitate the same precautions as those above indicated for securing perfect asepsis. In grave cases it is very difficult to prevent the disease from extending in infected stables, because the necessary precautions cannot be observed.

\section{CHRONIC MAMMITIS.}

Chronic inflammation of the udder may form a termination of ordinary acute mammitis, or it may result from infection with organisms of a lesș virulent type. 
In cases of acute mammitis, where severe injury of the interstitial tissue or mammary parenchyma has occurred, its physiological function is partially destroyed. The circulation is disturbed, the vessels undergo change, the layers of connective tissue are indurated and sclerotic, the epithelial tissue is not fully restored, and chronic inflammation of the udder persists.

Clinically, it is impossible to distinguish all the variations that may occur, because the constituent tissues are all injured, and the disease is of a mixed type, with very numerous variations.

Symptoms. When chronic mammitis follows the acute form its onset is only indicated by gradual diminution in the signs of acute inflammation. The appetite is regained, and all the important vital functions are properly performed.

Nevertheless, it is possible to distinguish two different clinical forms. In one, the secretion of milk is almost entirely suspended, the udder becomes atrophied, shrivelled and sclerotic; the hardening gradually continues, and the patients, being useless as milch cows, can only be fattened for slaughter. The induration may be partial, nodular, or diffused.

In the other form the udder is large, but the milk is replaced by a purulent secretion which permeates the acini and galactophorous canals. Although they eat well, the patients remain thin, and are with difficulty fattened for slaughter.

The suppuration may be diffuse or local. In rarer cases it is localised in the form of "cold abscess."

Treatment. No treatment can perfectly restore the udder after chronic mammitis with sclerosis and atrophy.

In the suppurating forms of chronic mammitis the morbid secretion may be checked by internal irrigation of the udder and antiseptic injections, but, as this treatment necessitates careful and continued attention, it is usually out of the question.

If only one quarter is diseased the animal may be kept for milking purposes, but where two or three quarters are attacked it should be prepared for the butcher.

In cows suffering from chronic purulent mammitis of all four quarters, Kroon suggests removing the teats in order to facilitate fattening. The teats are removed with the bistouri or scissors, by which means a large opening into the galactophorous sinus is produced, through which the purulent secretion escapes freely and continuously; the retention of pus and intoxication in consequence of resorption are prevented and the animal can be fattened, which would be difficult without the operation. 


\section{GANGRENOUS MAMMITIS OF MILCH EWES.}

This disease occurs in different parts of France, and has also been seen in Germany.

Causation. Lafosse in 1856 attributed it to the dark and dirty condition of the sheep-folds, a cause which certainly contributes to its propagation, though it is not the determining cause of the disease itself. The latter is a specific micrococcus discovered in 1875 by Rivolta, and thoroughly studied by Nocard in 1886 and 1887.

Symptoms. The course of the symptoms offers a certain analogy to that of septic engorgements and interstitial mammitis or mammary lymphangitis. As a rule only one gland is infected, but generally symptoms at once appear, indicating an extremely dangerous condition, viz., peracute mammitis. The patient suddenly becomes dull and entirely loses appetite, rumination ceases and respiration is short and jerky, although the bodily temperature does not always rise to any marked extent.

Local symptoms soon develop. The udder assumes a violet-red tint and becomes the seat of an erysipelatous swelling; the local temperature rises, but as the disease progresses it gradually falls again. Milk secretion ceases.

All these appearances rapidly become aggravated. The patient lies down; the æedematous swelling extends to the belly and even to the chest and thighs; the local temperature falls, indicating the imminence of gangrene; the teat becomes contracted, and the pulse is very frequent and almost imperceptible. From time to time the animal grinds its teeth.

The bodily temperature next falls to $98^{\circ}$ or even $96^{\circ} \mathrm{Fahr}$. $\left(37^{\circ}\right.$ or $36^{\circ}$ C.), and the animal shows extreme prostration. The subcutaneous swelling extends as far as the sternum in one direction and the quarters and perineum in the other. The udder crackles under the finger. Death occurs without a struggle.

All these symptoms follow as a rule in barely more than twentyfour hours. Nevertheless, in certain cases, the disease lasts for three, four or five days. Cases of spontaneous recovery are exceptional. The gangrenous part may become delimited and slough away, leaving an enormous suppurating wound, which slowly cicatrises. Even though the animals survive, they never recover condition, but remain weakly, so that, from a monetary standpoint, death would have been preferable. Moreover, the lambs are starved and require a fostermother.

Lesions. Post-mortem examination reveals œedematous infiltration of the udder and surrounding connective tissue, and often extensive, 
diffuse gangrene. The serosity is of a reddish colour, and sections of the diseased udder of a violet tint. The tissues of the udder and the serous liquid contain the specific micrococcus.

It is very small, and stains readily by the Gram-Nicolle method. It grows rapidly in liquid and solid media, liquefies gelatine, and quickly renders neutral media alkaline.

The injection of a few drops of culture into the udder of a milch ewe reproduces the typical disease. In any other species it is without effect. Infection occurs through the open extremity of the teat, or the medium of a wound, and the micro-organism is so virulent that it rapidly invades all the tissues.

Treatment. No antiseptic treatment appears capable of checking the course of the disease.

Surgical treatment alone is of any value, and consists in ablation of the udder, followed by antiseptic dressing. Only a portion of the gland is removed: an elliptical incision is made, including the diseased teat, the skin is then dissected free so as to form a flap; the diseased tissue is isolated; last of all, the vessels are ligatured. The consequences of operation are less grave than might be expected, considering the size of the wound, which heals with fair rapidity.

Moussu has frequently practised this radical method of treatment without losing a single case. The remaining portion of the udder becomes hypertrophied, and is often quite capable of secreting sufficient milk for the nourishment of one lamb.

\section{GANGRENOUS MAMMITIS IN GOATS.}

Goats suffer from a form of mammitis which presents symptoms precisely similar as regards development and termination to that of ewes. The disease occurs in a sporadic form in animals giving milk, whatever their breed or the conditions under which they are kept. It has been seen at Alfort, as well as at Lyons.

Moussu has seen it in an enzootic form in herds of milch goats near Bizerta (Tunis), and considers that contagion results from kids sucking healthy animals after having been suckled by diseased ones. Fifty milch goats out of a total of three hundred were affected at the time of Moussu's visit, and two had already succumbed.

The diagnosis presents no difficulty.

The prognosis is very grave.

The treatment is precisely similar to that of gangrenous mammitis of milch ewes, viz., ablation of the diseased udder.

Of the above-mentioned herd, twenty animals were operated on, and all recovered without accident. Among the others treated by less 
radical methods, such as scarifications, incisions, antiseptic injections, etc., eight died. The only justifiable treatment, therefore, is ablation.

\section{CYSTS OF THE UDDER.}

Cysts of the udder may assume one of two forms, milk or galactocelous cysts and serous cysts; possibly the latter are only hydatid cysts.

Both varieties are of the nature of retention cysts, and result from accidental obliteration of a milk conduit, which has been obstructed by coagulated casein, or from atresia due to contraction of inflamed connective tissue. The acini are isolated and become dilated, the milk undergoes changes in composition, the secretory epithelium degenerates, and the cyst is produced.

The serous or milk cysts may vary considerably in size, and always show a rapid tendency to suppuration.

The diagnosis is based on the detection of uniform fluctuation without excessive sensibility, and can be confirmed by an exploratory capillary puncture.

The prognosis is grave, for recovery can only occur after the lining membrane of the cyst has been destroyed.

Treatment. Puncture followed by irrigation is insufficient, whatever the nature of the cyst.

Free incision, or simple puncture with a bistoury, produces suppuration within the cystic cavity, which continues until the lining membrane is completely destroyed; but this takes a very long time.

Removal of the entire cyst, either with the bistoury or by tearing through the surrounding connective tissue, is the best method of treatment. The wound thus produced heals regularly and rapidly under an antiseptic surgical dressing.

\section{TUMOURS OF THE UDDER.}

Tumours of the udder have been little studied in the larger domestic animals, the reason being that immediately animals cease to yield milk they are fattened and despatched to the butcher. Clinically, therefore, these tumours are not of great practical importance.

Without entering into general considerations of a pathological anatomical character, by which different varieties of these tumours are differentiated, we may say that they assume one of three different forms. The first is sharply circumscribed, of clearly defined shape, and easily separated from neighbouring tissues, to which it adheres but slightly. Such tumours are benign, and have no tendency to return after removal.

The second is ill-defined, very adherent, and appears to infiltrate 
the surrounding tissue. It is malignant in character, is often impossible to completely remove, returns after extirpation, and infects neighbouring lymphatic glands.

Between these two varieties may be placed a third, occupying an intermediate position as regards both its characteristics and gravity.

Practically a knowledge of the above facts is a sufficient guide in dealing with lesions of this character.

Canalicular Papillomata.-There exists another variety of tumours which is of much more frequent occurrence, and which is apt to cause mistakes. This variety consists of inter-canalicular papillomata.

These show no sign of their existence on external examination of the diseased udder, and can be diagnosed only by reasoning based on the signs observed.

They are of very small size, and grow from the internal wall of the galactophorous sinus or excretory canal. They increase in length, finally extending for some distance along the sinus, where they remain unrecognised until some external manifestation arouses suspicion as to their existence.

Under the mechanical contraction due to milking they easily become excoriated, and their existence is then suggested almost solely by the fact that the milk is tinted with blood, for palpation of the udder very seldom gives more than negative results.

The diagnosis is always doubtful. The prognosis is grave, because it is impossible to remove the tumour, which is often deeply placed within the udder. There is no curative treatment.

\section{VERRUCOUS PAPILLOMATA OF THE UDDER.}

In cows suffering from cutaneous papillomata the udder is often covered with a varying number of little sessile warts, which are spread over a considerable surface, are very sensitive, and bleed at the lightest touch. Milking is thus rendered extremely painful and difficult; the animals struggle, kick, and become dangerous.

The condition is extremely troublesome, and in spite of every precaution the milk is always soiled.

The best method of treatment consists in removing the warts individually with fine, sharp-bladed curved scissors.

The animals must be firmly secured, if necessary cast, and the operator should avoid removing more of the skin covering the udder than is absolutely necessary. The bleeding which follows is trifling, and stops of itself. After washing the parts with an antiseptic solution, the little wounds may be powered with a mixture in equal proportions of tannin and boric acid, 


\section{CHAPTER VI.}

\section{DISTURBANCE IN THE MILK SECRETION AND CHANGES IN THE MILK.}

Changes in the milk are so common and play so important a part in the milk industry that it is absolutely necessary to mention the more important, the country veterinary surgeon being frequently consulted on this point.

The udder acts as a natural emunctory, just like the kidney, by which are eliminated, in consequence of special selective properties, certain natural principles (the active principles contained in the forage and other food, vegetable alkaloids, etc.), drugs (alcohol), and poisons (nicotine). This physiological peculiarity explains the influence of changes of diet on the composition of the milk in mothers and on the condition of their offspring. In the human species it also explains congenital alcoholism in children, and a number of diseases whose cause was formerly unknown.

Agalaxia.-This term implies a temporary or definite arrest of the milk secretion. In many instances it represents nothing more than a disturbance in the physiological function of the gland, but in others it forms a true diseased condition.

Thus it is beyond dispute that psychical influences may produce disturbance in this respect. Temporary suspension in the secretion as a consequence of removal of the offspring from its mother, especially when the young animal has been sucking for several days or weeks, is a fact very well known to all breeders.

Under ordinary circumstances, however, the reasons are quite different, and when the secretion of milk is diminished or suppressed it is due to the ingestion of plants, substances, or drugs which check the secretion of milk. All the solanaceæ (belladonna, hyoscyamus, stramonium, woody nightshade, etc.), certain umbelliferæ (hemlock), colchicum, etc., have this effect.

As to agalaxia of a true pathological character, it occurs in debilitating and grave diseases, and sometimes follows certain contagious forms of mammitis complicated with sclerosis of the udder (infectious agalaxia of goats). 
Diagnosis. The diagnosis of agalaxia presents no difficulty.

Prognosis. The prognosis varies, according to the determining cause. In cases of accident and in temporary agalaxia, it is sufficient to change the food in order to restore the secretion. Cooked food and warm drinks, with an allowance of roots such as turnips or mangolds, have excellent results. Where restoration of the secretion is delayed the use of what are called galactogogues has been recommended, comprising fennel, carraway, cummin, aniseed, juniper, sulphur, etc., mixed in equal parts and given in doses of 6 to 8 drachms per day for a cow.

They act principally through the stimulating effects of their aromatic principles.

\section{MICROBIC CHANGES IN MILK.}

\section{LACTIC FERMENTS.}

Microbic changes in milk are much commoner than changes of a chemical nature. Milch animals differ very markedly one from another, and, according to circumstances, give milk of ordinary composition, milk of a very rich character, or watery milk; but the most important changes in composition are those due to microbic agents.

During the milking, and according to whether this is performed in a low, dirty byre, in a clean, roomy byre, or in the open air, various numbers of germs obtain entrance to the milking vessels, and develop there with extraordinary rapidity. The milk may even become infected by non-pathogenic germs while still within the udder, in the sinus and galactophorous canals. The cleanliness of the milking vessels also has a considerable influence on the number and variety of the microbes which may eventually germinate in the liquid.

Among the micro-organisms usually found in milk there are some, however, which always preponderate and play the part of organised ferments, viz., the lactic ferments and the organisms which cause coagulation of the casein; these may be regarded as normal constituents. The others are more or less foreign, and may cause important changes in the milk or cream.

The lactic ferments are numerous, comprising the lactic bacilli of Hueppe and Grotenfeld, the micrococci of Hueppe and Marpmann, and the bacilli and micrococci of Freudenreich. These different agents act on the lactose of the milk, decomposing it into carbonic and lactic acids, which coagulate the milk.

Another group of micro-organisms which were well studied by Duclaux comprises those acting on the casein, among others Tyrothrix tenuis, filiformis, turgidus, scaber, virgula, etc.

These organisms secrete principles having similar effects to those of 
rennet, and are capable of coagulating enormous quantities of milk. After a certain time, they also secrete a second diastase, viz., casease, which acts in the ripening of cheese.

Clotted Milk.-This term is used in dairies to indicate milk which coagulates in lumps immediately after being withdrawn from the udder, or which coagulates spontaneously a few hours later.

The change may be of a chemical nature, depending on conditions of keep or feeding. More frequently, however, it is related to a latent non-pathogenic infection of the udder, or to immediate infection of the milk after removal by lactic ferments contained in the milk vessels or the atmosphere.

It is necessary, according to circumstances, either to modify the diet or disinfect the milk vessels, and immediately pasteurise the milk.

Milk without Butter.-Less commonly the diseased condition is indicated by marked diminution in the quantity of cream.

Churning only produces a poor kind of butter, particles of which do not readily cohere. This peculiarity is due to the presence of microorganisms, which have not yet been fully identified. It can be prevented by disinfection of the milking vessels, as well as of the dairy itself, and by the use of centrifugal separators.

Putrid Milk.-This milk is characterised by its odour. It cannot be used for making butter. In fact, as soon as the cream separates, little bubbles of gas form at various points and break, leaving small cavities. These little separate cavities reunite very rapidly, and the cream becomes reabsorbed as fast as it is formed. Afterwards oily drops formed of butyric, capric, and caprylic acids appear in the depressions and give the milk a repulsive odour (rancidity).

This change is seen during mammitis, but most commonly results from uncleanliness in byres and dairies. In the latter case putrefaction occurs about twenty-four hours after milking, and is due to the growth in the milk of Bacterium termo, lineola, etc. These organisms are present in the dust which falls into the milking pails in the byre; when milk so contaminated is stored in the dairy the changes occur.

Putrid odour may also be due to the presence of ammoniacal gas in the byre, or to special toxins liberated by microbes which have found their way into the milk. It is most marked during the warm seasons of the year.

The occurrence of putrid milk can be prevented by disinfecting the dairy and the milking pails daily for a certain time.

Mucous, viscous, or thready Milk.-These terms are applied to a condition which usually appears twenty-four or thirty-six hours after the milk has been withdrawn. The milk seems thick and viscous, and can be drawn out into threads like mucus. It sticks to neighbouring objects, 
and adheres to milk vessels like molasses. It coagulates imperfectly on standing, gives little cream, and even this cream only furnishes a mawkish, ill-flavoured butter.

In certain parts of Switzerland the production of mucous milk is favoured, because it is employed in making cheeses.

The change is due to the presence of various micro-organisms. Those which have been best studied are Schmidt-Mülheim's micrococci, the Actinobacter polymorphus of Duclaux, the Bacillus lactis pituitosi of Löffler, the Bacillus lactis of Adametz, the Streptococcus hollandicus, and, finally, three others which are much commoner, Guillebeau's bacillus, the Micrococcus Freudenreichii, and the Bacterium Hessii. These microorganisms act on the lactose, decomposing it and causing the formation of a kind of filamentous mucilage, which can be isolated by the addition of alcohol.

The mucilaginous change in milk can be prevented by ordinary methods of disinfection.

Red Milk.-Milk which becomes red some hours after withdrawal, or within forty-eight hours after milking, should be distinguished from milk which on withdrawal from the udder is tinted red in consequence of hæmorrhage within the udder itself. When the milk is of a hæmorrhagic tint the blood corpuscles are soon deposited on the bottom of the vessel if the milk is allowed to remain undisturbed.

The tint which the milk assumes is due to the growth of chromogenic organisms, the best known of which are as follows:-1. B. prodigiosus, which produces large red patches on the surface. It grows readily on potato and gelatine, which it liquefies. 2. The Sarcina rosea, which develops first of all in the cream and afterwards invades the milk. It grows in sterilised milk, on alkaline potato, and on gelatine. 3. The Bacterium lactis erythrogenes, which liquefies gelatine and produces a reddish coloration. Casein can be precipitated and peptonised by means of its cultures. It develops in the milk below the cream, the serum alone becoming red, and only when shaded from the light.

Blue Milk.-In this case the milk appears normal when withdrawn, but some days afterwards shows blue patches, which gradually increase in size, and by uniting produce a distinct blue tint at the surface.

This change is connected with the presence of the B. cyanogenus. The organism grows in sterilised milk, but in this case merely produces greyish patches, the blue tint only occurring when a certain quantity of lactic acid is added or when the ordinary lactic ferments are present.

Yellow Milk.-A yellow tint occurs in ordinary milk and cream, particularly in certain breeding districts-in Normandy, for example, where the butter produced is greatly valued on account of this appearance. Pathological yellow milk is the result of the growth of 
B. synxanthus Schröter, which secretes a substance resembling rennet, curdles the milk, and finally dissolves the clot, at the same time producing the yellow colour.

Bitter Milk.-Milk which is of a normal character on being withdrawn from the udder may acquire a bitter taste some hours later. At rest, this milk produces a small quantity of yellowish, frothy cream. The organisms which produce the change have been studied in Germany, Switzerland, and Auvergne. We may mention Weizmann's bacillus of bitter milk, Conn's micrococcus of bitter milk, and Duclaux's Tyrothrix geniculatus.

Medicated Milk.-Medicated milk may be divided into two kinds: Firstly, medicated milk proper, which differs from normal milk inasmuch as it contains a certain proportion of drugs, which, when swallowed by milch cows are partly eliminated through the udder. When taken by a young animal or child such milk has a distinct therapeutic effect, depending on the principles employed.

It does not appear, however, that up to the present any very great success has followed this system. It is possible to increase the richness of the milk in phosphates, but as regards mercurial or iodine preparations the failure has been complete.

Secondly, fermented milks, which in addition to their nutritive action are made more digestible.

Fermented milk is easily digested, and is better borne by the weakest stomachs.

In human practice the fermented forms of milk are two, viz., kephyr and koumiss.

Kephyr is prepared in Afghanistan and Persia from camel's milk, but for some years past it has been made in England with cow's milk. A certain quantity of cow's milk is placed in a bottle and the ferment, consisting of kephyr grains, is added. The lactose is converted into carbonic acid and alcohol in consequence of the action of certain lactic microbes.

This milk after ingestion does not require to be coagulated and then digested before absorption, a fact which considerably diminishes peptic digestion.

Koumiss is a milk preparation resembling kephyr; it is made by the Kirghizes with mare's milk according to the same principles, but the ferment employed gives more alcohol.

Preseryation of Milk.-On account of the importance of preserving milk for use in large towns, in hospitals, and in the army during war, the question of its preservation has long been studied.

Chemical Processes.-The principle of preserving milk by chemical action consists in preventing, or at least retarding, the changes which 
inevitably follow exposure to the air. For this purpose, chemical substitutes are added which in themselves have no injurious action. Those most commonly employed are :-

\begin{tabular}{|c|c|c|c|c|c|c|c|c|}
\hline \multirow{2}{*}{\multicolumn{2}{|c|}{$\begin{array}{l}\text { Carbonate of soda } \\
\text { Bicarbonate of soda }\end{array}$}} & $\cdots$ & $\cdots$ & $\cdots$ & $\cdots$ & \multicolumn{3}{|c|}{45 grs. per quart. } \\
\hline & & . & . & . & . & . & 45 grs. & $"$ \\
\hline Boric acid.. & .. & . & . & .. & $\ldots$ & 15 to & 30 grs. & ," \\
\hline Salicylic acid & . & . & . & . & . & . & 12 grs. & , \\
\hline Borax & .. & $\therefore$ & . & .. & $\cdots$ & . . & 60 grs. & ", \\
\hline Lime & .. & . . & . & . & . . & . & 20 grs. & " \\
\hline
\end{tabular}

The results obtained are of comparatively little value; the milk only keeps for a few hours, or at the most for three or four days.

Cold.-Refrigeration, which is so valuable in preserving all kinds of animal products for long periods, has also been used for preserving milk. Unfortunately, although cold impedes the development of bacteria, it also has the grave inconvenience of causing the cream to separate from the milk, and it being impossible to mix them again satisfactorily, milk preserved in this way is more or less unfit for consumption.

Heat.-The principle of preserving milk by heat is based on the destruction of the micro-organisms at a high temperature. In this respect again, one meets with obstacles, for, if the heat be applied direct, some of the principles of the milk are converted into caramel, and if the temperature rises beyond $157^{\circ}$ Fahr. $\left(70^{\circ}\right.$ C.) the composition of the milk is changed.

Preservation by Oxygen.-Within the last few years the use of oxygen at a pressure of about two atmospheres has been recommended. When the milk is to be used it is only necessary slightly to relieve the pressure and allow the oxygen to escape, the liquor which remains having all the characters and qualities of fresh milk. The method appears excellent, but is too costly for every.day use.

Pasteurisation.-The pasteurisation of milk aims at destroying the greater proportion of the ferments above mentioned. The milk is heated at atmospheric pressure, and is kept for a time at a tempera-

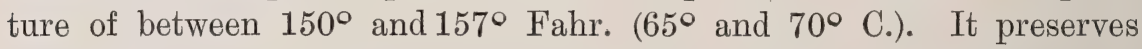
its properties and composition, but sterilisation is not complete, and the milk cannot be kept indefinitely.

Concentrated Milk.-Concentrated milk is obtained by prolonged heating to $157^{\circ}$ Fahr. $\left(70^{\circ}\right.$ C.) in a vacuum, when it becomes syrupy by evaporation and its composition is not greatly modified. It is then drawn off into bottles, which are hermetically sealed and subjected to a higher temperature to complete the destruction of all the germs. Condensed milk keeps for a very long time. To prepare it for use it is mixed with a certain quantity of water, and then yields a liquid similar to normal milk. 
Sterilisation.-Sterilisation necessitates the use of special apparatus in which the milk is heated in a water or steam bath sheltered from the action of the air, the temperature rising to $212^{\circ}$ to $240^{\circ} \mathrm{Fahr}$. $\left(100^{\circ}\right.$ to $115^{\circ}$ C. $)$; all the ferments are destroyed, and the milk will keep indefinitely, but its composition is slightly modified.

Diseases Transmissible to Man through the Medium of Milk.Tuberculosis.-The history of tuberculosis contains numerous facts proving the possibility of contagion by milk from cows suffering from tuberculous mammitis, though it seems necessary that the milk should be taken for a certain time to produce these effects.

Foot-and-Mouth Disease.-Observations recorded by veterinary surgeons prove that this disease affects the teats. It may be transmitted to man. The milker may be directly inoculated, but the milk is the ordinary vehicle of contagion. Chauveau saw an epidemic in a school at Lyons where milk was obtained from cows suffering from foot-and-mouth disease. In a similar way 205 persons were inoculated at Dover in 1884, and suffered from vesicles about the mouth.

Although foot-and-mouth disease is extremely benign in men, it is well to take every precaution against it.

Gastro-Intestinal Infections.-Cases have been recorded of gastrointestinal infection in young animals and children in consequence of consuming milk which had undergone abnormal changes. Milk containing various kinds of micro-organisms may at first produce lactic indigestion and afterwards diarrhœic enteritis. 


\section{CHAPTER VII.}

\section{MALE GENITAL ORGANS.}

The scrotum, the vaginal sheath, the testicles, the vas deferens, the vesiculæ seminales, the prostate, and Cowper's glands may all become the seat of disease.

The scrotum and testicles seldom reveal more than mechanical injuries of external origin, producing wounds and cuts and, in the case of severe contusions, hæmatoma of the scrotum, of the vaginal sheath, and of the testicles. Inflammation of the testicle, that is to say, traumatic orchitis, is rare; on the other hand, Moussu has several times seen tuberculous orchitis, for which he has operated. This, however, was in the boar. These different lesions, the last named excepted, usually heal with rest and the application of antiseptic dressings, anodyne and resolvent lotions.

Breeders seldom retain more entire animals than are strictly necessary for reproduction. The others are castrated, and this alone explains why the treatment of genital diseases in male animals of any species is rare.

\section{TUMOURS OF THE TESTICLE.}

Of the genital diseases which possess real clinical interest the most important are tumours of the testicle. These occur not only in male animals, but also in those which have undergone the operation of bistournage.

It might à priori be believed that in an animal of the latter class the testicle had been completely destroyed, not only from the physical, but from the pathological point of view. This, however, is by no means the case, and Cruzel has described, under the erroneous designation of sarcocele, tumours of the testicle which develop in oxen of various ages.

Moussu has had a similar experience with animals of from four to six years of age which had been carefully operated on.

These tumours, the origin of which is unknown, develop at the expense of the rudiments of the atrophied testicle. They vary in 
character, and Moussu has only observed tumours of a type different from that of the testicle itself, containing tracts of carcinomatous, sarcomatous, and fibrous tissue. They also appear to vary greatly in gravity, for, although the cases seen by Moussu had become generalised in a few months, Cruzel states that these tumours may remain stationary for several years.

The symptoms consist in progressive enlargement of the scrotal region and the appearance of a tumour surrounded by cedematous or lardaceous tissue.

The tumour, which is confined to one side, increases in size, is bosselated and adherent at points to the surface of the skin, while it is insensitive or only slightly painful to the touch.

In a few months it may increase to the size of a child's head. The animals have difficulty in moving or lying down, the hind limb on the affected side is abducted, and the animal usually lies on its chest and abdomen. The patient rapidly loses flesh, although the appetite remains good.

When the growth is removed, it is easy to prove that the tumour has developed in the atrophied mass of testicular tissue, and that it remains suspended from the end of the cord.

The diagnosis of tumour

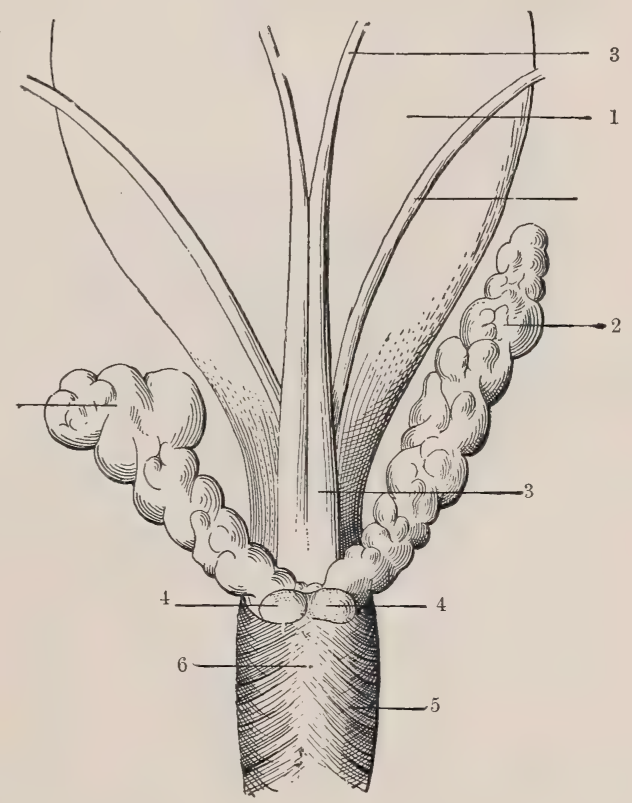

FIG. 239.-Upper surface of the neck of the bladder and origin of the urethra in the ox. 1, Bladder;-2, vesiculæ seminales; 3 , vas deferens; 4, principal portion of the prostate gland extending beneath the sphincter ; 5, sphincter; 6, aponeurosis of the sphincter, clothing the upper portion of the urethra and the flattened portion of the prostate. (After Barrier.)

of the testicle is extremely easy, for the condition cannot be mistaken for a hæmatoma or for a scrotal abscess.

The prognosis necessarily depends on the nature of the tumour, but as it is impossible to determine this point before extirpation, the prognosis should always be regarded as very grave.

Treatment. Whatever the nature of the tumour, ablation is advisable. If it is of a benign character, recovery may be complete and 
permanent, but if it is malignant, generalisation will rapidly ensue. Before interfering surgically, however, it is essential to examine the inguinal, pelvic, and sublumbar lymphatic glands, to make sure that

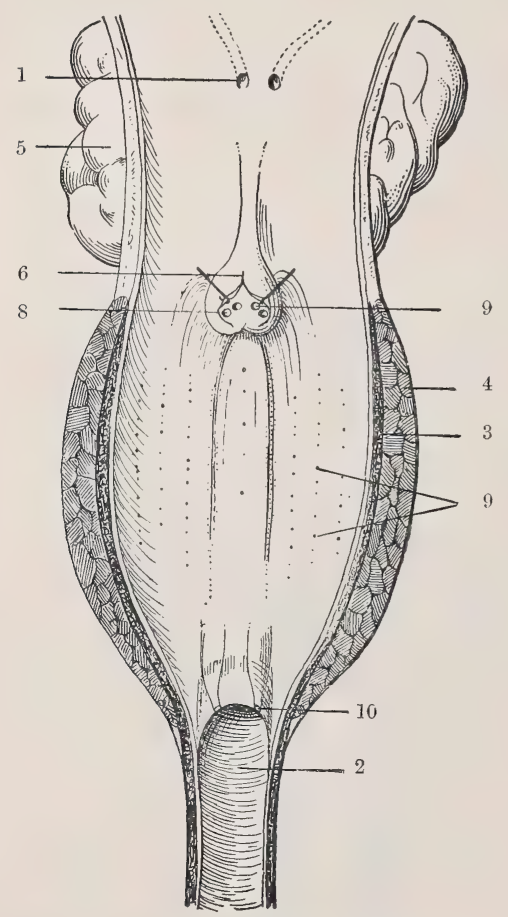

FIG. 240. - Roof of the neck of the bladder and first part of the urethra (internal surface). 1, Orifice of the ureter; 2 , urethra at a point beyond the urethral valve; 3, submucous erectile tissue; 4, urethral sphincter; 5, vesiculæ seminales ; 6 , veru montanum; 7 , orifices of the vesiculæ seminales; 8 , orifice of the vas deferens; 9 , orifices of the prostate; 10 , urethral valve, showing on its free margin the point-like openings of the ducts of Cowper's gland. (After Barrier.)

Local new growths assume the form of ulcerating swellings. In practice, if the general condition can be improved for a short time, it is advisable to slaughter the animal. they are not affected. If they prove to be already invaded, an operation should be avoided.

Even despite such precautions and the apparent absence of any glandular infection, operation may be followed by generalisation in a few months, although for a short time the condition may seem to have greatly improved. No special indications need be given beyond those suggested as necessary in dealing with all kinds of tumours, whatever their nature.

The skin should be freely divided, that the tumour may be thoroughly dissected out and no fragments be overlooked. The mass of the tumour having been dissected free, the écraseur is applied to the pedicle or the cord. Enucleation of the tumour may be very difficult on account of the presence of numerous ramifications or attachments, but the closest attention must be given to removing every fragment if a fresh local growth is to be avoided. In the event of fragments being left, a fresh growth is certain to occur.

Generalisation, which may extend to all the viscera (lymphatic glands, liver, spleen, lungs, pleura, peritoneum, heart, etc.), is indicated by rapid wasting, fever, acceleration of breathing, digestive disturbance, and sometimes coughing, ete. 


\section{ACCESSORY GLANDS OF THE GENITAL APPARATUS،}

(PROSTATE, VESICULȦ SEMINALES, COWPER'S GLANDS.)

Inflammatory or other diseases of the above glands are most frequent in entire animals. As a group they are uncommon, and are still ill-recognised and ill-described, being seldom identified except on post-mortem examination.

The cause of inflammation of the prostate, of the vesiculæ seminales, and of Corper's glands is probably an ascending infection of the urethra and neighbouring channels, a fact which explains the comparative rarity of such conditions in castrated animals.

The pathogenic germs penetrate from the urethra into the excretory ducts of the glands, obtain a lodgment in the culs-de-sac of the glands themselves, and thus produce simple or suppurative inflammation.

The symptoms may easily be mistaken, to some extent at least, for those of acute cystitis or cystitis due to the presence of a calculus, and it is only by rectal examination that the diagnosis can be made.

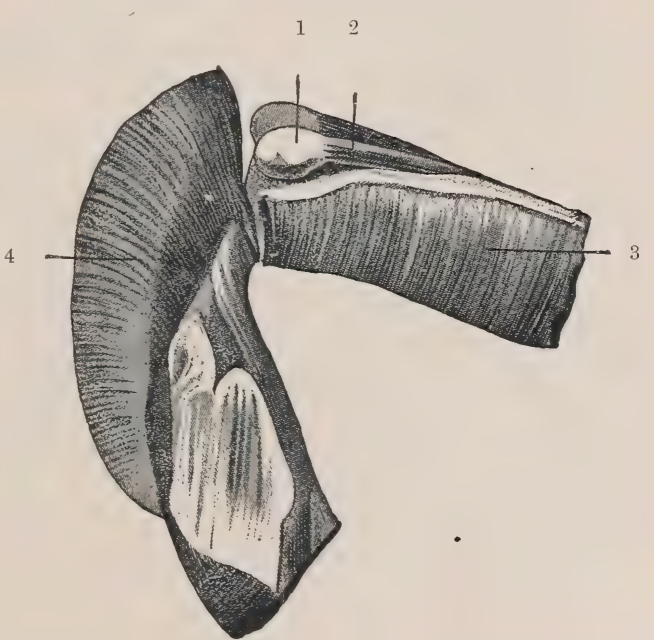

Fig. 241.-View of Cowper's gland and of its compressing muscles. (After Cocu.) 1, Cowper's gland; 2, compressing muscle; 3 , accelerator urinæ muscle turned back to expose the gland; 4, sphincter of the urethra.

The first-symptom is defective micturition, which occurs in jets, is intermittent and painful, and sometimes causes slight groaning. It is accompanied by vesical colic, spasmodic lifting of the hind legs, and more or less unsuccessful expulsive efforts.

These symptoms are of reflex or mechanical origin, and are due either to direct compression of the urethra by an hypertrophied and inflamed gland, or to spasm of the vesical sphincter. The urine passed, unlike that in cases of acute cystitis or in cystitis due to calculus, is of a normal character. Rectal examination usually reveals distention of the bladder, and hypertrophy and exceptional sensitiveness at certain points.

If the painful and hypertrophied area is over the neck of the 
bladder, this indicates that the lobes of the prostate are affected. If, however, the affected parts are situated on the sides of the neck and along the posterior pointed end of the bladder (Fig. 239), the vesiculæ seminales are affected; finally, if the painful points are directly above the ischial arch and below the sphincter ani, Cowper's glands are the seat of disease (Fig. 240).

To diagnose these conditions requires very close and careful examination. They may easily be mistaken for acute cystitis, though the character of the urine should suggest doubts and lead to rectal examination.

Although they do not endanger the animal's life, these conditions give rise to such serious inconvenience as often to render entire animals useless for stud purposes.

Local treatment is impossible, and the practitioner is restricted to the use of anodynes, balsamic preparations and diuretics. The treatment, in fact, differs little from that of cystitis. In cases of suppuration of Cowper's glands, the abscess usually forms just below the anus and close to the median line. Thus the diagnosis may be confirmed by capillary puncture, the part being laid open if necessary. 


\title{
SECTION VIII.
}

\section{DISEASES OF THE SKIN AND SUBCUTANEOUS CONNECTIVE TISSUE.}

\author{
CHAPTER I.
}

ECZEMA.

UNDER the name of eczema may be grouped a series of cutaneous diseases characterised by pruritus, by a discharge from the skin, or simply by epidermic proliferation, without any apparent parasitic or accidental cause.

These diseases have been referred to a special constitutional condition termed a diathesis, although during the last few years we have come to recognise certain microbic and toxic influences in their production. In the ox eczema assumes different appearances. Accordingly, the disease has been divided into the acute, chronic, sebaceous, and toxic forms.

\section{ACUTE ECZEMA.}

Causation. In the ox, as in all other species, the determining cause is to be found in bad hygienic conditions, improper feeding, and in a special individual condition of the animal affected, that is to say, a diathesis.

Symptoms. During a preliminary period, which might be termed a prodromic period, only general symptoms can be detected, such as fever, loss of appetite, digestive disturbance, constipation, etc. Local signs are still absent, or at least are not noticeable, and are only represented by congestion of the skin.

The second phase is characterised by a papulous eruption which is difficult to detect, inasmuch as it occurs in the depths of the coat. Nevertheless, the skin is distinctly sensitive, and at numerous small points the hairs appear to stand upright.

The third phase consists in the development of more or less confluent vesicles, with exudation and discharge. The disease is not really visible externally until after the hair has become agglutinated 
by the discharge. This discharge is seldom as abundant as in eczema in the dog or horse. It is produced slowly and dries rapidly.

As the crusts fall, carrying with them a portion of the hair, the general symptoms disappear, but the sites of these vesicular patches now show cracks extending as deep as the dermis and often complicated by secondary infection involving suppuration, adenitis, abscess formation, or diffuse subcutaneous suppuration.

Acute eczema is generally confined to the limbs. It may develop fully in from twenty-four to forty-eight hours. The acute stage is attained in a few days, and the condition disappears in two to three weeks provided it does not assume the chronic form as the result of complications.

Diagnosis. The absence of parasites enables the condition to be distinguished from phthiriasis and acariasis, whilst the history prevents its being confounded with toxic eczema.

Prognosis. The condition is troublesome rather than grave. Suppuration is often persistent, and despite careful attention the discharge may only diminish slowly, while the disease is always liable to return.

The treatment is local and general. Local treatment consists in emollient and antiseptic washes and the application of drying powders. The former comprise glycerole of starch, bran water, boric ointments, camphorated vaseline, iodine and glycerine. At a later stage the skin can be washed with decoction of oak bark or a weak iodine solution, followed by the application of talc or starch powder. As far as possible this external treatment should be supplemented by the frequent use of mild purgatives and various diuretics, which seem to have a special action on the arthritic diathesis.

\section{CHRONIC ECZEMA.}

Comparatively few cases of chronic eczema have hitherto been described in oxen, and the details given are extremely meagre. Chronic eczema may assume that form from the first or may succeed acute eczema. 'The causes are probably the same in both conditions.

The symptoms appear to be those of the acute form, but are much less severe. They consist in papulation, a miliary vesicular eruption, pruritus, and the formation of crusts and epidermic scales.

In a case seen by Mégnin the crusts separated and fell away, leaving bare spots. The disease re-appeared for several years in succession. The spots finally remained bare, but showed no thickening of the skin and no microscopic changes.

Diagnosis. Microscopical examination is necessary to distinguish this disease from scabies or ringworm. 
The prognosis is grave, because large areas of the skin may be invaded in succession.

Treatment. As in acute eczema, the condition is improved by the prolonged administration of small doses of salines and diuretics. Arsenical preparations are also valuable, but should only be employed for two or three weeks together, with intervals of equal duration. The dangerous complications which sometimes result from accumulations of this drug in the system are thus avoided.

\section{SEBACEOUS OR SEBORRHCEIC ECZEMA.}

Whilst the two preceding forms of eczema result from vascular disturbance of the skin or dermis that now under consideration seems

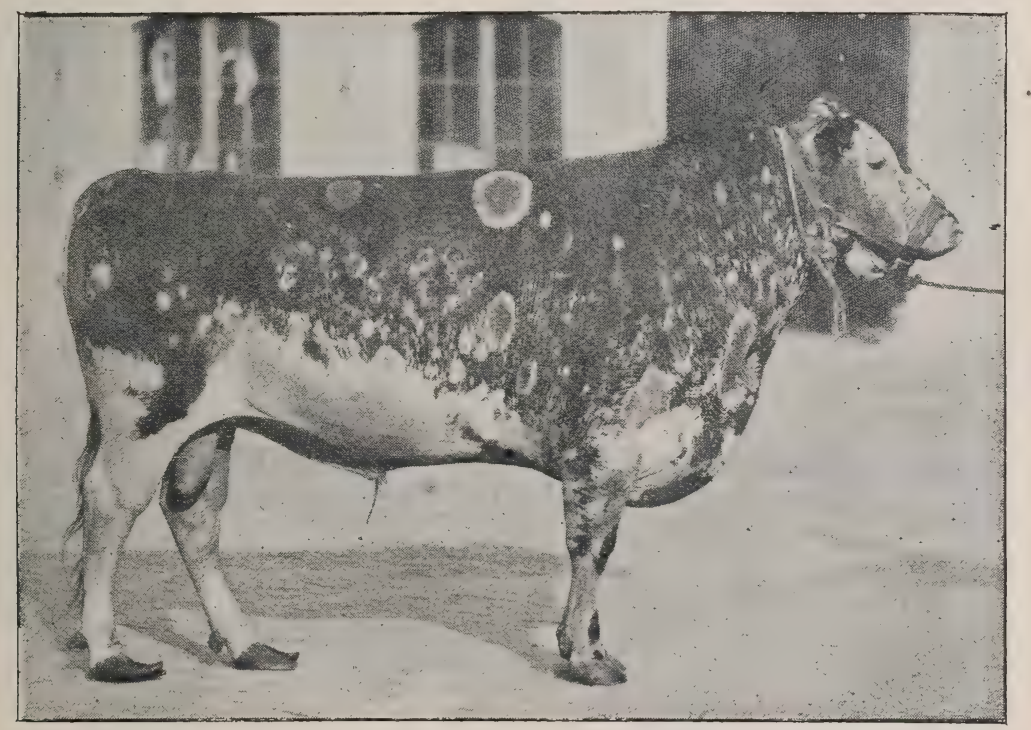

FIG. 242.-Sebaceous eczema, first stage. Depilation of the extremities.

due to vascular and secretory troubles in the accessory structures of the skin, and more particularly in the sebaceous glands.

Symptoms. The disease develops slowly. At first circular or elliptical patches of skin, distributed regularly over the body, more especially round the natural orifices, become deprived of hair. The surface of these patches is covered either with thick crusts of a peculiar greyish-brown tint which display numerous superficial cracks, or parts normally free from hair exhibit shining epidermic crusts arranged in layers, which are shed on the slightest touch.

This depilation has a strong tendency to spread, and so at first it often resembles ringworm. It results from changes in the hair 
follicles, with atrophy of the papillæ and complete loss of hair. The changes in the skin, however, are little marked. The skin

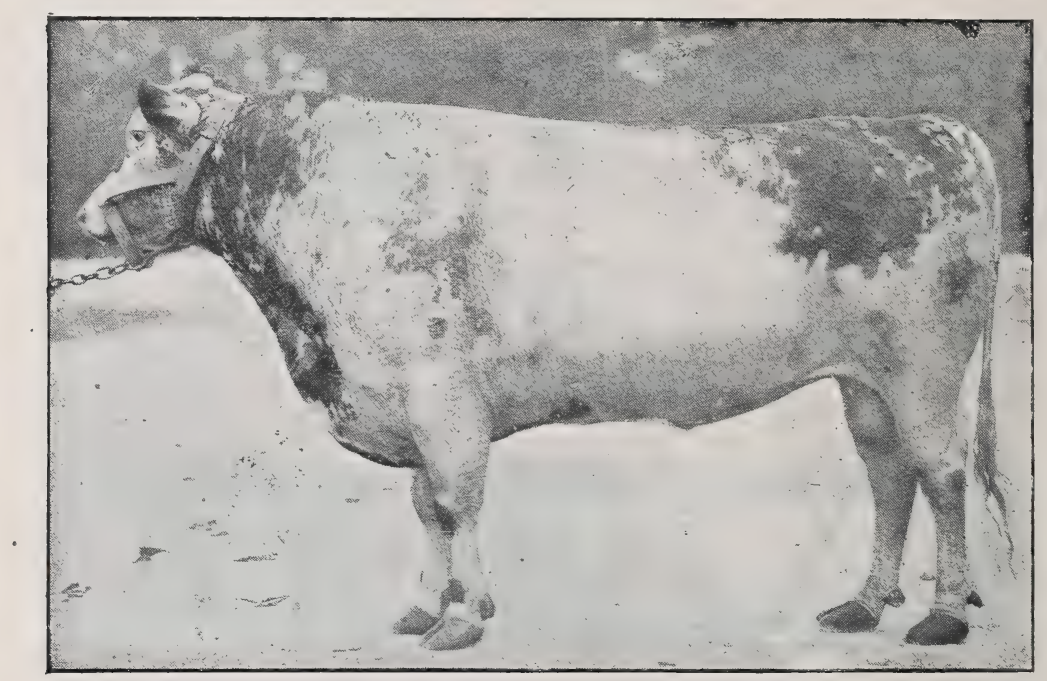

Frg. 243.- Sebaceous eczema, second stage. Partial alopecia.

itself is but slightly thickened and preserves its usual suppleness, while the subcutaneous tissue is not œdematous.

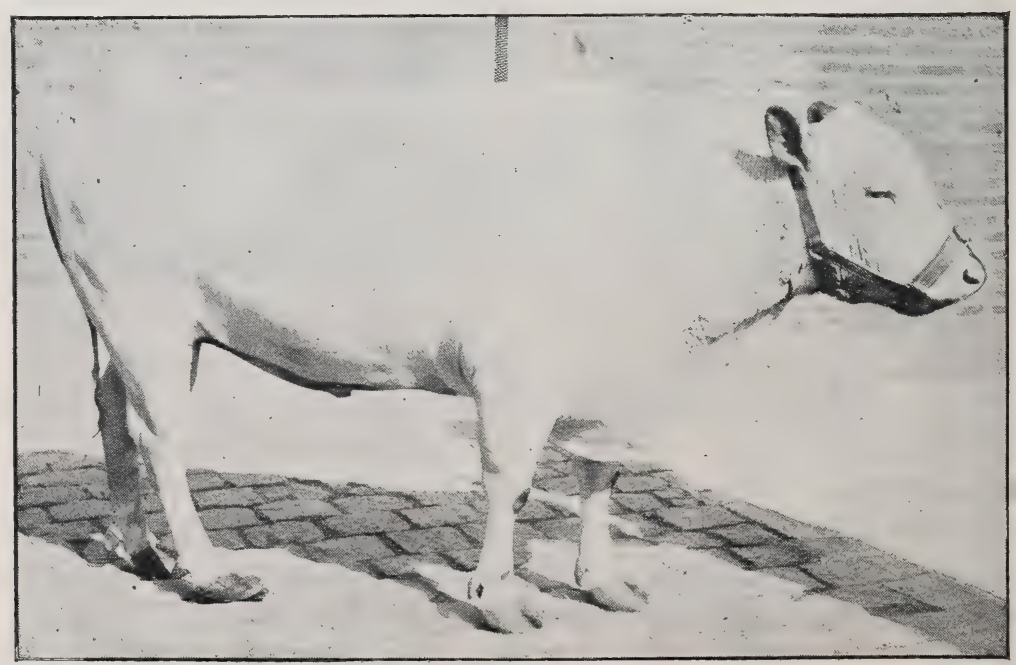

FIG. 244.-Sebaceous eczema, third stage. Total alopecia.

The hairs, however, when examined microscopically are found to be thickened at their roots, though otherwise throughout their whole 
length they remain normal. The condition is a consequence of disturbance in the circulation and nutrition of the papilla and the root of the hair.

Finally, the congestive process extends to the sebaceous glands, the secretion of which it modifies, and sets up seborrhœa, which is responsible for the formation of the crusts and epidermic scales above-mentioned.

The pathogeny of the disease is difficult to explain, but the stages in the clinical development are as follows: the skin undergoes repeated attacks of congestion, followed by seborrhoeic folliculitis, epidermitis and loss of hair.

The diagnosis is easy, the absence of ringworm being proved by the absence of the spores of trichophyton and by the non-contagious character of the discharge.

The prognosis is not grave from the point of view of general health, the chief functions being perfectly regular, but it is otherwise as regards possible terminations, viz., partial or total loss of the coat, which may be irremediable.

Treatment. It is difficult to lay down a really satisfactory method of treatment, because the exact cause of seborrhœa is still unknown. As it is probably to be sought in some individual constitutional peculiarity, the feeding and method of life should be changed and the skin stimulated by washing with lukewarm water and soap and by dry friction. Locally, if the alopecia threatens to be permanent stimulating applications may be tried. These comprise lotions containing alcohol, solutions of chloral, salicylate of soda, etc., and they should be applied with smart friction.

The sufferers should not be used for breeding purposes.

\section{ECZEMA DUE TO FEEDING WITH POTATO PULP.}

Among the forms of eczema due to toxins, several varieties may be recognised. Some result from the administration of drugs such as iodine, others from special forms of food. The only one of clinical importance is that following the consumption of excessive quantities of potato pulp.

Causation. The disease is seen in all districts in which potatoes are employed for the manufacture of alcohol and starch, more especially in Germany. The disease seems to be a direct consequence of feeding with residual products of distilleries and starch manufactories.

Spinola states that $160 \mathrm{lbs}$. of potato refuse per day to $1,000 \mathrm{lbs}$. of body weight would certainly produce eczema: 60 to $80 \mathrm{lbs}$. only produce it in rare cases and 20 to $40 \mathrm{lbs}$. are harmless. 
There is a further point to consider as regards the variation of potato pulp in toxicity. Potatoes yield a more or less active residue, according to the year in which they are grown, their state of germination, and the variety to which they belong. Raw or cooked potatoes may produce eczema if given in large quantities or for long periods.

Numerous theories have been advanced as to the nature of the morbid disturbance producing eczema. Some authorities declare the appearance of the disease to be due to the presence of a toxic principle contained in potatoes, viz., solanin. It might be objected that this chemical principle only exists during germination while potatoes that have not undergone germination produce the disease. Further, the symptoms of poisoning by solanin differ from those of this form of eczema, among which loss of appetite, for instance, or stupefaction, or narcosis is never observabłe.

According to other writers eczema is due to the action of the lower alcohols contained in the refuse pulp, but again these properties exist in brewers' and distillers' grains, the consumption of which produces no bad results. Similar objections might be made regarding the suggested action of the acids of fermentation (lactic, butyric, and acetic acids, etc.).

Johne blames the salts of potassium, which, however, can only act as digestive irritants, and Zürn suggests mycosic inflammation.

Whatever the toxic principle, its effects are most marked in animals undergoing fattening, and are rarely found in working oxen, still more rarely in milch cows. In the last named the injurious principle appears to be eliminated in the milk, and this theory is supported by the fact that the liquid has purgative properties; the calves which consume it suffer from diarrhoea, which ceases when the feeding is altered.

Finally, it has been proved that different animals show different degrees of susceptibility to the action of potato pulp.

The symptoms do not appear until after two or three weeks' feeding on the potato pulp. Then the animals walk stiffly, rise with difficulty, and display redness, swelling and sensitiveness of the limbs. When the odematous infiltration arid reddening have become distinctly marked there appear, not only between the claws, as in footand-mouth disease, but over the entire limb and principally near the folds of skin about the joints, numerous closely-packed small papules, which in one or two days become transformed into vesicles through exudation below the epidermis.

This marks the eczematous phase properly so called. The vesicles then become ruptured, the exuded matter glues the hairs together, dries, and forms crusts, which have a peculiar and distinctive odour. 
The disease may extend towards the hocks, the knees, the 'stifle, the armpit, etc. In the folds of skin surrounding the joints deep cracks form, and sometimes become secondarily infected, thus leading to the development of lymphangitis.

General symptoms, such as fever, loss of appetite and constipation, always follow; they are afterwards succeeded by diarrhœa and progressive weakness, ending in death.

The disease is easily curable if seen in its earlier stages, but after all signs of the first attack have disappeared, the condition may return five or six times in a year if potato pulp is again given. Recovery is always very difficult in aged or enfeebled patients.

The mortality varies greatly; formerly it was as high as 20 per cent., but at the present time it is much lower.

Diagnosis. Provided the history of the case is borne in mind, the diagnosis is always easy.

The prognosis is not grave if the disease is treated early.

Treatment. This consists first of all in altering the diet and reducing the quantity of potato pulp, or, better still, in discontinuing it entirely. The food should consist of good hay, bran, oatmeal gruel, pollard, etc. Internally, diuretics are given to assist in the elimination of the toxic products.

This treatment arrests the course of the disease. Suitable local treatment will remove the existing lesions. It is precisely similar in character to that of acute eczema, but it must be borne in mind that it can only prove effective if the primary cause be removed.

\section{IMPETIGO IN THE PIG.}

The term impetigo is used to describe a disease characterised by an eruption of papules, the discharge from which forms yellowish crusts, which when dry are of a grey or brown colour. The point of origin of the eruption is unknown, but the crusts rapidly become infected on contact with the air, and the bodily lesions may end in suppuration. The disease is not frequent nowadays except in sucking pigs and in large or badly-kept piggeries.

Symptoms. The eruption usually appears between the ages of two and three months, and is accompanied from the first by moderate pruritus. The papules rupture after two or three days and discharge a lemon-coloured liquid, which is distributed over the surface, dries rapidly, and causes the bristles to stick together at the roots. The crusts formed in this way remain adherent to the skin, though their surface becomes cracked. They increase in thickness, cover the head and part of the body, particularly the belly and the inner surface of 
the thighs, and if removed, an operation of some difficulty, leave exposed a bleeding, sanious, or purulent wound. The animals lose condition and appetite, cease to grow, seem as though attacked with rachitis, and may die if the general conditions of their maintenance are not improved.

The diagnosis is not difficult, but the prognosis depends on how long the disease has existed and the bodily condition of the patients.

The treatment consists entirely in improving the hygienic conditions and the feeding. The patients must be repeatedly washed or bathed and carefully disinfected, and they must have better food.

The crusts should be softened before the animals are washed, so that bleeding may be avoided and the affected areas not be transformed into suppurating wounds. By applying oil or some fatty matter to the crusts it is possible to cleanse the parts with bran water. If considered necessary, this cleansing can be followed by dressing with boric or weak creolin solution. Open-air life and good food soon relieve the principal symptoms.

\section{ACNE IN SHEEP.}

Acne, that is to say, localised inflammation of the sebaceous glands and hair follicles, sometimes occurs in sheep apart from any parasitic invasion. The eruption is particularly seen after shearing, and it is probable that, as in the horse, irritation produced by the machine, and possibly by accidental infection, constitute the principal determining causes.

Symptoms. The disease is indicated by the appearance of cutaneous pustules, which are only slightly painful on pressure and which involve the entire thickness of the skin. The dermis is hardly congested, and no constitutional disturbance occurs.

Acne lesions may be more or less confluent, and may attain the size of a small hazel-nut.

The diagnosis presents no difficulty. Puncture or incision reveals the fact that the abscesses are filled with white sebaceous material and are quite free from parasites.

The prognosis is not grave. Recovery occurs spontaneously in a few weeks.

Treatment. Liquid emollient applications and the opening of the small follicular abscesses appear to represent the only means of hastening recovery.

\section{FAGOPYRISIM (BUCKWHEAT POISONING).}

Fagopyrism is a disease of toxic origin in sheep, and is due to eating buckwheat (Polygonum fagopyrum). 
The disease has also been entitled erysipelatous or gangrenous dermatitis, according to its form and gravity.

Causation. The cause is extremely simple, viz., the consumption of buckwheat and other food pertaining to a like species, such as Polygonum persicaria. The green plant and the straw give the same results, but the action of light and air are also necessary for the production of the disease, a fact which is somewhat difficult to explain.

According to German writers the disease occurs more frequently in white sheep and lambs than in those in which the skin is of a very dark colour.

Symptoms. When the sheep are fed in folds, with the green plant in summer or with the straw in winter, nothing unusual is seen, the herds being, to all appearance, in perfect health. On their being set at liberty, however, the first symptoms appear, perhaps in less than an hour. Some animals become restless, make peculiar movements of the head, and soon display intense congestion of the parts free of wool, together with redness and swelling of the ears, eyelids, face, throat, etc. The condition develops with extreme rapidity, the animals being immediately afflicted with pruritus over the affected regions. If they are not removed to the quiet and warmth of the fold the symptoms increase and papules appear, which may be transformed into vesicles and bullæ. In the fold, on the other hand, all the symptoms rapidly disappear.

The disease rarely assumes an erysipelatous form, but respiratory and cerebral symptoms, together with fever and vertigo, are not exceptional.

Treatment. The feeding on buckwheat should at once be discontinued, and the patients should be kept in the fold until the toxic principles have been eliminated, that is, for a month or more.

Bicarbonate of soda may be added to the drinking water. The local lesions about the head must be kept clean and dressed with antiseptic astringent lotions. 


\section{H A P T E R I I.}

\section{PHTHIRIASIS.}

The term phthiriasis is applied to infestation of the skin with lice.

Causation. These diseases are due to the presence of various parasites which live by destroying the epidermic scales, or by piercing the superficial layers of the skin. They are of a greyish-yellow colour, and belong to the genera Hcematopinus and Trichodectes.

The Hematopinus forms have pointed heads, and are equipped for

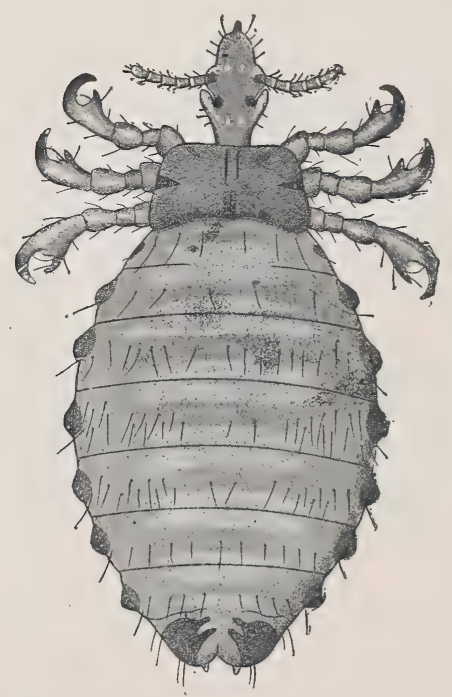

FIG. 245.-Hamatopinus eurysternus of the ox, magnified 20 diameters. (After Neumann.)

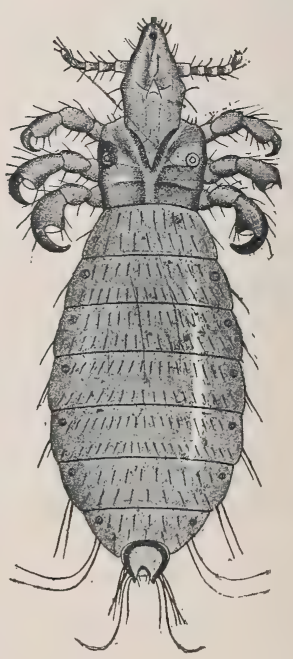

FIG. 246.-Homatopinus of the calf, magnified 20 diameters. (After Neumann.)

penetrating the skin by suction. The Trichodectes have a large flat head constructed for masticating.

The ox harbours two forms of Hamatopinus and one of Trichodectes, the Hematopinus eurysternus and tenuirostrus, and the Trichodectes scalaris.

The sheep suffers from Trichodectes spherocephalus and a Melophagus, 
the goat from the Hcematopinus stenops and the Trichodectes climax, and the pig from the Hcematopinus urius.

The symptoms are, with trifling variations, the same in all domestic animals, the principal being rubbing and itching. The animals scratch, bite and attempt to rub against hard objects, even abrading the skin when this is thin and the irritation is severe.

The parasites may, however, remain localised, and it rarely happens
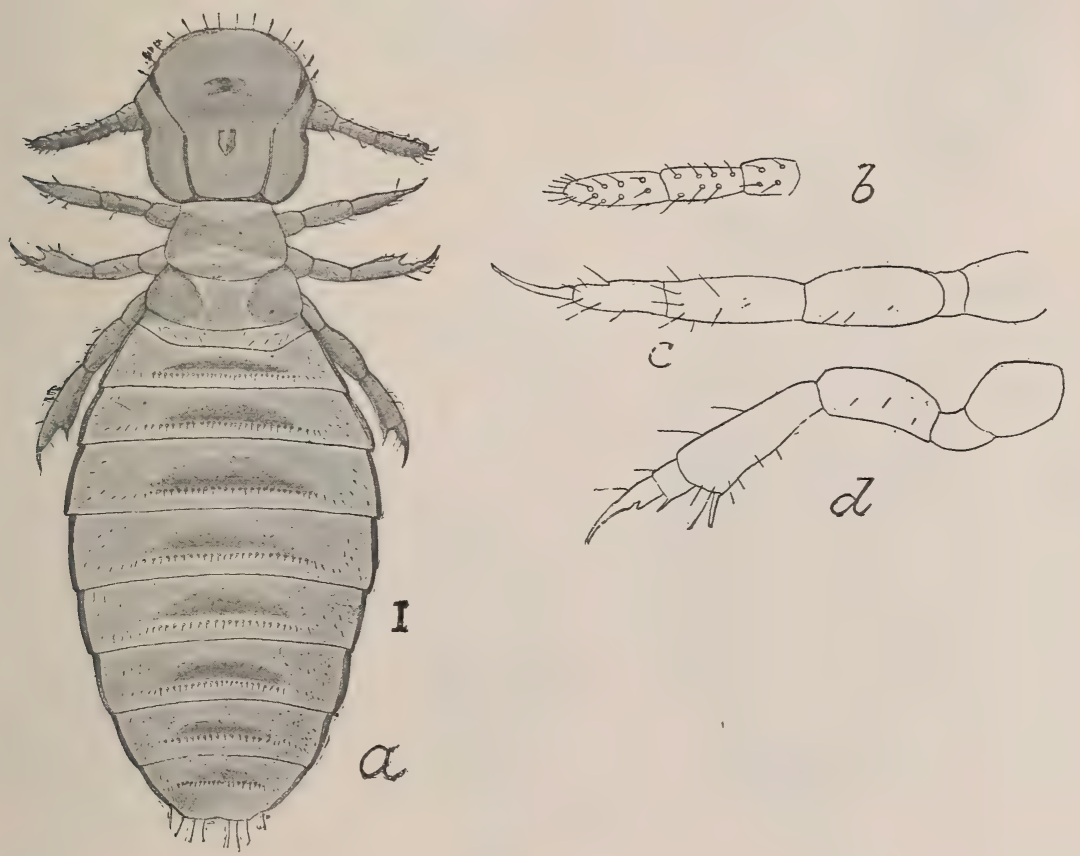

FIG. 247.-Sheep louse (Trichocephalus sphcrocephahus). a, Female; $b$, antenna; $c, d$, dorsal and side view of leg. Enlarged. (After Osborn, 1896; Bul. No. 5, Div. Entomology, Dept. Agr.)

that they are present in any considerable number in all parts of the body.

In the ox they are principally found in the depression at the back of the base of the horns, and in the upper margin of the neck and the back. In the absence of treatment phthiriasis may become generalised over the entire surface of the body.

The trichodectes and the melophagus of the sheep choose similar points, but when the wool is long they may be found nearly all over the body.

In the pig the hæmatopinus is found on the neck, in the region of the poll, about the armpits, and round the eyes and easr.

D.C. 
These parasites, whose powers of increase are astonishing, keep the patients in a continual state of irritation, causing them to lose condition and, in the absence of treatment, to die of exhaustion.

The diagnosis is very easy, the parasites being visible to the naked eye.

The prognosis is not grave unless the condition affects a large number of animals in herds. In young animals the prognosis is much graver, for the little creatures rapidly become anæmic and die in a state of exhaustion.

Treatment. When the byre, fold, or piggery is infested the first point is to remove the animals and thoroughly disinfect and cleanse all parts.
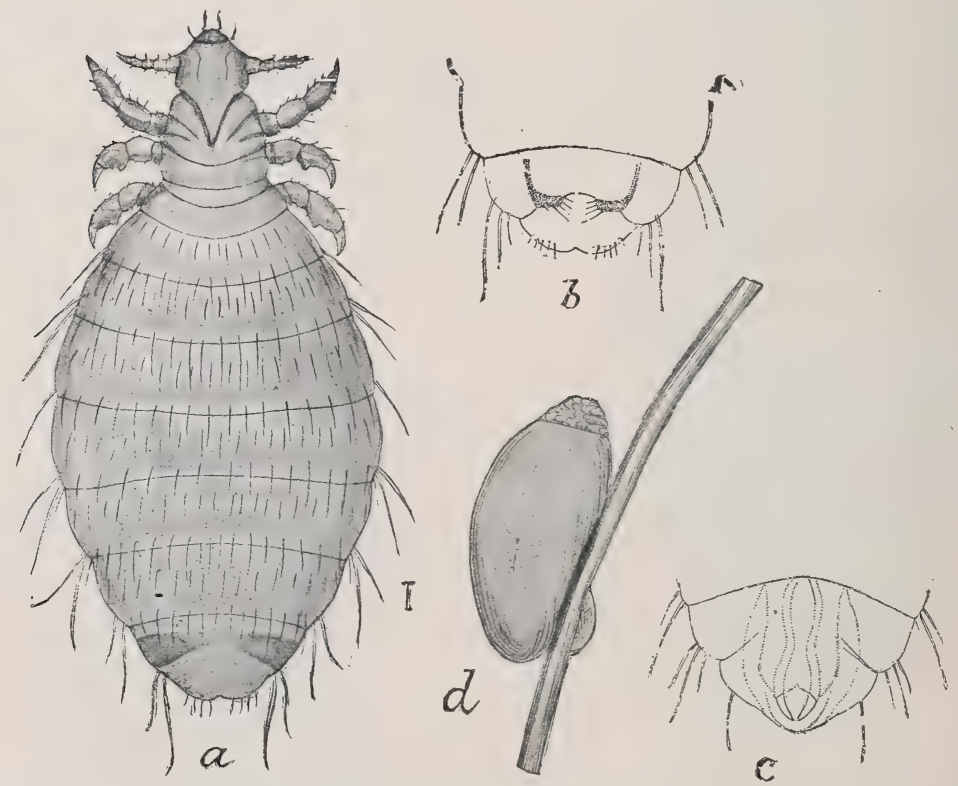

FIG. 248.- Sheep foot louse (Homatopinus pedalis). a, Adult female; $b$, ventral view of terminal segment of same, showing brushes ; $c$, terminal segments of male ; d, egg. Enlarged. (After Osborn, 1896; Bul. No. 5, Div. Entomology, Dept. Agr.)

After the manure has been cleared out, the walls, mangers, racks, etc., are washed with boiling water, or, better still, potash solution, and disinfected first with vaporised sulphurous acid, then, if necessary, with a washing of caustic lime.

The patients are afterwards clipped, washed with soft soap and dressed with anti-parasitic solutions, such as 1 per cent. tobacco juice, or a mixture of equal parts of benzine and oil or benzine and petroleum, etc., which give excellent results.

A 3 per cent. creolin solution is also a very active anti-parasitic and very easy to use. 
All these solutions, however, are more or less poisonous and need to be used with caution, weak solutions only being used at first, par-

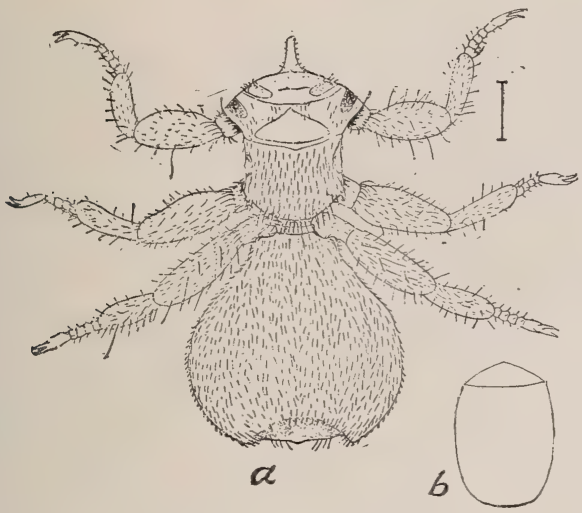

FIG. 249.-Adult sheep tick (Melophagus ovinus). (a) Tick; (b) puparium. Magnified. (Salmon and Stiles, Annual Report, U.S.A. Dept. of Agriculture, 1897, p. 103.)

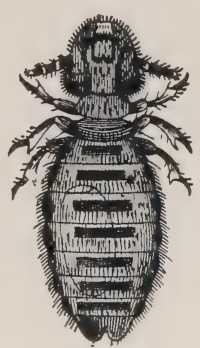

FIG. 250. -Trichodectes scalaris of the ox. Magnified 20 diameters. (After Railliet.)

ticularly in the case of animals, such as oxen, which are given to licking themselves.

\section{SCABIES-SCAB-MANGE.}

The term scabies is given to a group of diseases affecting man and all domestic animals. These diseases are produced by two classes of parasites, viz., sarcoptinæ, which live within the epidermis or on the surface of the skin, and demodectes (sing. demodex), which penetrate into the sebaceous glands and hair follicles.

Scabies, though known from the earliest times, has long been confounded with constitutional diseases characterised by cutaneous eruptions. The symptoms shown were formerly regarded as due to the elimination of "humours" which the organism was casting off, for which reason scabies was even treated with internal medicines.

At the present time the cause of the disease is perfectly well understood, as well as the mode of development of the different parasites. The rate at which these parasites develop is almost incredible, a fact which explains the highly contagious character of the disease.

Each species of animal may present several varieties of scabies, caused by different parasites, such as sarcoptes, psoroptes, chorioptes, demodectes, etc.

\section{SCABIES IN SHEEP.}

Scabies in sheep usually assumes one of three forms-sarcoptic, psoroptic, or chorioptic scabies. Follicular, or demodectic, mange affects 
the eyelids, and is very rare. It is produced by the Demodex folliculoin var. ovis.

\section{SARCOPTIC SCABIES.}

This scabies has long been recognised as affecting more especially the head, muzzle, etc. It was mentioned as long ago as the fourteenth century by Jehan de Brie, but, until Delafond's time, no one recognised that it was caused by an acarus. In 1858 Delafond discovered the parasite in Piedmont sheep.

Causation. Formerly, writers on the subject and shepherds attributed this disease to the wounds and excoriations which sheep receive in passing through brambles, holly, etc., or in rubbing against their racks. The true cause of the disease

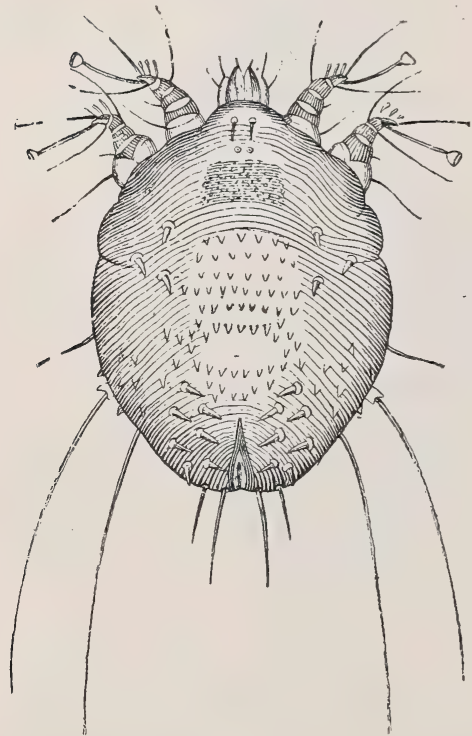

FIG. 251.-Sarcoptis, magnified 100 diameters. (After Railliet.) is the presence under the skin of the Sarcoptes scabei var. ovis, which passes from sheep to sheep by direct contact. The animals attempt to rub against everything about them, even against their neighbours. These parasites can be transferred from the goat to the sheep, and vice versâ.

Walraff, Roloff, Delafond, Gerlach and Railliet have described cases of infection in man, but the disease is rarely more than of a temporary character.

Symptoms. This form of scabies affects the head and the parts free from wool.

At first the parasites invade the upper lip and the tissues about the nostrils, sometimes, but more rarely, the eyes and ears. They cause the formation of vesicular papules, accompanied by violent itching. The animal, in rubbing itself, excoriates these papules, which discharge a fluid and soon become covered with yellowish-brown crusts.

The disease afterwards invades the face, forehead, jaws, and entire head. The skin becomes wrinkled and the brownish crusts thicker and more abundant. These are fissured and bleeding, and they give the face the appearance of one vast sore.

The parasite rarely attacks the region of the elbow, the belly, or the inside of the thigh. The disease never advances in parts covered by wool, although in breeds of sheep with thick wool, such as are found 
in Algeria and Tunis, the sarcoptic form of scabies may become generalised and attack the entire body. The extremities of the limbs, however, are usually attacked after all the head has become involved.

Throughout the course of the disease the patients scratch and rub themselves, thus tearing off the crusts and causing bleeding and the formation of new crusts of a blackish hue. This form of scabies about the head may become complicated with conjunctivitis, the inflammation extending from the extremity of the eyelids to the conjunctiva. This arises from the fact that the membrane is frequently injured by the animal rubbing the parts. Conjunctivitis may be so intense as to lead to purulent ophthalmia and the loss of the eye.

Diagnosis. Sarcoptic mange in sheep cannot be mistaken for any other disease on account of its localisation.

Prognosis. The disease is not very dangerous, for it is easy to treat, though if left to itself it might in time become fatal.

\section{Treatment.} Preventive treatment consists in

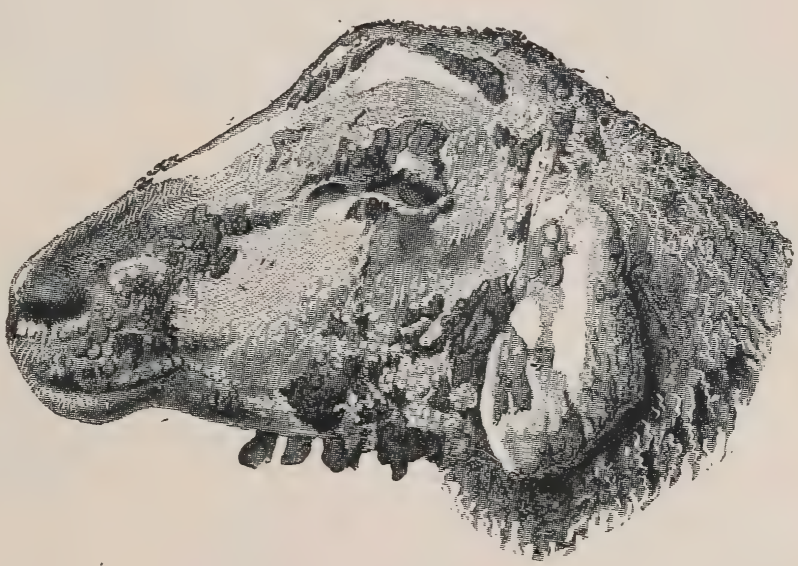

FIG. 252.-Sarcoptic mange of the sheep, showing the appearance of the head. isolating diseased animals, cleansing and disinfecting the folds, and preventing the introduction of diseased animals into healthy flocks.

Curative treatment. When the disease is detected at an early stage anti-psoroptic remedies may be directly employed.

If, however, it is of old standing, the crusts must first be softened and removed by the use of fatty substances, such as vaseline or oil, before any curative treatment can be undertaken.

The crusts can be removed in from twenty-four to forty-eight hours by vigorous washing with soft soap and the application of antiparasitic solutions. The omission to wash the parts causes drugs to lose much of their efficacy.

Helmerich's ointment, oil of cade, mixtures of oil, benzine and petroleum, and 3 per cent. to 4 per cent. of tobacco juice, are the commonest and most efficacious applications. 
A non-poisonous ointment may be made by taking 4 ounces of oil of turpentine, 6 ounces of flowers of sulphur, and $1 \mathrm{lb}$. of lard. Mix the ingredients at a gentle heat, and rub in well with the hands or with a brush, at the same time breaking the crusts. The simple sulphur ointment may be made of one part of sulphur and four parts of lard; one fourth part of mercurial ointment may be added. Few remedies are so useful as sulphur iodide, and it may well be given a trial on head scab.

In most countries this disease has been made the subject of special legislation.

\section{PSOROPTIC MANGE-SHEEP SCAB.}

This is probably the gravest form of mange. It was described by Cato the Censor in 160 в.c., by Virgil, Juvenal, Celsius, Columella, Pliny, Vegetius, etc. In 1787 Abildgaard first showed that psoroptic mange in sheep could be cured by simple external remedies, without internal medications. In 1809 Walz described the causes, nature, seat, and treatment of the disease.

Since then, the parasitic, contagious nature of mange or scabies has been more and more clearly recognised.

Causation. Experience and observation have long shown that the only cause is the presence of the Psoroptes communis (ovis). This disease is much more contagious than that just described. Psoroptic mange or scabies exclusively affects those portions of the body covered by the wool, and may for a long time remain unrecognised.

The parasite is visible to the naked eye, though most inspectors employ the microscope. The adult female is about $\frac{1}{40}$ of an inch long and $\frac{1}{60}$ of an inch broad; the male is $\frac{1}{50}$ of an inch long and $\frac{1}{80}$ of an inch broad. The mites are discovered more easily on a dark background, and if a portion of the wool and crusts is placed on black paper and exposed to the sun for a few minutes the parasites will generally be seen crawling about on the paper.

The disease is transmitted directly or indirectly by contact from diseased to healthy animals in the folds, fields, or sheep runs. One diseased sheep may contaminate an entire flock. The disease is extremely contagious, and may appear even within a week after exposure.

The parasites have exceptional vitality. It is generally stated that, kept at a moderate temperature on portions of scab, the adults may live from four to twenty days, but they will occasionally live much longer; cases are on record where they have lived three, four, or eren six weeks when separated from sheep; if the atmosphere is dry they will generally die in about fifteen days; but death is often only apparent, for the mites may sometimes be revived by warmth and moisture even after six or eight weeks; the fecundated females are especially tenacious of life. 
Experience has shown that in some cases apparently healthy sheep have become infected in places where no sheep have been kept for four, eight, twelve, or even twenty-four months. The conditions underlying
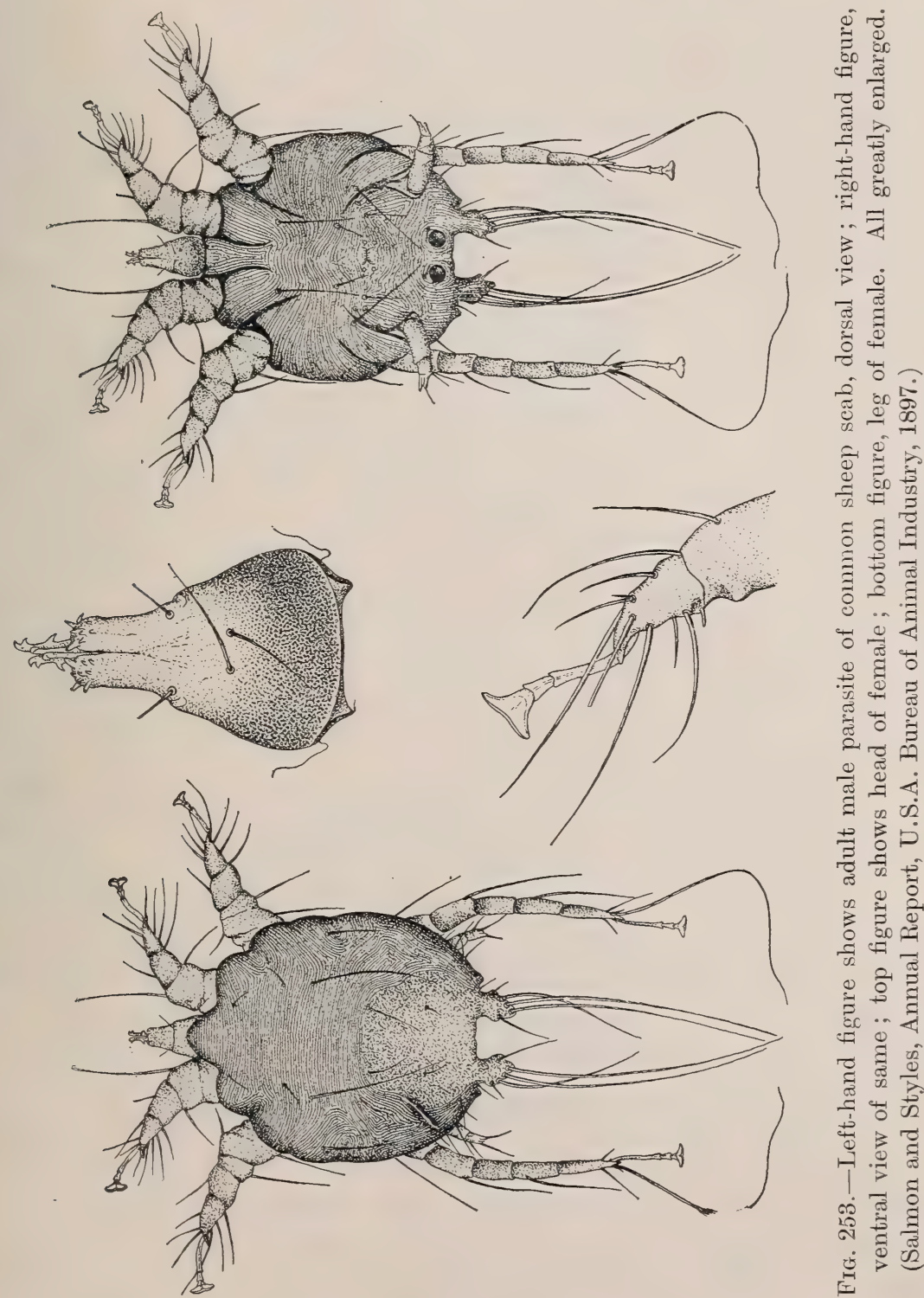

this infection are not thoroughly understood. Possibly some of the eggs have retained their vitality a long time and then hatched out; possibly the vitality of the fecundated female has also played a rôle; 
while it is not at all improbable that an entirely new infection has accidentally been introduced by birds or other animals. Certain authors of high standing scout the idea that birds can introduce an infection of scab, but there is no reason why birds should not do this, and there are some reasons for believing that they do. It has been noticed on the Experiment Station of the United States Bureau of Agriculture, for instance, that crows delight in perching on the backs of scabby sheep and picking at the scab; while so doing it is only natural that small tags of wool would adhere to their feet, and thus scatter scab.

Delafond's experiments show that psoroptic mange is most troublesome amongst thin, ill-nourished, weakly animals, whilst robust sheep in good condition may be cured simply by attention to cleanliness and abundant feeding.

In America this disease causes extremely heavy pecuniary loss second only in importance to that produced by hog cholera. It has also interfered very seriously with the export of American sheep.

The course of the disease is affected by the time of year and surrounding conditions. In autumn and winter, when sheep are in continual contact in a moist, warm atmosphere within the folds, the disease makes rapid progress. Young, weak, closely-inbred animals, and those with long, coarse wool, most quickly succumb. Unhealthy surroundings, damp, and bad ventilation favour the disease. Pure or mixed bred merino sheep suffer severely. In summer the animals are generally shorn and live in the open, and the disease then usually diminishes or may even be arrested.

A study of the life history of the scab parasite is necessary in order to determine several important points of practical value, such as the proper time for the second dipping, etc.

The female mite lays about fifteen to twenty-four eggs on the skin, or fastened to the wool near the skin; a six-legged larva is hatched; these larvæ cast their skin and become mature; the mites pair and the females lay their eggs, after which they die. The exact number of days required for each stage varies somewhat, according to the writings of different authors, a fact which is probably to be explained by individual variation, and by the conditions under which the observations and experiments were made. Thus Gerlach, in his well-known work (1857) estimates about fourteen to fifteen days as the period required for a generation of mites from the time of pairing to the maturity of the next generation. He divides this time as follows: Under ordinary. conditions the eggs hatch in three to four days, although two authors allow ten to eleven days for the egg stage; three or four days after birth the six-legged larvæ moult and the fourth pair of legs appears; this fourth pair is always present 
when the mites are two-thirds the size of the adults; when seven to eight days old the mites are mature and ready to pair; several (three
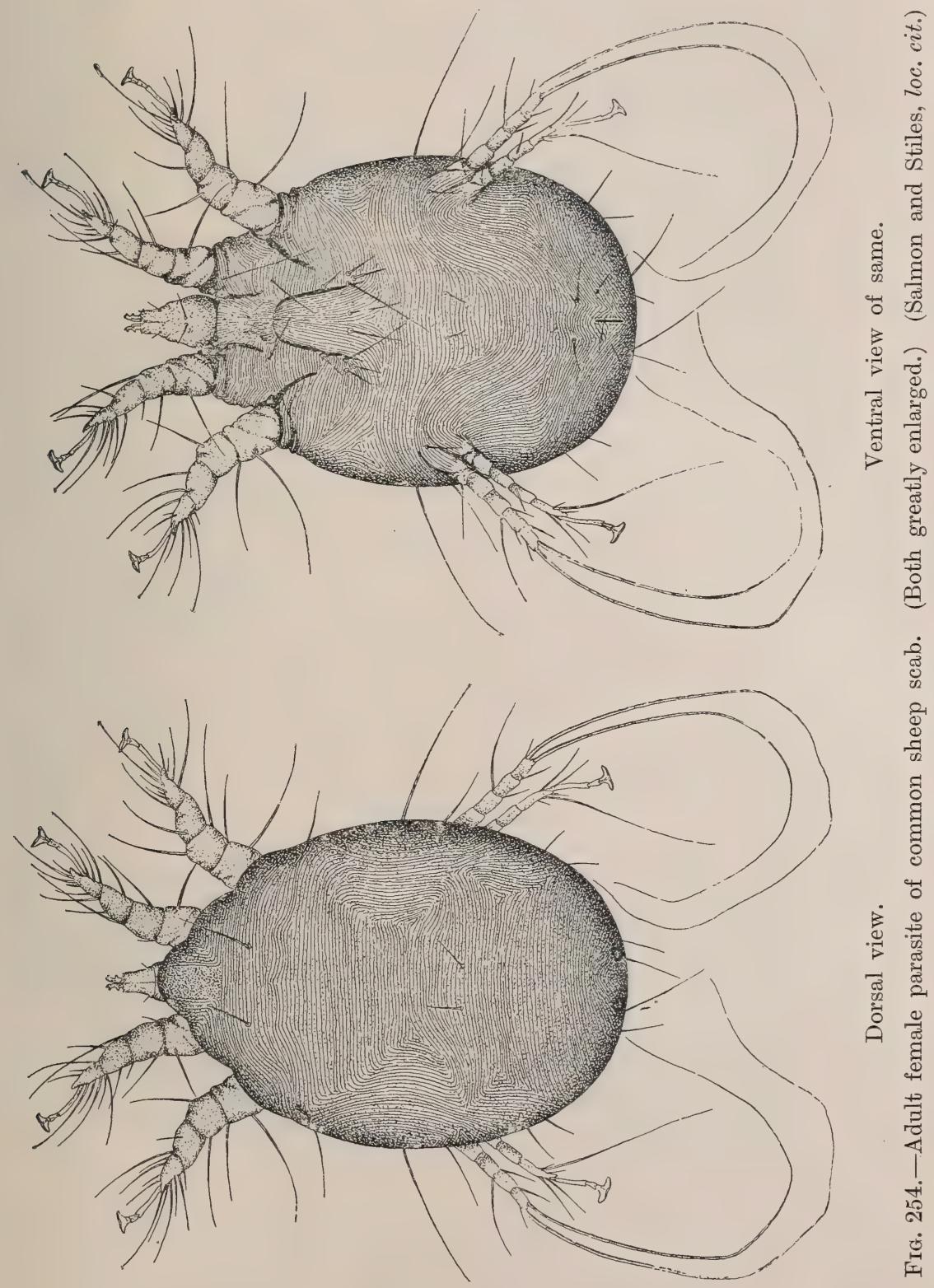

or four) days are allowed for pairing; another generation of eggs may be laid fourteen to fifteen days after the laying of the first generation of eggs. Without going into all of the other observations on 
these points, it may be remarked that the eggs may not hatch for six or seven days; the six-legged larvæ may moult when three to four days old, and become mature; after pairing, a second moult takes place, lasting four to five days; a third moult follows immediately, then eggs are laid and the adults die; in some cases there is a fourth moult, but apparently without any further production of eggs. Accepting Gerlach's estimate of fifteen days as an average for each generation of ten females and five males, in three months' time the sixth generation would appear and consist of about $1,000,000$ females and 500,000 males.

Several practical lessons are to be drawn from these figures: Firstly, it is seen that the parasites increase very rapidly, so that if scab is discovered in a flock, the diseased sheep should immediately be isolated; secondly, if new sheep are placed in a flock, they should either first be dipped, as a precautionary measure, or they should at least be kept separate for several weeks to see whether scab develops; thirdly, since the chances for infection are very great, the entire flock should be treated, even in case scab is found only in one or two animals; fourthly, as dipping is not certain to kill the eggs, the sheep should be dipped a second time, the time being selected between the moment of the hatching of eggs and the moment the next generation of eggs is laid. As eggs may hatch between three and seven, possibly ten or eleven days, and as fourteen to fifteen days are required for the entire cycle, the second dipping should take place after the seventh day, but before the fourteenth day; allowing for individual variation and variation of conditions, the tenth, eleventh, or twelfth day will be the best time to repeat the dipping.

Symptoms. Psoroptic mange attacks the parts covered with wool, so that attention is only drawn to the disease by some slight injury to the fleece, which becomes rough, matted, brittle, and liable to fall.

Scabies commences with intense pruritus. The animals scratch and bite themselves, and tear away the fleece. These symptoms become aggravated when the animals are hot, as, for instance, when travelling. If the sheep have travelled through mud, the fleece becomes matted on the neck, behind the shoulders, and at all points where they are able to scratch themselves with the hind limbs.

When a scabby sheep is touched at a diseased point, the animal shows pleasure by nibbling and moving the head up and down.

At an early stage, if the fleece is divided and the skin examined, little papules may be discovered somewhat less in size than a lentil. They are of a yellowish colour, and are distinctly visible against the reddish colour of the skin. These papules are due to the attacks of the psoroptes. 

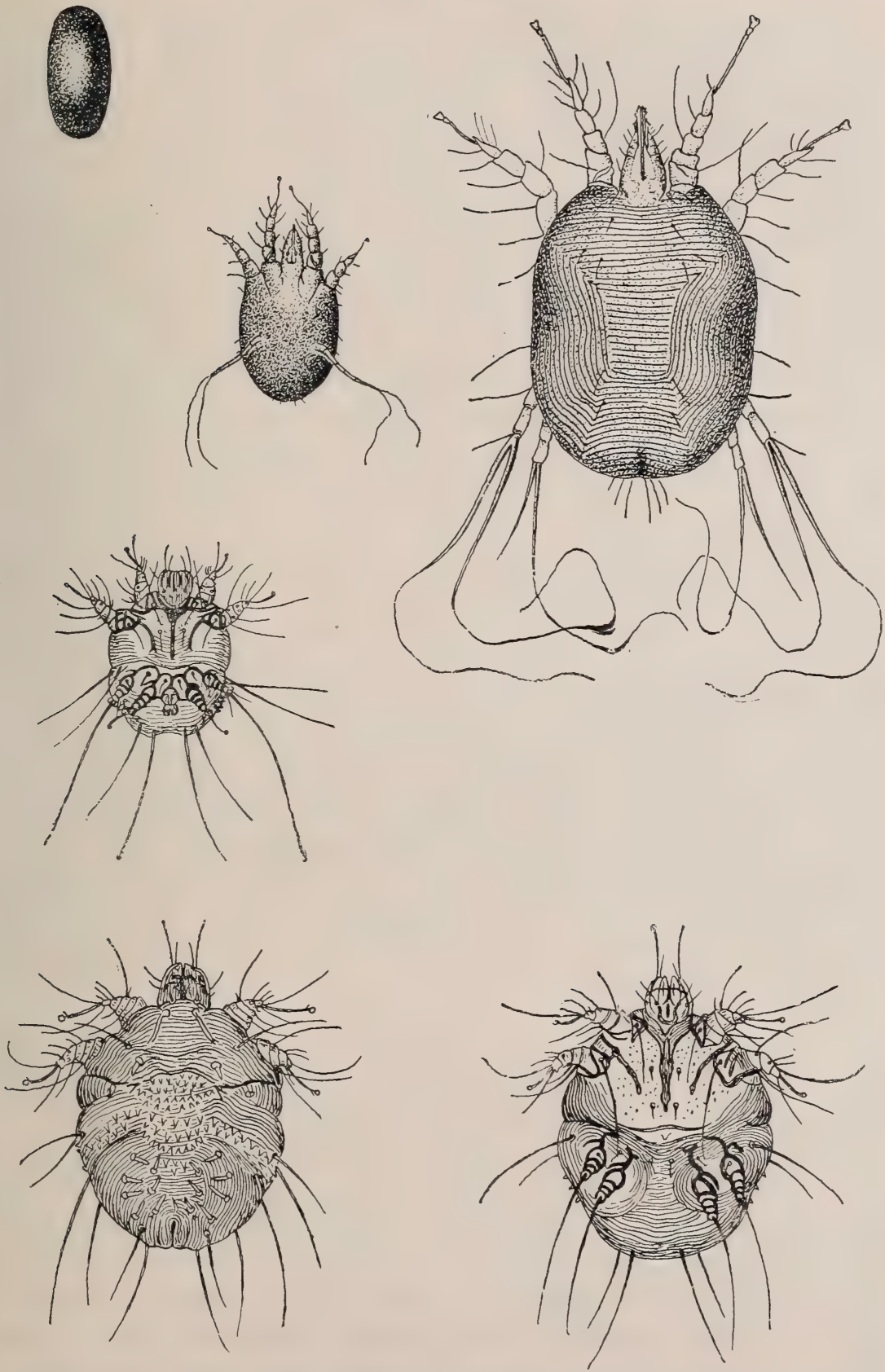

FIG. 255.-Left top figure, egg of mite which causes common sheep scab; middle top figure, six-legged stage of sheep scab mite; right top figure, young female before moulting for the last time, dorsal view; middle figure, adult male parasite of sarcoptic scabies of man (the corresponding parasite of sheep is very similar), ventral view, $\times 250$ (after Blanchard); left bottom figure, adult female parasite of sarcoptic scabies, dorsal view, $\times 250$ (after Blanchard); right bottom figure, same, ventral view (after Blanchard). All greatly enlarged. 
They soon become more numerous and even confluent, break and discharge, become converted into pustules, and cause the formation of crusts. In a few days the diseased points are covered with a squamous, yellowish, sticky covering, under which the psoroptes lie hidden and which affords them nourishment.

The crusts steadily grow thicker and lift the individual fibres of wool, tearing them from their follicles, so that patches of skin become bare. The patches thus formed increase in diameter, for the acari leave the centre, where crust-formation is replaced by abundant desquamation of the epidermis. The skin is thickened, assumes the character of parchment, and in old-stariding cases becomes wrinkled.

The disease always commences along the back, withers, loins, and the upper part of the quarters. Thence it spreads to the flanks and sides of the chest. The psoroptes are almost exclusively confined to recently affected points on the edges of the scabby patches. They are visible to the naked eye, and appear as little whitish-brown points.

Scab is specially liable to attack a flock containing lambs and yearling sheep, whose skin is thin, fine and supple, and therefore more susceptible to their attacks. If a portion of a scabby flock be shorn, the shorn animals will probably recover on account of the psoroptes transferring themselves to the animals with long fleeces.

The diagnosis is easy. Psoroptic mange cannot be mistaken for sarcoptic mange, on account of the different points affected.

Psoroptic mange only attacks regions covered by wool, and sarcoptic mange those free of wool. A microscopical examination of acari removed from the diseased animals will, however, immediately remove
all doubt.

Nor can the disease be confounded with phthiriasis, the trichodectes being immediately distinguished from the psoroptes by their greater size and the shape of their head. Moreover, they are usually to be found on the front portions of the shoulders.

It is more likely to be mistaken for another disease, termed by some writers seborrhœa and studied and described by Delafond under the name of sebaceous folliculitis. This disease appears mostly in autumn, and attacks arimals much exposed to the weather and on moist, cold soils. It begins with very violent pruritus, followed by biting and loss of portions of the fleece. The skin is red, inflamed and painful, and the wounds are covered with large quantities of yellowish acid discharge of a sticky and offensive nature. The treatment of this disease consists in placing the sheep in clean, dry, wellventilated sheds. Recovery is assisted by clipping and the application of some emollient dressing to the diseased parts. 
The ease with which the disease can be cured and the absence of parasites enable one to readily distinguish it from psoroptic mange (scab).

Prognosis. The disease is not specially grave, so long as only a few animals are affected, for it is not difficult to cure by isolation, good feeding, and proper external treatment; but if scab appears in a flock, the freedom with which the animals intermingle is such that all are rapidly attacked, and the irritation produced at once checks their growth and causes loss in condition. Many ewes give birth to small, feeble lambs, which are almost certain to die, and in any case the wool is considerably diminished in value.

Delafond estimated that psoroptic mange formerly attacked one thirty-fifth of all the sheep in France every year, causing damage to the extent of five francs per head. At the present time, and since proper sanitary laws have been instituted, it has become much less common.

During the bad season of the year the mortality is greater, and may reach as much as from 40 to 50 per cent. In cases where scab is accompanied by some other disease, such as distomatosis, it may even rise to 80 per cent.

The treatment is preventive and curative. Preventive treatment consists in separating the healthy from the diseased animals and in disinfecting the folds, sheds, etc.

Curative treatment. The first point in this treatment consists in improving the diseased animal's food both as to quality and quantity.

It is to be observed that the parasite has more difficulty in living on robust and well-nourished animals. Moreover, observation shows that transference from poor land to rich pastures is sometimes in itself sufficient to bring about a spontaneous cure. Such, at least, is the belief of the Spanish sheep farmers in Estramadura and of the French shepherds.

The shepherd can do a great deal to arrest the course of the disease. If he is careful, zealous, intelligent and observant he will quickly note the first indications of the disease and, by isolating the animals, check its spread.

The second point consists in shearing the diseased animals, and this must be carried out at any season of the year. The money loss is sometimes important, but must be met, for otherwise treatment is impossible. In cases of localised scab, empyreumatic oil, oil of cade, solutions of sulphuret of potassium, decoctions of black hellebore (water 1 quart, fresh rhizome 4 ounces or dry rhizome 2 ounces), decoction of tobacco and diluted tobacco juice 6 ounces in 1 quart of water) have been recommended. Such local treatment, however, is often useless, because incomplete. 
When scab is generalised and it is impossible to define the parts attacked, general treatment is indispensable and the diseased sheep should be dipped.

As a preliminary, however, and in order to make sure that the application will produce its effect, the animals after shearing should be passed, twenty-four hours before the medicinal bath, through a warm bath containing soap in order to soften and remove the scabs. Applications of oil or some fatty substance will also soften the scabs, which may afterwards be removed with a scraper without producing bleeding. One pound of soft soap may be dissolved in fifty quarts of water and each sheep plunged into this and scrubbed with a brush for a few minutes. Washing alone removes a large number of the parasites.

Whatever bath be used it should not be given until four or five hours after the last feeding. The dips most popular in France are as follows:-

\section{Tessier's Bath (1810).}

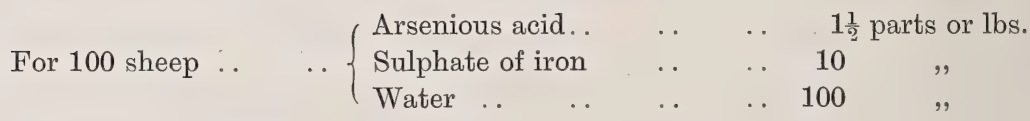

The above materials should be boiled for ten minutes, and, as a consequence of the chemical fusion which occurs in the process, the proportion of arsenious acid dissolved amounts to about 2 drachms per quart instead of $3 \frac{3}{4}$ drachms.

In this bath the arsenious acid acts as a parasiticide and the sulphate of iron as an astringent, the latter checking the absorption of toxic principles by the skin and sores, and preventing the sheep from licking themselves.

Absorption by the skin is not so dangerous as has been believed. Rossignol has shown that poisoning need not be feared in chemical baths unless the proportion of dissolved arsenic is above 150 grains per quart, especially if the period of immersion does not exceed five minutes: Even pure solutions of arsenic, free from any astringent, may be used, provided the quantity in the bath does not exceed 120 grains per quart.

The bath should, if possible, be kept warm $-85^{\circ}$ to $95^{\circ}$ Fahr. $\left(30^{\circ}\right.$ to $35^{\circ}$ C.).

Four men are generally employed for the operation. One drives in the sheep, two others hold and brush them in the bath, and the fourth holds the head of the animal above the liquid. Tessier recommended gloves for the use of the operators, but experience has shown that such a precaution is unnecessary. The udder, and particularly the teats, of ewes with young may, if necessary, be smeared 
with some fatty substance, such as vaseline or oil, in order to guard against the astringent action of the liquid.

Each sheep is plunged in the bath for one or two minutes, or five minutes at the most. All the diseased spots must be brushed, rubbed and cleansed; but care must be taken not to make them bleed.

Tessier suggested leaving the animals for twenty-four hours in some disinfected place, without straw or food, to prevent these materials from being wetted by the liquid which runs from the fleece, and which, if afterwards eaten, might have a poisonous effect. Here, again, the danger has been exaggerated. Delafond has shown that sheep may be given a fluid ounce of Tessier's bath for eight days running without producing the slightest unfavourable sympiom.

Tessier's bath is excellent from the therapeutic standpoint, but it imparts a yellow tint to the fleece, which is thus rendered less valuable. The mixture has therefore been modified in various ways.

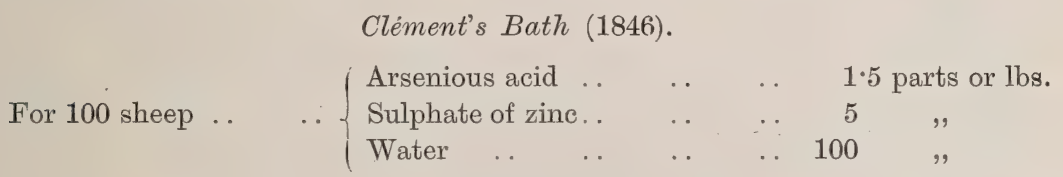

In this bath every quart contains about $2 \frac{1}{2}$ drachms of arsenious acid. Its toxic power, therefore, is considerably greater than that of Tessier's bath.

Clément's formula has one drawback. The sulphate of zinc may be mistaken for a non-astringent alkaline sulphate (sulphate of soda), and as a consequence poisoning may occur, as experience has shown.

Mathieu of Sèvres replaced the sulphate of iron by an equal quantity of alum. In this case, each quart of the bath contains $2 \frac{1}{2}$ drachms of arsenious acid.

Clément's and Mathieu's formulæ have given just as good results as Tessier's.

A last formula is that of Professor Trasbot. The aloes is of very little use, however, because it is almost insoluble.

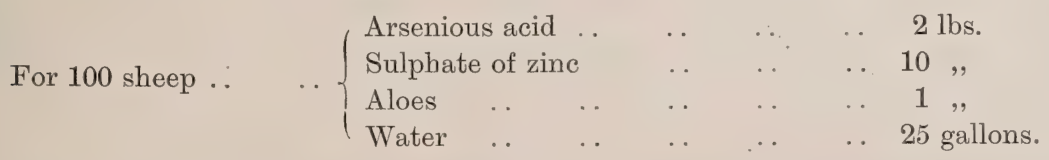

Condition of the animals after the bath.-On leaving the bath the abraded parts are slightly cauterised. During the five or six following days the skin is stiff, and covered with adherent crusts over the points attacked by the parasites. The animals no longer scratch or bite themselves. 
Towards the eighth day the crusts fall, the skin appears supple and of a pink colour, and the wounds cicatrise. In animals which have suffered for a long time recovery is much slower, and may extend over from thirty to fifty days. The wool again grows soft and bright, while the sheep rapidly regain their spirits and condition. The cicatrisation of the wounds is often accompanied by intense itching, which must not be taken as a sign of the persistence of the disease. It is well, however, to keep the animals under observation at this period.

Under any circumstances, six weeks or two months should always be allowed to elapse before giving a second bath. Should a few spots appear to be attacked secondarily, they may be moistened with a little of one of the bath liquids.

In Germany the creolin bath is generally employed:

For 100 sheep .. .. $\left\{\begin{array}{ccccc}\text { Water } & \ldots & \ldots & \ldots & 250 \text { gallons or parts. } \\ \text { Creolin } & \ldots & \ldots & \ldots & 6 \cdot 5\end{array}\right.$

Each sheep is bathed a second time after an interval of one week, the animal being immersed for three minutes in the bath and thoroughly scrubbed with a brush. The efficacy of this method, however, is less certain.

In America, where the flocks are large and scabies is frequent, sulphur baths are employed, the baths themselves being of great size. The animals are forced to pass through them and remain there for some minutes.

The size and value of American, Australian, and New Zealand flocks demand that the modes of treatment practised and the experience gained in these countries should receive something more than passing notice. We therefore purpose giving a short résumé of some parts of the very valuable monograph on sheep scab issued in 1897 by Dr. Salmon and Mr. Stiles, for the American Bureau of Animal Industry.

In selecting a dip the question of expense will naturally arise; next, the question as to whether or not scab actually exists in the flock to be dipped. The facilities at hand, the set-back to the sheep, and the length of the wool are also matters for consideration, as well as the pastures into which the dipped sheep are to be placed.

Expense.-In estimating the expense one should consider not only the actual outlay for the ingredients of the ooze, but the cost of fuel and labour, the injury, if any, to the sheep, and the liability of not curing the disease. It is much more economical to use an expensive dip and cure scab, than it is to use a cheap dip and fail to cure it. 
Does scab exist in the flock? - If scab does not actually exist and the wool is long, the dipping in this case simply being a matter of precaution, it is best not to select a dip containing lime.

The facilities at hand for preparing dip.-If fuel is very scarce, so that it is impracticable to boil the mixture for at least two hours, the lime-and-sulphur dips should not be selected.

The pastures.-In case it is necessary to place the dipped sheep on the same pastures they occupied before being dipped, it is always

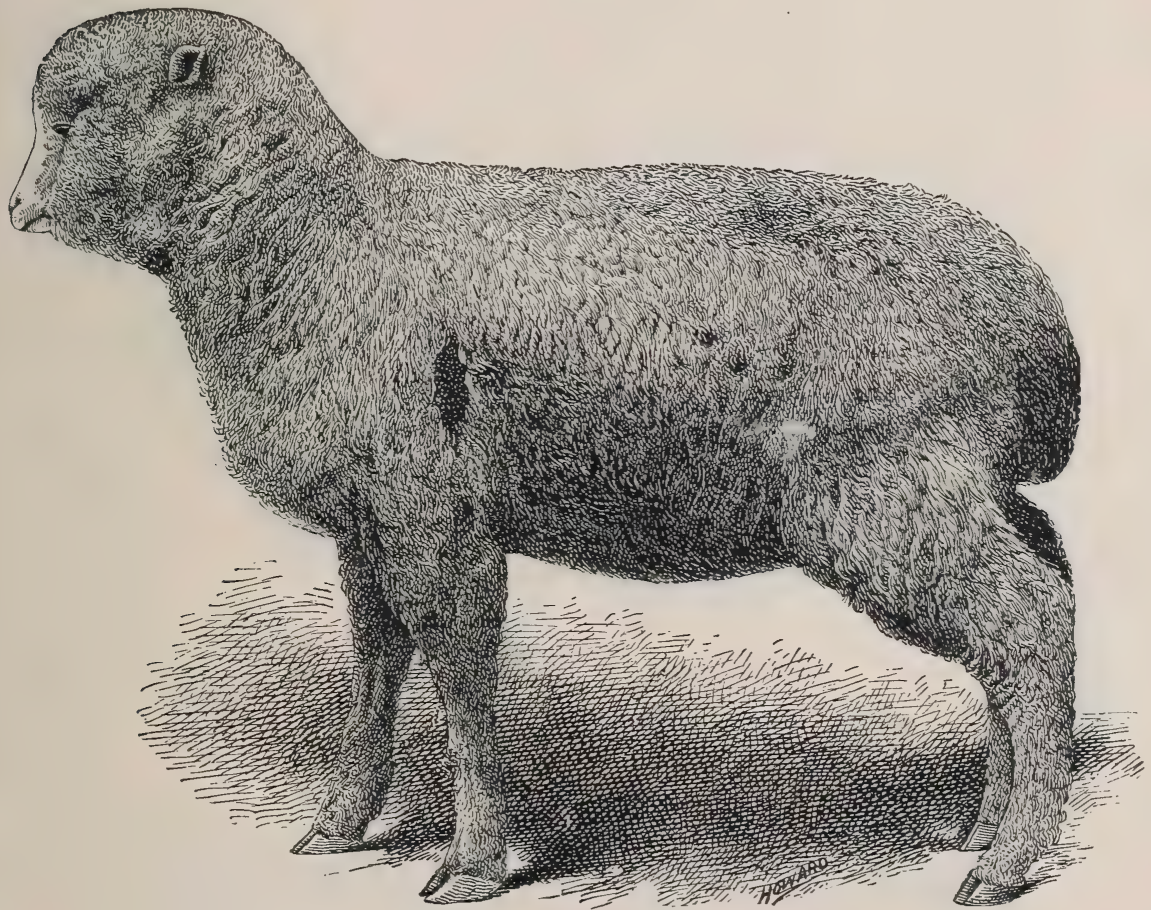

FIG. 256.--A comparatively early case of common scab, showing a bare spot and tagging of the wool.

best to use a dip containing sulphur. If a proprietary dip is selected under those circumstances, it is suggested that sulphur be added, about $1 \mathrm{lb}$. of flowers of sulphur to every 6 gallons of dip. The object in using sulphur is to place in the wool a material which will not evaporate quickly, but will remain there for a longer period of time than the scab parasites ordinarily remain alive away from their hosts. By doing this the sheep are protected against reinfection.

Sulphur is one of the oldest known remedies for scab; its use is best known in the tobacco-and-sulphur dip and in the lime-andsulphur dip. These home-made mixtures are the two dips which D.c. 
have played the most important rôles in the eradication of scab from certain English colonies, and their use is extensive in America.

\section{The Tobacco-and-Sulphur Dip.}

The formula as given here, and as adopted by the New South Wales sanitary authorities, appears to have first been proposed in

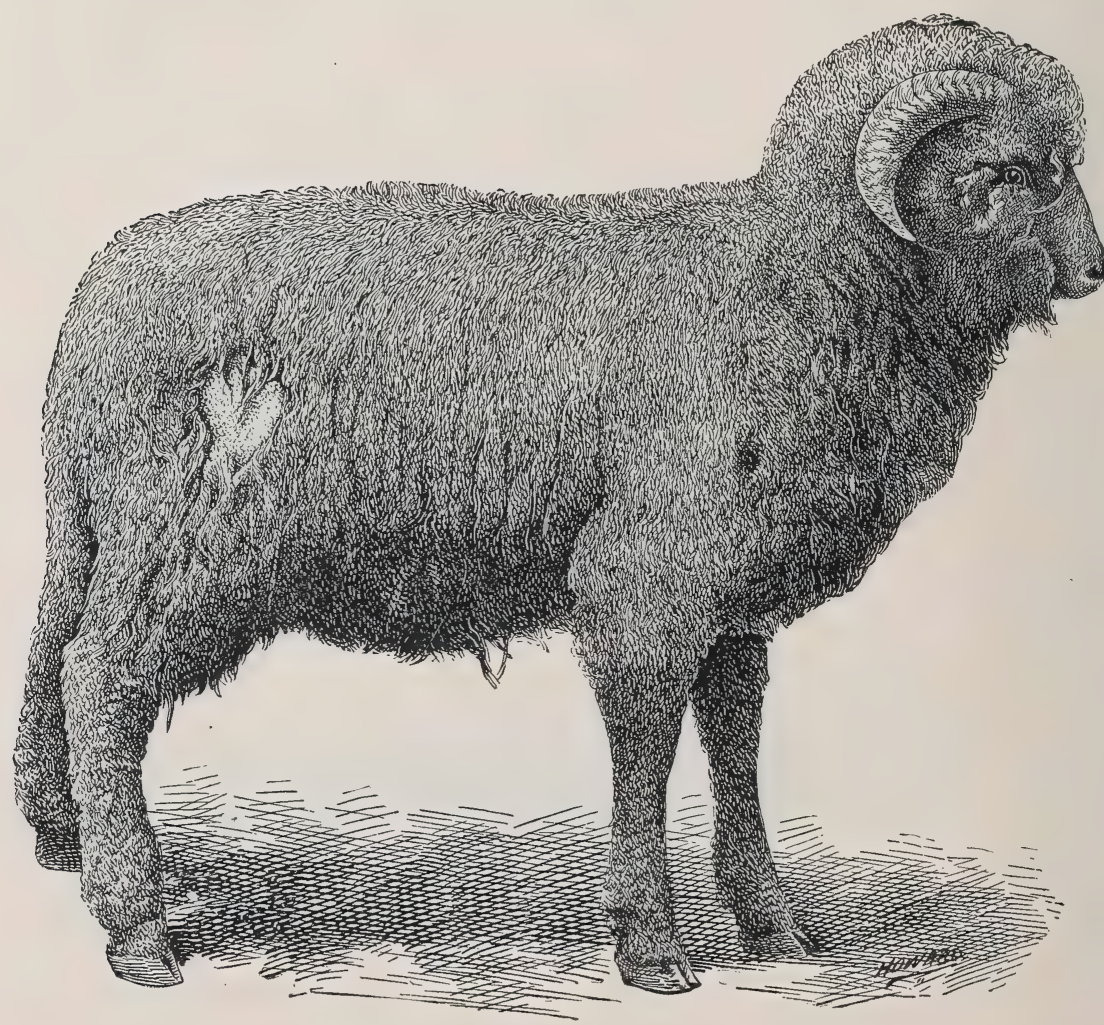

FIG. 257.-A slightly advanced case of common scab.

1854 by Mr. John Rutherford. "On the Hopkins Hill Station Mr. Rutherford, with two dressings of these ingredients, then cured over 52,000 sheep which had been infected for eighteen months. Since then millions of scabby sheep have been permanently cured in Victoria in the same way, and in South Australia and New South Wales hundreds of thousands of scabby sheep have also been cleansed with tobacco and sulphur. Judging, therefore, from the experience of the three colonies, there is no medicament or specific yet known that can be compared with tobacco and sulphur as a thorough and lasting 
cure for scab in sheep." (Dr. Bruce, Chief Inspector of Sheep for New South Wales.)

The proportions adopted by Rutherford, and afterwards made official by the scab sanitary authorities, are-

$\begin{array}{lllllllll}\text { Tobacco leaves } & \ldots & \ldots & \ldots & \ldots & \ldots & \ldots & \ldots & 1 \text { lb. } \\ \text { Flowers of sulphur } & \ldots & \ldots & \ldots & \ldots & \ldots & \ldots & 1 \text {, } \\ \text { Water } & \ldots & \ldots & \ldots & \ldots & \ldots & \ldots & \ldots & 5 \text { gallons. }\end{array}$

The advantage of this dip lies in the fact that two of the best scab remedies, namely, tobaceo (nicotine) and sulphur, are used together, each of which kills the parasites, while the sulphur remains in the wool and protects for some time against reinfection. As no caustic is used to soften the scab, heat must be relied on to penetrate the crusts.

Directions for preparing the dip.-Infusing the tobacco:-Place $1 \mathrm{lb}$. of gold-leaf or manufactured tobacco for every 6 gallons of dip desired in a covered boiler of cold or lukewarm water, and allow to stand for about twenty-four hours; on the evening before dipping bring the water to near the boiling point (212 ${ }^{\circ}$ Fahr.) for an instant, then remove the fire and allow. the infusion to stand overnight.

Thoroughly mix the sulphur $(1 \mathrm{lb}$. to every 6 gallons of dip desired) with the hand in a bucket of water to the consistency of gruel.

When ready to dip, thoroughly strain the tobacco infusion from the leaves by pressure, mix the liquid with the sulphur gruel, add enough water to make the required amount of dip, and thoroughly stir the entire mixture.

\section{Lime-and-Sulphur Dips.}

Under the term "lime-and-sulphur dips" is included a large number of different formulæ requiring lime and sulphur in different proportions.

To give an idea of the variety of the lime-and-sulphur dips, the following list is quoted, the ingredients being reduced in all cases to avoirdupois pounds and United States gallons:

(1.) The original "Victorian lime-and-sulphur dip," proposed by Dr. Rowe, adopted as official in Australia:

Flowers of sulphur

Fresh slaked lime

Water

$$
\begin{array}{lllllll}
\ldots & \ldots & \ldots & \ldots & \ldots & \ldots & 20 \frac{5}{6} \mathrm{lbs} . \\
\ldots & \ldots & \ldots & \ldots & \ldots & \ldots & 10 \frac{5}{12}, \\
\ldots & \ldots & \ldots & \ldots & \ldots & \ldots & 100 \text { gallons. }
\end{array}
$$

(2.) South African (Cape Town) official lime-and-sulphur dip, February 4th, 1897 :

Flowers of sulphur

Unslaked lime

Water

$$
\begin{array}{ccc}
\ldots & \ldots & 20 \frac{5}{6} \mathrm{lbs} . \\
\ldots & \ldots & 16 \frac{2}{3}, \\
\ldots & \ldots & 100 \text { gallons. } \\
& & \multicolumn{2}{c}{\text { S S } 2}
\end{array}
$$


(3.) Fort Collins lime-and-sulphur dip :

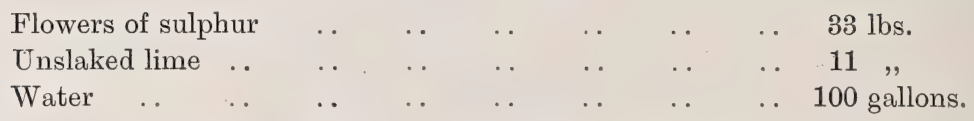

(4.) A mixture which, used to some extent by the Bureau of Animal Industry, contains the same proportions of lime and sulphur (namely, 1 to 3 ) as the Fort Collins dip, but the quantities are reduced to-

Flowers of sulphur

$24 \mathrm{lbs}$.

Unslaked lime ..

Water

8 ,

100 gallons.

In case of fresh scab Formula No. 4 will act as efficaciously as the dips with a greater amount of lime, but in cases of very hard scab a stronger dip, as the Fort Collins dip, should be preferred; or, in unusually severe cases, an ooze with more lime in proportion to the amount of sulphur, such as the Victorian (No. 1) or the South African (No. 2) dip might be used.

\section{Prejudice against Lime-and-Sulphur Dips.}

There is at present great prejudice (a certain amount of it justified, no doubt) against the use of lime and sulphur, emanating chiefly from the agents of patent or proprietary dips and from the wool manufacturers.

In the first place, it is frequently asserted that lime and sulphur does not cure scab. Experience in Australia and South Africa, as well as in America, has shown beyond any doubt that a lime-andsulphur dip, when properly proportioned, properly prepared, and properly used, is one of the best scab eradicators known.

It is claimed by some that it produces "blood poisoning." But the cases of death following the use of lime-and-sulphur dips have been infinitesimally few when compared with the number of sheep dipped in these solutions, and when compared with the deaths which have been known to follow the use of certain proprietary dips. The details of such accidents, so far as they have been reported, have not shown that death was due to any properly prepared and properly used lime-and-sulphur dip. It is highly probable that the cases of so-called "blood poisoning" of shear-cut sheep are generally due to an infection with bacteria in stale dip containing putrefying material.

The greatest objection raised against the use of lime-and-sulphur dip is that it injures the wool. This objection is raised by many wool manufacturers, and echoed with ever-increasing emphasis by the manufacturers of prepared dips; while, after years of extensive 
experience with properly prepared dip, its injury to the wool is strongly and steadfastly denied by the Agricultural Department of Cape Colony.

It is believed that a certain amount of justice is attached to this objection to lime and sulphur as generally used; unless, therefore, lime and sulphur can be used in a way which will not injure the wool to an appreciable extent, we should advise against its use in certain cases; in certain other cases the good accomplished far outweighs the injury it does. Let us, therefore, examine into this damage and its causes.

The usual time for dipping sheep is shortly after shearing, when the

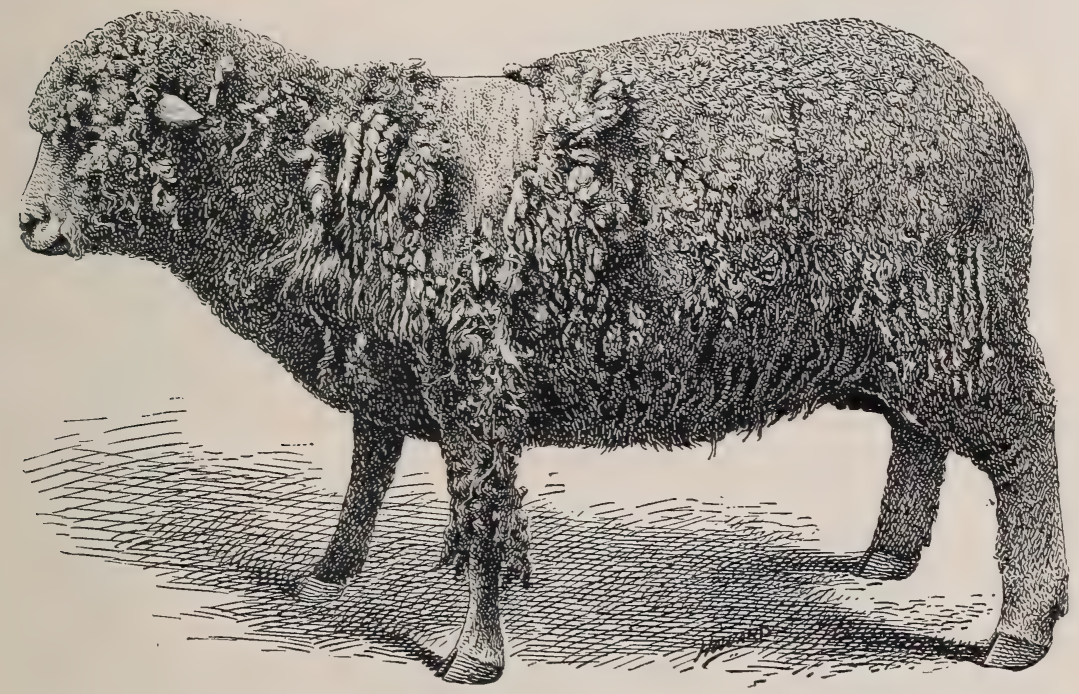

FIG. 258.-A more advanced case of common scab.

wool is very short; whatever the damage at this time, then, it can be only slight, and the small amount of lime left in the wool will surely do but little harm.

In full fleece lime and sulphur will cause more injury. In Australia the deterioration was computed by wool buyers at 17 per cent., although in Cape Colony the Department of Agriculture maintains that if properly prepared, and if only the clear liquid is used, the sediment being thrown away, the official lime-and-sulphur formula will not injure the long wool. The. United States Bureau of Agriculture have found some samples of wool injured by dipping, while on other samples no appreciable effect was noticeable.

If a lime-and-sulphur dip is used, care must be taken to give the 
solution ample time to settle; then only the clear liquid should be used, while the sediment should be discarded. In some of the above tests on samples of wool it was found that the dip with sediment had produced very serious effects, even when no appreciable effects were noticed on samples dipped in the corresponding clear liquid.

Experience has amply demonstrated that a properly made and properly used lime-and-sulphur dip is one of the cheapest and most efficient scab eradicators known, but its use should be confined to flocks in which scab is known to exist, and to shorn sheep, with the exception of very severe cases of scab in unshorn sheep. It should only be used when it can be properly boiled and settled. The use of lime-and-sulphur dips in flocks not known to have scab, especially if the sheep are full fleeced, cannot be recommended; in such cases tobacco, or sulphur and tobacco, is safer and equally good.

All things considered, where it is a choice between sacrificing the weight of sheep and to some extent the colour of the wool by using tobacco and sulphur, and sacrificing the staple of the wool by using lime and sulphur, the owner should not hesitate an instant in selecting tobacco in preference to lime. The loss in weight by using tobacco and sulphur is not much greater than the loss in using lime and sulphur, while the loss in staple is of more importance than a slight discoloration.

Preparation of the mixture.-Take 8 to $11 \mathrm{lbs}$. of unslaked lime, place it in a mortar-box or a kettle or pail of some kind, and add enough water to slake the lime and form a "lime paste" or "lime putty." *

Sift into this lime paste three times as many pounds of flowers of sulphur as of lime, and stir the mixture well.

Be sure to weigh both the lime and the sulphur. Do not trust to measuring them in a bucket or to guessing at the weight.

Place the sulphur-lime paste in a kettle or boiler with about twentyfive to thirty gallons of boiling water, and boil the mixture for two hours at least, stirring the liquid and sediment. The boiling should be continued until the sulphur disappears, or almost disappears, from the surface; the solution is then of a chocolate or liver colour. The longer the solution boils the more the sulphur is dissolved and the less caustic the ooze becomes.

Pour the mixture and sediment into a tub or barrel placed near the

* Many persons prefer to slake the lime to a powder, which is to be sifted and mixed with sifted sulphur. One pint of water will slake $3 \mathrm{lbs}$. of lime if the slaking is performed slowly and carefully. As a rule, however, it is necessary to use more water. This method takes more time and requires more work than the one given above, and does not give any better results. If the boiled solution is allowed to settle the ooze will be equally safe 
dipping vat and provided with a bung-hole about 4 inches from the bottom, and allow ample time (two to three hours, or more if necessary) to settle.

When fully settled draw off the clear liquid into the dipping vat, and add enough water to make a hundred gallons. Under no circumstances should the sediment be used for dipping purposes.

To summarise the position of the United States Department of Agriculture on the lime-and-sulphur dips:-When properly made and

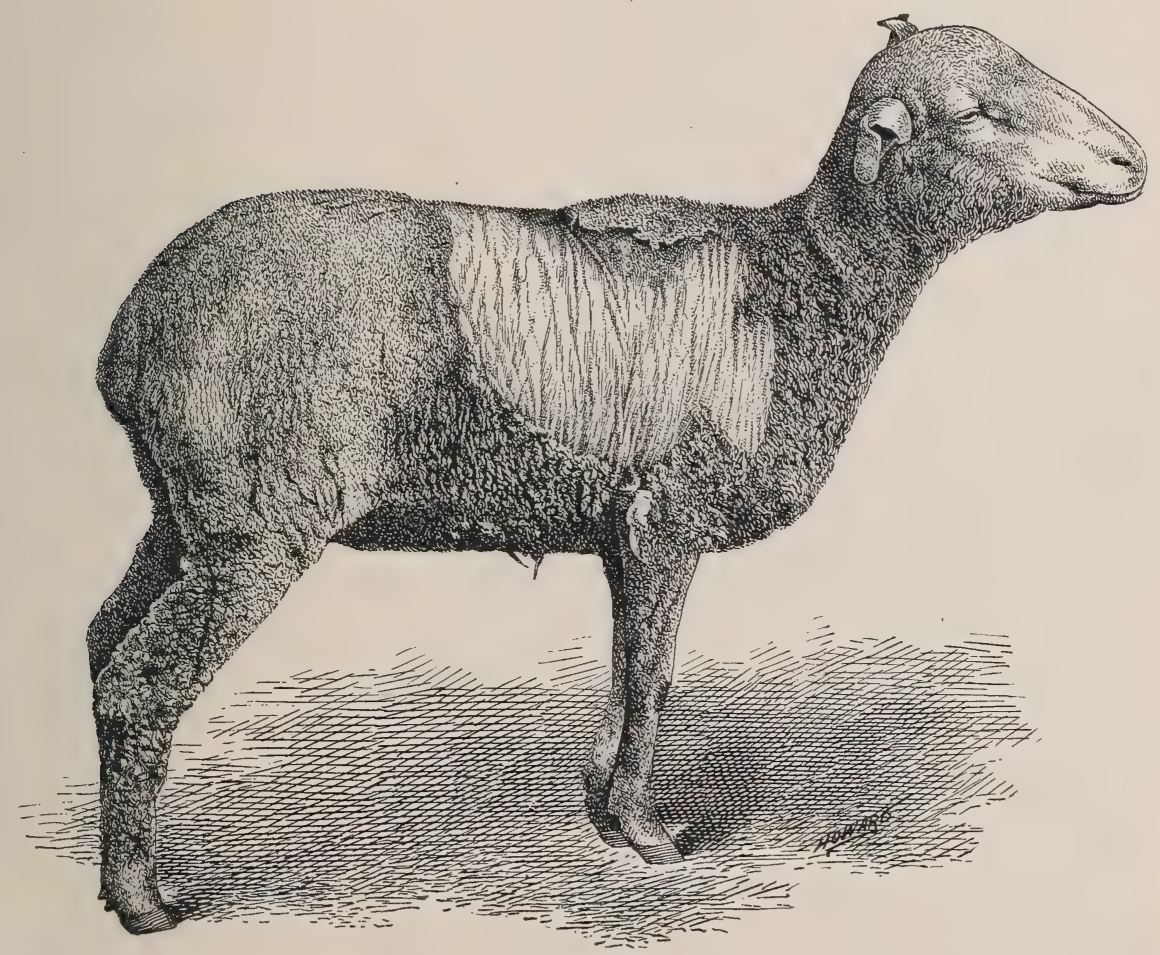

Fig. 259.-A shorn sheep with large bare area due to scab.

properly used these dips are second to none and equalled by few as scab eradicators. There is always some injury to the wool resulting from the use of these dips, but when rroperly made and properly used upon shorn sheep, it is believed that this injury is so slight that it need not be considered; on long wool the injury is greater and seems to vary with different wools, being greater on a fine than on a coarse wool. This injury consists chiefly in a change in the microscopic structure of the fibre, caused by the caustic action of the ooze. When improperly made and improperly used the lime and sulphur dips are both injurious and dangerous, and in these cases the cheapness of the ingredients does not 
justify their use. In case scab exists in a flock and the farmer wishes to eradicate it, he cannot choose a dip which will bring about a more thorough cure than will lime and sulphur (properly made and properly used), although it will be perfectly possible for the farmer to find several other dips which will, when properly used, be nearly or equally as effectual as any lime-and-sulphur dip. There is no dip to which objections cannot be raised.

\section{Arsenical Dips.}

There are both home-made arsenical dips and secret proprietary arsenical dips. It is well to use special precautions with both, because of the danger connected with them. One of the prominent manufacturers of dips, a firm which places on the market both a powder arsenical dip and a liquid non-poisonous dip, recently summarised the evils of arsenical dips in the following remarkable manner :

"The drawbacks to the use of arsenic may be summed up somewhat as follows: (a) Its danger as a deadly poison. (b) Its drying effect on the wool. (c) Its weakening of the fibre of the wool in one particular part near the skin, where it comes in contact with the tender wool roots at the time of dipping. (d) Its not feeding the wool or stimulating the growth, or increasing the weight of the fleece, as good oleaginous dips do. (e) The danger arising from the sheep pasturing, after coming out of the bath, where the wash may possibly have dripped from the fleece, or where showers of rain, after the dipping, have washed the dip out of the fleece upon the pasture. ( $f$ ) Its occasionally throwing sheep off their feed for a few days after dipping, and so prejudicing the condition of the sheep. (g) Its frequent effect upon the skin of the sheep, causing excoriation, blistering, and hardness, which stiffen and injure the animal, sometimes resulting in death."

Although this manufacturer has gone further in his attack upon arsenic than the United States Bureau of Agriculture would have been inclined to do, it must be remarked that when a manufacturer of such a dip cannot speak more highly of the chief ingredient of his compound than this one has done in the above quotations, his remarks tend to discredit dips based upon that ingredient. Bruce, the Chief Inspector of Live Stock for New South Wales, speaking of arsenical dips, says: "Arsenic and arsenic and tobaceo (with fresh runs) cured 9,284 and failed with 9,271."

It may be said, on the other hand, that arsenic really has excellent scab-curing qualities; it enters into the composition of a number of the secret dipping powders, and forms the chief ingredient in one of the oldest secret dips used. This particular dip has been given second place (with some qualifications) among the officially recognised dips in South Africa. 
Formule for arsenical dips.-Finlay Dun recommends the following:-Take 3 lbs. each of arsenic, soda ash (impure sodium carbonate) or pearl ash (impure potassium carbonate), soft soap, and sulphur. A pint or two of naphtha may be added if desired. The ingredients are best dissolved in 10 to 20 gallons of boiling water, and cold water is added to make up 100 gallons. The head of the sheep must, of course, be kept out of the bath.

A mixture highly endorsed by certain parties consists of the following ingredients :

$\begin{array}{lllllll}\text { Commercially pure arsenite of soda } & . & & \ldots & \ldots & 14 & \text { lbs. }\end{array}$

$\begin{array}{llllllll}\text { Ground roll sulphur } \quad \ldots & \ldots & \ldots & \ldots & \ldots & 34 \frac{1}{2} & \text {, }\end{array}$

$\begin{array}{lllllllll}\text { Water } & \ldots & \ldots & \ldots & \ldots & \ldots & \ldots & \ldots & 432 \text { gallons (U.S.) }\end{array}$

The arsenite of soda is thoroughly mixed with the sulphur before being added to the water.

Precautions in use of arsenical mixtures.-Any person using an arsenical dip should bear in mind that he is dealing with a deadly poison. The following precautions should be observed:

(1) Yards into which newly-dipped sheep are to be turned should first be cleared of all green food, hay, and even fresh litter ; if perfectly empty they are still safer. (2) When the dipping is finished, the yard should be cleaned, washed, and swept, and any unused ooze should at once be poured down a drain which will not contaminate food or premises used by any animals. (3) Dipped sheep should remain in an open, exposed place, as on dry ground. (4) Overerowding should be avoided, and every facility given for rapid drying, which is greatly facilitated by selecting fine, clear, dry weather for dipping. (5) On no account should sheep be returned to their grazings until they are dry and all risk of dripping is passed.

The feeling of the United States Bureau of Agriculture towards arsenical dips is shown by the following:

Suggestion as to danger.-The formulæ given above are copied from the writings of men who have had wide experience in dipping, but this Bureau assumes no responsibility for the efficacy of the dips given, or for their correct proportions. Furthermore, as long as efficacious nonpoisonous dips are to be had, we see no necessity for running the risks attendant upon the use of poisonous dips.

\section{Carbolic Dips.}

This class of dips kills the scab mites very quickly, but unfortunately the wash soon leaves the sheep, which is consequently not protected from reinfection in the pastures. If, therefore, a carbolic dip is selected, it is 
well to add flowers of sulphur ( $1 \mathrm{lb}$. to every 6 gallons) as a protection against reinfection.

The advantages of carbolic dips are that they act more rapidly than the tobacco or sulphur dips, and that the prepared carbolic dips are very easily mixed in the bath. They also seem, according to Gillette, to have a greater effect on the eggs of the parasites than either the sulphur or the tobacco dips. The great disadvantages of this class of dips are-first, in some of the proprietary dips, that the farmer is uncertain regarding the strength of material he is using; second, the sheep receive a greater set-back than they do with either lime and sulphur or tobacco.

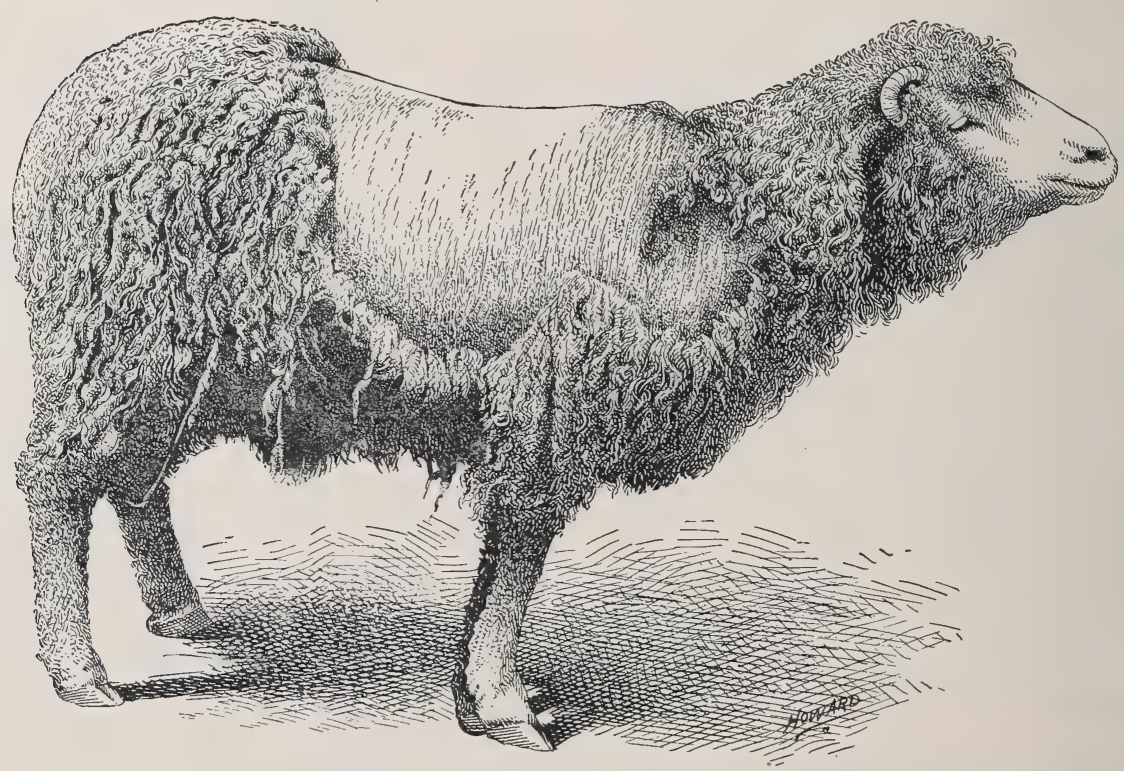

Fig. 260.-An advanced case of common scab.

The United States Bureau of Agriculture is inclined to be extremely conservative in regard to them, and to advise their manufacturers to prepare them in a guaranteed strength with more explicit directions for use than are to be found in the present circulars.

One of the prominent proprietary carbolic dips was formerly recognised as one of the three official dips in New South Wales, but it has now been erased from the list. In Cape Town carbolic dips are not much used, and in the official reports little is said concerning them.

The United States Bureau of Animal Industry gives the following advice as regards dipping:

(1.) Select a dip containing sulphur. If a prepared "dip" is used which does not contain sulphur, it is always safer to add about 
$16 \frac{1}{2} \mathrm{lbs}$. of sifted flowers of sulphur to every 100 gallons of water,

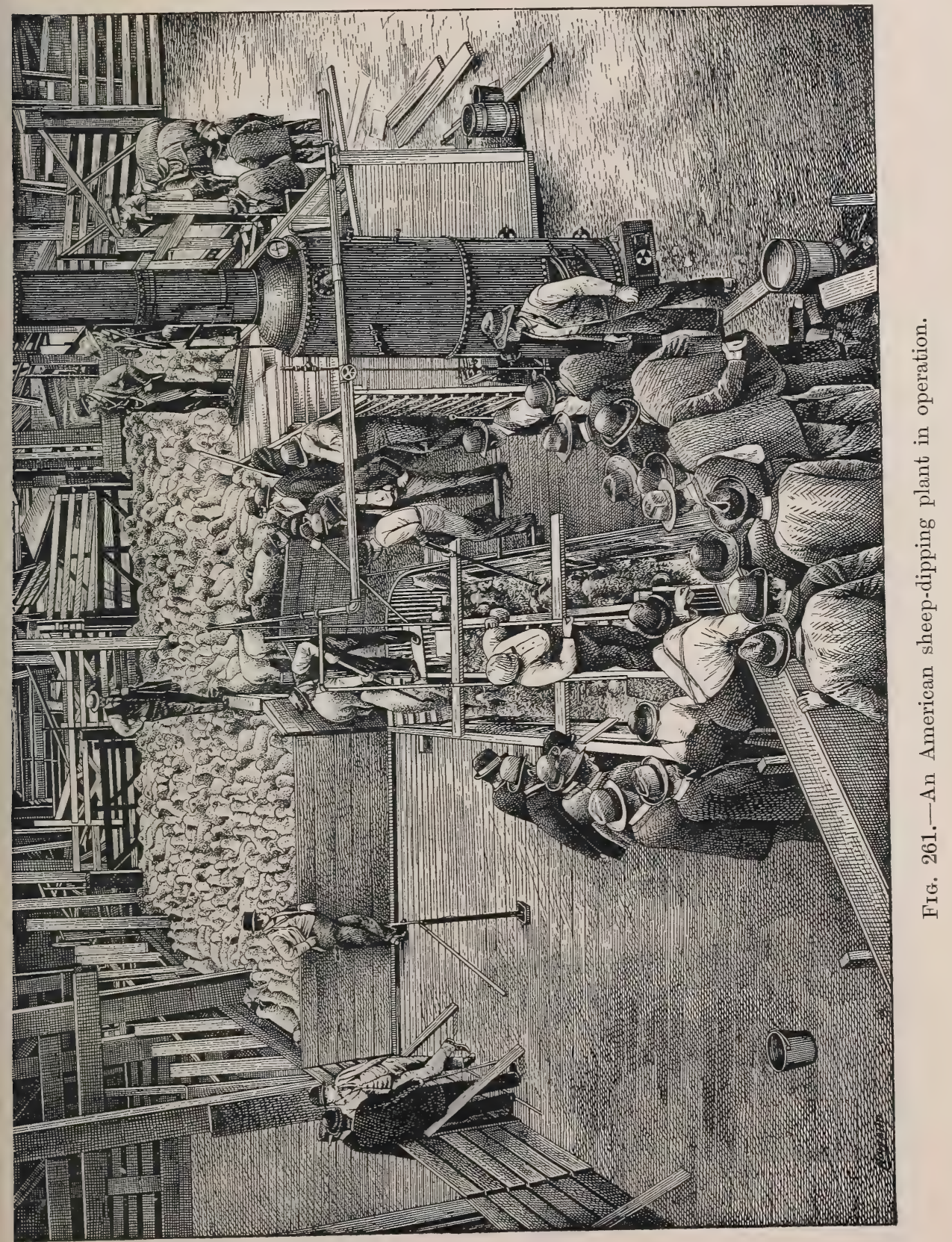

especially if, after dipping, the sheep have to be returned to the old pastures. 
(2.) Shear all the sheep at one time, and immediately after shearing confine them to one half the farm for two to four weeks. Many persons prefer to dip immediately after shearing.

(3.) At the end of this time dip every sheep (and every goat also, if there are any on the farm).

(4.) Ten days later dip the entire flock a second time.

(5.) After the second dipping, place the flock on the portion of the farm from which they have been excluded during the previous four or five weeks.

(6.) Use the dip at a temperature of $100^{\circ}$ to $110^{\circ} \mathrm{Fahr}$.

(7.) Keep each sheep in the dip for two minutes by the watchdo not guess at the time-and duck its head at least once.

(8.) Be careful in dipping rams, as they are more likely to be overcome in the dip than are the ewes.

(9.) Injury may, however, result to pregnant ewes, which must on this account be carefully handled. Some farmers arrange a stage, with sides, to hold the pregnant ewes, which is lowered carefully into the vat, and raised after the proper time.

(10.) In case a patent or proprietary dip, especially an arsenical dip, is used, the directions given on the package should be carried out to the letter.

CHORIOPTIC MANGE, SYMBIOTIC MANGE, FOOT SCAB.

This disease was studied in Germany by Zürn in 1874, and by Schleg in 1877. It has not yet been seen in France.

Causation. The sole cause is the presence of Chorioptes scabiei $(v$. oris). Contagion is favoured by the animals being in poor condition. The disease extends very slowly. Only 2 to 3 per cent. of the animals are affected, and the sufferers are usually those with fine skins.

German shepherds consider this disease to be due to an excessive allowance of salt, because it is most common during the winter, when the sheep are housed. Needless to say, this theory is incorrect.

Symptoms. Sometimes this form of mange attacks the limbs and develops very slowly. It commences about the pasterns, and gradually extends upwards towards the knee or hock. It really advances beyond these points. The parasites are much smaller than those of common scab, and are often overlooked. The sheep stamp their feet and scratch and bite the infected parts, sometimes transferring the disease to the lips and face, where it may persist for a time.

In very old standing cases which have been entirely neglected, it may be met with in the region of the armpit and thigh, the 
CHORIOPTIC MANGE, SYMBIOTIC MANGE, FOOT SCAB.

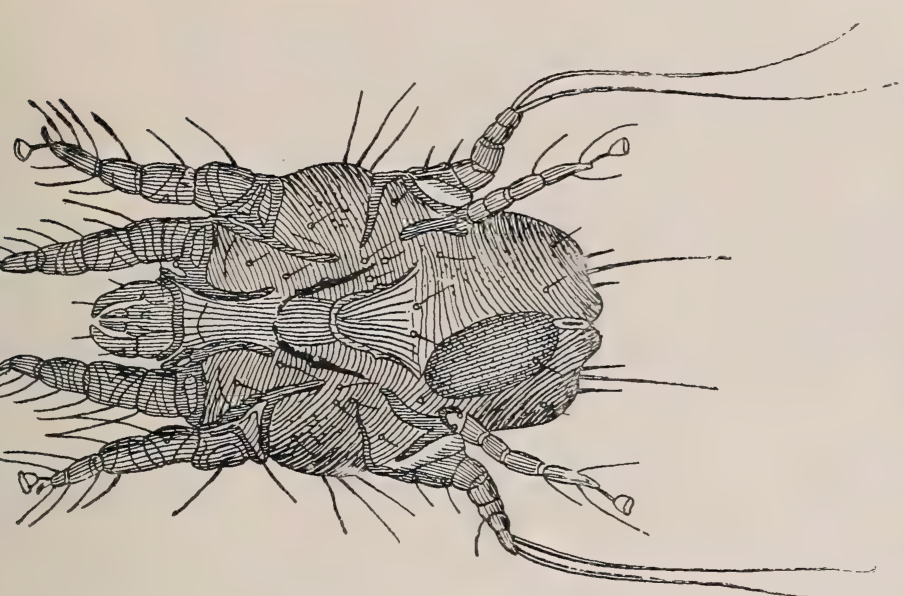



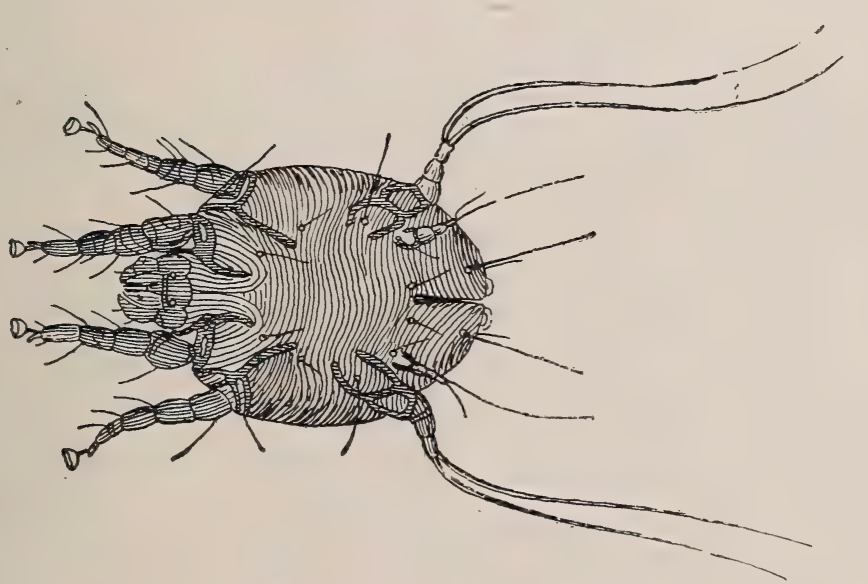

$\frac{0}{0}$

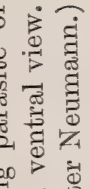

if î

$\frac{0}{3}<$

o

8 응 $x$

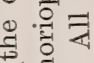

$0{ }^{0}<$

$00 \cdot \frac{9}{5}$

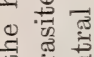

+ สี

की ल0

है।

ర્ల

○

궁

엉 임

ए है

ㅇ - 담

चี

क्षै

ब.

$\doteq \circlearrowleft$

त्ठु है

क्षे

व2

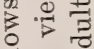

वै तु के

0 进 $\frac{8}{12}$

$50>$ क

$\Leftrightarrow \hat{=}$

ए]

党泀

의

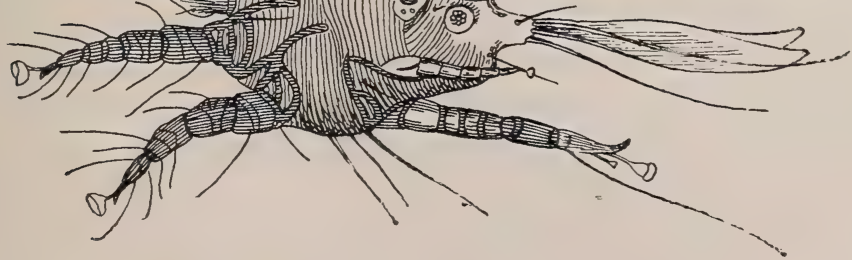

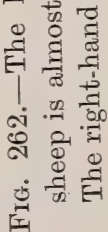


limbs becoming swollen so as to suggest lymphangitis. In the folds of the hock and pastern the thickening of the skin may lead to the formation of yellowish or brownish crusts, according to whether the exudation is merely of a serous character or accompanied by bleeding.

The diagnosis is easy. The parasites are found in the thickness of the crusts, and are readily recognised under the microscope.

The prognosis is not grave, because of the comparative rarity of the disease and its mildly contagious character. This disease, moreover, never attacks the head or body, but remains localised in the lower portions of the limbs.

Treatment. The crusts should be removed by washing or by standing the animals for a time in warm water. Simple cleanliness ofter suffices, but, to save time, some anti-parasitic may be used. The condition is easily cured even without disinfecting the folds.

\section{MANGE IN THE OX.}

Bovine animals may be attacked by three varieties of mange, all of which have long been known.

\section{SARCOPTIC MANGE.}

This is not of any great clinical importance, as it is purely accidental, and only results from the conveyance of sarcoptes from other animals, such as the horse, sheep, dog, goat or cat, to an animal of the bovine species. That the disease does occur, however, is shown by the following excerpt from a report by Professor McFadyean : The animal showed no symptom of skin disease at the time of purchase, but soon after it was brought home it was noticed to be rubbing, and the skin began to assume an unhealthy appearance. When seen in January (four months after purchase) it was rather poor, although it had been in very good condition when sold. Almost the entire skin had become affected, including that of the legs down to the top of the hoofs. There had been extensive loss of hair, and the skin was thick, grey, wrinkled, and dry. At some places it had become thrown into thick folds. Only a few scabs or crusts had formed where the animal had recently rubbed itself. In some scurf scraped from what seemed to be the parts most recently invaded, numbers of acari were without much difficulty found with the microscope.

A good many cases of mange of undetermined character have been reported as occurring among cattle in various parts of England during the last year or two, and in a number of instances the disease was believed to have been contracted during exhibition at a show. It is 
not improbable that some or all of these were cases of this sarcoptic mange, and in view of its very contagious character it is desirable that care should be taken to exclude from shows animals exhibiting any symptom of the disease.

\section{PSOROPTIC MANGE.}

This condition has also been termed dermatodectic mange. This is very rare, and, like the former, of trifling clinical importance.

Causation. It is due to infection with Psoroptes communis ( $v$. boris). Poor condition, want of grooming, bad hygienic surroundings and general neglect, facilitate its spread.

Symptoms. It commences at the base of the neck, in rarer cases at the sides of the neck, along the withers, and at the root of the tail; thence it gradually attacks the croup, loins, back, shoulders, sides of the chest, and finally all the body with the exception of the limbs.

It produces violent itching, the animal continually scratching itself, even causing raw sores. At first the epidermis is elevated in little miliary points, which may be isolated or confluent, and are filled with serosity. This fluid discharges, gluing together the hairs; it then hardens and produces adherent crusts, which increase in number and size. The skin exhibits large numbers of bare, mangy points; these have irregular margins, and are covered with thick, grey, scaly crusts. The psoroptes are found under these crusts.

The skin becomes hard, dry, fissured and cracked, and sometimes forms large folds on the sides of the neck, shoulders and chest.

The influence of the seasons on the development of this disease has been well shown by Gerlach and Muller. The disease commences about the end of autumn, when the animals are stabled. It continues to extend until February, but diminishes as soon as the animals return to the fields in spring. The crusts fall, the hair again grows, and the animal appears to be cured, but the disease again revives during the autumn. The psoroptes lie hidden during the summer round the poll and the horns. The disease seldom attacks animals at grass and in good condition, or those over three years old. Calves, yearlings, and two-year-olds in poor condition suffer most.

When the disease is very extensive, the animals lose condition and may even die.

Diagnosis. Psoroptic mange in the ox may be mistaken for several cutaneous diseases, to which it has a certain resemblance, as for instance phthiriasis and the first stage of ringworm.

These different diseases, however, show their own distinctive symptoms on a careful examination. 
The prognosis only becomes grave when the disease has been neglected and the animals are greatly reduced in condition. When recent, this form of mange can readily be cured by the application of parasiticides.

Treatment. As in dealing with all transmissible diseases, the animals must be isolated and washed with soap, and the diseased parts must be dressed with some parasiticide, such as sulphur ointment. The best preparations are: Benzine and petroleum in equal quantities; concentrated solution of sulphuret of potassium (8 ounces to the quart); Helmerich's ointment; diluted creolin solution; decoctions of tobacco; ointment of pentasulphuret of potassium, and oil of cevadilla.

One or two applications are generally sufficient to effect a cure, and relapses are not likely to take place if the stable is disinfected. In America the disease is common in the West and North-West, where it is treated by the lime-and-sulphur bath recommended for sheep-scab (which see). Large vats are constructed, and the oxen are lowered into these by means of a wooden cage controlled by machinery, which is operated either by a small engine or more frequently by a horse.

The food should be of good quality, for good general health plays a great part in resisting parasitic invasions.

\section{CHORIOPTIC MANGE.}

This disease has also been termed dermatophagic and symbiotic mange.

It was first described in 1835 by Kégélaar, and has been the subject of investigation by Hering, Gerlach, Delafond, and Mégnin.

Causation. It is produced by the Chorioptes bovis, and is transferred with difficulty, even by co-habitation.

Symptoms. This form of mange in the ox does not affect the same parts as in other animals. In the horse, etc., it attacks the limbs, whilst in the bovine species it is usually found at the base of the tail. It causes slight itching. The diseased region becomes covered with numerous little pellicles, the hairs gradually fall, crusts form, and the skin shows deep cracks. When the disease is completely neglected, it may extend to the loins, back, sides of the body and shoulders. It may also affect the perineal region, the inner surface of the thighs, and in fact the whole of the body if the animals are young and in poor condition.

Diagnosis. At the outset it is difficult to distinguish between chorioptic and psoroptic mange, and the use of the microscope is necessary. On a superficial examination chorioptic mange may be mistaken for phthiriasis when the latter attacks the posterior portions of the body, 
particularly the base of the tail, and when it is accompanied by eruptions, loss of hair, and intense itching.

The distinction between the two conditions, however, is extremely easy after an examination of the two parasites.

Prognosis. The disease is of slight gravity, and does not threaten the animal's health unless neglected for so long a time that the parasites invade all parts of the body. In that case the disease may cause anæmia and loss of condition.

The treatment of this form of mange comprises nothing special, it being sufficient to proceed as directed in the previous article.

The stables ought always to be thoroughly disinfected.

\section{MANGE IN THE GOAT.}

The goat suffers from the attacks of sarcoptes, psoroptes, and chorioptes, but up to the present time only two forms of mange have been described, sarcoptic and chorioptic mange.

Psoroptes have only been found about the eve, where the disturbance they produce is comparatively trifling.

\section{SARCOPTIC MANGE.}

This disease was noticed in 1818 in goats imported into France from Thibet.

Henderson published the history of a Persian goat which conveyed sarcoptic mange to men and horses. In 1851 Walraff noticed an epizootic mange which attacked the goats in the Prattigau valley of Switzerland, which was transmitted to men and sheep and which exhibited the clinical characters of sarcoptic mange.

Causation. This disease is due to the presence of Sarcoptes scabiei (v. capre).

It sometimes occurs in an epizootic form, as Walraff's observations show, but it seems specially to attack goats in Asia and Africa.

This mange may be transmitted by the goat to sheep, in which animals it attacks the head and muzzle; it is particularly contagious in sheep having coarse, dry fleeces.

Similarly sarcoptic mange of sheep may be conveyed to the goat, in which animal it extends all over the body.

Symptoms. This mange causes intense itching. It first attacks the head and ears, then the trunk, belly, udder, and limbs. If the disease is neglected it becomes generalised very rapidly, and the animals waste away and die in a very short time.

At the commencement little crusts, which discharge a viscous liquid, are found about the head. The goats rub themselves raw, and, as in D.C. 
facial mange of sheep, there appear dry, scaly, branlike patches. After a time the diseased area extends, the wool falls, and the skin becomes dry, thick and wrinkled. The appearance is exactly like that of sarcoptic mange in sheep, the lower part of the head being seldom invaded. The animals lose condition, waste and die of exhaustion.

Diagnosis. The parasite is readily recognised, and the practitioner, moreover, is often put on his guard by the epizootic character assumed by the disease.

The prognosis is grave. Walraff declared the mortality in Grisons (Switzerland) to be as high as 20 per cent.

The treatment is identical with that of psoroptic mange in sheep. After the animals have been sheared and washed with soap, they should be completely immersed in a bath of the character mentioned in connection with sheep scab. If only one animal is infected, it may be sufficient to dress it repeatedly with an ointment containing some parasiticide.

\section{CHORIOPTIC MANGE.}

This form of mange was noted by Delafond in 1854 at the Jardin des Plantes (Paris) in some angora goats, and by Mollereau in 1889. The disease studied by Delafond had invaded both sides of the neck, the eyes, withers, back, loins, and base of the tail. It was characterised by partial loss of hair, the finer hair falling and the coarser remaining in position.

In Mollereau's case the disease was located in one of the hind pasterns, and assumed the form of a thickened band, which produced an œedematous swelling. The chorioptes were discovered in a thick crust formed by the drying on the hair of the discharge due to their punctures.

Diagnosis. The parasites can easily be found under the crusts, and, once recognised, distinguish the disease from any other infection.

Treatment. Ointments containing some parasiticide and solutions of sulphuret of potassium generally suffice, the disease having little tendency to become generalised.

\section{MANGE IN THE PIG.}

The pig suffers from one variety only of mange. It was described by Viborg, Gurlt and Spinola, who found a sarcopt in the mange of wild boars in 1847. Hertwig and Gerlach made a similar observation some years later. Delafond in 1857 discovered the sarcopt of mange in the pig.

Causation. Sarcoptic mange in the pig is due to the presence of Sarcoptes scabiei (v. suis), although the pig may contract (temporarily) the sarcoptic mange of goats.

Contagion is favoured by poor condition, over-crowding, dirt and bad hygienic surroundings, 
The primitive races of pigs resist the disease better than the improved races. This mange can be conveyed to man and to other animals.

Symptoms. It usually commences about the head, ears, and eyes, and extends to the quarters, internal surface of the thighs, etc. In the early phases it is impossible to discover the little galleries under the epidermis, but closely placed reddish papules may be seen. The active proliferation of the epidermis, together with discharge, causes the formation of dry crusts of a greyish-white, silvery tint, adherent while still thin, easy to detach at a later stage, and sometimes $\frac{3}{8}$ of an inch in thickness. The skin becomes wrinkled, the bristles are shed or loosened in their follicles, and are glued together in little bunches before falling. As these patches extend over the whole surface of the body, the animal appears to be bespattered with dry guano (Muller).

Under the crusts the skin is rough, excoriated, and, about the thorax and abdomen, is indurated, and sometimes measures 1 to $1 \frac{1}{2}$ inches in thickness. In other parts, particularly at the base of the ears, the papillæ are hypertrophied; they become as large as a pea, or even a bean, and, lifting the crusts which cover them, assume the appearance of the warts sometimes found on the cheeks of dogs or the teats of cows. Sarcoptes may be found under these epidermic growths, though in order to obtain them the skin must be scraped until it almost bleeds.

The dimensions of these parasites render them visible to the naked eye. They are the largest variety of the sarcoptinæ, the egg-bearing female being half a millimètre in length. Guzzoni has found in the ears specimens of smaller size.

Mange in pigs develops slowly. When it affects the whole body, it prevents fattening and causes loss of condition.

Diagnosis. This is the only parasitic disease which affects the entire surface of the body and presents these peculiar powdery crusts.

Treatment. All the styes should first be carefully disinfected. Treatment is commenced by vigorously scrubbing the animal with a brush dipped in soap and water, and thus getting rid of the crusts as far as possible.

The animals are afterwards dressed with decoctions of tobacco, with Helmerich's ointment, or the other mixtures above mentioned.

\section{DEMODECIC MANGE.}

This mange is produced by parasites of the family Demodecidæ (Demodex folliculorum), which live in the hair follicles and sebaceous glands of several species of mammals. 
DEMODECIC MANGE IN THE OX.

This was described in 1845 by Gros, and in 1878 was found by Faxon in Illinois in the skins of cows prepared for tanning. It has not been met with in France. The skins examined by Faxon showed numerous rounded enlargements, resulting from dilatation of the hair follicles in the regions of the neck and shoulders.

By pressing on these enlargements a whitish, greasy, sebaceous material was ejected, very rich in demodectes.

\section{DEMODECIC MANGE IN THE GOAT.}

This was first noticed by Niederhaüsern, at the Bern Veterinary College, in a goat which showed little nodosities over different parts of the trunk, varying in size between

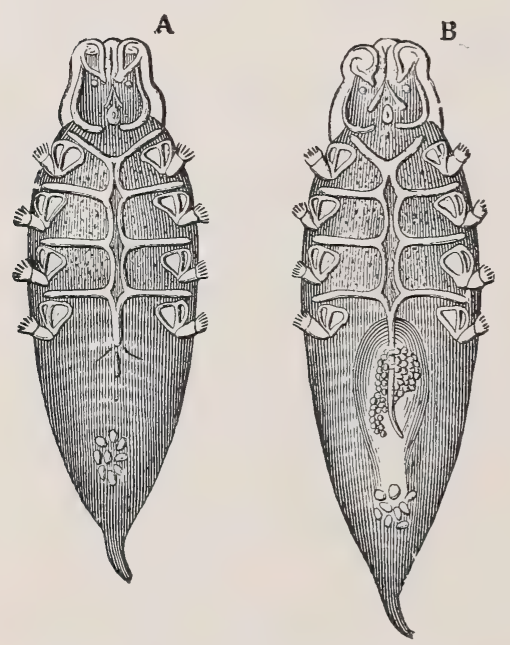

FIG. 263.-Demodex of the pig, magnified 250 diameters. (After Railliet.) that of a pea and that of a hazelnut. By forcibly compressing these enlargements a yellowish-grey semisolid material, containing a considerable number of demodectes, was caused to exude.

In 1885 Nocard and Railliet found the same parasite in a young hegoat; the pustules were spread over the sides and flank. Treatment consists in opening the pustules and dressing them a few times with an antiparasitic lotion.

DEMODECIC MANGE IN THE PIG.

This was well described for the first time by Csokor. It was afterwards seen by Neumann and Lindqvist.

The isolated pustules are of the size of a grain of sand, but when confluent may reach the size of a hazel-nut. They are sometimes dark in colour, often deep-seated, are surrounded by a zone of inflammation, and appear in places where the skin is fine (the groin, neck, belly, etc.). The demodex becomes lodged and multiplies, not in the hair follicles, but in the sebaceous glands. Csokor regarded this disease as contagious; in a herd of one hundred he found twenty-two pigs affected with it. Lindqvist, however, found but one case in a herd of two hundred. 


\section{NON=PSOROPTIC FORMS OF ACARIASIS.}

These are produced in farm animals by arachnide belonging to the families of Trombidiidæ and Ixodidæ.

(1.) The Leptus autumnalis is considered to be the larva of the Trombidium Holosericeum, or silky trombidion. It lives in late summer and autumn, in the grass.

Symptoms. The animals show intense itching, and cannot sleep owing to burning sensations. They continually rub themselves, and thus, secondarily, produce excoriated papules and patches resembling those of eczema. When the papules are very numerous, particularly if the animals are thin-skinned, more or less extensive erythema may be produced.

At the points attacked the skin swells, becomes red, and sometimes even violet, and exhibits irregular, isolated or confluent swellings, $\frac{1}{4}$ to $\frac{3}{8}$ of an inch in diameter.

The parasite most commonly becomes fixed round the lips, the forehead, the cheeks, the sides of the neck, and the extremities.

The diagnosis is easy, the discovery of the parasite removing all doubt.

The condition is of slight importance. The parasites do not live for more than a few days on the animal's skin, so that they only produce temporary disturbance.

Treatment consists in bathing the parts with some lotion, such as 2 to 3 per cent. creolin or 2 per cent. chloral, or in applying mixtures of oil and petroleum, etc.

(2.) Ixodes hexagonus, I. ricinus, and other species of the tick family (Ixodidæ) attack sheep, goats, and oxen in France.

Symptoms. In sheep the ixodidæ usually affix themselves at points where the skin is tender and unprotected by wool, as for instance the thighs, armpit, and upper part of the neck. Their bites produce irritation, followed by an intense burning sensation, and the formation of a red blush round the point bitten.

In the ox the ticks fasten on the neck, behind and within the ears, and also wherever the skin is tender. Until the last few years little importance was attached to their development, but since it has been proved that Rhipicephalus anmulatus is the active factor in desseminating Texas fever, ticks have attracted much attention.

It seems, moreover, to be proved by the researches of Lignières that a form of piroplasmosis exists in France, and it seems possible that the Ixodes ricinus may be a means of propagation.

The diagnosis of acariasis produced by ticks is easy, for the parasites attain large dimensions. 
Prognosis. It is difficult at present to say what importance should be attached to this form of acariasis, but its existence and possible consequences should be noted.

Treatment. Some authors have recommended killing the ticks by touching them with benzine, petroleum, essence of turpentine, etc., but these methods do not always succeed. Applications of concentrated solution of chloral are more effective. When the parasites are so large as to render this possible it is better to remove them by hand, taking care at the same time to remove the rostrum, which, if left in place, might cause more or less suppuration. In countries where ticks are

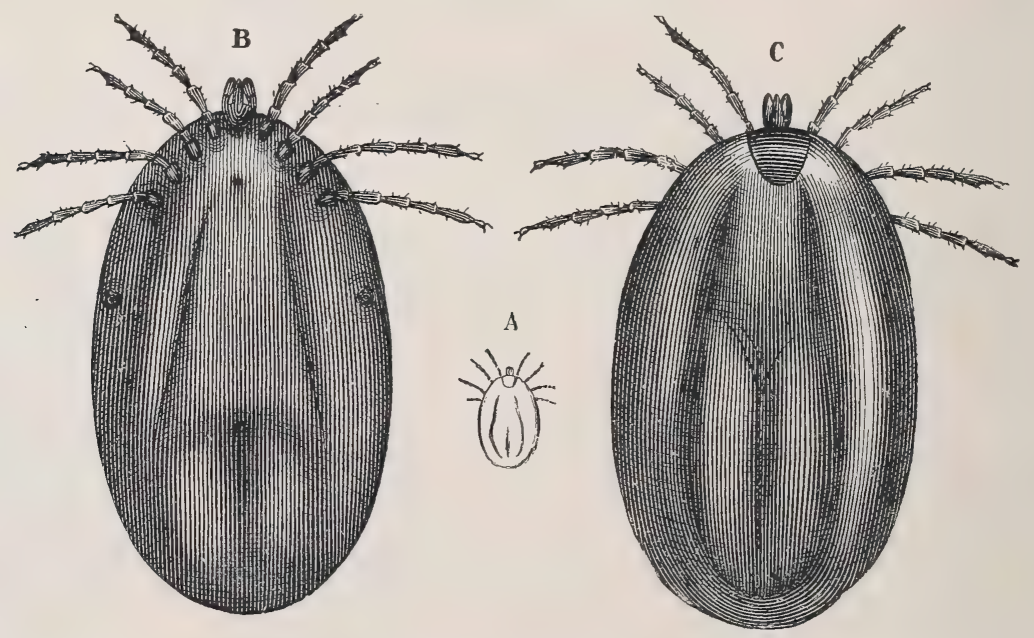

- FIG. 264.-Ixodes ricinus. (After Railliet.) A, Natural size; B, ventral surface ; C, dorsal surface.

numerous and large numbers of cattle are infested, the parasites are destroyed by smearing and dipping.

(The cattle ticks of America are of especial importance in relation to the disease known as Texas fever. Those who wish to study the entomology of this subject are referred to the masterly account and fine coloured illustrations of Salmon and Stiles, "Cattle Ticks of the United States," Ann. Rep. U.S.A. Bureau of Agriculture, 1900, p. 380.)

\section{HYPODERMOSIS IN THE OX (WARBLES),}

Causation. This is a parasitic disease characterised by subcutaneous swellings due to the presence of larvæ of the Hypoderma bovis. The larva is met with throughout Europe. It attains the perfect stage during the summer, from the middle of June to the commencement of September. 
The female deposits her eggs on animals with fine skins. These eggs are elliptical, and provided with a kind of tail of a brownish colour. They soon become converted into larvæ, provided with rows of little spines.

The manner in which the eggs are laid is not exactly understood, nor are we better informed regarding the hatching of the young larvæ. Until recently it was believed that the larva perforated the skin as soon as it quitted the egg, and then penetrated as far as the subcutaneous connective tissue. Recent observations, however, have upset this view. It is probable that this larva, like other gastrophili, is swallowed by animals of the bovine species, and passes through the intestine into the surrounding tissues by a path which is yet unknown, possibly by the blood-vessels, whence it makes its way after a longer or shorter interval into the subcutaneous connective tissue.

Certain recent observations seem to support the latter view, which
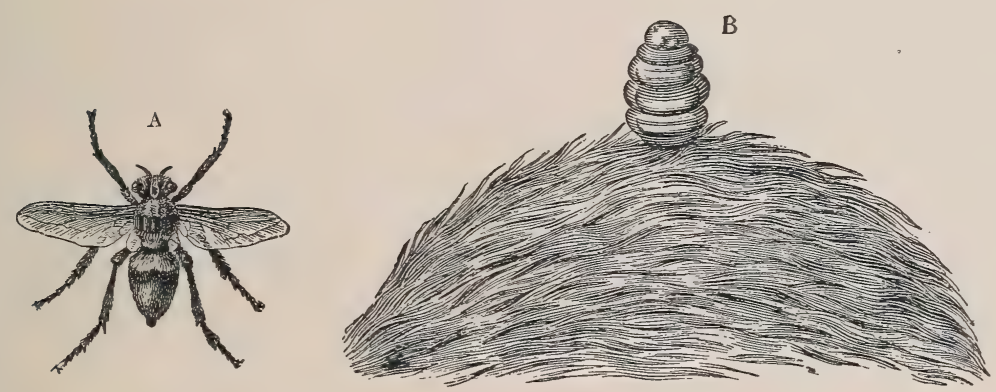

FIG. 265.-A, Hypoderma bovis, natural size. B, larva of the hypoderma escaping from a "warble." (After Railliet.)

is also corroborated by known facts regarding hypodermic myiosis in man. The eggs are laid in summer, and the swellings indicating the presence of the larvæ only appear during the winter. Henrichsen found young larvæ in the fatty tissue situated between the periosteum and spinal dura-mater, between the period from December to March.

Symptoms. Whatever the mode of development of the larvæ, cutaneous swellings appear between the months of February and March on the back, lumbar region, quarters, shoulders and ribs, and, less frequently, over the chest, belly and thighs.

They vary in number. Commonly there are from ten to twenty, and it is only in rare cases that less than four or five are found. As soon as they attain the subcutaneous connective tissue they act as foreign bodies, causing a circumscribed inflammation, and finally suppuration. In this way the so-called "warbles" are produced. 
Each larva is surrounded by a thick wall, forming a cavity, which communicates with the outer air by a minute aperture.

When the swelling is sufficiently advanced the larva may be extricated by pressing with the fingers around the base of the warble. A few days before it leaves its shelter the larva enlarges the little opening by thrusting its last rings into it. Soon after the larva has escaped the discharge of pus ceases, and the skin wound heals.

Diagnosis. The times at which the swellings appear and the larvæ are present render the diagnosis easy.

Prognosis. This is seldom grave, for the larvæ rarely cause death In cases where they are present in very large numbers, however, they may set up purulent infection.

Treatment. No really effective treatment against warbles is known. Curative treatment consists in squeezing out or kiliing the larvæ when in the subcutaneous tissue, but this is practically useless, as the dead larvæ then set up prolonged suppuration. 


\section{CHAPTER III.}

\section{RINGWORM.}

The old term "ringworm" is still used to indicate a well-marked skin disease due to parasitic fungi which grow at the expense of the epidermis. Other names, such as dermatophytis and epidermophytis, have been suggested, to indicate the mode in which the parasite grows. The term dermatomycosis suggests a cutaneous vegetable parasite.

The dermatomycoses of the domestic animals are caused by fungi belonging to six distinct genera:-

Trichophyton (horse, ass, ox, dog, pig); Eidamella (dog); Microsporum (horse, dog); Achorion (dog); Lophophyton (fowl); Oospora (dog).

Ringworm is common in animals of the bovine species, but very rare in other domesticated animals, except, perhaps, the horse. It is caused by the growth of a parasite, Trichophyton mentagrophytes (Robin), of the genus Trichophyton, family Gymnoascea, order Ascomycetes.

The ascosporaceous form of reproduction is still unknown, but the mode of reproduction by conidia is characteristic. In cultures the mycelium is represented by growing filaments branching off at right angles, and by separate superficial aerial reproductive filaments of the conidian form. There is some reason for believing that these fungi may lead a saprophytic as well as a parasitic existence, i.e., that they can exist and multiply apart from the animal body.

Their vitality is marked. Various experimenters have transmitted the disease with crusts kept for eighteen months. Thin declares that in two and a half years the spores had lost all power of germination. They resisted immersion in water for two days, but were dead after eight days. Soft soap and 1 per cent. acetic acid kill them in an hour.

Symptoms. The disease most frequently attacks young animals and milch cows - very rarely adults or old animals. This peculiarity is very difficult to explain.

In calves, ringworm seems specially to attack the head, the 
neigbourhood of the lips, the nostrils and sub-maxillary region, as well as the throat and neck. It assumes the form of circular patches, over which the hair stands erect.

Gruby in 1842 discovered the parasite of tinea tonsurans, or herpes, and thus proved that the cutaneous lesions were not due to any constitutional condition, as was long thought, although dirt, bad hygienic conditions, and crowded stables favoured the spread of ringworm.

Direct contact between healthy and diseased animals and the transport of spores, by combs, brushes, etc., favour contagion. The disease may not only be conveyed from one animal to another of the

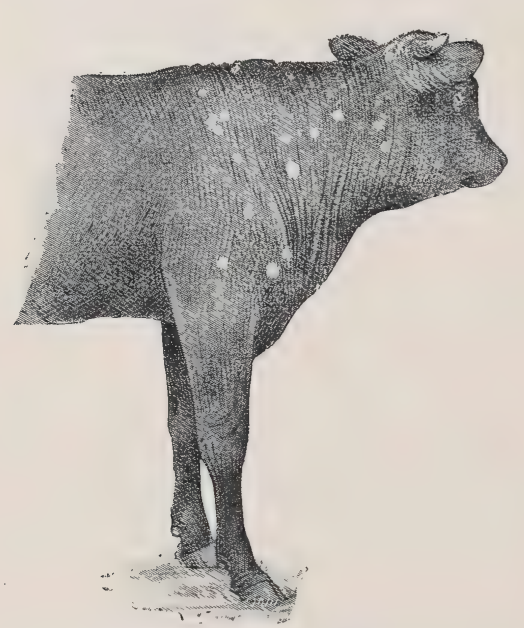

FIG. 266.-Calf suffering from ringworm. same species, but from the ox to man, and, with somewhat greater difficulty, from the ox to the horse. Cases of transmission from the ox to the sheep, pig, and dog have also been recorded.

Megnin in 1890 attempted to prove that all the trichophytons producing ringworm in animals do not belong to the same species, and gave the name of Trichophyton epilans to that usually found in the ox, because it causes absolute loss of the hair by growing in the follicle, whilst he named the parasite found in the horse Trichophyton tonsurans, because it only grows on the surface of the skin and in the thickness of the hair, without causing inflammation of the hair follicle and without invading it.

The epidermis soon undergoes proliferation, and becomes covered with crusts, which adhere to the hairs, gluing them together, and finally causing them to be shed, leaving bare patches the size of a shilling or a florin. The lesion extends in an ever-widening circle, until it attains, perhaps, the dimensions of a five-shilling piece or more.

The affected hairs break off level with the free surface of the skin, rendering the patches more apparent. White hairs are less affected, and some always remain projecting above the crusts, causing the patches, when on a white skin, to retain a certain amount of covering.

At first the crust is closely adherent to the skin, and, if forcibly 
detached, exposes the dermis, which is swollen and bleeding. Gradually the centre becomes detached, whilst the periphery, representing a more recent lesion, continues to adhere. The crusts then rest on a thin layer of pus, and the dermis, whilst still inflamed, is punctuated with numerous minute apertures, representing the roots of the detached hairs. The pus lifts the crust; gradually it dries up and forms superposed layers, which may or may not prove adherent to the parasitic products, and which form a new crust. The latter is purely inflammatory in character, and is left after the fall of the first. It no longer contains any parasites, at least within its deeper layers.

This second crust dries up in its turn, falls away or breaks up, leaving a smooth spot, over which the hairs again appear, either at once, or at least after a short period of desquamation.

The disease is accompanied by well-marked pruritus, more marked at the commencement and towards the end than during the intermediate period, but, nevertheless, much less acute than in scabies.

Ringworm may undergo spontaneous cure in from six weeks to three months. It is more obstinate in calves than in adults, and the want of grooming tends to increase its duration. If it extends over a large part of the body the disease may seriously affect the animal's health, and the cases described by Macorps prove that where pruritus is violent it seriously affects the animal's general condition.

The patches may finally become confluent and the disease extend over the whole of the neck, shoulder and back, or it may attack the entire body, leaving it practically hairless.

When the hair has been shed, the crusts and discharge seen at the outset disappear, and the bare spots are covered with a scaly coating, due to excessive production of epidermic cells.

According to Gerlach, such crusts are thicker where the skin is black, and often exhibit a greyish-white, fibrous, starchy appearance. On unpigmented portions of the skin, which are usually thinner, the crust is less dense, and is slightly yellowish. Gerlach failed to reinoculate the bare patches of skin left after a primary eruption of ringworm. Where the hair had again grown an eruption could again be produced, though it was usually of a feeble character.

In a second form of the disease, the spots may be of very small dimensions. The hair falls away, but there is no exudative inflammation, and no formation of crusts. In this second form the animals simply show characteristic circular bare spots about the head, neck, or shoulders.

Causation. The disease is due to the growth of germs on the skin of animals which are in a receptive condition. The parasite 
thereafter develops in the hairs, the hair follicles and the epidermis, causing lesions which vary according to the species.

At the present time three groups of ringworm are recognised as occurring on animals, Trichophytic, Microsporous, and Favus.

Clinically the trichophytons are divided into the T. ectothrix, which lives outside the hair, and both outside and inside the hair follicles; $T$. endothrix, which penetrates the thickness of the hair itself, rendering it brittle and easily destroyed; and $T$. endo-ectothrix, which both surrounds and invades the hair.

The study of artificial growths of these fungi will probably afford valuable information on the above points. In the ox the particular parasite is invariably the T'richophyton mentagrophytes, whatever may be the characteristics of the clinical lesion.

In France ringworm is particularly common in Auvergne and Normandy, where hygienic precautions are neglected, but cases may also be found throughout the country.

After affecting cows throughout the winter, the disease often disappears in the spring. Throughout the winter cows are kept in dark and often filthy sheds, where the parasite propagates rapidly, whilst in spring they are sent to grass, where the conditions are inimical to contagion.

Diagnosis. The diagnosis of ringworm seldom presents any difficulty. 'The appearance of the lesions (Fig. 266), their particular tendency to spread and contagious character, facilitate the diagnosis. They entirely differ from those of eczema or mange, and should any doubt exist, the slightest microscopic examination is sufficient to dissipate it. In ringworm in the ox the base of the hairs is covered with enormous numbers of spore chains, which do not extend into the depths.

To detect the parasites it is best to shave off a thin fragment of skin from the periphery of the patch, place it on a slide with a drop. or two of 30 per cent. caustic potash solution, and heat it for a few seconds almost to boiling point. By applying a cover glass with firm pressure the epidermal cells are spread out and the parasites can be seen, especially around the roots of the hairs. Sometimes they form little dirty-yellow masses, consisting almost exclusively of spores.

When ringworm has attacked the entire surface of the body, it is much more difficult to distinguish from sebaceous eczema, and a microscopic examination or experimental inoculation may become necessary. Inoculation with ringworm material always succeeds with calves.

Prognosis. Ringworm is not dangerous in itself. In time it may disappear spontaneously, but when it extends over the whole body it 
may be dangerous. Owing to their bare condition the animals easily catch cold, while the epidermic proliferation is very great, and makes great demands on the animal's bodily powers. Under such circumstances it is better to slaughter early.

Spontaneous recovery from small lesions may occur in two or three months.

Treatment. The patients should be isolated, and any brushes, combs, etc., with which they have been in contact must be disinfected.

The diseased areas should then be dressed with some fatty substance, to soften the crusts and enable them to be removed without injury.

Dressings are useless unless these crusts have been removed, for the spores are always in the deepest recesses and in the follicles, so that the drugs employed never come in contact with them.

Once the skin is cleansed, numerous chemical substances may be utilised, the solutions being applied for several days in succession. Amongst them may be mentioned tincture of iodine, oil of cade, 10 per cent. solution of sulphate of iron, and solution of perchloride of iron. Such drugs, however, must not be used for a long time, as they all attack the skin to some extent.

When the patches are small and well defined the following mixture proves very efficacious:-

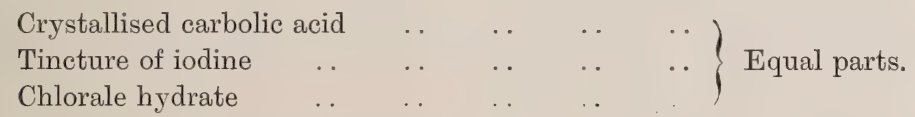

Two or three applications generally prove successful.

Despite the natural activity of the spores, which are capable of germination after three months' desiccation, some medicines are useful, although it is necessary, in order to judge of their effects, to wait for the renewal of the skin, hair follicles, and hair.

Where the disease is generalised this form of treatment is scarcely practicable, or at least it becomes more difficult.

Nevertheless, by dressing with soft soap the disease may be cured in a few months. The soap should remain on the skin for some hours and then be washed off, after which dressings of creolin, lysol, or chloral solution, etc., should be employed.

\section{RINGWORM IN THE SHEEP, GOAT, AND PIG.}

Little information regarding the dermatomycoses of the sheep, goat, and pig is available. Allowing for modifications due to the nature 
of the coat, the symptoms of recorded cases seem to indicate a close relationship with ringworm in the horse and ox.

In sheep suffering from ringworm, the wool is at first matted into small irregular tufts, which grow larger and more numerous. The coat appears felted together at various points. The neck, chest, shoulders and back exhibit crustaceous patches covered with branlike epidermal scales, and the animals suffer from marked pruritus, which causes them to rub and injure the coat.

Ringworm is very obstinate in the goat.

Two pigs described by Siedamgrotzky showed irregular, rounded patches, due to trichophyton, from 1 inch to 2 inches in diameter, reddish in tint, without exudation, but covered with abundant scales.

In the pig ringworm attacks the croup, sides of the chest, flanks and sides of the abdomen, but is commonest on the back and outside of the quarters. It forms red isolated patches, from 1 to 2 inches in diameter, covered with miliary vesicles, which in turn are replaced by brown crusts. The bristles remain unchanged, and are not shed or broken. There is no pruritus. Contagion from pig to pig occurs readily. The disease may be conveyed from oxen to pigs through the medium of litter removed from the cowsheds to the piggery.

Gerlach was unable to inoculate sheep or pigs with ringworm from the ox. Perroncito mentions a case of contagion from the ox to a lamb. Schindelka has seen sheep contract ringworm as a result of confinement to sheds previously occupied by oxen suffering from the disease.

Siedamgrotzky successfully inoculated two pigs and two sheep with ringworm from the horse and a goat with the bovine form of the disease. The two pigs inoculated two others by contact. Contagion from the goat to the ox was noted in the canton of Zürich in 1852.

Fuller particulars on these heads will be found in a series of articles by Neumann in the Revue Vétérinaire, January to June, 1905.

In 1876 Laillier communicated to a French medical society a letter written by Lespiau describing an endemic of trichophyton disease in the cantons of Céret and Arles-sur-Tech. Thirty-four persons, including twenty-eight children, were affected. A dog was first attacked and seems to have inoculated a pig, which in these districts often lives with the human family. The pig inoculated the human beings. A moist season appears to have favoured the development of the disease. The parts principally attacked were the head, eyebrows, cheeks, and neighbourhood of the genital organs. The subjects showed considerable pruritus. 


\section{CHAPTER IV.}

\section{WARTS IN OXEN.}

WARTS are cutaneous tumours, real papillomata, which most commonly attack young animals such as heifers. As a rule they are pedunculated, smooth, wrinkled or deeply cracked on the surface, but in some cases they are sessile.

Causation. The cause is difficult to ascertain. It has been referred to the growth of bacteria (Bacterium porri) in the superficial layers of the skin. It is at least certain that warts can be transmitted by inoculation or through the medium of cutaneous injuries.

Symptoms. On their first appearance warts consist in hypertrophy of the cutaneous papillæ, which become covered with layers of actively growing epidermis and end by projecting above the general surface. The lesions may remain isolated, or they may become confluent or unite at their base. This form is fairly common, the warts attaining the size of a man's fist or more.

The disease attacks the most tender portions of the skin, such as that covering the udder, internal surface of the thighs, lower abdominal wall, region of the elbow, posterior surface of the ears, etc. In rarer cases warts may be seen on the limbs.

When they extend over a considerable surface they become infected, suppurate and give rise to various complications, the most serious being pyæmia. The patients lose condition and value.

Diagnosis. The diagnosis is easy. It has been proved that warts are contagious, not only as between animal and animal, but as between animal and man.

Prognosis. Warts are not dangerous to life, but they diminish the value of the animals, particularly that of milch cows when the teats are affected.

Treatment. Leaving out of account internal medication with calcined magnesia, many medicines that are still recommended are of comparatively little value.

Peuch and Cruzel recommend friction with oil of cade. Repeated cauterisation with nitric acid is declared to give good results by destroying the new tissue. 
These modes of treatment, however, are impracticable in dealing with large multiple lesions, nor does the elastic ligature give much better results.

Total removal with the scissors or bistoury, or simply tearing out by hand, is preferable to any other course. Troublesome bleeding may follow, but is rarely of great importance. It usually stops in a few minutes, even where small arteries of the size of several millimètres in diameter have been divided. As a measure of precaution, however, the little wounds may be touched with the red-hot blade of the thermo-cautery.

The écraseur is rarely required. The smaller warts are generally sessile, and can be removed with a bistoury or a sharp curette. The

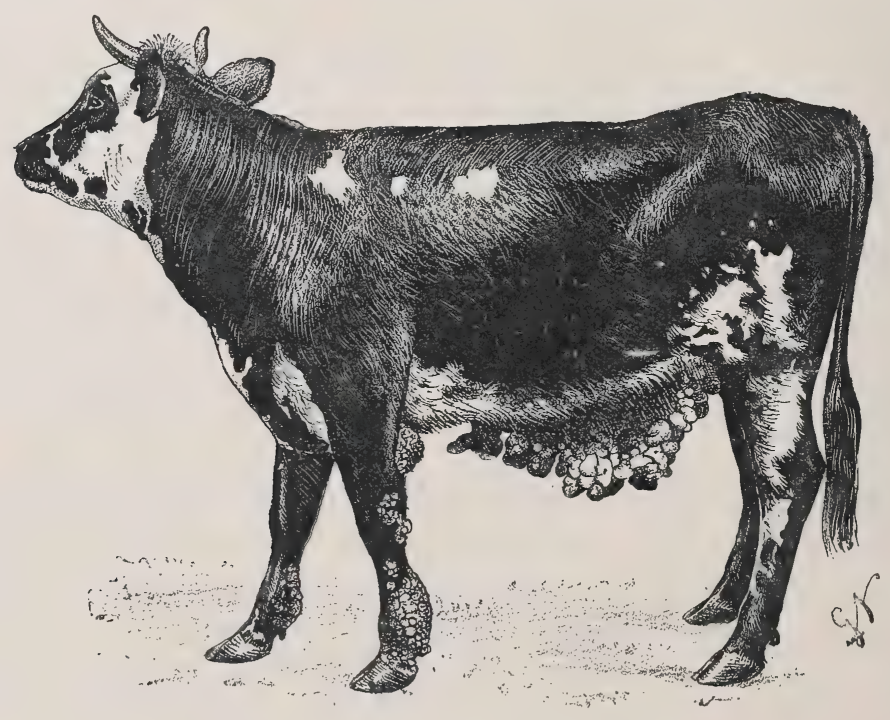

FIG. 267.

removal of those about the udder requires considerable precaution to avoid injuring the teats.

All the growths are usually removed at one operation; Moussu has thus taken away 30 lbs. weight without the slightest ill-effect. After the wounds have been washed with an antiseptic, the raw surfaces are powdered with a mixture of equal parts of boric acid, tannin, and calcined alum; cicatrisation occurs in a few days.

\section{URTICARIA IN THE PIG.}

This disease usually attacks pigs during the spring or summer, producing characteristic cutaneous lesions, which, however, are of a 
benign character. It seems to arise from some form of alimentary intoxication.

Symptoms. At first the dominant symptoms point to disturbance of digestion. The appetite is lost, and the bowels may be confined or there may be diarrhœa. This is sometimes accompanied by vomiting, and by fever.

The symptoms may develop fully in from six to twelve hours; sometimes the skin is covered with slightly prominent reddish patches, varying in size from $\frac{1}{3}$ of an inch to $1 \frac{1}{4}$ inches in diameter.

The patches may also become confluent and form large, irregular red or violet flattened swellings, sensitive to the touch and spread over the upper and lateral portions of the body. Only in exceptional cases is there any oozing of blood.

In favourable cases recovery takes place in forty-eight hours, and even in grave cases in from five to six days.

Diagnosis. It is sometimes very difficult to distinguish this condition from swine erysipelas, particularly in the first few cases, although the congested or hæmorrhagic patches occupy different positions.

The prognosis is usually favourable.

Treatment. The disease being unquestionably of digestive origin, the animals should be kept without food and receive repeated doses of mild purgatives according to their age and condition, sulphate of soda, 4 to 12 drachms, or calomel, $1 \frac{1}{2}$ to 8 grains. Recovery is rapid.

\section{SCLERODERMA.}

This term is applied to a disease characterised by thickening and hardening of the skin. Up to now it has been described only in the pig, and principally in male animals or old animals of either sex.

The symptoms are difficult to detect, and in many cases are only discovered after slaughter. Without any change in external appearance, the skin becomes thick, hard and sclerosed over limited or extensive areas, and is thus transformed into hard, rigid, inextensible and inelastic plates, sometimes as much as 1 to 2 inches in thickness. The change usually commences about the dorsal region, and extends irregularly towards the chest and sometimes towards the limbs.

The patient thus becomes imprisoned in a kind of cuirass, which interferes with its movements and causes unaccountable stiffness. Palpation of the skin gives the impression of a piece of wood, for it is hard and resistant over the affected regions, whilst over the belly, inner surface of the thighs, and region of the elbow, it retains its usual pliability.

D.C.

U U 
The patient exhibits no other symptoms, the principal functions of the body appearing to be properly performed. There is no fever, but in time the animals lose condition and waste away.

Causation. This disease is also well known in man, but no general agreement exists regarding its nature. Some refer it to disturbance of the thyroideal function, though scleroderma is quite different to myxœdema. Others attribute it to changes in the cutaneous blood-vessels, others, again, to peripheral neuritis accompanied by atrophic disturbance. Nothing, however, is proved.

The apparent lesions are limited to hypertrophic sclerosis of the dermis, with progressive atrophy of the layers of subcutaneous adipose tissue.

The diagnosis is comparatively easy.

The prognosis is grave, because it is never known how rapidly the disease may develop.

No method of treatment being known, the animals should at once be slaughtered. 


\section{CHAPTER V.}

\section{SUBCUTANEOUS EMPHYSEMA.}

Br subcutaneous emphysema is meant the condition produced by the entrance of air or gas into the subcutaneous and interstitial connective tissue. Emphysema may remain localised or it may become generalised, according to the nature and extent of the lesion which causes it, and the points where emphysema is developed. Subcutaneous emphysema is common in the sheep and ox.

Symptoms. Sometimes the symptoms of subcutaneous emphysema are extremely well defined. They consist in the presence of diffuse or limited crepitant swellings which may appear at various points-in the flank or the entrance to the chest; more rarely in the region of the elbow, etc.

The limits of crepitation may be ascertained by palpation, while percussion produces a peculiar abnormal sound. The subcutaneous tissue and very often the interstitial tissue appear as though blown out.

Emphysema may be generalised. Such an accident is rare, but may occur in the ox as well as in the sheep and goat.

Provided the emphysema remains confined to the subcutaneous tissue, the animals are not necessarily in danger. Where, however, it also extends to the interstitial tissue, and particularly if the cause to which it is due continues, death may result in a very short time. This occurs, for example, when the emphysema extends into the mediastinum, and thus gains the pleura and lung.

The symptoms of emphysema are then complicated with respiratory and circulatory disturbance and with signs of asphyxia.

Causation. Subcutaneous emphysema may be produced in many different ways.

If, for example, in puncturing the rumen the canula be carelessly withdrawn so that the skin is slightly separated from the subjacent tissues, gas may pass from the rumen into the channel produced by the instrument. It then becomes distributed throughout the subcutaneous tissue, and if the cutaneous opening is displaced its escape is confined to the connective and interstitial tissues in the region of 
the flank. Diffuse suppuration may then be set up in these parts, and may extend far beyond them.

In the ox emphysema rarely becomes generalised, but in the sheep and goat extension is more common; the patients perish of intoxication, caused by reabsorption of septic gases.

Under other circumstances emphysema may be due to an injury in some region where the connective tissue is loose and pliable, as for instance the region of the elbow, the internal surface of the shoulder, or the fold of the flank. Every time the animals move the tissues are displaced, and air being drawn in, it is imprisoned by the valvelike action of the injured part and gradually finds its way into the subcutaneous tissue.

Accidental injuries to the trachea, particularly injuries prođuced by dogs biting sheep or goats, are always accompanied by local emphysema, unless the wounds in the skin and trachea correspond, which rarely happens. At every respiration a portion of the air expelled passes into the peritracheal tissue, from which it gradually invades neighbouring parts, and may attain the mediastinum, etc. The injured animal thus inflates its own tissues and dies from asphyxia.

The open lesions due to pulmonary echinococcosis, and the accidents associated with pneumo-thorax, tuberculous caverns and abscesses, or pulmonary emphyserna may become points of departure for local, general, interstitial or subcutaneous emphysema.

The diagnosis of accidental emphysema presents no difficulty, for the local swellings can only be mistaken for those of blackquarter. In the latter disease, however, fever is a constant accompaniment, whilst in simple emphysema it is absent.

Nevertheless, it is well to remember the possibility of complications due to compression, asphyxia, and even intoxication.

The prognosis may be very hopeful or very grave. Everything depends on the primary lesion, and it is therefore important that the practitioner should know how to interpret the course of affairs.

Treatment. In slight cases the best method is to immobilise the parts and await developments, but in grave cases, for instance where the trachea is much injured, the animal should at once be slaughtered.

Scarification, cutaneous incisions; and massage were formerly recommended as a means of aiding the escape of gas accumulated in the tissues. Such methods, however, are useless, and have the disadvantage of causing numerous suppurating wounds.

Provided the initial wounds are not seriously infected and the animals are kept quiet, in a well-ventilated place, the gas gradually becomes reabsorbed, and healing may take place in a fortnight or three weeks. 


\section{SECTION IX.}

\section{DISEASES OF THE EYES.}

IN domesticated animals, apart from parasitic diseases, the diseases of the eye which particularly deserve description and offer a special clinical interest are very few. These are the diseases that affect the globe of the eye or the organs annexed to it.

\section{FOREIGN BODIES.}

Foreign bodies become lodged on the internal surface of the eyelids, in the folds of the conjunctiva, in the thickness of the cornea, and sometimes, though rarely, in the anterior chamber, the lens, or the vitreous humour. They include particles of grit or dust, the awns and glumes of grain, etc.

The eyes are half closed and the conjunctiva is swollen, whilst the eye weeps and the animals dread the light.

Diagnosis. This is somewhat difficult, for the parts rapidly become very sensitive, and the animals violently resist examination. When the foreign body penetrates the anterior chamber or the lens, it produces suppuration or traumatic cataract.

Before anything can be done it is often necessary to render the parts anæsthetic by instilling a few drops of cocaine solution into the eye.

The foreign body may then be discovered by close observation. If the pain is very intense, and great resistance is offered to opening the eye, the practitioner may confine himself to passing a soft camelhair brush saturated with cocaine solution over the surface of the eye and into the conjunctival sacs. The brush loosens, and often removes, the offending body. In the absence of a camel-hair brush, the little finger, covered with a piece of fine linen, may be used.

Where the parts cannot be touched owing to the resistance of the animal, lukewarm solutions of antiseptics such as boric acid may be occasionally injected into the eye by means of a syringe, but care must be taken to prevent the animal injuring itself against the syringe by sudden movements. 


\section{CONJUNCTIVITIS AND KERATITIS.}

Inflammation of the conjunctiva and inflammation of the cornea almost always occur together, and reciprocally induce one another when of a certain degree of intensity. They may be simple, that is to say, produced by simple causes, or they may be specific, and of a contagious character.

Simple inflammation is caused by the action of cold, draughts, dust, or mechanical injuries. Specific inflammations, the nature of which is still little understood, occur in the ox and goat. They are very contagious, and may successively attack all the animals of a herd.

The symptoms of acute and specific inflammation differ very little. They comprise congestion, lachrymation, chemosis, a certain amount of suppuration, and sometimes superficial ulceration of the cornea. The patients suffer very acute pain, avoid the light, present all the symptoms of photophobia, and are affected with spasm of the orbicularis muscle.

In simple cases these symptoms frequently disappear, provided the byres are kept clean and astringent eye-washes are applied.

In contagious keratitis, however, the cornea may suppurate and even become perforated after a few weeks.

Treatment. The chief object of treatment under any circumstances must be to insure the most perfect cleanliness both of the globe of the eye and the conjunctival sacs.

The eye must, therefore, be irrigated with lukewarm water, the stream being injected beneath the lids. Each irrigation is followed by the use of an anodyne and astringent eye-wash containing borate of soda or sulphate of zinc, combined if necessary with cocaine.

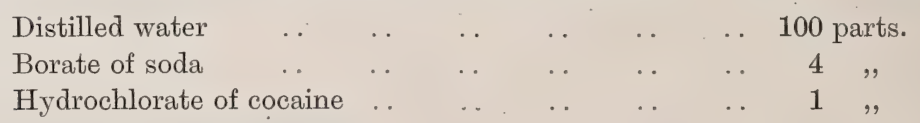

But saturated solution of boric acid is simple, and no less effective.

In contagious keratitis the eye lotion may contain 2 to 3 per cent. of nitrate of silver, the excess of silver being neutralised by washing out with a weak solution of common salt. After three or four applications this should be changed for a saturated solution of boric acid.

\section{VERMINOUS CONJUNCTIVITIS.}

This form of conjunctivitis, described by Rodes in 1819, is due to the presence of the Filaria lachrymalis, which varies in length between $\frac{3}{8}$ of an inch and 1 inch. 
Like ophthalmia, the disease attacks cattle reared in wet localities.

Symptoms. The symptoms are those of acute conjunctivitis, and consist first of lachrymation, then of injection of the blood-vessels of the conjunctiva, together with swelling of the eyelids and photophobia. The animals keep the eye closed, and display extreme sensitiveness. Here again cocaine proves of value.

Examination is rather difficult, the worms being sometimes displaced towards the cornea or membrana nictitans, although more commonly they remain hidden in the folds of the mucous membrane towards the point where the membrana nictitans is inserted. It is, therefore, necessary to thoroughly expose the folds of the mucous membrane in order to discover them.

In time conjunctivitis becomes complicated with diffuse ulceration, keratitis, and sometimes with ophthalmia and suppuration of the eye.

Diagnosis. The diagnosis is rather troublesome, and cocaine is of great assistance.

Prognosis. This is rather grave.

Treatment. The object of treatment is the complete removal of the parasites. This can sometimes be attained by means of the finger, a pair of forceps, or a very clean feather.

Treatment is completed by injecting an antiseptic and antiparasitic eye lotion for several days, lest some of the worms should remain hidden in the folds of the mucous membrane. One per cent. creolin or 1 in 2,000 sublimate solution may be used.

If in some exceptional case it is difficult to remove the parasites, they may be got rid of by injections or free irrigation. The stream of liquid, pointed in different directions, distends the mucous membrane and washes away the foreign bodies on its surface.

\section{VERMINOUS OPHTHALMIA OF THE OX.}

This ophthalmia is due to the presence of a small worm, $\frac{2}{3}$ of an inch to $1 \frac{1}{4}$ inches in length, which has been regarded as the larval form of the Filaria cervina of the serous cavities.

It is very common in animals which are kept permanently in lowlying meadows particularly in some parts of France, as for instance in Normandy, in the departments of the Sarthe and the Mayenne. Not infrequently it occurs as an epizootic, and is then regarded as a contagious ophthalmia. Verminous ophthalmia occurs chiefly during the spring and autumn.

Symptoms. The disease is accompanied by lachrymation, signs of conjunctivitis, and fear of light. Very soon the media of the eye become turbid, the sclerotic and cornea are injected, and finally exhibit marked opalescence. 
On examination the eye appears extremely sensitive; in fact, it can scarcely be touched unless cocaine solution is previously applied.

The parasites, two or three as a rule, but in exceptional cases from five to seven in number, are seen rolled up within the anterior chamber of the eye. A week after the beginning of the attack, however, they begin to move about, and are then found close behind the cornea, upon the lens, or suspended in the aqueous humour.

The irritation produced sets up inflammation of Descemet's membrane and the cornea, together with iritis, and, secondly, keratitis and changes in the lens.

Unless treatment is adopted verminous ophthalmia inevitably ends in cataract.

Diagnosis. Diagnosis is always uncertain on account of the difficulty of examination. When the cornea is very opaque examination necessarily gives a negative result.

The prognosis is grave.

Treatment. Eye lotions containing tincture of aloes, creolin, corrosive sublimate, etc., have been suggested, but are practically useless, because they can have no action on a parasite enclosed within the globe of the eye. The most logical treatment consists in aseptic puncture of the anterior chamber of the eye towards its lower border with a cataract needle.

The escaping liquid carries with it the parasites, and recovery is then only a matter of time, provided the wound does not become inflamed. The great danger consists in inflammation and suppuration of the eye. This, however, can be avoided by antisepsis and by applying a surgical wool dressing, which can be left in place for a few days. 


\section{SECTION $\mathrm{X}$.}

\section{INFECTIOUS DISEASES.}

\section{COW-POX-VACCINIA.}

The name cow-pox, or vaccinia, is employed to describe a special disease which in animals of the bovine species is characterised by the development of pustules at points where the skin is fine, and more particularly the mammary region.

It can be conveyed both to man and the domestic animals.

This disease has been known from time immemorial, and it would appear that first of all in the East and later in England it was a general belief that its attacks rendered human beings proof against small-pox. Medical men, it must be admitted, long regarded this belief as a popular delusion, as is proved by their continuing to practise inoculation with true small-pox material.

Jenner in 1770 was the first to declare the truth of this popular opinion, and by his wise foresight to confer on humanity one of the most beneficent discoveries ever made, although the weight of modern opinion is in favour of the identity of cow-pox and human variola. Having observed that milkmaids who happened to have small cuts or sores about the hands sometimes contracted the disease in a mild form, and that they did not afterwards suffer from small-pox, he was struck with the advantages consequent on such a discovery, and having proved the possibility of inoculating human beings artificially, he immediately formulated the principles of vaccination. A child eight years of age was vaccinated with cow-pox, and afterwards inoculated with pus from a small-pox patient. It contracted vaccinia in consequence of the first inoculation, but entirely resisted the attempt to inoculate it with small-pox. Vaccination had been discovered.

Jenner furthermore proved that cow-pox was transmissible from cow to cow and from man to man, but it seemed to him that the original disease was to be sought elsewhere, and that the pustular affection originated primarily with the horse. The horse is sometimes the subject of a pustular disease called horse-pox; this disease when inoculated in man confers immunity against small-pox, just as does cow-pox, and Jenner believed that the disease did not attack 
cows unless they had been accidentally inoculated through the medium of the people about the farm. Unfortunately, he named the pustular disease of the horse which he had studied "sore heels," and for a long time all those who busied themselves with the question of vaccine confounded "sore heels" with a number of different diseases, although as early as 1802 Loy had experimentally proved that so-called "grease" (in reality horse-pox) was transmissible by inoculation to the cow, in which it produced cow-pox.

Loy's "grease" and Jenner's "sore heels" only represent forms of horse-pox, but for more than fifty years the origin of vaccine was sought in grease, lymphangitis, and other diseases which attack the extremities of horses' limbs. Pételard (1845-1868) rediscovered and redescribed horse-pox and proved its transmissibility to man; Lafosse and U. Leblane discovered it in an epizooty which broke out at Rieumes; and Bouley in 1862 furnished a synthetical description of it under the designation of horse-pox. He shows that horse-pox is always a pustular disease, but that it may sometimes appear in the form of a discrete eruption around the lips and nostrils, sometimes of an eruption limited to the pasterns or extremities of the limbs when inoculation has been effected in this region, sometimes of lymphangitis, and sometimes of a more or less confluent and generalised eruption.

Symptoms. The disease as discovered and described by Jenner was soon rediscovered and redescribed on all sides-by Sacco in Italy, Hering in Germany, etc.

The pustular eruption usually appears on the udder in the case of cows, and on the muzzle, nose, and lips in that of calves. In exceptional cases the eruption may become generalised.

The pustules are round or slightly elliptical, and are preceded by the appearance of red congested patches, followed by infiltration and thickening of the skin.

The pustule is moderately prominent, and after some days there is exudation at its centre, transforming it into a vesico-pustule. The exuded liquid collects under the thickened layer of epidermis, which it raises, and on examination it appears as a white or transparent little central patch, with a thin grey periphery surrounded by a reddish inflammatory zone. This liquid becomes thicker and the pustule is flattened at its centre, then, towards the eighth or ninth day, the pustule is ruptured, owing to tearing of the epidermic patch. The vaccine thus escapes.

In what is termed spontaneous vaccinia the udder is covered with a varying number of pustules, usually in different stages of development. Some are very small, whilst others have attained the size of sixpence and are already in course of cicatrisation. 
When cow-pox is accidental or the result of inoculation, the eruption occurs exactly at the point of inoculation, whether the latter has been through an abrasion, a puncture, an incision, or any other skin injury, and the eruption may assume the most varied appearances, according to the nature of the primary lesion, although the mode in which the pustules themselves form never varies. Pustules experimentally produced by puncturing the parts may be taken as a type of inoculation. On the day following the operation nothing abnormal appears. On the third day there is a slight swelling around the point punctured, and this increases until the fifth day, when there is exudation, which converts the primary lesion into a vesico-pustule. On the sixth day the vesico-pustule becomes umbilicated at its centre, the exudation is abundant, and already vaccine might be collected.

This may be termed the period of crisis; the appearances are most characteristic. During the following days the vesicle is ruptured; the discharge continues from the ninth to the twelfth day, when the pustule diminishes in size and dries up. After the fifteenth day crusts of a brownish colour form; these separate between the twentieth and twenty-fifth days, leaving hard whitish-looking cicatrices, which permanently remain.

Moderate itching accompanies the development of the eruption, the principal functions are not disturbed, and fever only appears in the event of the eruption becoming confluent or extending over a large area. When the eruption is generalised the pustules or vesico-pustules are found mostly in the region of the elbow, the lower border of the neck, the flank and the inner surface of the limbs. They present exactly the same appearance as the pustules on the surface of the udder, but, being covered with hair, are less open to inspection.

In certain rare cases the eruption extends to the perineum and lips of the vulva. Signs of inflammation then develop on one or both sides, the tissues display œdematous infiltration and disseminated or confluent pustules. The lymphatic glands and vessels in the neighbourhood of the pustules are always swollen.

Causation. Cow-pox, or vaccinia, is a virulent disease transmissible by accidental or intentional inoculation. The discharge from the vesicopustules and the crusts which afterwards cover them are virulent, and inoculation can be performed by simply scratching the skin. A first attack confers prolonged and sometimes perfect immunity, the operation being successful if only one pustule develops.

The disease is transmitted to healthy animals by milkers, by calves in sucking, or by the conveyance in whatsoever form of virulent material to sores or cuts.

The nature of the parasitic or microbic agent which produces the 
disease is still unknown. Some investigators have described intracellular parasites, others extra-cellular parasites, others, again, blood parasites, etc., but the exact cause has always eluded research.

It is, however, known that filtration of vaccine, pure or diluted, through porcelain removes the active material, which remains in the residue arrested by the filter.

Prolonged exposure to a temperature above $104^{\circ}$ Fahr. $\left(40^{\circ}\right.$ C.) greatly diminishes the activity of the vaccine. Simple desiccation has no action. Warming to $140^{\circ} \mathrm{Fahr}$. $\left(60^{\circ} \mathrm{C}\right.$.) for fifteen minutes also destroys its action completely.

These facts explain why it has always been so difficult to preserve and cultivate vaccine in tropical regions.

Mixed with equal parts of neutral glycerine, the virulent material preserves its activity unimpaired for from six to eight months.

The disease develops equally in man, the horse, the ox, the buffalo, the goat, and the camel. Its development is less typical in the pig, sheep, dog, and rabbit. Young animals are best adapted for its cultivation.

The blood and serum of animals suffering from cow-pox possess immunising properties, but only when administered in very large doses, say from 6 to $12 \mathrm{lbs}$. of blood, or $\frac{1}{2}$ to $1 \mathrm{lb}$. of serum.

The curative action of this serum against small-pox is comparatively trifling.

The diagnosis of cow-pox is not very difficult.

At first the disease might be mistaken for false cow-pox, the eruptions of foot-and-mouth disease, or gangrenous coryza.

In false cow-pox, the nature of which is also little understood, although it is known to be contagious and is regarded by some as true vaccinia, the pustules are smaller and thinner, while the vesicle is more developed, and the disease runs a more rapid course.

In foot-and-mouth disease the eruptions are of the nature of vesicles or bullæ, not pustules. The eruption occurs in twenty-four hours or less, and can only be mistaken for vaccinia during the period of desiccation and the formation of crusts.

Finally, as regards gangrenous coryza, the hardened pustules do not produce vesicles.

The prognosis is generally favourable. The disease runs its course within relatively fixed periods, according to the development of the pustules, and recovery occurs without complications.

Treatment. No curative treatment can be laid down, the development of the disease being perfectly regular and tending to recovery. Simple hygienic precautions and cleanliness are sufficient to avoid complications due to suppuration. 
COW-POX AND HUMAN VARIOLA-PREPARATION OF VACCINE.

Time and experience having proved that inoculation with cow-pox or vaccinia protected human beings against small-pox, the question arose as to the connection between the two diseases, whether or not they were identical and whether vaccinia in the bovine animal might not merely represent an alternative form of small-pox. The importance of the question will at once be understood by bearing in mind the danger to which human beings would be exposed by vaccination with small-pox virus unmodified by passage through the calf.

Nevertheless, at the present time the opinion of the early writers appears to prevail, and the theory of identity is accepted by the great majority of scientific men. The reason why experimenters in the second group came to believe in duality is that the method of inoculation chosen (by puncture) was not entirely reliable. The inoculations proved too slight, and it is only after inoculation by scarification or incision that typical eruptions can be reproduced in series.

Preparation of vaccine. Whether cow-pox and small-pox are or are not identical, the benefits resulting from vaccination are none the less real, and it is to be hoped that vaccination and revaccination will soon be made obligatory in all countries. We should then no longer have to deplore those epidemics of small-pox which periodically cause consternation in large cities and colonies.

The preparation of vaccine has been the subject of such minute care in every country that neglect of vaccination is astonishing. The material is obtained from calves or cows. In France the vaccine is prepared from animals of five to eight months old, free from disease. The old system of inoculation by puncture has been completely abandoned, the yield being insufficient, scarifications or incisions being now employed.

The animal is secured or, better still, laid down on a suitable table, and is shaven over a sufficient surface. The inoculations are made on the sides of the chest, over the thorax or elsewhere, but preferably over the flank and thorax, as being most readily accessible. The region of operation is rendered aseptic as far as possible, and scarified in lines about 1 to 2 inches in length, the lines of one horizontal row alternating with those in the next. It is imperative that the slight bleeding which may result should entirely cease before inoculation is attempted.

The scratches are inoculated with the purest vaccine obtainable, preferably with glycerinated pulp which has been kept for six weeks or two months. From the third day the lines of inoculation become prominent, and an indurated longitudinal swelling, with all the characteristics of a pustule, soon projects above the neighbouring portions 
of skin. On the fifth day exudation commences, and from the sixth to the seventh day a large quantity of vaccine lymph may be collected. The line of inoculation appears slightly umbilicated and surrounded by a greywish-white zone and a hard peripheral swelling.

Vaccine may be collected from the fifth day in summer to the eighth day in winter.

The inoculated area having been cleansed with boiled water and carefully dried, the little crusts covering the inoculation wounds are loosened and the wounds themselves gently scraped with a special curette of small size. The exuded liquid is very active.

The base of each swelling is then grasped in a little special clamp, which acts like a pressure forceps and causes the discharge of a further large quantity of active vaccine lymph. All the material thus obtained is mixed; an equal quantity of neutral glycerine is added, the whole is finely triturated, passed through a cloth, and stored in little sterilised glașs tubes, which are hermetically sealed.

The vaccine thus prepared retains its activity for from five to eight months, if kept from the action of heat and light. Accidental germs which may have developed in the wounds and thus gained entrance to the vaccine gradually lose their activity. After from forty to sixty days the vaccine may be regarded as absolutely pure and incapable of producing accidental suppuration, as sometimes occurs when fresh vaccine is employed.

The old electuaries, dried vaccines, vaccine pastes, etc., have been almost entirely given up, the above method always yielding a pure and active vaccine. Vaccination with calf lymph should always be preferred to vaccination from arm to arm, in view of possible transmission of grave disease, such as syphilis.

\section{TETANUS,}

Tetanus is a disease characterised by tonic contraction of the muscles of one or more limbs or of all the muscles of the body.

Causation. It is due to the growth of Nicolaier's bacillus in some part of the body (in accidental wounds, in the uterine cavity after parturition, etc.), and the contraction of muscles is due to toxins (elaborated by the microbe), which have a selective affinity for the nervous centres.

These toxins, secreted by bacilli localised in wounds, are absorbed and carried away by the lymphatic and vascular channels and distributed throughout the body. They seem chiefly to affect the cells of the central nervous system. Infection is due to microbes capable of living as saprophytes outside the animal body. 
Nicolaier's bacillus assumes the form of a straight rod, one end of which is swollen by the presence of a spore. It is anerobic, grows in a number of different media, most rapidly at a temperature of $100^{\circ}$ to $102^{\circ}$ Fahr. ( $38^{\circ}$ to $39^{\circ}$ C.), and stains well by Gram's method.

Though quite common in the horse, tetanus is rare in other domestic animals.

In the ox it may result either from mechanical injuries, suppurating sores, or surgical operations. In the cow, goat, and sheep it sometimes assumes the form of a true enzooty after parturition if the byres, etc., are not disinfected. In male animals it principally follows castration by one of the cutting methods, and in lambs is seen after amputation of the tail. A large number of animals belonging to one flock may be affected, and Moussu has known two-thirds of a given number of castrated lambs to die of tetanus.

Despite the sensitiveness of domesticated animals to tetanic infection they may all be protected, either by injections of cultures, or by gradually increasing injections of specific toxin. The latter, however, are more efficacious when modified by the addition of terchloride of iodine or of iodine water. The blood of immunised subjects rapidly acquires antitoxic powers, which may be greatly increased for the purpose of obtaining anti-tetanic serum.

The symptoms of tetanus are the same in all species.

In the first stage the animal appears stiff, walks in a jerky way and holds the head high, with the ears pricked. The eyes are slightly withdrawn into the orbits, and the animal shows marked general excitability.

In the second stage there are muscular contractions, together with trismus, stiffness of the neck, limbs and vertebral column, spasm of the spinal muscles and muscles of the limbs (tonic contraction), and the animal has a peculiar, staring look.

In the third stage mastication becomes difficult or impossible, respiration is impeded, and the animal suffers from spontaneous attacks of muscular contraction or from attacks due to external stimulation (noises, sudden movements, changes from darkness to light, etc.).

In the fourth stage the animal is liable to fall, asphyxia threatens, and death occurs from respiratory syncope.

Recovery is quite exceptional in the sheep, goat, and ox; death usually occurs between the second and sixth days.

Diagnosis. Tetanus being less cummon in the ox, sheep, and goat than in the horse, the diagnosis is not so easy in these animals, but as it develops under different conditions, and as it usually attacks several animals in one byre or fold, the diagnosis is rarely very 
difficult. At the worst some hesitation may be felt at first, the condition being mistaken for disease of the brain.

The prognosis is extremely grave.

The treatment is, above all, of a preventive nature, investigation having proved that injections of anti-tetanic serum, before the first appearance of tetanus, are invariably effectual.

If, therefore, one case of tetanus appears in a byre at calving time or in a flock at the season when the lambs are castrated, no hesitation should be felt in preventively inoculating all the castrated animals and the cows which have calved. The quantities required are, for a cow 10 cc. (about 3 fluid drachms) and for a sheep 5 cc. of anti-tetanic serum.

This treatment, however, should be supplemented by general hygienic precautions and internal treatment, such as irrigation and disinfection of the parts affected.

Curative treatment has little chance of success. Experiments have also proved that when the first symptoms of tetanus appear, anti-tetanic serum is powerless to prevent the development of the disease. Nevertheless, as its gravity is in direct ratio to the quantity of toxin absorbed, and as the degree of this absorption depends on the length of time that the place remains infected, the first thing to be done is to disinfect and, in certain cases, curette the wounds which are believed to be the source of mischief. Although antiseptics have little action on Nicolaïer's bacillus, they may be used. Solutions of iodine appear most active, both as regards ordinary wounds and infection of the uterus.

General tonics, diuretics, and lukewarm gruels can be given. Unfortunately the patients are often unable to swallow them. In such cases both liquids and medicines may be directly introduced into the rumen by puncturing the parts with a trocar and canula, the latter being left in position.

Intravenous injections of large quantities of normal salt solution are also of considerable value, 4 to 6 quarts per day for an ox and 20 to 40 fluid ounces per day for a sheep.

\section{ACTINOMYCOSIS.}

Actinomycosis is a disease produced by a fungus belonging to the group of oomycetes (Actinomyces bovis) which develops in the depths of living tissues in man and the ox, producing grave and sometimes incurable lesions, most commonly in and about the jaws.

Actinomycosis is very common in America, and is also met with in all parts of Europe.

Symptoms. The disease assumes many different clinical forms, but 
it more frequently attacks some parts of the body than others, and by far the greater number of cases occur in the ox.

ACTINOMYCOSIS OF THE MAXILLA.

Actinomycosis of the maxilla attacks young animals, and its usual seat is in the molar region, although occasionally it affects the incisors.

The earliest symptoms consist in swelling of the bone, which may be overlooked if within the mouth, but the outline of the jaw soon becomes deformed, generally in the middle region of the row of molars. Somewhat tender and firm to the touch at first, the tumour gradually increases in size, invades the deeper regions of the skin, and displays fluctuation at one or two points, followed by abscess formation. The pus discharged maỹ be white, creamy, and inoffensive, but the cavity of the abscess shows no tendency to cicatrise, and the opening through which the pus has escaped is transformed into a fistula. From this moment the pus discharge is of a greyish, sanious nature, and contains a greater

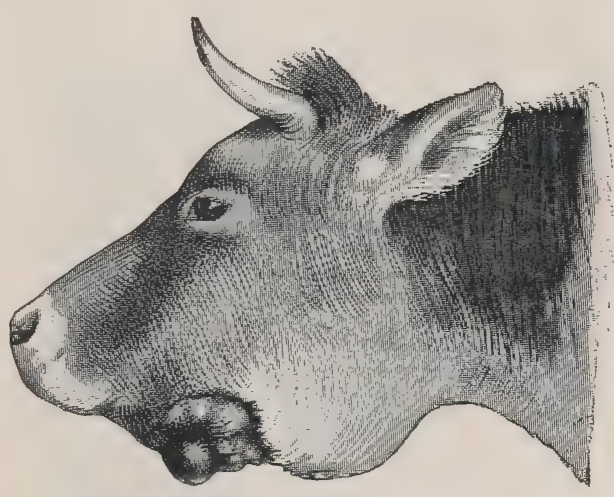

FIG. 268.-Actinomycosis of the jaw. or less number of little yellowish grains. It soon acquires an offensive odour, and the fistulous opening is surrounded by exuberant granulations, forming a fungoid mass.

The neighbouring tissues become hardened and lose their sensitiveness, the jaw becomes completely deformed, and a condition is set up which the old writers considered as true cancer of the jaw or maxillary osteosarcoma (Fig. 268).

A probe passed into the fistula penetrates deeply, usually into the thickness of the jaw itself, and however carefully manipulated injures the diseased tissues and causes free bleeding.

If neglected, these lesions become steadily worse, mastication is more difficult, being possible only on the healthy side, and the animals lose condition and eventually die of exhaustion. The external lesion, represented by the fungoid mass, increases in size, assumes a blackish colour, and discharges an offensive liquid. Portions of it undergo mortification and give off a characteristic and extremely fœetid odour. The molars become loose and in some cases fall out, but development is D.C. 
usually slow, and some weeks or months elapse before this stage is reached.

When the disease attacks the region of the incisors the symptoms are much sooner apparent, and treatment is much easier. The parasitic invasion results from an injury to the jaw caused by shedding of the milk teeth. Swelling of the body of the jaw thrusts the lower lip downwards, interferes with the prehension of food, and calls for prompt treatment. The disease is rarely allowed to attain the degree of development shown in Figs. 269 and 270.

As in the preceding instance, the animals die of exhaustion unless relieved.

For reasons difficult to explain, but probably because inoculation

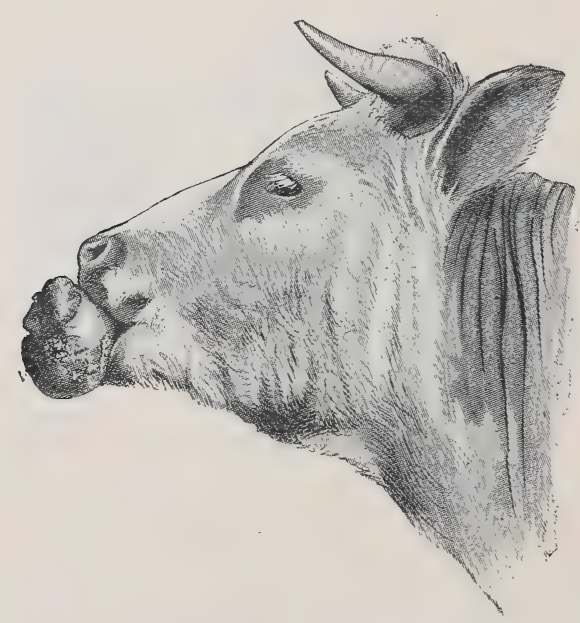

FIG. 269.-Actinomycosis in the region of the incisors.

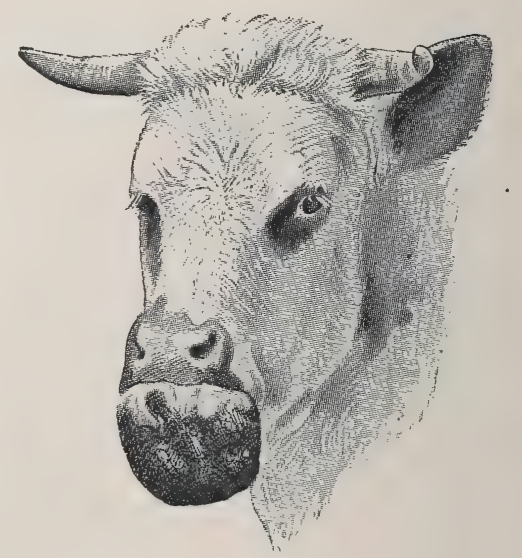

FIG. 270.-Actinomycosis in the region of the incisors.

is less easy, actinomycosis is much rarer in the upper than in the lower jaw. The disease develops exactly as above described, but shows much less tendency to external ulceration. It invades the maxillary sinus and the region of the palate, and fistulæ are found opening into the buccal cavity, while at the same time the region of the forehead is often deformed.

\section{ACTINOMYCOSIS OF THE TONGUE.}

Actinomycosis attacks the tongue apart from any lesion of the jaws, and produces what is commonly called "wooden tongue."

The disease develops in the tongue itself, generally in the submucous zone, and causes chronic interstitial inflammation, infiltration 
of the connective tissue, and, in time, changes in the muscular structures themselves.

The tongue shows progressive hypertrophy, and becomes hard, sensitive, rigid, and incapable of free movement. As a result the patients first have difficulty in grasping food, then in swallowing their saliva, which dribbles from the mouth, and finally are quite unable to feed themselves.

The tongue is enlarged and indurated, and fills the entire cavity of the mouth. Sometimes it projects beyond the incisors, excoriated and bleeding. On passing the hand into the mouth it is found that the surface is covered with little yellowish or red ulcerated nodules, varying in size from that of a large pin's head to that of a lentil.

In eating, the animals seize food between the lips and lift the head high, so as to allow the food to fall between the rows of molars. The motion is very similar to that of a fowl drinking.

ACTINOMYCOSIS OF THE PHARYNX, PAROTID GLANDS AND NECK.

Actinomycosis may sometimes leave the mouth and tongue unaffected and attack the pharynx, from which it extends in the direction of

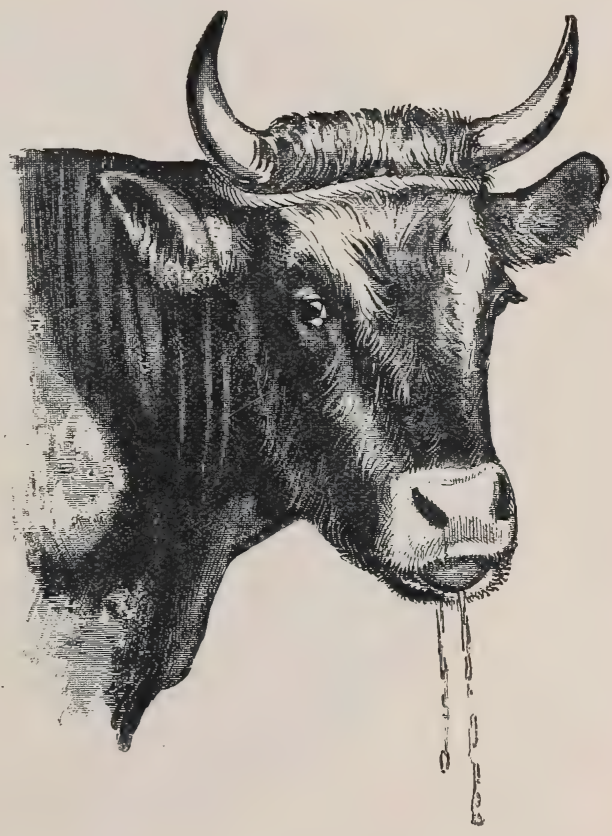

FIG. 271-Actinomycosis of the tongue. the parotid glands and external surface of the neck. In these cases, however, the inoculations are more localised than when the surface of the tongue is attacked, and the lesions consist of vegetations, polypi, or actinomycomata.

The growths develop on the posterior pillars of the fauces, on the sides of the pharynx, or near the entrance to the œsophagus. They interfere with swallowing, and produce symptoms which are easy to detect and interpret.

The lesions may also affect deeper-seated tissues and produce growths in the parotid or subparotid region, or lead to the development of fistulæ in the region of the neck. Most fistulæ, however, in 
this region are due to specific inoculation of external injuries. Fistulæ originating in the parotid region and in the upper part of the neck usually resemble in appearance the maxillary fistulæ. The external fungoid growth, however, is less exuberant, suppuration is less abundant, and the surrounding induration less extensive.

Yarious localisations. Although the disease generally attacks the mouth, tongue or pharynx, it may invade the osophagus, rumen,

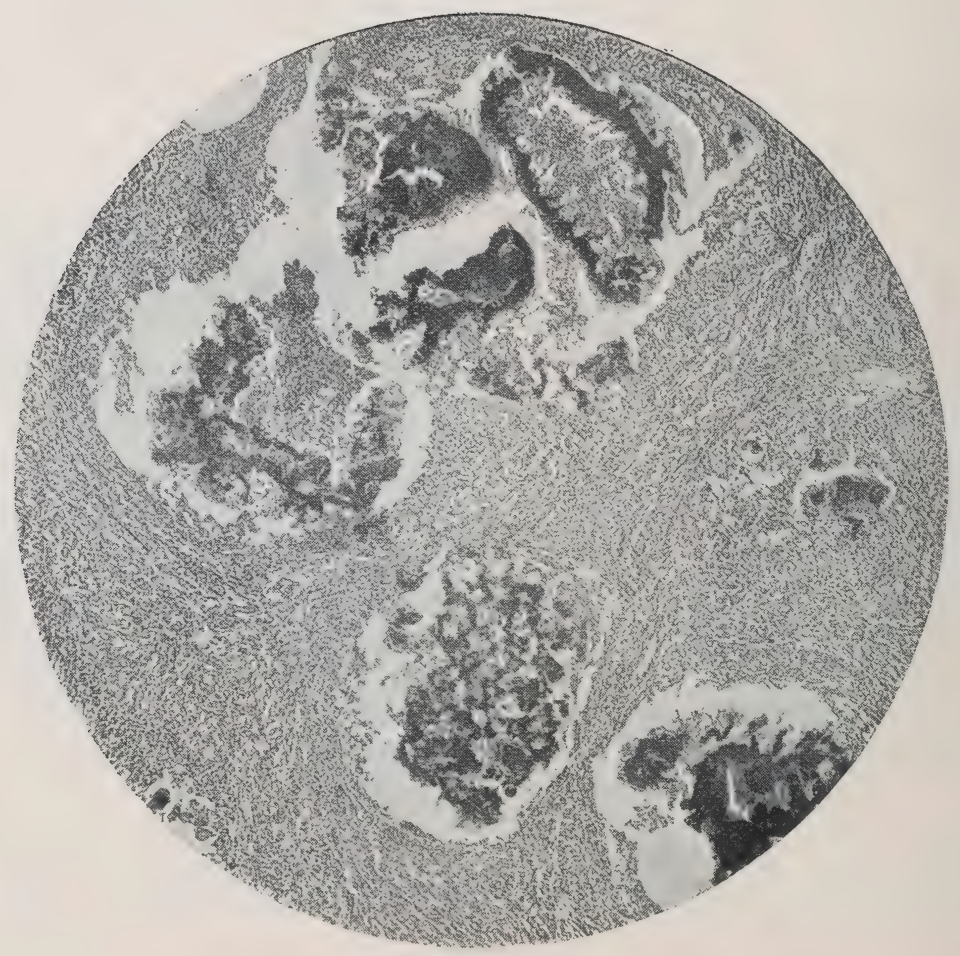

FIG. 272.--Actinomycosis of mammary gland (cow).

reticulum, liver and intestine, larynx, trachea, lung, peritoneum, epiploon, and even the udder.

Localisations in the udder and peritoneum are commonest in pigs, and it is believed that inoculation occurs either through the galactophorous sinuses or through the abdominal wounds made for purposes of castration.

Causation. The cause of actinomycosis is to be sought in the development of Actinomyces bovis within living tissues. It seems problematical whether the germs to be found in the pus or saliva of affected animals ever directly infect new hosts, and it is difficult to carry out infection in this manner even in very sensitive experimental 
animals. Nevertheless, the persistence of the disease in certain brres would seem to support the view of direct infection.

On the other hand, it is proved that the actinomyces is a parasite affecting vegetables, principally the graminaceæ, and that domestic animals are most commonly infected through injuries caused by vegetable substances. This is suggested by the discovery of the débris of grain at the point where the lesions have originated.

Inoculation is commonest in the mouth and on the surface of the

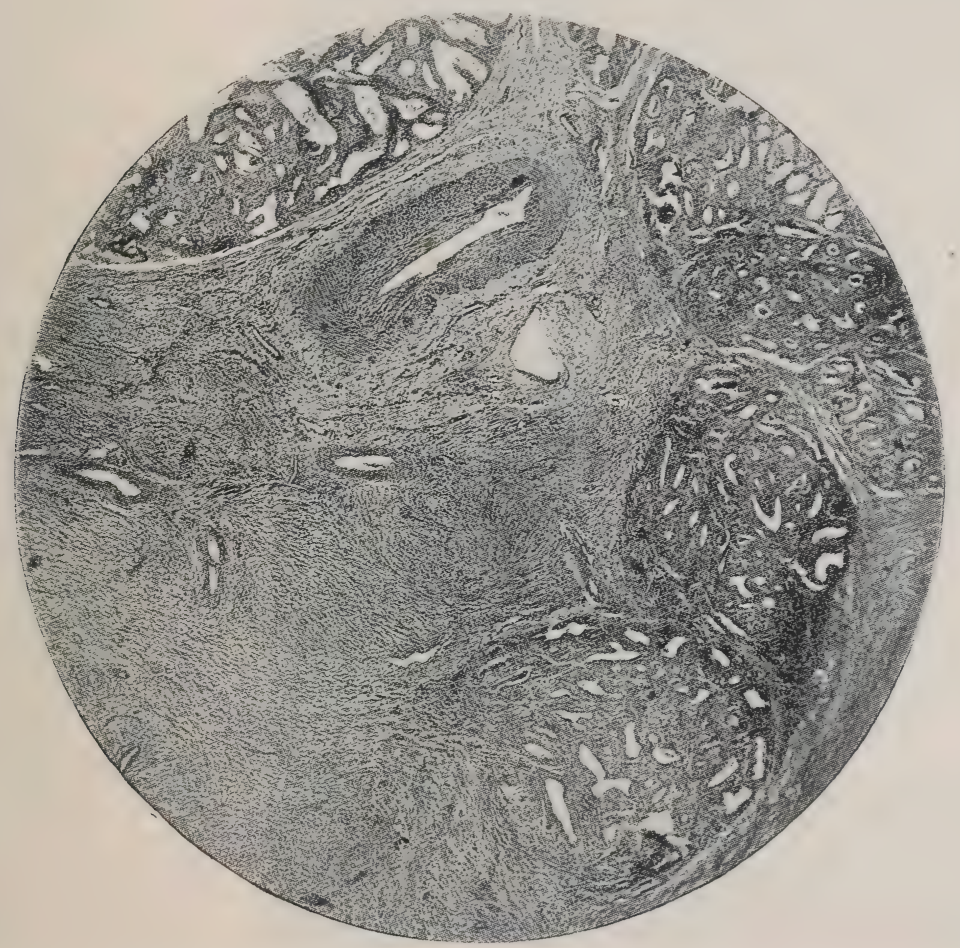

FIG. 273.-Actinomycosis of mammary gland (cow), showing growth invading lobules. (Figs. 272 and 273 are from blocks kindly supplied by Mr. Gilruth, F.R.C.V.S.).

tongue, parts which are, so to speak, permanently excoriated. The shedding of the temporary molars favours such accidents, and this is why actinomycosis of the jaw is, relatively, so common. The incisor region may also be inoculated during the shedding of the milk teeth, but as the infected food comes more closely and for much longer periods in contact with the molars, it is easy to understand why actinomycosis is rarer in the incisor region.

The conditions are less favourable for inoculation of the pharynx, because food does not remain in position there for more than a 
second or two, but when the epithelium has been shed as a consequence of laryngitis or pharyngitis, infection may occur.

As regards cutaneous inoculation, the parasite only seems dangerous when the skin is excoriated or injured either accidentally or as the result of surgical interference.

Actinomycosis of the lung is probably caused by the germs being inhaled along with the inspired air.

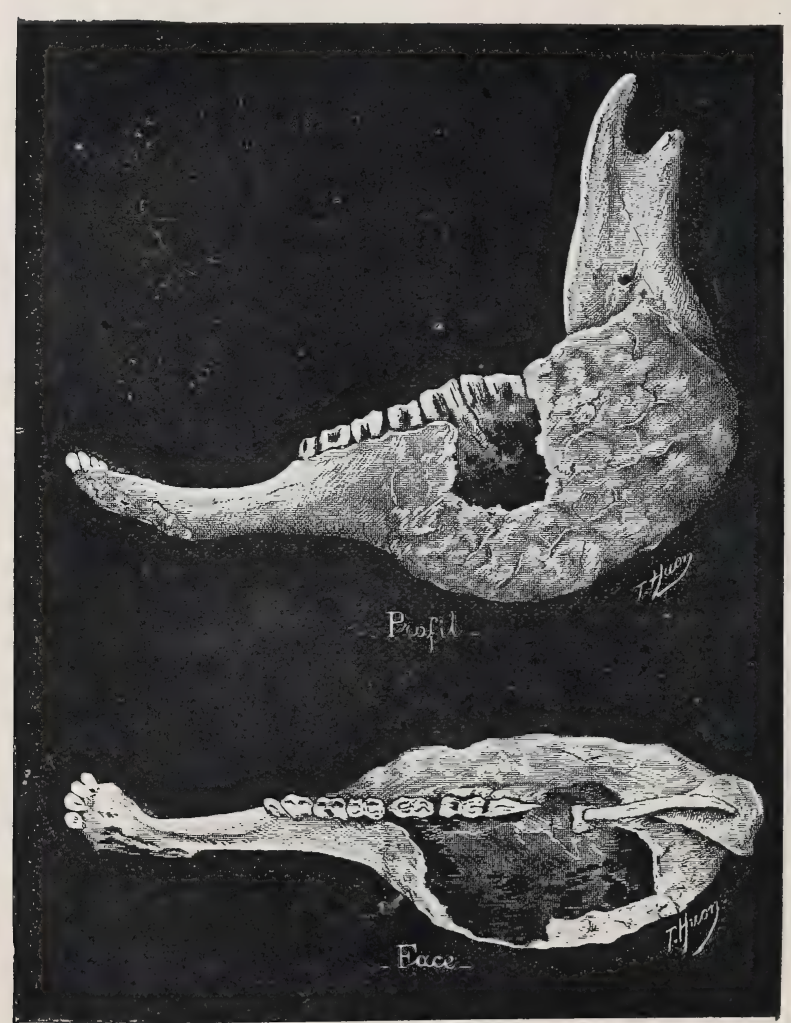

FIG. 274.-Old-standing bone lesions in a case of actinomycosis of the jaw.

Lesions. The lesions are very peculiar in character, and end in completely destroying the tissues invaded.

Once lodged within an organ, the disease shows a. tendency to extend in all directions, and, despite the defensive reaction of the tissues, it soon form s numerous parasitic centres.

In bones, for example, actinomycosis invades the spongy tissue with the greatest ease. It causes subacute ostitis, which leads to diffuse suppuration and local hypertrophy of the bone, destruction of the compact layers, and the development of an abscess with fungoid, exuberant, granulating walls which show no reparative tendency whatever.

The pus of the abscess and the liquid from the fistula contain varying quantities of yellowish grains, representing clusters of actinomyces. The surrounding tissues, muscles, tendons, skin, etc., are all involved before long in the inflammatory process, and the granulating masses themselves are invaded by the yellowish parasitic tufts. All the fistulæ are surrounded by enormous zones of infiltration, which on 
incision exhibit a lardaceous appearance. On section it may appear that the lesion is confined entirely to the bone, though this is exceptional (Fig. 274). Ordinarily the neighbouring tissues are also destroyed, and not infrequently there is communication with the external air. Sections then display a fungoid tissue, interspersed with perforated lamellæ of bone and lardaceous tissue containing cavities crammed with actinomyces.

The lesions in the parotid regions, the neck or other parts attacked always present the same appearance, viz., wide, tortuous, bifurcated fistulæ, with exuberant granulations both in the direction of the cavities and of the exterior, together with lardaceous induration of the tissues and abundant foetid liquid pus.

When it affects the tongue the parasite is to be found in the submucous region, where it causes little swellings, which, when superficial, rapidly undergo ulceration. The subjacent regions, the interstitial connective tissue, and the muscular tissue become infiltrated, hardened and progressively sclerosed. The tongue is gradually hypertrophied, and soon it becomes as hard as wood, whence the term "wooden tongue."

Actinomycosis of the lung may easily be mistaken for tuberculosis, for the centres, although

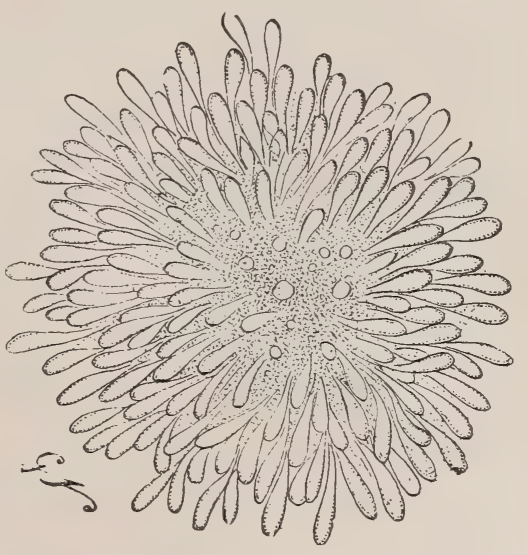

FIG. 275.-Highly-magnified clump of actinomyces. usually confined to one lobe, may also be disseminated. The lesions, however, are surrounded by an abundant fibro-sclerous inflammatory tissue.

In the abdominal cavity, particularly in sows, actinomycotic lesions occur as little masses varying in size between that of a pea and that of a haricot bean, attached to the epiploon and peritoneum and filled with pus containing mycosic grains.

Diagnosis. Actinomycosis is usually easy to recognise, both on account of the special character of the lesions and the presence of the little grains formed by the parasite. The practitioner will rarely fail to recognise at once the signs of actinomycosis of the jaw, but actinomycosis of the tongue is more apt to be mistaken for deep-seated sclerosing glossitis, although a careful examination will always enable the different symptoms to be distinguished. 
It is otherwise with regard to growths in the pharynx and œsophaguis, for, until after removal, simple polypi cannot be distinguished from actinomycotic growths. In such cases the administration of iodide of potassium affords valuable indications.

The prognosis is grave, whatever the clinical form of the disease. Important advances, it is true, have lately been made, and the iodide of potassium treatment is of great value, but too much must not be expected of it, and its benefits have certainly been exaggerated. Clinical experience suffices to prove that only actinomycosis of soft tissues can be cured by drugs, bony lesions being amenable only to medical and surgical treatment combined. Even combined treatment is often unsuccessful.

Treatment. Thomassen in 1885 first explained the favourable action of iodide of potassium on actinomycotic growths, and Nocard in 1892 again directed public attention to the advantages attending the use of this drug both in man and the lower animals. As too frequently happens, however, the benefits of this treatment have been exaggerated, and iodide of potassium has been held out as a specific even against lesions in bone. With very few exceptions this is incorrect, and, as Moussu has shown, when the disease affects bone tissue it only yields to mixed treatment.

The treatment of actinomycosis may therefore be considered under two heads.

Firstly, the treatment of actinomycosis of soft tissues; and, secondly, that of bone.

Actinomycosis of soft tissues, muscle, skin, lymphatics, serous membranes, etc., comprises the most common forms of actinomycosis, viz., those of the tongue, pharynx, parotid glands, neck, etc.

The second form comprises actinomycosis of the lower jaw (molar region), the upper jaw, region of the incisors, etc.

Iodide of potassium in daily doses of from 2 to 3 drachms is almost a specific in dealing with the first form of disease.

In lingual actinomycosis, for example, the effects may be seen a few days after treatment is begun. The tongue becomes softer and more mobile, can be protruded beyond the mouth and retracted into it, and day by day tends progressively to resume its normal appearance.

The patients, which were previously slowly dying of inanition because they were unable to feed themselves, again take to their food and begin to put on flesh. To ensure the treatment being efficacious it should, as a rule, be continued for three or four weeks.

During the course of this treatment the system becomes saturated with the drug, but no bad effects follow. The patients suffer from lachrymation, coryza, bronchorrhæa, and especially iodic eczema, but 
all these symptoms diminish and disappear soon after the administration of the drug is discontinued.

Recovery, however, is not always permanent, and even when the tongue has resumed its normal appearance a relapse may occur. IVe have seen several such cases after treatment extending over more than six weeks, and it is therefore often advisable to fatten the animals as rapidly as possible and prepare them for slaughter.

If no relapse occurs, and recovery is regarded as permanent, another complication may make its appearance, viz., sclerous atrophy of the tongue. This is almost as dangerous as the primary lesion, because it prevents the animals from feeding, and constitutes an additional reason for following the course above suggested.

Other lesions of soft tissues, such as disease of the parotid or cervical glands, etc., yield to the same treatment, but it is advisable first of all to clean out the fistulæ, scrape off exuberant granulations, cleanse the irregular culs-de-sac, and thoroughly curette all accessible parts.

Treatment is much longer than in the case of actinomycosis of the tongue, but it is not always necessary to push the remedy to extreme limits. As soon as symptoms of iodism appear only a drachm or two of the drug need be given daily.

Actinomycosis of Bone.-As a general rule, actinomycosis of bone resists the administration of iodide of potassium, a fact probably explained by the much less abundant blood supply in bone as compared with very vascular tissues, such as the tongue.

To have any chance of success the iodide treatment must be supplemented by surgical interference. As regards the surgical aspect of the case, the affected bone should be removed as far as possible, together with all broken-down tissue. Should this be neglected, the disease returns in a little while.

In actinomycosis of the region of the incisors the method is radical when adopted in time. The body of the maxilla can be partially removed with a fine saw, two cuts being made disposed thus: < (the letter $\mathrm{V}$ sideways). The upper and lower layers of compact tissue should be spared as much as possible, so that the body of the bone may not afterwards break. Recovery is only a matter of time.

A very small local iodoform dressing is applied, and, when healthy granulations appear, cicatrisation can be left to natural means.

Cases of actinomycosis of the jaw are much more troublesome. If, as usually happens, the lesion is ulcerated before the practitioner is called in, the external fungoid growth should be removed by means of an elliptical incision through the skin, the axis of the ellipse being parallel with the branch of the maxilla. The bony fistula is then exposed. 
In following up this fistula care must be taken not to injure the facial artery, the facial vein, or Stenon's duct. Once the bone is exposed the disease can be attacked in the depths. The diseased interior is cut away by means of a special curette, all affected portions being removed, and an iodine or iodoform dressing is then applied.

The operation is extremely troublesome, owing to the enormous bleeding, and sometimes it is impossible to carry out successfully, as in the case of old-standing and extensive lesions. To ensure recovery under such circumstances, it is necessary to remove a portion of the branch of the jaw, and this, though quite possible from the scientific standpoint, would not be worth while in an animal, the value of which is usually small.

Curettage of the bone is only of value in dealing with recent lesions, and even then should not be practised except in the case of animals which the owners particularly desire to keep.

In cases of actinomycosis of the upper jaw surgical treatment is just as difficult as in the lower jaw, and calls for similar precautions.

The diseased portions of bone having been removed, the cavity is plugged with iodoform or cotton-wool, or a dressing saturated with boric acid and iodoform.

In all surgical operations it is important not to injure the dental arteries or nerves, or the alveolo-dental periosteum.

\section{TUBERCULOSIS.}

Tuberculosis is a contagious disease produced by the action of Koch's bacillus. It is common to man and all domesticated animals, but it specially affects animals of the bovine species. Its existence has long been recognised, although in oxen it was formerly confounded with the lesions of peri-pneumonia and echinococcosis.

It was not until the beginning of the nineteenth century that Laënnec (1811) described the tuberculous lesion from the anatomical and pathological standpoint. Gürlt pointed out for the first time in 1831 the similarity, the identity in fact, of tuberculous lesions in man and the ox.

In 1865 Villemin showed that tuberculosis could be conveyed from animal to animal, always producing similar lesions, and in 1868 Chauveau proved that, in the calf, infection might arise simply from the eating of tuberculous material.

At a somewhat later date doubts were entertained regarding the identity of human and bovine tuberculosis. Virchow denied the identity of the two diseases on the basis of a comparative study of the lesions. His opinion, however, has not prevailed, and the doctrine 
of the identity of tuberculosis in mammals still appears probable, in spite of the recent declarations of Koch (1901).

Causation. Tuberculosis is due solely to the activity of the tubercle bacillus. In 1884 Koch isolated and cultivated this bacillus in living animals, and always reproduced typical tuberculous lesions by injecting cultures. In 1887 Nocard and Roux described a rapid method of cultivating the bacillus, and in 1890 Koch announced the discovery of tuberculin.

The tubercle bacillus assumes the form of a little rod, five or six micro-millimètres in length, and 03 to $05 \mu$ in thickness. It has a special staining reaction when treated with Ehrlich's or Ziehl's solution. It grows between $98^{\circ}$ and $104^{\circ}$ Fahr. (37 ${ }^{\circ}$ and $40^{\circ}$ C.) in various artificial media containing glycerine.

Healthy subjects become infected by the accidental entrance of germs into their bodies, either by the respiratory and digestive tracts, or through solutions of continuity in the skin.

The material from tuberculous centres is virulent, whether consisting of sputum or discharge, saliva, fæces, urine, milk, etc., or tuberculous tissues derived from the different viscera.

The blood and muscular tissues are not always virulent, even in cases of generalised tuberculosis.

The virulent organisms usually enter the body through the lymphatic system; invasion proceeds from the point inoculated towards the nearest lymphatic glands and thence along the chain of lymphatic vessels, and the lesions extend, attacking the internal organs imore or less rapidly. The body does not necessarily become fatally infected as a consequence of accidental or even experimental infection, for the bacillus may itself be destroyed by the phagocytes, or the lesion may remain purely local.

Although tuberculosis is the gravest and most widespread disease on the surface of the globe, its contagious character is relatively little marked, a fact which has unfortunately led to its receiving little attention in ordinary life.

Contagion is usually the result of cohabitation, although contact between diseased and healthy subjects for a period of some days or even weeks does not seem sufficient to produce the disease. Nocard has fixed a mean period of five to six months as necessary for the contraction of the disease by bovine animals, and Moussu has arrived at almost identical results by placing tuberculous and healthy cows together in a byre reserved for such researches. In this connection, however, very great differences of individual susceptibility exist, and these are difficult to appreciate in the present state of our knowledge. It thus happens that an animal of vigorous appearance and in good 
condition may easily contract tuberculosis, whilst a thininer and less vigorous one will resist it for a comparatively long time.

Speaking generally, it may be said that young animals contract tuberculosis by cohabitation in infected places more easily than adult or aged ones, and the fact that old animals contribute the larger number of cases is to some extent due to their having in the course of their lives been more exposed to continued or successive infection.

Contagion does not occur in byres unless as the result of the presence of animals with open tuberculous lesions, such as caverns in the lungs, tuberculous bronchitis with ulceration of the mucous membrane, tuberculous metritis, enteritis, etc. The virulent germs are expelled in the saliva, nasal discharge, excrement, etc., and are distributed over the forage, manure, litter, and in the drinking water; after desiccation they may be spread by currents of air.

The mangers, racks, drinking pails, and various stable utensils become permanently contaminated, the air of the cow-sheds contains virulent dust, and the animals there confined are continually exposed to infection either through the respiratory or digestive passages.

Contamination through the respiratory tract is by far the most frequent cause of the evil, and recent experiments at Pouilly-le-Fort (1900) have shown how easy it is to convey the disease experimentally by inhalation.

Patients suffering from closed tuberculous lesions of the pleura, pericardium, spleen, peritoneum, etc., do not spread the bacilli. Healthy animals may remain in contact with them without danger, but it is well to remember that such cases are quite exceptional. As a rule the lesions are of a mixed character, and the general principle may be laid down that cohabitation of any duration with tuberculous subjects is dangerous.

Contagion spreads more easily, in proportion to the number of tuberculous subjects in a given byre, to the total number of animals in a herd, and to the neglect of cleanliness, good feeding, ventilation, etc.

Life in the open air and at grass greatly diminishes the chances of contagion. 'The virulent products are then disseminated in all directions and are soon destroyed by the general atmospheric conditions. Close confinement in ill-ventilated stables, on the contrary, strongly tends to the propagation and development of tuberculosis.

In calves infection may occur through the alimentary tract by means of tuberculous milk, whether such milk is obtained directly from the udder or out of a pail. The same may be true of young pigs fed with skimmed milk.

Goats contract tuberculosis somewhat readily by confinement in 
byres with tuberculous cows, and Moussu declares that contagion afterwards spreads just as rapidly among goats as among cows. The vaunted great resistance of goats to tuberculosis, formerly so often spoken of, and by some wrongly considered as a condition of immunity, is deceptive, and if tuberculosis is less frequently seen in goats, this is solely because goats enjoy the greatest liberty at all seasons.

On the other hand, the disease is very rarely conveyed to sheep, even when they are kept for long periods with tuberculous cows. Moussu found that two years of close cohabitation were necessary for its development under these conditions.

Heredity is a factor of the highest importance in determining the causation of tuberculosis. At the present time a tendency exists to deny this, but such a view is erroneous.

Observation has clearly shown that tuberculosis is rarely conveyed from the mother to the fœetus, and that practically none of the calves borne by tuberculous mothers react to tuberculin (95 per cent.: Nocard and Bang); but even if this is absolutely correct, it only shows that great benefits might be derived if proper sanitary organisation and intelligent hygienic conditions in byres were found everywhere in the country. Unfortunately in practice this is far from being the case. These non-tuberculous calves are left in common contaminated byres, where they rapidly become infected and perpetuate the disease.

Physiologically these facts are easily explained. The placenta resists the passage of microbes, or at least only allows them to pass under quite exceptional conditions, and practically only when the blood-vessels are affected. As, on the other hand, tuberculosis of the ovaries, Fallopian tubes or uterus generally prevents pregnancy and causes sterility, there is nothing extraordinary in the fact that tuberculosis is not hereditary in the strict sense of the term. The influence of the sire has been invoked, but it has been proved that direct paternal infection is only possible where ulcerating tuberculous lesions of the testicle, prostate, or vesiculæ seminales exist. Such conditions seldom or never occur in the sires of domestic animals.

As a general rule, therefore, it may be said that tuberculosis is not hereditary. New-born animals become infected during the months following birth, either directly through the alimentary tract when the mothers are suffering from mammary tuberculosis, or, perhaps more frequently, through the respiratory and digestive tracts.

But although microbic infection is not hereditary, it by no means follows that the offspring of tuberculous subjects are as well prepared for the struggle of life as the descendants of healthy subjects. What is transmitted is a greater tendency to contract the disease. 
This aptitude or predisposition is of such importance that in Moussu's opinion it should be regarded as one of the essential factors in the development of tuberculosis. The cause of tuberculosis is Koch's bacillus. It does not always produce its full effects in animals born of healthy parents; but in one that suffers from a tuberculous hereditary taint tuberculosis appears.

Physiological and pathological researches cast considerable light on this question. In tuberculous mothers the organism not only suffers from the infection, but from a permanent intoxication which interferes with normal metabolism in the vital organs and the exchanges between mother and foetus. If the microbes remain confined to the system of the mother, their poisons are conveyed by the blood and pass through the placental barrier. In a greater or less degree they saturate the tissues of the little creature in process of development, and communicate to it a peculiar hereditary taint. The effects of this taint are often noticeable from the moment of birth, for comparative physiological and pathological investigations have shown that the tissues of tuberculous animals assimilate given foods less perfectly and are the seat of greater losses of all kinds than those of healthy subjects.

Although the disease itself, therefore, is not hereditary, it is otherwise with the organic taint which plays so important a part in its development. This organic taint consists in a special condition of the tissues or cells of the parents, which show a diminished power of resistance to the action of the germs of tuberculosis; it is therefore easy to understand how important a part these influences may play under certain conditions.

Without doubt, in the case of bovine animals, the predisposition could be neutralised in carefully managed studs by the immediate isolation of the new-born under conditions which shield them from tuberculous infection, and experiment has shown the benefits derived from such precautions; but it must not be forgotten that intelligently managed studs are the exception, and that for a long time to come we must in practice take cognisance of the actual conditions under which the disease develops.

The lesions of tuberculosis vary greatly in appearance, according to the organs affected, though the method of development is always identical.

The primary lesion corresponds to what has been termed tuberculous granulation, or anatomical tubercle properly so called; this, the macroscopical, pathological entity, assumes the form of a small prominent centre, semi-transparent, greyish, opaque or yellowish, according to its age. 
These tubercles, produced by the presence of colonies of bacilli, are due to the defensive reaction of the invaded tissues, which gradually undergo change and are destroyed in a direction radiating from the centre towards the periphery. The tubercle in itself has no very specific character-only the bacillus.

The elementary lesion may remain isolated, but very frequently it is closely surrounded by other similar tubercles, and becomes enveloped in a common inflammatory area. A large portion of an organ may appear as if riddled with tubercles of different age and size, while the interstitial connective tissue reacts and forms fibrous separating partitions. The general appearance is that described under the term "diffuse tuberculous infiltration."

At a still more advanced stage in the development of the disease conglomerations are produced, consisting of tuberculous masses the size of a hazel-nut, a walnut, an egg, a man's fist, or even larger. These lesions, irrespective of size, undergo caseous degeneration from the centre towards the periphery.

In exceptional cases the tubercles remain fibrous. More frequently, particularly in animals of the bovine species, they become infiltrated with lime salts. Caseous degeneration not only invades the centre of the tubercles but also the peripheral layers, and sometimes the whole of a conglomerated mass.

Steadily pursuing their course of pathological development, the tuberculous masses become softened and are transformed into tuberculous abscesses, which open towards any free passage, leaving behind sometimes ulcerations, sometimes caverns of varying sizes, or blind simple or bifurcated fistulæ.

Recent experiments by Nocard and Rossignol (1900) prove conclusively that a certain time (always more than a fortnight) elapses between the moment of entry of the contagion into the organism and that at which its effects become manifest by furnishing a reaction to tuberculin. Calcification or softening of the lesions, moreover, never occurs in less than fifty days.

According to the organs studied, these tuberculous lesions assume certain appearances, which in each locality seem almost always to be identical.

Thus, as regards the larynx, trachea, and bronchi, the tubercles develop in the depths of the mucous membrane, rapidly undergoing caseous transformation, softening and purulent degeneration, and producing numerous isolated or confluent ulcerations in the air passages.

According to the case and the kind of animal affected, the lung presents either disseminated tuberculous formation, tuberculous infiltration, tuberculous conglomeration, or eavern formation. 
The lung may be affected to such a degree that it appears incredible that the blood can have been sufficiently aerated to support life.

The lungs may be transformed into yellowish, caseous, calcareous, or softened masses enveloped in thick, fibrous, resistant walls. The intervening pulmonary tissue may be healthy in appearance, or reddened, congested, and sometimes hepatised.

The pleural, pericardial, and peritoneal membranes may be covered with exuberant tuberculous lesions, like ripe mulberries, in consequence of fusion and massing of the tuberculous growths. The primary tubercles are surrounded with fibrous walls, which granulate when on the surface of a serous membrane, and impart to the membrane a vegetative, sometimes villous appearance, and a colour varying from pink to light or dark red.

The collective lesions lining the cavities are described by butchers under the significant term of "grapes." In the interior of these exuberant masses, which sometimes form layers an inch or more in thickness, the tuberculous lesions undergo the usual developmental changes, that is to say, they become caseated or infiltrated with lime salts, but they do not so readily undergo softening as those of the lung. The parietal and visceral serous membranes readily become adherent at numerous points, setting up union between the lung and the walls of the chest, or the intestine and the walls of the abdomen, etc.

In the pericardium the vegetations are frequently of a fungoid character.

T'uberculosis of lymphatic glands sometimes assumes a disseminated, discrete form or that of a diffuse infiltration, or, again, in old-standing cases it constitutes a massive tuberculous conglomeration. In point of fact, the lymphatic glands as such no longer exist, their tissue having undergone total degeneration; they are represented only by an enlarged, thick, fibrous shell, forming the envelope which encloses caseated and calcareous masses of a more or less soft nature.

Tuberculous infiltration of the sub-maxillary and sub-parotideal lymphatic glands interferes with swallowing and breathing, compresses the pharynx, œesophagus and larynx, and deforms the head.

Compression of the arteries, veins, nerves, etc., at the entrance to the chest may cause various symptoms which are not difficult to interpret. The glands at the entrance to the chest and the whole of the anterior mediastinum may form a single mass. Lesions in the posterior mediastinum, however, are of even greater importance and explain certain symptoms, such as difficulty in swallowing, spasm of the osophagus, mechanical contraction of the osophagus, permanent tympanites, etc., for which the state of the lungs alone would not account. 
Even when the lungs are unaffected it may happen that the lymphatic glands of the mediastinum (superior or inferior œsophageal lymphatic glands) and the bronchial lymphatic glands may be so diseased that the osophagus is completely surrounded and compressed by them, and its function thus seriously impaired (Fig. 276).

In the abdomen the mesenteric glands are most exposed to disease, and when infected through the intestinal tract they assume the form of large flattened masses arranged along the mesentery.

In the digestive tract, as in the trachea and bronchi, tuberculosis has a marked tendency to assume the ulcerative form. Disseminated or aggregated tubercles develop in the thickness of the mucous membrane, and, after rapidly softening, become ulcerated. The nature of

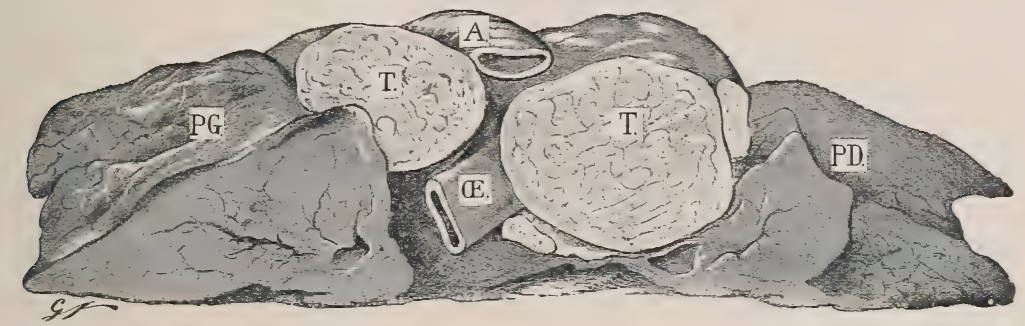

FịG. 276.-Tuberculosis of lymphatics. PG, Left lung; PD, right lung; TT, tuberculous œsophageal lymph glands; A, aorta; $\mathrm{E}$, œsophagus (the lung is divided transversely near its centre).

these lesions can only be determined by noting their character and examining the discharge.

The ulcerations are localised in the mouth and pharynx, in the second half of the small intestine towards the ileum, and in Peyer's patches.

Tuberculous lesions develop in the vaginal sheath of the male genital organs exactly in the same way as in an ordinary closed serous cavity; tubercles may also develop on the surface or in the substance of the testicle. They become aggregated, undergo softening, spread towards the interior, and may break down, thus forming abscesses. In the female genital passages the disease invades the thickness of the walls, but shows a marked tendency to ulceration, as in the intestine or trachea.

In the udder tuberculosis is generally diffuse, shows a tendency to hypertrophy and the free formation of fibrous or sclerous tissue; only tubercles in the glandular layer of the acini become ulcerated. In time the whole of the secreting structure undergoes diffuse tuberculous suppuration, fibro-caseous masses form in the depths of the tissue and may soften, producing deep-seated tuberculous "cold abscesses."

D.c. 
The mammary lymphatic glands are affected in the same way as other lymphatic glands.

In the joints tubercles appear either on the synovial membrane or in the thickness of the bony epiphyses, very often at both points simultaneously. The synovial membrane is covered with vegetations and villous growths, the ends of the bones are attacked by a destructive ostitis, tubercles or tuberculous centres form in the thickness of the spongy tissue, the articular cartilages are destroyed, the ends of the bones become deformed, and in the last stages fungoid arthritis in various forms may be produced.

In bones the tubercles originate in the depths of the spongy tissue. They produce destructive hypertrophic ostitis, in which the bony tissue is replaced by tuberculous centres or masses divided by fibrous partitions. On section, these lesions exhibit the same yellowish caseated or calcified appearance as the lesions of other affected organs. The compact layer may sometimes be perforated at several points before being destroyed.

In tuberculosis of the brain the primary lesions develop at the expense of the serous layers of the arachnoid and on the pia-mater, towards the base of the brain and the fissure of Sylvius, or at the expense of the small vessels which penetrate the depths of the nerve substance itself. Some tubercles remain isolated, become confluent or are collected in masses of different sizes, and provoke symptoms which vary with the locality attacked.

Symptoms. Tuberculosis is the most protean of all diseases, and at first sight it often seems impossible to assign to one group, clinical conditions presenting such essentially different appearances. All the tissues may be attacked, from the bones to the most delicate of the viscera, a fact which explains why all aspects of tuberculosis cannot be described. Certain forms, however, occur very frequently, and may be regarded as classic; these will be considered in the order of their frequency.

\section{TUBERCULOSIS OF THE RESPIRATORY APPARATUS.}

Without doubt this form of tuberculosis is by far the most frequent. It assumes the form either of bronchitis, laryngo-bronchitis, or pulmonary tuberculosis.

Tuberculous Bronchitis.- The symptoms of tuberculous bronchitis do not essentially differ from those of ordinary bronchitis, though the disease develops more insidiously and slowly, and is seldom accompanied by fever. At first the cough is dry and suppressed; later it becomes paroxysmal, and at a still more advanced period liquid and 
rough. The least irritation brings on these attacks of coughing; changes from the warmth of the stable to the coldness of the outer air or vice versâ, the presence of dust or the action of liquids when drinking, etc., etc. During the first stage coughing is not followed by expectoration, but later yellowish-grey, glairy mucus may be discharged: more frequently it is coughed into the pharynx and swallowed.

These symptoms continue for weeks or months without showing any tendency to abate. If the larynx is attacked inspiration becomes rattling and difficult, while the neck and head are held extended, and the least pressure over the larynx produces coughing.

Tuberculosis of the larynx, trachea, and bronchi is usually accompanied by disease of the lung, but may occur by itself.

When there is a discharge it consists of thick, viscous, sticky mucus of a peculiar greyish-yellow colour. Microscopical examination shows it to contain tuberculous bacilli.

Pulmonary Tuberculosis usually assumes the chronic form, and is almost always preceded by specific bronchitis. The patients retain their appearance and condition for a longer or shorter time, and, without the experience resulting from continued observation, it would be difficult to believe them to be suffering from the slow development of a serious disease.

Frequent coughing without any apparent reason is the only symptom likely to arouse suspicion.

At a later stage these animals lose condition, feed less eagerly or exhibit capricious appetite, and sometimes well-marked and repeated digestive disturbance, such as slight tympanites with constipation or diarrhea, moderate impaction of the rumen, relative atony and slackening of peristaltic movements. The wasting gradually becomes more marked or, in the case of pregnant or milch cows, makes intermittent progress, until the animals become anæmic and finally cachectic. The cough is more frequent and more severe, and is followed by discharge from the nose or by swallowing movements. From this time phthisis, properly so called, exists.

The course of the disease is not invariable. Certain animals may appear ill for years without clinically showing the least apparent aggravation; others on the contrary, though living under similar conditions, are rapidly attacked, and in six to twelve months exhibit all the signs of advanced phthisis. Pregnancy, suckling, and prolonged lactation favour the development of the disease by taxing the physical resources of the animal.

Animals suffering from phthisis exhibit a peculiar appearance. They are extremely thin, all their soft tissues are wasted, the limbs are dragged in moving, respiration is rapid and sometimes jerky, 
the mucous membranes are pale and discoloured, and the skin is tight and adherent to the subjacent tissues.

These general signs, however, would not warrant a diagnosis, for, apart from the cough, certain other diseases present all the external appearances of the last period of tuberculosis (chronic diarrhœea, chronic forms of poisoning-bacterial or otherwise-dyspepsia, etc.).

In cases of doubt it is essential to discover by percussion and auscultation that the external signs are really the result of lesions of the lung, and that the lung disease has developed gradually in accordance with the signs shown by simple external inspection.

The symptoms presented during the development of the pulmonary lesions may be divided into three phases.

In the first phase percussion gives no information, though auscultation reveals rough respiration, inspiration and expiration being also unequal. Expiration, which, in the healthy subject, is silent, becomes clearly perceptible, not over the whole lung, but usually over the anterior lobes, particularly the cardiac lobes. This sign is the result of tuberculous infiltration and of the neighbouring pulmonary tissue having lost its elasticity.

Inspiration is rough and rasping, and sometimes occurs in several stages, the act being interrupted or jerky; expiration lasts longer than inspiration, is rough and prolonged, but never blowing in character. These peculiarities are only found in one other condition of the lung, viz., emphysema.

The patients appear little affected in this, the first, stage of tuberculosis. But for the cough they may seem perfectly healthy.

In the second phase the tuberculous infiltration extends and ends in the massing, by fusion or centrifugal growth, of the tuberculous masses.

Percussion may now indicate localised dulness, but this is not invariable, because the diseased anterior and middle lobes of the lung are concealed beneath the muscles of the shoulder. When dulness is noted, it is usually over the lower part of the posterior lobes, very rarely at any higher point on the side of the chest. Frequently the dulness is only partial.

On auscultation the signs met with during the first stage become much more marked. Inspiration is always rough, rasping, painful and difficult at certain points, particularly in the anterior zones. In this region expiration is rough, prolonged and sometimes of a clearly marked blowing character. This is particularly the case in the sub-scapular zone and the auscultation zones 2 and 3 (Fig. 166). In the dorsal region and in zone No. 1, respiration may appear normal. Nevertheless, the sounds are propagated to a distance, the 
infiltrated lung steadily loses its elastic qualities, the vesicular murmur entirely disappears from the affected regions, and the sounds noted are of bronchial origin.

Like the first, the second phase may vary in intensity, extent, and in the diffusion or localisation of the tuberculous lesions. Blowing respiration may be noted over different areas, accompanied by sibilant, snoring and migratory mucous râles. The vesicular murmur is exaggerated in the healthy parts, coughing, accompanied by expectoration or followed by swallowing movements, is frequent, the appetite becomes capricious, and the general condition suffers. In this second phase almost the whole of one lung may be diseased and exhibit the signs described.

The third phase corresponds to the softening of the tuberculous masses, and the formation of ulcers and caverns. The zones of dulness or partial dulness may be more extensive, though cavern formation is usually confined to the anterior or middle lobes. Percussion still affords no precise information.

As the tuberculous masses undergo softening and ulceration, their contents are gradually passed into the bronchi, and auscultation reveals signs indicative of the existence of caverns, which signs vary with the dimensions of the caverns themselves. On auscultation the respiration is always found to have at certain points a blowing character, and it may even develop into a true tubal souffle. In other areas, where the caverns are merely in course of formation, gurgling sounds are all that are heard, but where true caverns exist there is an incessant cavernous souffle.

The lesions peculiar to the third phase are seldom seen in practice; because the animals become anæmic, exhausted and cachectic, they are usually slaughtered early. Nevertheless, the third stage occasionally develops in an astonishingly short time, six to eight months at most.

Very frequently the patients, although cachectic and even phthisical, do not yield on auscultation the sounds described as peculiar to the third stage, because the tendency to softening is not very marked in bovine animals. The lungs exhibit massive infiltration, and, whilst pulmonary consumption is not uncommon, the development of caverns is comparatively rare.

The expectoration or discharge in this third form is puriform, glairy, viscous, and of a dirty-yellow or even greenish-yellow colour. Bacteriological examination reveals the presence of tubercle bacilli and adventitious organisms.

These conditions are always associated with various complications, and the second and third stages of chronic tuberculosis are frequently 
accompanied by lesions of the pleura, of the mediastinal lymphatic glands, of the liver, etc.

Digestive disturbances often occur; the appetite is capricious or in abeyance, there is atony of the rumen and chronic dyspeptic tympanites. These disturbances are easily understood where there are lesions of the liver, intestine, and mesenteric lymphatic glands, but not when the lung alone appears the seat of the disease. In this condition the patients probably suffer from permanent complex intoxication, due to toxins elaborated by the tubercle bacillus and other microbes which multiply on or in the lesions, and this chronic intoxication reacts on the vital functions (innervation, secretion, digestion and nutrition). Nor are the effects limited to these appearances; the heart's action is also accelerated, and the temperature rises. During the first and part of the second phase there is comparatively little fever, but afterwards this is continuous or of a peculiar intermittent character. In the morning the patient's temperature may be normal; in the evening it has risen from 1.5 to as much as $9^{\circ} \mathrm{Fahr}$. $\left(1 \cdot 1^{\circ}\right.$ to $5 \cdot 2^{\circ} \mathrm{C}$.) above normal, and this recurs day by day. These attacks coincide with softening of the lesions, and when suppurating caverns exist they are more marked and more nearly continuous, assuming the characters of the hectic fever shown in consumption.

Often during the febrile periods the urine is albuminous.

In chronic tuberculosis of bovine animals bleeding from the lung is rare even when caverns exist, and Moussu, in spite of extensive experience, has seen only two cases. This is in striking contrast with the condition in human sufferers from pulmonary tuberculosis, twothirds of whom bleed at the lungs.

\section{TUBERCULOSIS OF SEROUS MEMBRANES.}

After pulmonary tuberculosis, tuberculosis of the pleural and peritoneal serous membranes is the most frequent clinical form of this disease. Sometimes both forms exist, and although the pleural and peritoneal lesions predominate or alone attract attention, there are also lesions in the lung or mediastinal lymphatic glands.

It is difficult to explain how the pleural and peritoneal serous membranes can be seriously invaded without the lung becoming affected, though in point of fact such a state of things frequently exists.

Tuberculosis of the pleura without pulmonary lesions is suggested by very obscure symptoms. The general signs consist in diminution of appetite, loss of condition, tachycardia, elevation of temperature, 
and progressive organic wasting. These are always present, though in themselves they have no specific significance.

The local symptoms are still more vague. Percussion causes pain, and the practitioner might at first suspect peri-pneumonia. The patient edges away, and tries to avoid the application of the pleximeter hammer. Firm pressure over the intercostal spaces sometimes causes struggling, and produces indications of abnormal sensitiveness. There is generally extensive partial dulness, sometimes complete dulness towards the lower regions of the chest.

On auscultation the lung may reveal the different indications of chronic pulmonary tuberculosis, or simply diminution of the respiratory murmur at points, accompanied by crepitant, sibilant râles, and moist, crackling sounds. As the anterior portions of the pleural sacs are most commonly invaded, the anterior vena cava is compressed, causing some difficulty in the return circulation, and producing venous pulse, which may extend as high as the parotid gland; there is, however, no swelling of the dewlap.

Respiration is frequent and difficult in consequence of adhesions between the pleura and lungs, which are connected by bands of fibrous tissue of varying extent. Coughing is rarely absent, and if the lung is diseased may be followed by discharge containing numerous bacilli. Otherwise the cough exhibits the pleuritic character, that is, it remains slight, dry, paroxysmal, and painful. The pericardium may be affected as well as the pleura; if the conditions occur simultaneously the venous pulse in the jugulars will be particularly apparent.

The symptoms of tuberculous pericarditis are similar to those of ordinary pericarditis, except that the exudation is less abundant; in a word, the symptoms are those of rather trifling exudative pericarditis.

Tuberculosis of the peritoneum is frequently accompanied by that of the pleura or the abdominal viscera. The lesions are localised on the parietal peritoneum and epiploon, producing in time adhesions between the viscera and walls of the peritoneal cavity, which affect the action of the digestive organs, gradually causing interference with the peristaltio movement both of the rumen and the intestines. The stagnation of alimentary matter favours fermentation, so that the rumen becomes permanently distended. The right flank also is swollen, and the abdomen exhibits a change in shape similar to that in peritonism, which is a constant symptom of tuberculous peritonitis.

As in the thorax, the tuberculous lesions seldom produce extensive liquid exudation, so that ascites does not occur, but on palpation the abdominal walls appear to have entirely lost their pliability and 
to be unyielding and greatly thickened, a point which is the more remarkable as the animals are thinner.

The wall of the abdomen is stiff, incapable of being depressed as in ordinary subjects, and gives to the fingers the sensation of a thick hard covering, through which the subjacent organs and their contents, that is, the rumen, intestine and alimentary material, can no longer be felt. This rigidity is always most marked in the lower abdominal region. The digestive peristaltic movement can no longer be detected, and on auscultation the normal sounds are manifestly much slower than usual.

\section{TUBERCULOSIS OF LYMPHATIC GLANDS.}

It might perhaps have seemed more logical to place tuberculosis of the lymphatic glands at the commencement of these clinical divisions of tuberculosis, as when tuberculous lesions, of whatever kind, occur in the lung, pleura, abdomen, etc., the lymphatic glands in the neighbourhood are invariably invaded. In such cases, however, the lesions in question are not the dominant features.

Under this heading must be classed tuberculous lesions which, on the contrary, affect the lymphatic glands in so marked a manner that lesions in other organs may be regarded as secondary. This occurs somewhat frequently, because at the present day there is a tendency to believe that inoculation takes place mainly through the mucous membrane of the pharynx, and thence extends towards the neighbouring lymphatic glands. At any rate, it is unquestionable that tuberculosis of the lymphatic glands may exist quite apart from any other lesion visible to the naked eye.

Two forms are very common, tuberculosis of the retro-pharyngeal region and of the neck, and tuberculosis of the mediastinal lymphatic glands.

Tuberculosis of the Retro-pharyngeal Glands.-In addition to the retro-pharyngeal glands the cervical chain of lymphatic glands, the sub-glossal, sub-atloid, pre-parotid, and even the pre-scapular lymphatic glands and those at the entrance to the chest, may also be invaded more or less.

This form of tuberculosis may remain latent for a long time, attention being attracted to it only when deglutition is impeded and local deformity becomes apparent.

Swelling of lymphatic glands resulting from tuberculous infection is slow and progressive, differing entirely from that which accompanies suppurative adenitis. The neighbouring connective tissue is certainly somewhat thickened or infiltrated, but the glands themselves can always be detected. The region of the gullet is enlarged, the 
depression marginating the lower jaw is filled up, the sub-atloid space disappears, the sub-glossal glands occupy the space beneath the tongue, and in cases where the lesions are very pronounced the oesophagus and larynx may even be pushed downwards.

Swallowing is difficult, in consequence of compression of the upper part of the oesophagus, and, as the laryngeal nerves may be included in the swelling, dyspnoea or roaring not uncommonly results.

By palpation with one or both hands, it is easy to identify the glands and detect enlargement, hardness and sensitiveness. In exceptional instances the caseous masses they contain undergo softening and conversion into purulent material.

When the cervical lymphatic glands are attacked the jugular furrows disappear, and the whole of the pretracheal and lateral regions of the neck exhibit doughy swellings.

These swellings are rarely symmetrical, a fact which admits of this . condition being distinguished from lesions due to lymphadenitis, without examining the blood.

The prescapular glands are rarely attacked, but those at the entrance to the chest, which may be found on either side of the trachea by passing the fingers between the two first ribs, are frequently enlarged to the size of a fowl's egg.

\section{Tuberculosis of the Mediastinum.} -Whenever the lungs are much involved, the bronchial glands are also

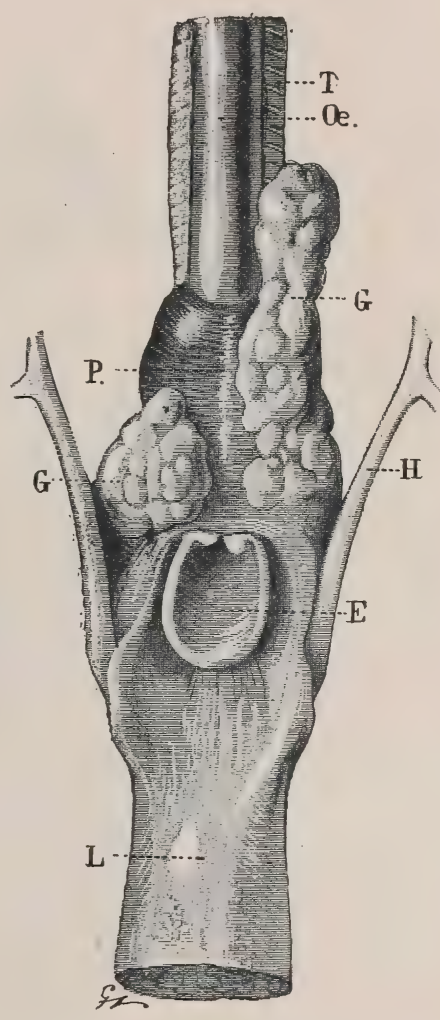

Fig. 277.-Lesions in retro-pharyngeal tuberculosis. T, Trachea; (E, œsophagus ; P, pharynx ; $\mathrm{H}$, hyoid bone; E, epiglottis ; $\mathrm{L}$, tongue; $\mathrm{G}$, tuberculous retro. pharyngeal glands. invaded, though the glands of the anterior and posterior mediastina may escape. On the other hand, the mediastinal glands are sometimes much involved, whilst the lung remains intact.

The lymphatic glands, particularly those of the mediastinum, may be enormously enlarged, and the various accidents which result are due as much to mechanical interference with the functions of adjacent organs as to the lesions themselves. 
When the glands of the anterior mediastinum are affected, they cause compression of the anterior vena cava, with stasis of blood in the jugular vein and venous pulse, then compression of the œesophagus and trachea, and of the nerves at the entrance to the chest, producing difficulty in swallowing, respiration and circulation.

If, as often happens, the glands of the posterior mediastinum are

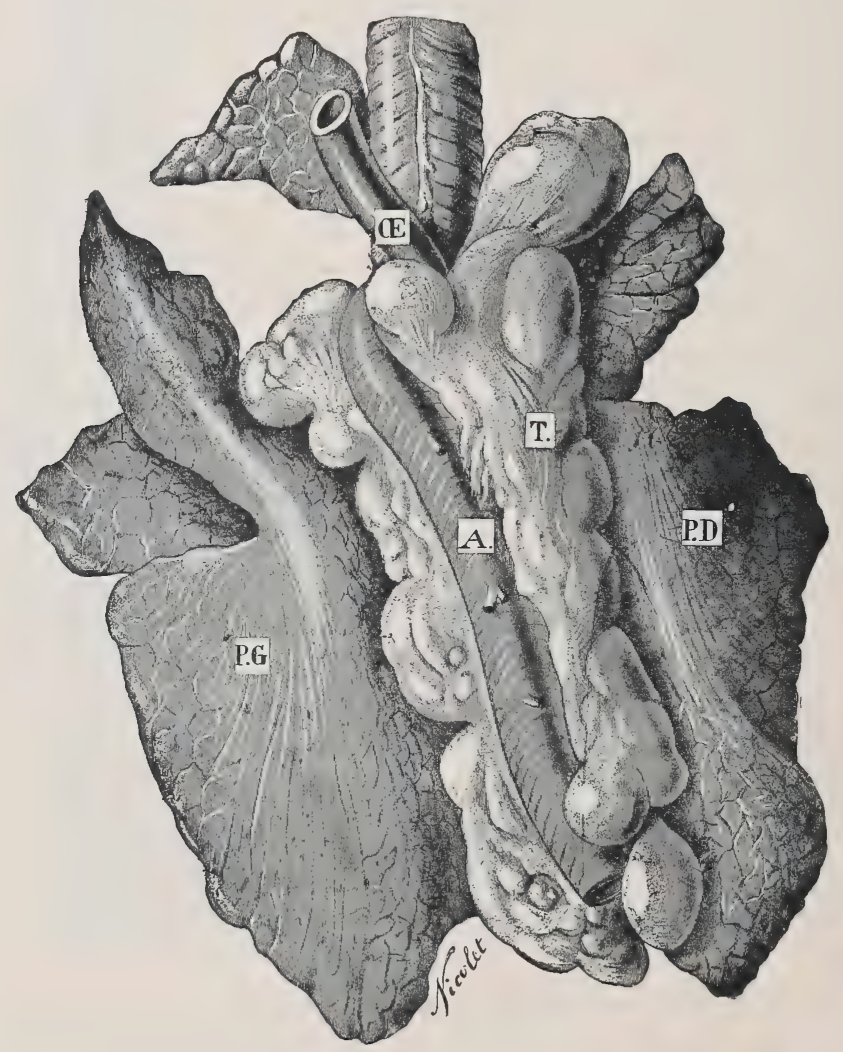

FIG. 278.-Well-developed tuberculosis of the mediastinal lymphatio glands. PG, Left lung; PD, right lung; E, œsophagus; A, posterior aorta; T, tuberculous lymphatic glands.

affected and greatly enlarged, they may involve the oosophagus and the œesophageal nerves, interfere with deglutition and rumination, and thus produce marked disturbance. The animals only swallow with difficulty, and later rumination becomes impossible, the anti-peristaltic movement not being powerful enough to overcome the resistance. Soon after eating, the patients exhibit tympanites, certainly only to a moderate extent, but the swelling is long in disappearing. This 
tympanites is due to the difficulty in eructation and to the impossibility of rumination. The contents of the rumen pass slowly towards the intestine until the onset of a fresh attack.

On account of digestive difficulties, the animals rapidly lose flesh, just as though they were suffering from extensive visceral lesions.

\section{TUBERCULOSIS OF THE DIGESTIVE TRACT.}

Tuberculosis of the digestive tract is rarer than tuberculosis of the lungs or lymphatic glands, and occurs in two well-differentiated clinical forms, that is to say, tuberculosis of the buceal and pharyngeal membrane and tuberculosis of the intestines. Tuberculosis of the liver, which is less easily recognised, is indicated by signs of dyspepsia.

Bucco-Pharyngeal Tuberculosis.-This may be primary or secondary, and occurs in the form of local or general glossitis or superficial ulcerative stomatitis.

In the former case the glossitis may be regarded as due to actinomycosis; in the latter it can only be mistaken for simple ulcerative stomatitis.

It is accompanied by difficulty in mastication, and still more, where the pharynx is invaded, in deglutition; abundant frothy salivation in feeding; sometimes by rrue spasm of the pharynx and rejection of masticated balls of food.

Locally the buccal mucous membrane (cheeks, tongue, pillars of the fauces, etc.) exhibit ulcerations, with festooned borders, in size something between a florin and a five-shilling piece, covered with a greyish-yellow, earthy-coloured exudation adhering firmly to the subjacent parts. The margin of the ulceration is but slightly indurated, and the tongue preserves its mobility, except in cases of general deep-seated glossitis.

The condition may continue for weeks and months without improvement.

Intestinal Tuberculosis.-Tuberculous enteritis is always accompanied by tuberculosis of the mesenteric glands and of the sub-lumbar lymphatic chain. It is indicated at first by chronic tympanites and peritonism, unaccompanied, however, by atony of the rumen; at a later period by intermittent diarrhœa, which attains a maximum and is followed by constipation. Finally, when there exist numerous intestinal ulcers, the diarrhœa is profuse and intractable, the animals rapidly grow exhausted, and the disease spreads to other organs with startling rapidity.

The food is ill-digested and the fæces have a repulsive odour, as has also the gas which escapes from the rumen when it is punctured. 


\section{TUBERCULOSIS OF THE GENITAL ORGANS.}

In males, tuberculosis may attack the testicle and neighbouring organs and tissues; in females, the ovary, uterus, vagina, or udder.

Tuberculosis of the testicle is rare. Moussu appears only to have seen one case, namely, in the boar. The disease produces specific vaginitis and specific orchitis, the serous surfaces of the vaginal tunic becoming adherent, and tubercles forming in the depths of the testicle, whilst in time the testicle becomes the seat of fungoid growths.

Tuberculosis of neighbouring glands has only been observed in the case of the prostate. Clinically it is distinguished by symptoms which suggest difficulty in urination, and comprise frequent straining, efforts to micturate, dysuria, etc. Examination by the rectum reveals changes in the prostate, but affords no exact information as to their nature.

Tuberculosis of the genital tract in the female is usually a delayed complication of a preceding visceral tuberculosis, though it may occur as a primary disease, in which case the lesions are localised about the vulva or the vagina. Tuberculosis of the ovaries, Fallopian tubes, and uterus is much more common than that of the two organs just mentioned. It seems certain that the disease may be transmitted directly from the male to the female by copulation, in cases where the male animal has a lesion on the penis.

Tuberculosis of the vulva is rarer, and is indicated by swelling, sclerotic changes, and the presence of tuberculous nodules, varying in size between a lentil and a hazel-nut; after ulceration of these growths, a thick yellowish pus containing the specific bacilli is discharged.

Tuberculosis of the vagina is also indicated by hardening of the walls, sclerous infiltration, and the presence of deep-seated tuberculous nodules, which may or may not become ulcerated. It may follow uterine tuberculosis, the discharge from the uterus continually soiling and at length infecting the floor of the vagina. In such cases the lower wall of the vagina is thickened and infiltrated to a greater extent than the roof, and is sometimes intersected by transverse ulcerated suppurating folds. Bacteriological examination reveals the presence of bacilli.

Tuberculous invasion of the ovaries, Fallopian tubes, and uterus is externally indicated by signs of chronic metritis accompanied by a purulent discharge, which may or may not be foetid, but always possesses special characteristics. The neck of the uterus is half open, and the discharge is continuous. The pus is of a greyishyellow colour, ill-formed, grumous, or more frequently granular, and it sometimes accumulates in large quantities in the depressions of 
the vagina. Examination by means of the speculum is of great value in diagnosing such lesions. On examining the parts through the rectum, the walls of the uterus are found to be greatly thickened, sometimes indurated, bosselated or totally deformed.

The Fallopian tubes and ovaries may have attained enormous dimensions, and the normal anatomy of the parts is greatly altered both as regards dimensions and relations.

The almost inevitable consequences of tuberculosis of the genital organs are hypertrophy, induration or caseation of the subsacral and sublumbar lymphatic glands.

Udder. - Tuberculosis of the udder may be primary or secondary. When the infection is slight the results may escape notice for weeks or even months, the patients appearing to suffer only from sub-acute or chronic mammitis, while at the same time the milk preserves its ordinary appearance. In time, however, the mammitis be-

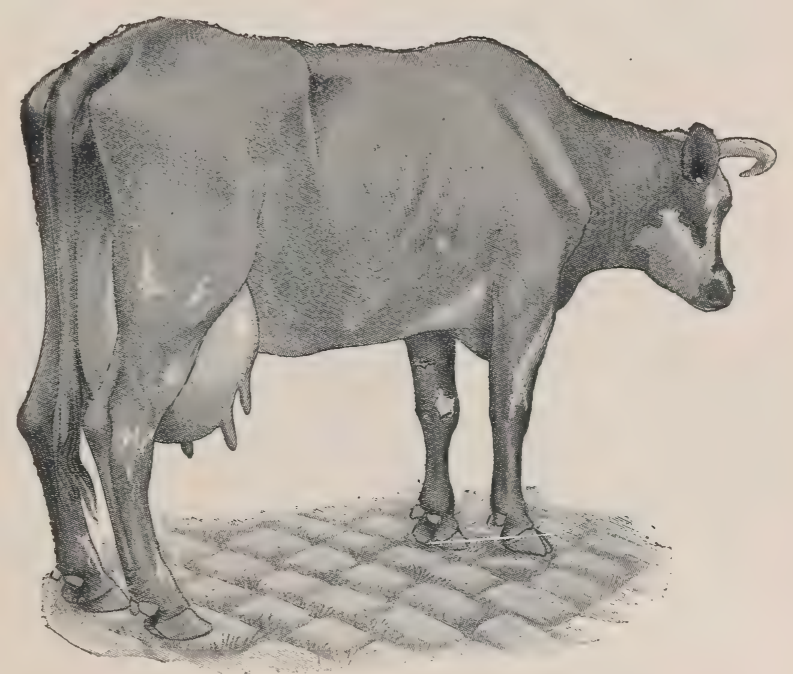

FIG. 279.-Hypertrophic tuberculous mammitis. comes aggravated, the infected regions are enlarged, and the secretion becomes grumous, serous, curdled, and of a yellowish colour, afterwards ceasing altogether. In some cases one quarter only is attacked, though total mammitis is more common.

These forms of tuberculous mammitis tend towards hypertrophy, local hardening, and the formation of deeply-seated cavities containing pus, the gland itself sometimes acquiring enormous dimensions. The retro-mammary lymphatic glands are invaded even before the gland itself is seriously attacked. For a longer or shorter time the udder may externally appear healthy, although on manual examination these lymphatic glands are found to be indurated and bosselated.

\section{TUBERCULOSIS OF BONES AND ARTICULATIONS.}

Tuberculosis of the bones is seen only in young animals, and chiefly affects the vertebral column and the bones of the head. The 
limb bones are attacked as a rule only in the vicinity of diseased articulations.

The vertebral lesions corresponding to those in Pott's disease in human beings are very difficult to discover before they produce complications, such as depression of the spine, compression of the spinal cord, paralysis, etc.

Lesions of the bones of the head or of the limbs are characterised by local deformity, destruction of osseus tissue, invasion of surrounding tissues, and by local symptoms peculiar to tumours originating in the periosteum.

Tuberculosis of joints produces special symptoms resembling those seen in the "white swellings" of man, that is, diffuse, œdematous,

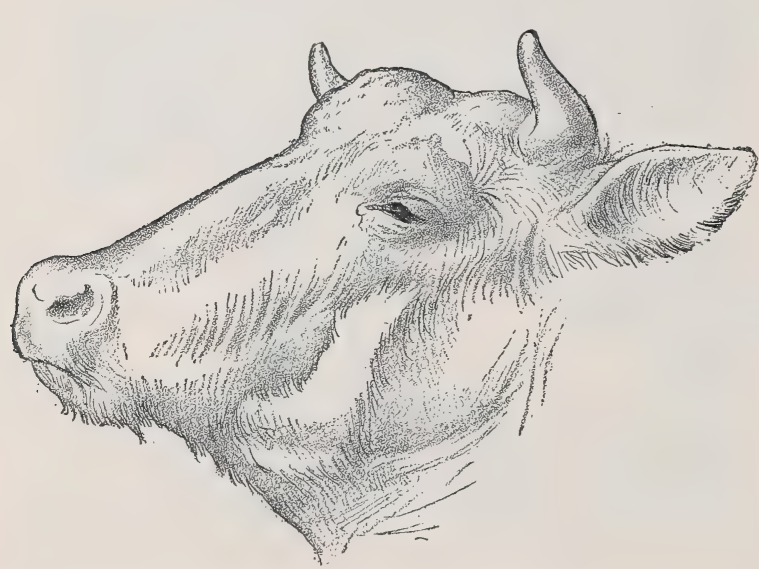

FIG. 280.-Perforating tuberculosis of the right frontal region. warm and moderately painful swelling of adjacent parts, accompanied by lameness of varying intensity. According to Guillebeau and Hess, many conditions described as strain or rheumatic arthritis are really tuberculous in character. They may remain stationary for a long time, or even recede under treatment. As a rule, however, these forms of tuberculous arthritis assume the fungoid type and prove incurable.

They are clinically distinguished from ordinary arthritis by the enormous swelling, which involves the extremities and a portion of the shafts of the bones. The adjacent muscles are chronically contracted, and the diseased joint is held semi-flexed. In course of time, if the patients are kept alive, abscess formation may occur, but this is seldom seen in practice, because the animals are slaughtered.

\section{TUBERCULOSIS OF THE BRAIN.}

Tuberculosis of the nervous centres, localised either in the meninges or the brain proper, may attack both young and old animals, not as a primary condition, but as a sequel to visceral disease, which, however, may have produced no outward indications, a fact that renders the diagnosis extremely difficult. 
When localised in the meninges, the disease produces the symptoms of ordinary meningitis, general weakness, vacillating, staggering or irregular gait, disturbed vision, variation in the size of the pupils, difficulty in swallowing, muscular twitching, cramp of the muscles along the upper margin of the neck, etc.

Tuberculosis of the brain proper seems more commonly to affect the anterior convolutions and the depths of the frontal and temporal lobes. It produces some of the symptoms of meningitis or symptoms suggestive of the existence of cœnurosis, as, for instance, walking in circles, lameness of central origin, without appreciable lesions of the limbs, prolonged kneeling, disturbed vision, generalised attacks of epilepsy or of Jackson's epilepsy, spasm of the pharynx, general

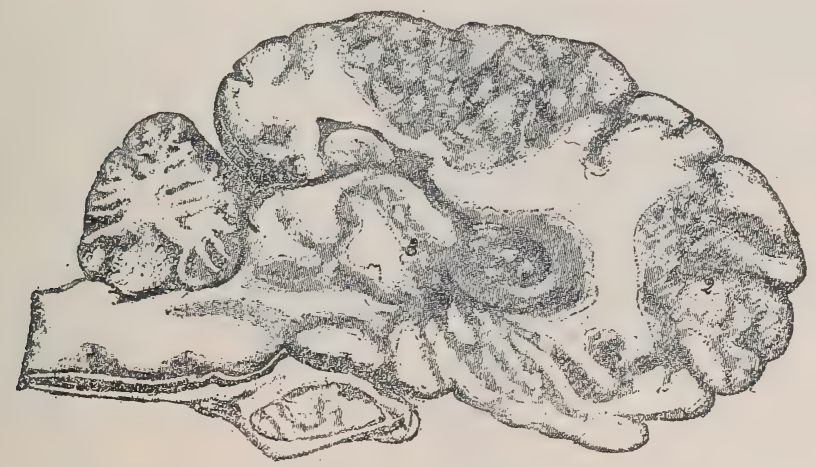

FIG. 281.-Tuberculosis of the brain. 1, 2 and 3, Tuberculous centres.

signs of compression of the brain or cerebral dropsy, dulness, coma, etc.

In the absence of pulmonary lesions it is extremely difficult to arrive at an exact diagnosis except after injection of tuberculin, for the above symptoms very closely resemble those of cœnurosis, brain tumours, and even tumours in the frontal sinuses.

\section{TUBERCULOSIS OF THE SKIN.}

Cutaneous tuberculosis is one of the rarest forms of the disease. It is distinguished by the formation beneath the skin of little hardened swellings varying in size between a hazel-nut and a walnut, and containing caseous or calcareous material. These swellings have no connection with the superficial lymphatic glands. They may be found grouped together within certain areas, or distributed irregularly over the whole body, particularly towards the base of the tail.

The condition may be mistaken for generalised sarcomatosis, from 
which, however, it is readily distinguished by microscopic examination of the contents of the swellings.

ACUTE TUBERCULOSIS-TUBERCULOUS SEPTICEMIA.

However rapidly the above-described forms of tuberculosis may develop, the disease as a whole is always of long duration, and continues for months, or even for years. The development of these chronic forms may, however, be interrupted by various influences which cause it to assume an acute character, either for a time or continuously. Each intermittent attack aggravates the condition of

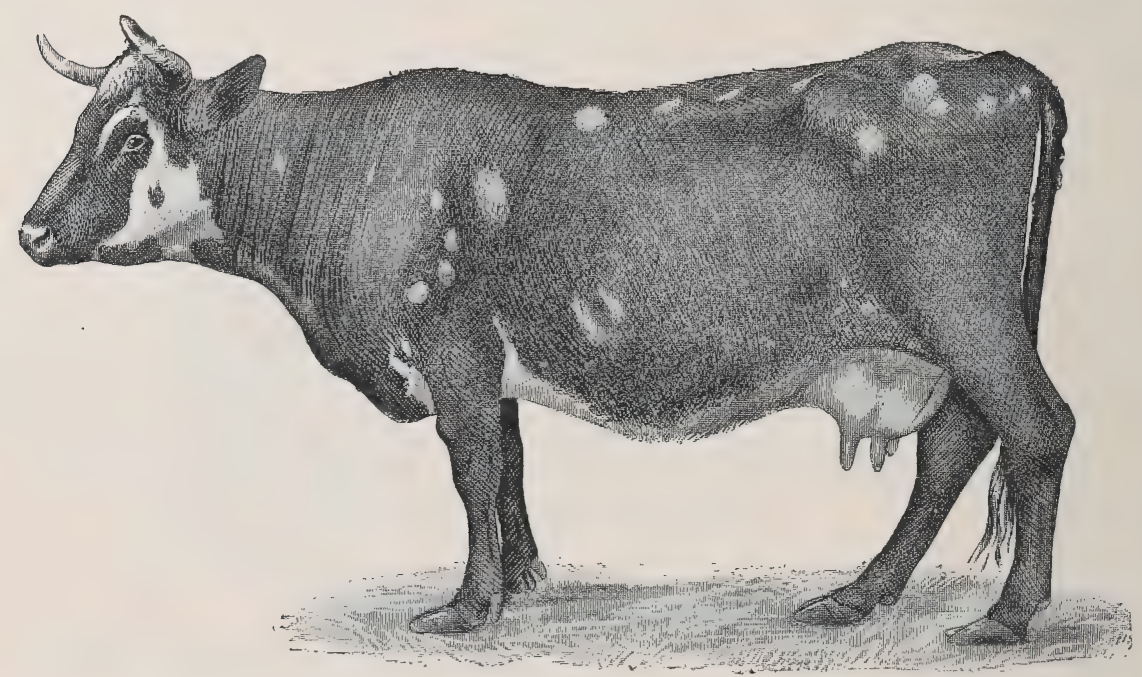

FIG. 282.-General appearance of a case of cutaneous tuberculosis.

the patient, but gradually subsides, with or without treatment. Continued attacks, however, rapidly lead to death; they may be seen in animals previously unsuspected of any grave disorder.

The dominant symptom is continuous fever, accompanied by signs of disturbance of any or all of the chief bodily functions.

The temperature rises to $102^{\circ} \mathrm{Fahr}$. $\left(39^{\circ} \mathrm{C}\right.$.), or even to $104^{\circ}$ or $105^{\circ}$ Fahr. $\left(40^{\circ}\right.$ or $41^{\circ} \mathrm{C}$.), with morning and evening remissions of some hours. The respiration is accelerated. On auscultation it is often difficult to discover signs of chronic tuberculosis. The lung is the site of repeated congestive changes, resembling those of bronchopneumonia or contagious pleuro-pneumonia. The pleura and walls of the chest become extremely sensitive, as in the last-named disease, and the abdomen may exhibit signs of peritonism, as at the beginning of acute peritonitis. The pulse rises to $80,90,100$, or even 
120 beats per minute, and the urine contains albumen in notable quantities. This condition continues for weeks without apparent diminution, the patients refuse food, lose flesh with startling rapidity, and finally die of exhaustion.

It would be impossible from these peculiarities alone to identify the nature of the disease which causes such progressive organic wasting, as the continued presence of fever prevents the use of tuberculin, but fortunately the preliminary changes in the lungs, lymphatic glands, genital tract, etc., are sufficient in most cases for the purposes of diagnosis.

Sheep, Goats, and Pigs.-In the other domestic animals tuberculosis is only of secondary importance to the practitioner.

It has been seen in the sheep and goat, but almost exclusively as the result of experiment. It must be understood, however, that prolonged co-habitation with diseased oxen or lengthened sojourn in contaminated places may easily produce tuberculosis in the goat, though the sheep continues to resist for a somewhat longer period.

Clinically such tuberculosis presents little interest on account of its rarity.

The same remark applies to pigs; nevertheless, an entire herd may become infected, and it may be necessary, after making a preliminary post-mortem, to examine the other patients. All forms of the disease occur in pigs, the lung being most frequently affected, but tuberculosis also attacks the intestine, udder, lymphatic glands, joints, etc. The pig, in fact, is extremely susceptible to this disease, whilst the sheep is only subject to it in a comparatively trifling degree.

Diagnosis. The clinical manifestations of tuberculosis are so numerous and so various that it is often an extremely hard task to form a diagnosis. Without doubt detection is relatively easy in wellmarked forms, such as tuberculosis of the lungs, lymphatic glands and genital apparatus, but even in such cases the symptoms must be reasonably well-marked.

At first, unless the lesions produce externally visible signs, diagnosis is impossible, and in the case of hidden forms, such as tuberculosis of the serous membranes, mediastinum, intestine, testicle, etc., all that can be done is to take into account the probabilities.

Clinical diagnosis is therefore possible, but only in exceptional cases can it be absolutely relied upon. Fortunately, methods of investigation increase and become more exact every day, so that the points which clinical examination is incapable of deciding are often cleared up in the laboratory. Bacteriological examination of morbid products, such as the nasal discharge, the products of suppuration, the milk or the diseased tissues, is a valuable means in many cases

D.C.

Z Z 
of determining the presence of the organism which causes the disturbance. In all cases, in fact, the tubercle bacillus should be sought for in order to confirm the diagnosis.

If this method cannot be employed, as for example in tuberculosis of the liver, brain, etc., and the diagnosis is uncertain, the use of tuberculin constitutes

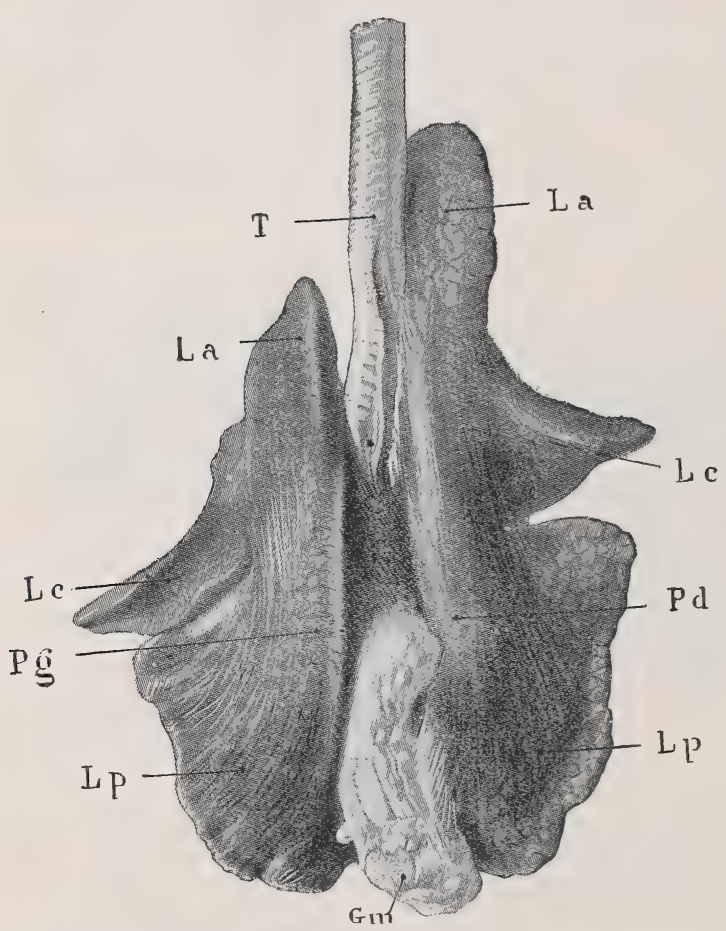

FIG. 283.-Tuberculosis of the posterior mediastinal lymphatic glands in a sheep which had been kept for two years in company with some tuberculous cows. PG, Left lung; PD, right lung; $\mathrm{T}$, trachea; $\mathrm{L} a$, anterior lobes; $\mathrm{L}$, middle cardiac lobes; $\mathrm{L} p$, posterior lobes; $\mathrm{G} m$, tuberculous and enlarged posterior mediastinal lymphatic glands. the surest and easiest method of coming to a conclusion. Nocard's researches have shown the precautions to be observed. A minimum febrile re-action of $2^{\circ} \mathrm{Fahr}$.

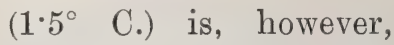
necessary before the existence of the disease can be affirmed.

Lastly, there remains a less rapid method, which aims at transmitting the disease to specially susceptible animals by inoculating with suspected materials, such as the nasal discharge, pus, milk or pulp of internal organs. This method is most valuable when bacteriological examination has failed and tuberculin has produced only doubtful results. The guinea-pig is the subject usually chosen, but some weeks, or even months, may elapse before definite results are obtained.

In those forms where ordinary methods of investigation prove sufficient it is well to bear in mind the symptoms which differentiate this disease from others closely resembling it.

Pulmonary tuberculosis, for example, should always be suspected whenever there exists frequent coughing, nasal discharge and poor bodily condition; if in addition to this respiration is found on auscultation to be rough, inspiration interrupted or rasping, expiration 
prolonged or blowing and the vesicular murmur non-existent, suspicion becomes almost a certainty. The diagnosis is even more assured if the respiration is of a blowing character at certain points, accompanied by snoring and sibilant râles and gurgling or cavernous souffles.

The distinction between pulmonary tuberculosis and chronic bronchitis or simple pulmonary emphysema is based on the increased resonance revealed by percussion in the latter case; the different character of expiration; the existence of a double breathing movement; the external appearance of the animal; the absence of bacilli from the nasal discharge, and the failure to react to tuberculin.

The condition cannot be mistaken for verminous broncho-pneumonia if the information furnished by auscultation and the results of microscopical examination of the discharge are taken into account, the eggs or embryos of strongyles being extremely easy to detect.

Tuberculosis of the pleura may be mistaken for peri-pneumonia if the observer trusts to percussion alone, but the auscultation sounds are then different, and an injection of tuberculin will remove any doubt.

Tuberculosis of the peritoneum is often suggested by the indications afforded by careful palpation (thickening of the walls of the abdomen, rigidity and sensitiveness), and is distinguished from ordinary acute peritonitis by the difference in the appearance of the animals and the absence of much fluid. Chronic exudative peritonitis and ascites also exhibit sufficiently well-marked characteristics to enable them to be differentiated from tuberculosis of the peritoneum, but this is certainly not true of adhesive peritonitis, and in cases of the latter kind tuberculin is the only means of confirming the diagnosis.

External tuberculosis of the retro-pharyngeal and cervical lymphatic glands resembles, at a first glance, simple inflammation of lymphatic glands, but in the last-mentioned disease the lesions are symmetrical and the glands' still retain a certain amount of elasticity, whilst in tuberculosis they are bosselated, hard, and sometimes fluctuating.

Tuberculosis of the mediastinum is suggested by difficulty in swallowing, especially if tympanites follows soon after eating, eructation is absent, and rumination is arrested.

A careful study of the development of the ulcerations will also in most instances make clear the difference between tuberculous stomatitis and simple stomatitis or stomatitis due to actinomycosis. Intestinal tuberculosis and tuberculous enteritis are sufficiently characterised by persistent intractable diarrhœa accompanied by tympanites, 
by the fœtid character of the fæces, and by moderate but continued fever. Chronic diarrhœa, which alone presents some analogy with this condition, is never accompanied by permanent tympanites.

Tuberculosis of the genital organs in male animals is always liable to be mistaken for simple orchitis and the development of tumours in the testicle; an injection of tuberculin will, however, indicate the nature of the lesion.

When the symptoms of genital disease or chronic mammitis in female animals suggest that the disease is of a specific character, the diagnosis can frequently be confirmed by a microscopical examination of the pus or milk.

Finally, should the practitioner hesitate as to the nature of the lesions which are the cause of arthritis, deformity of bones, cerebral symptoms, etc., tuberculin again will in most cases settle the question.

The prognosis in cases of tuberculosis is extremely unfavourable, whatever the form of the disease or its manifestations. Clinically the disease should be regarded as incurable in the strict sense of the word, however limited may be the lesions. The affected animals are not all doomed to immediate death: some may be kept alive, and may even serve an economic purpose without necessarily endangering others; it is sometimes possible to fatten them, though the risks probably far outweigh the advantages, but one can never rely on recovery in any particular case.

The gravity of this disease is the greater inasmuch as it assumes so many forms, any one of which may result in the infection of other animals.

All those forms of the disease, such as tuberculosis of the respiratory, digestive and genital tracts, in which virulent material containing bacilli is discharged realise these conditions. The patient becomes a source of infection to others of its kind, a fact which more than anything else renders the disease so dangerous to the farmer and breeder.

Only in cases where the lesions are closed (as in tuberculosis of the lymphatic glands, serous membranes, joints, etc.) can the sufferers be regarded as innocuous, and-as these lesions are exceptional or at least, as animals suffering from them are very frequently afflicted with open lesions from which bacilli are continually being discharged-every tuberculous animal must be regarded from a clinical standpoint as a constant danger to its neighbours.

This, however, must not be understood to mean that there are not different degrees of danger. It is quite certain that a patient with pulmonary caverns which are constantly throwing off enormous 
quantities of material full of bacilli is much more dangerous than another suffering only from slight bronchial or tracheal lesions, though the danger in the latter case is none the less always present.

Treatment. There is no really curative treatment of tuberculosis.

It must not be thought, however, that we are completely helpless and that the present condition of affairs must be allowed to continue indefinitely. Nocard and Leclainche have minutely laid down the lines to be followed as regards prophylaxis, though unfortunately the measures recommended cannot always be carried out.

Tuberculin having been proved an exact means of detecting tuberculous lesions in animals even where none were suspected, it is desirable, firstly, to test all the animals in a given establishment with tuberculin; and, secondly, to separate into classes (1) all animals which have reacted, and (2) those which have resisted.

The stables, etc., should then be completely disinfected by sweeping, washing first with hot water, then with strong antiseptic solutions, brushing over the walls with quick-lime solution and fumigating with sulphurous acid or formic aldehyde. The healthy animals should then be placed in one shed and the diseased animals in another. In order to render this system of isolation really efficacious the isolated animals and the healthy animals should have nothing in common, and the persons tending the two classes of animals, the buckets and other utensils, the watering places, etc., should be kept rigorously apart.

The animals known to be tuberculous should as rapidly as possible be prepared for slaughter, and if pregnant cows are included in the number the calves should be removed to the healthy stable immediately after birth and brought up either on boiled milk or by a healthy mother, experience having shown that congenital tuberculosis is of rare occurrence.

After the tuberculous animals have left the shed, this should again be thoroughly disinfected, in order to make it fit for the reception of healthy subjects.

Unfortunately such precautions can only be observed in model establishments. They necessitate expenses and immediate sacrifices of a very" serious character, and breeders too often view only the sacrifice without regard to the after benefits. For this reason the above system has only been practised in certain of the best known and best managed farms.

T'o ensure the full benefit of these precautions, and to prevent a fresh introduction of tuberculosis into the herd, every new animal introduced should be subjected to the tuberculin test. Unless this 
precaution is taken, there is always a risk of introducing a tuberculous subject, thus nullifying all the precautions previously taken.

The problem is therefore still very complex, and the system can only give good results when rigorously observed and followed out.

As, however, in spite of all precautions, animals regarded as healthy are always, under normal conditions of existence, more or less exposed to accidental infection, it is desirable to subject the entire herd to the tuberculin test annually. This would cause the immediate detection of any animals with latent infection, so that they could be removed from the herd.

These wise precautions might, if understood and observed, eliminate the disease from the country, but they depend on individual initiative, and have not as yet been grasped by the mass of small breeders, farmers, etc. This class only see the difficulties in the way of realising the idea, without appreciating the constant benefit which they would derive from it.

\section{SWINE FEVER-VERRUCOUS ENDOCARDITIS AND PNEUMONIA OF THE PIG.}

Although it is not contemplated in this work to deal with those disorders which, on account of their highly contagious or infectious character, can only be dealt with by legislative action and by processes of "stamping-out," it may be permissible to make certain exceptions. While we have made no reference to contagious pleuropneumonia of cattle, foot-and-mouth disease, rinderpest, anthrax and black-quarter we have devoted some space to Texas fever and tuberculosis, and give herewith a summary of the present state of knowledge regarding swine fever or hog cholera and a hæmorrhagic septicæmia of cattle known under various names in different countries and of very wide distribution.

\section{SWINE FEVER.*}

Swine fever may assume two distinct forms, viz., the acute and fatal and the non-acute or slowly progressive.

Symptoms. In the acute form all those symptoms which are indicative of a severe febrile affection are present. The animals are disinclined to feed; they present evidence of great prostration and lie about their dwellings in a listless manner sheltering themselves from cold; their skins are hot, their eyes partially closed, and they are obviously suffering from some severe constitutional disturbance. Within

* Report of the Departmental Committee re Swine Fever. (Annual Report of Board of Agriculture, 1896.) 
a very few hours after these premonitory symptoms hare set in the pigs become rapidly worse; they may or may not have a deep-red blush on the skin, which is more particularly noticeable on those parts of the body where there is an absence of hair, such as the inside of the thighs, the point of the axilla, and over the abdomen. Choleraic evacuations, having a most offensive odour, succeeding upon constipation, follow later on, and the animals die perhaps as early as the third or fourth day after the symptoms have first been observed.

In some instances the disease proceeds with great rapidity through a herd, the symptoms being of a most aggravated and pronounced character, and the outbreak attended with great fatality.

Generally speaking, the above description depicts the symptoms of swine fever in the acute form, more especially when it breaks out in a herd of young pigs.

In the non-acute form the disease progresses slowly, the clinical evidence is extremely obscure, the reddening of the skin, formerly regarded as being invariably present in swine fever, is absent, and beyond the fact that the animal is unthrifty, develops slowly, and perhaps has a constantly relaxed condition of the bowels, it may be asserted that there are no symptoms which could be regarded as absolutely indicative of swine fever, and nothing short of a post. mortem examination will enable even an expert to satisfy himself that the animal was affected with the disease.

As a general rule swine fever assumes this non-acute and slowly progressive form in pigs which have arrived at an age when their powers of resistance to disease are materially increased, i.e., in animals of eight or more months old; on post-mortem examination they are found to have been extensively diseased, more particularly in the large intestine, a portion of the digestive apparatus which does not appear to perform any very important function in connection with the nutrition of the animal, and so long as the stomach and small intestines remain healthy, pigs with a considerable amount of disease in the large. intestine may still keep up their condition for a considerable time.

Etiology. As regards the etiology of the form of swine fever prevalent in England no question now exists. It has been proved to demonstration by the bacteriological inquiry conducted by Professor McFadyean that it is due to a special pathogenic organism, a bacillus, which, after cultivation in artificial media, will produce in the healthy pig fed with the pure cultures the typical ulcerations which are found in the intestines of pigs affected with swine fever contracted in the ordinary way. 
The observations made by the veterinary officers of the Board of Agriculture caused them to doubt whether there was any disease of the lungs of pigs which, in the absence of lesions in the intestinal tract, could be accepted as evidence of swine fever.

The bacillus which produced swine fever when introduced in the healthy pig did not induce any special disease of the lungs.

Pathology and morbid anatomy. Swine fever, like typhoid fever in man, is essentially a disease of the digestive system, its chief characteristic being certain morbid changes of a well-marked nature which are found upon the surface of the mucous membrane in some part of the alimentary canal.

The changes referred to consist of what have been commonly described as the formation of a series of ulcers, single or confluent, distributed upon some part of the intestinal tract, varying in size and shape, of a yellowish-grey to black colour, and assuming as a rule a circular form. In some instances the lesions consist of diphtheritic exudations with necrosis of the lining membrane of the bowels.

These ulcers or necrotic patches may be found upon the tongue, tonsils, epiglottis, stomach, and small intestines, but they are more constant in the large intestines, especially the cæcum and colon. The lesions may involve the whole thickness of the mucous membrane, but seldom penetrate the other coats of the intestine; in fact, perforation of the peritoneal covering of the bowel is very rare in even prolonged cases of swine fever.

In cases where swine fever assumes the more acute form and death supervenes rapidly, it is usual to find that the small intestines are largely involved.

In the non-acute or slowly progressing form the lesions are more abundant in the large intestines, and in some instances the walls of the intestines become so thick as a result of infiltration into their structure and the excessively thick deposits upon the lining membrane, that it becomes a matter of surprise that the passage of the ingesta has been possible and that the animal has lived so long.

Next to the intestinal lesions the congested condition of the lymphatic glands, especially those of the mesentery, may be considered as most prominent among the pathological changes which occur in swine fever. Occasionally centres of necrosis are observed in the liver, and some writers refer to changes in or upon the spleen and kidneys.

The only lesions which can be characterised as absolutely typical of swine fever are those present in the bowels, the absence of which will justify any observer in declining to accept the case as one of swine fever without some further evidence or inquiry. It must, 
however, be distinctly understood that in the case of very young pigs which have died shortly after infection, there is often an entire absence of the lesions described, the only changes present being inflammation of the stomach or some part of the intestines.

Further, there are instances where older pigs have been slaughtered in the early stage of the disease in which no definite lesions have been found, and in such cases inquiry into the condition of the rest of the herd becomes necessary.

One most important feature in connection with the morbid anatomy of swine fever is the disposition which many animals have to recover from the disease; evidence of the reparatory process having often been detected in the intestines after they had been carefully washed.

Dr. Klein also maintained that many pigs took the disease in the mild form, and recovered without presenting any of the marked symptoms of swine fever.

It was found that, whether infected in the ordinary way or by direct inoculation, in some pigs killed only a few days after being infected the ulcers were occasionally seen gradually detaching from the surface of the intestines, and cicatrisation had already commenced.

\section{VERRUCOUS ENDOCARDITIS OF THE PIG.}

In the report of the Board of Agriculture for 1894 reference was made to the numerous instances in which the heart of the pig had been found affected with verrucous endocarditis.

This form of disease of the heart was known to veterinarians in Great Britain as far back as the year 1847. For reasons given in that report it became obvious that this diseased condition of the valves of the heart was not produced by swine fever. The question arose whether in addition to swine fever another disease existed, known on the Continent under the name of swine erysipelas. The importance of this question will be appreciated when it is explained that on the Continent swine erysipelas is classed among the contagious diseases of the pig.

The clinical evidence of the disease called swine erysipelas on the Continent appears to be more or less discoloration of the skin, similar to that which is frequently observed in swine fever, together with the occasional presence within the warty growths upon the valves of the heart of a bacillus which is regarded by Continental authorities as the cause of the disease.

Early in the inquiry it was ascertained that a bacillus identical with that found in swine erysipelas was also present in the diseased portion of the valves of the heart of the pigs in this country. 
But the inquiries made did not corroborate or favour the suggestion that the disease which produced these morbid growths was in any way infectious or contagious. Such inquiries as could be made led to the opposite conclusion, since in every instance where the cases could be followed up it was ascertained that the deaths had been quite sudden, were limited to a single animal, and that those in contact remained in perfect health.

At this stage the all-important point to determine was whether the disease which existed in this country, "verrucous endocarditis," was communicable from pig to pig, and with this object numerous experiments have been conducted to discover whether the bacilli found within the hearts of diseased pigs were pathogenic to healthy swine.

A large number of healthy pigs have been fed or inoculated with the blood, the diseased portions taken from the valves of the heart, and with artificial cultures of the bacilli obtained from the heart, but in no instance has the attempt to produce this disease been successful.

\section{PNEUMONIA OF THE PIG.}

The occasional association of pneumonia with or without pleurisy in cases of swine fever has led many veterinarians in England to regard lung complications as one of the lesions produced by that disease.

In the Board of Agriculture's report for the year 1894 a description was given of the various diseases in the lungs of swine which had come under notice, and it was therein stated that the Board had been unable to discover any special lesion of the lung which would warrant them in stating that it was indicative of swine fever or due to contagion.

It is an indisputable fact that pigs are extremely liable to pneumonia and pleurisy. But as the clinical appearances present in the lungs examined in no wise differed from those which take place in the lungs of other animals which have been exposed to cold or septicæmia and other causes, the Board's officers have never accepted these lesions as being specific.

It is well known that both in Germany and the United States outbreaks of pneumonia of a contagious nature attributed to the presence of a bacillus pathogenic to the pigs of those countries are reported to occur. Indeed, contagious pneumonia of swine under the names of schweinesuche in Germany and swine plague in America are regarded as one and the same disease.

In view of the fact that in a large number of cases pneumonia, more or less extensive, sometimes associated with pleurisy, was found 
among the specimens forwarded to London, it was considered desirable that the departmental committee should institute a series of experiments to decide whether we had in this country a form of pneumonia in the pig which was communicable from one pig to another.

Accordingly a series of bacteriological experiments were conducted by Professor McFadyean with a view to isolate, if possible, a microorganism which would be capable of inducing pneumonia in healthy pigs. A number of diseased lungs, some of which were taken from pigs affected with swine fever, were examined microscopically by him, and, as was to be expected, several organisms were isolated, but they proved to be morphologically and culturally different from the bacillus of swine fever. Inoculations were carried out with these organisms not only subcutaneously but directly into the lung through the walls of the chest, and feeding experiments were also conducted. The results of these experiments were entirely negative; a certain amount of local injury was caused to the lungs at the seat where they had been punctured, but in no case was either pneumonia or swine fever induced.

The experiments have therefore demonstrated that the pneumonia found in the lungs of pigs affected with swine fever is not due to the swine fever bacillus.

The departmental committee arrived at the conclusion that the pneumonia which is occasionally encountered as an independent disease of the pig or in association with swine fever is not ascribable to contagion, but to the presence of organisms which are generally saprophytic in their mode of life, and which only in particular circumstances (such as lowered vitality and diminished resistance on the part of the pig) are able to multiply in the air passages and lung tissue and thus induce pneumonia ; and it appeared to the departmental committee that in this country pneumonia of the pig is sporadic and not contagious or epizootic.

\section{CONCLUSIONS.}

There is now no reason whatever to believe that there exists at the present time in Great Britain any disease of a contagious nature affecting pigs other than swine fever. The disease of the heart, "verrucous endocarditis," and the pneumonia which are so frequently met with in pigs cannot be regarded as lesions indicative of an attack of swine fever.

As regards verrucous endocarditis and pneumonia, it may safely be said that they do not exist in England in a contagious form.

Considering all the evidence, it may reasonably be concluded that the departmental committee were correct in their views when they 
stated that "the evidence obtained during the whole inquiry justifies the conclusion at which they have arrived, viz., that there is no epizootic of swine except swine fever in any part of the United Kingdom which requires to be dealt with under the provisions of the Act of 1894."

Finally, it may be said that the great factors in perpetuating swine fever will always be pigs which are affected with that disease in the less fatal and unrecognisable form. These animals are constantly distributing the germs of swine fever through their highly infective evacuations wherever they may be taken during the whole period of their illness, and the final extinction of the malady must depend upon the possibility of enforcing measures which will have the effect of preventing the movement of pigs affected with sivine fever in this particular form.

\section{HEMORRHAGIC SEPTICEMIA IN CATTLE.}

In 1902 Drs. Wilson and Brimhall, of the State Board of Health of Minnesota, U.S.A., described under the title of "hæmorrhagic septicæmia of cattle" a widespread infectious disease of bovines which has the following general characteristics:-The disease is distributed the world over, but is apparently most common in low-lying regions, and most general in wet seasons. The animals attacked are of all ages. The onset of the disease is sudden, its course rapid, and its termination usually (90 to 98 per cent.) fatal. Thirty to 90 per cent. of all animals in an infected herd die. The clinical symptoms are refusal of food, cessation of rumination and lactation, initially increased temperature $\left(107^{\circ}\right.$ to $109^{\circ} \mathrm{F} .: 42^{\circ}$ to $43^{\circ} \mathrm{C}$.), rapid, laboured breathing, sometimes bloody discharge from nostrils, bladder, and bowels, and non-crepitant swellings in the throat region, back of shoulders, or about the fetlocks. The most striking pathological lesions are hæmorrhages from 1 millimètre to 20 centimètres in diameter, throughout the subcutaneous, submucous, subserous and intermuscular connective tissue, infiltrating the lymphatic glands, and involving several or all of the internal organs. The spleen is neither enlarged nor darkened. The causative bacteria, which may be isolated from the larger hæmorrhagic areas, lymph glands, heart's blood, lung, spleen, etc., have the following distinguishing characteristics:

Ovoidal bacilli, with rounded ends of 0.5 to 0.8 microns in transverse diameter, and 1.0 to 1.5 microns in length; sometimes paired and sometimes in chains of three to six individuals. The bacilli in the tissues exhibit polar staining with an unstained "belt" or "middle piece." They are non-capsulated, non-spore-forming, non-Gramstaining, and non-motile. They grow best aërobically at $98.5^{\circ} \mathrm{F} .\left(37^{\circ} \mathrm{C}.\right)$, 
though capable of developing anaërobically and at room temperature; prefer the depths rather than the surfaces of media; grow feebly, if at all, on potato; fail to liquefy gelatine; produce acid, but no gas in glucose media, neither acid nor gas in lactose media; and develop varying amounts of indol and phenol in peptone solution. The organisms have been named Bacillus borisepticus. The lesions of the disease are reproduced in cattle and other animals by inoculation of pure cultures of the organism.

It should be insisted upon that the identification of the disease in a locality in which it has not been previously described, or by veterinarians not having had previous experience therewith, shall take into consideration-(a) the essential clinical symptoms; (b) the pathological lesions as observed before the onset of decomposition; and (c) the morphological and biological identification of the specific bacilli.

The following is a tabulated list of the principal epidemics so studied and reported to January 1st, 1901 :

Table showing the Principal Epidemics of Homorrhagic Septiccemia in Bovines due to Bacillus bovisepticus.

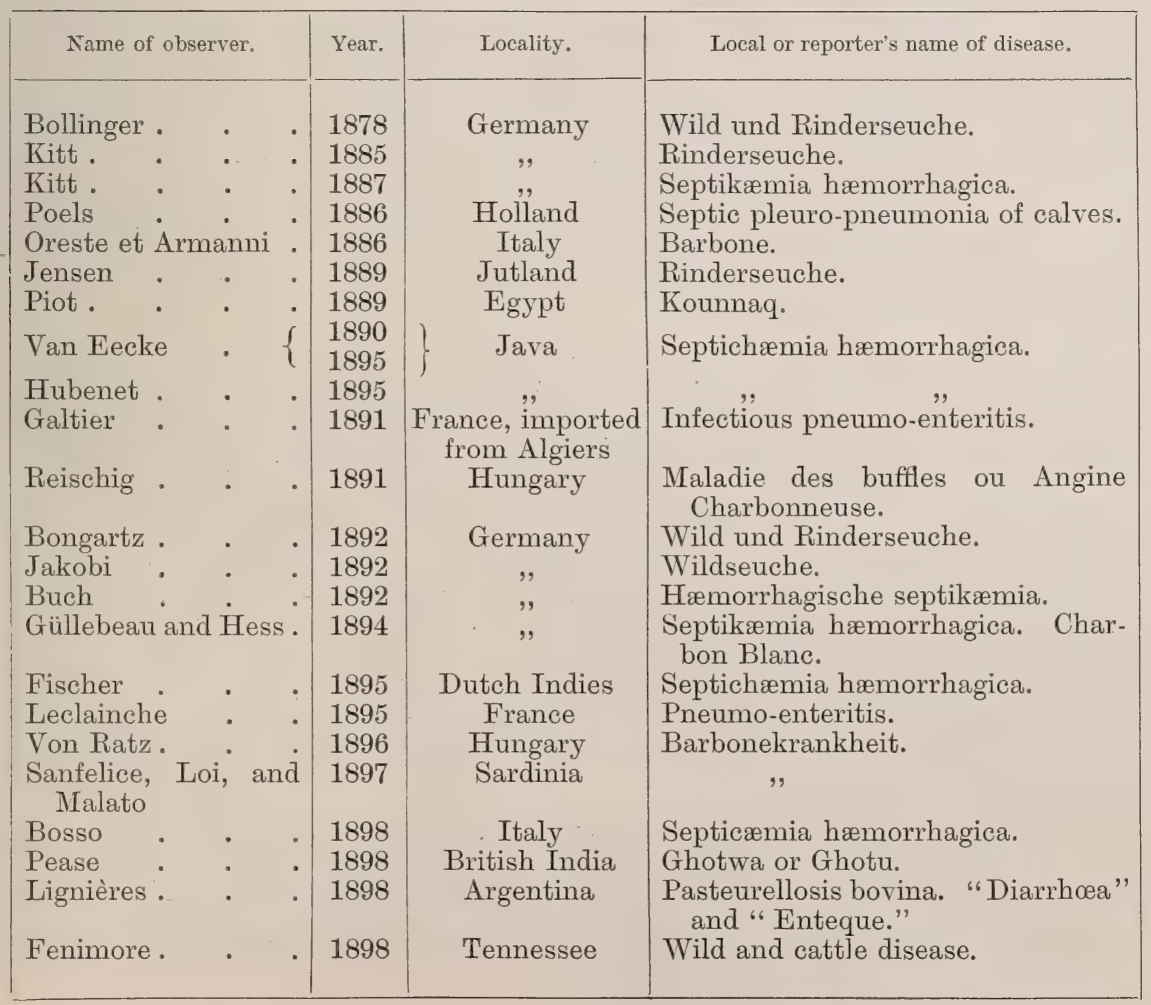


It may not be out of place to give at this point a short list of the principal infective diseases of definitely known ætiology, with which hæmorrhagic septicæmia in cattle has been, and may readily be, confused:

Name of disease.

Anthrax .

Black-quarter.

Septicpneumo-enteritis of calves (Galtier)

Septicæmia of calves

\section{Diagnostic points.}

Altered condition of blood, enlarged spleen, presence of $B$. anthracis, etc.

Usually localised lesion, crepitant tumour, presence of $B$. anthracis symptomatici.

Due to a spore-bearing bacillus-_" Pneumobacillus septicus" - which grows rapidly on potato.

Due to typhoid-like bacilli.

\section{CONCLUSTONS.}

(1.) Eight outbreaks of hæmorrhagic septicæmia in cattle due to B. borisepticus occurred in Minnesota from August to December, 1900.

(2.) So far as can be determined, the only other outbreak of this disease hitherto published as occurring in America was one near Knoxville, Tenn., in 1898. The foci of the disease have also apparently been present in Texas and the district of Colombia. No relation can be traced between the disease elsewhere and the present outbreaks, nor between any two of the present ones.

(3.) Of 160 animals in the eight herds, sixty-four showed symptoms of the disease, and all such died-a mortality of 40 per cent. of all the animals in the herds, and of 100 per cent. of those showing symptoms.

(4.) The chief symptoms were loss of appetite, fever, stiffness, swelling of the legs and throat, and a black, tarry, or bloody discharge from the bowels. Bloody urine and bloody nasal discharge were present in some cases. Death occurred usually in from six to twenty-four hours after the first appearance of symptoms.

(5.) The chief lesions discovered at autopsy were ecchymoses, and small and large hæmorrhagic areas in the subcutaneous connective tissues, muscles, lymph glands, and throughout the internal organs. The cervical lymph glands, heart muscle, and alimentary canal were most affected. The spleen was not enlarged nor darkened (except after onset of decomposition).

(6.) From the twenty-two animals on which autopsies were made the same bacillus was obtained from all the tissues examined. Where the examination was made immediately after death-nine cases-it was unmixed with any other organism. 
(7.) The bacillus was identified as belonging to the hæmorrhagic septicæmia group of Hüppe, best specifically designated as B. borisepticus; and besides causing hæmorrhagic septicæmia in cattle (synonyms-rinderseuche, buffleseuche, barbone, khounnaq, charbon blanc, ghotra, pasteurellosis bovina, etc.), closely resembles, if, indeed, it is not identical with, the bacilli causing wildseuche, swine plague, schweineseuche, rabbit septicæmia, chicken cholera, grouse disease, duck cholera, etc. The organism was studied in direct coverglass preparations, parallel cultures in and on various media, and by inoculation of animals in which the characteristic lesions were reproduced, and from the tissues of which the inoculated bacilli were recovered in pure culture.

(8.) An attempt was made to immunise cattle by the injection of filtered, and later of the killed, cultures of the bacillus. 'The chief difficulties met with were in maintaining the virulence of the bacillus on artificial media, and in determining the proper dosage. The experiments were too few, and the results not sufficiently tested to warrant conclusive statements as to the protective value of the inoculations, but it would appear that a fairly high degree of immunity was produced.

(9.) The prompt removal of the dead animals and isolation of sick ones, accompanied by thorough disinfection by fire, carbolic acid, corrosive sublimate, and freshly-slaked lime, apparently served to check each outbreak within a short time after the measures were instituted. 


\section{SECTION XI.}

\section{OPERATIONS.}

\section{CHAPTER I. \\ CONTROL OF ANIMALS.}

\section{CONTROL OF OXEN.}

The safe and efficient performance of surgical operations renders it necessary that the animal should first of all be placed under complete control. This precaution, therefore, is the first to merit attention. Animals are either secured completely or to a more or less limited extent, according to circumstances. The ox, for example, may be secured by the head, one or more limbs may be fastened, or, by being placed in a trevis, the whole of the animal may be secured.

\section{PARTIAL CONTROL.}

(1.) The simplest method of securing the ox is to grasp the nostrils or lower extremity of the septum nasi between the thumb and index finger of the right or left hand (Fig. 57).

(2.) This method may be rendered more complete by the assistant passing his arm from behind forward over the animal's head between its horns, and then grasping the nostrils as above described, whilst one of the horns is firmly held with the other hand (Fig. 58).

(3.) A third method consists in fixing the head to a post, tree, fence, or other solid body, by passing a rope round the base of the horns and tying it to the object selected.

\section{CONTROL OF THF LIMBS.}

To prevent the animal kicking or moving about during an operation it is sometimes necessary to fix the limbs. A front leg may be lifted as for shoeing, or may be kept lifted by means of a rope passed around the fetlock, over the withers, downward between the front legs, outside the forearm, in front of the chest and inside the pastern, after which one or more turns may be $\mathrm{m}$ de around the pastern to prevent the rope slipping. 
A hind limb may be secured in a simple way by passing the end of the tail round it in front of the hock (Fig. 284); any violent and extensive movement of the limb is then necessarily painful to the animal owing to the tension of the tail.

Greater security, however, is given by passing a rope backward and forward around the hind legs, above the hocks, in the form of

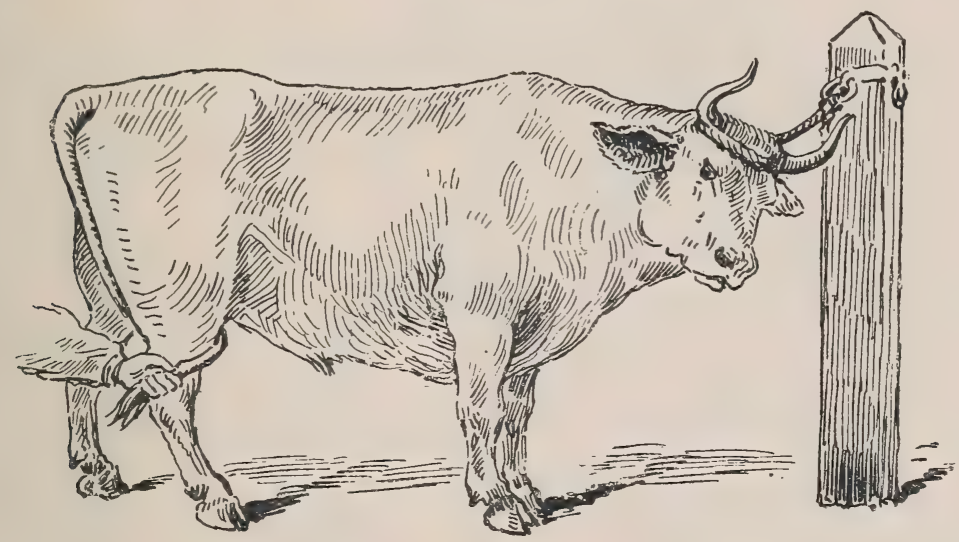

Fig. 284.-Fixing the head and a hind limb.

a figure of eight (Fig. 285). This does not prevent all movements of the hind limbs, but it limits them and secures both animal and operator from danger.

Should it become necessary to examine the hind foot or interdigital space, it is useful at times to pass a loop of cord round the leg in the region of the tendo Achillis, and to twist and tighten this loop by a short, stout stick passed through it; this is the leg twitch described in Dollar's "Operative Technique," p. 7.

For such examinations, however, the animal is usually placed in the trevis, or is secured to the side of a long waggon (Fig. 286).

To secure the limbs beneath the abdomen a strip of webbing or a rope is fastened round

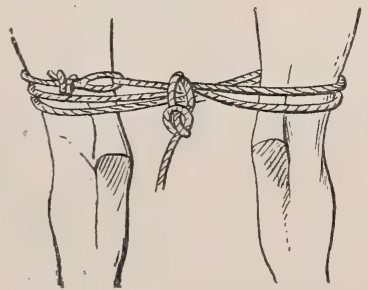

FIG. 285.-Fixing the hind limbs. the pastern, passed between the fore limbs, then in front of the shoulder of the opposite side, over the withers and beneath the elbow of the same side, being secured with a slip-knot.

For castration in the standing position a hind and a fore limb may be fastened together, as shown in Fig. 287.

In examining the sole of the hind claws, the animal's head may D.C. 
be secured to a tree, and the hind limb lifted by a strip of webbing or rope fixed to the body of a waggon (Fig. 288).

GENERAL CONTROL.

General control in the standing position can only be made really

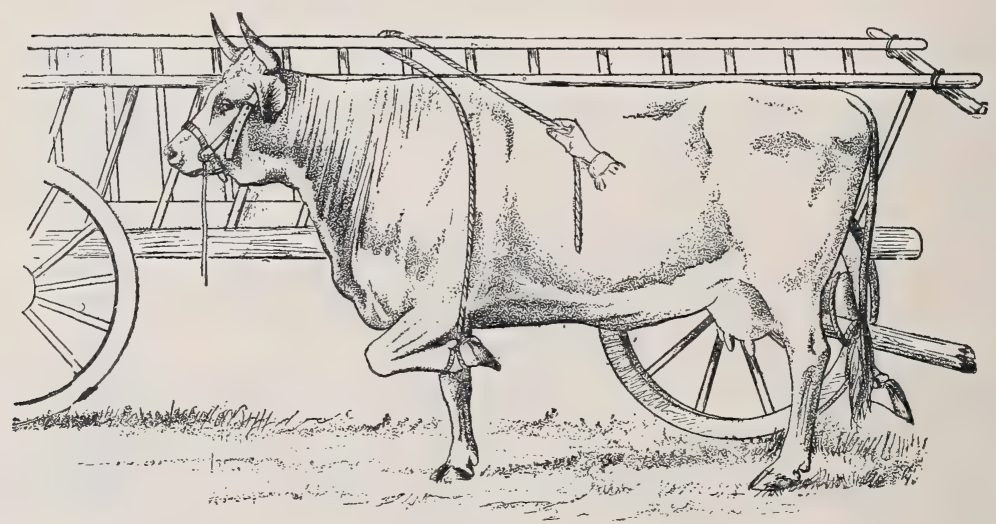

FIG. 286.-Fixing a front limb. Examining a hind foot.

effective by using the trevis, in which both the head and the fore and hind limbs are secured.

The practitioner, however, is often content with much less perfect

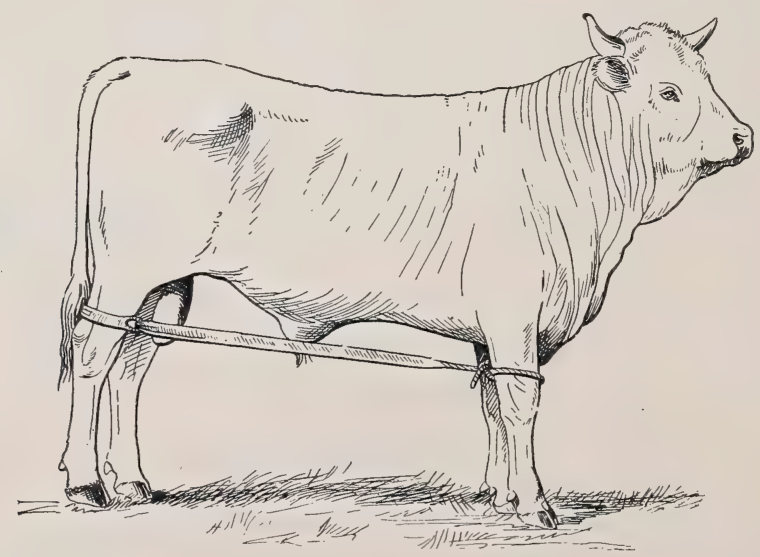

FIG. 287.-Fixing two limbs of one side for the operation of bistournage.

methods, using either the so-called "bulldogs" or the nose ring, which may usually be so manipulated as to control all but the most dangerous animals. The nose ring is seldom employed except for bulls, which 
are usually ringed at an early age; the "bulldogs" can be employed and removed at will.

In inserting a nose ring the head is securely fixed, the centre line of the muzzle is grasped with the left hand, and the septum nasi is pierced with a straight bistoury, the cutting edge being turned in a backward direction; the aperture being thus prepared, the ring is introduced and fixed. Some operators prefer to use a trocar, slightly exceeding in diameter the ring to be worn; in that case the manual technique is the same. The puncture is made, the trocar alone is withdrawn, and the canula remains in situ; one extremity of the ring is then inserted into the canula, and both

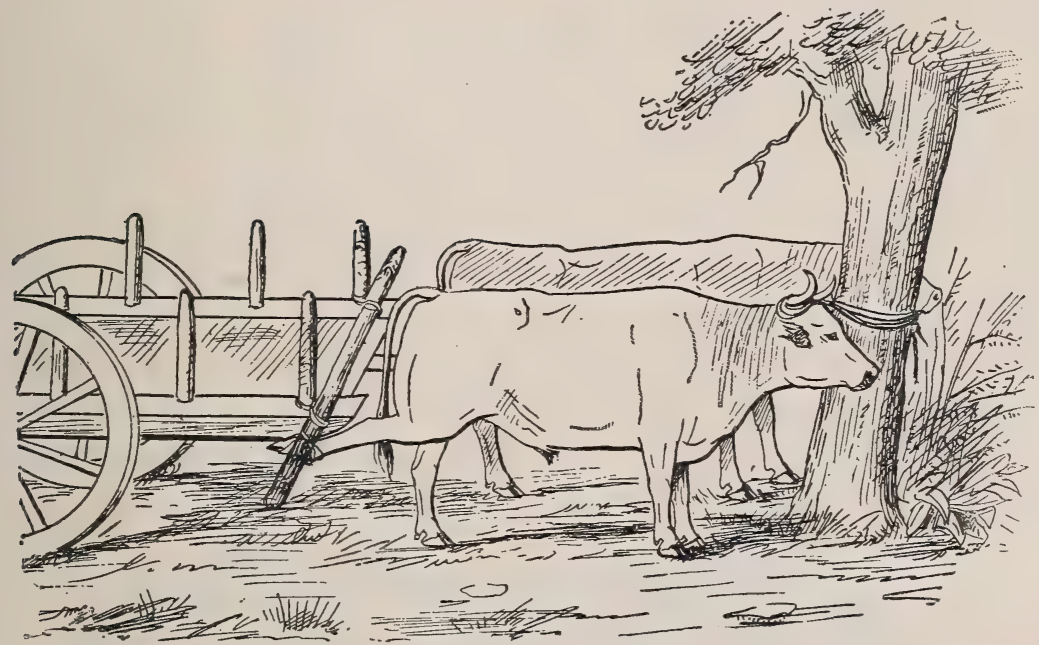

FIG. 288.-Fixing an ox for the examination of a hind limb.

are drawn through the septum together. There is no difficulty in thus inserting the ring, though puncture with the trocar is perhaps rather less convenient than with the bistoury.

Relative control without casting may also be effected by attaching the end of the tail to the base of the horns or the head to the cannon bone of a front or hind limb. These methods, however, are of little value except to prevent animals at grass from escaping entirely during operation.

\section{CONTROL BY CASTING.}

Oxen should always be cast on a thick straw bed to avoid fracturing the horns.

The simplest method consists in using hobbles and ropes, as for the horse. 
Another, though less practical, method consists in using two long ropes, each fixed to the base of the horns and passed first between the front, then the hind limbs, round the hind pasterns from within

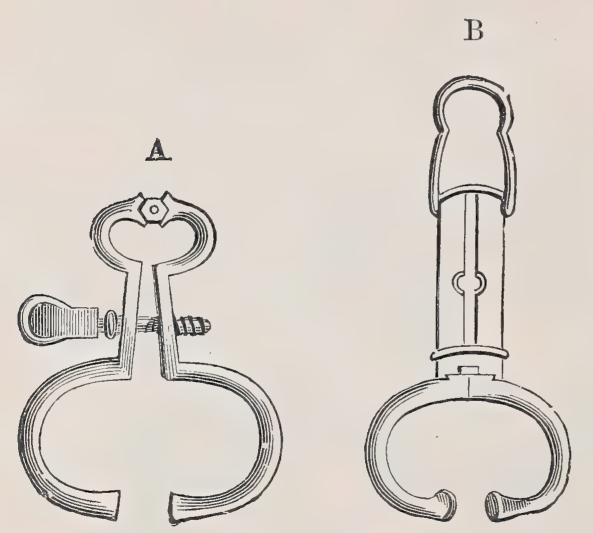

Fig. 289.-A, "bulldogs" operated by a screw ; B, "bulldogs" with a sliding ring. outwards and then brought forward (Fig. 292). When the ropes are drawn tight by assistants standing in front of the animal, the latter usually falls on its hocks and rolls over to the right or left, according to the direction given to it.

A third method is particularly useful in young or feeble animals. It consists in fixing around the horns a rope about eight to ten yards in length, the free end of which is carried along the upper margin of the neck and back. A loop is formed embracing the base of the neck, a second behind the shoulders in the region occupied by the girth, and a third around the flanks (Fig. 293). By pulling in a backward direction the rope is drawn tight, and the animal first backs and afterwards falls on its hocks, subsiding either to the right or left, as in the case previously mentioned.

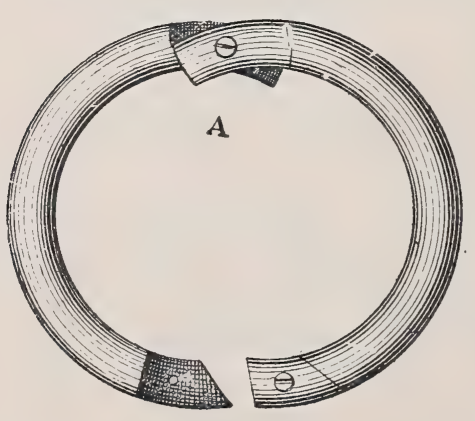

FIG. 290.-Nose ring open for insertion.

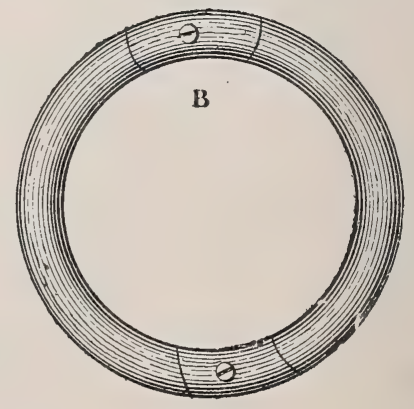

FIG. 291.-Nose ring closed.

In using either of these two methods it is very important, as soon as the animal falls, to secure the limbs in a certain way, according to the nature of the operation to be performed. This can be effected with hobbles and ropes passed round the hocks, above the knee, etc. 


\section{CONTROL OF SHEEP AND GOATS.}

The above animals may be secured, whilst standing, by grasping the head and neck or, when cast by crossing the front and hind

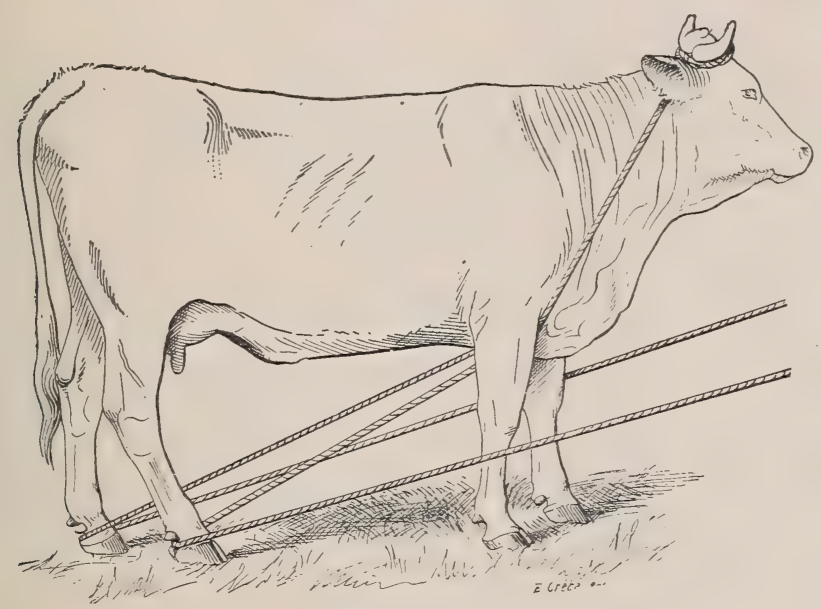

FIG. 292.

limbs in the form of an $\mathbf{X}$ and tying a soft rope or piece of webbing round the crossing of the limbs.

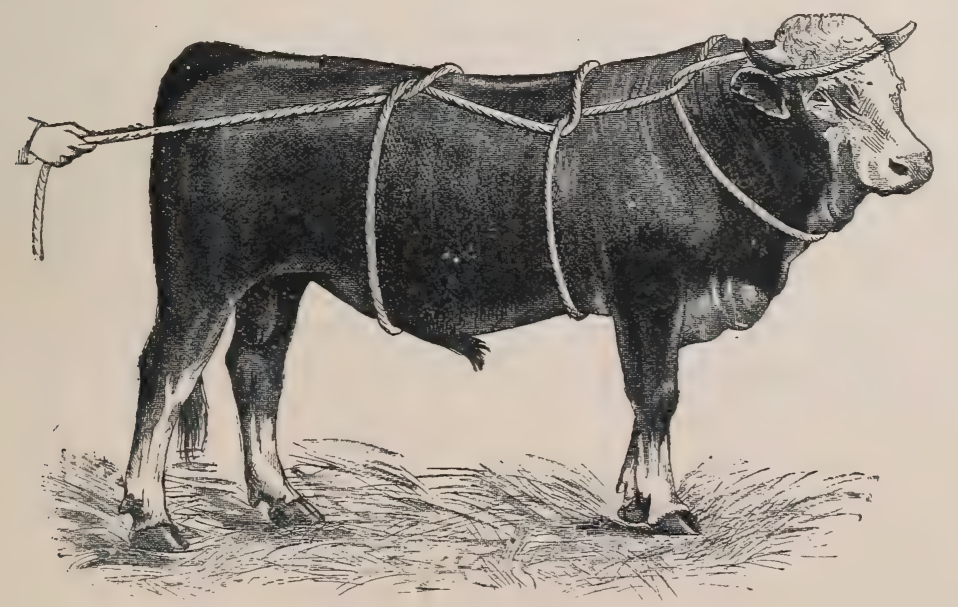

FIG. 293.

\section{CONTROL OF PIGS.}

No difficulty is usually experienced in controlling young pigs, either when standing or cast, only one or two assistants being required, but 
aged animals are more difficult and more dangerous to deal with, and by their tusks sometimes inflict severe wounds.

In the standing position they can be partially fixed by passing a running loop behind the canine teeth "of the upper jaw, but should the examination to be carried out prove to be of a difficult character it is best to cast the animal.

A strong assistant grasps one of the hind limbs by means of a running loop, fixed, for example, above the right hock. He rapidly slides his left knee towards the front of the left side of the chest, passes his left hand over the withers, and by the combined use of his knees and arms throws the animal on its left side, controlling as far as possible the struggles of the right front and hind limbs, which he grasps with his hands.

The animal is then further secured by rapidly passing a thin rope in figures of eight around the front and hind limbs. If necessary all four legs may be brought together and fastened by a rope passed round the region of the pastern; a muzzle can afterwards be applied to prevent biting.

\section{AN/ESTHESIA.}

Oxen rarely receive general anæsthetics, though in certain obstetrical cases they may be necessary. Ether and chloroform are given by inhalation, and chloral of 10 to 20 per cent. strength by intravenous injection. In utilising the latter method the injection should be made slowly, the pulse and heart being closely scanned to prevent cardiac syncope. The dose of chloroform varies with the size of the animal, 2 ounces often sufficing for a full-grown ox. The same methods may be used for sheep, goats, and pigs, the doses being suitably altered. (For fuller particulars see Dollar's "Operative Technique," pp. 44 to 70 .)

Most frequently, however, the surgeon contents himself with producing local anæsthesia by the injection of a 4 to 10 per cent. solution of cocaine. 


\section{CHAPTER II.}

\section{CIRCULATORY APPARATUS.}

\section{BLEEDING.}

Bovine animals are usually bled from the superficial jugular, or the mammary vein.

Bleeding from the Jugular.-The animal having been suitably fixed, the jugular is raised by means of a cord drawn tightly round the base of the neck, and the vessel is opened with a fleam about the middle of the neck.

The skin of the ox being thick, a long-bladed instrument is necessary. When the bleeding ceases, the cord is removed: some practitioners take no precautions as regards the wound; it is better to insert a pin suture.

Bleeding from the jugular may also be performed with the trocar, particularly in animals with fine, thin skin.

Bleeding from the Mammary Vein.-The mammary vein may be opened with the fleam, the straight bistoury, or the lancet. The head is firmly fixed and the hind limbs controlled by a rope passed in a figure of eight above the hocks.

In bleeding on the left the operator places himself at an angle to the animal's side, opposite the hypochondriac region, with his back towards the animal's head, and holds the fleam in his right hand. To operate on the right-hand side the fleam is held in the left hand.

This method of bleeding always causes thrombus formation, on account of the low position of the opening in the vein. The animal's bed should be kept very clean, in order to prevent any local infection which might cause hæmorrhagic or suppurative phlebitis. The lancet or bistoury can only be used in animals with very fine skin.

In bovine animals small quantities of blood are sometimes taken from the facial vein or the veins of the ear or tail.

\section{BLEEDING IN SHEEP.}

On account of the quantity of fatty tissue and wool covering the jugular furrow in the sheep, bleeding is scarcely practicable at that 
point. The operation is usually performed on the angular vein of the eye, the external saphenous vein, or the subcutaneous vein of the forearm.

In operating on the facial vein the animal's head is firmly held, the operator compresses with the fingers of his left hand the facial vein at the point where it passes into the maxillary fissure, and with a lancet opens the angular vein of the eye or one of the other branches of origin which project prominently beneath the skin. Bleeding ceases as soon as the pressure is relaxed.

In the case of the external saphenous vein, the vein is raised by compressing the middle region of the limb and the vessel is opened with a lancet, a little above and towards the outside of the hock.

The subcutaneous vein of

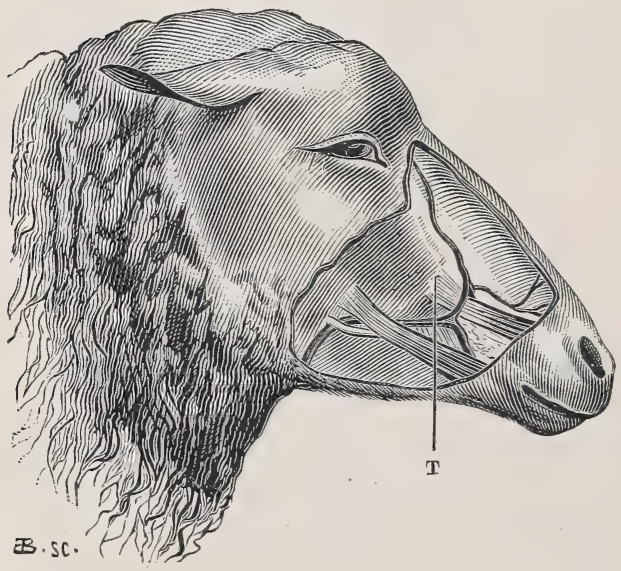

FIG. 294.-Angular vein of the eye and facial vein. the forearm can be raised by compressing the fore limb below the elbow. The vein is visible throughout the length of the inner surface of the radius, and can easily be opened with a lancet.

It is also possible to withdraw small quantities of blood by opening the marginal veins of the ear.

BLEEDING IN THE PIG.

Breeders sometimes bleed by slitting one of the animal's ears or cutting the tail. It is preferable to bleed with a lancet from the marginal veins of the ear, the external saphenous vein a little above the hock, or the subcutaneous vein of the forearm.

\section{SETONS, ROWELS, PLUGS, OR ISSUES.}

Although the application of setons is still practised in horses, that of "issues" has largely been given up in bovine animals, although some practitioners still regard issues as of considerable value and as producing effects similar to, or better than, those of sinapisms.

They are usually inserted in the region of the dewlap; the materials employed comprise black and white hellebore, veratrine and stems of clematis. 
Two methods are practised.

In the first, a transverse fold is raised in the skin of the dewlap, which is divided with a stroke of the bistoury, leaving a little aperature in the skin. By introducing the rounded ends of a pair of curved scissors the subcutaneous connective tissue is broken down, leaving a little space beneath the skin, into which the plug is introduced. Swelling takes places very rapidly-in twenty-four to forty-eight hours it is very considerable-and if the substance. - employed is violent in its action, like hellebore, it must be withdrawn, as otherwise considerable sloughing takes place. To facilitate this object a thread or piece of string is usually attached to the plug before it is inserted.

In the second method, the irritant material is attached to, or smeared on, a strip of broad linen tape which is passed in precisely the same manner as in the horse (see Dollar's "Operative Technique," pp. 107-111). 


\section{CHAP'TER IIII.}

\section{APPARATUS OF:LOCOMOTION.}

THe customary operations on the apparatus of locomotion are

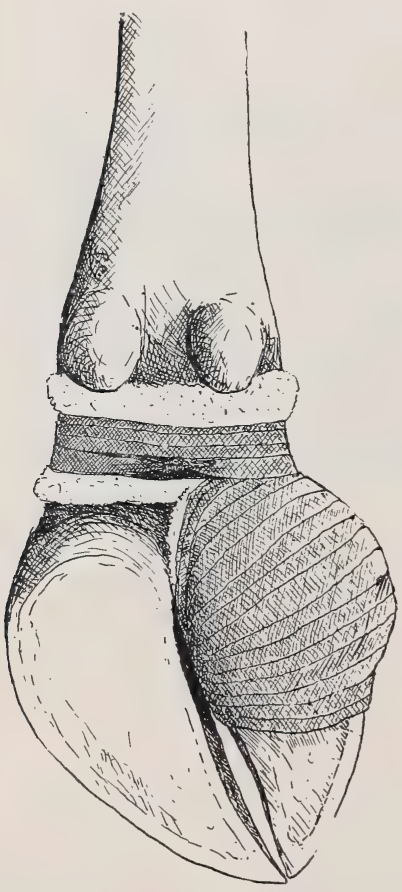

FIG. 295.-Dressing for claw after operation.

almost entirely confined to the feet. They consist in operations for sand-crack, pickedup nail, stabs by nails and bruising of the sole, elsewhere mentioned. As they call for no special precautions they need not be further mentioned here.

\section{SURGICAL DRESSING FOR A CLAW.}

The surgical dressing necessitated by the operation for sand-crack, picked-up nail, or injury to the heels is often very difficult to fix in the ox, and necessitates a support round the pastern. It can, however, be secured in the following way:

The seat of operation is covered with small antiseptic pads, which are also applied round the pastern and in the interdigital space. A bandage is then passed twice round the pastern and over the posterior two-thirds of the claw, as in fixing the dressing used after removal of the lateral cartilage in the horse. The bandage is then passed repeatedly round the pastern in an upward direction and tied above the interdigital space.

\section{AMPUTATION OF THE CLAW OR OF THE TWO LAST PHALANGES.}

It sometimes happens that certain grave diseases in the foot or pastern (stabs or picked-up nails, panaritium of the interdigital space, necrosis of the ends of the flexor tendons, etc.) are accompanied by 
AMPUTATION OF THE CLAW OR OF THE TWO LAST PHALANGES. 731

necrosis of the bones, suppurative synovitis, and even suppurative arthritis of the second and first inter-phalangeal joints.

If carefully treated these forms of arthritis may disappear, leaving the joints anchylosed, but unfortunately the application of the necessary antiseptic injections (free injection with warm boiled water, injection of 10 per cent. iodised glycerine, 3 per cent. carbolic glycerine or $\cdot 1$ per cent. sublimate) is difficult and costly.

It is better, in such cases, to remove the claw or the two last

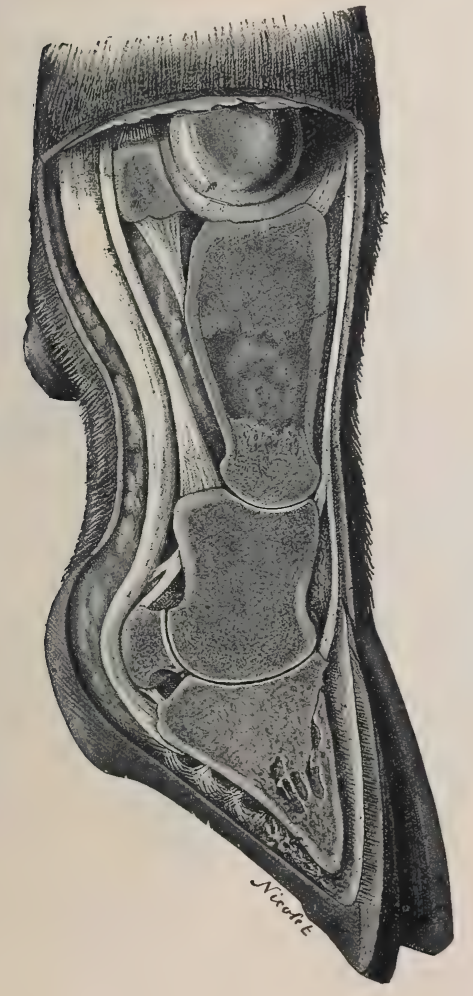

FIG. 296.-Anatomical relations of the inter-phalangeal joints.

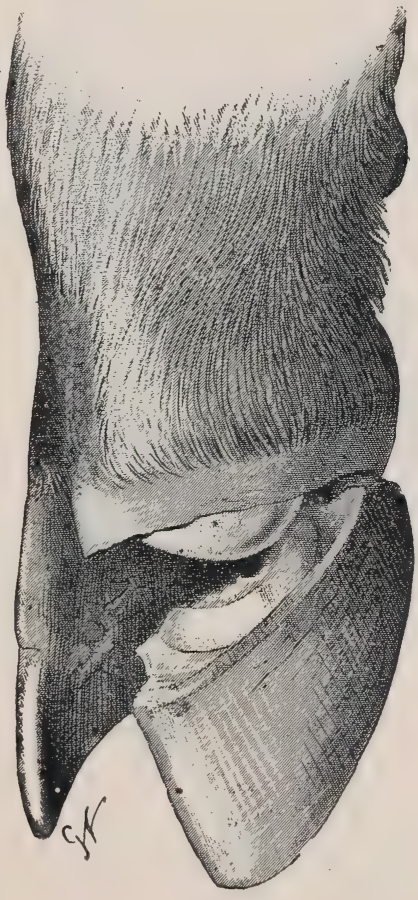

FIG. 297.-Disarticulation of the claw and third phalanx.

phalanges. With antiseptic precautions the stump heals, and recovery takes place without the interminable suppuration and pain which otherwise cause such grave loss of condition.

(1.) Disarticulation of the Claw and Third Phalanx.-The patient is cast and suitably fixed. The horn-secreting coronary band of the claw must be preserved.

First stage. The horny wall immediately beneath the coronary band is thoroughly thinned and the tissues are divided as far as the bone. 
Second stage. Disarticulation: The tendon of the extensor pedis is divided and the joint opened. The claw is pressed backwards, and first the external and internal ligaments, then the flexor tendons of the phalanges, are divided.

This operation is of no great use, because, on account of the position of the joint and the arrangement of the articular surfaces, the end of the second phalanx extends beyond the line of section. To avoid complications, therefore, it is better to remove the lower extremity

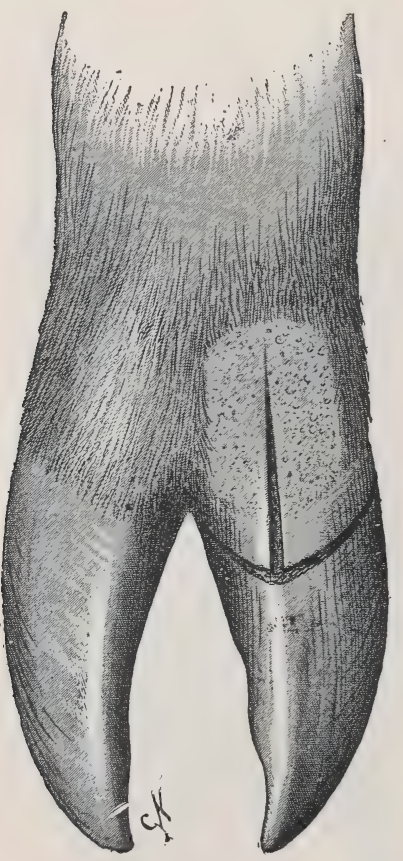

Fig. 298.-Amputation of the two last phalanges. First and second phases.

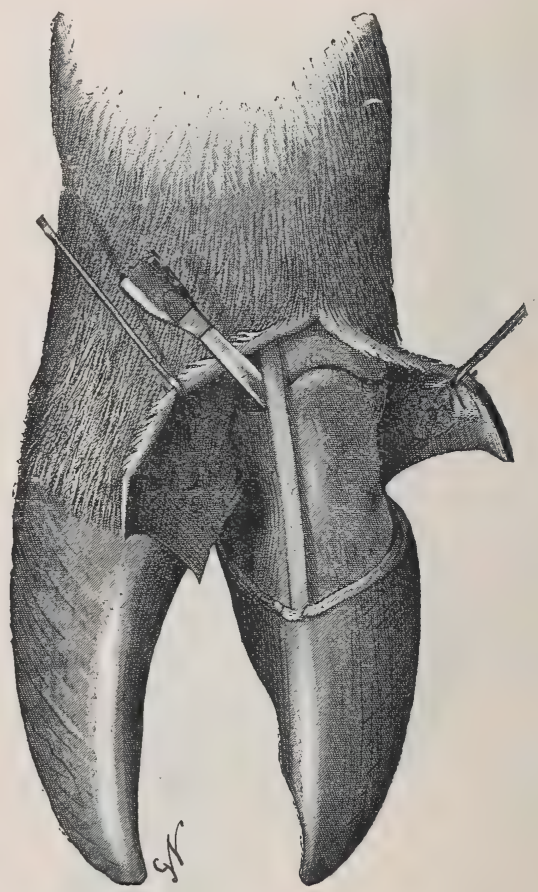

FIG. 299.-Amputation of the two last phalanges. Third phase.

of the second phalanx, which, moreover, is always injured to a greater or less extent in cases of pedal arthritis. To effect this it is only necessary to draw back the flap of skin a little and rapidly divide the second phalange at its upper third with a fine saw. The points of section of the tendons and ligaments must be carefully examined, and if they exhibit necrosis should be further shortened.

The stump is enveloped in a surgical dressing fixed to the pastern.

Amputation of the two First Phalanges.-When necrosis is very serious and has extended a long way upwards, it is often better immediately to resort to amputation of the two last phalanges. 
The region is first shaved and thoroughly cleansed. The coronary band of the claw is also preserved in this case.

First stage. The horn below the coronary band is thoroughly thinned and the tissues are divided as far as the bone.

Second stage. The skin covering the front of the limb is vertically incised from the lower third of the first phalanx (Fig. 298) to the coronary band; the skin is separated and external and internal flaps are formed.

Third stage. The extensor pedis tendon is divided, the first inter-phalangeal joint opened, the internal and external lateral ligaments are divided, the claw is pressed backwards, and the flexor tendons are also divided.

To facilitate disarticulation, and particularly to facilitate section of the lateral ligaments, the claw is rotated successively outwards and inwards.

According to circumstances, the lower extremity of the first phalange is either scraped or divided and the stumps of the tendons are carefully trimmed to a regular shape.

A surgical antiseptic dressing is applied over the whole of the seat of operation.

Several other methods of performing this operation will be found in Möller and Dollar's "Regional Surgery," pp. 831-835. 


\section{CHAPTER IV. \\ DIGESTIVE APPARATUS.}

\section{RINGING PIGS.}

ThIs operation is customary in countries where pigs are allowed to roam more or less at liberty, and it is necessary to adopt some precaution to prevent them from uprooting the soil and thus causing damage, but the practice tends nowadays to disappear. It simply

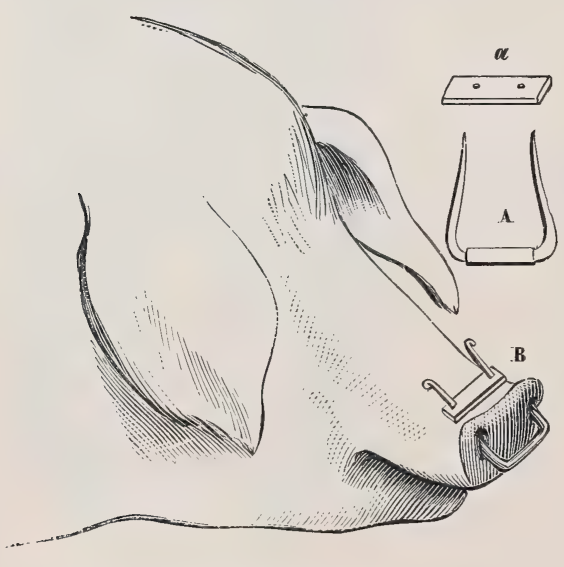

FIG. 300.-_"Ringing" the pig. consists in passing through the nose some object which on being rubbed against anything causes pain and thus checks the animal's natural proclivity.

Numerous methods have been suggested. One of the simplest is as follows: The animal having been cast, suitably secured and muzzled, two thick iron wires sharpened at the ends are passed through the snout, and the two ends are then twisted together in the form of two rings. These can, if necessary, be united.

Another method, perhaps even more efficacious, consists in bending a thick wire into the shape of the letter $U$, and preparing a small metal plate with two holes corresponding in position to the distance between the two nostrils. The ends of the wire, being sharpened, are passed through the nostrils and securely united to the metal plate by being bent into a spiral or simply at right angles.

\section{CESOPHAGUS.}

The operations practised on the œsophagus comprise passage of the esophageal sound or probang, taxis, crushing of foreign bodies within the œsophagus, and œsophagotomy. 
PASSING THE PROBANG.

Passage of the probang is called for in cases of marked tympanites, suspected dilatation or contraction of the œsophagus, and accidental obstruction. Special or improvised instruments may be used, according to circumstances.

The animal is secured in a standing position with the head extended on the neck and in a straight line with the body. A gag is placed in the mouth and the tongue is grasped and withdrawn by an assistant, whilst the operator, having carefully oiled the probang, passes it through the gag towards the back of the pharynx. Violence should be avoided, the probang being gently slid along the centre of the vault of the palate. When the animal makes swallowing movements, the apparatus is slowly pushed onwards.

This manipulation, though simple, requires some dexterity, because at the moment when the instrument enters the pharynx the animal often thrusts it to one side or the other with the base of its tongue, bringing it between the molar teeth, and so crushing, or at least injuring it.

The passage of hollow probangs gives comparatively little relief in cases of tympanites, because the probang is almost always obstructed by semi-digested material from the rumen, or plunges into the semi-solid masses of food contained therein.

When the œsophagus is dilated at a point within the thorax, the progress of the probang is checked by the accumulated food material, and it becomes possible to determine approximately the place where the dilatation occurs. In the same way, should a slender probang be arrested at a given point in the osophagus, this indicates that there is contraction of the tube at that point.

In cases of obstruction the cupped probang is always arrested by the foreign body. Efforts to thrust the latter onwards should always be made with great caution, otherwise the œsophagus may be greatly distended or its walls even ruptured.

CRUSHING THE FOREIGN BODY.

No attempt should be made to crush a foreign body within the cervical portion of the oesophagus unless it is quite certain that that body is of comparatively soft character. Crushing may be performed by lateral pressure with the fingers within the region between the two jugular furrows, or mechanical means may be adopted.

In the latter case a small piece of board is applied to one side of the neck behind the foreign body, whilst gentle blows are given from 
the opposite side with a little wooden mallet. Whatever precautions may be taken, however, this method cannot be recommended.

The same remark applies to the use of forceps, the jaws of which are so fashioned as to escape pressing on the trachea whilst they grasp directly the foreign body through the walls of the œesophagus.

CESOPHAGOTOMY.

Esophagotomy, or incision of the esophagus, is an operation which, though sometimes necessary, should only be regarded as a last resort after all other methods have failed. Unfortunately it can be performed only in the region of the neck, and even then the most favourable point (viz., the lower third of the jugular furrow) cannot always be selected, the operation having to be performed directly over the foreign body.

The animal may be either standing or lying down. The seat of operation should be thoroughly cleansed and disinfected.

First stage. Incision through the skin and subcutaneous connective tissue above the level of the jugular vein and opposite the foreign body.

Second stage. Isolation of the oesophagus by dissection and tearing through of the connective and fibro-aponeurotic tissue at the base of the jugular furrow.

Third stage. Incision through the oesophagus for a distance just sufficient to enable the foreign body to be extracted.

Fourth stage. Suturing of the mucous membrane, suturing of the muscular walls of the œsophagus, suturing of the skin, precautions being taken to allow of drainage at the lower part of the operative wound.

SUB-MUCOUS DISSECTION OF THE FOREIGN BODY.

As œesophagotomy, despite every precaution, often leads to fistula formation, Nocard has recommended sub-mucous dissection of the obstructive body, such body being usually semi-solid. This method has considerable advantages.

The first and second stages of the operation are exactly the same as those above mentioned.

The third stage consists in puncturing the walls of the œsophagus with a straight tenotome immediately behind the foreign body, as in tenotomy. A curved, button-pointed tenotome having next been introduced and passed with the blade flat between the foreign body and the mucous membrane of the cesophagus, it is turned on its axis, and attempts are made to divide the obstruction. A few moments are often sufficient to effect this, after which the substance may be further broken up by the. fingers. 
These various methods may lead to delayed complications, such as dilatation or contraction of the mucous membrane of the osophagus, muscular atrophy of the œsophageal walls, asophageal fistula, and, sometimes, abscess formation.

\section{RUMEN}

Two operations are currently performed on the rumen, puncture and gastrotomy.

\section{PUNCTURE OF THE RUMEN.}

Puncture of the rumen is essentially an urgent operation for the relief of acute and rapidly progressive tympanites. It is performed in the left flank, at an equal distance between the last rib and the angle of the haunch, and an inch or two beyond the transverse processes of the lumbar region.

First stage. Incision of the skin to the extent of about one inch (not absolutely necessary).

Second stage. Puncture with a sharp trocar directed forwards, downwards, and inwards. In making this puncture the point of the trocar is passed through the incision, and a sharp push is given. The sensation of resistance overcome indicates that the trocar has penetrated the cavity of the rumen. Gas then escapes. When the operation is completed, and the canula is being withdrawn, care should

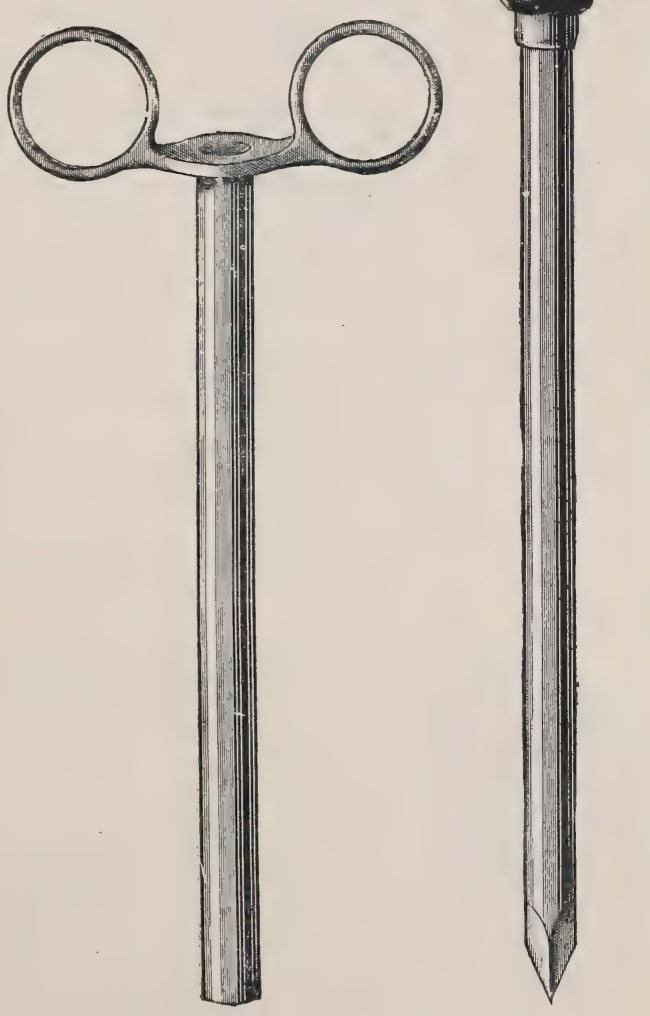

FIG. 301.-Trocar for puncture of the rumen. be taken to press down the skin on either side with the fingers of the left hand, to prevent accidental lifting and laceration of the connective tissue. Even so slight an accident as this might cause serious complications at a later stage.

In the absence of a trocar, and in cases of extreme urgency, the D.C. 
rumen may be directly punctured with a straight bistoury, and after the punctured wound is slightly enlarged, but before the blade of the bistoury is withdrawn, an improvised canula, consisting of a hollow elder twig, may be introduced. Were the blade of the bistoury withdrawn before the introduction of the canula, the rumen would be displaced, and the points punctured would no longer correspond.

Complications, such as respiratory or circulatory syneope, attacks of vertigo, etc., have been noted, but these in reality are very rare.

Subcutaneous Emphysema.-When the canula is carelessly removed, and the subcutaneous connective tissue is torn, local emphysema may occur if the pressure of gas in the rumen is very great. This gas enters the puncture, proceeds along the connective tissue, particularly the subcutaneous connective tissue, and causes crepitant subcutaneous emphysema, very easy to recognise. This emphysema may remain localised in the neighbourhood of the puncture and gradually become absorbed. It may, however, extend to the whole of the flank or even beyond, and in exceptional cases bring about generalised subcutaneous emphysema. Such very extensive emphysema as this rarely becomes reabsorbed without complications.

The suppuration which follows puncture of the rumen may assume one of two forms:-

(a) That of a little local abscess at the point of puncture, when foreign matter or the microbes of suppuration have been left in the path made by the withdrawal of the canula. Such abscesses are of little importance. They rapidly heal if opened and treated with antiseptic injections.

(b) That of diffuse subcutaneous or interstitial suppuration following accidental emphysema.

The pressure of gas forces fragments of food material between the layers of tissue, and suppuration is set up, the pus escaping by a fistula at the point of puncture. Such suppuration is decidedly dangerous, because it may result in necrosis of the aponeurotic layers of the small oblique muscle, in which case recovery is tedious and uncertain.

Treatment consists in laying open the orifice and fistula, and making a counter opening at the lowest point of the swelling. Free drainage and abundant irrigation with boiled water at the body temperature, followed by antiseptic injections, complete the treatment.

Peritonitis is not altogether exceptional as a sequel to puncture of the rumen, if ordinary precautions are neglected or if infective material or fragments of food pass into the peritoneal cavity.

At first the condition is usually local, but it may extend and assume the form of general peritonitis two or three weeks later. The symptoms are those of acute peritonitis. 
Speaking generally, however, puncture of the rumen in cattle and sheep is seldom followed by any complication.

\section{GASTROTOIMY.}

Gastrotomy is performed for the relief of impaction of the rumen and to remove foreign bodies, such as linen, nails, bits of leather, etc., which have been swallowed.

A vertical or slightly oblique incision is made in the left flank,

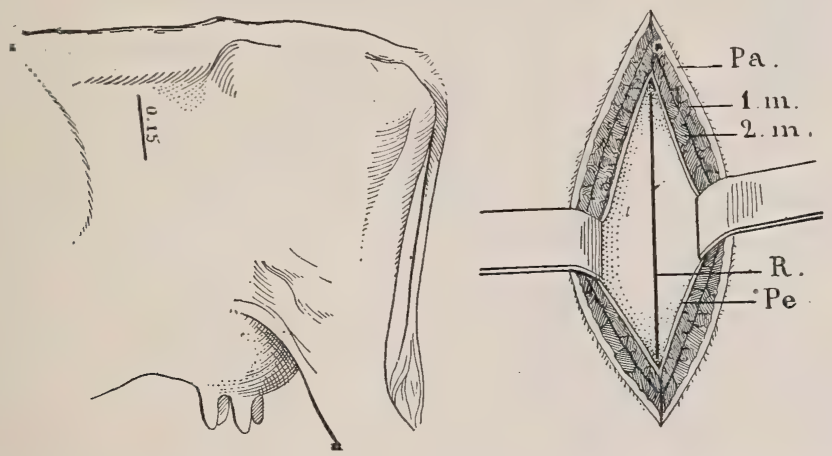

FIG. 302.-Gastrotomy. Pa, Skin; $1 m, 2 m$, muscular layers; $\mathrm{P} e$, peritoneum ; $\mathrm{R}$, rumen, showing line of incision.

extending from the fourth transverse process of the lumbar vertebræ towards the last rib. The operation comprises the following stages:-

First stage. Incision through the skin for a distance of from 6 to 10 inches, according to the size of the animal.

Second stage. Incision through the muscles and peritoneum and torsion of any small muscular arterioles, which may be divided.

Third stage. Fixation and immobilisation of the rumen with from four to six sutures (Fig. 303).

Fourth stage. Vertical incision into the rumen; manual examination of the cavity and its contents.

Formerly the operation was confined to these stages. In such cases localised adhesive peritonitis follows, causing the rumen to adhere to the internal surface of the abdominal wall, and the fistula con-

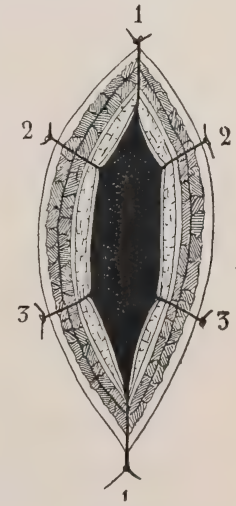

FIG. 303. tinues in existence for months before complete cicatrisation. It is better, therefore, to insert sutures in the rumen, in order to secure. more rapid and complete closure.

Fifth stage. Suture of the rumen with carbolised silk. The lips 
of the wound should be brought together face to face, or they can be slightly inverted, but the sutures should only pass through the peritoneum and muscular coats, avoiding the

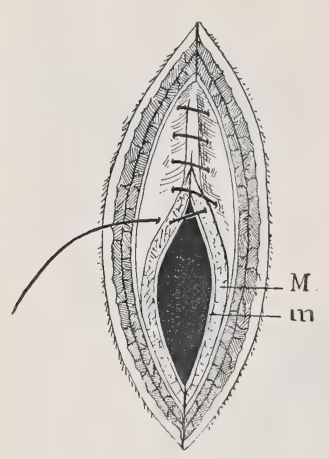

FIG. 304. mucous coat. If the silk threads pass through the mucous membrane and come in contact with the gas in the upper zone of the rumen they are rapidly macerated, and the sutures tear out before the wounds can heal. The rumen should always be kept fixed to the abdominal wall towards the upper and lower extremities of the operative wound, in order to avoid displacement and occurrence of peritonitis. For a similar reason the passing of the silk sutures should be preceded by careful disinfection of the operative wound, and free washing of the parts with boiled water.

The operation is concluded by bringing the skin together with a few silk sutures and inserting a strip of iodoform gauze into the lower portion of the wound, to serve as a drain.

\section{LAPAROTOMY.}

Laparotomy is comparatively. seldom performed on animals of the bovine species, though it may become necessary in dealing with cases of hernia, uterine torsion (where direct taxis is called for), Cæsarean section, invagination or strangulation of the intestine, and under a few other exceptional circumstances.

If simple exploration is aimed at, the operation is most conveniently performed from the right flank with the animal in a standing position, but should a prolonged operation be contemplated the animal should be cast. The incision varies in length, according to circumstances, from 8 to 16 inches, and, like that in gastrotomy, should correspond in direction with the fibres of the small oblique abdominal muscle; the seat of operation should previously be washed, shaved, and disinfected.

The operation comprises the following stages:-

First stage. Incision of the skin.

Second stage. Incision through the muscles and peritoneum.

Third stage. Exploration, inspection, palpation, extraction or ablation, etc.

Fourth stage. Suture of the peritoneal opening, the lips being brought together face to face.

Fifth stage. Suture of the muscles and the skin. It is sometimes advisable to insert a drain of iodoform gauze under the skin. 
In small animals, such as the sheep, goat, and pig, laparotomy is more easily practicable, and can be performed either in the right flank or towards the white line. The stages of operation are exactly the same, but after operating near the white line it is extremely important to use numerous and strong sutures, and afterwards to apply a suspensory bandage around the abdomen, securing it above the loins.

\section{HERNIE.}

The situation and nature of the hernia determine whether or not a radical cure should be attempted.

When a decision has been arrived at the seat of operation must first of all be thoroughly cleansed and disinfected. The animal is cast in a convenient position, and a general anæsthetic is given or a subcutaneous injection of 1 per cent. cocaine solution administered.

The operation comprises:-

First stage. Incision through the skin covering the hernial sac, opposite the orifice of the hernia.

Second stage. Isolation of the hernial sac.

Third stage. Reduction of the hernia and breaking down of any adhesions that may exist.

Fourth stage. Resection of the sac and obliteration of the peritoneal orifice by suture and ligature.

Fifth stage. Suturing of

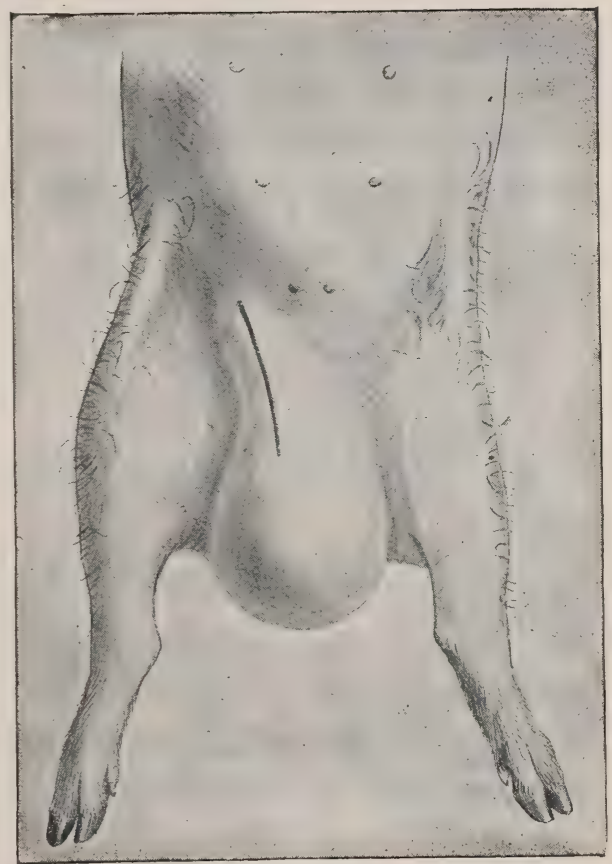

FIG. 305.--Inguinal hernia in a young pig. the muscles and skin, and application of a surgical dressing.

In practice, the deep sutures should be of bichromatised catgut or silk, and the skin sutures of catgut ligature or aseptic silk.

INGUINAL HERNIA IN YOUNG PIGS.

One of the most frequent forms of hernia which the practitioner is called on to treat is inguinal hernia in young pigs. Although this shows little tendency towards strangulation it is always desirable to 
operate, as otherwise the patients develop badly. There is no difficulty in this, though the animals must be cast and placed on their backs, the hind quarters being raised (Fig. 305).

First stage. A longer or shorter cutaneous incision over the neck of the hernia and along its greater curvature.

Second stage. Isolation of the hernial sac, consisting of the dilated internal sheath.

Third stage. Direct reduction of the hernia without opening the sac, provided no adhesions occur, or, in the event of adhesions, after incision of the sac.

Fourth stage. Torsion of the hernial sac and of the testicular cord up to the inguinal ring. Application of a catgut or silk ligature around the sac and cord at the level of the inguinal ring.

Fifth stage. Fixation of the ligature to the lips of the ring. Suture of the skin wound, and drainage of the wound with a strip of iodoform gauze.

\section{IMPERFORATE ANUS.}

This anomaly of development, which is not uncommon, presents two different degrees of development.

In the first degree the rectum is well formed, and extends as far as the skin below the base of the tail.

In the second the rectum is incomplete or non-existent, the floating colon terminating in a blind end at the entrance to the pelvis.

In calves, lambs, and young pigs very often imperforate anus is not diagnosed until the second or third day after birth. Defæcation cannot occur, and death is inevitable unless an artificial anus be established.

First Degree.- - The patient loses appetite, the abdomen remains distended, and on examination of the anal region a doughy swelling is felt, which projects backwards when the animal strains. The operation is quite elementary, and always proves successful.

First stage. The skin beneath the tail is incised vertically; the rectal cul-de-sac projects towards the incision.

Second stage. The rectal cul-de-sac is punctured, the contents are removed, and the rectum and skin united by a few sutures. An anus is thus established, though there is no sphincter.

Second Degree.-The general symptoms are similar, though very often the little patient shows symptoms of atrophy or arrest in development. The operation is somewhat complicated.

First stage. Vertical incision through the skin at the base of the tail, 
Second stage. Digital exploration of the cavity of the pelvis after breaking down of the layers of connective tissue, and search for the blind end of the floating colon. When discovered, the colon is grasped between the jaws of a clamp or large forceps with smooth jaws, and gently drawn towards the opening.

Third stage. Puncture of the blind end of the colon, and suture of the latter to the cutaneous wound, as in the former case.

A third condition may exist, where the extremity of the colon remains within the abdomen. Operation by way of the pelvis then proves unsuccessful. If considered advisable, an opening may be made through the right flank, so that the floating colon may be brought to the surface and an artificial anus produced in this region.

An incision 1 or 2 inches in length is made below the haunch, to allow of the introduction of the index finger, with which the loop is
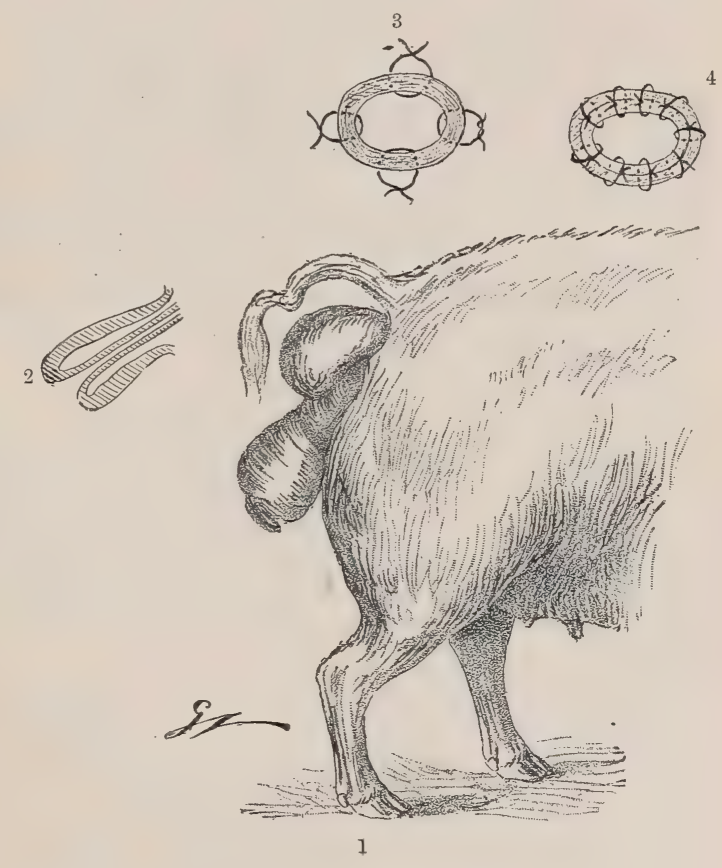

FIG. 306.-1, Prolapse of the rectum and vagina; 2 , schema showing the relations of the layers of the rectum in prolapse; 3 , first phase, showing manner of fixing the superposed layers of tissue by inserting four sutures-the left index finger is inserted into the rectum in order to manipulate the parts; 4 , interrupted sutures inserted around the bowel after amputation.

sought. The colon is withdrawn, and the operation thenceforth is as above described.

\section{PROLAPSUS AND INVERSION OF THE RECTUM.}

This condition occurs in young. pigs in various degrees, The necessity for reduction depends on the extent to which tearing or gangrene of the mucous membrane has progressed. The inverted portion is carefully washed, freely dressed with some non-irritant fatty substance such as vaseline, and progressively pushed back with the thumbs and index fingers of both hands applied flat on either 
side of the anus. To facilitate reduction it is best to check the animal's expulsive efforts by placing a gag in the mouth.

In more aggravated cases, when prolapsus of the rectum has returned several times and the mucous membrane is gangrenous in places so that such a complication as peritonitis of the pelvic cavity is to be feared, it is better to amputate the prolapsed portion.

The animal is secured either standing or lying down, and a large enema is administered to remove the contents of the rectum. The herniated portion of bowel is carefully examined, for it sometimes happens that loops of intestine have become lodged in the dilated peritoneal sac, produced by displacement of the rectum. In such cases reduction should be effected before anything more is done, and for this purpose the patient's hind quarters should be lifted or even suspended.

The operation for removal comprises two stages:

(1.) Fixation of the two layers of bowel by the passage of either two or four sutures about $\frac{1}{2}$ an inch behind the anus.

(2.) Circular amputation of the sutured tissues; insertion of interrupted silk sutures through the lips of the wound; reduction. The patient is restricted to milk diet for a week. Laxative gruels, etc., may then be given.

The complication to be feared is peritonitis of the pelvic cavity owing to the sutures tearing out and allowing infective material to pass from the bowel into the cavity.

Slight cases of prolapsus might possibly be treated by the injection in lines of melted paraffin wax beneath the mucous membrane of the last part of the bowel. The injection is made by means of a large syringe provided with a long needle, the needle being gradually withdrawn as the melted wax is expressed. Four "pillars" of wax are usually injected at equidistant points. As they solidify they support the bowel and prevent the recurrence of the prolapse. The operation, however, is delicate, and scarcely to be recommended in pigs. Moreover, in man, in whom it has chiefly been practised, the deferred results have not always proved satisfactory. 


\section{CHAPTER V.}

\section{RESPIRATORY APPARATUS.}

\section{TREPHINING THE FACIAL SINUSES.}

THIs operation is necessary when pus, tumours, or parasites exist within the sinuses, and in some cases where tumours form within the nasal cavities, etc.

TREPHINING THE HORN CORE.

This cavity is opened in front, at the base of the horn, about $\frac{3}{4}$ of an inch above the keratogenous band.

FRONTAL SINUS.

The frontal sinus may be trephined at one of two points, that is, either towards its highest or lowest extremity.

In the former case-the point selected is in the direction of the axis of the horn core, about $\frac{3}{4}$ of an inch nearer the middle line than the base of the horn itself.

The animal should be cast.

First stage. A V-shaped incision $\frac{3}{4}$ of an inch long on each side is made through the skin and subjacent tissues, exposing the bone.

Second stage. The skin and periosteum are dissected away and reflected upwards.

Third stage. Trepanation.

The lower portion of the cavity is trephined within the angle formed by a transverse line uniting the upper margin of the orbits and the inner margin of the super-orbital foramen.

The stages are precisely the same as those above described.

\section{MAXILLARY SINUS.}

In adult animals the maxillary sinus is opened immediately above the maxillary tuberosity. In the young the point selected is $\frac{3}{4}$ of an inch higher. 


\section{TRACHEOTOMY.}

In bovine animals tracheotomy is only performed in urgent cases, in order to ward off asphyxia or to facilitate some other operation on the upper air passages. It is performed exactly as in the horse, the animal either standing or lying down. In the former case, the animal may be placed in the trevis, but two strong assistants holding the animal's head and nose by means of "bulldogs" are often sufficient.

To prevent the animal from striking out with the front legs, a rope is passed above and around the knees in the form of the figure 8 ; the animal is backed into a corner, and operation is then quite safe.

Large animals must be cast or placed in the trevis.

The seat of operation should be washed, shaved, and disinfected. The operation may be divided into four stages.

First stage. Vertical median incision about 2 inches long through the skin at the height of the upper third of the trachea.

Second stage. Separation with a blunt director of the muscles covering the trachea. Incision through the pretracheal connective tissue.

Third stage. Circular or elliptical opening through the trachea of a size corresponding to that of the tracheotomy tube.

Fourth stage. Insertion of the tracheotomy tube. 


\section{CHAPTER VI. \\ GENITO-URINARY ORGANS.}

IN the domestic ruminants the penis exhibits a peculiar $\mathbf{S}$-shaped curve, situated in the sub-pubic region (Fig. 226), so that when operation on the urethra, or even on the extremity of the penis, becomes necessary the organ must first of all be withdrawn.

The manipulation is as follows:-

The animal having been fixed by the head and front legs in a standing position, and if possible thrust against a wall, the operator stands on its left side. With his right hand he seizes the penis and the skin immediately in front of the scrotum and pushes them forward in the direction of the opening of the sheath.

The extremity is nipped between the first fingers of the left hand, and to prevent the glans slipping or escaping when the right hand is removed (for the purpose of taking a fresh hold of the body of the penis further back) the operator may reverse the free extremity of the penis so that it forms a loop, and thus secure a firmer hold. With the right hand the skin is thrust backward, a new portion of the sheath fixed, and the organ again pushed forward. In this way the penis is gradually extended. When the animal is cast, this manipulation is much easier.

\section{URETHROTOMY IN THE OX.}

Urethrotomy consists in incising the urethra, usually for the purpose of extracting a foreign body or calculus which impedes micturition. In the ox, calculi may become fixed either in the intra-pelvic portion of the urethra, though this is very rare; in the ischial curvature, or more commonly at some point in the $\mathbf{S}$-shaped curve of the penis; or sometimes even within the sheath itself.

Urethrotomy is performed in the ischial or scrotal region, according to the point where the obstruction exists.

ISCHIAL URETHROTOMY.

Urethrotomy is performed in the ischial region either to displace or indirectly to abstract a foreign body fixed in the membianous 
portion of the urethra, or directly to remove one from the spongy portion opposite the ischial curve.

Calculi fixed in the intra-pelvic region are detected by rectal exploration.

The exact position of the foreign body is determined by inspection and palpation, whilst distension of the urethra by urine may be noted even before more striking symptoms appear.

The urethra can be incised by one of three methods.

The animal should be secured, if possible, in the standing position.

The first method, which dates back to very early times, consists in puncturing the urethra at one stroke with the fleam or lancet, and opening it more freely, after introducing a grooved director. This method is very useful where rupture of the bladder is imminent.

The extraction of a calculus fixed in the ischial region, or the manipulation of an obstruction at any other point, can afterwards be undertaken.

Second method. A second method consists in incising the subcutaneous tissues, layer by layer, until the urethra is reached at the ischial arch.

The operation is terminated by puncturing the urethra and enlarging the incision in an upward direction after passing a grooved director. This method minimises hæmorrhage and urinary infiltration. By previously injecting cocaine, the operation may be made practically painless.

Third method. Puncture of the urethra by a single stroke with a straight bistoury at the ischial arch.

The opening is enlarged in an upward direction with the same instrument.

\section{SCROTAL URETHROTOMY.}

Scrotal urethrotomy is necessary when the calculus is situated in one of the $\mathbf{S}$-shaped curves of the penis or nearer the glans.

The operation is facilitated by casting the animal and withdrawing the penis from the sheath, but as there is considerable danger of rupturing the bladder when casting an animal with marked distension of that organ, the more serious operation should be preceded by puncturing the urethra with a fleam at the ischial arch.

By repeated moderate traction on the extremity of the glans, the S-shaped curve can be obliterated and the anterior portion of the penis withdrawn beyond the sheath.

One of two conditions may exist. 
First case. Where the calculus is in the anterior, extra-prepubic portion, it is removed through an incision made directly over it. After extraction and disinfection, one or two sutures are inserted.

Second case. Should the calculus be situated in that portion of the penis which remains within the sheath after the fullest withdrawal of the organ, it is necessary to proceed as follows:-

(1.) The skin covering the sheath, the subcutaneous tissue, and the mucous membrane are first incised for a length of from $1 \frac{1}{4}$ to $1 \frac{3}{4}$ inches.

(2.) The penis is drawn through this opening; an incision is made directly over the calculus, dividing the fibrous layer, erectile tissue and mucous membrane of the urethra; the parts are disinfected and the wounds closed with sutures.

With ordinary antiseptic precautions little danger is to be feared.

Even should infiltration of urine occur, the operator need not be unduly anxious, for, provided the parts are punctured or scarified early, recovery usually follows.

\section{PASSAGE OF THE CATHETER AND URETHROTOMY IN THE RAM.}

Obstruction of the urethra in rams is more commonly caused by deposits of gravel than by single large calculi. It is generally found in highly-fed animals, in which gravel accumulates and becomes massed together at some point in the canal, often near the free extremity, where it forms a plug, causing complete retention of urine. In other cases this retention is due to a mass of sediment formed by vesical mucus and fine gravel which collects about the neck of the bladder.

Three operations have been advised for the removal of this condition :-

(1.) Section of the Appendix of the Penis.-When the disease is just appearing the sedimentary material may be collected at the anterior extremity of the penis behind the appendix. The shepherds in such cases remove the extremity of the penis. The resistance disappears, the plug formed of gravel yields to the pressure of urine, and micturition occurs as usual. Excision of the appendix, however, incapacitates the ram for service.

(2.) Passage of the Catheter--Passage of the catheter has been recommended for the removal of deposits of gravel in the urethra, but it seems a very questionable method.

Should it be determined on, the animal must be placed on its back. The penis is then withdrawn and the double $\mathbf{S}$-shaped curve is obliterated. An incision is made over the canal behind the 
appendix and a soft gutta-percha sound is passed. The sabulous accumulation is thus dispersed. the ox.

(3.) Urethrotomy.-Scrotal urethrotomy may be performed as in

Ischial urethrotomy is impracticable in very fat animals, but when

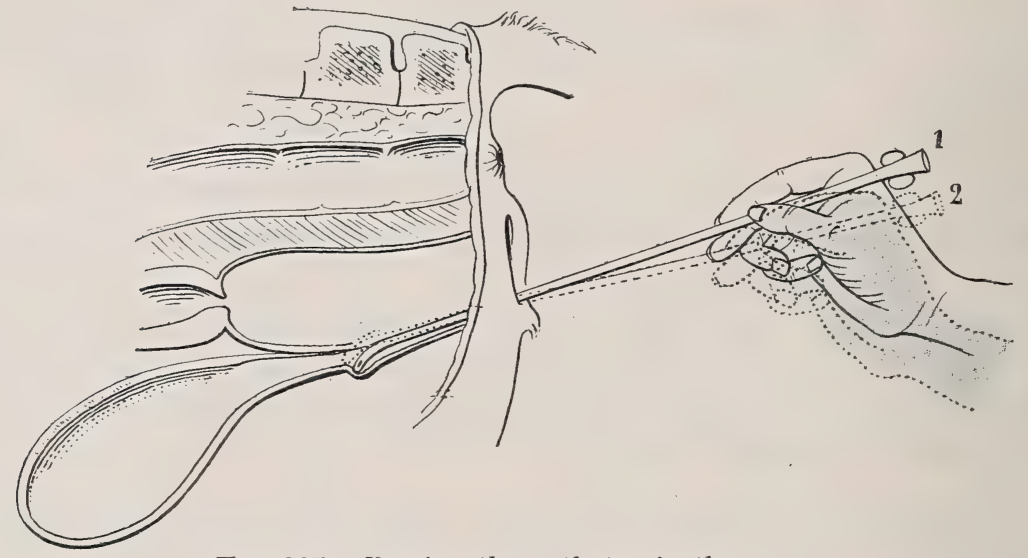

FIG. 307.-Passing the catheter in the cow.

the obstruction is about the neck of the bladder, and the animal's condition admits of it, this operation may be performed.

The patient is fixed on its back, and a metallic or gutta-percha sound is passed into the urethra. The tissues are incised layer by layer in the direction of the sound. Once the urethra has been

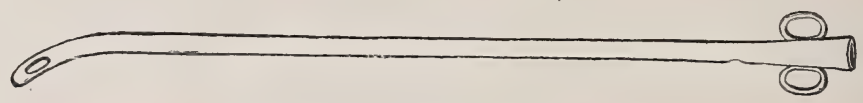

FIG. 308.-Catheter for cows.

opened the soft magma may be washed out of the bladder by a free injection of boiled water or similar aseptic liquid.

\section{PASSAGE OF THE CATHETER IN THE COW.}

It sometimes becomes necessary to examine the bladder of the cow.

There is an obstacle, however, to the introduction of the sound into the urethral canal. The meatus urinarius is covered by a little valve which springs from the lower wall and forms behind the real opening of the urethra a cul-de-sac, into which the point of the catheter is apt to pass. The instrument usually employed is of gutta-percha, glass, or, better still, of metal, as more easily sterilised (Fig. 308). It is held like a pen, and is directed along the floor of 
the vagina as far as the opening of the meatus, being guided by the index finger of the left hand, which has previously been introduced. The point being very slightly depressed, it enters the cul-de-sac. It is then only necessary to reverse the movement, that is to say, raise the point, whilst gently pressing forward; a slight resistance is felt and the sound enters the bladder. If necessary the little valve may be held down by gently pressing on it with the point of the left index finger.

It is sometimes an advantage to expose the seat of operation. In such cases the lips of the vulva and the walls of the vagina may be separated by retractors or by the use of a speculum.

\section{CASTRATION.}

Castration is performed for the purpose of removing the reproductive porver, either by obliterating the testicle or ovary or by suppressing their functions.

In ruminants, the testicles are elongated and placed in a vertical position, the upper portion of the scrotum presenting a constriction and the whole scrotal mass resembling in shape a cone with its base downwards.

\section{CASTRATION OF THE BULL AND RAM.}

These two animals, when destined for slaughter, are usually castrated either at birth or at latest two or three months afterwards. In Normandy, in Franche-Comté, and in England breeders castrate young bulls by torsion of the cord.

Two incisions about $1 \frac{1}{4}$ to $1 \frac{3}{4}$ inches in length are made on the lower extremity of the scrotum. The testicles are enucleated and the testicular cords seized with two pressure clamps, with which torsion is effected. In the South of France, in Auvergne, and in the Limousin, bulls intended for working are not castrated until after the lapse of some months, on account of the influence which the testicles have on the development of the bones and muscles. Such animals are only operated on at the age of from six months to a year, and as a rule the method employed is that of bistournage.

\section{BISTOURNAGE.}

This method of castration has been practised from time immemorial. It consists essentially of torsion of the testicular cord, and aims at obliterating the vessels which it contains, and thus bringing about atrophy of the organs served by them.

The Bull.-The animal is operated on in the standing position. The head is fixed to a post or ring somewhat high up, in order to 
check movement of the hind legs. The hind legs are also partially

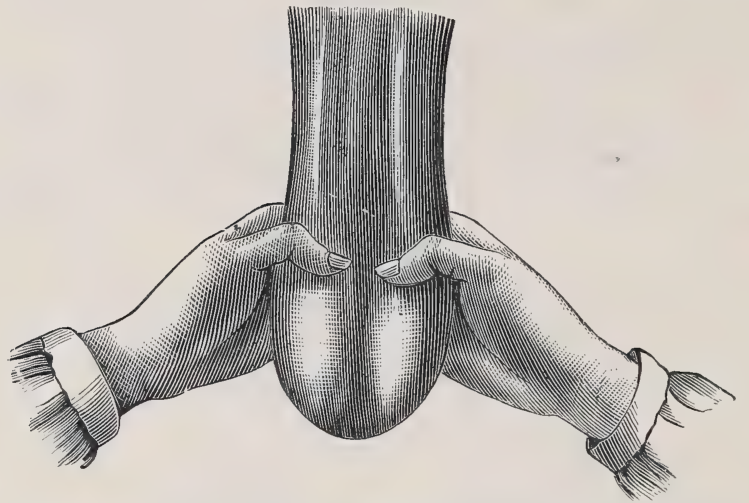

FIG. 309.-Bistournage. First phase. Manipulating the scrotum. secured by means of ropes or two pieces of webbing passed in a running noose about the hocks and fixed above the knee. No preliminary disinfection is practised, because no wound is made.

Manual Technique. The operation comprises four stages:

First stage. 'The operator, standing behind the animal's hocks, grasps the testicular cords with the hands, immediately above the testicles, and by exercising strong pressure, thrusts the latter to the extreme base of the scrotum. The movement is next reversed; seizing the base of the scrotum with the right hand, he draws it smartly downwards, whilst he places the left hand above the right, and thrusts the testicles towards the abdomen. If the testicles do not rise sufficiently high, the right hand is slipped between these and the left hand, and the testicles are thus thrust upwards towards the lower inguinal rings, slightly dilating the latter.

After this manipulation has been repeated two or three times, the scrotum, etc., become more pliable and the testicles more easily displaced. The second stage of the operation is thus facilitated.

Second stage. The second stage of operation may be effected by one of two methods.

Old method: The oldest method

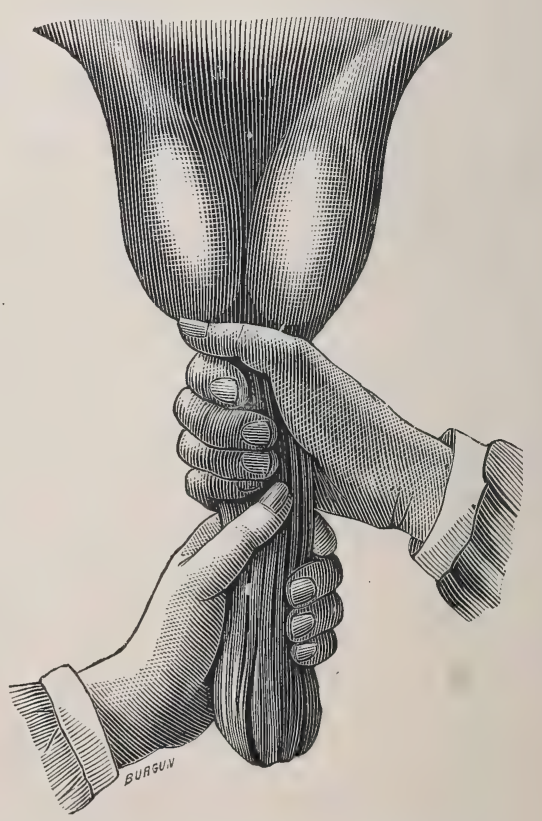

FIG. 310.-Bistournage. First phase. Thrusting the testicles upwards; manipulating the scrotum. consists in allowing one of the testicles to rise towards the inguinal 
ring and to turn the other in a vertical plane. If, for instance, it is desired to turn the right testicle, the cord is grasped between the
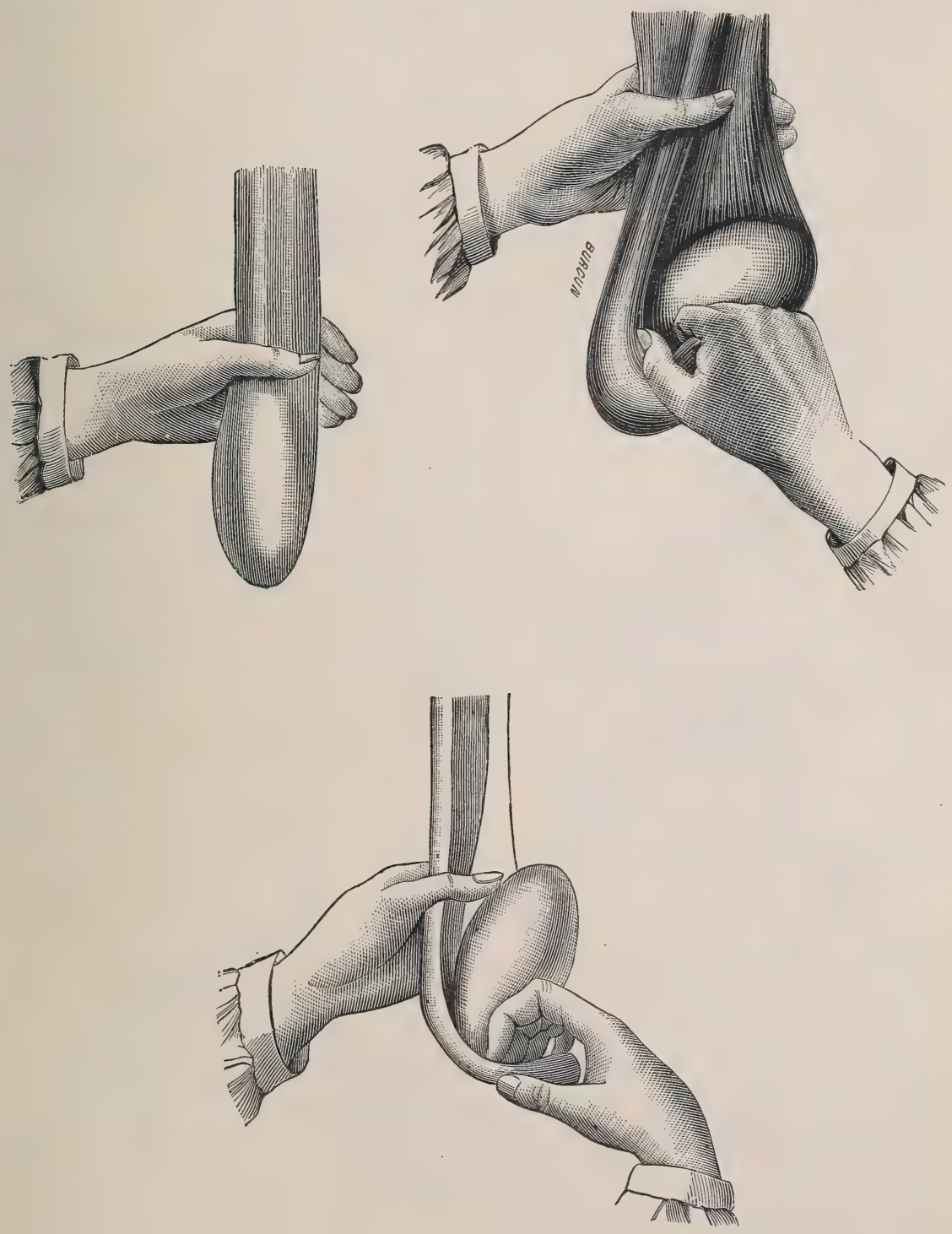

FIG. 311.-Bistournage. Second phase.

thumb and index finger of the left hand (Fig. 311), the lower part of the scrotum is seized with the right hand, and the object then is to slide the point of the testicle along the dorsal surface of the D.C. 
fingers (Fig. 311). Simultaneously the operator presses on the base of the testicle with the thumb of the left hand, thus causing a rotary movement in a vertical plane; the tail of the epididymis becomes uppermost. A certain empty space separates the testicle from the base of the scrotum.

Third stage. Torsion of the cord. The testicle having been rotated, the cord must be twisted so that the vessels may be obliterated. The left hand continues grasping the eord, which is then brought in front of the testicle, whilst with the right hand the testicle is pushed backwards and is made to describe a semi-circle. The cord was previously on the left side; it is now on the right, and simultaneously the testicle passes from right to left.

In completing the turn the

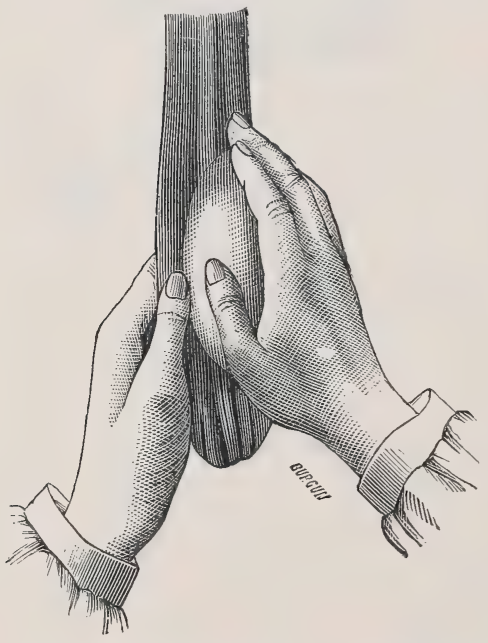
hands must not be changed, and, above all, must not let go their hold; and the cord is pushed forwards and towards the right with the right hand, whilst the testicle is pushed backwards and to the left with the left hand. The cord and the testicle resume their original position; one complete turn has been effected. These manipulations are repeated several times, and the cord soon assumes the appearance of a large, hard, tense string. To ensure obliteration seven or eight turns should be made in the case of the bull and four or FIG. 312-Bistournage. Third phase. five in that of the ram.

Torsion of the right testicle being complete, the gland is thrust towards the upper part of the scrotum and the left testicle is submitted to the same manipulation, the position of the hands, however, being reversed.

Fourth stage. Fixation of the testicles in the inguinal region. Both testicles having been thrust upwards as far as possible into the inguinal region, the scrotum is ligatured below them. Tape or thick cord should be used, to guard against gangrene of the lower portions of the scrotum. A considerable œedematous swelling soon occurs, and when at the end of twenty-four or forty-eight hours infiltration is well developed, the ligature should be removed.

Dubourdieu has described a different method, in which the testicle is rotated in a horizontal plane. The position of the hands is 
then different. The left testicle, for instance, being at the base of the scrotum, the cord is grasped with the right hand opposite the base of the testicle, and the tail of the epididymis and the testicle are held with the whole hand whilst being rotated. If care is taken to fix the cord with the right hand, rotation is more rapid and easier than in the preceding method.

Difficulties in Operation. -Bistournage is highly commended in France on account of its avoiding all the complications resulting from sanguinary operations. Nevertheless it presents great diffculties, particularly in bulls of

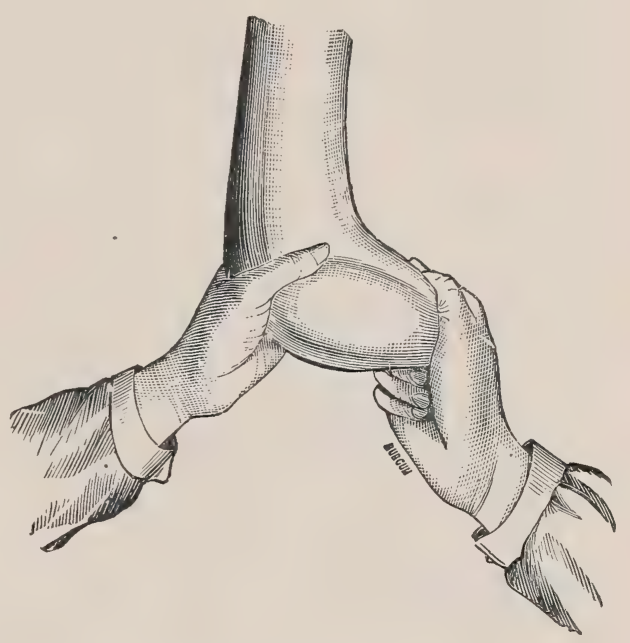

FIG. 313.--Bistournage. Second phase. Dubourdieu's method. from two to three years of age, in which the testicles are hard to manipulate on account of their size, the thickness of the connective

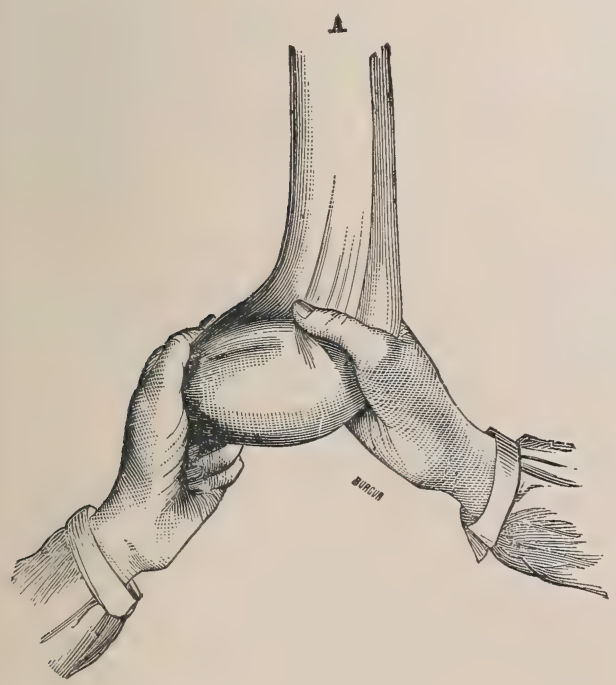

Fig. 314.-Bistournage. Second phase.

Dubourdieu's method. tissue, and sometimes because of abnormal adhesions. In such cases the preliminary manipulation alone sometimes extends over half an hour.

Bistournage is of doubtful efficacy when the testicles are small and round, because after the ligature has been applied the testicular cord tends to untwist, and the shape of the testicles readily lends itself to such movements. If untwisting occurs, the operation fails.

\section{Consequences of the Opera-} tion.-The operation is often followed by more or less violent attacks of colic; the animal may suffer for five or six hours, after which it recovers. 
If torsion has been clumsily performed, or if the ligature becomes displaced, the testicle may descend and the cords untwist; the latter then appear to have lost the firm, tense consistence which they presented after operation. To prevent slipping of the ligature and untwisting of the cord, Guittard suggests the use of an iron needle, with which the scrotum is pierced through the median line, just beneath the testicles when at their highest position; above this is placed the ligature, which then cannot possibly slip.

The Basque operators, in order to avoid untwisting, exercise vigorous traction from above downwards after rotating the testicle. In this way ruptures occur which diminish the elasticity of the cord and the epididymis, and tend to check the untwisting of the former.

When the operation has succeeded the testicles gradually atrophy. They do not disappear completely, and may sometimes be found several years later of the size of a hazel-nut or a chestnut and of fibrous consistence. It need scarcely be said that in the event of bistournage failing, cutting operations can always be resorted to.

\section{MARTELAGE.}

The process of martelage consists in mutilating with a mallet the testicular cord whilst still covered by all its envelopes. This mutilation injures the walls of the arteries, causing the formation of a clot, which cuts off the supply of blood to the testicle and causes the gland to atrophy.

The practice is very ancient.

The animal is fixed by the horns as if for bistournage, and the limbs are secured by two strips of webbing or two ropes, as in the former case, though some practitioners neglect the latter precaution.

Two cylindrical rods the size of broomsticks and a wooden mallet or farrier's hammer are the instruments employed.

The method, however, is barbarous, cruel, and of doubtful value. It would never be countenanced in England.

CASTRATION BY CLAMS.

Castration of bulls by means of clams has been practised in many different forms. 
Castration by the Exposed Method.-The operation is the same as in the horse, the scrotum being incised on either side, and the dartos, connective tissue, tunica vaginalis scroti, and tunica vaginalis testis being divided. Short clams are applied to the cord, and the lumen of the arteries is completely obliterated in five to six days, when the clams can be removed.

Instead of an incision being made for the removal of each testicle, the scrotum and dartos may be divided in the middle line, after which incisions may be made to the right and left respectively, exposing the fibrous tissue and enabling the testicles to be enucleated. A clam may then be applied to each cord, or the two cords may be included in one pair

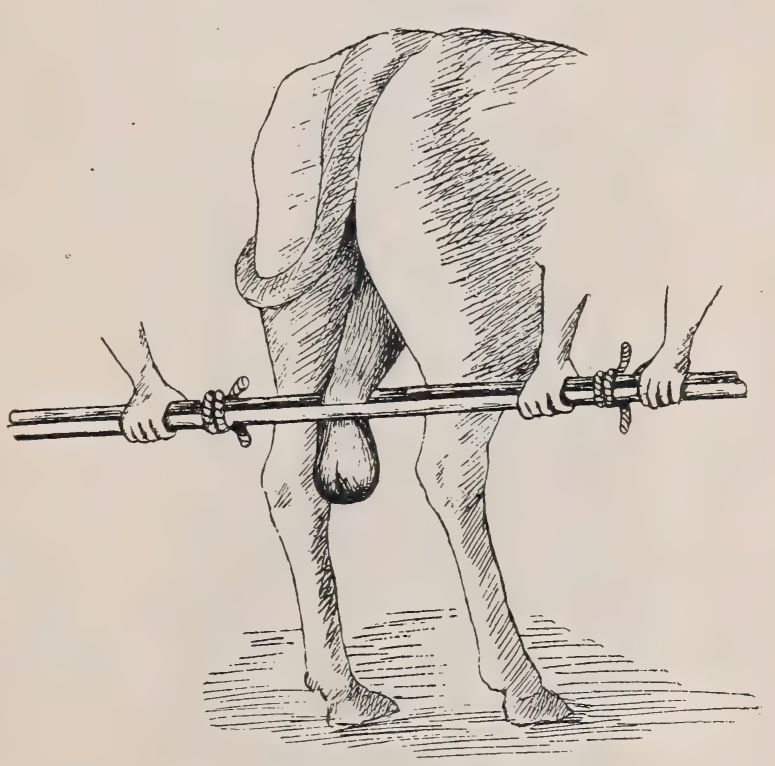

FIG. 315.-Crushing the testicular cord. (This method is to be strongly condemned.) of clams.

This method has the advantage of inflicting less injury on the scrotum, a point which is of some importance in animals destined for slaughter.

Castration by the Covered Method.-This operation is identical with the preceding, except that the incision of the scrotum does not involve the cremaster and fibrous tunic, or the tunica vaginalis scroti.

CASTRATION BY TORSION.

The cord may be twisted throughout its entire length or torsion may be limited to a part of the cord, hence the two methods hereafter described.

(a) Limited Torsion.-The testicles are exposed as in castration by the open method. The cord is then drawn forward and fixed by 
means of forceps applied just outside the scrotum; $\frac{3}{4}$ to 1 inch below this point the torsion forceps are applied. The cord is slowly twisted, and usually ruptures about the centre of the fragment included between the two pairs of forceps.

(b) Direct or Unlimited Torsion.-First stage. The testicle is ex-

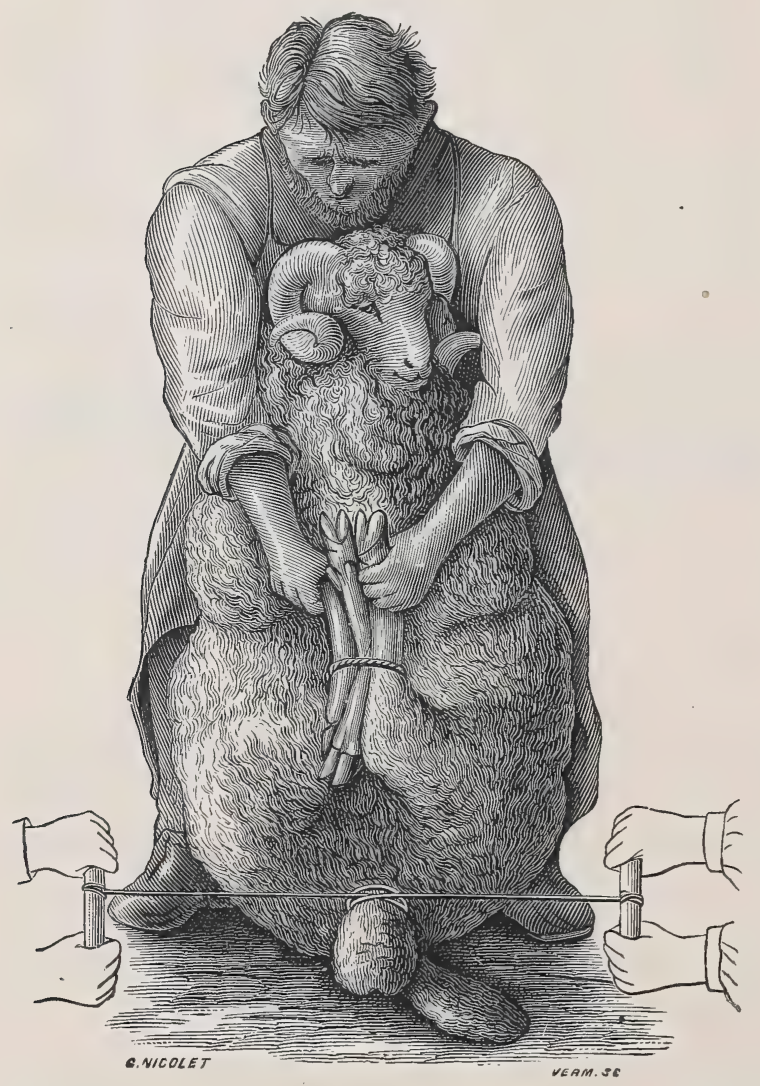

FIG. 316.-Castrating the ram by ligature. (This method is to be strongly condemned.) posed, as in castration by the covered method.

Second stage. The cord and vaginal tunic are twisted by grasping the testicle, which is protected with a piece of clean linen.

CASTRATION WITH THE ACTUAL CAUTERY.

The testicles are exposed, the cords are seized with clams and divided separately or together with a sharpbladed cautery at a white heat. The eschar so produced is sufficiently dense to obliterate the vessels.

\section{CASTRATION BY THE}

ELASTIC LIGATURE.

This method has been largely employed during the last ten or fifteen years. It consists in applying to the upper part of the scrotum several turns of a tensely stretched round or square rubber thread. The two ends of the rubber thread are crossed and tied with string. About the seventh or eighth day the testicles may be removed with a knife close to the ligature, provided the process of delimitation is well advanced.

The chief objection to this method lies in the fact that the scrotum is destroyed, which lowers the value of the animal from a commercial point of view. 


\section{CASTRATION OF THE RAM.}

Most of the preceding methods may be used in castrating rams, but certain special methods are more generally employed: These we shall shortly mention.

Castration by Bistournage. - The method is exactly similar to that in the bull. The animals are placed in the position shown in the figure, except that the hind limbs are extended and held in that position by the operator's knees or feet. The process is only applicable to animals of four or five months old.

Castration by Tearing.--This method is only practised by shepherds, and on animals a few days or at most a month old. The base of the scrotum is snipped off with scissors, the testicles are

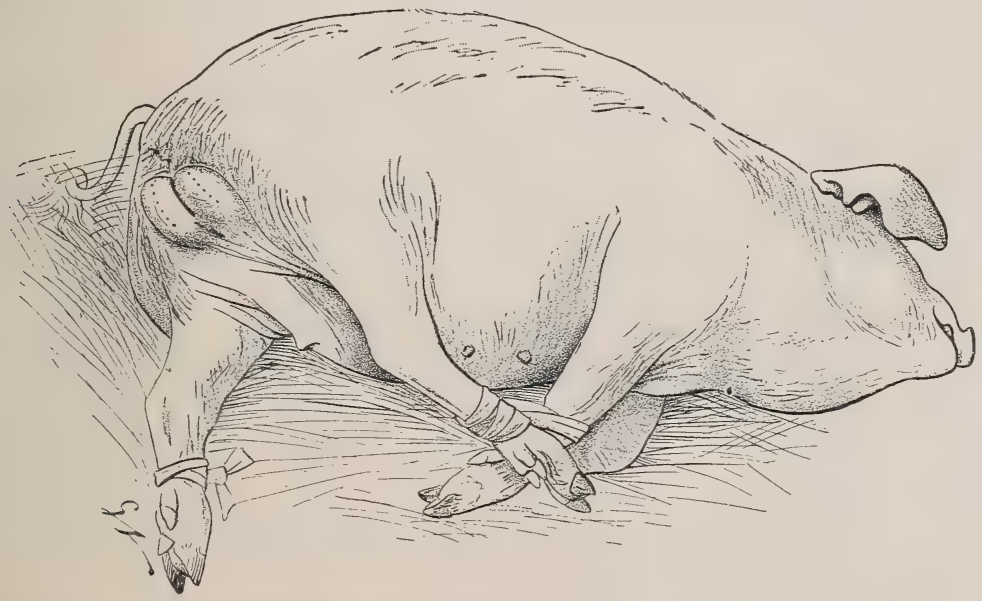

FIG. 317.-Castration of young pigs.

enucleated, and each in turn is seized and torn away with a sudden snatch. Even though a considerable length of cord is removed with the testicle, bad results seldom follow, provided the subject is not of greater age than that mentioned.

Castration by Ligature.-This method consists in passing a stout ligature in the form of a running knot over the neck of the scrotum and, by means of two short pieces of wood, drawing it tight. The method, however, is not to be recommended, as it not infrequently leads to tetanus.

\section{CASTRATION OF BOARS AND YOUNG PIGS.}

For castration boars should be cast on the left side, and three legs at least firmly secured together (Fig. 317). The best method is that of limited torsion. Care should be taken to avoid dragging the 
cord downwards during the operation, for the testicular artery ruptures readily, and fatal abdominal hæmorrhage may follow.

As the subjects are apt to wallow in the litter after the operation a strip of iodoform gauze should be applied and secured by one or two sutures. This is removed on the third or fourth day.

On young pigs the operation is simpler. The animal is cast on the left side and firmly held, the left hind limb being extended and the right drawn towards the right shoulder.

The testicles are grasped each in turn with the left hand, whilst with the right they are exposed by a single sweep of the bistoury. The testicles are removed by torsion with artery forceps. Many laymen simply use the hands, the cord being grasped between the left thumb and index finger, whilst torsion is effected by the right index finger thrust between the vas deferens and the body of the testicle.

\section{CASTRATION OF CRYPTORCHIDS.}

Cryptorchids are very rare amongst cattle and sheep, as the testicles enter the scrotum during intra-uterine life. The internal inguinal ring in the pig being of very small size, the condition is more common in that animal.

The same process is employed in castrating cryptorchid bulls, rams, or boars. The animal is thrown on one side and securely fixed. A vertical incision is made in the region of the flank, varying in length from 4 to 5 inches in the bull, 2 to $2 \frac{1}{2}$ inches in the ram, and 4 to 5 inches in the pig. The abdominal cavity is examined, the testicle found, and a ligature applied to the cord, after which the testicle is removed.

Another method consists in employing the écraseur for division of the cord.

To avoid subsequent complications antiseptic precautions should be taken.

Complications after Castration.-Whatever the method employed, swelling of a more or less abundant character always develops during the few days immediately succeeding the operation. It is, however, of little importance.

Should antiseptic precautions be neglected, suppuration, septicæmia, tetanus, and sometimes scirrhous cord may follow.

FEMALE GENital ORgans.

Examination with the Speculum.-In the cow certain diseases of the vagina, bladder, neck of the uterus, and even of the uterus itself, may necessitate visual examination in addition to the manual 
examination commonly employed. Under such circumstances a special speculum is introduced in a closed condition, being afterwards opened and dilated to the required extent.

Before inserting the speculum, however, the genital passages should be cleaned, and the speculum itself smeared with vaseline. It penetrates readily with moderate pressure.

Heifers and similar animals require a special (small) instrument (Fig. 318).

In certain circumstances, moreover, it is preferable to use retractors, with which local examination is easier. These can be applied at either side of the vagina and drawn apart, thus exposing the depths of the genital tract.

\section{CASTRATION OF THE COW.}

The operation of castrating the cow is very old, and was mentioned by both Aristotle and Pliny. Many other descriptions of it have since been given. But more recently the manual technique has been considerably simplified and very fully described.

Utility.-The operation is practised for the cure of nympho-mania; also to prolong the period of milk-yielding and to facilitate fattening.

As regards nympho-mania, it is only of value where the excessive excitement is due to disease of the ovaries.

Under ordinary conditions the secretion of milk diminishes more or less, and becomes very slight after eight or nine months from calving. If, however, the cow is castrated

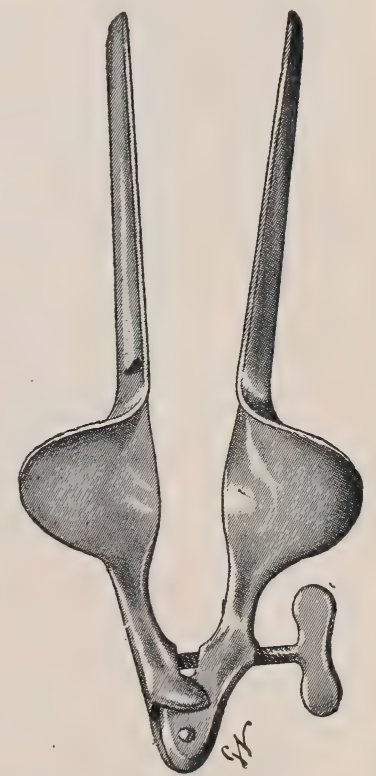

FIG. 318._Vaginal speculum for heifers. under favourable circumstances, lactation continues for several months, sometimes for several years, beyond this period. It is said that castrated cows yield milk of a more constant composition and richer in butter fat, casein, and mineral salts than those which are not castrated, although the point cannot be said to have been fully established.

To obtain the best results the subjects should have attained their maximum yield of milk and be from five to seven years old. The best period is six weeks to two and a half months after calving. Neglect of these considerations is liable to be followed by inappreciable, doubtful, or bad results. 
The influence of castration on fattening is explained by the suppression of œstrum.

Manual Technique.-An ovariotome with a hidden blade and an ecraseur with an extra long stem are the only instruments required. The animals should be prepared for some days by diminishing their food supply and administering gentle laxatives.

Acute or chronic lesions of the genital tract should be held to contra-indicate operation, and it should be noted that tuberculous animals are particularly liable to awkward complications.

On the day of operation an abundant enema is given, to empty the rectum, after which the vagina is washed out freely with a lukewarm solution of some non-irritant antiseptic. The hind quarters, and particularly the neighbourhood of the anus, vulva, base of the tail, etc., should be carefully washed and disinfected with a solution of lysol, cresyl or carbolic acid.

The patients are secured in a standing position, a rope being passed in the form of the figure 8 around the hind limbs above the

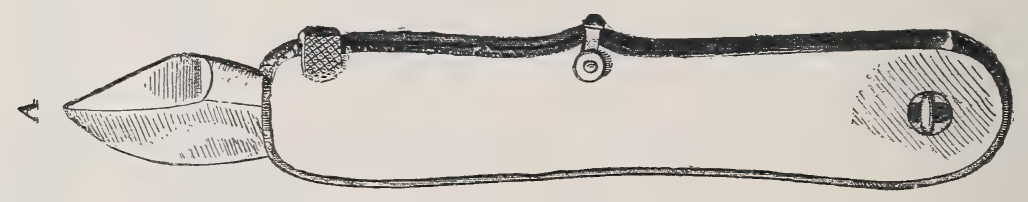

FIG. 319.--Ovariotome.

hocks, and the animal is then firmly thrust against a wall by several strong assistants.

'The operator's hands and instruments must be rigorously disinfected.

The operation comprises three stages:

First stage. Puncture of the vagina.

Second stage. Finding and securing the ovaries.

Third stage. Ablation.

The hand is smeared with sterilised oil, and, grasping the ovariotome, is passed into the vagina, which contracts on it and on the forearm. Within a short time, however, which may vary between two or three minutes and a quarter of an hour, the vagina becomes distended and its walls rigid, so that the operator is able to continue his manipulations more easily. This is the moment for effecting puncture, after an examination of the pelvic organs through the vaginal walls.

The blade of the ovariotome is advanced until fully exposed, and the point is brought directly above the neck of the uterus, about $\frac{3}{4}$ to $1 \frac{1}{4}$ inches from it. By a sharp movement the instrument is then thrust directly forward, dividing the wall of the vagina in the median line. 
The blade is next retracted into its sheath and the instrument dropped on to the floor of the vagina. The right index finger is at once passed through the orifice so made into the peritoneal cavity, in order to malie certain that all the membranes have been divided. By pressing on and slightly tearing the tissues the middle finger is then introduced alongside the index. Only these two fingers should be passed into the peritoneal cavity.

In order to secure the ovaries it then suffices (Fig. 321) to thrust forward the base of the vagina, allowing the two fingers to glide over the body of the uterus and thence downward over its side to the point where the horns of the uterus originate. Here the fingers meet the ovary, which is readily recognisable on account of its size and shape (those of a walnut). The gland is nipped between the index and middle fingers, and is drawn into the vagina through the operative opening.

In order to remove the ovary the operator seizes the écraseur with the

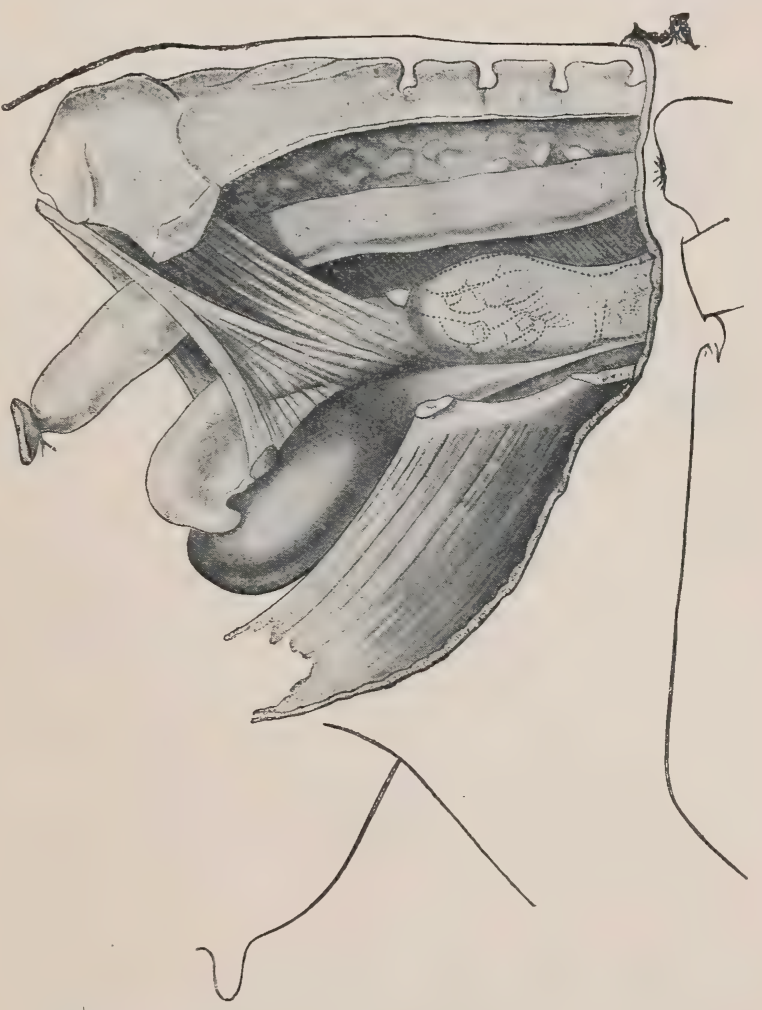

FIG. 320.-Castration of the cow. First phase. left hand, a loop of chain about an inch long projecting, and slides the instrument along his right forearm. The arm must be retained in the vagina, the fingers grasping the ovary. The organ is slipped into the loop of the écraseur, which is then manipulated with the left hand until the pedicle is divided. To prevent hæmorrhage the screw of the écraseur should only be turned at the rate of about twice a minute. The ovary when separated is left on the floor of the vagina, the fingers being again introduced into the abdominal cavity to secure the second one. This is removed in precisely the same way. 
The pedicles of the ovaries are released, and at once return to the peritoneal cavity, while the operator, in withdrawing his hand, brings with it the knife and the ovaries themselves. The lips of the operative incision come together again spontaneously as the vagina contracts.

This operation is followed by slight colic, which, however, need not cause alarm.

Complications: Hæmorrhage.-If the incision is unskilfully performed, it may in exceptional cases result in injury of the terminal

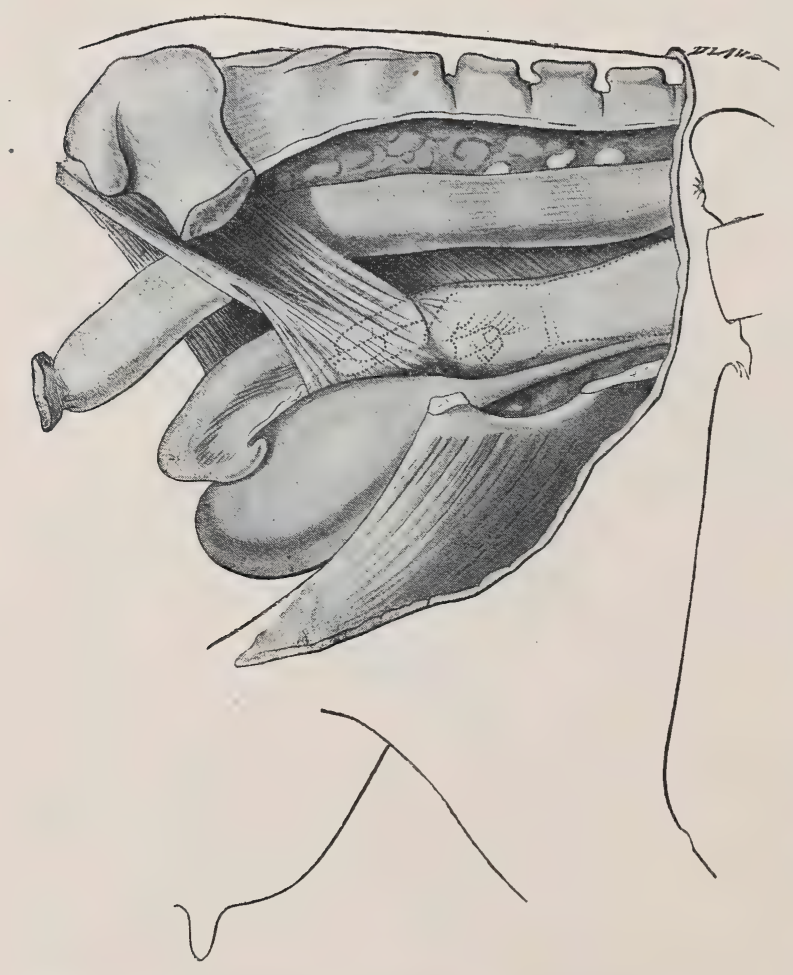

FIG. 321.-Castration of the cow. Second phase. portion of the aorta or the iliae arteries. The blood then streams past the operator's hand, and the animal dies of internal hæmorrhage in a few minutes; nothing can be done.

If there is undue haste in manipulating the écraseur, the pedicle of the ovary is cut rather than crushed, the vessels close imperfectly, and grave hæmorrhage may occur.

In cases where hæmorrhage is slight the peritoneal clot is readily re-absorbed; but should the animal happen to be tuberin. any other way diseased, free hæmorrhage may occur and may
eventually be followed by fatal eventually be followed by fatal peritonitis.

Hernia of the intestine through the vagina is a rare complication nowadays, on account of the small dimensions of the perforations. In former times, when larger incisions were made, it occurred rather
frequently.

Abscess Formation.-Suppuration of the wound and peritonitis or vaginitis are caused solely by the neglect of antiseptic precautions.

Pelvic peritonitis following an operation is indicated, like ordinary acute peritonitis, by loss of appetite, peritonism, colic, etc. 
Even where peritonitis is avoided a local abscess often forms in the vaginal wall owing to infection of the operation wound. The symptoms are delayed for several days, sometimes for a fortnight after operation, and consist in straining efforts, moderate peritonism, diminution in appetite, etc. Vaginal or rectal examination reveals the character and extent of the disease. The abscess should be punctured through the vagina.

Finally, it may happen that castration does not prevent the recurrence of restrum. The ovarian pedicle may have been divided too close to the glandular tissue, a fragment of which has remained adherent to the pedicle.

Certain other operative complications are also possible in dealing with cows suffering from nympho-mania which have developed cysts, tumours, or abscesses of the ovaries. It is then necessary to enlarge the incision in the vagina and take particular precautions not to rupture the cysts or abscesses in the peritoneal cavity. The operator must proceed cautiously and modify his technique according to circumstances.

\section{CASTRATION OF THE SOW.}

Castration of the sow has been practised since very ancient times. The operation is performed on animals intended for fatten. ing, and at all ages between six weeks and maturity.

Anatomical Arrangement of the Genital Organs.-Before performing ovariotomy in the sow it is indispensable to understand the special arrangement of the genital organs. The uterine horns are very long and folded on themselves, forming convolutions which give them somewhat the appearance of small loops of intestine. Nevertheless they can readily be distinguished by the touch, for they are much smaller in size than the latter.

In young sows, two to three months old, they are of about the thickness of a small pencil. Differentiation is more difficult in sows that have borne litters, but as the ovaries alone are withdrawn, leaving the horns of the uterus uninjured, this distinction is unimportant.

The horns of the uterus are suspended in the peritoneal cavity by means of very extensive, well-developed, and very lax ligaments, and, as the horns of the uterus lie at a very acute angle one to the other, the ovaries are very close to the median line of the abdomen. The length and yielding character of the parts and the close apposition of the ovaries explain why the latter can be found and extracted through a single incision in the flank, either on the right or left side. 
An ordinary convex bistoury or a special knife and two artery forceps are the instruments employed.

Manual Technique.-The animal is cast on the right or left side, preferably on the right, so that the right index finger can be employed. Full-grown sows should always be muzzled. In the case of young animals, the limbs should be grasped by assistants, the hind limbs being crossed one over the other and drawn backwards.

Antiseptic applications are highly desirable, although they are usually neglected when a layman directs the operation.

The operator places himself close to the animal's back. The incision may be made in one of three different places.

Certain practitioners recommend a vertical incision about 2 to

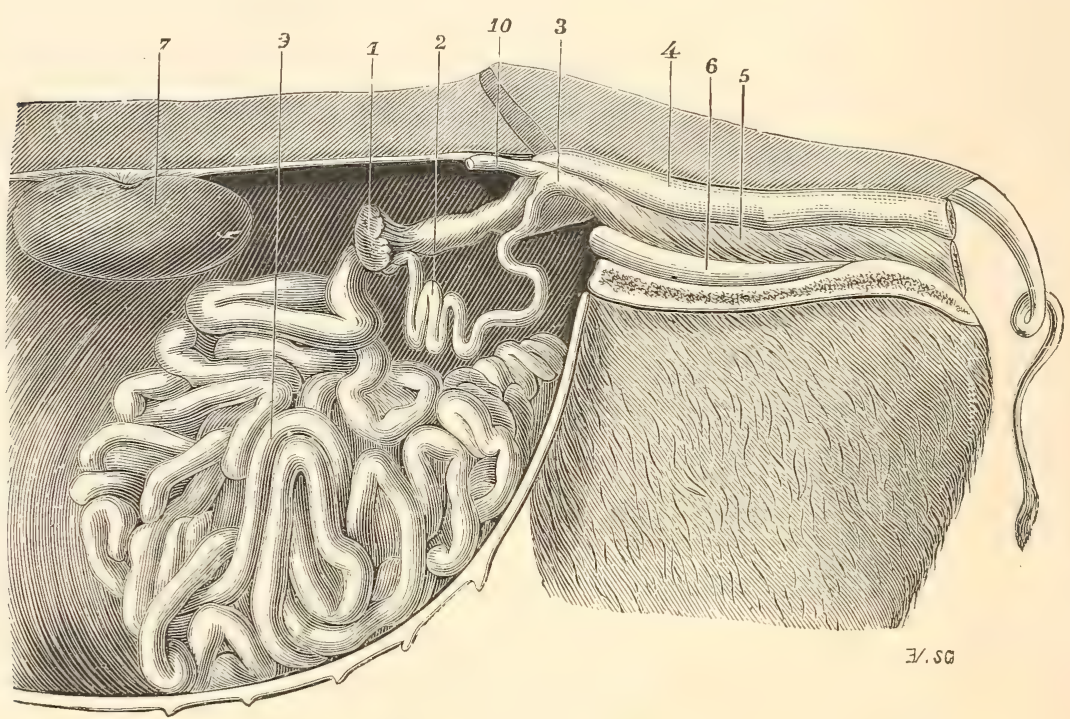

FIG. 322.-Genital organs of the sow. 1, Ovary; 2, horn of the uterus;

3 , uterus ; 4 , rectum ; 5 , vagina ; 6 , bladder ; 7 , kidney ; 9 , intestine.

$2 \frac{1}{4}$ inches in length, commencing at the external angle of the ilium, or $\frac{1}{3}$ of an inch in front of it, and prolonged downwards; others make a horizontal incision parallel with the vertebral axis; and, lastly, some believe that an oblique incision following the direction of the "cord of the flank" is just as advantageous. A vertical or oblique incision is probably the best.

'The operation comprises four stages:

First stage. Incision of the skin and subjacent muscles.

Second stage. Perforation of the peritoneum and discovery of the ovaries. 
Third stage. Ablation of the ovaries or of the ovaries and horns of the uterus.

Fourth stage. Suture of the wound.

The tissues are divided layer by layer. The skin is formed into a longitudinal fold and divided in a vertical direction, and the subjacent muscular layers are then divided with the bistoury. The tissues are next broken through layer by layer with the index finger until the parietal peritoneum is reached. This membrane is then fissured, or at least scraped with the nail, and perforated with a sudden sharp thrust of the index finger.

This practice, however, has the disadvantage of sometimes causing the parietal peritoneum to strip away from the wall of the abdomen, which greatly increases the difficulties of operation. It is better, therefore, to grasp the peritoneum with a small pair of forceps, draw it outwards, and secure it so. as to puncture it with more certainty. When experience has been acquired this precaution will be unnecessary.

The incision being made and the finger introduced into the abdomen, the operator, who kneels against the animal's back, searches for the ovaries with his index finger. The upper ovary of the side in which the incision has been made will be found immediately in contact with the parietal peritoneum, and the operator must take care not to displace it by untimely or careless manipulation, which may thrust it away among the loops of intestine. The finger being doubled up in the form of a hook, the ovary is seized and drawn out. Sometimes it may be

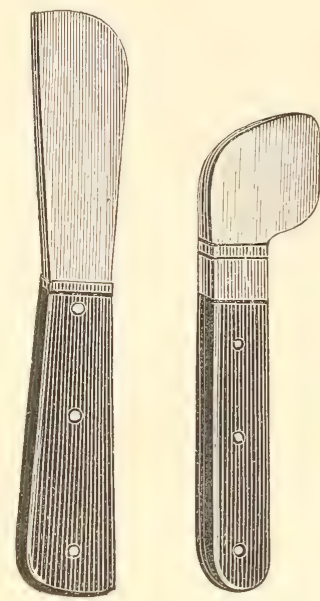

Fig. 323-Castrating knives. easier to withdraw the uterine horn, leaving the search for the ovary until a little later.

The first ovary having been discovered, its pedicle is seized between the left thumb and index finger or the jaws of pressure forceps, and then the search is continued for that of the opposite side. In young sows the horn of the uterus may be followed up from its extremity (ovarian extremity) towards its origin (bifurcation of the body of the uterus) and the search continued along the horn of the uterus of the opposite side, which is followed in the reverse direction from its base towards its extremity until the second ovary is found.

The most difficult stage is that at which the change is made from one horn to the other, for this is the moment when the contraction 
of the parts is most violent, the animal's struggles most energetic and the pain most acute, so that great care must be taken not to let go the horn which has already been secured.

When the second ovary appears at the external orifice, it is secured like the preceding, and both are removed by torsion. The horns of the uterus are then freed and returned to the abdominal cavity, the wound is thoroughly disinfected and united with from one to three interrupted sutures passed through the skin. The animal is then allowed to rise. Complications are rare.

In small females the uterine horns are often removed by torsion along with the ovaries. In adults, only the ovaries are removed.

Subsequent Precautions: Operative Accidents.-The patients are kept on low diet for some days after operation. Accidental stripping away of the peritoneum at the seat of operation may sometimes result in the formation of a little abscess when the wound has been infected. This is diagnosed by direct examination or palpation. The lips of the wound are then opened in order to permit the pus to escape and avoid peritonitis. Should the horns of the uterus or the broad ligaments be roughly manipulated, they may be torn to some extent, but this rarely causes grave complications.

Bleeding from the incision in the abdominal wall is of little importance.

Hernia rarely occurs, for the opening in the peritoneum is of very small size.

In rare cases, and when care is not used, a portion of the intestine may be sutured to the margin of the wound. The intestine then becomes adherent to the abdominal wall, but grave results seldom follow.

\section{SUTURE OF THE VULVA}

In cases of recurrence after reduction of an inversion of the uterus or the vagina it may become necessary to suture the vulva in order to control the effects of straining.

Several forms of suture are employed; the best are probably those of Rainard and Strebel.

Simple Suture.-Simple suture may be formed of very flexible copper wires. Three are usually inserted, one at the base, one about the middle, and one near the upper third of the vulval opening. The ends of each suture are knotted and drawn moderately tight over the opening, then one of the ends of the highest knot is united vertically to an end of the middle knot, and the latter in its turn is similarly secured to the lowest knot. 
To be reliable, sutures should embrace the entire thickness of the lips of the vulva.

Rainard's Suture.-Rainard's suture consists only of two oblique stitches, crossed in the form of the letter "X," starting from the upper third of one of the lips of the vulva and terminating in the lower third of the opposite lip. The ends are tied opposite the centre of the vulval opening.

Strebel's Suture.-Strebel's suture consists of three stitches inserted transversely. The material employed is galvanised wire, sharpened at one end and rolled into a flat spiral at the other. Each wire,
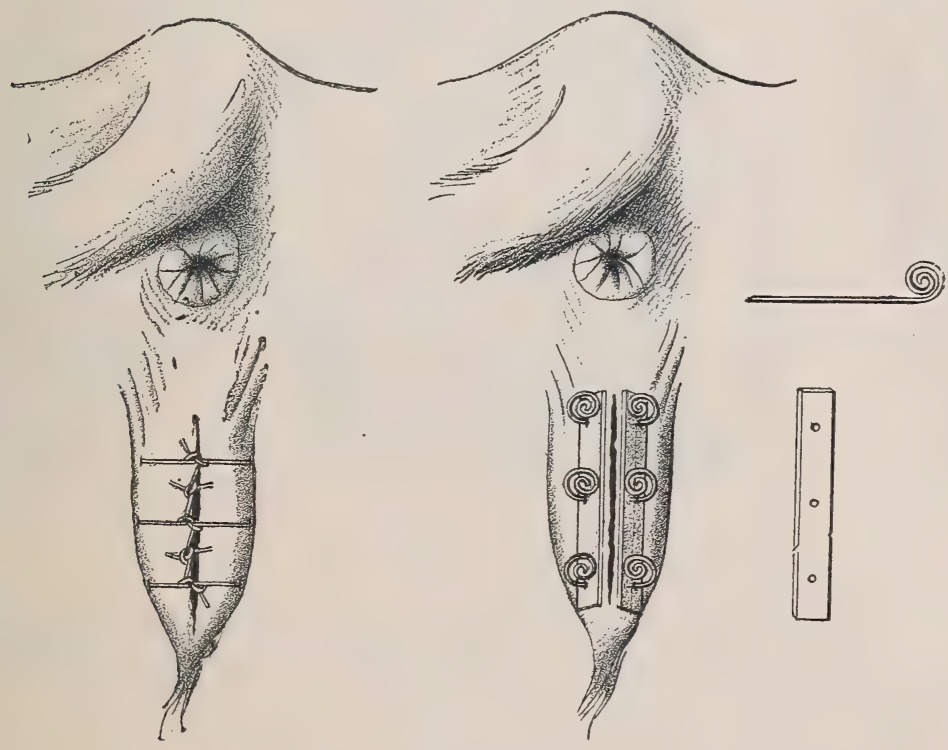

FIG. 324.

which plays the part of a needle, is inserted separately, and is afterwards twisted into a spiral by means of special forceps, the excess of length being thus taken up until the lips of the vulva are brought closely in contact.

In practice, in order to avoid the cutting and irritant effect of such sutures and to increase their efficiency, two rectangular pieces of leather are applied on either side through holes in which the actual metallic sutures are passed. West's vulval clamp is better than sutures. It is very popular in England

\section{TRUSSES.}

Trusses applied for the purpose of preventing prolapsus of the genital organs are now almost entirely given up, as they very imperfectly achieve their object.

D.C. 
Lund's truss, shown in the illustration (Fig. 325), is perhaps the most efficient of those which survive. The essential portion of this is of metal, and is approximately of the shape of the letter "V." The two ends may be separated and drawn together at will by means of a cord. This apparatus is held in contact with the vulva by

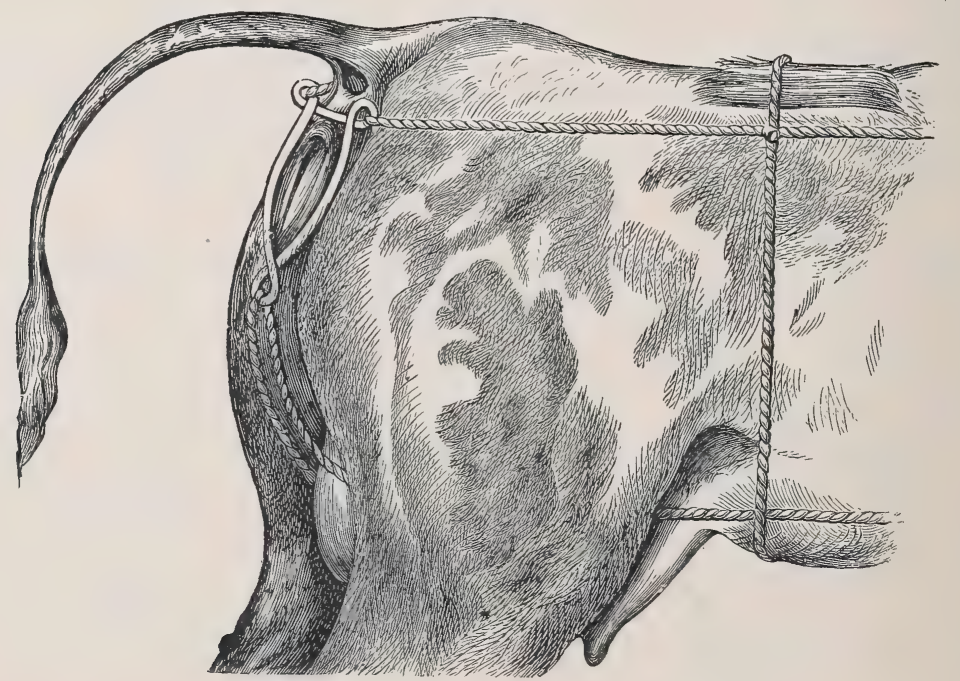

H'IG. 325.-Lund's truss.

means of strings which pass through eyes in the metallic triangle and are secured to a collar placed on the neck or to a girth secured round the chest.

\section{SECTION OF THE SPHINCTER OF THE TEAT.}

This operation is performed to render easily pervious the ends of teats which have undergone contraction as a consequence of changes in the sphincter or from some other cause.

The animals are secured, and the teat to be operated on having been grasped between the index finger and thumb of the left hand, the point of the little cutting instrument shown in Fig. 326 is introduced into the opening of the teat, and it is then thrust in as far as the shoulder on the cutting edges.

\section{DILATATION OF THE ORIFICE OF THE TEAT.}

The results of the above operation not always proving permanent, and cicatricial contraction often following after a few weeks' time, 
forced dilatation by means of the conical tubes shown (Fig. 327) has been generally substituted for it. Three instruments of varying thickness are usually sufficient. Forced dilatation may be effected at a single operation, and has not the drawback of causing cicatrices.

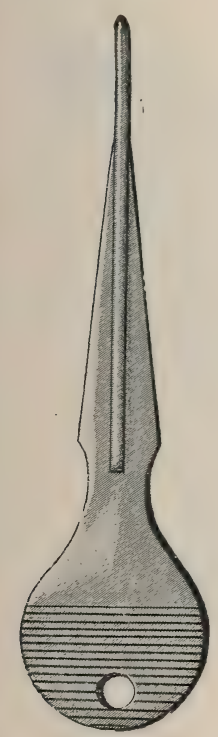

FIG. 326.

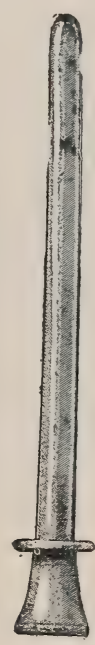

ABLATION OF THE MAMME.

Ablation of the mammæ is rather frequently necessary, principally in cases of gangrenous mammitis, of prolonged, continuous suppuration, or of grave mammitis, where death would otherwise follow.

Provided the anatomical structure of the parts is known (Fig. 237) ablation does not present any insuperable difficulty.

In the cow, either two lateral quarters or the entire udder must be removed. The lines of incision through the skin should first of all be traced.

One half of the udder may be removed by the following method:

First stage. Elliptical incision through the skin, including the two teats of one side.

The lines of incision should extend backwards sufficiently high to enable the vessels at the base of the gland to be easily ligatured.

Second stage. Dissection and breaking down of the subcutaneous and intermammary connective tissue. The anterior mammary vein must be ligatured.

Third stage. Isolation of the mamma from the front backivards, and ligature of the vessels of supply. Extirpation. 
Fourth stage. Suture and drainage of the operative wound with iodoform gauze.

This operation appears to cause formidable injuries, the abdominal

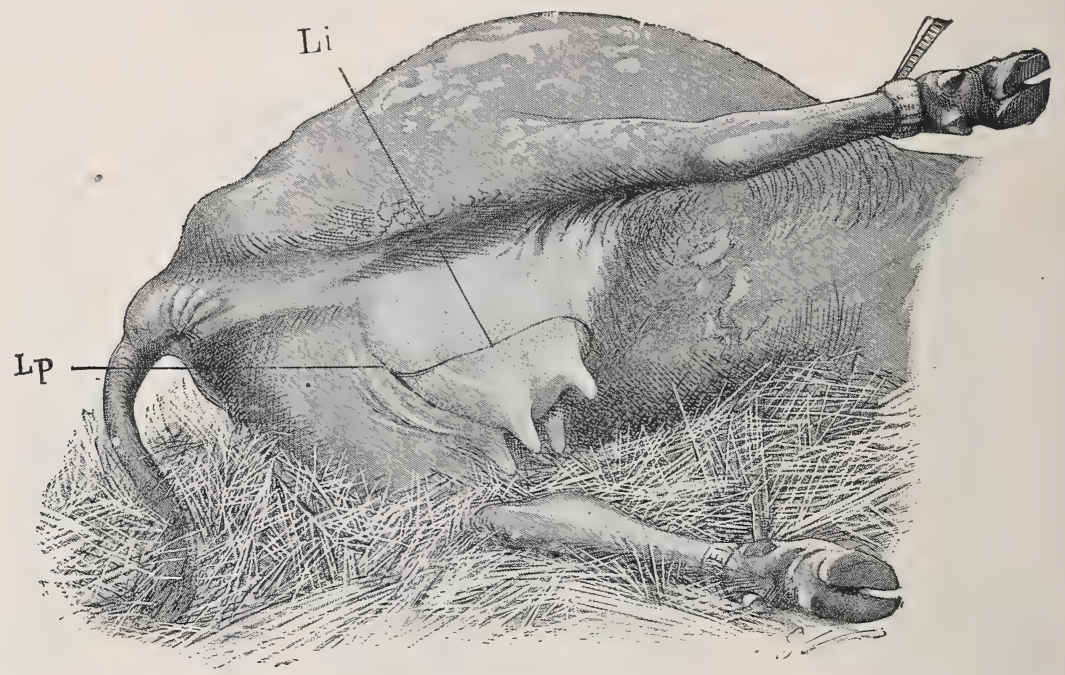

FIG. 328 - Ablation of the udder in the cow. Li, Line of incision;

$\mathrm{L} p$, prolongation backwards.

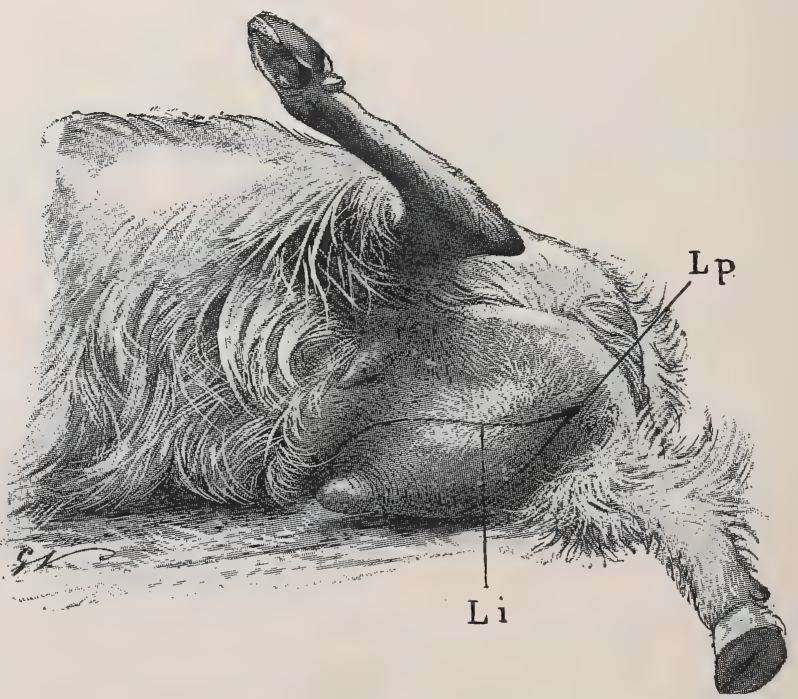

FIG. 329.--Ablation of the udder in the goat. Li, Line of incision; L $p$, prolongation backwards.

tunic and the muscles of the flat portion of the thigh being largely exposed, but in reality the wound is less grave than might be supposed, 
and healing occurs in a comparatively short time, provided none of the diseased tissue is left.

The dressing is renewed at intervals of two or three days, and when cicatrisation proceeds regularly it may be omitted and replaced by antiseptic irrigation.

In the ewe and she-goat the operative technique is identical:

First stage. Elliptical incision, including the teat.

Second stage. Breaking through of the intermammary connective tissue partition and the subcutaneous tissue. Ligature of the anterior mammary vein.

Third stage. Isolation of the mamma from the front backwards. Ligature of the vessels of supply. Extirpation.

Fourth stage. Suture and drainage. 



\section{INDEX.}

A.

ABDominal cavity, diseases of Ablation of the mammæ

Abomasal indigestion

Abomasum

$$
\text { " }
$$

hernia of the

obstruction of the

strongylosis of, in the ox

Abortion, epizootic, in cows

Acariasis, non-psoroptic forms of .

Accessory glands of the genital apparatus

Accidental phlebitis

Acid theory in diseases of bones

Acids, caustic, poisoning by .

Acne in the sheep .

Acorns, poisoning by . . . . 228

Acquired herniæ . . . . . 489

Actinomycosis . . . . . 672

Actual cautery, castration with the .758

Acute cystitis . . . . 511

" deep-seated glossitis . . . 131

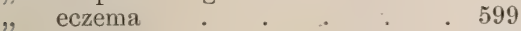

"enteritis . 203

" gastric indigestion in swine . 1 .

" gastritis

" inflammation of the gastric com-

" partments . . . 186

". laryngitis . . . . . . 333

" mammitis . . . . 573

" metritis . . . . 550

" nephritis $: 528$

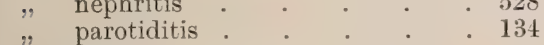

" peritonitis . . . . 478

" peritonitis.$\quad \cdot \quad \cdot{ }^{\prime} \quad \cdot \quad \cdot 361$

" tuberculosis. : : : $: 704$ " vaginitis

Agalaxia

Agaricaceæ

Alkalies, caustic. poisoning by . . 217

Aloes, poisoning by . . . . 221

Alsinaceæ $\quad . \quad . \quad 229$

Ambrosiaceæ .

Amputation of the claw $\quad .730$

Anæmia in cattle. 268

" lambs: $\quad . \quad \cdot \quad . \quad \cdot \quad 268$

$\begin{array}{ll}\text { Anæsthesia } & \text { sheep * * } \cdot 726\end{array}$

Annual mercury, poisoning by . $\quad 256$

Anomalies, physiological . . 567

Anus . . 116

" imperforate.
Apiaceæ. PAGE

Apocynaceæ : $\quad . \quad+\quad . \quad 5 \quad$. $\quad .251$

Apparatus of locomotion . . . 730

Aragallus spicatus, poisoning by . . 237

Arsenic, poisoning by . . . . 218

Arsenical dips . . . . . . 632

Arteries, examination of . . . 371

"Arthritis of mileh cows" . . . . 99

Articular rheumatism . . . . 89

" $\quad$ " causes . . 89

", complications . 91

Articulations, diseases of . . . . 4.)

Ascites . . . . . 483

Asclepiadaceæ . . . . . 252

Aspergilli, pneumo-mycosis due to . 350

B.

BARBERry family. . . . . . 235

Beech family. . . . . . 228

Beef measles . $\quad . \quad$. $\quad . \quad$. $\quad .79$

" $"$ cause $\quad . \quad . \quad . \quad . \quad 79$

" $"$ symptoms . . . 81

" where prevalent . . 79

Bell-flower family . . . . . . 255

Berberidaceæ . . . . . 235

Biceps femoris . . . . . . 70

Bile ducts, cancer of . . . . . 282

Bilharziosis in cattle and sheep . . $\quad 439$

Bistournage . . . . . 751

Bitter milk . . . . . . . . 591

Bladder, diseases of the. . . . 511

". eversion of the . . . . 519

" paralysis of the $\quad . \quad$. $\quad .519$

Bleeding . . . . . 727

, in the pig . . . 728

" in sheep . . . . $\quad .727$

Blood, diseases of the . . . . 406

" examination of . . . . 372

" poisoning in sheep and lambs in New Zealand. . . 415 -vessels, diseases of . . $\quad .396$

Blood-wort family . . . . . 228

Bloody flux in calves and lambs . . 271

Blue milk . . . . . 590

Boars, castration of $\quad . \quad 759$

Bog spavin in the ox . . . $\quad . \quad 46$

Bone, actinomycosis of . . . . 681

" tumours . . . 30

Bones and articulations, tuberculosis of

the. 701 
Bones, diseases of .

$$
\text { " } \quad \text { " } \quad \text { acid theory } \quad \text { theory of insufficiency }
$$

Bovine animals, calculi in

$$
\text { piroplasmosis }
$$

Box family

Brain, tubereulosis of the

Braxy

Bronchi

Bronchitis

$$
\begin{aligned}
& \text { chronic } \\
& \text { pseudo-membranous } \\
& \text { simple acute } \\
& \text { verminous, in sheep and } \\
& \text { cattle }
\end{aligned}
$$

\begin{tabular}{|c|c|c|}
\hline " & $"$ & infectious \\
\hline$"$ & $"$ & of sucking calves . \\
\hline$"$ & $"$ & $\begin{array}{c}\text { sclero-caseous, of } \\
\text { sheep }\end{array}$ \\
\hline
\end{tabular}

Broncho-pneumonia, gangrenous, due to foreign bodies.

Bryony, poisoning by

Buckwheat poisoning

Bull, castration of the

Bunch-flower family

Bursal sheath of the flexor tendcns, distension of

Butneriaceæ .

Butter, milk without

Buxaceæ

\section{C.}

CALCULT in bovine animals . . . . 515

urinary, in sheep . . . 518

Calculus formation

Calves, depraved appetite in .

$$
\text { diarrhœic enteritis in }
$$

dysentery in

goitre in

intestinal coccidiosis of

lumbricosis of

mycotic stomatitis in

necrosing stomatitis in

Calving, dropping after.

Campanulaceæ

Cancer of the bile ducts

$$
\text { " " liver }
$$

Cancerous pericarditis .

Canker

$$
\text { treatment . }
$$

Capillary system, examination of .

Carbolic acid poisoning .

$$
\text { dips. }
$$

Cardiac anomalies.

Carduaceæ

Carrot family

Caseous lymphadenitis of the sheep

Casting, control of oxen by

Castor-oil cake, poisoning by

Castration

$$
\begin{array}{ll}
" & \text { complications after } \\
" & \text { by clams } \\
" & \quad \text { " the covered method } \\
& \quad, \text { elastic ligature } .
\end{array}
$$

PAGE

3

3

416

246

702

435

333

336

337

339

337

351

354

356

358

256

606

751

227

49

235

589

246

518

160

212

210

453

271

267

124

123

461

255

282

282

375

40

41

372

221

633

374

256

247

453

723

257

751

760

756

757

758
Castration by the exposed method

PAGE

" of boars and young pig. 757

" $\quad$ the bull and ram . . 751

". . cow. . . 761

" " cryptorchids . . . 760

" " the ram . . . 759

$" \quad " \quad, \quad$ " by bistournage . 759

" " " , ligature . . 759

" $\quad " \quad " \quad$ " $" \quad$ " tearing . $\quad 759$

" sow". 765

", with the actual cautery . 758

Catarrhal gastritis in swine . . 190

, stomatitis in sheep . . 122

" general, in swine . 126

Catheter, passage of the, in the cow . 750

" " ram . 749

Cattle, anæmia in . . . . . . 268

" bilharziosis in . . . . 439

". diarrhœea in . . . . . 268

, hæmorrhagic septicæmia in . 716

" parasitic gastro-enteritis in . 268

,$\quad$ pseudo-membranous pharyngitis

in . . . 141

., verminous bronchitis in . . 340

Caustic acids, poisoning by . . . 217

, alkalies, poisoning by . . 216

Cerebral congestion . . . . 456

. tumours . . . . . 459

Changes in the milk $\quad . \quad . \quad 587$

Chaps . . 568

Chemical dyspepsia $\quad . \quad \cdot \quad . \quad \cdot 195$

Chenopodiaceæ . . . . . 229

Chorioptic mange . $\quad . \quad 636,640,642$

Chronic bronchitis . . 337

cystitis $\quad: \quad \cdot \quad \cdot \quad 513$

" diarrhœa . $\quad . \quad . \quad .207$

" eczema . . . 600

" enteritis . . . . 207

". . . . : 194

" glossitis : $\quad . \quad+\quad . \quad+132$

" indigestion . . . . 194

" mammitis. $\quad . \quad$. $\quad . \quad 581$

, metritis . . . . 552

" nephritis . $\quad . \quad 530$

. parotiditis . . . . 136

" pericarditis $\quad . \quad$. $\quad .389$

" peritonitis . . . . 481

" pleurisy . $\quad . \quad$. $\quad .362$

"simple synovitis, forms of . 45

". tympanites . . . 194

vaginitis . 546

Circulation, organs of the . . . $\quad .370$

Circulatory apparatus . . . . 727

Clams, castration by . . . . 756

Claw and third phalanx, disarticulation of the

731

amputation of the $\quad . \quad 730$

", surgical dressing for a . . . . 730

Claws, congestion of the . . . 31

Clement's bath . . . . . $\quad .623$

Clotted milk . . . . . . . 589

Cœnurosis . . . . . . . 467

Colchicum poisoning . . . . 256

Cold water, colic due to ingestion of . 162

Colic . . . 116

,$\quad$ as a result of strangulation . $\quad 167$ 
Colic due to intragination

in the

Common salt, poisoning by $\quad \cdot \quad \cdot \quad \cdot 217$

Complications after castration . . 760

Condylomata. . . . . 38

Congenital herniæ.

Congestion, pulmonary . of the claws

Congestive colic

$$
\text { " liver }
$$$$
\text { udder }
$$

Conjunctivitis

Contagious disease (takosis) of goats "mammitis in milch cows vaginitis

"Contagious foot disease"

Contraction of the sphincter

Control of pigs

$$
\begin{aligned}
& " \quad \text { sheep and goats } \\
& " \quad \text { oxen by casting } \\
& " \quad \text { general, of oxen }
\end{aligned}
$$

Contusions of the sole

Convallariaceæ

Copper poisoning .

Coryza, gangrenous

" simple

570

162

662

$+12$

580

545

41

567

725

$7 \cdot 5$

720

723

722

31

228

221

320

319

Cotton cake, poisoning by $\quad 257$

Covered method, castration by the . 757

Cow, castration of the

" " " complications in " passage of the catheter in the

Cows, epizootic abortion in

Cowper's glands

Cow-pox

Cracks

and human variola

Creolin bath.

Croupal vaginitis

Crowfoot family $\quad 230$

Crushing a foreign bod 5 in the osophagus 735

Cryptorchids, castration of . . . 760

Cysticerci, infection with . . . 73

Cysticercosis . . . . 290

" . . . . 485

Cysticercus disease of the pig . $\quad 73$

Cystic parasites of animals, table of . 73

Cystitis, acute . . . . . . 11

, chronic . . . . . . 513

Cysts of the udder. . . . . . . 585

\section{D.}

DEF æCATION : examination of fæcal material

Demodecic mange

Depraved appetite.

in $\cdot 158$

$" \quad$ in calves and lambs. 160

Diaphrage " the ox 158

Diarrheamatic herniæ . . . . 496

$$
\text { " in cattle } \quad . \quad \text {. } \quad . \quad \text {. } 268
$$$$
\text { " lambs }
$$

" . sheep . . . . . 268

Diarrhœic enteritis in calves. $\quad . \quad 212$
Digestive apparatus $\quad$ PAGE

diseases of

" $"$ fistulæ of : : $\quad$ : 500

$" \quad$ " parasites of . . 263

",$\quad$ semiology of . . 106

Digestive tract, tuberculosis of the $\quad 699$

Dilatation of the œesophagus . . . 149

$, \quad, \quad$ orifice of the teat . 770

Disarticulation of the claw and third phalanx . 731

$$
\text { , } \quad \text { two first pha- }
$$

Diseases of the bladder . . . . 511

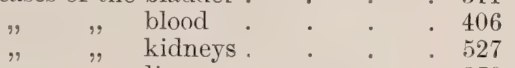

" " kidneys. . . . 527

" $\quad$ " lyver $\quad$. $\quad$. 449

" " mouth. . 106

$"$ " $"$ cesophagus : . 109

$"$ " pharynx . . 108

$" \quad$ salivary glands : 108

$"$ stomach $" .110,169$

$"$ " rumen . . . 110

$" \quad$ " peritoneum and abdominal cavity . 478

" . urinary apparatus . . 502

$" \quad$ produced by distillery and sugar factory pulp

" transmissible to man through the medium of milk

Distillery and sugar factory pulp,

diseases produced by . . . . 259

Distomatosis . . . 293

Disturbance in the milk secretion . . 587

Dogbane family . . . . . 251

Dropping after calving . . . . . 461

Dysentery in calves . . . . 210

,,$\quad$ and lambs : . 271

Dyspepsia ." . . . 194

" motor $\quad \cdot \quad 195$

E.

ECHINOcOcCosis of the liver . . 283

suppurative . . . 288

Ectopia of the heart . . . . 374

Eczema . . . 599

" acute . . . . $\quad . \quad 599$

" chronic . . 600

" due to feeding with potato pulp . . . 603

sebaceous or seborrhœic . $\quad 601$

Elastic ligature, castration by the . 758

Emphysema, pulmonary 359

" subcutaneous . . . 659

Encephalitis .

Endocarditis . .394

Enteritis . . . . . 203

" acute . 203

" chronic . . . . 207

". diarrhœic, in calves . . 212

". hæmorrhagic . . . 206

Epizootic abortion in cows . . . 553

Equisetaceæ . . . . . . 225

Ergot family . . . . . . . 223 


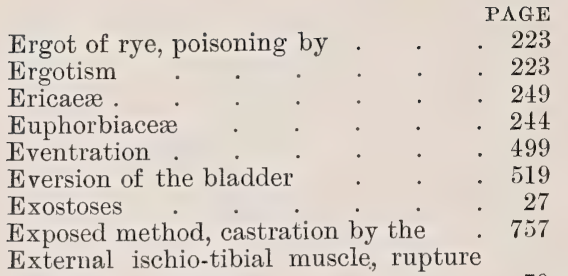
of the.

Exudative pericarditis due to foreign bodies.

Eyes, diseases of the

\section{F.}

FACIAL sinuses of sheep, œstrus larvæ in the

trephining the

Fæcal material, examination of

Fagaceæ

Fagopyrism

False sturdy

Fasciola hepatica.

Felon

Female genital organs, examination of, with the speculum

Femoro-tibial articulation, luxation of the.

, , $\quad$ symptoms.

Femur, luxation of " treatment.

" " " symptoms

" "treatment

Fennel, poisoning by

Ferments, lactic

Fern family

Fetlock joint, distension of the synovial capsule of the strain of

Figwort family

Fistula, parotid

Fistulæ, milk

Flax family

$$
\text { of the digestive apparatus }
$$

Flexor metatarsi, rupture of the

"tendons, distension of the bursal sheath of

Food, poisoning due to .

Foot, diseases of the

$$
\begin{aligned}
& \text { " rot } " \text { symptoms } \\
& " \text { " treatment }
\end{aligned}
$$

Foreign bodies causing diseases of the eyes. . . exudative pericarditis due to. gangrenous brouchopneumonia due to gastric disturbance due

migration of, from the reticulum, pneumonia due to
Foreign bodies, pneumonia due to Foreign body in the œesophagus, crushing a

$$
\text { " } \quad \text { " } \quad \text {, } \begin{array}{r}
\text { sub-mucous } \\
\text { dissection }
\end{array}
$$
of . $\quad 736$

Fractures . 20

France, bovine piroplasmosis in . . 424

Frontal sinus, trephining the . . 745

",$\quad$ purulent collections in . 327

G.

GANGRENous broncho-pneumonia due to foreign bodies

corvza $\quad \cdot 320$

" mammitis in goats $\quad 584$

" $\quad$ mammitis in goats $\quad$ of milch ewes $\quad 583$

Gaseous indigestion" . . . 170

Gastric compartments, acute inflamma-

$$
\begin{array}{cc}
\text { tion of } & 186 \\
\text { tumours of the } . & 202
\end{array}
$$

". disturbance due to foreign bodies 198

, indigestion, acute, in swine . 185

Gastritis . . 186, 188-194

Gastro-intestinal strongylosis in sheep . 263

Gastrotomy . . . . . . 739

"Gathered Nail" . . . . . . 37

General diseases : $\quad . \quad$. $\quad . \quad 4$

Genital apparatus . . . . . . 542

\section{H AEMATURIA}

accessory glands of the

" malformations .

organs, male

tuberculosis of the $\quad .700$

Genito-urinary organs . . . . 747

" regions . . . . 502

mammary, diseases

Glossitis $\quad . \quad 130$

, acute, deep-seated . . 131

" chronic . . . . . 132

"nodular sclerosing : . . 133

superficial $\quad 130$

Goat, demodecic mange in the . . 644

, mange in the . . 641

ringworm in the . . . . 653

Goats, control of . . . 725

, gangrenous mammitis in . . 584

Goitre in calves . . . . . . 453

" lambs . . . . . 453

Goosefoot family . . . . . . 229

Grass family . . . . . . . . 226

" tick, life history of . . . 432 Grease

\section{$\mathrm{H}$}

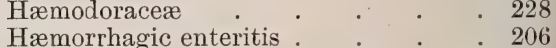


Hæmorrhagic septicæmia, infective dis-

eases confused with

Haunch, hygroma of . . . 67

Heart, ectopia of the . . . . 374

Heat stroke . . . . . . 442

Heath family 249

Hellebore, poisoning by . . . 234

Helminthiasis, intestinal, in ruminants 275

Hemlock, poisoning by .

Hepatitis, nodular necrosing .

Hernia, inguinal, in young pigs . . 741 " of the abomasum . . . 493

$"$ " intestine . . . 494

". rumen . . . 490

Herniæ perineal, of young pigs $\quad 487,741$

" acquired : $\quad: \quad . \quad 489$

congenital

" diaphragmatic .

treatment of

Hock, hygroma of the point of the joint, distension of the synovial capsule of the

, , strain of

$"$ region, distension of the tendon sheaths in

Hoose

Horn core, trephining the

Horns, anatomy of

"detachment of :

". fissuring of

". fractures of

" splints for

"treatment of

Horse-chestnut family

Horsetail family

Human variola and cow-pox

Husk

Hydro-nephrosis . $\quad . \quad 531$

Hydro-pneumo-thorax . . . . 366

Hygroma of the haunch $\quad . \quad 67$

" knee. . . 65

$" \quad$ " point of the hock : 68

$" \quad$ " point of the sternum . 69

",$\quad$ stifle . . 67

" " trochanter of the femur

487

496

68

46

Hygromas

Hypericaceæ

Hypocreceæ

Hypodermosis in the ox. . . . . 646

\section{I.}

IMPACTION of the omasum . . : : 179

" " . . . 175

Imperforate "anus . . . . . . 742 " condition of the teat . 567

" vagina . . . . 560

Impetigo in the pig . . . . . 605

Indigestion . . . . . . 170

abomasal : . $\quad . \quad 182$

acute gastric, in swine $\quad 185$

as a result of over-eating . 175

chronic . . . 194

Infectious broncho-pneumonia : 354
665

" pyelo-nephritis . $\quad 533$

" pseudo-rheumatism in adults 99

". ", symptoms 100

" $\quad "$ treatment 103

,. rheumatism in young animals 94

$" \quad " \quad$ " causes . 94

" $\quad$ " symptoms 95

Infective diseases, confused" with hæmor-

rhagic septicæmia

Inflammation of the sheath . $\quad .506$

sub-maxillary sali-

Inflammatory diseases

Inguinal hernia in young pigs $\quad . \quad 541$

Insolation . 460

Interdigital space, inflammation of the 38

Internal infectious phlebitis . . 398

Interstitial mammitis . . . . $57 t$

Intestinal helminthiasis in ruminants . 275

Inte tuberculosis . . . . 699

116

hernia of the .

Inversion and prolapsus of the rectum . 743

Iodine poisoning . . . . . 222

Iodism . . . . . . 222

Iodoform poisoning . . . . 222

Ischial urethrotomy . . . . . 747

Ischio-tibial muscle, external, rupture of the

Issues

J.

JoINTs, luxation of

56

K.

KERATITIS : : . . . 662

Kidney worm of swine . $\quad$. $\quad$. $\quad$. 5339

Kidneys, congestion of the . . . 527

Knee, distension of tendon sheaths in the region of . . . . 49

, hygroma of . . . . . 65

$"$ joint, distension of the synovial capsule of the . . . 47

, strain of . . . . 53

\section{L.}

LACTIC ferments . . . . . 588

Lambs, anæmia in . . 268

$"$ blood poisoning in, in New Kealand 415 depraved appetite in $\quad . \quad 160$ diarrhœa in . . . . 268 goitre in . .453 intestinal coccidiosis of . .271

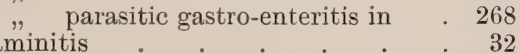

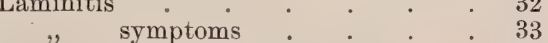

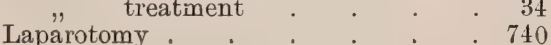


Larkspur poisoning in sheep.

Laryngitis

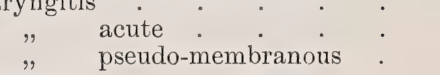

Larynx

examination of
tumours of

Lathyrism

Lead poisoning

Licking habit

Lily of the valley family

Lime and sulphur dips .

" " prejudiceagainst

Linaceæ

Liver

$\Rightarrow$ cancer of
$"$ diseases of
$"$ echinococcosis of
rot

Liver-fluke (Fasciola hepatica)

" disease

$$
\text { disease }
$$

Locomotion, apparatus of

$$
\begin{array}{ll}
\text { organs of, diseases of } \\
\text { gait in }
\end{array} .
$$

Louping ill percussion in

Lumbar prurigo, or "trembling," in sheep .

Lumbricosis of calves

Lund's truss .

Lungs

Lupines, poisoning by

Luxation of the femoro-tibial articula-

$$
\text { tion . }
$$

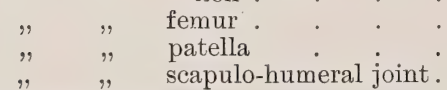

Lymphadenitis scapulo-humeral joint.

Lymphatic glands, tuberculosis of $\quad-696$ " glandular apparatus, topography of

$" \quad$ system, diseases of . 445

Lympho-cythæmia

Lymphogenic diathesis .

M.

\section{MAGNOLIACE $E$}

Magnolia family

Maize, poisoning by the male tufts of .

Male genital organs

Malformations, genital .

Malignant œdema .

Mammæ, abiation of the

Mammary glands, diseases of

Mammitis

$$
\text { toxæmia }
$$

$$
\text { acute }
$$

chronic

contagious, in milch cows
PAGE

231

333

333

333

313

335

243

220

228

627

628

244

282

280

279

283

293

294

293

20

730

1

1

1

429

475

267

770

343

$2+1$

61

56

58

63

448

696

444

448

448

Mammitis, gangrentis

mitis, gangrenous in goats - 584

" interstitial of milch ewes . 583

". parenchymatous : : $\quad . \quad 575$

611

" chorioptic. . . $636,640,642$

, demodecic. . . . 643

" in the goat . . . . 641

", ox. . . . . 638

" $\quad$ " pig . . . . . . 642

" psoroptic . . . . 614,639

" sarcoptic . . . . 638,641

" symbiotic . . . . 636

Manual technique in bistournage . . 752

" $" \quad$ castration of the cow 762

" " " " sow 766

. . . 756

Maxilla, actinomycosis of the $\quad . \quad$. 673

Maxillary sinus, purulent collections in 329

" trephining the . . 745

Teasles, beef . 79

" pork. . . . . . . 78

Mechanical pneumonia . . . . . 347

Mediastinum, diseases of structures enclosed within the . 368

" tuberculosis of the $\quad 697$

Medic" tumours of . 369

anthaceæ. . . . 227

Meliaceæ . . . . . . . 244

Meningitis . . . . 456

Mercurial poisoning . . . . $\quad$. 219

. stomatitis . . . 128

" nature of . - 129

Tetritis . . 547

" acute $\quad . \quad . \quad . .550$

" chronic . . . . . 552

" septic . . . . . 547

. • 588

foreign bodies from the

reticulum, pneumonia due to . . 348

Milch cows, contagious mammitis in : 580

" ewes, gangrenous mammitis of . 583

Milk, bitter . . . . . 591

" blue . . . . . . 590

" changes in the . . . $\quad .587$

" clotted . . . . . . 589

"diseases transmissible to man

through the medium of . . 593

fever... . 461

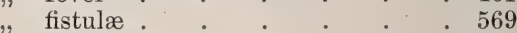

" medicated . . . . 591

" microbic changes in . . . 588

" mucous, viscous, or thready . $\quad$. 589

" preservation of . . . . 591

" putrid. . . . . . 589

" red . . . . 590

"secretion, disturbance in the 587

" thready . . . 589

" viscous . . . . . . 589

" without butter . . . . 589

" yellow. $: 590$

Milkweed family . . . . . . 252

Molasses refuse, poisoning by $\quad . \quad 258$

Motor dyspepsia . . . . . . 195

Mouth, diseases of . . . . . 106, 121

Mucous milk . $\quad$. $\quad$. $\quad$. $\quad$. 589 
Muguet .

Muscles and tendons, diseases of . 70 parasitic diseases of . . . 73

Theurism

Mushroom family .

Mycotic stomatitis in calves.

Myelo-cythæmia

\section{N.}

NAILs, picked-up .

Nasal cavities

$"$ gleet tumours of : :

sinuses, purulent collections in . 326

Neck, actinomycosis of the . . . 675

Necrosing stomatitis in calves : . 123

Nephritis, acute . . . . 528

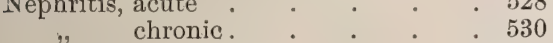

suppurative . . . . 537

Nervous system . . . . . 456

Nettle family . . . 229

New-born animals, septicæmia of . $\quad 406$

New"Zealand, umbilical phlebitis of 399

Nitrates of potash and soda, poisoning by

Nodular necrosing hepatitis .

, sclerosing glossitis . . . 133

Non-psoroptic forms of acariasis . . 645

Nympho-mania

562

\section{O.}

OBSTRUCTION of the abomasum

Esophageal obstructions

194

Esop" sounds . . .

Esophagitis .

Esophagotomy

Esophagus

dilatation of

diseases of

ruptures and perforations of 157 stricture of .

Estrus larvæ in the facial sinuses of sheep .

Oleaceæ

Olive family

Omasum

Omphalo-phlebitis .

"Open arthritis"

"Open synovitis"

Operations

Ophthalmia, verminous, of the ox.

Organs of circulation, semiology of locomotion, diseases of .

of "examination in " methods

Orifice of the teat, dilatation of the

Osseous cachexia.
$9-11$

. 8,11

18

Ostitis, suppurating $\quad-29$

Ovary, tumours of the . . . . . $\quad 559$

Over-eating, indigestion as a result of .175

Over-exertion . . . . . . 442

Ovine pasteurellosis . . . . . 263

$"$ piroplasmosis . . . . 425

$\mathrm{Ox}$, colic in the.$\quad 162$

" demodecic mange in the . . 644

, depraved appetite in the . . 158

", hypodermosis in the . . . 646

mange in the . . . 638

" strongylosis of the abomasum in the 268

urethrotomy in the . . 747

" verminous ophthalmia of the . . 663

Oxen, canker in 40

" control of . . . . 720

" by casting : . 723

$" \quad$ " the limbs . . . 720

warts in .655

P.

Panaritium . . . . . 41

Pancreas . . . 119

Papaveraceæ . . . . . . 235

Papillomata, verrucous, of the udder . 586

Paralysis of the bladder . . . 519

Parasites, cystic, of animals, table of . 73 ,$\quad$ of the digestive apparatus : 263

Parasitic diseases of muscles . . . $\quad 73$ " gastro-enteritis . . . 268

" $\quad$ in cattle $\quad 268$

" $\quad$ in lambs $\quad$. 268

Parenchymatous mammitis . $\quad \cdot 575$

Parotid fistula . . . . 136

glands, actinomycosis of the 675

Parotiditis (Parotitis) . . . 134, 136

Parturient apoplexy . . . . 461

Passage of the catheter in the cow $\quad 750$

" " , ram . 749

Passing the probang . . . . 735

Patella, luxation of . . . . . $\quad .58$

",$\quad$ bandage for . . 60

, $\quad$ " symptoms $\quad . \quad 59$

$\begin{array}{ccc}\text { treatment } & & \\ \text { Patellar synovial capsule, inflammation of } & 40\end{array}$

Pea family . . . . . . . 236

Pelvis, fractures of . . . . 20

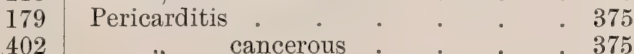

$51 \quad$ " $\quad$ chronic $\quad . \quad$. $\quad 389$

49 " exudative, due to foreign

bodies . . . 376

simple acute . . . $\quad .375$

specific . . . . 375

Perineal hernia of young pigs . . 487

Perinephritis . . . . 537

Peripneumonia and pneumonia, differ-

ences between . . . . . 347

Perisporaceæ . . . . 223

Peritoneal cysticercosis . . . . $48 \tilde{5}$

Peritoneum, diseases of . . . 478 


Peritonitis.
acute
chronic

Phosphorus poisoning

Phthiriasis poisoning

Physiological anomalies . . . . 567

Phytolaccaceæ . . . . . 229

Pica . . . . . . . 158

Picked-up nails . . . . 37

Pig, cysticercus disease of the . . . 73

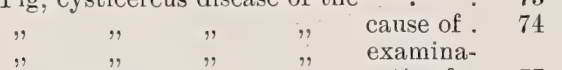

" " " $\quad, \quad \begin{gathered}\text { tion for } \\ \text { symptoms } \\ \text { of }\end{gathered}$

". "

"demodecic mange in the . . 644

", impetigo in the . . . . 605

mange in the 642

pneumonia of the . . . 710,714

ringworm in the . . . . 653

, urticaria in the . . . 656

" verrucous endocarditis of the. 710,713

Pigs, control of . . . . . . 725

" ringing . . . . . 734

" tonsilitis in . . . . . 138

"young, castration of . . . $\quad 759$

" $"$ inguinal hernia in . $\quad 741$

" " perineal hernia of . . 487

Pink family . . . . . . 229

Piroplasmosis . . . . . 416

Plantar aponeurosis, injury to . . $\quad 38$

Plants poisonous to stock . . . 22:

Pleura, diseases of . . . . . 361

Pleuræ . . . . . . 343

Pleurisy, acute . . . . . 361

" chronic . . . . . 362

Plugs . . . . : : $\quad$ : 728

Plum family . . . . . . 236

Pneumonia due to foreign bodies . $\quad 347$

, migration of foreign bodies from the reticulum . . 348

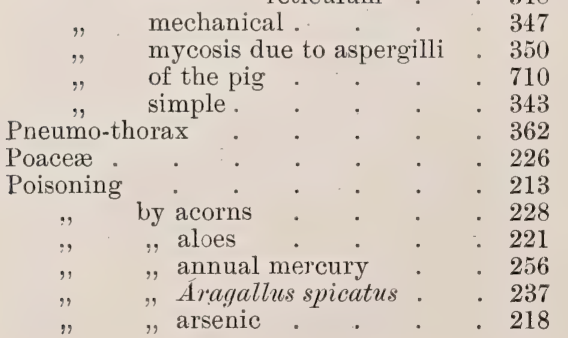

Poisoning by bryony PAGE

256

" castor-oil cake : $\quad 257$

., caustic acids . . . 217

" " alkalies . . 216

common salt . . : 217

" cotton cake . . . 257

", ergot of rye . . . 223

", fennel . . . . 249

" hellebore. . . . 234

" hemlock . . . . 248

"lupines . . . . 241

" male tufts of maize. $\quad 226$

" molasses refuse . . 258

"nitrates of potash and soda . . . . 217

" poppies . . . . 235

"ranunculaceæ . . 234

"s smut of barley . . 224

"St. John's-wort - 246

", sweet sorghum grass _ 226

. tartar emetic . . . 218

"vetches . . . . 243

", white loco weed . 237

„, wild chervil . . . 248

carbolic acid . . . . 221

colchicum . . . . 256

copper . • • . 221

due to food . . . . 215

jodine . . . . . 222

iodoform . . . . . 222

larkspur, in sheep. . . 231

lead . . . . 220

mercurial . . . . 219

phosphorus . . . . 219

strychnine $\quad 222$

tobacco. . . . . 254

Pokeweed family. . . . . 229

Polypi of the glans penis and sheath . 506

" pharyngeal . . . . 143

Polypodiaceæ . . . . . . 225

Poppies, poisoning by . . . . 235

Poppy family . . . . . 235

Post-partum paralysis . . . . . 461

Potato family $\quad . \quad . \quad . \quad . \quad 252$

" pulp, eczema due to feeding 603

Preservation of milk . . . . 591

Pricks and stabs in shoeing . . . $\quad 36$

Primrose family . . . . . 251

Primulaceæ. . . . . 251

Probang, passing the . . . 73 5

Probangs . . . . . 155

Prolapsus and inversion of the rectum . 743

Prostate. . . . 597

Prunaceæ . . 236

Pseudo-membranotis bronchitis : . 339

," laryngitis $\quad$ pharyngitis in 333

cattle. 141

pharyngitis in sheep . . 142

Pseudo pericarditis $\quad . \quad$. $\quad . \quad$. 390

Pseudo-rheumatism .94

, $\quad$ infectious, in adults 99

Psoroptic mange . . . . 614, 639

Psorospermosis in calves and lambs 271

Pulnonary congestion . . . . 343 


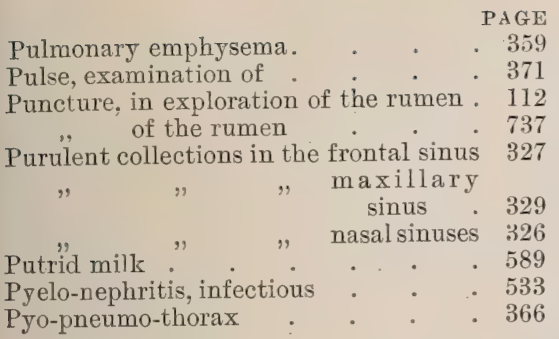

\section{R.}

\section{RACHITIS}

Ragweed family

$$
\text { symptoms of }
$$

treatment of

Ram, castration of the

" passage of the catheter in the

" urethrotomy in the

Ranunculaceæ

$$
\text { poisoning by : } \quad \text {. } \quad .234
$$

Rectal exploration . . 116

Rectum, prolapsus and inversion of the 743

Red milk

Respiratory apparatus . . . 311, 745

examination of . 311

tuberculosis of the . . 690

Reticulitis . . . . . . 186

Reticulum $\because . \quad . \quad 113$

Retro-pharyngeal glands, tuberculosis of 696

Rheumatism

articular . . . . 89

forms of

" infectious forms of . . $\quad 94$

Ring-bone

muscular . . . 92

Ringworm . 649

in the sheep, goat, and pig . 653

Rot-mould family. . . 223

Rowels . . . . . . 728

Rumen . 737

examination of the . 110

" hernia of the . . 490

" impaction of the . . 175

" puncture of the . . . 737

Rumenitis . . . . 186

Ruminants, intestinal helminthiasis in . 275

Rupture of the external ischio-tibial muscle . 70

flexor metatarsi $\quad 72$

Ruptures and perforations of the œsophagus

\section{S.}

"SALIVARY abscesses". Salivary glands, diseases of . $\quad 108,13 t$ Salpingitis Salpingo-ovaritis
Salt common, poisoning by . . . . 217

Sand crack . 34

symptoms . . . . 35

treatment . 35

612

638,641

Saturnism . $\cdot \quad \cdot \quad . \quad 611,614$

Scab dips, arsenical $\cdot \cdot \quad \cdot 632$

". .633

" " Carbolic $\quad \cdot \quad \cdot \quad \cdot 623$

" " . . . . 624

" $\quad$ " lime and sulphur. $\quad . \quad$. $\quad .627$

" " . Tessier's bath . . 622

" " $\quad$ tobacco and sulphur : . $\quad 626$

" ". . . 623

" foot $\quad$. . $\quad . \quad$. $\quad .636$

Scabies . . 611

. in sheep . 611

Scapulo-humeral joint, luxation of the . 63

Sclero-caseous broncho-pneumonia of

sheep. . . . . . 358

Scleroderma . . . . . 657

Sclerostoma pinguicola (kidney worm) . 539

Scorbutus . . . . . $10 \pm$

Scrophulariaceæ . . . . . 25.

Scrotal urethrotomy . . . . . 748

Scurvy . . . . . 104

Sebaceous or seborrhœic eczema . . 601

Secretory dyspepsia . . . . . 195

Section of the sphincter of the teat $\quad .770$

Semiology of the digestive apparatus . 106

organs of circulation. 370

$\begin{array}{lll}\text { Septic metritis } \cdot \text { - } & \cdot & 547 \\ \text { Septicæmia of new-born animals } & \cdot & 406\end{array}$

Serous membranes, tuberculosis of . 694

Setons . . . 728

Sheath, inflammation of . . . 506

Sheep, acne in the . . . . . 606

" anæmia in . . . . . 268

", bilharziosis in . . . . . 439

" blood poisoning in, in New Zealand. . 415

caseous lymphadenitis of the . 453

catarrhal stomatitis in . . 122

control of . . . . . 725

diarrhœa in . . 268

gastro-intestinal strongylosis in . 263

larkspur poisoning in . . 231

". œstrus larvæ in the facial sinuses of . . . 330

parasitic gastro-enteritis in 268

" pseudo-membranous pharyngit is

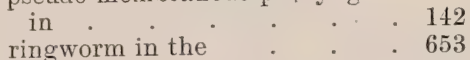

scab. . . 614

", scabies in . $\quad . \quad . \quad . \quad 611$

"sclero-caseous broncho-pneumonia of . . . 358

"trembling,", or lumbar prurigo, in 475

ulcerative stomatitis in $\quad . \quad 125$

" urinary calculi in . . 518

". verminous bronchitis in . . $\quad .340$

Shoeing, stabs and pricks in . . $\quad 36$

Shoulder, strain of . . . . 52

Simple acute bronchitis $\quad \therefore \quad$ : 337 
Simple acute pericarditis

coryza

., pneumonia

. stomatitis

Sinuses, examination of

Skin, diseases of

tuberculosis of the

Smut of barley, poisoning by

, family.

Solanaceæ

Sole, contusions of the .

Sow, anatomical arrangements of the - genital organs in the

" castration of the

" operative accidents in

Spavin in the ox

Specific pericarditis

Speculum, examination of female genital organs with the .

Sphincter of the teat, contraction of the " " section of . .

Spurge family"

Stabs and pricks in shoeing

Sternum, hygroma of the point of the

Stifle, hygroma of the

Stink-horn family
joint, strain of

St. John's-wort family . poisoning by

Stock, plants poisonous to

Stomach, diseases of the

Stomatitis

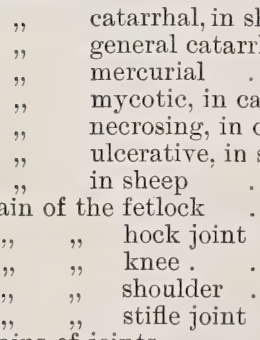

Strains of joints

Strangulation, colic as a result of

Strawberry-shrub family . . . $\quad .235$

Stricture of the œesophagus . . . . 148

Strongylosis of the abomasum in the ox 268

Strychnine poisoning . . . . . 222

Sturdy

Subcutaneous connective tissue, diseases of the

emphysema - .

Submaxillary salivary gland, inflammation of

Sucking calves, broncho-pneumonia of

Sugar factory pulp, diseases produced by Superficial glossitis

Suppurating ostitis

Suppurative echinococcosis

nephritis and perinephritis

Surgical dressing for a claw.

Suture of the vulva

$\begin{array}{llll}" & \text { " } & \text { Rainard's suture } \\ , & \text { " } & \text { simple suture } \\ \text {, } & \text { " } & \text { ", } & \text { Strebel's suture }\end{array}$

765

765

768

375

760

567

770

244
36

69

288

537
Sweet sorghum grass, poisoning by

Swine, acute gastric indigestion in

PAGE

226

catarrhal gastritis in

, fever

stomatitis in $\quad 126$

" ulcerative stomatitis in . . 127

"kidney worm of

Symbiotic (chorioptic) mange . . 636

Synovial capsule of the hock joint, distension of . 46

67

48

222

Synovitis memt

fetlock joint, distension of knee joint, distension of

T.

TAKOSIS

Tartar emetic, poisoning by . $\quad \begin{array}{r}412 \\ -\quad\end{array}$

Taxaceæ 226

Taxus baccata $: 226$

Teat, dilatation of the orifice of the $\quad 770$

" imperforate condition of the .567

" section of the sphincter of the . 770

Tendon sheaths, distension of . 48

" , , in the hock region . 46 in the region
of the knee $\quad 49$

Tendons and muscles, diseases of . . $\quad 70$

Tessier's scab dip . . . . . . 622

Testicle, tumours of the $\quad . \quad . \quad .594$

Tetanus $\quad . \quad . \quad . \quad .670$

Thorax, examination of $\quad . \quad \cdot \quad 315$

Thistle family $\quad . \quad+\quad . \quad+256$

Third stomach, impaction of the : $\quad 179$

Thready milk . . . . . 589

Thrush . . . . . . . 124

Tobacco and sulphur dip . . . $\quad .626$

Tobacco poisoning . . . . . 254

Tongue, actinomycosis of the $\quad .674$

Tonsilitis in pigs . . . . . 138

Tonsils, diseases of $\quad . \quad$. $\quad . \quad 134$

Torsion, castration by . . . . . . 757

Trachea of the uterus . . . . 556

. . . . 333

Tracheotomy

Trasbot's . . . . . 746

Traumatic arthritis $\quad \cdot \quad \cdot \quad \cdot 623$

$\begin{array}{llr}, \quad \text { articular synovitis } \quad: & 51 \\ & \text { lesions } & 568\end{array}$

" lesions . . . 568

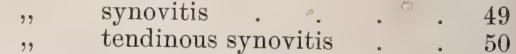

"Trembling," or lumbar prurigo, in sheep 475

Trephining the facial sinuses . . 745

$130 \quad, \quad$ frontal sinus . . . 745
Trichiniasis-trichinosis sinus · · 745

Trochanter of the femur, hygroma of the 67

'Truss, Lund's . . . . 770

Trusses . . . . . . . $\quad 769$

Trypanosomata, diseases produced by . 426

Tuberculosis. . . . 682 


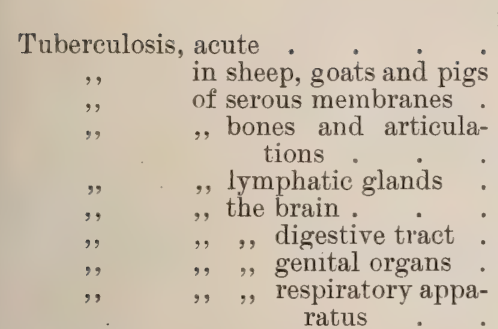

PAGE

704

705

694

Tube, ,, , skin.

• . 704

Tumors, bone . • . . 30

,, cerebral . * 459

,, of the gastric compartments. 202

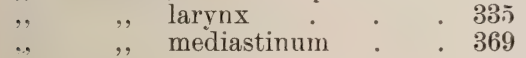

., $\quad$, nasal cavities . . 325

, , , ovary . . . . 559

,, , testicle . . . 594

,, udder . $\quad . \quad$. $\quad .585$

", " uterus . . . 559

Turn-sick . • . . . . 467

Tympanites, chronic . . . . . 194

U.

UDDER, congestion of the . . . 570

,, cysts of the . . . . 585

", tuberculosis of the $\quad . \quad 701$

,, tumors of the . . . 585

,$\quad$ verrucous papillomata of the. 586

Ulcerative gastritis . . . . 191

,$\quad$ stomatitis in sheep . . 125

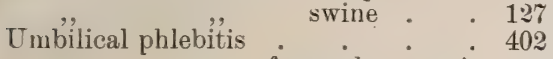

,, ,, of new-born animals . 399

Umbrella-tree family . . . . . 244

Urachus, persistence of the . . 508

Urethrotomy in the ox . . . $\quad 747$

," ram . . . 749

Urinary apparatus, diseases of the . 502

,, calculi in sheep . . . 518

, lithiasis

Urticaceæ $\quad$ PAGE

Urticaria in the pig : : : $\quad: 656$

Ustilaginaceæ : . . . 224

Utero-ovarian phlebitis . . . 398

Uterus, torsion of the . . $\quad 556$ , tumors of the . . . 559

V.

VACCINE, preparation of . . 669

Vaccinia . . . . . . 665

Vagina, imperforate . $\quad . \quad \therefore 560$

Vaginitis . . . . . . 543

,, acute . . . . . 544

,, chronic . . . $\quad 546$

,, contagious . . . . 545

, croupal . . . 545

Veins, examination of $\quad \cdot \quad \cdot \quad 372$

Verminous bronchitis in sheep and
cattle. 340

Verminous conjunctivitis : $\quad \cdot 662$ ,, ophthalmia of the ox $\quad 663$

Verrucous endocarditis of the pig 710, 713

Verrucous papillomata of the udder 586

Vesiculæ seminales . . . . 597

Vetches, poisoning by $\quad . \quad$. 243

Viciaceæ . . . . . 236

Viscous milk $\quad . \quad$. $\quad$. $\quad 589$

Vulva, suture of the : $\quad . \quad 768$

W.

WARBLES . . . . . 646

Warts in oxen . . . . . 655

Whitlow • . . . . 41

White loco weed, poisoning by . . 237

Wild chervil, poisoning by . . 248

Wounds or traumatic lesions $\quad$ • $\quad 568$

Y.

YELLOW milk . . . . . 590

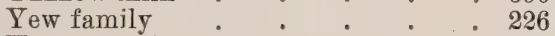

Yew poisoning : $\quad \cdot \quad \cdot \quad \cdot \quad \cdot 226$ 



\title{
CATALOcue of WILLIAM R. JENKINS'
}

\author{
Works Concerning
}

HORSES, GATTLE, SHEEP, SWINE, Ełc.

\section{5}

(*) Single asterisk designates New Books.

(**) Double asterisk designates Recent Publications

ANDERSON. "Vice in the Horse" and other papers on Horses and Riding. Bv F. L. Anderson. Size, $6 \times 9$, cloth, illustrated.....................1 75

ARMSTEAD. "The Artistic Anatomy of the Horse." A brief description of the various Anatomical Structures which may be distinguished during Life through the Skin, By Hugh W. Armstead, M.D., F.R.C.S. With illustrations from drawings by the author. Cloth oblong, $10 \times 12 \frac{1}{2} \ldots \ldots \ldots \ldots \ldots \ldots \ldots \ldots \ldots \ldots$

BACH. "How to Judge a Horse." A concise treatise as to its Qualities and Soundness; Including Bits and Bitting, Saddles and Saddling, Stable Drainage, Driving One Horse, a Pair, Four-in-hand, or Tandem, etc. By Capt. F.W. Bach. Size, $5 \times 7 \frac{1}{2}$, clo., fully illus.1 00

(*)BANHM. "Anatomical and Physiological Model of the Cow." Half life size. Composed of superposed plates, colored to nature, showing internal organs, muscles, skeleton, etc., mounted on strong boards, with explanatory text. Size of Model opened, $10 \mathrm{ft} . \times 3 \mathrm{ft}$., closed $3 \mathrm{ft} . \times 1 \frac{1}{2} \mathrm{ft} \ldots \ldots \ldots \ldots \ldots \ldots 750$

- "Anatomical and Physiological Model of the Horse." Half life size. By George A. Banham, F.R.C.V.S. Size of Model $38 \times 41$ in.................... 750 
BANHAM (continued)

- "Tables of Veterinary Posology and Therapeutics," with weights, measures, etc. By Geo. A. Banham, F.R.C.V.S. New edition. Cloth, size 4 x 5 1-2, 192 pages................................. 00

BAUCHER. "Method of Horsemanship." Including the Breaking and Training of Horses. By F. Baucher........................... 00

$\left.{ }^{*}\right)$ BELL. "The Veterinarian's Call Book (Perpetual)," By Roscoe R. Bell, D.V.S., editor of the American Veterinary Review. Revised every year.

A visiting list, that can be commenced at any time and used until full, containing much useful information for the student and the busy practitioner. Among contents are items concerning: Veterinary Drugs; Poisons; Solubility of Drugs ; Composition of Milk,Bile, Blood, Gastric Juice, Urine, Saliva; Respiration; Dentition; Temperature, etc., etc. Bound in flexible leather, with flap and pocket ..........1 25

\section{BITTING. "Cadiot's Exercises in Equine Surgery."} See "Cadiot."

BRADLEY. " "Outlines of Yeterinary Anatomy." By O. Charnock Bradley, Member of the Royal College of Veterinary Surgeons; Professor of Anatomy in the New Veterinary College, Edinburgh.

The author presents the most important facts of veterinary anatomy in as condensed a form as possible, consistent with lucidity. $12 \mathrm{mo}$.

Complete in three parts.

PART I.: The Limbs (cloth)...............1 25

PART II.: The Trunk (paper) ..............125

PART III.: The Head and Neck (paper).........125

The Set complete ...................... 25 
CADIOT. "Exercises in Equine Surgery." By P. J. Cadiot. Translated by Prof. A. W. Bitting, D.V.M. Edited by Prof. A. Liautard, M.D.V.M. Size, $6 \times 91 / 4$, cloth, illustrated..................... 250

- "Roaring in Horses." Its Pathology and Treatment. This work represents the latest development in operative methods for the alleviation of roaring. Each step is most clearly defined by excellent full-page illustrations. By P. J. Cadiot, Professor at the Veterinary School, Alfort. Translated by Thos. J. Watt Dollar, M.R.C.V.S., etc. Cloth, size 5 1-4 x 7 1-8, 77 pages, illustrated.................... 75

- "Studies in Clinical Veterinary Medicine and Surgery." By P. J. Cadiot. Translated, edited, and supplemented with 49 new articles and 34 illustrations by Jno. A. W. Dollar, M.R.C.V.S. Cloth, size $7 \times 93.4,619$ pages, 94 black and white illustrations............. 25

(*)-_6 A Treatise on Surgical Therapentics of the Domestic A nimals:" By P. J. Cadiot and J. Almy. Translated by Prof. A. Liautard, M,D., V.M.

Part I.-Restraint of horses; cattle, etc.; general anæsthesia ; surgical antisepsis and asepsis; hemostasis and cauterization; firing. 45 illus ...... 00

Part II.-Inflammations, gangrene, foreign bodies, traumatic lesions, chilblains, frost bites, congelations, complications of traumatic lesions, etc...1 00

Part III.-Granulations, cicatrices, mycosis, virulent diseases, tumors, diseases of skin and cellular tissue, tendons, etc................... 100

Part IV. (completing the volume) nearly ready.

CHAPMAN. "'Manual of the Pathological Treatment of Lameness in the Horse," treated solely by mechanical means. By George T. Chapman. Cloth, size $6 \times 9,124$ pages with portrait............200

CHAUVEAU. "The Comparative Anatomy of the Domesticated Animals." By A. Chauveau. Revised by G. Fleming, F.R.C.V.S. 8vo, cloth, 585 illus., 625 
CLARKE. "Chart of the Feet and Teeth of Fossil Horses." By W. H. Clarke. Card, size 9 1-2 × $12 . .25$

- "Horses' Teeth." Fourth edition, re-revised, with second appendix. Cloth, size 5 1-4 x 7 1-2, 322 pp., illus..2 50

CLEAVELAND. " "Pronouncing Medical Lexicon." Pocket edition. By C. H. Cleveland, M.D. Cloth, size $31.4 \times 41-2,302$ pages................. 75

CLEMENT. "Veterinary Post Mortem Examinations." By A. W. Clement, V.s. The absence in the English language of any guide in making autopsies upon the lower animals, induced Dr. Clement to write this book, trusting that it would prove of practical value to the profession. Cloth, size 5 × $71-2,64$ pages, illustrated........................ 75

(**)COURTENA Y. "Manual of the Practice of Veterinary Medicine." By Edward Courtenay, V.S. Revised by Frederick T. G. Hobday, F.R.C.V.S. Second edition. Cloth, size 5 1-4 x 7 1-2, 573 pages ..........2 75

COX. "Horses : In Accident and Disease." The sketches introduced embrace various attitudes which have been observed, such as in choking; the disorders and accidents occurring to the stomach and intestines; affection of the brain ; and some special forms of lameness, etc. By J. Roalfe Cox, F.R.C.V.S. Cloth, size $6 \times 9,28$ full page illustrations.............. 50

CURTIS. "Horses, Cattle, Sheep and Swine." By Geo. W. Curtis, M.S.A. Cloth, size $71-4$ × 10, 343 pages, 117 illustrations....................250

$\left.{ }^{* *}\right)$ DALR YMPLE. "Veterinary Obstetries." A compendium for the use of advanced students and Practitioners. By W. H. Dalrymple, M. R. C. V. S., principal of the Department of Veterinary Science in the Louisiana State University and A. \& M. College; Veterinarian to the Louisiana State Bureau of Agriculture, and Agricultural Experiment Stations. Cloth, sire 6 × $91-4,162$ pages, 51 illustrations...2 50 
DALZLEL. “Breaking and Training Dogs." Part I, by Pathfinder. Part II, by \#lugh Dalziel. Cloth, illustrated............................ 50

— “The Collie." By Hugh Dalziel. Paper, illustrated... 40

- "The Diseases of Dogs." Causes, symptoms and treatment. By Hugh Dalziel. Cloth, illustrated...........100

— "Diseases of Horses." Paper ................... 40

- "The Fox Terrier." By Hugh Dalziel. Paper, 40 ; clo.1 00

- "The Greyhound." Cloth, illus..................1 00

- "The St. Bernard." Cloth, illustrated..............100

DANA. “Tables in Comparative Physiology." By Prof. C. L. Dana, M.D. Chart, $17 \times 17 \ldots \ldots \ldots \ldots .25$

DANCE. "Veterinary Tablet." By A. A. Dance. Chart, $17 \times 24$, mounted on linen, folded in a cloth case for the pocket, size $33-4 \times 6$ 1-2. Shows at a glance the synopsis of the diseases of horses, cattle and dogs; with their cause, symptoms and cure............ 75

(")DE BRUIN. "Bovine Obstetrics." By M. G. De Bruin Instructor of Obstetrics at the State Veterinary School in Utrecht. Translated by W. E. A. Wyman, formerly Professor of Veterinary Science at Clemson A. \& M. College, and Veterinarian to the South Carolina Experiment Station. Cloth, size $6 \times 9,382$ pages, 77 illustrations....................5 00

Synopsis of the Essential Features of the Work

1. Authorized translation.

2. The only obstetrical work which is up date.

3. Written by Europe's leading authority on the subject.

4. Written by a man who has practiced the art a lifetime.

5. Written by a man who, on account of his eminence as bovine practitioner and teacher of obstetrics, was selected by Prof. Dr. Frōhner and Prof. Dr. Bayer (Berlin and Vienna), to discuss bovine obstetrics both practically and scientifically.

6. The only work containing a thorough differential rijas.. nosis of ante and post partum diseases, 
DE BRUIN, “Bovine Obstetrics" (continued)

7. The only work doing justice to modern obstetrical surgery and therapeutics.

8. Written by a man whose practical suggestions revolutionized the teaching of veterinary obstetrics even in the great schools of Europe.

9. The only work dealing fully with the now no longer obscure contagious and infectious diseases of calves.

10. Absolutely original and no compilation.

11. The only work dealing fully with the difficult problem of teaching obstetrics in the colleges.

12. The only work where the practical part is not overshadowed by theory.

. A veterinarian, particularly if his location brings him in contact with obstetrical practice, who makes any pretence toward being scientific and in possession of modern knowledge upon this subject, will not be without this excellent work, as it is really a very valuable treatise.-Prof. Roscoe $R$. Bell, in the American Veterinary Review.

In translating into English Professor De Bruin's excellent textbook on Bovine Obstetrics, Dr. Wyman has laid British and American veterinary surgeons and students under a debt of gratitude. The works represents the happy medium between the booklets which are adapled for cramming purposes by the student, and the ponderous tomes which, although useful to the teacher, are not exactly suited to the requirements of the everyday practitioner . . We can strongly recommend the work to veterinary students and practitioners.--The Journal of Comparative Pathology and Therapeutics.

(*)DOLLAR. "Diseases of Cattle, Sheep, Goats and Swine." By G. Moussu and Jno. A. W. Dollar, M.R.C.V.S. Size $6 \times 9$ 1-2, 785 pages, 329 illustrations in the text and 4 full page plates in colors.,.....8 75

$\left({ }^{* *}\right)$ - "A Hand-book of Horse-Shoeing," with introductory chapters on the anatomy and physiology of the horse's foot. By Jno. A. W. Dollar, M.R.C.V.S., with the collaboration of Albert Wheatley, F.R.C.V.S. Cloth, size $6 \times 8$ 1-2, 433 pages, 406 illustrations ..4 75

- "Operative Technique." Volume 1 of "The Practice of Veterinary Surgery." Cloth, size $63-4 \times 10,264$ pages, 272 illustrations......................... 75

- "General Surgery." Volume 2 of "The Practice of Veterinary Surgery." In preparation.

(*)- "Regional Veterinary Surgery." Volume 3 of "The Practice of Veterinary Surgery." By Drs. Jno. A. W. Dollar and H. Möller. Cloth, size 6 1-2 x 10853 and xvi pages, 315 illustrations.............6 25 
DOLLAR-(continued)

- "Cadiot's Clinical Veterinary Medicine and Surgery." See " Cadiot."

- "Cadiot's Roaring in Horses."

See "Cadiot."

DUN. "Veterinary Medicines, their Actions and Uses." By Finlay Dun, V.S., late lecturer on Materia Medica and Dietetics at the Edinburgh Veterinary College, and Examiner in Chemistry to the Royal Coliege of Veterinary Surgeons. Edited by James Macqueen, F.R.C.V.S. Tenth revised English edition. Cloth, size $6 \times 9 \ldots \ldots \ldots \ldots \ldots \ldots \ldots \ldots \ldots \ldots \ldots \ldots \ldots$

DWYER. "On Seats and Saddles." Bits and Bitting, Draught and Harness and the Prevention and Cure of Restiveness in Horses. By Francis Dwyer. Cloth, size $5 \times 7,304$ pages, gilt, illustrated........... 50

FLEMING. “Animal Plagues." Their History, Nature, and Prevention. By Geo. Fleming, F.R.C.V.S., etc. First Series. Chronological History from B.C. 1490 to A.D. 1800. Cloth, size $6 \times 9,548$ pages.......6 00 Second Series. Chronological History from A.D. 1800 to 1844 . Cloth, size $6 \times 9,539$ pages........3 00

- "The Comparative Anatomy of the Domesticated Animals." By A. Chauveau. Translated by Dr. Fleming. See "Chauveau."

- "The Contagious Diseases of Animals." Their in fluence on the wealth and health of nations and how they are to be combated. Paper, size $5 \times 71-2,30$ pages .... 25

- "Human and Animal Variolæ." A Study in Comparative Pathology. Paper, size 5 1-2 x 8 1-2, 61 pages.... 25

- "Parasites aud Parasitic Diseases of the Domesticated Animals." By L. G. Neumann. Translated by Dr. Fleming. 


\section{FLEMING (continued)}

- "Operative Veterinary Surgery." Vol. I, by Dr. Geo. Fleming, M.R.C.V.S. This valuable work, one of the most practical treatises yet issued on the subject in the English language, is devoted to the common operations of Veterinary Surgery ; and the concise descriptions and directions of the text are illustrated with numerous wood engravings. Cloth, size $6 \times 9$ 1-4, 285 and $x$ viii pages, 343 illustrations.............2 75

$\left({ }^{*}\right)$ Vol. II, edited and passed through the press by W. Owen Williams, F.R.C.V.S. Cloth, size $6 \times 91-4$, 430 and xxxvii pages, 344 illustrations........... 25

Both volumes bound in one................5 25

- "Roaring in Horses." By Dr. George Fleming, F.R.C.V.S. Its history, nature, causes, prevention and treatment. Cloth, size 5 1-2 x $83-4,160$ pages, 21 engravings, 1 colored plate.................. 50

- "Tuberculosis." From a Sanitary and Pathological Point of View. By Geo. Fleming, F.R.C.V.S. Paper, size $51-2 \times 81-2,39$ pages......................25

- "Veterinary Obstetrics." Including the Accidents and Diseases incident to Pregnancy, Parturition, and the Early Age in Domesticated Animals. By Geo. Fleming, F.R.C.V.S. Cloth, size $6 \times 83-4,758$ pages, illus. 625

(*)GOTTHIEL. "A Manual of General Histology." By Wm. S. Gottheil, M.D., Professor of Pathology in the American Veterinary College, New York; etc., etc.

Histology is the basis of the physician's art, as Anatomy is the foundation of the surgeon's science. Only by knowing the processes of life can we understand the changes of disease and the action of remedies; as the architect must know his building materials, so must the practitioner of medicine know the intimate structure of the body. To present this knowledge in an accessible and simple form has been the author's task. Second edition revised. Cloth, size 5 1-2 x 8, 152 pages, 68 illustrations...100 
851-853 Sixth Avenue (cor, 48th St.), New York.

GRESSWELL. "The Bovine Prescriber." For the use of Veterinarians and Veterinary Students. Second edition revised and enlarged, by James B and Albert Gresswell, M.R.C.V.S. Cloth, size, 5 × 7 1-2, 102 pages................................75

- "The Equine Hospital Prescriber." For the use of Veterinary Practitioners and Students. Third edition revised and enlarged, by Drs. James B. and Albert Gresswell, M.R.C.V.S. Cloth, size $5 \times 71-2,165$ pages...............................75

- "Diseases and Disorders of the Horse." A Treatise on Equine Medicine and Surgery, being a contribution to the science of comparative pathology. By Albert, Jas. B. and Geo. Gresswell. Cloth, size 5 3-4 x 8 3-4, 227 pages, illustrated.................. 175

- Manual of "The Theory and Practice of Equine Medicine." By James B. Gresswell, F.R.C.V.S., and Albert Gresswell, M.R.C.V.S. Second edition revised. Cloth, size 5 1-4 × 7 1-2, 539 pages............2 75

- "Veterinary Pharmacopæia and Manual of Comparative Therapy." By George and Charles Gresswell, with descriptions and physiological actions of medicines, by Albert Gresswell. Second edition revised and enlarged. Cloth, 6 × $83-4,457$ pages........... 50

HASSLOCH. "A Compend of Veterinary Materia Medica and Therapeutics." By A. C. Hassloch, V.S., Lecturer on Materia Medica and Therapeutics, and Professor of Veterinary Dentistry at the New York College of Veterinary Surgeons and School of Comparative Medicine, N. Y. Cloth, size $51-4$ x 7 1-2, 225 pages............................... 50

HEATLEY. " "The Stock Owner's Guide." A handy Medical Treatise for every man who owns an ox or cow. By George S. Heatley, M.R.C.V.S. Cloth, size $51-4$ × 8,172 pages...................... 25 
(**)HILL. "The Diseases of the Cat." By J. Woodroffe Hill, F.R.C.V.S. Cloth, size $51-4$ × 7 1-2, 123 pages, illustrated.............................. 25

Written from the experience of many years' practice and close pathological research into the maladies to which our domesticated feline friends are liable-a subject which it must be admitted has not found the prominence in veterinary literature to which it is undoubtedly entitled.

- "The Management and Diseases of the Dog." By J. Woodroffe Hill, F.R.C.V.S. Cloth, size $5 \times 71-2$, extra fully illustrated....................200

HINEBAUCH. "Veterinary Dental Surgery." By T. D. Hinebauch, M.S.V.S. For the use of Students, Prac. titioners and Stockmen. Cloth, size $51-4$ × 8, 256 pages, illustrated........................200

HOARE. "A Manual of Veterinary Therapeutics and Pharmacology." By E. Wallis Hoare, F.R.C.V.S. Cloth, size $51-4$ × $71-4,560$ pages...............2 75

(")HOBDAY. "Canine and Feline Surgery." By Frederick T. G. Hobday, F.R.C.V.S. Cloth, $53-4$ x 8 3-4, 152 pages, 76 illustrations....................200

$\left({ }^{*}\right)$ "The Castration of Cryptorchid Horses and the Ovariotomy of Troublesome Mares." By Frederick T. G. Hobday, F.R.C.V.S. Cloth, size 5 3-4 × 8 3-4, 106 pages, 34 illustrations,.........1 75

(**)HUNTING. The Art of Horse-shoeing. A manual for Horseshoers. By William Hunting, F.R.C.V.S., ex-President of the Royal College of Veterinary Surgeons. One of the most up-to-date, concise books of its kind in the English language. Cloth, size $6 \times 91-4$, 126 pages, 96 illustrations..................1 00

${ }^{(* *)}$ JENKINS. " Model of the Horse" and "Model of the Cow."

See "Banham." 
KEATING. "A New Unabridged Pronouneing Dictionary of Medicine." By John M. Keating, M.D., LL.D., Henry Hamilton and others. A voluminous and exhaustive hand-book of Medical and scientific terminology with Phonetic Pronunciation, Accentuation, Ftymology, etc. With an appendix containing important tables of Bacilli, Micrococci, Leucomaines, Ptomaines; Drugs and Materials used in Antiseptic Surgery; Poisons and their antidotes; Weights and Measures; Themometer Scales; New Officinal and Unofficinal Drugs, etc., etc. Cloth, 818 pages ...5 00

(**)KOBERT. "'Practical Toxicology for Physicians and Students." By Professor Dr. Rudolph Kobert, Medical Director of Dr. Brehmer's Sanitarium for Pulmonary Diseases at Goerbersderf in Silesia (Prussia), late Director of the Pharmacological Institute, Dorpat, Kussia. Translated and edited by L. H. Friedburg, Ph.D. Authorized Edition. Practical knowledge by means of tables which occupy little space, but show at a glance similarities and differences between poisons of the same group. Also rules for the Spelling and Pronunciation of Chemical Terms, as adopted by the American Association for the Advancement of Science. Cloth, $61.2 \times 10,201 \mathrm{pp..2} 50$

КОСН. "Etiology of Tuberculosis." By Dr. R. Koch. Trauslated by T. Saure. Cloth, size $6 \times 91-4,97$ pages...............................1 00

\section{LAMBERT. "The Germ Theory of Disease."} Bearing upon the health and welfare of man and the domesticated animals. By James Lambert, F.R.C.V.S. Paper, size 5 1.4 × 8 1-4, 26 pages, illustrated.... 25

$\boldsymbol{L A} \boldsymbol{W}$. "Farmers' Veterinary Adviser." A Guide to the Prevention and Treatment of Disease in Domestic Animals. By Prof. James Law. Cloth, size $51-4 \times 7$ 1-2, illustrated....................3 00 
${ }^{(* *)} \boldsymbol{L E G G E . ~ " C a t t l e ~ T u b e r c u l o s i s . " ~ A ~ P r a c t i c a l ~ G u i d e ~}$ to the Farmer, Butcher and Meat Inspector. By T.M. Legge, M.A., M.D., D.P.H., and Harold Sessions, F.R.C.V.S. Cloth, size $51.2 \times 81-2,77$ pages....1 00

("*)LIAUTARD. "Animal Castration." A concise and practical Treatise on the Castration of the Domestic Animals. The only work on the subject in the English language. By Alexander Liautard, M.D., V.S. Having a fine portrait of the author. T'enth edition revised and enlarged. Cloth, size $51-4$ × 7 1-2, 165 pages, 45 illustrations....................200 . . The most complete and comprehensive work on the subject in English veterinary literature.-American Agriculturist.

- "Cadiot's Exercises in Equine Surgery." . 'Translated by Prof. Bitting and edited by Dr. Liautard.

\section{See "Cadiot."}

- ${ }^{64}$ A Treatise on Surgical Therapeutics of the Domestic Animals." By Prof. Dr. P. J. Cadiot and J. Almy. Translated by Prof. Liautard.

See "Cadiot."

- "How to Tell the Age of the Domestic Animal." By Dr. A. Liautard, M.D., V.S. Standard work upon this subject, concise, helpful and containing many illustrations. Cloth, size $5 \times 71-2,35$ pages, 42 illustrations............................ 50

- "Lameness of Horses and Diseases of the Locomotory Apparatus." By A. Liautard, M.D.,V.S. This work is the result of Dr. Liautard's many years of experience. Cloth, size $51-4 \times 7$ 1-2, 314 pages.......2 50

— "Manual of Operative Veterinary Surgery." By A. Liautard, M.D., V.M. Engaged for years in the work of teaching this special department of veterinary medicine, and having abundant opportunities of realizing the difficulties which the student who earnestly strives to perfect himself in his calling is obliged to encounter, the author formed the deter- 
LIAUTARD (continued).

mination to facilitate his acquisition of knowledge, and began the accumulation of material by the sompilation of data and arrangement of memorandum, with the recorded notes of his own experience, the fruit of a long and extended practice and a careful study of the various authorities who have illustrated and organized veterinary literature. Cloth, size $61-4 \times 9,786$ pages, 563 illustrations.......... 500

- "Pellerin's Median Neurotomy in the Treatment of Chronic Tendinitis and Periostosis of the Fetlock." Translated by Dr. A. Liautard.

See "Pellerin,"

- "Vade Mecum of Equine Anatomy." By A. Liautard, M.D.V.S. For the use of advanced stutents and veterinary surgeons. Third edition. Cloth, size $5 \times 71-2,230$ pages and 10 full page illustrations of the arteries. .......................... 00

- Zundel's "The Horse's Foot and Its Diseases."

See "Zundel."

LONG. "Book of the Pig." Its selection, Breeding, Feeding and Management. Cloth............400

$\left(^{* *}\right)$ LOWE. "Breeding Racehorses by the Figure System." Compiled by the late C. Bruce Lowe. Editerl by William Allison, "The Special Commissioner," London Sportsman, Hon. Secretary Sporting League, and Manager of the International Horse Agency and Exchange. With numerous fine illustrations of celebrated horses. Cloth, size $8 \times 10,262$ pages............................. 750

LUDLOW. "Science in the Stable"; or How a Horse can be Kept in Perfect Health and be Used Without Shoes, in Harness or under the Saddle. With the Reason Why. Second Edition. By Jacob R. Ludlow, M.D. Late Staff Surgeon, U. S. Army. Paper, size $41-2 \times 53-4,166$ pages..................... 50 
LUPTON. "Horses: Sound and Unsound," with Law relating to Sales and Warranty. By J. Irvine Lupton, F.R.C.V S. Cloth, size 5 3-4 × 71 1-2, 217 pages, 28 illustrations.................... 25

MAGNER. "Standard Horse and Stock Book." By D. Magner. Comprising over 1,000 pages, illustrated with 1756 engravings. Leather binding. .......600

McBRIDE. "Anatomical Ontlines of the Horse." By J. A. McBride, M R C.V.S. Second edition revised and enlarged. Cloth, size $51-4 \times 71-4$, illus ....2 50

(")M'FADYEAN. "Anatomy of the Horse." Second edition completely revised. A Dissection Guide. By John M'Fadyean, M.B., B.Sc., F.R.S.E. Cloth, size $6 \times 834,388$ pages, illustrated...........5 50

This book is intended for Veterinary students, and offers to them in its 48 full-page colored plates, 54 illustrations and excellent text, a valuable and practical aid in the study of Veterinary Anatomy, especially in the dissecting room.

- "Comparative Anatomy of the Domesticated Animals." By J. M'Fadyean. Profusely illustrated, and to be issued in two parts.

Part I-Osteology, ready. Size 51-2 x 81 -2, 166 pages, 132 illustrations. Paper, 2 50; cloth....2 75 (Part II in preparation.)

MILLS. "How to Keep a Dog in the City." By Wesley Mills, M.D., D.V.S. It tells how to choose, manage, house, feed, educate the pup, how to keep him clean and teach him cleanliness. Paper, size $5 \times 71-2$, 40 pages............................. 25

(*)MÖLLER - DOLLAR. “Regional Veterinary Surgery." See "Dollar."

MOHLER. "I'andbook of Meat Inspection." By Robert Ostertag, M.D. Translated by Earley Vernon Wilcox, A.M., Ph.D. With an introduction by John R. Mohler, V.M.D, A.M. See " Ostertag." 
MOSSELMAN-LIENAUX. "Manual of Veterinary Microbiology." By Professors Mosselman and Liénaux, Nat. Veterinary College, Cureghem, Belgium. Translated and edited by R. R. Dinwiddie, Professor of Veterinary Science, College of Agriculture, Arkansas State University. Cloth, size $51.2 \times 8,342$ pages, illustrated............................2 00

$\left({ }^{*}\right)$ MOUSSU. " $\quad$ Diseases of Cattle, Sheep, Goats and Swine." By G. Moussu and Jno. A. W. Dollar, M.R.C.V.S.

$$
\text { See "Dollar." }
$$

NEUMANN. "A Treatise on Parasites and Parasitic Diseases of the Domesticated Animals." A work to which the students of human or veterinary medicine, the sanitarian, agriculturist or breeder or rearer of animals, may refer for full information regarding the external and internal Parasites-vegetable and animal-which attack various species of Domestic Animals. A Treatise by L. G. Neumann, Professor at the National Veterinary School of Toulouse. Translated and edited by George Fleming, C. B., L.L. D.,F.R.C.V.S. Cloth, size $63.4 \times 10,873$ pages, 365 illustrations ....................... 50

NOCARD. “The Animal Tuberculoses, and their Relation to Human Tuberculosis." By Ed. Nocard, Prof. of the Alfort Veterinary College. Translated by H. Scurfield, M.D. Ed., Ph. Camb. Cloth, $5 \times 7$ 1-2, 143 pages...1 00

Perhaps the chief interest to doctors of human medicine in Professor Nocard's book lies in the demonstration of the small part played by heredity, and the great part played by contagion in the propagation of bovine tuberculosis. It seems not unreasonable to suppose that the same is the case for human tuberculosis, and that, if the children of tuberculous parents were protected from infection by cohabitation or ingestion, the importance of heredity as a cause of the disease, or even of the predisposition to it, would dwindle away into insignificance. 
OSTERTAG. "Handbook of Meat Inspection." By Robert Ostertag, M.D. Authorized Translation by Earley Vernon Wilcox, A.M., Ph.D. With an introduction by John R. Mohler, V.M.D., A.M. The work is exhaustive and authorative and has at once become the standard authority upon the subject Clotb, size $63-4$ × $93-4,920$ pages, 260 illustrations and 1 colored plate................................ 50

PALLIN. "A Treatise on Epizootic Lymphangitis." By Capt. W. A. Pallin, F.R.C.V.S. In this work the author has endeavored to combine his own experience with that of other writers and so attempts to give a clear and complete account of a subject about which there is little at present in English veterinary literature. Cloth, size $53-4 \times 81-2,90$ pages, with 17 fine full page illustrations..................... 25

PEGLER. "The Book of the Goat." Third edition rewritten and enlarged. Cloth, 223 pages, illus....175

PELLERIN. "Median Neurotomy in the Treatment of Chronic Tendinitis and Periostosis of the Fetlock." By C. Pellerin, late repetitor of Clinic and Surgery to the Alfort Veterinary School. Translated, with Additional Facts Relating to It, by Prof. A. Liautard, M.D., V.M. Having rendered good results when performed by himself, the author believes the operation, which consists in dividing the cubito-plantar nerve and in excising a portion of the peripherical end, the means of improving the conditions, and consequently the values of many apparently doomed animals. Agriculture in particular will be benefited.

The work is divided into two parts. The first covers the study of Median Neurotomy itself; the second, the exact relations of the facts as observed by the author. Boards, $6 \times 9$ 1-2, 61 pages, illustrated..1 00

PETERS. "A Tuberculous Herd-Test with Tuberculin." By Austin Peters, M. R. C. V.S., Chief Inspector of Cattle for the New York State Board of Health during the winter of 1892-93. Pamphlet....25 
REYNOLDS. "An Essay on the Breeding and Management of Draught Horses." By R. S. Reynolds, M.R.C.V.S. Cloth, size $51-2 \times 83-4,104$ pages. .1 40

ROBERGE. "The Foot of the Horse," or Lameness and all Diseases of the Feet traced to an Unbalanced Foot Bone, prevented or cured by balancing the foot. By David Roberge. Cloth, size $6 \times 9$ 1-4, 308 pages, illustrated.............................. 00

SEWELL. "The Examination of Horses as to Soundness and Selection as to Purchase." By Edward Sewell, M.R.C.V.S. Paper, size 51-2 × 81.2 , 86 pages, lllustrated with 8 plates in color............ 150

.... It is a great adrantage to the business man to know something of the elements of law, and nobody ought either to buy or own a horse who does not know something about the animal. That something this book gives, and gives in a thoroughly excellent way....

-Our Animal Friends.

\section{SMITH. "A Manual of Veterinary Physiology." By} Veterinary Captain F. Smith, M.R.C.V.S. Author of "A Manual of Veterinary Hygiene."

Throughout this manual the object has been to condense the information us much as possible. The broad facts of the sciences are stated so as to render them of use to the student and practitioner. In this second edition-rewritten-the whole of the Nervous System has been revised, a new chapter dealing with the Development of the Ovum has been added together with many additional facts and illustrations. About one hundred additional pages are given. Second edition, revised and enlarged. Cloth, size 6 × $83-4$, 673 pages, 102 illustrations.................. 75

- "Manual of Veterinary Hygiene." Second edition revised. Cloth, size 5 1-4 × 7 1-2, 477 pages, 93 illus.....2 75

(") STRANGEWAY. "Veterinary Anatomy." Edited by I. Vaughan, F.L.S., M.R.C.V.S. New edition revised. Cloth, size 61.4 × 9 1-2, 625 pages, 224 lllus.....5 00 
SUSSDORF. "Six Large Colored Wall Diagrams." Ву Prof. Sussdorf, M.D. (of Göttingen). Text translated by Prof. W. Owen Williams, of the New Veterinary College, Edinburgh. Size, 44 inches by 30 inches.

$$
\begin{array}{ll}
\text { 1.-Horse. } & \text { 4.-0x. } \\
\text { 2.-Mare. } & \text { 5.-Boar and Sow. } \\
\text { 3.-Cow. } & \text { 6.-Dog and Bitch. }
\end{array}
$$

The above are printed in eight or nine colors. Showing the position of the viscera in the large cavities of the body.

Price, unmounted...................1 75 each

“ mounted on linen, with roller......3 50 “

(") THOMPSON. "Elementary Lectures on Veterinary Science." For agricultural students, farmers and stock keepers. By Henry Thompson, M.R.C.V.S., lecturer on Veterinary Science at the Aspatria Agricultural College, England. It is complete yet concise and an up-to-date book. Cloth, 397 pp., 51 illus..3 75

VAN MATER. "A Text Book of Veterinary Ophthalmology." By George G. Van Mater, M.D., D.V.S., Professor of Ophthalmology in the American Veterinary College; Oculist and Aurist to St. Martha's Sanitarium and Dispensary; Consulting Eye and Ear Surgeon to the Twenty-sixth Ward Dispensary; Eye and Ear Surgeon, Brooklyn Eastern District Dispensary, etc. Illustrated by one chromo lithograph plate and 71 engravings. Cloth, $6 \times 91-4,151$ pages...3 00 book. We intend to adopt this valuable work as a text book.-E. J. Creely, D.V.S., Dean of the San Francisco Veterinary College.

VETERINARY DIAGRAMS in Tabular Form. Size, $28 \frac{1}{2} \mathrm{in} . \times 22$ inches. Price per set of five...4 00 Mounted and folded in case................. 700 Mounted on roller and varnished............10 00

No. 1. "The External Form and Elementary Anatomy of the Horse." Eight colored illustrations1. External regions; 2. Skeleton; 3. Muscles (Superior Layer); 4. Mruscles (Deep Layer); 5. Respiratory Ap- 
VLTERINARY DIAGRAMS (continued).

paratus ; 6. Digestive Apparatus ; 7. Circulatory Apparatus ; 8. Nerve Apparatus ; with letter-press descrip-

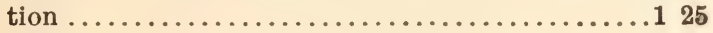

Mounted on roller and varnished............2 25

No. 2. "The Age of Domestic Animals." Forty-two figures illustrating the structure of the teeth, indicating the Age of the Horse, Ox, Sheep, and Dog, with full description .........................75 Mounted on roller and varnished............200

No. 3. "The Unsoundness and Defects of the Horse." Fifty figures illustrating-1. The Defects of Conformation; 2. Defects of Position; 3. Infirmities or Signs of Disease ; 4. Unsoundnesses; 5 . Defects of the Foot; with full description..................... 75 -Mounted on roller and varnished............200

No.4. "The Shoeing of the Horse, Mule and 0x." Fifty figures descriptive of the Anatomy and Physiology of the Foot and of Horse-shoeing...........75 Mounted on roller and varnished.............2 00

No. 5. "The Elementary Anatomy, Points, and Butcher's Joints of the 0x." Ten colored illustrations -1. Skeleton; 2. Nervous System: 3. Digestive System (Right Side); 4. Respiratory System ; 5 . Points of a Fat Ox; 6. Muscular System; 7. Vascular System; 8. Digestive System (Left Side); 9. Butcher's Sections of a Calf ; 10. Butcher's Sections of an Ox; with full description ............................. 25 Mounted on roller and varnished............2 25

WALLEY. "Four Bovine Scourges." (Pleuro-Pneumonia, Foot and Mouth Disease, Cattle Plague and Tubercle). By Thomas Walley, M.R.C.V.S. With an Appendix on the Inspection of Live Animals and Meat. Quarto, cloth.....................6 40 
WALLEY (continued).

$(")$ - "A Practical Guide to Meat Inspection." By Thomas Walley, M.R.C.V.S., late principal of the Edinburgh Royal (Dick) Veterinary College; Professor of Veterinary Medicine and Surgery, etc. Fourth Edition, thoroughly revised and enlarged by Stewart Stockman, M.R.C.V.S., Professor of Pathology, Lecturer on Hygiene and Meat Inspection at Dick Veterinary College, Edinburgh. Cloth, size 5 1-2 x 81 -4, with 45 colored illus., 295 pages.... 300

An experience of over 30 years in his profession and a long official connection (some sixteen years) with Edinburgh Abattoirs have enabled the author to gather a large store of information on the subject, which he has embodied in his book.

While Dr. Stockman is indeed indebted to the old for much useful information, this up-todate work will hardly be recognized as the old "Walley's Meat Inspection."

WILCOX. "Handbook of Meat Inspection." By Robert Ostertag, M.D.

$$
\text { See "Ostertag." }
$$

WILLIAMS. "Principles and Practice of Veterinary Medicine." Author's edition, entirely revised and illustrated with numerous plain and colored plates. By W. Williams, M.R.C.V.S. Cloth, size $53-4$ × 8 3-4, 865 pages............................ 50

- "Principles and Practice of Veterinary Surgery." Author's edition, entirely revised and illustrated with numerous plain and colored plates. By W. Williams, M.R.C.V.S. Cloth, size $61-2$ × 9 1-4, 756 pages.............................. 50 


\section{THE MOST COMPLETE, PROGRESSIVE AND}

SCIENTIFIC BOOK ON THE SUBJECT IN

\section{THE ENGLISH LANGUAGE}

\section{(*)WINSLOW. "Veterinary Materia Medica and Therapen-} tics." By Kenelm Winslow, B.A.S., M.D.V., M.D., (Harv.); formerly Assistant Professor of Therapeutics in the Veterinary School of Harvard University; Fellow of the Massachusetts Medical Society ; Surgeon to the Newton Hospital, etc.

\section{Second Edition Revised.}

Cloth, size $61.4 \times 91-4,761$ pages.. ..........6 00

Your letter received and I am pleased to know that we are to have an American Materia Medica.-J. H. Wattles, Sr., M.D., D.V.S., The Western Veterinary College, Kansas City, Mo.

. . Am delighted vith it. It is remarkably correct, complete and up-to-date and is bound to supersede any other work on the same subject heretofore before the profession.

No practitioner's library is complete without it and it will be indispensable for students, as it does away with the necessity of their having a number of collateral books on the subject.

It will be adopted as the text book in the Chicago Veterinary College.-Dr. E. L. Quitman, Chicago Veterinary College.

. . The book is of admirable merit and full of valuable information from beginning to end, very explicit, rich and interesting, and should be in the hands of every student as well as practitioner of the art of Veterinary Medicine.-Thurston Miller, M.D., Professor of Materia Medica, Therapeutics and Chemistry, San Francisco Veterinary College.

I consider it the only work on materia nedica and therapeutics suitable to the American veterinary practitioner. It deserves a wide distribution among veterinarians. I have recommended it to my students.-John J. Repp, V.M.D., Iowa State College, Ames, Iowa. 
(*) W $\boldsymbol{Y M A} \boldsymbol{N}$. "Bovine Obstetrics." By M. G. De Bruin. Translated by W. E. A. Wyman, M.D.V., V.S.

See also "De Bruin."

(")- "Catechism of the Principles of Veterinary Surgery." By W. E. A. Wyman, M.D.V.,V.S. Cloth, size $6 \times 9$,

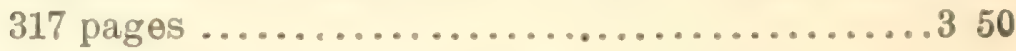

Concerning this new work attention is called to the following points:

1.-It discusses the subject upon the basis of veterinary investigations. 2.-It does away with works on human pathology, histology, etc.

3.-It explains each question thoroughly both from a scientific as well as a practical point of view.

4.--It is writen by one knowing the needs of the student.

5.-It deals exhaustively with a chapter on tumors, heretofore utterly neglected in veterinary pathology.

6.-The only work in English specializing the subject.

7.- The only work thoroughly taking into consideration American as well as European investigations.

8.-Offering practical hints which have not appeared in print, the result of large city and country practice.

$\left({ }^{* *}\right.$ - "The Clinical Diagnosis of Lameness in the Horse." By W. E. A. Wyman, D.V.S., formerly Professor of Veterinary Science, Clemson A. \& M. College, and Veterinarian to the South Carolina Experiment Station. Cloth, size $6 \times 9$ 1-2, 182 pp., 32 illus....2 50

$\left({ }^{\circ}\right.$ - "Tibio-peroneal Neurectomy for the Relief of Spavin Lameness." By W.E. A. Wyman, M.D.V., V.S.

Boards, size 6 × 9,30 pages, illustrated ........ 50 Anvone wanting to perform this operation should procure this little treatise; he will find it of considerable help.-The Veterinary Journal.

ZUNDEL. "The Horse's Foot and Its Diseases." By A. Zundel, Principal Veterinarian of Alsace Lorraine. Translated by Dr. A. Liautard, V.S. Cloth, size $5 \times 73-4,248$ pages, illustrated.............2 00

ZUILL. "Typhoid Fever; or Contagious Influenzs in the Horse." By Prof. W. L. Zuill, M.D.,D.V.S. Pamphlet, size $6 \times 9$ 1-4, 29 pages...........25

Any book sent prepaid for the price

WILLIAIT R. JENKINS, 








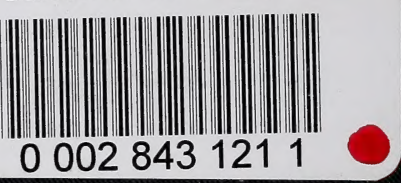

